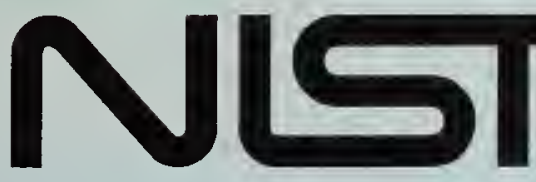 \\ United States Department of Commerce Technology Administration \\ National Institute of Standards and Technology
}

NIST Special Publication 923

Seventh International Conference on

Computer Technology in Welding

July 8-11, 1997

San Francisco, CA

Edited by

T. Siewert

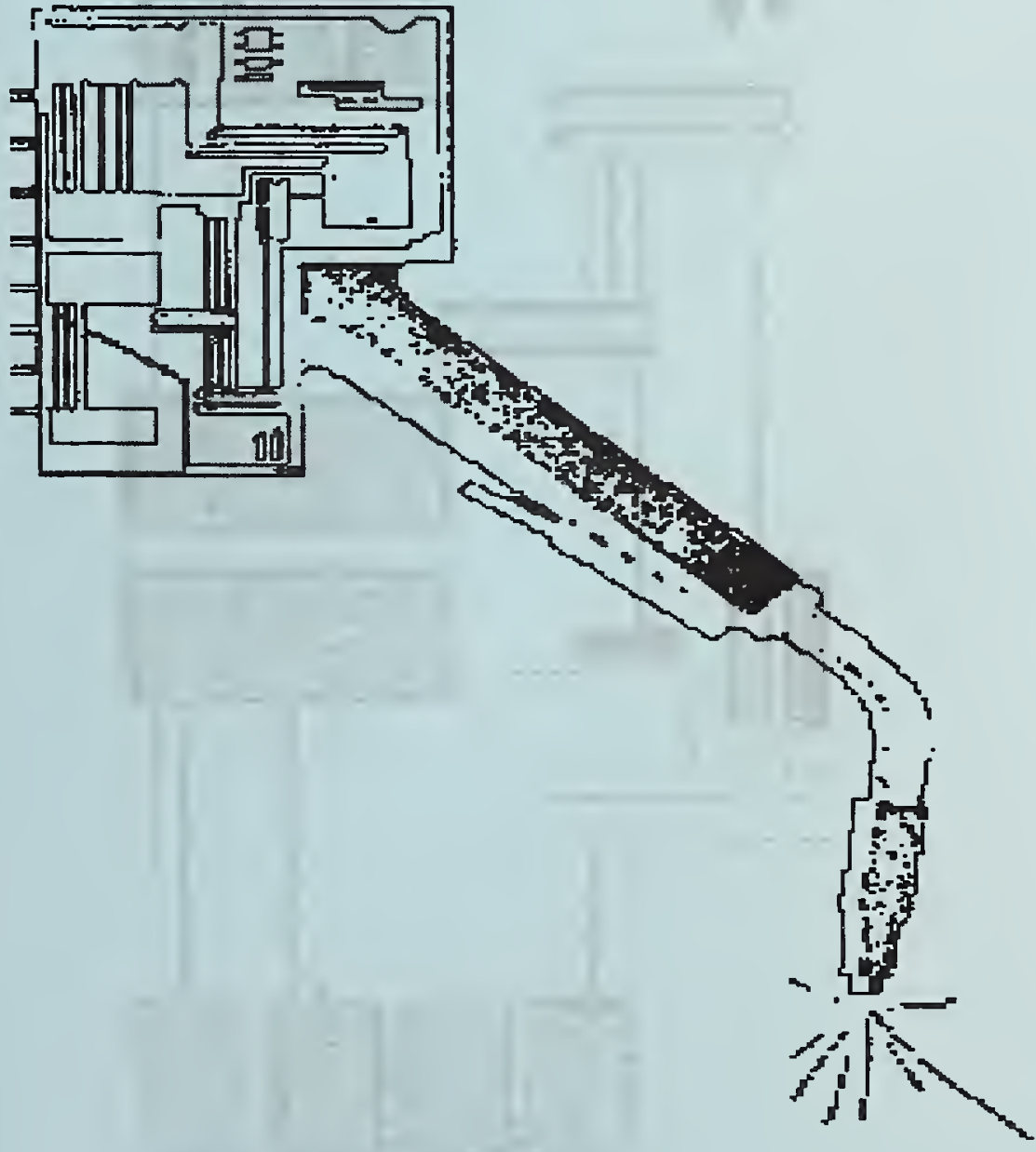

QC

100

.057

N0.923 
$T$ he National Institute of Standards and Technology was established in 1988 by Congress to "assist industry in the development of technology ... needed to improve product quality, to modernize manufacturing processes, to ensure product reliability ... and to facilitate rapid commercialization . . . of products based on new scientific discoveries."

NIST, originally founded as the National Bureau of Standards in 1901, works to strengthen U.S. industry's competitiveness; advance science and engineering; and improve public health, safety, and the environment. One of the agency's basic functions is to develop, maintain, and retain custody of the national standards of measurement, and provide the means and methods for comparing standards used in science, engineering, manufacturing, commerce, industry, and education with the standards adopted or recognized by the Federal Government.

As an agency of the U.S. Commerce Department's Technology Administration, NIST conducts basic and applied research in the physical sciences and engineering, and develops measurement techniques, test methods, standards, and related services. The Institute does generic and precompetitive work on new and advanced technologies. NIST's research facilities are located at Gaithersburg, MD 20899, and at Boulder, CO 80303. Major technical operating units and their principal activities are listed below. For more information contact the Publications and Program Inquiries Desk, 301-975-3058.

\section{Office of the Director}

- National Quality Program

- International and Academic Affairs

\section{Technology Services}

- Standards Services

- Technology Partnerships

- Measurement Services

- Technology Innovation

- Information Services

\section{Advanced Technology Program}

- Economic Assessment

- Information Technology and Applications

- Chemical and Biomedical Technology

- Materials and Manufacturing Technology

- Electronics and Photonics Technology

\section{Manufacturing Extension Partnership Program \\ - Regional Programs \\ - National Programs \\ - Program Development}

\section{Electronics and Electrical Engineering} Laboratory

- Microelectronics

- Law Enforcement Standards

- Electricity

- Semiconductor Electronics

- Electromagnetic Fields'

- Electromagnetic Technology'

- Optoelectronics ${ }^{1}$

\section{Chemical Science and Technology Laboratory \\ - Biotechnology \\ - Physical and Chemical Properties ${ }^{2}$ \\ - Analytical Chemistry \\ - Process Measurements \\ - Surface and Microanalysis Science}

Physics Laboratory

- Electron and Optical Physics

- Atomic Physics

- Optical Technology

- Ionizing Radiation

- Time and Frequency

- Quantum Physics ${ }^{1}$

Materials Science and Engineering Laboratory

- Intelligent Processing of Materials

- Ceramics

- Materials Reliability

- Polymers

- Metallurgy

- NIST Center for Neutron Research

\section{Manufacturing Engineering}

Laboratory

- Precision Engineering

- Automated Production Technology

- Intelligent Systems

- Fabrication Technology

- Manufacturing Systems Integration

Building and Fire Research

Laboratory

- Structures

- Building Materials

- Building Environment

- Fire Safety Engineering

- Fire Science

Information Technology Laboratory

- Mathematical and Computational Sciences ${ }^{2}$

- Advanced Network Technologies

- Computer Security

- Information Access and User Interfaces

- High Performance Systems and Services

- Distributed Computing and Information Services

- Software Diagnostics and Conformance Testing

'At Boulder, CO 80303.

${ }^{2}$ Some elements at Boulder, $\mathrm{CO}$. 


\section{Seventh International Conference on Computer Technology in Welding}

July 8-11, 1997

San Francisco, CA

\section{Edited by}

\section{T. Siewert}

Materials Reliability Division

Materials Science and Engineering Laboratory

National Institute of Standards and Technology

Boulder, Colorado 80303-3328

\section{Sponsored by}

American Welding Society

The Welding Institute

National Institute of Standards and Technology

November 1997

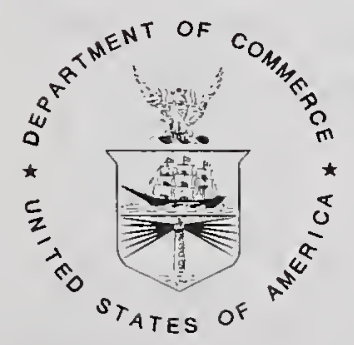

U.S. DEPARTMENT OF COMMERCE, William M. Daley, Secretary TECHNOLOGY ADMINISTRATION, Gary R. Bachula, Acting Under Secretary for Technology NATIONAL INSTITUTE OF STANDARDS AND TECHNOLOGY, Raymond G. Kammer, Director 
National Institute of Standards and Technology

Special Publication 923

Natl. Inst. Stand. Technol.

Spec. Publ. 923

596 pages (November 1997)

CODEN:NSPUE2
U.S. Government Printing Office

Washington: 1997
For sale by the Superintendent of Documents

U.S. Government Printing Office Washington, DC 20402-9325 


\section{CONTENTS}

Preface $\quad \ldots \ldots \ldots \ldots \ldots \ldots \ldots \ldots \ldots \ldots \ldots \ldots \ldots \ldots \ldots \ldots \ldots \ldots \ldots$

Conference Steering Committee $\ldots \ldots \ldots \ldots \ldots \ldots \ldots \ldots \ldots \ldots \ldots \ldots \ldots$

\section{OVERVIEWS}

Trends in Welding Software $\ldots \ldots \ldots \ldots \ldots \ldots \ldots \ldots \ldots \ldots \ldots \ldots \ldots \ldots \ldots$

T.A. Siewert, National Institute for Standards and Technology; S. Roosen, German Welding Society

Applications of DSP in Welding

D.J. Newman, SIGNALWARE Corporation

\section{CASE STUDIES}

Using TURBO-FMC (The Electronic Filler Metal Catalog) in Power Plant

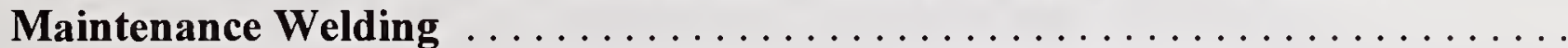

C.L. Foster, Pacific Gas and Electric Company

Deep Water Pipeline Welding and Repairs Using Modern Computer Technology to Create A Diverless Future for Statoil $\ldots \ldots \ldots \ldots \ldots \ldots \ldots \ldots \ldots$

T. Håbrekke, SINTEF Materials Technology; M. Armstrong, Isotek

Electronics Limited; J.O. Berge, Statoil PRS

Implementation of Computer Systems for Production and QA/QC in the

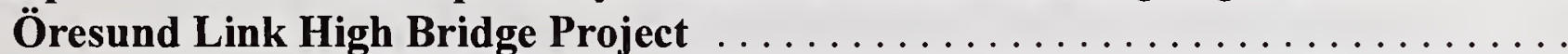

T. Johnsson, 4X Software, Norway

Gas Metal Arc Penetration Welding Development Utilizing Neural Nets . . . . . .

J. Noruk, Tower Automotive

Spreadsheet Technology Advances Pipefitting to the Next Generation . . . . . . . .

G.D. Simpson, Wayco, Inc.

\section{CONTROLS and CONTROLLERS}

Method for Preparation of Laser Welding Control . . . . . . . . . . . . .

H.J. Andersen and H. Holm, Aalborg University 
Scan Welding: Thermal Modeling and Adaptive Control $\ldots \ldots \ldots \ldots \ldots \ldots$

C.C. Doumanidis, Tufts University

A Method for Automatic Voltage Tuning of Conventional GMAW . . . . . . . .

G.C. Carvalho, GRACO - University of Brasilia; T.E.B. Ogunbiyi and

R. McMaster, SIMS - Cranfield University

SCIAKY W2000, A Multiple Weld Process CNC Machine Controller . . . . . . . .

S.R. Potter and R. Thompson, Sciaky Inc.

\section{DATABASES and KNOWLEDGE BASES}

An Information Model Based Weld Schedule Database $\ldots \ldots \ldots \ldots \ldots \ldots \ldots$

S.D. Kleban, G.A. Knorovsky, and G.K. Hicken, Sandia National Laboratories;

G.A. Gershanok, University of New Mexico

Managing Welding With the Proweld Welding Information System . . . . . . . .

W. Scholz and T. Pinn, New Zealand Welding Centre; A. Hunter, formerly with New Zealand Welding Centre

A Computerized System for Prequalified Structure Welding (AWS D1.1.94) ... .

A. El-Ashram and E.A. Shaaban, Alexandria University; H.I. Shaaban, Atomic Energy Authority, Alexandria, Egypt

Welding Databases - Experiences from Implementation in U.S., Canada,

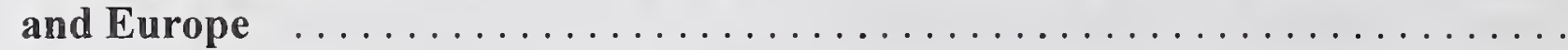

E. Engh, 4X Software, Norway

A Computer Accessible Welding Procedure Database System for Use with Intelligent Off-Line Planning Systems . . . . . . . . . . . . . . .

P. Sewell, N.B. Gomez, C. Sakata, J.E. Jones, and V. Rhoades, N.A. Technologies

Use of an Expert System for On-Line Process Optimization In GMA Welding ...

S. Roosen, German Welding Society; U. Dilthey, Welding Institute (ISF), Aachen University, Germany

\section{INTERNET and NETWORKED SYSTEMS}

The Joining Information Network: Build It and They Will Come . . . . . . .

R.G. Citterberg, Edison Welding Institute 
The NIST Automated Arc Welding Testbed

W.G. Rippey and J.A. Falco, National Institute of Standards and Technology

\section{MODELING - HEAT FLOW and FLUID FLOW}

Prediction of Laser Spot Weld Shape by Numerical Analysis and

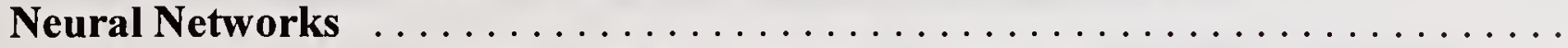

W.-S. Chang and S.J. Na, KAIST; H.-S. Moon, Hyundai Heavy Industries;

S.-J. Hong and H.-S. Kang, LJ Electronics

Nonlinear Analysis of Weld Thermal History $\ldots \ldots \ldots \ldots \ldots \ldots \ldots \ldots$

M. Becker, Oregon Graduate Institute

Computationally Efficient Weld Analysis $\ldots \ldots \ldots \ldots \ldots \ldots \ldots \ldots \ldots$

L. Zhang, X. Chen, M. Becker, L. Meekisho, and E. Atteridge,

Oregon Graduate Institute

SOAR: An Extensible Suite of Codes for Weld Analysis and Optimal

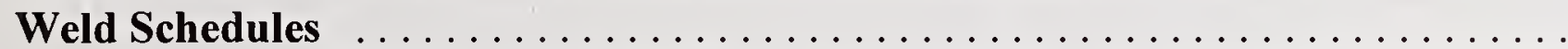

G.R. Eisler and P.W. Fuerschbach, Sandia National Laboratories

Heat and Mass Transfer in a Molten Droplet During GMA Welding $\ldots \ldots \ldots \ldots$

V.A. Nemchinsky, ESAB Welding and Cutting Products

Numerical Model of Short-Circuiting Transfer Process in GMA Welding . . . . . .

Y. Hirata, T. Osamura, and T. Ohji, Osaka University; N. Goto, Tsu Labs

\section{MODELING - PHASE TRANSFORMATION}

Creating New Welding Materials on the Basis of Metallurgical

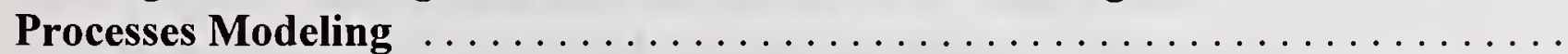

M. Zinigrad, N.i. Zalomov, V. Mazurovsky, and O. Aksyutin, College of Judea and Samaria

Microstructures in the Heat-Affected Zones Based on Dilatometer Data

E.A. Metzbower, G. Spanos, R.W. Fonda, R.A. Vandermeer,

U.S. Naval Research Laboratory, Physical Metallurgy Branch

Mathematical Model of Prediction of Phase Composition, Structure, and Properties of Weld Metal . . . . . . . . . . . . . . . . . . .

N.V. Korolev, V.N. Boronenkov, and O.V. Pimenova, Ural State Technical University 
Prediction of Ferrite Content in the Heat Affected Zone of Duplex and

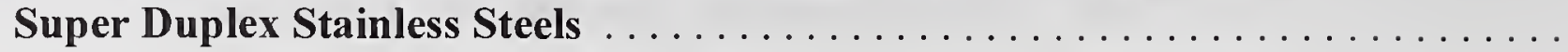

S.M. Khafagy, J.C. Suárez, and F. Molleda, Universidad Politécnica de Madrid

Computational Modeling of Microstructure Evolution in Weld Heat

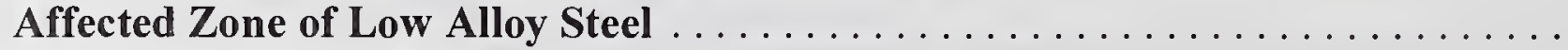

M.V. Li, Battelle Memorial Institute; L.L. Meekisho and D.G. Atteridge,

Oregon Graduate Institute

\section{MODELING - RESIDUAL and MECHANICAL STRESSES}

Computer Simulation of Welded Connections in Seismic Tests $\ldots \ldots \ldots \ldots \ldots$

O. Blodgett and T. Spear, The Lincoln Electric Company

Measurement of Restraining Forces for a Gas Metal Are Welded

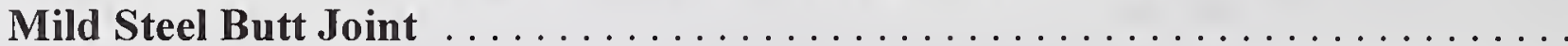

M.A. Wahab and P.E. Stafford, The University of Adelaide;

M.J. Painter, CSIRO-DMT

Finite Element Modeling of Spot Welding Similar and Dissimilar Metals

W. Zhang, H.H. Jensen, and N. Bay, Technical University of Denmark

Two Dimensional Finite Element Analysis of Residual Stress in

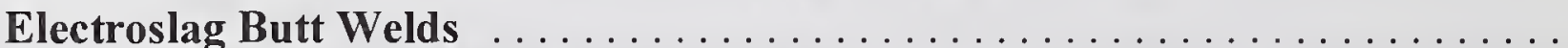

L. Zhang, D.G. Atteridge, L. Meekisho, and M. Becker,

Oregon Graduate Institute of Science and Technology

\section{MODELING - THERMOMECHANICAL EFFECTS}

Finite Element Modeling of Resistance Spot Welding of Galvanized Steel ......

M.V. Li and P. Dong, Battelle Memorial Institute; M. Kimchi,

Edison Welding Institute

The Numerical Simulation of Welding: Description of Required Functions and Industrial Applications with the Software SYSWELD $+\ldots \ldots \ldots \ldots \ldots \ldots$

F. Boitout, G. Mangialenti, and J.M. Bergheau, Framasoft, France

\section{PROCESS AUTOMATION}

Fully Automatic GMAW System $\ldots \ldots \ldots \ldots \ldots \ldots \ldots \ldots \ldots \ldots \ldots \ldots \ldots$

A. Kolasa and P. Cegielski, Department of Welding Engineering,

Warsaw University of Technology 
Intelligent Automatic Pipe Welding $\ldots \ldots \ldots \ldots \ldots \ldots \ldots \ldots \ldots \ldots \ldots$

O. Madsen, Aalborg University; R. Larsen, L. Overgaard, and N.J. Jacobsen, AMROSE, Ltd.; and I. Hafsteinsson and C.B. Sørensen, Odense Steel

Shipyard Ltd. Odense, Denmark

\section{RESISTANCE WELDING}

Modeling of Contact Resistance during Resistance Spot Welding $\ldots \ldots \ldots \ldots$

M.V. Li and P. Dong, Battelle Memorial Institute; M. Kimchi, Edison Welding Institute

Modeling of Nugget Formation Process in Resistance Spot Welding ..........

K. Matsuyama, Osaka University

The Coupled Electrical-Thermal-Mechanical Process Associated

With Aluminum $\mathbf{R S W} \ldots \ldots \ldots \ldots \ldots \ldots \ldots \ldots \ldots \ldots \ldots \ldots \ldots$

X. Sun and P. Dong, Battelle Memorial Institute; M. Kimchi,

Edison Welding Institute

\section{SENSING}

GMA Process Identification Using Are Acoustics $\ldots \ldots \ldots \ldots \ldots \ldots \ldots$

A.M. Mansoor and J.P. Huisson, University of Waterloo, Canada

Fast Detection of Essential Changes in GMAW Processes $\ldots \ldots \ldots \ldots \ldots \ldots$

E. Blumschein, IELE, Otto von Guericke University

Nugget Size Sensing of Spot Weld Based on Neural Network Learning .......

K. Matsuyama, Osaka University

Weld Defect Decisions Using Through-the Arc Sensor Data .............

T.P. Quinn and R.B. Madigan, National Institute of Standards and Technology;

C.B. Smith, Tower Automotive; E.G. Blachowiak, A.O. Smith Corporation

Through-the-Wire Sensor for Seam Tracking in GMA/CO $\mathrm{C}_{2}$ Automatic Welding .

P. Orszagh, Slovak Technical University; Y.C. Kim and K. Horikawa,

Joining and Welding Research Institute, Osaka University

\section{WELD PLAN SIMULATION}

Design of a Welding Application Programming Interface $\ldots \ldots \ldots \ldots \ldots$

T.E. Doyle, McDermott Technologies Inc. 


\section{Estimating Optimal Welding Parameters Using Artificial Neural}

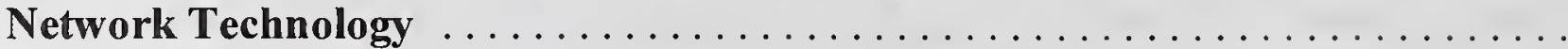

B. Chan, MIL Systems Engineering; J. Pacey, Northern College; M. Bibby,

Carleton University

Computer Aided Hardfacing Technology Planning $\ldots \ldots \ldots \ldots \ldots \ldots \ldots$

B. Palotas, L. Beres, B. Keskeny and R. Pap, Technical University of Budapest

Automatic Extraction of CAD Geometry Data in an Automated Intelligent

Weld Off-Line Planning System . . . . . . . . . . . . . . . . . . . .

P.A. Oberly, P.T. Sewell, V.L. Rhoades, and J.E. Jones, N.A. Technologies

MAGSIM and SPOTSIM - PC Aided Simulation of GMA and

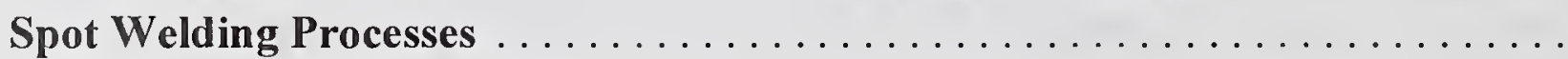

U. Dilthey, Welding Institute (ISF) Aachen University; S. Roosen,

German Welding Society (DVS); V. Sudnik, Welding Institute

The Robotic Easy Teaching System in Computer Aided Welding

H. Handa, S. Okumura, and S. Nio, Yaskawa Electric Co.

Appendix A: Participants in Seventh International Conference on Computer Technology in Welding

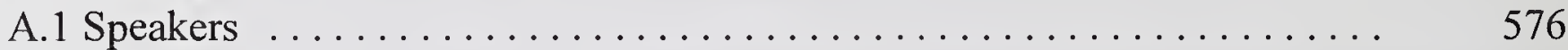

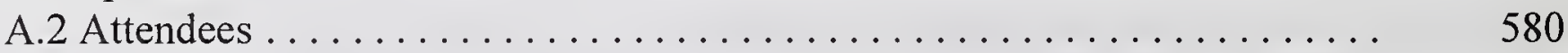

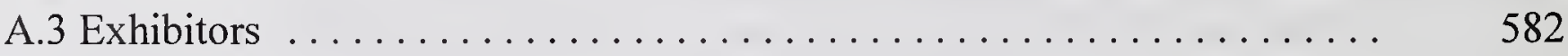

Author Index $\ldots \ldots \ldots \ldots \ldots \ldots \ldots \ldots \ldots \ldots \ldots \ldots \ldots \ldots \ldots \ldots \ldots$ 


\section{Preface}

The first U.S. Workshop on computerization of welding data was held in 1986, under the sponsorship of the American Welding Institute (AWI) and the then National Bureau of Standards (now, National Institute of Standards and Technology-NIST). The workshop produced a list of national needs in welding data, a list designed to guide database developers. The proceedings of that first workshop are available from the Government Printing Office as NIST Special Publication 742.

There had been sufficient advances in database activity by 1988 to justify a second meeting, this time as a joint workshop-conference under the sponsorship of AWI, NIST, and the American Welding Society (AWS). The scope was expanded to include a conference on the latest developments and a preconference tutorial to provide novices with a background in common computer applications. The conference was attended by 61 managers, welding engineers, and computer professionals. The proceedings of the workshop and conference are available from the Government Printing Office as NIST Special Publication 781.

By 1990 , the welding database activity had grown large enough to justify another conference on this topic, again sponsored by AWI, AWS, and NIST. It consisted of a series of tutorials, a keynote presentation, and technical sessions on the topics: off-line planning, real-time welding information, data systems and standards, and industrial applications. The conference included demonstrations of welding software, and was followed by a meeting of the AWS Committee on Computerization of Welding Data.

The fourth conference, held during November 1992 in Orlando, Florida, was truly an international conference; speakers representing ten countries presented papers on the topics of standards, applications, quality and NDE, sensing, control, and databases. Once again, a preconference tutorial was organized and taught by AWI personnel covering PC networks, expert systems, neural networks, Windows and the Excel Spreadsheet, and databases. The conference also included a keynote presentation, tabletop exhibits, and hands-on demonstrations of welding software. The AWS Committee on Computerization of Welding Data met following the Conference. These proceedings are available from AWS (Code: CP-1192).

The fifth conference, held August 1994, in Golden, Colorado, continued the trend of growth in size and scope. It consisted of 31 papers (by 69 authors) on the topics of quality control, off-line planning and simulation, commercial software systems, control and automation, welding optimization, data acquisition and sensors, application case studies, welder and procedure qualification systems, weld prediction and control, and large-scale systems. This year the preconference tutorials were on the topics of computing platforms, sensing and data acquisition, and line planning for welding automation. These tutorial were taught by experts from AMET, NIST, CSM, Ford Research Laboratory, and ABB Robotics. The conference was cosponsored by AWS, the Colorado School of Mines, NIST, and AWI. The conference proceedings are available from AWS (code: CP-794). 
The sixth conference was held June 9-12, 1996, in the 'Heart of Europe,' where Belgium, Germany, and the Netherlands converge in the Limberg region. The Technical Program was aimed to embrace all known facets of computing practice, as applied to welded fabrication and manufacturing. Expert advice was provided in process selection, consumable selection, and welding procedure generation and interpretation of standards. Proceedings are available through Woodhead Publishing Ltd. (see below).

This is the report of the seventh conference, held July 8-12, 1997, in San Francisco, California, and was presented in conjunction with the American Welding Society, The Welding Institute and the National Institute of Standards and Technology. Attendees were able to observe how computers can be used for more than just databases. In addition to tutorials, the following were covered: case studies, controls and controllers, sensing, process automation, modeling heat and fluid flow, modeling thermomechanical effects, and modeling residual stress or mechanical effects. Also covered were the Internet and networked systems in the welding industry. Additional copies are available from the Government Printing Office.

To order the NIST Special Publications, contact:

Superintendent of Documents

or

Government Printing Office

Washington, DC 20402

(202) 512-1800

http://www.access.gpo.gov/
National Technical Information Service

Springfield, VA 22161

(703) 487-4650

http://www.ntis.gov/

To order the 1992 and 1994 proceedings, contact:

Order Department

American Welding Society

550 N.W. LeJeune Road

Miami, Florida 33126

(800) 334-9353 (or (305) 443-9353, Ext. 280)

To order the 1996 proceedings, contact:

Woodhead Publishing Ltd.

Abington Hall, Abington

Cambridge CB1 6AH

England

$+44(0) 1223891358$

Except where attributed to NIST authors, the content of individual sections of this volume has not been reviewed nor edited by the National Institute of Standards and Technology. NIST therefore accepts no responsibility for comments or recommendations therein. The mention of trade names in this volume neither constitutes nor implies an endorsement or recommendation by the National Institute of Standards and Technology. 


\title{
Conference Steering Committee
}

\author{
Dr. Jerald E. Jones \\ Chief Scientist \\ N.A. Technologies Co. \\ Golden Colorado \\ Dr. Dawn White \\ Principal Engineering Specialist \\ Materials System Reliability \\ Ford Research Laboratory \\ Dearborn, Michigan \\ Dr. Thomas A. Siewert \\ Group Leader \\ Materials Reliability Division \\ NIST \\ Boulder, Colorado
Ms. Valerie Rhoades
Software Engineer Golden, Colorado \\ N.A. Technologies Co.
Mr. Darren M. Barborak
Research Engineer
Edison Welding Inst.
Columbus, Ohio
Mr. Matthew W. White
Navy Joining Center
Columbus, Ohio

Acknowledgment: The editor wishes to express appreciation to those who helped prepare and produce these proceedings: Nannette Zapata, Gladys Borges, Marilyn Levine, and Nina Zeno, American Welding Society. 


\section{OVERVIEWS}





\title{
TRENDS IN WELDING SOFTWARE \\ T. A. Siewert* and S. Roosen**
}

\begin{abstract}
This report reviews the worldwide growth and trends in welding software over the past 10 years. It discusses these trends in terms of the changes in computer resources (rapid acceptance of the personal computer and advances in operating systems) as well as the identification and development of sophisticated welding applications for these computer resources. The trends have been somewhat different by country (the U.S. had an initial emphasis on welding databases, while Europe had an initial emphasis on applications software), but there has generally been a rapid growth in the development and acceptance of welding software during this period.
\end{abstract}

\section{KEYWORDS}

computerization; databases; software trends; welding information; welding software

\section{INTRODUCTION}

Moore's Law, the observation that the number of elements (both memory and processor circuits) on a chip doubles every 18 months or so, has been followed for about 20 years, and is expected to slow only slightly in the coming decade (Refs. 1-3). In turn, this continues to drive the tremendous growth in microcomputer systems, so we now produce weekly more computers than existed in the whole world in 1975 (Ref. 4). In spite of their ubiquity, Gordon Moore feels that the impact of microcomputers is just starting to be felt in our lives (Ref. 1). It therefore seems very appropriate to review what has happened in welding over the past 10 years, to prepare us for the changes (and rate of change) that might occur in the next 10.

The acceptance of personal computers in a broad range of business and professional activities is well known. This growth is usually attributed to the simple combination of a rapid increase in the capabilities of computers and a decrease in cost per unit of capability over the past 10 years. Of course, equal credit must go to the developers of operating systems and development languages for providing easier access to the power of computers. A third aspect of the wide acceptance of computers is the availability of application software that is directed to specific tasks. Both operating system and application software shield the average user from the complexities of the binary logic used in the actual hardware of the computer, and display the data in a form that is familiar to the technical user. This report investigates the history of software specific to welding. Information on this subject is mostly from the reports of workshops and conferences in various countries.

Contribution of NIST, not subject to copyright.

*Materials Reliability Division, NIST, Boulder, CO 80303

**DVS, Duesseldorf, Germany 


\section{A HISTORY OF WELDING SOFTWARE IN THE U.S.}

In the U.S., the first national meeting that addressed the application of computers to welding information was a workshop held in Knoxville, Tennessee in 1986 (Ref. 5). It was sponsored by the National Bureau of Standards and the American Welding Institute, and was supported by the U.S. Navy's David Taylor Research Center. This meeting drew forty-two engineers, welding managers, and computer scientists. This workshop was organized because a number of the planners had recognized that computer technology had matured to where it was able to address a broader range of welding tasks. In particular, the planners accurately anticipated the onset of a period of more rapid growth in computer technology. The workshop was designed to identify welding applications for software developers and to produce a ranked list of database needs.

The workshop included a series of overview and background talks on software and its application to welding. F. Smith, in the keynote lecture, talked about the development of computer databases during the previous 25 years. He described the evolution of commercial software from primarily business and financial applications, to include engineering and scientific applications. Also, he described the attributes that a computerized system must have (such as low cost, reliability, and fast and easy accessibility) to compete with other forms of data storage. Later, $\mathrm{J}$. Jones described the traditional alphanumeric data that were associated with mainframe computers and the nontraditional data that can be included as storage and display capabilities increase. J. Rumble reviewed the terminology of databases and emphasized the planning and implementation steps needed to make a successful database.

Other speakers at the workshop came from welding backgrounds, and described the progress and needs from their perspectives. F. Breismeister described the broad data needs of Bechtel Corporation, a large engineering and design company. Data needed for design include the physical and mechanical properties of the material, welding procedure details and results, information about residual stresses, and analysis of weld discontinuities. The quality of this data is very important, since faulty data would reduce rather than enhance productivity. As a practical application, he described their use of a procedure qualification database with about 1200 records to produce welding procedure specifications.

Four other speakers described existing welding database efforts. J. Sims described a mainframebased database at Chicago Bridge and Iron with 10,000 welding procedure specifications, and some improvements that had made it more useful. G. Olejniczak described a computerized database that the National Training Fund had developed to match sheet-metal welders of various skill levels to the changing job opportunities around the country. H.G. Kaufman described the National Materials Property Data Network, a gateway to a variety of sources of materialproperty data. J. Jones described the Welding Information Network being developed by the American Welding Institute and the application of artificial intelligence techniques to make it more useful. 
In addition, A. Kuhne described a small expert system designed to help a welder to diagnose and control welding defects, and $\mathrm{H}$. Cary of Hobart Brothers predicted the role of computers in complex automation with closed-loop control.

Based on these presentations, the workshop participants (in small groups) identified the major data needs in welding. When these lists were combined, the most important common needs for welding data were: general welding procedures, properties of materials, procedure qualification records, and welding variables.

At that first workshop, some expected that a national welding database would emerge, which would reside on a mainframe computer and would be accessed by dumb terminals. Other than this underestimation of the domination of personal computers, the trends and observations of data needs served to guide the early developers of welding software and many of the observations are still true today.

\section{$\underline{1988}$}

By 1988 , the organizers of the 1986 U.S. conference perceived a need to review the progress in welding software development. However, the growth in the use of computers in welding suggested a change to a conference structure with a short workshop at the end. This provided a greater opportunity for developers and users to report their experiences, while still providing a chance to refine the list of needs. This change in emphasis was supported by requests from those who wanted to describe their experiences. A reduced emphasis on the ranking of needs was confirmed when the workshop at the end of the conference developed a list of national needs that was almost identical to the one in 1986, even though only about $20 \%$ of the attendees were the same (Ref. 6). Another change was the recognition of the broad interest within the welding industry, as the American Welding Society joined as a sponsor.

The 1988 conference was attended by 61 managers, welding engineers, and computer professionals. A higher level of computer knowledge for this group was indicated by a preconference survey which showed a median of 12 years of computer experience, and that $97 \%$ of the attendees had access to a microcomputer (primarily 8088 to 80386-based systems) at work. Although data were not gathered at the 1986 workshop, most felt that only $50 \%$ of those attendees had access to computers. In spite of their familiarity with computers, $90 \%$ of the attendees in 1988 took advantage of a preconference tutorial on software packages that a welding engineer might use, including word processing, spreadsheets, CAD, and generation of welding procedures from a database.

The papers were divided into three main sections: Overviews of Computers and Databases, Welding Application Software, and Welding Case Studies. The overviews section built on the presentations at the 1986 conference by adding databases that had been missed. The application software session highlighted welding software that was commercially available, while the case studies looked at the software from a user's perspective. Both the application and case studies sections had more microcomputer than mainframe applications, starting a trend that would continue in future years. 


\section{0 and Following}

In the U.S., subsequent conferences on the computerization of welding information have typically followed the biannual schedule set in the 1980's, being held in 1990, 1992, and 1994. The attendance has generally increased, while the number of papers specifically directed toward software and its application in welding (not including keynote, overview, and concept papers) and the number of software programs (both commercial and research) mentioned in these papers have generally increased as well (Refs. 7-9); see Table 1. The total number of programs in the table is sometimes greater than the number of papers, because some authors described multiple programs.

Table 1. Data on papers and programs at previous conferences.

\begin{tabular}{||c|c|c|c|}
\hline Year & $\begin{array}{c}\text { Papers on software and its } \\
\text { application }\end{array}$ & $\begin{array}{c}\text { Programs } \\
\text { for } \\
\text { Mainframes }\end{array}$ & $\begin{array}{c}\text { Programs } \\
\text { for PCS }\end{array}$ \\
\hline 1986 & 9 & 5 & 1 \\
\hline 1988 & 9 & 5 & 12 \\
\hline 1990 & 10 & 5 & 8 \\
\hline 1992 & 33 & 3 & 24 \\
\hline 1994 & 31 & 3 & 35 \\
\hline
\end{tabular}

One additional trend is that the ratio of programs that run in a client-server mode (mainframe or minicomputer) to the programs that run on a local computer (PC or workstation) has continued to drop. This trend is probably driven as much by the engineer's desire to be in full control of the system as by a desire to increase the performance. There is some uncertainty in these data since some software and modeling concepts could be used either way. When we were uncertain of the platform, a vote was added to each column.

\section{HISTORY OF WELDING SOFTWARE IN THE U.K.}

The first International Conference on Computer Technology in Welding was organized by The Welding Institute and held in London in 1986. This conference included 43 papers, divided roughly equally into sessions on principles, applications design and analysis, information technology, and knowledge-based and expert systems (Ref. 10). In fact, these conference proceedings refer back to what is perhaps the first use of a PC in welding. It contains a reference to W. Lucas' 1979 paper on the use of a PC to control gas tungsten arc welding.

Since 1986, The Welding Institute has held these conferences on a biennial schedule. 


\section{HISTORY OF WELDING SOFTWARE IN GERMANY}

The first steps towards the development and distribution of welding software in Germany were made in 1989, when the German Welding Society (DVS) founded a service center for welding information, the so-called "Informationsverbund Schweißtechnik". The purpose of this center was to gather information about the use of software in welding and about tools which could be applied to store information. Until then, only preliminary steps had been made by individual computer enthusiasts, who programmed welding software, mostly for their own use.

The primary goal of the "Informationsverbund Schweißtechnik" was to promote the development and the use of welding software in Germany. At the time, PC-based welding software was accepted only poorly by industry, especially by small- and medium-sized enterprises and the skilled trades. But these companies are the ones who dealt with welding technology in Germany. The problem with the acceptance of welding software continues to a smaller degree, even today.

With time, the level of research on the use of computers for technological application increased. These research projects were funded mostly by the welding and cutting research association of DVS. As more programs became available, better distribution systems were needed.

The publishing house of the German Welding Society started to offer third-party welding software for PCs in 1990. Initially, most programs calculated the various parameters associated with welding. Today, documentation and quality-control systems (according to EN standards) are most popular. Another trend is the updating of the various programs to run on the latest operating systems.

To help customers find the software that best fits their needs and to give them the opportunity to evaluate the software, 15 demonstration centers for software have been established all over Germany since 1992. They make it possible to give individual advice to potential software users and to solve problems with the acceptance of software.

Programs, even on the same topic, differ widely in content, handling of data, and results. An individual evaluation with competent advice by independent people who are familiar with all the available programs can help to decide which program will best solve a company's problem.

Furthermore, the use of software is promoted in conferences organized by the publishing house of the German Welding Society, where welding software is presented not only by papers, but also by on-line presentations on computers.

The development of welding software in Germany can be traced through the software presented at the software conferences in Essen, Germany, in September 1993 and in Graz, Austria, in April 1996. The next conference on welding software is scheduled for May 1999 in Halle, Germany. 


\section{INTERNATIONAL REVIEWS OF WELDING SOFTWARE}

Several authors have collected information on and categorized the various welding software programs. In 1990, Howard Cary prepared two reports for Commission XII of the International Institute of Welding (documents XII-1190 and XII-1190-2) in which he listed the welding software available in 1990. This list was subsequently published in the January 1991 issue of the Welding Journal. It listed 29 software programs that support the needs of a welding engineer, including welding procedure generation and welder tracking, code interpretation, cost and material estimation, ferrite prediction, electrode selection, and weld symbol generation. In addition, he listed related software for optimization of cutting (nesting of parts) and for calculating loads in beams and structures.

Commission XII continued to follow the developments in welding software, and in 1992, hosted a workshop in conjunction with the IIW Assembly in Madrid. Staffan Budgifvars, on behalf of the working group on welding software, gave an overview of the status of welding software around the world (Ref. 11). He stated that they had located over 100 programs around the world to support the needs of the welding engineer. Many of these programs are available in English, and the rest are available in a variety of other major languages. He also reported the results of a survey on the sales of these programs, with many companies reporting sales of over 100 units, and some reporting sales of over 1000 units. He observed that there was rapid growth in the computer and welding software industries, in spite of a general recession at this time, and concluded that most companies recognized the critical importance of automating their procedures so they could remain economically competitive. The rest of the workshop was devoted to 15 developers of software from around the world who described the features of their products.

In 1995, Commission XII reviewed the progress in welding software. This time there were four presentations, one each for the Americas, the U.K., Japan, and Europe. I described 29 programs offered in North America specifically for welding applications, together with another 25 associated programs for cutting optimization, gas cylinder inventory control, and structural engineering calculations (Ref. 12). W. Lucas reported on 11 programs offered in the U.K. specifically for welding applications, together with 4 more programs for related needs such as storing and sorting NDE records, facility design, and fracture assessment (Ref. 13). M. Kutsuna took a different approach on welding software in Japan (Ref. 14). He characterized the 158 responses to a survey of welding software use in Japan industry. Almost half of the companies were using welding software, but the majority $(80 \%)$ of the programs were internally developed within the companies. S. Budgifvars described 21 programs offered in Europe specifically for welding applications, together with 6 more programs for related needs such as cutting optimization, NDE, and robot planning (Ref. 15).

\section{THE PRESENT}

The interest in software for welding continues, with many other meetings on this topic occurring, primarily in the U.S., U.K., and Europe, although these meetings have drawn users and developers from other continents. These meetings have usually been structured as 
conferences, with presentations on individual software packages by developers or users, and have often included demonstrations sessions and tutorials. The major players in these meetings have been the American Welding Society (together with other interested U.S. organizations), The Welding Institute (in Cambridge, U.K.), and Commission XII of the International Institute of Welding.

As an example of the growth, 1996 saw three major venues for meetings and conferences on welding software. These were a May conference in Lanaken, Belgium organized primarily by TWI, a September Workshop sponsored by GTE (the Hungarian Welding Society), and the September Meeting of International Institute of Welding Subcommission XII-C devoted to welding automation.

\section{ACKNOWLEDGMENTS}

We appreciate the assistance of $\mathrm{W}$. Lucas who provided information on the workshops organized by TWI.

\section{REFERENCES}

1. Schmit, J. 1996 A conversation with Gordon Moore, USA Today, November 8, p. 12B.

2. Matey, J. 1997 Moore's Law and the PC Revolution, Computers in Physics, 11, 2, p. 141.

3. Brenner, A. 1997 Moore's Law, Science, 275, 5306, p. 1552.

4. Eubanks, G. 1997 Stamp Out Digital Homelessness, Computerworld, February 24, p. 37.

5. Computerization of Welding Information - a Workshop Report, August 5-6, 1986, Knoxville, Tennessee, T.A. Siewert and J.E. Jones, Editors, NBS Special Publication 742, National Bureau of Standards, 1988.

6. Computerization of Welding Data - Proceedings of the Conference and Workshop, October 19-21, 1988, New Orleans, Louisiana, T.A. Siewert, J.E. Jones, and H.G. Ziegenfuss, Editors, NIST Special Publication 781, National Institute of Standards and Technology, 1990.

7. Computerization of Welding Information - III, September 12-14, 1990, Upsilanti, Michigan, T.A. Siewert, J.E. Jones, and H.G. Ziegenfuss, Editors, available from the American Welding Society, Miami Florida, 1992. 
8. International Conference on Computerization of Welding Information - IV, November 3-6, 1992, Orlando, Florida, T.A. Siewert, Editor, Available from the American Welding Society, Miami, Florida, 1994.

9. Fifth International Welding Computerization Conference, August 9-12, 1994, Golden, Colorado, T.A. Siewert, Editor, available from the American Welding Society, Miami, Florida, 1996.

10. First International Conference on Computer Technology in Welding, W. Lucas Ed., London, 3-5 June 1986, published 1987 by The Welding Institute, Cambridge, U.K.

11. S. Budgifvars, 1992. Computer Aided Welding (CAW) - 1992, IIW Document XII1295-92, available from the American Council of the IIW, American Welding Society, Miami, Florida.

12. T.A. Siewert, 1995. CAW '95: Computer Aided Software in the Americas, IIW Document XII-1396-95, available from the American Council of the IIW, American Welding Society, Miami, Florida; also published in the Welding Journal, 74, p. 39.

13. W. Lucas, 1995. Computer Aided Software in the U.K., IIW Document XII-1397-95, available from the American Council of the IIW, American Welding Society, Miami, Florida.

14. Kutsuna, N., et al. 1995. CAW 95 in Japan, IIW Document XII-1398-95, available from the American Council of the IIW, American Welding Society, Miami, Florida.

15. Budgifvars, S. 1995. Computer Aided Welding (CAW) in Europe, IIW Document XII1399-95, available from the American Council of the IIW, American Welding Society, Miami, Florida. 


\title{
DIGITAL SIGNAL PROCESSING
}

\section{Applications of DSP in Welding}

\author{
Dr. Donald J. Newman \\ President \\ SIGNALWARE Corporation \\ wWw.signalware.com/DSP
}

July 1997

\section{OUTLINE}

What are DSP's?

Applications in Welding

* Offline Weld Planning

* Online Welding Data Analysis

* Online Welding Station Control

\section{DSP in the Future}

\section{Questions}




\section{DIGITAL SIGNAL PROCESSING}

\section{COMPARISON of DSP's and OTHER PROCESSORS}

\begin{tabular}{|c|c|c|c|}
\hline & Micro Controller & DSP & PC-CPU \\
\hline Examples & $\begin{array}{c}\text { INTEL 805' } \\
\text { MOT 68000 }\end{array}$ & $\begin{array}{c}\text { TI TMS320C40 } \\
\text { MOT DSP5600 1 }\end{array}$ & $\begin{array}{c}\text { INTEL 486 } \\
\text { Cyvix 686 }\end{array}$ \\
\hline TYPE & RISC & WISC & RISC based \\
\hline $\begin{array}{c}\text { Clock Cycles Per } \\
\text { Added Instruction }\end{array}$ & $4-16$ & $1-2$ & $6-20$ \\
\hline $\begin{array}{c}\text { Concurrent } \\
\text { Instruction }\end{array}$ & 1 & $2-20$ & $1-2$ \\
\hline $\begin{array}{c}\text { Arithmetic } \\
\text { Precision }\end{array}$ & $8-16$ & $16-32$ & $16-64$ \\
\hline Optimized & Logic & Computation & Input / Output \\
\hline $\begin{array}{c}\text { Peripheral Support } \\
\text { Central Lines / }\end{array}$ & $\begin{array}{c}\text { AD's \& DA's } \\
\text { Sideo / Disk }\end{array}$ \\
\hline $\begin{array}{c}\text { Processor } \\
\text { Organization }\end{array}$ & Standalone & Multiple Parallel & Central Processor \\
\hline $\begin{array}{c}\text { Processor } \\
\text { Interconnect }\end{array}$ & Serial Port & $\begin{array}{c}\text { Parallel / Serial } \\
\text { Port } \\
\text { Shared Bus } \\
\text { Cross Bar Switch }\end{array}$ & Shared Bus \\
\hline Memory Cache & None & Mostly Cache & Some Cache \\
\hline
\end{tabular}




\section{DIGITAL SIGNAL PROCESSING}

\begin{tabular}{|l|l|l|l|l|}
\hline \multicolumn{4}{|c|}{ Table 1: Widely Used DSP Programmable Chip Parts } \\
\hline \multirow{4}{*}{ Company } & \multicolumn{1}{|c|}{ Part } & \multicolumn{1}{c|}{$\begin{array}{c}\text { MAC } \\
\text { time (ns) }\end{array}$} & $\begin{array}{c}\text { No. bits } \\
\text { fixed } \\
\text { point }\end{array}$ & $\begin{array}{l}\text { No. bits } \\
\text { floating } \\
\text { point }\end{array}$ \\
\hline \multirow{4}{*}{ AT\&T } & DSP16 & 55 & $16 / 36$ & - \\
\cline { 2 - 5 } & DSP16A & 33 & $16 / 36$ &,- \\
\cline { 2 - 6 } & DSP32 & 160 & 16 & $32 / 40$ \\
\cline { 2 - 6 } & DPS32C & 80 & 16 or 24 & $32 / 40$ \\
\hline \multirow{4}{*}{$\begin{array}{l}\text { Texas } \\
\text { Instrumenta }\end{array}$} & DSP56001 & 74 & $24 / 56$ & - \\
\cline { 2 - 6 } & DSP96002 & 75 & $32 / 64$ & $44 / 96$ \\
\hline & TMS320C10 & $114-280$ & $16 / 32$ & - \\
\cline { 2 - 6 } & TMS32C25 & $80-100$ & $16 / 32$ & - \\
\cline { 2 - 6 } & TMS320C50 & $25-50$ & $16 / 32$ & - \\
\cline { 2 - 6 } & TMS320C30 & $50-75$ & $24 / 32$ & $32 / 40$ \\
\cline { 2 - 6 } & TMS320C40 & $40-50$ & - & $32 / 40$ \\
\hline \multirow{4}{*}{$\begin{array}{c}\text { Analog } \\
\text { Devices }\end{array}$} & ADSP2100 & 125 & $16 / 40$ & - \\
\cline { 2 - 6 } & ADSP2100A & 80 & $16 / 40$ & - \\
\hline
\end{tabular}

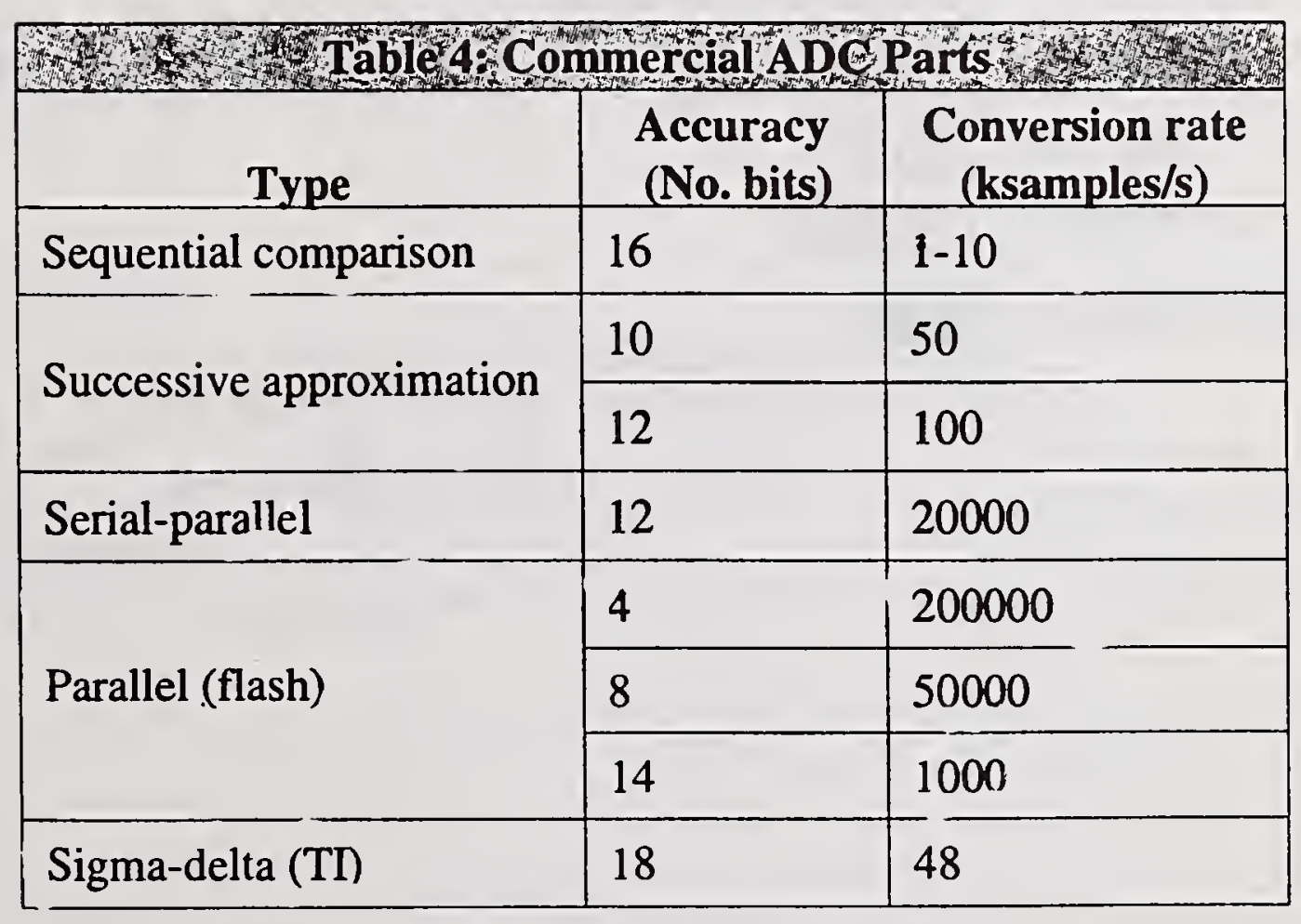




\section{DIGITAL SIGNAL PROCESSING}

\section{Cost Performance FFT Benchmark}

Analog Devices ADSP-2171

ADSP-21062

ADSP-21062-C

Lucent Technologies DSP1627

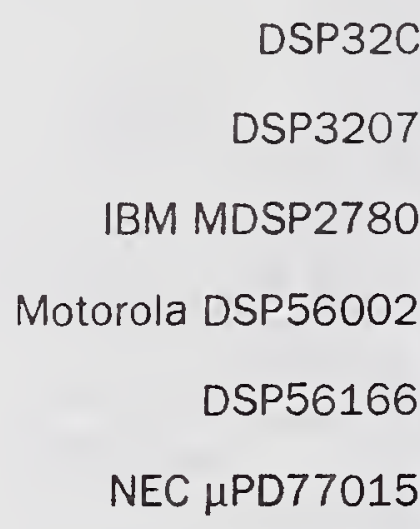

Texas Instruments TMS320C209

TMS320C31

TMS320C44

TMS320C52

TMS320C541

Zoran ZR3800x

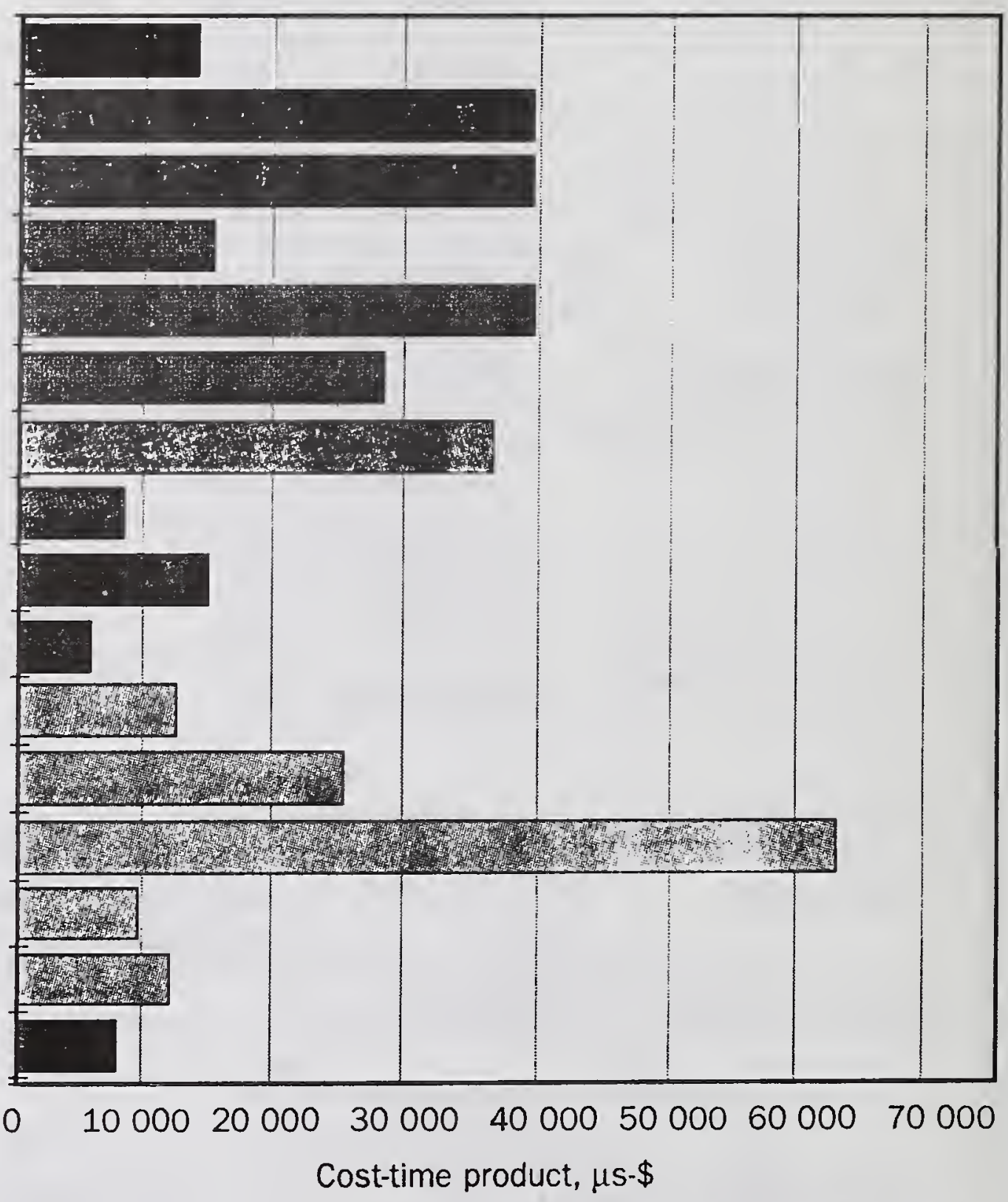

Source: Berkeley Design Technology 


\section{DIGITAL SIGNAL PROCESSING}

\begin{tabular}{|c|c|c|}
\hline Function & Description & Application examples \\
\hline $\begin{array}{l}\text { Real block finite impulse } \\
\text { response (FIR) filter }\end{array}$ & $\begin{array}{l}\text { FIR filter that operates on a } \\
\text { block of real (not complex) data }\end{array}$ & $\begin{array}{l}\text { G. } 728 \text { speech encoding, } \\
\text { other speech processing }\end{array}$ \\
\hline Complex block FR filter & $\begin{array}{l}\text { FIR filter that operates on a } \\
\text { block of complex data }\end{array}$ & Modem channel equalization \\
\hline $\begin{array}{l}\text { Real single-sample } \\
\text { FIR filter }\end{array}$ & $\begin{array}{l}\text { FIR filter that operates on a } \\
\text { single sample of real data }\end{array}$ & $\begin{array}{l}\text { Speech processing, general } \\
\text { filtering }\end{array}$ \\
\hline $\begin{array}{l}\text { Least-mean-square } \\
\text { adaptive FIR filter }\end{array}$ & $\begin{array}{l}\text { Least-mean-square adaptive FIR } \\
\text { filter that operates on a single } \\
\text { sample of real data }\end{array}$ & $\begin{array}{l}\text { Channel equalization, servo } \\
\text { control, linear predictive encoding }\end{array}$ \\
\hline $\begin{array}{l}\text { Infinite impulse response } \\
\text { (IIR) filter }\end{array}$ & $\begin{array}{l}\text { IIR filter that operates on a } \\
\text { single sample of real data }\end{array}$ & Audio processing, general filtering \\
\hline Vector dot product & $\begin{array}{l}\text { Sum of the pointwise } \\
\text { multiplication of two vectors }\end{array}$ & $\begin{array}{l}\text { Convolution, correlation, matrix } \\
\text { multiplication, multidimensional } \\
\text { signal processing }\end{array}$ \\
\hline Vector add & $\begin{array}{l}\text { Pointwise addition of two } \\
\text { vectors producing a third vector }\end{array}$ & $\begin{array}{l}\text { Graphics, combining audio } \\
\text { signals or images, vector search }\end{array}$ \\
\hline Vector maximum & $\begin{array}{l}\text { Discovery of the value } \\
\text { and location of a vector's } \\
\text { maximum value }\end{array}$ & $\begin{array}{l}\text { Error-control coding, algorithms } \\
\text { using block floating-point } \\
\text { arithmetic }\end{array}$ \\
\hline Convolutional encoder & $\begin{array}{l}\text { Application of convolutional } \\
\text { forward error-correction code } \\
\text { to a block of bits }\end{array}$ & $\begin{array}{l}\text { North American digital } \\
\text { cellular telephone equipment } \\
\text { (IS-54 standard) }\end{array}$ \\
\hline Finite-state machine & $\begin{array}{l}\text { A contrived series of control } \\
\text { operations (test, branch, push, } \\
\text { pop) and bit manipulations }\end{array}$ & $\begin{array}{l}\text { Control operations appear in } \\
\text { nearly all digital signal-processing } \\
\text { applications }\end{array}$ \\
\hline $\begin{array}{l}\text { 256-point, radix-2, } \\
\text { in-place fast Fourier } \\
\text { transform (FFT) }\end{array}$ & $\begin{array}{l}\text { FFT conversion of a normal } \\
\text { time-doman signal into the } \\
\text { frequency domain }\end{array}$ & $\begin{array}{l}\text { Radar, MPEG audio } \\
\text { compression, spectral analysis }\end{array}$ \\
\hline
\end{tabular}




\section{DIGITAL SIGNAL PROCESSING}

\section{Offline Weld Planning}

OBJECTIVES:

* Use more sophisticated simulations/models in planning.

* Keep the planning time reasonable.

${ }^{* *}$ Target is $5 \%$ of teach pendant time

* Have very accurate process simulation

** No "touch up" at workcell

${ }^{*}$ Keep trouble shooting of shop floor

APPLICATION OF DSPs:

*Workcell Simulation - kinematics, collision avoidance, etc.

*Process Simulation - dynamics of the process.

* Simulation faster than real-time - parallel processors on desktop

\section{TECHNIQUES:}

* Finite Element Modeling - multiple DSP's for simultaneous processing of thermal, fluid flow, and mechanical/distortion portions of the model

* Neural Networks - Multiple DSP's for simultaneous processing of nets for weld shape, strength/fatigue, and cost analysis

* Optimal Search Methods - find the "best" operating parameter values and conditions (e.g. weld position, torch angles, etc.)

DSP HARDWARE: TI C3x, C4x; AD Shark;

Boards - off-the-shelf for PCs 


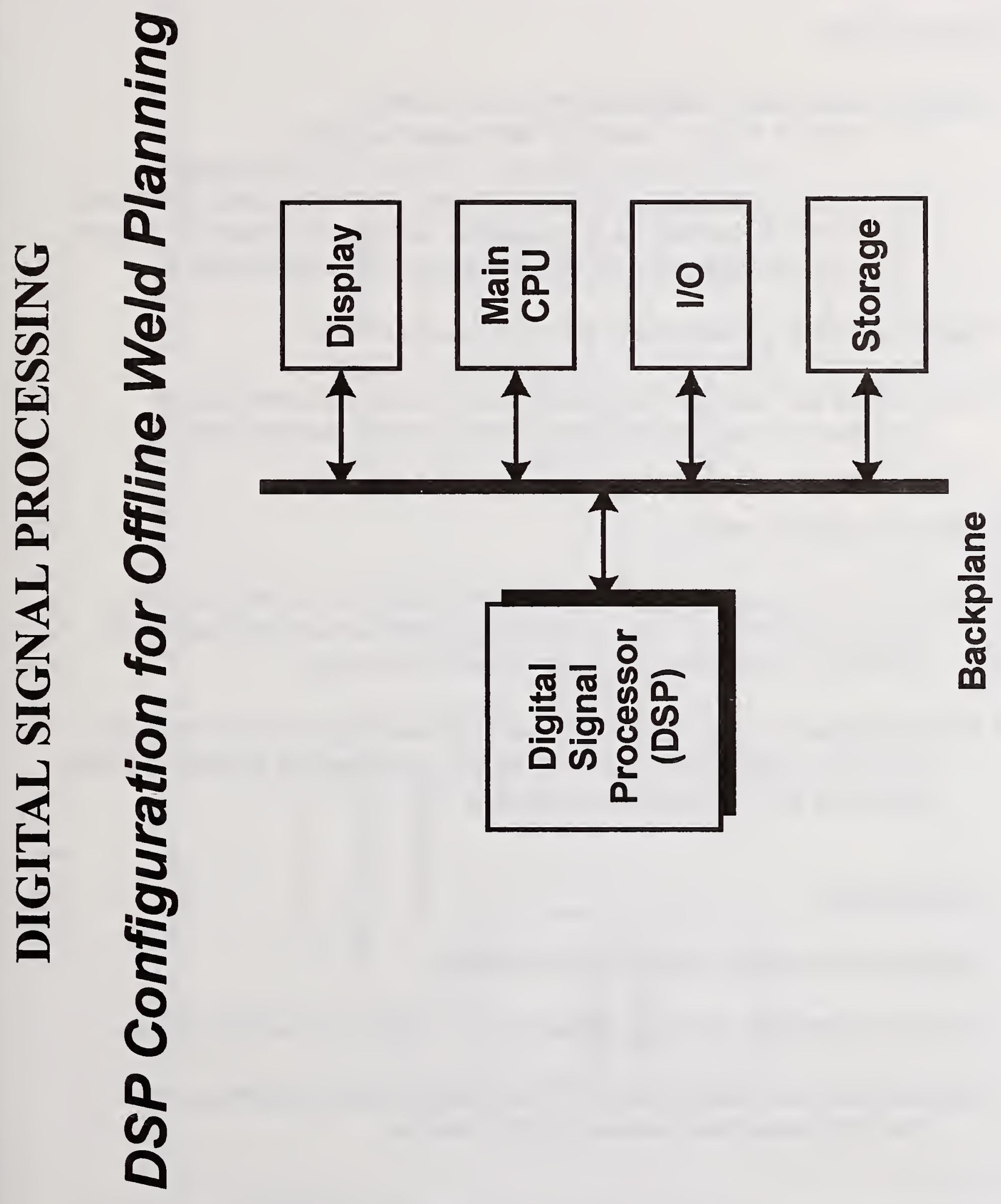




\section{DIGITAL SIGNAL PROCESSING \\ Online Welding Data Analysis}

OBJECTIVES:

* Quality Assurance - Statistical Process Control

-- Out of Range - coupled with expert system,

e.g. low wire feed rate $=$ wire jam in feed system

-- $\quad$ Trend Analysis - real-time analysis for immediate feed-back

-- $\quad$ Error Detection - e.g. excessive voltage fluctuations = poor arc stability (loss of shielding gas or contamination)

* Data Reduction - reasonable storage requirements

" Have ability to "tap into" real-time data stream and provide engineer/management with on-going quality assessment

\section{APPLICATION OF DSPs:}

* Weld Pool Dynamic Analysis - Real-time feedback of sensor data fusion operations - e.g. combining multiple sensor streams to determine weld shape, or other weld properties

* Sensor System Data Analysis - Smart processing of data from laser scanners, acoustic sensors, multiple sensor data streams, vision systems, IR, arc spectral analyzers, etc.

TECHNIQUES:

* Statistical Analysis - vector procecessing

"Numerical Models, look-up tables, etc. - "smart" reporting of data

* Neural Networks - reporting of weld shape, size, properties, etc. through real-time processing of a model

DSP HARDWARE: TI C4X, C62, C80; AD Shark - Boards available for $P C$ and VME, more difficult to select 


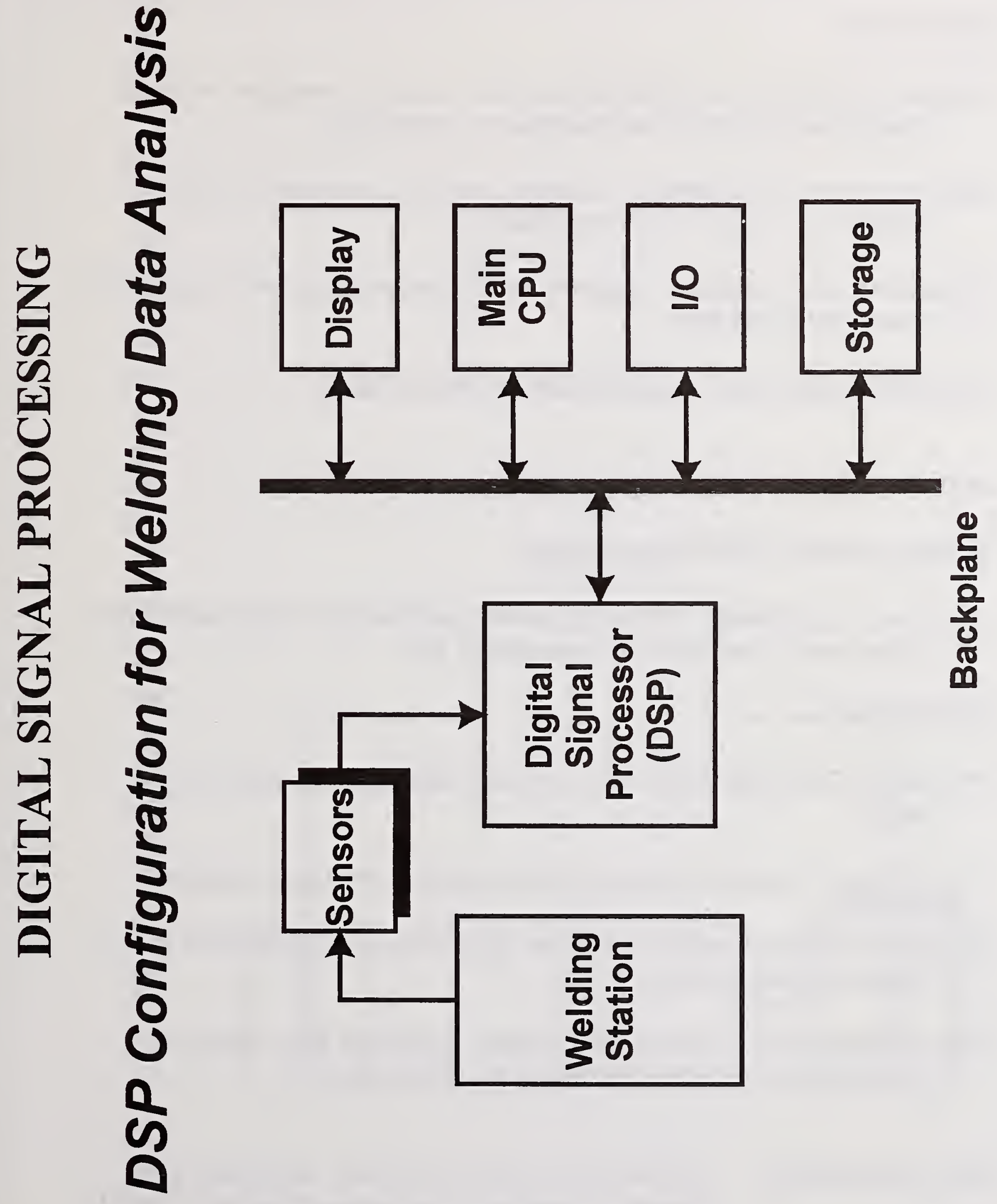




\section{DIGITAL SIGNAL PROCESSING \\ Online Welding Station Control}

\section{OBJECTIVE:}

* Single- and Multi-pass Weld Shape/Size Control - requires multiple data stream analysis and feedback control loop

* Weld Penetration Control - requires real-time analysis of complex sensor data and "smart" decisions

* Weld Distortion Control - requires model processing and sensor data analysis in real-time

....and otherwise Continuously Maintain Weld Quality

\section{APPLICATION OF DSPs:}

* Robot Guidance with Vision System

* Process Adjustment - including power parameters, high speed pulse adjustment, manipulator movement, etc.

\section{TECHNIQUES:}

* Predictive Feedback Control - complex data analysis with look-up tables

* Fuzzy Logic Control - complex data analysis with logic feedback

* Knowledge Base Control - multiple data stream analysis with rules for control decisions

* Neural Networks - multiple data stream input and fast response function inversion for feedback to control loop

DSP HARDWARE: TI C2xx, C4x, C5x, C62, C80; AD Shark, 21xx Boards - custom or carrier+module for PC and VME, require detail design 


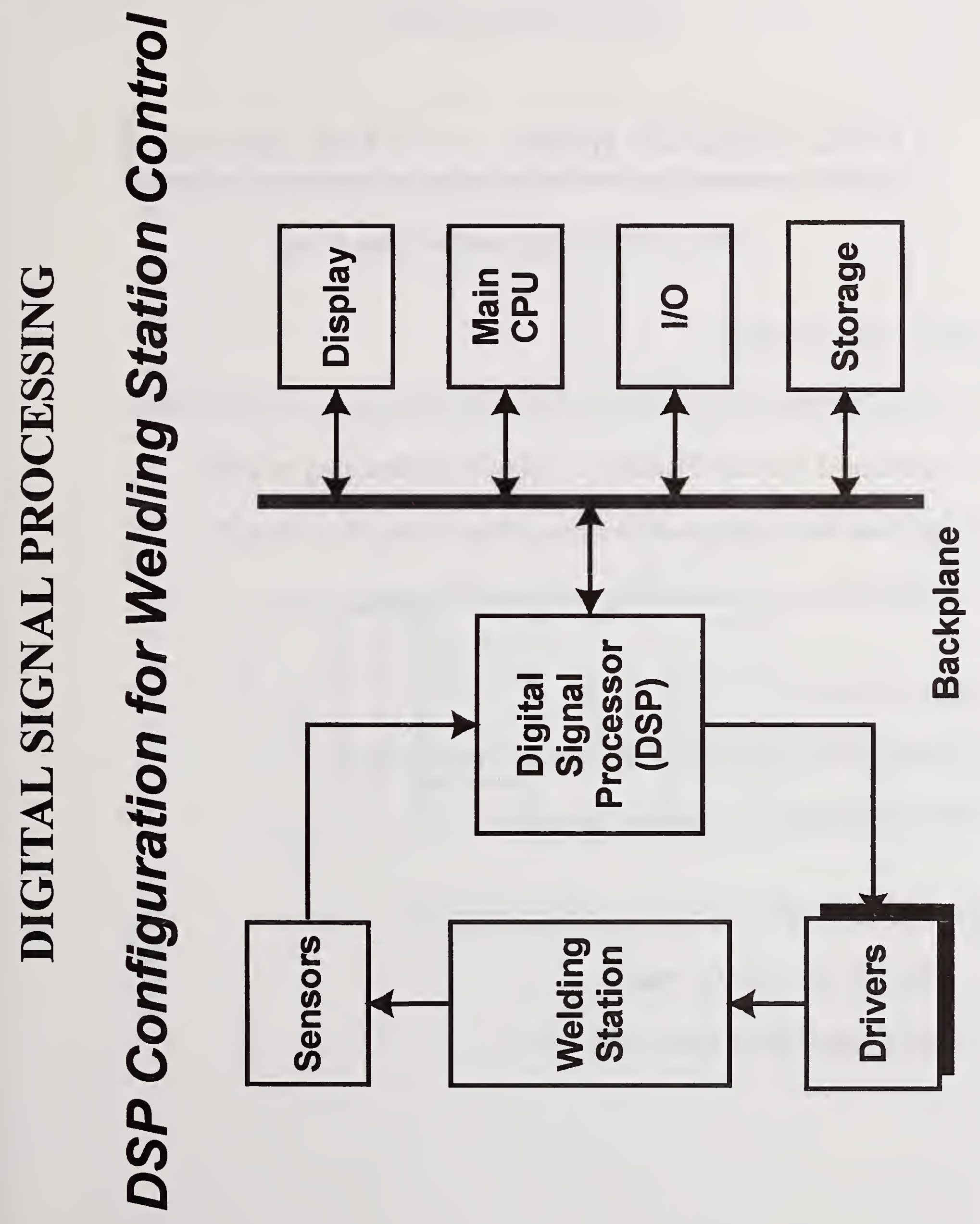




\section{DIGITAL SIGNAL PROCESSING}

\section{DSP in the Future}

\section{PROCESSOR (now) --> FPGA (future)}

FPGA - Field Programable Gate Array

\section{WHAT ARE FPGAs?}

* Large Collection of Logic and Arithmetic Hardware in One Device

* High Speed Parallel Hardware - parallel processing of data

${ }^{*}$ Any Desired Configuration without Removing from Circuit

* Rapidly Reconfigurable Any Number of Times

\section{WHY FPGAs?}

* Order of Magnitude Faster than DSP Processors

* More Adaptable to Various Sensors

WILL FPGAS REPLACE DSP PROCESSORS?

* In Certain Situations - Yes

* Most Likely will be Used Together 


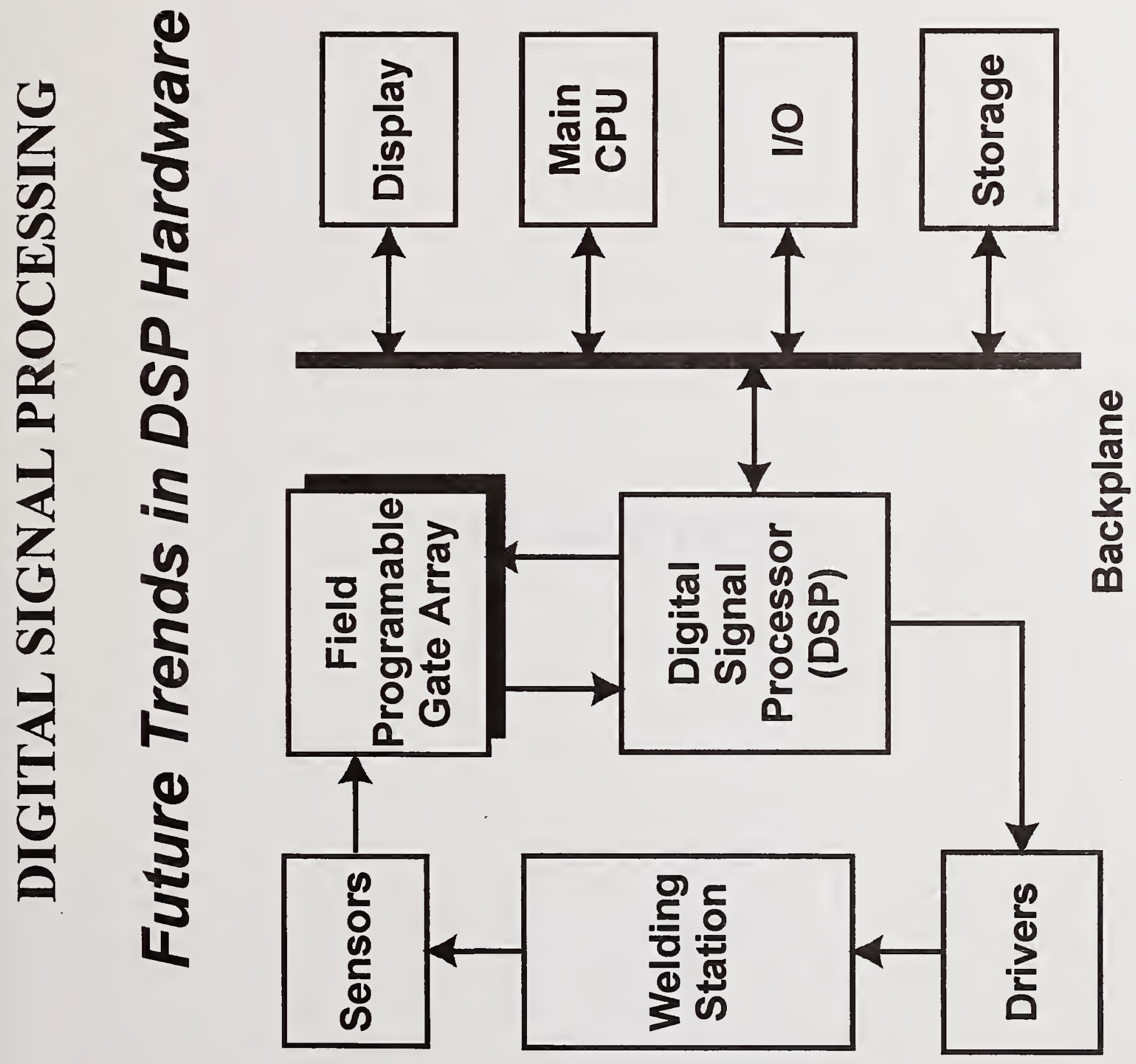


CASE STUDIES 



\title{
USING TURBO-FMCTM (THE ELECTRONIC FILLER METAL CATALOG) IN POWER PLANT MAINTENANCE WELDING
}

\author{
C. L. Foster ${ }^{*}$
}

\begin{abstract}
Turbo-FMCtm database was compiled and developed by C-Spec. The program lets the user quickly and easily search and display over 10,000 trade names, over 2,000 AWS classifications, 135 vendors and 2,000 filler metal characteristic descriptions. Additional useful information included are the UNS numbers, the ASME assigned F-numbers and, when applicable, the A-numbers for ferrous filler metals. The descriptions of filler metal characteristics closely match those contained in the usability description paragraphs of the Filler Metal Specifications published by the American Welding Society. The contents of the database are indexed and thoroughly cross-referenced so it is very simple to find information about an equivalent competing product. This product is a practical tool for engineers, inspectors, procurement personnel, filler metal manufacturers, vendors and distributors.
\end{abstract}

\section{INTRODUCTION}

Welding is a vital part of any power plant maintenance program so selecting or specifying appropriate filler materials becomes critical to quality, code compliance, and cost effectiveness. The information that is necessary for specifying appropriate filler materials is dispersed throughout many documents and is not summarized or organized in any straightforward manner in a single source. Most codes specify ranges for filler and base material combinations based upon chemistry and/or mechanical properties. Codes refer to filler materials either by classification and/or specification. Codes seldom directly address the usability characteristics of filler materials and certainly never mention trade names, common names or manufacturers. In addition, trade names and product lines frequently change ownership. So we are faced with the daunting task of sequentially looking up two or more tables and then manually cross referencing them so appropriate filler materials may be selected.

The need for a comprehensive and concise source of this information has existed for years. There have been attempts to compile this information into charts or documents. Unfortunately the filler material market is a rapidly changing market with processes, specifications, classifications, filler metals, and manufacturers coming into and leaving the market on a regular basis, therefore written charts and documents become out dated as soon as they are printed.

Computer databases are well suited for compiling, cross referencing diverse data and reporting the data in a useful format. As will be demonstrated later, any database for filler materials needs to be as current as possible and should lend itself to being easily and frequently updated. This paper and demonstration will show how such a database is used in the daily maintenance welding of power plants.

\footnotetext{
* Pacific Gas \& Electric Company, San Francisco, California
} 


\section{SELECT, SPECIFY AND PURCHASE}

Selecting, specifying and purchasing appropriate filler material are some of the steps that must be taken in order to keep a power plant's welding activities code compliant and productive. There is a relatively large range of materials that must be welded in a power plant including carbon steel, all three categories of stainless steel, nickel alloys, various grades of $\mathrm{Cr}$. Mo. steels, Copper/Nickel, aluminum and various hardfacing applications. At least four different welding processes are used routinely. Welding applications range from performing fillet welds on 1/8" carbon steel to full penetration welds on thirty inch diameter Cr. Mo. pipe with a three inch thick wall or applying a $1 / 8$ " thick hardface pass on a two inch diameter valve seat. Codes refer to filler material by classification or specification. Power plant OEM specifications often refer to filler materials by trade name or common names. This is particularly true of hardfacing materials. Using a filler metal database helps in taking the differing terminology from the varying sources used to describe the same product and determine a correlation. The following four examples are typical applications where a filler metal database saves time and money.

\section{$\underline{\text { Troubles With Selecting, Specifying and Purchasing Filler Metal by Classification }}$}

Recently a pipe bridge needed to be constructed of carbon steel, most of the structure would be field fabricated. FCAW was selected as the process and E71T-11 as the filler metal. This selection was included in the job specification with no mention of trade name or manufacturer. The warehouse ordered four rolls of E71T-11 from a local supplier and welding commenced on the job. Immediately the welders began complaining that they could not weld vertical or overhead. A quick check of the filler wire indicated that the wire was E71T-11. Visual inspection of the welding indicated that flat and horizontal welds were acceptable, however vertical and overhead welds were visually unacceptable. A call to the local supplier was of no help. The manufacturer of the wire was a large and very reputable company and the classification was clearly stamped on the cardboard shipping box and the reels of wire. However the trade name of the wire was not familiar and include a suffix of "MC". A quick search of the database confirmed that E71-T11 was an all position electrode, but no reference to the trade name was found. It did, however, give me a direct telephone number to the manufacturer. Within five minutes I was talking with the product development engineer who explained that the product I was using was a brand new product and that the $\mathrm{MC}$ stood for metal core. He also explained that while the AWS classification E71-T11 was the nearest classification match available, his product was not capable of all position welding. According to the engineer, AWS was working on a classification for metal core FCAW filler metals that would be available soon. The MC filler metal was immediately returned to the local supplier and a classification E71T-11 wire with a familiar trade name was purchased. The database provided quick information that helped keep this incident from becoming a productivity issue with the loss of two man/days and instead a total of only two man/hours were lost.

\section{Keeping The Troops Happy By Purchasing From Classification and Trade Name}

Welders develop a preference for certain brands and products. When they request replacement filler material they most often use trade names or product names because they don't want just any 
E7018. They want a specific material such as Jetweld-LH $78 \mathrm{MR}$, for example. I personally know that certain trade name fillers work better for me than others, having experienced lower stub wastage, lower splatter and a more stable arc with certain brands and trade names. All of these factors affect productivity. Warehousemen and purchasing agents will generally purchase the least expensive filler metal, if given only a classification for ordering. This does not always mean that the cost of the completed weld will be less expensive if the performance of the material is not what could have been achieved using the specific filler requested. Matching the request with the correct material may additionally be complicated by the fact that the welder may only identify the trade name or even part of a trade name, then the codes and engineering document specify and refer to filler metals by classification. How do you easily correlate a trade name to a classification? Without a database it can be a time consuming effort requiring telephone research or pouring over one or several documents to find the correlation and because of the time factor may be omitted. However, a computer search of a database can show exact correlations or even show possible correlations based upon a partial trade name.

\section{Purchasing Needs A List Of Filler Metal Manufacturers To Bid On List Of Classifications}

The purchasing department gets a new directive that all purchases above a certain dollar amount must be competitively bid. You are ordering filler metal for a job and the estimated dollar amount exceeds the competitive bid criteria. Purchasing calls you back and presents you with the good news that you must provide them with a list of five sources for each filler metal classification that you have specified. How long would it take you to compile an accurate and cost competitive list for the purchasing department? A minimum of an hour, maybe much longer. The job can be done using a computer and database within five minutes. Search the database for the classification cross referenced to manufacturers, then select the first five names on the menu list or be more selective and hand pick the manufacturers you prefer the most. Print the report for each manufacturer and send the collection of manufacturer data pages to purchasing. Job done in less than five minutes.

\section{$\underline{\text { A Valve Manufacturer Specifies Seat Replacement With Stellite }}$}

A situation arises where the valve seat in a valve must be corrected in some manner. To complicate the situation, the valve is forty years old and the manufacturer is out of business. It would be very expensive to replace the valve and would take three months lead time. Operations Dept. needs the valve now so we choose to weld repair the seats. We do have an OEM drawing which identifies the seat material as stellite. End of problem, or is it just the beginning? Now comes the hard part. Are there grades of stellite? Who makes it? Does it have an AWS classification? Once again you could spend hours on the phone or in documents researching the usability notes for the filler metal, researching the specification and classification numbers and finding who now makes the product line. By searching the database for the trade name first then looking for all of the cross references I found that it is manufactured by Stoody in three grades, 1,6 , and 12, the specification is 5.13, the classifications are ECoCr-A, ECoCr-B and ECoCr-C, then searching from the classification I also found that there are numerous manufacturers who produce equivalent hardfacing filler metals. This was all done in a matter of approximately five minutes. Some telephone work was required to understand the significance of the grades which 
applied to our particular application, but this was done by using the manufacturers data page from the database. There were no local vendors with stellite stock on hand. However, in this particular case, I was able to find and procure an equivalent locally within one hour and the repairs were completed in four hours.

\section{CONCLUSION}

A filler metal database program is a helpful tool which has reduced the amount of time required by engineering, supervision or technical support personnel in selecting, specifying and purchasing filler metals. The database has been a useful tool in troubleshooting as well. The increase in my productivity far offsets the cost of the database. The time/money I saved in just two of the four applications cited above was enough to purchase the database. I currently use the database at least once a week and sometimes more than once a day. 


\title{
DEEP WATER PIPELINE WELDING AND REPAIRS USING MODERN COMPUTER TECHNOLOGY TO CREATE A DIVERLESS FUTURE FOR STATOIL
}

\author{
T. Håbrekke *, M. Armstrong \#, J.O.Berge †
}

\begin{abstract}
Harsh weather conditions and exploration of oil and gas on deep water in the North Sea demanded new underwater repair / installation technology. Due to this, the Norwegian oil companies have gradually increased their effort to remove divers from the operations when the business seems to approach deeper waters. Experience has shown that human interface during the production welding will also improve the job performance and minimize the risk of weld defects. Further, the high costs involved in such operations forces the companies to perform the installations as efficient as possible. With the consortium PRS (Pipeline Repair Spread), significant sums have been invested to reach the Norwegian oil companies goals. The technology is based upon solutions around PC based computers, and welding procedure qualifications are performed in a small pressure chamber equipped identically as the full scale welding gear. The development has now come so far that all wet operations can be remotely operated from surface without help of divers. In parallell, a mechanical connector is worked out. However, such connectors are so far not used in the North Sea for the main transport pipelines (diameters up to $42 "$ ).
\end{abstract}

\section{INTRODUCTION}

The recovery of oil and gas resources from the north sea, is a challenge in which new technology is continually required. The subsea pipelines have to cross deeper waters, and the oil platforms are drilling at greater depths. These challenges demand new technology, not only for exploring the oil and gas, but also for transportation. To deliver oil and gas to central Europe, the Norwegian oil companies have had to plan for huge export pipelines from the North Sea down to France, Belgium and Germany. The outer diameter of these pipelines can be as large as 42 inches. These pipelines need to be joined to subsea connection points or platforms. The joining method is either to use a welding process or to use a mechanical coupling. In most cases welding has been chosen, and in the Norwegian sector, dry, remote controlled, hyperbaric welding.

Dry hyperbaric welding is welding performed in a subsea habitat, where the water is evacuated by pumping down gas until the habitat pressure is equal to the outside water pressure. The gas is Heliox, i.e. Helium mixed with $\mathrm{O}_{2}$. The welding is performed by remote control from the topside on board the deployment vessel. The welding process is Gas Tungsten Arc Welding (GTAW) This process is suitable for mechanisation, and is considered to be a process creating fewer weld defects and less fumes than many other welding processes. The latter is an important factor as divers are currently present in the habitat.

* SINTEF Materials Technology, 7034 Trondheim, Norway

\# Isotek Electronics Limited, Claro House, Servia Road, Leeds LS7 1NL, UK

† Statoil PRS, Kårstø, 5500 Haugesund, Norway 
The development of a remotely controlled, dry, hyperbaric welding spread began in 1984 by Norsk Hydro and Statoil, using SINTEF to develop the process requirements and Isotek Electronics Limited (of the UK) to develop the control system. Trials began in 1986 using a specially built habitat to house the equipment. Following the successful trials a consortium agreement was made between Norsk Hydro and Statoil to supply a contingency pipeline repair system, for the North Sea, using the technology. A number of oil companies have subsequently signed up to this agreement paying an annual fee. Although the equipment was primarily developed for contingency repair, it is now used routinely for scheduled subsea connections and since 1988 the following hyperbaric welds have been performed:

- 1988, Oseberg A, 2 x 28 " welds.

- 1989, Gullfaks C, 6 welds of between 10" and 36" diameters.

- 1990, Oseberg C, 8 16" welds.

- 1991, Snorre-Statfjord, 8 welds of 10" and 20" diameters.

- 1992, Zeepipe 1, 6 welds of 30 " and 40" diameters.

- 1995, Troll-Zeepipe, 7 welds of 30" and 40" diameters.

- 1996, Sleipner Vest / Haltenpipe, 5 welds of 16" and 20" diameters.

In the first years of this system the installation of the habitat and pipe support equipment was installed by the use of divers in the water and only the welding phase and habitat telemetry were computerised. Since 1994 all of the subsea equipment, including its deployment, installation and removal has been computerised and is remotely controlled from the deployment vessel. Currently divers are still required to assist inside the habitat; to hook up equipment, replace tungsten during welding, grind out errors, make 'ad hoc' measurements, and perform Non Destructive Testing (NDT) after completion of welding. In the longer term, the intention is to automate these remaining tasks and make the operation fully diver-less.

\section{DRY, HYPERBARIC WELD OPERATION}

A mechanised hyperbaric weld is different to welds performed on the surface as it introduces a number of extra complications. First and foremost, the weld is performed at a pressure in excess of surface pressure. This has a fundamental effect on the arc and the voltage required to sustain the arc. Secondly the magnetic effects on the arc can be considerable, causing large deflections from the normal arc path. To compound this, the visibility of the arc and supporting tasks is restricted. The operator is remote and can only see the arc from cameras. The environment inside the habitat is a Helium $/ \mathrm{O}_{2}$ gas mixture with high humidity. The supporting divers performing set-up tasks have a distorted voice due to the Helium atmosphere and communications to the surface is often difficult to interpret. The performance of the supporting divers is also less than optimum due to the high pressure effect on humans. The space inside the habitat is limited when the pipes, diving equipment, weldheads and other support equipment is installed, leaving very little room to manoeuvre. The divers have a depth and working time limitation and therefore many divers and shift changes are needed to have a continuous operation to complete a large diameter hyperbaric weld. In addition to these difficulties, the preparation prior to welding involves the deployment and manipulation of large subsea structures. 
Before a hyperbaric weld can be performed, the two pipes to be joined have to be lifted up from the seabed, and any concrete coating removed. A computerised concrete removal machine has been developed to perform this task. This and other equipment in the spread can be deployed 'live' with an umbilical providing power and communications, or 'dead' with power and communications being delivered via an ROV (with a 'docking skid') docking onto the equipment via a docking panel as shown in Figure 1. The valves on the panel are backup manual over-rides in the case of equipment failure. A picture of the docking skid with its power and communications wet mateable connectors is shown in Figure 2. The skid is also capable of providing backup hydraulics in the event of equipment failure. All the subsea units contain exactly the same control pod containing a computer, $\mathrm{V} / \mathrm{O}$ cards and power supplies. A picture of this pod is shown in Figure 3. The configuration and information dictating the equipment to be controlled is all held in a database on the topside computer. The operator selects the equipment under control and the software loads the relevant data to allow panel, switches and joysticks to operate the subsea equipment, Figure 4.

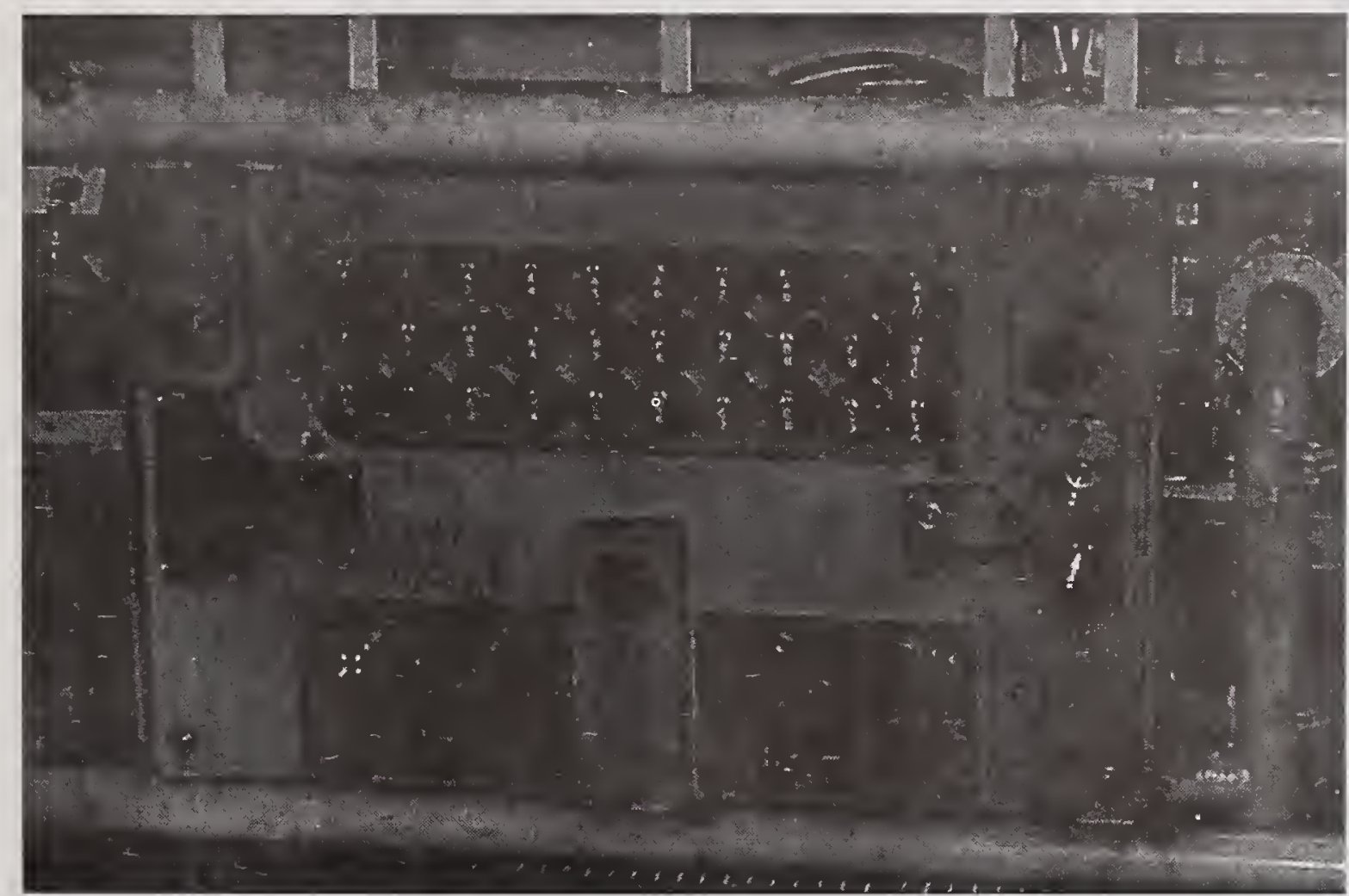

Figure 1. ROV docking panel. 


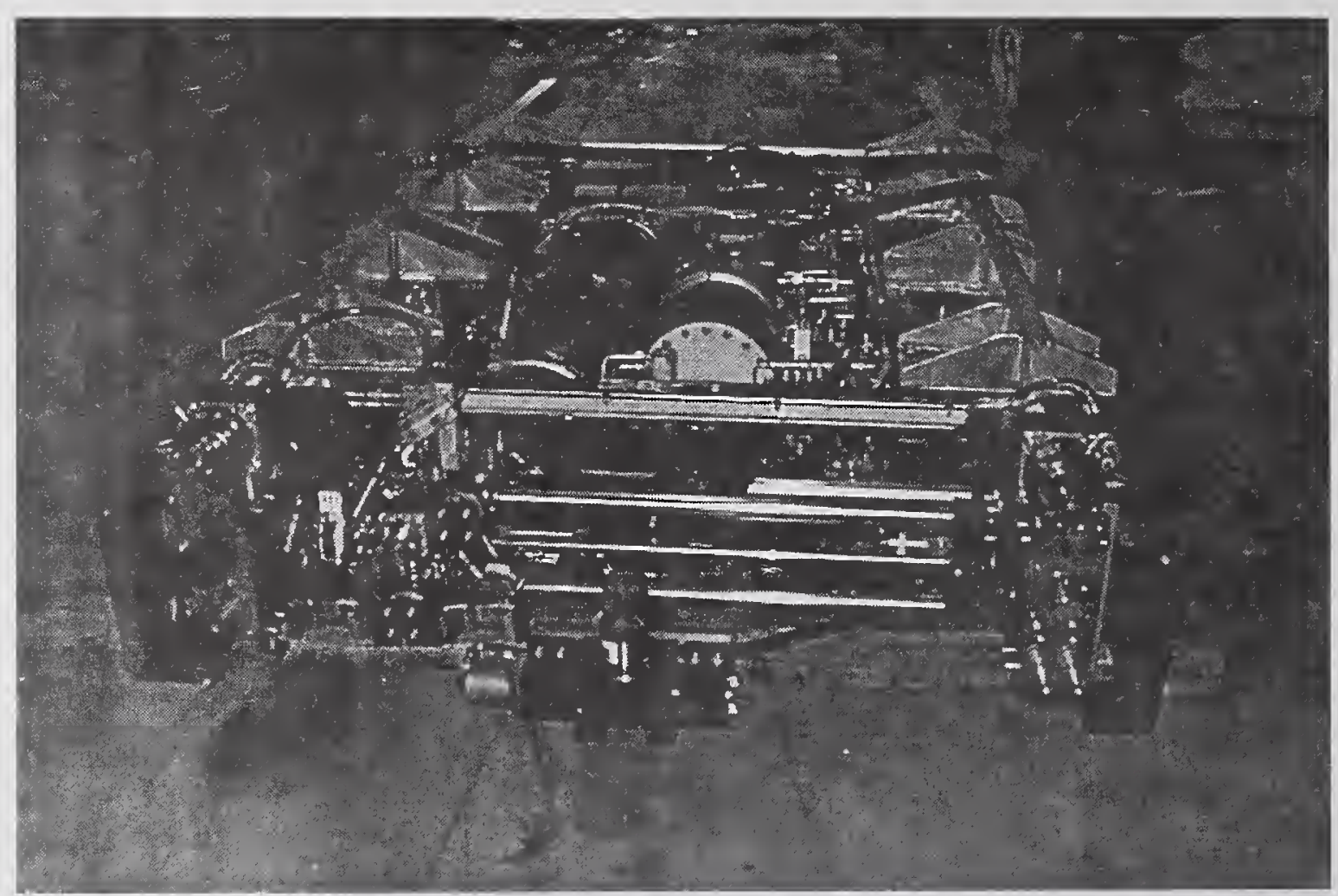

Figure 2. ROV docking skid.

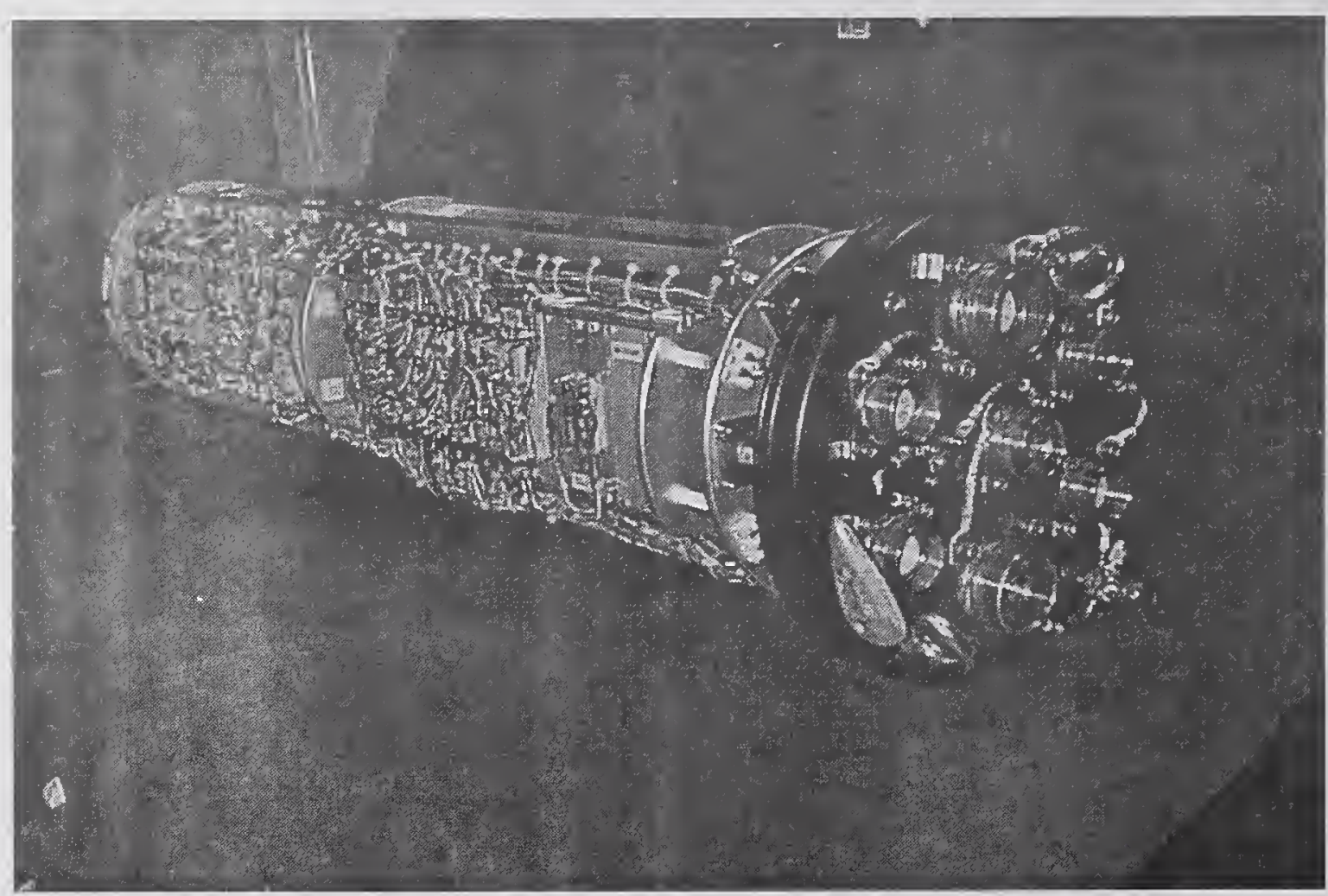

Figure 3. Control pod installed on the subsea equipment. 


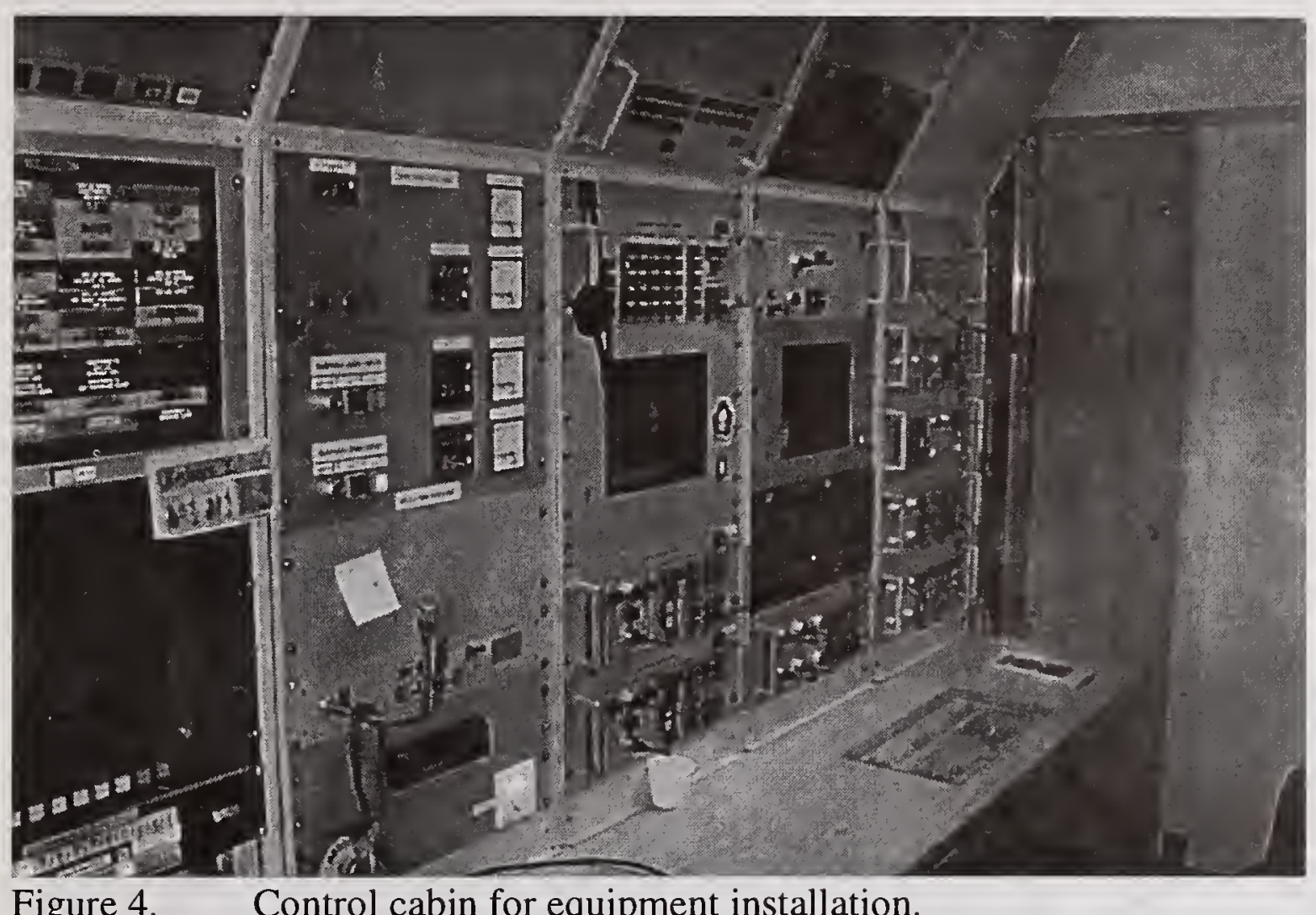

Figure 4.

Control cabin for equipment installation.

After the pipes are clean, to allow a proper grip for the claws of the alignment frame, the lifting frames (H-frames) are installed, see Figure 5. These help with the alignment of the pipe ends. Since the pipelines can be up to 42 " in diameter with heavy wall thickness (typically $25-35$ $\mathrm{mm})$,these frames are heavy and difficult to handle .

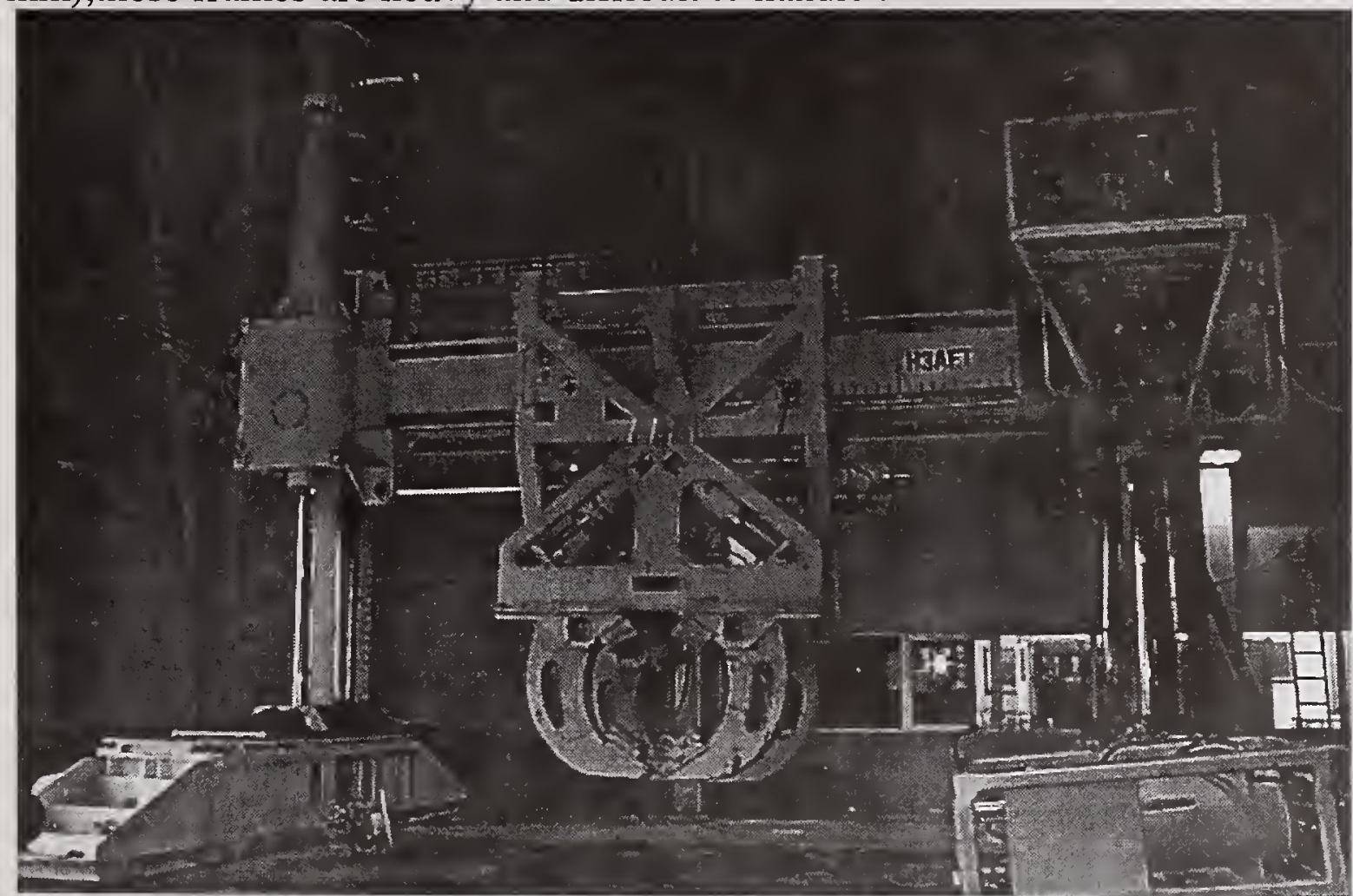

Figure 5. Dry PRS lifting H-frame. Total weight, 70 metric tonnes with a lifting capacity of 120 metric tonnes. 
The H-frames are 70 metric tonnes with a lifting capacity of 120 metric tonnes using a sea water hydraulic power unit. These pipe handling units are designed for heavy pipe operations and are capable of providing vertical, lateral and axial movements of the pipe.

When the H-frames are in place, at some distance from the end of each pipe end to be joined, the pipes are roughly aligned and the habitat is installed over the pipe ends. The habitat with its alignment frame has a weight of 90 metric tonnes in air, see Figure 6. The habitat is lifted off the vessel with cranes and lowered to the seabed, while ROV's survey the placement operation. After the habitat is in place, the POwer and COntrol module (POCO) is launched. The POCO is the control unit on the seabed containing the computers for the Weld Control System (WCS), the habitat and POCO Telemetry System (PTS), welding / preheat power sources and gas /hot water supply for welding and diving. All supplies and signals between the welding habitat and the support vessel are via an umbilical and the POCO.

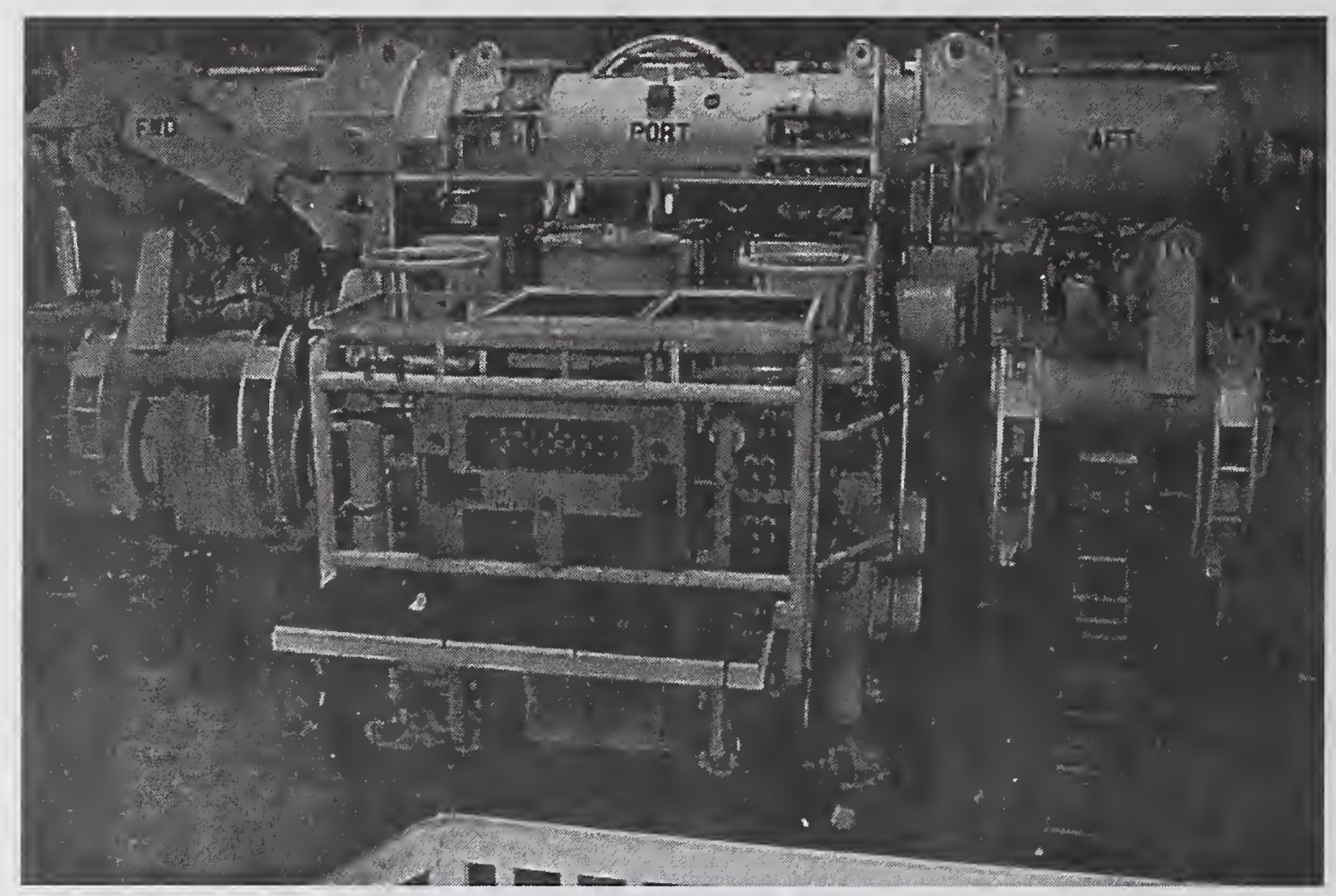

Figure 6. The subsea habitat with alignment frame on top.

Before the habitat is dry and ready for the entry of divers, it must be sealed around the pipes entering the habitat. These doors are remotely operated from the surface. When the doors are sealed, the water can be evacuated by blowing down the habitat with Heliox diving gas. Since the pressure inside is equal to the surrounding seawater, no water will come inside the habitat.

The next phase is to prepare the pipe ends for welding. The bevelling of the welding groove is normally started after the habitat is dry, and the machining is performed with a machining tool hooked on the Integrated Modular Tool (IMT). For pipes with a diameter under 20", the machining is performed using a Roberts \& Brothers lathe machine. For larger pipes the IMT is used which goes down wet with the habitat, and is installed with the assistance of divers. The divers close the IMT (which is a split clamp with rotating faceplates surrounding the pipe, see 
Figure 7) around the pipe and it is secured by the topside operator activating 8 hydraulic jacks in sequence that can squeeze the pipe circular if necessary. A computerised machining tool is then mounted on the IMT and the "J" preparation bevel milled. The machine tool computer is contained in a subsea atmospheric container as shown dismounted in Figure 6.

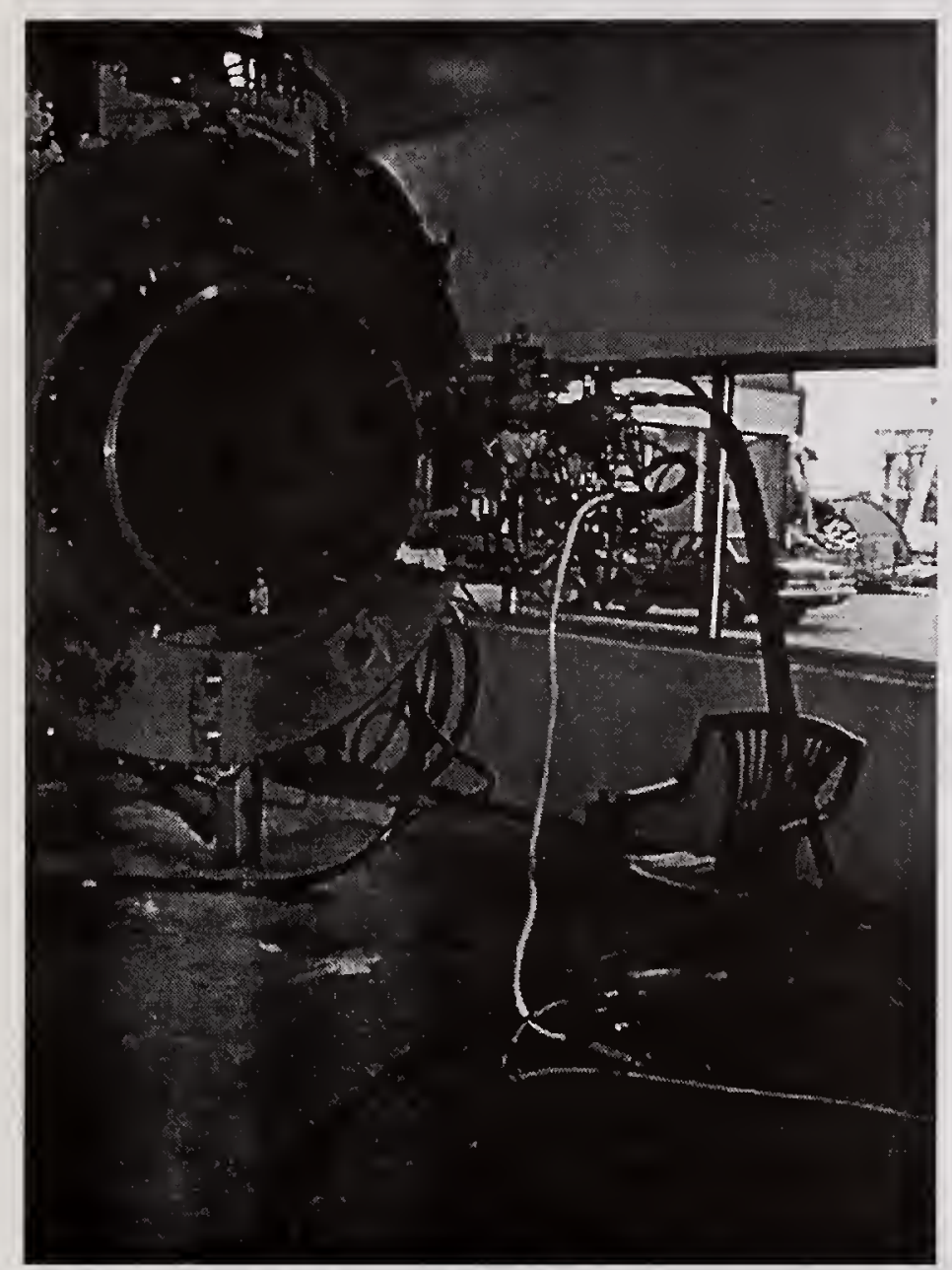

Figure 7. The Integrated Modular Tool (IMT) with automatic machining tool.

After machining, the weldhead installation takes place. The weldhead is sent down to the habitat in a special designed TRansport COntainer (TRACO) to keep the equipment dry. The weldhead is then attached to the same faceplate on the IMT as the machining tool. After this installation the fine alignment of the pipe ends is performed. The weldhead has been specially designed to reduce umbilicals (items most likely to cause problems in a subsea environment). All head motors and drive systems (torch, wireguide and cameras) are placed at the head so that only power and communications cables are required between the weldhead and the POCO. Computers are installed in atmospheric containers at the weldhead to control all motions in response to commands sent from the automatic weld controller computer in the POCO. The intelligent weld head is shown in Figures 8 and 9. 


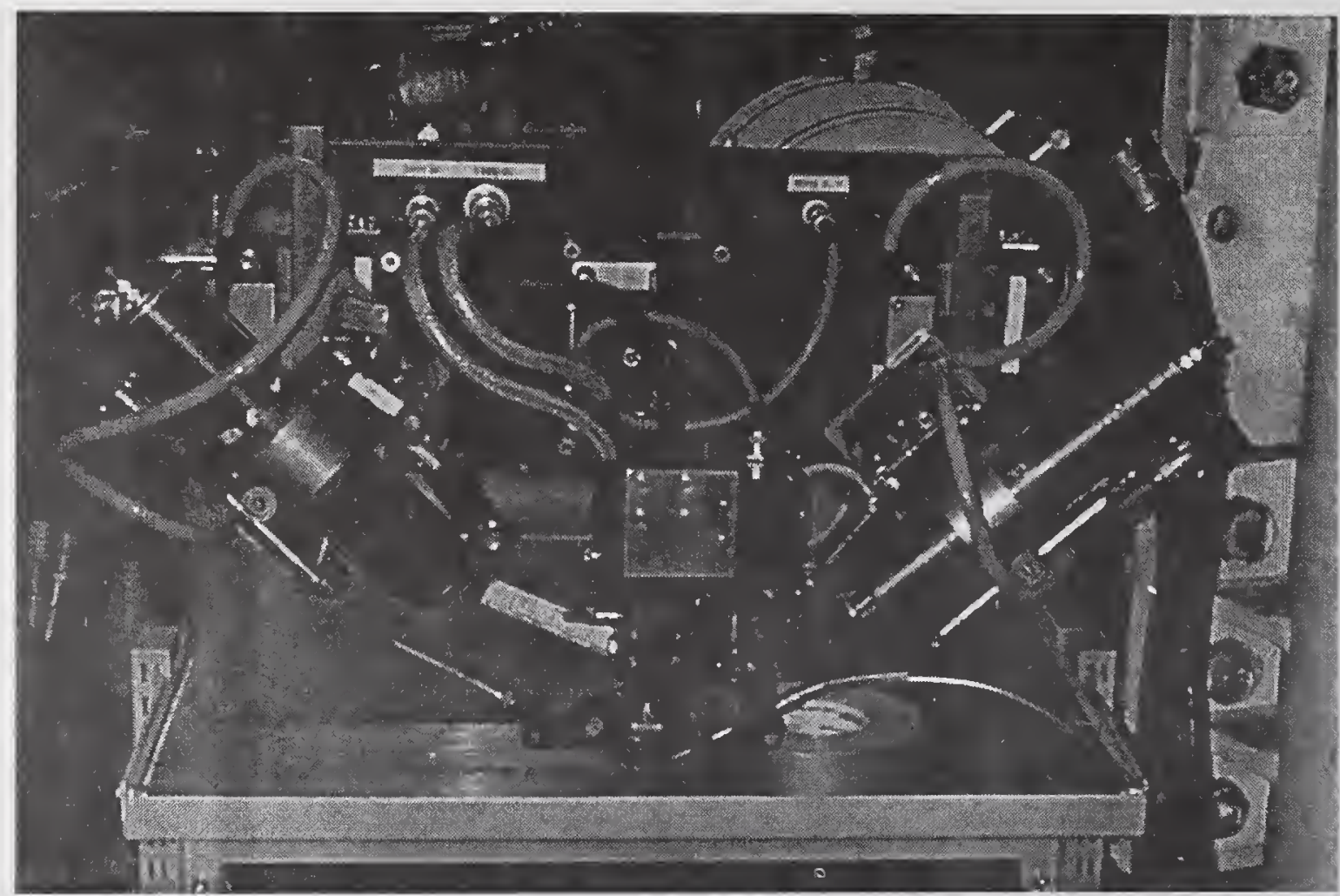

Figure 8. Front view of the weld head.

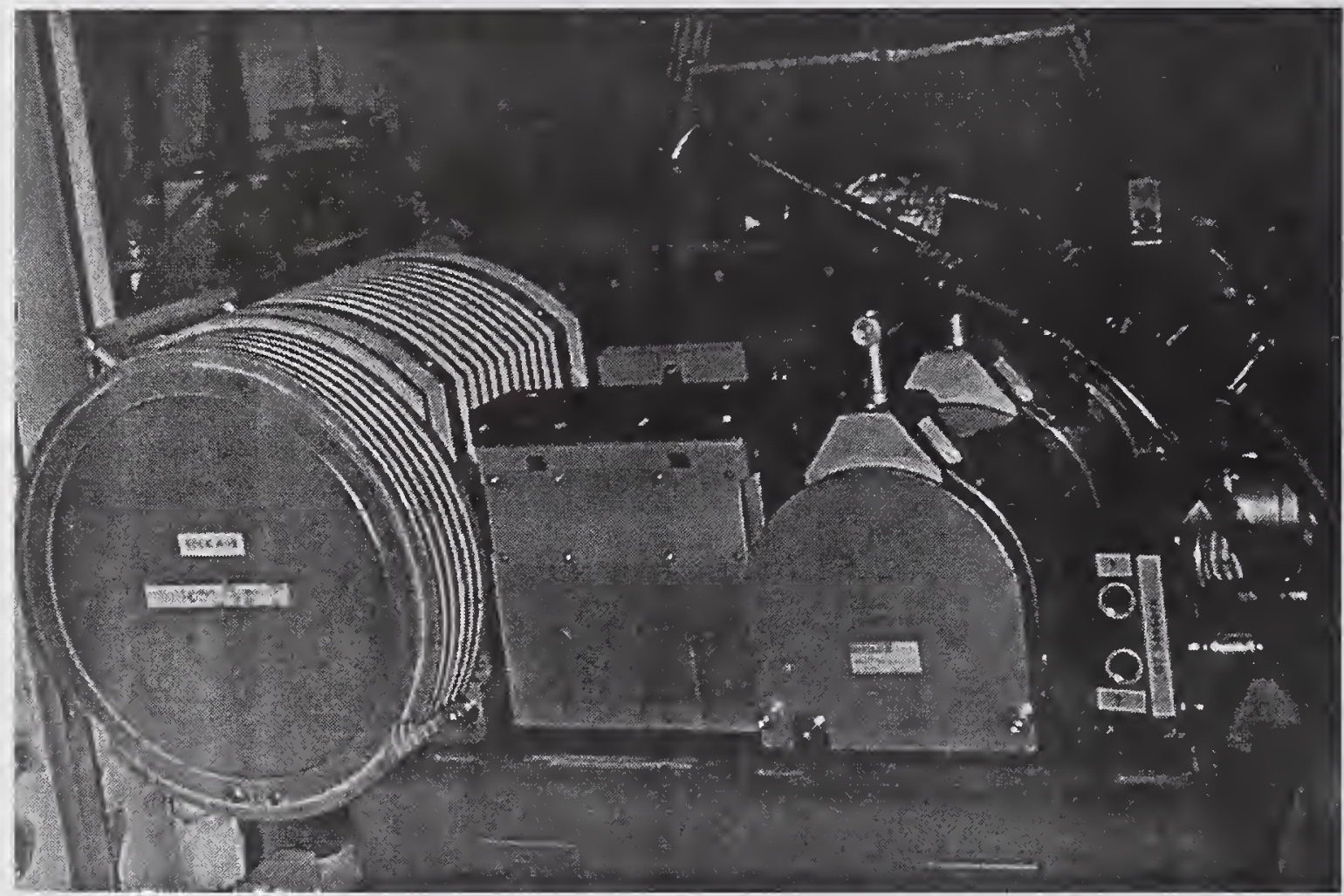

Figure 9. Rear view of the weld head.

Before welding, demagnetising coils are installed. These are mounted on each side of the welding groove, such that the surface operator can minimise the magnetism in the groove prior to welding. Magnetism will cause arc deflections which need to be carefully monitored by the cameras, and the torch and wireguide adjusted to offset any such deflections not taken out by the demagnetising coils. 
Since the atmosphere inside the habitat has a high humidity, preheat coils are also installed as a part of the standard equipment. Normally for mild steels, the temperature is set to $+50^{\circ} \mathrm{C}$ to prevent condensation of water on the pipe. The preheating is also controlled from the weld control system, and temperatures are logged continuously in the computer and displayed on a video overlay. Four thermocouples on the pipe are sending signals back such that the computer is able to switch on/off the power for the preheating induction coils.

At this point welding can start. The welding is controlled from the surface by a qualified operator and a welding engineer. Two weldhead cameras are surveying the weldpool, one in front of the electrode and the other one behind. The camera in front will also allow the operator to see where the wire enters the weld pool. The cameras are linked to a computer on the weldhead, and allow the operator to have a real time control of the aperture, lights, XY movement, tilt and filters.

The welding is controlled via a 3 stage process. The topside operator has overall control via the topside computer. This contains a prequalified database of possible welding parameters for all eventualities (root, filling, capping and repairs) and tables of equipment calibrations for all possible equipment permutations in case of emergency equipment changes on the seabed. From this station the operator selects the welding pass to be performed and sends the information to an automatic weld controller inside the POCO attached to the habitat. Once loaded the operator can initiate the subsea computer to start and stop its automatic sequence which can be over-ridden at any stage by operator intervention via keyboard or joystick panel. The parameters and the changes that can be performed by operator intervention are part of the qualified procedure. The subsea computer then communicates with the weldhead computers to achieve the motions required. At all stages during the process, the status of the equipment is communicated back to the surface and displayed to the operator.

The weldhead and wire feeders are built as modules, such that if a computer unit, camera or power supply should fail, it is easy to replace them. The damaged unit can easily be hooked off, recovered to surface, and be replaced with one of the spare units for the system. During welding tungsten tips have to be replaced frequently, and therefore 15 complete welding torches are available so that a torch can be quickly replaced by the divers. The torches have a quick release from the weldhead torch holder, and a torch-change takes about 2 minutes from the moment the arc is extinguished until it is re-established again.

After weld completion, the weldheads are detached from the IMT faceplate and a remote controlled ultrasonic testing (UT) head installed. This provides on-line quality control of the weld. The UT equipment is remotely rotated around the pipe and corresponding signals interpreted by a computer at the surface. The computer notifies of any unacceptable defects based on pre-programmed levels. 


\section{PHILOSOPHY.}

\subsection{Welding operators and divers.}

One of the key features of the PRS system, is that it aims to reduce the qualification requirements and stress level on welding operators and divers. This is done by extensive use of computers to create equipment that can be operated after a short training period. The welding operators and divers are not required to be skilled welders as procedures are developed and preprogrammed in detail in advance, although a welding engineer is available to manage the task. In the future, it is also planned to remove divers altogether from the operation as the Norwegian oil companies are aiming for reduced diving and avoiding all diving deeper than 180 meters. To fulfil this, further computerisation will be required, thus increasing the current computer count from an already staggering 23 in the system!

\subsection{Welding procedure qualification.}

All machining and welding procedures are developed in detail prior to the offshore project. The developed procedures also include contingency parameters such as hole repairs. The procedure development and qualifications for the PRS are based on scaled laboratory development, and not on manned full scale onshore dives. The PRS laboratory is situated at SINTEF, Trondheim, Norway. The laboratory is equipped with equivalent offshore welding equipment, but all welding is performed in a small (185 litres) hyperbaric chamber, see Figure 10. The torch is fixed inside the chamber, which in turn rotates about the pipe. The chamber is pressurised, with gas equivalent to that used offshore, to a pressure representative of the depth of the offshore weld. In addition, actual samples of the pipeline steel for the particular project are used in the parameter development. The chamber is capable of holding pipes up to a maximum of 12 " outer diameter (OD). Since most of the offshore projects operate with OD's from 28 " - 40", the qualification pipes have to be reformed to a smaller diameter before welding in the laboratory pressure chamber.

There are significant benefits in this approach. The reduced size coupons can be welded quicker and cheaper than full scale mock-ups. Due to the reduced costs and time, many more welds with different groove combinations can be tested before an offshore production procedure is accepted. At the same time experience is gained on the equipment performing the task in a production environment and observations can be fed back to optimise the equipment for the offshore task. Any new modifications can be tested on the laboratory equipment before the modifications are transferred to the offshore equipment. In addition the laboratory equipment acts as emergency spares for the offshore equipment. As the laboratory equipment is in daily use, this is a cheap and efficient way of performing a rigorous quality control on the equipment and operation as the offshore equipment is only likely to be used for a few welds per year. 


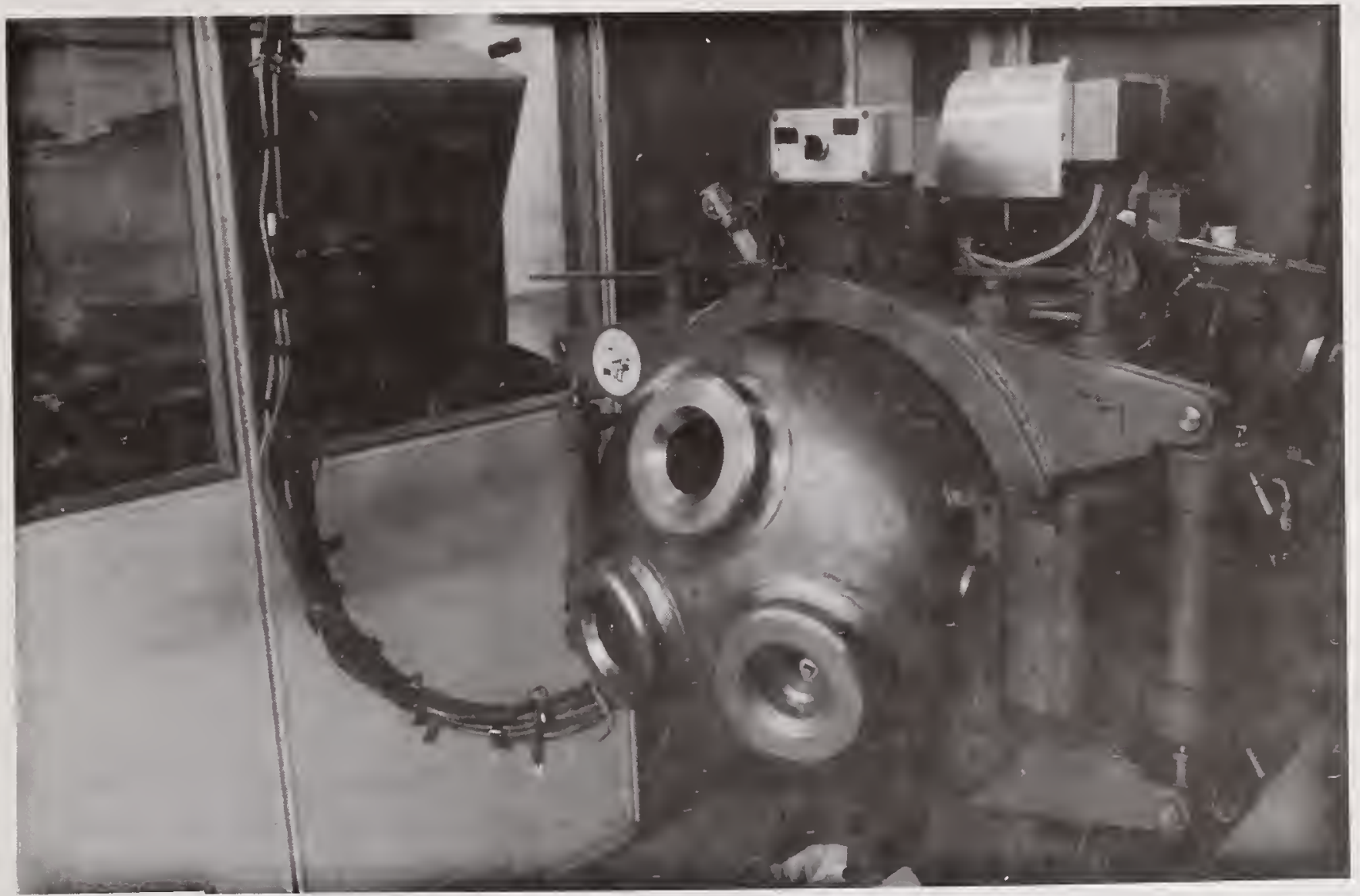

Figure 10. The hyperbaric chamber at SINTEF.

The hyperbaric GTAW procedures are qualified as closed gap procedures, where the accuracy of the alignment and machining is very strict. Closed gap procedures are preferred since it is easier to perform alignment, and magnetism effects are reduced.

\subsection{Future enhancements.}

Today all activities inside the habitat require diver assistance. This will in future restrict the operating depth to 180 meters in Norwegian waters. It also restricts the flexibility of the operation and adds to the cost as divers require saturation support facilities. The current development program is gradually being extended to ultimately eliminate the role of the diver. Future programs are likely to encompass the following:

1. A further reduction of umbilicals within the habitat, increasing the distributed intelligence, and an optimisation of the equipment to make it suitable for remote installation and set-up. 


\title{
IMPLEMENTATION OF COMPUTER SYSTEMS FOR PRODUCTION AND QA/QC IN THE ÖRESUND LINK HIGH BRIDGE PROJECT
}

\section{T. Johnsson ${ }^{1}$}

\begin{abstract}
For the Öresund High Bridge, the main project contractor Karlskronavarvet AB, uses computer systems for quality assurance of production, of inspection of welded structures and of other methods of inspection. The applications are installed on computers at subcontractors and on assembly sites connected with ISDN and modem lines to a central database server. The server stores up-to-date production and inspection data, giving the project management team excellent data material for progress statistics, planning and cost estimates. The full documentation, including summary lists, inspection reports and deviation records can be printed at the end of the project. This documentation is derived from as-built documentation and from the production and inspection data which have been entered in the system.
\end{abstract}

\section{BACKGROUND}

\section{The Öresund Link}

The Öresund Bridge runs between Malmö in southern Sweden and Copenhagen in Denmark. The whole Öresund Link consists of a bridge 4.9 miles long, a 2.5 mile long artificial island, a 2.2 mile long tunnel under sea and an artificial penninsula. The total project is estimated at US\$ 3 billion.

The central bridge section, called Öresund High Bridge, is being built by Karlskronavarvet AB. The High Bridge is approximately 0.9 miles long and has a width of 100 feet. It consists of two levels: the top level holds a 4 lane highway and the lower holds 2 railway tracks. Figure 1 shows the cross section of the high bridge with the highway and the railways. The total weight is 16000 tons and when in place, the high bridge will be approximately 600 feet above the water surface.

\section{The 4X Software A.S. concept}

The requirements for welded structures in the offshore, nuclear and boilermaking industry place big responsibilities on the producer in areas of traceability, testing and documentation. For instance, as-built documentation should contain:

* Welding Procedure Specification (WPS)

* Procedure Qualification Record/Welding Procedure Approval Record (PQR/WPAR) with Test Records

* Production Test Documents

* Non Destructive Desting (NDT) documentation including extent and results (reports if necessary) 
The documentation shall be fully traceable - identifying WPSs, welders, NDT methods, material and weld repairs. 4X Software A.S. has, with its experience and knowledge of the Norwegian offshore industry, long recognized the need for a computer system that would allow producers to fulfill these requirements without consuming too many resources of the organization. For the Öresund High Bridge Project, Karlskronavarvet AB realized the need for a flexible system for QA/QC and together with $4 \mathrm{X}$ Software A.S. enhanced the QMWeld system and developed the XQuality system.

\section{QUALITY REQUIREMENTS}

The contractors of the Öresund High Bridge are required to comply with many national and international standards and requirements set forward in the contract. Among them are EN-288 for Welding Procedures Specification, EN-287 for Welder Certificates, EN-1090 for NDT extents and EN-25817 for Defect Categories to mention the most important ones for welding.

In the Öresund High Bridge Project NDT starts out with $100 \%$ requirement. After presenting documentation that the required quality has been reached and can be maintained, the customer and the contractor decide on a new testing level - usually about $10 \%$ Ultrasonic Testing (UT) and 5\% Magnetic Particle Testing (MT). 100\% Visual Inspection is maintained throughout the whole project. For special joints Lamination Testing is also required.

In addition, all material is inspected by the material supplier and, upon arrival to the contractor, by the contractor himself, and the production is subject to random inspection, for example, that the correct WPS is used and that welders have the correct certificates. All components are subject to thorough dimensional inspection as they are assembled, for example, from a joint to a full railway deck. All parts are painted and are thereafter given a coating and surface inspection.

\section{INFORMATION FLOW}

A traditional and typical pattern for the information flow can be seen in figure 2. Engineering design drawings are sent to production via the Planning department to make detail design "welding drawings". These include weld numbers, weld lengths and extent and type of NDT. They are submitted to the Production department with copies to the welding engineer and Quality Control/Quality Assurance department (QA/QC). The welding engineer applies the applicable WPS, standard procedures and instructions to the welding foreman. On completion of the weld(s), the foreman notifies the QA/QC department; an inspection request is established and the specified NDT is performed. Where welds are found acceptable the report is re-submitted to the QA/QC department for as-built documentation. If, on the other hand, defects are found, a control report is made and a repair request implemented. The documents are, in turn, sent to Planning department for the actual planning and implementation of the works. This sequence of manual reporting and planning often takes from 3 days to a week.

In the following presentation, a more efficient route of implementation by the use of a computer system is presented. 


\section{USE OF COMPUTER SYSTEM}

Karlskronavarvet $\mathrm{AB}$ uses two computer systems in the project: QMWeld for documenting welding activities and welding related inspection activities and XQuality for additional testing and inspection.

\section{Welding activities}

Using the QMWeld system, information for the project is entered by each department. The information flow is shown in figure 3. The Engineering department enters information about each weld: weld number, length, material, NDT category, etc. This data is based on the weld or inspection drawings. When all weld information has been entered and verified by the system, the weld is ready for production (welding).

The weld is then available for shop floor foremen or welders who can obtain listings of welds to be welded - a sort of work order - for each drawing. After the welds have been welded, this work is reported by the foreman or by the welders themselves. Welder IDs, material IDs, WPSs used and production date and time are reported. Figure 4 shows information entered by production. After being reported the welds are available for NDT.

The QA/QC department or the individually-assigned inspector can then obtain NDT requisitions. Each NDT method has its own requisition and contains a list of welds that are available for testing (for instance, taking 48 hours delay into account) with production information, weld length and test extent. After the operator has performed the NDT, he or she reports tested length and test result and signs off, see figure 5. If the test result is not acceptable, an NDT report for the method is automatically generated and the operator fills in the test data (see figure 6). The operator may also generate a Non-Conformance Notice (NCN) from which the welding engineer may create a Weld Repair Request (WRR). In the WRR he or she specifies type of defect, corrective actions, new WPS to be used and generates a repair weld which is automatically entered into the system.

\section{$\underline{\text { Additional testing and inspection }}$}

The bridge also involves other tests in which Karlskronavarvet AB uses a computer system. This includes, for example, extensive dimensional testing and coating inspection. To ensure that all inspections needed for the bridge are performed and accepted, this is done through Inspections Plans. The QA/QC department creates:

1) A list of Inspection Plans,

2) Main Inspection Plans (MIP)

3) Detailed Inspection Plans (DIP).

The Inspection Plan List is a document, listing the Inspection Plans that must be performed and from this the MIPs are created. A MIP is a short description of the tests or inspections to be performed and the QA/QC department enters the type of test (for instance material reception, function, marking, or dimensional), test levels (for instance full, random or a per cent level), the responsible department, ref. specifications, ref. drawings and a short description. The MIP should be enough for the customer to approve the Inspection Plans. An example is shown in figure 7. 
From each line in the MIP, the QA/QC department creates a DIP. The DIP holds a more detailed specification of the tests or inspections to be performed or even a short description on how to perform the inspection. The QA/QC Department may also specify whether a report is required and which report type should be used, for example dimensional report, fiber content report, NDT reports or pressure report.

The DIP is then issued and the person on the shop floor performs the test or inspection. He or she then signs off the result of the test - and if required or necessary (for instance if the test was not acceptable) fills in the inspection report and NCN. The DIP through which the users report, is shown in figure 8. When all the tests of the DIP have been performed, the QA/QC department signs off the DIP. This signature is then transferred back to the MIP and the test in the MIP is regarded as OK.

The systems automatically generates inspection numbers to ensure unique references to inspection plans in reports and other related documents. Since each MIP and DIP refers to existing documents the structure and relations between the plans are always correct and up-todate.

\section{QMWELD and XQUALITY}

By using these two computer systems (QMWeld for welding and NDT quality assurance and XQuality for other inspection and testing activities) a correct information flow and work method is ensured. In addition, a separate program for handling WPSes is used (Xweld) that is integrated into the application.

The QMWeld system also has a module for Welder Certificates in which the company can enter details of all welders and their qualifications to ensure that only welders with the correct qualifications are used.

\section{NETWORK AND EXTERNAL USERS}

The network in use is showed in figure 9. The main server is the database server for all applications. It is also used as application server in the internal network. It runs an Oracle database and has the applications installed. The server holds important data and therefore has disk mirroring and back-up routines.

The suppliers and external sites are connected to this server with modem lines or ISDN lines. They run the application on their own workstations and the modem or ISDN lines are used only to transfer data from the application to the database and vice verse. This solution thus limits the load on the lines. Since all parties have access to the central server they can share information, for instance all the WPSs used in the project are shared among all parties. 


\section{PROJECT MANAGEMENT}

Since all information is stored in a database on a central server, the information is instantly available throughout the organization when the user has saved his information. All information from production and quality inspection is entered into the database which then holds the asbuilt documentation. This can be viewed in different weld summary lists as part of the day-today project management. Figure 10 shows an example of a graphical presentation for defect levels for used WPSes.

The project management team can also continuously view and follow up the project progress through a set of statistical reports that can summarize production and inspection data: what has been produced/inspected, what remains to be produced/inspected, error levels, etc. This can be viewed from a project level down to drawing/weld level and be checked against WPS, test methods, defect categories etc. to locate critical points and errors in production. An example of a status report is shown in figure 11 .

Through a batch print module, all documentation can be printed as one package. This package then includes weld summary lists with NDT reports, inspection lists and statistics, NCNs and WRRs. This can then be handed over to the client as the product documentation at the end of the project.

\section{BENEFITS}

The main benefit of the system is that the database at all times holds correct and up-to-date data. It also holds all the production and inspection data as it was entered by the welding foremen and the inspectors, in other words the as-built documentation. The system also forces the organization to work systematically and in a well-structured way.

Since the information put into the system is entered by the person who performed the task, for example the UT inspector, these values are certain to be more correct and accurate. Also the user will feel greater responsibility for the data put in. This in turn will increase the accuracy and hence quality of the data.

The savings gained by using these two systems, can be summarized as:

* Less work obtaining information (the system forces certain information to be entered before the next set can be entered, and vice-verse; once information is entered one can be certain previous information is entered in the proper way)

* Reduced inspection requirements (due to better control over inspection activities)

* Reduced certification costs (for example, the production data can also be used to automatically update welder certificates thus saving requalification costs)

* Reduced reparation costs. Savings of 5-10\% have been reported.

* Reduced costs for planning (information about the up-coming tasks as well as centrally captured production/inspection data is in the system). 
* Greatly reduced costs in producing final documentation at the end of the project or at critical milestones, for instance payment milestones. Through the batch print module all necessary information is available for printing at any time.

* Offers management tools for monitoring production and inspection.

* Automatically makes the fabricator comply with ISO-3834 (welding requirements under ISO-9001).

\section{CONCLUSION}

The use of the computer based systems from 4X Software A.S. has allowed Karlskronavarvet AB to comply with the very strict quality requirements defined by the customer in an economic way and to free resources for other more important tasks. For example, the QA/QC Department spends more time on quality control than running around the shop floor collecting and systemizing production and inspection data. It has also given Karlskronavarvet $\mathrm{AB}$ the opportunity to closely monitor and support their subcontractors and on-site teams.

\section{ACKNOWLEDGMENTS}

A special thanks to Karlskronavarvet $\mathrm{AB}$ for the help in providing valuable background material for this article. 


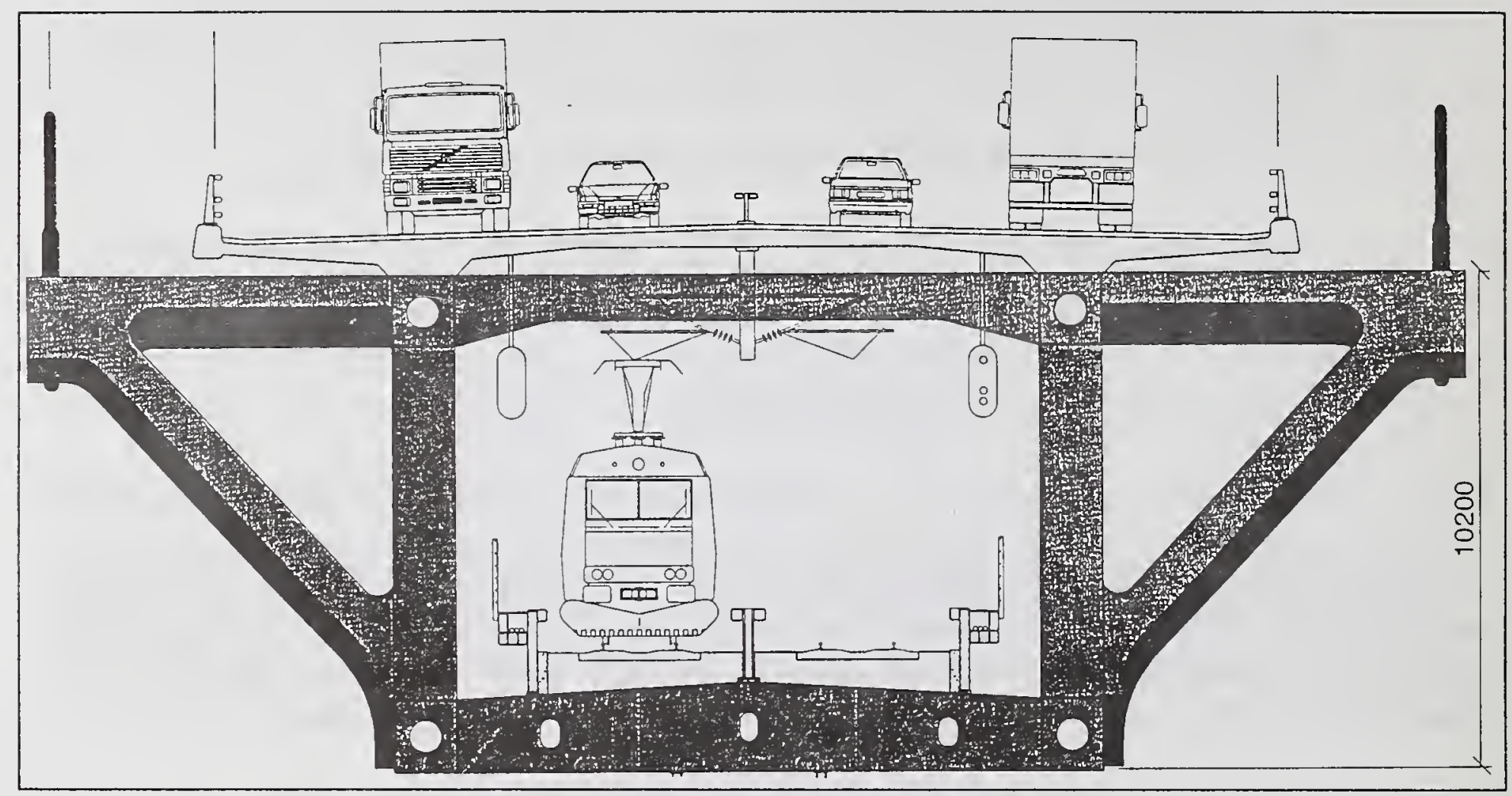

Figure 1: Cross section of the high bridge.

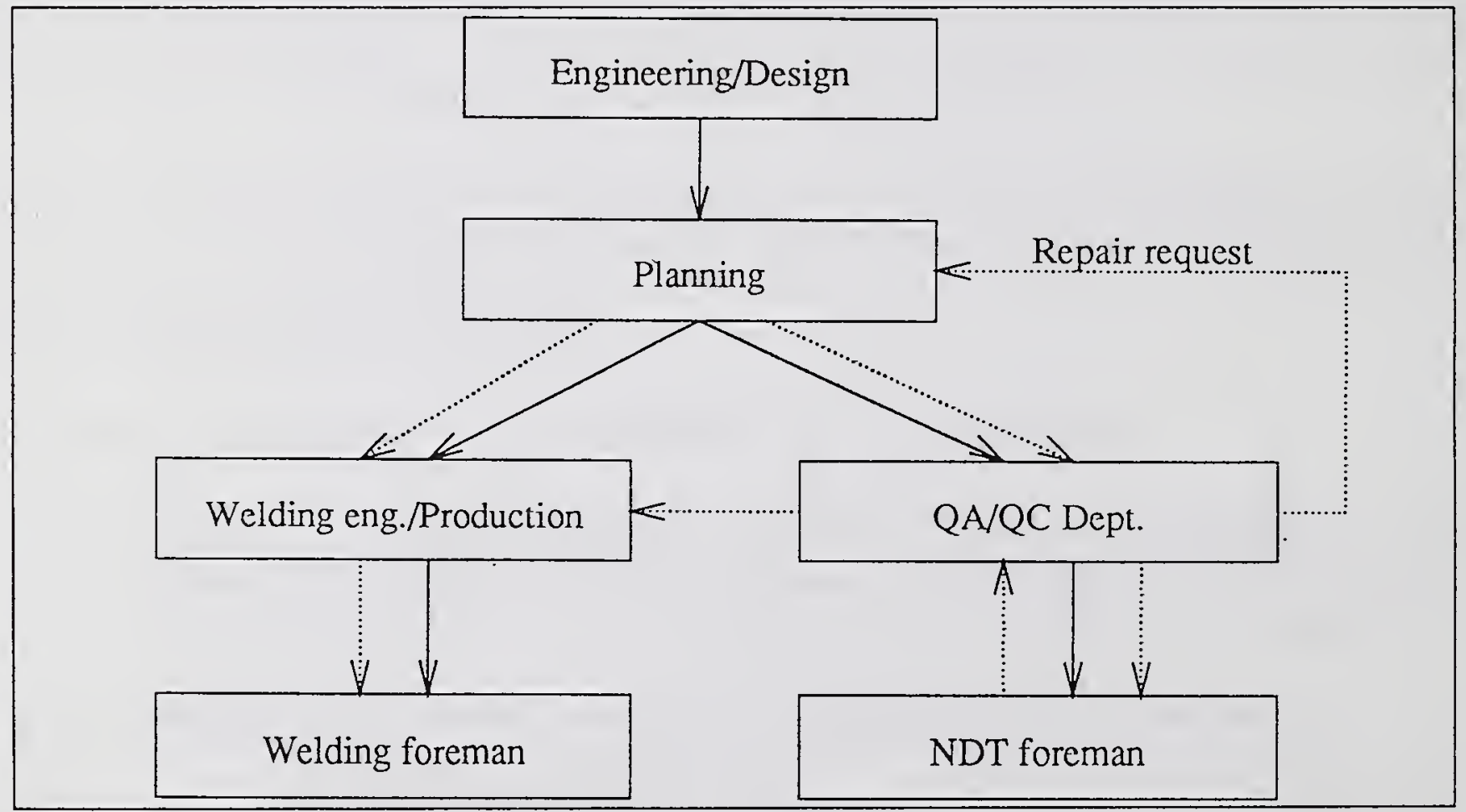

Figure 2: Traditional information flow. 


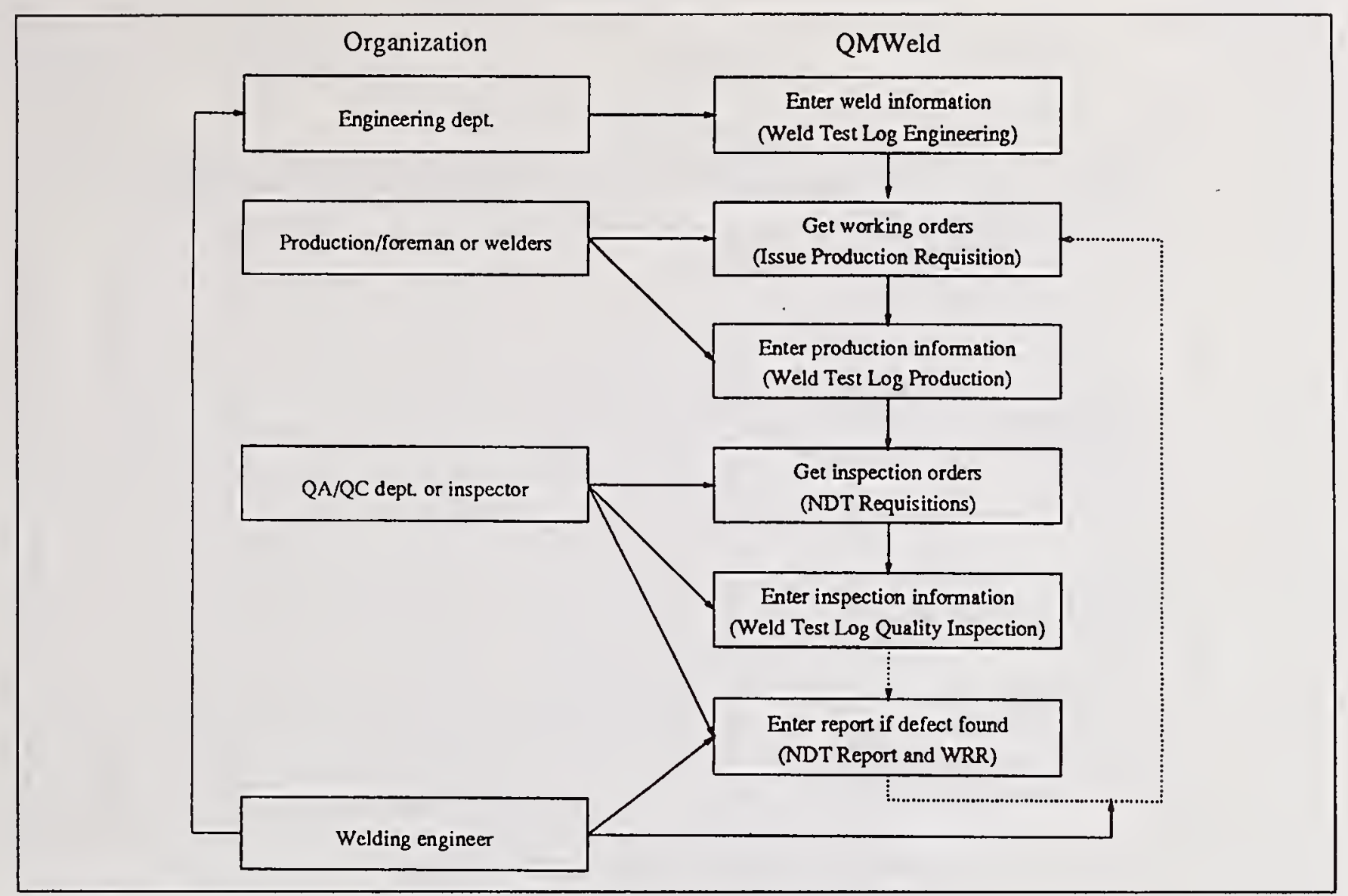

Figure 3: Information flow in the QMWeld system.

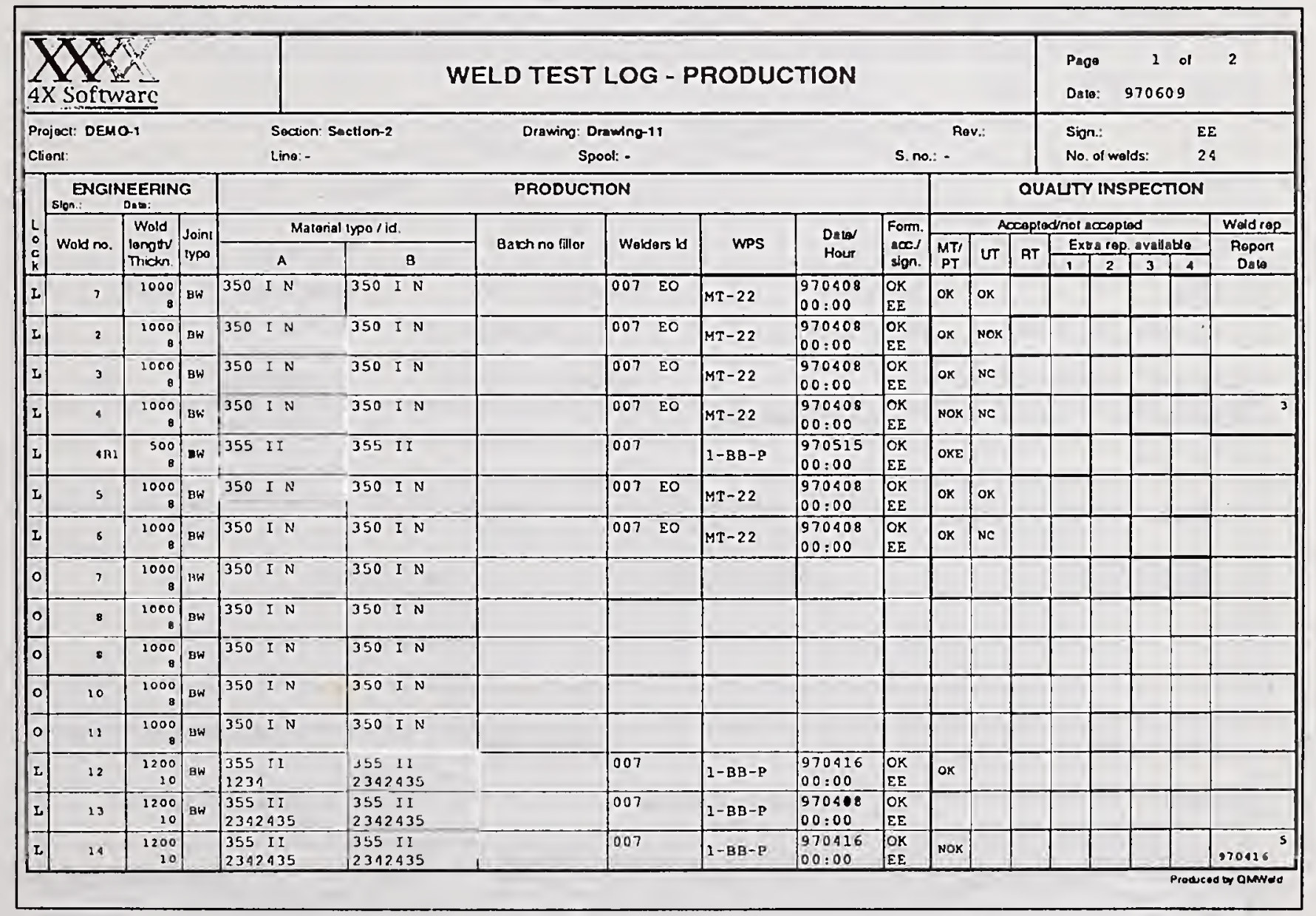

Figure 4: Production information entered into the QMWeld system. 


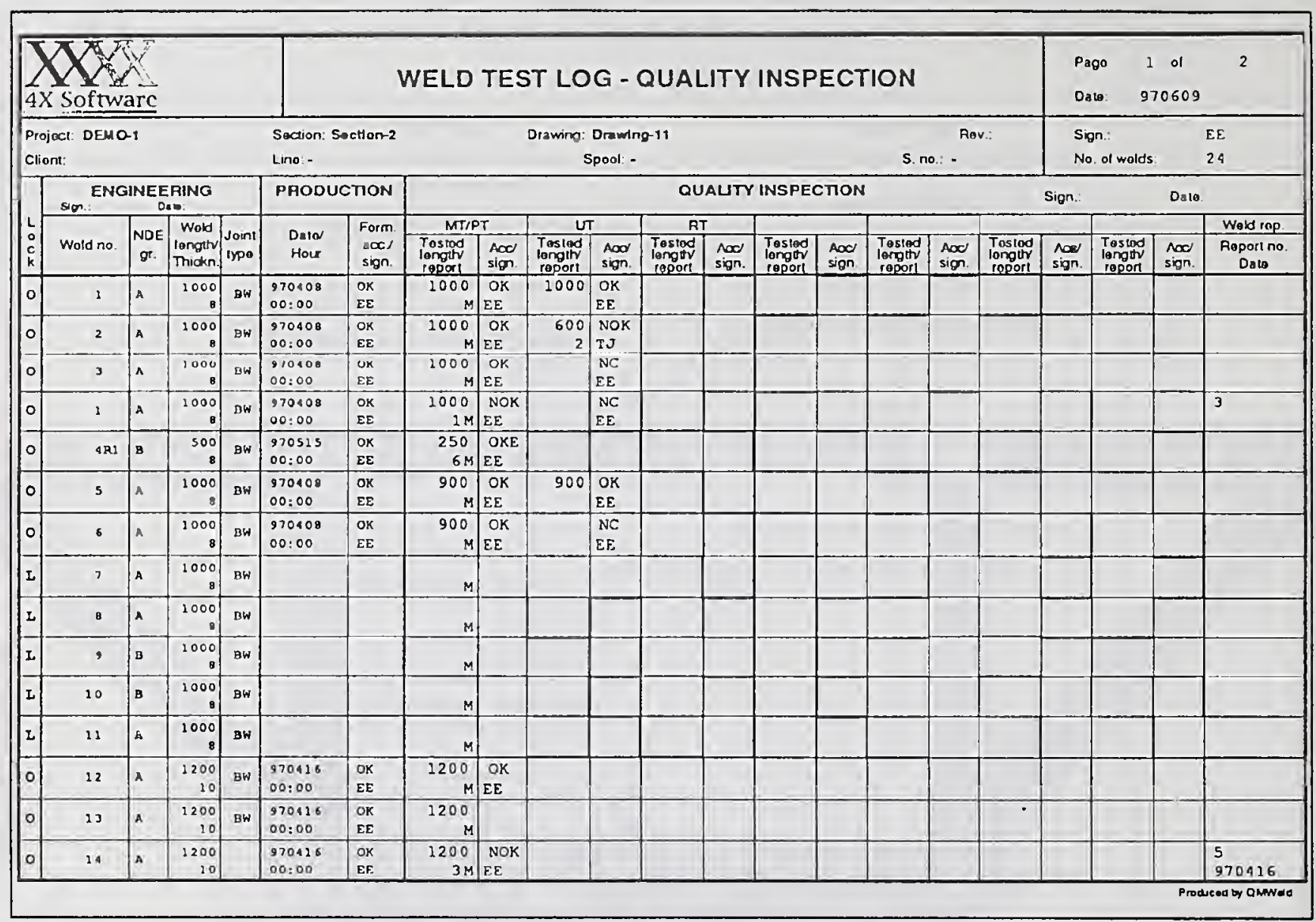

Figure 5: Inspection data entered into the QMWeld system.

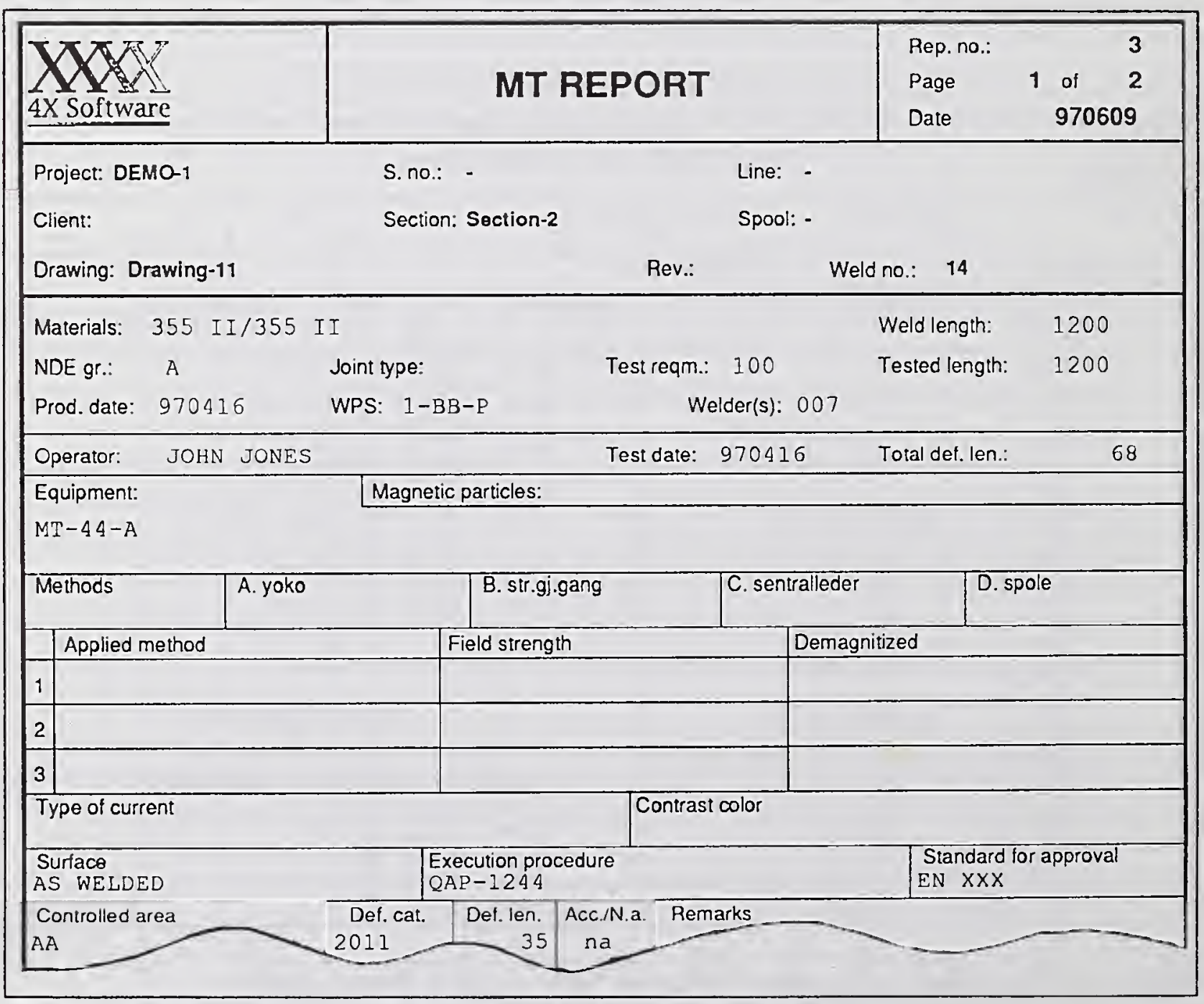

Figure 6: Example of a NDE Report entered by the inspector. 


\begin{tabular}{|c|c|c|c|c|c|c|c|c|c|c|c|}
\hline \multirow{2}{*}{\multicolumn{3}{|c|}{$\begin{array}{l}\text { XXky } \\
\text { Ax Software }\end{array}$}} & \multirow{2}{*}{\multicolumn{2}{|c|}{$\begin{array}{ll}\text { Insp plan: } & \text { B0005-1 } \\
\text { Object: } & \text { DEMO }-1\end{array}$}} & & & \multirow{2}{*}{\multicolumn{2}{|c|}{$\begin{array}{l}\text { Ordor no: } \\
\text { Det } \\
\text { Acivily: Pre-EAT activity }\end{array}$}} & \multirow{2}{*}{$\begin{array}{ll}\text { Prep by: } & \text { EE } \\
\text { Rev: } & 0\end{array}$} & \multirow{2}{*}{\multicolumn{2}{|c|}{$\begin{array}{l}\text { Page: } 1 \text { of } 4 \\
\text { Peudace: } 970410\end{array}$}} \\
\hline & & & & & & & & & & & \\
\hline PBS & \multicolumn{2}{|c|}{ Insp code } & Insp no & Pert by & $\begin{array}{l}\text { Rea } \\
\text { code }\end{array}$ & Specification & Drawing, vald documets) & \multicolumn{2}{|c|}{ Desalpion, method and scope of insp } & Week & Report \\
\hline 0.1 & A & UP & 1001 & oc & 18 & $\mathrm{KA}-650$ & $1-201417$ & \multicolumn{3}{|c|}{$\begin{array}{l}\text { Contar Soction } \\
\text { Verification of activities } \\
\text { which shall be completed and } \\
\text { verifled before EAT }\end{array}$} & $\square$ \\
\hline 02 & & 111 & 1002 & o: & $m$ & $k \wedge-650$ & $1-201417$ & \multicolumn{3}{|c|}{$\begin{array}{l}\text { Mudrate } \\
\text { Veriflcation of activities } \\
\text { which shall be completed and } \\
\text { verifled before } F A T\end{array}$} & $\square$ \\
\hline 03 & & HP & 1003 & Qcc & $1 \mathrm{~B}$ & $K A-650$ & $1-201417$ & \multicolumn{3}{|c|}{$\begin{array}{l}\text { Hoga } \\
\text { Verification of activities } \\
\text { which shall be completed and } \\
\text { verified before EAT }\end{array}$} & $\square$ \\
\hline 04 & & HP & 1004 & oc & $1 B$ & $k n-650$ & $1-201417$ & \multicolumn{2}{|c|}{$\begin{array}{l}\text { Tripla porch } \\
\text { Verification of activities } \\
\text { which shall be completed and } \\
\text { verified before FAT }\end{array}$} & 42 & $\square$ \\
\hline & & & & & & & & & & Prrobures & $1 \times 0$ \\
\hline
\end{tabular}

Figure 7: Main Inspection Plan (MIP).

\begin{tabular}{|c|c|c|c|c|c|c|c|c|c|}
\hline \multirow{3}{*}{\multicolumn{2}{|c|}{$\begin{array}{l}X \times x^{\top} \\
\text { XX Sofuare } \\
\end{array}$}} & \multirow{3}{*}{\multicolumn{2}{|c|}{$\begin{array}{l}\text { Insp plan: } \\
\text { Object: }\end{array}$}} & & \multirow{3}{*}{\multicolumn{2}{|c|}{$\begin{array}{ll}\text { Order no: } & \\
\text { Devact: } & \text { Structural steel } \\
& \text { Pre-FAT activity } \\
& \text { Center Section } \\
\end{array}$}} & \multirow{2}{*}{\multicolumn{2}{|c|}{$\begin{array}{l}\text { Prep by: EE } \\
\text { Rev: } \quad 0\end{array}$}} & \multirow{2}{*}{$\begin{array}{l}\text { Page: } 2 \text { of } 2 \\
\text { Revdaso: } 970410\end{array}$} \\
\hline & & & & & & & & & \\
\hline & & & & & & & \multicolumn{2}{|c|}{ Daw: } & Appr: \\
\hline $\begin{array}{l}\text { PBS } \\
\text { OIC }\end{array}$ & Insp code & Insp no & Pertormed by & Specificaton & Drawing, valid document(s) & Week & Report & Approved & Date. Sign \\
\hline & A & $1001-1$ & oc & $K A-650$ & $1-201417$ & 41 & $\square$ & ox & 970527 EE \\
\hline \multicolumn{10}{|c|}{$\begin{array}{l}\text { Description, mathod and scope of inspection } \\
\text { Structural steel in accordance with } \\
\text { generaI arrangement and detailed } \\
\text { drawings. }\end{array}$} \\
\hline 2 & A & $1001-2$ & Oc & $K A-650$ & $1-201417$ & 41 & 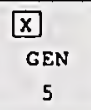 & ok rem & $970527 \quad E E$ \\
\hline \multicolumn{4}{|c|}{$\begin{array}{l}\text { Desciption, method and scope of inspection } \\
\text { Welding completed according to } \\
\text { procedures. }\end{array}$} & $\begin{array}{l}\text { Remarks } \\
\text { Verify that }\end{array}$ & \multicolumn{4}{|c|}{ the procedure survey is enclosed in documentation } & \\
\hline & A & $1001-3$ & oc & $\mathrm{KA}-650$ & $1-201417$ & 41 & $\square$ & & \\
\hline \multicolumn{4}{|c|}{$\begin{array}{l}\text { Descripon. method and scope of inspecton } \\
\text { NDE carried out and documented. }\end{array}$} & $\begin{array}{l}\text { Remarks } \\
\text { verify We Id }\end{array}$ & Sumary List & & & & \\
\hline Inappetdon $P$ & Ten- Daces Reopart & Fonm & & & & & & & Produces of XOun if \\
\hline
\end{tabular}

Figure 8: Detailed Inspection Plan (DIP). 


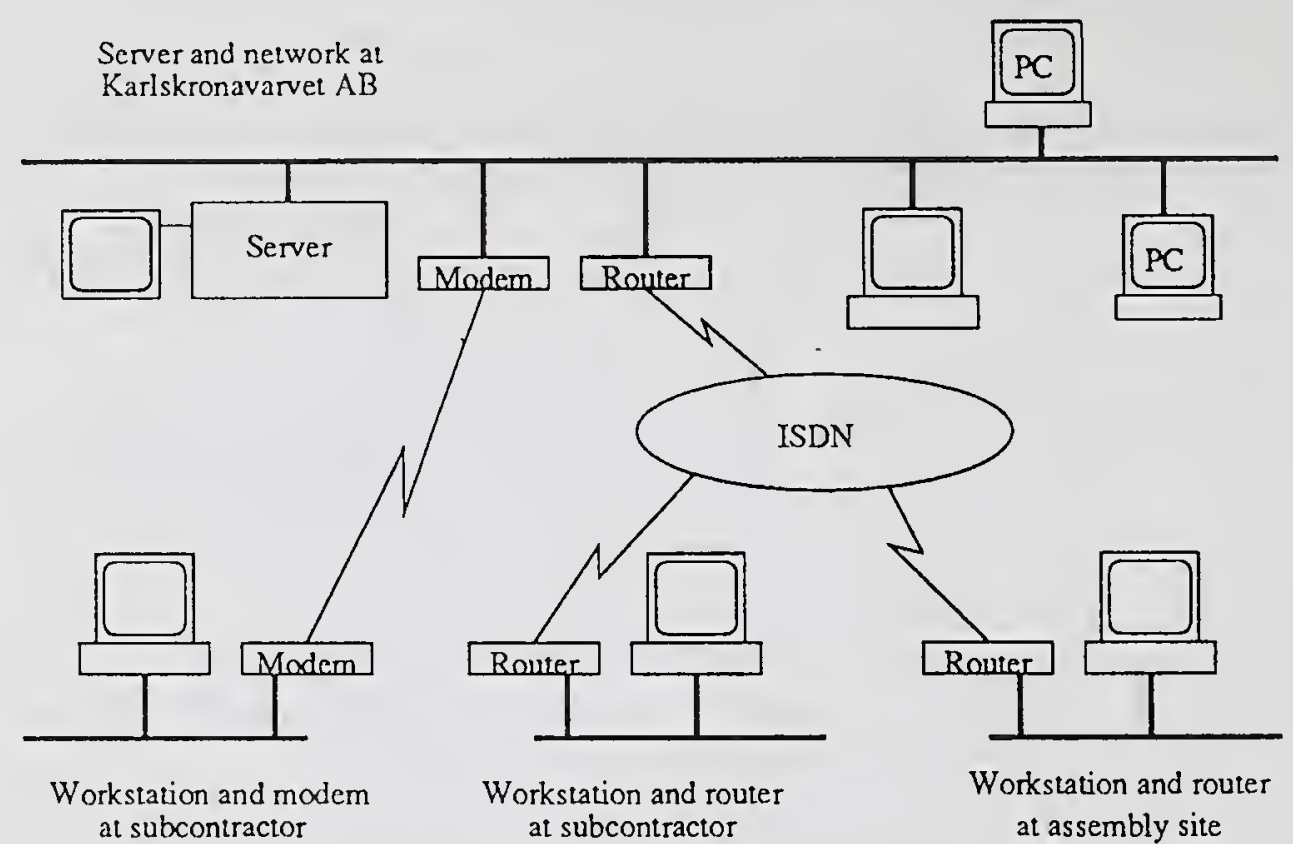

Figure 9: The network used by Karlskronavarvet $A B$ (shop floor and site) and subcontractros.

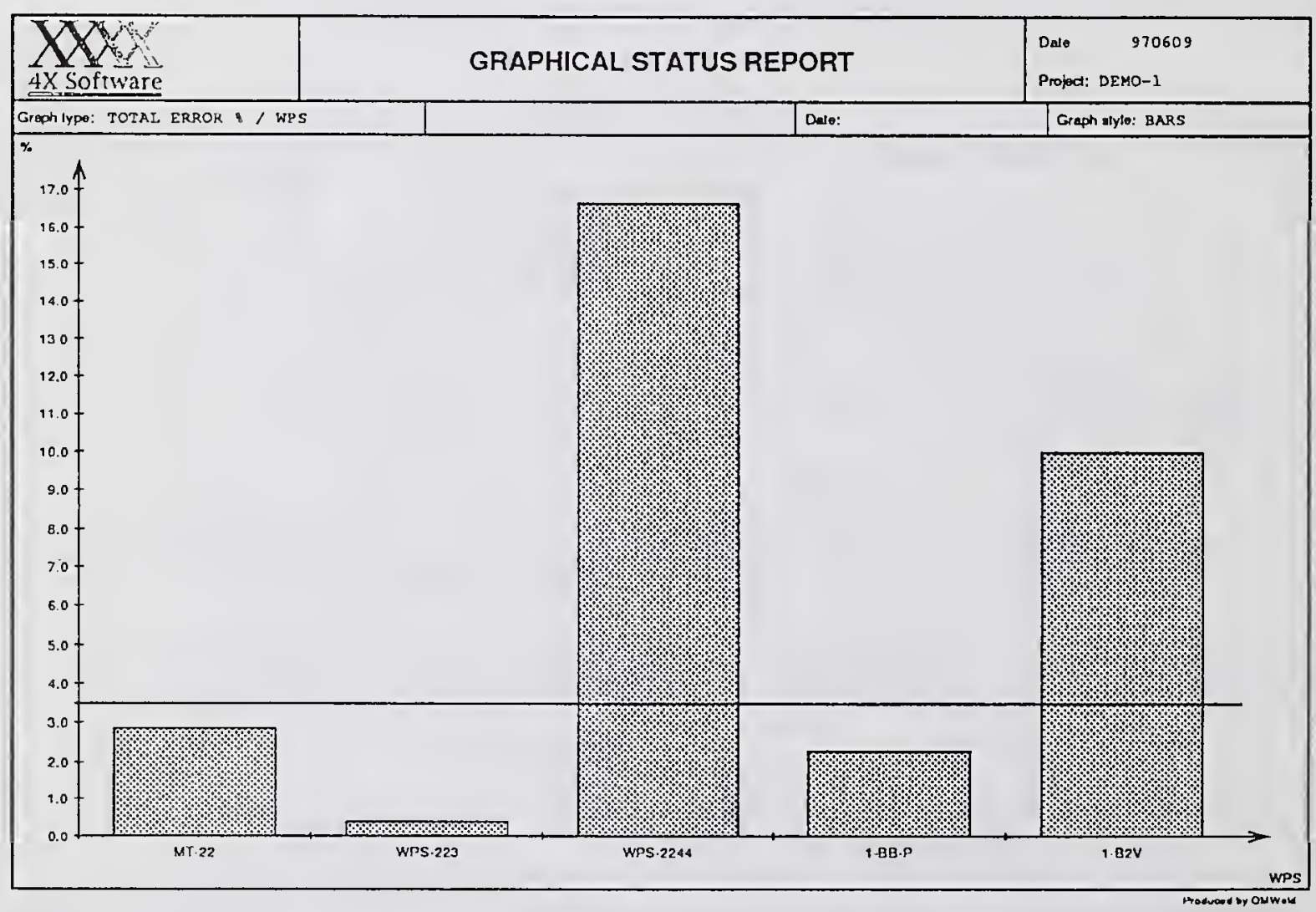

Figure 10: Graphical presentation of error levels. 


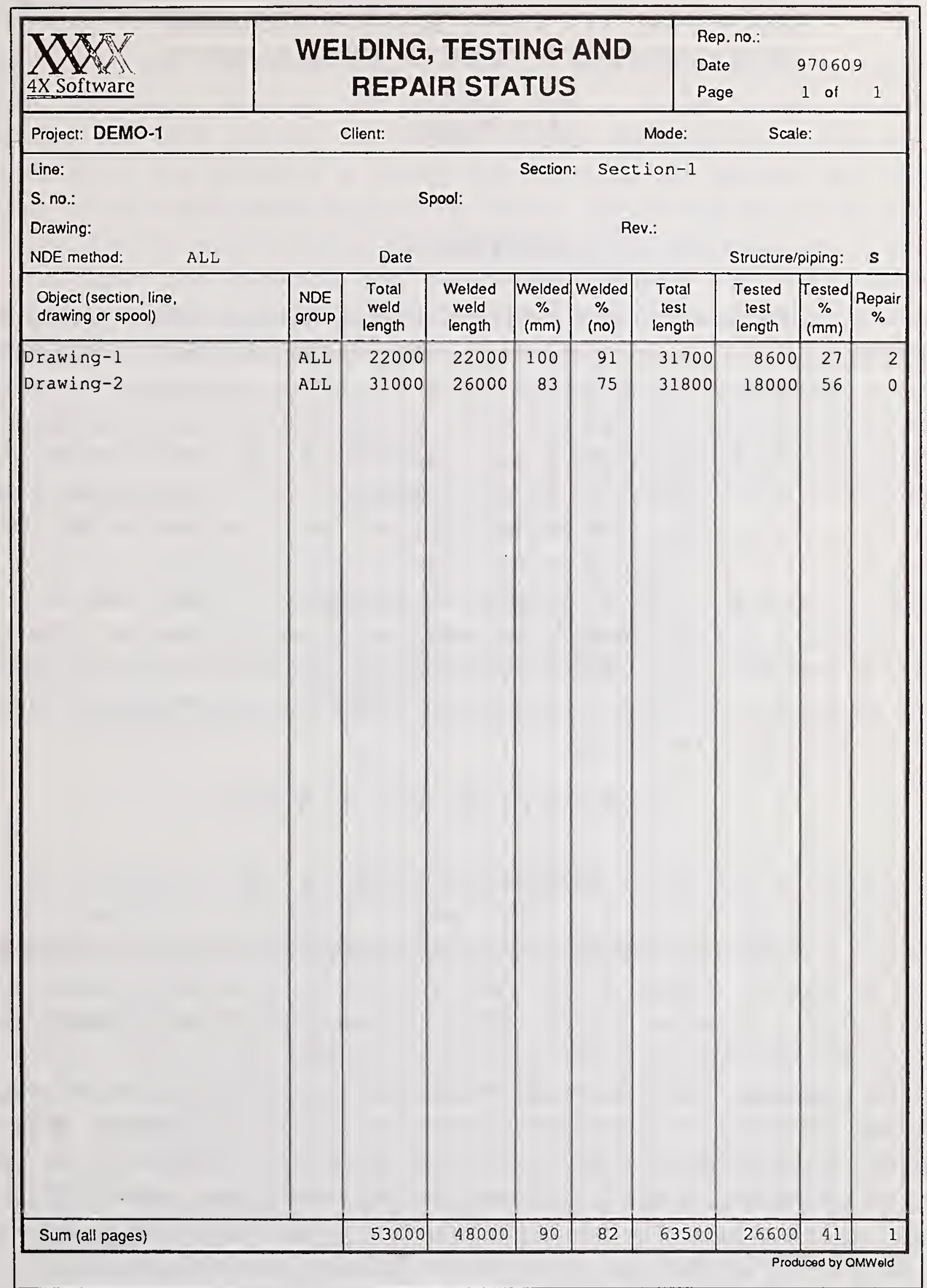

Figure 11: Status report showing total weld length and test length, work progress (per cent) and repair per cent. This shows for all the sections in the project. 


\title{
GAS METAL ARC PENETRATION WELDING DEVELOPMENT UTILIZING NEURAL NETS
}

\author{
J. S. Noruk *
}

\begin{abstract}
Prior to the introduction of a new welding technique into high volume automotive manufacturing, it was important to have a complete understanding of its process capability. Historically, one has had to rely on a multitude of lab trials, or at best a Taguchi style design of experiments. The results of such experiment are normally not sufficient in defining the true process capability or allowing one to properly train people on the intricacies of the new technology. As an alternative, a new robotic arc welding process was developed, proven, and transferred to the factory by using the power of a Neural Network. This allowed the complete process to be computer modeled to within 5\% accuracy of the real world. Response surfaces allow one to pick the most suitable operating conditions that will be resilient to normal real world variation. This visual model demonstrates how the change in welding parameters will affect the actual weld. This visual model helped transfer this new technology to the factory.
\end{abstract}

\section{INTRODUCTION}

The simple operation of the finished neural net belies the detailed work required to produce an accurate realistic model. The work begins by defining exactly what one hopes to have as usable outputs. This is best accomplished in a brainstorming session where one defines all the inputs that can affect the process. Once the inputs are listed, one must select and ensure the extremes of the selected ranges are realistic. In the case of our screening test, we found too many burn throughs occurred, which meant no useful data was produced. In response to this, the ranges were altered such that only a couple of poor welds were created using the extreme variable values. The first draft of the Net revealed fair results, but several areas were found to have too much error. Running more tests in these areas, combined with a study to correlate the inherent process variation, has resulted in a very accurate finished Net.

* Tower Automotive, 3353 N. 27"t Street, Milwaukee, WI 53216 
The success of this Net led us to begin work on four more areas including laser welding, aluminum welding, stir welding, and a design project aimed at automating the FEA process for chassis bracket optimization. This Neural Network effort has provided a strong link between the process and design functions of our Simultaneous Engineering Teams. The Neural Net can be utilized in initial design for manufacturing to ascertain things like allowable travel speeds, total heat input, and allowable part positioning. Finally, the Net is used to establish manufacturing welding procedures and to train the technicians to take advantage of the robustness of the process.

Neural Networks are a vital part of our efforts to improve our Design for Manufacturing skills, thus reducing costs and time to market while improving overall weld quality. Weld modeling using Neural Networks is a necessity in meeting our customer's demand for up front virtual prototyping.

This paper was written to provide a guideline to other companies considering using artificial neural nets to improve their manufacturing process. The methodology followed and the lessons learned are included. This experience was gained developing a welding neural net.

\section{NEURAL NET DEVELOPMENT}

There are five primary steps involved with developing artificial neural nets (ANN) in a manufacturing environment. Figure 1 describes the development sequence and proper check points. The following discussion refers to this figure.

\section{Process Selection Criteria}

The primary reason to develop a net is that there is a fundamental lack of know how. This is especially true when a company is trying to use a new process. The process to be modeled must have a high capability index (Cp) and be under control. The net is especially helpful when nonlinear responses are present. 


\section{Development Sequence}

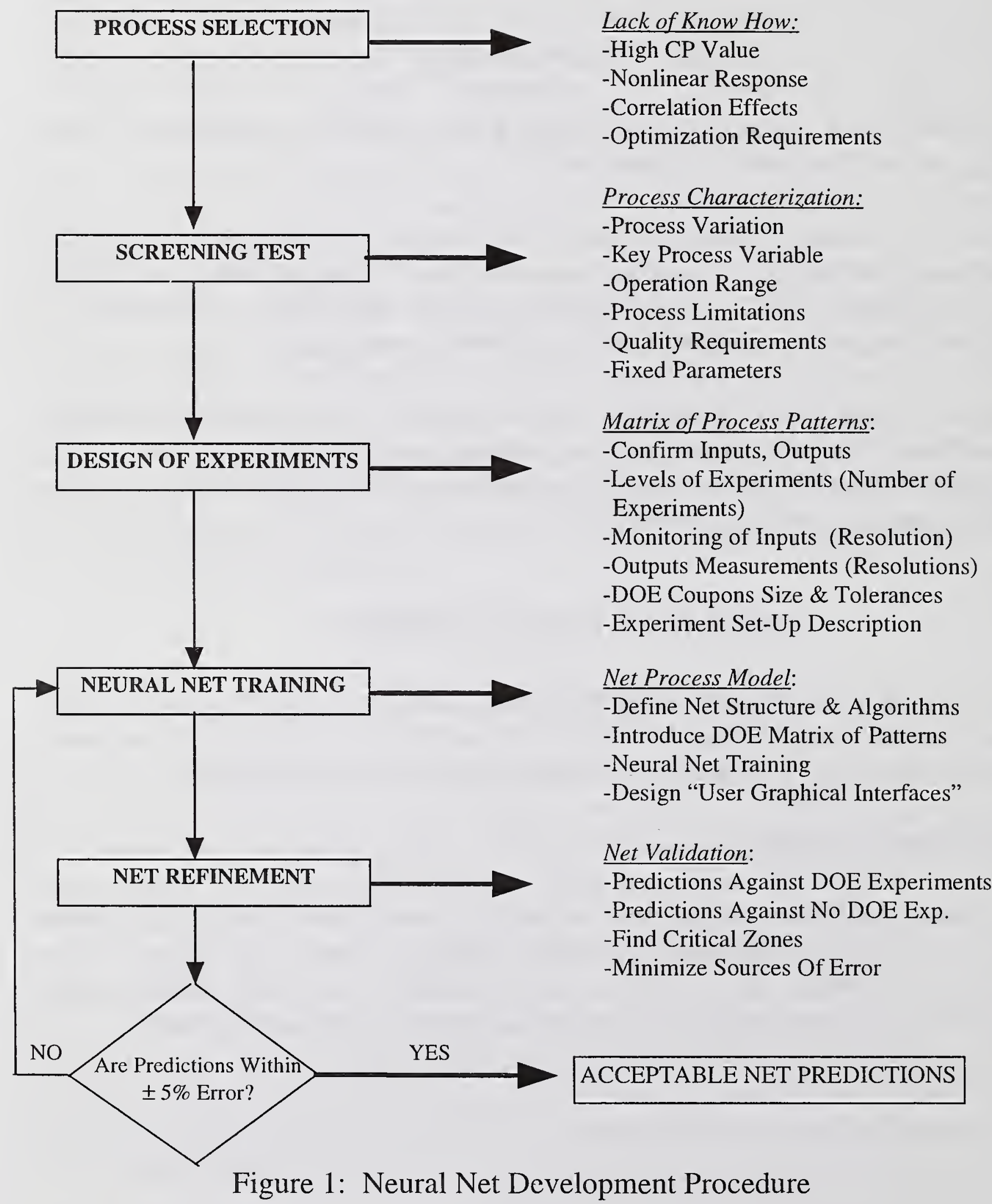




\section{Screening Test $\rightarrow$ Process Characterization}

First one must identify the key process inputs involved. In this net, welding parameters such as current, voltage, and travel speed were important. The acceptable operating range for each parameter and the overall process limitations must be considered in depth early on. To insure a valid DOE, it is recommended that one run the extremes of the parameters to make sure that $90 \%$ of the weld samples will be valid and measurable. In this experiment, a complete lack of fusion or burn-through would represent a wasted sample. Figure 2 details the input parameters and output parameters of this specific neural network.

Other items to be considered at this stage include what to measure on the finished product and which of the input parameters should be fixed. Figure 3 shows a magnified view of a weld cross section and the six output measurements and their tolerances for clarity.

\section{Design of Experiments $\rightarrow$ Matrix of Process Parameters}

Prior to producing the DOE one must do the following:

* Confirm the inputs and outputs

* Determine the levels for each parameters. For example the voltage was $24,26,27$ and $28 \mathrm{~V}$. for this DOE.

* Plan to monitor as many input variables as possible to insure accuracy.

* Insure that the output measurement resolution is appropriate.

* Experiment set-up and the DOE coupon size. 


\section{Process Parameter (Inputs)}

Fixed:

Wire

Machine

: ER70S-3, 1/16" Diameter

Mode

Stickout

: Constant Voltage

: Buried ARC ("Key Hole")

Torch Angle

: $3 / 4 "$

Gas

: Perpendicular, i.e., $90^{\circ}$ off Horizontal

Steel

: $100 \% \mathrm{CO} 2$

: AOS APC Grades 1110 or 1058

Variables:

WPS

Voltage

Part Position

Top Sheet Thickness

Bottom Sheet Thickness

Gap

Travel Speed
: $200,230,250 \& 280$ IPM

: $24,26,27, \& 28 \mathrm{~V}$

: $0 \& 15 \mathrm{Deg}$

: $2 \& 3 \mathrm{~mm}$

: $2,3, \& 4 \mathrm{~mm}$

: $0,030, \& .045 "$

: $50,75 \& 100$ IPM

Quality Measurements (Outputs):

Strength

Penetration

Bottom Width

Fatigue

Undercut

Reentry Angle

Appearance

Top Width

Top Height

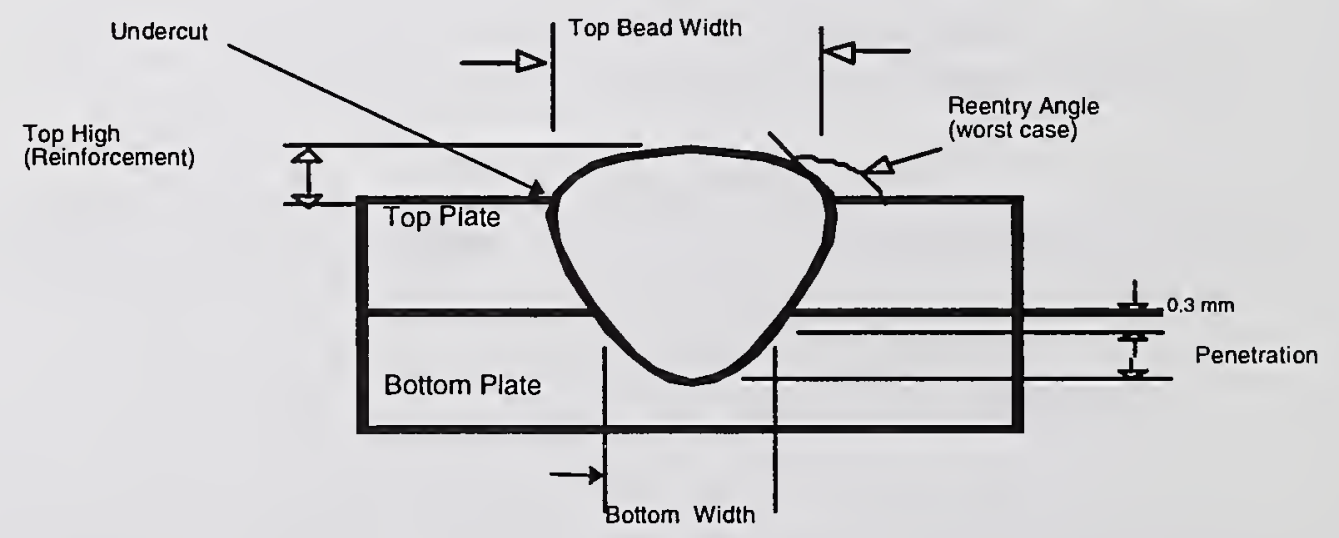

Penetration Weld Cross - Section

Figure 2: GMAW Penetration Welding DOE Information 


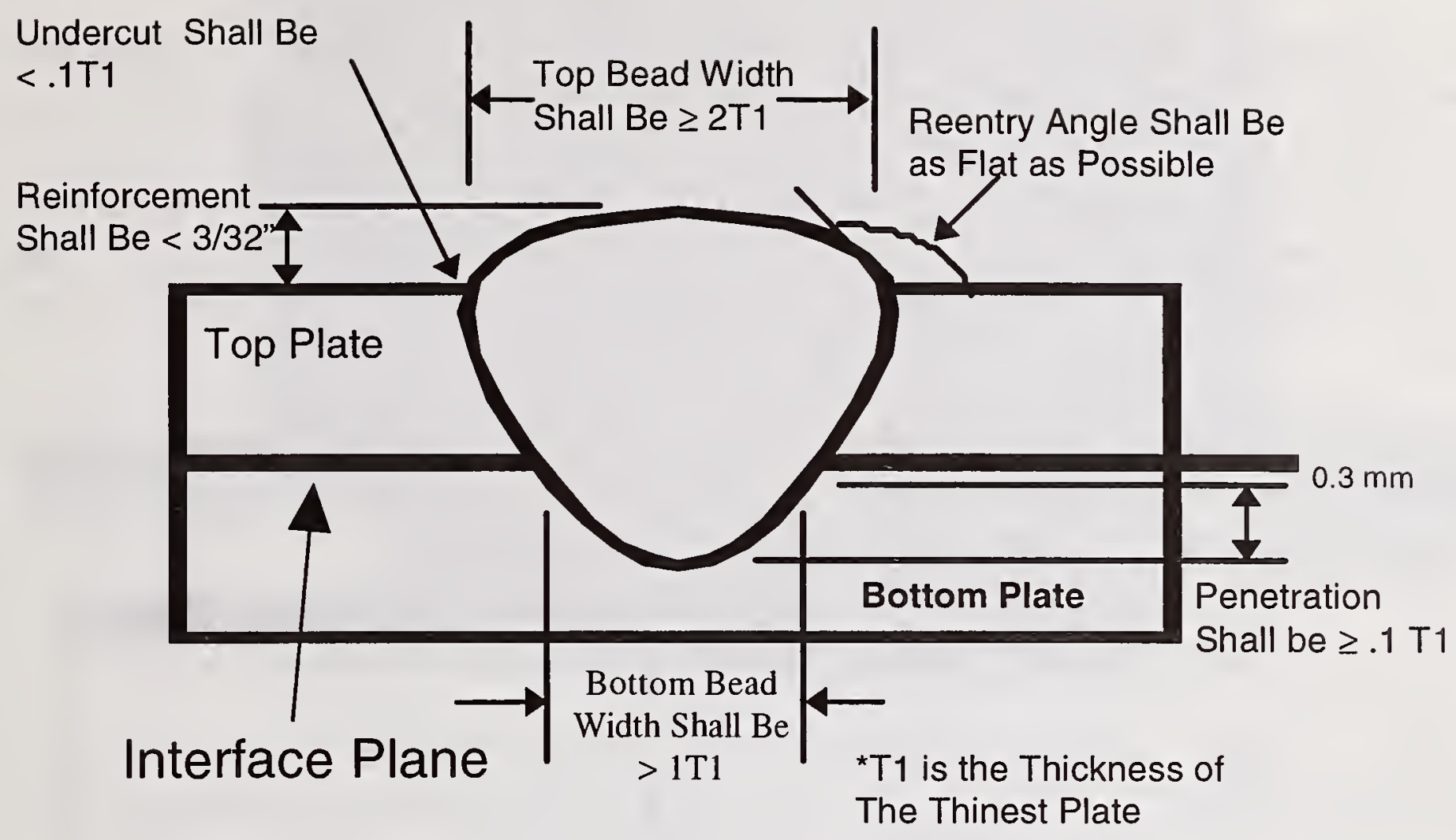

Figure 3: Penetration Weld Cross Section

\section{Neural Net Training $\rightarrow$ Process Modeling}

Once the experiments are done and the results measured it is time to develop the "net". One must define the net structure, introduce the DOE results, and then complete the training. Training of this specific net took several weeks to complete. Another key area at this stage is to design the Graphical User Interface to be easy to use and to provide the most information possible.

\section{Net Refinement $\rightarrow$ Net Validation}

In this final phase the validity of the net is determined. Tests are run to compare predictions against actual DOE tests and then tests are done with arbitrarily selected parameters. During this exercise, areas of large error will be found. To correct for this, further tests are run and retraining is done, until overall average error is less than $\pm 5 \%$. 


\section{SUMMARY}

The resulting neural network software successfully simulated the Gas Metal Arc Penetration welding process and predicted weld bead shape to within the desired five percent. A screen dump of the neural network interface is shown in Figure 4. The software has toggle bars to vary the input parameters on the bottom, and the resulting weld bead shape is shown graphically in the upper right corner. The various output parameters are displayed numerically in the upper left corner of the figure.

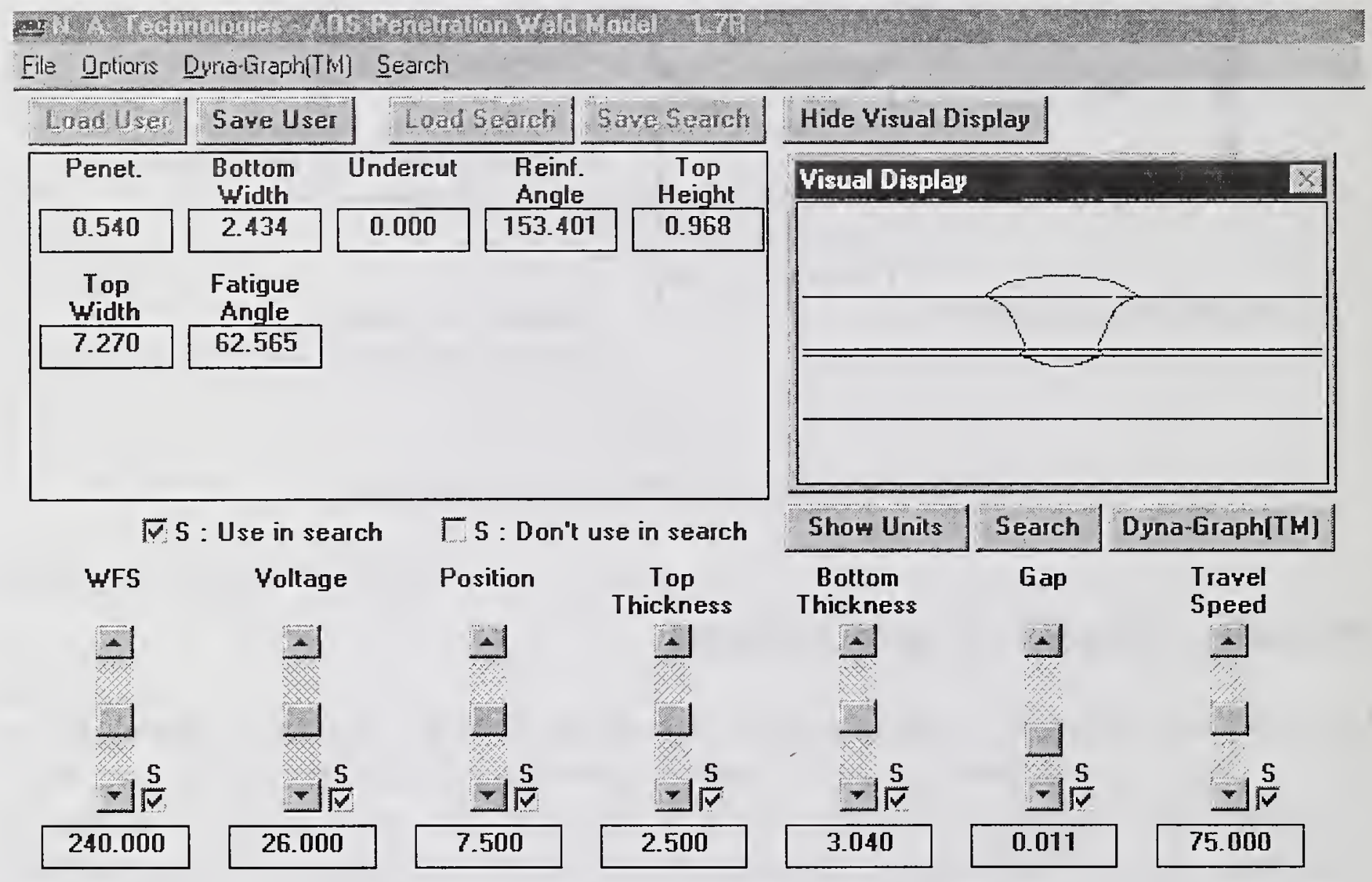

Figure 4: Neural Network Interface

Another output of this neural network software is a response surface. One example is shown in Figure 5, where the penetration depth is plotted against the wire-feed speed and the voltage. For a manufacturing process, it is desirable to have a relatively flat response surface; that is, the desired output varies little with changes in the input parameters. Therefore, typical variations in the manufacturing environment will not cause unacceptable changes in the output variable. 


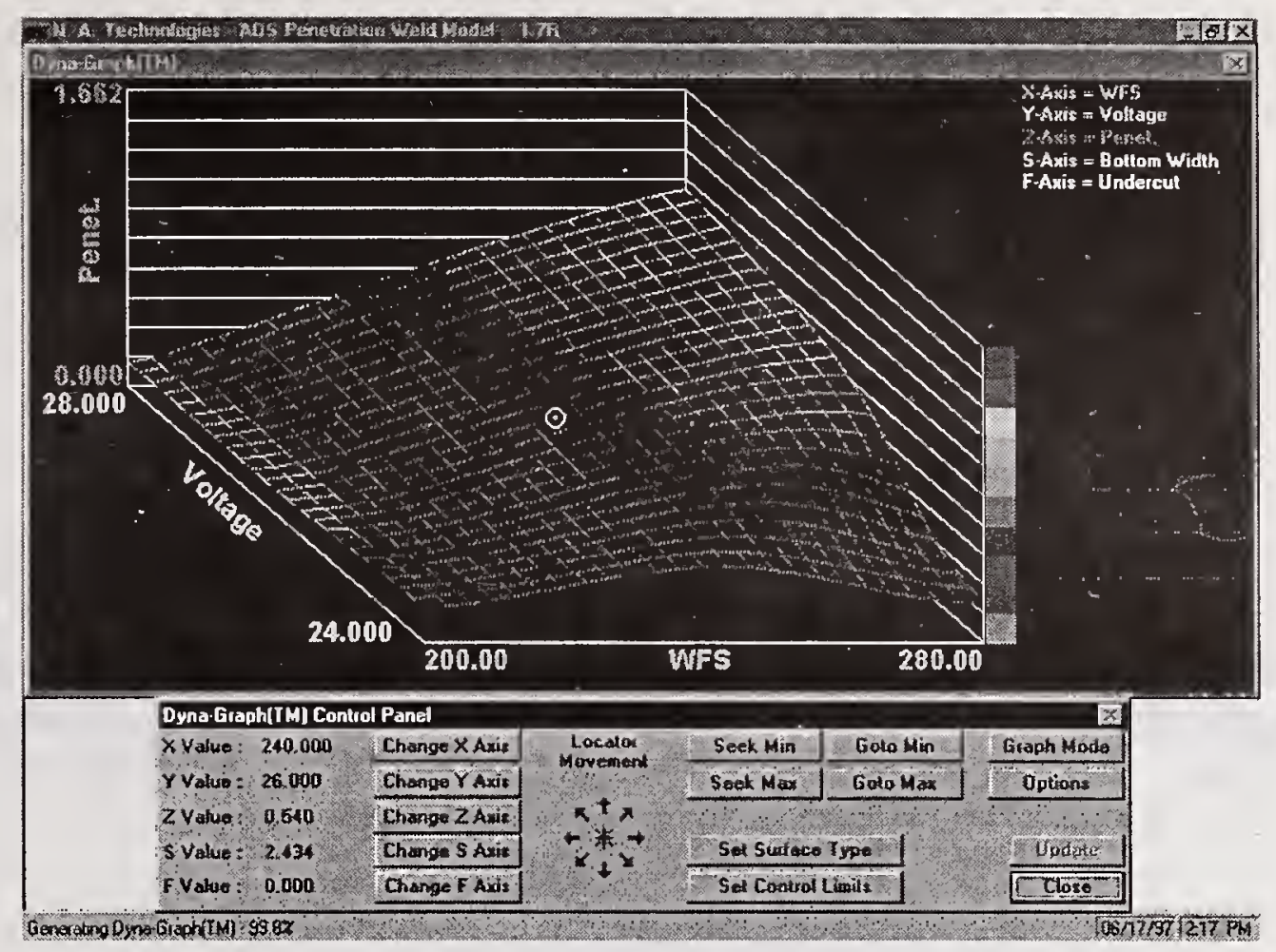

Figure 5: Neural Network Response Surface

\section{CONCLUSIONS}

It was demonstrated that the ANN successfully simulates the Gas Metal Arc (GMA) penetration welding process and predicts with an acceptable accuracy the weld bend shape. Error introduced into the net could be caused by,

1) The standard deviation of the welding process itself,

2) The measurement procedure of the weld bead shape, or

3) The equipment setup conditions and welding procedure.

A list of lessons learned during this work includes:

* Take your time on initial stage of project, i.e. defining objectives of program.

* Limit scope of project so neural net does not span too large of range making the DOE tough to manage.

* Measure and document everything! Keep a running log of all activities. Measure parameters using a weld monitor. 
* Make sure neural net developer understands enough about process to be aware of obvious inconsistencies in data.

* Be patient! Your first net will take longer than you think it should but rushing will insure an unsuccessful effort. Close doesn't count.

\section{REFERENCES}

1. Martinez, A., GMAW Penetration Welding Project Neural Net Development Report \#1475, A. O. Smith Automotive Products Company, Oct. 1, 1996. 


\title{
SPREADSHEET TECHNOLOGY ADVANCES PIPEFITTING TO THE NEXT GENERATION
}

\author{
G.D.Simpson
}

\begin{abstract}
This paper traces the history of pipefitting calculations and shows that the next logical advancement in the trade is to use computer spreadsheet technology to solve piping offsets and layout. Piping offsets, fitting layouts, and pipe intersections are set up in a spreadsheet format with simple prompts for pipefitter input, thus eliminating the need for the pipefitter to apply trigonometric formulas.
\end{abstract}

\section{INTRODUCTION}

Welding technology has made tremendous advancements in the last 15 years, from robotic welders with computer controlled parameters to orbital welders capable of code welds in spaces too tight for a manual weld. As a result, we have experienced productivity and quality levels unimaginable just 20 years ago. As welding intensive industries take advantage of these advancements, so also must the support trades up-grade with computer-enhanced technologies to maximize their productivity and quality. Pipefitting is a prime example.

In the 1970's, before the advent of the pocket scientific calculator, pipefitters calculated offsets by longhand using trigonometric tables found in the back of a pipefitter's handbook. Obviously, this was a tedious and time-consuming task, which resulted in careless errors and untold man-hours of rework.

By the mid 80 's most pipefitters were using scientific calculators for their calculations, referring to their handbooks for formulas and layout dimensions. Certainly this was a time-saving and error reducing advancement.

In the future, pipefitters will be able to solve odd angle rolling offsets to a cut pipe length as quickly as they now solve a simple 90 degree offset. In fact, even the layout dimensions of oddangled fittings, mitered pipe turns, and pipe intersections will be determined instantaneously.

The next generation of pipefitting is at hand. Current computer spreadsheet software brings an elegance to the pipefitting trade never thought possible, and it's hardware [such as a palmtop computer] now fits in the shirt pocket. Even the most difficult offset problems can be solved using iterative solutions. Difficult pipefitting calculations can be performed immediately without errors. Obviously there will be less rework, more productivity, and higher quality due to more exact layout. 


\section{DISCUSSION OF SPREADSHEET APPLICATION FOR PIPEFITTING}

The following spreadsheet application, PIPEPRO(c) 1996-1997 G.Simpson, [Table 1] solves the most common long radius butt-weld piping offsets, layouts, and pipe intersections with a few pipefitter friendly prompts and is compatible with the major spreadsheet programs. The column headings of Pipe Dia., Angle (of fitting or intersection), Set (offset dimension), and Degree (of fitting) serve as prompts for pipefitter input. Cut length, fitting layout, takeout, or angle is instantly displayed, depending on the solver used. Any change to any of the prompts, Pipe Dia., Set, or Angle yields an immediately recalculated cut length for the pipe. Rolling offsets are just as easily handled by entering dimensions for Roll (A) and Roll (B). Mitered pipe turn layout is solved by entering pipe O.D. and cut angle at the prompts. Concentric pipe intersection layout is solved by simply entering Header O.D., Branch I.D. and Angle of intersection.[See corresponding sketches Figures 1- 8]. The traditional offset terms of run and travel and their dimension are shown for reference but are rarely needed by the pipefitter because the spreadsheet has already determined and processed their values in arriving at cut length and advance.

\section{CONCLUSION}

Spreadsheet technology will usher in the next generation of pipefitters. And the palmtop computer now puts the technology in the shirt pocket. In view of the current cost of labor and piping materials, the first error the pipefitter doesn't make as a result of using this new spreadsheet application may easily pay for the cost of the technology. 


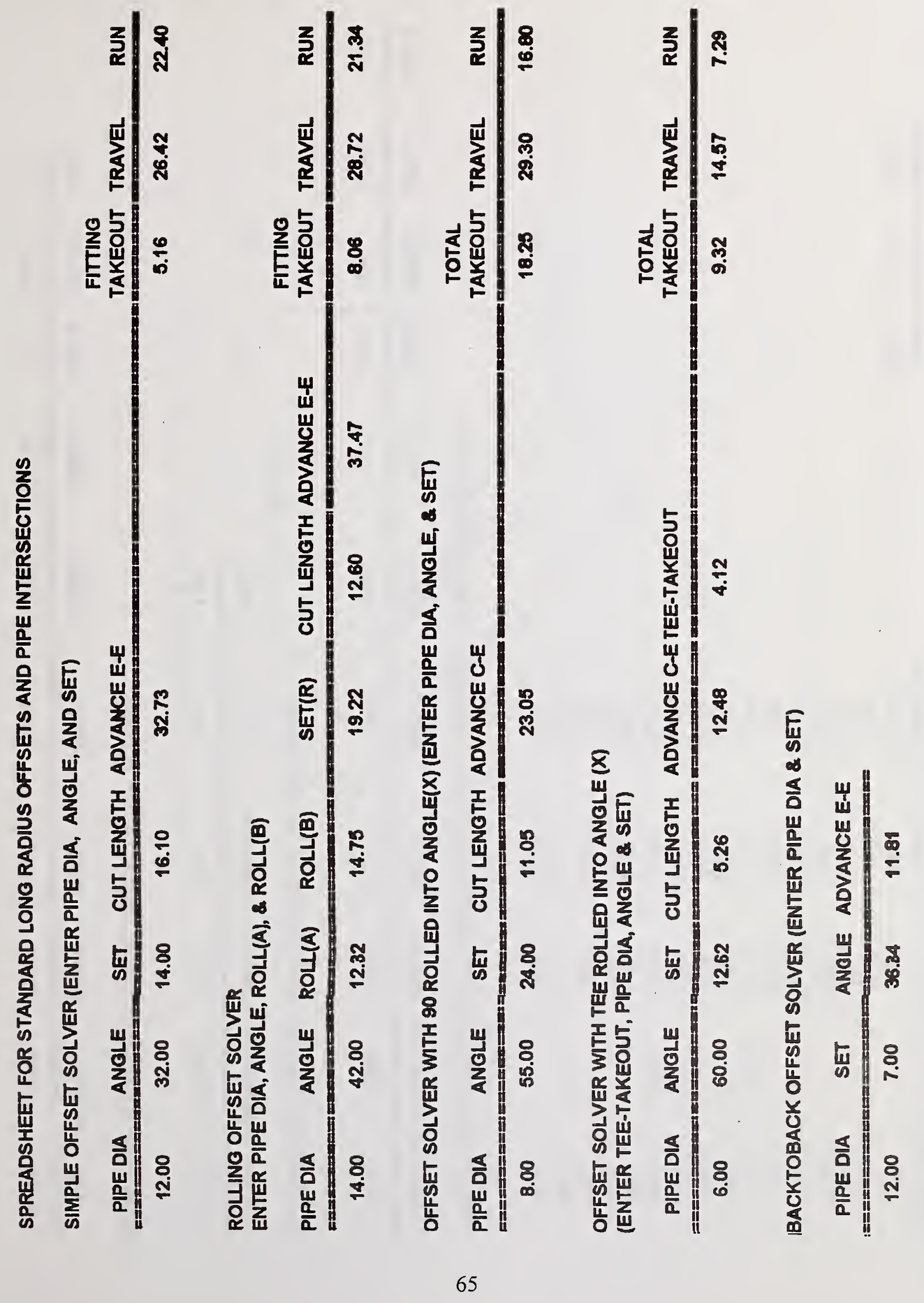



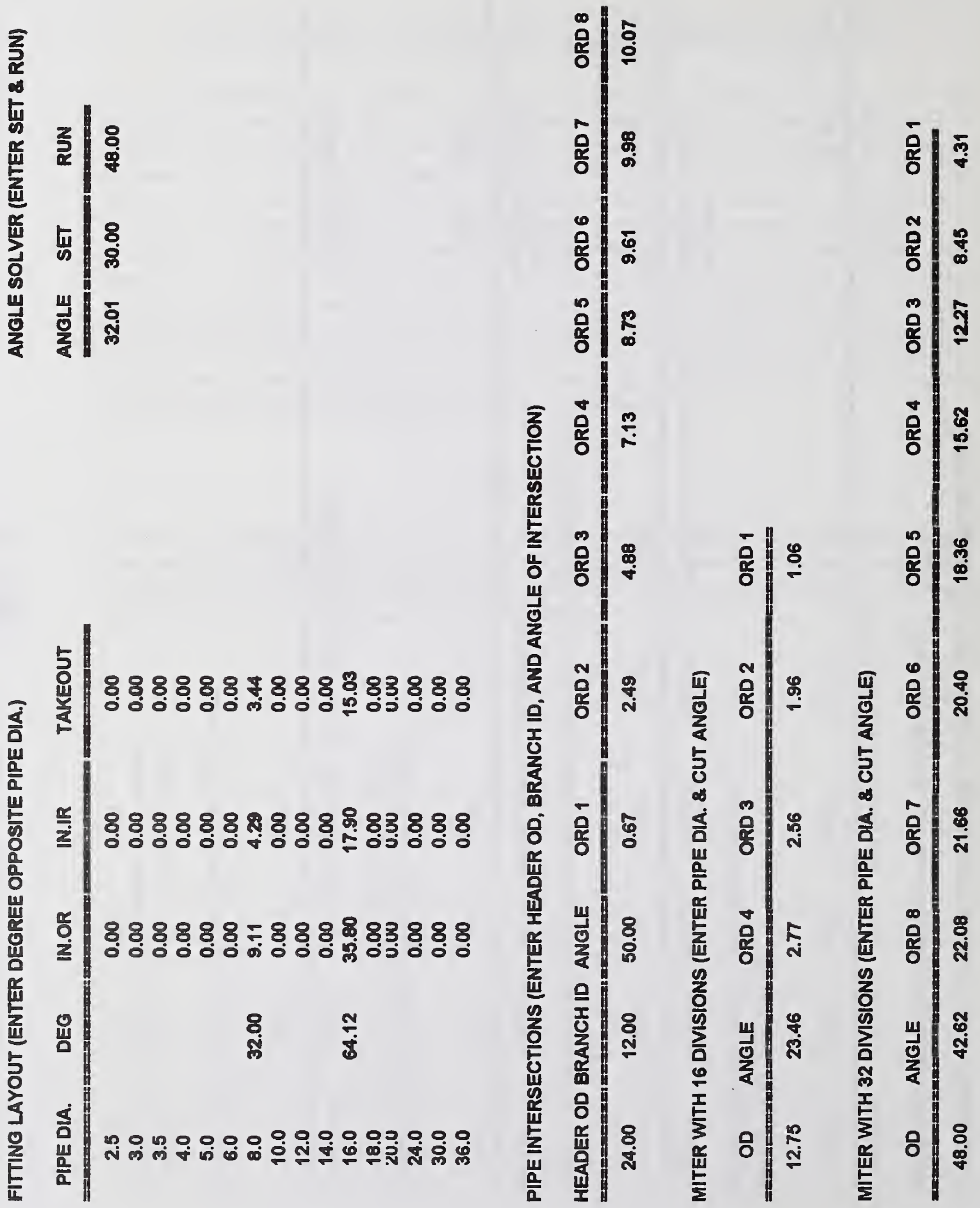


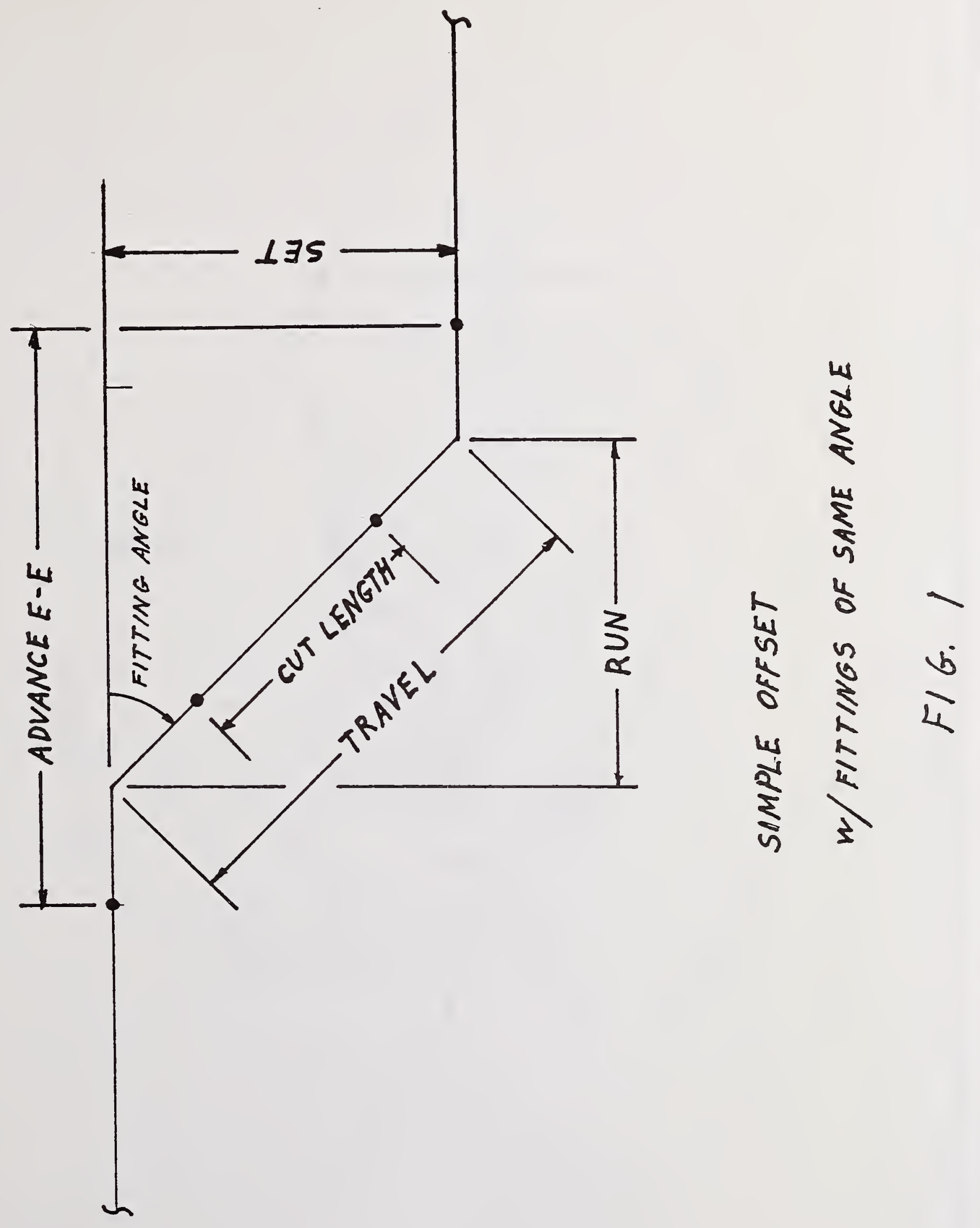




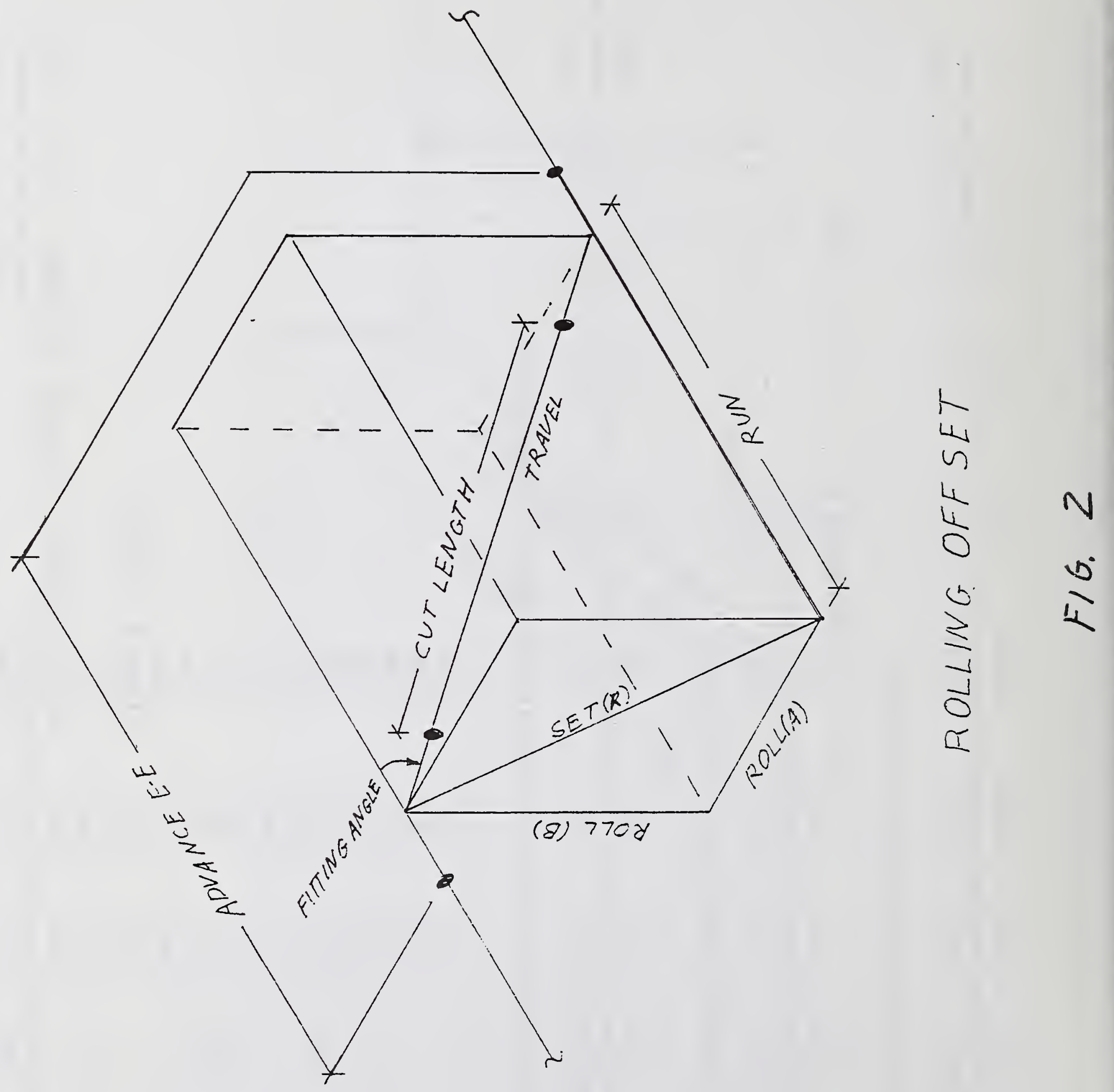




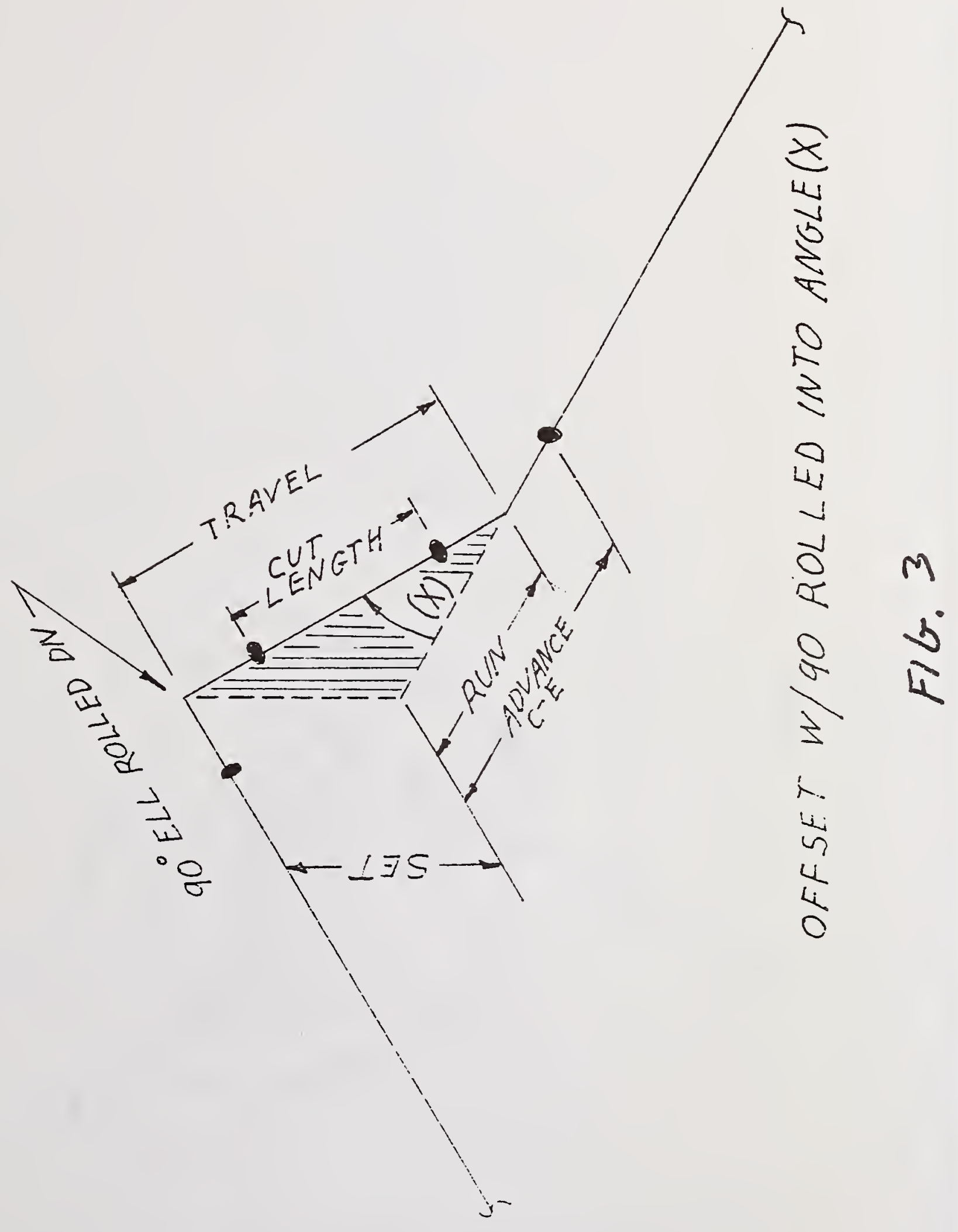




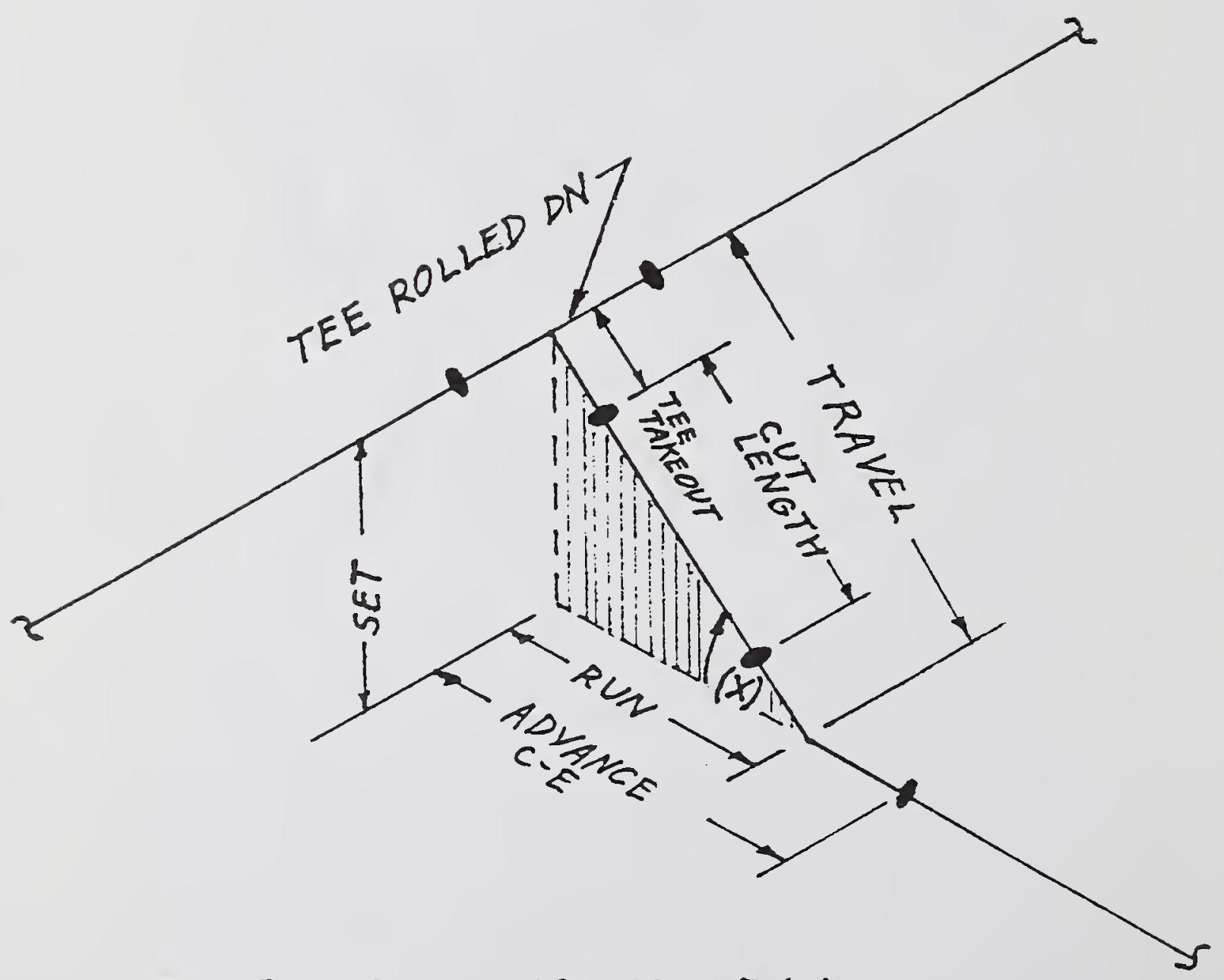

TEE ROLLED INTO ANGLE (X)

FIG. \& 


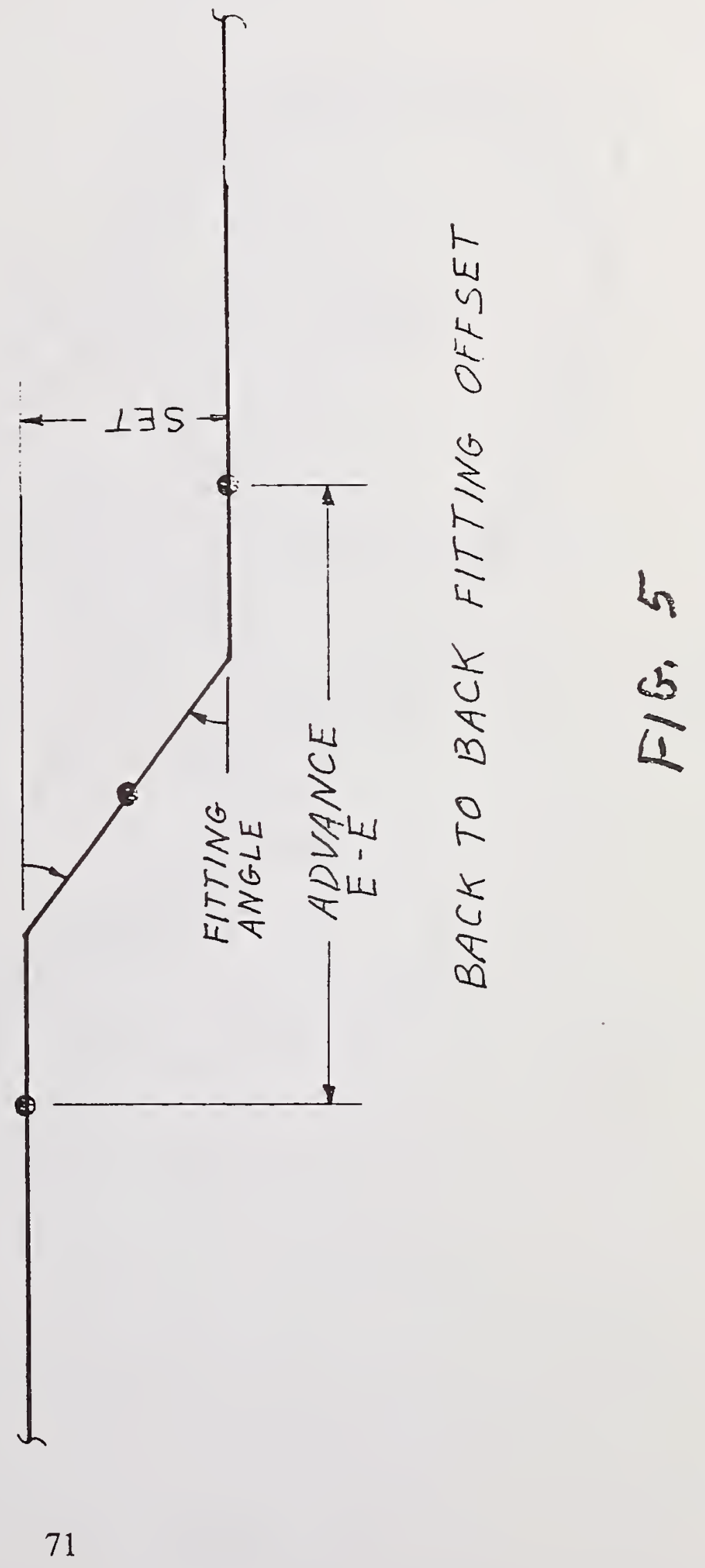




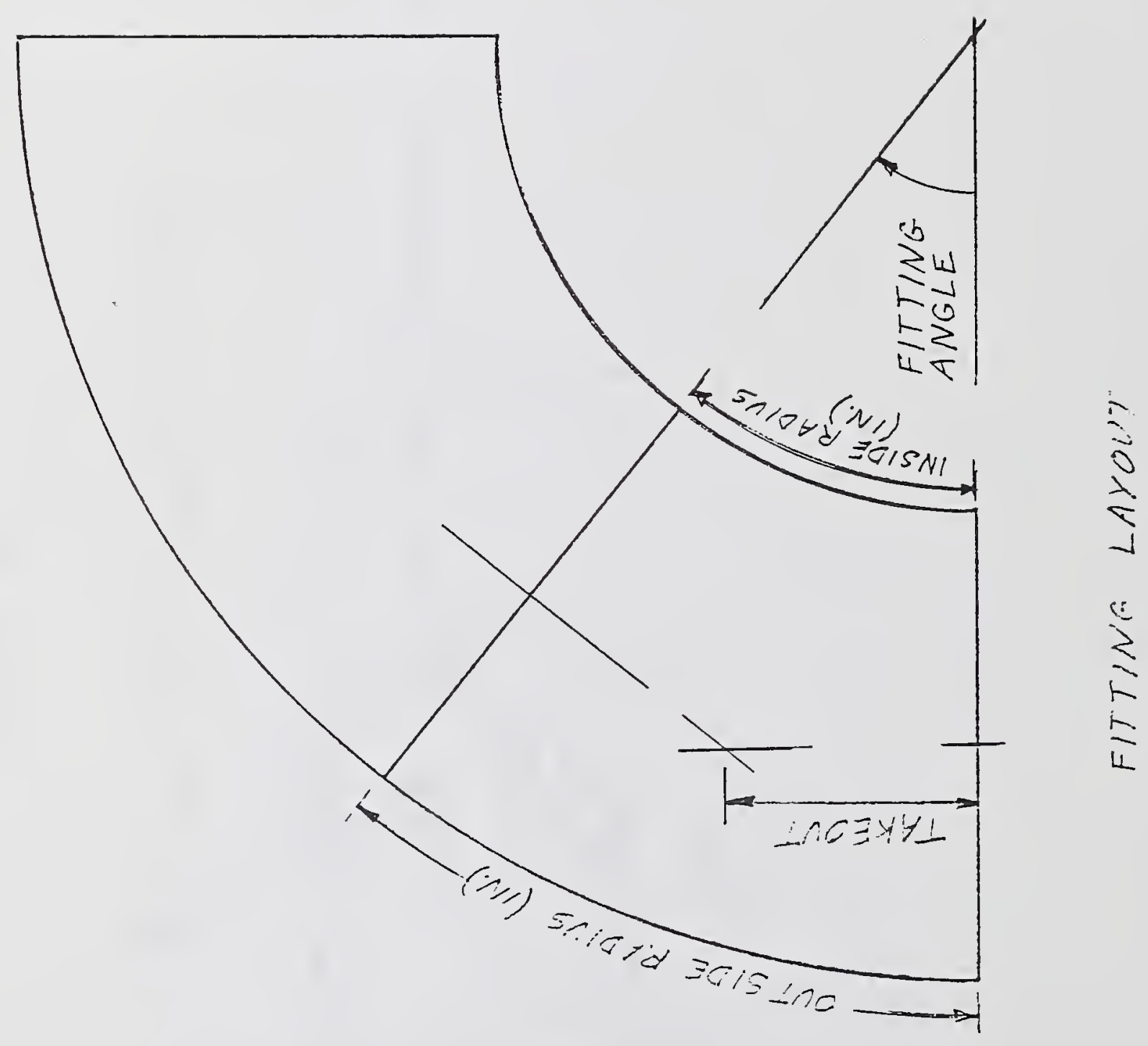

72 


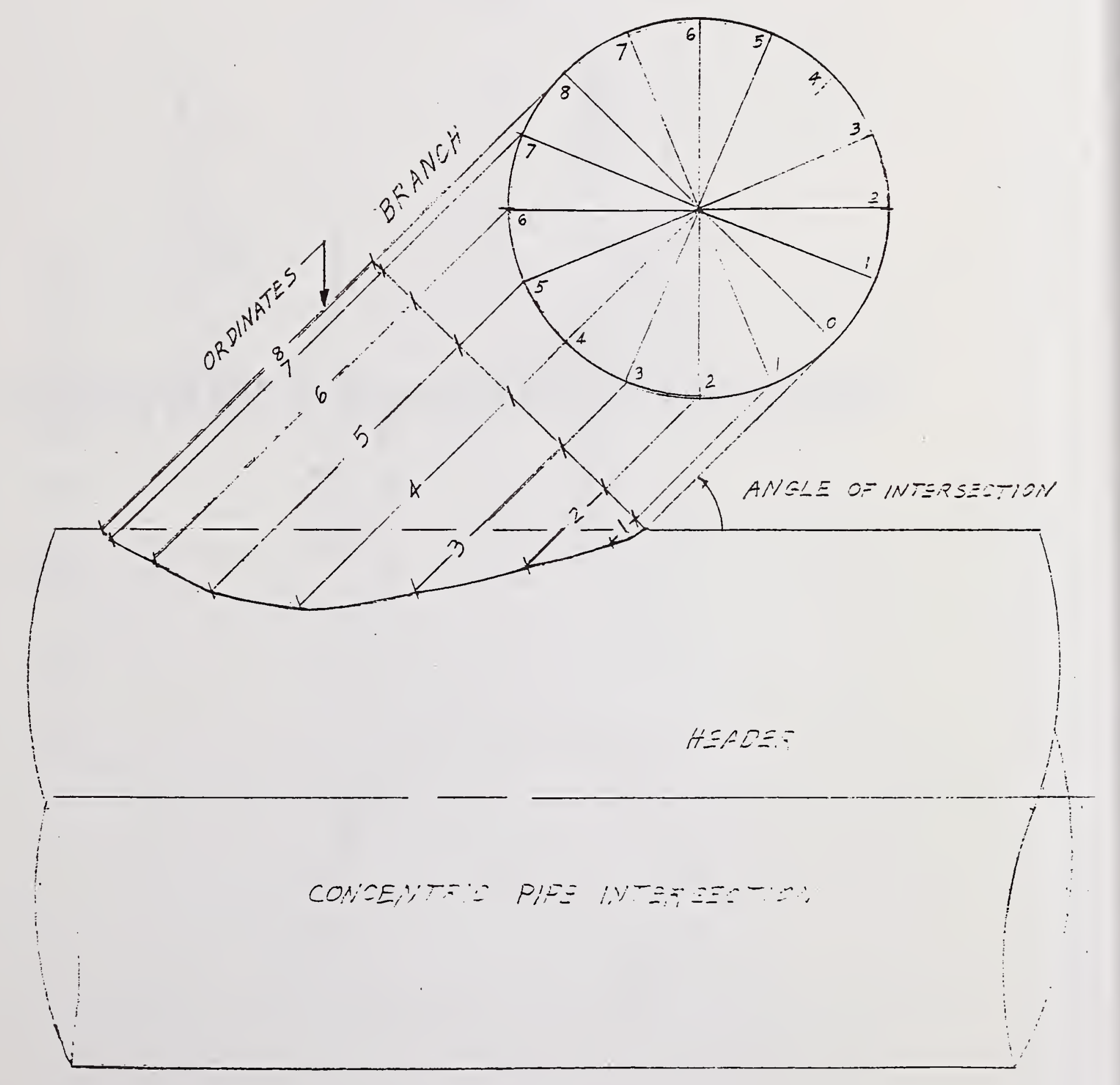

$73 \quad$ FIG. 7 


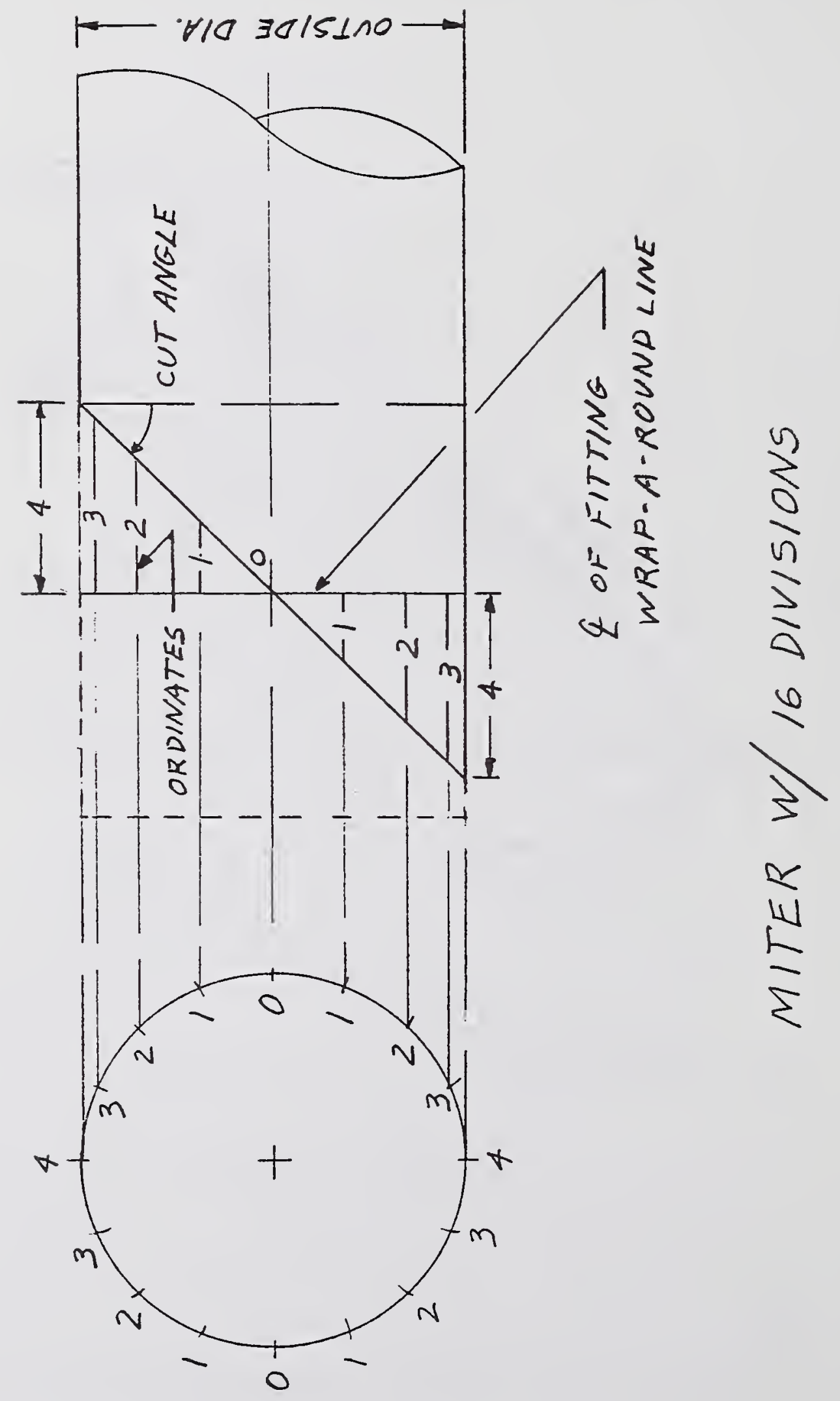

$\frac{\infty}{6}$ 


\section{CONTROLS and CONTROLLERS}





\title{
METHOD FOR PREPARATION OF LASER WELDING CONTROL \\ H. J. Andersen", H. Holm"
}

\section{KEY WORDS}

Laser Welding, Weld Control, Weld Preparation, Process Modelling, Automation.

\begin{abstract}
One of the most important characteristics of the laser welding process is the shape of the heat distribution, which only affects a very deep and narrow area near the weld groove. Hence to achieve a satisfactory weld quality, it is necessary to compensate the process parameters (e.g. welding speed) in order to compensate for even small disturbances in the workpiece geometry (e.g. in the gap size).

This paper presents a generic method for preparing the control of laser welding processes. The control is in this case prepared to satisfy the specified quality for laser welded Tee joints. The main control strategy is here to transform the geometric parameters of the workpieces into a set of welding parameters. In order to apply this control strategy to industrial production of laser welded joints it is necessary to explicitly define the preconditions and the specific methods of the process control system.
\end{abstract}

\section{INTRODUCTION}

In literature several types of models are stated to be applicable for controlling the quality of laser welded joints. One of these strategies includes the establishment of values for the welding parameters on the basis of measured internal process states, such as weld pool area (ref. 1). Another control strategy, which is described thoroughly in the present paper, is to adapt the welding parameters to known characteristics of the workpieces (ref. 2) and (ref. 3).

Experimental work has shown that it is possible to re-produce the quality of laser welded joints by establishing homogeneous pre-conditions and welding parameters for the process execution. Additionally it has been proved by (ref. 4) and (ref. 5) that it is possible to find a robust dependence between some particular welding parameters and the resulting output of the process. These circumstances are utilized by (ref. 2) to establish a model for controlling the weld quality from the laser welding process while the gap is in the range of 0 to $1 \mathrm{~mm}$. The investigations in (ref. 2) covers $\mathrm{C} / \mathrm{Mn}$ steel with plate thickness from 6 to $14 \mathrm{~mm}$.

The method used in (ref. 2) is to establish a set of welding procedures for different values of gap size. These welding procedures includes different welding speeds and rates of wire feed. All other

\footnotetext{
"Aalborg University, Department of Production, Fibigerstraede 16, Dk-9220 Aalborg, Denmark
} 
parameters are held constant for the weld tasks. In this manner the weld quality is maintained by adapting the welding speed and wire feed rate to the actual size of the gap.

A set of welding procedures which are available for determination of the welding parameters for a specific set-up of workpieces and laser welding equipment will from now be denoted as an inverse process model (IPM).

The methods which are presented in the present paper is the result of the work performed in order to develop and implement a control system for automatic control of the laser welding process in shipbuilding. The control system is based on utilization of IPMs in order to maintain the process quality in dispite of disturbances in the workpiece geometry.

In ship building, as in a number of industries, the production includes a large number of different weld tasks. In order to cope with these different weld tasks it is necessary to apply a number of different IPMs which covers the scope of different preconditions for the weld tasks, such as e.g. different types of materials and weld grooves.

The present paper deals with a generic method for preparing the control of laser welding tasks in a production in which different weld tasks occur. This includes specifications of the control system and the establishment of inverse process models.

When different weld tasks must be handled by the control system, it is necessary to keep track of the precondition for execution of each weld tasks. These preconditions are constraining the control system to use the proper IPMs and the proper control strategies. In the following we have categorized the types of preconditions, process parameters etc. in order to be able to describe the control system.

\section{Categorization of Process Control Components}

The characteristics which has a significant influence on the quality of laser welded joints are in the following denoted as the process control components of the laser welding process. These components are organized in 6 categories with respect to their ability to be controlled during preparation and execution of the process. This is in order to facilitate a structured preparation and control of laser welding tasks. The categorization is inspired from (ref. 6) in which similar categories are established for Gas metal Arc Welding.

During the categorization we will use the terms variables and parameters. The variables are the control components which are controllable during the process execution. The parameters are the control components which are not controllable by the process control system.

Equipment Parameters are determined by the characteristics of the equipment only. Among these are the dynamics of the equipment and the range of control variables. These parameters are controlled by choosing a specific set of process equipment.

Workpiece Parameters are a result of the design and manufacturing of workpieces and of occurred process dependent heat distortions. These are e.g. the type of material and the geometry of workpieces. 
Control Parameters are the parameters which are chosen for the specific weld task and which can not (or are chosen not to) be changed during execution of the laser welding process. These are e.g. the type and diameter of wire and the type of shielding gas.

Control Variables are the variables which may be controlled during process execution. Typical control variables are the welding speed and the wire feed rate.

Internal Process state variables are the present process states during process execution. They are functions of the composition of parameters and variables which are mentioned in the preceding four categories. Examples of internal process state variables is: the temperature distribution in weld metal and in the heat affected zone, the acoustic and luminary emission from the weld zone and the chemical composition of the plasma. The internal process state variables must be measured in real time during process execution.

Quality Variables defines the resulting output quality of the process, denoted by geometrical, mechanical and metallurgical characteristics of the laser welded joints. The process Quality variables are, like the internal process state variables, functions of the first 4 categories in this section and by the rate of changes in these. Though we can say that the quality variables are measured after the welding process is finished.

All of the categories contains several parameters or variables. Therefore a set of parameters or variables within a category is denoted as a vector of variables/parameters, like e.g. a vector of control variables or a vector of internal process state variables.

Some process parameters or variables may shift from one category to another, depending on the set-up of the manufacturing system. This may be illustrated by the angles between the weld groove and gravity. These characteristics are denoted as control variables if the workpiece is fixed at a workpiece positioner and the mentioned angles are manipulated during process execution. In contrary these angles are workpiece parameters if the workpiece position is not manipulated during process execution.

\section{GENERAL STRUCTURE OF THE PROCESS CONTROL SYSTEM}

Transformation of workpiece parameters into a set of control variables is in our case performed by use of an inverse process model (IPM). The "inverse" is because the input vector specifies a set of quality variables, which is an output from the physical process. In opposite the output vector specifies a set of control variables, which is an input to the physical process.

The general structure of a control system using IPMs is shown in figure 1. The welding equipment is here dotted because the inverse process model provides specifications of the control variables which actually are outputs from the welding equipment. Of course the welding equipment is an important part of the control system since the equipment parameters, as well as all other parameters which are mentioned in the introduction, affects the welding process. 


\section{Control System Structure}

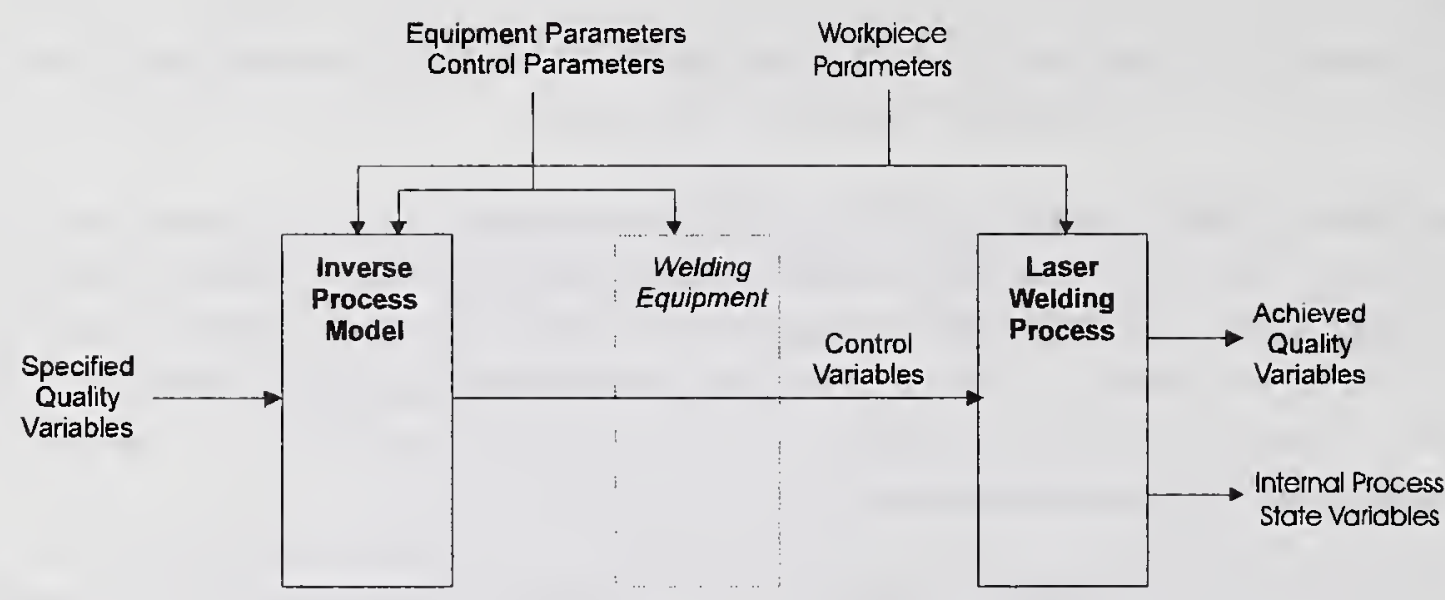

Figure 1.

Specified quality variables for the particular laser welding task are by use of an inverse process model transformed into a trajectory of control variables. The IPM is in this case using information about the workpiece parameters in order to choose the proper approximation to the established weld procedures. The composition process control components will maintain the specified quality variables by use of the IPM. For this control system structure it is important that the workpiece parameters which are known by the IPM corresponds to the actual workpiece parameters which affects the laser welding process. This is further discussed later in the present paper.

In case we had a thorough understanding of the connection between the internal process state variables and the achieved quality parameters we might be able to establish an observer based control system. This would enable us to measure some internal process state variables and by that means estimate the unmeasurable achieved process quality variables in real time (ref. 7). This will not be treated further in the present paper.

\section{PREPARING THE CONTROL SYSTEM FUNCTIONALITY}

The functionality of the control system should be chosen in accordance with the range of different weld tasks to be performed and with the types of disturbances which occurs in the weld tasks. The steps which must be performed in order to define the functionality of the control system are outlined below. Additionally we have to some detail explained the choices which are made during implementation of the actual control system.

1) Classification of weld joints into classes which is covered by the same input/output structure of the inverse process models.

2) Choice of the specified quality variables for each class of joint types.

3) Choice of the parameters and/or variables which creates the background for calculation of the control variable values for the laser welding equipment. 
4) Choice of the method for obtaining the chosen variables and or parameters, such as e.g. sensing or extraction of information from CAD models.

5) Choice of the control method for the laser welding procesi, such as closed loop, open loop and/or adaptive control.

6) Choice of the process control strategy, i.e. the process control variables and their dependence of the parameters/variables mentioned in 3 ).

7) Choice of laser welding equipment and set-up.

The choices which are made for the actual control system as a consequence of following the outlined steps, are described in the following sections.

\section{Re point one: Classes of Joint Types for Development of Models for Laser Welding}

During the considerations for development of inverse process models for laser welding, different classes of weld grooves are defined. This will ensure that the relevant workpiece parameters can be defined uniquely for each class of weld grooves (see figure 2).

\section{Classification of Joint Types}

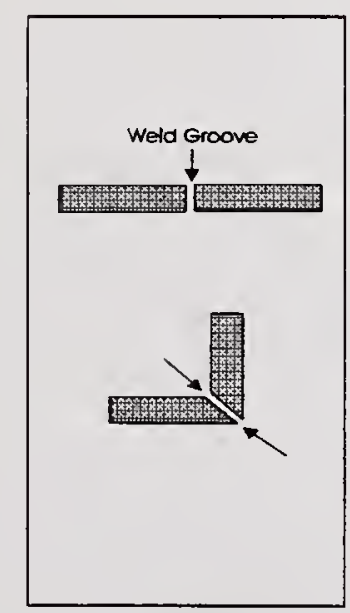

Class of Joint type A

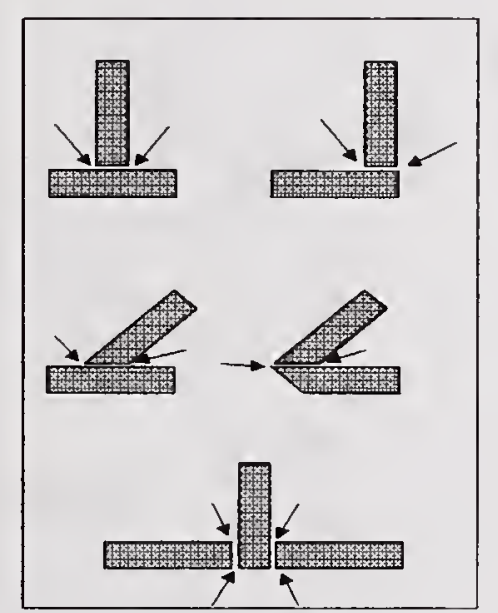

Class of Joint ty'pe B

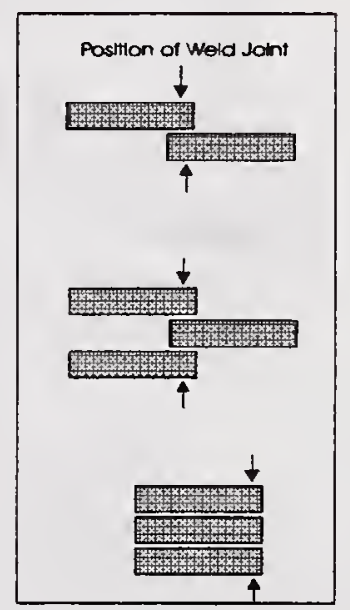

Class of Joint type C

Figure 2

The inverse process models has an input vector which provides all the workpiece parameters required for calculation of control variables for any weld task within the particular class of weld grooves. 


\section{Re point two: Choice of Quality Variables for Joint type B}

In this section we will define a set of geometric and mechanical quality variables for joint type B in figure 2 . The geometric quality variables are:

- Binding depth which is defined as the length of the penetrated weld metal in workpiece 1. This length is measured perpendicularly on the surface of workpiece 1 (see figure 3 ),

- Concavity (shown in figure 3 ).

- Maximum Notch size (shown in figure 3).

- Maximum length and area of cracks (including crater cracks).

- Maximum length and area of porosities and gas pores.

- Maximum length and area of solid inclusions.

Geometric Quality Variables for Joint type B
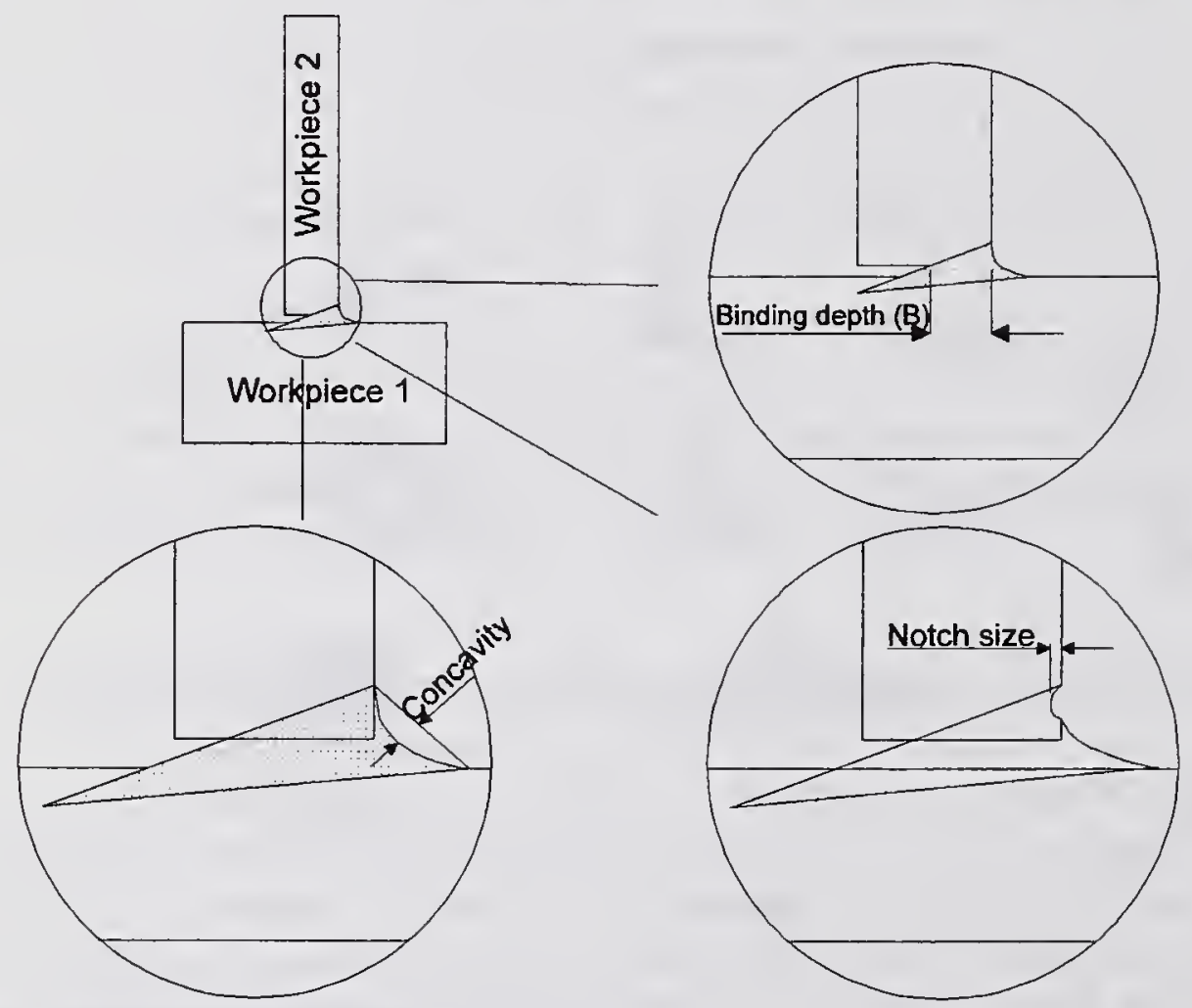

Figure 3.

The mechanical quality variables are:

- Maximum Hardness of weld metal

- Minimum Elongation capability of laser welded joint. 
- Minimum value of Charpy test.

The quality variables which are mentioned in this section are supposed to define the most commonly used quality specifications for laser welded joints. Additional quality variables may be defined. On the other hand one may only make use of a subset of the defined variables in order to set the constraints for the inverse process models.

\section{Re point three: Choice of Constraining Workpiece Parameters}

The resulting quality variables of laser welded joints are controlled by the distribution of heat and weld metal in the weld groove during process execution. The distribution of heat and weld metal is influenced by a number of workpiece parameters. The values of these parameters must be provided in order to calculate suitable control variables by use of inverse process models. The IPMs which are used for calculation of control variables must be valid within the constraints which are set by these workpiece parameters. The geometric workpiece parameters are shown in figure 4 .

\section{Constraining Workpiece Parameters}

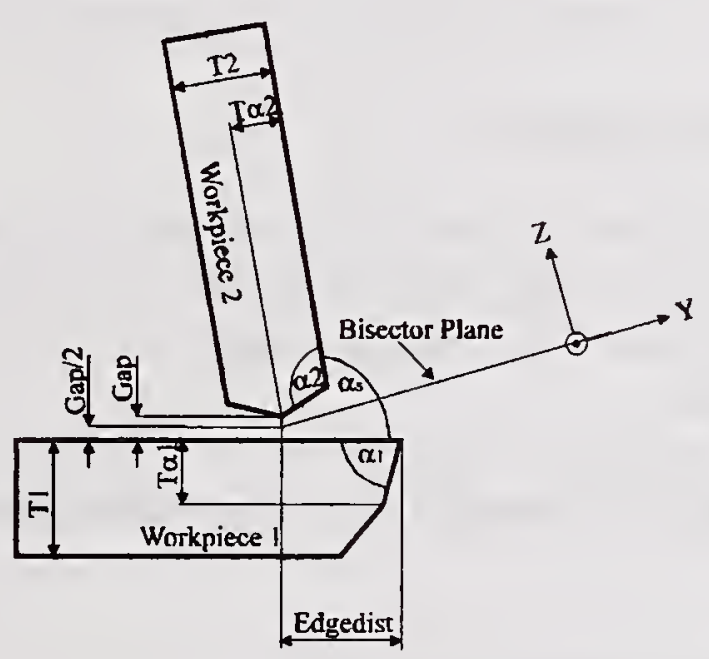

Figure 4

The distribution of weld metal is furthermore a consequence of the angles between the weld groove and gravity. These angles are defined by $\psi$ and $\phi$. Where $\psi$ is the angle between the XZplane (see figure 4) and a vertical plane which is parallel to the direction of the weld grooved, and $\phi$ is the angle between the XY-plane and a horizontal plane. The distribution of heat in the weld groove is further more a result of the types of workpiece materials and of the initial temperature of the workpieces. The heat distribution is additionally affected by the emission of laser power due to the surface condition of the workpieces, such as the roughness and the type of surface coating.

In order to assure that the equipment is capable of adapting to the changes in workpiece parameters, it may be necessary to include the amplitude and frequency of some geometric changes (such as e.g. the size of the gap). 
All of the illustrated and mentioned workpiece parameters will be included in the calculation of control variables, i.e. these are used as inputs to the inverse process models. Though some of the workpiece parameters are constant for all weld tasks which are covered by the models. In this case an exclusion of these parameters in the calculations will be natural. These parameters will the serve as constraints for the model. If these parameters are changed we will have to establish a new model.

\section{Re point four: Method for Acquisition of Constraining Workpiece Parameters}

For the actual process control system it has been chosen to acquire some data from a CAD-model and measure some other data by use of a vision sensor. The data which is acquired from CAD models are the data which are known by a sufficient accuracy for control purposes. These are the type of material, Plate thickness, groove angles, bevel shape and surface coating. These parameters are supposed to be relatively well defined for the specific weld tasks and therefore no measurements are necessary.

The workpieces are designed to fit each other without a gap between the workpieces. During manufacturing and tacking of the workpieces some disturbances of the designed workpiece parameters will occur. These disturbances, which includes a small gap between the plates and a displacement of the weld groove, are measured by use of a vision sensor.

\section{Re point five: Choice of Control Method}

In this particular case the control system is controlled adaptively in open loop. The adaptive part of the control system is explained by the fact that the control variables are adapted to changes in the workpiece parameters. The open loop part is explained by the difference between the controlled and the measured variable.

In case we wanted a closed loop process control system we should measure the resulting quality of the process.

\section{Re point six: Choice of the Process Control Strategy}

The process control strategy may be defined as the specific set of rules, by which the control variables are calculated on the basis of chosen workpiece parameters. This includes the rules for handling of both steady state and dynamic disturbances in the workpieces parameters.

In the actual process control system we have chosen to adapt the control variables welding speed and wire feed rate to the size of the gap in the weld groove. The handling of dynamic changes in the gap size is handled by adapting the control variables to discrete steady state values of the gap.

The adaptation of control variables to values of the gap is performed by use of an inverse process model. This IPM contains a set of welding procedures which are constrained by different values of the gap size, as shown in figure 5 . 


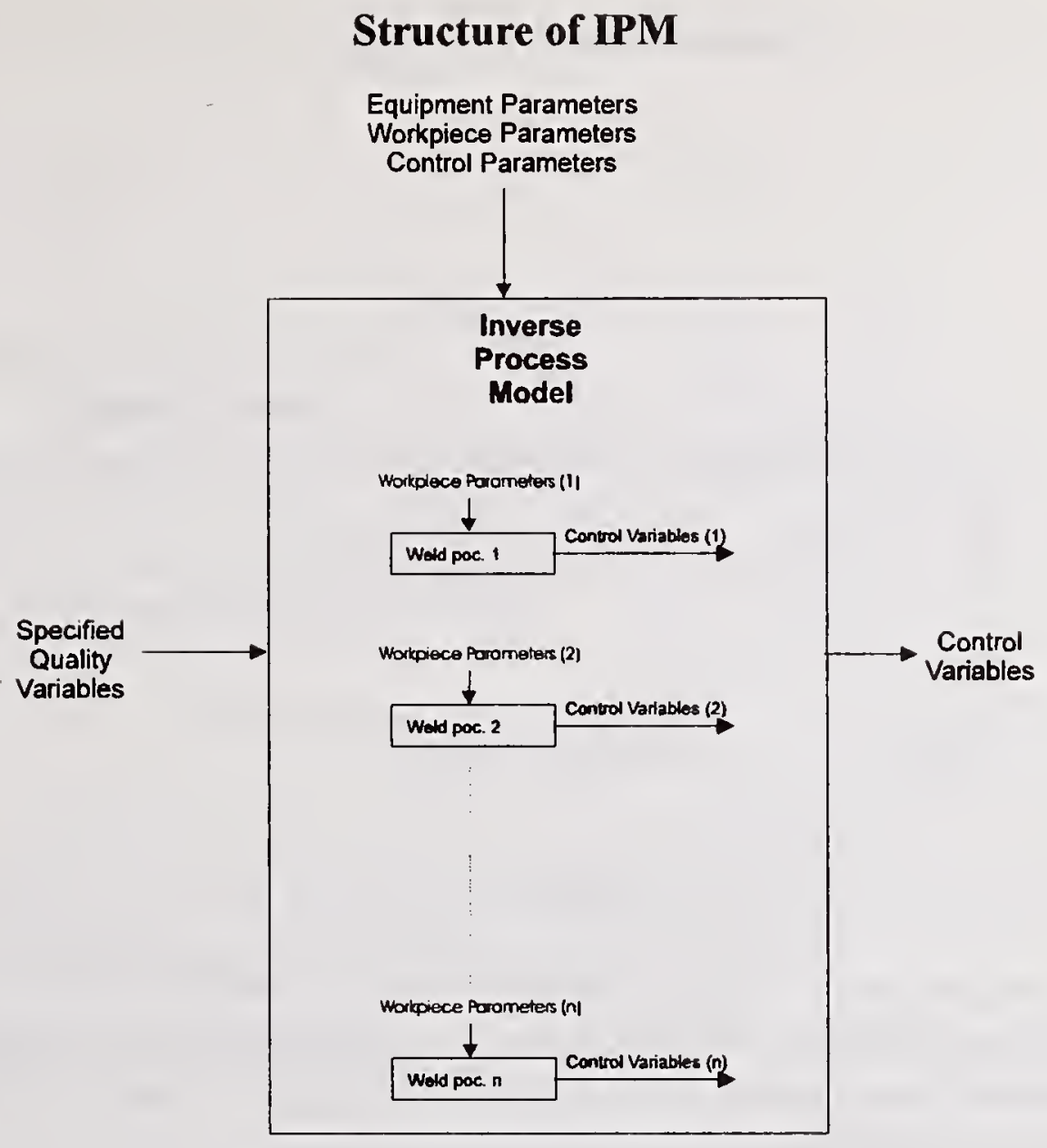

Figure 5 .

The control variables must be defined in a manner that makes them applicable to any weld task within the specific class of weld joints. For the class of joint type B the control variables includes:

- Welding speed.

- Wire feed rate.

- Defocus length (L), which is the distance between the surface of workpiece 2 and the focus point of the incident laser beam (shown in figure 6).

- Angle of incidence $(\Omega)$, which is the angle between the incident laser beam and a vector which is normal to the welding direction and lying in the surface of workpiece 1 (shown in figure 6).

- Distance from flange (D), which is the distance between the focus point of the incident laser beam and the surface of workpiece 1 (shown in figure 6). 


\section{Process Control Variables}

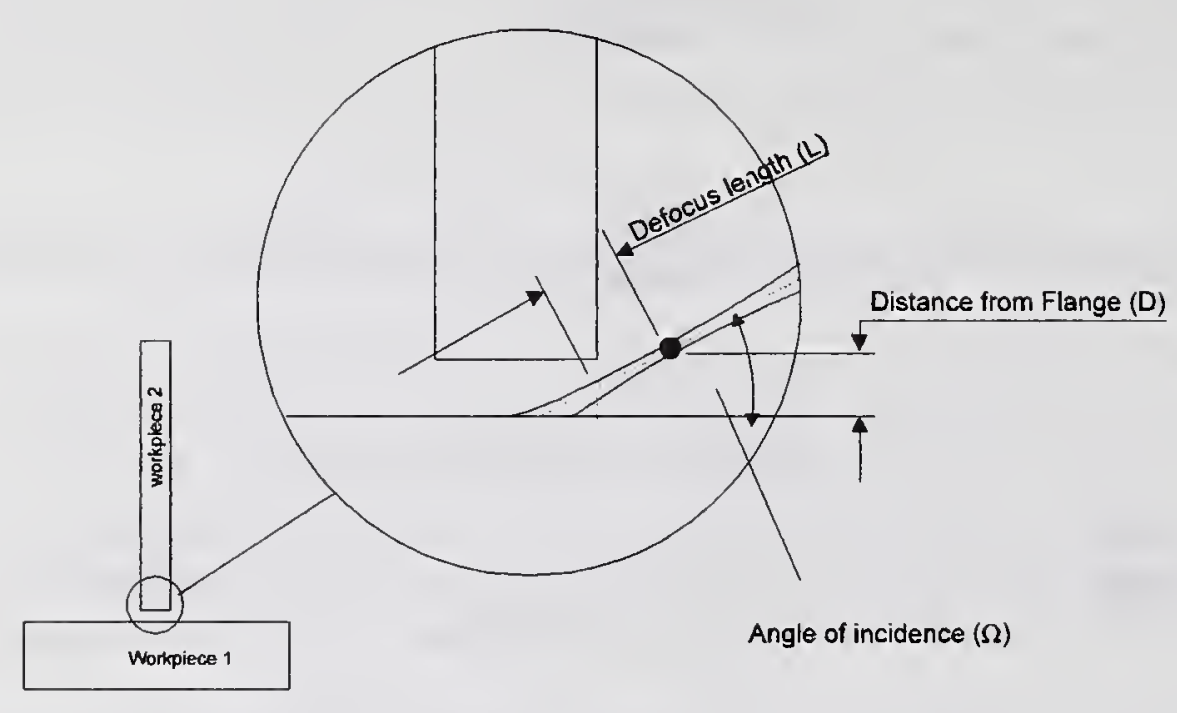

Figure 6.

As long as the size of the gap varies from 0 to $1 \mathrm{~mm}$ it has been possible to use the welding speed and the wire feed rate as control variables. The rest of the mentioned variables are possible control variables because our equipment allows us to change them during process execution. Though in our case they are held constant.

\section{Re point seven: Choice of Equipment and set-up}

The equipment parameters are fixed for a specific set-up of equipment. The validity of inverse process models will be constrained to these equipment parameters and therefore it is necessary to specify these parameters by the inverse process models. The most obvious method for this specification is to specify the physical equipment, at which the specific model is developed.

Contrary to the control variables it will be necessary to be aware of all of the equipment parameters, either explicitly or implicitly by using a specific set-up of equipment.

Control parameters are the constant parameters which are chosen before each individual weld task. These parameters are:

- Type of filler wire.

- Diameter of filler wire.

- Position of wire feeding mechanism relative to focus point of laser beam.

- Angle between the wire feed direction and the welding direction. 
- Angle between the wire feed direction and a vector which is perpendicular on the welding direction and lying in the bisector plane.

- Plasma control gas type.

- Plasma gas flow rate.

- Dimensions of the plasma gas nozzle.

- Angle between the plasma control gas direction and the welding direction.

- Angle between the plasma control gas direction and a vector which is perpendicular on the welding direction and lying in the bisector plane.

\section{CONCLUSIONS}

The most important result of the present paper is a generic method for development of a process control system which controls the quality of laser welded joints by use of inverse process models. The structure of such a process control system is shown in figure 1 .

By use of the methods in the present paper it is possible to establish control systems which are capable of handling different types of weld tasks. The categorization of process control components and the structural description of the process control system provides the basic concept for establishment of adaptive control systems for laser welding.

In the paper seven steps are described for preparing the process control system. These steps covers the most important considerations one should take into account in order to establish a generic process control system for laser welding. Taking these into account it is possible to establish a control system which covers the chosen classes of weld joints under the specified constraints.

At this particular point in time we still need to perform the final tests of our control system, but the results which is achieved until now indicates that the remaining test will turn out successful. The tests has until this point shown that it is possible to control the weld quality when the gap is either growing from 0 to $1 \mathrm{~mm}$ or decreasing from 1 to $0 \mathrm{~mm}$.

The control system which is established in our case is delimited to control the weld quality of Teejoints in plate thicness $25 \mathrm{~mm}$ for workpiece 1 and $12 \mathrm{~mm}$ for workpiece 2 (see figure 6). Furthermore is the gap between the workpieces allowed to be in the range 0 to $1 \mathrm{~mm}$ and the control variables are only adapting the welding speed and the wire feed rate to changes in the size of gap.

It may be possible to extend the limits of the gap in case we also adapted the remaining three process control variables to the disturbances in the workpiece geometry. 


\section{REFERENCES}

1. Dr. Claus Bagger, Flemming Olsen, On-line Process Control of the $\mathrm{CO}_{2}$ Laser Welding Process with the Aid of Vision Technology, Proceedings of the Conference, The Laser and the Electron Beam in Welding, Cutting and Surface Treatment State of The Art 1991.

2. Steen Erik Nielsen, Seam Tracking and Adaptive Control Based on Vision Technology Used for High Power Laser Welding, 5th. NOLAMP Conference, Oslo, Norway, 1995.

3. Henrik John Andersen et al., Application of Inverse Process Models for Laser Welding, Proceedings of the eleventh IPS Research Seminar, Fuglsø, Denmark $15_{\text {th }}-17$ Th. April 1996.Distributed by Aalborg University, Department of Production, Fibigerstraede 16, 9220 Aalborg.

4. Steen Erik Nielsen, Svejsning med højeffektlaser, grundlæggende undersøgelser, 1992, Force institutterne, københavn, ISBN 87-7784-009-7.

5. Claus Bagger, Parameteroptimering ved ND:YAG lasersvejsning, AP 19.35, LASRAM, IPU-019, MIL, Instituttet for Produktudvikling, building 425, DTU, DK-2800 Lyngby.

6. Ole Madsen, Sensor Based Robotic Multi-Pass Welding, ISBN NR: 87-89867-02-5, Aalborg University, Department of Production, Fibigerstraede 16, 9220 Aalborg, Denmark.

7. Jesper Højland Høyer, Hans Holm, State Modelling of Welding for closed loop Welding Control, ISBN nr: 87-89867-29-7, Proceedings of the tenth IPS Research Seminar, 1995, Aalborg University, Department of Production, Fibigerstraede 16, 9220 Aalborg, Denmark. 


\title{
SCAN WELDING: \\ THERMAL MODELING AND ADAPTIVE CONTROL
}

\author{
C. C. Doumanidis*
}

\begin{abstract}
This article introduces scan welding as a redesign of classical joining methods, employing computer technology to ensure the composite morphologic, material and mechanical integrity of the joint. This is obtained by real-time control of the welding temperature field by a proper dynamic heat input distribution on the weld surface. This distribution is implemented in scan welding by a single torch, sweeping the joint surface by a controlled reciprocating motion, and power adjusted by feedback of infrared temperature measurements in-process. An off-line numerical simulation of the thermal field in scan welding is used for this purpose, as well as a linearized multivariable model with real-time parameter identification. An adaptive thermal control scheme is thus implemented and validated both computationally and experimentally on a robotic Plasma-Arc Welding setup. The resulting productivity and quality features of scan welding are comparatively analyzed in terms of material structure and properties of the joint.
\end{abstract}

\section{KEYWORDS}

Scan Welding, Adaptive Control, Infrared Thermometry, Temperature Model, Robotic Welding.

\section{INTRODUCTION}

Use of computers in the modern welding technology, both for off-line analysis and for real-time control of the process, has not only improved the performance of classical methods, but it also leads to the development of completely novel welding techniques. These operations must be designed to optimize the product quality in its totality, including the weld bead geometry, the material structure in the heat affected zone (HAZ) and the mechanical properties of the joint. The combination of desirable weld characteristics in all these aspects is imperative: A weld may have an ideal bead geometry but may suffer from a martensitic structure in steels, an overaged $\theta$-phase in precipitation-hardened aluminum alloys, a sensitization zone in stainless steels (Ref. 1) or extensive residual stresses (Ref. 2) rendering it useless, as the fate of the Liberty ships in World War II has demonstrated (Ref. 3). But since the bead morphology, metallurgical microstructure and thermal stress or distortion fields are all time-dependent, spatially-distributed features, they may not be simultaneously controllable by the few, lumped process variables of localized, sequentially moving heat sources such as a plasma arc or a Laser beam. Clearly a distributed, simultaneous heat input on the entire accessible weld surface is desired to regulate the bead profile, metal structure and property distributions in the joint volume. To expand the control authority of conventional, concentrated heat sources, hardware solutions involving multiple torches distributed in space have been tested in the literature (Refs. 4, 5). However, such arrangements are plagued by the complexity and cost of multiple power sources, and by resolution and interference problems between torches.

An alternative software approach, in which a single point thermal source is shared in time to generate the necessary heat input distribution for composite weld quality control, is enabled by computer technology in the newly invented scan welding process (Ref. 6): A traditional welding

* Department of Mechanical Engineering, Tufts University, Medford, MA 02155. 


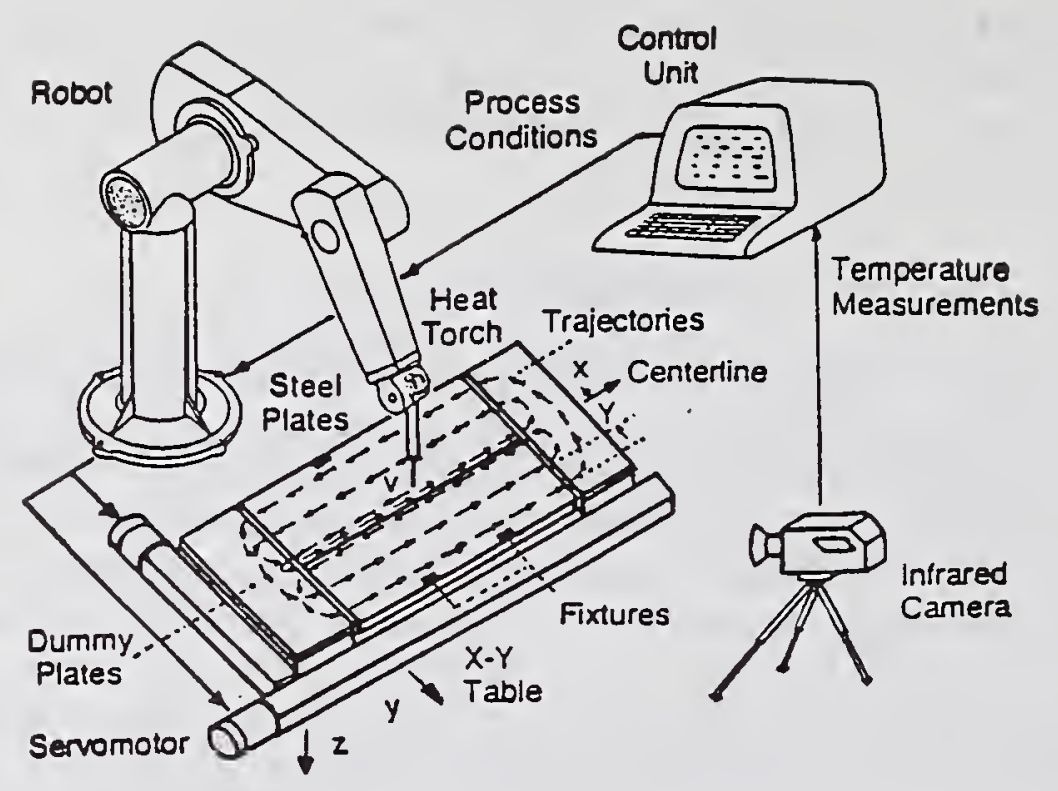

Figure 1. General configuration of the scan welding process.

torch or beam, guided by a high-speed robotic manipulator, sweeps rapidly the full part surface, to provide the right amount of thermal power at each surface element, needed to develop a specified local temperature cycle and thus the desirable thermal quality distributions in the weld (Figure 1). The heat source power and trajectory is dynamically modulated in real time by the control computer, using feedback of thermal measurements on the joint by a non-contact sensor. However, such technology of non-destructive, in-process sensing of internal material characteristics (e.g. ultrasonic or X-ray imaging) is currently costly and impractical, and it involves long time delays in measuring features such as residual stresses, which are finalized as the part cools down after the process is completed. Fortunately, these characteristics are deterministically determined by the dynamic temperature field during the operation, which can be readily measured on the external surface e.g. by non-invasive infrared pyrometry (Ref. 7).

Thus, scan welding can be visualized as a computer-controlled two-dimensional weaving of a point heat source with thermal feedback, capable of generating any desirable distribution of the preheating, welding, and/or postheating cycle in the processed joint. The experimental testing and parametric analysis of the scan welding technique, both for planar, orthogonal metal sheets and for circular flange and pipe arrangements, has been reported in the literature (Ref. 8). The computer role in thermal modeling and real-time control of the process conditions, i.e. the source power and motion, of the new welding technique is the focus of this article. Thus, after a short reference to a computational simulation of the temperature field in scan welding, an in-process thermal regulation strategy will be established on its basis, and implemented by the control computer of the scan welding configuration in the laboratory.

\section{IMPLEMENTATION AND SIMULATION}

The thermal actuation in scan welding can be implemented in two different modes: Highbandwidth sources, such as electron or Laser beams (EBW, LBW) can be scanned on a raster pattern on the weld surface in a shawtooth (row-wise) fashion similar to the beam scanning in a CRT monitor, while their intensity is simultaneously modulated for each raster element (pixel). This raster scanning can be obtained by a two-dimensional scanner system, such as an electrostatic (capacitive) deflector for an electron beam, an electromagnetic galvanometer beam positioner of a Laser beam (Ref. 9) or an electromechanical digital micromirror device (DMD) of reflector 
elements on a single micro-electromechanical system (MEMS) chip (Ref. 10). Alternatively, for slowly-moving sources, such as arc or plasma-arc torches, a more efficient actuation is obtained by vector scanning of the heat source on a real-time guided trajectory, similar to the beam scanning on an oscilloscope screen. In this case a general mechanical two degree-of-freedom positioning system of the torch relative to the part is needed (e.g. a servodriven positioner or a robotic manipulator).

Such a vector-scanning technique is illustrated on the experimental scan welding station in Figure 2. It consists of a water-cooled Plasma-Arc Welding (PAW) torch, rated at $300 \mathrm{~A}$ and powered by a multifunction $\mathrm{CC} / \mathrm{CV}$ transformer supply rated at $400 \mathrm{~A}$, through a plasma console unit and a closed-circuit coolant recirculator. The PAW system is capable for both transferred and nontransferred (pilot) plasma arc modes for metals as well as non-metallic materials. In the experiments, consisting of bead-on-plate scan welding of stainless steel (304) coupons, measuring $30 \times 24 \times 3 \mathrm{~mm}$, the arc voltage was $15 \mathrm{~V}$, the arc length $3 \mathrm{~mm}$, and the plasma and shielding gas (Ar$2 \% \mathrm{O}_{2}$ ) flow is $0.5 \mathrm{l} / \mathrm{s}$. The PAW torch is guided by an articulated 6 degrees-of-freedom process robot, with a positioning accuracy of $0.15 \mathrm{~mm}$, maximum speed of about $1 \mathrm{~m} / \mathrm{s}$ and payload up to $6 \mathrm{~kg}$. The manipulator is powered by AC servomotors with optical encoder feedback hardened for high-frequency arc initiation interference in welding, and its control unit is programmable in highlevel code through an embedded terminal and teach pendant. The robot controller as well as the PAW supply are connected to the central microcomputer of the setup through a serial and AD/DA converter board respectively, ensuring software control of the torch motion and power in real time.

The same system computer is used to monitor the temperature field on the top surface of the weld by an infrared pyrometry camera. This consists of a high-speed 2-D electromechanical galvanometer scanner, a set of focusing lenses and filters, and a liquid nitrogen-cooled HgCdTe sensor element, sensitive in the $8-12 \mu \mathrm{m}$ wavelength range. The temperature resolution of the infrared camera is $\pm 2 \mathrm{~K}$ on a $1000 \mathrm{~K}$ window range, and the spatial resolution of the scanner is 0.2 mrad, equivalent to about $0.2 \mathrm{~mm}$ on the weld surface. A microprocessor-based control unit converts the measured infrared emission values to temperatures based on user-defined surface emissivity values. The dynamic thermal image, besides being monitored in-process by the control computer through a high-performance frame grabber, is also stored in standard composite video format by a tape recorder for off-line thermal analysis by dedicated image processing software.

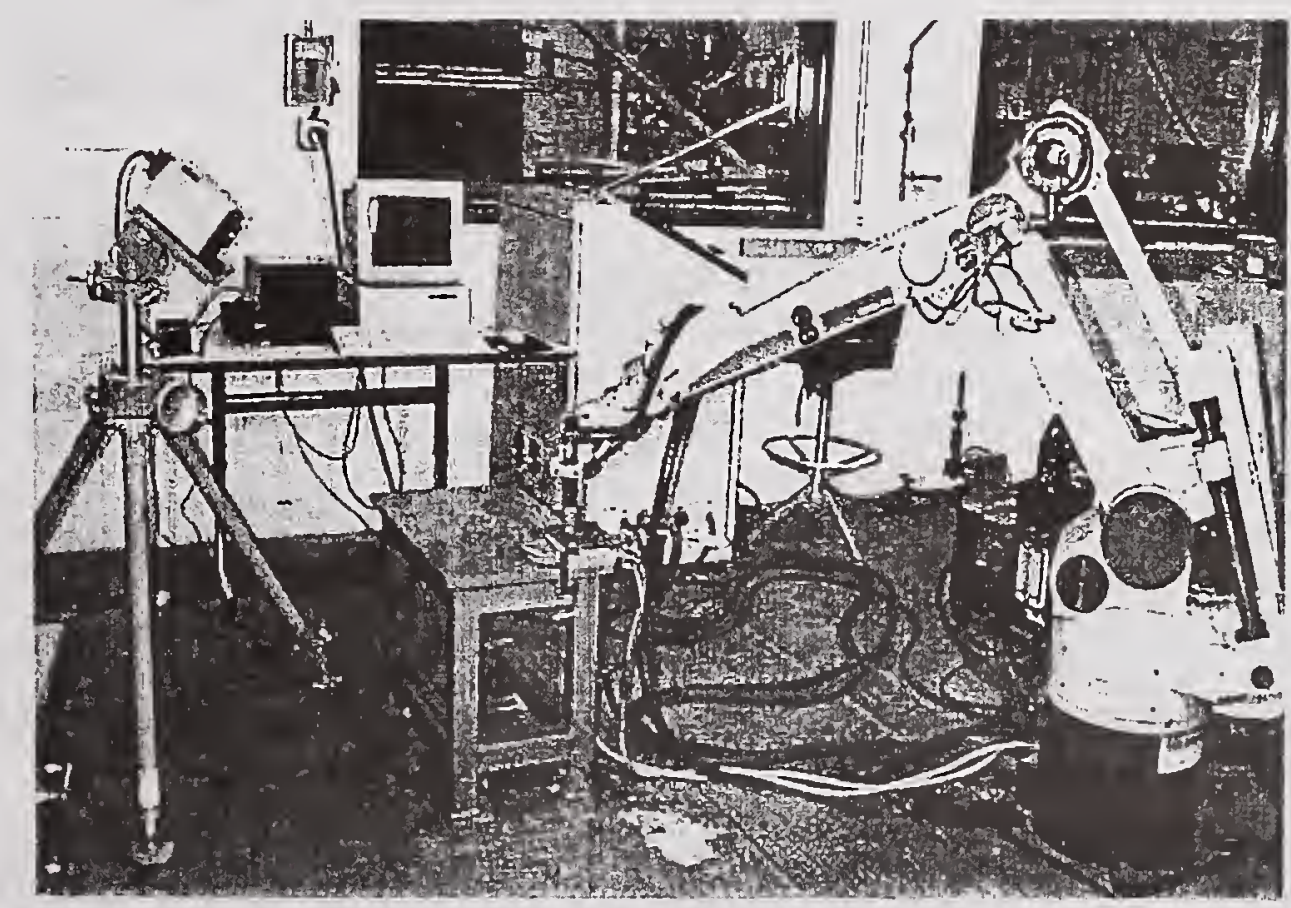

Figure 2. Laboratory setup of robotic plasma-arc scan welding station. 

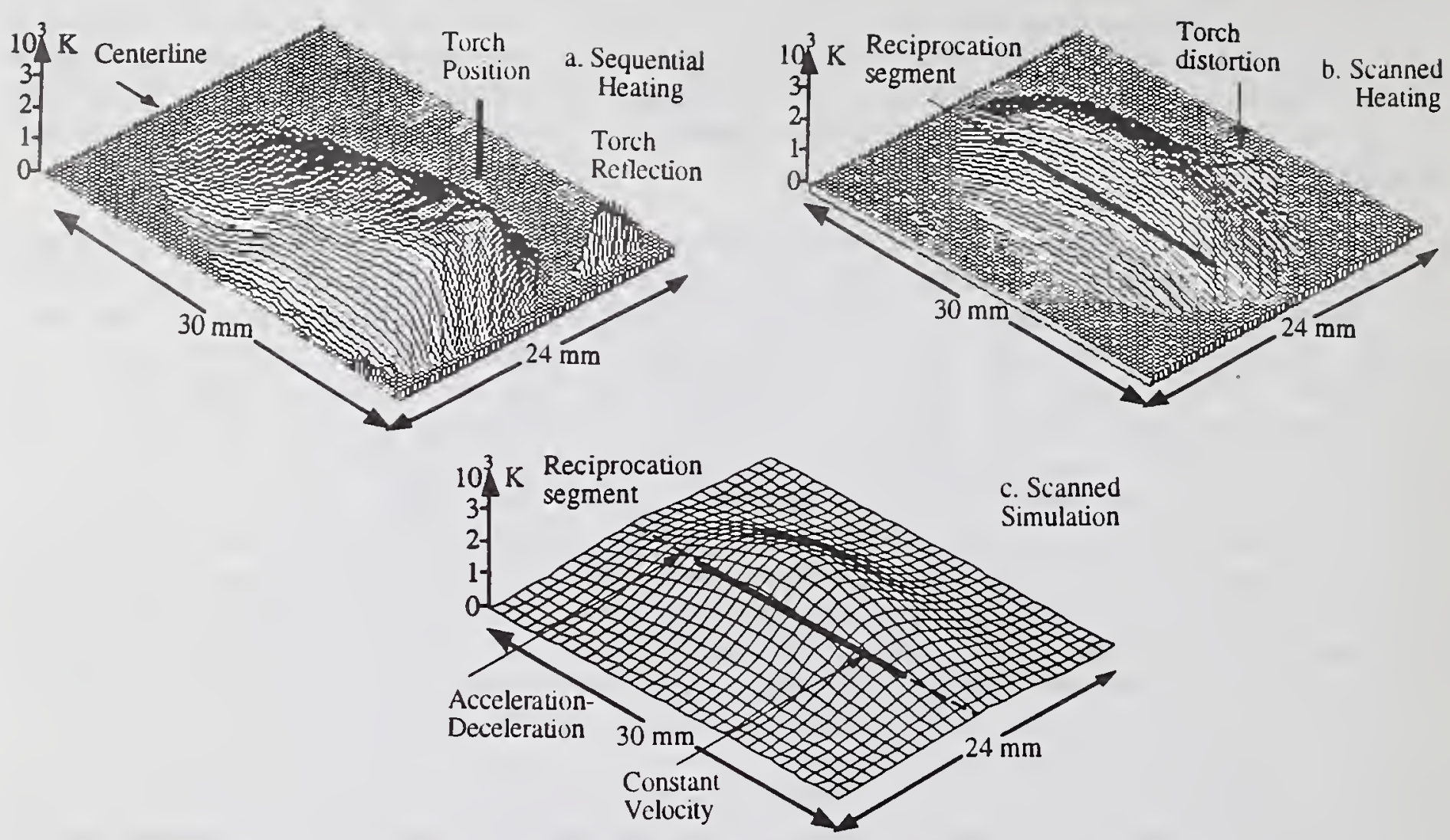

Figure 3. Temperature hills on top surface in traditional and scan welding of stainless steel (304).
a. Classical welding
b. Scan welding
c. Numerical simulation of scan welding.

Figure $3 \mathrm{a}$ and $3 \mathrm{~b}$ illustrates typical infrared images of the temperature hills on the top weld surface, generated by traditional welding at an arc current of $80 \mathrm{~A}$ and speed of $2 \mathrm{~mm} / \mathrm{s}$, and by scan welding at a reciprocation frequency of $2 \mathrm{~Hz}$ along the coupon centerline respectively. As expected, unlike the concentrated temperature hill of classical welding, the scanned process yields a more uniform thermal distribution along the joint. At the two plate ends, near the robot deceleration/acceleration region of the scanned torch path, smearing of the temperature hill yields ellipsoidal thermal isotherm endings. Note also the apparent local distortion of the thermal field at the torch location and its reflection on the polished weld surface. The noise of the experimental data is attributed to emissivity variations of the weld surface.

Besides laboratory experimentation, computer simulation of scan welding is necessary for the design of a thermal control system for manufacturing applications. This is because the variety of heat transfer phenomena in welding must be repeatably and accurately reproduced and studied over the full range of their important parameters, without laboratory constraints and uncertainty. Also, computational simulation overcomes the difficulty or inability of non-intrusive measurements of certain features, such as internal temperatures, weld penetration geometry, material structure and properties and stress distribution during the process. These reasons have historically contributed to the proliferation of such models since the days of analog computers, when the thermal distribution in welding was simulated by physical analogy to electrical charge dissipation in carbon specimens (Ref. 11). However, despite the wealth of the welding literature in empirical (Ref. 12), analytical (Ref. 13) and numerical models (Ref. 14) of classical welding methods, there is no direct modeling precedent for the new scan welding technique.

Thus, a numerical finite-difference simulation of the temperature and phase field was developed as an off-line process description. This integrates the transient solid conduction (Fourier) equation in discrete time steps and space elements of a grid of nodes, using an explicit Eulerian formulation 
(Ref. 15). The simulation employs a large, coarse grid of nodes encompassing the full heat affected zone of the part, and a small, finer grid for better resolution in the vicinity of the molten region. These grids can be stationary, spanning the full part size, or relocatable, following the torch motion. The simulation code covers various process arrangements, such as planar sheets and cylindrical welds, and is easily expandable to more general geometry configurations. The simulation can handle heat sources with arbitrary power density distributions and trajectories in traditional and scan welding. The numerical model provides for flexible initial preheat and boundary heat transfer conditions, and temperature-dependent material properties with latent transformation effects, such as fusion and solidification. The simulation output includes maps of the 3-D thermal and phase field, as well as 2-D temperature hill and isotherm surface sections. Figure $3 \mathrm{c}$ compares the top surface temperature hill developed during scan welding, as predicted by the numerical simulation, to the measurements of the infrared camera in Figure $3 \mathrm{~b}$, under the same process conditions as in the previous section. Clearly, despite certain deviations near the solid-melt interface and the plate edges because of uncertainty in the assumed material and heat transfer parameters, the thermal data in the laboratory confirm the results of the simulation model.

\section{THERMAL FEEDBACK CONTROL SYSTEM}

The experimental infrared images and the simulated temperature distributions of Figure 3 demonstrate the spatially distributed nature of heat transfer in scan welding. However, for such an infinite-dimensional formulation of the scan welding model, distributed-parameter systems theory has not yet yielded practical controller design tools applicable to thermal manufacturing problems (Ref. 16). In addition, the off-line numerical simulation above can not offer a computationally efficient basis for thermal process control in real time. A succinct alternative description, which can serve as an in-process reference model to the control algorithm, must be established through lumping of the continuous thermal distribution to vectors of distinct temperature outputs $\underline{T}$ and heat inputs $\underline{Q}$ at the nodes of a mesh of element size Ds in the weld, which are updated periodically every sampling period Dt. Such a discrete-time, finite-dimensional modeling approach is illustrated in the two-dimensional thermal distribution on the thin metal sheet of Figure 4, in which the temperature is assumed uniform in the thickness direction. On such an orthogonal mesh of dimensions $1 \mathrm{xm}(\mathrm{l}=6, \mathrm{~m}=5$ in Figure 4$)$, the localized, sampled values of temperatures $\mathrm{T}(\mathrm{i} ; \mathrm{k})$ and heat inputs $Q(i ; k)$ in vectors $\underline{T}(k)$ and $Q(k)$ respectively, are defined at each node $i=1 . .1 x m$ and time instant $\mathrm{k}$. By linearization of the heat transfer equations, the multivariable dependence of the resulting temperature outputs $\underline{T}(\mathrm{k}+1)$ at the next sampling period on the current heat inputs $\underline{Q}(\mathrm{k})$ can be expressed in a discrete-time, autoregressive moving average (ARMA) form (Ref. 17) as:

$$
\underline{\mathrm{T}}(\mathrm{k}+1)=\Theta^{\mathrm{T}}(\mathrm{k}) \cdot \underline{\mathrm{S}}(\mathrm{k})
$$

where the augmented thermal vector $\underline{S}$ contains the present and a number $\mathrm{n}$ of past values of the lumped temperatures $\underline{T}$ and heat inputs $\underline{Q}$, concatenated in a single column:

$$
\underline{\mathrm{S}}(\mathrm{k})=\left[\underline{\mathrm{T}}^{\mathrm{T}}(\mathrm{k}): \underline{\mathrm{T}}^{\mathrm{T}}(\mathrm{k}-1): \ldots: \underline{\mathrm{T}}^{\mathrm{T}}(\mathrm{k}-\mathrm{n}): \underline{\mathrm{Q}}^{\mathrm{T}}(\mathrm{k}): \underline{\mathrm{Q}}^{\mathrm{T}}(\mathrm{k}-1): \ldots: \underline{\mathrm{Q}}^{\mathrm{T}}(\mathrm{k}-\mathrm{n})\right]^{\mathrm{T}}
$$

The parameters in matrix $\Theta$ in the linearized, multiple-input, multiple-output (MIMO) formulation of Eq. 1 express the thermal dependence of each local, instantaneous temperature $\mathrm{T}(\mathrm{i} ; \mathrm{k}+1)$ at node $\mathrm{i}$ and period $k+1$, on the temperature $T(j ; h)$ or heat input $Q(j ; h)$ at another node $j$ and past period $\mathrm{h}=\mathrm{k} . \mathrm{k}-\mathrm{n}$. The simulated temperature hill in Figure 3, generated by the concentrated torch, shows that the conservativeness of the dominant conductive heat transfer in the solid region results in a decaying transient, localized thermal field in the vicinity of the heat input, with almost no effect at remote points. Thus, the temperatures $\mathrm{T}(\mathrm{i} ; \mathrm{k}+1)$ are contributed only by recent and neighboring node temperatures $T(j ; h)$ and heat inputs $Q(j ; h)$, yielding a sparse matrix $\Theta$ with a limited number of nontrivial elements, and with all the others being negligible. In addition, the radial symmetry of a thermal distribution around the source location indicates that the parameters $\Theta(i, j)$ are determined 


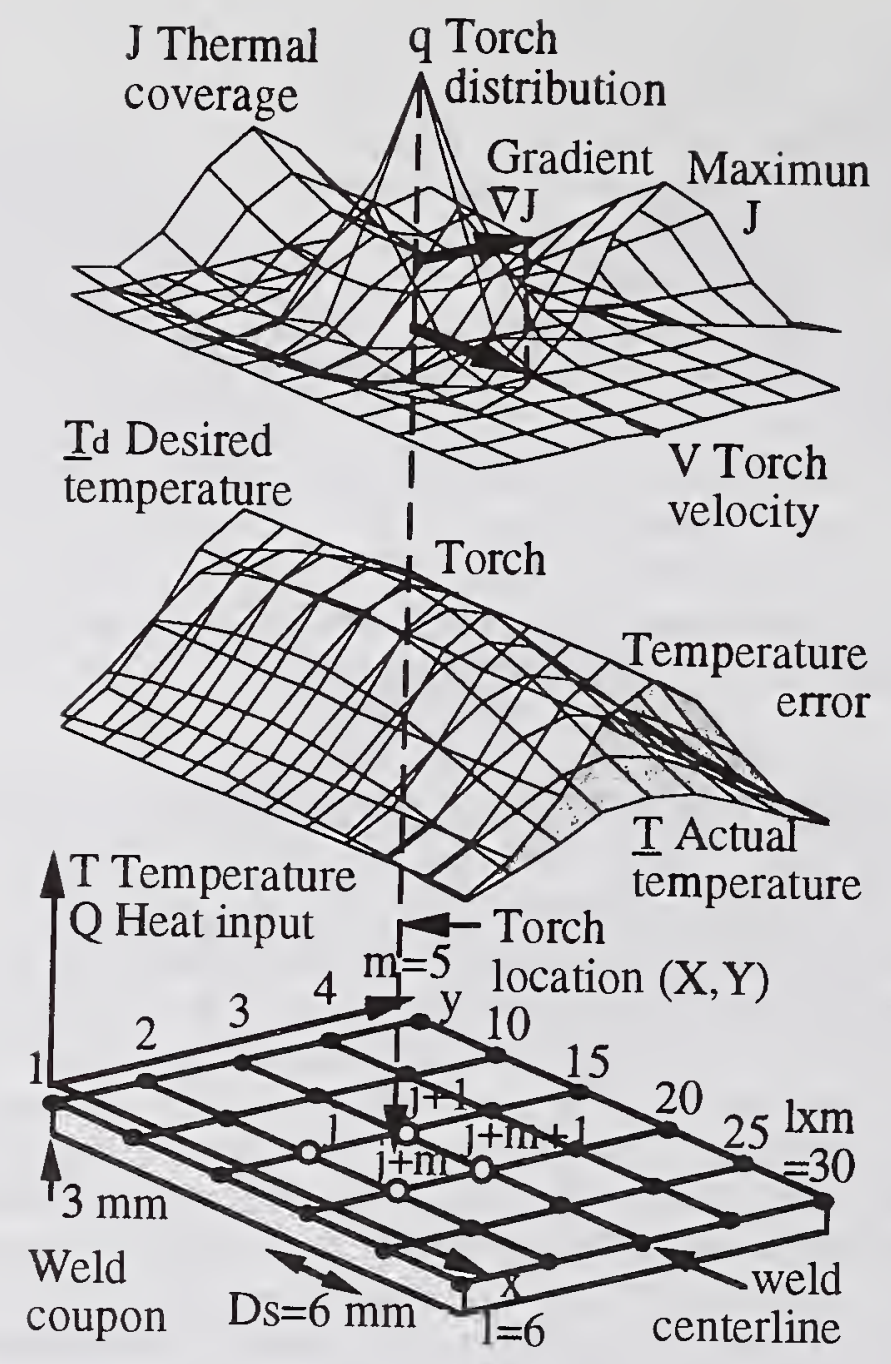

Figure 4. Linearized MIMO thermal model with mesh of input/output nodes on the top surface, actual $\underline{T}$ and desired temperature hill $\underline{T} \mathrm{~d}$, thermal coverage index $\underline{\mathrm{J}}$ and heat source distribution $\mathrm{q}$.

only by the radial distance of the correlated nodes $i$ and $j$. Thus, matrix $\Theta$ contains only a few of independent parameters with numerically significant values. The shawtooth raster (row-wise) parametrization of vectors $I$ and $Q$ in Figure 4 results in a diagonally banded structure of matrix $\Theta$. Finally, if the part material is homogeneous and isotropic, i.e. the heat flow conditions are uniform over the weld, and the thermal losses from its edges are equivalent to its internal conductive heat flux, as for example when the coupon of Figure 4 is part of a larger plate, then matrix $\Theta$ is diagonally uniform, i.e. its subdiagonals contain identical elements. This special structure of matrix $\Theta$ facilitates greatly the real-time computation of the MIMO model of Eq. 1, through sparse matrix techniques involving order reduction and vector algebra (Ref. 18).

The values of the independent parameters in matrix $\Theta$ can be conveniently estimated by the numerical simulation of scan welding at a thermal equilibrium corresponding to nominal conditions $\underline{T}_{d}, Q_{d}$ of linearization for Eq. 1, through unit perturbations of the temperatures and heat inputs at the respective locations. Thus, the temperature changes $\mathrm{T}(\mathrm{i} ; \mathrm{k}+1)$ computed at various nodes $\mathrm{i}$ and sampling periods $\mathrm{k}$, after the temperature $\mathrm{T}(\mathrm{j} ; \mathrm{h})$ or heat input $\mathrm{Q}(\mathrm{j} ; \mathrm{h})$ at node $\mathrm{j}$ is perturbed by $1 \mathrm{~K}$ or $1 \mathrm{~J}$ respectively, reflect the related parameters in $\Theta$. However, scan welding involves nonlinearities and time variations of heat transfer mechanisms. These include radiation effects, thermal drift of the temperature-dependent material properties, especially in the presence of structural transformations with latent phenomena (e.g. fusion and solidification), and alterations of the torch characteristics and part morphology. These effects yield time-dependent dynamic 
parameters $\Theta(\mathrm{k})$ in the linearized model of Eq. 1, during the thermal transients or process disturbances from the operating conditions. Thus, the initial off-line estimates of the essential time-varying parameters in $\Theta$ must be updated in-process, using temperature measurements $I$ for the applied heat inputs $\underline{Q}$. Such a real-time parameter identification is performed by a recursive adaptation law, based on orthogonal projection of the augmented vector $\underline{S}$ of Eq. 1 on its measured error, using an adaptation gain $\beta$ (Ref. 17):

$$
\Theta(\mathrm{k})=\Theta(\mathrm{k}-1)+\beta \cdot \underline{\mathrm{S}}(\mathrm{k}-1) \cdot\left[\underline{\mathrm{T}}(\mathrm{k})-\Theta^{\mathrm{T}}(\mathrm{k}-1) \cdot \underline{\mathrm{S}}(\mathrm{k}-1)\right]^{\mathrm{T}}
$$

As explained at the introduction, the objective of regulating the composite quality of scan weldments, i.e. its morphology, structure and properties is obtained through real-time control of the dynamic thermal field $\mathbf{T}$ to a specified temperature distribution $\underline{T}_{d}$, dictated by the desired metallurgical and thermomechanical characteristics of the joint. This reference field $\underline{T}_{d}$ can be stipulated through an off-line parametric optimization of the process conditions using the computer simulation above. Alternatively, the desired $\underline{T}_{d}$ can be designated directly through experimental measurement on the weld surface, during an optimal off-line pilot welding test in the laboratory. In this reference test, the temperature hill is recorded by the infrared camera, in order to be reproduced subsequently by the thermal controller in real-time processing. This feedback control scheme uses in-process temperature measurements $I$ in order to determine the heat input distribution $Q$ that is required to obtain the specified thermal distribution $\underline{T}_{d}$ (Figure 4 ), repetitively in discrete time. A closed-loop regulation algorithm is necessary to compensate for process disturbances in the ambient heat transfer and the heat source performance, certain welding parameter alterations such as thermal drift of the material properties, as well as transient effects at the start an end of the operation (arc spatter, molten craters etc.). For such a thermal regulation strategy, if the sampling period Dt is long enough to cover the measurement, computation and process delays in each discrete-time control iteration, the MIMO linearized model of the ARMA Eq. 1 can be inverted to form the basis of a stable one-step adaptive control law. With the specified temperature values $\underline{T}_{d}(k+1)$ in place of the anticipated $T(k+1)$, and with the feedback thermal measurements $\mathrm{T}$ in place of the temperature data $\underline{T}(\mathrm{k}) . . \underline{\mathrm{T}}(\mathrm{k}-\mathrm{n})$ in the augmented vector $\underline{\mathrm{S}}(\mathrm{k})$, as well as the applied past input values $\underline{Q}(\mathrm{k}-1)$.. $\underline{\mathrm{Q}}(\mathrm{k}-\mathrm{n})$, Eq. 1 can be solved for the necessary heat inputs $\underline{\mathrm{Q}}(\mathrm{k})$ in $\underline{\mathrm{S}}(\mathrm{k})$ :

$$
\underline{\mathrm{T}}_{\mathrm{d}}(\mathrm{k}+1)=\Theta(\mathrm{k}) \cdot \underline{\mathrm{S}}(\mathrm{k}) \quad \Leftarrow \text { solve for } \underline{\mathrm{Q}}(\mathrm{k})
$$

This multivariable adaptive control scheme, further elaborated in Ref. 5, has been adapted from the original MIMO deadbeat algorithm in Ref. 19. In this reference, the stability of the thermal input and output responses $\underline{Q}(\mathrm{k}), \underline{T}(\mathrm{k})$, the asymptotic tracking of the reference temperature field (i.e. $\mathrm{T}(\mathrm{k}) \rightarrow \mathrm{I}_{\mathrm{d}}(\mathrm{k})$ as $\left.\mathrm{k} \rightarrow \infty\right)$ and the convergence of parameters $\Theta(\mathrm{k})$ is generally shown for systems with segment-bounded nonlinearities, such as the thermal dynamics of scan welding.

The resulting heat input distribution $\mathrm{Q}(\mathrm{i} ; \mathrm{k})$ could in principle be induced directly at each pixel element of the part surface in raster-scanned welding by a rapidly deflected Laser or electron beam, as already mentioned. However, in vector-scanned welding by a mechanically driven, concentrated torch, this heat flux surface (Figure 4) must be implemented by properly guided sweeping and scaling of the localized power density distribution of the heat source, manipulated through the torch trajectory $\mathrm{X}(\mathrm{k}), \mathrm{Y}(\mathrm{k})$ and intensity $\mathrm{Q}(\mathrm{k})$. As it appears in Figure 4, the point heat source introduces a radially symmetric Gaussian thermal distribution $\mathrm{q}$ at its current location, which can generate the required heat input $\mathrm{Q}(\mathrm{i} ; \mathrm{k})$ by spatial and temporal superposition as the torch moves. Thus, the torch motion should be guided to ensure an optimal fitting of the heat input surface $\underline{Q}$ by the torch power distribution $q$, i.e. towards the dynamic maximum of a thermal coverage index $J(k)$ of the Gaussian-weighted heat inputs $Q(i ; k)$ :

$$
\mathrm{J}(\mathrm{k})=\sum_{\mathrm{i}=1}^{\mathrm{lxm}} \frac{\mathrm{f} \cdot \mathrm{Q}(\mathrm{i} ; \mathrm{k})}{\mathrm{Ds} \mathrm{s}^{2}} \exp \left[-\frac{(\mathrm{x}(\mathrm{i})-\mathrm{X}(\mathrm{k}))^{2}+(\mathrm{y}(\mathrm{i})-\mathrm{Y}(\mathrm{k}))^{2}}{2 \mathrm{~s}^{2}}\right]
$$


where $f, s$ are the efficiency and radius of the Gaussian power density distribution $q$, and $x(i), y(i)$ the coordinates of each heat input location i. Thus, guidance of the torch is posed as dynamic optimization of the thermal coverage distribution J, i.e. driving the heat source along the locus of the moving maximum of the J surface. This is obtained by a steepest-ascent method, by aligning the torch velocity to the current gradient of the coverage distribution (Figure 4):

$$
\underline{\mathrm{V}}(\mathrm{k})=\mathrm{K}_{\mathrm{V}} \cdot \nabla \mathrm{J}(\mathrm{k}) \Rightarrow \mathrm{X}(\mathrm{k})=\mathrm{X}(\mathrm{k}-1)+\mathrm{K}_{\mathrm{V}} \cdot \mathrm{DJ} \mathrm{J}_{\mathrm{x}}(\mathrm{k}) \cdot \mathrm{Dt} \text { and } \mathrm{Y}(\mathrm{k})=\mathrm{Y}(\mathrm{k}-1)+\mathrm{K}_{\mathrm{V}} \cdot \mathrm{DJ} \mathrm{J}_{\mathrm{y}}(\mathrm{k}) \cdot \mathrm{Dt}
$$

with

$$
\begin{array}{ll}
D J_{x}(k)=\frac{1}{D s}\left([J(j+m ; k)-J(j ; k)] \cdot\left(1-n_{y}\right)+[J(j+m+1 ; k)-J(j+1 ; k)] \cdot n_{y}\right), & n_{y}=\frac{Y(k)-y(j)}{D s} \\
D J_{y}(k)=\frac{1}{D s}\left([J(j+1 ; k)-J(j ; k)] \cdot\left(1-n_{x}\right)+[J(j+m+1 ; k)-J(j+m ; k)] \cdot n_{x}\right), & n_{x}=\frac{X(k)-x(j)}{D s}
\end{array}
$$

where the node $j$ with coordinates $x(j), y(j)$ is the one of the smallest number $j$ among the four nodes surrounding the torch position $X(\mathrm{k}), \mathrm{Y}(\mathrm{k})$ (Figure 4). Eq. 6 actually represents a linear interpolation of the gradient of the thermal coverage index $J$ at the source location, on the basis of the J values at these neighboring four nodes. In the same way, the torch power $\mathrm{Q}(\mathrm{k})$ is modulated proportionally to the linearly interpolated value of thermal coverage $J(k)$ at its current position:

$$
\mathrm{Q}(\mathrm{k})=\mathrm{K}_{\mathrm{Q}} \cdot \mathrm{J}(\mathrm{k})
$$

with $J(k)=J(j ; k) \cdot\left(1-n_{x}\right) \cdot\left(1-n_{y}\right)+J(j+1) \cdot\left(1-n_{x}\right) \cdot n_{y}+J(j+m) \cdot n_{x} \cdot\left(1-n_{y}\right)+J(j+m+1) \cdot n_{x} \cdot n_{y}$ with $\mathrm{n}_{\mathrm{x}}, \mathrm{n}_{\mathrm{y}}$ defined as before (Eq. 6) and $\mathrm{K}_{\mathrm{V}}, \mathrm{K}_{\mathrm{Q}}$ the velocity and power gains. Eqs. 6 and 7 define the control law of the torch motion from point $X(k-1), Y(k-1)$ to $X(k), Y(k)$ and torch power $\mathrm{Q}(\mathrm{k})$ during the $\mathrm{k}$-th sampling period $\mathrm{Dt}$. Implementation of this heat source transition in the laboratory setup by a servosystem or robot, as in Figure 2, ensures a smooth torch trajectory between its two successive locations, consisting of a straight, constant velocity segment with parabolic blends at its ends, due to the acceleration and deceleration of its motion. Finally, the heat source power $\mathrm{Q}(\mathrm{k})$ follows a rapid first-order (exponential) transient to its final value during the sampling period $\mathrm{Dt}$, because of the fast dynamics of the welding transformer.

\section{REAL-TIME THERMAL CONTROL TESTS}

The thermal regulation strategy above has been implemented in scan welding both by off-line computation, with the simulation software in place of the process hardware, and by real-time experimentation on the plasma-arc laboratory setup, with feedback of infrared thermal images to the control computer, under identical conditions. The scan welding tests involve processing of the stainless steel coupons of Figure 4 along a composite weld centerline consisting of three segments as in Figure 5, with process parameters similar to the open-loop configuration of Figure 3. The desired dynamic reference field $\underline{T}_{d}(t)$ is specified so as to ensure a longitudinally uniform thermal distribution, with a smooth normal temperature profile across the weld bead (Figure 5a), and with a uniform exponential rise in time from the ambient to the final thermal conditions. This nominal distribution $T_{d}$ was developed through numerical simulation, by a constant, uniform line heat source along the weld centerline segments, with power density $30 \mathrm{~kW} / \mathrm{m}$.

In the computational model, the material parameters correspond to those of stainless steel (304). The PAW torch characteristics, i.e. its efficiency $\mathrm{f}=0.71$ and distribution radius $\mathrm{s}=1.2 \mathrm{~mm}$, are calibrated by comparison of the simulated solidus $\mathrm{T}_{\mathrm{S}}$ isotherm dimensions to the size of the weld nugget section. The torch power is limited by saturation to the amperage range $20-120 \mathrm{~A}$, and the manipulator motion is constrained within the coupon area, when is occasionally commanded to a location off the weld edges. The computer regulation routine implements the control laws of Eqs. 6 and 7 for the torch motion $\mathrm{X}(\mathrm{k}), \mathrm{Y}(\mathrm{k})$ and power $\mathrm{Q}(\mathrm{k})$ respectively, on the basis of the deadbeat 

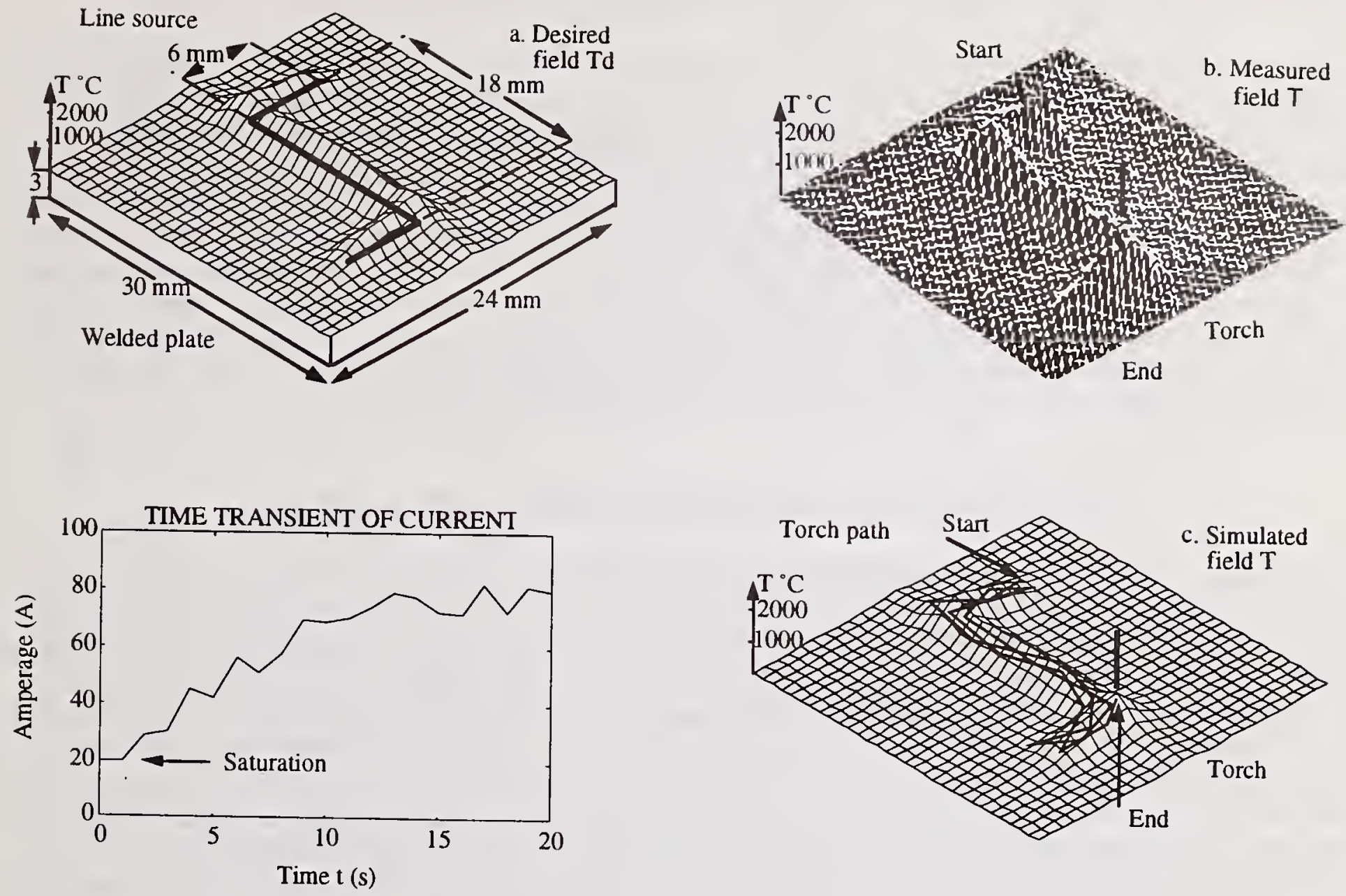

Figure 5. Closed-loop thermal control of scan welding. a. desired temperature field $\underline{T}_{d}$ (simulated) $\mathrm{d}$. Time response of torch heat input $\mathrm{Q}(\mathrm{k})$

b. Experimental infrared temperature hill $\mathrm{I}$ c. Simulated temperature hill $\mathrm{T}$ with torch path.

MIMO algorithm of Eq. 4. The parameters $\Theta(k)$ in this linearized formulation are updated by the adaptation law of Eq. 3 from their initial estimates, obtained by unit thermal perturbations of the numerical simulation at the initial conditions $\mathrm{Q}_{\mathrm{d}}=0, \mathrm{~T}_{\mathrm{d}}=\mathrm{T}_{\mathrm{a}}$, as already explained. The adaptation gain is empirically determined to $\beta=0.8$, the control gains $\mathrm{K}_{V}=0.92 \cdot 10^{-6} \mathrm{~m}^{2} / \mathrm{J}, \mathrm{K}_{\mathrm{Q}}=1.4 \cdot 10^{-3} \mathrm{~m}^{2}$, and the sampling period of the control iterations is $\mathrm{Dt}=1 \mathrm{~s}$.

Figure $5 b$ illustrates the eventual temperature hill $I$ on the top surface of the scan welded coupon after $\mathrm{t}=20 \mathrm{~s}$, as measured by the infrared pyrometer, while Figure $5 \mathrm{c}$ shows the respective prediction of the thermal field $T$ by computer simulation. As in the open-loop tests of Figure 3, their comparison validates the utility of the numerical model as an off-line computational testbed for the development of real-time thermal control systems in scan welding. The RMS temperature deviation of the experimental from the simulated field is $28 \mathrm{~K}$, while its respective error with respect to the reference distribution $\underline{T}_{d}$ is $61 \mathrm{~K}$. This larger thermal deviation of the actual from the desired temperature field is attributed to speed limitations of the closed-loop system due to the prolonged sampling period $\mathrm{Dt}$, which is needed to provide for the time-consuming multiple temperature measurements at the weld surface nodes. Despite the bandwidth constraints of the infrared sensor, the feasibility of successful regulation of the two-dimensional thermal distribution in scan welding by the previous control strategy is clearly demonstrated in Figure 5. As in the off- 
line experiments (Figure 3), the deviations of the thermal hill $I$ from $I_{d}$ are only significant at the torch location because of plasma-arc effects, as well as at the ends of the weld bead on the coupon edges, because of uncertainties in the assumed heat transfer conditions to the environment.

The computed path of the heat source during this closed-loop test is also shown in Figure 5c, and consists of a zig-zag line defined by the successive torch locations $\mathrm{X}(\mathrm{k}), \mathrm{Y}(\mathrm{k})$, within a band of width about $\pm 3 \mathrm{~mm}$ along the weld centerline. As expected, this jagged torch trajectory attempts to track the moving peak of the thermal coverage surface $J$ in real time, i.e. the weld region with the highest heat input demand. For example, as the torch operates near one of the weld centerline ends, the other end finds the time to cool down yielding a larger deviation from the reference field, and thus the controller directs the heat source towards that end. This results in a continuous reciprocation of the torch along the weld joint, in conformity to the scan welding motion. The respective time response of the applied heat source power $\mathrm{Q}(\mathrm{k})$ is depicted in Figure $5 \mathrm{~d}$. It appears that this transient of the current follows the exponential evolution of the specified thermal reference $\mathrm{T}_{\mathrm{d}}$, while its noise peaks can be correlated to the crooked torch trajectory.

\section{COMPARATIVE EVALUATION AND CONCLUSION}

This special morphology and dynamics of the temperature field in computer-controlled scan welding gives rise to peculiar thermal characteristics of the joint relative to traditional welds. Figures 3 and 5 illustrate the different features of the two arrangements. In traditional welding, the concentrated temperature hill results in a localized ellipsoidal weld pool, which melts the base material at its front part and solidifies at its back part in the longitudinal direction of the joint, as it proceeds along with the torch motion. Thus, in classical serial welding the sequential torch motion produces the weld bead in progressive longitudinal steps as the torch moves. On the contrary, in scan welding the longitudinal uniformity of the temperature hill along the weld direction yields an elongated pool covering the full length of the joint, and growing and solidifying in the crosssectional (transverse) directions. Thus, the torch reciprocation in scan welding deposits the entire weld bead length simultaneously, in gradual cross-sectional increments. As a result, scan welds do not display the familiar ripples on the bead surface, and eliminate craters and strikes where the arc is initiated or extinguished, since this position is then overlapped by torch reciprocation. As already mentioned, these defects are a major cause of failures (Ref. 3)

More importantly, this difference in the liquid-solid interface geometry and the dissimilar thermal boundary conditions from the heat source in the two methods, result in different melt circulation patterns in the pool. In conventional welding, the steady-state geometrical configuration of the pool following the torch motion, yields at equilibrium a fully developed, constant 3-D flow field with intense convective heat transfer and rapid melting and freezing of the metal at the leading and trailing interface fronts respectively. This vigorous circulation of the melt results in steep temperature gradients in the pool and fast solidification in the mushy zone, causing solidification defects (Ref. 2). On the other hand, in scan welding the distributed heat input produces much smoother thermal slopes on the pool surface, and thus considerably weaker surface tension and torch momentum-induced flow streams, yielding a conduction-dominated heat flow. The elongated puddle with changing cross sectional geometry results in a transient 2-D circulation scheme, agitated periodically at each torch pass and causing fusion and solidification in progressive steps at the lateral weld interface. The controlled melt freezing and slow development of the scan weld bead limit drastically solidification defects. The solidification rate of scan welds is no longer dependent on the travel speed of the torch as in classical joints, but can be separately controlled by the diminishing source power at the end of welding. Thus, cooling of the scanned molten bead can be specified as slow as needed, resulting in the desired microstructure of the weld.

Thus, in the heat affected zone the controlled cooling rates and smooth thermal gradients of scanned joints ensure the development of the desired material phase distribution. The continuous, 


\section{Weld Bead width for TRaditional and Scan Weldinc}

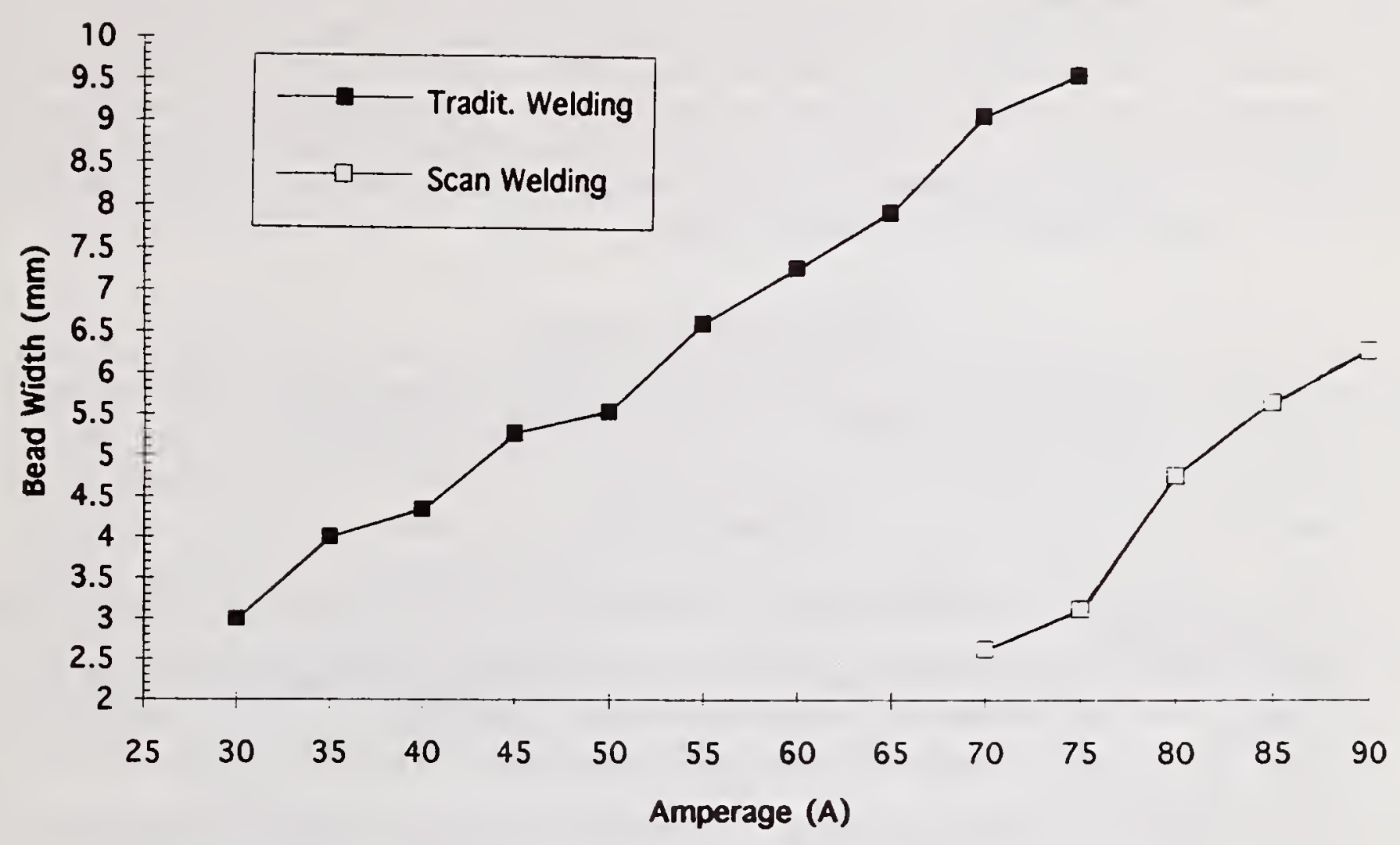

Figure 6. Dependence of traditional and scan weld widths on torch power.

gradual nature of thermal effects in scan welding results in better regulation of material equilibrium transformations, i.e. sensitization (depletion of $\mathrm{Cr}$ by intergranular formation of $\mathrm{Cr}_{4} \mathrm{C}$ ), grain growth and possible formation of $\sigma$-phase (Ref. 1). Moreover, the controlled thermal cycles to which the scan weld material is subjected, provide close regulation of cooling rate-dependent, nonequilibrium phase transformations, such as the nucleation of $\delta$-ferrite and carbon supersaturation. These also limit thermal cracking because of residual stresses and possible distortion effects of the joint. Thus, scan welding reduces or completely eliminates the necessity for initial preheating, or subsequent remedial thermal processing of the weld, as these operations can be performed inprocess by a proper heat input distribution of the scanned torch. This favorable metallurgical structure influences the fracture toughness, as well as the fatigue and creep strength, the oxidation, corrosion and stress-corrosion cracking resistance of scanned weld joints, and thus contributes to the mechanical properties of the joint. In fact, a comparative static tensile strength testing (ASTM standard A 370-92) perpendicular to the joint on a universal testing machine has shown that scan welding resulted in a slightly but consistently higher (by about 5\%) fracture load than traditional welding (Ref. 8).

In summary, the previous discussion revealed the weld quality benefits of introducing computer hardware technology, as well as control software methodology, to the redesign of traditional sequential techniques to scan welding methods. These advantages are coupled to several productivity benefits, such as higher welding throughput and/or lower processing cost. These can indeed be realized, despite the fact that more power is needed in scan welding to obtain the same weld bead width as in classical operations, as illustrated in Figure 6 . One reason for improved production speed or efficiency is that the heat source can be operated closer to its maximum or optimal power rating in its scanned motion by the thermally regulated setup. More important, automated realization of any desired thermal distribution leads to elimination of auxiliary stages of 
the welding operation, i.e. preheating and postheating steps, since these can be conveniently implemented in-process by the thermally controlled scanning system. Moreover, simplification or obviation of certain weld inspection requirements can be realized by the higher level of confidence to the weld quality characteristics. These benefits make thermal control in scan welding particularly valuable to critical applications, such as near-net shape and agile manufacturing of thermally sensitive materials, with demanding specifications in geometrical tolerances, structure requirements and resulting properties. As demonstrated in this paper, both in off-line numerical modeling and in real-time thermal control of scan welding, the computer has been instrumental in transforming the primitive practices of the human smiths of the past into the modern automated welding equipment and know-how in the manufacturing industry.

\section{ACKNOWLEDGMENT}

This research is supported by NSF Grant DMI-9553038.

\section{REFERENCES}

1. Linnert, G.E. 1968. High Alloy Steels Fundamentals, Metals Engineering Institute, ASM, Metals Park, $\mathrm{OH}$.

2. Hayden, H.W.; Moffatt, W.G.; and Wulff, J. 1965. The Structure and Properties of Materials, Vol. III, Mechanical Behavior: NewYork: J. Wiley.

3. Masubuchi, K. 1980. Analysis of Design and Fabrication of Welded Structures: New York: Pergamon Press.

4. Kannatey-Asibu, E. 1989. Split-Beam Laser Welding. Trends in Welding Research: 443451. Gatlinburg, TN: ASM International.

5. Doumanidis, C.C. 1995. Thermal Regulation of Material Transformations in MultipleSource Arc Welding. Welding Journal (June): 185s-196s.

6. Doumanidis, C.C. 1996. Scan Welding Method and Apparatus. U.S. Patent No. 5,552,575: Medford, MA: Tufts University.

7. Lukens, W.E.; and Morris, R.A. 1982. Infrared Temperature Sensing of Cooling Rates for Arc Welding Control. Welding Journal (June): 27-39.

8. Doumanidis, C.C. 1993. Scan Welding: A New Distributed Thermal Process. AWS Intl Conference on Modeling and Control of Joining Processes: Orlando, FL: 273-280.

9. Steen, W.M. 1991. Laser Material Processing. London, UK: Springer-Verlag.

10. Younse, J.M. 1993. Mirrors on a Chip. IEEE Spectrum (November): 27-31.

11. Paschkis, V. 1943. Establishment of Cooling Curves of Welds by Means of Electrical Analogy. Welding Journal 22: 462-469.

12. Cristensen, N.; Davies, V.L.; and Gjermundsen, K. 1965. The Distributions of Temperatures in Arc Welding. British Welding Journal 12 (February): 54-75.

13. Rosenthal, D. 1941. Mathematical Theory of Heat Distribution During Welding and Cutting. Welding Journal 20 (5): 220s-234s.

14. Hibbitt, H.D.; Marcal, P.V. 1973. A Numerical Thermomechanical Model for the Welding and Subsequent Loading of a Fabricated Structure. Computers \& Structures 3: 1145-1174.

15. Marquis, B.P.; Doumanidis, C.C. 1993. "Distributed-Parameter Simulation of the Scan Welding Process", IASTED Intl. Conf. Modelling \& Simulation. Pittsburgh, PA: 146-149.

16. Tzafestas, S.G. 1982. Distributed Parameter Control Systems. Oxford, UK: Pergamon Press.

17. Astrom, K.J.; Wittenmark, B. 1995. Adaptive Control. Reading, MA: Addison-Wesley.

18. Duff, I.S.; and Stewart, D.J. 1979. Sparse Matrix Proceedings. Philadelphia, PA: SIAM.

19. Goodwin, G.C.; Ramadge, P.J.; and Caines, P.E. 1980. "Discrete-Time Multivariable Adaptive Control. IEEE Trans. on Automatic Control AC-25/3 (June): 449-456. 


\title{
A METHOD FOR AUTOMATIC VOLTAGE TUNING OF CONVENTIONAL GMAW
}

\author{
G.C. Carvalho", T.E.B. Ogunbiyi ${ }^{\dagger}$, R. McMaster ${ }^{\dagger}$
}

\begin{abstract}
Published works have shown that on-line tuning of welding voltage for achieving process stability is feasible if the mode of metal transfer and the stability of the process can be objectively assessed and quantified. This paper presents a method of attaining process stability in conventional gas metal arc welding by automatic voltage tuning (AVT). The AVT algorithm utilises fuzzy production rules, which use as inputs the values of four monitoring indices calculated from the welding current and voltage waveform features, the output of which is the voltage control instruction. The control objective is to ensure that the welding voltage is not too low or unnecessarily high, thereby preventing unstable arc and minimising the level of fume and spatter generated.
\end{abstract}

\section{INTRODUCTION}

Current research work (Ref. 1) has shown that when welding procedures, that were established in a previous work (Ref. 2) as giving good weld quality and stable welding arcs on one specific power source, were transferred to another from the same manufacturer, problems such as bad ignition, excessive spatter and fume generation occurs. These problems were eliminated by trimming the welding voltage to a suitable level.

The effectiveness and consistency of the trimming process, however, depends on the skill and subjective judgement of the welder(s). This led to the development of the automatic voltage tuning (AVT) algorithm presented in this paper.

There is already a growing trend in equipping welding power sources with on-line automatic voltage control to ensure process stability (Refs. 3,4). This is achieved by monitoring and objectively assessing the welding process stability in real time and then controlling the welding voltage (Refs. 3, 5) and current (Ref. 4) based on the result of the assessment.

\footnotetext{
" GRACO - University of Brasília - UnB - Brasília, DF, Brasil

${ }^{\dagger}$ SIMS - Cranfield University - Cranfield, Bedford, MK43 0AL, UK
} 
Process stability is generally assessed by using descriptive statistical measures such as the standard deviation and the coefficient of variation. For example, in dip transfer the standard deviation of variables such as the arc voltage, the short-circuiting and arc current, the arc and short circuiting period are used as a measure of process stability. The smaller these values, the more stable the process is (Ref. 6). It should be noted that most of the published works on the stability of gas metal arc welding, mainly focused on dip transfer; globular transfer is generally considered to be unstable while spray transfer is considered naturally stable. However, in contrast to the established practise of using descriptive statistical measures for stability assessment, some authors are using ratios developed from the features of the welding current and voltage transient waveforms (Ref. 7).

The objective assessment of the stability of a welding process is critical to the on-line tuning of welding voltage. The tuning objective is to ensure good arc stability while assuming that the travel speed and wire feed speed are adequate.

The first published work (Ref. 5), seen by the present authors concerning on-line voltage tuning, used the arc stability index developed by Mita et al. (Ref. 8) for assessing the arc stability of $\mathrm{CO}_{2}$ welding. The Mita's stability index was a regression model developed from the normalised standard deviation of short-circuiting time, arcing time, average short-circuiting current and average arcing current, correlated with the subjective assessment of the arc stability provided by experienced welders. Won and Cho (Ref. 5) used the index as input into a fuzzy-logic based controller, that iteratively seeks a stable arc condition for dip mode metal transfer by automatically adjusting the welding voltage until stability is achieved. The same authors (Ref. 5) used the minimisation of the Mita's stability index as their objective function. Overall, the technique presented was computationally time consuming, the authors reported that it takes about 15 cycles (90 seconds) to obtain a stable welding condition. In a cycle, the welding voltage and current were sampled at $1 \mathrm{kHz}$ for 2 seconds and the computation of the index and voltage correction took about 4 seconds.

In a similar work, Mita et al. (Ref. 3), used fuzzy logic method to automatically set the welding voltage in $\mathrm{CO}_{2}$ gas metal arc welding. The fuzzy rules used were developed from the standard deviation of short circuiting and arcing times. It should be noted that, previous work by Mita et al. (Ref. 8) has shown that the correlation between the stability of the welding arc and the standard deviation of these parameters becomes poor as the welding current increases. Hence, they divided the welding current into three ranges, namely: a) low range (80-200 amps); b) medium range (210-290 amps); and c) high range (greater than $300 \mathrm{amps}$ ), and used different production rules for each of the current ranges (for example 20 fuzzy production rules were used for the low current range and 25 rules, for the medium current range). Mita et al. (Ref. 3) work, compared to the work carried out by Won and Cho (Ref. 5), is a simpler approach of automatic voltage tuning; the main concern is the number of rules used. 
It should be noted that, the automatic voltage tuning method presented in this paper is conceptually similar to the work carried out by Mita et al. (Ref. 3) and Won and Cho (Ref. 5), in the sense that the process is first monitored, the stability assessed and, based on the assessment, voltage control commands are issued. The present approach is a more simplified, robust and fast automatic voltage tuning method. The whole process is controlled with only 15 fuzzy production rules (Ref. 1).

\section{THE AUTOMATIC VOLTAGE TUNING (AVT) ALGORITHM}

This section introduces the Automatic Voltage Tuning algorithm. The algorithm is implemented in two stages: process monitoring and voltage control.

The process monitoring is carried out using indices calculated from the features of the welding current and voltage transient waveforms (Ref. 7). The monitoring indices - Transfer Index (TI), Transfer Stability Index (TSI), Dip Consistency Index (DCI) and Power Ratio (PR) - give qualitative interpretation of the mode of metal transfer, an indication of the arc ignition behaviour and of the stability of the welding arc. Equations ( 1 ) to (4) define the indices.

$$
\begin{gathered}
T I=\frac{I_{\text {mean }}-I_{\text {min }}}{I_{\text {mean }}} \\
T S I=\frac{I_{\text {max }}}{I_{\text {mean }}} \\
D C I=\frac{V_{\text {mean }}-V_{b k}}{V_{\text {mean }}} \\
P R=\frac{I_{b k} V_{b k}}{I_{\text {mean }} V_{\text {mean }}}
\end{gathered}
$$

where

$I_{\text {mean }}$ is the arithmetic average of the welding current transient samples collected in a fixed time period;

$I_{b k} \quad$ is the arithmetic average of all the welding current transient samples less than or equal to $I_{\text {mean }}$;

$I_{\min }$ is the minimum value in the welding current transient samples;

$I_{\max }$ is the maximum value in the welding current transient samples: 
$V_{\text {mean }}$ is the arithmetic average of the welding voltage transient samples collected in a fixed time period;

$V_{b k} \quad$ is the arithmetic average of all the welding voltage transient samples less than or equal to $V_{\text {mean }}$

The indices have been proven to provide an accurate classification of the process stability and the mode of metal transfer, and are described in detail by Ogunbiyi and Norrish (Ref. 7). Figure 1 shows a schematic summary of how they are used to classify the state of the arc and the mode of metal transfer. The indices are however not valid for use with pulsed arc welding. Research is ongoing to develop similar indices for pulse welding.

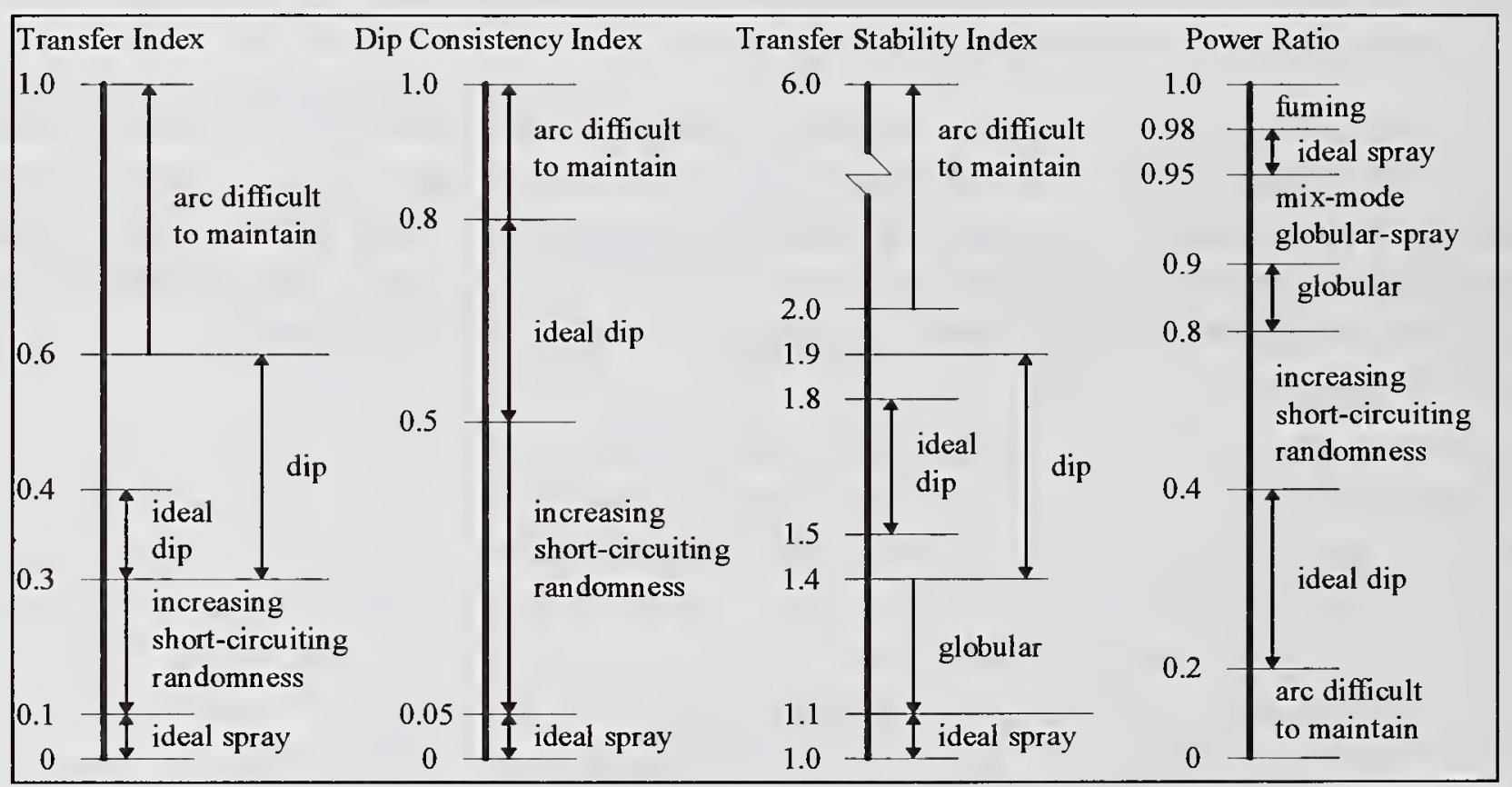

Figure 1 - Metal transfer and stability assessment chart (ref. 2)

The voltage tuning algorithm used fuzzy rules developed from the four monitoring indices above. The output of the fuzzy rules is the voltage correction value, which is added to the current value of the set-up welding voltage. The voltage tuning control equation is:

$$
V_{i+2}=V_{i}+\beta \Delta V
$$

where

$\beta \quad$ is the smoothing factor;

$V_{i} \quad$ is the set-up welding voltage in the monitoring cycle $i$;

$V_{i+2}$ is the set-up welding voltage in the monitoring cycle $i+2$;

$\Delta V \quad$ is the voltage correction value. 
The algorithm and rules for obtaining the values of $\beta$ and $\Delta \mathrm{V}$ are fully described by Carvalho (Ref. 1). It was decided that globular metal transfer mode would be prevented, since it is considered to be very unstable. Depending on the level of current, the controller moves the process to either dip or spray mode of metal transfer. A threshold welding current of $240 \mathrm{amps}$ was used for the switch. The power ratio (PR) was used as the major index for monitoring the state of the process while the other indices (TSI, TI and DCI) were found to be useful in giving indication about short term instabilities and spatter generation, mainly in dip and globular transfer modes.

The automatic voltage tuning (AVT) algorithm was designed to keep the process operating within the empirically established "stable zones" for the indices (see Figure 1) by increasing or decreasing voltage as necessary. Figure 2 shows the schematic summary of the automatic voltage tuning algorithm, showing the voltage tuning direction. The maximum voltage correction allowed in a control cycle was constrained to -1.5 or 1.0 volts. A control cycle comprises of the monitoring/control period, and the power source response time. A sampling rate of $2.5 \mathrm{kHz}$ was adopted, resulting in a monitoring/control cycle of about 250 milliseconds ${ }^{\ddagger}$. This sampling rate was used in all trials carried out in this work. It was established experimentally that the response time of the power source (that is, the time taken by the process to respond to a 2.0 volt step change) was around 200 milliseconds. Therefore the control cycle was fixed at 500 milliseconds (that is, twice the monitoring/control cycle), hence making the assumption that the process would have reached the voltage level required within a monitoring cycle. Also, the algorithm takes into consideration the time needed to establish the arc at the weld start; this was fixed at 1 second.

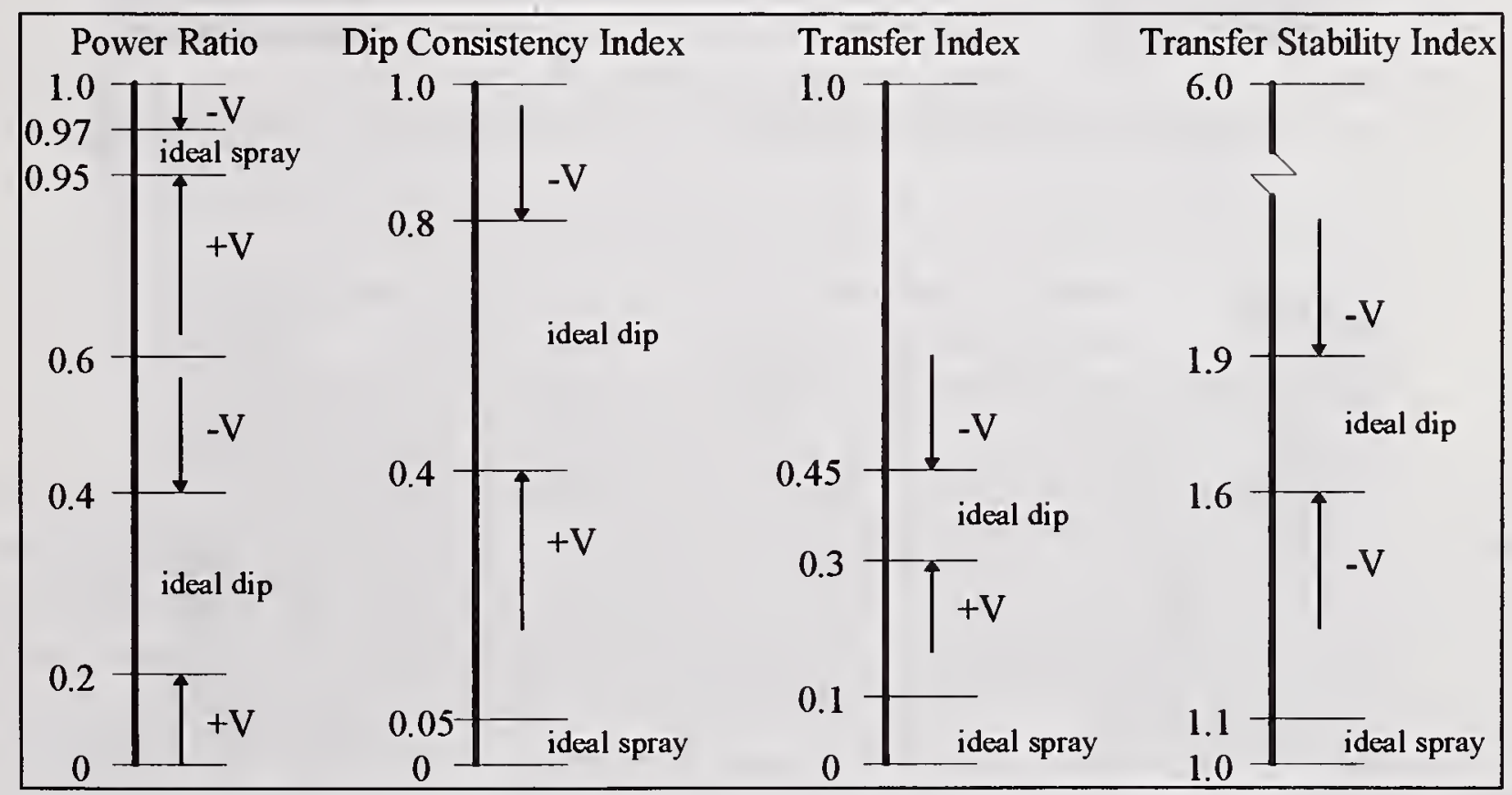

Figure 2 - AVT voltage control directions

‡ At $2.5 \mathrm{kHz}$ sampling rate, it takes 205 milliseconds for acquiring 512 samples. Some time for data transfer and computer processing elevated the monitoring cycle to 250 milliseconds approximately. 


\section{EXPERIMENTAL RESULTS}

The AVT method was implemented on a monitoring and control system, consisting of a personal computer, equipped with $\mathrm{A} / \mathrm{D}$ and $\mathrm{D} / \mathrm{A}$ channels and signal conditioning, a power source with $\mathrm{I} / \mathrm{O}$ capabilities, and voltage and current sensors. The welding trials were performed using a Migatronic BDH-550 inverter-based power source, $1 \mathrm{~mm}$ mild steel solid wire (BS $2901 \mathrm{pt} .1 \mathrm{GR}$ $\mathrm{A} 18)$ and $\mathrm{BOC}$ Argonshield $5\left(\mathrm{Ar} 5 \% \mathrm{CO}_{2} 2 \% \mathrm{O}_{2}\right)$ shielding gas.

To demonstrate the effectiveness of the tuning algorithm, several welding trials were performed with intentionally inadequate and excessive welding voltages. In all the trials carried out the welding process was quickly stabilised by the AVT algorithm in less than three seconds. Figures 3 to 9 show the welding voltage plots and the resulting bead appearance.

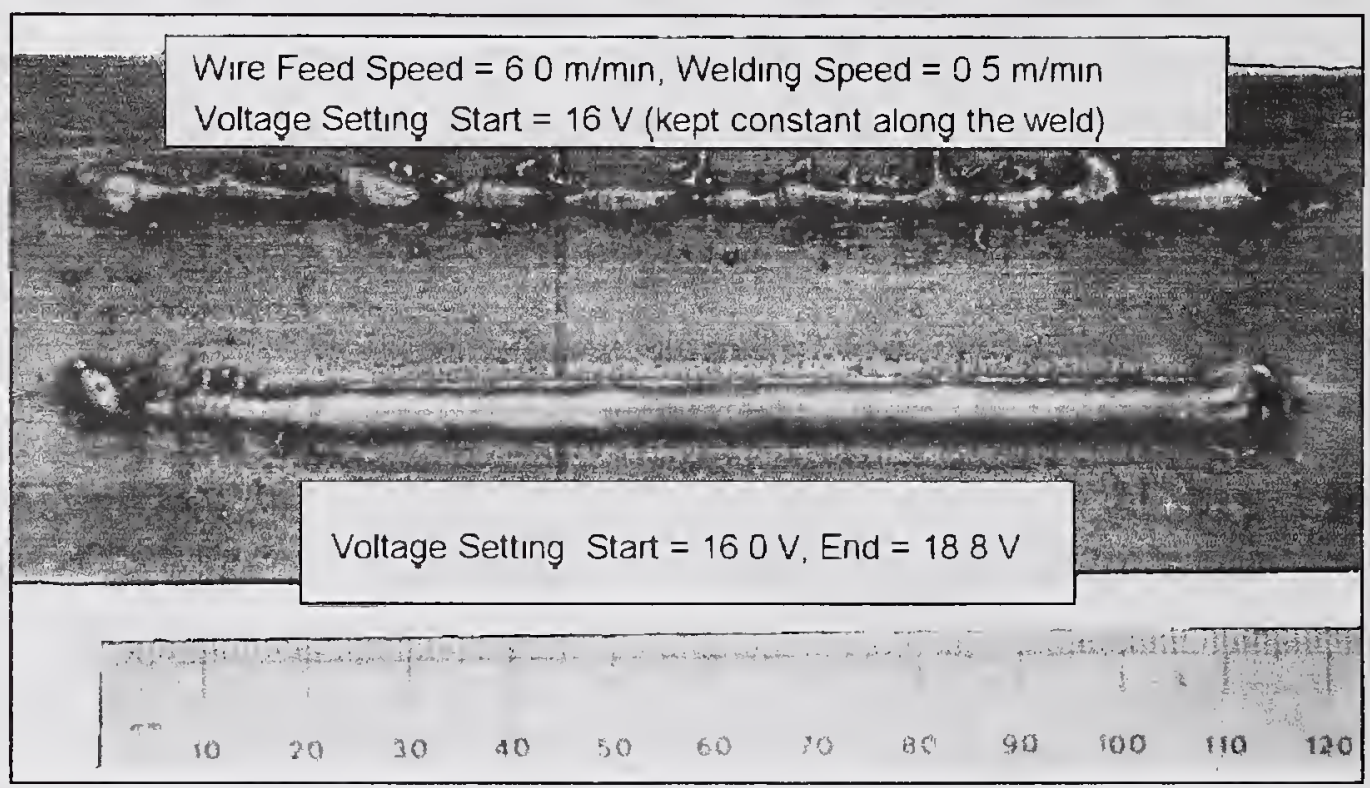

Figure 3 - Bead appearance for uncontrolled and controlled trials using insufficient initial welding voltage

Figure 3 shows a welding scenario where the set-up welding voltage was set so low that it was difficult to maintain a stable arc thus resulting in a poor quality weld. The average values of the indices, PR, TI, TSI and DCI were $0.13,0.97,3.21$ and 0.53 respectively when the process was not controlled, thus indicating and confirming the process instability in accordance to Figure 1. Under control, the AVT algorithm brings to the process to a stable state in less than three seconds and maintains the stable arc. It can also be seen from Figure 3 that, the weld quality was also quickly corrected.

Figure 4 shows the comparison between the set-up welding voltage, measured voltage and the power ratio (PR) for the controlled and uncontrolled welding trials. For clarity of the Figure, PR was multiplied by 50 and the set-up voltage, by 3 


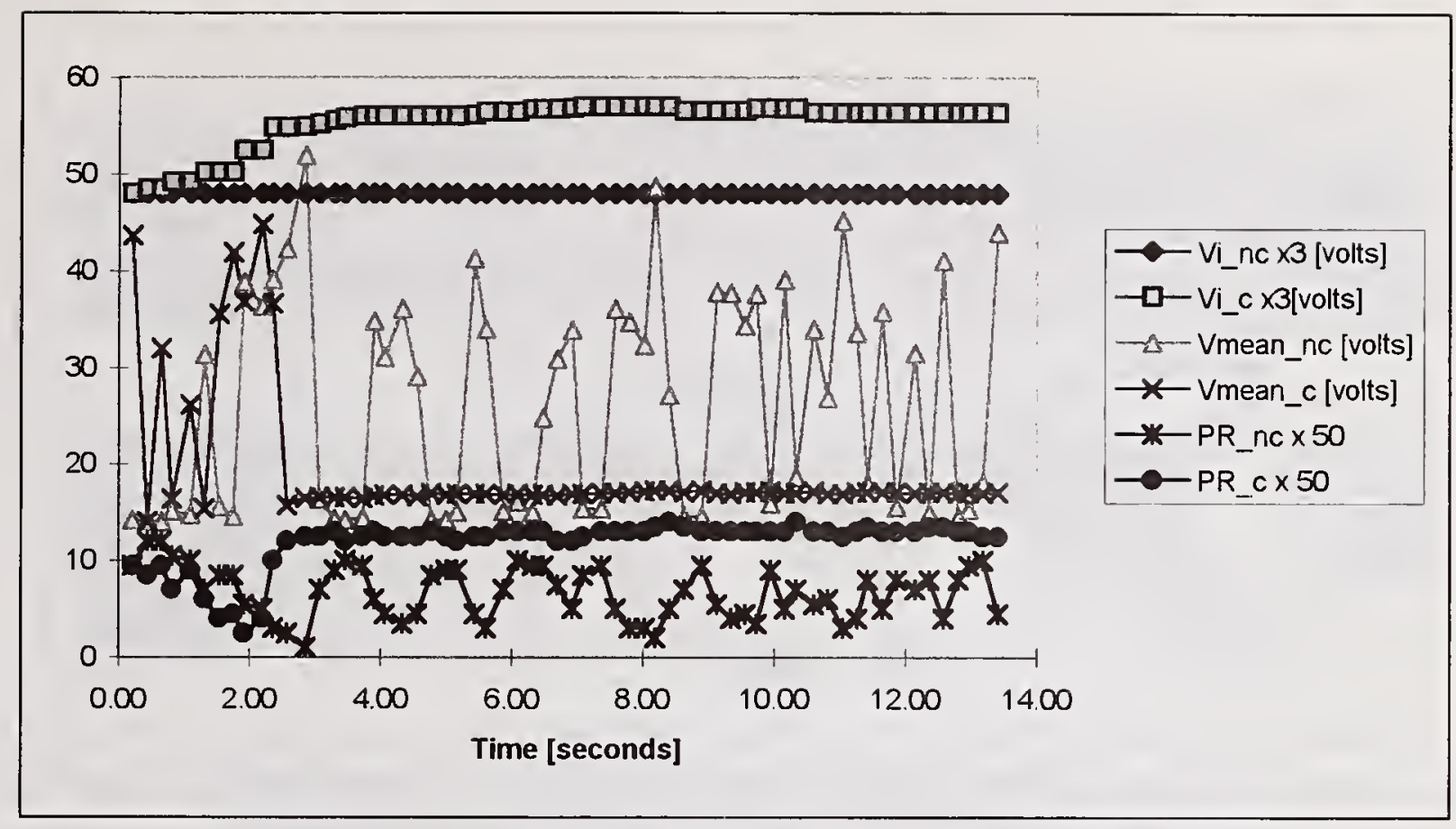

Figure 4 - Comparison between uncontrolled and controlled welding data for welds shown in Figure 3 ( $\mathrm{nc}=$ voltage not-controlled; $\mathrm{c}=$ voltage controlled)

Figure 5 shows a situation were excessive welding voltage was used for the set-up wire feed speed. The mode of metal transfer was classified as mix mode of dip and globular transfer. The indices, PR, TSI, TI and DCI were $0.65,2.68,0.27$ and 0.23 respectively. The measured current during welding was less than $240 \mathrm{amps}$, therefore the mode of transfer was changed by the algorithm to dip transfer. A stable dip transfer was achieved in less than two seconds (see Figure $6)$.

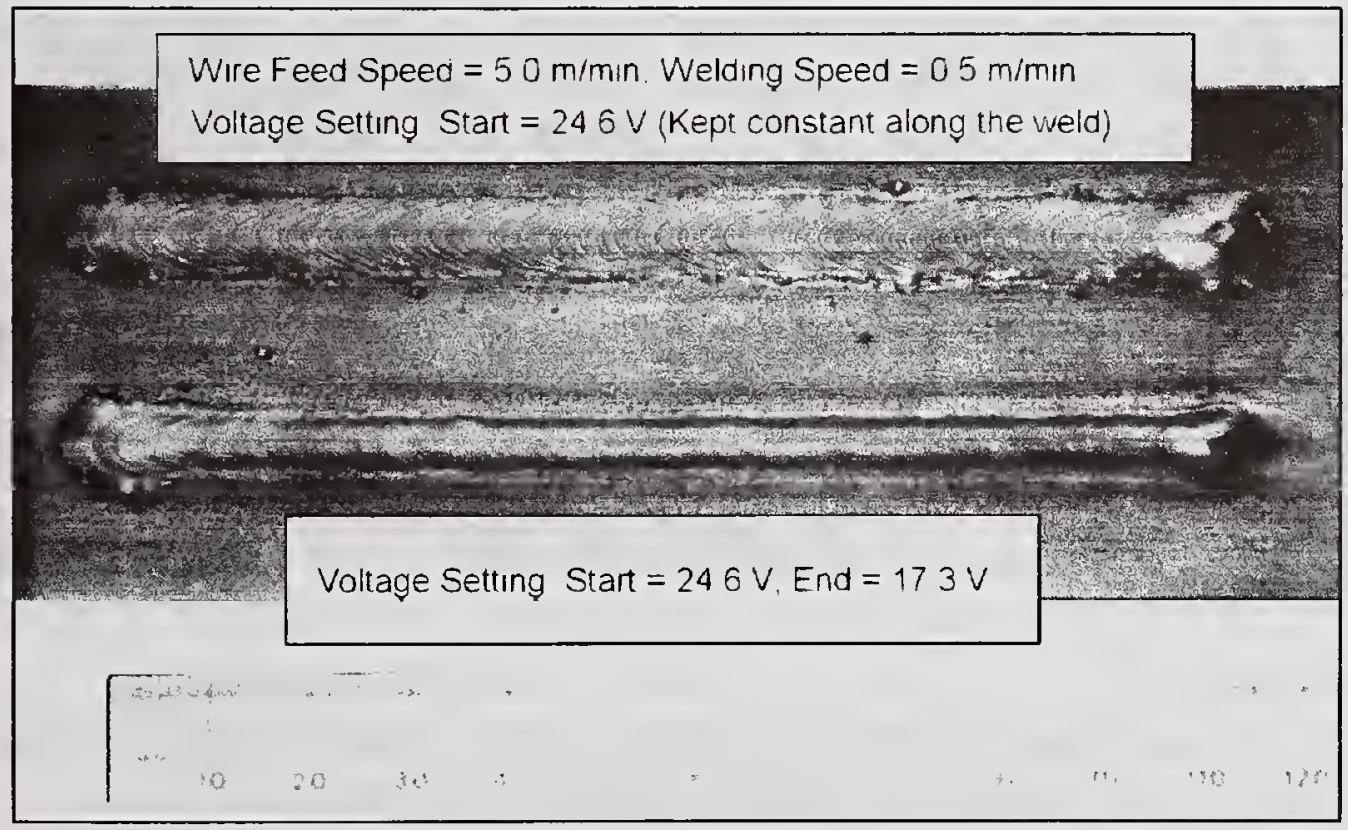

Figure 5 - Bead appearance for uncontrolled and controlled trials using excessive initial welding voltage 


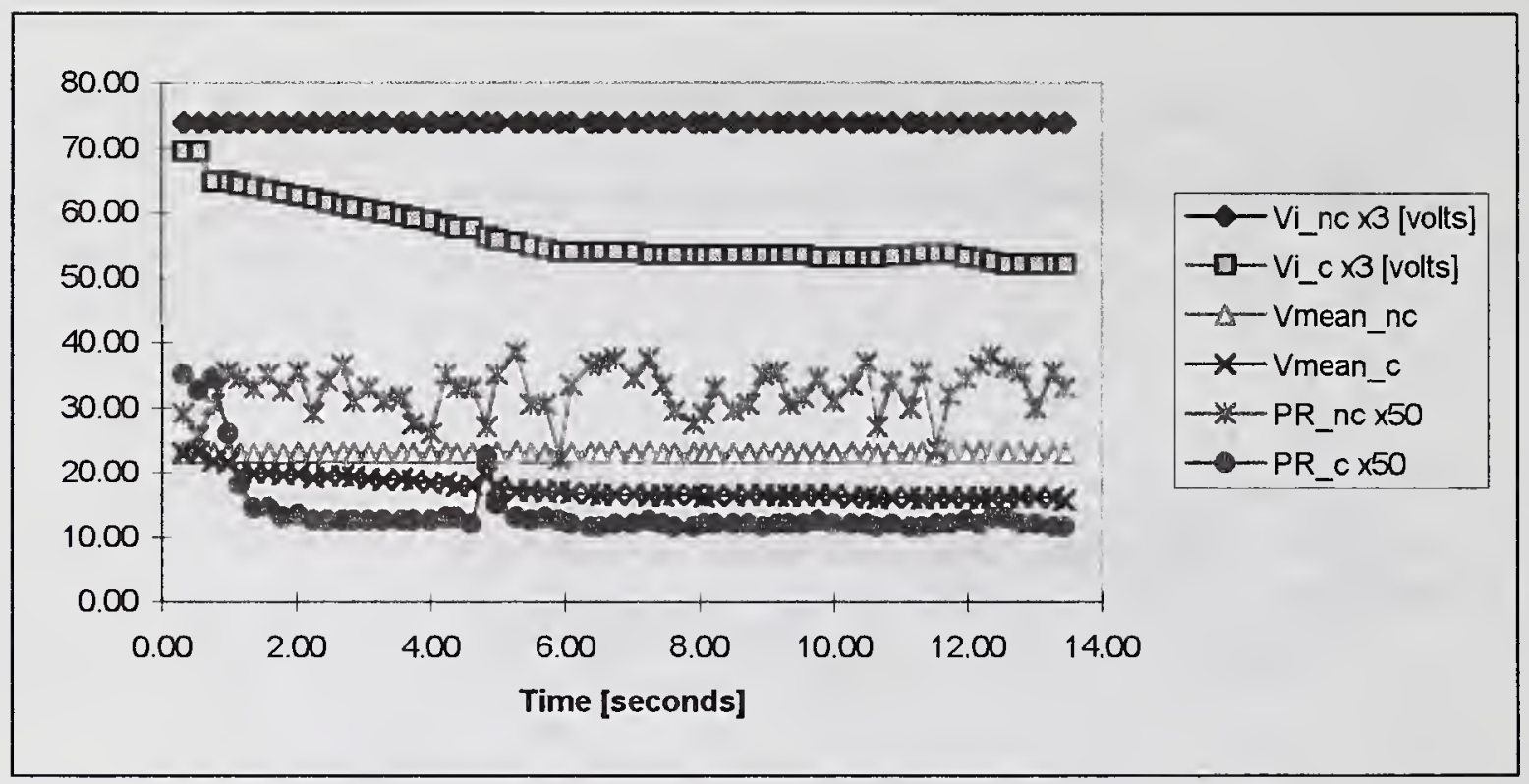

Figure 6 - Comparison between uncontrolled and controlled welding data for welds shown in Figure $5(\mathrm{nc}=$ voltage not-controlled; $\mathrm{c}=$ voltage controlled)

In spray transfer mode, welding with excessively high voltage usually leads to fumes, porosity and undercut. Figure 7 shows the situation where spray transfer with excessive voltage was used to produced a fillet weld. Porosity and undercut occurs, however, under AVT control, the porosity was eliminated and the undercut, significantly reduced (see Figure 8).

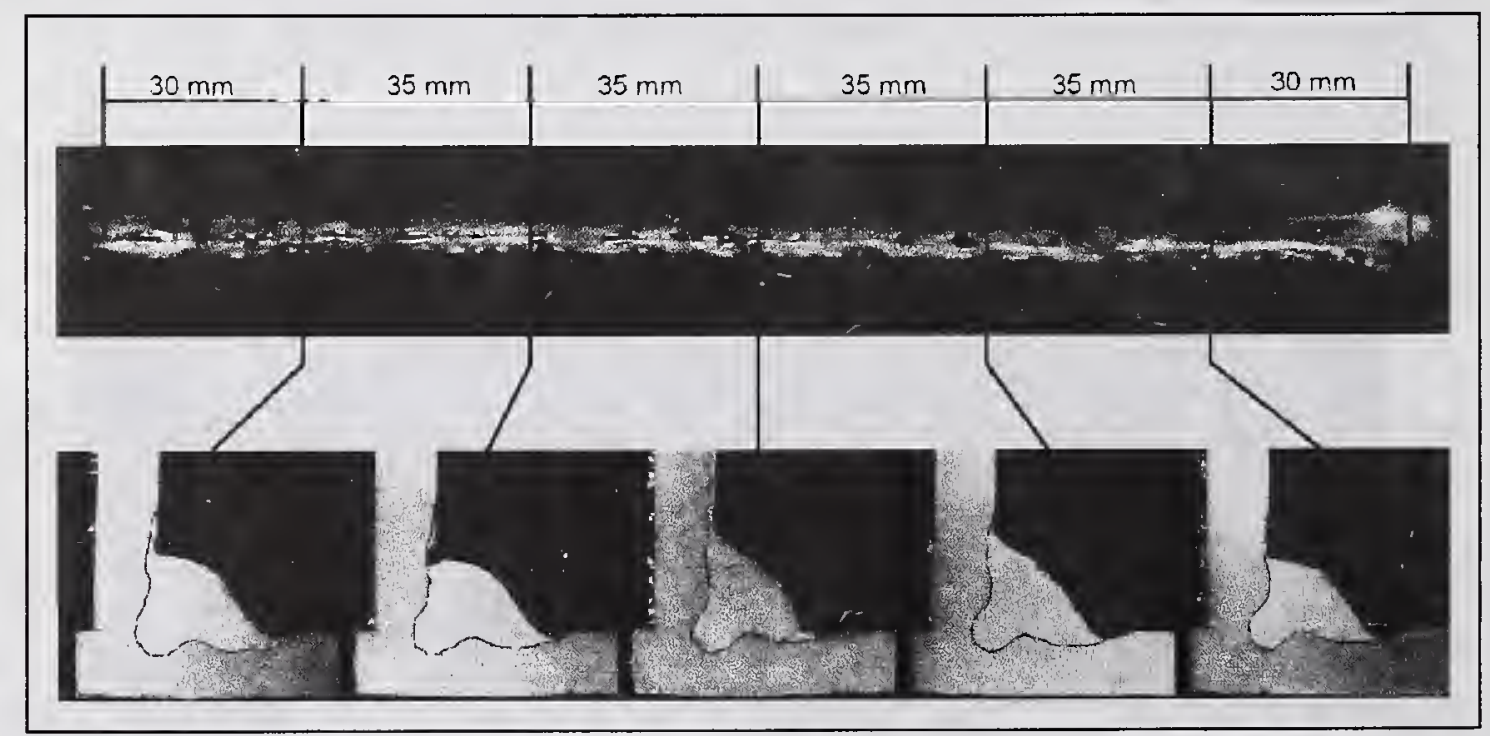

Figure 7 - Welding trial carried out with excessive voltage in spray transfer $(3.2 \mathrm{~mm}$ thick mild steel sheet in flat position with: wire feed speed $=11 \mathrm{~m} / \mathrm{min}$; welding speed $=0.8 \mathrm{~m} / \mathrm{min}$; initial welding voltage $=32.9$ volts, kept constant along the weld) 


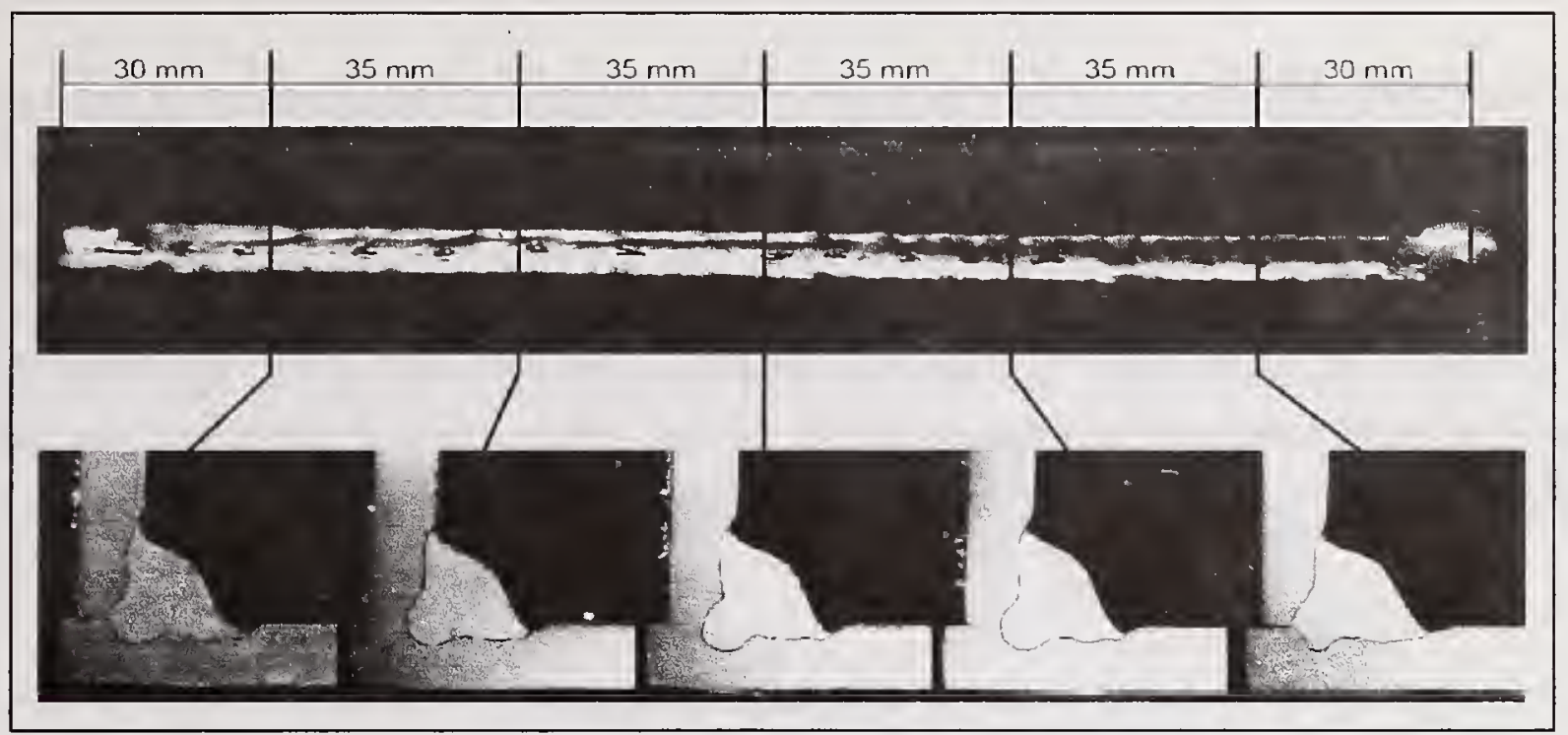

Figure 8 - Welding trial carried out with voltage control for an excessive initial welding voltage in spray transfer $(3.2 \mathrm{~mm}$ thick mild steel sheets in flat position with: wire feed speed $=11 \mathrm{~m} / \mathrm{min}$; welding speed $=0.8$ $\mathrm{m} / \mathrm{min}$; initial welding voltage $=32.9$ volts, reduced to 29.13 volts by the controller in less than 3 seconds)

Figure 9 also shows a fillet weld produced using spray transfer with excessive voltage. The resulting weld has a finger-like penetration, however, under control, this was eliminated.

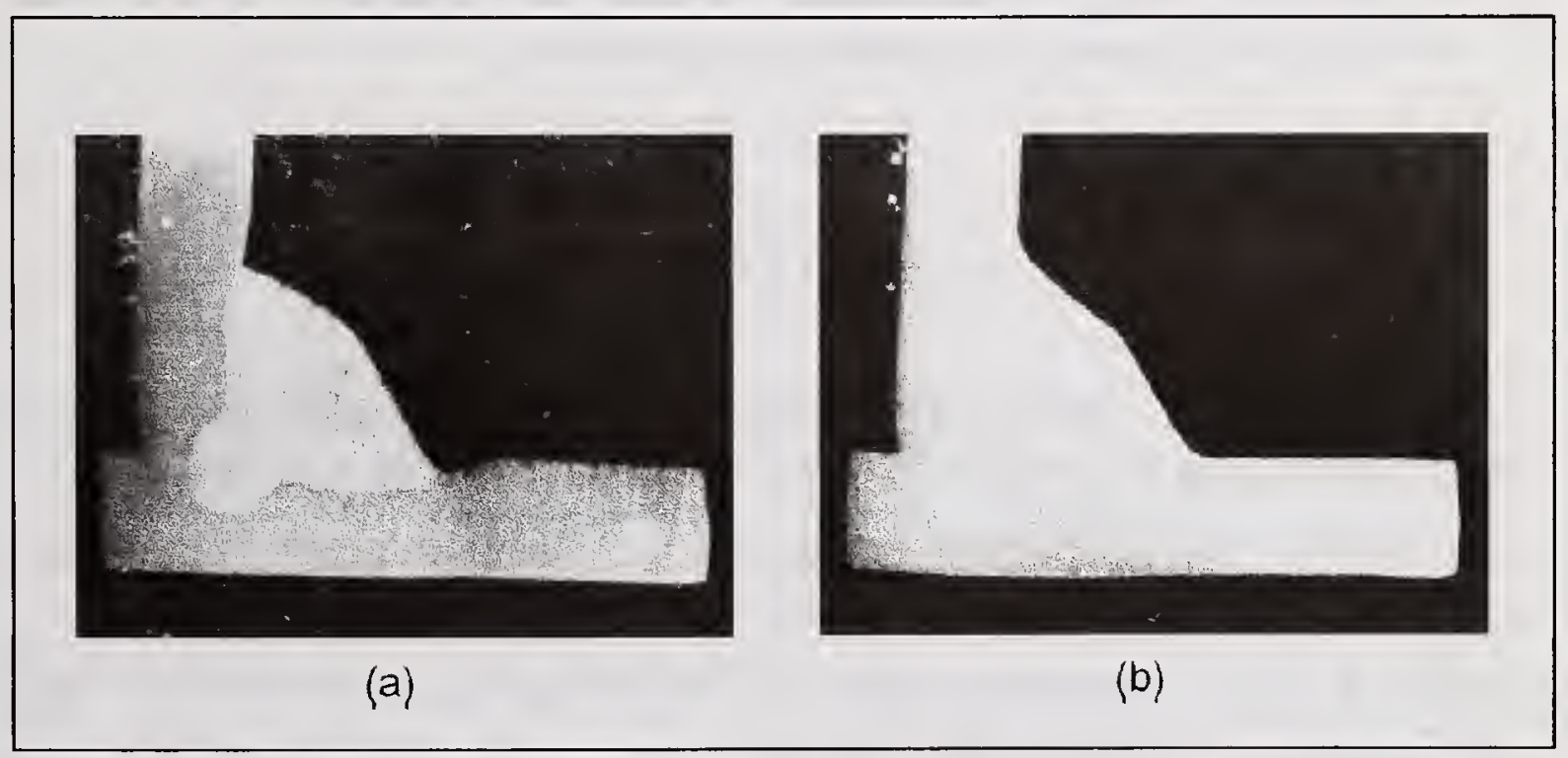

Figure 9 - Comparison between bead profiles from uncontrolled (a) and controlled (b) fillet welding trials, carried out in flat position (3.2 $\mathrm{mm}$ mild steel sheets, wire feed speed $=13 \mathrm{~m} / \mathrm{min}$, welding speed $=0.7 \mathrm{~m} / \mathrm{min}$, initial welding voltage $=33.2$ volts, reduced to 29.4 volts by the controller in less than 3 seconds) 


\section{THE BENEFIT OF CURRENT WORK}

The obvious industrial benefit of the automatic voltage tuning method presented is that it could reduce, for the power source manufacturers, the time and effort used in developing synergic welding curves for different gases and wires, by automatically setting up the welding voltage. While, it is noted that most power sources already have voltage trim facility for this; the effective use of which requires (and depends on) skilled welder(s). The results of this research work shows that the AVT method could be used to replace the trim facility. The simplicity of the monitoring rules and the algorithm meant that the method can easily be built into the controller of new generation of gas metal arc welding power sources.

\section{CONCLUSIONS}

The following conclusions can be drawn from this work:

1. if the wire feed speed and travel speed are set at an appropriate level then by controlling the voltage using AVT a stable arc and good quality weld would be obtained;

2. simple indices calculated from the welding current and voltage waveform features can be used to make quick and robust decision on how to tune welding voltage; and

3. the automatic voltage tuning method presented in this work could be used to replace the trim facility in gas metal arc welding power sources.

\section{REFERENCES}

(1) CARVALHO, G.C. Strategy for off-line programming and adaptive control of robotic gas metal arc welding. Cranfield University, PhD thesis, 1997 (not yet published).

(2) OGUNBIYI, T.E.B. Process monitoring and adaptive quality control for robotic gas metal arc welding. Cranfield University, PhD thesis, 1995.

(3) MITA, T. et al. Automatic setting of arc voltage using fuzzy logic. Welding Review International, November 1996, 130-132.

(4) YAMAMOTO, H. et al. Automatic setting of welding conditions by fuzzy controlled $\mathrm{CO}_{2} / \mathrm{MAG}$ welding machine. IN: Automatic Technology of Arc Welding, Technical Commission on Welding Processes, Japan Welding Society, IIW-DOC. XII-1471-96, 1996, II-127 to II-132.

(5) WON, Y.J. and CHO, H.S. A fuzzy rule-based method for seeking stable arc condition under short-circuiting mode of GMA welding process. Proceedings of the Institution of 
Mechanical Engineers Part 1 - Journal of Systems and Control Engineering, 206, 1992, 117-125.

(6) NORRISH, J. Advanced Welding Processes. Bristol: Institute of Physics Publishing, 1992, chapter 10.

(7) OGUNBIYI, T.E.B. and NORRISH, J. GMAW metal transfer and arc stability assessment using monitoring indices. IN: TWI Conference on Computer Technology in Welding, 9-12 June 1996, Lanaken, Belgium, paper 11.

(8) MITA, T., SAKABE, A., YOKOO, T. The estimation of arc stability on $\mathrm{CO}_{2}$ gas shielded arc welding. IN: Houldcroft, P.T. (ed.) TWI International Conference on Advanced Welding Systems, 19-21 Nov 1985, London, 261-271. 


\title{
SCIAKY W2000, A MULTIPLE WELD PROCESS CNC MACHINE CONTROLLER
}

\author{
S.R. Potter*, R. Thompson*
}

\begin{abstract}
A manufacture of Resistance, Fusion and Electron Beam welding machines for over fifty years, Sciaky Inc. has been a leader in employing sophisticated CN coordinated motion and real-time process control in many of these systems. The advent of small "mini" computers into the industrial arena during the 1970's provided the opportunity to satisfy an increasing demand for weldment production requiring complex path motions combined with highly variable welding parameters using the Electron Beam Welding process. Successful development of a sophisticated CNC controller for Electron Beam was followed by systems employing GTAW and resistance welding processes. By the mid 1980's, industrial computer technology was rapidly changing and it was realized that in order to avoid constant hardware obsolescence a long term view of control technology needs was required.
\end{abstract}

\section{INTRODUCTION}

The W2000 Control was conceived as a physically modular controller capable of being configured to meet application requirements ranging from basic velocity loop single axis control, to full CNC multi-axis systems independent of the physical machine configuration (physical machine configurations are rectilinear). In all instances of the control hardware, real-time process control was to be an integral function, while providing the user degrees of programming sophistication. In order to meet this criteria, a long term view of the potential applications was undertaken. The evaluation identified that control technology, particularly in welding applications, requires broad capability and constant development of features, interfaces and functionality. It was realized that to satisfy this continual development process the control needed two fundamental components. Modular computer hardware that would allow for system improvement without constant obsolescence, and a software language that offered high level function in industrially popular format.

The paper describes the hardware selected, the system arrangement and software development from initial concept philosophy through current day developments. Numerous application aspects are described showing the versatility of the controller.

* Sciaky Inc., 4915 W. $67^{\text {th }}$ Street, Chicago, IL 60638 


\section{CONTROL DESCRIPTION}

\section{Hardware Selection}

During the mid 1980's there was a proliferation of welding control systems. In almost all instances they were based on discrete processor systems. The result over time being wholesale obsolescence and generational replacement. When considering hardware, PC technology and commercial CNC controllers were reviewed. PC's were discounted as at that time they lacked the capability to be hard real-time controllers. CNC's in all instances were considered discrete control systems and would automatically fall victim to obsolescence by virtue of increasing CPU capability and the integrated and dedicated software arrangements needed to run those types of systems. It was necessary therefore to identify hardware that could evolve.

VME (Versa Modular Europa) hardware was finally selected. The hardware is a Bus based system utilizing a card cage and bus back plane. The hardware is based on formalized international standards requiring manufacturers of Printed Circuit Boards (PCB's) to conform to an operational standards. By following internationally agreed standards, the selection of the VME standard offered multiple sources of similar products performing at comparable and consistent levels, thereby ensuring competitive pricing and evolution.

The most persuasive issue in the selection was the perceived longevity. The format, the international standard and the numerous manufacturers of products for VME systems were indicators that a VME system could have a reasonable expectation of fifteen years or more without being overtaken by hardware obsolescence. When adopted as the hardware of choice, the target of foreseeable use was the year 2000, hence W(Weld)2000.

Figure 1 shows the basic arrangement of the control which comprises of three major hardware systems. The core hardware which resides on the backplane along with the system specific hardware such as $\mathrm{I} / \mathrm{O}$ and servo systems, the user interface where programming functions occur and the portable pendant where operational and in process functions required for real-time system operation are effected.

The modular arrangement of the control allows the addition or subtraction of $\mathrm{I} / \mathrm{O}$ and axis related cards as well as the Network Communication (NCP) microprocessor. The NCP processor is dedicated to XMMI Man Machine Interface (MMI) functions on either a SPARC (Sun) or X86 (Intel) platform. The RTP microprocessor provides real-time control of system function during operation. ICP provides communication to the portable pendant and the user computer interface. This separation between processors allows high level critical control functions to occur without interruption from requested or non-critical status and information data flows, which would burden a general purpose processor if those functions of communication were resident. The processors used in all instances of the control have been Motorola 680X0. In the first control units 68010 processors were employed. The development of the hardware has resulted in the current generation being 68040 processors. The processor speed improvement is approximately a factor of four over the original 68010 's. In all cases, 68040 processors can directly replace prior generations with only minor software patches and with no other hardware substitutions. 


\section{COMPUTER CONTROL \& INTERFACE VME BUS}

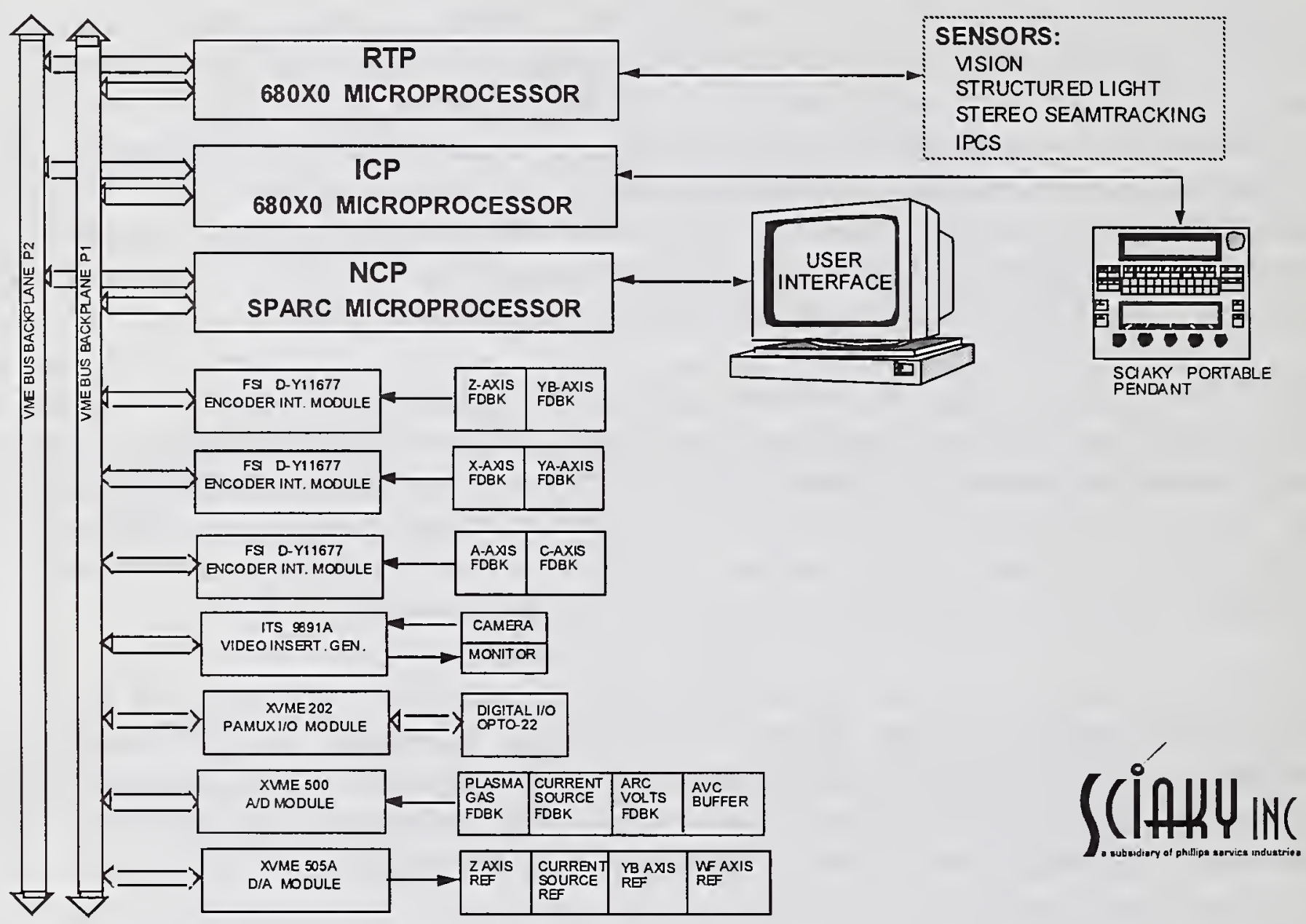

FIGURE 1

Figure 1 also shows several cards with specific functions; encoders providing axis position feedback (two axes per card); a video text insertion card for overlaying welding or system status data on CCTV images of the weld; I/O modules; D/A modules providing process reference signals and $A / D$ modules providing process feedback signals. In each instance of the control, the PCB population of the card cage may vary greatly depending on the actual application, but always reflects some number of the primary cards (encoder, I/O, A/D, D/A). With the exception of the encoder cards which are of proprietary design, almost all other cards are selected from commercial sources.

\section{Man Machine Interface}

There are two Man Machine Interface (MMI) types offered with the controller, a DOS based system and a UNIX based system. The most common unit for the programming interface is the DMMI $(D=D O S)$. Based on an industrialized PC platform, the DMMI stands alone from the controller. Utilizing DOS, three programming formats have been created for the user to select 
from. All formats are resident in the system and can be selected based on program complexity or operational preference.

For single one axis programs (linear or circumferential type welds) a graphic interface can be employed. The user selects a parameter set to be programmed such as Weld Current, and then completes a pull down table by numeric input to the specific parameter field. Initial current, run current, final current, slopes, pulse rates, etc., are completed in this manner. The user repeats this programming effort for the remaining discrete parameter sets that compose the weld and the controller complies the program. The compiled format is displayed as a multi-channel time or distance based graphic that allows the user to readily see the relationship of parameters in time/distance and scale to one another. This is a simple, quick and intuitive format designed for use by operators and engineers alike.

A second format referred to as "Blocks" offers the ability to program multi-axis positional welds where more complex axis paths are required. Presented in a menu format, up to seven axes of coordinated motion can be programmed. A "Block" is a page of the program that describes the axis moves (from one special point within the machine envelope to another) and the associated welding parameters as well as certain machine functions that the user can selectively employ. Block 1 of a program would represent pre-weld information that is preparatory in nature (positional moves to a start point and/or turning on gas flow or other system functions). Subsequent blocks then represent various stages of a weld program. Depending on complexity of the weld as few as 3 or more than 100 Blocks may be used. Each block presents three columns of operating parameters to the user. The first column contains welding related set-up information (AVC response etc.), (welding process dependent) and numeric values are input to each parameter field. The second column provides motion data, again specific to the actual machine configuration. Each axis is designated with appropriate $\mathrm{CNC}$ nomenclature and again requires a numeric input into a fixed field. The programmer can select the mode of the axis move; absolute, incremental, APT (Axis Position Table) or time based. Move modes can be intermixed within one block, thus one axis can be moved to an absolute position while another moves an incremental amount. This column also allows programming of actual welding parameters that correspond with the motion. The third column presents machine functions and allows features to be selectively turned on or off at points throughout the program (gas flow, AVC, wire feed, etc.).

The third programming format is based on a generic NC language (G, F and $M$ code) developed from a prior generation of control (Mark VII), and is more sophisticated and powerful than Graphic or Block formats. Designed for multi-axis machines where the user desires or needs to create programs using abbreviated welding terminology for complex tasks, it offers a high degree of flexibility. The interface employs function keys to access pull down menus to assist the programmer. The format allows the creation of master programs (referred to as "schedulers") and sub-routines that can be called directly from the scheduler or can be activated on a conditional basis. The format employs $G$ codes to set function type (G1, G90, etc.) which determine the function activity and $\mathrm{F}$ codes which relates to feedrate. The feedrate can be speeds or time based as designated by the $G$ code. $M$ codes are available to set special functions. The welding commands are derived from common welding terms (Wirefeed = WIR ON/OFF, Arc 
Voltage - AV, etc.). The format offers more than two-hundred abbreviated word functions to create programs. Other programming constructs such as for/next loops, schedule registers, IF logic, re-entrancy and arithmetic functions are available to accommodate a wide variety of programming application tasks. Numerous function keys are provided to assist the programmer and the user may also employ search/find functions, where used/common expressions and copy sample text from context sensitive help files directly into program files.

With the XMMI system, the interface is based on a UNIX system running X windows. Only the NC generic language format is offered for this system providing the most powerful programming and display interface. As such, it has become the interface of choice for high quality/sophisticated users. Being based on X Windows the system uses a keyboard and mouse, and employs a point and click style of operation. The UNIX system is a true multi-tasking unit and allows multiple windows to be opened for display of the welding system status as well as program status. The system is designed to allow factory wide networking via an Ethernet connection. Programs can be downloaded from a central host system providing work scheduling directly to the factory floor. Conversely, the system can transport quality assurance data and back to the host for archiving of weld results. In critical service applications this capability and the traceability it provides are essential functions of process control.

Regardless of which programming interface is used, the result is a compiled form of code that is then downloaded to the controller. This native code can be executed directly by the controller without any additional interpretation resulting in less foreground cycle and memory overhead.

Figures $2,3,4$, and 5 below show examples of the programming formats available.

The portable pendant as shown in Figure 6 provides local control and status information to the operator. The system can be run directly from the pendant once programs have been loaded into controller memory. The pendant provides jog functions, axis control, proportional process overrides via digital encoders, and segregated information displays where the operator can clearly see machine position and weld process information on the two LCD displays on a realtime basis.

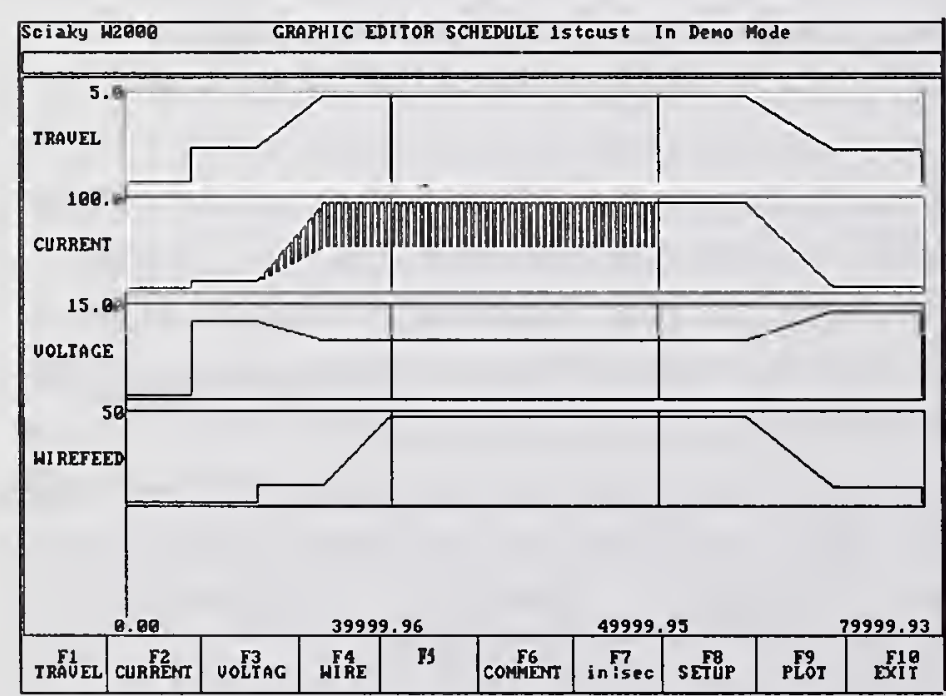

FIGURE 2

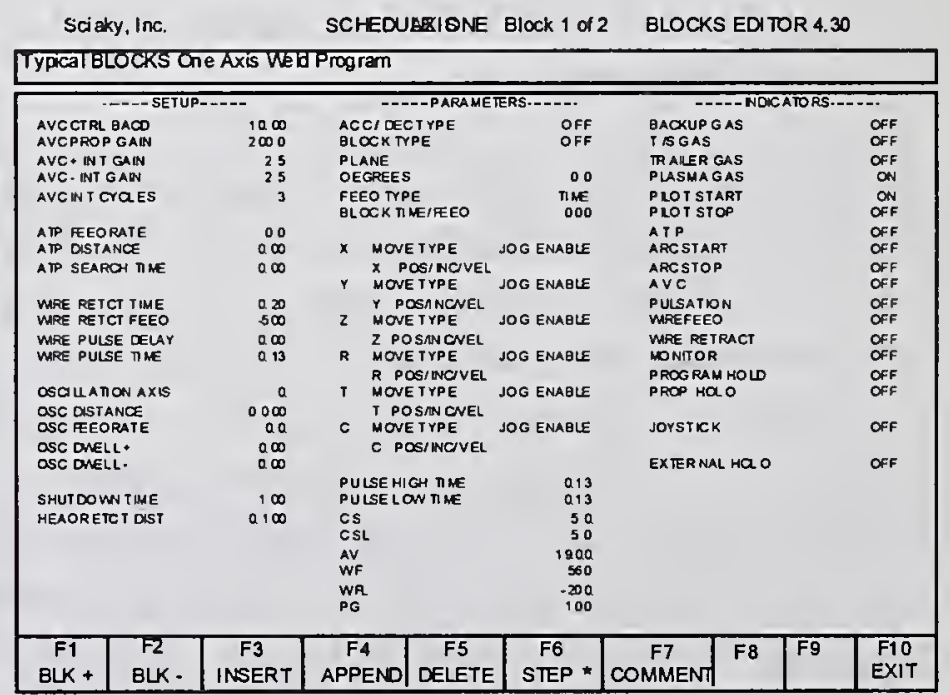

FIGURE 3 


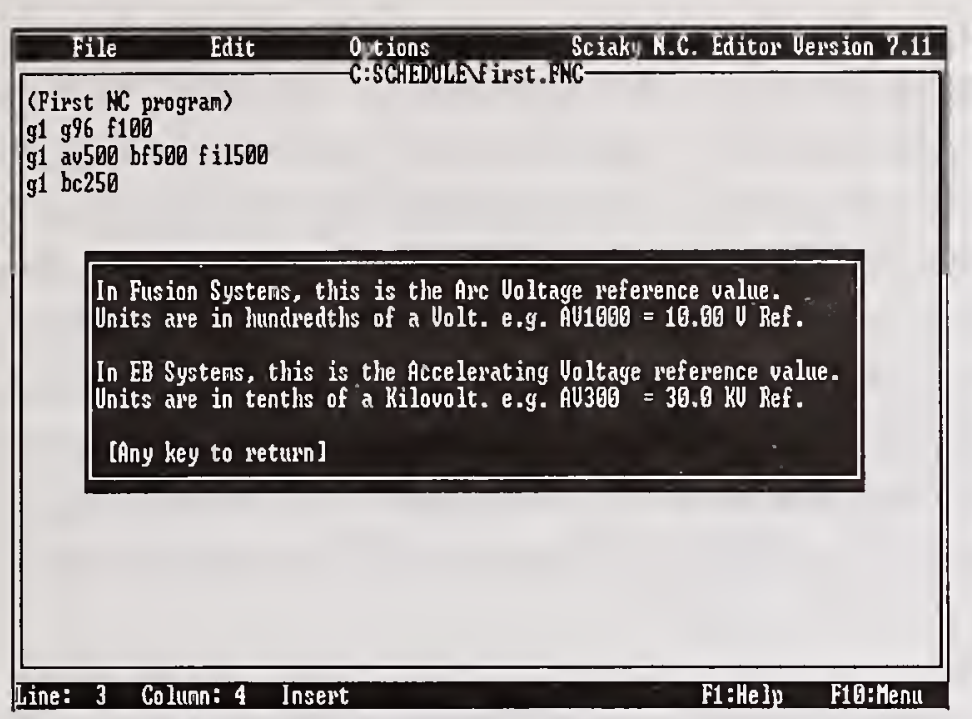

FIGURE 4

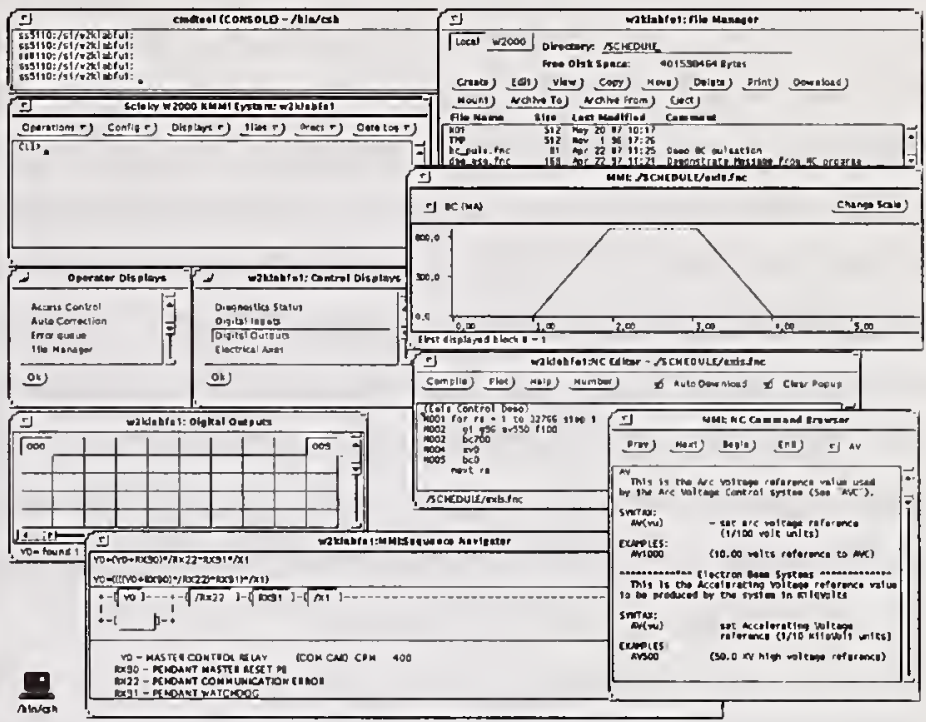

FIGURE 5

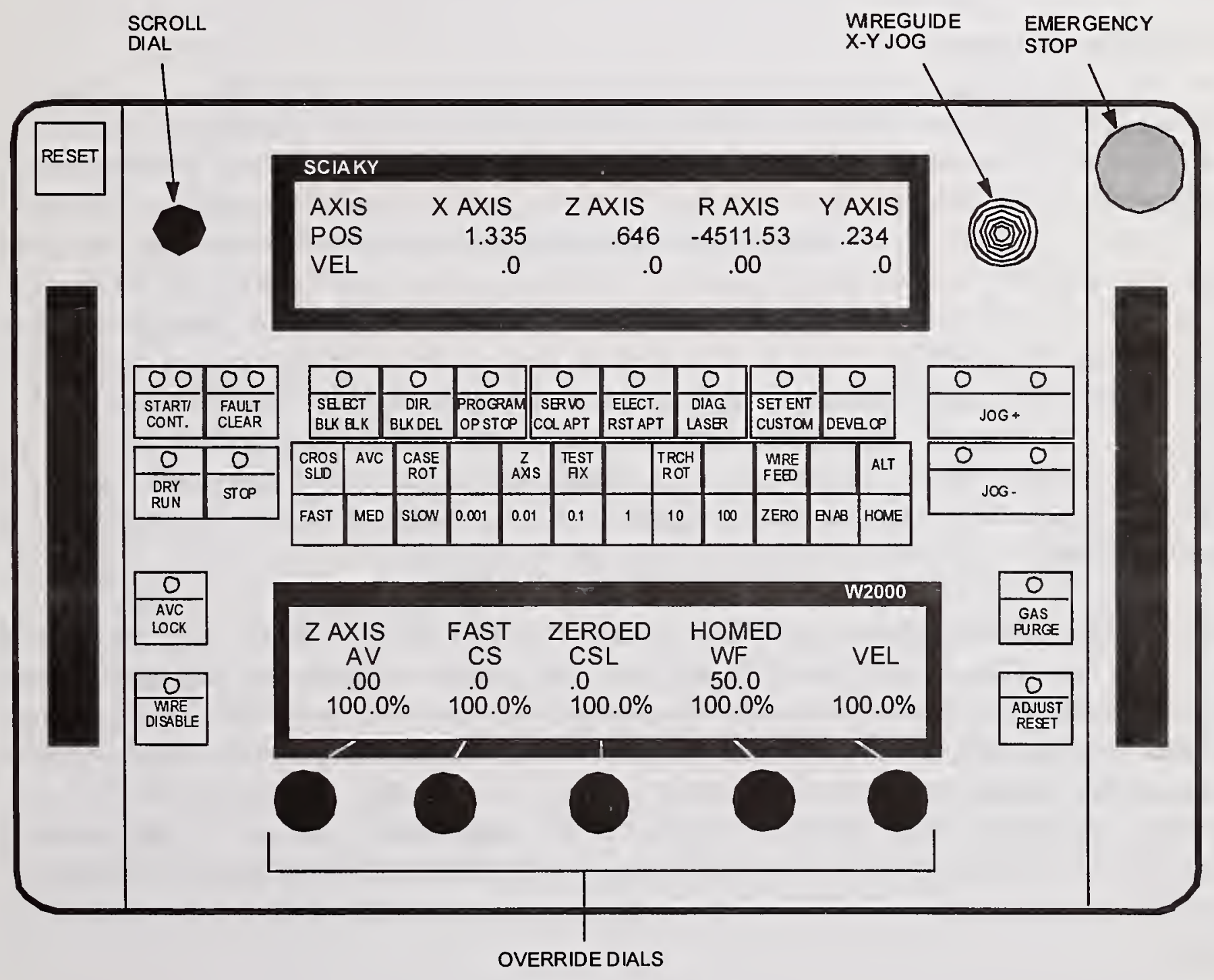

FIGURE 6 


\section{Software}

The software is best described as a loosely coupled user interface architecture. This has allowed development of customer required features and interfaces by virtue of adding and tailoring of software, as opposed to substantive rewriting of the code to accommodate such requests. A secondary benefit of this arrangement is that the DMMI can communicate with the control via three separate communication schemes; RS-232, Arcnet, or VMEbus techniques. The XMMI utilizes both Ethernet and VMEbus communication. This latter interface, being X Windows, also provides the user the advantage of remotely logging into the local interface (at the machine) and running another instance of the XMMI at the remote location. In addition to monitoring of machine operations, remote trouble-shooting and/or operation assistance can be accomplished at distance.

With the exception of the interface operating systems (DOS and X Windows) the code has all been generated in house. Over the thirteen years of existence, the code has been continually developed and now represents in excess of one-hundred thousand man hours of effort. There are major advantages to generating code internally, complete documentation control, the ability determine prevailing philosophy and immediate modification and test capability.

\section{Controller Development}

The initial controller was developed based on the direct input of an aerospace gas turbine manufacturer. The unit included the Graphic and Block modes of programming with the NC language planned for inclusion in later units. Operating methods and information presentation were defined by the initial user and have been continually refined by the company and users through later systems. The welding processes of the first system were GTAW and PAW. The PAW process was required for circumferential welds using the keyhole mode and therefore required the use of a massflow meter to accurately control gas flows for the opening and closing of the keyhole. This was the first use of a programmable mass flow meter for this application.

Shortly after the first control was developed, a standard machine configuration was designed for GTAW/PAW operations and the NC language software was ported over from the prior generation control.

In that same period, a number of machines were acquired for both domestic and international space launch manufacturing. The domestic user required that additional quality assurance systems be implemented in the controller. The quality assurance feature was enhanced to capture all process feedback signals and compare them to the reference values. By employing user programmable warning and error bands the control assured that any deviation from the programmed value would be captured to a quality file and that the operator would receive a warning signal. If the excursion from preset values continued and the error band threshold met, the controller would automatically end the program in a controlled fashion to prevent part damage. 
Several sensor based systems have offered process enhancement, of major interest are the vision based sensors. Numerous applications have required that the control be able to find a component within the work envelope and offset pre-programmed positions prior to welding. To accomplish this, software has been developed for several different vision based systems. The most common application has been High and Low Pressure Turbine blades for jet engines. These components exhibit sufficient variability from part to part that without a vision system they would require constant operator intervention. In the most advanced version of a vision system, the SPARC station interface hardware was employed to analyze the digital vision image of the component surface and automatically generate welding parameters most suited for that individual component. Unlike several other systems that have relied on a data library to piece together weld parameters, this system applied an "intelligent" algorithm based rule set. A variant of this system was created eliminating the vision system hardware. By presenting the programmer with some preset formats, the program guides the process of parameter development. This interface known as the "Z" notch developer, has allowed weld programs to be optimized in several hours as opposed to traditional developments that usually extended several days or even weeks.

Another aerospace application for solid rocket motor casings caused the development of a special XMMI feature, the "Procedure Manager". In this feature the user describes the important elements of the process, (special tooling or set-up, required operator actions, etc.) and causes the operator to interact with the control confirming that process requirements have been fulfilled. Figure 7 is a depiction of the machine hardware in which the procedure manager was first included. Due to the large scale hardware being welded it was imperative that strict adherence to process operation procedure was assured. The procedure manager provided the interactive interface and as the operation progressed offered the operators a continuous menu of the operation activity as well as providing a record of adherence.

\section{Applications}

While GTAW/PAW were the first users for the control, Electron Beam Welding systems and resistance welding systems share the same hardware platform with differing PCB population, and in some instances the software heritage of the control. GTAW/PAW machines have been designed and built from small (bench size) through systems exceeding 120 feet long and 30 feet high (see figure 7). EB controllers are currently being developed to provide immediate diagnostic "health" checks on beam output along with other physical performance monitoring exercises which will be performed immediately prior to production welds. These "systems" health checks will involve real-time evaluation of the system against a known nominal performance. Software and systems programs are also being developed that will provide indepth checks which will be made on a weekly or monthly basis. After each system check, a report indicating a pass or fail status will be posted within the system and also reported to a maintenance file. The maintenance file will in turn post messages calling for preventive or corrective maintenance action. 


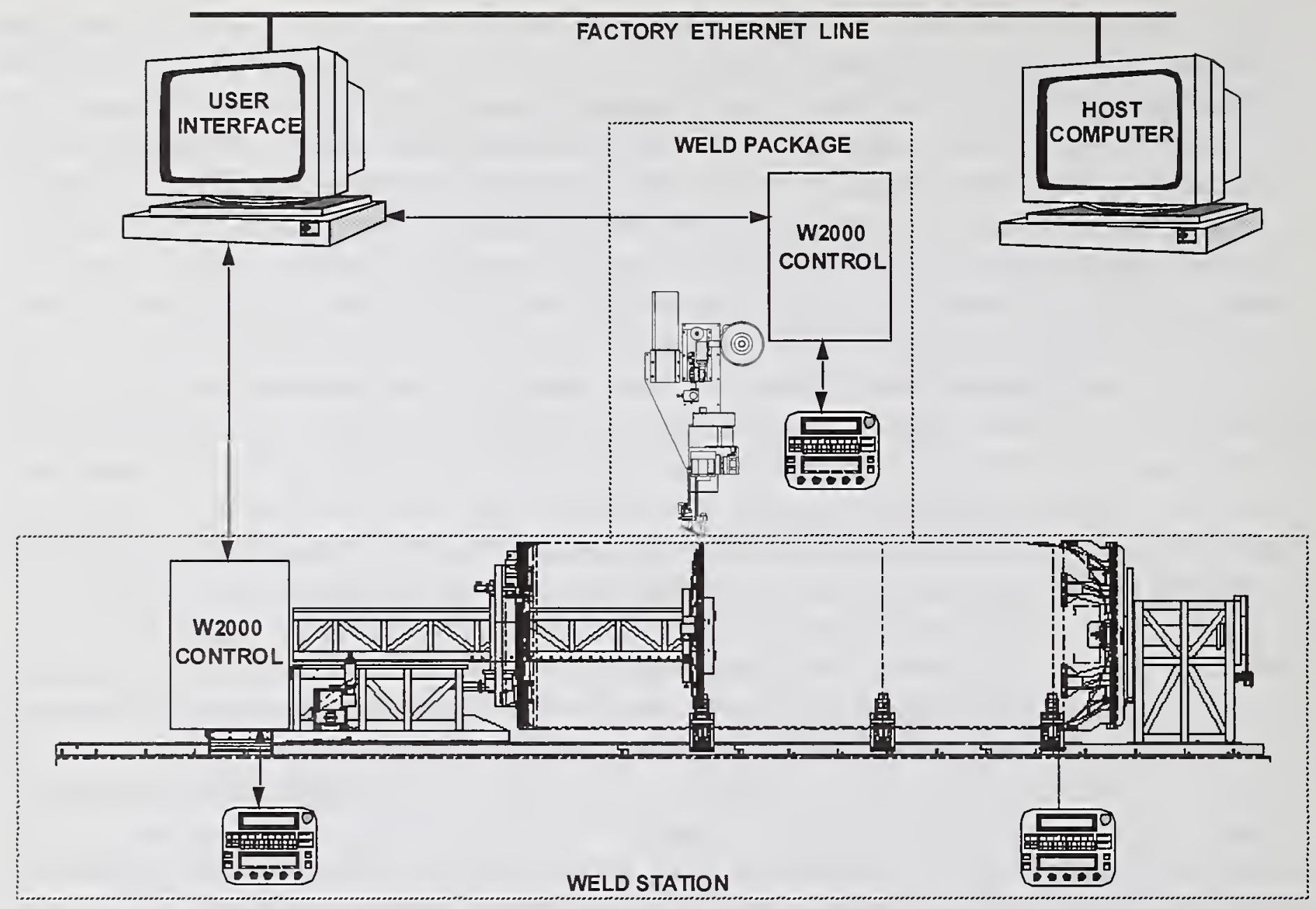

FIGURE 7

A new High Speed Data Acquisition (HSDA) system has also been developed for Electron Beam Applications. This system continually monitors Electron Beam power supply systems and buffers the feedback data. The gathering rate is $250 \mathrm{~K} \mathrm{~Hz}$ across eight channels and allows the user to gather data on "Micro Arc" events. These events have previously not been seen on conventional data acquisition systems due to high speeds at which they occur. Analysis with HSDA systems will allow users to identify precursor events that may lead to a major arc out and the resulting defect. It will also allow rapid identification of areas requiring repair by capturing event position data, or that same information could be supplied to an NDE file for close examination of suspect weld areas to determine if repair work is required.

\section{SUMMARY}

The control hardware has met and exceeded every goal originally set. On-going software development of custom applications continues to extend the range of the control and is expected that more sensor technologies will be integrated as they are developed. A new resistance welding control that employs Windows NT as it's operating system has been developed and will be the subject of a future paper. The control has also been employed for Electron Beam Physical Vapor Deposition (EB PVD) where six full controllers have been integrated into a shared Bus 
scheme. Because on the complexity of running six controls on a single operation, custom graphic interfaces have been created providing instant depiction and control of the process while complex programs run in parallel in the background. It is expected that multiple controllers will be employed on future welding systems as operations and data assurance demands become increasingly complex.

VME hardware continues to offer improved hardware performance with an extended range of products. With this continued growth it is now anticipated that VME will continue to be the platform of choice for at least another seven to ten years. 



\section{DATABASES and KNOWLEDGE BASES}




\title{
AN INFORMATION MODEL BASED WELD SCHEDULE DATABASE ${ }^{1}$
}

\author{
S. D. Kleban", G. A. Knorovsky", G. K. Hicken" , G. A. Gershanok
}

\begin{abstract}
As part of a computerized system (SmartWeld) (Ref. 1) developed at Sandia National Laboratories to facilitate agile manufacturing of welded assemblies, a weld schedule database (WSDB) was also developed. SmartWeld's overall goals are to shorten the design-to-product time frame and to promote right-the-first-time weldment design and manufacture by providing welding process selection guidance to component designers. The associated WSDB evolved into a substantial subproject by itself. At first, it was thought that the database would store perhaps 50 parameters about a weld schedule. This was a woeful underestimate: the current WSDB has over 500 parameters defined in 73 tables. This includes data about the weld, the piece parts involved, the piece part geometry, and great detail about the schedule and intervals involved in performing the weld.

This complex database was built using information modeling techniques. Information modeling is a process that creates a model of objects and their roles (i.e. data \& relations) for a given domain (i.e. welding). The Natural-Language Information Analysis Methodology (NIAM) (Ref. 2) technique was used, which is characterized by: 1) elementary facts being stated in natural language by the welding expert, 2) determinism (the resulting model is provably repeatable, i.e. it gives the same answer every time), and 3) extensibility (the model can be added to without changing existing structure). The information model produced a highly normalized relational schema that was translated to Oracle ${ }^{\mathrm{TM}}$ Relational Database Management Systems for implementation.
\end{abstract}

\section{INTRODUCTION}

This database system has two primary purposes. The first purpose is to provide a compatible database for the SmartWeld Advisor (Ref. 3) to search for plausible weld schedules. The second purpose is to provide a mechanism for storage and recovery of historical information about weld schedules used in the Department of Energy complex. Previously, such weld schedules were recorded on paper and stored in file cabinets or engineering notebooks. Developmental schedules in particular were notoriously difficult to retrieve. Both production and developmental weld schedules are intended to be recorded.

\footnotetext{
${ }^{1}$ Sandia is a multiprogram laboratory operated by Sandia Corporation, a Lockheed Martin Company, for the United States Department of Energy under contract DE-AC04-94AL85000

* Sandia National Laboratories, Albuquerque, NM 87185

${ }^{*}$ Sandia National Laboratories, Livermore, CA 94551

' University of New Mexico, Albuquerque, NM 87131
} 
The WSDB can be accessed directly, for entering data or browsing by welding engineers, or in an intelligent, automated fashion through the knowledge-based SmartWeld Advisor. The WSDB is designed to store weld schedules for a number of welding processes including: 1) arc (GTAW,

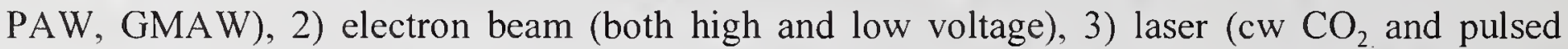
Nd:YAG), 4) friction (continuous drive and inertia), 5) upset and pinch solid state resistance welds, and 5) spot and seam fusion resistance welds.

The WSDB provides a user-friendly, systematic, and comprehensive interface for weld schedule data storage and retrieval. The graphical user interface consists of hierarchical layers of screens. These begin at the part description level, and then follow a natural progression in developing the information storage. For example, after the part has been identified, the weld process is identified, followed by characteristics of the weld itself (and the weld preparation), followed in turn by the process parameters needed to achieve it (power, travel speed, equipment, etc.). Other types of information are also included at logical points, such as required heat treatments, preweld cleaning, post-weld inspection, and specifications. Finally, catch-all comment fields are provided at every level to capture any information which is not explicitly noted.

In this paper, we will: 1) show the hierarchy of screens which are encountered for a typical database entry, 2) describe the logical structure of the database, 3) describe the many types of search procedures which may be conducted and 4) discuss how the database fits into the overall SmartWeld system.

\section{AN OVERVIEW OF THE WSDB}

After the user starts the weld schedule database tool (if not started from within SmartWeld), a login dialog window appears to authenticate the user. After completing this preliminary step, the first screen appears, giving the user the option to enter data for a weld schedule. There are five initial sections where the user can enter data, as depicted by the five buttons along the top of the screen in Figure 1.

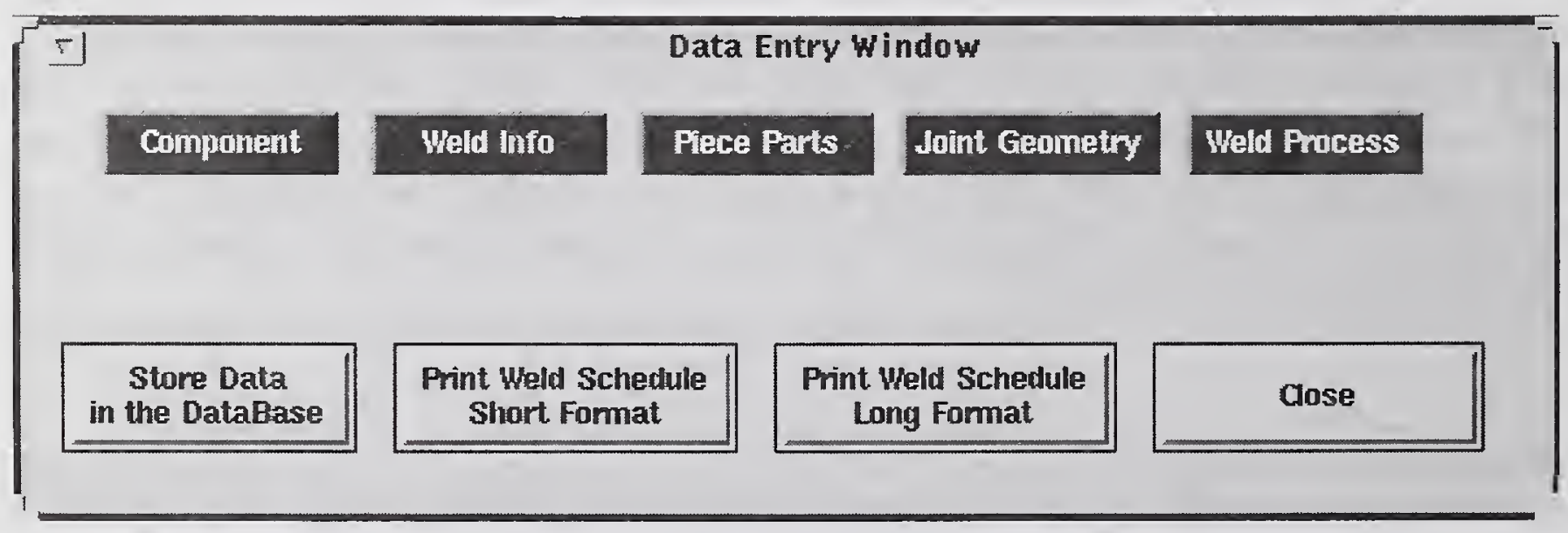

Figure 1. Initial data entry screen. 
The buttons along the bottom store the data to the ORACLE database, print the weld schedule in short or long format (see details below), or close the application.

By clicking on the "Component" button, entry fields appear for information about the component being welded as well as information about the subassembly it is contained in (Figure 2.)

F) Data Entry Window
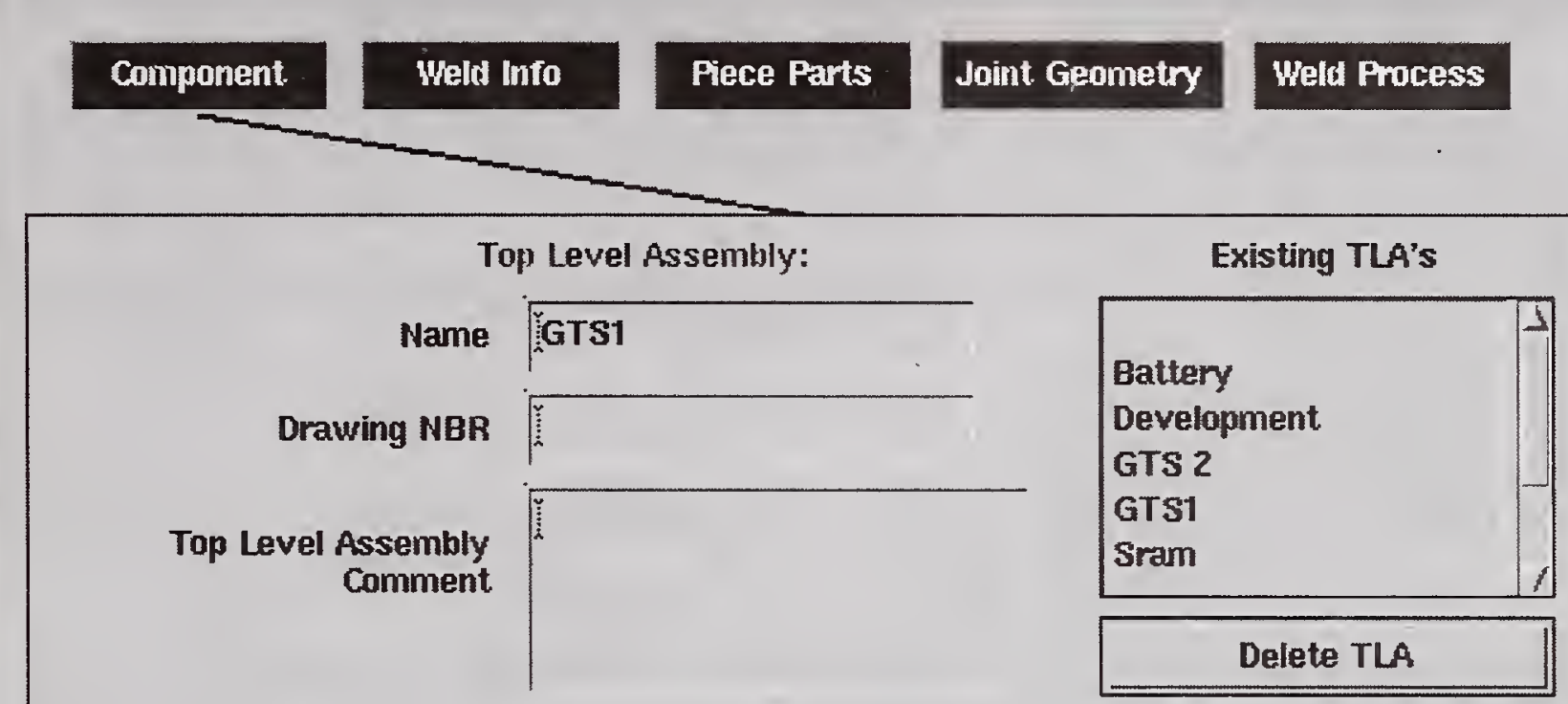

Component Level Assembly:

Existing Components
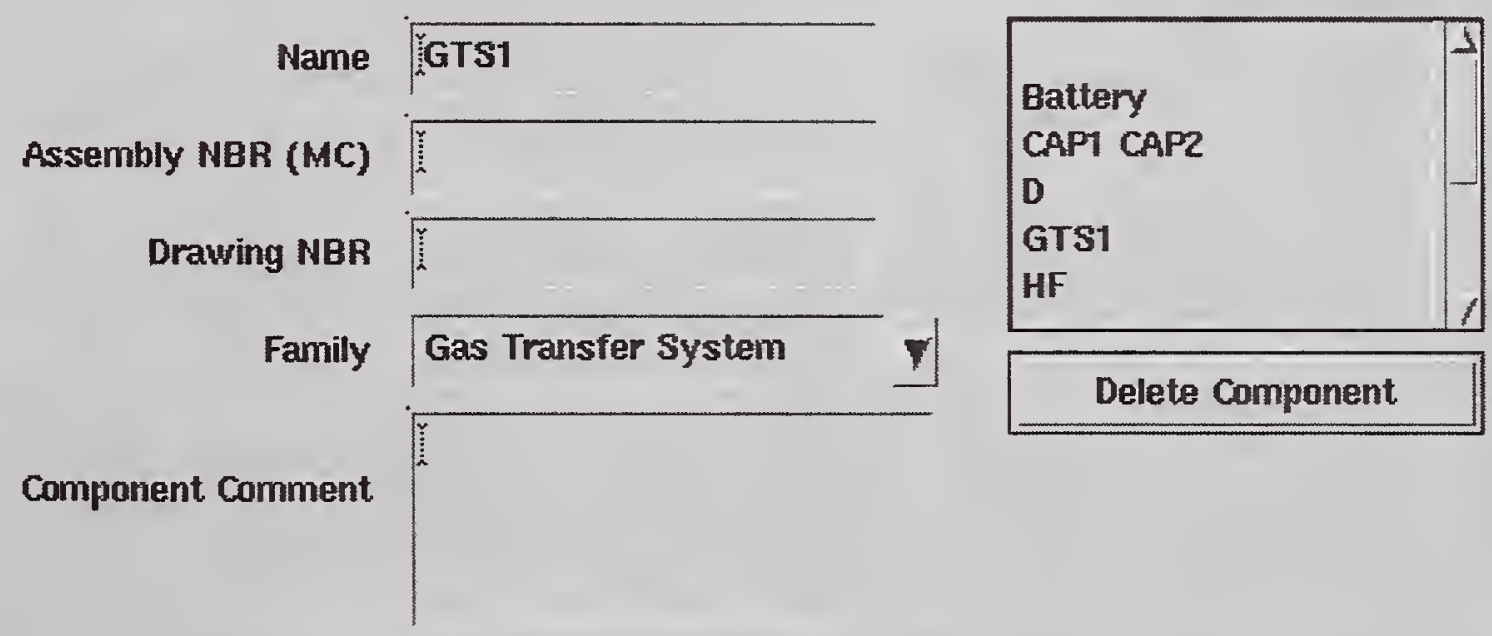

Delete Component.

Store Data in the DataBase
Print Weld Schedule Short Fomat.
Print Weld Schedule Long Format.

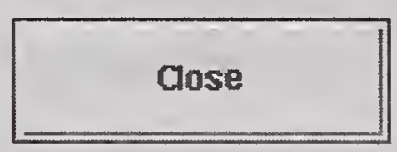

Figure 2. Database entry screen for component and subassembly information. 
To enter or obtain information about the weld, one clicks on the "Weld Info" button and the screen in Figure 3 appears.

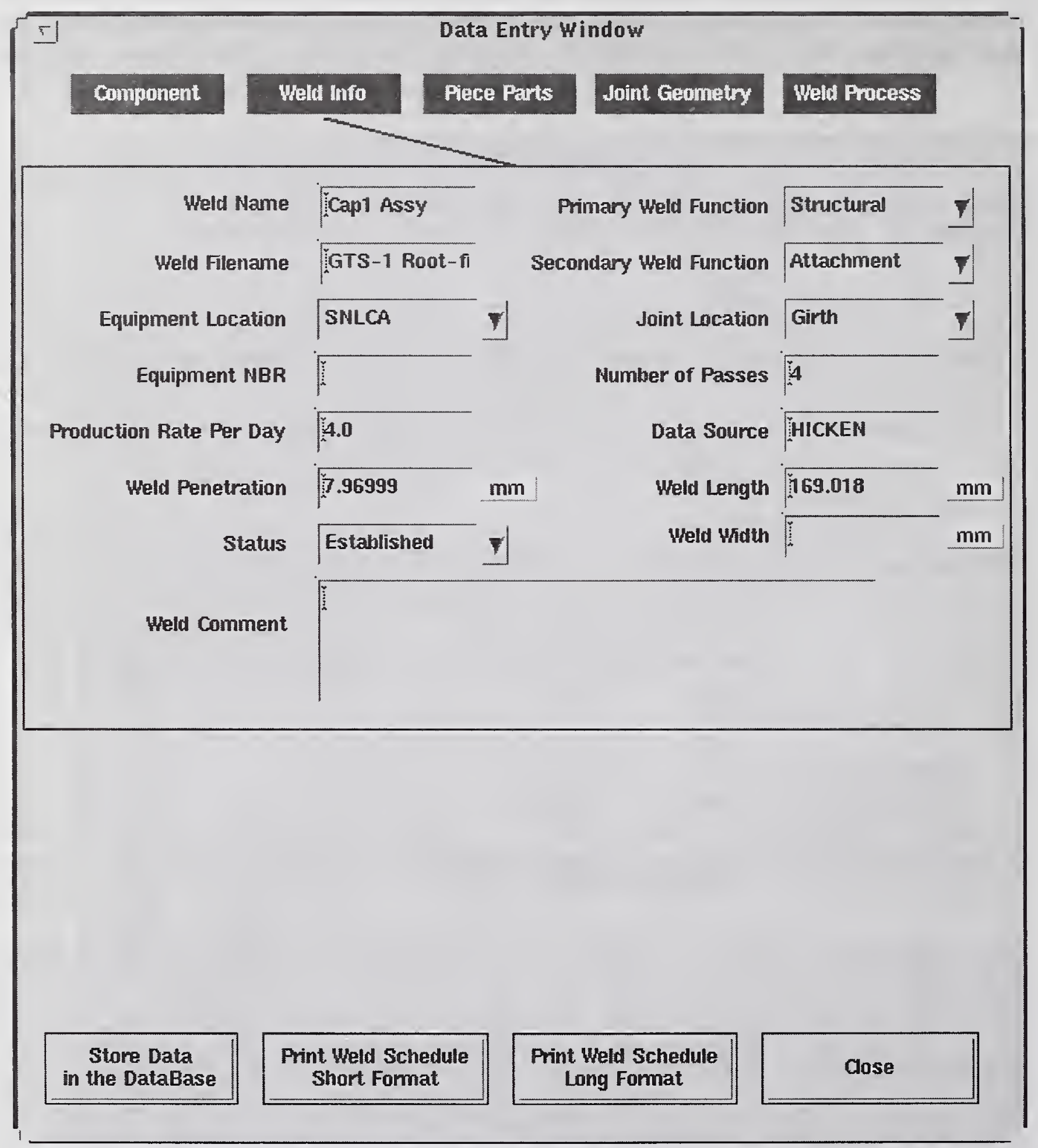

Figure 3. Weld Information.

For piece part information, one clicks on "Piece Parts" and obtains the screen illustrated in Figure 4. 


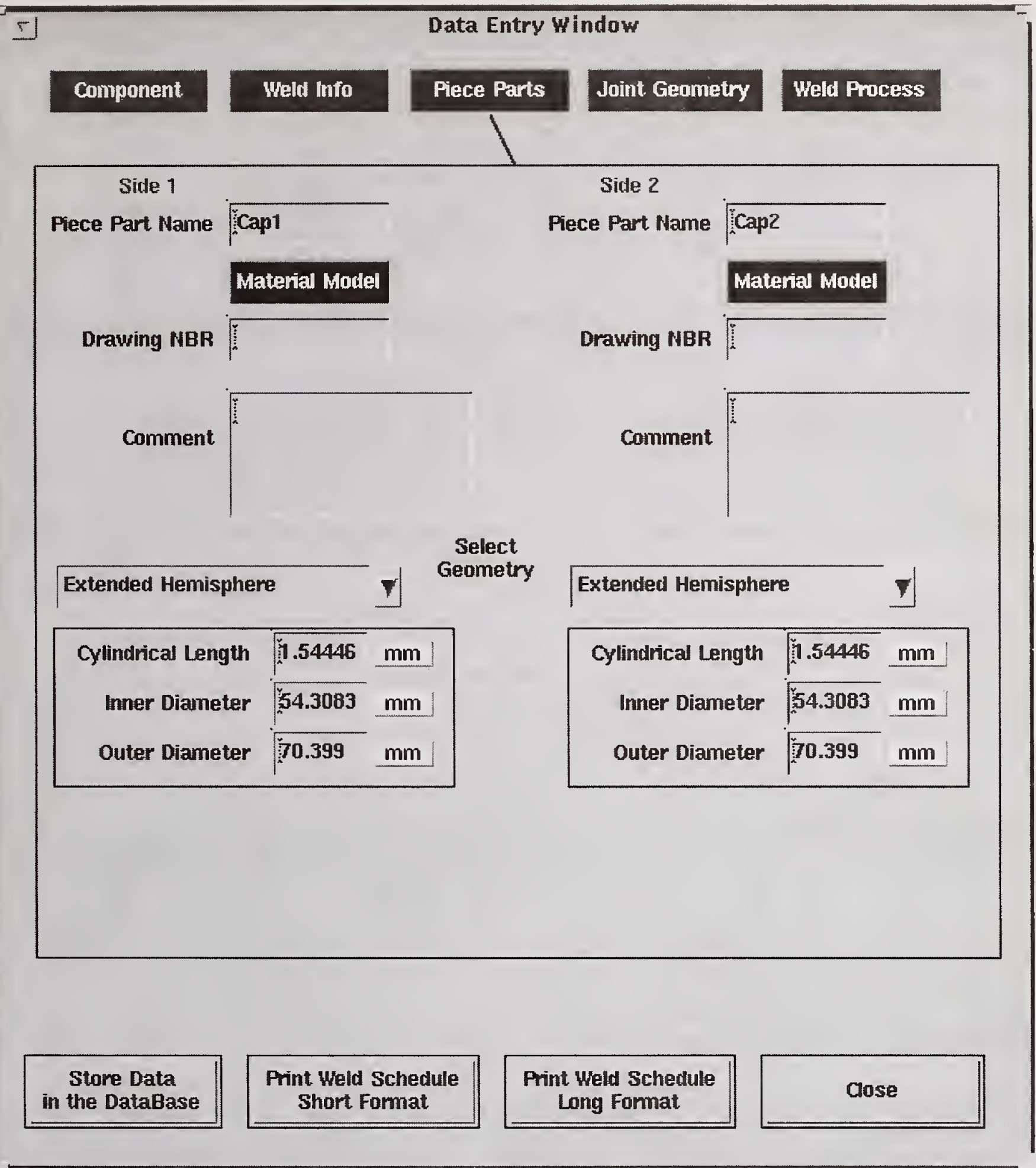

Figure 4. Piece part information.

Note that information for both piece parts is entered as well as material chemistry information (by clicking on the "Material Model" button.) Figure 5 shows the joint geometry screen. In this case it is a U-groove butt weld with reinforced shoulders. 


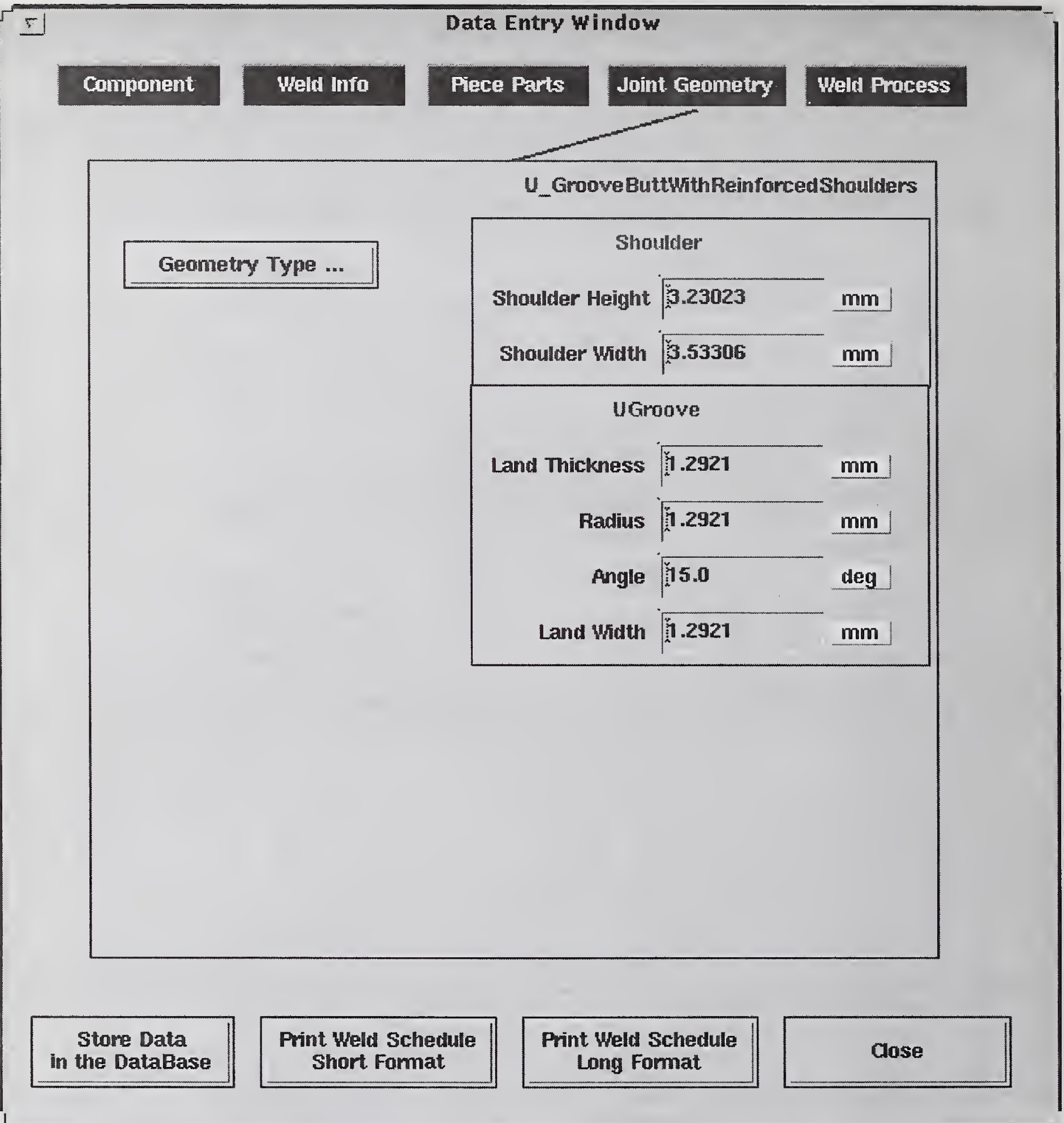

Figure 5. Joint geometry information.

Finally, by clicking on "Weld Process", the hierarchy of weld processes is presented and the user is led through a series of screens particular to the process chosen. For GTAW, the series starts with the most general Fusion information (Figures 6, 7), then Arc specific information (Figure 8), followed by Non-consumable electrode information until we end with the most specific information pertaining only to GTA welds. Fusion information includes data about the weld fusion zone, such as its top and bottom surface contours, size and shape, associated shrinkage and distortion, cleaning and inspection data, temperature (thermocouple data), filler 
(Figure 7), travel speed, and heatsink information. The Arc data includes voltage and current information (Figure 8) and the Non-consumable screen includes data about the electrode and torch. Current, voltage, filler, and speed are entered as interval data, that is, for a given interval number beginning at time $t_{1}$ and ending at time $t_{2}$ the current went from Amps to $\mathrm{Amps}_{2}$.

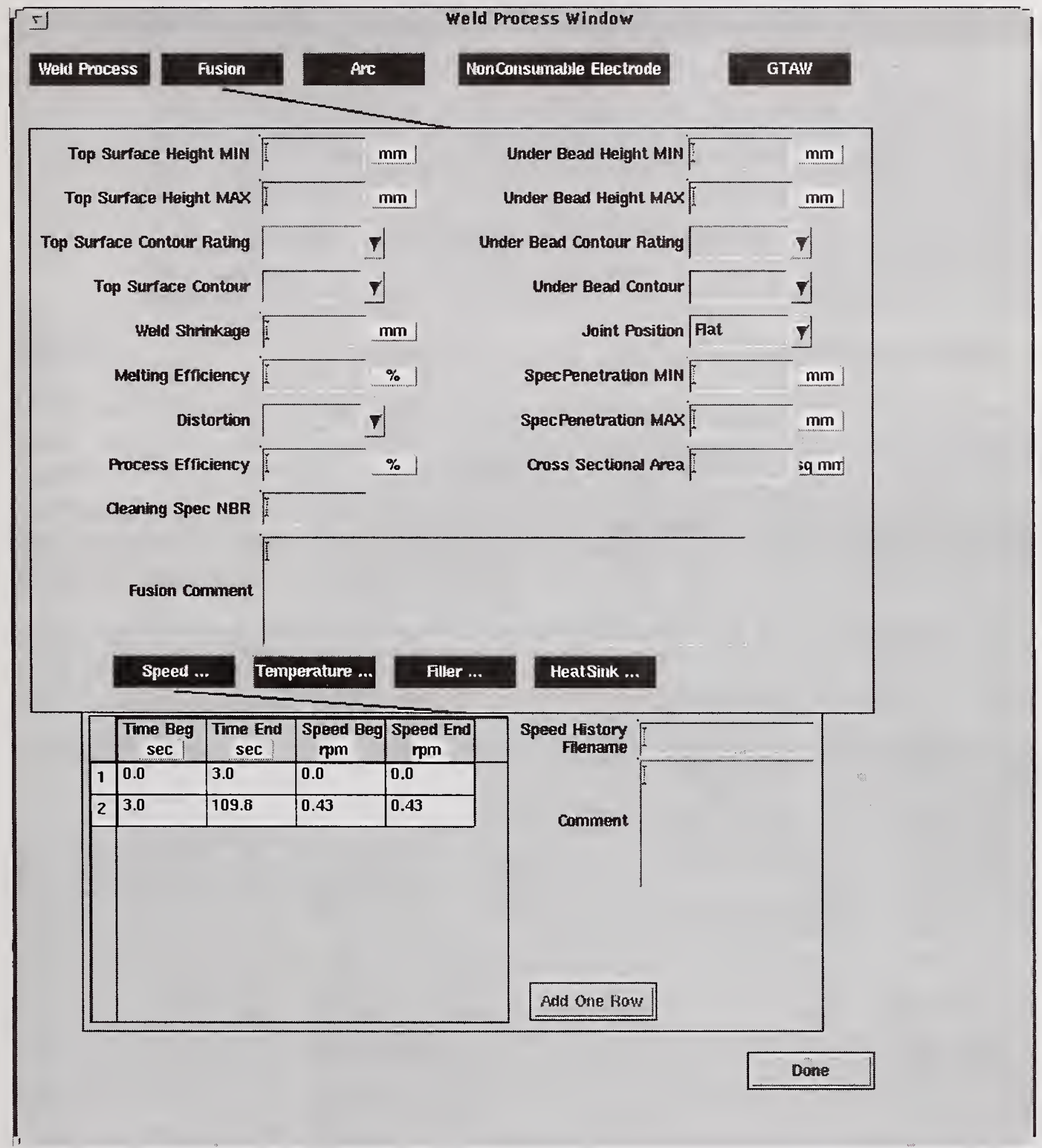

Figure 6. Fusion zone information screen showing general fusion information and the Speed interval values. 


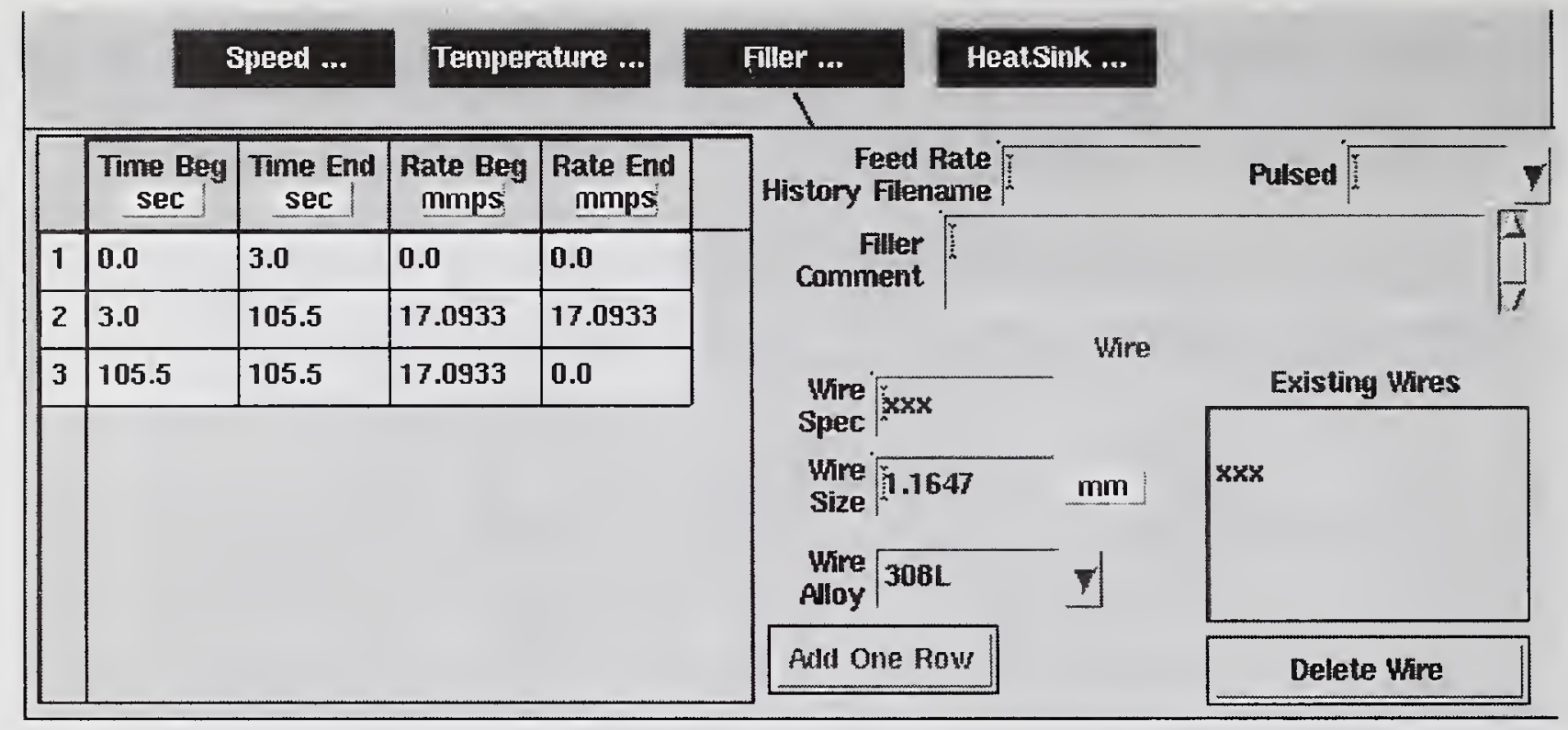

Figure 7. Filler interval information including wire specifications.

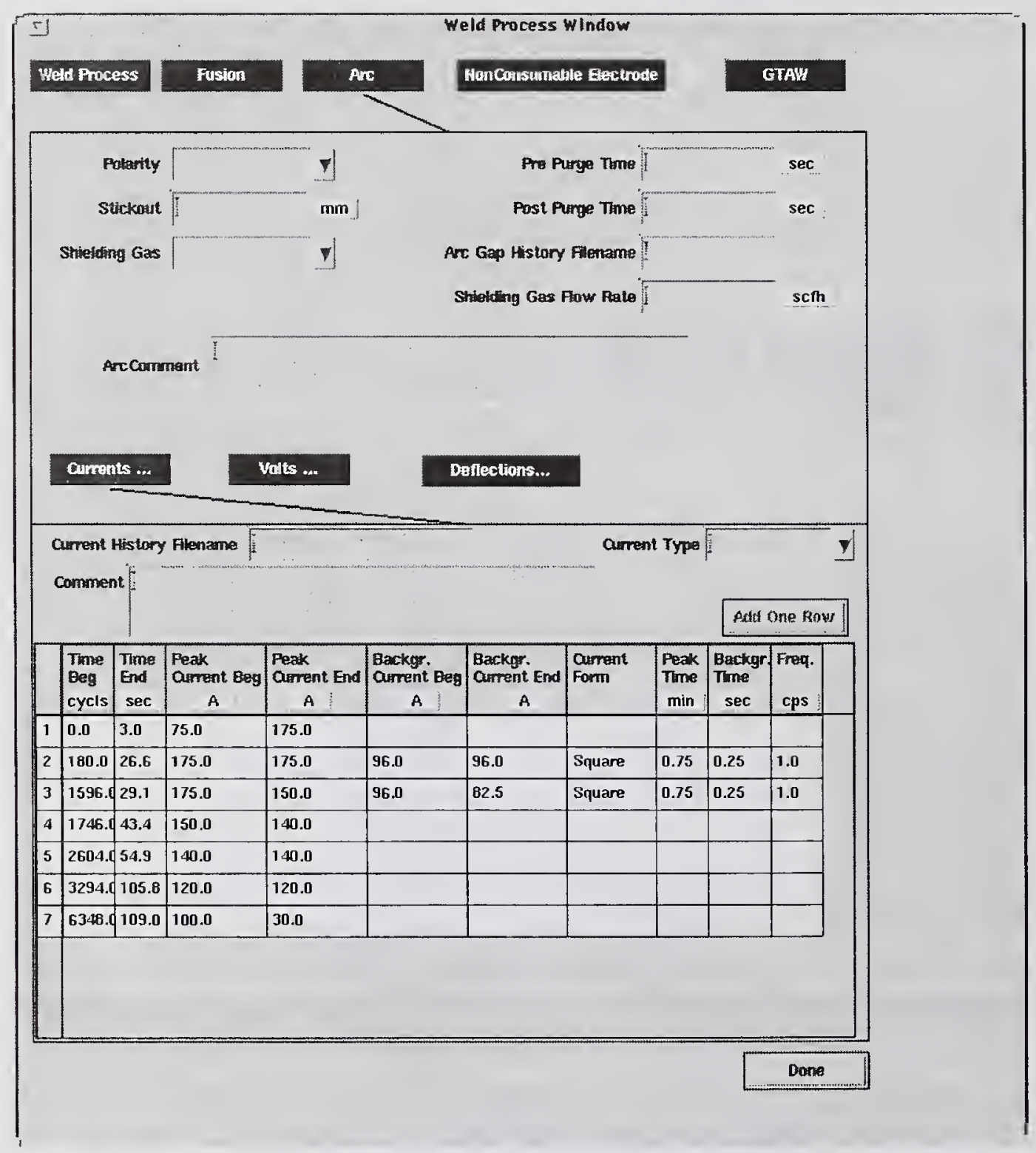

Figure 8. Arc information including Current interval information. 
Due to the logical structure followed, by the time the user reaches the last screen (in this case labeled GTAW) all the salient information needed has already been filled in; thus, only a comment field is included (not shown for reasons of brevity).

When all the information available is typed in, the user simply clicks the insert button on the first screen to insert the weld schedule into the database. The WSDB does not expect all slots to be filled.

\section{DATABASE UTILITIES}

\section{$\underline{\text { Search }}$}

Anyone with access can search the database since this function only requires read access to the data. The user is presented with a search criteria screen where material, a thickness range, welding process, joint geometry, data source, and weld process expert can be specified as shown in Figure 9. Once this is done a search is performed and the resulting abbreviated weld schedule are presented in a table. The user can now select a particular schedule from the table of search results to examine in more detail.

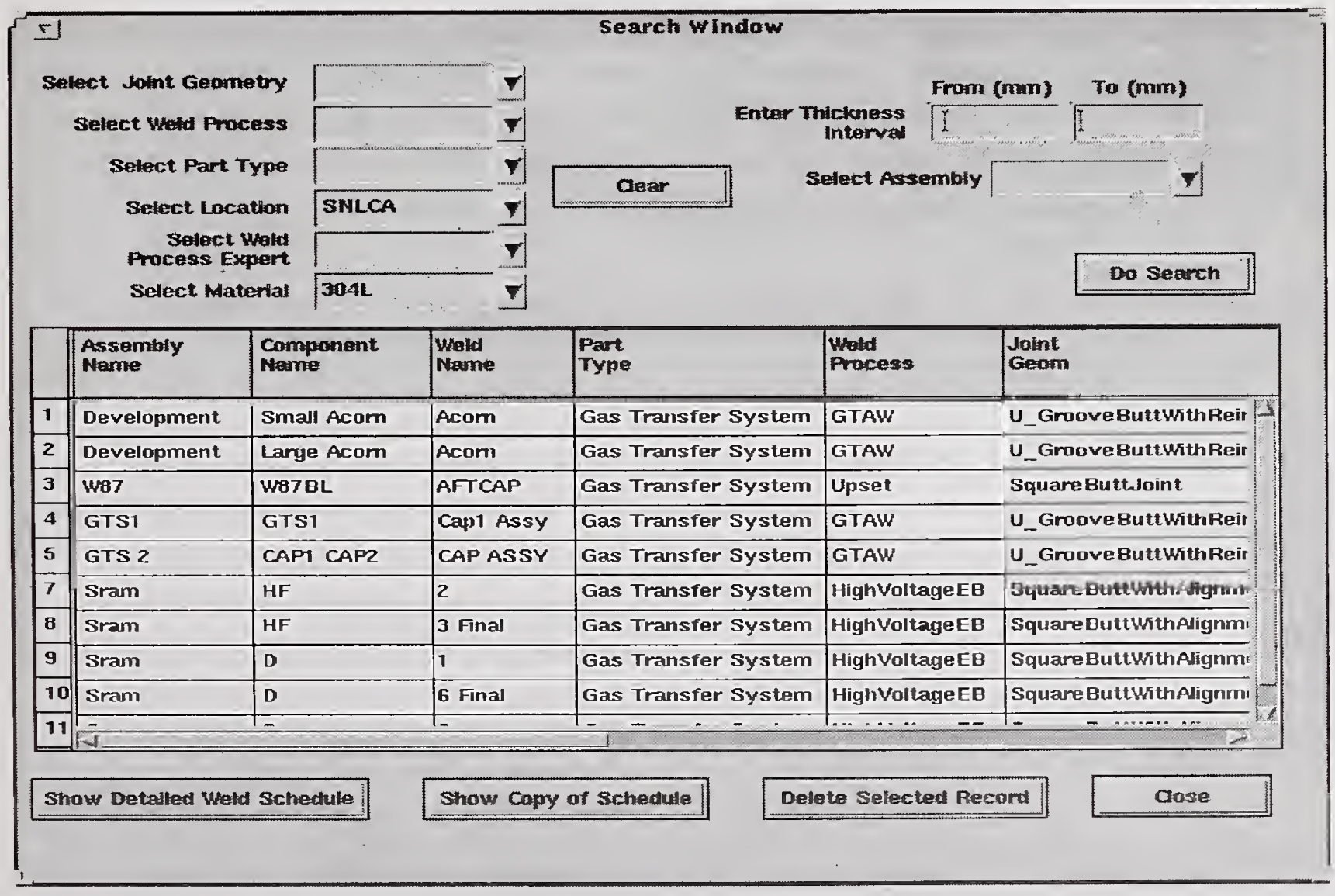

Figure 9. The results of a search for welds done at SNLCA using 304L stainless steel. 


\section{Update}

Requiring more extensive access privileges, this feature works much the same as search, except that once the user is examining a weld schedule in detail, an update or change of the data is allowed. Also, the user can make a copy of a weld schedule. This is useful if one weld schedule is very similar to another with only a few modifications. Instead of entering a hundred parameters that are almost the same as an existing weld schedule entry, the user just copies the existing schedule under a new name, and then changes the few values that are different.

\section{Printing}

On the top level data entry screen, the user can select a weld schedule for printing. Two choices are given: 1) Print the schedule in short format, which only prints the parameters that have values, 2) Print in long format, which prints all parameters whether or not data exists. The blank fields are helpful to manually record information for subsequent data updates. Once the printing is invoked, a dialog screen will appear asking the user which printer to use. The schedule is printed in landscape format and usually takes three or four pages.

\section{STRUCTURE OF DATABASE}

The database was designed using the NIAM methodology which uses atomic facts, i.e. data and relations, about the domain to produce an information model. This model was developed using Asymetrix's InfoModeler ${ }^{\mathrm{TM}}$ on a personal computer. The model maps directly to a relational schema that was implemented in the ORACLE relational database management system. The four types of relationships between data objects used in NIAM are shown below in Figure 10.
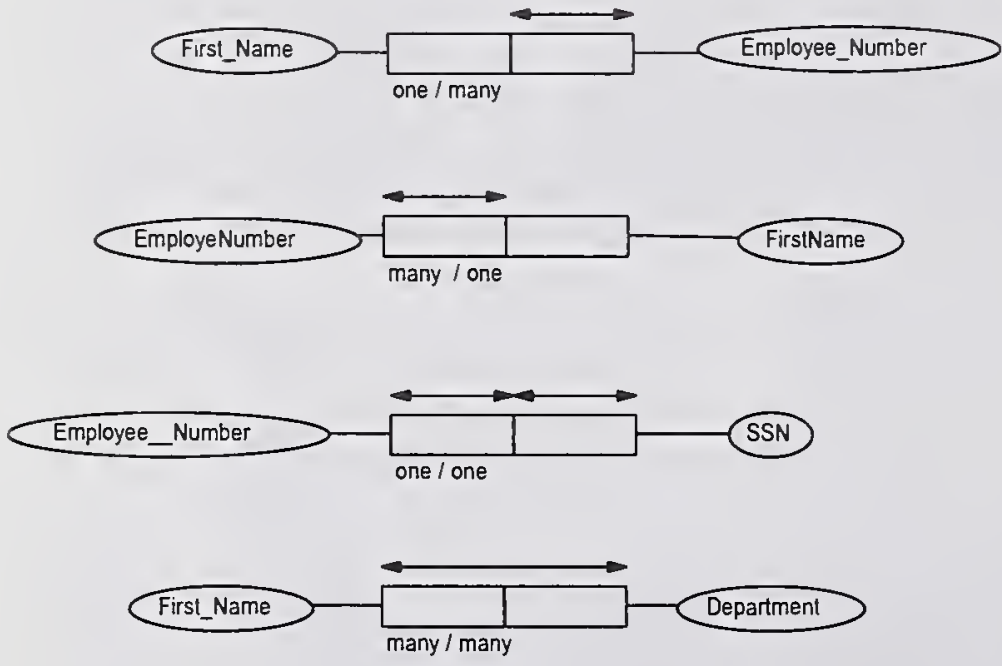
A FirstName can be had by many Employees
(one-to-many)

Many Employees can have the same FirstName (many-to-one)

Each Employee has one SSN (one-to-one)

A FirstName can be in many Departments A Department can have many FirstNames (many-to-many)

Figure 10. The fundamental relationships in information modelling. 
An overview of the information structure using these relations is shown in Figure 11. The weld itself is the heart of the structure and a unique internal identifier gets assigned to the weld by the system (WeldID). A component can have one or more welds (one-to-many) but each weld is uniquely identified. This overview diagram also shows that a weld can be performed by one or more welding processes (one-to-many). That is, a root pass can be done with an arc process and the remaining passes with a low voltage electron beam. The weld has a one-to-one relationship with its PieceParts, i.e. a weld has one set of PieceParts and a set of PieceParts belongs to a single weld (likewise with its JointGeometry). The other relationships are one-to-many; for example, the equipment number (EquipmentNBR) that identifies a piece a equipment can be used for many welds.

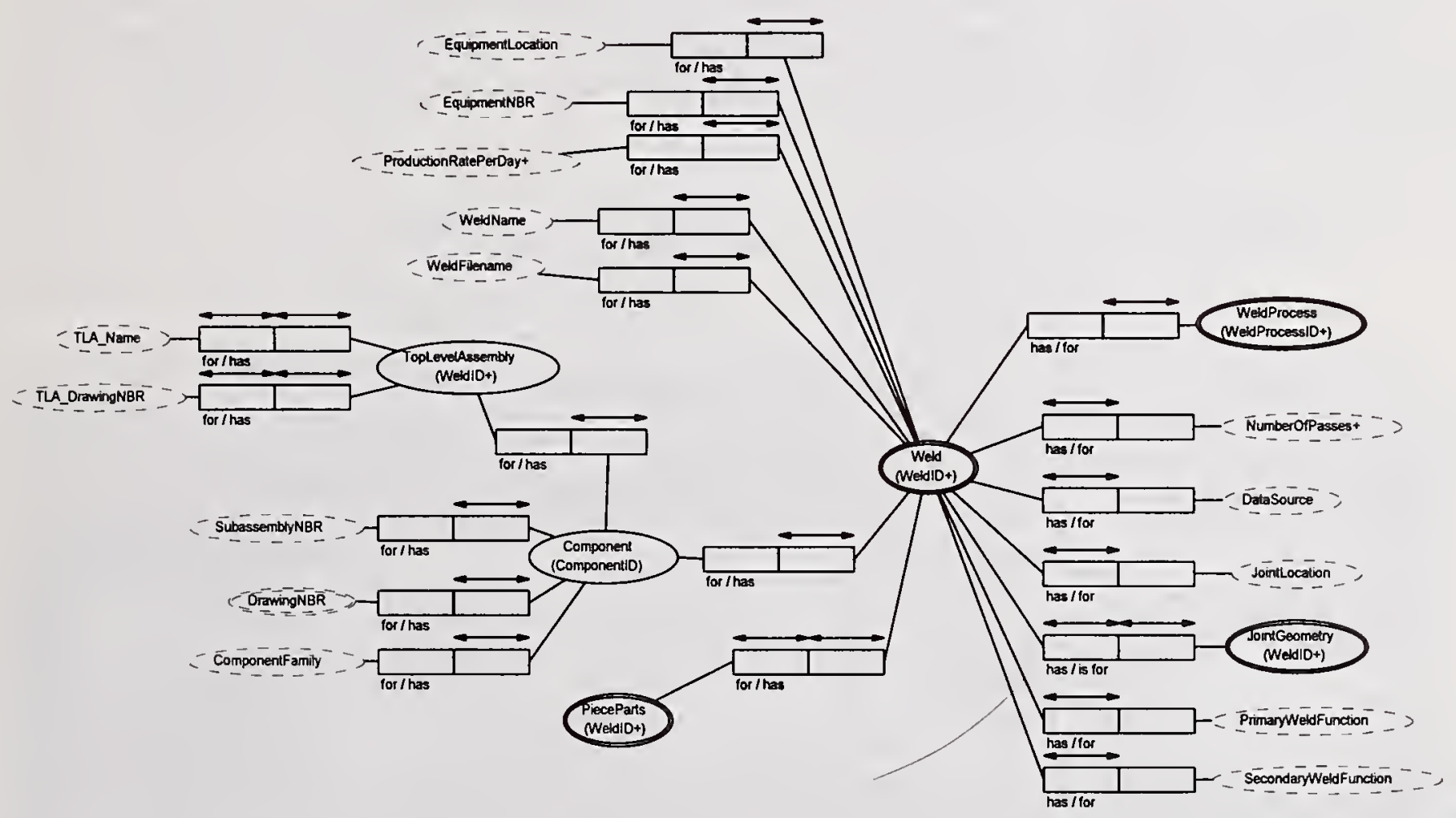

Figure 11. Overview of the information model for weld schedules.

The Welding Process object shown in the top right hand corner of Figure 11, with a double oval, shows that another entire information structure for welding processes exist. Each welding process has a set of parameters that are common to all welds regardless of process and a set of data specific to that welding process. A hierarchical information structure of the welding processes is shown in Figure 12. Items near the top are more general and represent information for all the welding process below them. The information increases in detail and specificity as you traverse the tree depthwise. The dashed ovals represent actual data for the weld schedule and for this information amounts to more than 500 elements that span 50 pages of the information modeling diagrams.

This information model maps directly to a highly normalized relational schema (the information cannot be expressed in fewer tables without loss of information or introduction of redundant data). Once you have the information modeled, the implementation to a relational database is 
trivial and actually amounts to selecting the type of database management system (Oracle, Ingres, Sybase, Informix, etc) and pushing a button in the information modeling tool. The code that gets generated is called SQL (a database definition and query language) and it captures the data and relations defined in the model and ultimately is used to create the database to store the information.

The rest of the implementation is done using a database product and a third party tool for the Graphical User Interface (GUI) implementation. For the weld schedule database, we used Oracle's version of SQL to implement the data and relations and Kappa, an object-oriented toolkit, to implement the graphical user interface for data entry, browsing, and update. For a database of this magnitude, the GUI development turned out to be a sizable project. There are commercial tools that make this implementation easier than using Kappa, but we chose Kappa because the underlying object representation that is used for the GUI can also be used for manipulating the weld schedule information in the Advisor, which is also written in Kappa.

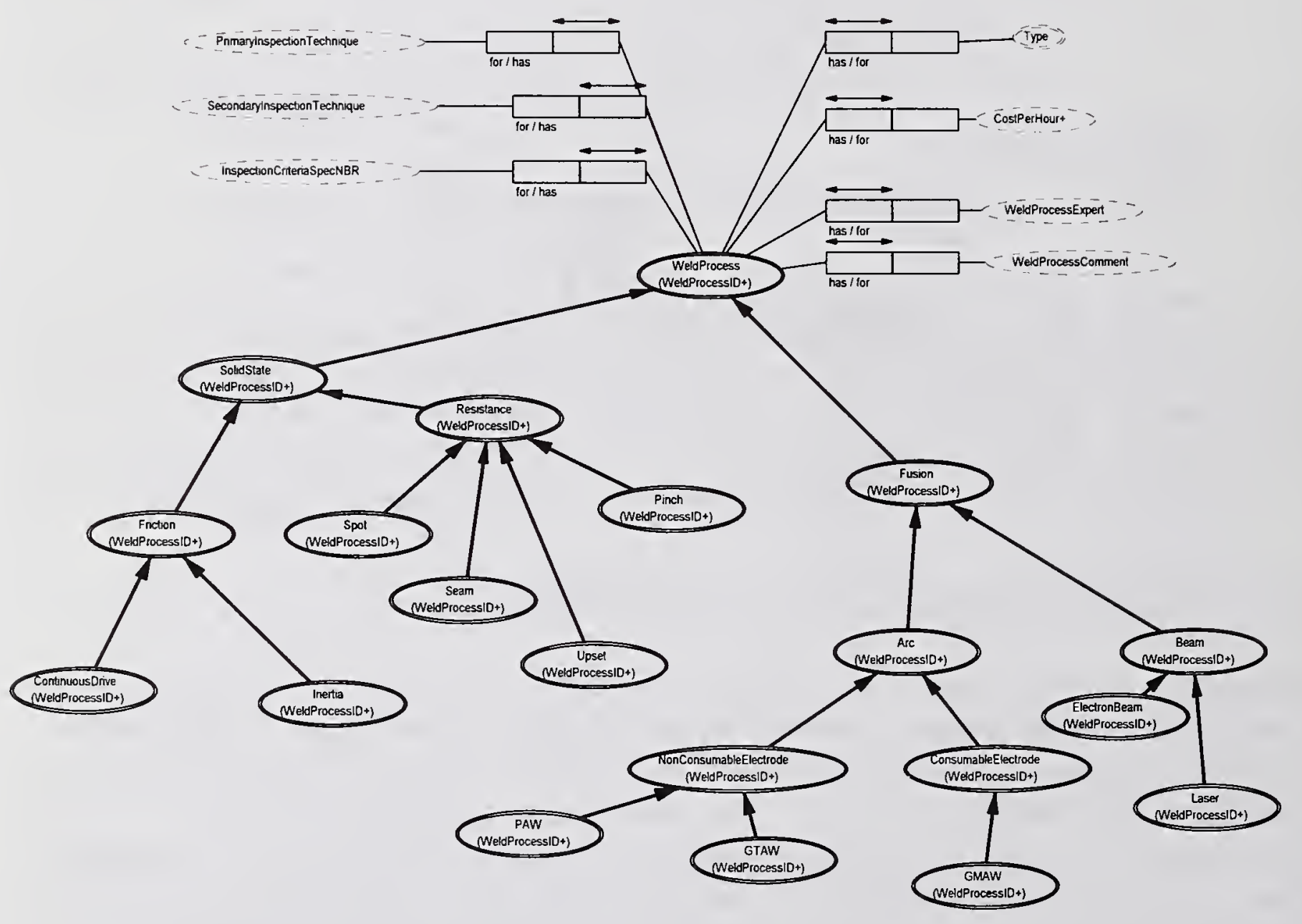

Figure 12. A hierarchical representation of welding processes.

The mapping from relational tables to Kappa objects is an automated procedure provided by Kappa. The tool is called DataAccess and maps any subset of a relational table to an object. The advantage of using objects is that now the data from the database is in objects and we can attach methods directly to each object to perform operations on the data. For data manipulation this 
becomes very attractive when using a relational database management system. Typically this is done with a programming language specific to the database provider which can be cumbersome.

\section{INTEGRATION WITH SMARTWELD}

SmartWeld, developed at Sandia National Laboratories, is a concurrent engineering system that integrates product design and processing decisions within an electronic desktop environment (Ref. 1). The WSDB can be accessed for search, browse, and update directly by selecting the "Weld Schedule DB" button from the toolbar at the bottom of the main SmartWeld screen, Figure 13. The WSDB is also seamlessly integrated into the information flow of the guided SmartWeld environment (the center buttons in Figure 13.) After defining the part to be welded (materials and dimensions) and selecting a weld process and joint geometry as suggested by the Weld Advisor, the next step is to determine a weld schedule. This can be done in SmartWeld by either running an optimization code, SOAR (Ref. 4), to derive the weld schedule parameters or by accessing the WSDB and searching for any similar weld configurations. In either case (SOAR or the WSDB), the pertinent information required to determine the schedule (material, penetration depth, weld process, joint geometry, etc) is passed by SmartWeld.

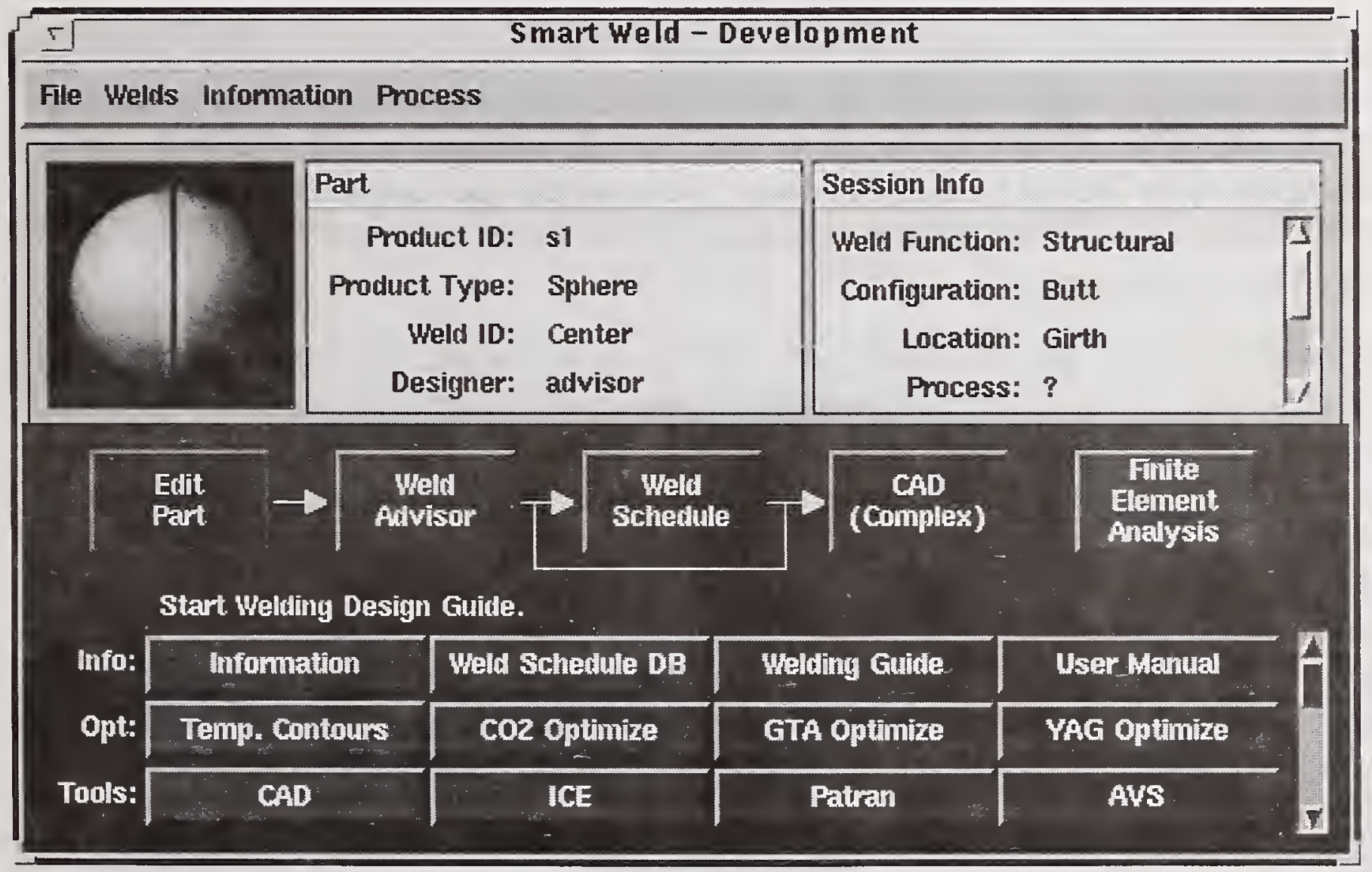

Figure 13. Top level SmartWeld screen.

Since SmartWeld and the WSDB are both written in Kappa, the same software is reused for searching the database and manipulating the data. Once a weld schedule is selected from the advisor, the data is extracted from either source and mapped into an object representation for use 
downstream in the analysis codes that require the details of the weld schedule intervals including the settings of power, speed, voltage, current, and filler wire parameters to model the welding heat source.

\section{SUMMARY}

The WSDB described herein is complete and deployed for use at several DOE sites for manual weld schedule data entry and search. It is anticipated that historical weld schedules will be inserted into the WSDB on a gradual basis and current and newly developed schedules will be input regularly. Future work includes enabling intelligent welding machine controllers to transfer a major percentage of the weld schedule information to the WSDB with minimal human intervention. Likewise, once SmartWeld and the user concur on a schedule, it will be downloaded from the database to the machine controller.

\section{ACKNOWLEDGMENTS}

We wish to acknowledge the efforts of K.W. Mahin, without whose vision the SmartWeld system would never have happened. We also need to acknowledge the efforts of Larry Claussen in the actual construction of the database model. We also acknowledge the role played in testing and development of the WSDB by John Mitchiner. The USDOE and Sandia Corporation do not wish any mention of commercial software contained in this report to be construed as a product recommendation or endorsement.

\section{REFERENCES}

1. Mitchiner, J. L., Kleban, S. D., Hess, B. V., Mahin, K. W., and Messink, D. 1996. SmartWeld : A Knowledge-based Approach to Welding. Proceedings Artificial Intelligence and Manufacturing Research Planning Workshop. AAAI Press.

2. Kleban, S. D. 1996. Design Issues of a Knowledge-Based Welding Advisor. Proceedings Artificial Intelligence and Manufacturing Research Planning Workshop. AAAI Press.

3. Wintraecken, J. V. R. 1990. The NIAM Information Analysis Method. Dordrecht/Boston/London. Kluwer Academic Publishers.

4. Eisler, G. R., and Fuerschbach, P. W. 1996. SOAR: Smartweld Optimization and Analysis Routines. Sandia Report SAND96-2378, Sandia National Laboratories. 


\title{
MANAGING WELDING WITH THE \\ PROWELD INFORMATION SYSTEM
}

\author{
Scholz W. . , Pinn T. , Hunter A.*
}

\begin{abstract}
The New Zealand Welding Centre has investigated the requirements of welding procedure development and costs associated to welding within several research projects with the overall aim to increase welding productivity and weld quality. As a result of these consecutive projects, a new approach of modelling welding procedures and providing essential information for cost assessment and quantity surveying purposes has been developed and implemented in the computer based PROWELD information system.
\end{abstract}

This paper describes the approach of welding procedure development, welding procedure management, cost determination and cost comparison as implemented in the welding information system.

\section{KEYWORDS}

Welding, software, information system, welding procedure specification, welding procedure management, welding cost, quantity surveying, project cost, arc welding processes.

\section{INTRODUCTION}

Everyone involved in welding related project planning, design, job scheduling, manufacturing, or quality assurance comes across these typical situations:

- A new product with a short delivery time is ready for welding, but no procedures and work instructions are available.

- A quality assurance system is installed and requires detailed documentation.

- A specified welding consumable is not available and an alternative has to be identified.

- A suggested welding procedure does not perform satisfactorily. An alternative is needed.

- There exist several welding process alternatives, which is the most cost effective?

- How can project specific welding procedures be quickly prepared?

- What electrodes comply to the required classification? For which applications are they recommended?

- What quantities of electrodes and gases are required for a project?

- What are the welding man hour requirements for a project ?

- What are the total welding cost of a project?

\footnotetext{
"New Zealand Welding Centre, HERA, 17-19 Gladding Place, Manukau City, New Zealand

\# formerly with New Zealand Welding Centre
} 
The welding information system described in this paper was specifically developed to handle the above tasks. Based on several research projects conducted by the New Zealand Welding Centre and the Manufacturing Systems Group at the University of Auckland (Ref 1-6), the system design utilises a modular structure that allows an evolutionary development of further modules gradually enhancing the systems functionality in order to meet user demands.

The system is designed and written using sophisticated methodologies and engineering principles. All modules provide a state of the art user interface, with extensive on-line help information. The modules run under Windows 3.1 or higher, on PCs with a $486 \mathrm{CPU}$ or higher and a minimum of 8MB RAM (recommended Windows 95, 16MB and Pentium CPU).

\section{THE PROWELD EXPERT AND INFORMATION SYSTEM STRUCTURE}

Figure 1 shows the system modules and their interactions to assist in solving a welding task .

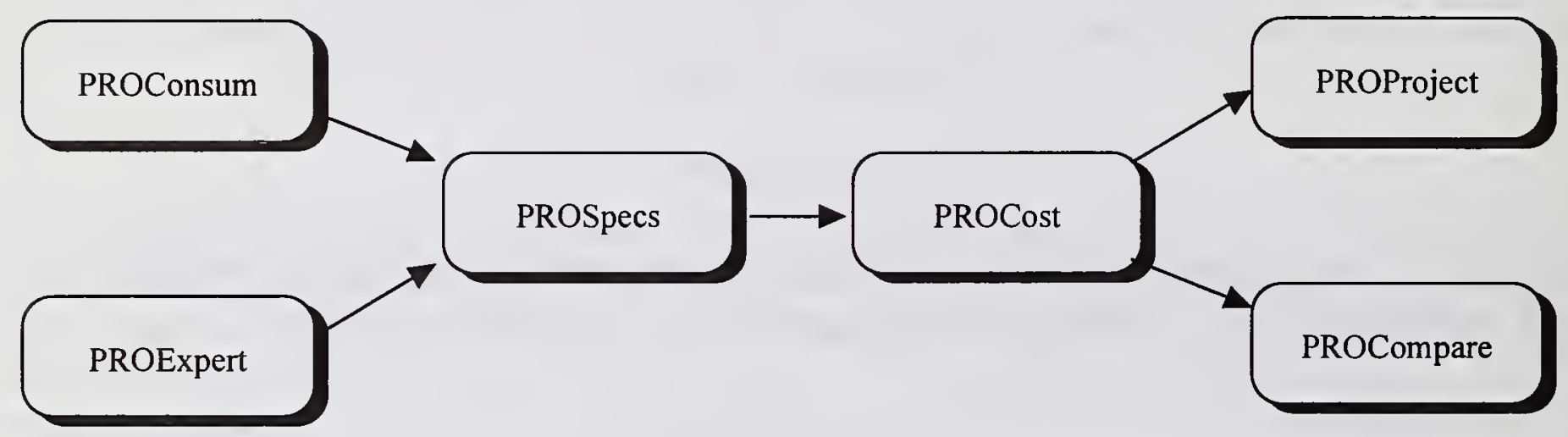

Figure 1 Module Structure of PROWELD

The modules are applied as follows:

\section{PROConsum - consumable information}

PROConsum is the consumable database filled with consumable information provided and maintained by the major consumable suppliers.

\section{PROSpecs - Welding Procedure Management}

Welding procedures are entered and maintained in the PROSpecs module.

PROExpert - expert advised generation of prequalified Welding Procedure Specification Recommended joint preparation details following prequalified details of AS/NZS 1554.1 are generated with the PROExpert module. The results of the generation are send back to the PROSpecs module to add other procedure details.

\section{PROCost - cost calculation}

The cost of a welding procedure is calculated in the PROCost module. 


\section{PROCompare - cost comparison}

Cost analysed welding procedures can be compared in the PROCompare module. The comparison shows the cost differences of alternative solutions and helps to identify where the specific differences are.

\section{PROProject - project summary}

In the PROProject cost module procedures can be grouped as required by a welding project. Cost of welding as well as quantities of consumable and labour requirements can be calculated by this module.

The interactions between the modules will be controlled by the PROWELD management module.

\section{USING PROWELD IN PRACTICE}

The use of the welding information system is straight forward and is explained in the following task descriptions related to managing welding.

\section{Welding procedures development and maintenance}

Not only are procedures to relevant fabrication standards often needed quickly, but they may also include specific client requirements. For example a company wants to qualify their procedures to more than one standard as is frequently the case in New Zealand.

The system handles the formats of most standards commonly used in Australasia and indeed the world e.g. AS/NZS 1554, ASME IX, EN288 (ISO 9656), ANSI/AWS D1.1. The information system is particularly suited to search existing procedures to find those suitable for a new welding task. This can be done effectively by using the mouse as the system uses the Windows@ interface in a very efficient manner. New welding procedures can be developed interactively within minutes.

Figure 2 shows the opening window for the procedure specification module. The system learns from the previous input and also shows as option the previously inputted data making repetitive data input simple. Figure 3 shows this option with the welding standards window open.

Figure 4 shows the procedure search window highlighting search options such as specification no, job/project, specification type (WPS, Preliminary WPS, PQR), welding standard, material, welding process.

Also the corresponding procedure qualification documentation such as the PQR's can be established and maintained effectively in the system. 


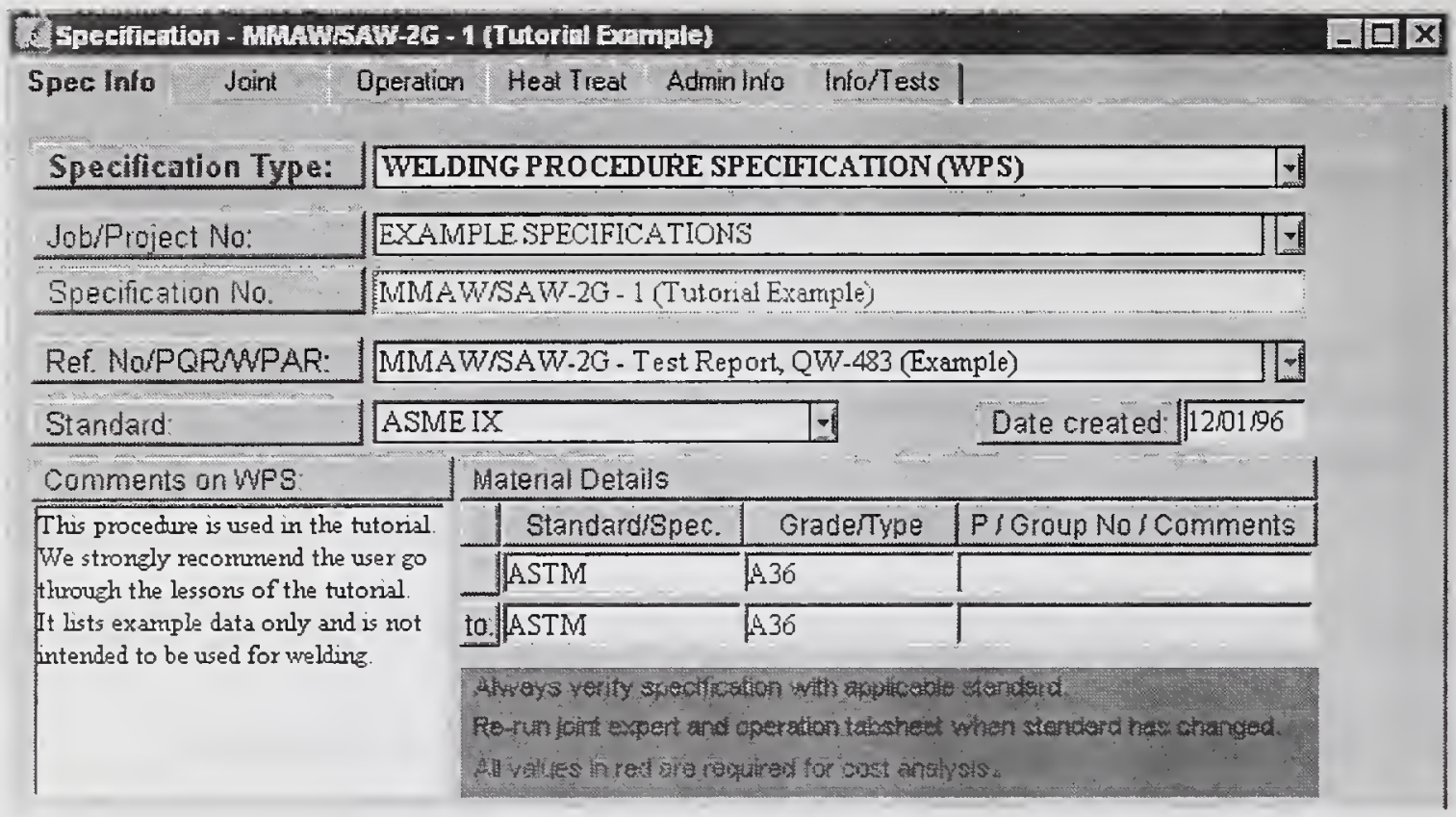

Figure 2 Welding Procedure Specification Window

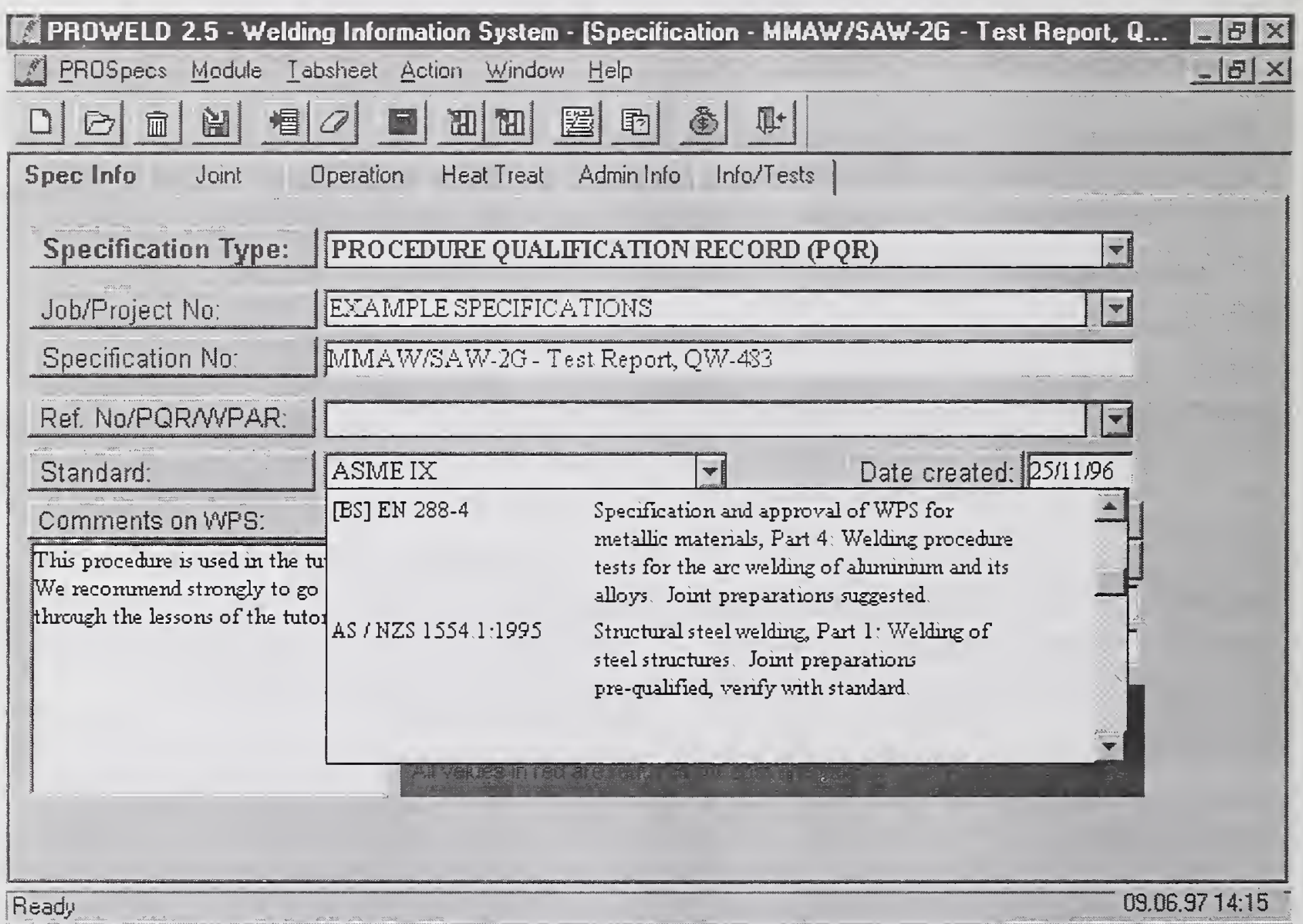

Figure 3 Welding Procedure Specification Window with Welding Standard Option 


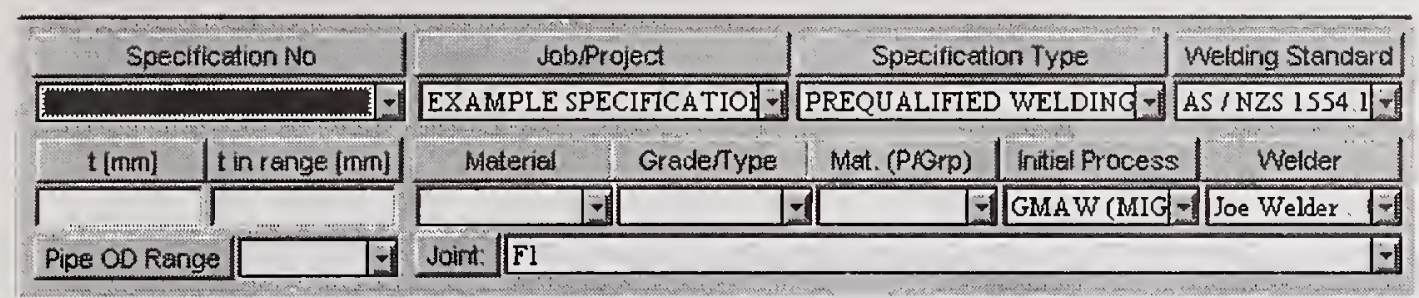

Figure 4 Welding Procedure Search Option Window

Figure 5 shows the joint preparation window which allows to show preparation details in form of sketches and the corresponding numerical input. A large number of frequently used preparation details are stored in the database reducing the time required for drawing the joint preparation sketches.

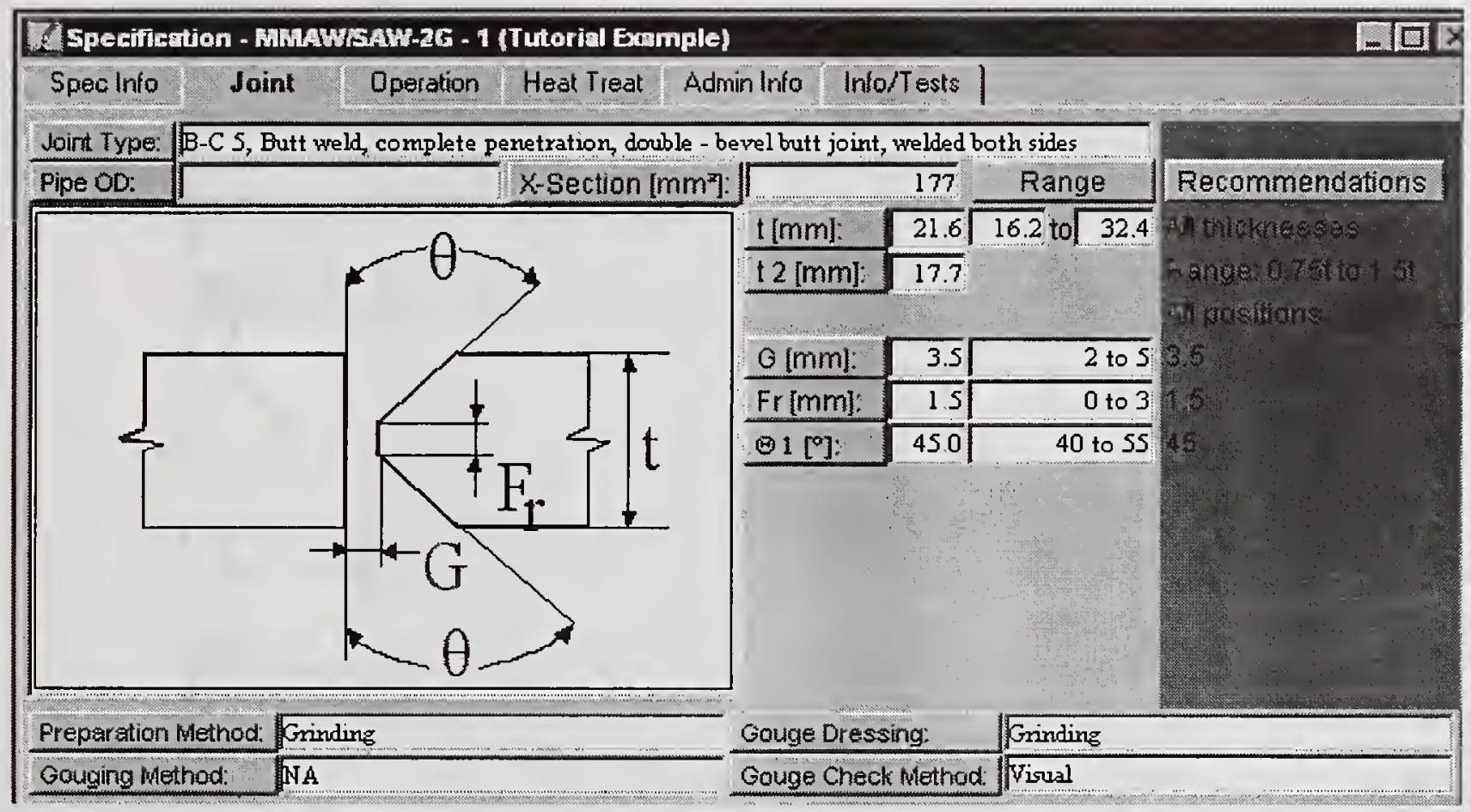

Figure 5 Joint Preparation Window 
PROWELD uses a very logical format for the development of specifications. An example is the development of the individual welding operations which describe the run sequence and details of the welding task. Figure 6 shows an example of a filled in operations window.

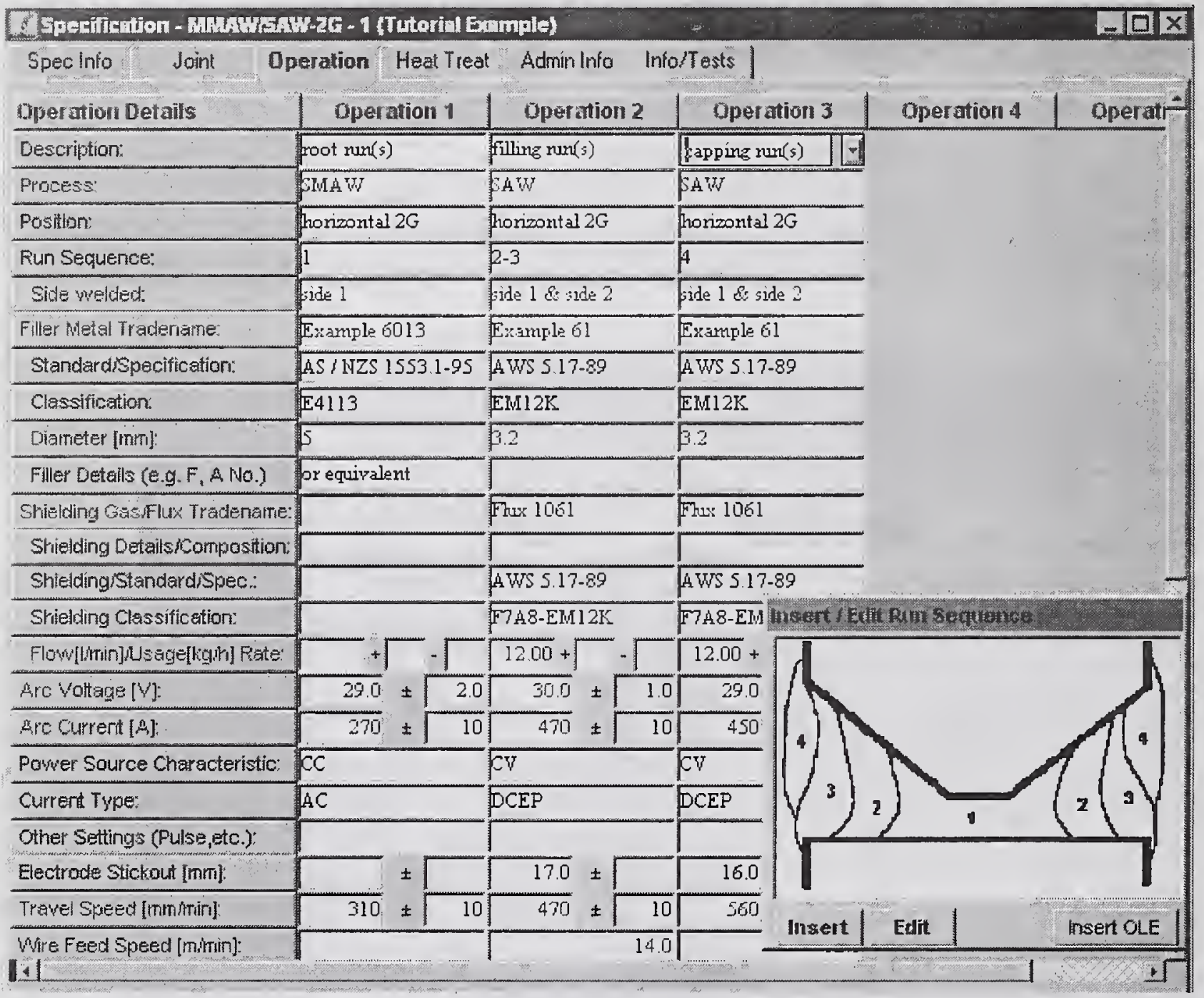

Figure 6: Welding Operations Window

Additional windows are available guiding through the topics of heat treatment, administrative information such as qualified welders, and corresponding information such as qualification testing.

Printed documentation of the procedures is well structured and clear. A printout of the first page of a completed example procedure is shown in Figure 11.

\section{Expert Advice - PROExpert}

PROExpert assists in generating welding procedures to meet the requirements of welding codes and standards with the aid of stored expert knowledge. Currently joint preparations to AS/NZS 1554.1 are proposed by the system. Figure 7 shows an example of the "joint expert" which proposes suitable joint preparations including dimensions: 
Select position(s) for specification:

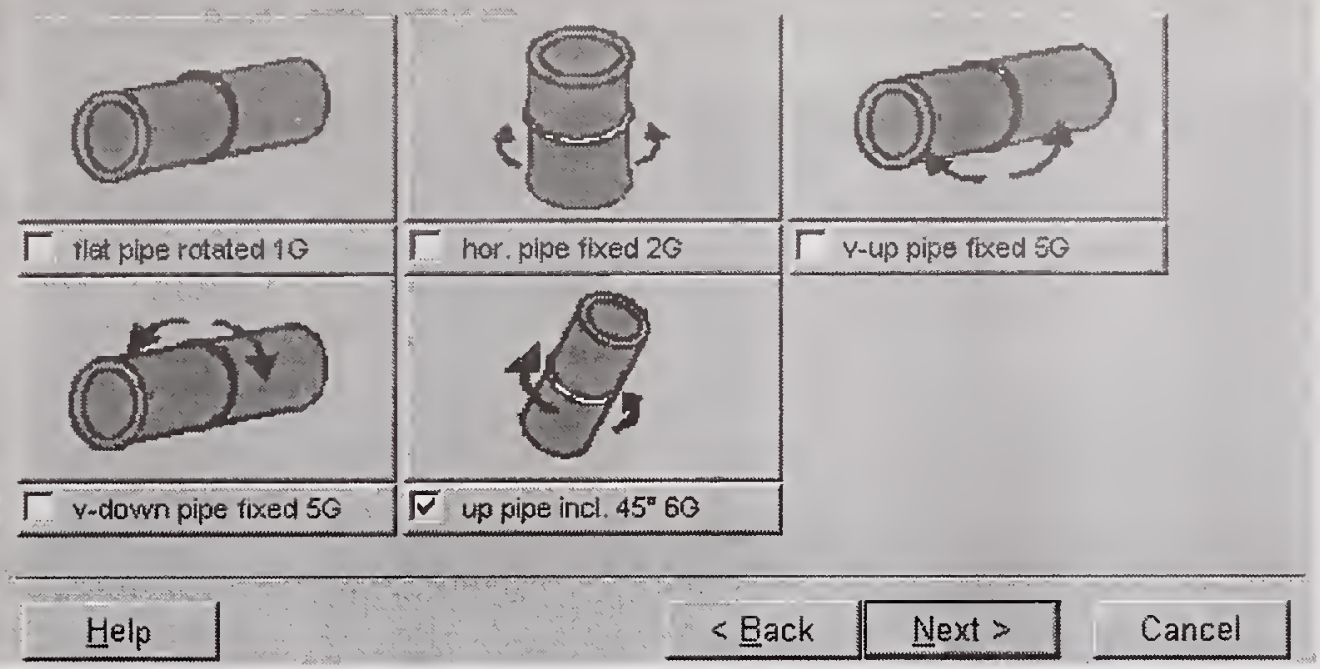

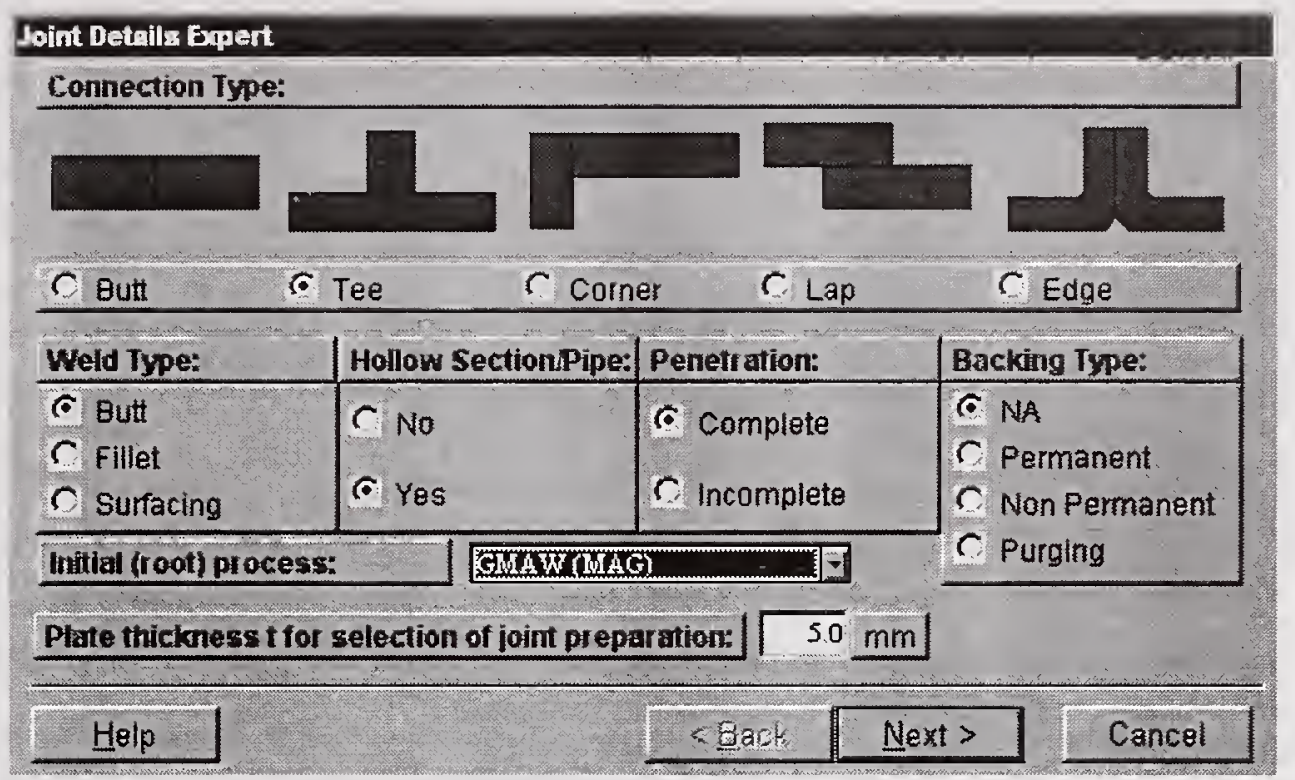

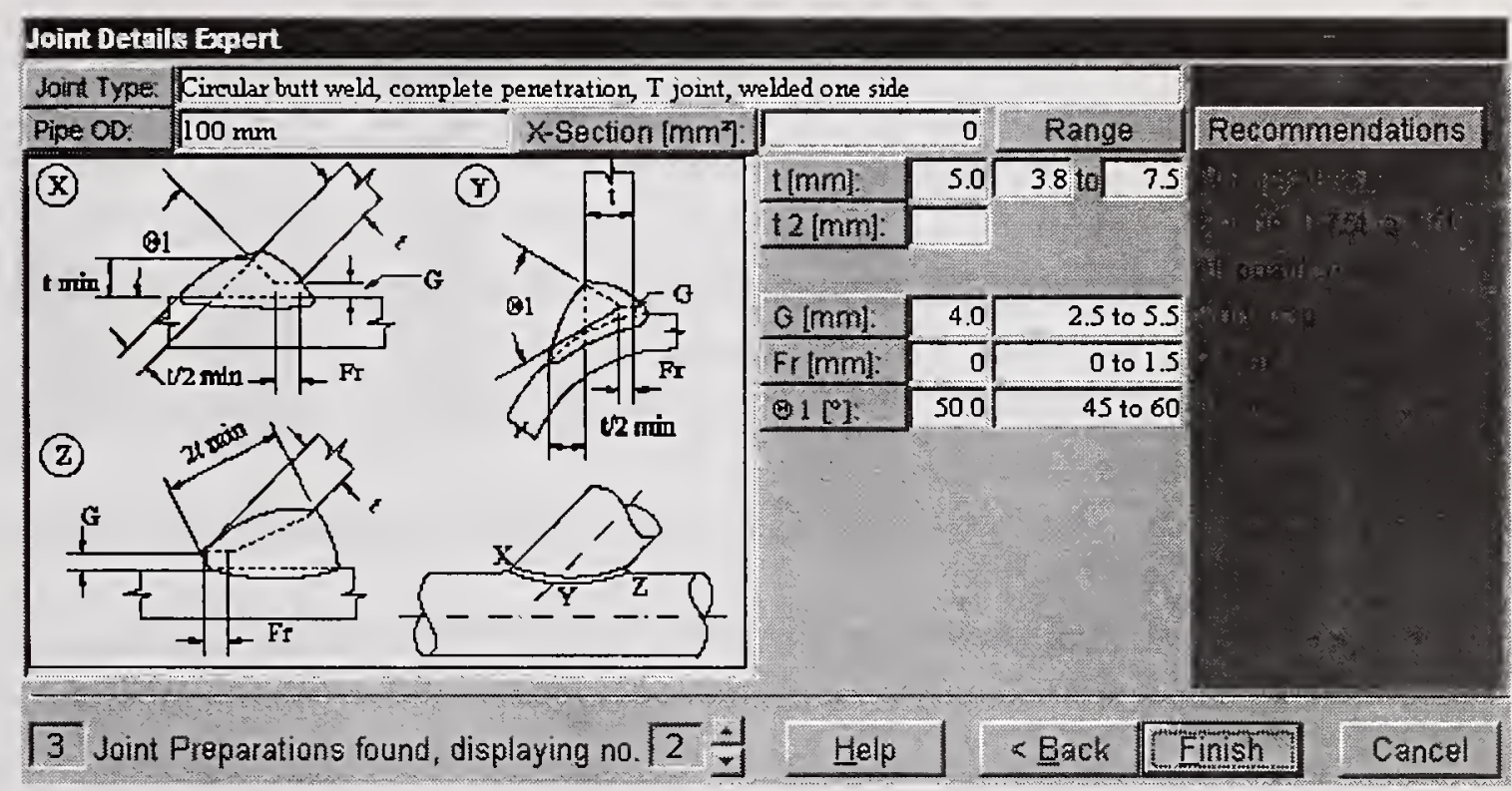

Figure 7 Joint preparation details as recommended by PROExpert 


\section{Welding Consumable Information - PROConsum}

A comprehensive, up to date database with detailed consumable information covers electrodes, wires, non consumable electrodes, fluxes and gases. The system contains detailed consumable information from the major suppliers of Australasia. The filler metal consumable data can be searched by material group, supplier, tradename and classification (Figure 8). The user can add its own welding consumable information in PROConsum. The search through different supplier catalogues is usually not needed.

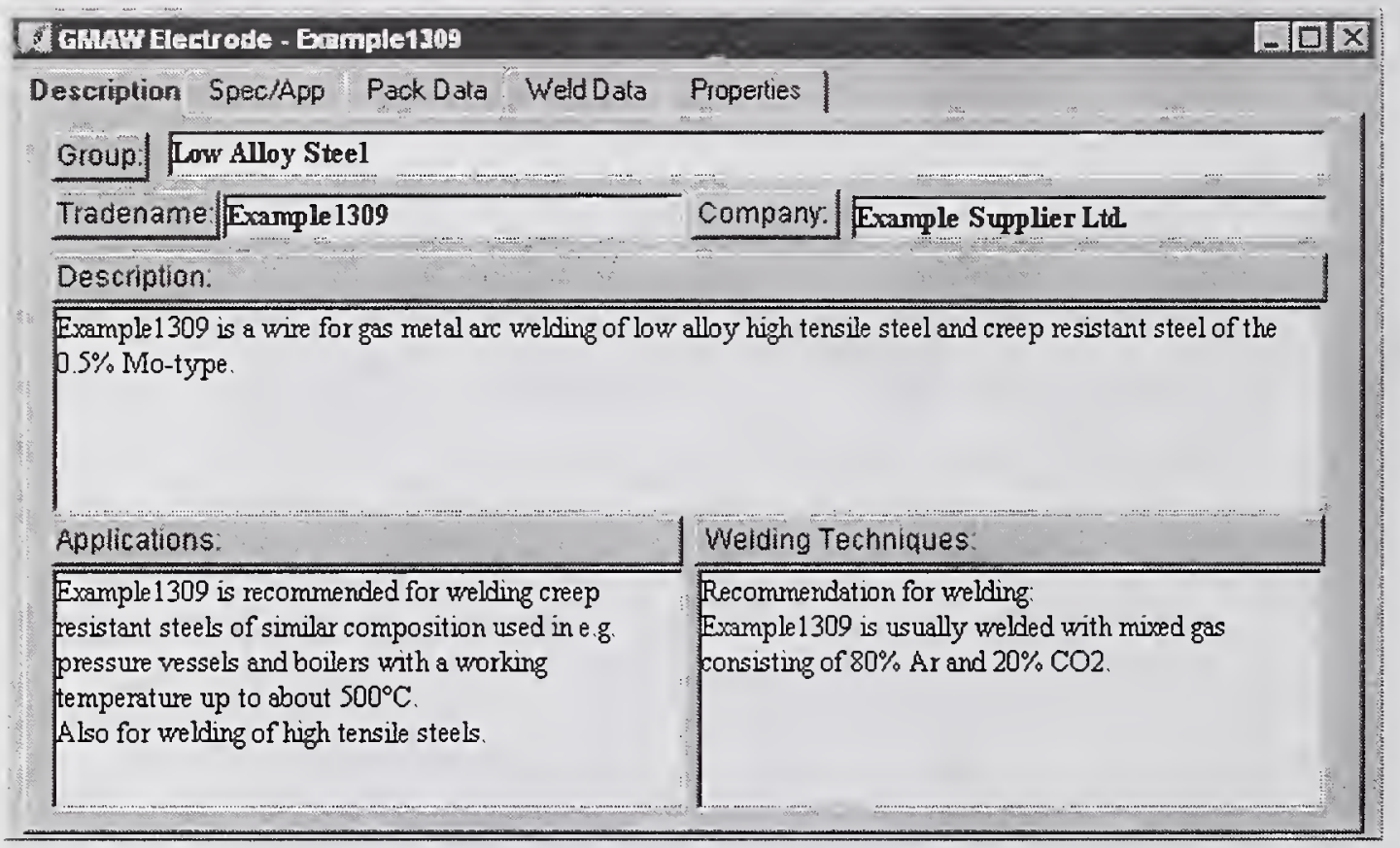

Figure 8 Example of the Consumable Main Window

\section{Productivity Assessment - PROCost , PROcompare and PROProject}

There are frequently situations in which more than one process could be used satisfactorily to achieve a required weld quality.

- A detailed cost analysis and comparison allows improvement of welding procedures and identification of the most economic solutions.

- The cost analysis also considers all ancillary operations, as well as preparation, heat treatment and equipment costs.

- The result of the cost analysis contains detailed information on required consumable quantities and labour hours.

- Welding procedures can be attributed to a project to determine project cost and project quantities.

The costing can be analysed based on default data or the user can easily customise it to particular situations. As the module follows the welding procedure specification, costs calculated are accurate provided the welding procedure information used is representing the true situation. Results are presented as standard business graphics and in table form to allow easy analysis. 
Figure 9 shows as an example the cost analysis of a particular procedure.

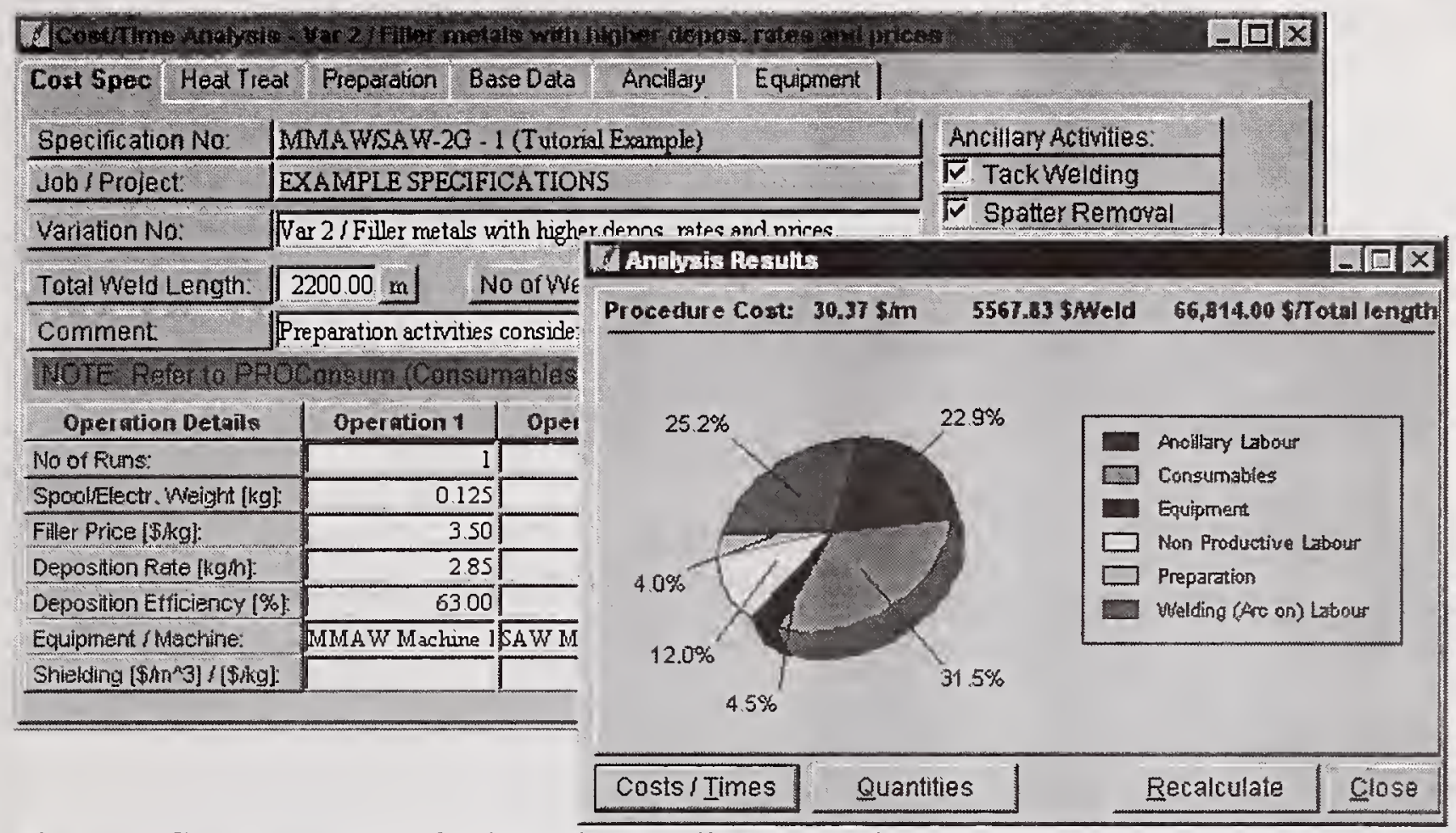

Figure 9 Cost Example of a Sample Welding Procedure

PROCompare compares costs of alternative welding procedure solutions. The comparison displays the cost differences in a graphical form, allowing an effective interpretation of the cost structures (Figure 10).

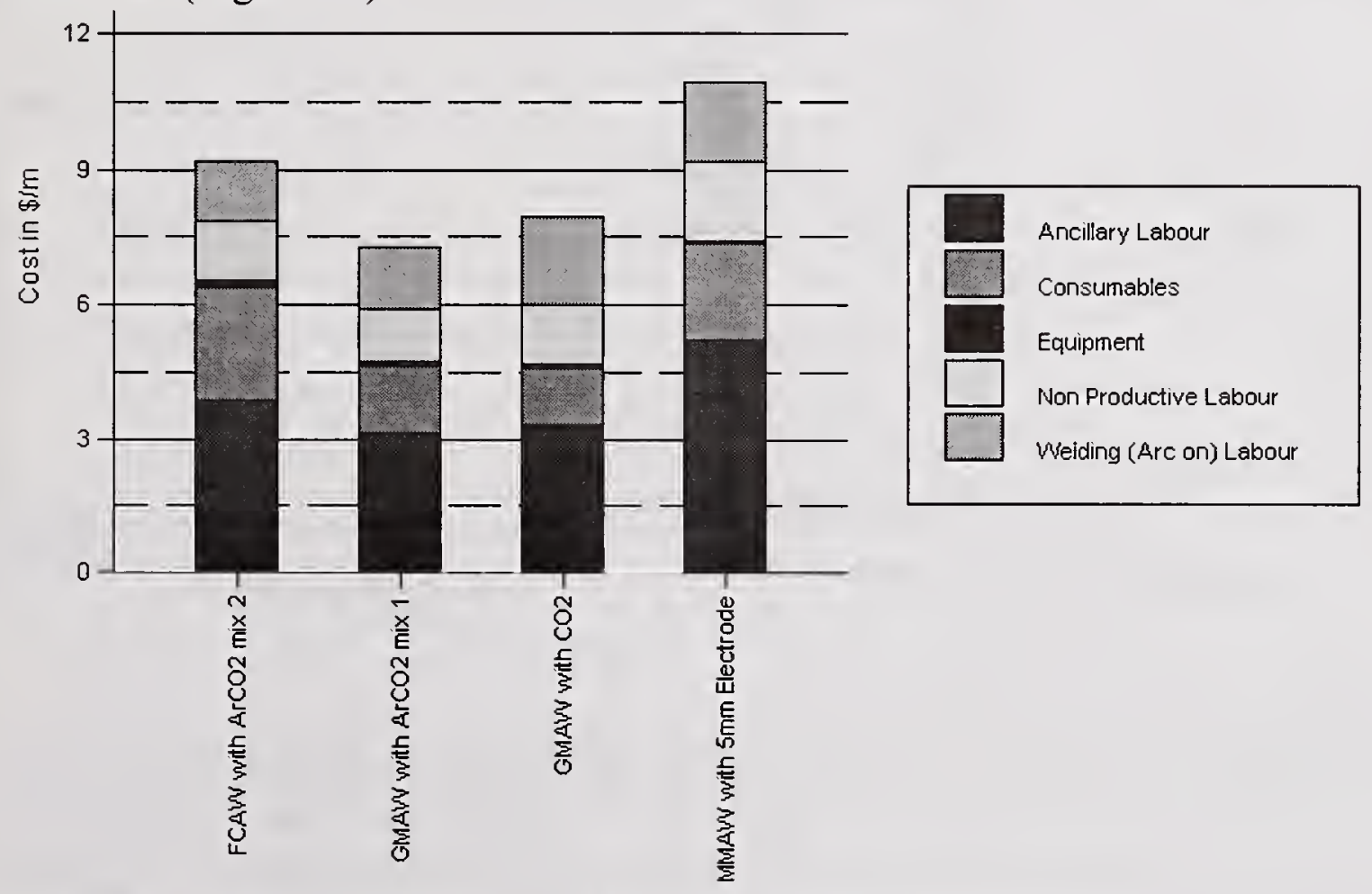

Figure 10 Cost Comparison of Alternative Welding Procedures

Figure 11 shows as an example the printout of the first page of a welding procedure. Figure 12 is from the corresponding welding consumable quantities and cost report. Figure 13 shows the corresponding project summary. 
WELDING PROCEDURE SPECIFICATION (WPS)

Welding Standard: ASME IX

Specification No: MMAW/SAW-2G - 1 (Tutorial Example)

Ref. No /PQRMPAR: MMAW/SAW-2G - Test Report (Tutorial Example)

Now Zemend mang Conters PERA HOUE: Merexten Now Zeavend

Tex. $+64-8-2622805$ Fax $+54-9-262285$

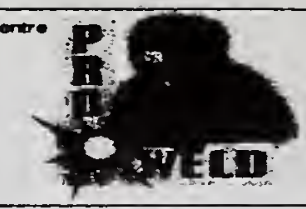

Joint Details:

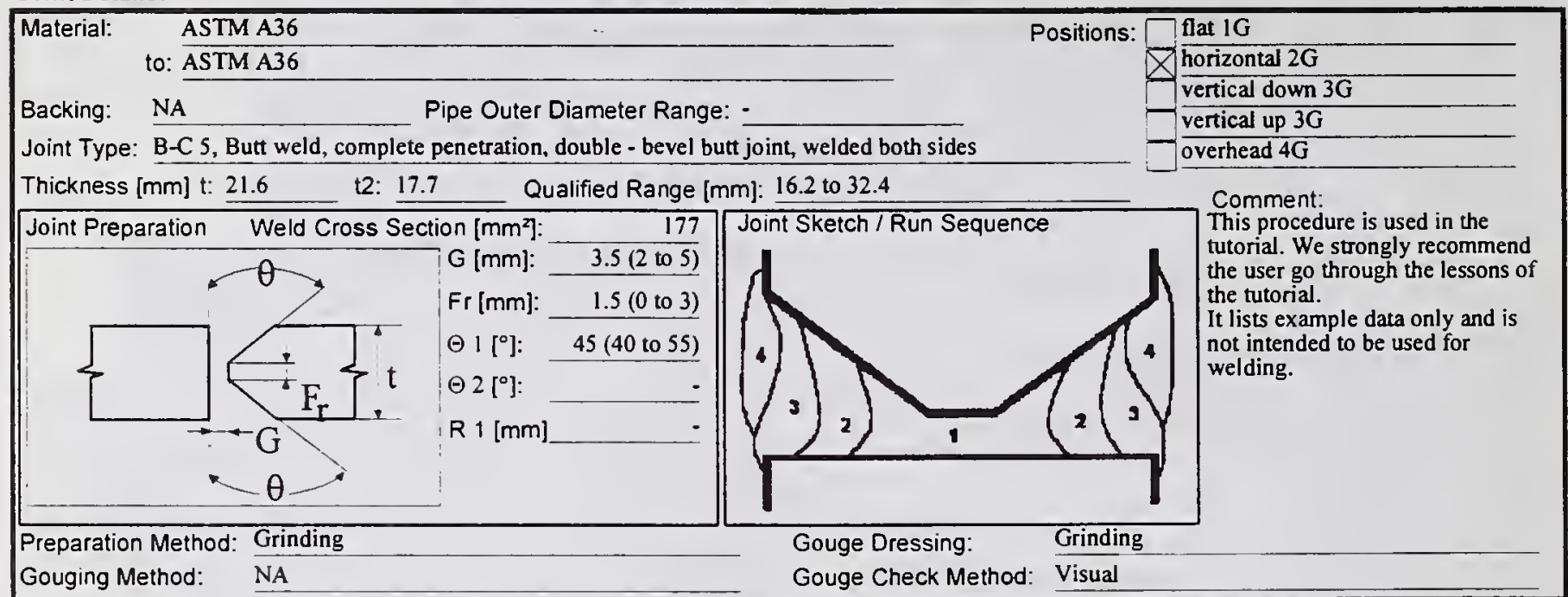

\begin{tabular}{|c|c|c|c|}
\hline Operation Details & Operation 1 & Operation 2 & Operation 3 \\
\hline Description: & root run(s) & filling run(s) & capping run(s) \\
\hline Process: & SMAW & SAW & SAW \\
\hline Position: & horizontal $2 \mathrm{G}$ & horizontal $2 \mathrm{G}$ & horizontal $2 \mathrm{G}$ \\
\hline Run No.(s) / Side(s) Welded: & $1 /$ side 1 & $2-3 /$ both & $4 /$ both \\
\hline Filler Metal Tradename: & Example 6013 & Example 61 & Example 61 \\
\hline Standard/Specification: & AWS A5.01 & AWS 5.17-89 & AWS 5.17-89 \\
\hline Classification: & E6013 & $\overline{E M} 12 \mathrm{~K}$ & EM12K \\
\hline Diameter [mm]: & 5 & 3.2 & 3.2 \\
\hline Filler Details (e.g. F, A No.) & or equivalent & - & - \\
\hline Shielding Gas/Flux Tradename: & $=$ & Flux 1061 & Flux 1061 \\
\hline Shielding Details/Composition & - & - & - \\
\hline Shielding/Standard/Spec.: & - & AWS 5.17-89 & AWS 5.17-89 \\
\hline Shielding Classification: & - & F7A8-EM12K & F7A8-EM12K \\
\hline Flow[l/min]/Usage[kg/h] Rate: & $=$ & 12 & 12 \\
\hline Arc Voltage [V]: & $29 \pm 2$ & $30 \pm 1$ & $29 \pm 1$ \\
\hline Arc Current [A]: & $270 \pm 10$ & $470 \pm 10$ & $450 \pm 10$ \\
\hline Power Source Characteristic: & $\overline{C C}$ & $\mathrm{CV}$ & $\mathrm{CV}$ \\
\hline Current Type: & $\mathrm{AC}$ & $\overline{\text { DCEP }}$ & $\overline{\text { DCEP }}$ \\
\hline Other Settings (Pulse, etc.): & - & - & - \\
\hline Electrode Stickout [mm]: & $\therefore$ & 17 & 16 \\
\hline Travel Speed $[\mathrm{mm} / \mathrm{min}]$ : & $310 \pm 10$ & $470 \pm 10$ & $560 \pm 20$ \\
\hline Wire Feed Speed $[\mathrm{m} / \mathrm{min}]$ : & $=$ & 14 & 12 \\
\hline Arc Energy Input [kJ/mm]: & 1.52 & 1.8 & 1.4 \\
\hline Preheat/Min. Interpass T. $\left[{ }^{\circ} \mathrm{C}\right]$ : & . & $=$ & - \\
\hline Max. Interpass Temp. $\left[{ }^{\circ} \mathrm{C}\right]$ : & - & $=$ & - \\
\hline Torch Angle [ $\left.{ }^{\circ}\right]$ : & - & - & - \\
\hline Technique: & - & - & - \\
\hline Interrun Cleaning: & Grinding & $=$ & - \\
\hline Additional Information: & - & Two Welding Heads & Two Welding Heads \\
\hline
\end{tabular}

Heat Treatment:

Preheatıng Method: required if temperature below $20 \mathrm{C}$

Heat Treatment Comment

Temp. Check Method: NA

Warm to touch

Temp. Check Location: NA

Postweld heat treatment / Cooling Rate: NA

Fabricator:

Welder: Joe Welder . 007

Revision: Date: :

Representative: Your representative

Signature:

Created: $1 / 12 / 96$

Printed: 6/5/97
Principal's Representative / Inspection Authority:

Contract: $\quad$ Contract Number / Description

Organisation: The Boss' Inspection Company Ltd.

Approved by: The Boss

Signature:

File Ref: EXAMPLE SPECIFICATIONS IMMAWISAW-2G - 1 (Tutonal Example) PROWELD Welding Information System

Figure 11 First page of an example welding procedure printout 


\section{Consumables Quantities and Cost Report}

Specification No.: MMAW/SAW-2G - 1 (Tutorial Example)

Scenario No.: $\quad$ Var 2 / Filler metals with higher depos. rates and prices

Job / Project: EXAMPLE SPECIFICATIONS

Total Weld Length: $\quad 2,200.00 \mathrm{~m}$

No of Welds:

12

Average Weld Length:

$183.3 \mathrm{~m}$

Comment: Preparation activities considered

\section{Electrode:}

\begin{tabular}{|c|c|}
\hline Tradename: & Example 61 \\
\hline Standard/Classification: & AWS 5.17-89 : EM12K \\
\hline Diameter: & $3.2 \mathrm{~mm}$ \\
\hline
\end{tabular}

Quantities and Cost of Example 61

$1.10 \mathrm{~kg}$ per metre

$201.67 \mathrm{~kg}$ for each weld

$2,420.00 \mathrm{~kg}$ over total weld length

$\$ 3.26$ per metre

$\$ 597.81$ for each weld

$\$ 7,173.76$ over total weld length

Electrode:

$\begin{array}{ll}\text { Tradename: } & \text { Example } 6013 \\ \begin{array}{l}\text { Standard/Classification: } \\ \text { Diameter: }\end{array} & \text { AWS A5.01: E6013 } \\ \text { 5 mm }\end{array}$

Quantities and Cost of Example 6013
$0.27 \mathrm{~kg}$ per metre
$49.50 \mathrm{~kg}$ for each weld
$\$ 0.95$ per metre
$\$ 173.25$ for each weld

$594.00 \mathrm{~kg}$ over total weld length

$\$ 2,079.00$ over total weld length

Total quantities of Filler(s) required for welding procedure:
$1.37 \mathrm{~kg}$ per metre
$251.17 \mathrm{~kg}$ for each weld
$3,014.00 \mathrm{~kg}$ over total weld length
$\$ 4.21$ per metre
$\$ 771.06$ for each weld
$\$ 9,252.76$ over total weld length

Gas / Flux:

Tradename:

Flux 1061

Composition / Comment:

Standard/Classification: AWS 5.17-89 : F7A8-EM12K

\section{Quantities and Cost of Flux 1061}

$2.41 \mathrm{~m}^{\wedge} 3(\mathrm{~kg})$ per metre $\quad 441.83 \mathrm{~m}^{\wedge} 3(\mathrm{~kg})$ for each weld

$\$ 4.82$ per metre
$5,302.00 \mathrm{~m}^{\wedge} 3(\mathrm{~kg})$ over total weld length $\$ 10,604.00$ over total weld length

Quantities are listed in $\mathrm{kg}$ for $100 \% \mathrm{CO} 2$ and SAW Flux otherwise in $\mathrm{m}^{\wedge} 3$

Figure 12 Example of printout of consumable quantities and cost report 


\section{Project Summary}

Project No: Tutorial Example Project

Comment: PROWELD example procedure variations combined as project

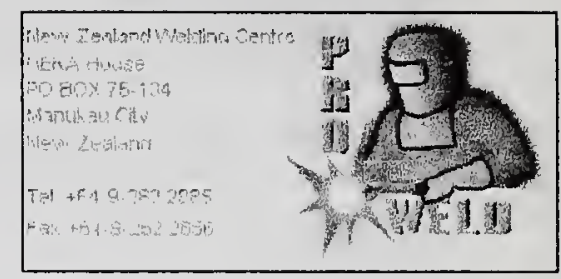

\section{Variation No}

Cost of GMAW hollow section butt weld

Var 2 / Filler metals with higher depos. rates and prices

\section{Total Cost of Project: \$196,534}

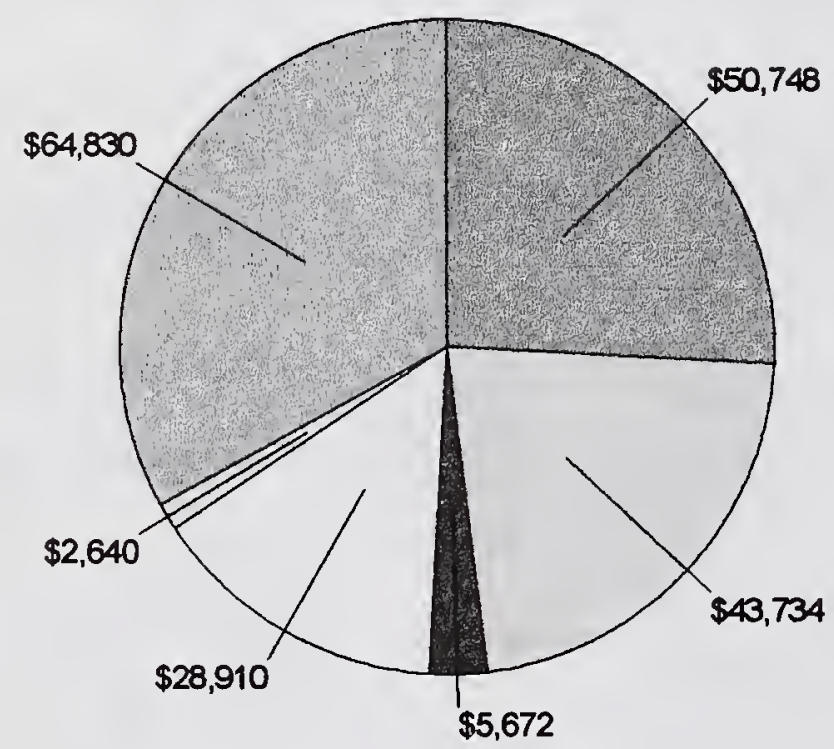

Total Time of Project: 4,925 hours

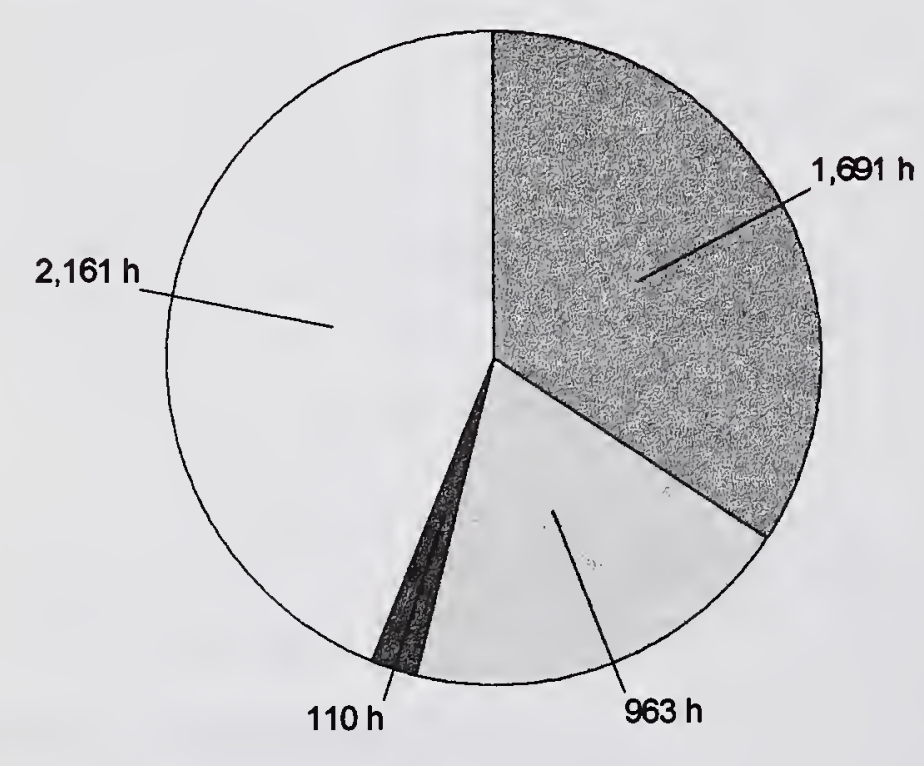

Figure 13 Print out of example project summary

\section{Specification No}

GMAW hollow section butt weld example MMAW/SAW-2G - Plate - 001
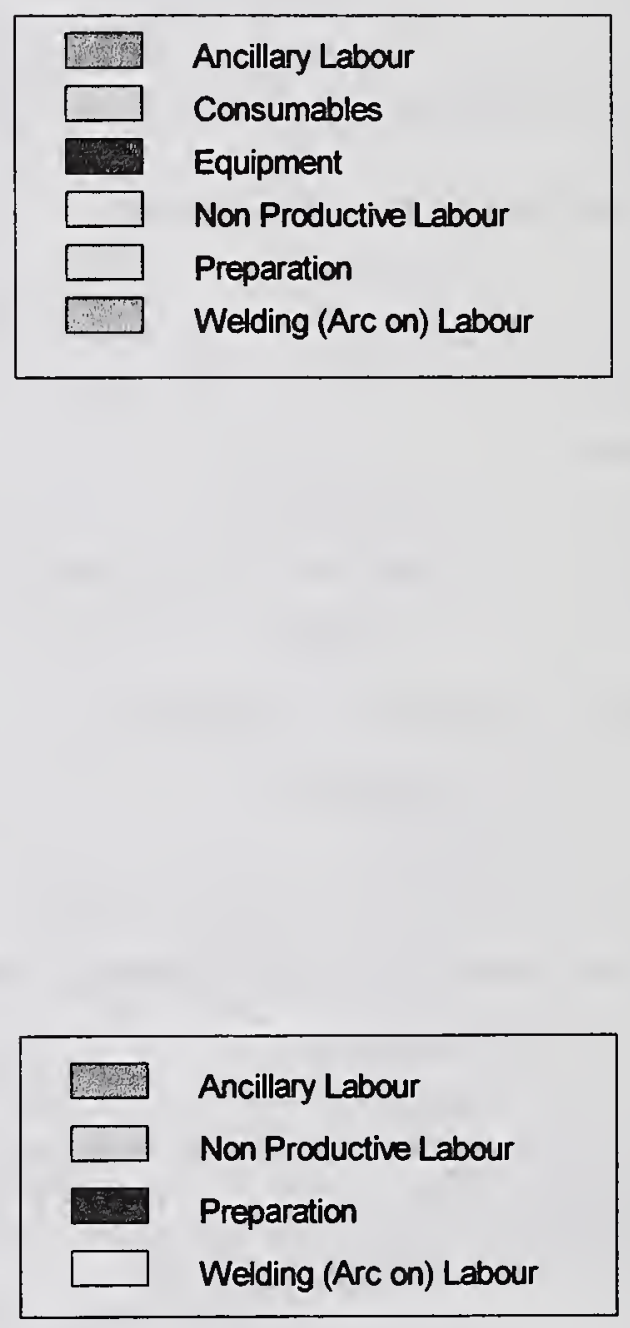


\section{CONCLUSIONS}

PROWELD is a easy to use, Windows ${ }^{\circledR}$ based welding information system. Using the welding information system provides the following benefits:

- Simplifies development and maintenance of welding procedures related records.

- Improves productivity of welding and administration.

- Project estimation is made easier and more accurate.

- Improvement and simplification of welding procedure data management.

- Helps to find more economic welding solutions.

- Development of a knowledge base that is available within an organisation.

- A higher standard of quality assurance is achieved.

\section{REFERENCES}

1. Scholz W 1988. Strategies to Improve Welding Productivity, Proceedings IIW Asian Pacific Regional Welding Congress, Hobart.

2. Seidel R and Scholz W 1990. Productivity Improvement of Arc Welding Processes using Artificial Intelligence. Proceedings of the 3rd International Conference on Computer Technology in Welding, Brighton.

3. Rieck O 1989. Development of a Concept for a Computer/Based Expert System for Arc Welding Process Selection. Master thesis at the Department of Mechanical Engineering, University of Auckland.

4. Pook R W 1991. Concepts for an Expert System for Arc Welding Productivity Improvement. Master thesis at the Department of Mechanical Engineering, University of Auckland.

5. Frank C 1991. Development of the Logical Structure for an Optimal Goal-Generation in Expert Systems. Diploma thesis Institut A für Mechanik, Universität Stuttgart and University of Auckland.

6. Pinn T 1994. Wissensakquisition und -strukturierung für Expertensysteme: Anwendung der KADS-Methode auf ein Problem der Schweißtechnik (Knowledge acquisition applying the KADS method for a welding expert system). Final year project, New Zealand Welding Centre and Fachhochschule Niederrhein. 


\title{
A COMPUTER SYSTEM FOR PREQUALIFIED STRUCTURE WELDING (AWS D1.1.94)
}

\author{
A. El-Ashram *, E.A. Shaaban * and H.I. Shaaban**
}

\begin{abstract}
The American National Standard Structure Welding Code (ANSI / AWS . D1.1) is different from other welding codes (e.g., ASME, API .... etc.) .It presents prequalified welding procedures if some requirements are fulfilled. These requirements include : joint type, workmanship and welding techniques which are specified in details in various Chapters of this Code.

The Present work aimed at transforming the available required data for prequalified welding procedure into a computer software that facilitates the use of the data. A complete set of logic decision trees were developed. Such set of trees remove most of the decision making problems upon using the Code.

All decision trees were collected to form one computer software, Which is a type of intelligent and user friendly system. The user is asked some specific questions about the welding procedure being used. The final decision if this procedure is preqelified or not is given.
\end{abstract}

\section{INTRODUCTION}

Welding codes and standards have been developed to provide public health, Safety and high quality of the welded joints. Since the early development of the welding codes and standards, all of which have been continually edited and revised to comply with the demands of the growing technology. One of the most effective methods of technology transfer is to apply a code or standard, either in welding or in any other industry.

It was found in Egypt that applying the welding codes in industry will be much easier if these codes have been simplified for use. Thus a project have been developed to transfer some of the most important welding codes to a simply used computer software. This project is a joined work between researchers from Egyptian Atomic Energy Authority and Alexandria University. In this project three welding standards have been dealt with . These standards are:

a) The American Petroleum Institute Standard No. API-1104 concerned with welding

\footnotetext{
* Alexandria University, Alexandria, Egypt.

** Atomic Energy Authority, Alexandria, Egypt.
} 
petroleum pipelines and related facilities.

b) The American Society of Mechanical Engineers Boiler and Pressure Vessel Code, Section IX concerned with welding procedure and welder qualification.

c) The American Welding Society Code No. AWS D1.1-94 concerned with welding of steel structures such as buildings, bridges and tubular structures.

It has been noticed that AWS D1.1-94 Code is different from other codes in that welding procedures can be considered prequalified, i.e. prequalification by tests is not required. In this case, welding procedure variables must meet the prequalified conditions of the code. In this paper, these prequalified conditions and requirements have been transformed into a set of logic flow charts (LFC's). Then, these LFC's have been transformed into a simply used computer software. Such software could be used to simply determine in advance what are the conditions required for prequalification. Also, it could be used to check any welded joint if it is prequalified or not.

The generation of the computer software as an aid in the welding technology has been developed extensively in the recent years. Together with the specialized conferences an computer technology in welding, most of the general conferences on welding have sessions related to using of computer in welding (Ref. 1,2). However, in the fields of WPS (Welding Procedure Specifications) preparation or qualification most of the commercially available software are just data bases (Ref. 3,4 ). Very little of the published work in this specific field in considered artificial intelligent (Ref.5). The present authors had some publications in this field (Ref. 6,7).

\section{CONTENTS OF THE SYSTEM}

According to AWS D1.1-94 Code a prequalified welding procedure must comply with Sections One through Four of the Code, as well as the applicable Sections of Eight, Nine and Ten. Section One provides general requirements that pertain to the type of weldment governed by this Code, which is restricted to welded steel structure. Also, this section specifies the shape and type of material welded, which include carbon and low alloy steels. The Section also specifies the prequalified welding process.

Section Two deals with the design of the welded connections. The section also contains information about minimum and maximum weld size, length and spacing. A detailed set of Tables are provided which describes prequalified partial and complete penetration groove welds. Each Table includes : welding process, joint designation, base metal; thickness, groove preparation, position, shielding gas, together with some notes.

Section Three provides requirements for workmanship, which includes production and inspection of the welded assemblies. These requirements includes: preparation of base metal prior to welding i.e. cleanliness, inspection of edges and sometimes repairs in accordance with Code requirements. After preparation, assembly is discussed with regard 
to the fit-up of members to be welded, control of distortion and shrinkage of dimensional tolerances. Acceptable and unacceptable profiles for fillet and groove welds (such as overlap, undercut, ..etc.) are also discussed. Other requirements dealing with penning, caulking, arc strikes, weld cleaning and weld termination are given in this Section. These requirements are not specific to a particular process but apply to the weldment as a whole. For a welding procedure to be prequalified the procedure must meet the requirements of Section Three.

Section Four is process oriented, dealing with the prequalified processes which include : SMAW, SAW, GMAW and FCAW; except in short circuit transfer. Each process is discussed with regard to selection of filler metals for base metals listed in the Code. Care and storage of filler metals, fluxes, and shielding gases requirements are also specified. Other requirements such as weld size, maximum single pass fillet weld sizes, size of electrodes, ambient temperature (the temperature in the area adjacent to the weld) and other requirements are also discussed in this Section.

\section{THE LOGIC FLOW CHARTS AND THE SOFTWARE}

A complete set of logic flow charts (LFC's) were developed and collected to form a computer software, the following is a brief description of these LFC's

The main stages for obtaining prequalified welded joints are illustrated in Fig. 1. This main LFC consists of three main modules including joint requirements, workmanship requirements and technique requirements. The first module consists of two submodule: fillet weld and groove weld submodules. Fig.2 includes data and information collected from Section Two of the Code. The main part of this submodule is how to identify the Table containing all the information needed, by choosing from 10 weld types (square groove, single-v-groove, double$\mathrm{v}$-groove, ...etc.) and 3 joint types (Butt joint, Corner joint and T-joint). The program displays the drawing of the joint, maximum base metal thickness, root opening, tolerances, permitted welding positions, gas shielding for FCAW and some other notes.

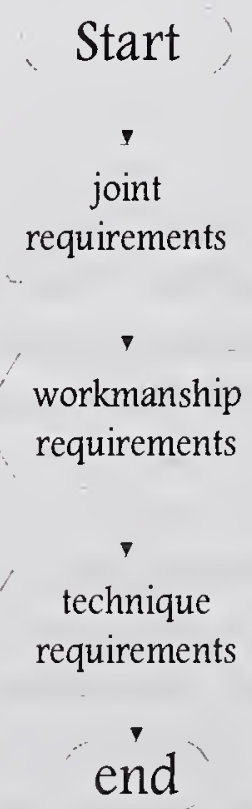

Fig. 1 : The main stages for obtining prequalified welded joint according to AWS - D1.1 


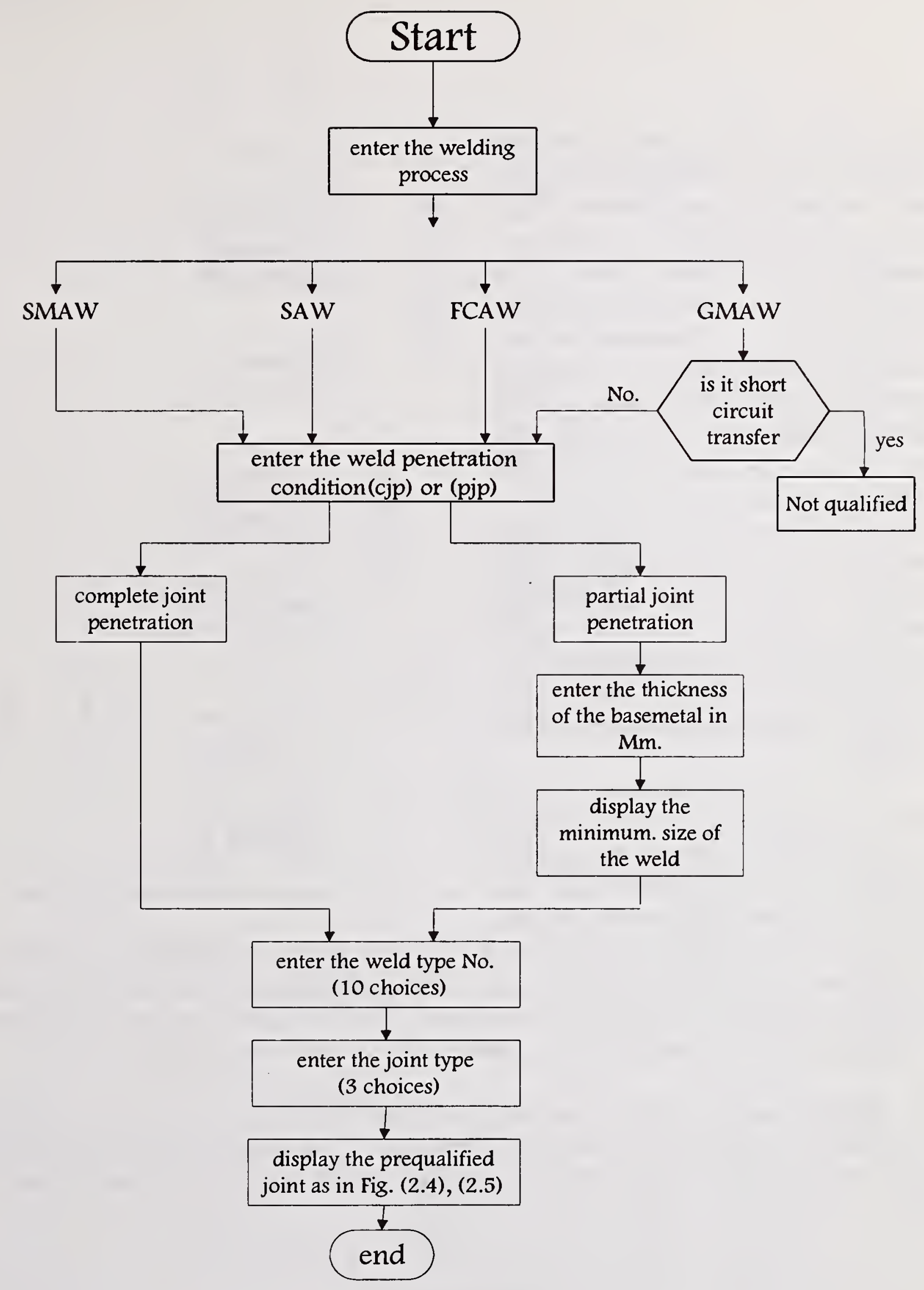

Fig. 2: Groove weld submodule as a part of the joint requirements module 
The second stage (second module) consists of five submodules discussing the workmanship requirements. Fig.3 shows these requirements which are collected from Chapter Three of the Code. The five submodules include: prequalification requirements for base metal preparation, assembly, distortion control, welded structure tolerances and accepted weld profile. As an example, Fig.4 shows the assembly submodule which is divided into five types of welds and joints. One of them is the groove weld which contains three different types of joints. According to backing conditions the groove angle, root opening and root face permitted are determined in addition to some notes needed to fulfill the requirements of the assembly.

The last stage (module) for prequalified welds consists of several submodules illustrated in Fig.5 and based on information given in Chapter Four of the Code. As an example of these trees a logic flow chart for electrode requirements in SMAW are given in Fig.6. This logic flow chart is explaining the electrode requirements which is a part of the SMAW submodule. The LFC shows the electrode storage temperature, period for getting dry, exposure time, moisture content accepted ... etc.

The software was developed using the Turbo-Pascal language. The system could be considered as intelligent and user friendly system. It is an interactive system with the user. Some examples of the obtained screens during the consultation process are given in Fig.7.

\section{CONCLUSIONS}

1. The developed computer software could be considered as an intelligent system with two main objectives. The first objective is to determine if a specific joint is prequalified or not. The second objective is to determine in advance (before welding) all the prequalification requirements for any joint.

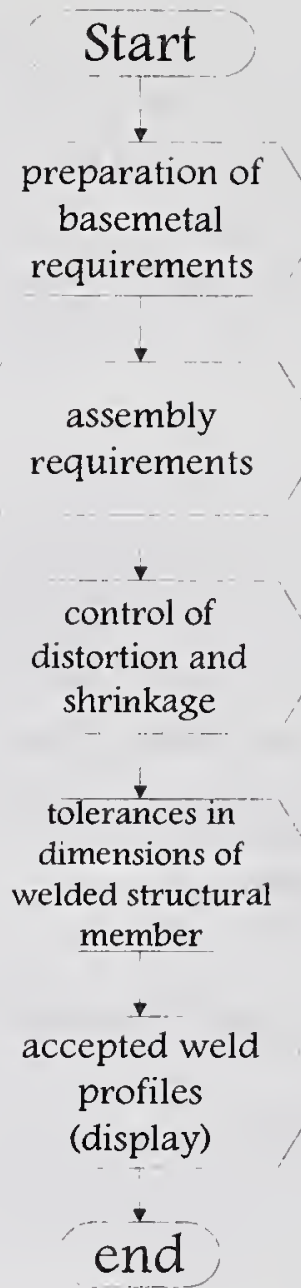

Fig. 3 : Stages of workmanship requirements 


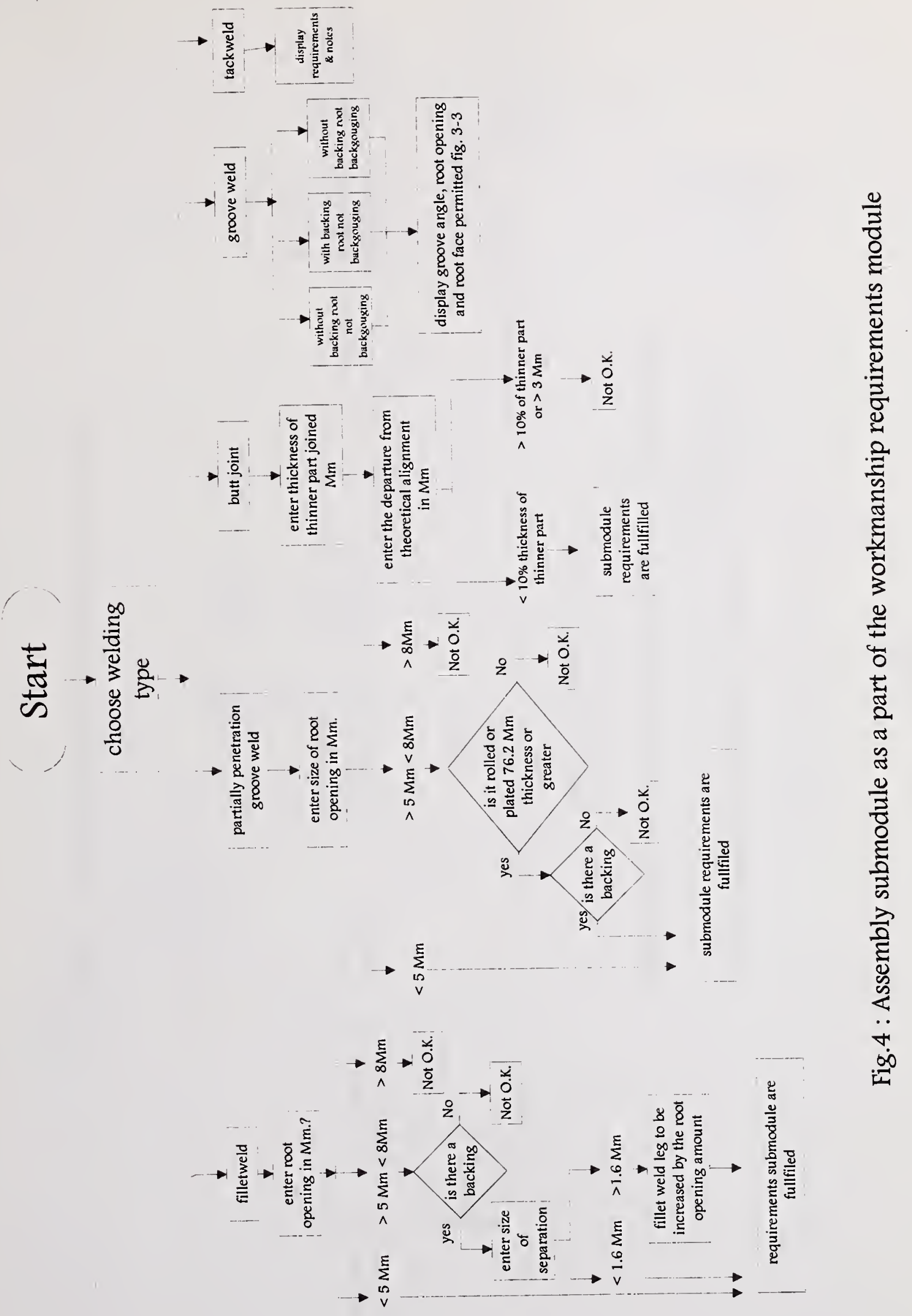




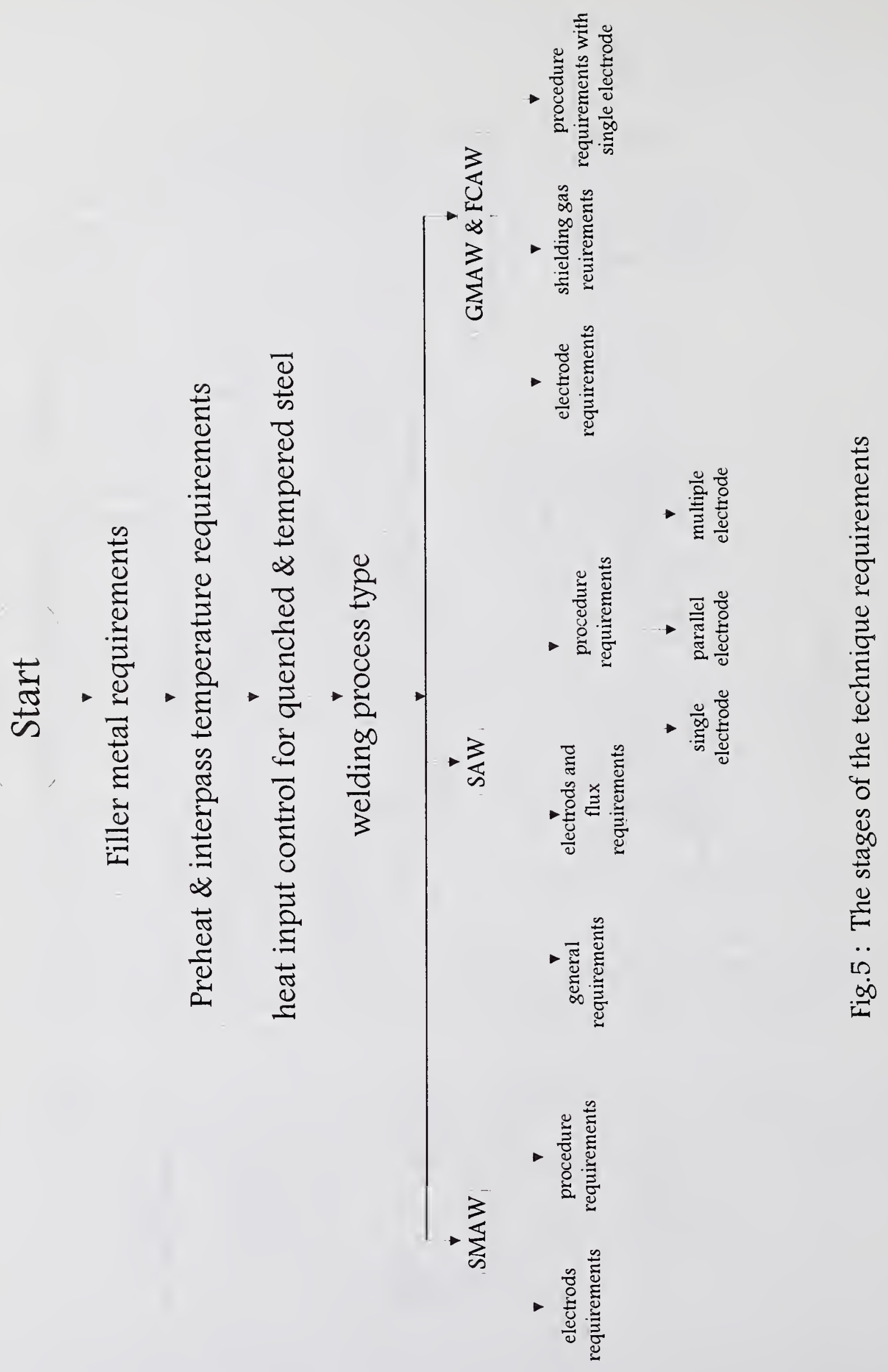




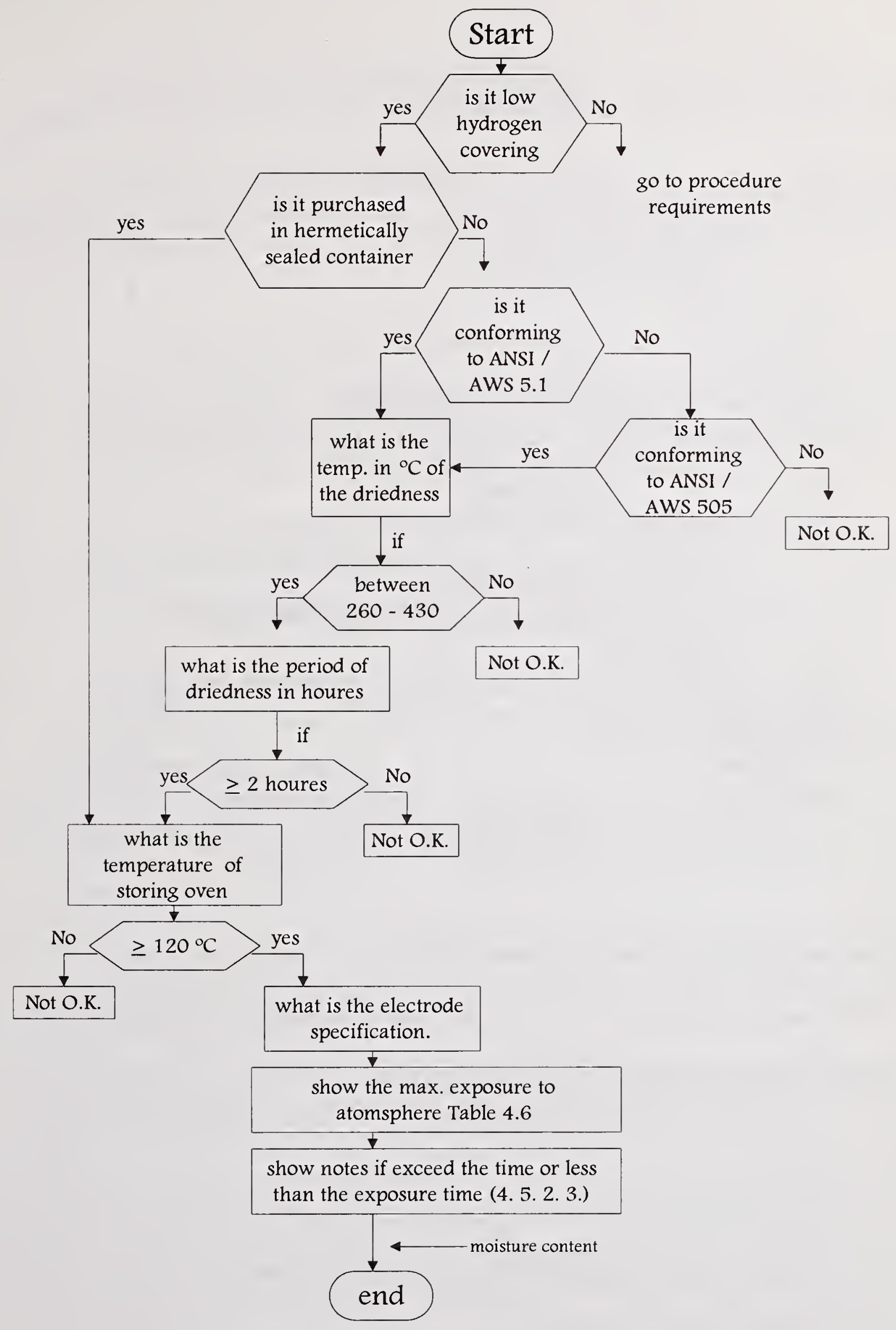

Fig.6 : Logic flow chart for SMAW electrode requirements. 


\begin{tabular}{|c|}
\hline $\begin{array}{c}\text { Prequalified joints } \\
* * * * * * * * * * * * * * * * *\end{array}$ \\
\hline $\begin{array}{l}\qquad \text { Make your selection : } 2 \\
\text { 1. Joint requirements } \\
\text { 2. Workmanship requirements } \\
\text { 3. Technique requirements } \\
\text { 4. Exit }\end{array}$ \\
\hline
\end{tabular}

Workmanship requirements
Make your selection : 2
1. Preparation of base metal
2. Assembly
3. Control of distortion and shrinkage
4. Tolerances in dimension of welded structure member
5. Weld profiles
6. RETURN

Choose one of there weld types

1. Fillet weld

2. Partial joined penetration

2. Butt joint

5. Tack weld

4. Groove weld

3

Enter the thickness of thinner part joined in Mm?

50

Enter the departure from the optical alignment $\mathrm{Mm}$ ?

5

The joint is not a prequalified joint you have to qualify it

Press any key to continue

Fig. 7 : Typical screens during running of the program. 
2. The system could be used for training of personnel in using the AWS - D1.1 Code, specifically in the part concerned with prequalified joints.

3. The work developed in this article is a part of a more comprehensive work concerned with several welding codes and standards as a part of applying welding codes in some Egyptian industries.

\section{REFERENCES}

1. Kannaty - Asibu, E.Jr.; Cho, H.S. and Fukuda, S. (eds.) 1991. Welding and Joining Processes. Proc. of the Winter Annual Meeting of ASME, Atlanta, Georgia, December 1-6, 1991, PED-Vol.51, ASME, U.S.A.

2. The European Institute for the Joining of Materials, 1997. JOM-8. Proc. Intl. Conf. on the Joining of Materials, JOM, Helsingor, Denmark.

3. C-Spec. Catalog, 1997. ASME and Welding Software. C-Spec., U.S.A.

4. Luces, W. and Brightmore, A.D. 1987. Information Software for Welding Engineers. Proc. 1st Intl. Conf. in Computer Technology in Welding. AWS, U.S.A.

5. Fukuda, S.; Morita H.; Yamauchi, Y. and Tsuji, S. 1991. Expert System for Producing WPS. Proc. Winter AnnualMeeting ASME, December 1991 eds. Kannatey - Asibu, E. Jr.; Cho, H.S. and Fukuda, S.; PED- Vol. 51, 91-97, ASME

6. Shaaban, H.I.; Shaaban, E.A. and Abou-Ali,M.G. 1997. An Intelligent ComputerBased System for Welding Qualification According to ASME - Section IX. Proc. JOM-8 Intl. Conf. Joining of Mat. : 470-475. JOM, Helsingor, Denmark.

7. Abdel-Moatty, M.S.; Abou-Ali, M.G. and Shaaban, H.I. 1997. A Computerized Practical Guide to API-1104 Welding Qualification. ibid : 476-481. 


\title{
WELDING DATABASES - EXPERIENCES FROM IMPLEMENTATION IN US, CANADA AND EUROPE
}

\author{
E.Engh 1
}

\begin{abstract}
A number of welding databases have been implemented on various hardware and software platforms over the years. From a marketing point of view these databases should have a similar approach and functionality and it should be able to implement them all over the world. However, in practice, the implementation and requirements differ greatly between US and Canadian fabricators and European fabricators. Historical background and culture as well as fabrication experience and difference in standards, has led to different requirements in this field. The understanding of what a database is, as well as the requirements for integration with other systems, are different.

This paper summarizes the experience gained through the implementation of welding databases in Europe, US and Canada and discusses some of the differences between these markets. Some important questions are raised for the fabricator that wants to work in a international environment with changing requirements.
\end{abstract}

\section{BACKGROUND}

4X Software has been working with the development of welding databases since 1992 both in Europe as well as in US and Canada. The experience gained during this time is the basis for this presentation. The company has been working with demanding clients in the offshore business, as well as with fabricators building nuclear submarines, high quality boilers and power plants, bridges, etc. In addition, the company has been developing the Norwegian Quality Manual for ISO 3834 (EN 729) 'Quality Requirements.......'

\section{HISTORY}

In the early 80 's the first computer systems for managing welding documentation appeared. These were either proprietary systems developed on mini- machines or mainframes in-house, or in the latter part of the 80 's, PC based systems running under MS-DOS.

Most of these systems were file-oriented systems, but due to the nature of the programs, they were called databases or database systems. When we first investigated these systems, we found that they had a limited functionality, especially when you wanted to store a welding procedure. The procedure is typically stored with a file name in the file structure of MS-DOS. The proprietary file structure resulted in difficulties if you needed to modify the structure. It 
is also nearly impossible to have a flexible way of searching for the properties or the essential variables in these procedures.

These systems were designed to be a tool for the welding engineer and they were not intended to be used for integration with other systems, if ever thought of.

Most of these systems were developed through Institutes or Universities in conjunction with local fabrication industry. A limited number of systems were developed commercially by private organization in US.

Later developments in the mid 90's started to use PC based database systems like Access, Paradox and so forth and they moved to Windows 3.11 and later Windows 95. Still these developments have been designed as a tool for the welding engineer only. Looking at the possibilities for integration and flexible connections to other systems today, they still lack some of the basic functionality.

\section{STANDARDS (CODES)}

One of the basic problems when developing software for the welding industry is that there are a number of welding standards available. US standards and Canadian standards which differ and European standards which differ even more.

The information required in a WPS (Welding Procedure Specification) is quite different when you look at ASME IX and EN 288. The recommended document layout is also different for these two standards. The same is true for the WPAR (Welding Procedure Approval Record) or PQR (Procedure Qualification Record) as it is called in US. To make it even worse, the test documentation required in ASME IX and in EN 288 are completely different. The documentation itself (or the document layout) is also quite different.

Apart from the national standard, other requirements coming from the MIL standards as well as the NORSOK standards for the offshore industry, makes life difficult from a documentation point of view.

If we look at the boiler industry, the same problems will appear and special industry-related requirements have been applied making it difficult to establish a common documentation.

The file oriented systems from the late 80 's could not cope with this situation. The results have been that a number of systems have been developed in US and Canada for this market and the same has happened in Europe. Since all these systems have a proprietary data structure, exchange of information has been impossible. The document layouts that these systems have are also proprietary and have been the result of a supplier's understanding of the standard. 


\section{SOFTWARE DEVELOPMENTS}

When we started the development of the XWELD system in early 90's, the challenges were to overcome these barriers from a standardization point of view.

However, we ran into a number of other problems that we did not foresee, but at the end, these were overcome:

\section{Method dependent parameters.}

As a welding engineer knows, the essential variables for a welding procedure will vary depending on the welding process. This means that the welding parameters and the other variables can change completely depending on the welding process that has been selected. A typical example can be welding parameters for FCAW compared with the parameters for GTAW Hot Wire. Neither ASME IX nor EN 288 covers this aspect from a documentation point of view. The result is that the different fabricators choose their own solution to the documentation problem.

One solution is the development of different process-oriented welding procedures where a procedure is so general that it can cover more than one process or a range of processes.

A creative welding engineer, Lee Piggot O'Connell has selected another approach. He developed a general purpose steel structure and piping welding procedure, each covering all processes. These procedures are not suitable for production because they contain too much information for the welders. When selecting a welding process, the system automatically selects the correct output welding procedure layout which contains the correct parameters for that very process only. By doing so he, at the same time, makes it simple for the welders to read and understand the procedures.

\section{Industry dependent requirements.}

Many of the requirements in a procedure can be industry dependent. Offshore specifications will require more information in a procedure than a normal steel structure of general nature. The same happens on nuclear structures as well as in other industry types. Examples can be that PCM, CE values and so forth can be required for these industries, but not directly required in the standards. This kind of industry related information can vary from country to country.

\section{Standard related information.}

When studying the ASME IX code and comparing it with EN 288, one will easily see that the requirements for information are different. The way this is documented is also different.

If one looks at the ANSI standard for database contents one will not find that information in any EN standard.

The document layout and the content in the documents for ASME IX and EN 288 are not completely defined from a data type point of view. We very often see that the documentation and its information content has been developed differently from company to company. 
A flexible system must cover both standards and the user should be able to select which standard or code he would like to work according to.

\section{$\underline{\text { Terminology. }}$}

One of the major problems that can occur comes from the terminology and the use of technical units. For example, what is really meant by the phrase "layer" or "pass" or "string" ? What is "weaving" and "stringing"?

What is "as welded" ? Can you have used wire brush, cleaning or grinding to remove spatter before ? What is a repair? If you have flushed the weld by grinding, is that a repair ?

Questions like these create problems from a database point of view.

The documents that are designed should therefore have an explanation that can be uniquely defined. If the expressions are different but the technical content is equal, the system should store the information in the same place in the database.

\section{Technical units.}

Although standards have been defined, one must admit that old habits still hang around. Most of the European countries now use the metric system, but some companies still use English units, especially on pipe work. The Canadians still use decimal imperial, but a lot of the US companies still use imperial units.

From a system point of view this causes problems. How shall you store the information in the database and how accurate shall the values be stored ? If you use decimals, how many decimals are appropriate for the different information? What technical units shall be used for the test data?

\section{Language.}

The US and Canadian market has one advantage, the same language (except for Quebec in Canada).

In Europe we have a number of languages. Although a lot of the welding information can be written in English, one has to face the language problem and admit that procedures have to be generated in a multilingual environment.

This can be solved through multilingual procedures, which will be so filled with information that it is not practical to use in production, or it can be solved through a switch mechanism that allows the user to select which language to work with.

The language problem can be of high importance specially when the job has been specified in one country and carried out in a different country. 


\section{SOLUTION}

In an international environment, a system has to address and solve these problems. An analysis of the requirements in the standards combined with an analysis of the above problems has resulted in the following solution. This was to develop a database structure on top of a relational database system. This allowed us to be flexible with regard to later extension of the database itself. It allows us be flexible in the retrieval and search for information and it lets us be open to interface and data exchange.

The other solution was to separate the data from the document layout. This means that the documents as seen on the screen only represents the data that have been selected by the document itself. This allows us to separate the data and the presentation. This will solve two problems: the format according the standards or welding methods and the problem with the presentation language.

The solution gave us full flexibility to meet any documentation requirements and any language requirements.

From a software support point of view we have also solved another problem by selecting this solution. The standard system could be isolated, the database could be standardized and the customization could be added at the presentation level.

\section{IMPLEMENTATION}

The implementation of a database system for welding can be a straight forward job since it influences only a limited number of persons in the company. A standard implementation can be carried out within a couple of days including training. Such a system would typically be used as a stand-alone system for the welding engineer and the welding foremen. In some cases the test laboratory is also affected.

In larger companies this can grow into a company network with a number of users.

Two new areas are however growing:

An intranet welding network.

Such networks can either consist of a number of welding databases that are connected so that the different users can communicate and select procedures from the different databases. Or a corporate database is established and the different users are working from the corporate database.

From experience, such network can reduce the qualification of new welding procedures by approximately $20 \%$ to $30 \%$, saving a huge amount of money and time.

Such intranets will require a standardization of the terminology throughout the company to be able to use the correct search parameters for the database in order to be able to retrieve information. 


\section{Project oriented databases.}

Such networks, established for projects only, are also growing. Because the new standards often allow procedures to be used at different production facilities due to common welding supervision or common quality requirements, we see an increasing use of project related networks. Networks between a main supplier and a sub-supplier also fall into this category. The sub-supplier can be approved and verified as a supplier and through the verification process automatically gets access to the common welding database.

Again this saves time and reduces the need for requalification of procedures.

\section{New business opportunities.}

Most of the new standards require that the fabricator shall be responsible for the WPS. It does not say too much about the WPAR (PQR). In some cases a fabrication can use prequalified procedures or procedures based on experience.

This presents opportunities for a new market, the trade of welding procedures. If the procedure (WPAR/PQR) is of such a kind that it can be used directly for generating a WPS, it simplifies the whole process.

On the other hand, if a fabricator has to weld the WPAR (PQR), then a procedure that has been welded before, containing acceptable test data, will be extremely valuable, because using those data gives you the possibility to have a "hit" at the first try. For unusual material or material properties, this can save enormous amount of money and time.

\section{Integration with expert systems.}

A welding database solves only the problem when you have done the welding before. New technologies, neural nets and expert systems can be very attractive complementary technologies. Integration of databases and these technologies should be of great interest for the welding industry.

How can this be used?

\section{Expert Systems.}

When you do not have experience, an expert system could lead the way through a number of questions to a solution or a proposed solution which may be presented as a welding procedure.

In some cases such a welding procedure can be used directly if there is no special requirements for a WPAR (PQR).

Or, such a procedure can be used as a pWPS (preliminary Welding Procedure Specification). A welding foreman or even a welder could generate this before welding the WPAR (PQR) itself. Such a use can reduce the resource requirements of the welding engineer. 


\section{Neural net.}

The net could be trained to give a solution to a specific task or process. Thus, the net could generate the required welding parameters as described above.

However, one could imagine a solution where a net was directly connected to the welding process itself and was used in conjunction with a welding procedure to give feedback and corrections to the welding parameters during the welding process itself.

\section{Integration with robotics welding.}

Robotic welding creates new opportunities for a technologist. Firstly is the problem related to the geometrical path, pattern recognition and correction, adjustment of the welding parameters according to changes in the fitup of the parts, compensation for welding distortion and so forth.

The most common way of solving the settings of the welding parameters today in robotic welding has been to develop a separate program or data entry in conjunction with the robot itself.

From an isolated welding point of view this will work, but it creates some basic problems as well. Firstly, it normally allows the operator to manually enter the data. From experience this may result in data entry errors or alterations caused by individual reasons. Some of the most common errors have been that the welding process "sound" is not correct and adjustments have been made so that the correct "sound" appears. If there is any changes in the wire characteristics, this can cause huge problems.

Secondly, the requirements in ISO 3834 for traceability of the welding process are normally not met by such an approach.

One solution that may prove successful, was implemented at KARLSKRONAVARVET AB in Sweden, welding military bridges in Weldox $1100(1100 \mathrm{MPa})$ material. A welding database was used, showing a normal welding procedure according to the EN 288 requirements. Such a procedure does not contain all the parameters necessary for robotic welding and consequently a secondary datasheet was created to fulfill the requirement of the robotic welding equipment.

The welding database was directly connected to the welding equipment and it was possible for the operator to transfer the welding procedure, and consequently the robot data, directly into the unit itself. This approach solved the documentation requirements, as well as the QA aspect of using the correct data for the welding.

A two way communication was established from the operator to the welding engineer to allow the operator to communicate instantly with the welding engineer if alterations or modifications have to be made to the process itself, or if new procedures must be generated.

The production results have been outstanding with excellent quality and high productivity in production. 
A welding database should not be a stand-alone system, although in most cases this is the situation. The welding data should be of interest to the designer who should be able to retrieve information from such databases to be able to make an optimum design. From a QA and QC point of view, the welding database is essential for extracting data for other areas of the information flow through production.

The welding foremen should have access to such a database for planning purposes. The QC department needs to have access to welding information for reference purpose for the NDT reports.

The document control department need access to the welding database when creating the asbuilt documentation and final delivery documentation.

Welders certificates can be updated based on successful production welding. This require reference both to the weld itself and the welding procedures.

In an integrated quality assurance system this means that the welding data will be accessed for other purposes than the physical welding itself. The database must therefore be open for integration with other database structures.

Examples of utilizing the welding database for other purposes which we have seen have been:

$+\quad$ Generating statistics for welding defect type based on WPS

$+\quad$ Generating defect rates for WPS

$+\quad$ Generating filler requirements based on WPS and weld length

$+\quad$ Generating Job - Cards based on WPS

$+\quad$ Generating purchase order for filler based on weld length and WPS

$+\quad$ Update of welders certificates based on WPS, weld number and NDT result

$+\quad$ Download WPS data to robotics welding equipment

$+\quad$ Analyzing differences in WPS over a range of methods and essential variables

\section{CONCLUSIONS}

From a historical point of view the term Welding Database has been used to cover a number of technologies, from pure file system and text editing systems to real databases of different types, hierarchical, relational and so forth. This has and still does, create a lot of confusion in the industry.

However, a database system, based on relational or similar technology has a great opportunity in the industry if implemented correctly.

A welding database should not be the solution, but it should be one of the building blocks in an integrated system for quality control and quality assurance.

Special care has to be taken when implementing such databases in the company. Some of the 
key questions you have to ask is:

$+\quad$ Shall we work internationally and cover different languages and standards ?

$+\quad$ Shall we adopt a TQM strategy for welding ?

$+\quad$ Do we want to meet the ISO 3834 (EN 729) requirements ?

+ Do we foresee a changing environment for the company, and do we need to be flexible for the future?

$+\quad$ Do we need to cooperate with other welding fabricators?

If your answers are positive, an open approach must be taken when implementing a welding database in the company. 


\title{
A Computer Accessible Welding Procedure Database System for use with Intelligent Off-line Planning Systems
}

\author{
P. Sewell, N. B. Gomez, C. Sakata, J. E. Jones, V. Rhoades \\ N. A. Technologies Co. \\ Golden, Colorado
}

\section{Summary}

Today's welding procedure databases have three primary shortcomings within the purview of recent advances in computer technology. First, there are no provisions for connectivity across computer networks and directly into other applications software; second, most systems do not store weld cross section images, dynamic measurements of the weld, or the resulting quality parameters; third, full multimedia support is usually not included.

N. A. Tech's N-Base ${ }^{\mathrm{TM}}$ welding procedure database addresses these issues. The actual database can be stored in a central location as part of a LAN'; it is integrated with the $\mathrm{NAMeS}^{\mathrm{TM}^{2}}$ weld measurement software; and it includes multimedia decision support for off-line planning of welding processes (in the case of a Welding Procedure Specification). N-Base ${ }^{\mathrm{TM}}$ was developed as part of a joint effort between the U. S. Navy, N. A. Technologies Company, and several commercial organizations. N-Base ${ }^{\mathrm{TM}}$ supports multiple standards such as $\mathrm{AWS}^{3} \mathrm{~A} 9$ \& $\mathrm{D} 1, \mathrm{UNS}^{4}, \mathrm{ASME}^{5} \mathrm{IX}$, and military welding specifications. $\mathrm{N}-\mathrm{Base}^{\mathrm{TM}}$ was developed to work with standard PC computers using the Windows ${ }^{\mathrm{TM}} 95$ and NT operating systems. 


\section{Introduction}

Computer Accessible Welding Procedure Database: There are approximately 150 separate fields available in the database for storing welding procedures. The database is divided into two areas: Weld Records, and Weld Procedure Specification. The Weld Records area is designed to hold information about individual welds. When a weld record has been appropriately signed off by an engineer according to applicable Code, it becomes a Procedure Qualification Record. The database is accessible by authorized users through a graphical user interface that is designed for easy data entry, search, and record retrieval.

Special provisions were made so that the database would be computer accessible. The objective was to make it possible for another computer or computer software system to be able to enter the database and retrieve information that was useable by a computer program without a human being having to interpret the data. Each field in the N-Base ${ }^{\mathrm{TM}}$ interface contains a list of possible entries. These lists are called pick lists. As an example, when entering the electrode used in a $\mathrm{GMAW}^{6}$ process, the pick list displays many standard electrode wire classifications and specifications. If a new electrode is developed or is not in the pick list, the welding engineer with the required password can modify the pick lists. Since N-Base ${ }^{\mathrm{TM}}$ is built on this library of pick lists which are used to populate each field in the $\mathrm{PQR}^{7}$, it is impossible to enter a non-recognizible base metal, electrode, joint type or any other PQR field item. This ensures recognizable data for the computer, and makes the decision-making ability of the PC an asset to the welding engineer.

$\mathrm{N}$-Base ${ }^{\mathrm{TM}}$ incorporates standard networking protocols such as $\mathrm{TCP} / \mathrm{IP}^{8}$ to allow the addition, modification and recall of PQR's from anywhere on a network. This allows for a single database location that can be accessed from any password supplied client and prevents the growth of multiple databases in the corporation. The database uses Microsoft ${ }^{\mathrm{TM}}$ standard communications tools and is completely compliant with Windows ${ }^{\mathrm{TM}} 95$ and NT.

Intelligent Off-line Planning Systems: N-Base ${ }^{\mathrm{TM}}$ was designed to work cooperatively with the N. A. Tech's AiCES ${ }^{\mathrm{TM}}$ (Automated intellegent Concurrent Engineering System) environment. AiCES ${ }^{\mathrm{TM}}$ migrates an initial CAD drawing of a particular part to the production floor by defining weld placements, process procedures and automation programs. All of the functions of AiCES ${ }^{\mathrm{TM}}$ are accomplished automatically by the computer. The computer understands, for example, what a T-Fillet weld is, and has functions that plan the robot path and select welding procedures accordingly. For this level of computer functionality, it is imperative that the databases that are queried return information that the computer can understand. That 
is, the database must understand that the weld type is T-Fillet (which to a computer is different than " $t$ fillet", or "tee fillet"). The N-Base ${ }^{\mathrm{TM}}$ system can be used from inside the AiCES system to define welding procedures for welds on a particular part. This gives the engineer a powerful automated reference tool right at his/her desktop.

\section{$\underline{\text { N-Base }}$ - $^{\text {TM }}$}

\section{N-Base ${ }^{\mathrm{TM}}$ Main Objectives:}

- Provides electronic accessibility to data from any location on a network and other $\mathrm{DAO}^{9}$ or $\mathrm{OLE}^{10}$ compliant applications.

- Provide a more compatible database for using the PC as a valuable tool by providing information in a standard form and including information about the welding hardware.

- Contains pick lists that are populated with AWS, ASME, UNS and Military standards information

- Fields sufficient to support the following welding codes: ASME IX, AWS D1.1, D1.2, D1.3, and D1.5, AWS B31.1, AWS A9.1 and A9.2.

$\mathrm{N}-$ Base $^{\mathrm{TM}}$ stores information on the entire welding process. This information is categorized into the following sections for ease of addition, modification and recall.

Joint Detail

Base Metal Detail

Backing Detail

Heat Treatment Detail

General Detail

Process Detail
Filler Detail

Gas Detail

Insert Detail

Flux Detail

Power Detail

Testing Results

$\mathrm{N}-\mathrm{Base}^{\mathrm{TM}}$ is more complete than previous welding databases. With the addition of pick lists that contain items with which to populate the PQR fields, it is essentially impossible for the user to enter incorrect data (figures $1 \mathrm{a}, \mathrm{b} \& \mathrm{c}$ ). N-Base ${ }^{\mathrm{TM}}$ also contains information about the welding hardware. Items such as the type of power supply, wire feeder, and robot are an integral part of the database. This information is also necessary, because the uses of this data, to go from CAD-to-production with a first time good part necessitate that the welding procedures work correctly in the specific welding cell selected for production. Since N-Base ${ }^{\mathrm{TM}}$ leaves little room for errors and contains such complete lists of $\mathrm{PQR}$ variables, it has the ability to make 
intelligent decisions about the $\mathrm{PQR}$. The information contained in the pick lists comes from standards such as AWS A9 and D1, ASME IX, UNS, and Military Specifications.

In order to facilitate the entering of PQR data, $\mathrm{N}-\mathrm{Base}^{\mathrm{TM}}$ features a PQR wizard. This wizard guides the engineer through the appropriate steps for creating a proper and complete $\mathrm{PQR}$ record. The PQR wizard assists all the way from entering company information, process information, and test results to saving the data in the database. Not only are the PQR's less time consuming with $\mathrm{N}-\mathrm{Base}^{\mathrm{TM}}$, but they are a lot easier to create.

\section{Program Features}

System Requirements: Although the database software may run on less than the minimum system, it has been tested and will run on the minimum system indicated below. However, the graphics will run faster and provide improved response if the recommended system configuration is used, or one that is upgraded from the recommended system.

Recommended System

Pentium 75 Processor (or better)

25 Mbytes available on Hard Disk

32 Mbytes RAM (or greater)

31/2" Floppy Drive

VGA Monitor and Graphics Card

Microsoft Windows ${ }^{\mathrm{TM}} 95$

(Also works with Windows ${ }^{\mathrm{TM}}$
Minimum System

486 DX/66 Processor

20 Mbytes available on Hard Disk

8 Mbytes RAM

31/2" Floppy Drive

VGA Monitor and Graphics Card Microsoft Windows ${ }^{\mathrm{TM}} 95$

NT)

(Also works with Windows ${ }^{\mathrm{TM}}$

NT)

Record Access Modes: Weld records may be accessed through three different modes (figure 2): viewing, adding or editing. Access type 1 lets the user view weld records. Access type 2 lets the user add a weld record to the database, as well as view a record. Access type 3 allows the user full access, the previous two conditions, plus the ability to edit existing records. Both levels, type 2 and type 3 , require passwords for access.

Adding a record consists of several series of information entry screens. Each series is grouped according to a specific topic, shown in the main view screen (figure 3) adjacent to each magnifying glass. The first series of screens pertain to general weld record information. The user should enter information into each window. Three types of windows exist: 1.)The user may choose from a list by clicking on the down arrow button to the right of the window. 2.)The user may choose from a list or enter information from the 
keyboard. 3.)The user will not be able to choose from a list and must enter information through the keyboard. Some windows require the user to enter information in order to proceed to the next screen.

The record-editing mode executes the identical sequence of screens as adding a record with one exception. Current information in the record being edited will appear in the corresponding windows of each screen.

\section{Conclusions}

A new generation database has been created that, unlike common welding . databases today, is completely computer accessible and provides for future uses of welding data.

The database has been successfully integrated into an intelligent automated weld planning system, and is capable of supplying information necessary to automatically plan a robotic welding process. 


\section{Figures}

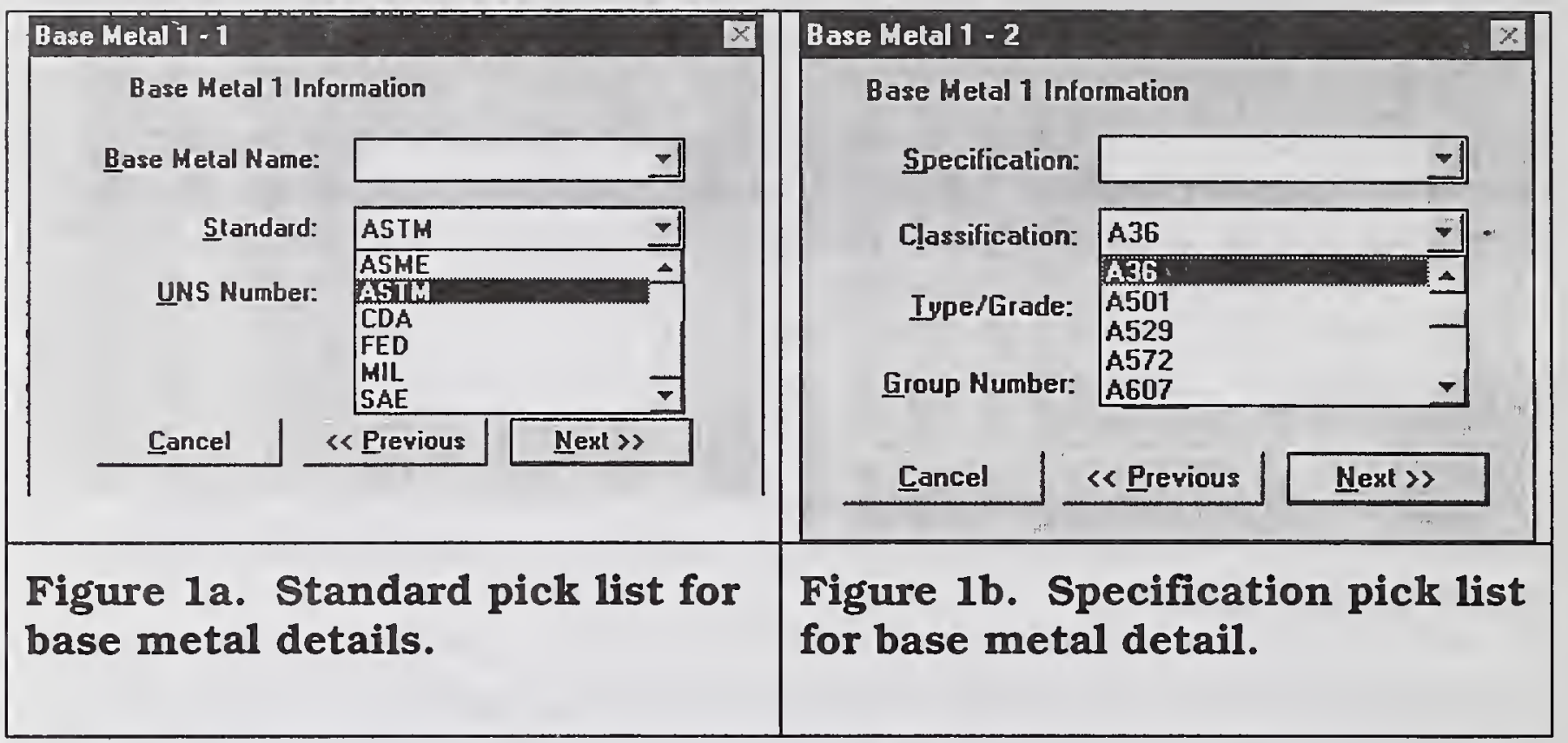

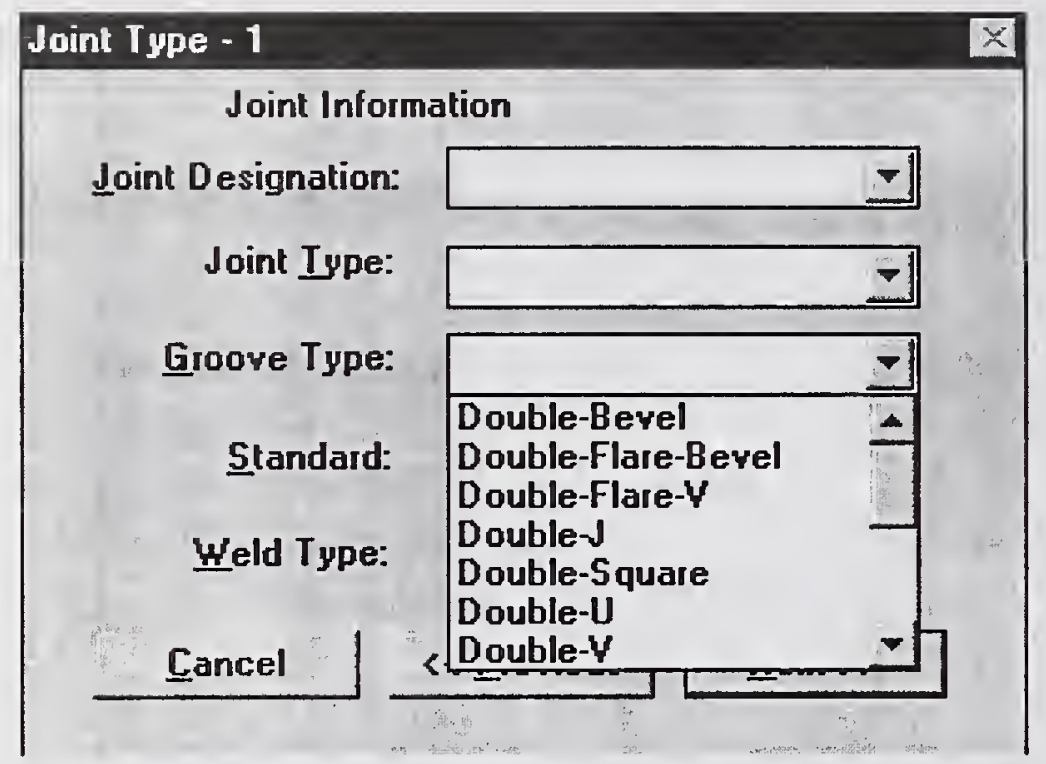

Figure 1c. Groove type pick list for joint detail.

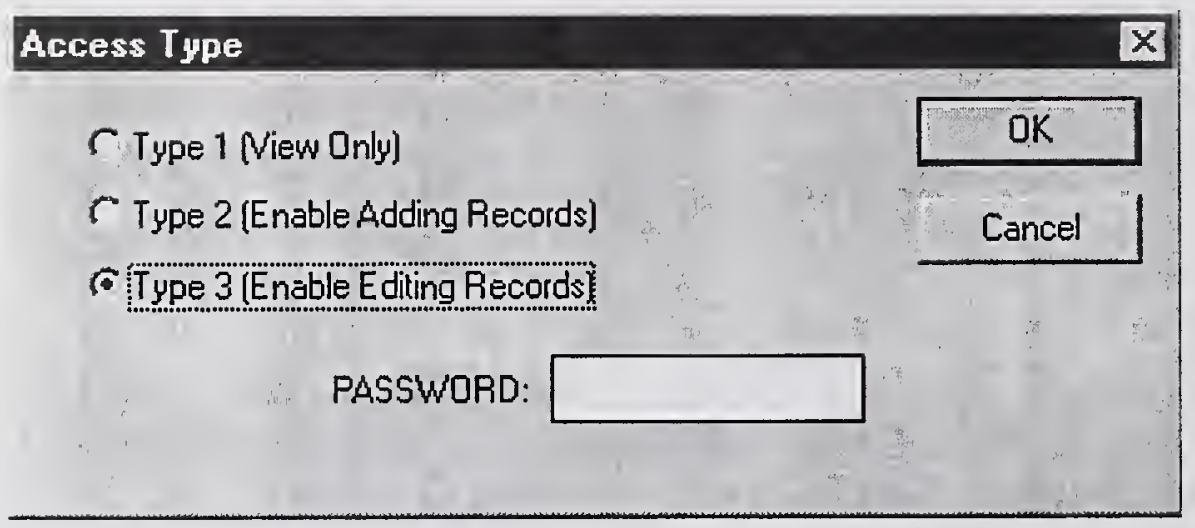

Figure 2. Record Access Modes Dialog 
File Édit View Help

\begin{tabular}{|c|c|}
$\begin{array}{c}\text { General Information } \\
\text { Record \# NA001T }\end{array}$ Date 6/24/97 \\
Revision
\end{tabular}

Code: AWS
Joint Design

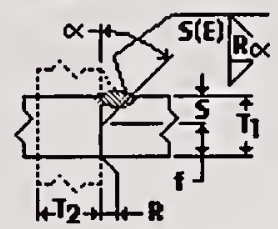

Joint TEE

Base Metal 1 Mild Steel (A36)

Base Metal 2 Mild Steel (A36) ? ? ?

Base Metal 3

Backing Type NONE

Heat Treatment

PWHT Required

NO
Prequired

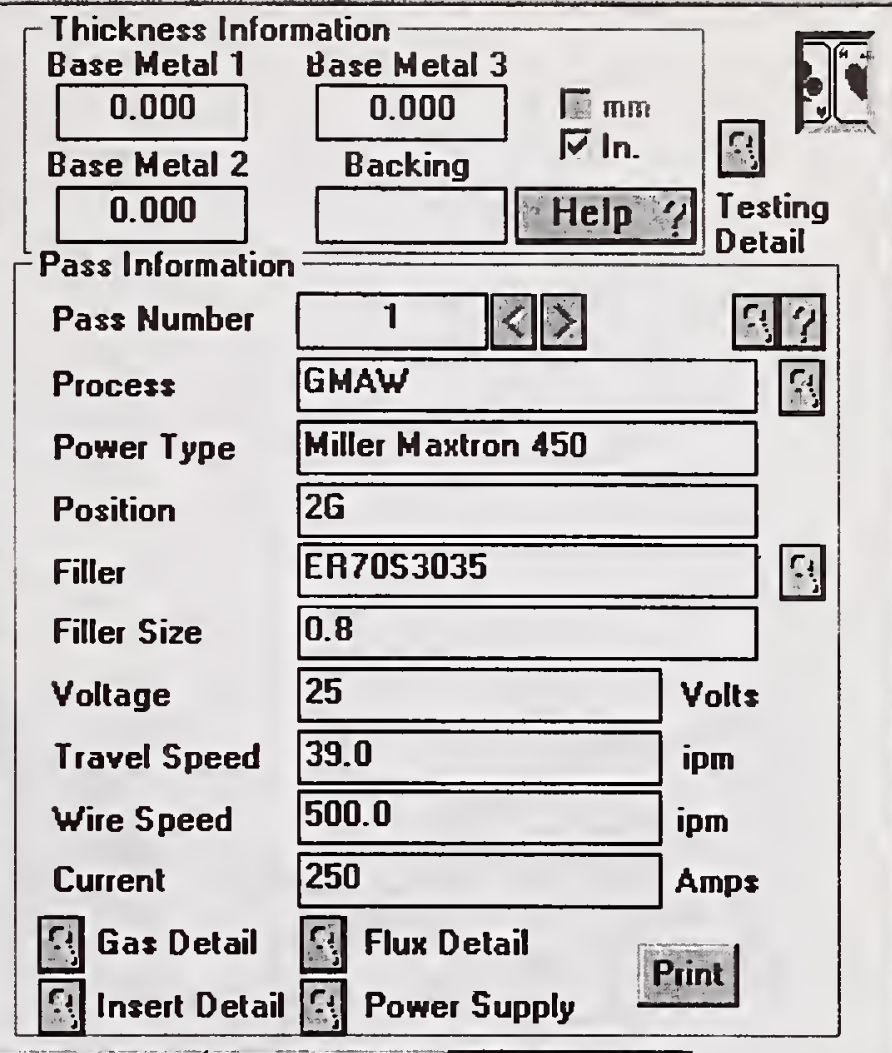

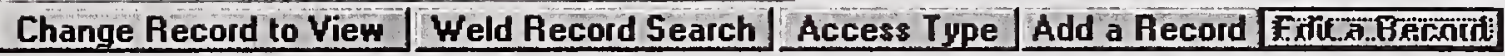

Figure 3. N-Base ${ }^{\mathrm{TM}}$ main screen

\footnotetext{
${ }^{1}$ LAN - Local Area Network

${ }^{2}$ NAMeS - Native American Technology, Inc. Measuring System

${ }^{3}$ AWS - American Welding Society

${ }^{4}$ UNS - Unified Numbering System

${ }^{5}$ ASME - Ameican Society of Mechanical Engineers

${ }^{6}$ GMAW - Gas Metal Arc Welding

${ }^{7}$ PQR - Procedure Qualification Record

${ }^{8}$ TCP/IP - Transmission Control Protocol/Internet Protocol

${ }^{9}$ DAO - Data Access Object

${ }^{10}$ OLE - Object Linking and Embedding
} 


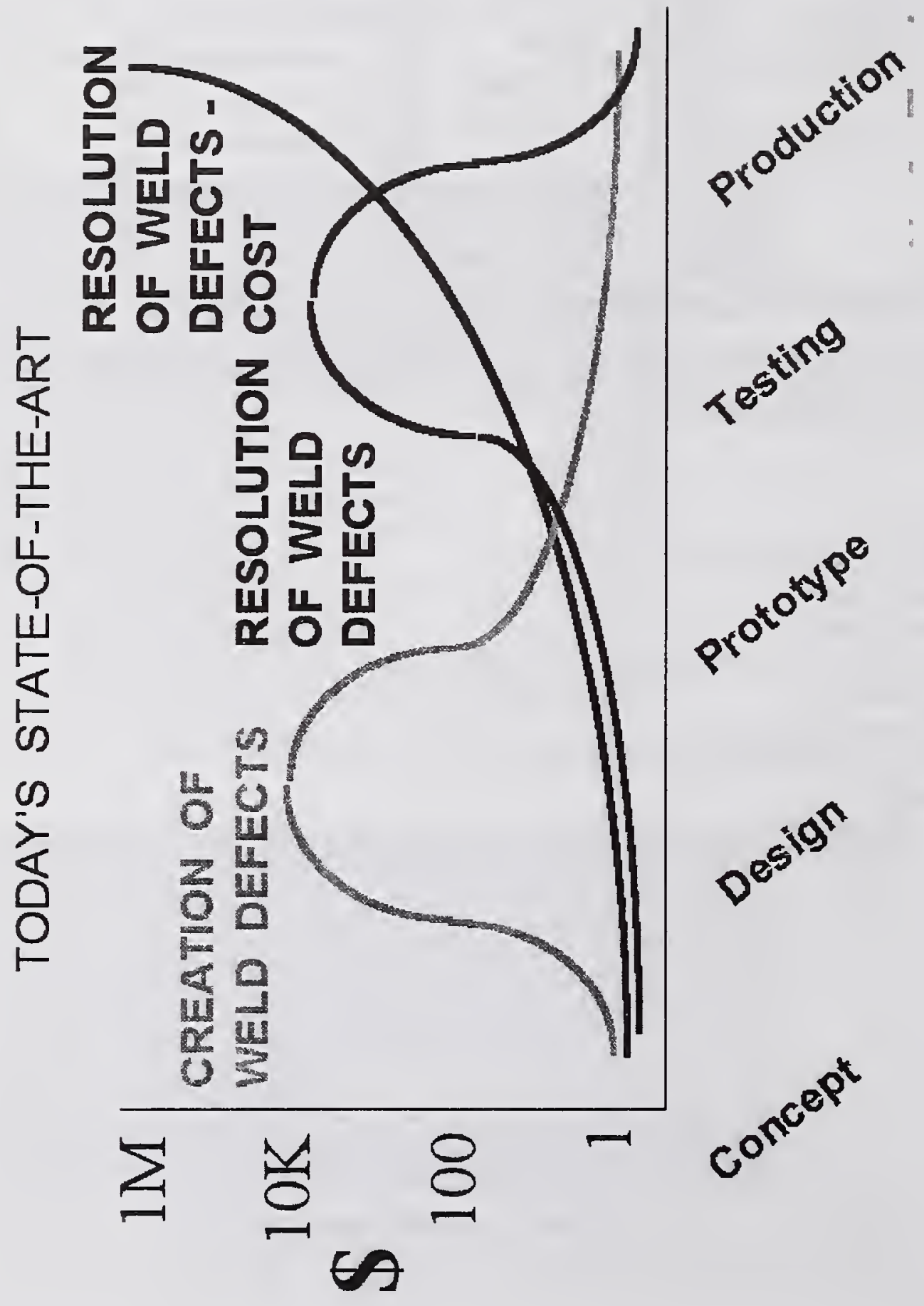




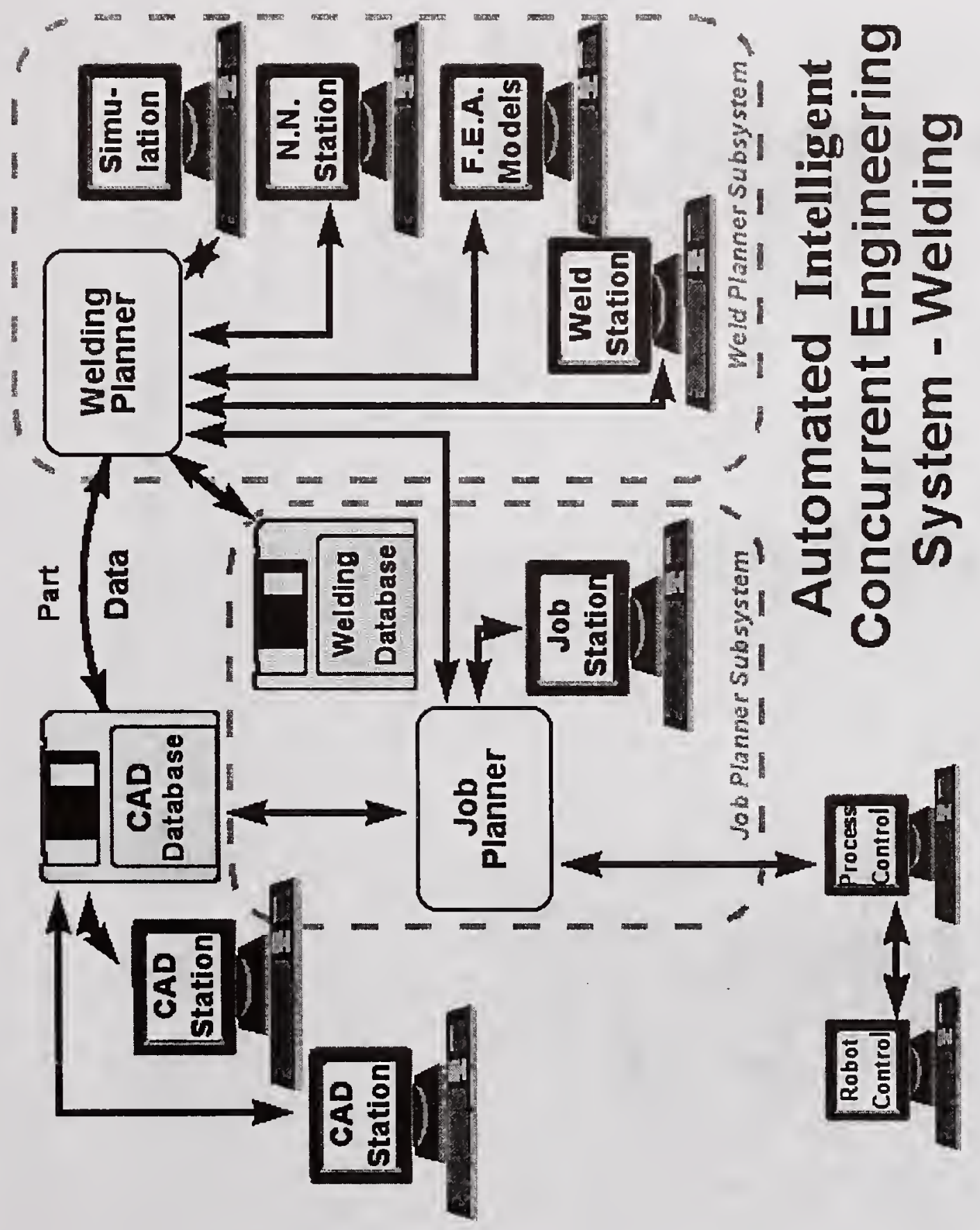




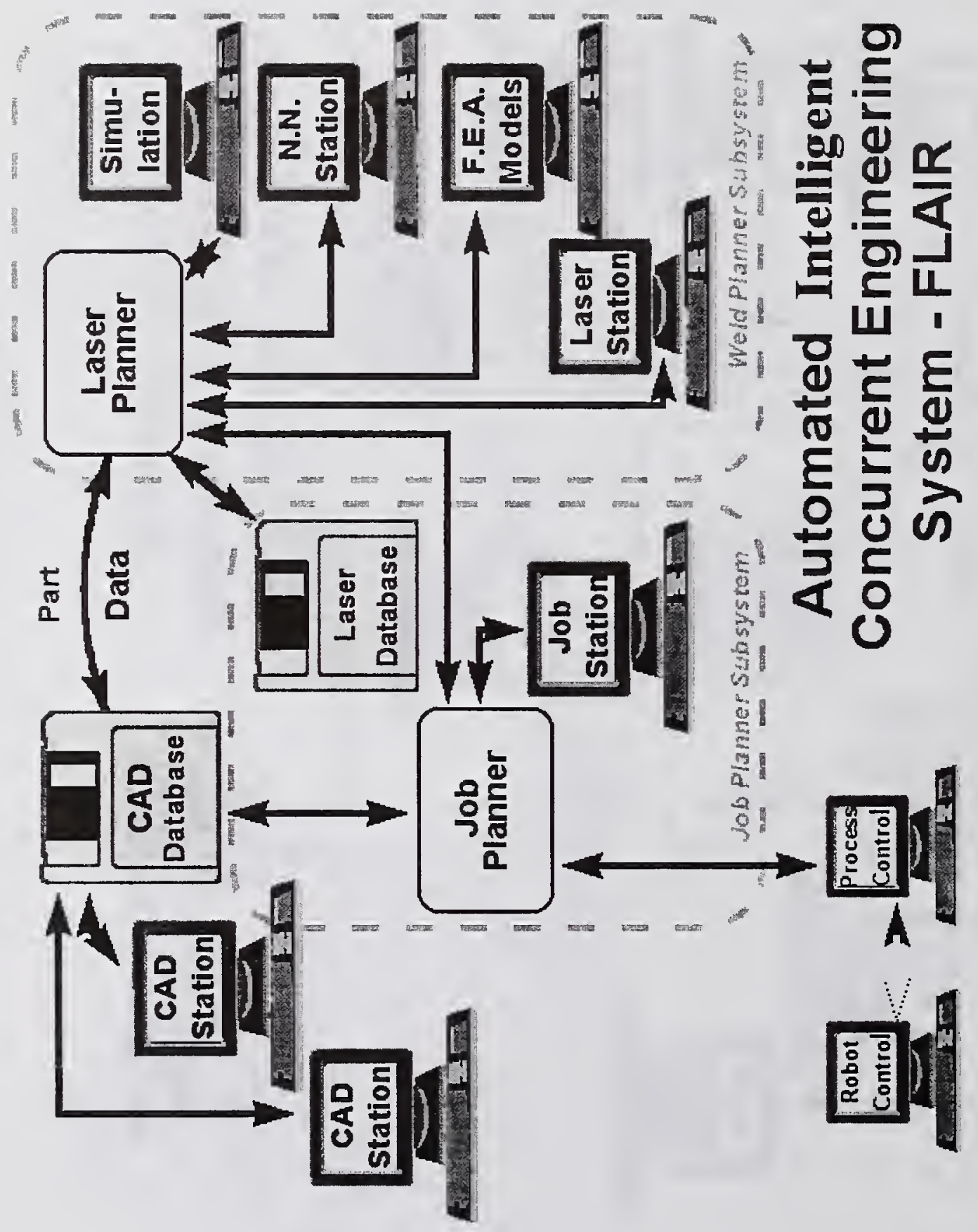



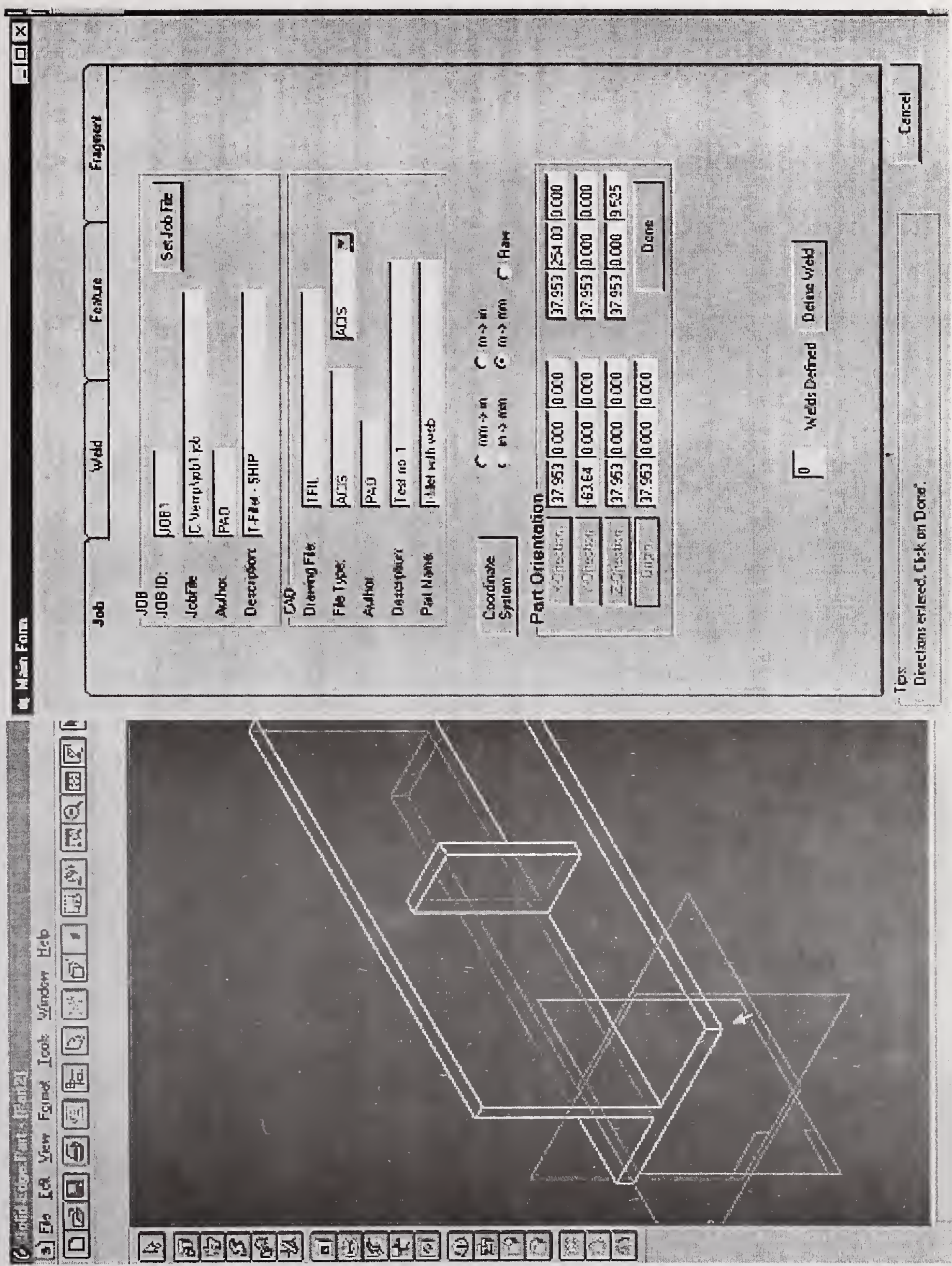


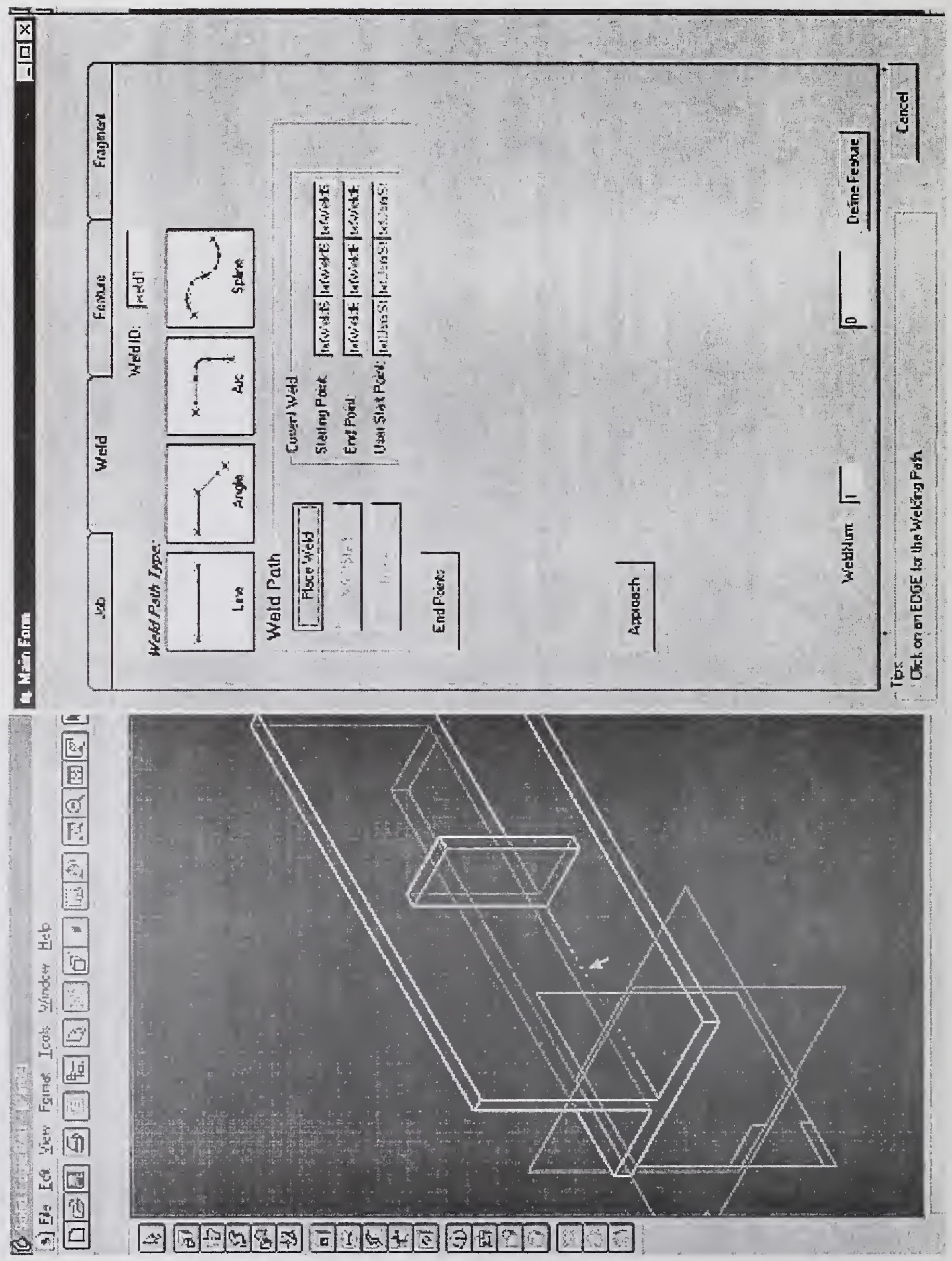



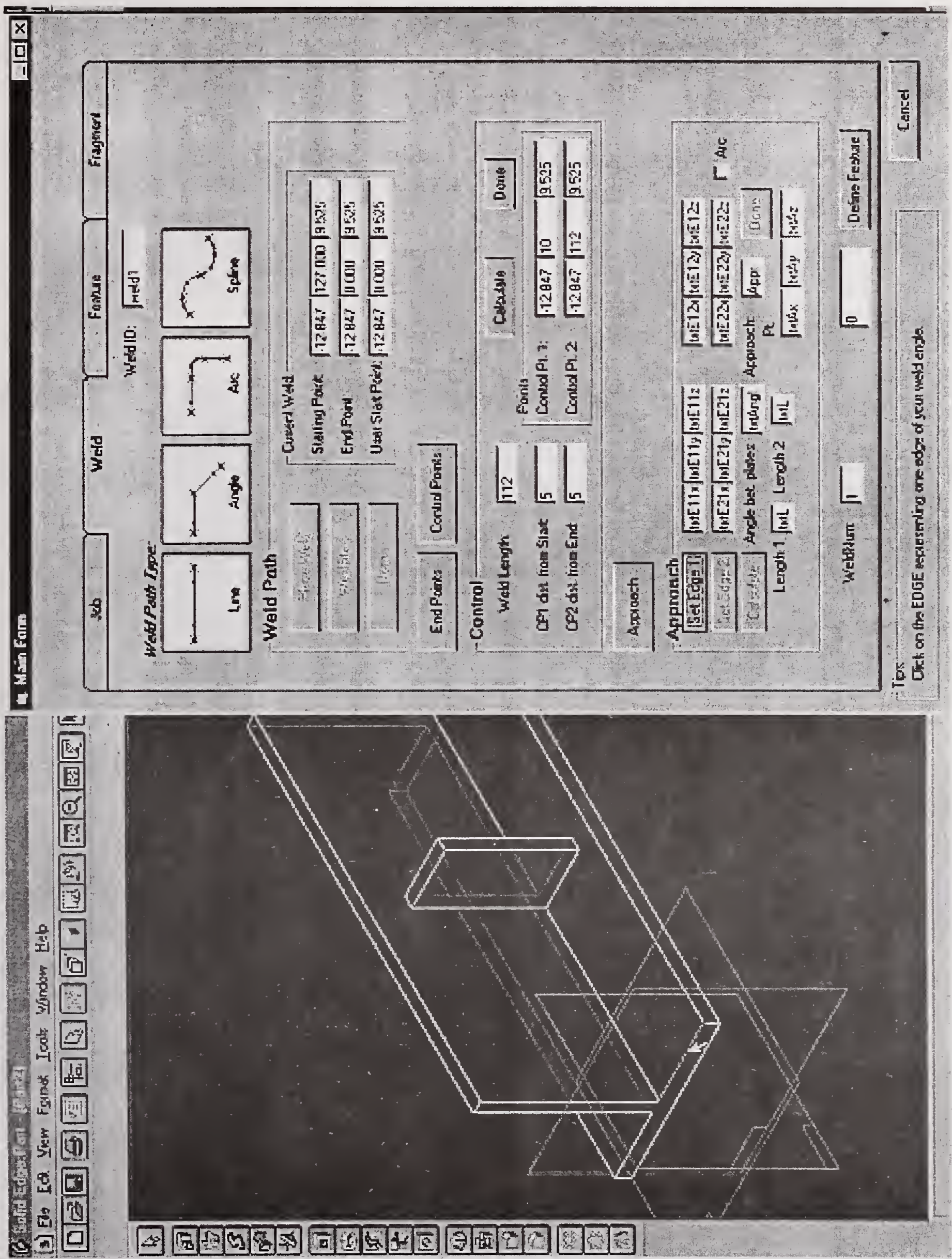


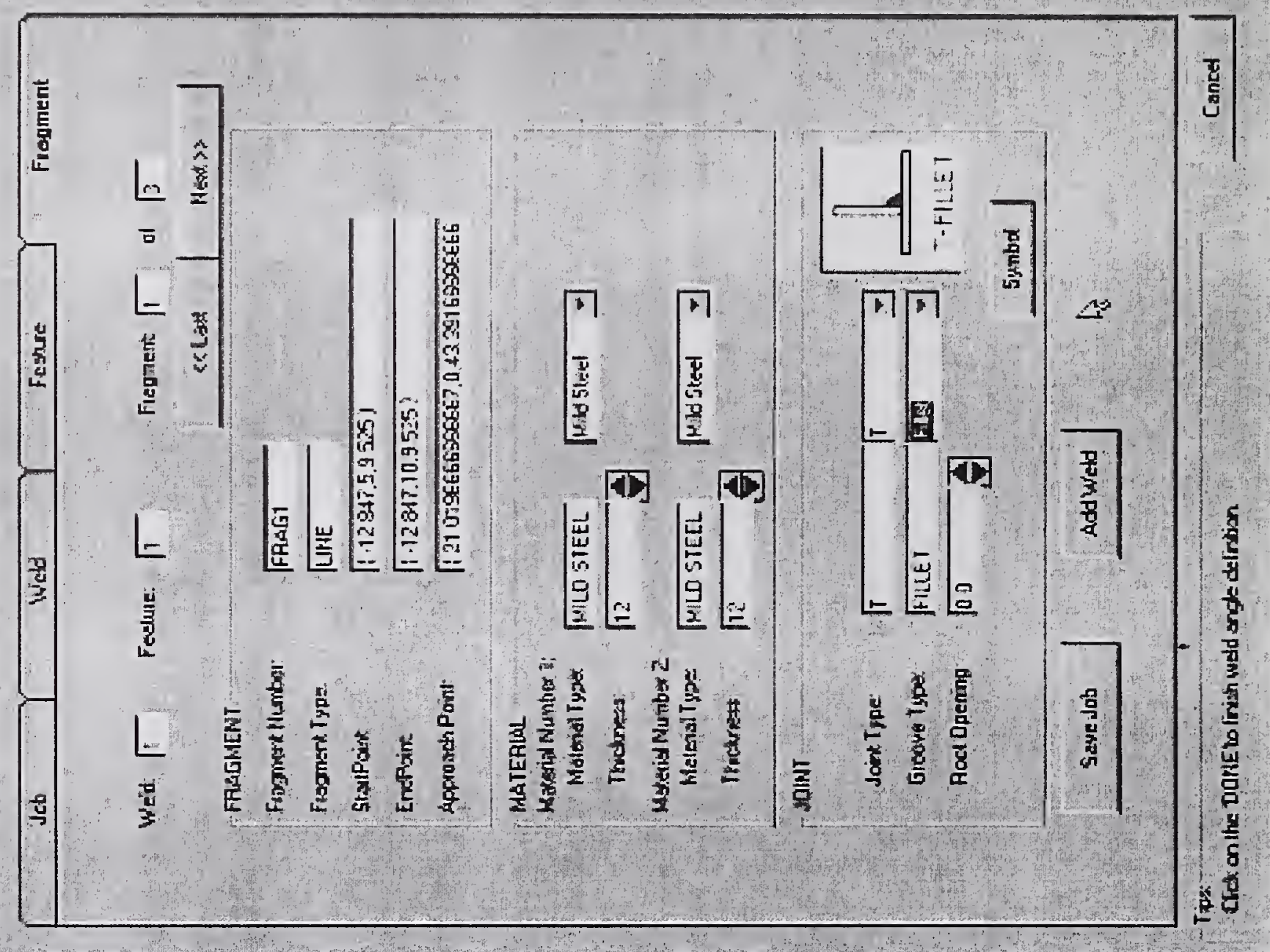




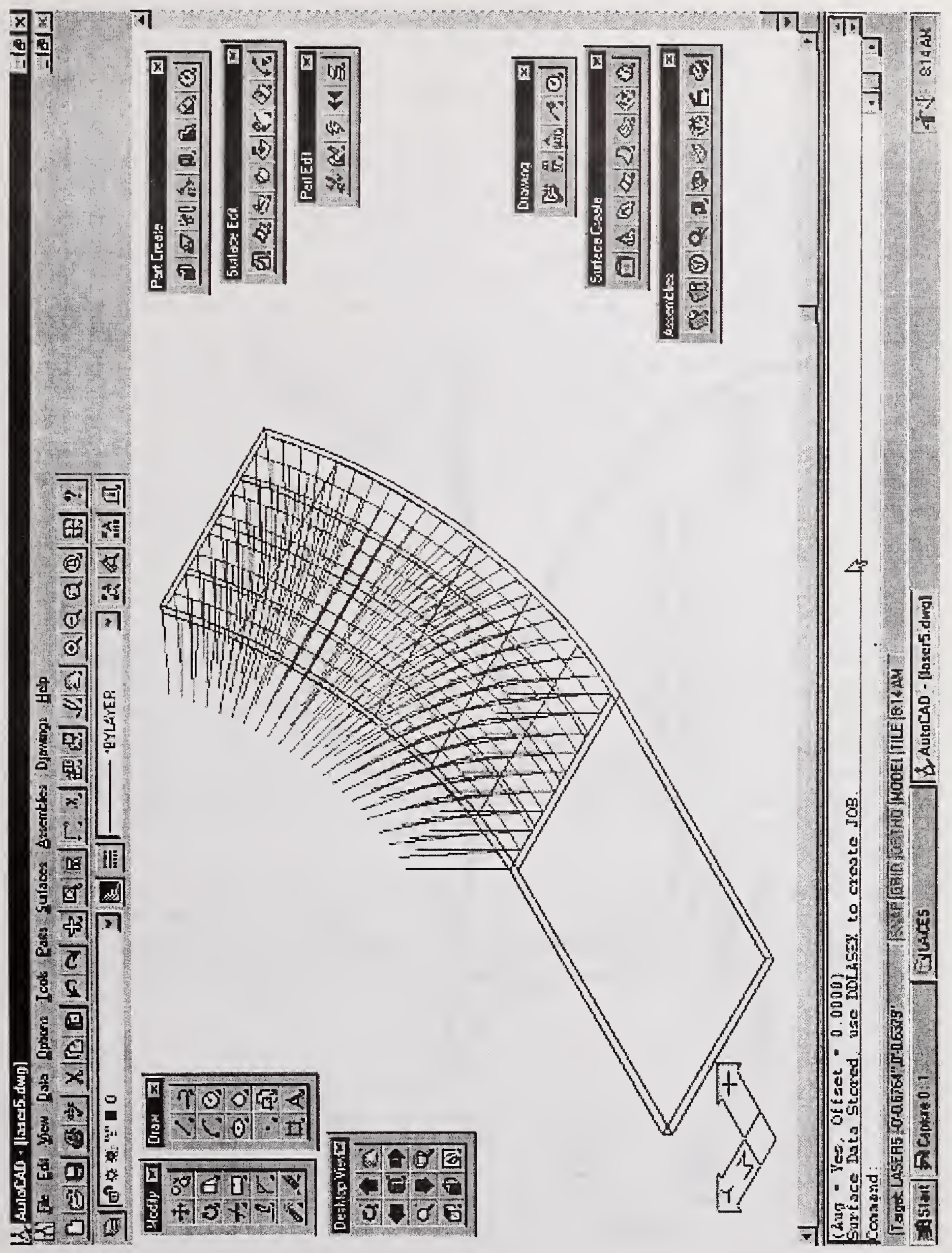




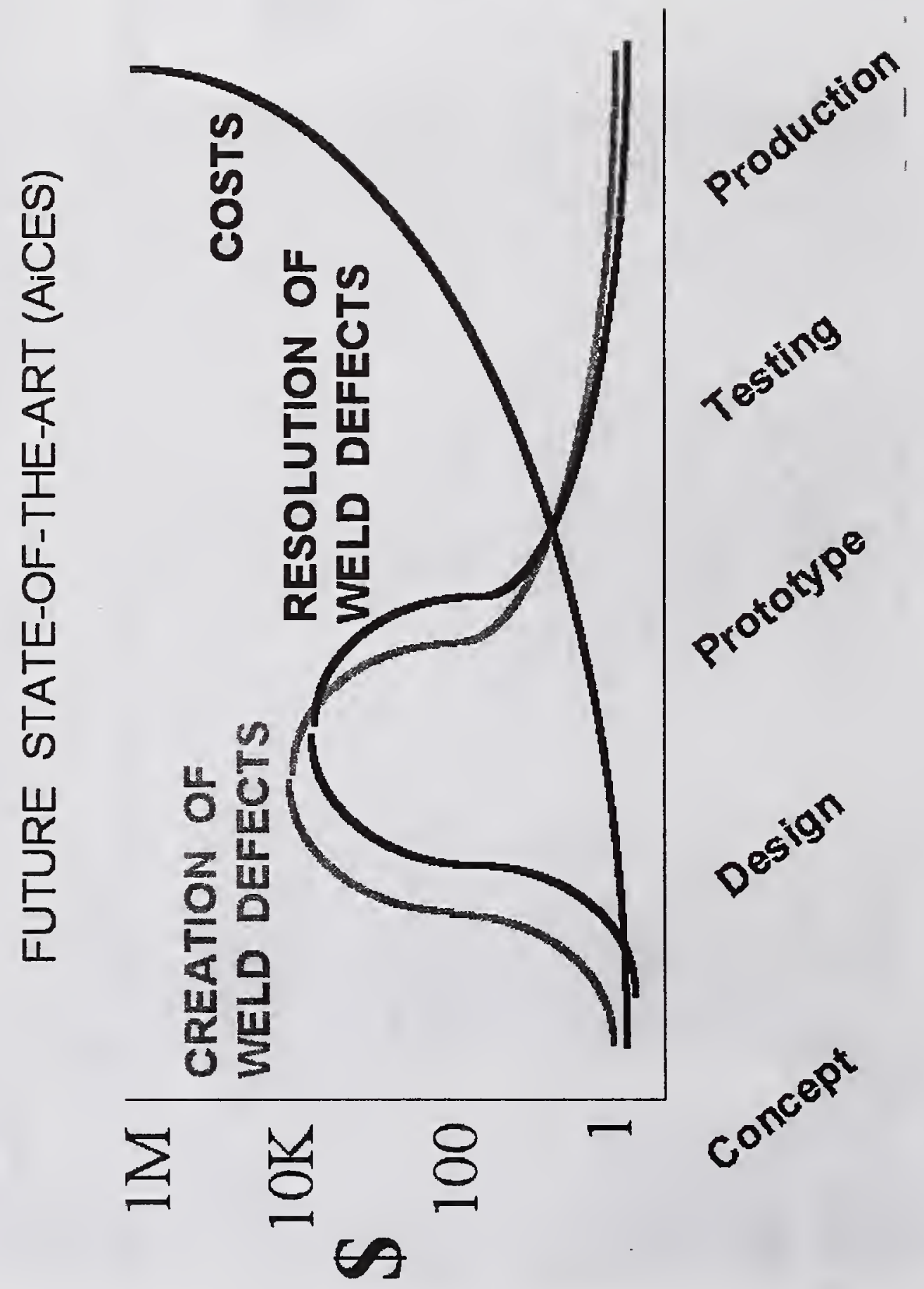




\title{
USE OF AN EXPERT SYSTEM FOR ON-LINE PROCESS OPTIMIZATION IN GMA WELDNG
}

\author{
S. Roosen*, U. Dilthey ${ }^{\#}$
}

\begin{abstract}
The Expert System MAGXPERT for parameter determination and process optimisation for GMA-Welding was developed for offline operating by the Welding Institute at Aachen University (ISF). Based on this system, AIXPERT was developed. With this new Expert system online controlling for the optimization of the process parameters in gas metal arc welding is possible. The just finished weld surface is measured online by a laser-scanning system positioned directly behind the welding torch and determines information on the weld seam appearance including the penetration pattern by using fuzzy logic based on the knowledge of the weld seam preparation. This information and the actual process parameters are input for the expert system. With these input parameters the system will determine the new process parameters as input for the power source and the welding speed control. AIXPERT requires DOS as an operating system. Further development of the expert system MAGXPERT is the fuzzy logic based version using Windows, MAGWIN, which will be integrated to operate online next.
\end{abstract}

\section{INTRODUCTION}

Today, expert systems are applied as soon as technical knowledge can no longer be described by fixed algorithms but only by rules and facts (Ref. 1). This problem is well known in welding operations, in particular if - together with the increasing automation brought about by the use of industrial robots - broad-based training of the operator needs to be augmented by specific training in arc welding techniques (Ref. 2). Although the operator may usually be able to programme the robot, any lack of enhanced knowledge in adjusting and optimizing the arc welding process may lead to long periods of disuse of the expensive investment goods.

This is the reason why the ISF developed the expert system MAGXPERT to determine and optimize the parameters for the process of GMA-welding (Ref. 3). The expert system allows the user to optimize the welding process in interactive communication with the computer, and it offers a set of process parameters from the integrated database prior to the first welding test (Ref. 4).

\footnotetext{
*German Welding Society, Germany

${ }^{\#}$ Welding Institute (ISF), Aachen University, Germany
} 
However, this interactive procedure used in the process optimization is very time consuming as repeated cross sections have to be prepared for the evaluation of the weld seam. Moreover, the evaluation of the weld seam depends on the subjective assessment of the operator. Thus, an automated optimization of the process during welding seems to be desirable. To that end the expert system was integrated into a computer system which allows automated and rapid optimization of the GMA-welding process even by an untrained user.

\section{EXPERT SYSTEM FOR ADAPTIVE PROCESS CONTROL}

With a view to achieving the on-line connection to the welding process the expert system was first of all adapted to meet arising demands; this included, above all, adjusting the boundary conditions and a highly efficient programming of the inference mechanism. Furthermore, the expert system was integrated into the entire system.

Taken into consideration at present are square groove and $\mathrm{V}$ groove joints on unalloyed steels of 2 to $10 \mathrm{~mm}$ in thickness and with varying weld seam geometries as well as different shielding gases, wire types and wire diameters. Different kinds of arcs like short arcs, spray arcs or long arcs can be employed here.

As in the off-line system, the process characteristics and technological boundary conditions are determined interactively between user and computer prior to the start of the welding process. Afterwards, both the user-defined data and the process parameters defined by the system are transmitted to computer software which takes over the system control, including both the control of and regulation of the power source. The computer initiates the start-up of the process as well as the subsequent steps for the process optimization. This procedure allows the user to define the welding task at his work place independent of the process computer, provided his $\mathrm{PC}$ and the process computer are connected within a network.

\section{DESIGN OF THE ON-LINE OPERATION}

The software system ALXPERT developed at the ISF for automated process optimization during GMA-welding is consisting of the following modules: an expert system, process computer software, a laser-scanning process controller and fuzzy-logic-based welding seam classification. The system runs on any commercial PC with a 486 processor and $16 \mathrm{MB}$ RAM equipped with the required $\mathrm{A} / \mathrm{D}$ and $\mathrm{D} / \mathrm{A}$ transducer boards for the connection to the power source. The present system works alternatively by anlog and digital control of the power source.

The computer software handles the power source and the feed device as well as control of the power source in order to maintain the adjusted process parameters. During the welding process the process data are monitored and average values are regularly determined which, if necessary, are transferred to the expert system for process optimization. In order to determine the weld seam geometry achieved, the weld seam surface is detected on-line by laser-scanner. 
To that end, the sensor is adjusted to its maximum angle so that delay times can be as short as possible because of the close distance to the welding torch, figure 1 . This procedure required the development of special software to record the scanner data. Nevertheless, a minimum distance had to be kept when welding with the hot spray arc with high radiant heat emission of arc and hot seam in critical wave length ranges where the measurement of the laserscanner is located.

The average values of weld seam width and weld reinforcement is calculated from the measured geometry data and compared with the target values determined on the basis of joint shape and process parameters. This is carried out within a fuzzy-logic based classification of the weld seam shape, which, in the case of a non-optimum weld seam, is fed into the expert system in order to allow process optimization. The course of this process is also controlled by the process computer software. The inference mechanism thus initiated recommends adjustment to the welding parameters of voltage, wire feed and welding speed which are further transmitted to the power source and the feeding device, fig. 2. The procedure described above is repeated until the process is adjusted to its optimum. Finally, off-line documentation is made of the welding data and the corresponding classification that has been carried out, fig. 3.

\section{BEHAVIOUR OF THE SYSTEM DURING TESTING PHASE}

Testing of the system revealed stable control behaviour, in particular where it has to contend with external disturbances. With further optimizing of the control algorithm, the performance of the system could be further increased. Appropriate timing of the process optimization and a stable state of the already optimized process can be determined with both the severe permanent as well as with momentary disturbances of the welding process.

\section{SUMMARY AND FUTURE WORKS}

AIXPERT, the modular on-line expert system developed at the ISF and described above, allows an automated on-line optimization of the GMA-welding process during the welding operation.

Further developments of the system are being tailored to include an integrated control of side and height of the torch position during welding as well as implementation of the on-line system under WINDOWS, as a new WINDOWS-based off-line system has already been developed (Ref. 5). This fuzzy-logic-based system also allows optimization of the impulse arc process which, due to the multitude parameters, can currently be adjusted only with great difficulty. Together with the optimized impulse arc process, first results have been carried out with the on-line operation of this system. Of course, rapid development of computer techniques will contribute to this process whose complexity and scope of application is currently still restricted for real time data processing by the computer memory available under the DOS-system. 
Another interesting aspect of the future development of the system token into consideration is the integration of the learning capability so that optimizations can, once gained, be stored without having to be repeated.

\section{ACKNOWLEDGEMENT}

These investigastions were achieved within a programme of co-operative research, administered by the 'Arbeitsgemeinschaft Industrieller Forschungsvereinigungen' (Association of Industrial Research Organizations) on behalf of the 'Bundesminister für Wirtschaft' (German minister of trade and commerce) and the support of DVS (German Welding Society). The authors wish to record their thanks for this funding and the generous assistance of Cloos Schweißtechnik, Haiger, Germany and Delft Instruments, Delft, The Netherlands.

\section{REFERENCES}

1 Puppe F: 'Introduction to Expert systems' (Einführung in Expertensysteme), Springer Verlag, Germany, 1988

2 Borutzki U: 'Use of Expert systems in Welding' (Einsatz von Expertensystemen in der Schweißtechnik), Schweißtechnik, Vol 38 (1988), No 8, p340

3 Dilthey U, Habedank G and J-Y Park: 'MAGXPERT - A Knowledge Based Advisory System for MAG Welding', Proceedings of the Workshop Computer Aided Welding, IIW COM XII, 08.Sept.1992, p77

4 Dilthey, U and S Roosen: 'MAGXPERT - A Knowledge Based Advisory System for MAG Welding' (MAGXPERT - Ein wissensbasiertes Beratungssystem für das MAGSchweißen), DVS-Berichte (reports of DVS), No 156, p98

5 Park, J-Y: 'Fuzzy-Logic-based advisory system for process optimization and defect analysis in GMA Welding' (Fuzzy-Logic-basiertes Beratungssystem zur Prozeßoptimierung und Fehlerdiagnose beim MAG-Schweißen), thesis Technical University of Aachen, Germany, 1993, published by Verlag Shaker, series Aachener Berichte Fügetechnik (reports of joining technology from Aachen), No 6/93 


\section{FIGURES}

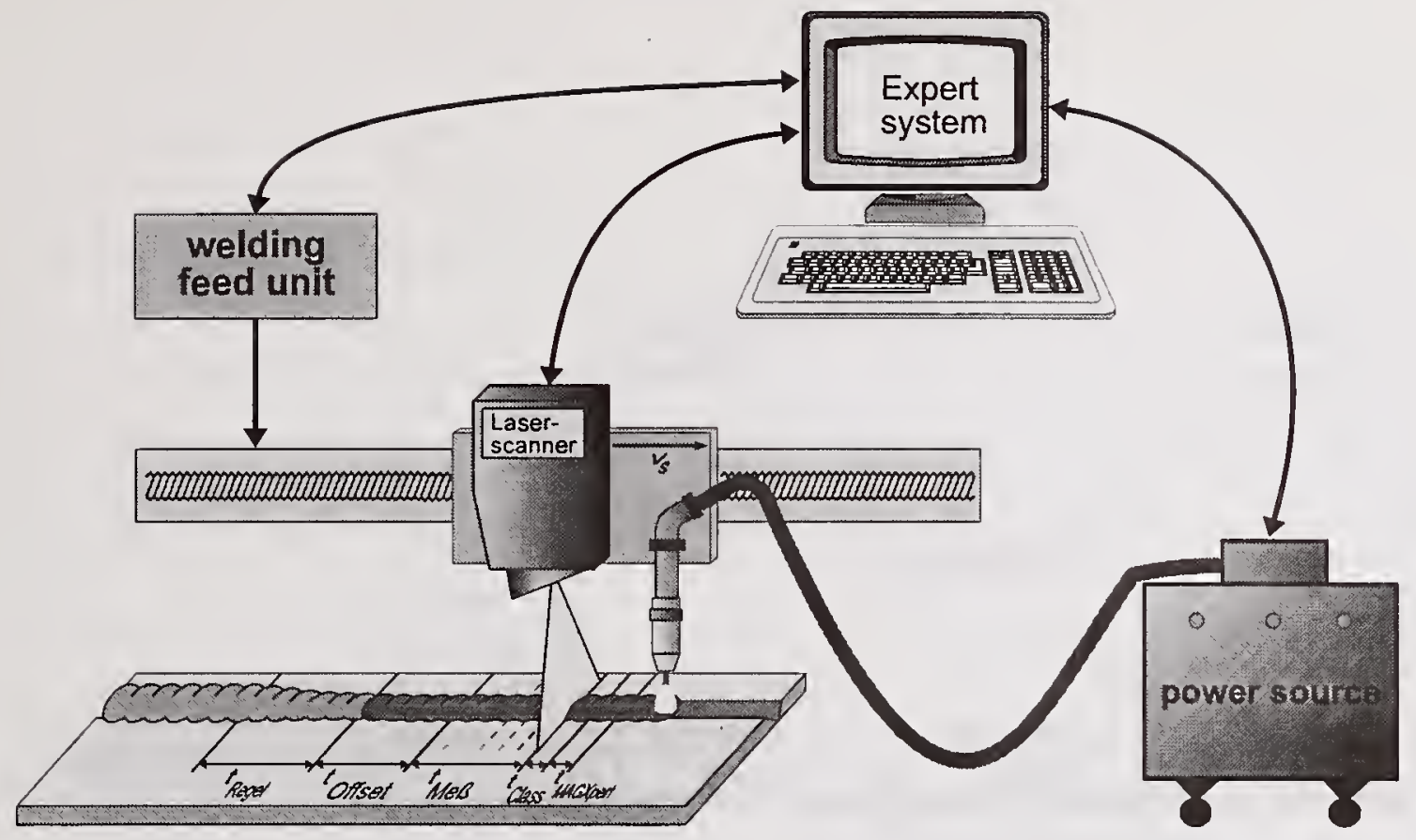

Figure 1: Experimental set-up for the expert system AIXPERT

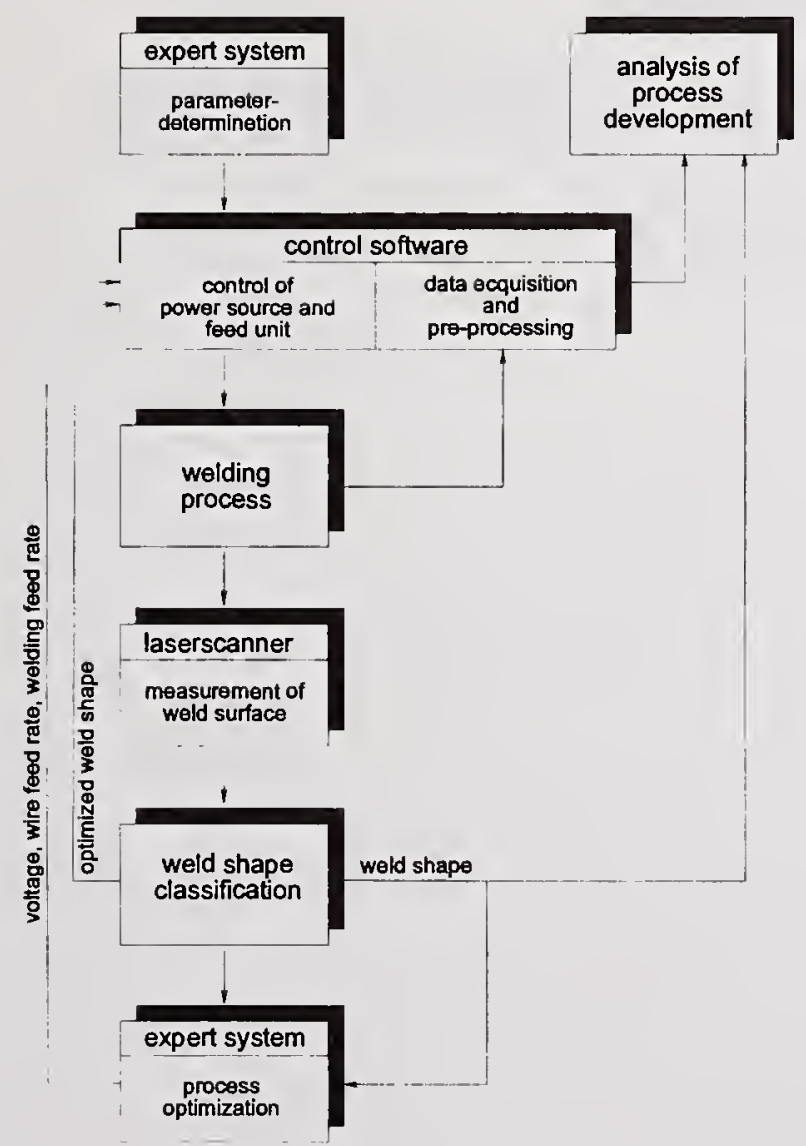

Figure 2: System modules AIXPERT 


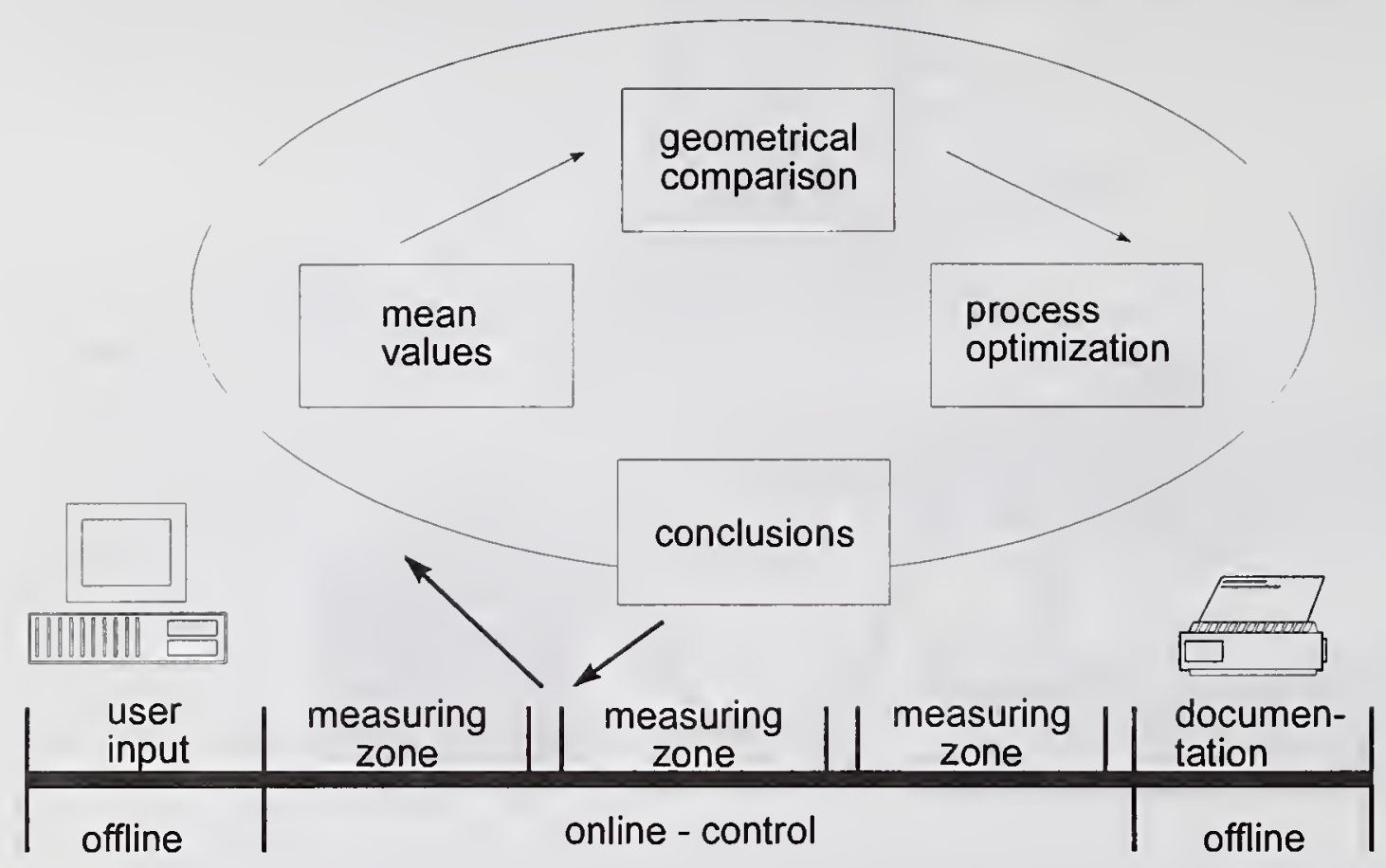

Figure 3: Chronological sequence of the on-line operation 


\section{INTERNET and NETWORKED SYSTEMS}





\title{
THE JOINING INFORMATION NETWORK BUILD IT AND THEY WILL COME
}

\author{
Ronald G. Citterberg*
}

\begin{abstract}
Materials joining processes are high-impact operations, and significant sources of value added. Information on current welding technologies, procedures, and practices is critical to manufacturing. More broadly, successful implementation of modern manufacturing and organizational concepts is based on ready access to information on manufacturing technology, capabilities, and requirements. Lead organizations must be aware of technologies, uses, and providers - each partner in the development/production chain must have information on the impact of their activities on the product cycle. Similarly, the prime's standards, specifications, and recommended manufacturing practices must be readily accessible. Acquiring this information is time-consuming and expensive - one manufacturer estimated costs of over $\$ 1,000$ per engineer per project to identify information resources for materials joining. Mechanisms are required to streamline the information gathering process, reducing design, development, and implementation costs.
\end{abstract}

To meet this need, Edison Welding Institute, operates the WeldNet ${ }^{\circledR}$ Joining Information Network. WeldNet is a communications network serving the materials joining community to ensure advancements in joining technology are quickly disseminated to U.S. industry and that the joining capabilities of potential partners can be identified. WeldNet will also provide a platform through which prime contractors can communicate designs, procedures, and recommended practices to their supplier chains.

A "home page" has been established on the World Wide Web, allowing Internet access with ease of navigation. The Web also allows greater capabilities in the transmission of graphic and multimedia products, and serves as the interface for more sophisticated database activity. The site is searchable to allow rapid access to the electronic contents.

WeldNet is a "one-stop shop" for joining information in electronic form; outside resources are accessed via links and gateways. Levels of security are incorporated, allowing protection of business sensitive information, while providing carte blanche access to public domain resources. The system utilizes a simple user interface and relies on off-the-shelf components and software for maximum reliability and ease of use.

Since its launch in 1994, WeldNet has grown into a leading manufacturing resource on the Internet. The system is averaging over 3500 accesses per week. This paper describes the

*Edison Welding Institute, Columbus, OH 43221

${ }^{\circledR}$ WeldNet is a Registered Trademark of the Edison Welding Institute 
development of the various databases, and how they interface with the WWW. During the presentation, the system will also be demonstrated.

\section{INTRODUCTION}

The Edison Welding Institute (EWI), the nation's largest independent materials joining technology center, through the Navy Joining Center (NJC), a Navy Manufacturing Science and Technology Center of Excellence and the Alliance for National Excellence in Materials Joining (NEMJ), an ARPA-TRP program, operates the WeldNet Joining Information Network (JIN). This network provides an extensive network of linkages with the materials joining community to ensure that the latest advancements in joining technology are quickly disseminated to U.S. industry and that the joining capabilities of potential agile partners can be identified.

\section{Edison Welding Institute (EWI)}

Founded in 1984, EWI is the largest nonprofit industrially-driven engineering organization in the U.S. dedicated to advancing and applying materials joining technology to benefit industry. Established in Columbus $\mathrm{OH}$, this resource center links industry, government, and academic organizations to promote industrial competitiveness.

\section{Navy Joining Center (NJC)}

The Navy Joining Center is a Center of Excellence for the Navy Manufacturing Science and Technology (MS\&T) Program. The Mission of the NJC is to provide state-of-the-art material joining solutions, develop leading edge materials joining technology, and disseminate joiningrelated technology and information to the Navy, DOD, and US industry. Through its technology transfer activity, the NJC launched and operates the Joining Information Network to accelerate the rate at which state of the art joining information is disseminated.

\section{The Alliance For Excellence In Materials Joining (NEMJ)}

The Alliance for Excellence in Materials Joining, a program funded through the Technology Reinvestment Program (TRP) links a nationally recognized technology center, EWI, with thousands of small- and medium-sized businesses through a variety of mechanisms. This direct support expands the materials joining technology base of these manufacturers, and creates a structured technical assessments assistance delivery network. NEMJ's PrimeNet, is a pilot program bringing industry leaders together for the advancement of materials joining. PrimeNet is an assessment and technical assistance program designed to improve materials joining capabilities of the supplier base of key defense and commercial industries. By joining forces with prime manufacturers such as Chrysler, Caterpillar Inc, Allison Engine Co. and GE Aircraft Engines, PrimeNet is able to provide proved materials joining assessment methods from which all manufacturers can benefit. NEMJ Advanced Communications develop the required functionality to electronically distribute materials developed by the primes to the various suppliers which make up these agile networks. 


\section{COMPONENTS OF THE WELDNET ON-LINE SERVICE}

\section{WeldNet Operating Principles}

WeldNet was developed with certain basic operating concepts in order to maximize its responsiveness to the small and medium businesses which comprise the majority of manufacturing enterprises.

1) WeldNet is designed to be a "one-stop shop" for joining information in electronic form. If manufacturers are required to access multiple resources to achieve their objectives, the utility of the information technology is diminished. Other existing resources are access via links and gateways. New services or enhancements are developed where necessary, but an attempt is made not to reproduce existing services.

2) The system utilizes a simple user interface. This reduces the risk of new users being frustrated with complex offerings and ensures wide access. The system also relies off-theshelf components and software. The use of custom code is minimized to provide maximum compatibility with outside systems. Upgrades are designed to be backward-compatible whenever possible.

3) Levels of access to the system are incorporated. This allows private corporations to protect proprietary or business sensitive information, while providing open access to public domain resources.

\section{System Components}

The system began alpha and beta site testing in July 1994, and was rolled out to public users on October 1, 1994.

The Web server is an HP9000 series model 715/100 UNIX workstation, connected to the Internet on a T-1 link via a fiber optic link. To maintain databases of new material, WeldNet employs the Texis database software, which has strong capabilities to process textual and numeric data, and output HTML compatible documents.

World Wide Web: The EWI "home page" has been established on the World Wide Web which is accessible from the URL address http://www.ewi.org/. The Web allows Internet access with a greater ease of navigation, and provides the capability of providing new and innovative hypertext mark-up language (HTML) documents prepared specially for the welding community. Conversion of other electronic textfiles to HTML is relatively straight-forward, so data can be accepted in a variety of formats. The Web also allows greater capabilities in the transmission of graphic and multimedia products, and serves as the interface for more sophisticated database activity.

Dial-in access: Modem speeds up to $28.8 \mathrm{kbs}$ are supported. As a direct dial-in system, it provides the easiest access to WeldNet services. It only assumes prospective users have access to 
a PC with a modem and phone line, and are able to use them to make the call. Therefore, a prospective user can access WeldNet and make an assessment of the value of the Joining Information Network with a minimal investment. Once convinced of the utility of the tools, the user can obtain Internet access through a local provider and migrate to the WWW home page.

Database Capability: To maintain databases of new material, the WeldNet service employs Texis software by Thunderstone Corporation. Texis has strong capabilities to process both textual and numerical data. Its output is compatible with the hypertext mark-up language, HTML, required for the Web. The database software is resident on the UNIX workstation. Linked to the network are several Pentium based PC's and a Macintosh workstation for document preparation and peripheral functions.

\section{WELDNET FUNCTIONS}

\section{Electronic Communications}

WeldNet provides an e-mail "home" for WeldNet participants in order to communicate with EWI staff and each other. The WeldNet electronic mail gateway provides an Internet presence for the staffs of NJC and NEMJ. Internet e-mail is processed by a private provider into format compatible with EWI's local area network, while maintaining network security.

Significant effort has also been expended in assisting the PrimeNet prime-supplier networks in establishing e-mail connectivity. This has proven to be challenging - many of the large organizations have a legacy of internal e-mail systems which may not provide consistent access to the outside world. It has been necessary for WeldNet system specialists to work closely with systems administrators in these organizations to establish system compatibility. In other cases there may be established policies which prohibit sending/receiving electronic mail outside the organization. On the other hand, the smaller business on the other side of the prime-supplier network have no existing systems. In most cases, suppliers have been directed to local resources or national providers, such as CompuServe or America Online, to acquire reliable e-mail capability.

\section{On-Line Materials Joining Databases And Information Resources}

This capability allows the generation of on-line databases of NJC and other research, profiles of technology providers and educators. Initial applications are databases of project abstracts and technology providers, but may grow into standardized process procedures and property data. Similarly, on-line programs of interest to the welding community can be demonstrated and run. It is important to note that classified information will not be stored on the network. Sensitive or proprietary information is password and access-protected. These resources are categorized as follows:

\section{News Bulletins:}

Continuous posting of topical information such as industry news, announcements for upcoming events, lists of seminars, schedules for EWI, NJC or NEMJ sponsored training or 
demonstrations, announcements of recent technology developments at the EWI and elsewhere, and listings of key EWI contacts for help in specific materials joining technologies.

\section{Industry Links and Material Joining Resources on the Internet}

EWI continuously searches the Internet for other relevant materials joining links on the Internet and maintains a database of these links on WeldNet. These links are segregated into the following categories:

Associations, Societies and Institutes

Commercial

Educational

Events

Processes

Projects

Publications

Software

Standards

Technical

Other welding and material joining associations, societies and institutes

companies offering welding equipment, services and information to the welding community

educational institutions offering degrees, courses or continuing education classes relating to welding

listing of conferences, seminars, meetings, educational offering and other events by organizations interested in welding and materials joining

welding and other material joining processes

welding and material joining projects underway at various societies and institutes

welding publications

listing of engineering software relating to joining activities

standards resources and databases as they relate to materials joining

companies, laboratories, universities, and other organizations active in joining research and development, or providing engineering services relative to joining

other information or activities related to materials joining 


\section{Inquiries}

Nearly two years ago, EWI setup an electronic registration form on WeldNet with the sole purpose of trying to gain some insight about visitors to our website. The registration form has always been completely voluntary and simply asks for the user's name, organization and their welding and materials joining interests. To date we have received more than 1500 registrations and the demographics mirror what is happening on the Internet today. The majority of respondents are from commercial (.com) accounts, approximately $20 \%$ are international and a good percentage access our website from home.

It did not take long for users to start submitting a myriad of questions along with their registration information. Some of the questions are just requests for information, but others were requests for assistance with a real materials joining problem they were experiencing. We have now incorporated two additional forms on WeldNet. A simple "Ask Us" form to serve as a request for more detailed information on something they may have seen on the website. Secondly, a technical inquiry form, primarily for members, which prompts for very specific information (welding process, material types, etc.).

The forms have been a great customer service tool for EWI. It basically provides a mechanism for members and other users to submit inquiries and technical assistance requests 24 hours a day, 7 days a week.

\section{Suppliers Materials Joining Database}

As part of the effort to demonstrate how information technology can be used to support primes and suppliers in an agile relationship, a suppliers materials joining database is under development. The database, an effort of EWI, PrimeNet partner Allison Engine Company, American Welding Institute, and Objective Computing, contains data from existing joining design and fabrication information provided by Allison's Joining Development Group. Also contained in the database are documents detailing Allison's recommended best practices, plus welding procedure specifications for specified joints. Proprietary information will be contained and accessible only by Allison authorized individuals.

The access software will include the use of hypertext which will allow it to access through the WWW home page. Hypertext "hot buttons" and links will assist in browsing and searching data. While the initial data will be specific to aircraft engine fabrication, the design is modular to allow additions of other joining processes and information after its initial proving trials. Once accepted, the database architecture is adaptable to support the construction of other specific databases for PrimeNet consortium members. Lessons learned will be documented and made available through WeldNet, guiding other manufacturers in utilizing the technology. 


\section{UTILIZATION OF WELDNET}

\section{Current Usage}

WeldNet was rolled out to the public in October of 1994. Initial usage was numbered in the tens of accesses per week. Mirroring the explosion of WWW activity over the past year, utilization of the service has grown rapidly. Statistics for the first six months of calendar 1997 show over 81,000 accesses to the system, with a significant growth trend.

\section{Future Efforts}

EWI is monitoring the usage of the system on an ongoing basis, and will be making adjustment to the system to increase user-friendliness, outreach and more database driven content. The current system emphasizes documentation to technology developments, typically characterized by discrete projects. The future information content of the system will be shaped by Navy, DoD, and industrial needs. The NJC is already receiving guidance from its Navy Advisory Group and industry-led Technology Advisory Board, and is holding a series of workshops to discuss needs on topics such as weld-induced residual stress and distortion. These efforts have indicated a strong need to acquire and provide actual process, materials, and mechanical performance data in order to capture the complete state of the art and practice in welding and joining. These and other emerging requirements will determine the WeldNet of the future.

EWI also looks to expand its WeldNet service for members. EWI will soon be limiting access to certain new information to members and/or certain classes of users.

EWI, NJC, and NEMJ will continue to identify additional resources and providers to assure comprehensive service to the joining community. The Suppliers Materials Joining Database shell, currently under development, should begin to be populated with procedures and recommended practices specific to individual organizations. Links to major primes and government organizations to identify and compile specific requirements for future manufacturing needs.

\section{IS THE INTERNET STARTING TO CREAK?}

Both the number of Internet individual users and corporate users continues to explode. Practically every new movie has an established website and, in most cases, even before the movie is officially released. Every major league sports team has a website, and all the major television networks are constantly hyping their own sites. The growth is global, with nearly every country now having some type of Internet access. It has placed a huge burden on the infrastructure in some countries, and other countries are scurrying to expand their existing infrastructure. For example, China is building a colossal Intranet link .

To meet growing demand, the number of Internet Service Providers globally has expanded to several thousand. According to US News \& World Report, data traffic soared 300 percent in 
1996, and will easily exceed that again in 1977. The traffic jams you are encountering on the Internet are indeed real. The backbune is starting to creak in spots.

\section{Host Usage Statistics}

A recent survey from Network Wizards shows the number of Internet "hosts", or machines reachable through the Internet, reached 16,146,000 as of January 1997. That's an increase of 25 percent from July 1996 when 12,880,699 "hosts" were found, and a 94 percent increase over the previous year. In January 1993 there were a mere 1,313,000 hosts.

A host used to be a single machine on the net. However, the definitions of a host has changed in recent years due to virtual hosting, where a single machine acts like multiple systems (and has multiple domain names and IP addresses).

\section{Internet 2 and vBNS}

Two initiatives, Internet2 and vBNS, are already underway to expand the Internet to the next level. In 1993 the National Science Foundation decided to concentrate on creating a very high speed Backbone Network Service (vBNS) that would connect the Supercomputing Centers at speeds of up to 2.5 gigabits ( 2.5 billion bits) per second -- fast enough to transmit the contents of two public libraries per second. Achieving these tremendous speeds is one of the goals of the High Performance Computing and Communications (HPCC) program, a federal effort to develop advanced communications, computer and software technologies. To support the HPCC program, the NSF has restructured its data networking architecture by establishing network access points (NAPs) and by selecting NAP managers, a routing arbiter, and MCI as the very high speed Backbone Network Service (vBNS) provider.

Internet 2 is a high performance networking initiative among major universities whose mission is to: Facilitate and coordinate the development, deployment, operation and technology transfer of advanced, network-based applications and network services to further U.S. leadership in research and higher education and accelerate the availability of new services and applications on the Internet. Several major corporations have recently become part of the Internet2 program.

The merging of these two initiatives has already begun. In May of this year, Vice President Gore announced the High-Performance Connections for 24 Internet 2 Members. They were among the awardees of $\$ 12.3$ million in grants to 35 research institutions across the United States. The National Science Foundation (NSF) awards will allow them to connect to the very high speed Backbone Network Service (vBNS) and to communicate with other Internet2 members at speeds 10 to 100 times greater than is possible through today's Internet. This grant brings to 44 the number of Internet 2 institutions connected to the vBNS.

\section{References}

http://www.i-b-d.com/growth.htm

http:// www.nw.com

http://www.usnews.com/usnews/issue/30NET.HTM 


\title{
THE NIST AUTOMATED ARC WELDING TESTBED
}

\author{
W. G. Rippey*, J.A. Falco*
}

\begin{abstract}
The Automated Welding Manufacturing System (AWMS) is a research and development testbed for automated gas metal arc welding technology. Its activities are aimed at developing and validating standards that will contribute to increased use of automated welding technology by manufacturers. The National Institute of Standards and Technology (NIST) plans to work with technology suppliers and manufacturing users to test systems in the AWMS. Our experiments and control system designs will test the feasibility of interface standards and intelligent control technology to increase productivity, improve quality, and reduce the cost of system integration. Further, we will explore integration techniques that make multi-vendor system solutions more effective and easier to build, program, and operate.
\end{abstract}

Keywords: data acquisition, gas metal arc welding, open architecture, robotic arc welding, standards, welding automation, welding sensors.

\section{INTRODUCTION}

NIST's mission is to promote economic growth by working with industry to develop and apply technology, measurements, and standards. The Intelligent Systems Division (ISD) is part of NIST's Manufacturing Engineering Laboratory. ISD's goals are to foster the development and implementation of advanced manufacturing systems, processes, and equipment and to anticipate and address the needs of U.S. industry for the next generation of measurements and standards.

ISD began an effort to investigate technology in automated arc welding in 1995. ISD worked with NIST's Materials Reliability Division (MRD), a group that has experience in welding process research, to determine the initial approach of the project.

This document describes the current testbed hardware and the initial design for the control system and off-line planning system of the AWMS. The testbed, shown in Figure 1, includes a robot, arc welding power source, gun and wire feed, control computers and sensors, and robot simulation software. We emphasize modular software and hardware design to investigate opportunities for standards and to allow insertion of components and algorithms developed by others, for studying interface standards and doing performance testing.

\section{AWMS GOALS}

The three major goals of the AWMS testbed are to:

- validate and test standards

- incorporate new hardware and software components developed by others to investigate openarchitecture concepts

- develop advanced welding technology.

\footnotetext{
* National Institute of Standards and Technology, Gaithersburg, Md. 20899
} 


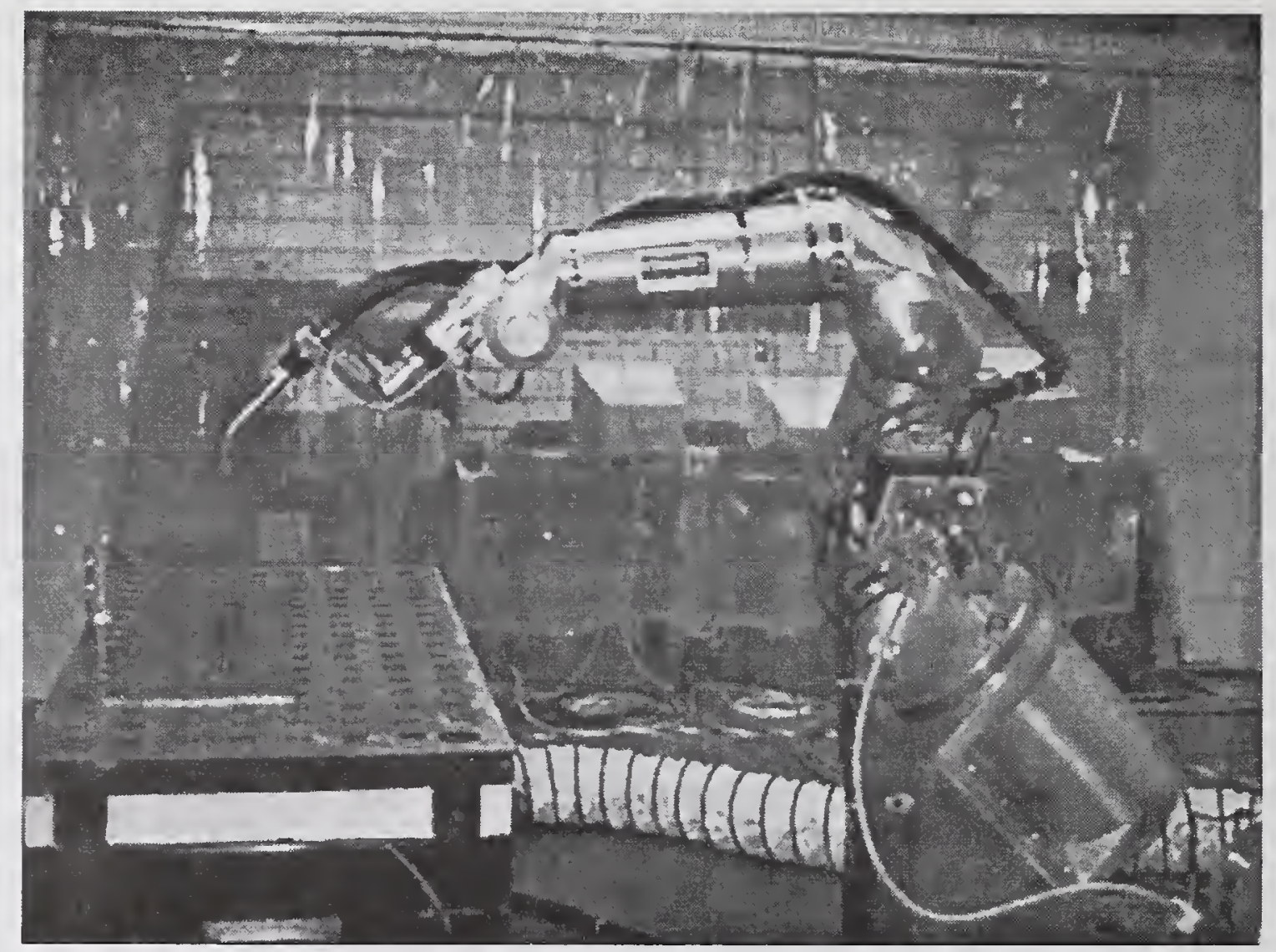

Figure 1. The robot arm of the AWMS testbed.

The scope of the standards work includes hardware and software interfaces for shop-floor computerized welding equipment, and data formats for exchange between Computer Aided Design Systems (CAD), welding procedure and knowledge bases, and off-line programming systems. The goals are pursued using experiments in integration of components such as robots, power sources, weld process sensors, and robot proximity and position sensors. Emphasis of the experiments is on system welding capabilities and the ease with which products from different vendors can be integrated into the testbed. The technology subsystems will primarily be imported from commercial companies and research organizations. The AWMS project continues ISD's efforts in open-architecture methods with focus on real-time control technology. Possible testbed activities may include off-line planning and cell programming.

Standards cannot be validated and tested if there is an insufficient host of technology subsystems to integrate. To accomplish our mission, we will work closely with commercial companies who supply technology and who buy welding systems. We will also leverage new technologies at the university and government laboratory level and are maintaining close relations with welding standards organizations.

AWMS integration experiments will test the feasibility of:

- standards for interfaces between components that free integrators from product-by-product interface engineering. This could allow them to offer their customers a wider range of equipment options. Further, component developers and potential developers would know that conforming to the standard would ensure compatibility of their products with other components. For the relatively small welding technology industry this ease of integration is essential to encourage companies to develop new products.

- open architecture interface standards that make it possible to access and store real-time weld process data. The data would be used in developing process models and in monitoring and controlling quality. 


\section{AWMS APPROACH}

The AWMS project emphasizes experiments in the testbed. ISD is maintaining testbed hardware and software and incorporating state-of-the-art products and evolving research results. AWMS experiments address welding techniques that apply to commercial manufacturing processes. The testbed can be used to test progressing technology and to anticipate the near-future needs for standards that arise from new technology development.

We are studying architectural designs and data formats developed by others, and will do validation testing of them where appropriate. Where possible, we plan to study system-level configurations and tools being developed by CYBO (Ref. 13), AWI (WeldExcel), PAWS, and Sandia National Labs (SmartWeld) (Ref. 8), to find commonality in ways to make components from each usable in other systems, or to make outputs of one system compatible with another. AWMS can be used to test and validate data formats such as those being developed by American Welding Society A9 Committee, CYBO (Ref.13) and NIDDESC (Ref.10).

AWMS control system technology emphasizes open-architecture of potentially distributed systems. The extent of distribution can range from using different computers on a local network for a cell's sensor, motion control, and power source control, to using the Internet to allow users to remotely program, control, and monitor a welding cell. The results of the open-architecture approach are interfaces that allow interoperability of products from different vendors.

Possible standards issues within the scope of the testbed include realtime hardware interfaces to robots and power sources, interfaces to sensors, open-architecture controller software APIs, data formats for weld geometry and weld parameters, welding program data including motion and weld parameters. We will address these issues in cooperation with industry research partners and AWS and Robotic Industries Association standards committees. AWMS government-industry research activities will identify standards needs for the near future and demonstrate new intelligent control systems for the arc welding industry. Current manufacturing application areas being considered include, but are not limited to, shipbuilding, automotive and heavy equipment manufacturing, and the building construction industry.

\section{TESTBED ARCHITECTURE}

Figure 2 shows the major components of the AWMS testbed control structure. This is a logical architecture -- components are processes that may run on different configurations of computers. The detailed architecture may change as we experiment with configurations or implement commercial product interfaces. Our current plan is to run the robot controller on a $486 \mathrm{PC}$ running Lynx OS, and the IWC and Cell Controllers on a dual-pentium PC running Windows NT ${ }^{\prime}$. The Cell to IWC communications will use NT shared memory, and communications to the robot controller will be via the ethernet TCP/IP protocol.

The CAD/OLP system will integrate commercially available computerized components for weld design and planning, and for motion planning with automation systems. An off-the-shelf CAD system will be used to create part, assembly and weld designs. This design data will then be extracted from the CAD system using an application layer and passed to an off-line planning

\footnotetext{
${ }^{1}$ Certain commercial equipment, instruments, or materials are identified in this paper in order to adequately specify the experimental procedure. Such identification does not imply recommendation or endorsement by NIST, nor does it imply that the materials or equipment identified are necessarily best for the purpose.
} 


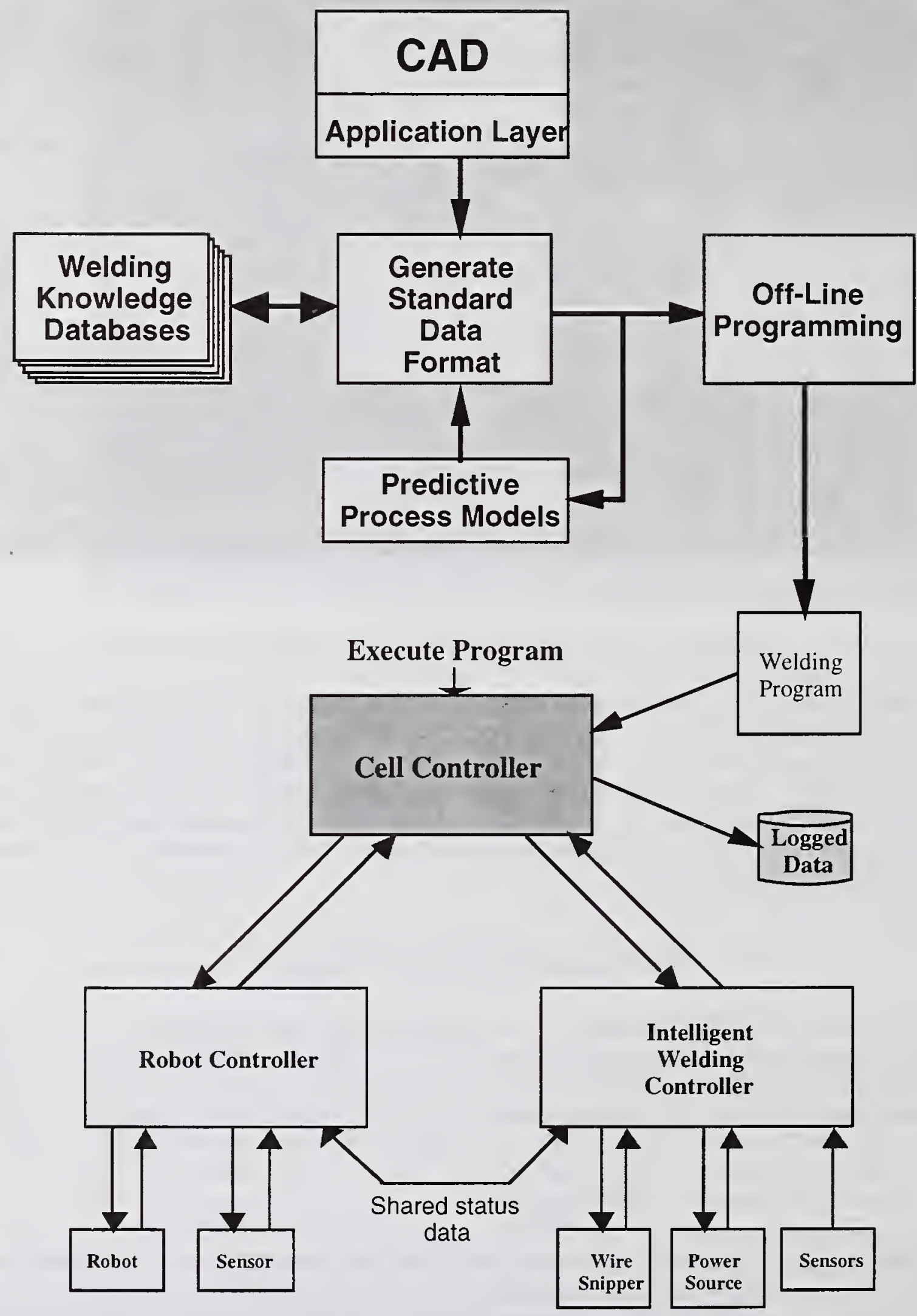

Figure 2. The AWMS logical architecture - control structure and data flow. 
system which will merge CAD data with existing welding knowledge bases. This merged data will be input to a commercial off-line programming package where it will be integrated with motion planning, producing a welding program specific to the AWMS testbed hardware including robot/torch, speed, orientation, and sensor-guided actions. The program generated by the AWMS OLP system will then be interpreted by the AWMS Cell controller. Ideally, predictive process models could be modified to accept this standard format to verify welding plans.

\section{Cell Controller}

The cell controller supervises both the Robot Controller and the Intelligent Welding Controller (IWC). The Cell controller interprets welding programs that it receives from the AWMS off-line planning system and passes commands based on the weld program to its subordinates. The commands are synchronized based on status updates that the cell controller receives from both the Robot Controller and the Intelligent Weld Controller.

\section{Robot Controller}

The robot controller interfaces to the AWMS robot and generates joint commands based on motion commands from the Cell Controller. The robot controller generates motion in either Cartesian or joint space. The robot controller calculates and reports the current torch pose and velocity.

\section{Robot Sensors}

The first sensor implementation will use the touch sensing capability of the commercial power source to measure part and weld-joint location. The commercial power source will notify the IWC controller when an object is touching the weld wire. Our initial architecture will have all IWC sensor status signals routed through the Cell controller. Robot touch sensing is implemented through a cell controller algorithm of commanding the IWC to report wire touch status, giving the robot a low speed move command, monitoring the IWC wire status until a touch is reported, sending a stop command to the robot. An alternate architecture is to have the IWC wire touch status conveyed directly to the robot controller and have the robot monitor it and generate its own stop. We are experimenting with both approaches.

\section{Intelligent Weld Controller}

The Intelligent Weld Controller (IWC) supervises the wire snipper and the commercial weld power source. It executes commands from the Cell controller and reports status about tasks and its equipment and sensors. The IWC hosts welding control algorithms to be tested, and interfaces to sensors and commercial power sources.

\section{Weld Process Sensors}

We will test weld process sensors from research and commercial sources, and investigate hardware and software issues of sensor integration into a cell. We hope this will lead to working with sensor vendors and users on hardware and software standards to make adding sensors, or replacing one with another, easy and inexpensive. Our initial sensors are a photocell, and arc current and voltage sensors. These inputs are used by the Arc Monitor System and the Arc Length Controller (Ref. 7), both hosted in the IWC.

\section{NIST BACKGROUND IN SYSTEMS ENGINEERING}

The Intelligent Systems Division has over 15 years of experience in the development and application of real-time control technology. We have developed and applied an engineering methodology called Real-Time Control Systems (RCS) to the analysis of problems and the subsequent development of computerized control hardware and software systems. Applications for RCS have included manufacturing control and integration, e.g., the Automated Manufacturing Research Facility (AMRF) (Ref. 14), control of autonomous vehicles, robotic deburring, and robotic crane technology. The scupe of the activities includes sensor integration, servo-level 
control, path planning, off-line task planning, and real-time image processing. (Ref.15)

Our most recent direct research involvement with Industry is the Enhanced Machine Tool Controller (EMC) project. (Ref. 1) (Ref.11)(Ref.12)(Ref.15) NIST engineers developed a controller for machine tools, based on open architecture application programming interfaces (APIs) and commercially available components. Two versions were installed on commercial machine tools: a machining center at the General Motors Powertrain Division and a milling machine in a one-person shop near Baltimore, MD. The NIST team collaborated with two controller vendors-Advanced Technology and Research Corp. and a unit of Hewlett-Packard--during the trials at General Motors. The goal is to demonstrate "plug and play" interoperability between such components as motion control and discrete input/output. The installations also help NIST evaluate whether EMC interface specifications are complete, perform as intended, and are compatible with the skills and preferences of factory personnel.

\section{ROBOCRANE}

NIST will use another robotic testbed, the RoboCrane (Ref. 5)(Ref. 2), to test concepts of portability and interoperability by moving welding components developed on AWMS to RoboCrane and its control system. The RoboCrane testbed, shown in Figure 3, has been constructed with a six degree-of-freedom work platform suspended by cables driven by winches under computer control. RoboCrane transforms the crane from a device for simply lifting and placing heavy loads into a robot capable of precisely manipulating objects and/or maneuvering power tools with position and force control in all six degrees of freedom. In addition the RoboCrane has a large work volume -- the 6 meter version has a work space of $20 \mathrm{~m}^{3}\left(686 \mathrm{ft}^{3}\right)$.

We have done a quick adaptation of an arc welding torch to the RoboCrane and have done some simple welds. Future experiments will use the RoboCrane to simulate a commercial welding system that will host sensor and control technology developed or integrated into the AWMS.

\section{PLANS}

Our 1997 experiments will include weld process monitoring and control, recording and display of weld quality data, sensory interactive robot motion for plate finding, and robotic simulation for program tryout. We plan to add commercial robot sensors for non-contact plate-finding, weld joint location, inspection and/or tracking. This will involve the software and hardware interfaces to integrate the devices as well as software motion and control algorithms to use them. Activities with other organizations and companies will be conducted under formal legal agreements called Cooperative Research and Development Agreements (CRADAs). (Ref. 6) 


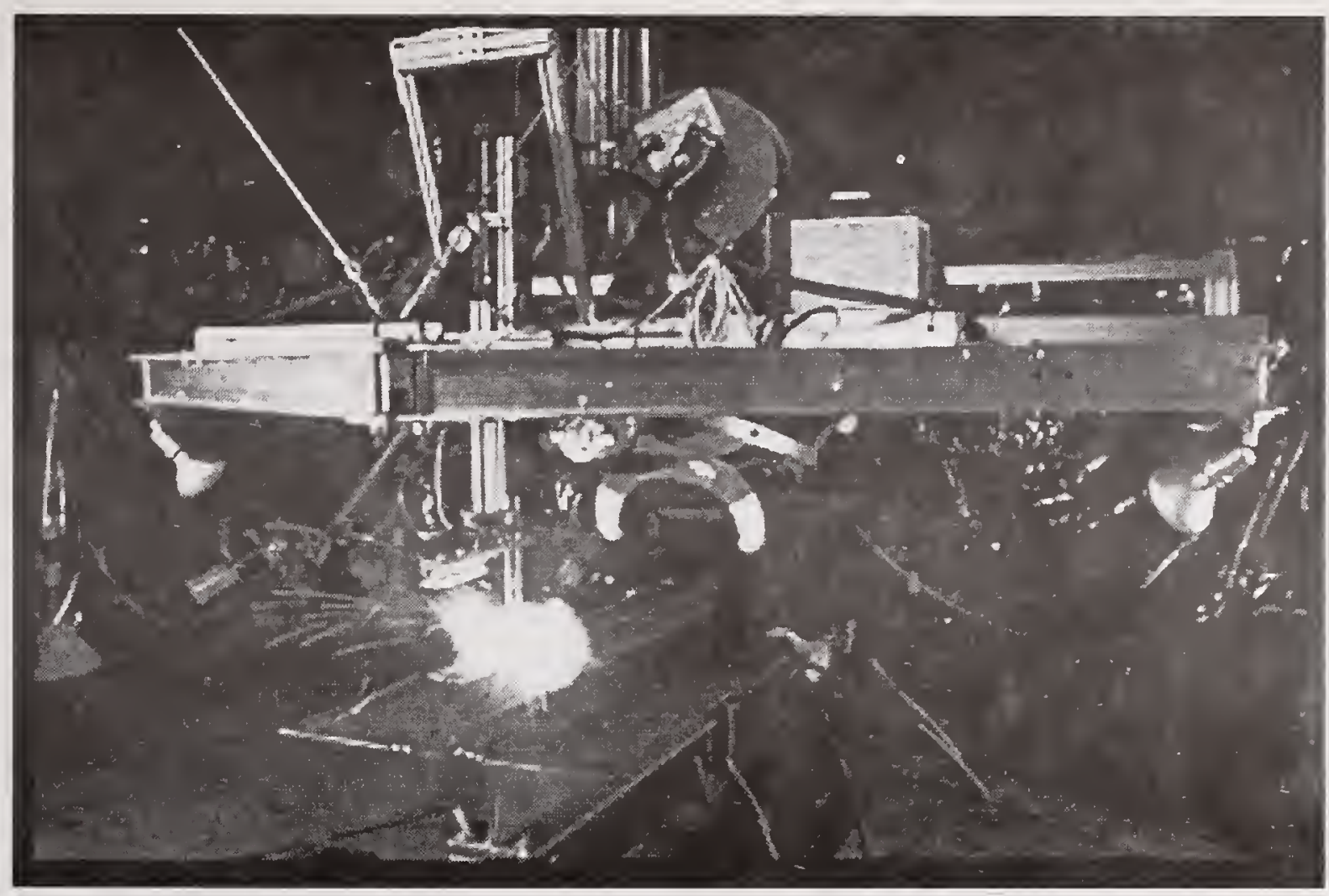

Figure 3. The welding torch is mounted beneath the platform of the Robocrane, the wire feed above. Two of the six winch driven cables can be seen at the upper left.

\section{REFERENCES}

1. Enhanced Machine Controller project - http://isd.cme.nist.gov/proj/enc/

2. RoboCrane project - http://isd.cme.nist.gov/brochure/RoboCrane.html

3. Welding Project - http://isd.cme.nist.gov/brochure/Welding.html

4. Bello, M. 1997. NIST "Open" Controller Gets Thumbs Up from One-man Company: NIST News Release (to be published): Public and Business Affairs Division, Gaithersburg, Md 20899-0001.

5. Bostleman, R. 1996. RoboCrane Project: An Advanced Concept for Large Scale Manufacturing. Proc. of the Association for Unmanned Systems International (AUVSI) Conference. Orlando, $\mathrm{Fl}$.

6. Model NIST CRADA agreement. Available from the NIST Industrial Partnership Program, (301) 975-5073.

7. Madigan, R. B.; Quinn, T. P.; Siewert, T. A. 1995. Control of Gas-Metal-Arc Welding Using Arc-light Sensing: NIST Internal Report 5037.

8. Mahin, K.W; Mitchiner, J.; Knorovsky, G.A.; Fuerschback, P.W. 1995. SMARTWELD: An Intelligent System for Design and Fabrication of Welded Assemblies. Proc. 1995 AWS Convention: Cleveland, Oh.

9. McGhee, S.; Nalluri, S.; Reeve, R.; Rongo, R. 1996. Automatic Programming System for Shipyard Robots. Proc. of 1996 Ship Production Symposium and Workshop: San Diego, Ca.

10. Milano, J.; Kassel, B.; Mauk, D. 1996. Development of a Welding Protocol for Automated Shipyard Manufacturing Systems. Proc. of 1996 Ship Production Symposium and Workshop: San Diego, Ca.

11. Proctor, F. M.; and Michaloski, J. 1993. Enhanced Machine Controller Architecture Overview: NIST Internal Report 5331.

12. Proctor, F.M.; Shackleford, W.; Yang, C.; Barbera, T.; Fitzgerald, M.L.; Frampton, N.; 
Bradford, K.; and Koogle, D. 1995. Simulation and Implementation of an Open Architecture Controller. Modeling. Simulation, and Control Technologies for Manufacturing. Proc. of the SPIE 2596.

13. Reeve, R.; Rongo, R.; Blomquist, P. 1996. Flexible Robotics for Shipbuilding. Proc. of 1996 Ship Production Symposium and Workshop. San Diego, Ca.

14. Simpson, J.A; Hocken, R.J.; Albus, J.S. 1983. The Automated Manufacturing Research Facility. Journal of Manufacturing Systems 4 (1).

15. ISD Internet URL - http://isd.cme.nist.gov. Click on "Active Projects".

16. Some of the above documents can be found in ISD's web site, http://www.isd.cme.nist.gov, under "ISD On-line Documents". 
MODELING - HEAT FLOW and FLUID FLOW 
PI 


\title{
PREDICTION OF LASER SPOT WELD SHAPE BY NUMERICAL ANLAYSIS AND NEURAL NETWORK
}

\author{
W.-S.Chang* ${ }^{*}$ S.-J.Na* ${ }^{*}$ H.-S.Moon ${ }^{* *}$, S.-J.Hong ${ }^{\#}$, H.-S.Kang ${ }^{\#}$
}

\begin{abstract}
Finite difference method and neural network were applied for predicting the bead shape in LSW(laser spot welding) of type 304 thin stainless steel sheets with thickness smaller than $1 \mathrm{~mm}$. The parameters of pulsed Nd:YAG laser spot welding such as pulse energy, pulse duration, sheet metal thickness and gap size between the sheets were varied to make various experiments and numerical simulations. At first the penetration depth and nugget size of spot welds measured for specimens with no gap were compared with the calculated results to verify the proposed finite difference model, and then three different combinations of process parameters were considered to find out the appropriate input variables to the neural network. It could be found that the finite difference model proposed for no gap and the neural network model proposed for various gap sizes can estimate the LSW bead shape fairly well. Finally the LSW bead shape calculated with no gap was used as the input variable of neural network. This combined model of finite difference analysis and neural network could be effectively applied for the prediction of LSW bead shapes.
\end{abstract}

\section{INTRODUCTION}

Laser beam welding offers a unique combination of high speed, precision and flexibility, compared with conventional resistance spot welding. This combination is especially attractive for electronic component joining. A wide range of research activities have been undertaken, including laser beam delivery systems and mechanical behavior of laser-welded sheet steels. However, research on the dimension of laser beam welds for given metal thickness remains virtually not extensively studied. The weld pool dimension and weld quality of spot welds produced by using a pulsed Nd:YAG laser welding machine depend on various process

\footnotetext{
Dept. of Mechanical Engineering, KAIST, Taejon, Korea

** Industrial Research Institute, HYUNDAI Heavy Industries, Ulsan, Korea

\# Production Technology Center, LG Electronics, Changwon, Korea
} 
parameters such as the spatial intensity distribution of incident laser beam, the peak power of pulse, the pulse energy, the pulse time, and the temporal shape of beam power during the pulse. When developing the welding procedure for a specific application, each of these parameters must be characterized and fully specified. This is usually done by using empirical techniques, since most analytical and numerical models for laser weld pool development ignore various aspects of the laser-material interaction(Ref. 1), thus making it difficult to accurately predict the weld pool shape. Limited experimental studies of the effects of $\mathrm{Nd}$ :YAG laser process parameters on laser spot weld dimensions and weld quality have been reported.(Ref. 2-3)

In this study, finite difference method and neural network were applied for predicting the bead shape in laser spot welding of thin stainless steel sheets with thickness smaller than $1 \mathrm{~mm}$. The penetration depth and nugget size of laser spot welds measured for the specimen with and without gap were compared with the predicted results to verify the proposed model.

\section{FINITE DIFFERENCE ANALYSIS}

\section{Governing Equation and Boundary Conditions}

Heat transfer problem of laser processing can be solved by applying the heat conduction theory. The thermal analysis of laser spot welding can be treated as an axisymmetric heat transfer problem, Fig. 1. Thermal energy changes due to the chemical reaction and evaporation of material during laser spot welding were not considered. The energy equation of the problem is :

$$
\begin{aligned}
& \nabla^{2} T=\frac{1}{\kappa} \frac{\partial T}{\partial t}-\frac{G}{k} \\
& G=\frac{2 \alpha(1-R) P}{\pi \delta^{2}} \exp \left[-2 r^{2} / \delta^{2}-\alpha z\right]
\end{aligned}
$$

where, $\quad T(r, z, t):$ temperature

$$
\begin{aligned}
& \kappa: \text { thermal diffusivity } \\
& \alpha: \text { absorption coefficient } \\
& \delta: \text { effective beam radius } \\
& P: \text { laser power }
\end{aligned}
$$$$
G \text { : heat source function }
$$

A special form of heat generation(Ref. 4) in the workpiece was adopted to simulate the laser beam heat source which has the characteristics of keyholing. The boundary condition is : 


$$
k \frac{\partial T}{\partial n}=-h\left(T_{s}-T_{\infty}\right)
$$

where, $n$ : unit vector outward normal to the boundary

$h$ : convection heat-transfer coefficient

$T_{S}:$ temperature of surface $\quad T_{\infty}:$ temperature of atmosphere

Initial condition for the transient analysis is $T(r, z, 0)=T_{0}(r, z)$ at $t=0$, where $T_{0}$ is the initial temperature. The solution domain of the finite difference model used for the thermal analysis is shown in Fig. 2.

\section{Material thermal properties}

The material used was type 304 stainless steel sheet. The thermal properties of the material were assumed to be homogeneous and independent of temperature. Thermophysical constants used in all calculations are presented in Table 1.

\section{NEURAL NETWORK}

\section{Back-propagation Algorithm}

The neural network based on the back-propagation algorithm consists of three layers, that is, the input, the output and the hidden layer. The purpose of the learning process is to decrease the error between the desired output and the actual one obtained from the output layer of the neural network, which can be expressed by a cost function as follows.(Ref. 5)

$$
J=(1 / 2) \sum_{D} \sum_{k}\left(d_{k}-a_{k}\right)^{2}
$$

where, $\quad p:$ the number of input patterns $d_{k}:$ desired outputs $k:$ the number of output patterns

$a_{k}:$ actual outputs from the output layer

The hidden layer, output layer and the weights between the layers are expressed as follows. 


$$
\begin{aligned}
& h_{j}=S\left(\sum_{i} w_{i j} s_{i}\right) \\
& a_{k}=S\left(\sum_{k} w_{j k} h_{j}\right) \\
& S(x)=1.0 /(1.0+\exp (-x-\theta))
\end{aligned}
$$

where, $h_{j}$ : output at the hidden layers $s_{i}$ : input according to each pattern

$w_{i j}$, $w_{j k}$ : weights

$\theta:$ internal offset value

$S(x)$ : Sigmoid function

\section{Selection of Appropriate Input Variables to Neural Network}

Three different combinations of process parameters were considered to find out the appropriate input variables to the neural network. Laser spot welding process parameters(focal length, energy, pulse time), sheet metal thickness(upper and lower plate), gap size and bead shape(penetration depth, nugget size) of the workpiece were selected as the input variables for the back-propagation learning algorithm of the neural network and the bead shape(penetration depth, nugget size) were considered as its output variables. In type 1, the focal length, pulse energy, pulse time, sheet metal thickness(upper and lower plate), gap size, penetration depth and nugget size were selected as the input variables, while in type 2 the sheet metal thickness(upper and lower plate), gap size, penetration depth and nugget size and in type 3 the gap size, penetration depth and nugget size were selected as the input variables.

\section{Combined model of FDM and Neural Network}

Various combinations of stainless steel sheet metal thickness were considered to calculate the laser spot weld bead shape of the workpiece with no gap, which was then used as the input variable of the neural network for predicting the bead shape in the case of sheet metals with various thicknesses and gap sizes.

\section{EXPERIMENTS}


Experiments were designed to test the finite difference model and to determine the input and output variables appropriate to the neural network. An experimental set-up for Nd:YAG laser spot welding is shown in Fig. 3. A pulsed type Nd:YAG laser welding machine was used for all experiments. The beam oscillator was capable of the maximum output power $400 \mathrm{~W}$. Type 304 stainless steel specimens with 5 different combinations of thickness $(0.3 \mathrm{~mm}+0.33 \mathrm{~mm}$, $0.33 \mathrm{~mm}+0.5 \mathrm{~mm}, 0.4 \mathrm{~mm}+0.6 \mathrm{~mm}, 0.6 \mathrm{~mm}+0.5 \mathrm{~mm}$ and $0.5 \mathrm{~mm}+0.5 \mathrm{~mm}$ ) were prepared for experiments.

\section{RESULTS AND DISCUSSIONS}

Figure 4 shows a typical example of cross-sections of experiments and calculations obtained for the stainless steel thickness combination of $0.3 \mathrm{~mm}+0.33 \mathrm{~mm}$ with laser energy of $2 \mathrm{~J}$ and pulse time of $4 \mathrm{~ms}$. In the calculated result(Fig. 4a), it is shown that the nugget size and penetration depth agree fairy well with the experimental result(Fig. 4b), while the experimental result shows a somewhat wider top bead size than the calculated one. It is thought that the temporal pulse shape, plasma formation and laser-material interaction influence the formation of top bead, which cannot be considered in the developed finite difference model. However, the top bead size is less important in determining the weld quality such as joining strength than the nugget size and penetration depth. Consequently, this study is mainly dealing with the prediction of nugget size and penetration depth.

Figure 5 shows that the error of predicted nugget size and penetration depth determined by comparing them with experimental ones is less than $15 \%$ for the type 1 of the neural network, where the focal length, pulse energy, pulse time, sheet metal thickness(upper and lower plate), gap size, penetration depth and nugget size were selected as the input variables.

Figure 6 and 7 show that the neural network model of type 2 and type 3 can estimate the bead shape of laser spot welds such as nugget size and penetration depth. From these results it could be revealed that the process parameters adopted in type 2 and type 3 are also appropriate input variables to the neural network.

Figure 8 shows the error of nugget size and penetration depth predicted by using the combined model of finite difference analysis and neural network. Predicted results were compared with the experimental ones of the specimen with thickness combination of $0.5 \mathrm{~mm}+0.5 \mathrm{~mm}$. It shows that the error was smaller than $10 \%$. From these results, the combined model could be effectively applied for the prediction of laser spot weld bead shape such as nugget size and penetration depth. Figure 9 shows the windows program developed for the simulation and display of the combined model of finite difference analysis and neural network. In this program, the user can select the 
process parameters such as sheet thickness, gap size, pulse time, pulse energy, focal length and the bead shape calculated for no gap and then the bead shape is simulated for various gap sizes by using the neural network and displayed.

\section{CONCLUSIONS}

From the results of finite difference analysis, neural network and experiments for laser spot welding of thin stainless steel sheets, the following conclusions may be drawn:

1) The proposed finite difference model can estimate the laser spot weld bead shape of thin stainless steel sheets considerably precisely, if they are layered tightly.

2) The developed neural network which uses the bead shape data of the specimen with no gap and a few data of specimen with gap can predict the laser spot weld bead shape of thin stainless steel sheets with various gap sizes fairly well.

3) The combined model of finite difference analysis and neural network could be effectively applied for the prediction of laser spot weld bead shapes, because the numerical analysis of laser spot weld bead shape for the workpiece with a gap between two sheet metals is highly limited.

\section{REFERENCES}

1. Mazumder, J., and Voekel, D. 1992. Challenges in modeling and measurement of laser material processing . Proc. LAMP '92. Eds. A Matsunawa and S. Katayama, pp.373-380. Japan High Temperature Soc.

2. Liu, J. T., Weckman, D.C., and Kerr, H. W. 1993. The effects of process variables on pulsed Nd:YAG laser spot welds, prat I: AISI 409 stainless steel, Metallurgical Transactions B, 24B (6): 1065-1076

3. Bransch, H. N., Weckman, D.C., and Kerr, H. W. 1994. Effects of pulse shaping on Nd:YAG spot welds in austenitic stainless steel, Welding Journal, 73(6) : 141-s to 151-s

4. Jae-Do Kim, 1990. Prediction of the penetration depth in laser beam welding, KSME journal, $4(1): 32-39$

5. S.C. Chapra and R.P. Canale, 1988 , Numerical method for engineers, $2^{\text {nd }}$ edition, McGrawHill Book Company, pp.358-361. 
Table 1. Thermophysical constants used in finite difference analysis

\begin{tabular}{c|c|c|c|c|c}
\hline Material & $k, \mathrm{w} / \mathrm{cm}^{\circ} \mathrm{C}$ & $\kappa, \mathrm{cm}^{2} / \mathrm{sec}$ & $\alpha, \mathrm{cm}^{-1}$ & $R$ & $T_{m},{ }^{\circ} \mathrm{C}$ \\
\hline $\begin{array}{c}\text { Type 304 } \\
\text { stainless steel }\end{array}$ & 12 & 0.053 & 6 & 0.3 & 1450 \\
\hline
\end{tabular}

where, $\quad k:$ thermal conductivity $\alpha$ : absorption coefficient

$T_{m}$ : melting temperature

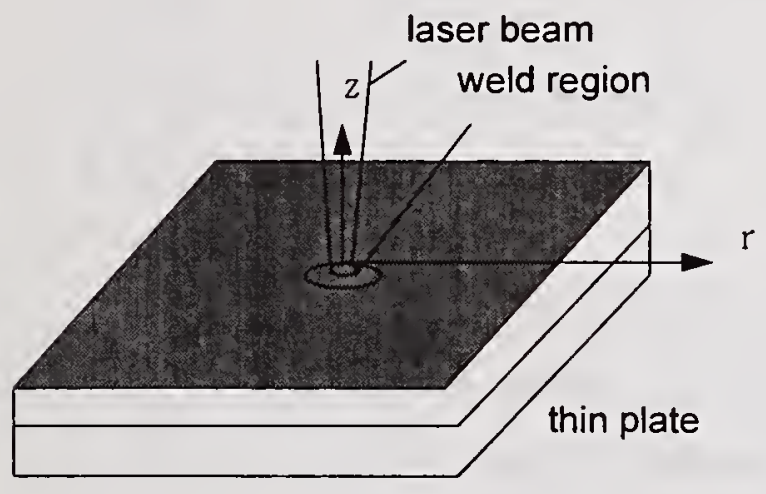

Figure 1. Schematic diagram of laser spot welding $\kappa:$ thermal diffusivity

$R:$ reflectivity

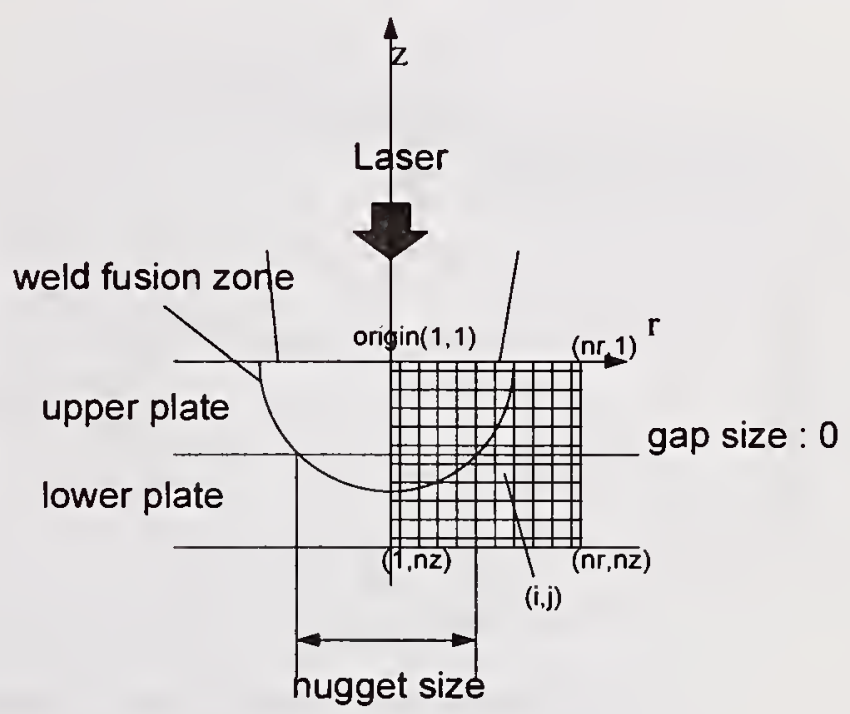

Figure 2. Solution domain of finite difference model for thermal analysis

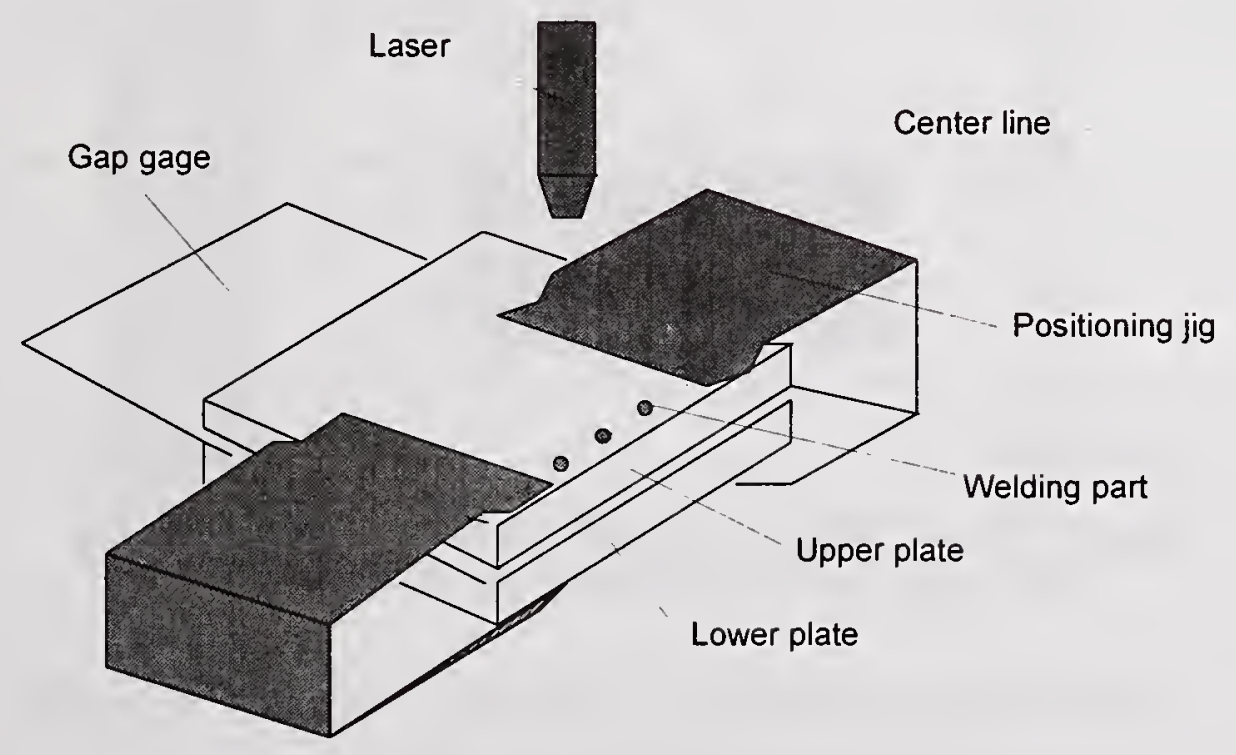

Figure 3. Experimental setup of laser spot welding 


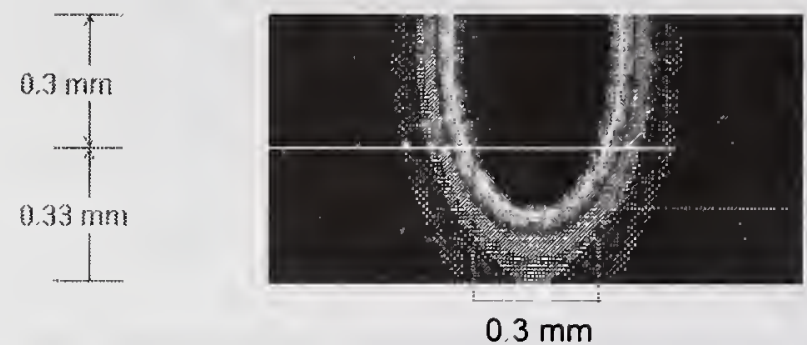

(a)

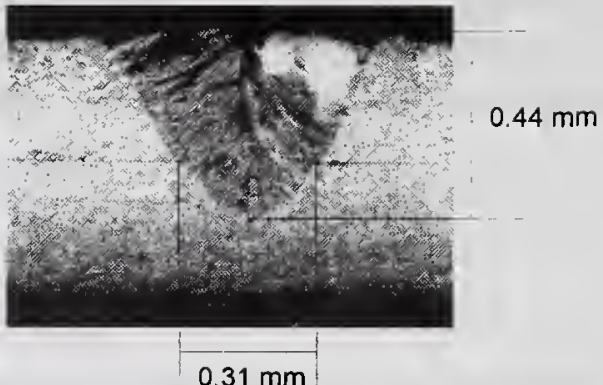

(b)

Figure 4. Comparison of calculated (a) and experimental (b) results (pulse time : $4 \mathrm{~ms}$, pulse energy : $2 \mathrm{~J}$ )
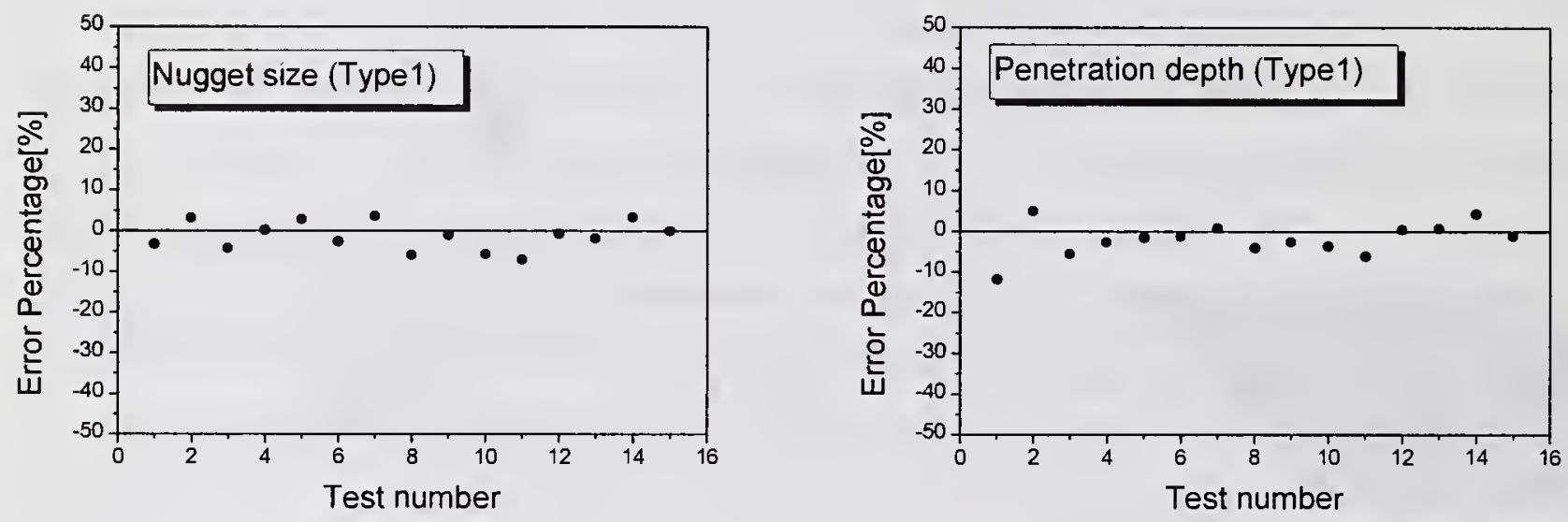

Figure 5. Estimated error of bead shape predicted in type 1
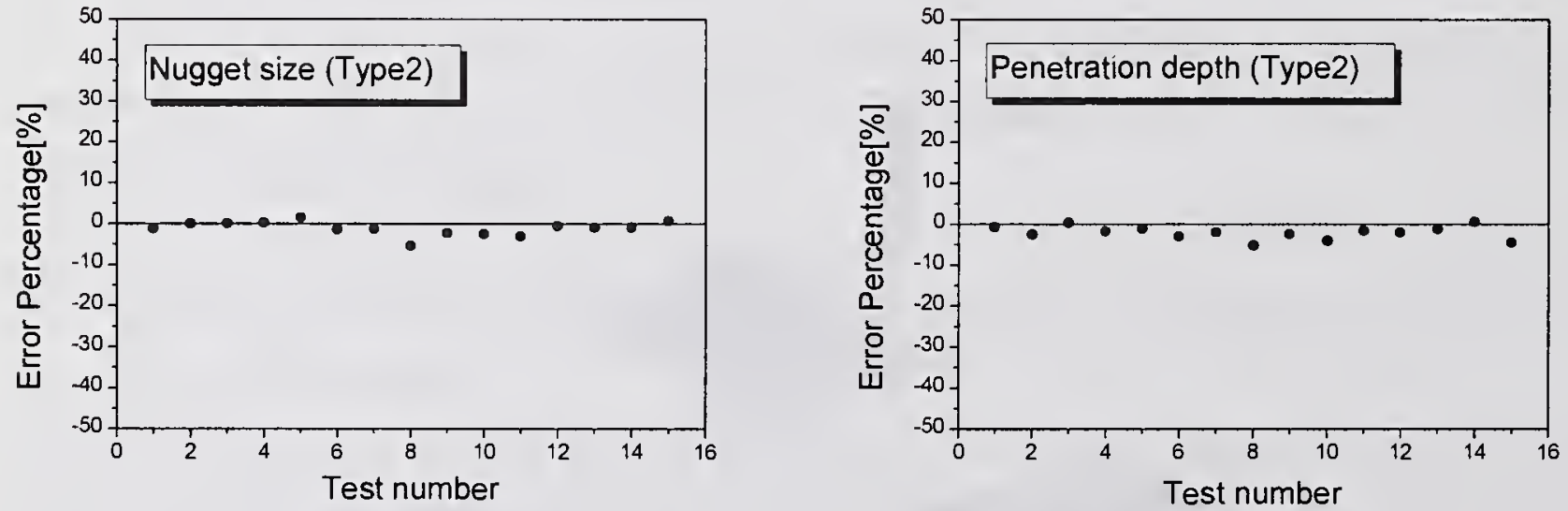

Figure 6. Estimated error of bead shape predicted in type 2 

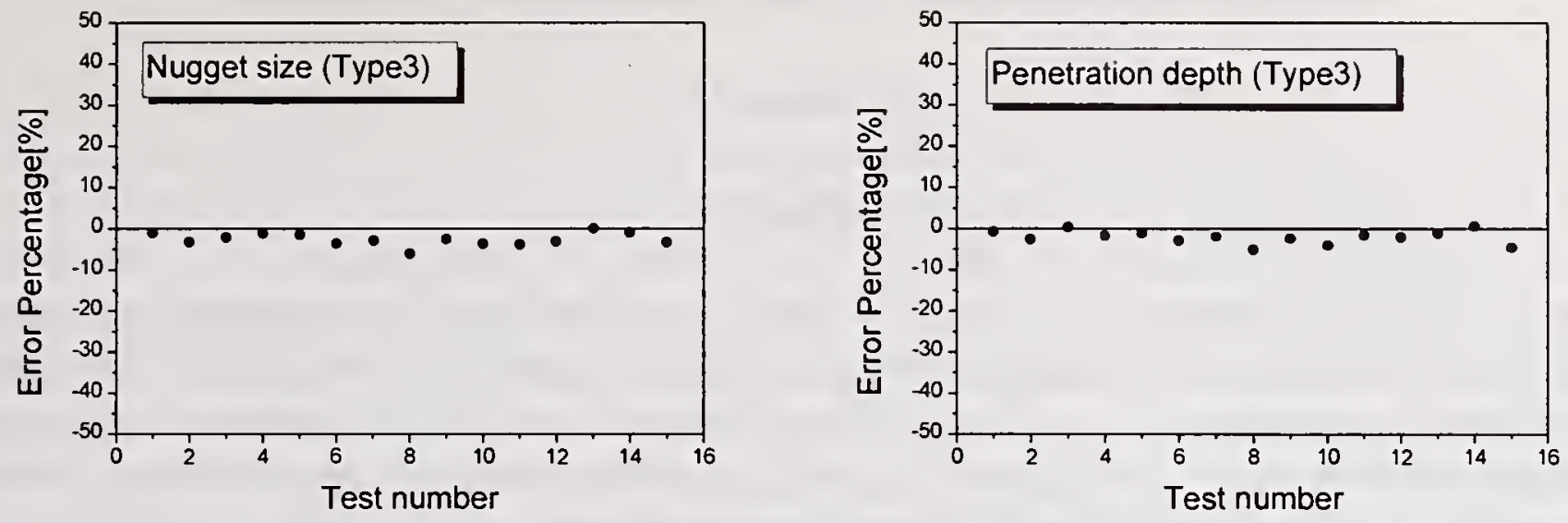

Figure 7. Estimated error of bead shape predicted in type 3
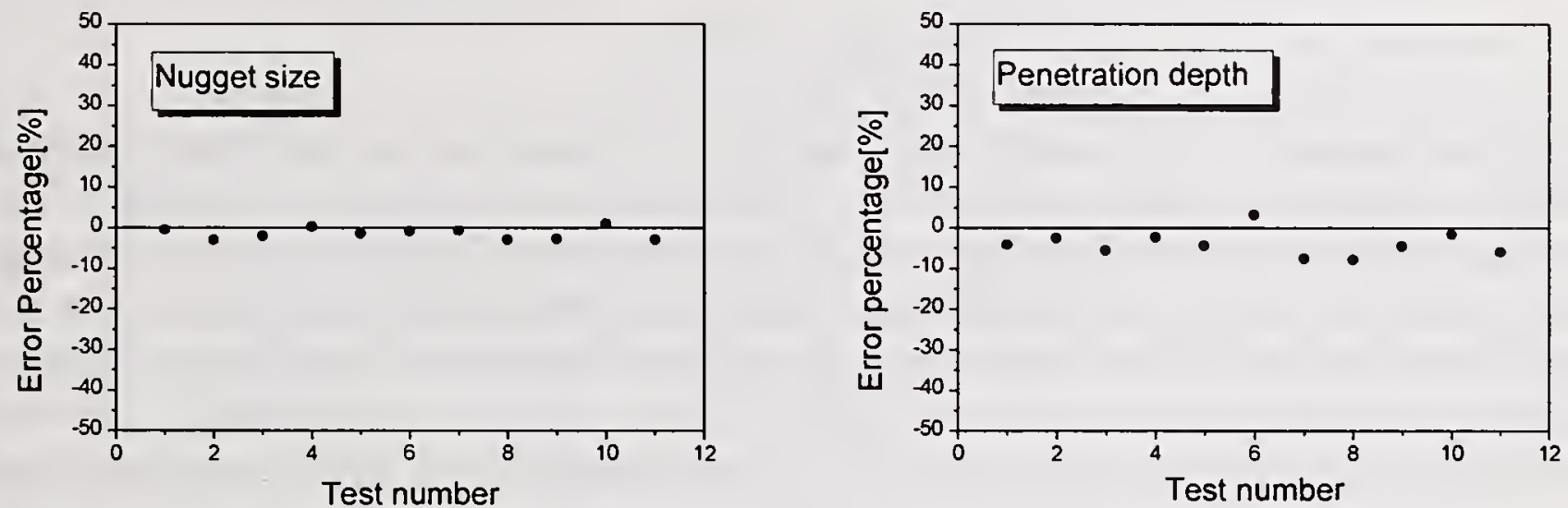

Figure 8. Estimated error of bead shape predicted by combined model of FDM and neural network

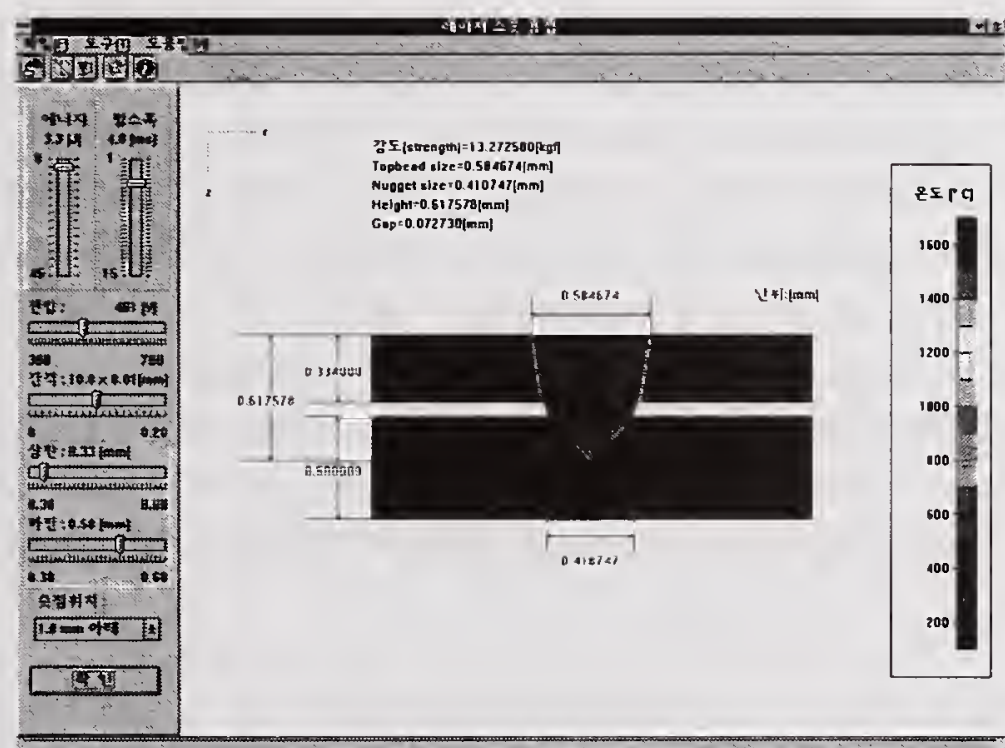

Figure 9. Windows program for simulations of bead shape by combined model of FDM and neural network 


\title{
NONLINEAR ANALYSIS OF WELD THERMAL HISTORY
}

\section{Becker ${ }^{1}$}

\begin{abstract}
Analytical models are developed for weld thermal history accounting for temperature dependence of thermal conductivity and heat capacity. It is shown that adjustments made to linear solutions in the literature are consistent with nonlinear solutions. Explicit nonlinear solutions are obtained for instantaneous and rapidly moving heat sources, and for one-dimensional moving sources. Approximate expressions are introduced for moving coordinates.
\end{abstract}

\section{INTRODUCTION}

Transient heat conduction associated with welding has been studied both analytically and numerically. Analytical solutions have been summarized by Radaj (Ref. 1), and a recent comprehensive solution has been presented by Kasuya and Yurioka (Ref. 2). Much of the analytical work traces back to early work by Rosenthal (Ref. 3) and Rykalin (Ref. 4). Almost all of the analytical work has been linear, i.e. assuming properties independent of temperature. Grosh and Trabant (Ref. 5) performed a nonlinear analysis based on a Kirchoff transformation, but had to assume that thermal conductivity and heat capacity varied in an identical manner with temperature. In actual welding situations, these two properties can vary in opposite directions (thermal conductivity decreasing, heat capacity increasing with temperature). Kasuya and Yurioka were able to obtain good agreement between theory and experiment, but had to use different values of thermal properties to predict different quantities. Moore, et. al. (Ref. 6) had found earlier that values suitable for certain predictions were not as good for others.

In this paper, nonlinear analysis is performed. When a particular form of property variation is fit to the data, explicit solutions are obtained for cases involving instantaneous or rapidly moving heat sources based on the method of similarity variables. These solutions are used to develop expressions for cooling times and cooling rates. The nonlinear solutions serve to explain the nature of the adjustments and observations made by Kasuya and Yurioka and Moore, et. al. In one dimension, moving source problems without restriction on magnitude of speed are addressed. These correspond to progressive melting of an electrode (Ref. 1). Analytical solutions are obtained with a variety of possible forms of property variation. For other moving source cases, analytical approximations are developed which reduce to exact linear and nonlinear solutions in appropriate limits.

\footnotetext{
' Oregon Graduate Institute, P.O. Box 91000, Portland, OR 97291-1000
} 


\section{INSTANTANEOUS AND RAPIDLY MOVING SOURCES}

Svensson (Ref. 7) observes that in most welding situations, arc speed is much faster than thermal diffusion. The solution of the moving heat source problem then becomes equivalent to the solution of an instantaneous heat source problem. A rapidly moving point source (thick plate case) becomes equivalent to an instantaneous line source, and a rapidly moving line source (thin plate case) becomes equivalent to an instantaneous plane source. In addition, for cooling of a thick plate directly behind the arc, the results for the rapidly moving source apply independent of the speed of the source, an important consideration in evaluation of cooling times. In this section, we will proceed from the perspective of instantaneous sources. Later in the paper, we will examine the relationship between the instantaneous and moving source perspectives. That examination will serve to demonstrate the range of applicability of instantaneous source solutions and to construct expressions for use in moving coordinate frames.

Nonlinear response to an instantaneous source can be studied using similarity variables. A detailed derivation, including review of earlier literature, has been presented (Ref. 8) and will be outlined here. Most attention in the literature has been for situations where properties increase with temperature. Zeldovich and Kompaneets (Ref. 9) present a solution for declining conductivity in plane geometry. Other authors have considered only properties increasing with temperature. For important materials, such as low carbon steels, thermal conductivity declines with temperature.

The time-dependent heat conduction equation is

$$
\rho c \frac{\partial T}{\partial t}=\left(1 / r^{m-1}\right) \frac{\partial}{\partial r}\left(r^{m-1} k \frac{\partial T}{\partial r}\right)+Q \delta(r) \delta(t)
$$

where $\mathrm{m}$ of 1,2 and 3 correspond respectively to plane, cylindrical and spherical geometry. The source term involves delta functions, so that the source is zero except at the origin at time zero, and so that the integral over volume and time of the source is $\mathrm{Q}$. Temperature is relative to the reference ambient temperature that prevails everywhere before the introduction of the source. Thus, the temperature is zero everywhere for $t<0$. In addition, the temperature must go to zero as $r$ goes to infinity for any $\mathrm{t}>0$.

Let the conductivity and the heat capacity depend on powers of temperature, i.e.

$$
\begin{gathered}
k=k_{0}\left(T / T_{0}\right)^{s} \\
\rho c=(\rho c)_{0}\left(T / T_{0}\right)^{p}
\end{gathered}
$$


Results will be useful to the extent that Eqs. 2 and 3, when fit to actual data, provide reasonable representations of those data. If a new variable $U$ is defined as (Ozisik, 1980)

$$
U=\left(T / T_{0}\right)^{p+1}
$$

then the heat conduction equation for $t>0$ becomes

$$
\frac{\partial U}{\partial \tau}=\left(1 / r^{m-1}\right) \frac{\partial}{\partial r}\left(r^{m-1} U^{-q} \frac{\partial U}{\partial r}\right)
$$

where the parameter $q$ is defined by

$$
q=(p-s) /(p+1)
$$

and where a modified time is defined in terms of thermal diffusivity $\alpha$ by

$$
\tau=t k_{0} /(\rho c)_{0}=\alpha_{0} t
$$

For cases where conductivity decreases with temperature and heat capacity increases with temperature, $\mathrm{p}$ will be positive, $\mathrm{s}$ will be negative and $\mathrm{q}$ will be positive.

Applying the method of similarity variables leads to a solution of the form (Ref. 8)

$$
\begin{gathered}
U(r, \tau)=\frac{1}{\tau^{m /(2-m q)}} \frac{A^{1 / q}}{\left\{1+\frac{q A}{2(2-m q)} \eta^{2}\right\}^{1 / q}} \\
\eta=\frac{r}{\tau^{1 /(2-m q)}}
\end{gathered}
$$

Eqs. 8 and 9 reduce to the familiar linear forms when q goes to zero.

The coefficient $\mathrm{A}$ is obtained by equating the integral of energy over all space with the amplitude of the source. This leads to (Ref. 8)

$$
A=\left[\frac{Q(p+1)}{4 \pi(\rho c T)_{0}}\right]^{q /(1-q)}
$$


for the instantaneous line source, and

$$
A=\left\{\frac{Q(p+1) \sqrt{q}}{2(\rho c T)_{0} \sqrt{2(2-q)}} \frac{2 \Gamma(1 / q)}{\Gamma(1 / 2) \Gamma(1 / q-1 / 2)}\right\}^{2 q /(2-q)}
$$

for the instantaneous plane source. The line source result is simpler because the gamma functions involved in the integration are easily evaluated.

\section{COOLING TIMES AND COOLING RATES}

Cooling times and cooling rates typically are evaluated by considering locations near the source, i.e. setting the variable of Eq. 9 to zero. Radaj (Ref. 1) observes that cooling intervals tend to be insensitive to the particular locations at which they are evaluated. Converting $U$ back to temperature from Eq. 4.

$$
\frac{T}{T_{0}}=\frac{A^{1 /(p-s)}}{\tau^{m /(2-m q)(p+1)}}
$$

For the instantaneous line source,

$$
\frac{m}{(2-m q)(p+1)}=\frac{1}{1+s}
$$

It then follows that

$$
\left(\frac{T}{T_{0}}\right)^{1+s}=\frac{k(T)}{k_{0}} \frac{T}{T_{0}}=\frac{A^{1 /(p-s)}}{\tau}
$$

where we have invoked Eq. 2 for conductivity. Using Eq. 10 for A, it follows that

$$
T=\frac{Q(p+1)}{4 \pi k(T) t}
$$

This is similar to the solution of a linear problem with two exceptions:

1. The actual temperature-dependent conductivity appears, instead of a constant value.

2. The factor of $(p+1)$ from heat capacity variation makes conductivity look smaller. 
This factor is pertinent to the observation of Moore et. al. that "unrealistically low" effective thermal conductivities have been required to predict the cooling from $800^{\circ} \mathrm{C}$ to $500^{\circ} \mathrm{C}$, commonly referred to as $\mathrm{t}_{8 / 5}$. Recasting Eq. 5 to cooling time yields

$$
t=\frac{Q(p+1)}{4 \pi k(T) T}
$$

Following a similar procedure for the instantaneous plane source leads to

$$
t=\frac{[Q(p+1) B]^{2}}{k(T) \rho(T) c(T) T^{2}}
$$

where the coefficient $B$ is given by

$$
B=\sqrt{\frac{q}{2(2-q)}} \frac{\Gamma\left(\frac{1}{q}\right)}{\Gamma\left(\frac{1}{2}\right) \Gamma\left(\frac{1}{q}-\frac{1}{2}\right)}
$$

As in the linear case, the cooling time depends inversely on the square of the temperature and on the product of conductivity and heat capacity, with the actual temperature-dependent quantities appearing instead of, as in the linear case, constant values.

The cooling rate from the instantaneous line source may be obtained by differentiating Eq. 15. After some algebra, one obtains

$$
\frac{d T}{d t}=-\frac{4 \pi}{(1+s)(p+1) Q} k(T) T^{2}
$$

This again is similar in form to linear solutions (Ref. 1), with the actual temperature-dependent thermal conductivity appearing. For the instantaneous plane source, analogous procedure leads to

$$
\frac{d T}{d T}=-\frac{4 \pi}{(2+s+p)(p+1) Q B} k(T) \rho(T) c(T) T^{3}
$$

Again, a form similar to that for the linear solution (Ref. 1) is obtained with properties evaluated at actual temperatures. 


\section{INSTANTANEOUS SOLUTIONS - POSITION DEPENDENCE}

The full solution of Eq. 8 is repeated for convenience as

$$
U(r, \tau)=\frac{1}{\tau^{m /(2-m q)}} \frac{A^{1 / q}}{\left\{1+\frac{q A}{2(2-m q)} \eta^{2}\right\}^{1 / q}}
$$

In the previous section, $\eta$ was set to zero to give results near the source. The deviation of the power of the time variable from its linear value was related to temperature, and thereby to the properties thermal conductivity and heat capacity. A similar approach is taken here. Details of the derivation are given in Ref. 8. It follows that

$$
A \eta^{2}=\frac{r^{2}}{\alpha_{r=0, t} t}
$$

Thus, the spatial dependence becomes similar to that of a linear problem if the thermal diffusivity is evaluated at the current time at the heat source. (The form of the spatial solution tends to the familiar Gaussian of linear solutions as q tends toward zero.)

\section{MOVING COORDINATES - ONE DIMENSION}

While instantaneous source problems provide much useful information for welding, the actual situation involves a moving source. In a moving Eulerian frame of reference, formulation can be as a static problem.. We will consider the nonlinear solution of the problem in plane geometry, which has been used to model the progressive melting of an electrode (Ref. 1). Previous nonlinear solution of this problem has been with the restriction of conductivity and heat capacity having identical variation with temperature (Ref. 5). It will be shown that several alternate approaches can be used to obtain general solutions.

The heat conduction equation with a source moving in the positive $\mathrm{x}$-direction is

$$
\rho c_{p} v d T / d x+d / d x(k d T / d x)=0
$$

In a linear problem, with coefficients independent of temperature, the solution when temperature is specified at the moving interface, is (Ref. 1)

$$
T=T_{c} \exp (-v x / \alpha)
$$


for positive $\mathrm{x}$ and constant for negative $\mathrm{x}$, where the $\mathrm{c}$ subscript denotes value at the source (center). Since positive $\mathrm{x}$ corresponds to negative time, this solution of temperature decaying with distance in the positive $\mathrm{x}$-direction is equivalent, in fixed coordinates, to temperature increasing with time as the heat source approaches.

Consider first the case where conductivity and heat capacity vary as powers of temperature, as treated above for instantaneous sources. With the same changes of variables used there, it follows that

$$
d / d x\left(U^{n} d U / d x\right)+\left(v / \alpha_{0}\right) d U / d x=0
$$

This equation can be integrated to obtain

$$
U=1 /\left[U_{c}^{-q}+\left(q v x / \alpha_{0}\right)\right]^{1 / q}
$$

Thus, for properties varying as powers of temperature, temperature rise in moving coordinates in plane geometry can be obtained.

For more general variations of properties, it is convenient to introduce the Kirchoff transformation

$$
\theta=\left(1 / k_{0}\right) \int^{T} k(T) d T
$$

Grosh and Trabant invoked this transformation in their solution. This leads to

$$
d^{2} \theta / d x^{2}+(v / \alpha) d \theta / d x=0
$$

They then assumed that conductivity and heat capacity varied identically with temperature. Thermal diffusivity consequently is independent of temperature, and the equation for this new variable is linear and can be integrated. The nonlinear character of the problem is recovered when transforming back to the temperature variable.

For the more general case where thermal diffusivity and thermal conductivity are different functions of temperature, let us make the substitution

$$
\zeta=d \theta / d x
$$

The second order equation becomes a first order equation

$$
\zeta d \zeta / d \theta+(v / \alpha) \zeta=0
$$


Let us now select a particular convenient functional form for thermal diffusivity

$$
\alpha=\alpha_{0} /(1+2 c \theta)
$$

Substituting and integrating leads to

$$
\theta /(1+c \theta)=\left[\theta_{c} /\left(1+c \theta_{c}\right)\right] \exp \left(-v x / \alpha_{0}\right)
$$

This can be rearranged to the form

$$
\theta=\theta_{c} \exp \left(-v x / \alpha_{0}\right) /\left\{1+c \theta_{c}\left[1-\exp \left(-v x / \alpha_{0}\right)\right]\right\}
$$

To transform back to temperature, a particular form for conductivity variation must be assumed for the Kirchoff transformation. Grosh and Trabant assumed linear variation with temperature. For conductivity declining with temperature, we consider

$$
k=k_{0} /(1+b T)
$$

This relation will stay positive even for large temperatures. The Kirchoff transformation becomes

$$
\theta=(1 / b) \ln (1+b T)
$$

The reverse transformation, yielding temperature, is

$$
T=[\exp (b \theta)-1] / b
$$

Thus, a nonlinear solution is obtained for one-dimensional moving coordinate problem allowing for separate variation of thermal conductivity and thermal diffusivity.

For analytical convenience, thermal diffusivity was expressed in terms of the new variable rather than in terms of temperature. For the particular Kirchoff transformation used, the diffusivity can be expressed in terms of temperature as follows

$$
\alpha=\alpha_{0} /[1+(2 c / b) \ln (1+b T)]
$$


An alternate approach to obtaining nonlinear solutions is to transform variables based on heat capacity rather than conductivity, i.e. to define a new variable

$$
\psi=\left[1 /\left(\rho c_{p}\right)_{0}\right] \int^{T} \rho c_{p}(T) d T
$$

This can be shown to lead to the equation

$$
d / d x(\alpha d \psi / d x)+v d \psi / d x=0
$$

This can be integrated directly, with the constant of integration again set to zero so that function and derivative vanish at infinity. Then expressing diffusivity in the form

$$
\alpha=\alpha_{0} /\left(1+2 c^{\prime} \psi\right)
$$

the resulting equation can in turn be integrated to yield

$$
\psi /\left(1+c^{\prime} \psi\right)=\left[\Psi_{0} /\left(1+c^{\prime} \psi_{0}\right)\right] \exp \left(-v x / \alpha_{0}\right)
$$

This is similar to the form of result obtained above with a transformation that was based on thermal conductivity.

It may be observed that the moving heat source problem in one dimension can be solved with a variety of assumptions about the behavior of thermal conductivity and heat capacity. While there are, as noted, applications of the one-dimensional problem, it is a restrictive case. Since after the heat source passes, the temperature remains constant, the problem is a study of the rapid temperature rise during heating. In welding thermal cycles, much of the interest is in the cooling part of the problem. In the next section, we consider more general geometries with moving heat sources.

\section{MOVING AND INSTANTANEOUS SOURCES}

In the previous section, explicit solutions were obtained for moving sources in one dimension. In more than one dimension, analytical solutions have not been obtained for nonlinear problems. As was noted above, in most welding applications the heat source moves more rapidly than heat diffuses, so that instantaneous heat source models apply. In this section, the relationships between instantaneous and moving source models will be examined in linear cases. These relationships will be used to infer the range of validity of instantaneous source models and to develop expressions that may be useful in nonlinear cases for moving sources. 
Consider a moving point source. The temperature distribution is given by (Ref. 1)

$$
\begin{gathered}
T=\left(Q^{\prime} / 2 \pi k R\right) \exp [-\nu(x+R) / 2 \alpha] \\
R^{2}=x^{2}+r^{2}
\end{gathered}
$$

where $\mathrm{R}$ is the distance from the point source and $\mathrm{r}$ is the distance from the line of motion of the source. Let $\mathrm{R}$ be expanded for small $\mathrm{r} / \mathrm{x}$

$$
R=|x|\left[1+\frac{1}{2}(r / x)^{2}-\frac{1}{8}(r / x)^{4}+\ldots\right]
$$

For negative $\mathrm{x}$ (cooling), this leads to

$$
T=\frac{Q^{\prime}}{2 \pi k|x|} \exp \left(-\frac{v r^{2}}{4 \alpha|x|}\right)+O\left[\left(\frac{r}{x}\right)^{2}\right]
$$

Noting that $\mathrm{x}=\mathrm{vt}$, this becomes the instantaneous source solution with $\mathrm{Q}^{\prime} / \mathrm{v}=\mathrm{Q} / 2$. Near the line of motion of the source (i.e. small r), the instantaneous source solution applies. Indeed, directly behind the source $(\mathrm{r}=0)$, it applies independent of the speed of the source.

Generally, there is interest in distances $r$ of up to the order of $10 \mathrm{~mm}$ (Note that Moore et. al. consider $38.1 \mathrm{~mm}$ to be thickness of a thick plate, i.e. to be an essentially infinite dimension.) A typical arc speed used in analysis by Moore et. al. is $3.81 \mathrm{~mm} / \mathrm{s}$. They cite cooling times $\mathrm{t}_{100}$ measured by several investigators to be on the order of 100 seconds for thick plates (over 600s for thin plates). The parameter $(\mathrm{r} / \mathrm{x})^{2}$ for $10 \mathrm{~mm}$ and 100 s equivalent to $381 \mathrm{~mm}$ is about .0007 . For such long cooling times, the instantaneous solutions should apply. For $\mathrm{t}_{8 / 5}$, Radaj (Ref. 1) shows temperatures in this interval occurring between $x$ distances of 30 and $40 \mathrm{~mm}$. The value of $(r / x)^{2}$ for $r$ of $10 \mathrm{~mm}$ and $x$ of $30 \mathrm{~mm}$ would be about .1 . The instantaneous model still should be reasonable, but deviations might not be considered negligible. For smaller values of r, Suarez, et. al. (Ref. 10) show much of the phenomena of concern taking place within $5 \mathrm{~mm}$, and $(\mathrm{r} / \mathrm{x})^{2}$ will be much smaller.

Consider the following expression for temperature

$$
T=\left[Q^{\prime}(p+1) / 2 \pi k R\right] \frac{1}{\{1+[q / 4(1-q)] v(x+R) / 2 \alpha\}^{1 / q(p+1)}}
$$

Properties are to be evaluated at the current time and at the source $(\mathrm{r}=0)$. This expression goes to two limits exactly. For small $r / x$ (small $r / v t$ ), expanding $R$ gives the nonlinear instantaneous source solution. For any $\mathrm{r} / \mathrm{x}$, for small $\mathrm{q}$ (i.e. in a linear problem) the expression becomes the linear solution. The expression applies to the nonlinear instantaneous solution a correction for actual source motion based on the linear solution. 
For a moving line source, the linear moving source solution is

$$
T=\frac{Q^{\prime}}{2 \pi k d} \exp \left(-\frac{v x}{2 \alpha}\right) K_{0}\left(\frac{v R}{2 \alpha}\right)
$$

where $\mathrm{d}$ is plate thickness and $\mathrm{K}_{0}$ is a modified Bessel function. With plate thickness incorporated into the source term, the problem becomes two-dimensional in $\mathrm{x}$ and $\mathrm{r}$. For small values of $\mathrm{R}$, expansion of the Bessel function and relation to the instantaneous source problem is complicated. As the argument of the Bessel function increases,

$$
K_{0}\left(\frac{v R}{2 \alpha}\right) \rightarrow \sqrt{\frac{\pi \alpha}{v R}} \exp \left(-\frac{v R}{2 \alpha}\right)
$$

$\mathrm{R}$ can be expanded as before to yield the instantaneous source solution plus a correction depending on $\mathrm{r} / \mathrm{x}$. An expression analogous to that used with point sources and thick plates can now be used which goes to the correct nonlinear instantaneous solution for small $\mathrm{r} / \mathrm{x}$, and goes to the correct linear moving solution for small q. Such an expression would be

$$
T=\frac{\left(Q^{\prime} / d\right)(p+1) B}{2 \sqrt{k \rho c v R}} \frac{1}{\left[1+\frac{q}{2(2-q)} \frac{v(x+R)}{\alpha}\right]^{1 / q(p+1)}}
$$

For distances such that the exponential approximation to the Bessel function is valid, this expression reduces to the linear solution for small q. For small $\mathrm{r} / \mathrm{x}$, it reduces to the nonlinear solution.

\section{APPLICATION TO REPORTED MEASUREMENTS}

Moore et. al. (Ref. 6) reviewed reports of conductivities used by various investigators in matching analytical solutions to experimental results. They noted that a variety of values between 20 and 41 $\mathrm{W} / \mathrm{m}^{\circ} \mathrm{C}$ had been used. They demonstrated that use of a value of $25 \mathrm{~W} / \mathrm{m}^{\circ} \mathrm{C}$ consistently gave the best predictions for $\mathrm{t}_{8 / 5}$. However, they noted that when this value was used, predictions of $\mathrm{t}_{100}$ were significantly in error. Kasuya and Yurioka used an empirical formula to select the thermal conductivity value for predicting $t_{8 / 5}$ for moderate heat inputs. While different values were obtained for different welding options, all were in the vicinity of $27 \mathrm{~W} / \mathrm{m}^{\circ} \mathrm{C}$. In addition, Kasuya and Yurioka used a value of $54 \mathrm{~W} / \mathrm{m}^{\circ} \mathrm{C}$ to predict $\mathrm{t}_{100}$. Kasuya and Yurioka stated that the linear solution should be used with high temperature properties to predict $\mathrm{t}_{8 / 5}$, and with low temperature properties to predict $t_{100}$. Moore et. al. commented that the value of $25 \mathrm{~W} / \mathrm{m}^{\circ} \mathrm{C}$ that they recommended was unrealistically low. It may be observed from Fig. 1 that thermal conductivity varies between 28 and $41 \mathrm{~W} / \mathrm{m}^{\circ} \mathrm{C}$ in the temperature range of $800^{\circ} \mathrm{C}$ to $500^{\circ} \mathrm{C}$. Moore et.al. noted that there are other factors (surface heat transfer, phase transformations, etc.) that are treated approximately or neglected in the analytical solution, and that the low conductivity at least in part represents compensation for 
these factors. The recommendations of Moore et. al. and of Kasuya and Yurioka, and the predictions with the nonlinear analysis are summarized in Table 1.

\begin{tabular}{|l|l|l|}
\hline & $\mathrm{t}_{8 / 5}$ & $\mathrm{t}_{100}$ \\
\hline Moore, et. al., Ref. 6 & 25 & \\
\hline Kasuya and Yurioka, Ref. 2 & $26.6-27.3$ & 54 \\
\hline Nonlinear Analysis, $\mathrm{k}_{\mathrm{c}}$ & 25 & 59 \\
\hline Nonlinear Analysis, $\mathrm{k}_{\mathrm{m}}$ & 21 & 59 \\
\hline
\end{tabular}

Table 1. Thermal Conductivities $\left(\mathrm{W} / \mathrm{m}^{\circ} \mathrm{C}\right)$ for Predicting Cooling Times in Linear Models

For the nonlinear analysis, power fits were made to thermal conductivity and heat capacity data from Ref. 11. These fits are shown in Figs. 1 and 2. They illustrate that power fits can represent data over significant temperature ranges, but tend to become unrealistic at zero ambient temperature. This is because fits for properties that decline with temperature, like thermal conductivity in this case, must go to infinity when the temperature goes to zero, and fits for properties that increase with temperature, like heat capacity, must go to zero. Since the power fits do match actual data over significant ranges, the trends predicted by the nonlinear analysis should provide useful guidance.

Noting the relationship between linear and nonlinear solutions, we select thermal conductivity according to

$$
k=k_{e f f}(T) /(p+1)
$$

where $k_{\text {eff }}(T)$ is the actual value $k$ from the power fit for cooling to a particular temperature (as for $t_{100}$ ) and is an effective value to use for an interval for cooling between two temperatures (as for $t_{8 / 5}$ ). The effective value is used because specifics in modeling and power fitting can lead to undue sensitivity to small differences of large numbers in applying Eq. 16 to an interval.

It may be observed from the results of Table 1 that the nonlinear analysis provides general agreement with the results recommended empirically by Moore et. al. and Kasuya and Yurioka. The factor $(p+1)$ serves to reduce the apparent thermal conductivity, and is consistent with the observation of Moore et. al. that the best value of $\mathrm{k}$ is unrealistically low. This factor, associated with the nonlinearity of heat capacity, arises from application of the boundary condition that the total energy (obtained by integrating the product of heat capacity and temperature) in the problem is a constant determined by the amplitude of the heat source. 
To evaluate $\mathrm{t}_{8 / 5}$, two options are considered in Table 1 for $\mathrm{k}_{\text {eff }}$. One is the use of $\mathrm{k}_{\mathrm{m}}=35 \mathrm{~W} / \mathrm{m}^{\circ} \mathrm{C}$, the mean value of $\mathrm{k}$ in the interval. The second is to use $k_{\mathrm{c}}=41 \mathrm{~W} / \mathrm{m}^{\circ} \mathrm{C}$, the value of $\mathrm{k}$ at the coldest temperature in the interval, $500^{\circ} \mathrm{C}$. This value is a mean value used by several investigators in the past. The second option yields the value of $25 \mathrm{~W} / \mathrm{m}^{\circ} \mathrm{C}$ recommended by Moore et.al. and is close to values obtained by Kasuya and Yurioka.

At low temperature, the power fit leads to a departure from realistic values of thermal conductivity. Consequently, there may be concern with range of validity of the analysis. However, the $(p+1)$ factor reduces that high value from the fit to one essentially equal to the actual conductivity and close to the value recommended by Kasuya and Yurioka.

\section{SUMMARY AND CONCLUSIONS}

Nonlinear analysis has been applied to weld thermal history. The analysis predicts the thermal conductivities adjusted empirically in the literature to predict important cooling times. Analysis has been based on instantaneous or rapidly moving heat sources, conditions suitable for most welding situations. Expressions also have been introduced to account for explicit effects of source motion.

\section{REFERENCES}

1. Radaj, D. (1992), Heat Effects of Welding, Springer-Verlag, New York

2. Kasuya, D. and Yurioka, N. (1993), "Prediction of Welding Thermal History by a Comprehensive Solution," Welding Journal 72 (3), 107s-115s

3. Rosenthal, D. (1941), "Mathematical Theory of Heat Distribution During Welding and Cutting,"Welding Journal, 20 (5), 220s-234s

4. Rykalin, N. N. (1957), Berechnung der Warmevorgange beim Schweissen, VEB Verlag Technik, Berlin

5. Grosh, R. J., and Trabant, E. A.(1956), "Arc Welding Temperatures," Welding Journal $35(8), 396-401$

6. Moore, J. E., Bibby, M. J., Goldak, J. A. and Santyr, S. (1986), "A Comparison of the Point Source and Finite Element Schemes for Computing Weld Cooling," in E. F. Nippes and D. J. Ball (ed) Welding Research: the State of the Art, ASM, Miami, pp1-9

7. Svensson, L. (1994), Control of Microstructures and Properties in Steel Arc Welds, CRC Press, Boca Raton, FL

8. Becker, M. (1997), "Nonlinear Transient Heat Conduction with Application to Welding," Proc. 1997 National Heat Transfer Conference, in press 
9. Zeldovich, Ya. and Kompaneets, A. S. (1950), "On the Theory of Propagation of Heat with Temperature-Dependent Thermal Conductivity," Izdat. Akad. Nauk SSSR, 61-71

10. Suarez, J. C. Molleda, F. and Gomez de Salazar, J. M. (1992), "Modeling of Grain Growth During Arc Welding of High Strength Low Alloy Steels," Materials Characterization 28, 3-13

11. Harvey, P. (1982), Engineering Properties of Steel, ASTM, Metals Park, Ohio 
Figure 1. Thermal Conductivity (Power Fit for Nonlinear Analysis).

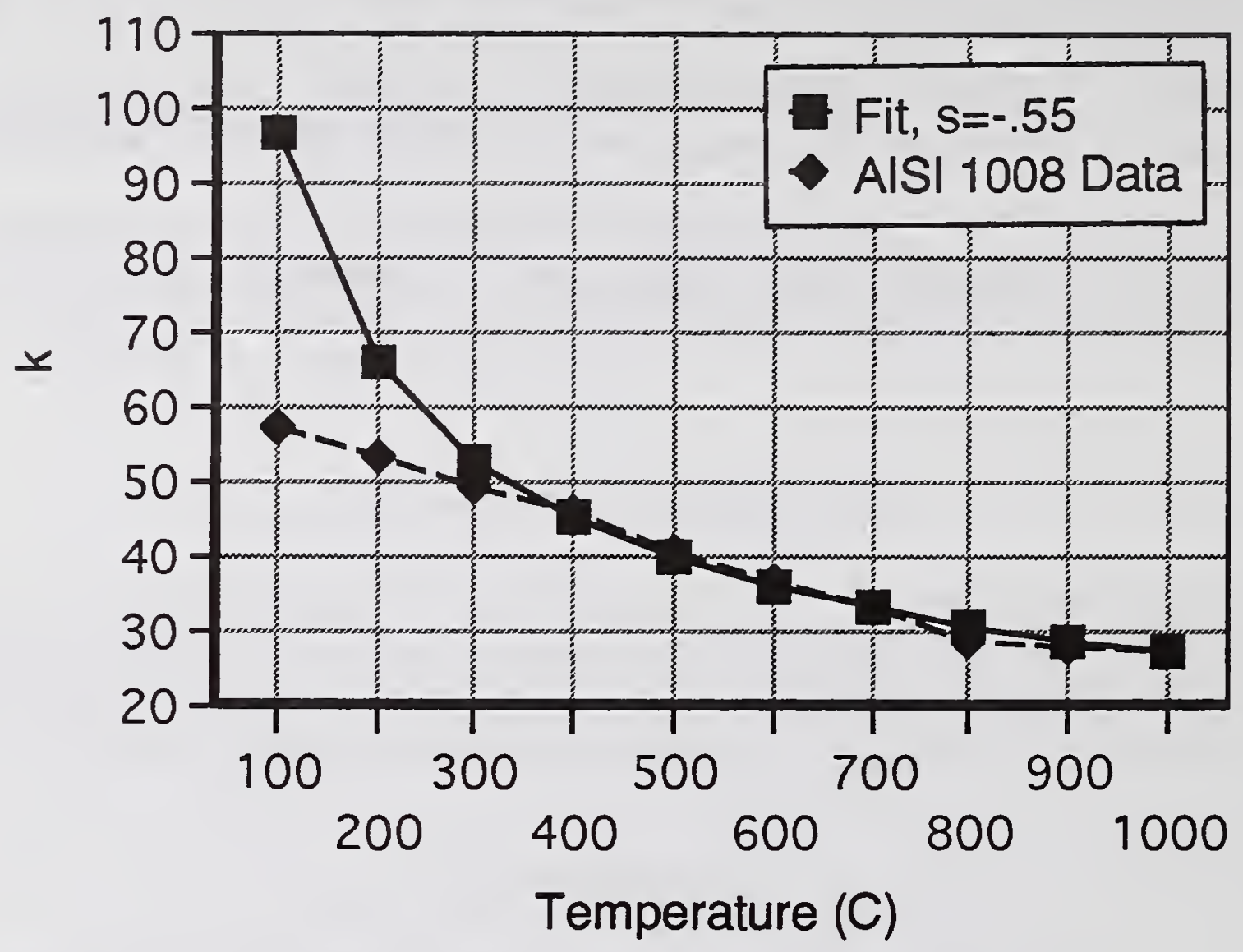

Figure 2. Specific heat (Power Fit for Nonlinear Analysis).

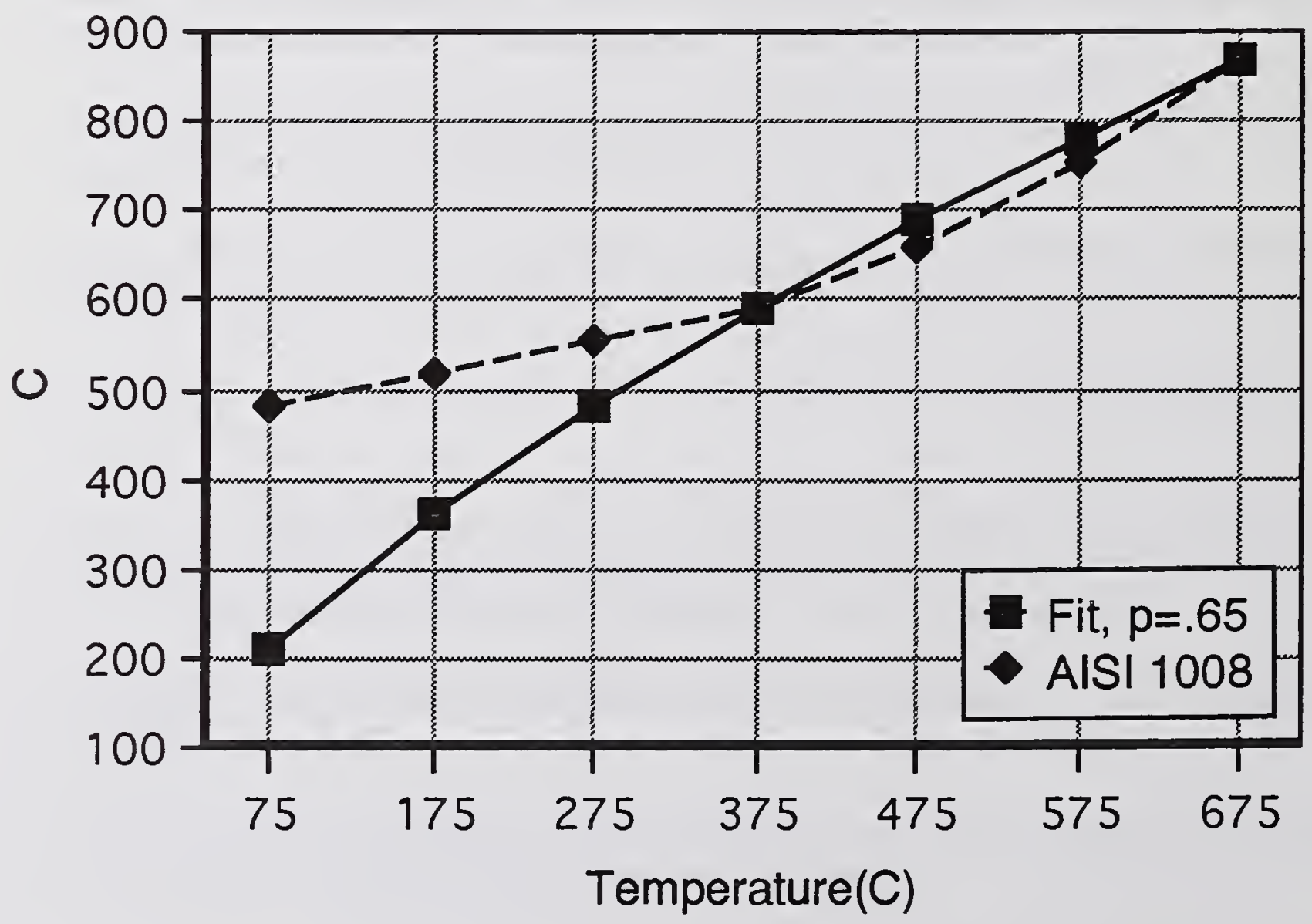




\title{
COMPUTATIONALLY EFFICIENT WELD ANALYSIS
}

\author{
L. Zhang ${ }^{1}$, X. Chen ${ }^{1}$, M. Becker ${ }^{1}$, L. Meekisho ${ }^{1}$, D. Atteridge ${ }^{1}$ \\ Oregon Graduate Institute of Science and Technology \\ Department of Materials Science and Engineering \\ P.O. Box 91000 \\ Portland, Oregon 97291-1000 \\ USA
}

(503)690-1313,(503)690-1432, mbecker@admin.ogi.edu

\begin{abstract}
Two procedures are used to reduce the time and storage associated with analyzing weld thermal histories. One is to utilize Eulerian moving coordinates in finite element analysis. It is shown that this procedure is actually more accurate than standard Lagrangian fixed coordinate analysis, and yields reductions in run time and in solution file data storage by a factor of 100 . The second, oriented to electroslag weld problems, is to incorporate three-dimensional effects into two-dimensional calculations.
\end{abstract}

\section{INTRODUCTION}

Analysis of weld thermal cycles can be very time-consuming. Realistic, three-dimensional time dependent heat conduction problems can take hours on modern work stations. Even when analysts are willing to spend the computer time needed, data storage requirements may be such that the problem cannot be handled by the computer. Welding has frequently been described as a moving source problem, and theoretical descriptions have been developed (Ref. 1) in moving coordinates (traveling in the direction and at the speed of the source). This approach is applicable to large plates without concern for starting and ending conditions. While analytical approaches have been applied in moving coordinates, numerical and computational approaches have been primarily in fixed Lagrangian coordinates. This may be because typical commercial computer codes are oriented to use in Lagrangian coordinate frames. There has been limited recent attention to Eulerian coordinates (Refs. 2,3), which demonstrate the possibility of substantial potential gain. However, very limiting assumptions were made - properties had to be independent of temperature and independent of position or region. A more extensive discussion of the literature is given in Ref. 4. This paper will demonstrate use of Eulerian coordinates with a commonly available commercial code (Ref.5), and will demonstrate major gains in running time and in data storage requirements.

Electroslag welding (ESW) presents unusual features that can make problems more difficult, but also that can motivate techniques for improving computational efficiency. ESW uses a slow-moving high-input heat source affecting a relatively large region as it passes. This provides incentive

\footnotetext{
${ }^{1}$ Oregon Graduate Institute of Science and Technology
} 
to economize by reducing the dimensionality of the problem. A simple procedure used to augment center plane calculations so as to estimate temperatures between the center plane and surface of the base plate.

\section{MOVING EULERIAN COORDINATES}

The heat conduction equation in moving Eulerian coordinates, for a source of magnitude Q moving at speed $\mathrm{v}$ through a material with conductivity $\mathrm{k}$, is

$$
\nabla \cdot k \nabla T+\rho c v \frac{\partial T}{\partial x}+Q=0
$$

In this moving coordinate frame, values of the $\mathrm{x}$-coordinate correspond to time. Positive values of $\mathrm{x}$ correspond to the heating portion of the problem, i.e. to times before the center of the moving source reaches a particular location. Negative values of $\mathrm{x}$ correspond to the cooling portion of the problem.

The ANSYS (Ref. 5) heat conduction equation, which allows for mass transport of heat, is

$$
\rho c\left(\partial T / \partial t+\{v\}^{T}\{L\} T\right)-\{L\}^{T}[K]\{L\} T=Q
$$

where $\{\mathrm{v}\}$ is the velocity vector, $\{\mathrm{L}\}$ is the gradient operator in matrix form, and $[\mathrm{K}]$ is the conductivity matrix. With appropriate selection of boundary conditions, the steady-state equation with mass transport becomes equivalent to the moving heat source problem.

Eulerian and Lagrangian formulations were compared on the basis of accuracy of results, running times and data storage. Cases considered are listed in Table 1, with results summarized in Table 2. It will be demonstrated below that the moving coordinate Eulerian formulation, for the same spatial detail, is more accurate than the fixed coordinate Lagrangian formulation. This is because the continuous motion of the source is treated exactly in the Eulerian coordinates, but has to be approximated by step wise discrete movements in the Lagrangian coordinates. Given this differential in accuracy, comparison of time consumption and data storage will be made for problems of comparable accuracy. These comparisons will show benefits of two orders of magnitude (factor of 100 ) in time consumption and in the result file portion of data storage.

\section{RESULTS - ACCURACY}

Fig. 1 shows results for a moving line source. Lagrangian results are displayed by converting the time variable to a position variable by $\mathrm{x}=\mathrm{vt}$. The temperature history is characteristic of welding thermal histories. This figure confirms that for the same spatial detail, the Eulerian result is more 
accurate than the Lagrangian result. Fig. 2 shows that the 50-interval Eulerian problem and the 80 interval Lagrangian problem are of comparable accuracy. These two problems will be used for comparisons of running time and data storage.

Fig. 3 shows results for a moving point source. The 60 -interval Eulerian problem and the 80 -interval Lagrangian problem are of comparable accuracy, and will be used for comparisons of running time and data storage. Also shown in Fig. 3 is an Eulerian result with 60 nonuniform intervals (i.e. putting more spatial detail where temperature varies more steeply, and vice versa). The nonuniform spatial intervals permits improving accuracy with essentially no cost. Because of the moving heat source, applying a nonuniform mesh is difficult because of need for detail in the vicinity of the source wherever the source may be. Ref. 3 recommends refining the mesh dynamically. That approach, while providing potential gains, is complicated. The option is not available in all codes, and typically is associated with in-house specialized programs.

\section{RESULTS - RUNNING TIME}

Figs. 4 and 5, as well as Table 2, show running times for the moving line and moving point source cases. The differences in running times are dramatic. For the comparable accuracy cases identified in the previous section (50-interval Eulaerian and 80-interval Lagrangian for the moving line source, 60 -interval Eulerian and 80 -interval Lagrangian for the moving point source), running time gains are approximately a factor of 100 .

Fig. 6 shows relative increase of running time as spatial detail is increased for the moving line source problem. This figure demonstrates that running time increases far more rapidly in fixed Lagrangian coordinates than in moving Eulerian coordinates.

\section{RESULTS - DATA STORAGE}

Figs. 7 and 8 show data storage requirements for the moving line and moving point source cases. It is interesting to observe that in the Eulerian problems, data storage requirements are comprised of two comparable files - the data base file and the results file. The data base file is actually the larger of the two. In the Lagrangian problems, the data base file is essentially the same as in the Eulerian problem. However, the result file is much larger than the data base file. For problems of comparable accuracy, the results files in the Lagrangian problems are about 100 times larger than the result files for the Eulerian problems.

Fig. 9 shows relative increase in data storage as spatial detail is increased. Analogous to running time, data storage requirements increase FAE more rapidly with spatial detail in fixed Lagrangian coordinates than in moving Eulerian coordinates. 


\section{ELECTROSLAG WELDING -REDUCED DIMENSIONALITY}

Electroslag welding has been modeled at OGI by finite element analysis. The heat source produces a large fusion zone. For calculational purposes, that zone provides a boundary condition of temperature equal to the melting temperature. The heat conduction problem is then solved for the temperature in the base plate. The weld area itself usually is covered with a moving shoe for shielding and containment. The shoe is cooled and provides strong surface cooling. At the base plate, surface heat transfer is by convection. Beyond the fusion zone in the plate, assume that the temperature is given by

$$
T(x, y, z, t)=\theta(x, y, t) f(z)=\theta(x, y, t) \cos B z
$$

Much of the interest for temperatures and stresses is in the plane, i.e in longitudinal (direction of heat source motion) and transverse (into the plate from the weld) directions. Eq. 3 provides for estimating temperatures between the central plane and the surface.

The heat conduction equation at the center of the plate $(\mathrm{z}=0)$ becomes

$$
\frac{\partial}{\partial x}\left(k \frac{\partial \theta}{\partial x}\right)+\frac{\partial}{\partial y}\left(k \frac{\partial \theta}{\partial y}\right)-B^{2} \theta=\rho c \frac{\partial \theta}{\partial t}
$$

This equation is of a standard form solved by commercial computer codes like ANSYS. With U as the overall boundary heat transfer coefficient, the surface boundary condition leads to a transcendental relation between $U$ and $B$ in a plate of thickness $D$ as follows

$$
\operatorname{Btan}(B D / 2)=\frac{U}{k_{s}}
$$

where the s-subscript denotes evaluation at the surface. For a thin plate, the relation reduces to

$$
B^{2}=\frac{2 U}{k_{s} D}
$$

\section{EVALUATION OF THE B-PARAMETER}

Several viewpoints are possible. One may assume that the overall surface coefficient $U$ is known and evaluate $\mathrm{B}$ from the transcendental equation. One may assume values of $\mathrm{B}$ and obtain results for a range of implied values for $U$. When experimental results are available, it may be possible to infer 
from examination of sections the shape of the temperature distribution in the thickness direction. Over the base plate, the heat transfer coefficient and plate thickness frequently are such as to make Eq. 6 valid

The authors have been concerned with assessment of existing structures containing electroslag welds. There consequently is some uncertainty as to the actual conditions prevailing when the welding was done. Plates cover a range of thicknesses. In addition, there may be uncertainties associated with heat transfer to the shoe. In the results to follow, representative surface heat transfer coefficients from Ref. 6 are used. Given the uncertainties, actual analysis calls for parametric variation.

\section{RESULTS}

The electroslag welding configuration is shown in Fig. 10. In addition to the two-dimensional central plane, two-dimensional cross-section (thickness $\mathrm{z}$ and transverse y directions) plane calculations were performed to provide another measure of the variation of temperature between center and surface. Fig. 11 shows the variation of surface temperature calculated by Eq. 4 and by the crosssectional plane finite element model. Surface temperatures are in good agreement in the base plate, with deviations occurring near the interface with the fusion zone, where the cooling shoe provides a step change in surface heat transfer. Table 3 shows these data and center temperature data. In general temperature variations with thickness are moderate. It thus is appropriate to perform twodimensional calculations and to use an estimating procedure for the third dimension.

\section{SUMMARY}

Two procedures for computational efficiency in weld analysis have been presented, each of which has the effect of reducing problem dimensionality. Use of moving coordinates was demonstrated to reduce running time and solution file data storage by two orders of magnitude. Except for analysis of edge effects (beginning and ending of arc motion), moving coordinates provides clear advantages over conventional fixed-coordinate finite element analysis. The approach is applicable to a variety of welding techniques. For electroslag welding, the B-parameter representation of variation with thickness provides an efficient procedure for reducing dimensionality. These two procedures were developed and tested simultaneously. There is potential for further gain in electroslag weld analysis by combining the two procedures.

\section{REFERENCES}

1. Rosenthal, D. (1941), "Mathematical Theory of Heat Distribution During Welding and Cutting,"Welding Journal, 20 (5), 220s-234s

2. Gu, M., J. A. Goldak and E. Hughes (1993), "Steady State Thermal Analysis of Welds with Filler Addition," Can. Metall. Q. 32, 49-55 
3. Goldak, J. A. and Gu, M. (1995), "Computational Weld Mechanics of the Steady State," in H. Cerjak (ed.), Mathematical Modeling of Weld Phenomena 2, Institute of Materials, London, UK

4. Chen, X. Becker, M., and Meekisho, L. (1997), "Finite Element Analysis in Moving Coordinates," Proc. 1997 Natl. Heat Trans. Conf., in press

5. Anon. (1997), ANSYS, Ansys, Inc., Houston, PA,

6. Debroy, T., Szekely,J., and Eagar, T. (1982), "Temperature Profiles, the Size of the Heat-affected Zone and Dilution in Electroslag Welding," Materials Sci. Eng. 56, 181-193 


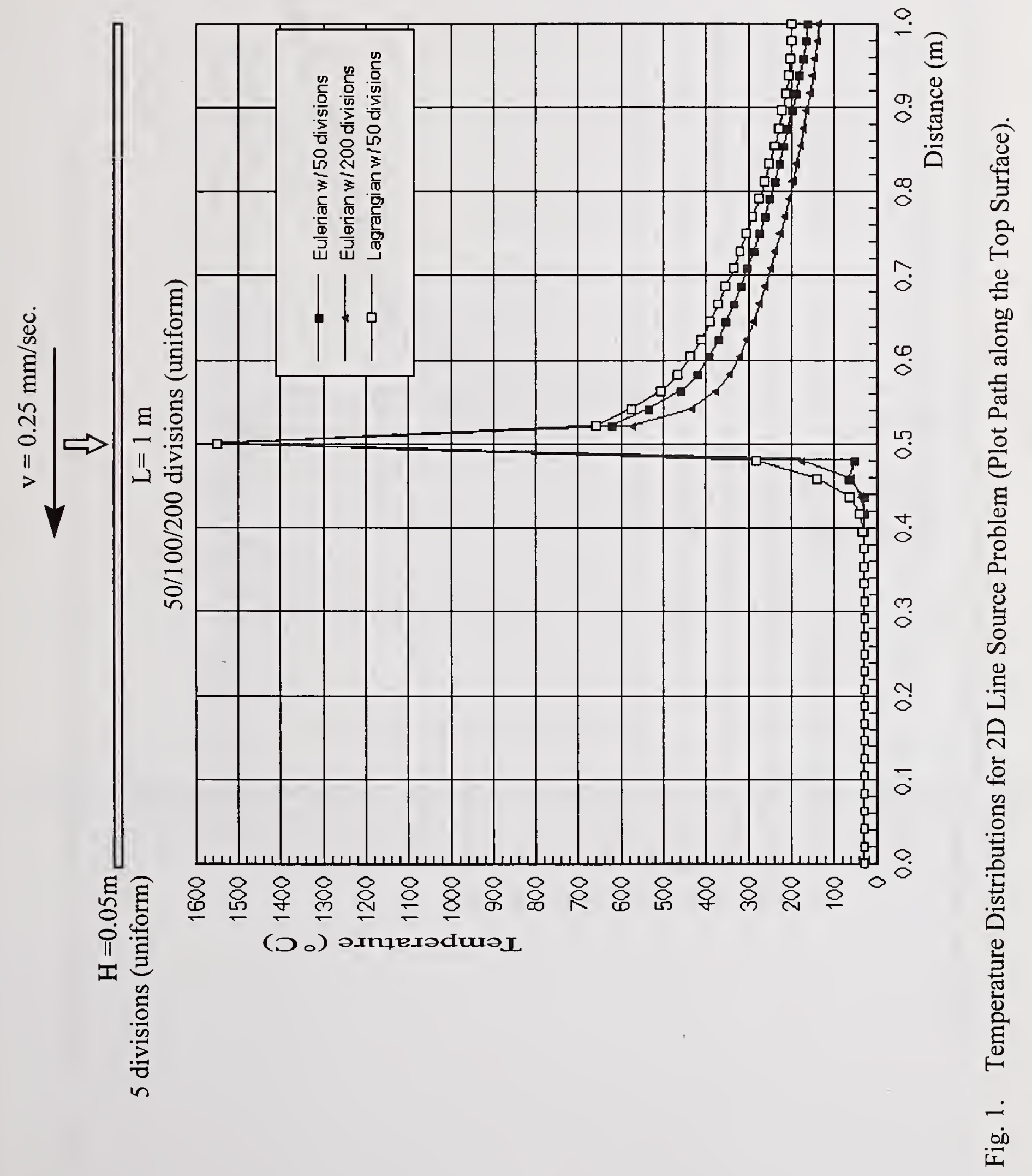




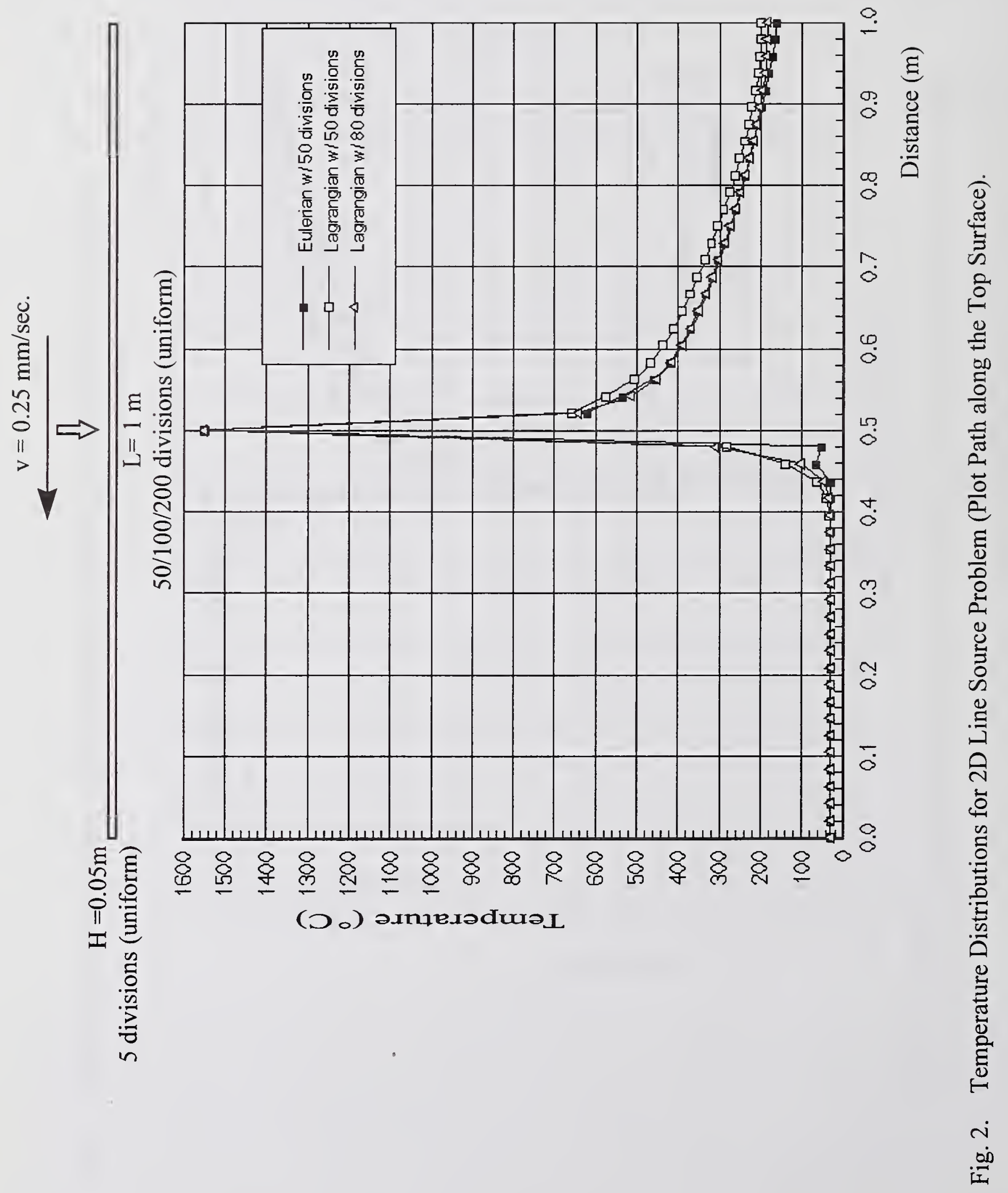




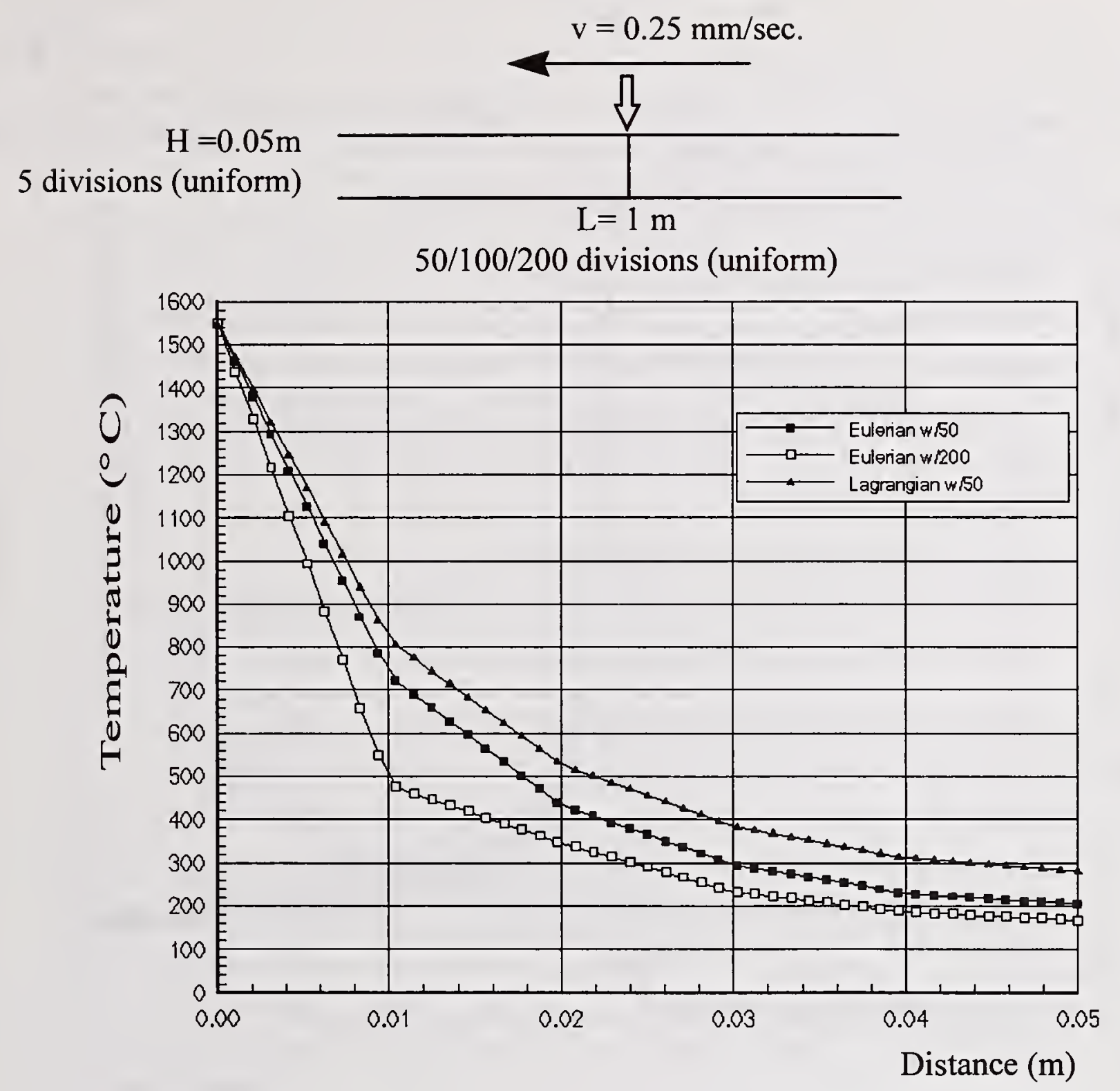

Fig. 3. Temperature Distributions for 2D Line Source Problem (Plot Path through the Depth as Indicated). 

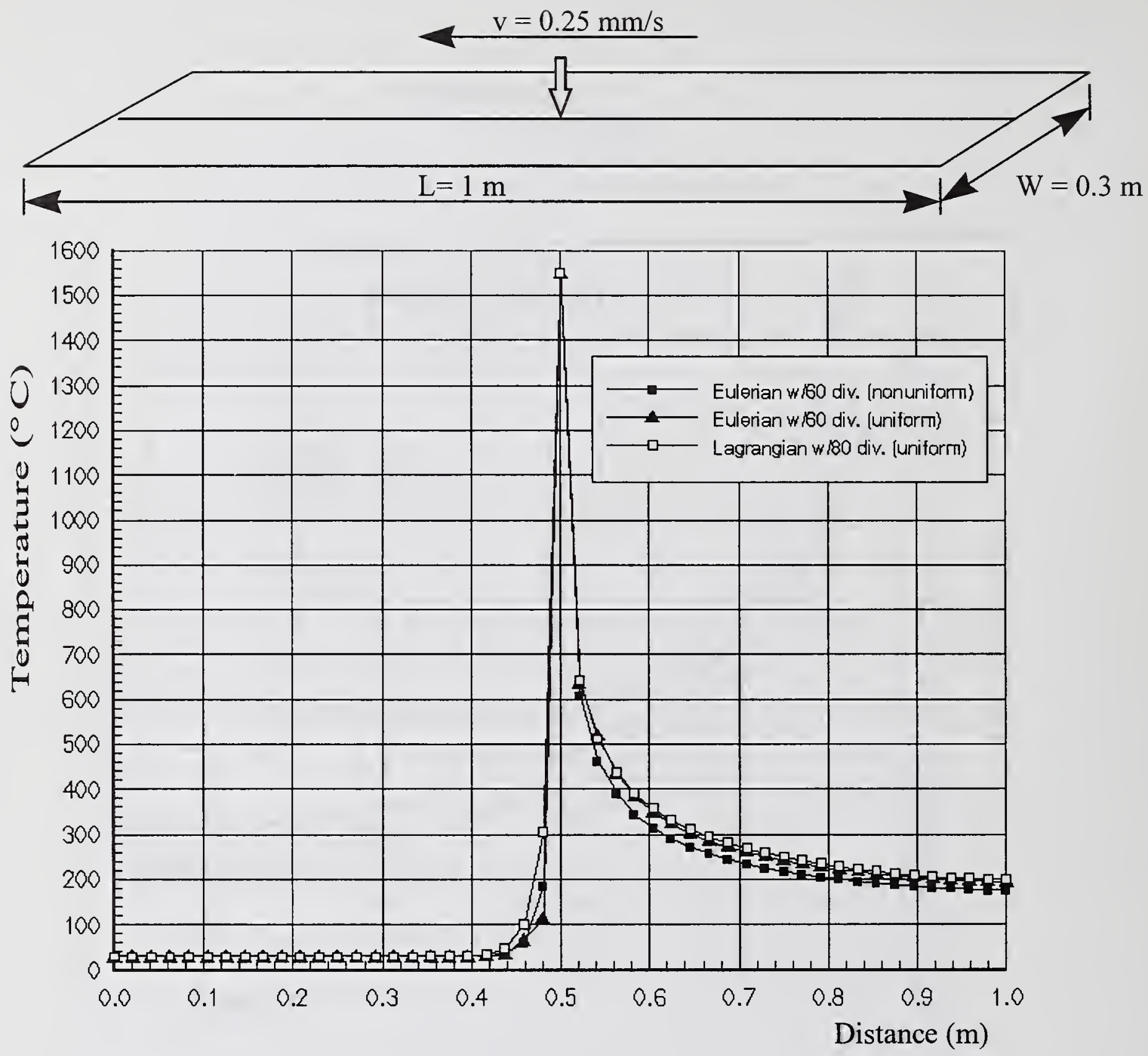

Fig. 4. Temperature Distributions for Point Source on Thin Plate Problem (Plot Path along the Centerline as Indicated). 

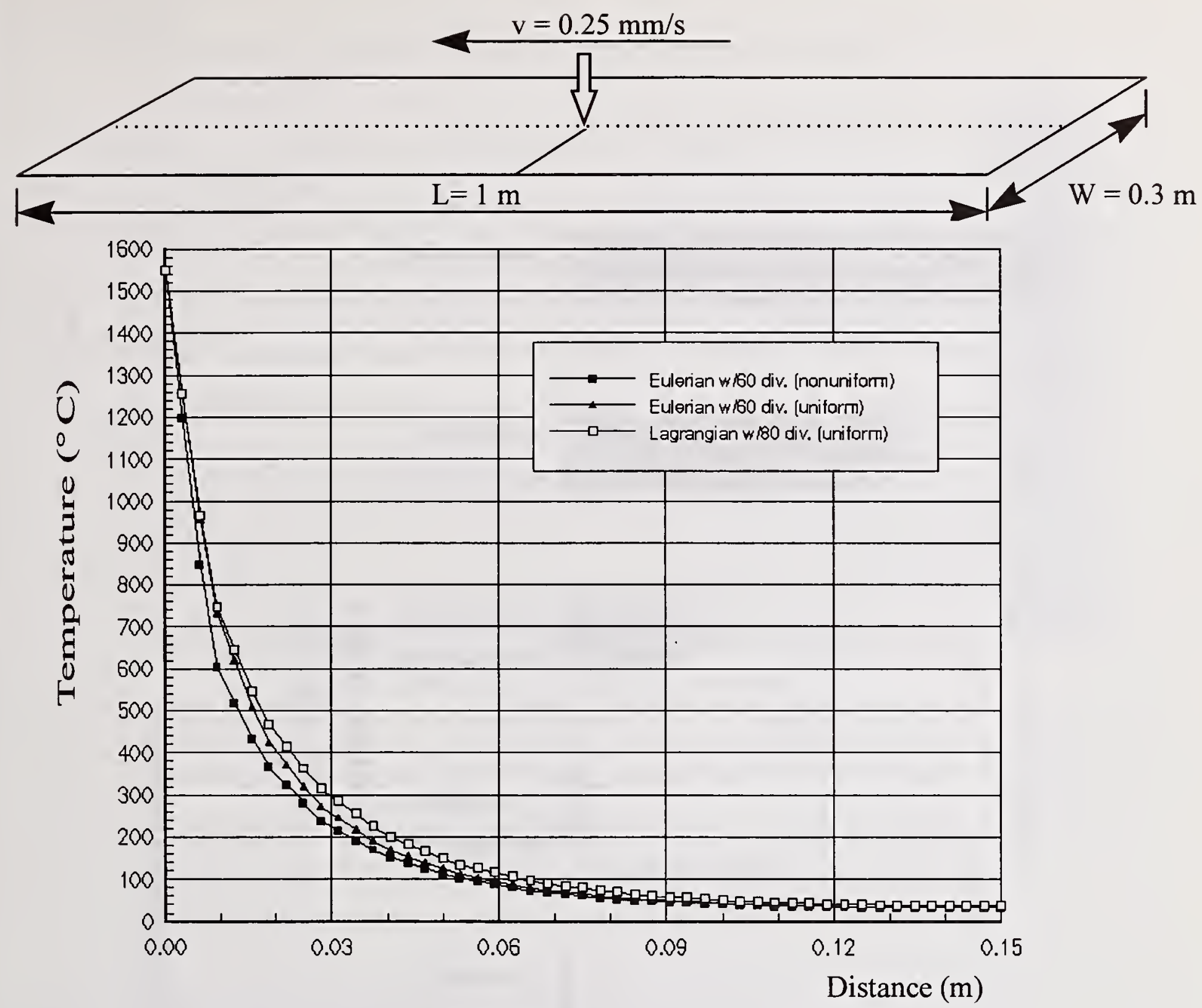

Fig. 5. Temperature Distributions for Point Source on Thin Plate Problem (Plot Path along the Line as Indicated). 


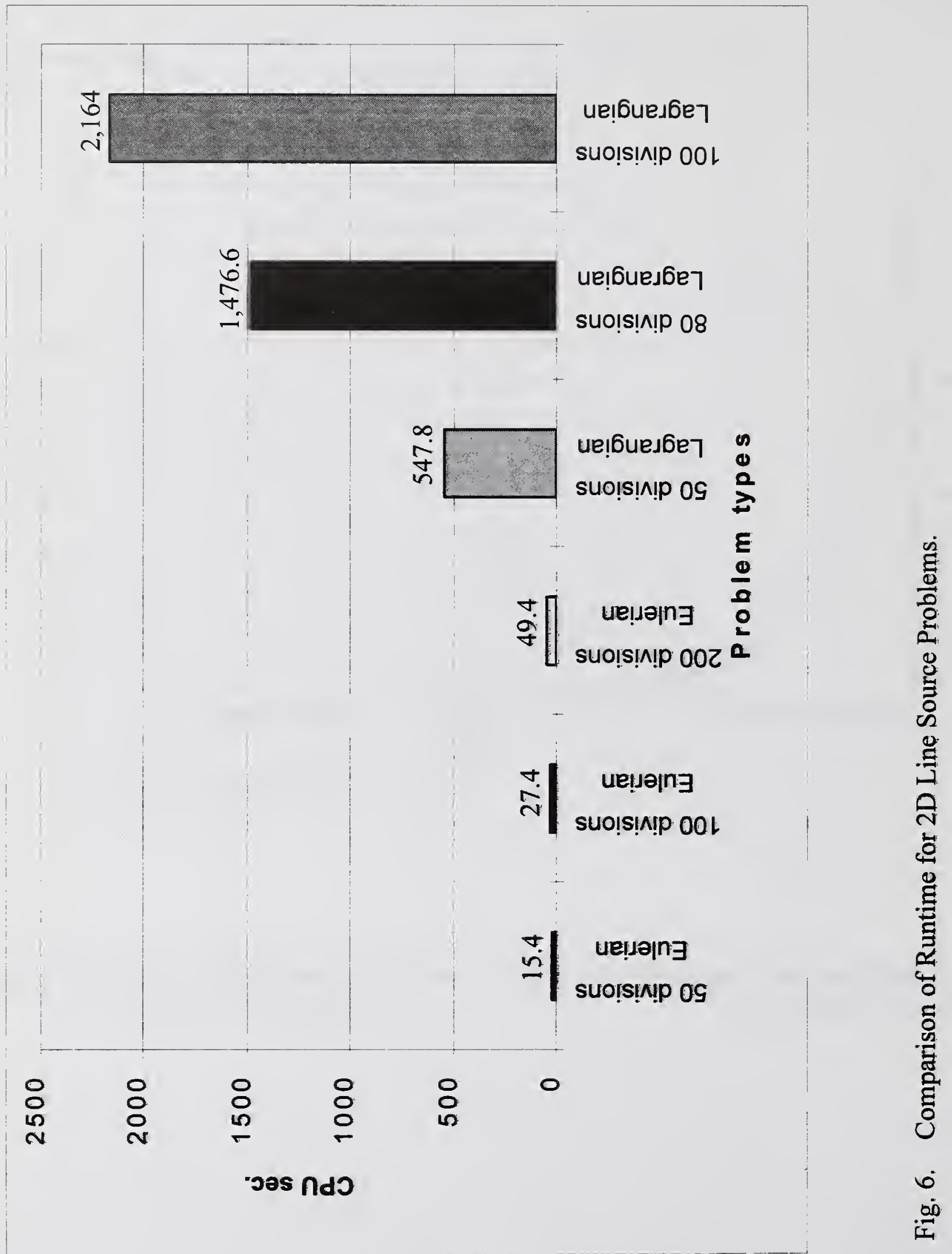




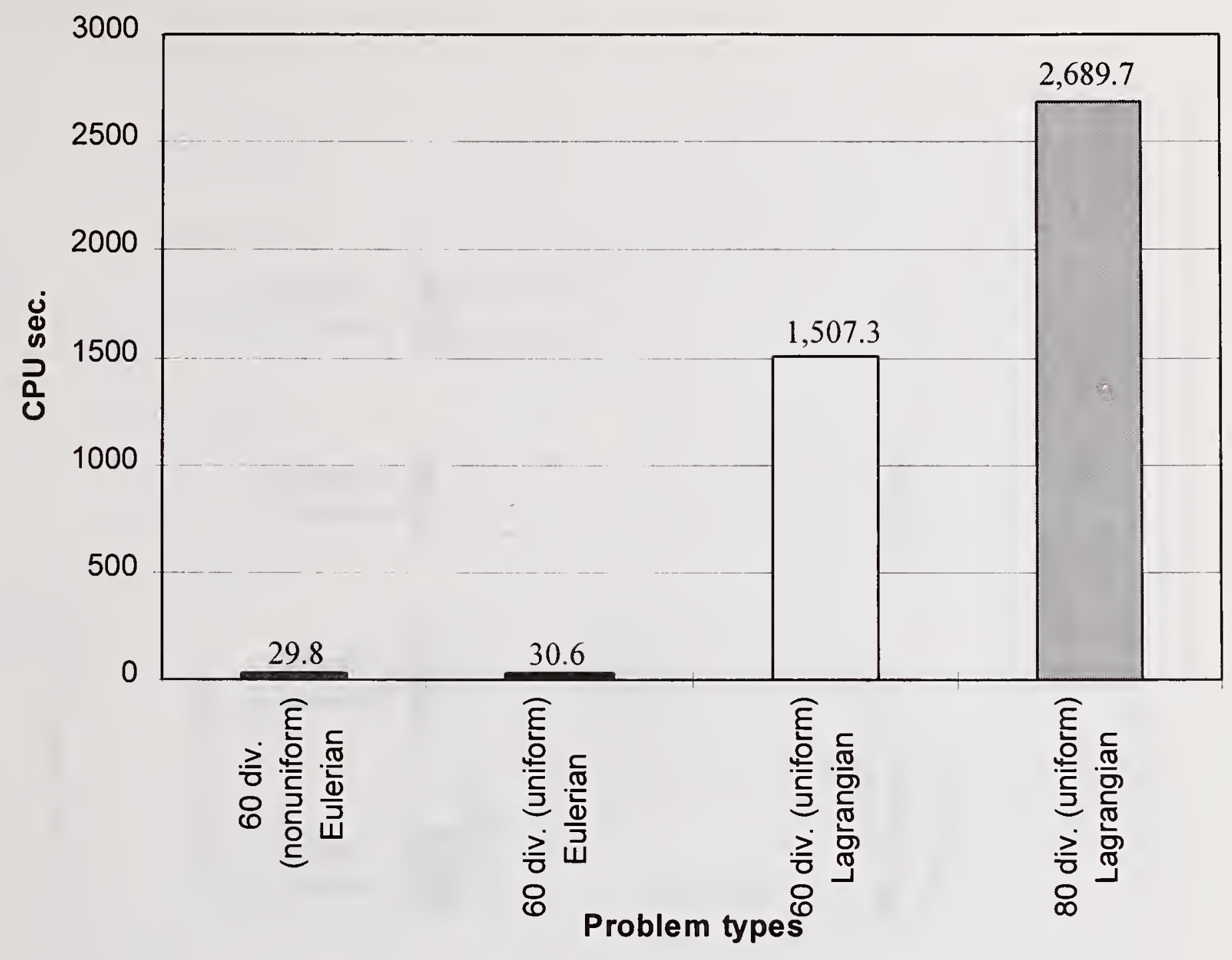

Fig. 7. Comparison of Runtime for 2D Point Source Problems. 


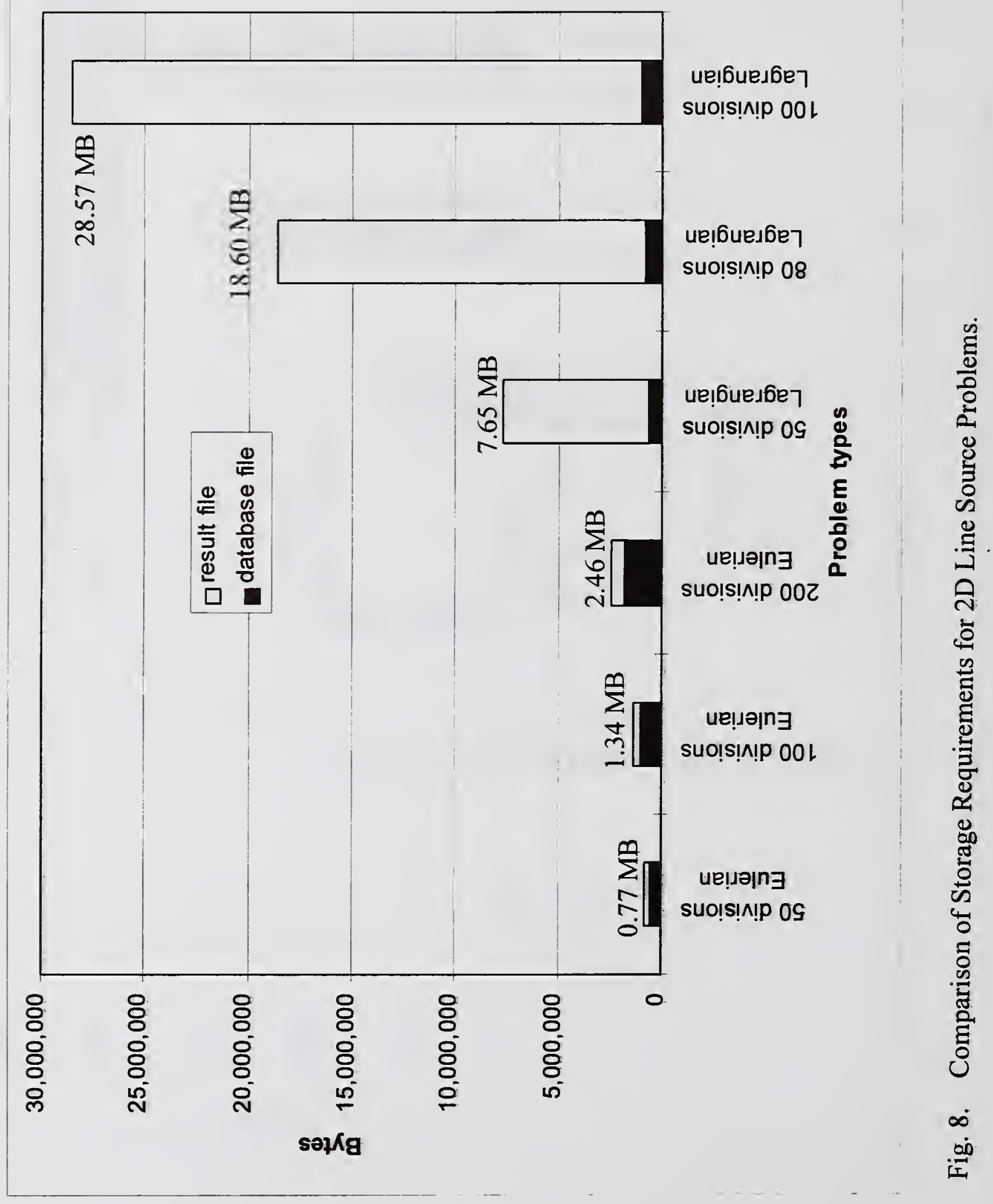




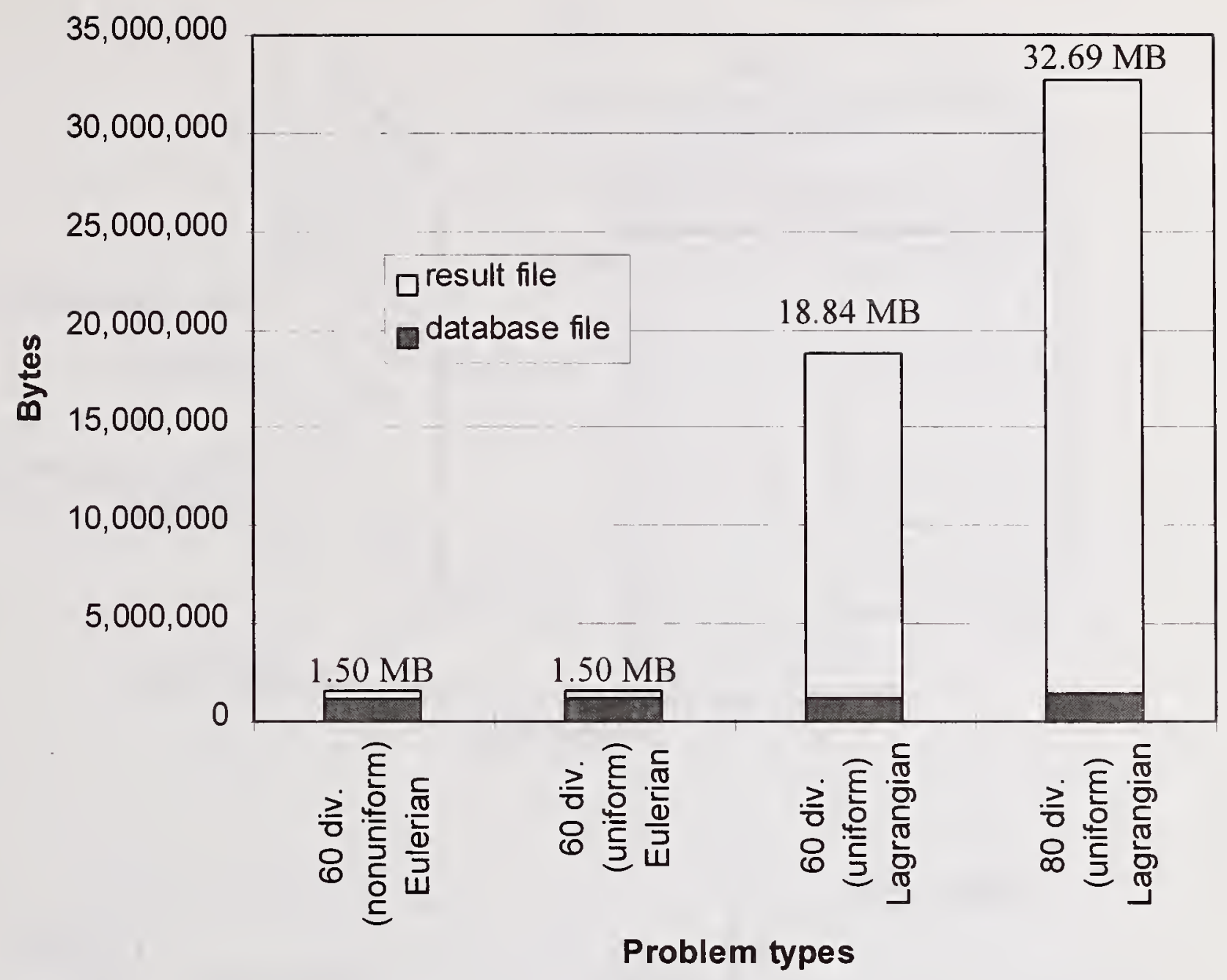

Fig. 9 Comparison of Storage Requirements for 2D Point Source Problems. 

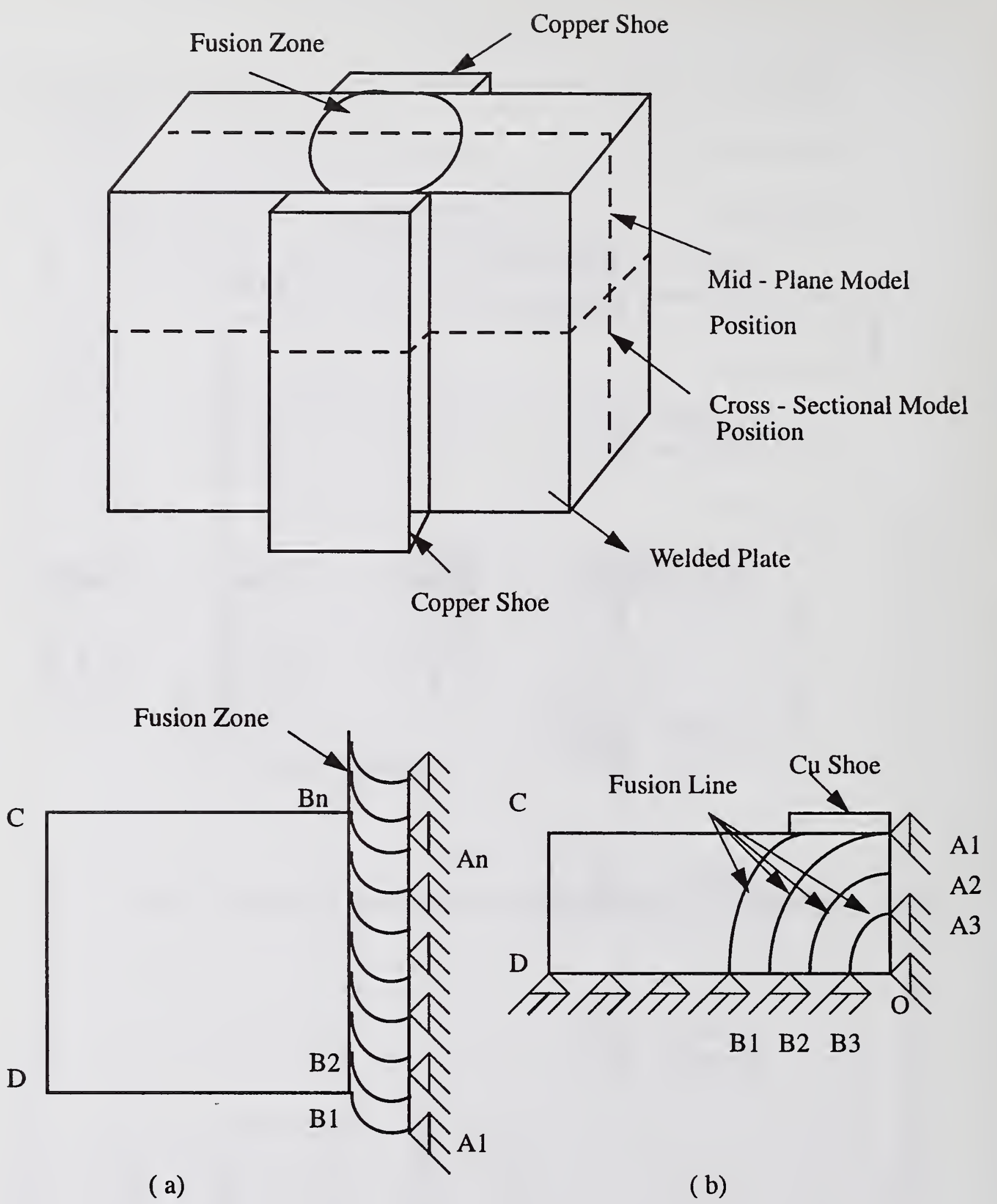

Fig. 10. Electroslag Weld Configuration (a) Mid-Plane, (b) Cross-Section. 


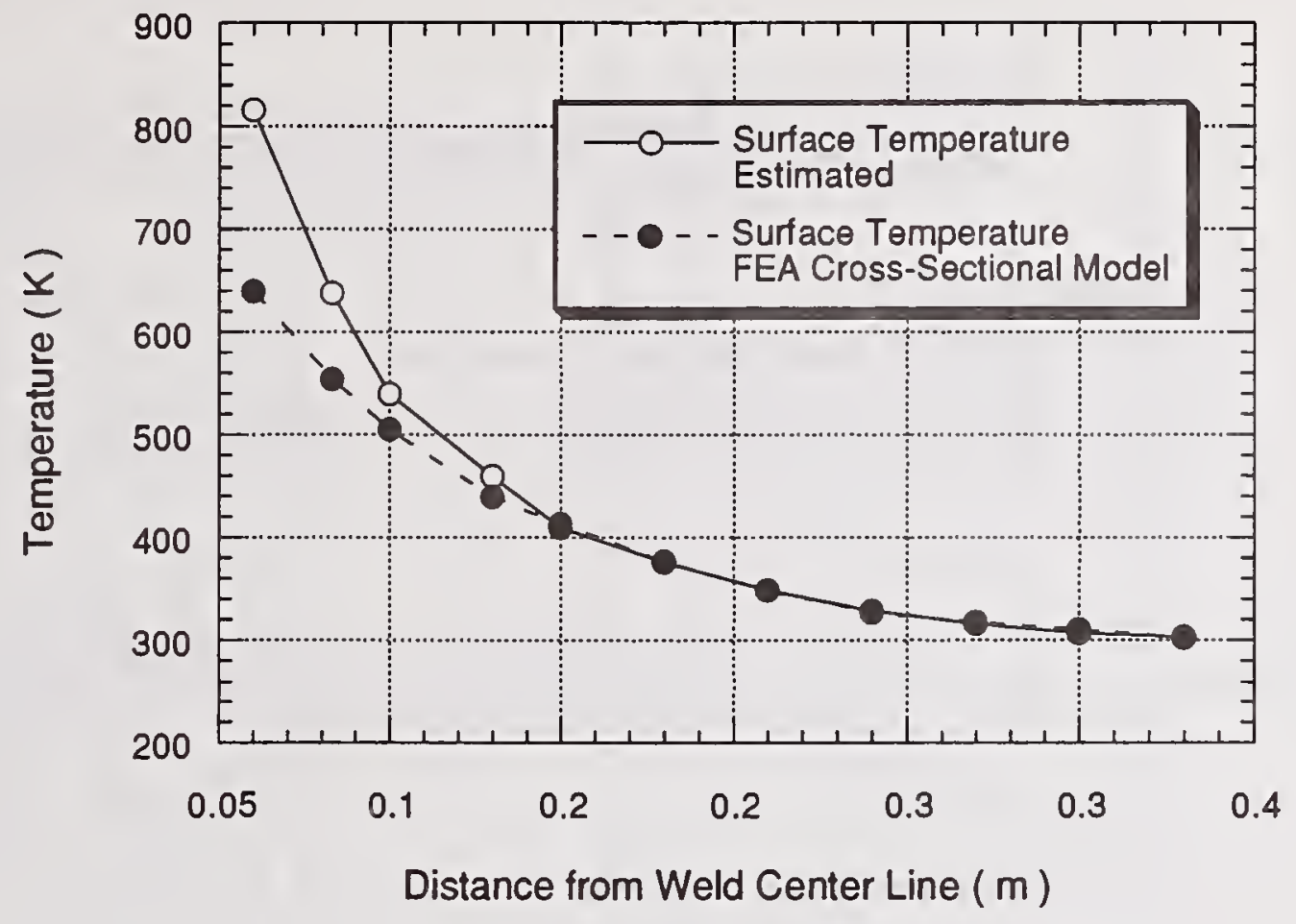

Fig. 11. Plate Surface Temperatures. 


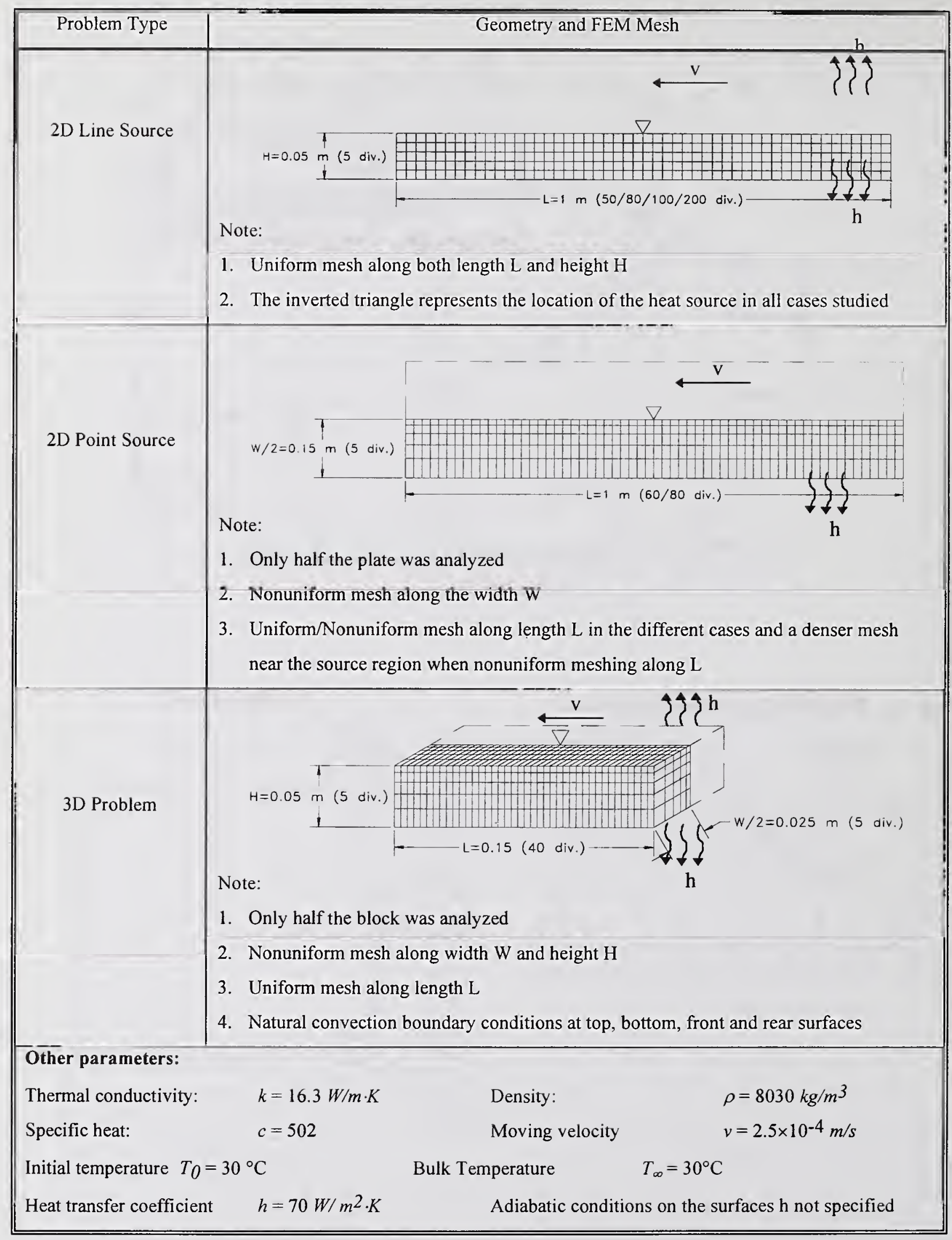

Table 1. Parameters for Difference Problem Types. 

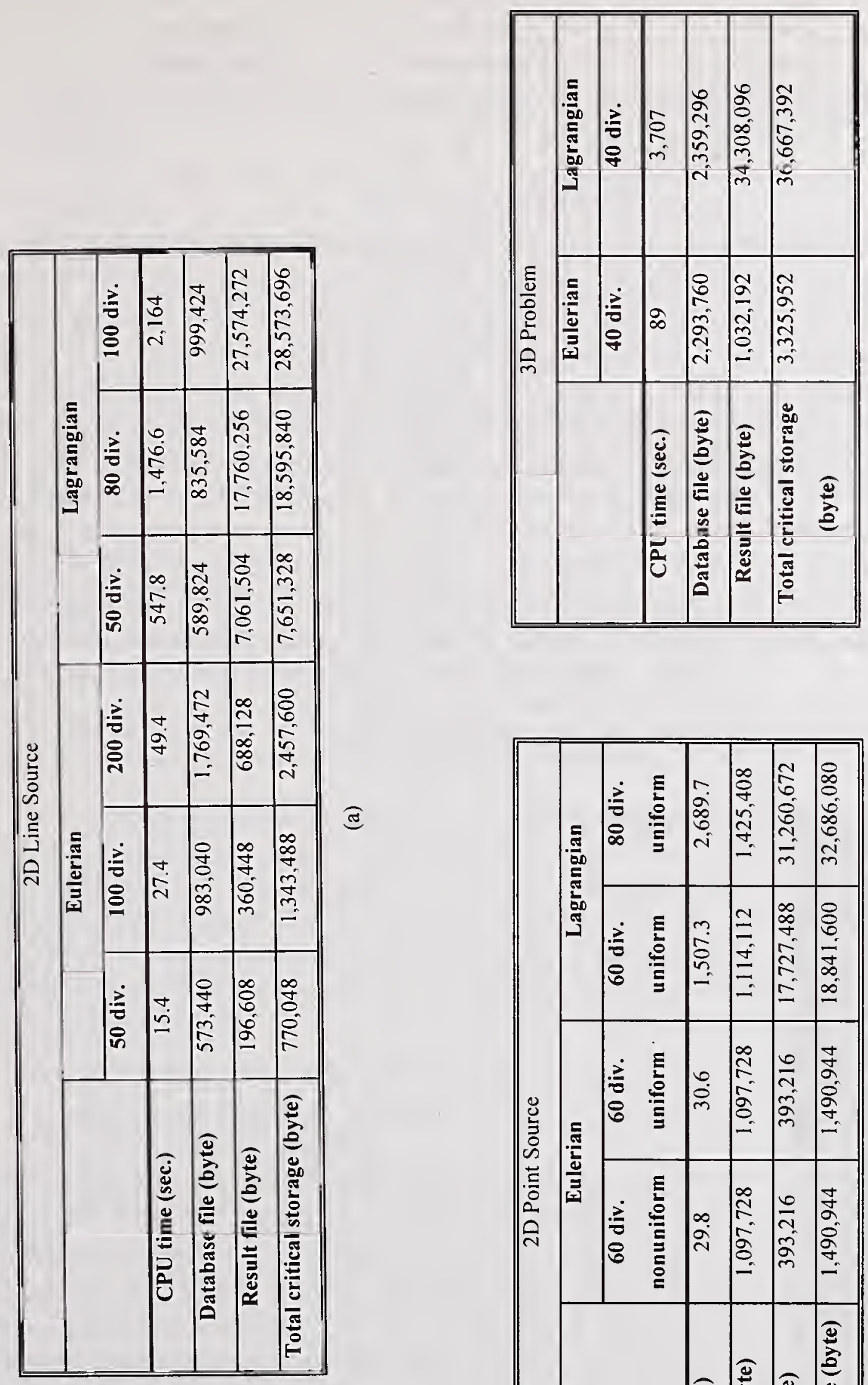

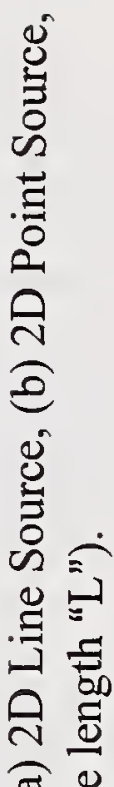

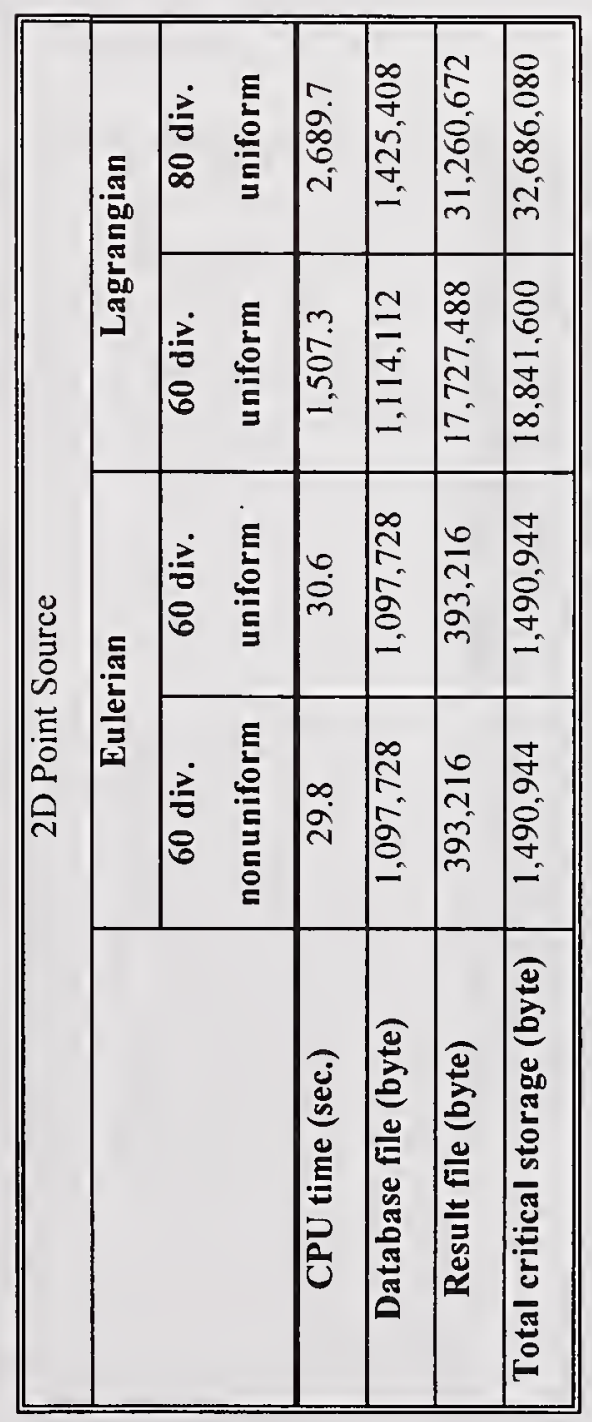

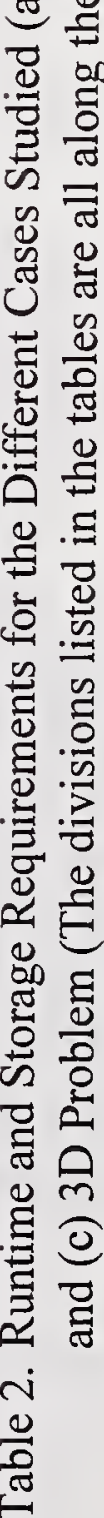




\begin{tabular}{|c|c|r|r|r|r|}
\hline & $\mathbf{X}$ & $\begin{array}{c}\text { Center } \\
\text { Temperature } \\
\text { (FEA Mid-Plan Model) }\end{array}$ & $\mathbf{B}$ & $\begin{array}{c}\text { Surface } \\
\text { Temperature } \\
\text { (Estimated) }\end{array}$ & $\begin{array}{c}\text { Surface } \\
\text { Temperature } \\
\text { (FEA Cross-Section Model) }\end{array}$ \\
\hline 0 & 0.060 & 847.00 & 6.8 & 815.00 & 640.00 \\
\hline 1 & 0.083 & 661.00 & 6.6 & 638.10 & 555.00 \\
\hline 2 & 0.100 & 558.00 & 6.4 & 539.82 & 506.00 \\
\hline 3 & 0.130 & 474.00 & 6.1 & 459.96 & 440.00 \\
\hline 4 & 0.150 & 422.00 & 5.9 & 410.30 & 414.00 \\
\hline 5 & 0.480 & 387.00 & 5.9 & 376.27 & 377.00 \\
\hline 6 & 0.210 & 359.00 & 5.9 & 349.38 & 349.00 \\
\hline 7 & 0.240 & 338.00 & 5.8 & 328.94 & 328.00 \\
\hline 8 & 0.270 & 325.00 & 5.8 & 316.59 & 318.00 \\
\hline 9 & 0.300 & 316.00 & 5.7 & 307.82 & 311.00 \\
\hline 10 & 0.330 & 312.00 & 5.7 & 303.93 & 303.00 \\
\hline
\end{tabular}

Table 3. Plate Surface and Center Temperatures. 


\title{
SOAR: AN EXTENSIBLE SUITE OF CODES FOR WELD ANALYSIS AND OPTIMAL WELD SCHEDULES
}

\author{
G. R. Eisler ${ }^{*}$, P. W. Fuerschbach ${ }^{\dagger}$
}

\begin{abstract}
Four MATLAB-based software applications called SOAR (Smartweld Optimization and Analysis Routines) have been developed to do weld analysis and provide optimal weld schedules for $\mathrm{CO}_{2}$ continuous wave laser welding, pulsed Nd:YAG laser welding, and for non-consumable arc welding processes. The optimization methodology consists of mixed genetic and gradient-based algorithms to query semi-empirical, nonlinear algebraic models. The optimization output provides heat-input-efficient welds for user-specified weld dimensions. User querying of all weld models is available to examine sub-optimal schedules. The inclusion of thermodynamic properties allows the extension of the empirical models to include materials other than those tested. In addition a heat conduction equation solver for 2-D heat flow is available to provide the user with an additional check of weld thermal effects. All solution methods are provided with graphical user interfaces and display pertinent results in two and three-dimensional form. The code architecture provides an extensible framework to add an arbitrary number of modules. The modules have been developed as integral parts of the Smartweld engineering system.
\end{abstract}

\section{INTRODUCTION}

It has been a goal of advanced manufacturing initiatives at Sandia to provide model-based applications to reduce the design cycle time. In pursuit of this objective, a set of MATLAB-based computer codes, SOAR (Smartweld Optimization and Analysis Routines) has been developed to aid the welding engineer. The contents cover solution methods to generate optimal weld schedules for:

- $\mathrm{CO}_{2}$ continuous wave laser welding (Fig. 1)

- Plasma or gas-tungsten arc welding

- Pulse Nd:YAG laser welding

For these applications, weld schedules represent the constant-value settings on the device for output power $\left(q_{o}\right)$ or energy delivered $(Q)$, part travel speed $(\mathrm{v})$, and in the laser-device cases the focusing lens size $(f)$. In addition a fourth application has been written to provide graphical solutions to a two-dimensional (2-D) steadystate, heat conduction equation. Results of the constant-value weld solutions provided by the "scheduling" applications can be entered into this application (dubbed $I S O$ ) to provide the engineer with a

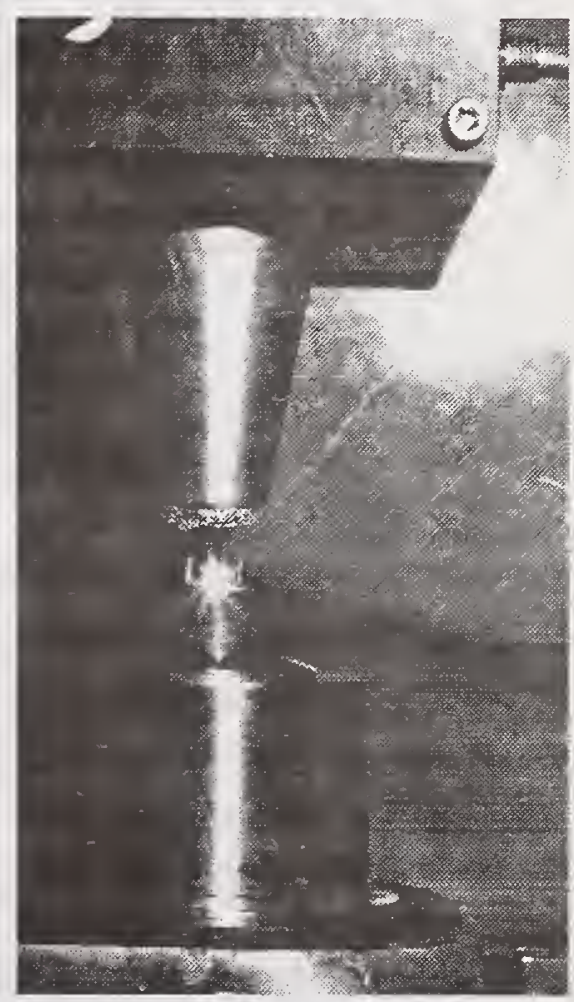
geometric check of weld thermal effects.

Figure $1 . \mathrm{CO}_{2}$ Laser weld in progress

*Structural Dynamics Dept., Sandia National Laboratories, Albuquerque, NM, USA, 87185-0439

$†$ Materials Joining Dept., Sandia National Laboratories, Albuquerque, NM, USA 87185-1134 
Since an important goal of Sandia's manufacturing initiatives is model-based design, it was felt that more software tools were necessary to make the specialized knowledge more accessible to the weld community. Smartweld is a concurrent engineering system developed at Sandia National Laboratories that integrates product design and processing decisions within an electronic desktop engineering environment. An application for $\mathrm{CO}_{2}$ laser welding (Ref. 1) was an initial component for this system. Based on models given in Ref. 2, additional weld schedule applications were able to be brought on-line. All have implemented the following approach:

1. Formulate a semi-empirical, input response model of the weld process. (A conceptual model is shown in Fig.2) These may consist of pure polynomial fits of experimental data, or may be parameterized extensions of relations developed in the literature to better fit experimental data. Model expansions to other than the experimentally-tested materials is accomplished via the embedding of thermodynamic properties within the model. The use of numerical scaling may be relied upon to achieve better data fits.

\section{Responses}

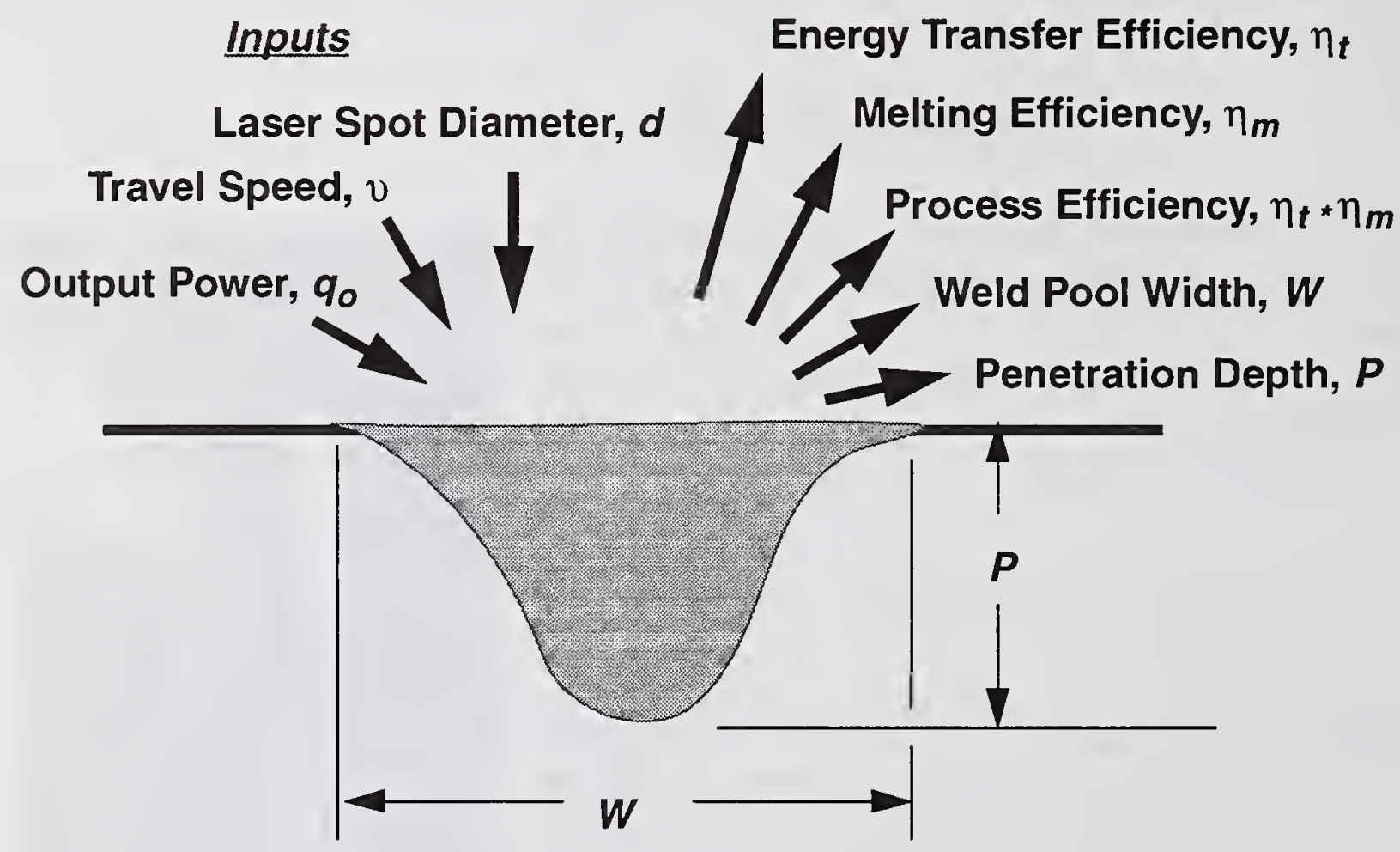

Figure 2. The Input-Response Model for $\mathrm{CO}_{2}$ Laser Welding

2. Use a combination of genetic and gradient-based optimization algorithms to invert the model by solving for the inputs to provide an "optimal" response. A surface of possible responses for a "grid" of inputs is shown in Fig.3. Typically, the optimal solution is one that maximizes an efficiency metric (i.e., Melting Efficiency, which can also be interpreted as minimizing heat input), while simultaneously yielding a weld of given depth/width dimensions. 
The use of a mix of optimization algorithms stems from the fact that genetic-based methods can survey a wide range of solution possibilities efficiently, and are thus better disposed towards finding the most promising region in the solution space from which a gradient-based scheme can converge quickly to the final optimal solution. Numerical scaling was used to aid the searching algorithms when various quantities were disparate in magnitude.

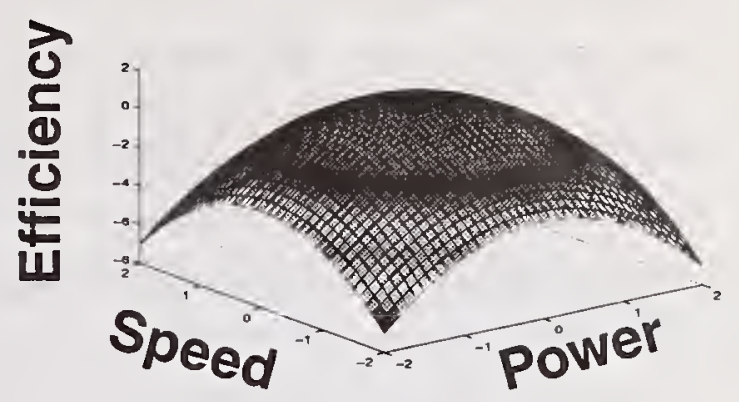

Figure 3. A Weld Response Surface

3. Provide 2-D and three-dimensional (3-D) presentations to allow the weld engineer to assess how this type of solution, which may not always be intuitive, fits in with qualitative metrics such as weld appearance, past experience, and equipment capability.

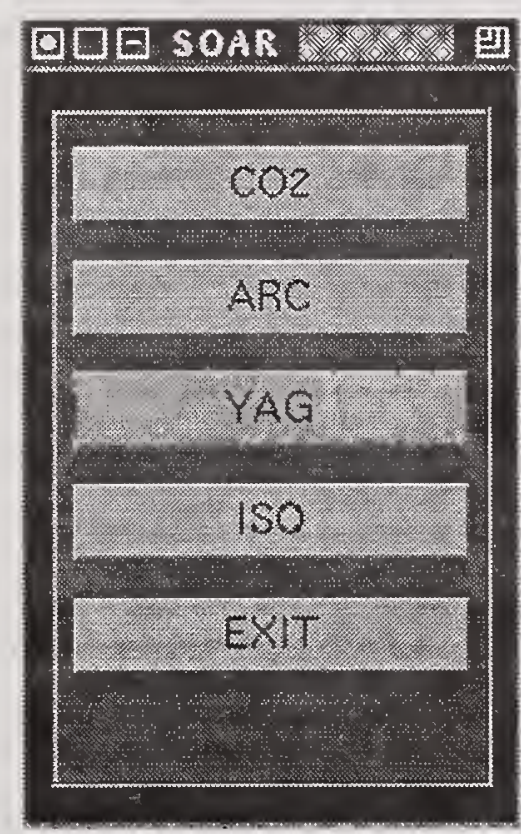

All applications were written in MATLAB (Ref. 3) which provides integrated "number-crunching", graphics, and graphical-user-interface (GUI) routines. The applications were separately launched from the SOAR "executive" control panel in Fig.4. The architecture of this panel allows the addition of an arbitrary number of analysis modules to cover future needs. The applications will be covered in the ensuing sections.

To access this panel:

\section{Enter MATLAB}

2.Type: soar (lower case) at the command prompt

Figure 4. The SOAR Executive Control Panel

\section{THE CO2 APPLICATION}

This application was the first developed and is explained in detail in Ref. 1. It will be summarized briefly here. For this study, the desired response characteristics are concerned with heat input efficiency on a given metal, while attaining a user-specified weld geometry. Weld schedules consist of constant values over a given weld for: 1) laser output power $\left(q_{o}\right)$ in watts, 2) part travel speed $(v)$ in millimeters $(\mathrm{mm}) / \mathrm{second}(\mathrm{sec})$, and 3 ) laser focusing lens focal length characterized by spot diameter $(d)$ in centimeters $(\mathrm{cm})$.

In the context of laser welding, $q_{o}$ and $v$ can be considered to vary continuously over a given range. However, spot diameter, $d$, corresponds to only a select few discrete lens focal lengths. It was decided to model four output responses or process variables as functions of the weld schedule (WS) parameters, $q_{o}, v$, and $d$. These quantities were: 
1. energy transfer efficiency $\left(\eta_{t}\right.$, dimensionless), defined as the ratio of net heat input to the part to the incident energy produced by the power source.

2. melting efficiency ( $\eta_{m}$, dimensionless), defined as the ratio of the amount of heat required to just melt the fusion zone to the net heat input deposited in the part. Slow travel speeds usually encountered in manual welding operations result in low melting efficiency.

3. top width of the weld $(W, \mathrm{~mm})$. Area of the weld is used for the thermodynamic calculation and a parabolic, weld shape approximation was used to map area to width.

4. weld penetration depth, $(P, \mathrm{~mm})$.

For $\mathrm{CO}_{2}$ laser welding, the weld schedule (WS) implies the triple, $q_{o}, v, d$. The method proposed for generating optimal weld schedules required a parameterized, algebraic model to relate the $W S$ to the responses, $\eta_{\mathrm{t}}, \eta_{m}, W$ and $P$. Experimental input-response data for 126 different welds for the 304 stainless steel, 1018 steel, and tin were fit with a nonlinear leastsquares algorithm. The parameters in the algebraic model were computed to minimize the sum of the squares of the errors (i.e., the least-squares fit) between experimental responses $\left(\eta_{t i}, P_{i}\right.$ for the $i$ th weld) and those from the model. Given $\eta_{\mathrm{t}}$ and $P$, then $\eta_{m}$ and $W$ were generated from known relations. Extensions to molybdenum, nickel, and titanium were done via the use of material thermal diffusivity, $\alpha$, and enthalpy of melting, $\delta h$, values embedded in the model. The final response model was given by the following:

$$
\begin{gathered}
P=\frac{c_{1} \alpha q_{o}}{v^{c_{2} d^{c_{3}}}} \quad \eta_{t}=c_{4}-c_{5} e^{\left[\frac{\pi}{2 \operatorname{atan}\left(c_{6} d / P\right)}\right]} \quad R y=\frac{q_{o} \eta_{t} v}{\alpha^{2} \delta h} \quad \eta_{m}=c_{7}+c_{8} e^{\left[\frac{-R y}{c_{10}}\right]}+c_{9} e^{\left[\frac{-R y}{c_{11}}\right]} \\
\text { Area }=\frac{R y \eta_{m} \alpha^{2}}{v^{2}} \quad \cdots \quad W=\frac{3 A r e a}{2 P} \text { (parabolic shape approximation) }
\end{gathered}
$$

Numerical constants, $c_{i}$, are those found via least-squares data fitting. $R y$ is the Rykalin parameter Ref. 4.

Given the parameterized model which provides the best "least-squares" fit to the experimental data, a genetic algorithm optimization method was used in consort with either a gradient-based optimization scheme or a nonlinear algebraic solver to find the WS to solve the following problems,

\section{Performance Metric Goal}

\begin{tabular}{lll}
\hline 1.Maximize $\eta_{\mathrm{t}}$ & $W_{\text {desired }}, P_{\text {desired }}$ & genetic w/nonlinear algebraic solver \\
2.Maximize $\eta_{\mathrm{t}}$ & $P_{\text {desired }}$ only & genetic $w /$ nonlinear optimization \\
3.Maximize $\eta_{m}$ & $W_{\text {desired }}, P_{\text {desired }}$ & genetic $w /$ nonlinear algebraic solver \\
4.Maximize $\eta_{m}$ & $P_{\text {desired }}$ only & genetic $w /$ nonlinear optimization \\
5.Maximize $\eta_{\mathrm{t}} * \eta_{m}$ & $W_{\text {desired }}, P_{\text {desired }}$ & genetic w/nonlinear algebraic solver \\
6.Maximize $\eta_{\mathrm{t}} * \eta_{m}$ & $P_{\text {desired }}$ only & genetic $w /$ nonlinear optimization \\
\hline \hline
\end{tabular}


or stated somewhat differently

$$
\begin{gathered}
\text { Maximize: } \eta_{\mathrm{t}}\left(q_{o}, v, d\right) \text {, or } \eta_{m}\left(q_{o}, v, d\right) \text {, or } \eta_{\mathrm{t}}{ }^{*} \eta_{m}\left(q_{o}, v, d\right) \\
\text { Subject to: } W_{\text {desired }}-W\left(q_{o}, v, d\right)=0 \text { and/or } P_{\text {desired }}-P\left(q_{o}, v, d\right)=0
\end{gathered}
$$

where the quantities followed by $\left(q_{o}, v, d\right)$ imply model responses resulting from the "constant" WS parameters. The optimization space is discontinuous due to the discrete variable, $d$, and would appear as separate surfaces in a vertical stack (one of which is shown in Fig.3). The largest lens $(f=$ $7.5 ", d=.0294 \mathrm{~cm}$ ) produces the lowest efficiencies and would be at the bottom of the stack. If $d$ had been continuous, this "space" would have appeared "solid". The gist of our optimization effort is to "jump" to the highest surface (which corresponds to the lowest value of $d$ ), which will simultaneously yield a bounded $W S$ solution to satisfy the ( $P_{\text {desired }}$ and $\left.W_{\text {desired }}, P_{\text {desired }}\right)$ constraints.

In the genetic algorithm (GA), the $W_{\text {desired }}, P_{\text {desired }}$ constraints were attached as a quadratic penalty function onto the performance metric to form a composite metric. The GA treats all values of $q_{o}$ $, v, d$ as discrete, makes up various combinations of them (members of the population), and evaluates the composite metric according to the response model. It then chooses the highest value after a designated number of population or "generation" changes. In Ref. 1 the entire problem was solved with the GA, but it was felt that the solution was too time-consuming for an adequate convergence of the search. However, it was found acceptable for narrowing the response space for initializing a gradient scheme.

Since gradient schemes necessitate continuous parameters (i.e., variables), it was necessary to reformulate the discrete optimization problem as a continuous one. The solution was to pose the following two types of problems:

1. $W_{\text {desired }}, P_{\text {desired }}$ specified: For each value of $d$, solve for the $q_{o}, v$ combination that algebraically solves the constraint equations

$$
W_{\text {desired }}-W\left(q_{o}, v, d\right)=0 \quad P_{\text {desired }}-P\left(q_{o}, v, d\right)=0
$$

Since $d$ is known, this reduces to solving two nonlinear algebraic equations in two unknowns. Then, sort the solutions that produce acceptably small residuals in the constraint equations to find the desired maximum according to whichever "efficiency" criterion (mentioned previously) was chosen. This was accomplished using a Newton-type solution algorithm. The analyst should note $->$ not all $W_{\text {desired }}, P_{\text {desired }}$ combinations are possible. An intersection of the contours for a given $d$ is needed to produce a solution.

2. $P_{\text {desired }}$ only specified: For each value of $d$, solve for the $q_{o}, v$ combination that

Maximizes: the efficiency criterion of interest (i.e. $\eta_{\mathrm{t}}, \eta_{m}$, or $\eta_{\mathrm{t}}{ }^{*} \eta_{m}$ )

Subject to: $P_{\text {desired }}-P\left(q_{o}, \mathrm{v}, d\right)=0$

Then sort the solutions that produce acceptably small residuals in the single constraint to find the desired maximum according to whichever "efficiency" criterion was chosen. This was accomplished using a MATLAB routine to do nonlinear programming.

The capability developed for optimization and graphical model output can be most effectively used by integrating them via GUI tools (available in the MATLAB system). The GUI panel in Fig.5 al- 
lows the user to:

1. Select any of presently seven metals (304 stainless steel, 304L stainless steel, 1018 steel, tin, nickel, titanium, molybdenum) to analyze.

2. Plot any of five "responses" from the model $\left(\eta_{\mathrm{t}}, \eta_{m}, \eta_{\mathrm{t}}{ }^{*} \eta_{m}, W, P\right)$. Surfaces are plotted as continuous functions of $q_{o}, v$ and as a discrete function of $d$. Contours appear as labeled iso-curves of the given response variable, continuous in $q_{o}, v$, and for a single value of $d$.

3. Obtain the nearest optimal weld schedule for user-specified, $W_{\text {desired }}$ and/or $P_{\text {desired }}$.

4. Display the assumed weld shape as it is changed by the user or the completed solution

5. Get continuous weld model output via "mouse-down" button clicks on the 2-D contour plots.

6. Get text help describing the application.

Item 5 provides the analyst with the flexibility to choose an alternative weld schedule. The optimization algorithms are configured to attain the extremum, be it minimum or maximum, regardless of the cost in terms of $q_{o}$, $v$. If an efficiency response can be further improved or "optimized" by a few percent for a large increase in $q_{o}$ or $v$, then that is what the algorithm will recommend. It is up to the analyst to decide whether this recommendation is practical.

\section{THE ARC APPLICATION}

Response characteristics for plasma-arc or gas-tungsten arc welding are concerned with minimizing heat input on a given metal, while attaining a user-specified weld depth for an assumed semi-circular weld cross-section. The schedules are produced by applying parameter optimization to the mathematical model in Ref. 4. Weld schedules consist of:

1. laser output $\left(q_{o}\right)$ power in watts with a range of 100-2000, and

2. part travel speed $(v)$ in millimeters $(\mathrm{mm}) / \mathrm{second}(\mathrm{sec})$, with a range of 1-50.

For arc welding, $q_{o}$ and $v$ can be considered to vary continuously over given ranges. It was not necessary to scale the model for this application.

Three output responses were modeled as functions of the WS parameters, $q_{o}, v$. These quantities were: 1$)$ melting efficiency $\left(\eta_{m}\right.$, dimensionless), 2)top width of the weld ( $W$, $\mathrm{mm})$, and 3)weld penetration depth, $(P, \mathrm{~mm})$. The weld cross-sections are assumed semicircular and therefore $W=2 P$. 


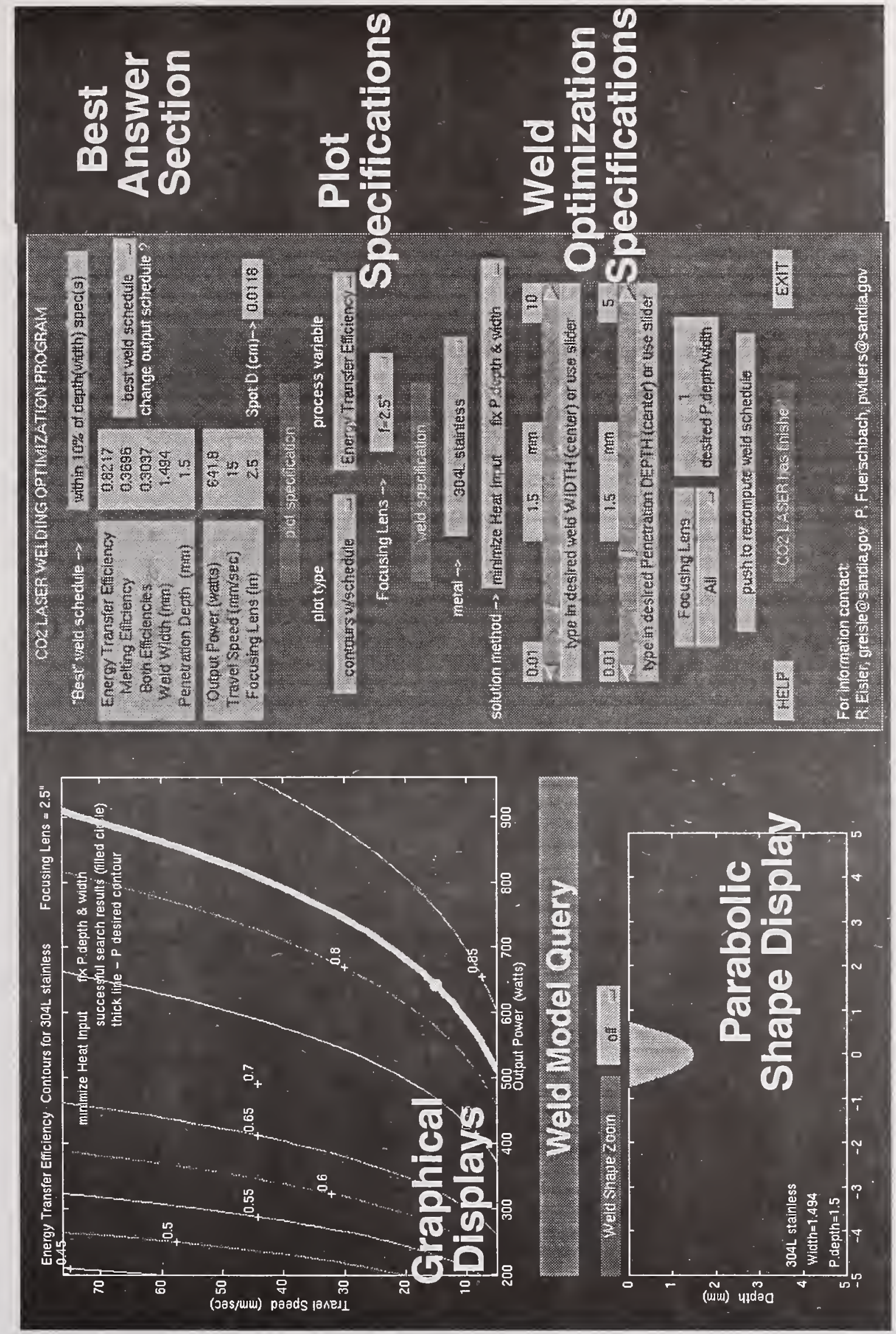

Figure 5. The $\mathrm{CO} 2$ Laser Application 
The following is the nonlinear algebraic, plasma-arc model

$$
\begin{gathered}
R y=0.8 \frac{q_{o} v}{\alpha^{2} \delta h} \quad \eta_{m}=c_{7}+c_{8} e^{\left[\frac{-R y}{c_{10}}\right]}+c_{9} e^{\left[\frac{-R y}{c_{11}}\right]} \\
\text { Area }=\frac{R_{y} \eta_{m} \alpha^{2}}{v^{2}} \quad P=2 \sqrt{(2 \text { Area }) / \pi} \text { (semicircular area) }
\end{gathered}
$$

Note that the $R y$ relation used here assumes a constant $\eta_{t}=0.8$. The gradient-based, nonlinear optimization algorithm (in MATLAB), described previously, was used to find the $W S$ to solve the single problem available for this application:

Maximize $\eta_{m}$ for $P_{\text {desired }}$ only.

To compensate for initial guess sensitivity, this single problem was solved for five different sets of initial conditions which represent the boundaries of the ranges on $q_{o}, v$. The solutions were sorted for those that produced acceptably small residuals in the single constraint and of these the one which maximized $\eta_{m}$ was chosen. The $A R C$ user-interface was patterned on that of the $\mathrm{CO} 2$ application and is not shown.

\section{THE YAG APPLICATION}

Response characteristics for Nd: Pulsed YAG welding are concerned with minimizing weld-induced temperature on a specific component, while attaining user-specified weld dimensions for an assumed parabolic-shape weld cross-section. The schedules are produced by applying parameter optimization to a mathematical model obtained in Ref. 5 for 304 stainless steel. Weld schedules consist of constant values over a given weld for: 1) peak power in watts $\left.\left(q_{p}\right), 2\right)$ energy in joules $(Q)$, and 3 ) lens focal length in $\mathrm{mm}(f)$

$q_{p}$ and $Q$ can be considered to vary continuously over given ranges. $f$ is discrete as in the CO2 application. It was necessary to scale the model for this application because of the magnitude disparity on $q_{p}$ and $Q$. Three output characteristics were modeled as polynomial functions of the $W S$ parameters, $q_{p}, Q, f$. These quantities were: 1$)$ temperature $\left.\left(T,{ }^{\circ} \mathrm{C}\right), 2\right)$ width of the weld ( $W, \mathrm{~mm})$, (converted from area via a parabolic shape approximation), and 3) weld penetration depth, $(P, \mathrm{~mm})$.

The polynomial functions, for a given $f$, are of the form

$$
\begin{gathered}
T=t_{1}+t_{2} Q+t_{3} q_{p}+t_{4} Q^{2}+t_{5} q_{p} Q+t_{6} q_{p}^{2} \\
\text { Area }=a_{1}+a_{2} Q+a_{3} q_{p}+a_{4} Q^{2}+a_{5} q_{p} Q+a_{6} q_{p}^{2} \\
P=p_{1}+p_{2} Q+p_{3} q_{p}+p_{4} Q^{2}+p_{5} q_{p} Q+p_{6} q_{p}^{2}
\end{gathered}
$$

where the coefficients $t_{i}, a_{i}, p_{i}$ are found via least-squares fitting of experimental data. Since $W$ is generated for a parabolic cross-section model, $W=(3$ Area $) /(2 P)$. Travel speed $(v$, $\mathrm{mm} / \mathrm{sec})$, pulse duration $(\tau, \mathrm{msec})$, pulse frequency $(v, \mathrm{~Hz})$ are also computed, based on an average power of 200 watts. These relations are: 


$$
v=50 \frac{W}{Q} \quad \tau=1000 \frac{Q}{q_{p}} \quad v=\frac{200}{Q}
$$

The gradient-based optimization and nonlinear algebraic solver schemes described previously for $\mathrm{CO}_{2}$ were used to find the WS to solve the problems

1. Minimize $T$, Subject to: $P_{\text {desired }}$ only (nonlinear optimization at each value of $f$ with sorting over lens $f$ for minimum $T$ ).

2. Minimize $T$, Subject to: $W_{\text {desired }}, P_{\text {desired }}$. (nonlinear algebraic solution at each value of $f$ with sorting over $f$ for minimum $T$ )

The solutions were sorted for those that produced acceptably small residuals in the constraints and of these the one which minimized temperature was chosen. Due to the fact that the ranges of $q_{p}, Q$ were discontinuous (i.e., depending on the value of $f$ ), the genetic algorithm was used to provide an initial guess for the sub-WS $\left(q_{p}, Q\right)$ each time a new value of $f$ was considered.The $Y A G$ userinterface was patterned on that of the $\mathrm{CO} 2$ application and is not shown.

\section{THE ISO APPLICATION}

This application computes constant temperature contours due to saturation from a heat source according to the 2-D steady-state, heat-flow equation as derived by Rosenthal (Ref. 6). The 2-D model arises from modeling the welding of thin sheets where the temperature variation is considered negligible in the thickness direction. The steady-state description is derived from welding a plate whose dimensions are large with respect to the size of the contours. A schematic is shown in Fig.6.

Easy access to temperature isotherms for a given set of welding conditions provides the optimization user with an additional check of weld thermal effects. While melting efficiency indicates how effective a given weld schedule is in minimizing heat input to the part, the Rosenthal analysis graphically presents the resulting temperature distribution and indicates the geometric extent of the weld heat input.

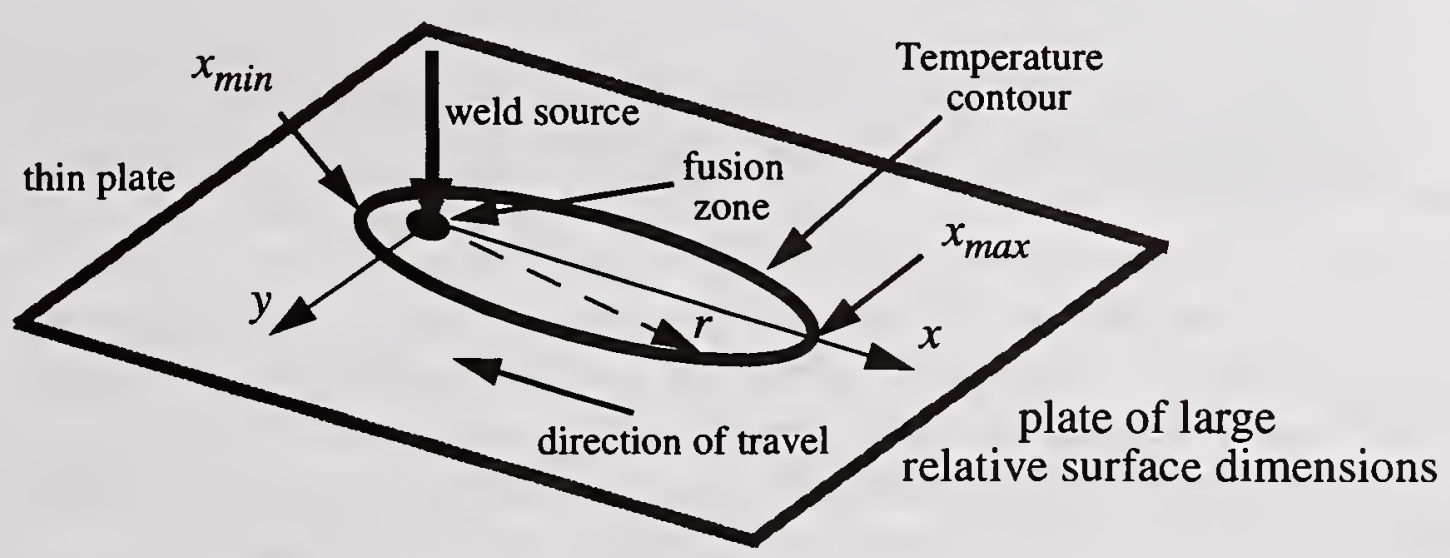

Figure 6. Schematic of the 2-D steady-state, heat-flow problem 
The Rosenthal analytical solution is

$$
\frac{2 \pi\left(T-T_{o}\right) k_{s} t}{Q}=e^{\left(\frac{v x}{2 \alpha_{s}}\right)} K_{o}\left(\frac{v r}{2 \alpha_{s}}\right)
$$

where the following are input by the user via the GUI-driven application,

$T=$ contour temperature of interest $(\operatorname{deg} \mathrm{C})$

$T_{o}=$ base metal temperature

$k_{s}=$ thermal conductivity of metal (Joules/(meter-sec-deg C))

$t=$ thickness of metal plate $(\mathrm{mm})$

$Q=$ energy (heat) input to the metal (Joules)

$v$ = welding speed $(\mathrm{mm} / \mathrm{sec})$

$\alpha_{s}=$ thermal diffusivity of metal (meters ${ }^{2} / \mathrm{sec}$ )

$K_{O}$ is the modified Bessel function of the second kind and zero order (a MATLAB function) and $e$ is the exponential operator. The variable, $r$, radial distance from origin $\left(\left(x^{2}+y^{2}\right)^{1 / 2}\right.$ in millimeters), is manipulated by the application.

The contour solution reduces to solving the above nonlinear algebraic equation as follows:

1. solve the equation: $c_{1}-e^{c_{2} x} K_{o}\left(c_{2} x\right)=0$ for $x=x_{\text {min }}, x_{\text {max }}$ using a nonlinear solver, where the constants $c_{1}=\left(2 \pi *\left(T-T_{o}\right) * k_{s}{ }^{*} t\right) / Q$ and $c_{2}=\mathrm{v} /\left(2 * \alpha_{s}\right) \cdot y\left(x_{\min }\right)=y\left(x_{\max }\right)=0$.

2. solve for the intermediate $x$ values on the top half of the contour by inputting a range of $r$ values, $a b s\left(x_{\min }\right)<r<x_{\max }$, and solving $x=1 / c_{2} * \ln \left(c_{1} / K_{o}\left(c_{2}{ }^{*} r\right)\right)$, and then the corresponding $y=\left(r^{2}-x^{2}\right)^{1 / 2}$. $\ln$ is the natural logarithm.

A complete contour is constructed by reflecting the top half and is approximately elliptical in shape. The ISO user interface is shown in Fig. 7.

\section{SUMMARY AND CONCLUSIONS}

Computational methods have been developed to provide optimal weld schedules based on semi-empirical mathematical models. The mathematical relations in the models originated from past research and were modified to provide best least-squares fits to experimental data. Model extension to other metals was provided through the use of thermodynamic constants. Genetic algorithms were used to provide initial guesses to gradient-based solution schemes. Gradient schemes were used to provide tight convergence on specified weld dimensions as well as optimize various performance indices. Solution algorithms work well provided that the required width and penetration specifications co-exist. In addition, the Rosenthal 2-D heat flow equation was solved to produce a geometric view of weld effects. GUI-driven input-output interfaces were provided for all applications. Future applications can be added in a straightforward manner to the MATLAB architecture. 


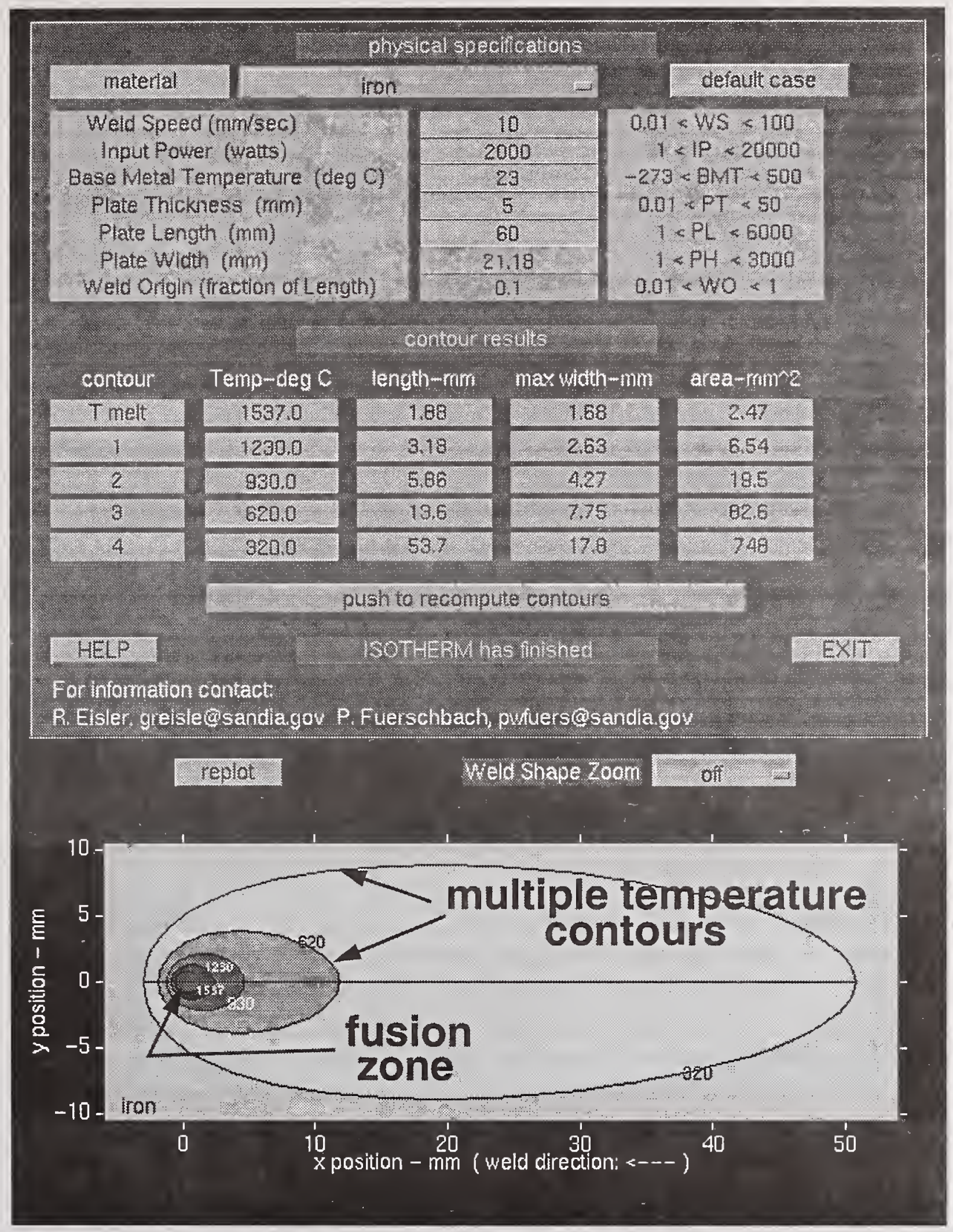

Figure 7. The ISO Application GUI 


\section{ACKNOWLEDGEMENTS}

This work was performed at Sandia National Laboratories, which is operated for the U.S. Department of Energy under Contract No. DE-AC04-94AL85000

\section{REFERENCES}

1. Eisler, G.R.; and Fuerschbach, P.W. 1995, A Computer Method to Provide Optimal Laser Weld Schedules, SAND 94-3241.

2. Fuerschbach, P. W. 1996, Measurement and Prediction of Energy Transfer Efficiency in Laser Beam Welding, Welding Journal 75(1):24s-34s.

3. MATLAB Optimization Toolbox 1995, The MATHWORKS, Inc., Natick, Mass.

4. Fuerschbach, P. W. 1995, A Dimensionless Parameter Model for Arc Welding Processes, 4th Intl. Conf. on Trends in Welding Research ASM Intl., Gatlinburg, Tenn

5. Fuerschbach, P. W.; Hinkley, D. A. 1996, Pulsed Nd:YAG Laser Welding of Heart Pacemaker Batteries with Reduced Heat Input, submitted to Welding Journal

6. Rosenthal, D. 1946, The Theory of Moving Sources of Heat and Its Application to Metal Treatments, Transactions of the ASME, 849-866. 


\title{
HEAT AND MASS TRANSFER IN A MOLTEN DROPLET DURING GMA WELDING
}

\author{
Valerian A. Nemchinsky*
}

\begin{abstract}
The motion of melted metal in a droplet hanging at the tip of an arc electrode during arc welding is considered. The motion is induced by a surface tension gradient due to non-uniformly heated surface of the droplet. It is shown that the melt flow is confined within a narrow boundary layer. The thickness of this layer and the melt velocity within it are estimated. The influence of the metal motion on heat transfer in the droplet is considered. A simple formula for effective thermal conductivity which takes into account the thermocapillary convection is obtained. Estimates show that for conditions typical for arc welding, the effective coefficient of thermal conduction exceeds the regular one approximately tenfold. Comparison of the calculated heat fluxes with those obtained from the observed electrode melting rates shows very good agreement.
\end{abstract}

\section{Introduction}

The tip of the electrode during GMA Welding is melted by heat from the surrounding plasma. Liquid droplets detach periodically from the electrode and become part of the welding pool. The effectiveness and the quality of the welding depend on droplet parameters: its size, speed, heat content. The processes of mass and heat transfer in the droplet, which determine these parameters, äre, therefore, very important.

Heat transfer in the solid portion of the electrode is relatively simple. The heat, the electrode receives from the surrounding plasma, and the heat generated within the electrode by resistance heating are transferred by thermal conduction to the cooled end of the electrode. Heat transfer in the liquid droplet is much more complex. A simple heat transfer model, based on the thermal conduction only, is insufficient to describe the observed heat fluxes. This was shown convincibly in paper (Ref. 1). The authors compared the heat flux, provided by the thermal conduction to the solid-liquid boundary, with the power necessary to melt the electrode at the observed rate. According to their estimations, the thermal conduction should be increased ten to twenty times to be able to supply the necessary heat fluxes (Our estimations give the similar result, see below.) After (Ref. 1,2) we conclude that heat convection should play a decisive role in heat transfer.

There are several forces which can set the melt into motion and thus intensify heat transfer: buoyancy, the Lorentz force (the force of interaction of the current with the magnetic field created by this current), and thermocapillary forces. Let us estimate the relative importance of different forces.

"ESAB Welding and Cutting Products. Florence, SC 29501 
For buoyancy we have estimation $F_{B} \sim 4 \pi R^{3} \rho g \beta \Delta T$, where $R$ is the droplet radius, $\rho$ is the melt density, $\beta$ is thermal expansion coefficient, and $\Delta \mathrm{T}$ is the temperature difference within the droplet. By putting typical data $\mathrm{R} \sim 1 \mathrm{~mm}, \Delta \mathrm{T} \sim 1200 \mathrm{~K}$ (Ref 3,4 ), $\rho \sim 710^{3} \mathrm{~kg}$ $\mathrm{m}^{-3}$, and $\beta \sim 10^{-4} \mathrm{~K}^{-1}$, we obtain $\mathrm{F}_{\mathrm{B}} \sim 310^{-5} \mathrm{~N}$.

Electromagnetic (Lorentz) force is due to interaction of the arc current with the magnetic field created by this current. Its value can be estimated as $\frac{\mu_{0}}{4 \pi} I^{2}$, where $\mu_{0}$ is the permeability of free space and I is the arc current. For $\mathrm{I} \sim 200 \mathrm{~A}, \mathrm{~F}_{\mathrm{L}} \sim 410^{-3} \mathrm{~N}$.

Thermocapillary force arises when there is a temperature gradient along the surface and, correspondingly, a gradient of the surface tension coefficient. This creates a stress $\nabla \sigma$ directed from the locations with a lower $\sigma$ towards those with the higher $\sigma$, i.e. to the coolest part of the surface. There is no force in immobile liquid that can balance this stress, so the thermocapillary motion arises in the liquid (Marangoni effect). The order of magnitude of the thermocapillary force is $\mathrm{F}_{\mathrm{TC}} \sim 2 \pi \mathrm{R} \frac{\mathrm{d} \sigma}{\mathrm{dT}} \Delta \mathrm{T}$, where $\mathrm{d} \sigma / \mathrm{dT}$ is temperature gradient of surface tension. For typical values do/dT $\sim 410^{-4} \mathrm{~N} \mathrm{~m}^{-1} \mathrm{~K}^{-1}$ (Rer. 5), $\Delta \mathrm{T} \sim$ 1200K, $\mathrm{R} \sim 1 \mathrm{~mm}$, we have $\mathrm{F}_{\mathrm{TC}} \sim 3^{\cdot} 10^{-3} \mathrm{~N}$.

We see that buoyancy can be neglected whereas the electromagnetic force $F_{L}$ has the same order of magnitude as the thermocapillary force. However, this does not necessarily mean that the melt velocities caused by these forces have the same order of magnitude. The distribution of the Lorentz force, even its sign, depends on the current distribution inside the droplet (Ref. 6) which in its turn depends on the processes in the adjacent plasma (Ref.7). Also, the Lorentz force, contrary to the thermocapillary force, can be compensated to some degree by the pressure gradient. This can be seen from the following expression for the volumetric Lorentz force $\mathrm{f}_{\mathrm{L}}$ :

$$
\mathbf{f}_{\mathrm{L}}=\mathbf{B} \times \mathbf{J}=-\mu_{\mathrm{o}} \mathbf{J} \times \operatorname{rot} \mathbf{J}=\mu_{\mathbf{o}}\left\{1 / 2 \operatorname{grad}\left(\mathrm{J}^{2}\right)+(\mathbf{J} \nabla) \mathbf{J}\right\}
$$

where $\mathbf{J}$ is the current density and $\mathbf{B}$ is magnetic flux. One can see that if current lines are parallel, the vortex component of $\mathbf{f}_{\mathrm{L}}$ is equal to zero. In this case the Lorentz force can be compensated by the pressure gradient completely.

The melt motion caused by the thermocapillary force is considered in this report in some more detail. Its influence on heat transfer is estimated. It is shown that thermocapillary convection is intensive enough to explain the observed electrode melting rates.

\section{Estimation of Flow Parameters and Heat Transfer Intensity}

The flow pattern is shown in Fig.1. The liquid moves from the hottest central part of the surface towards the liquid-solid interface, dives into the droplet and returns back through 
the droplet volume. During this circulation, the liquid transfers the heat from surface layer to the droplet volume adjacent to the solid-liquid interface. Intensity of this heat transfer depends on the speed of melt circulation and on the amount of liquid involved in the motion. Let us estimate these parameters.

As we will see later, the Reynolds number of the flow is high. This means that the melt moves within a relatively thin layer close to the surface and that the boundary layer approximation is applicable. This also means that one can neglect the droplet curvature.

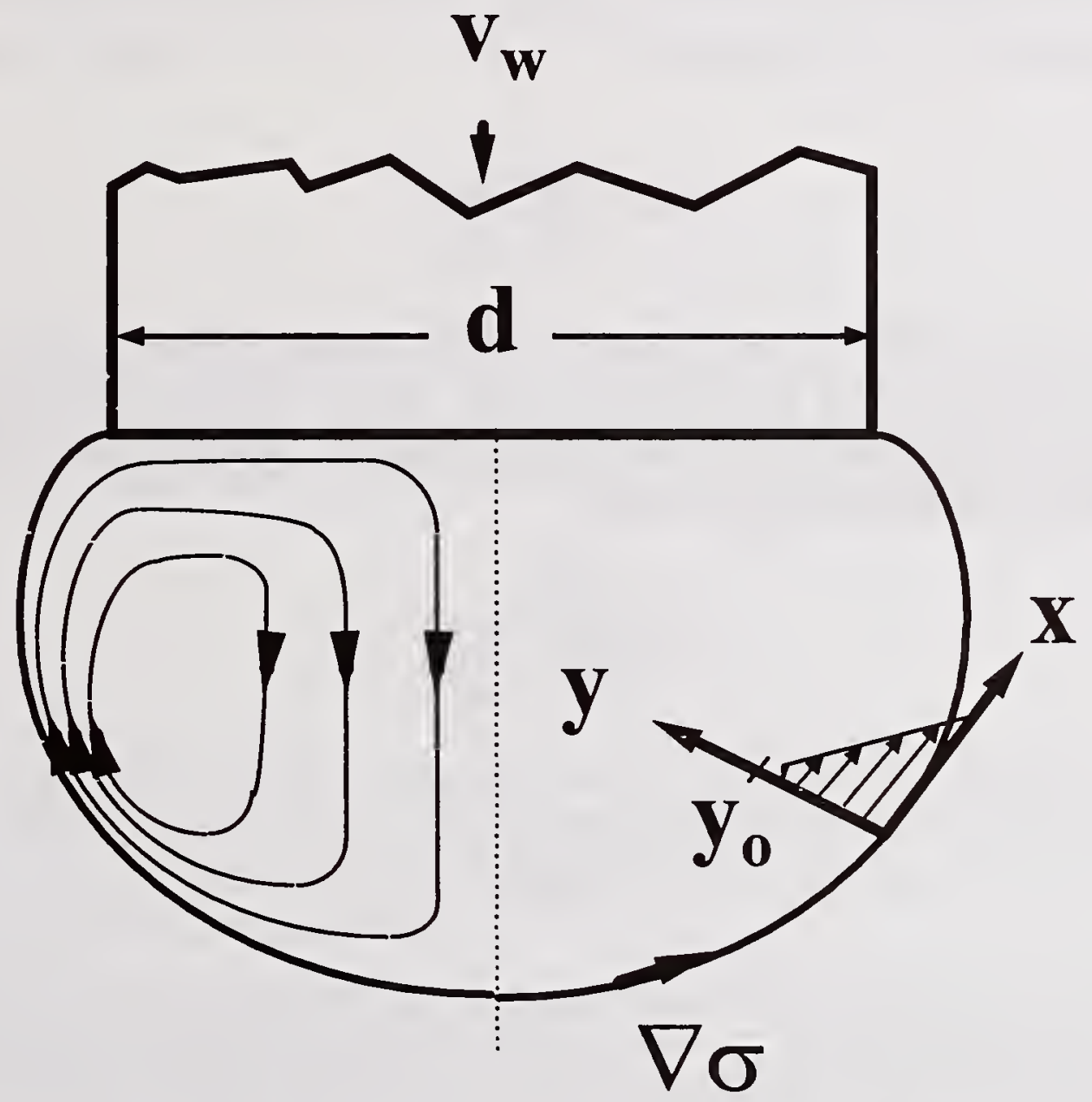

Figure 1. Schematic of the melt motion in a droplet. Left of the center shows the ftow pattern. Right of the center shows the coordinates used and the velocity distribution in the boundary layer. $\nabla \sigma$ shows the direction of the gradient of surface tension, $d$ is the electrode diameter and $v_{w}$ is wire feed rate.

There is no characteristic length in the problem. Therefore, all the flow parameters should depend on $x$, the distance along the surface from the center-line of the droplet. The Reynolds number is, therefore, $\operatorname{Re}=\mathrm{xv} / v$, where $v$ is kinematic viscosity. It is shown in the boundary layer theory (Ref. 8) that the layer thickness is $\delta \sim \mathrm{x} / \sqrt{\operatorname{Re}}$. At the surface the stress balance condition should be satisfied: 


$$
\rho \vee \frac{v}{\delta} \sim \frac{\mathrm{d} \sigma}{\mathrm{dx}}
$$

Here $\rho$ is the melt density. From (1) we obtain the estimate for velocity

$$
v \sim\left(\frac{x}{v}\right)^{1 / 3}\left(\frac{1}{\rho} \frac{d \sigma}{d x}\right)^{2 / 3}
$$

and the estimate for the layer thickness

$$
\delta \sim\left(\frac{\rho v^{2} x}{d \sigma / d x}\right)^{1 / 3}
$$

Let us substitute in (2) and (3) the gradient of surface tension $\mathrm{d} \sigma / \mathrm{dx}$ by $\Delta \sigma / \mathrm{R}$, where $\Delta \sigma$ is the $\sigma$ difference between its maximum value at the solid-liquid interface and its minimum value at the hottest spot of the surface. Here $\mathrm{R}$ is the radius of the droplet. We have the following estimations for $\mathrm{v}$ and $\delta$ :

$$
\begin{aligned}
& \mathrm{v} \sim\left(\frac{\mathrm{R}}{\mathrm{v}}\right)^{1 / 3}\left(\frac{\Delta \sigma}{\rho \mathrm{R}}\right)^{2 / 3} \\
& \delta \sim\left(\frac{\rho \dot{v}^{2} \mathrm{R}^{2}}{\Delta \sigma}\right)^{1 / 3}
\end{aligned}
$$

Let $\tau$ be the time the liquid particle at the surface is exposed to the heat flux from the plasma. One can estimate $\tau$ as $R / v$, the ratio of the particle path (in the order of the droplet radius $R$ ) to the particle velocity at about $R$ distance from the droplet center. We have

$$
\tau \sim\left(v \mathrm{R}^{2}\right)^{1 / 3}\left(\frac{\mathrm{R} \rho}{\Delta \sigma}\right)^{2 / 3}
$$

In order to determine how the motion of the liquid affects the heat transfer, the temperature distribution should be considered. For the sake of simplicity, as we have done with the flow problem, let us average the temperature distribution $\mathrm{T}(\mathrm{x}, \mathrm{y})$ over $\mathrm{x}$, the coordinate along the droplet surface : $\bar{T}(\mathrm{y})=\int \mathrm{T}(\mathrm{x}, \mathrm{y}) \mathrm{dx} / \int \mathrm{dx}$. To obtain $\mathrm{T}(\mathrm{y})$, consider a liquid particle moving along the surface of the droplet at the depth $y$. It takes this particle $\tau(\mathrm{y}) \sim \mathrm{R} / \mathrm{v}_{\mathrm{x}}(\mathrm{y})$ time to travel along the surface. During this travel, the particle accumulates an amount of heat $\rho C T(y)\left(J / \mathrm{m}^{3}\right)$, where $C$ is the specific heat and $\rho$ is the melt density. After its travel beneath the surface, the particle dives inside the droplet and 
there it is cooled by sharing its excessive heat with the rest of the droplet. The process of gaining and losing of heat by the liquid in the boundary layer can be described by the equation:

$$
\kappa \frac{d^{2} \bar{T}}{d y^{2}}-\frac{\rho C \bar{T}}{R} v_{x}(y)=0
$$

This equation should be solved with the following two boundary conditions: First, at the liquid-plasma boundary

$$
-\kappa \frac{d \bar{T}(0)}{d y}=q
$$

where $\mathrm{q}$ is the heat flux density at the surface. Second, beneath the viscous boundary layer, where there is no liquid motion along the surface, the heat is transferred by thermal conduction only. In our case, when convective heat transfer is much more intensive than conductive heat transfer, one may neglect the heat flux due to conduction and put

$$
\frac{\mathrm{d} \overline{\mathrm{T}}\left(\mathrm{y}_{0}\right)}{\mathrm{dy}}=0
$$

Here $y_{0}$ is the distance from the surface where velocity of the liquid vanishes. The boundary condition (9) can be justified in another way. There is a flow of the liquid in the core of the droplet toward the surface, although slow, which does not allow the heat to reach the central portion of the droplet .

Let us assume a linear distribution of the liquid velocity inside the boundary layer: $v_{x}(y)$ $=v_{x}(0)\left(1-y / y_{0}\right)$. The velocity profile inside the layer (the Blasius solution (Ref. 8)) can be well approximated by the linear dependence with $\mathrm{y}_{0}=3.16 \delta$, where $\delta=\mathrm{x} / \sqrt{\mathrm{Re}}$ is the boundary layer thickness. With this approximation, equation (7) can be rewritten in the following dimensionless form

$$
\begin{array}{r}
\frac{d^{2} \Theta}{d \eta^{2}}-\left(1-\frac{\eta}{\eta_{0}}\right) \Theta=0 \\
\Theta^{\prime}(0)=-1 \quad \Theta^{\prime}\left(\eta_{0}\right)=0
\end{array}
$$

where we introduced 
$\eta=y / \delta_{\mathrm{T}} \quad \delta_{\mathrm{T}}=\sqrt{\alpha \mathrm{R} / \mathrm{v}_{\mathrm{x}}(0)} \quad \Theta=\frac{\kappa \overline{\mathrm{T}}(\mathrm{y})}{\delta_{\mathrm{T}}^{\mathrm{q}}} \quad \eta_{0}=\frac{3.16 \delta}{\delta_{\mathrm{T}}}=3.16 \operatorname{Pr}^{1 / 2}$

Here $\alpha=\kappa / \rho C$ is thermal diffusivity. We are interested in $\theta(y=0)=\theta_{0}$, since with the given heat flux density at the surface, the heat transfer intensity is characterized by the surface temperature. To take convection into account, let us introduce the effective thermal conductivity $\kappa_{\text {eff }}$ by the formula

$$
q=\frac{\kappa_{\text {eff }} \bar{T}(y=0)}{R}
$$

We see that

$$
\frac{\kappa_{\mathrm{eff}}}{\kappa}=\frac{\mathrm{R}}{\delta_{\mathrm{T}} \Theta_{0}}=\frac{\sqrt{\mathrm{v}_{\mathrm{x}}(0) \mathrm{R}}}{\alpha \Theta_{0}}
$$

By putting $v_{x}(y=0)$ from (4) into (13) we have finally

$$
\frac{\kappa_{\mathrm{eff}}}{\kappa}=\left(\frac{\mathrm{R} \Delta \sigma}{\rho v^{2}}\right)^{1 / 3}\left(\frac{v}{\alpha}\right)^{1 / 2} \frac{1}{\Theta_{0}}
$$

Solution of the equation (10) with the boundary conditions (11) can be easily obtained by expanding in series. The calculated dependence $\theta_{0}\left(\eta_{0}\right)$ is shown in Fig. 2.

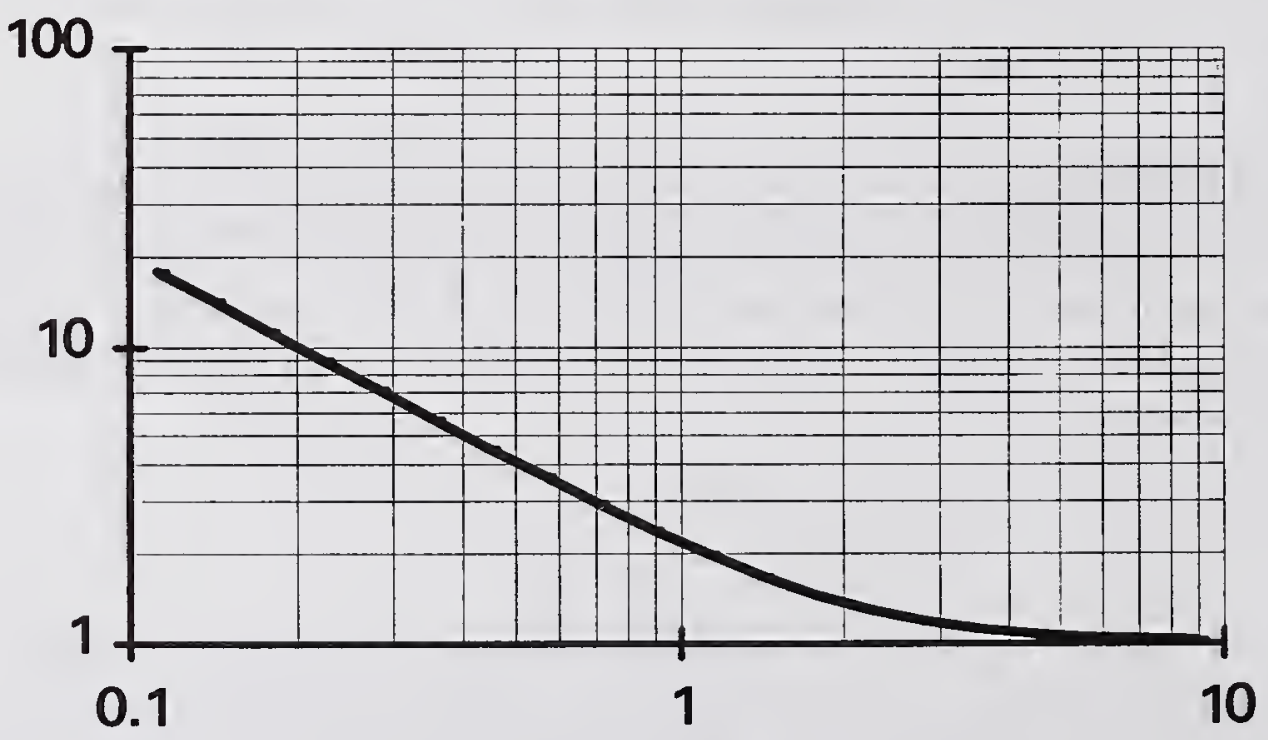

Figure 2. Calculated $\Theta_{0}\left(\eta_{0}\right)$ dependence 
For liquid iron, $\operatorname{Pr} \sim 0.1$, so that in our case $\eta_{\mathrm{o}}$ is close to unity. From Fig. 2 one can see that $\theta_{0}(1) \sim 2.2$.

\section{Comparison with the data obtained from the observed electrode melting rates}

The power delivered to the solid-liquid interface by thermal conduction can be calculated as

$$
\mathrm{Q}_{\mathrm{T}}=\mathrm{KS} \Delta \mathrm{T} / \mathrm{L},
$$

where $S$ is the arc attachment area and $L$ is the average distance from the heated surface to the interface boundary. When the droplet surface facing the arc is heated uniformly, $\mathrm{S}$ is about $2 \pi R^{2}$ and $L$ is about $3 R / 2$. In this case $Q_{T}$ is approximately $4 \kappa R \Delta T$, the value

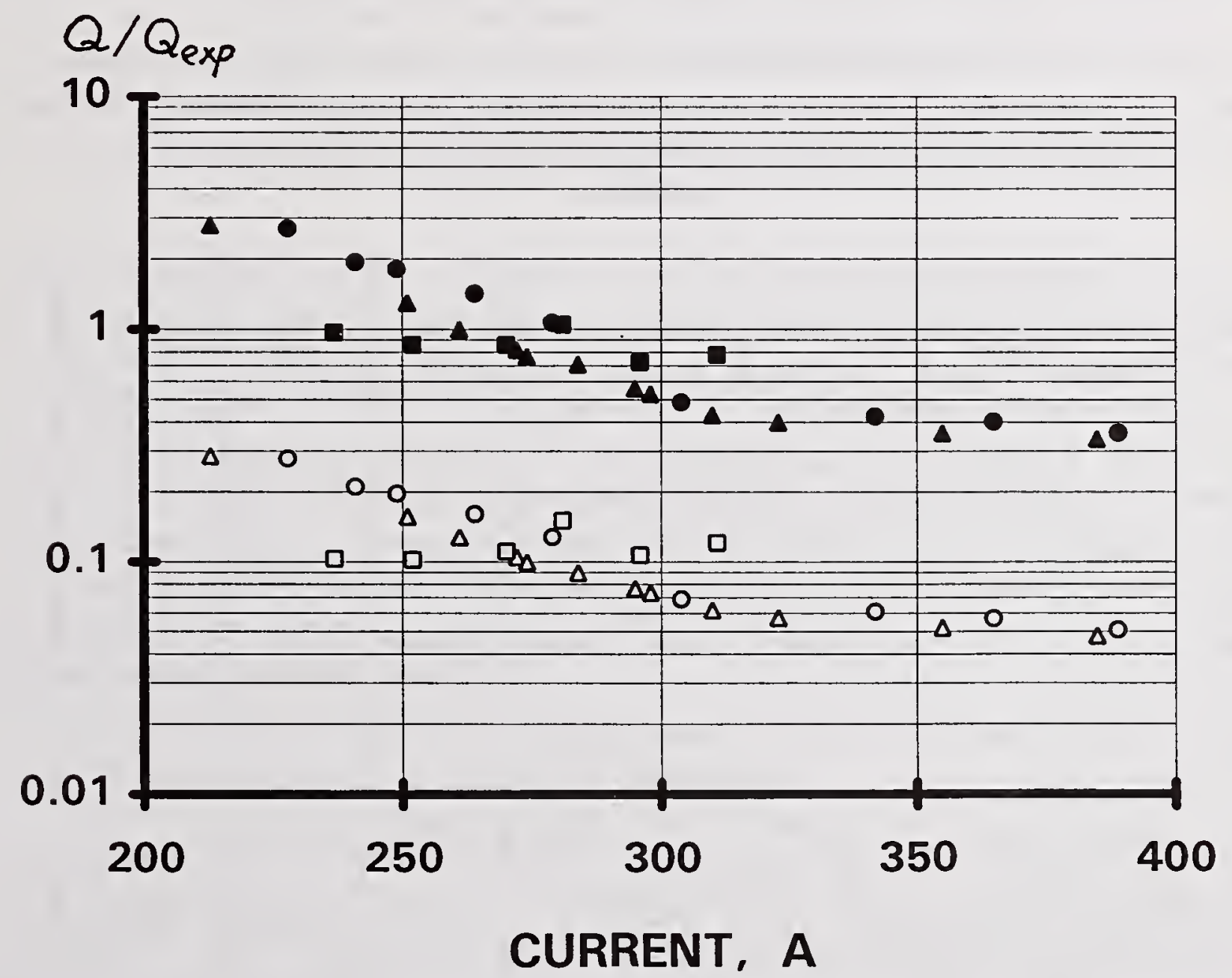

Figure 3. Comparison of the power consumed by electrode melting, as obtained from the observed melting rates, with the calculated power delivered to the melting boundary by thermal conductivity $\left(Q_{T} / Q_{\text {exp }}\right.$ open symbols) and by convection $\left(Q_{\text {eff }} / Q_{\text {exp }}\right.$ full symbols). Experimental data are taken from: $O,($ Ref. 11,12); $\square,($ Ref. 11, 12); $\Delta,($ Ref.13). 
we used in our computations. The power consumed by wire melting, $\mathrm{Q}_{\mathrm{exp}}$, is determined by the wire feed rate, $v_{w}$, by the wire diameter, $d$, and by the energy of heating and melting of the electrode material, $H\left(J / m^{3}\right): Q_{\text {exp }}=\frac{\pi d^{2}}{4} \rho v_{w} H \cdot{ }_{w}$.

For $\mathrm{Q}_{\exp }$ and $\mathrm{Q}_{\mathrm{T}}$ comparison we used data obtained (Ref. 9-11). These experiments were conducted with a steel electrode of $1.6 \mathrm{~mm}$ diameter and with plasma gases of Ar, $98 \% \mathrm{Ar}+2 \% \mathrm{O}_{2}$, and $95 \% \mathrm{Ar}+5 \% \mathrm{O}_{2}$. The $\mathrm{Q}_{\mathrm{T}} / \mathrm{Q}_{\text {exp }}$ ratio calculated for conditions (Ref. 911) with $\Delta \mathrm{T}=1200 \mathrm{~K}$ is shown in Fig. 3 with open symbols. Convection has been taken into account by multiplying $\mathrm{Q}_{\mathrm{T}}$ by the factor $\kappa_{\mathrm{eff}} / \mathrm{\kappa}$

$$
\mathrm{Q}_{\text {eff }}=\frac{\kappa_{\text {eff }}}{\kappa} Q_{T}
$$

The $\mathrm{Q}_{\mathrm{eff}} / \mathrm{Q}_{\mathrm{exp}}$ ratio calculated in this way is shown in the same Fig. 3 with filled symbols. One can see that convection increases heat transfer intensity approximately tenfold.

As an example, the details of calculations for the pure Ar case are shown in Table 1. It can be seen from this Table that the Reynolds number is in the range 1000 to 2000 , high

TABLE 1

\begin{tabular}{|c|c|c|c|c|c|c|c|c|c|}
\hline $\begin{array}{c}\text { current } \\
\mathbf{A}\end{array}$ & $\begin{array}{c}\mathbf{v}_{\mathbf{w}} \\
\mathbf{m} / \mathbf{m i n}\end{array}$ & $\begin{array}{c}\mathbf{R} \\
\mathbf{m m}\end{array}$ & $\begin{array}{c}\mathbf{f} \\
\mathbf{1} / \mathrm{s}\end{array}$ & $\begin{array}{c}\mathbf{v} \\
\mathbf{m} / \mathrm{s}\end{array}$ & $\mathbf{R e}$ & $\mathbf{f} \tau$ & $\begin{array}{c}\mathrm{Q}_{\exp } \\
\mathbf{k W}\end{array}$ & $\begin{array}{c}\mathrm{Q}_{\mathrm{T}} \\
\mathbf{k W}\end{array}$ & $\begin{array}{c}\mathbf{Q}_{\text {eff }} \\
\mathbf{k W}\end{array}$ \\
\hline 228 & 2.5 & 1.3 & 10 & 0.82 & 2100 & 0.016 & 0.701 & 0.196 & 1.91 \\
\hline 241 & 2.8 & 1.1 & 15 & 0.87 & 1900 & 0.019 & 0.787 & 0.166 & 1.53 \\
\hline 249 & 3.0 & 1.1 & 20 & 0.87 & 1900 & 0.025 & 0.843 & 0.166 & 1.53 \\
\hline 264 & 3.2 & 0.96 & 29 & 0.91 & 1700 & 0.031 & 0.903 & 0.145 & 1.28 \\
\hline 279 & 3.4 & 0.81 & 51 & 0.96 & 1600 & 0.043 & 0.955 & 0122 & 1.02 \\
\hline 304 & 3.8 & 0.49 & 260 & 1.13 & 1100 & 0.11 & 1.07 & 0.074 & 0.52 \\
\hline 342 & 4.2 & 0.47 & 320 & 1.15 & 1100 & 0.13 & 1.18 & 0.071 & 0.50 \\
\hline 365 & 4.5 & 0.48 & 331 & 1.14 & 1100 & 0.14 & 1.26 & 0.072 & 0.51 \\
\hline 389 & 5.0 & 0.48 & 360 & 1.14 & 1100 & 0.15 & 1.41 & 0.072 & 0.51 \\
\hline
\end{tabular}


enough for applicability of the boundary layer app:oximation, but not high enough for transition from laminar to turbulent flow to occur. Calculations showed that the droplet residence time is at least ten times larger than liquid particle circulation time: $f \tau<0.1$ (see Table). As for conditions for droplet uniform coverage with the arc attachment, it is not fulfilled at relatively low currents, under $\sim 250 \mathrm{~A}$ (see discussion below).

From Fig. 3 one can see that at currents above $250 \mathrm{~A}$ the ratio between $\mathrm{Q}_{\mathrm{T}}$ and $\mathrm{Q}_{\exp }$ is from 10 to 20 times. After the correction has been made, $Q_{e f f}=\kappa_{\text {eff }} / \kappa Q_{T}$ is in correspondence with $Q_{\text {exp. }}$ The difference of $\sim 100 \%$ should be considered as satisfactory for estimations valid within an order of magnitude. It can be seen that for lower currents $Q_{\text {eff }}$ substantially declines from $Q_{\exp }$. We believe that this is because formula (15) overestimates $\mathrm{Q}_{\mathrm{T}}$ for these currents. There are two reasons for this. First, $\Delta \mathrm{T}$ in the globular mode is substantially lower than $1200 \mathrm{~K}$, the value we used for $\mathrm{Q}_{\mathrm{T}}$ calculations. According to (Ref. 3) $\Delta \mathrm{T}$ is about $800 \mathrm{~K}$ in this mode. For this reason only, $\mathrm{Q}_{\mathrm{T}}$ is overestimated by a factor of $1.5^{4 / 3} \sim 1.7$. Also, in the globular mode, the arc does not cover the whole droplet surface, but only part of it (Ref. 10). Formally, it is equivalent to some decrease of $S$ and some increase of $L$ in formula (15). This means that $\mathrm{Q}_{\mathrm{T}}$ should be additionally decreased. It is likely that both factors cause $\mathrm{Q}_{\mathrm{T}}$ to be overestimated by several times. After taking this into account, the deviation of $\mathrm{Q}_{\mathrm{eff}} / \mathrm{Q}_{\exp }$ from unity can be decreased. Although this correction improves the agreement between $\mathrm{Q}_{\text {eff }}$ and $\mathrm{Q}_{\text {exp }}$, formula (14) does not describe the situation of partial coverage of the droplet by the arc.

At high currents, the shape of the suspended droplet declines from spherical : The higher the current, the more the droplet is elongated in the direction of the current. When obtaining the formula (14), the spherical shape of the droplet was not assumed. Therefore, this formula can be used for the elongated droplet as well. In this case, parameter R in (14) represents the droplet dimension in the direction of the temperature gradient. For high currents, when the droplet is elongated, the value of $\mathrm{R}$ obtained from the droplet volume measurements underestimates the suspended droplet dimension in the direction of the curreni. According to (14), this underestimates the correction factor $\kappa_{\mathrm{eff}} / \kappa$. Therefore, taking the droplet distortion at high currents into account leads to the increase in $Q_{\text {eff }} / Q_{\exp }$ and brings this ratio closer to unity.

\section{Conclusion}

In this paper, the simple formula describing the heat convection due to the Marangoni effect in a droplet is obtained. The effective coefficient of thermal conduction is expressed as a function of viscosity of liquid $v$ and the surface tension difference $\Delta \sigma$ along the droplet surface. The formula is applied to obtain the heat fluxes in the liquid metal droplet hanging at the tip of an electrode during welding. Both $v$ and $\Delta \sigma$ are not very well known under the very high temperatures existing at the droplet surface (nor is the temperature itself known). However, these parameters enter the formula with a small exponent only. Therefore, the result is not sensitive to the precise value of these 
parameters. Calculations based on this formula agree with data obtained from the observed electrode melting rates.

\section{References}

1. Waszink, Van den Hauvel G.J.P.M. 1984. "Heat Generation and Heat Flow in the Filler Metal in GMA Welding." Weld. J. 61, 269s - 282s.

2. Kim Y.-S., McEligot D.M. and Eagar T.W. 1991. "Analysis of Electrode Heat Transfer in Gas Metal Arc Welding." 70, 20s - 31s.

3. Tichelaar G.M., Jelmorini G. and Van den Heuvel G.J.P.M. 1977. "Droplet Temperature Measurements in Arc Welding." Int. Inst. Weld. Document 212-411-77

4. Pintard J. 1967. "Characteristiques de la Fusion et du Transfert dans la Procede MIG de Soudage de l'Acier sous Argon" Int. Inst. Weld. Document 212-111-67

5. Keene B.J. 1988. "Review of data for Surface Tension of Iron and Its Binary Alloys." Intern. Materials Rev. 33, N 1, 1-37.

6. Amson J C 1965 "Lorentz Force in the Molten Tip of an Arc Electrode." Brit. J. Appl. Phys. 16, 1169-1179

7. Nemchinsky V. A. 1996 "The Effect of the Type of Plasma Gas on Current Constriction at the Molten Tip of an Arc Electrode" J.Phys D.:Appl. Phys. 29, 1202-1208

8. Landau L.D. and Lifshitz E.M. Fluid Mechanics. Pergamon. 1959.

9. Rhee S., and Kannatey-Asibu E. Jr. 1992. "Observation of Metal Transfer during Gas Metal Arc Welding." Weld. J. 71, 381s - 386s.

10. Rhee S. Private Communication. 1994.

11. Kim Y-S and Eagar T W 1993 "Analysis of Metal Transfer in Gas Metal Arc Welding" Weld. J. 72, 269s-278s. 


\title{
NUMERICAL MODEL OF SHORT-CIRCUITING TRANSFER PROCESS \\ IN GMA WELDING
}

\section{Yoshinori Hirata*, Takafumi OSAMURA*, Naoyuki GOTO\#, Takayoshi Ohji*}

\begin{abstract}
In this report are described the effects of capillary action and electromagnetic pinch force on kinetics of bridging transfer of GMA welding. Time-dependent deformation of the liquid bridge, which is formed between the electrode and the weld pool by a contact of a pendent drop with the pool surface, is simulated with aids of the numerical solution technique based on finite-difference method. It is shown that both of the breakup time and the liquid volume bridging-transferred from the drop into the pool depend on the drop size before the contact in addition to the short-circuiting current level that serves electromagnetic pinch effect squeezing the bridge.
\end{abstract}

\section{INTRODUCTION}

In Gas shielded Metal Arc welding processes, short-circuiting transfer mode often serves for welding with short arc length in joining various kinds of steels, especially in such a case that welding operation in production is required in all position or with high speed. A cycle of the process in the short-circuiting transfer mode is consisted from a series of three stages as follows. In the first stage, a metal droplet is formed at the tip of the electrode wire which is melted by arc heat. In the second stage, the droplet comes into contact with the surface of weld pool, and subsequently the liquid bridge is formed between the wire tip and the pool. In the third stage, the melted metal bridge is broken up by both of the capillary action and electromagnetic pinch force induced by the transient high current at the instant of short-circuiting, and re-ignition of the arc occurs. The spatter generation, which deteriorates the weld quality and decreases the operation efficiency especially for mechanized or automated welding, is observed almost in both of the second stage and the third stage.

In the beginning of this research, regarding the stationary liquid bridge between the electrode and the pool, current and time required to breakup the bridge were theoretically and experimentally investigated(Ref.1). Secondly, the criteria for bridging transfer from the pendent drop to the pool were discussed focussing on the stability of liquid bridge(Ref.2).

Thirdly, phenomena associated with bridging transfer were cleared through model experiments using mercury and an analysis with aids of the numerical solution technique, the Marker And Cell(MAC) method(Ref.3). In the present study, modeling of the bridging transfer process in

\footnotetext{
* Welding and Production Department, Osaka University, 2-1, Yamada-oka, Suita, Osaka 565, Japan

\# Tsu Labs., Engineering Research Center, NKK, Japan(formerly graduate student of Osaka University)
} 
GMA welding is carried out. Both of the second stage and the third stage prescribed are focussed and replaced by a simple model for analysis(Ref.4), which involves the dynamics of the liquid bridge made by the pendent drop. In this paper are described the effects of capillary action and electromagnetic pinch force on the time and the volume of liquid bridging-transferred from the pendent drop into the pool.

\section{BRIDGING TRANSFER PROCESS CONTROLLED BY CURRENT WAVEFORM}

Recent advancements in power devices such as power transistor and/or FET, and microprocessors, have been making it possible for arc welding power supplies to give higher speed response of output welding current. And various high performance welding processes have been developed and realized. Figure 1 schematically shows a principle of current waveform control method for reduction of spattering in short-circuiting transfer mode, as described below;

(1)Forming a specific size of the metal drop at the tip by control of wire melting [(a)-(d)] ,

(2) Soft contact of the metal drop with weld pool surface after lowering current [ (e) ] ,

(3) Increase of current to break up the bridge, where current rise-up starts with a specific delayed time $T_{d}$ after the contact $[(f)]$,

(4) Squeeze of the bridge by electromagnetic pinch force of the higher current in the duration $\mathrm{T} s[(\mathrm{~g})]$,

(5) Breakup of the bridge by the inertia in the low level of current [ (h)(i) ] .
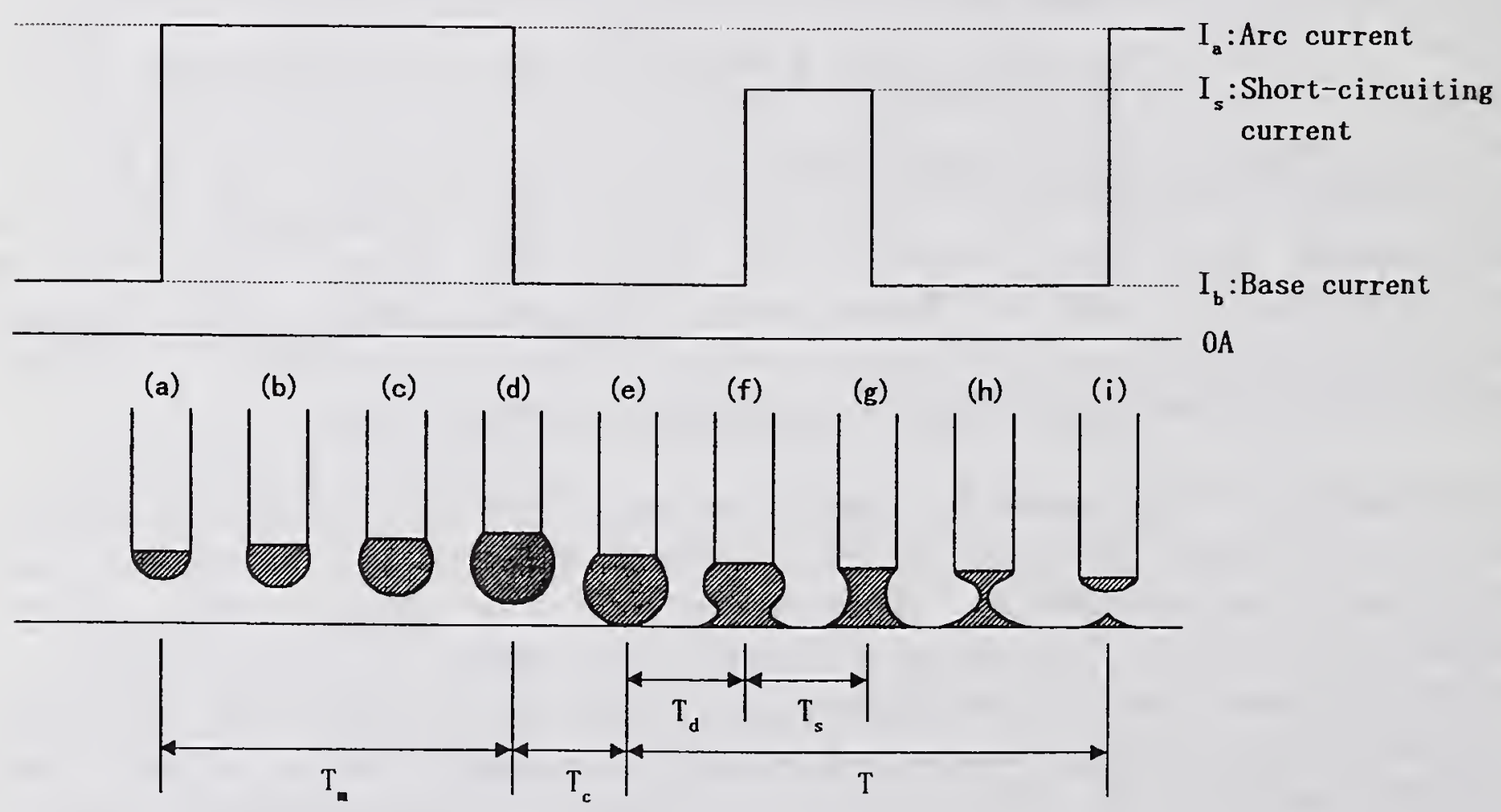

Figure 1 . Schematic explanation of bridging transfer process controlled by current waveform. 
The object of the present study is to simulate the bridging transfer process controlled by current waveform, given in the above model. The cycle time or the short-circuiting frequency is determined by the drop forming time $\left(\mathrm{T}_{\mathrm{m}}\right)$, the approaching time to $\operatorname{contact}\left(\mathrm{T}_{\mathrm{c}}\right)$ and the breakup time(T). The drop forming time depends on the drop size and the electrode wire melting rate, mainly governed by arc current level applied(I a ). As a matter of course, it requires longer time when the drop volume specified is larger and/or arc current is lower. The approaching time is varied by wire feeding speed, and the spacing between metal drop at the electrode wire and pool surface. The time to breakup is dependent on the drop size, short-circuiting current( $(\mathrm{I} s)$, delayed time( $\mathrm{T}$ d $)$ and fluid properties of the melting metal. In this report, the time required from contact to breakup and the metal volume transferred from the drop to the pool were estimated using a simple numerical model.

\section{NUMERICAL MODEL OF BRIDGING TRANSFER}

In the present numerical model, the basic equations governing the motion of the conductive liquid carrying electric current are used as follows(Ref.5);

$$
\begin{gathered}
\nabla \cdot \vec{v}=0, \\
\frac{\partial \vec{v}}{\partial t}+(\vec{v} \cdot \nabla) \vec{v}=-\frac{1}{\rho} \nabla p+\vec{g}+\nu \nabla^{2} \vec{v}+\frac{1}{\rho} \vec{J} \times \vec{B},
\end{gathered}
$$

where, $\quad \vec{v}$ :velocity vector $(\mathrm{m} / \mathrm{s}), \quad p$ :pressure $(\mathrm{Pa}), \nu$ :kinematic viscosity $\left(\mathrm{m}^{2} / \mathrm{s}\right), \vec{J}$ :current density vector $\left(\mathrm{A} / \mathrm{m}^{2}\right)$, $\vec{B}$ :magnetic field vector (T), $\nabla$ :nabra operator, and $\nabla^{2}$ :laplacian operator.

In order to simulate the change of bridging shape and the liquid metal flow, the dynamics of the bridging transfer are theoretically analyzed under the following assumptions;

[1] Surface tension, viscosity and density of the liquid are constant, respectively.

[2]Shape and motion of the bridging process are axisymmetric.

[3]The liquid velocity is uniform over the cross-section (plug flow), velocity component in axial direction becomes a function of two variables, position $Z$ and time $t$.

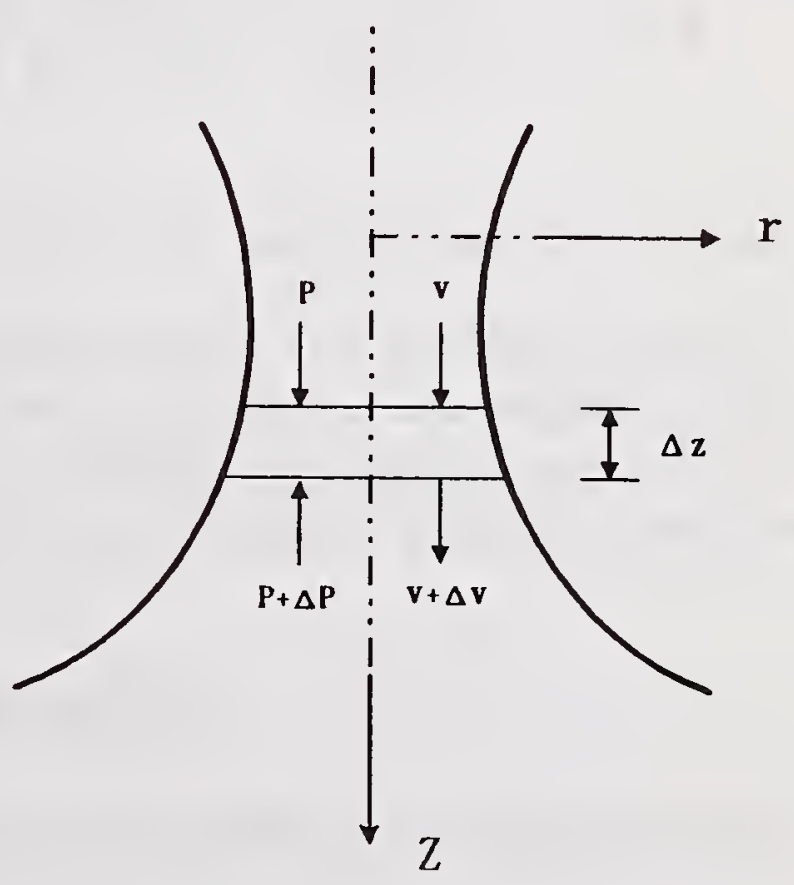

Figure 2. Coordinate system for calculation of bridging transfer process. 
[4]The electromagnetic force is given using magnetic field induced in a long cylindrical conductor having a diameter of $2 R \quad(\mathrm{~m})$ by an axial current $I \quad$ (A) as follows;

$$
\vec{J} \times \vec{B} \fallingdotseq-\nabla\left(\frac{\mu_{0} I^{2} r^{2}}{8 \pi^{2} R^{4}}\right)
$$

where, $\mu_{0}$ :permeability of free $\operatorname{space}\left(4 \pi \times 10^{-7} \mathrm{H} / \mathrm{m}\right)$.

From the assumption [2][3], velocity components $(u, v)$ in both of radial direction or $\mathrm{r}$-direction and $\mathrm{z}$-direction can be expressed as follows;

$$
\begin{gathered}
u=-\frac{1}{2} \frac{\partial \boldsymbol{v}}{\partial z} r, \\
\boldsymbol{v}=\boldsymbol{v}(z, t) .
\end{gathered}
$$

Using the coordinate system as shown in Fig.2, the following equations are derived from eqs.(1)-(5);

$$
\begin{gathered}
\frac{\partial R^{2}}{\partial t}+\frac{\partial v R^{2}}{\partial z}=0 \\
\frac{\partial v}{\partial t}+v \frac{\partial v}{\partial z}=-\frac{1}{\rho} \frac{\partial \bar{p}}{\partial z}+g+\nu \frac{\partial^{2} v}{\partial z^{2}}-\frac{1}{\rho} \frac{\partial}{\partial z}\left(\frac{\mu_{0} I^{2}}{16 \pi^{2} R^{2}}\right) \\
\bar{p}=\sigma+\frac{\mu_{0} I^{2}}{16 \pi^{2} R^{2}}-\frac{\rho R^{2}}{16}\left[\frac{\partial^{2} V}{\partial z \partial t}-\frac{1}{2}\left(\frac{\partial v}{\partial z}\right)^{2}+v \frac{\partial^{2} V}{\partial z^{2}}-\nu \frac{\partial^{3} V}{\partial z^{3}}\right]
\end{gathered}
$$

where, $\sigma$ :capillary pressure, $\sigma=\gamma\left(\frac{1}{r_{1}}+\frac{1}{r_{2}}\right)$ and $R$ :radius of the liquid surface.

The one-dimensional fluid equations given by L.E.Cram(Ref.6) and S.W.Simpson(Ref.7) were a little modified due to adoption of the mean pressure. Numerical computation was carried out by means of the finite-difference approximation to the continuity equation (6), the momemtum equation (7) and pressure equation (8).

\section{RESULTS AND DISCUSSION}

\section{Time-dependent deformation of the liquid bridge}

Figure 3 shows calculated results of time-dependent deformations for two sizes of the liquid drops at the wire tip in contact with the pool. Materials of electrode wire supposed is mild steel and its diameter is $1.2(\mathrm{~mm})$. As seen, in the case of the drop height $\mathrm{Z}_{\mathrm{p}}=0.92(\mathrm{~mm})$, the drop shape gradually changes after the contact and the stable bridge is forming between 
the wire and the pool. The bridge shape calculated is almost unchanged after $\mathrm{t}=2(\mathrm{~ms})$. On the other hand, as for the drop height $\mathrm{Z}_{\mathrm{p}}=1.72(\mathrm{~mm})$, the drop is approaching the bridge formation, but necking in the bridge occurs and it subsequently goes to the breakup. The drop has the internal pressure due to surface tension, whereas the pressure at the pool surface equals to atmospheric pressure. So, at the instant of the contact, the pressure difference generates at the contact region between the drop and the pool. Accordingly, the liquid in the bottom region of the drop is driven to flow into the pool by this capillary pressure, and then liquid bridging is formed. However, occurrence of the breakup or the bridging transfer depends on the drop size if short-circuiting current is not supplied. Because it is not possible to form a stable liquid bridge between the cylindrical rod and the pool beyond its critical height(Ref.1,2).

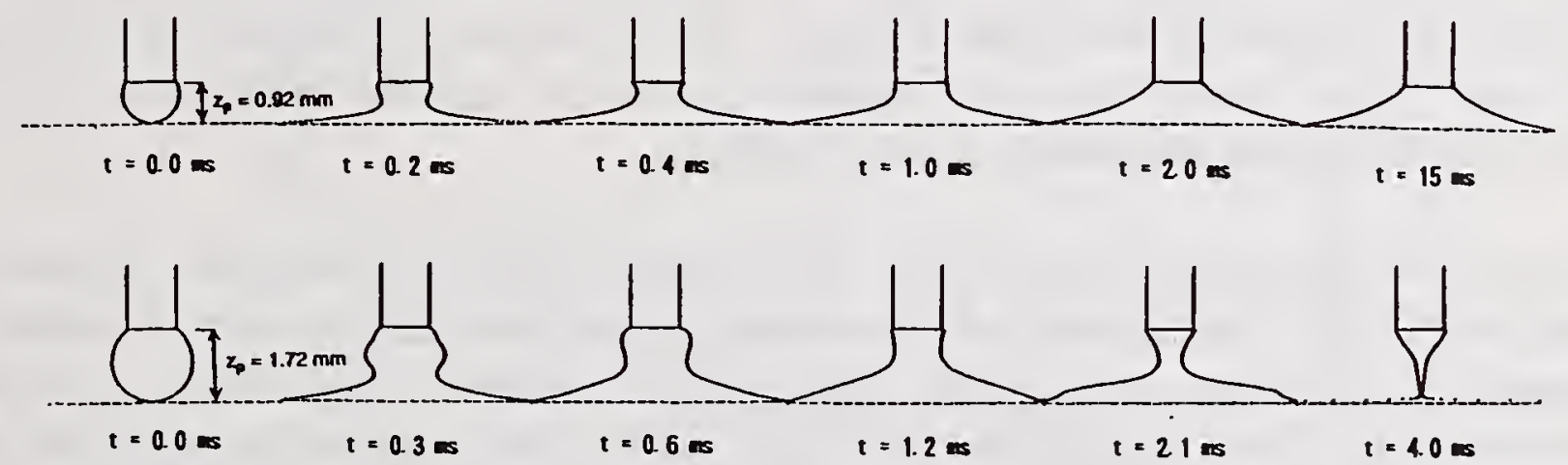

Figure 3. Time-change of the liquid bridge formed by a contact of the pendent drop with the pool surface for two sizes of the drops.

(Mild steel wire $1.2 \mathrm{~mm}$ in dia., $\gamma=1.2 \mathrm{~N} / \mathrm{m}, \rho=7.5 \times 10^{3} \mathrm{~kg} / \mathrm{m}^{3}, \nu=1 \times 10^{-6} \mathrm{~m}^{2} / \mathrm{s}$ )

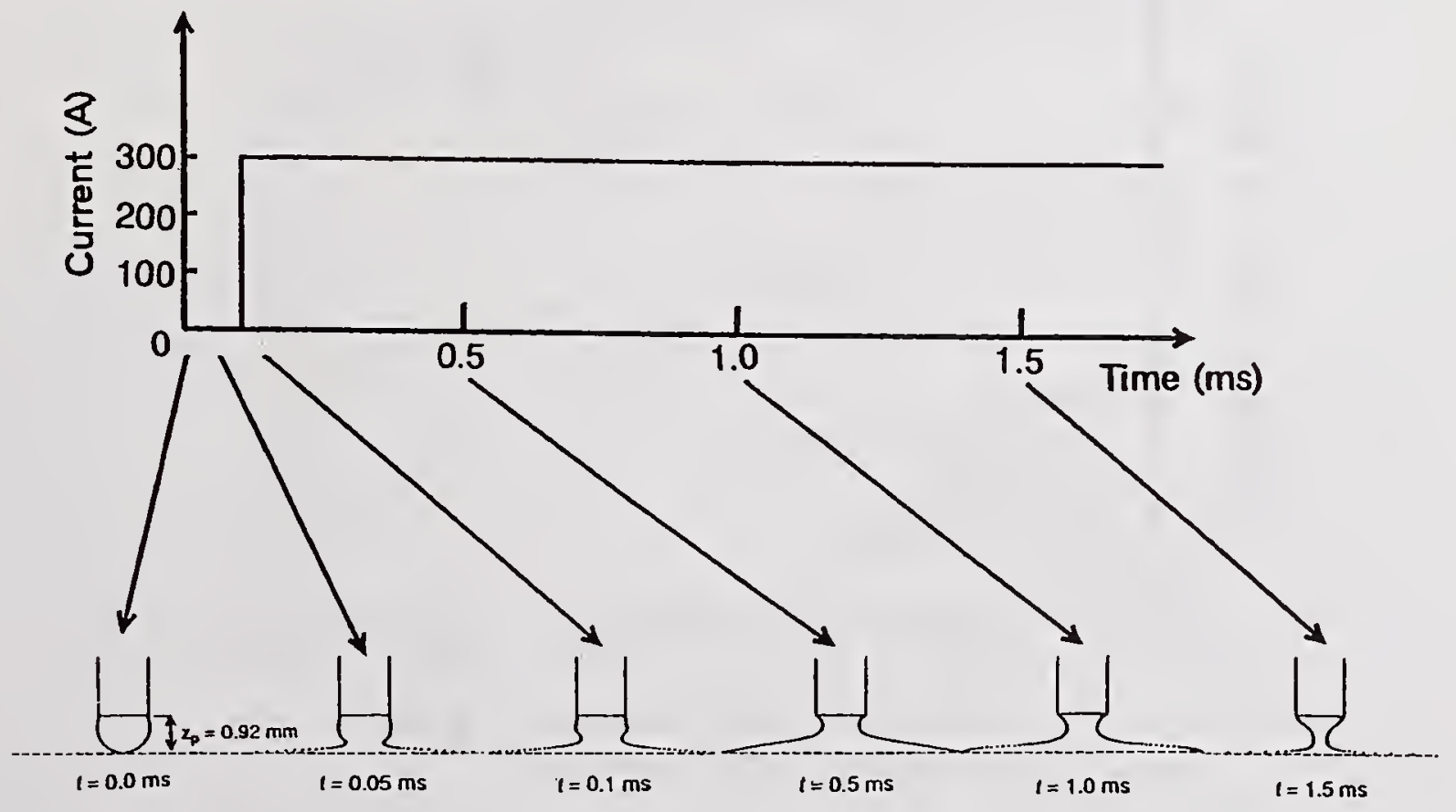

Figure 4. Time-change of the conductive liquid bridge squeezed by electromagnetic pinch force due to short-circuiting current supplied.

(Mild steel wire $1.2 \mathrm{~mm}$ in dia., $\gamma=1.2 \mathrm{~N} / \mathrm{m}, \rho=7.5 \times 10^{3} \mathrm{~kg} / \mathrm{m}^{3}, \nu=1 \times 10^{-6} \mathrm{~m}^{2} / \mathrm{s}$ ) 
A forced breakup of the bridge with short-circuiting current, which is caused by electromagnetic pinch effects, is shown in Fig.4. In this case, supplying electric current of 300 (A) with the delayed time of 0.1 (ms) squeezes the bridge and produces necking toward the breakup.

\section{Factors affecting on breakup time}

Figure 5 shows a relationship between the short-circuiting current, Is and the time required from the contact of the drop with the pool to the breakup, $T$. In these calculations the delayed time are settled to be $T_{d}=1(\mathrm{~ms})$ and supplying of short-circuiting current is not interrupted before the breakup. As seen, the time to breakup decreases with increase of short-circuiting current for both sizes of drop. As for smaller size of drop $Z_{p}=0.92(\mathrm{~mm})$, current level higher than 150 (A) is required in order to give the breakup or the bridging transfer, because a small drop makes a stable bridge.

In higher short-circuiting current region, the breakup time(T) decreases with decrease of the delayed time $\left(T_{d}\right)$. This implies that the bridge shape at the start of short-circuiting current dominates the distribution of the pinch force in the conductive liquid bridge. Intuitively, if the electric current flows immediately after the contact of the drop with the pool, bridging will be quickly broken up. Because higher current density, due to small diameter of the necking part in the bridge, makes the electromagnetic pinch force to act more effectively.

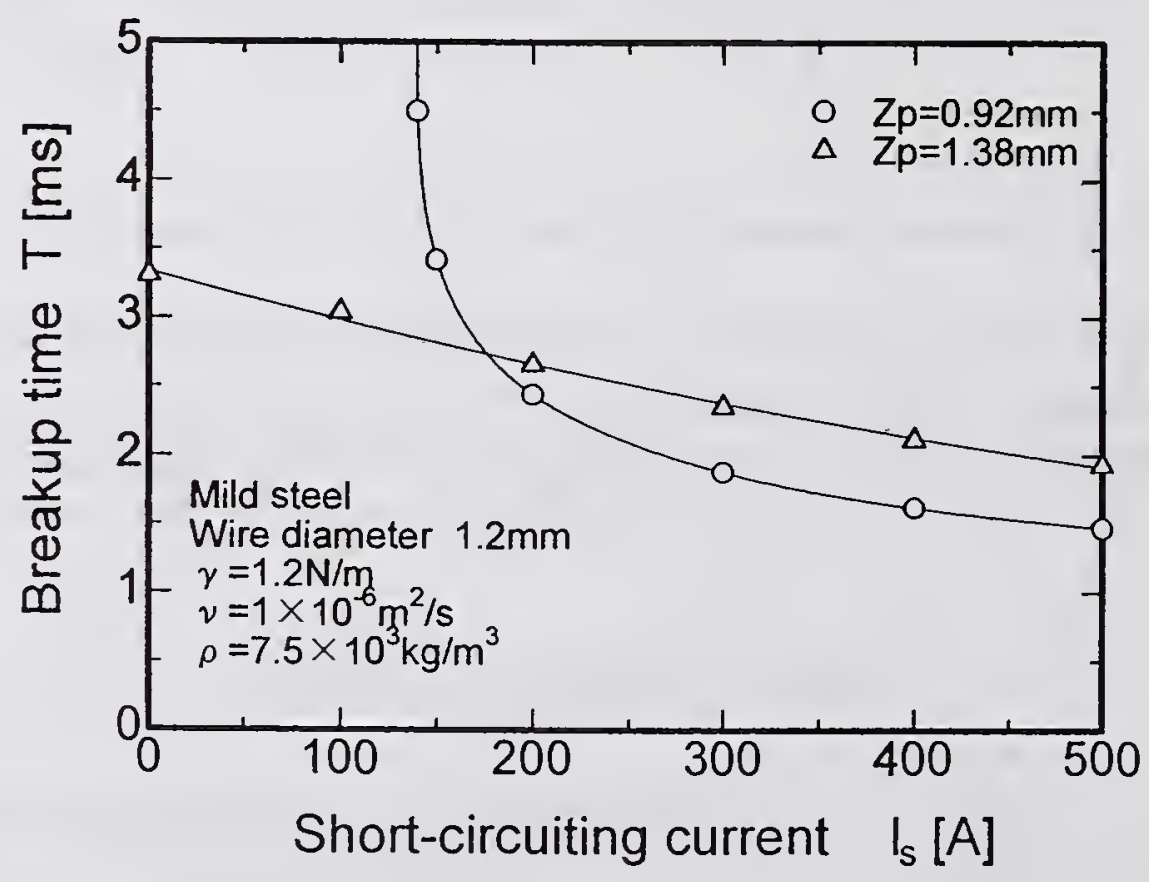

Figure 5. Relationship between short-circuiting current and time required from the instant of contact to breakup of the bridge. 
Figure 6 shows a relationship between the drop height before contact and the breakup time calculated. As seen, breakup of the bridge or bridging transfer occurs with larger size of the drop than the critical drop size which is dependent on short-circuiting current level, diameter of the electrode wire, and surface tension and/or density of the melting metal. In the bridging transfer region, the breakup time increases with increase of drop height.

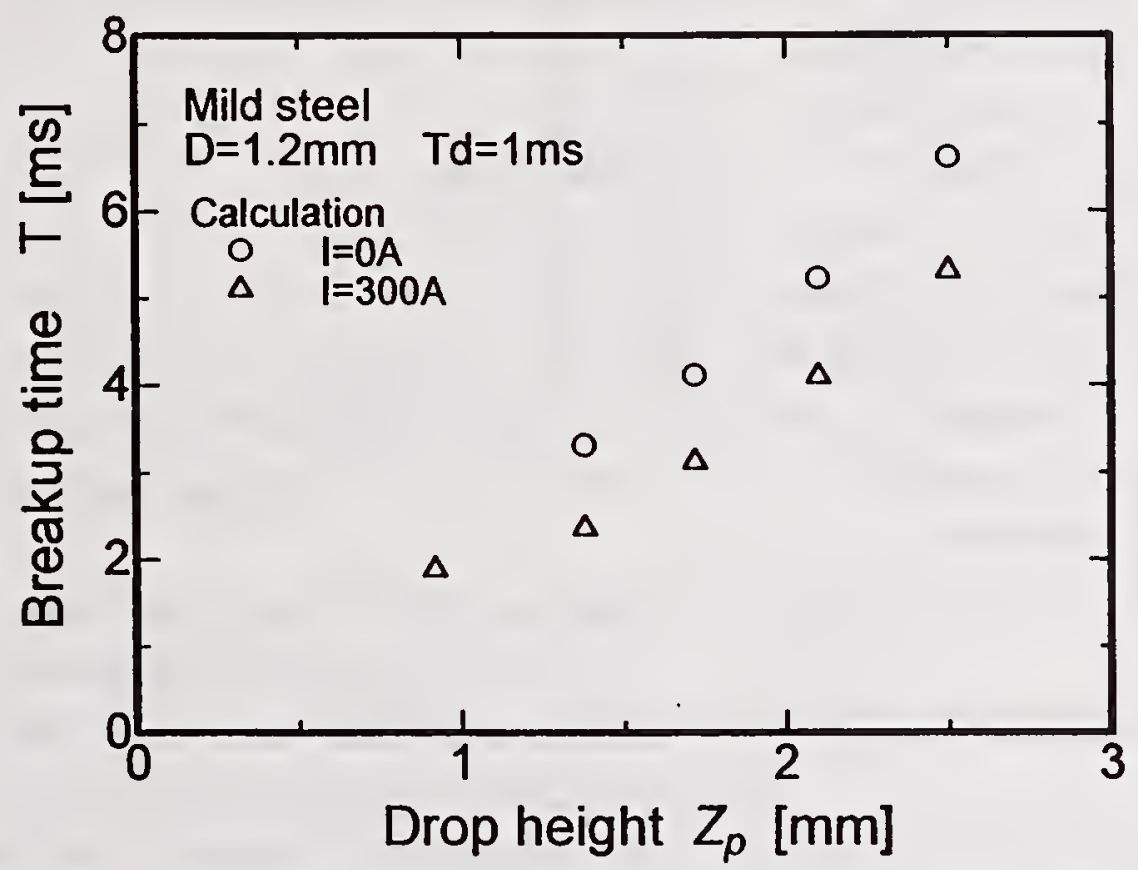

Figure 6. Relationship between drop height before contact with pool and breakup time.

(Mild steel wire $1.2 \mathrm{~mm}$ in dia., $\gamma=1.2 \mathrm{~N} / \mathrm{m}, \rho=7.5 \times 10^{3} \mathrm{~kg} / \mathrm{m}^{3}, \nu=1 \times 10^{-6} \mathrm{~m}^{2} / \mathrm{s}$ )

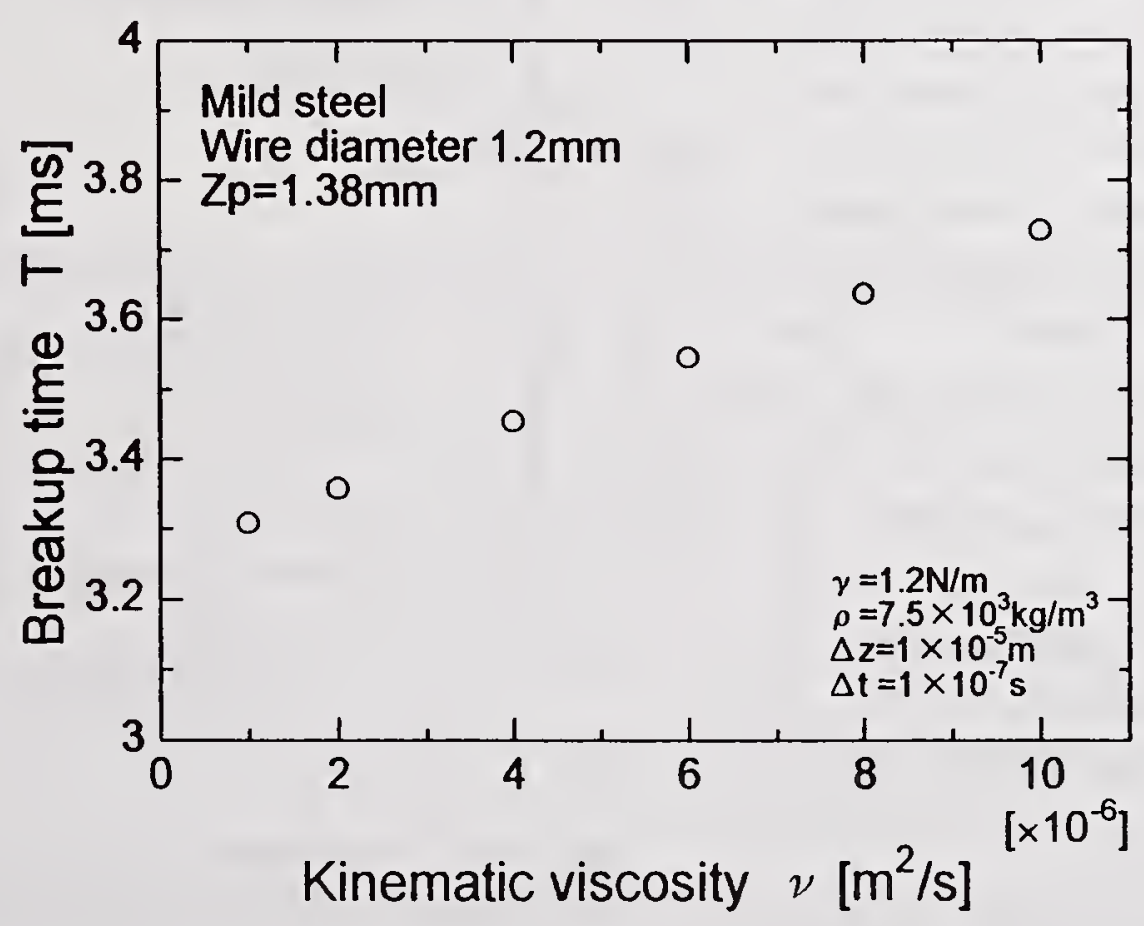

Figure 7. Relationship between kinematic viscosity of liquid metal and breakup time. 
As empirically known, surface tension and/or viscosity of the wire materials are varied with few amounts of chemical components, and affect on periodicity or smoothness of metal transfer in GMA welding.

Figure 7 shows a relationship between kinematic viscosity of the melted metal and the breakup time. As the velocity of liquid motion is lowered by viscous resistance, so increase of kinematic viscosity results in longer time for the breakup.

\section{Liquid volume transferred from drop to pool}

GMA bridging transfer mode welding is cyclic process.

Accordingly, the metal volume transferred per one contact is an important factor to determine the heat input for the electrode wire melting for the next cycle.

Figure 8 shows a relationship between the drop height and the transferred volume. The transferred volume seems to be strongly dependent on the drop size before the contact. It increases with increase of the drop height as shown but the ratio of the transferred volume to the volume of the pendent drop at the wire tip before contact was evaluated to be almost constant value of about $80(\%)$ through the calculation.

In order to examine the

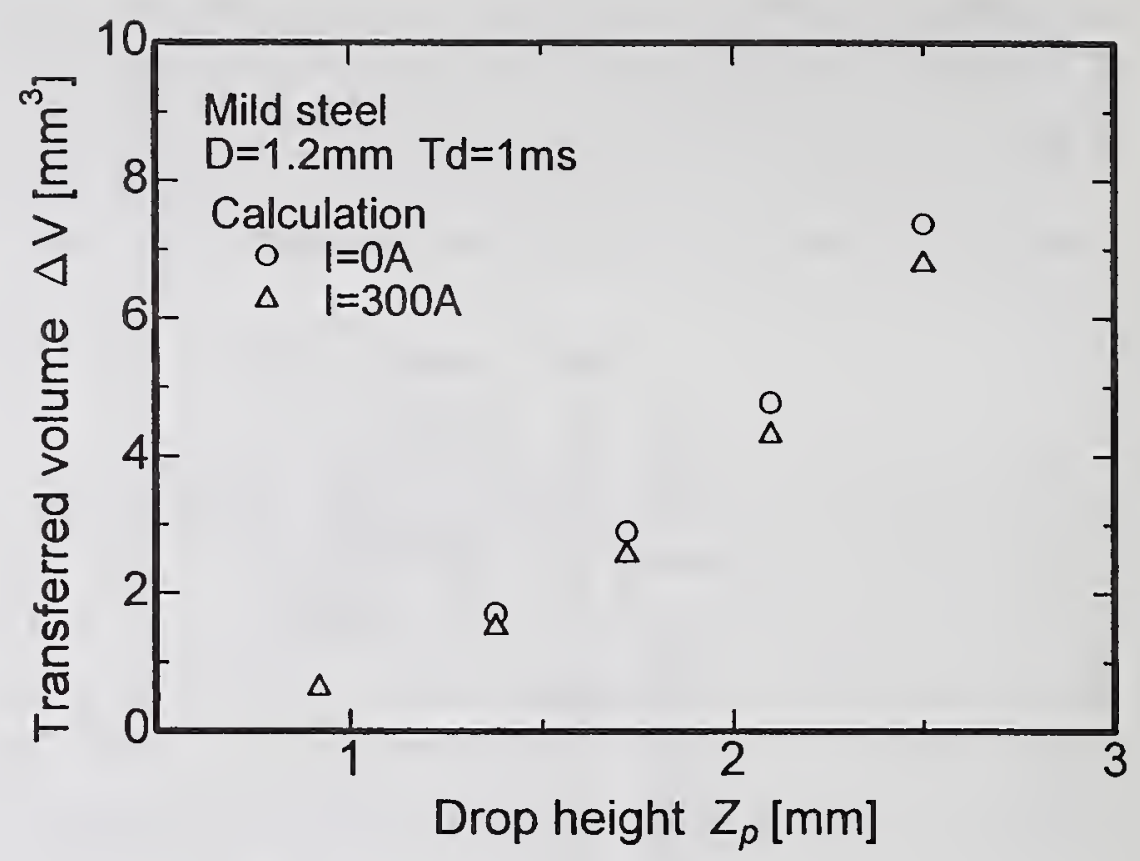

Figure 8. Relationship between drop height before contact with pool and liquid volume transferred from drop into pool.

(Mild steel wire $1.2 \mathrm{~mm}$ in dia., $\gamma=1.2 \mathrm{~N} / \mathrm{m}$, $\left.\rho=7.5 \times 10^{3} \mathrm{~kg} / \mathrm{m}^{3}, \nu=1 \times 10^{-6} \mathrm{~m}^{2} / \mathrm{s}\right)$

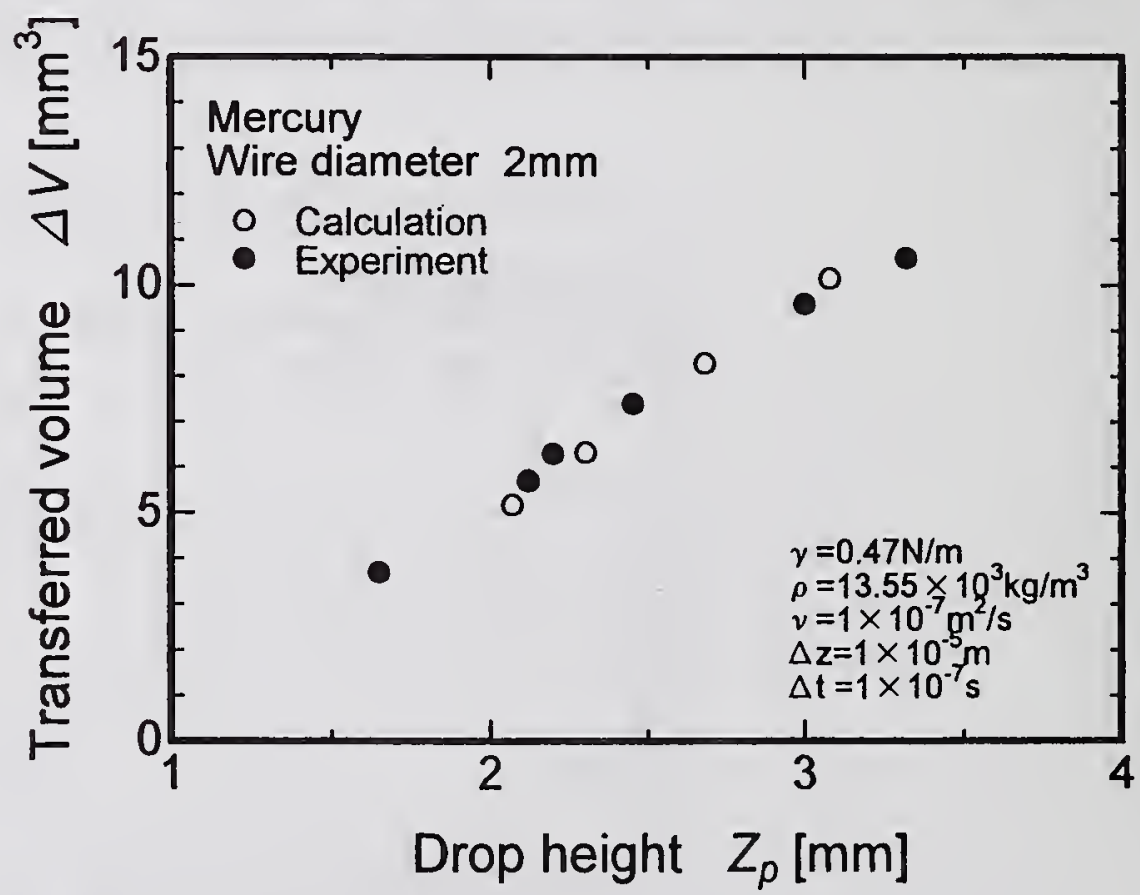

Figure 9. Comparison of calculation with experiment of mercury for liquid volume bridging transferred from pendent drop into pool. 
availability of the prediction by this calculation model, the model experiment was carried out using mercury. The comparison of calculation with experiment for the transferred volume is given in Fig.9. As seen, calculated results agree well with experimental. As for the breakup time the prediction was confirmed to correspond to the experiment. However, from observation of the high speed photography it was found that there exists a little difference in the bridging shape between the calculation and the experiment.

\section{CONCLUSIONS}

Time-dependent deformation of the liquid bridge, which is formed between the electrode wire and the pool by a contact of a pendent drop with the pool surface, is simulated with aids of the simple numerical solution technique based on finite-difference method. Results obtained in this series of research are summarized as follows;

(1)Bridging formation by a contact of the pendent drop with the pool results from the liquid flow induced by the capillary action.

(2)Time-dependent deformation of the bridge shape is varied with the drop size, the start timing and the level of short-circuiting current supplied.

(3)The time required from the contact to the breakup decreases with increasing short-circuiting current that serves electromagnetic pinch force squeezing the bridge. The breakup time is also dependent on the drop size, viscosity and surface tension of the melting metal.

(4)The liquid volume transferred from the drop into the pool depends on the drop size at the electrode wire tip before the contact.

\section{REFERENCES}

1. Maruo,H.;and Hirata,Y. 1989. Current and time required to break up a conducting liquid bridge. IIW Document 212-720-89

2. Maruo,H.;and Hirata,Y. 1990. Bridging transfer of pendent drop. Proc.of 5th Int'l Symp.of Japan Welding Society:471-476

3. Maruo,H.;and Hirata,Y. 1993. Bridging transfer phenomena of conductive pendent drop. IIW Document 212-833-93

4.Hirata,Y.;Osamura,T.;and Ohji,T. 1996. Numerical model of GMA bridging transfer process. IIW Document 212-897-96

5. Lancaster,J.F. 1986. Chap.3,Chap.4,Chap.7. THE PHYSICS OF WELDING : Pergamon Press

6. Cram,L.E. 1984. A numerical model of droplet formation. Computational Tech. \& Appl.(CTAC-83): Elsevier Science Publishers B.V.:182-188

7. Simpson,S.W.;Zhu,Peiyuan;and Rados,M. 1994. Study of the metal transfer process in gas metal arc welding. IIW Document 212-870-94 



\section{MODELING - PHASE TRANSFORMATION}





\title{
CREATING NEW WELDING MATERIALS \\ ON THE BASIS \\ OF METALLURGICAL PROCESSES MODELING
}

\author{
M. Zinigrad“ , N.I. Zalomov", V. Mazurovsky", O. Aksyutin*
}

\begin{abstract}
The main problem of welding technologies is the production of deposited metal (welds, coatings, est.) with required composition and assigned properties. At present time these problems are generally solved empirically, i.e. either by means of technological experiments using existing experimental data.
\end{abstract}

Alloying elements are introduced into welding materials either in the form of pure powders or in the form of iron alloys. Both forms are expensive and fairly difficult to obtain.

It's possibile to switch from the use of the expensive pure components to their oxide compounds which are found in many industrial wastes.

The mathematical modeling of real welding technologies based on the physico-chemical analysis of the interaction between the phases (metal, slag and gas) was employed in the work. The model was based on the fundamental equations of the thermodynamics and kinetics of high-temperature metallurgical reactions and factors which take into consideration the thermal and hydrodynamic conditions of the real process.

The model can be used for the forecast of the final composition of a weld metal or for finding the optimal ratio between the components in the welding materials, which need to obtain the assigned composition and properties of a weld metal.

Such approach permits optimization of the composition of the materials and the technology of the process already at the planning stage with minimal expenditures of time and materials.

\section{KEYWORDS}

Welding, new materials, thermodynamics, wastes, mathematical modelling.

Welding technology is one of the effective methods of worn-out parts restoration and creating on speciment surface a layer providing higher wear and heat resistance and other advantages of the alloy. Various welding materials allowing to obtain required metal properties due to the introduction of alloying additions in various welding materials, namely: electrode coatings, continuous and

\footnotetext{
" College of Judea and Samaria, Ariel, Israel
} 
flux-cored electrode wires and bands, fired and fused fluxes, powder mixtures (Ref.1-3) were used to harden any parts working under the conditions of abrasive and shock-abrasive exposure. In this process the components were introduced into welding materials mainly in the form of not readily available and expensive ferroalloys and alloying compositions, while alloying elements losses during built-up welding reached up to 30-40 percent.

At the same time these elements are included into composition of oxidic compounds which are industrial or ore concentrates and wastes which are sufficiently cheaper.

Thus the use of oxidic compounds with the purpose of extracting alloying elements with the help of oxidizers introduction into flux seems promising because of obvious decrease of the built - up materials cost and opportunity to involve into production local raw materials including various side products and wastes.

In this case the metals - slag interaction will be critical for built - up metal composition which determines its mechanical properties. Usually the degree of chemical processes completeness, both in metallurgy and welding, is defined by chemical analysis of metal samples. This way of searching for rational electrode materials compositions and built up welding technology is sufficiently difficult and time consuming. Therefore the existence of built - up metal composition prediction based on the phases interaction, physical - chemical and mathematical modeling will allow to perform the electrode materials compositions optimization using the modern computers technologies and with minimum of experiments (Ref.4).

Previously (Ref.5) equilibrium metal composition calculations have been carried out using computer. The metal was interacting with the slag containing iron, boron, chromium, vanadium, nickel and tungsten oxides.

In terms of thermodynamics the equilibrium in the metal - slag system can be described by any set of reactions possible with the given composition of the contacting melts. It's most advisable, however, to choose such reactions that contain one common reagent, for instance, $\mathrm{FeO}$. Such an approach allows to take into consideration mutual effects of concurrent processes since equilibrium concentration of iron oxide will be common for them.

Thus the interaction in the metal - slag system can be described by the reaction complex:

$$
\frac{n}{m}[E i]+(F e O)=\frac{1}{m}\left(E i_{n} O_{m}\right)+[F e]
$$

where $E \boldsymbol{E}$ is any element in iron and forming $E i_{n} \boldsymbol{O}_{m}$ oxide.

The number of these reactions will be equal to $\boldsymbol{E} \boldsymbol{i}$ elements number. In equations of (1) type there are $(2 i+1)$ unknown quantities: $i$ of the elements concentrations of the metal, $i$ of their oxides concentrations of the slag and the iron oxide concentration of the slag. 
To solve this problem it's necessary to set up $(2 i+1)$ equations relating independent quantities. Let us write down $i$ equations of equilibrium reagent concentrations relations:

$$
K_{E i}=\frac{\left(E i_{n} O_{m}\right)^{1 / m}}{(F e O)[E i]^{n / m}}
$$

It should be kept in mind that the values of $\boldsymbol{K}_{E i}$ in contrast to equilibrium constants depend not only the temperature but change with the phases compositions. Therefore it's possible to take equilibrium concentrations relationships from the literature sources only in case of similar phase compositions when there are grounds to neglect the change of activity coefficients with the composition.

Thus for the one complex unit, producing element containing melts activity of the elements was calculated on the base of the method described in (Ref.6, 7). Here the system of balance equations is used:

$$
\begin{aligned}
& \sum_{c=0}^{\infty} \sum_{i=c+1}^{\infty} \sum_{t=0}^{i+1-c} \ldots \sum_{k=0}^{i+1-c-t-\ldots-p} N_{i, c, t, \ldots, k}+\sum_{j=1}^{n} N_{o^{2-}}^{j}=1, \\
& \frac{\sum_{c=0}^{\infty} \sum_{i=c+1}^{\infty} \sum_{t=0}^{i+1-c} \ldots \sum_{k=0}^{i+1-c-t-\ldots-p}(i+1-c-t-\ldots-k) N_{i, c, t, \ldots, k}}{1+\sum_{c=0}^{\infty} \sum_{i=c+1}^{\infty} \sum_{t=0}^{i+1-c} \ldots \sum_{k=0}^{i+1-c-t-\ldots-p}(2 i-c) N_{i, c, t, \ldots, k}}=N_{M e^{I} O},
\end{aligned}
$$

$$
\frac{\sum_{c=0}^{\infty} \sum_{i=c+1}^{\infty} \sum_{t=0}^{i+1-c} \cdots \sum_{k=0}^{i+1-c-t-\ldots-p} t N_{i, c, t, \ldots, k}}{1+\sum_{c=0}^{\infty} \sum_{i=c+1}^{\infty} \sum_{t=0}^{i+1-c} \cdots \sum_{k=0}^{i+1-c-t-\ldots-p}(2 i-c) N_{i, c, t, \ldots, k}}=N_{M e^{H} O},
$$

$$
\frac{\sum_{c=0}^{\infty} \sum_{i=c+1}^{\infty} \sum_{t=0}^{i+1-c} \cdots \sum_{k=0}^{i+1-c-t-\ldots-p} k N_{i, c, t, \ldots, k}}{1+\sum_{c=0}^{\infty} \sum_{i=c+1}^{\infty} \sum_{t=0}^{i+1-c} \cdots \sum_{k=0}^{i+1-c-t-\ldots-p}(2 i-c) N_{i, c, t, \ldots, k}}=N_{M e^{k} O}
$$


where polarization constants for binary systems $\mathrm{FeO}-\mathrm{SiO}_{2}$ is $0.9, \mathrm{MnO}-\mathrm{SiO}_{2}$ is $0.2, \mathrm{MgO}-$ $\mathrm{SiO}_{2}$ is $0.03, \mathrm{CaO}-\mathrm{SiO}_{2}$ is $0.003, N_{i}$ - ions concentrations.

Table represents ion composition and components activity for melts of $\mathrm{FeO}-\mathrm{MnO}-\mathbf{M g O}$ $\mathrm{CaO}-\mathrm{SiO}_{2}$ type, which are to the highest extend close to welding fluxes.

Table 1. Ions concentrations and activities $\mathrm{FeO}$ in the $\mathrm{FeO}-\mathrm{MnO}-\mathrm{MgO}-\mathrm{CaO}-\mathrm{SiO}_{2}$ at $1873 \mathrm{~K}$

\begin{tabular}{|c|c|c|c|c|c|c|c|c|c|}
\hline \multicolumn{5}{|c|}{ Composition of Slag, $\%$} & \multirow[b]{2}{*}{$N_{S i O_{4}^{4-}}^{I}$} & \multirow[b]{2}{*}{$N_{o^{4-}}^{I}$} & \multirow[b]{2}{*}{$N_{o^{4-}}^{I I}$} & \multirow[b]{2}{*}{$N_{o^{4-}}^{I I I}$} & \multirow{2}{*}{$\begin{array}{l}a_{\mathrm{FeO}} \\
(\operatorname{Ref} .8)\end{array}$} \\
\hline $\mathrm{CaO}$ & $\mathrm{SiO}_{2}$ & $\mathrm{FeO}$ & $\mathrm{MgO}$ & $\mathrm{MnO}$ & & & & & \\
\hline 16.78 & 5.73 & 67.79 & 4.71 & 2.39 & $2.3210^{-4}$ & 0.745 & 0.026 & $7.4410^{-2}$ & 0.765 \\
\hline 0.32 & 2.52 & 62.24 & 1.82 & 33.01 & $2.3010^{-3}$ & 0.633 & 0.312 & $2.0910^{-2}$ & 0.581 \\
\hline 0.34 & 13.74 & 42.48 & 5.68 & 37.88 & $1.0110^{-2}$ & 0.490 & 0.248 & $2.4910^{-2}$ & 0.477 \\
\hline 0.28 & 22.12 & 28.63 & 12.76 & 36.47 & $4.3910^{-3}$ & 0.410 & 0.299 & $5.4910^{-2}$ & 0.396 \\
\hline
\end{tabular}

Calculations for oxide melts - metals and gas interaction modeling are based on thermodynamical equilibrium constants and oxide melts components activity values.

Also let us write down i equations of material balance for every element taking part in the reactions while bearing in mind that the element concentration changes in the metal and their oxides concentration change in the slag correspond to the stoichiometry of the forces under study:

$$
\begin{gathered}
\frac{m_{M e O}}{100 n M_{E i}}\left([E i]_{0}-[E i]\right)=\frac{m_{S l}}{100 M_{E i_{n} O_{m}}}\left[\left(E i_{n} O_{m}\right)-\left(E i_{n} O_{m}\right)_{0}\right] \\
\frac{m_{M e}}{100} \sum_{i=1}^{t}\left(\frac{n}{m} \frac{[E i]_{0}-[E i]}{M_{E i}}\right)=\frac{K m_{S l}}{100 M_{F e O}}\left[(F e O)_{0}-(F e O)\right]
\end{gathered}
$$

where it's taken into consideration that the sum of all elements moles quantities changes (meaning the elements taking part in the reactions(1)) equals the iron oxides moles quantities changes spent on the oxidation of these elemints.

Here $\boldsymbol{m}_{M e}$ and $\boldsymbol{m}_{\boldsymbol{s} \boldsymbol{l}}$ are masses of the metal and the slag, respectively; $M_{E i}, M_{E i_{n} o_{m}}$ and $M_{F e O}$ are atomic and molecular masses of the element, its oxide and iron oxide, respectively;

$[E i]_{0},\left(E i_{n} O_{m}\right)_{0},(F e O)_{0}$ and $[E i],\left(E i_{n} O_{m}\right),(F e O)$ are initial and equilibrium components concentrations, respectively; 
$t$ is quantity of the reactions of (1) type.

As it can be seen from equations (7) and (8), to make calculations it is important rather to know the liquid metal mass / slag relationship which should be present in terms of specific technological peculiarities of welding process, then to know absolute quantities of the said masses.

The calculation results of equilibrium metal and slag compositions for various conditions of their interaction are in good agreement with the experimental results (Ref.5). The suggested method is suitable to evaluate the completeness and flow direction of chemical reactions which determine the compositions of metal and oxidic melts. Even at this stage of the analysis it is possible to select the components concentration regions taken as a base of the alloying flux composition.

Therefore the estimation of build - up metal composition, formed in a real technological process, was necessary to choose a rational flux composition.

Advanced metal composition calculation methods for different technologies of welding and build up welding have been developed (Ref.4,9).

System of equations $(2-10)$ can be considered as the mathematical model of the chemical processes in welding. Initial input data for the calculation were: the melt and slag baths compositions, welding mode parameters, effective heating efficiency, thermal conductivity, heat capacity per unit volume, effective thermal capacity and parameters required for the physicochemical constants calculation. Output parameters were the compositions of weld metal and slag.

The metal composition calculation cited in (Ref.10) on the built - up welding with flux was carried out to check the adequacy of the developed mathematical model.

The mass of the molten metal was determined from the relationship (Ref. 11):

$$
m_{M e}=\left[F_{p}+F_{d}\right] V_{W} \gamma \tau
$$

where $F_{p}=\frac{U_{a} I_{W} \eta \eta_{T}}{V_{W} \gamma \Delta H_{m}}$ is the penetration area of the base metal; $F_{d}=\frac{K_{H} I_{W}}{V_{W} \gamma 3600}$ is the area of the deposited bead.

Here $\boldsymbol{U}_{\boldsymbol{a}}$ - arc voltage, $\mathrm{V} ; \boldsymbol{I}_{\boldsymbol{w}}$ - welding current, A; $\boldsymbol{V}_{\boldsymbol{w}}$ - welding velocity, $\mathrm{cm} / \mathrm{s} ; \boldsymbol{\gamma}$ - metal density, $\mathrm{g} / \mathrm{cm}^{3} ; \Delta \boldsymbol{H}_{\boldsymbol{m}^{-}}$specific melting enthalpy, $\mathrm{J} / \mathrm{g} ; \boldsymbol{\eta}_{\boldsymbol{T}}$ - thermal efficiency of penetration, assuming that the heat fraction consumed for the base metal melting was taken into consideration; $\eta$ - effective heating efficiency of the source, assuming that actual heat input into the speciment differed from the calculated patterns.

Having calculated the relative mass of the flux (Ref.9) : 


$$
K_{f}=17.8 I_{W}^{-1.43} U_{a}^{1.28} d^{0.72}
$$

where $d$ is the electrode wire diameter, one can determine the melted slag mass:

$$
m_{S l}=K_{f} m_{M e}
$$

The suggested method was used to develop the fired flux combination containing carbonic chromium, tungsten and vanadium containing slags. The aim was to obtain build - up metal alloyed with tungsten and vanadium with high content of carbon and chromium. High wear resistance of the alloys containing these elements working at both ambient and high temperatures with exposure to abrasive effects was convincingly demonstrated in (Ref.10). Lately, however, the output of the said electrode materials was decreasing due to the shortage and high cost of the main component. It seems promising to use relatively cheap materials such as tungsten and vanadium containing slags, carbonic chromium as flux components.

Mathematical model of the chemical processes occurring in submerged - arc built up welding has been developed, the program allowing to calculate metal and slag compositions depending on the process modes and initial input electrode material compositions has been prepared and debuged. A computer program based on the model developed for physicochemical interaction of the phases during welding was created for PC computers in Microsoft EXCEL 5.0 for WINDOWS software.

The rational composition of fired flux was chosen with the help of the program.

Furthermore the set up mathematical model allows to dame with computer different variants of technology effecting in such a way their optimization prior to their commercial testing. "Technology games" played in terms of the given variant of the mathematical model of built - up welding allow to resolve quite a lot of particular problems, namely built - up welding models optimization, choice of the electrode materials optimal composition, obtaining the built up metal with assigned composition and properties etc.

\section{REFERENCES}

1. Frumkin I. I. 1961. Automatical Electro-Arc Build-uping. 421 Kharkov: Mettalurgizdat.

2. Livshits L. S., Grinberg N. A., Kurkumelly E. S.Principles of Build-up Metal Alloying .188 Moscow: Mashinostroyenie.

3. Built-up Welding Materials of COMECON Countries Catalog 1979609 Kiev: MTSNTI.

4. Popel S.I., Sotnikov A. I., Boronenkov V. N. 1986. Theory of Metallurgical Processes: Moskow: Metallurgy.

5. Zinigrad M. I., Okolzdaev A. G., Flyagin A. A. Influence of Phase Composition and Temperature on the Equilibrium Distribution of Tungesten Bewteen Metal and Slag Melts. Rasplavy 5(1): 7-s to $27-\mathrm{s}$. 
6. Senday, 1992. Application of the Polymer Theory to Multicomponent Silicate and Alumosilicate Melts. Proc. 4th International Conference on Molten Slags and Fluxes. N. I. Zalomov, V. N. Boronenkov: 91 - 96. The Iron and Steel Inst. of Japan.

7. Zalomov N. I.; Boronenkov V. N. 1992. Activities and Ionic Composition Calculation in Multicomponent Silicate. Rasplavy 3: 33-s to 40-s.

8. Elliot D. F., Glayzer M., Ramacrichna V. 1969 Thermochemical Steel Processes : 252 Moscow: Metallurgy.

9. Shalimov M. P., Fefelov A. S., Zinigrad M. I. 1979. Metal Composition Calculation after Hardsurfacihg under Ceramic Flux.Avtomaticheskaya svarka 1.33 - $\mathrm{s}$ to 38 - $\mathrm{s}$.

10. Frolov V. V. 1988 Welding Processes Theory. 559 Moscow: Vischaya shcola. 


\title{
MICROSTRUCTURES IN THE HEAT-AFFECTED ZONES BASED ON DILATOMETER DATA
}

\author{
E. A. Metzbower, G. Spanos, R. W. Fonda, R. A. Vandermeer
}

\begin{abstract}
The objective of this study is to model the evolution of the microstructure and the resultant hardness in the heat affected zone (HAZ) of HSLA-100 steel weldments. The experimental HAZ results of Spanos et al., where four different weld thermal cycles, with two peak temperatures $\left(1400\right.$ and $\left.900{ }^{\circ} \mathrm{C}\right)$ and two cooling rates $\left(5\right.$ and $\left.60^{\circ} \mathrm{C} / \mathrm{s}\right)$, were emulated in a heating/quenching dilatometer, are compared to the results of three weld simulation models for the decomposition of austenite. The three models are those of Kirkaldy and co-workers, Ion, Easterling and Ashby, and Bhadeshia and co-workers. All of the models require the chemical composition, a thermal profile, and an austenite grain size measurement. All of the models gave excellent results for the fast cooling rates. Bhadeshia's model gave good results for one of the slower cooling rates, but not the other. Neither Kirkaldy's nor Ion et al. 's model were satisfactory for the slower cooling rate cases.
\end{abstract}

\section{INTRODUCTION}

The excellent mechanical properties of high strength, low alloy (HSLA) steels are achieved at low carbon (less than about $0.08 \mathrm{pct}$ ) content by the presence of fine precipitates of metal carbonitrides and copper. HSLA steels have the potential for being welded with little or no preheat, and with less stringent process control, thus significantly reducing fabrication cost (Ref. 1). The thermal cycling within the heat-affected zone (HAZ) as a result of the welding process has the potential to significantly modify the size and distribution of the austenite decomposition phases, the precipitates, and consequently the properties.

Spanos et al. (Ref. 2) have utilized a heating/quenching dilatometer to simulate the thermal cycles in the HAZ of HSLA-100 steel. In these experiments they used four peak temperatures $(675,750$, 900 and $1400{ }^{\circ} \mathrm{C}$ ) and two cooling rates $\left(5\right.$ and $60^{\circ} \mathrm{C} / \mathrm{s}$ from 800 to $500{ }^{\circ} \mathrm{C}$ ) to simulate the thermal cycles in the HAZ. They measured the prior austenite grain size, hardness and microstructure for each peak temperature and cooling rate. The microstructures were identified by extensive transmission electron microscopy, supplemented by optical microscopy, dilatometric analysis, and hardness measurements.

The experimental data obtained by Spanos et al. (Ref. 2) will be directly compared in this paper to the predictions of three models of the decomposition of austenite. Since the models assume that the alloy is completely austenite in the initial condition only the thermal cycles from two peak temperatures $\left(900\right.$ and $1400{ }^{\circ} \mathrm{C}$ ) will be analyzed; the peak temperatures of 675 and $750{ }^{\circ} \mathrm{C}$ resulted in only sub or partially re-austenization. Based on the Ac3 temperature $\left(770{ }^{\circ} \mathrm{C}\right)$ calculated from the composition by the formula from Andrews (Ref. 3), $900{ }^{\circ} \mathrm{C}$ is sufficient to completely reaustenitize the steel. This is confirmed by the data of Wilson et al. (Ref. 4) who show an Ac3 temperature of $825^{\circ} \mathrm{C}$ on their continuous cooling curve of a similar composition steel. The heating portion of the dilatometric data also indicated the complete transition from ferrite to austenite. Optical micrographs of a specimen subjected to each of the peak temperatures and cooling rates analyzed in this paper are shown in Fig. 1 Optical microscopy of these microstructures is unable to reveal the

U. S. Naval Research Laboratory, Physical Metallurgy Branch, Code 6320, Washington DC 20375-5320 USA 

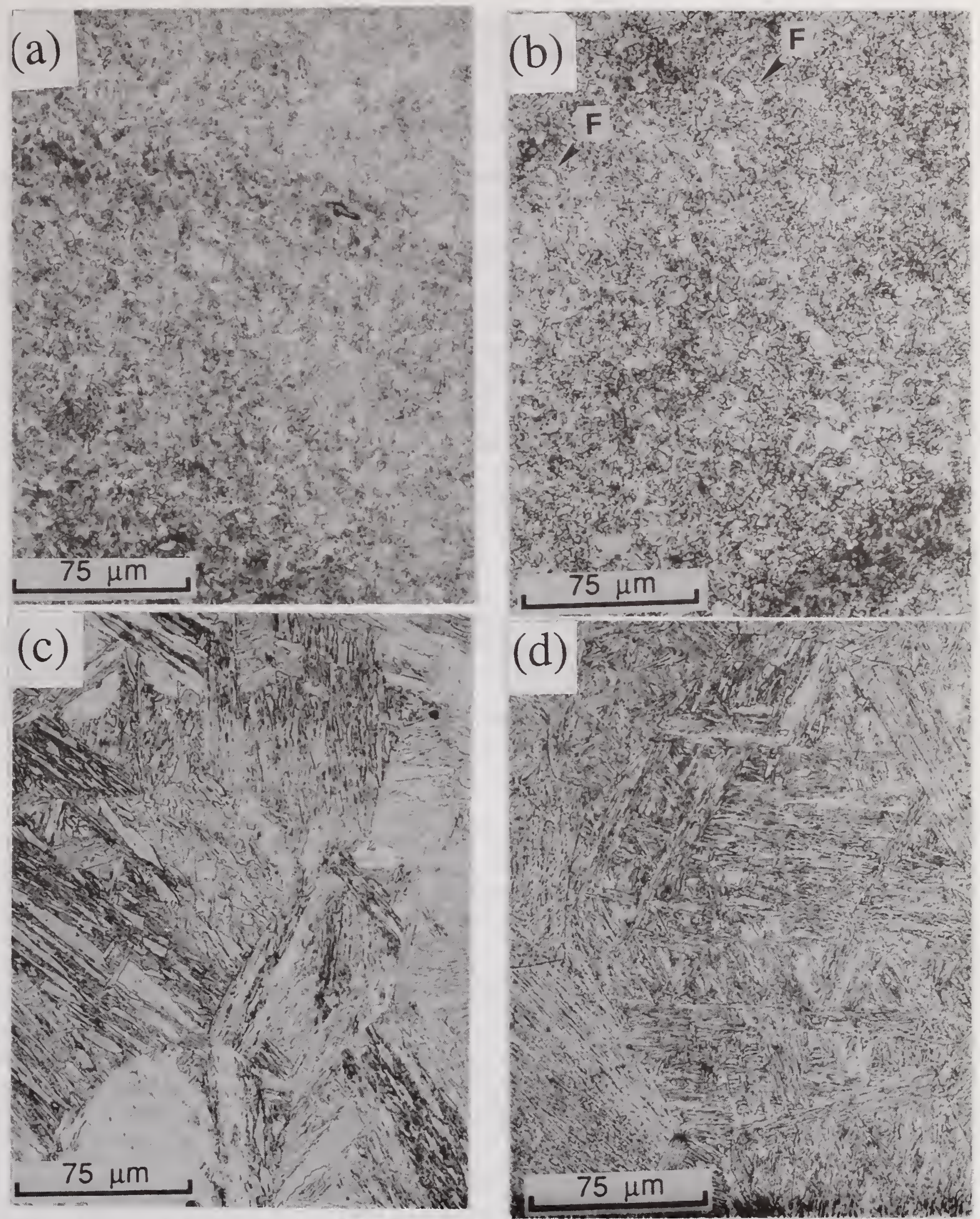

Fig. 1 Optical micrographs of HSLA100 specimens from the dilatometer subjected to the following thermal cycles: (a) $\mathrm{T}_{\mathrm{p}}=900^{\circ} \mathrm{C}, \mathrm{CR}=60^{\circ} \mathrm{C} / \mathrm{s}$; (b) $\mathrm{T} \mathrm{p}=900^{\circ} \mathrm{C}, \mathrm{CR}=5^{\circ} \mathrm{C} / \mathrm{s}$; (c) $\mathrm{T}_{\mathrm{p}}=1400^{\circ} \mathrm{C}, \mathrm{CR}=5^{\circ} \mathrm{C} / \mathrm{s}$; and (d) $\mathrm{T}_{\mathrm{p}}=1400^{\circ} \mathrm{C}, \mathrm{CR}=60^{\circ} \mathrm{C} / \mathrm{s}$. 
difference between the various forms of martensite, lath ferrite, and acicular ferrite. In Fig. 1b, equiaxed ferrite is indicated by the arrows. The details of the extensive microstructural

determination in the paper by Spanos et al. (Ref. 2) will not be reiterated in this paper, only the pertinent results will be stated.

\section{MODELS OF THE DECOMPOSITION OF AUSTENITE}

The three models for the decomposition of austenite into her daughter products are those of 1) Kirkaldy and co-workers, 2) Ion, Easterling, and Ashby, and 3) Bhadeshia and co-workers.

Kirkaldy et al. (Ref. 5-9) have developed hardenability algorithms that have been used by Watt $e t$ al. (Ref. 10) and Henwood et al. (Ref. 11) to calculate the volume fractions of the constituents of the microstructures in the HAZ of a microalloyed steel. In order to use these algorithms, the eutectoid temperature $\left(\mathrm{Ae}_{\mathrm{e} 1}\right)$, the $\mathrm{A}_{\mathrm{e} 3}$ temperature, the ferrite solubility temperature as a function of carbon, and the bainite and martensite start temperatures are required as inputs. These are usually estimated empirically from the composition of the alloy. Kirkaldy et al. (Ref. 5-9) have developed a set of equations which model the austenite decomposition reactions for each of the diffusional products of austenite. These equations assume that a single continuous function can describe both the nucleation and subsequent growth for each of the daughter phases. For each reaction, the general reaction rate is characterized as

$$
\frac{\mathrm{dX}}{\mathrm{dt}}=\mathrm{B}(\mathrm{G}, \mathrm{T}) \mathrm{X}^{m}(1-\mathrm{X})^{p}
$$

where $\mathrm{X}$ is the volume fraction of the daughter product, $\mathrm{B}$ is an effective rate coefficient which depends on $\mathrm{T}$, the temperature, and $\mathrm{G}$, the austenite grain size. The semi-empirical coefficients, $m$ and $p$, are set to less than one to assure convergence in a form that is derived from a point nucleation and impingement growth model (Ref. 12). $B(G, T)$ is a different function for each reaction product and expresses an empirical chemical dependence for each reaction. The explicit form of $\mathrm{B}(\mathrm{G}, \mathrm{T}), m$ and $p$ are stated by Watt et al. (Ref. 8) and will not be repeated here. The result of this algorithm is that the volume fraction of ferrite, pearlite, bainite, and martensite can be calculated. It should be noted that $\mathrm{B}$, the effective rate coefficient, and the semi-empirical parameters $m$ and $p$ were determined from data from the heat treatment of steels which usually have a higher carbon content than the weldable steels to which the equations are being applied.

Ion, Easterling and Ashby (Ref. 13) developed algorithms leading to models and calculation methods for precipitate dissolution, precipitate coarsening, grain growth and martensite formation in the HAZ. They calibrated their kinetic models against data from real welds and presented diagrams with axes of weld input energy and distance below the weld center line. They described a semi-empirical method for predicting the microstructure and hardness of the HAZ as a function of carbon-equivalent. Ion et al. (Ref. 13) used the data of Inagaki and Sekiguchi (Ref. 14) who obtained continuous cooling transformation diagrams for a wide range of structural steels. Inagaki and Sekiguchi (Ref.14) were able to determine that the volume fractions of the various phases depended on the time to cool from 800 to $500{ }^{\circ} \mathrm{C}$ and the carbon equivalent of the different steels. Ion et al. (Ref. 13) used this data to calculate the cooling time needed for a $50 \%$ martensite/50\% bainite microstructure, and for a $50 \%$ bainite $/ 50 \%$ ferrite/pearlite microstructure in terms of the carbon equivalent. They then used a form of the Johnson-Mehl equation (Ref. 15) to calculate the volume fraction of martensite and subsequently bainite and ferrite/pearlite. This equation may be written as

$$
V_{M}=\exp -\left\{0.69\left(\frac{\Delta t}{\Delta t_{1 / 2}^{M}}\right)^{2}\right\}
$$


where $V_{M}$ is the volume fraction of martensite after a time $\mathrm{Dt}$ (characterizing the cooling part of the weld cycle) and $\Delta t_{1 / 2}^{M}$ is the time required for half of the bainite/ferrite/pearlite transformation to occur and is a function of the carbon equivalent of the steel. Similar expressions were developed for the volume fractions of bainite. The volume fraction of ferrite/pearlite was taken to be one minus the sum of the volume fraction of martensite plus the volume fraction of bainite. Ion, Salminen, and Sun (Ref. 16) have recently modified the formula for the carbon equivalent in a study of the laser welding of carbon-manganese steels.

Bhadeshia et al. (Ref. 17-19) have developed a semi-empirical model for the decomposition of austenite based on thermodynamics. The model calculates the transformation start time for a timetemperature-transformation diagram for the steel. The original model developed for the fusion zone of weldments assumed an hexagonal prism to be a good representation of the grain shape resulting from epitaxial growth and heat flow in welding. Allotriomorphic ferrite is calculated based on the parabolic growth law. The volume fraction of allotriomorphic ferrite is then calculated taking into consideration the hexagonal prism morphology of the grains. Widmanstätten ferrite is then calculated based on Trivedi's analysis of the growth of parabolic cylinders, taking into account the strain energy associated with the transformation mechanism (Ref. 18). There are no models which allow the volume fraction of acicular ferrite to be calculated from first principles. For many welds it is still possible to estimate the volume fraction of acicular ferrite $\left(\mathrm{V}_{a}\right)$ by the remainder of the equation

$$
\mathrm{V}_{a}=1-\mathrm{V}_{a}-\mathrm{V}_{w}-V_{m}
$$

where $V_{a}$ is the volume fraction of allotriomorphic ferrite, $V_{w}$ is the volume fraction of Widmanstätten ferrite, and $V_{m}$ is the volume fraction of the microphases, martensite and retained austenite, which are calculated by the algorithm proposed by Takahashi and Bhadeshia (Ref. 20).

For all three models the hardness is estimated based on the rule of mixtures and the hardness of each phase as calculated by Maynier et al. (Ref. 21), based on the chemical composition and the cooling rate at $700{ }^{\circ} \mathrm{C}$.

None of the three models were directly applicable to the thermal profile prescribed by the heating/quenching dilatometer and thus had to be modified to some degree. Kirkaldy and Venugopalan (Ref. 5) demonstrated the applicability of their model to a Jominy bar, where the thermal profile is a function of time and distance in the bar. A computer program originally duplicated their results and then was tested by using the same cooling times from 800 to $500{ }^{\circ} \mathrm{C}$ and obtaining very similar results. The model of Ion et al. (Ref. 13) included using a modification of the Rosenthal (Ref. 22, 23) equation to describe the extended heat source and the "kinetic strength" of the thermal cycle. The kinetic strength of the thermal cycle, a concept developed by Ion et al. (Ref. 13) to represent the strength of the heating and cooling cycle, was determined by numerical integration of the heating/quenching thermal profile from the dilatometer. Allowance for grain growth was also utilized based on the austenite grain size data of Spanos et al. (Ref. 2). A third computer program was developed independently based on the paper by Bhadeshia et al. (Ref. 17) and its references and applied to the welding conditions in that paper. In order to apply the program to the thermal cycles from the dilatometer, the cooling curve was fitted to the formulation used by Bhadeshia et al. (Ref. 17). The hexagonal prism grain size was modified so that the c/a ratio was one and was more representative of the grain morphology in the HAZ. Copper was not an element considered in the original formulation by Bhadeshia et al. (Ref. 17) but was added by including the magnetic and non-magnetic contribution (Ref. 24) to the free energy change from austenite to ferrite. 


\section{EXPERIMENTAL VALUES}

An HSLA-100 steel of composition given in Table 1 was used by Spanos et al. (Ref. 2) for the dilatometry study. Table 2 gives the prior austenite grain size and the hardness values for each of the four thermal profiles. HSLA steels obtain their strength through the formation of precipitates, in the case of HSLA-100 the primary precipitates are a fine dispersion of $\varepsilon$-copper and carbonitrides (Ref. 2). The microstructure of the as-received, commercially processed steel was a mixture of tempered martensite and some ferrite. The minimum yield strength of HSLA-100 is $690 \mathrm{MPa}(100 \mathrm{ksi})$. This steel also has very good fracture toughness. The austenite fraction, determined by the change in length of the specimen as a function of temperature, is given in Fig. 2 for the $60^{\circ} \mathrm{C} / \mathrm{s}$ curves and in Fig. 3 for the $5{ }^{\circ} \mathrm{C} / \mathrm{s}$ curves for each peak temperature. The two cooling rates were chosen to reflect the fastest and slowest rates expected in the HAZ from 800 to $500{ }^{\circ} \mathrm{C}$.

Table 1 Composition of HSLA-100 Steel

$\begin{array}{lllllll}\mathrm{C} & \mathrm{Si} & \mathrm{Mn} & \mathrm{Ni} & \mathrm{Cr} & \mathrm{Mo} & \mathrm{Cu} \\ 0.07 & 0.37 & 0.80 & 3.49 & 0.57 & 0.58 & 1.61\end{array}$

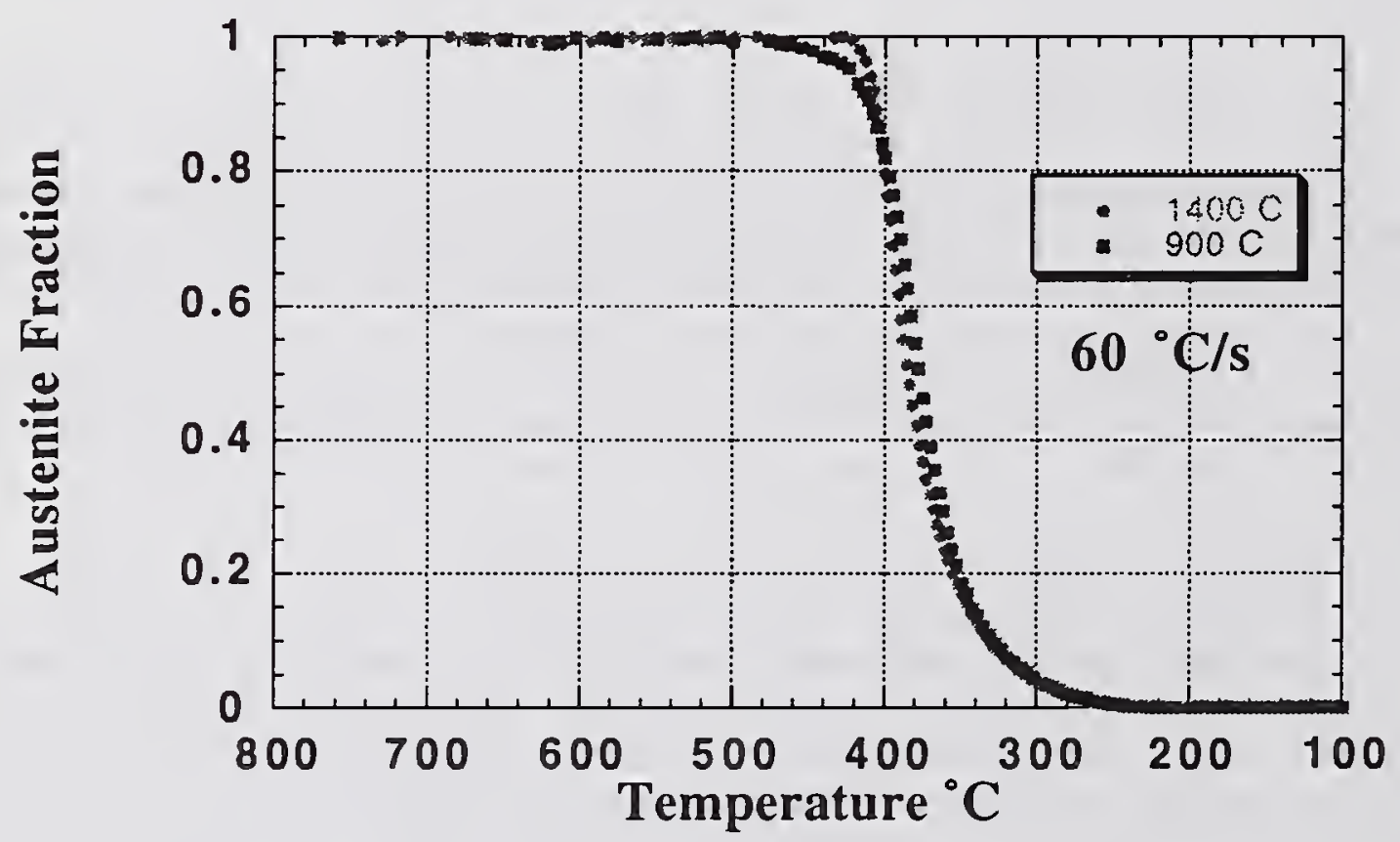

Fig. 2 Austenite fraction as a function of decreasing temperature for the cooling rate of $60^{\circ} \mathrm{C} / \mathrm{s}$.

Table 2 Prior Austenite Grain Size and Hardness Values

$\begin{array}{llll}\text { Peak Temperature }{ }^{\circ} \mathrm{C} & \text { Cooling Rate, }{ }^{\circ} \mathrm{C} / \mathrm{s} & \begin{array}{l}\text { Prior Austenite } \\ \text { Grain Size, microns }\end{array} & \text { Hardness, VHN } \\ \text { Base Plate } & & 11 & 270 \\ 1400 & 60 & 62 & 365 \\ 900 & 60 & 6 & 390 \\ 1400 & 5 & 54 & 365 \\ 900 & 5 & 6 & 315\end{array}$




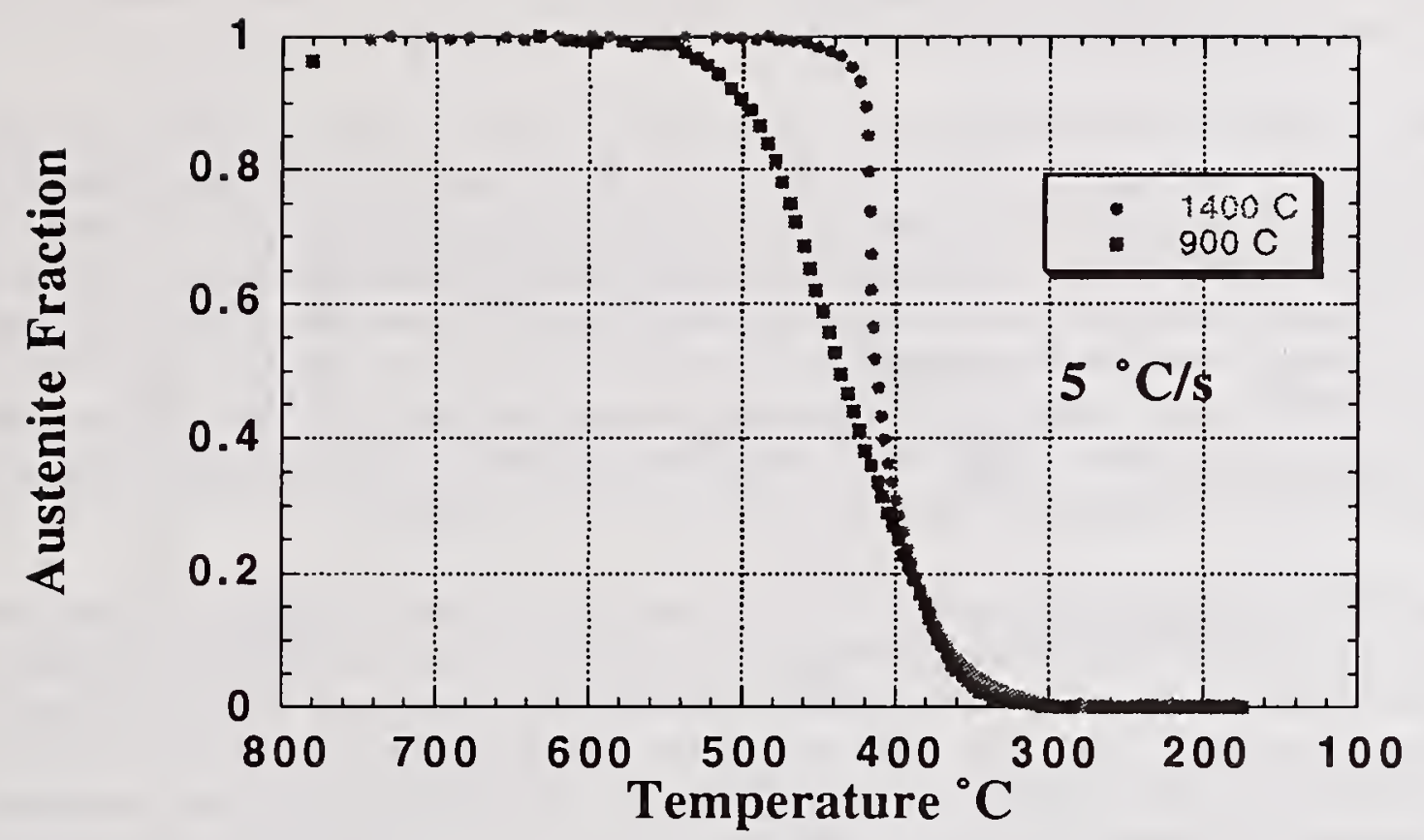

Fig. 3 Austenite fraction as a function of decreasing temperature for the cooling rate of $5{ }^{\circ} \mathrm{C} / \mathrm{s}$.

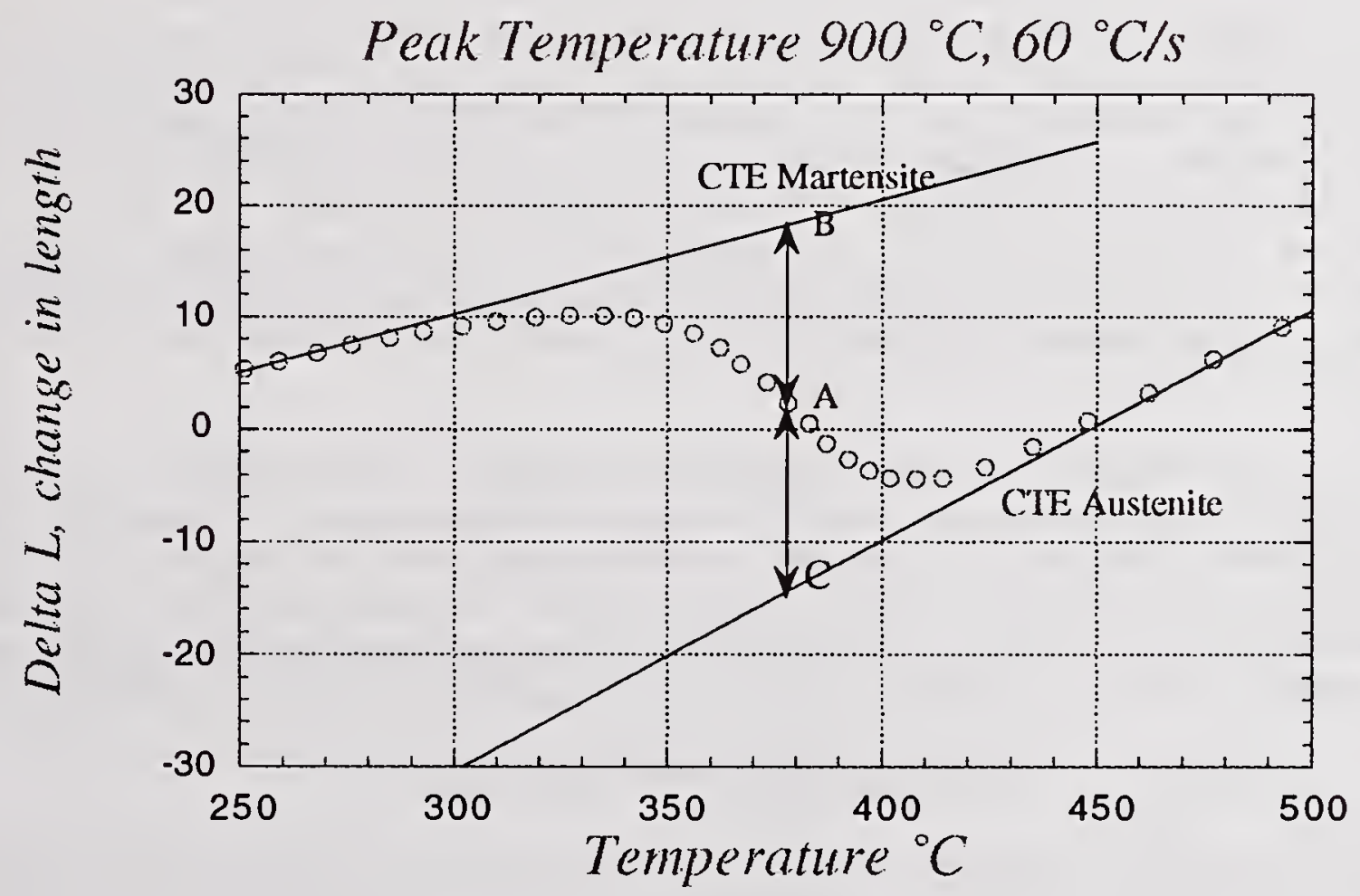

Fig. 4 Trace from the dilatometer showing the change in length of the specimen as a function of time.

The martensite fraction was determined using the method of Eldis (Ref. 25). Fig. 4 is a portion of the experimental dilatometer data from the peak temperature of $1400{ }^{\circ} \mathrm{C}$ and a cooling rate of 60 
${ }^{\circ} \mathrm{C} / \mathrm{s}$. The coefficients of thermal expansion (CTE austenite and CTE martensite) are the slopes of the solid curves and were determined from a linear least squares fit to the data. At any temperature the amount of austenite and the transformed product (martensite) can be calculated. Such a calculation is shown at the temperature denoted by the vertical line $\mathrm{BC}$, where the ratio of $\mathrm{AC}$ to $\mathrm{BC}$ is the volume fraction of martensite.

The optical microstructure of HSLA100 steel is very fine as shown in Fig. 1. The details of the microstructure can not be delineated at this resolution and it is only by utilizing transmission electron microscopy that the microstructure can be described. Even at the resolution of the electron microscope and employing selected area diffraction, it is difficult to distinguish between martensite and lath ferrite. The volume fractions were determined primarily from the dilatometer and the martensite start temperature of $420^{\circ} \mathrm{C}$. Analysis of the dilatometer traces as shown in Fig. 2 \& 3 indicate the volume fraction of austenite that had transformed above the $\mathrm{M}_{\mathbf{S}}$. The remaining austenite at the $\mathrm{M}_{\mathrm{S}}$ was assumed to transfer to martensite. Transmission electron microscopy was used to identify the actual microstructure that had transformed above the $\mathrm{M}_{\mathrm{S}}$.

In some cases the microstructural determinations by Spanos et al. (Ref. 2) differed significantly from the prediction of the continuous cooling diagram of Wilson et al. (Ref. 4) which is shown in Fig. 5. The cooling dilatometer curves are drawn such that time zero is set when the calculated $\mathrm{A}_{\mathrm{c} 3}$ of $770{ }^{\circ} \mathrm{C}$, as determined from the composition and the formula of Andrews (Ref. 3), is reached. These differences are attributed to the difference in austenite grain size, variations in precipitate distributions, and chemical banding effects (Ref. 2). By cooling at $60^{\circ} \mathrm{C} / \mathrm{s}$, regardless of the peak temperature, the microstructure was predominantly martensite. The peak temperature mainly influenced the type of martensite. With a peak temperature of $1400{ }^{\circ} \mathrm{C}$ and a cooling rate of $60^{\circ} \mathrm{C} / \mathrm{s}$, the microstructure was completely martensitic, a combination of untempered martensite and coarse plate autotempered martensite. With a peak temperature of $900{ }^{\circ} \mathrm{C}$ and a cooling rate of $60^{\circ} \mathrm{C} / \mathrm{s}$, the microstructure was predominantly untempered lath martensite with about $10 \%$ lath or acicular ferrite. At the slow cooling rate, the microstructures were more complicated. With a peak temperature of $1400^{\circ} \mathrm{C}$ and a cooling rate of $5^{\circ} \mathrm{C} / \mathrm{s}$, the resulting microstructure was determined to be untempered lath martensite with a small amount $(5 \%)$ of lath ferrite. With a peak temperature of $900{ }^{\circ} \mathrm{C}$ and a cooling rate of $5^{\circ} \mathrm{C} / \mathrm{s}$, the microstructure was predominantly ferrite (a combination of polygonal and lath ferrite, $65 \%$ ) with the remainder being lath martensite (35\%). The volume fractions were estimated by accurately determining from the dilatometer data the amount of transformation that had occurred above the martensite start temperature of $420^{\circ} \mathrm{C}$ and assuming that the remaining austenite transferred to martensite. Detailed transmission electron microscopy (Ref. 2) was used to determine the microstructures.

Examination of Fig. 5 indicates that at the fast cooling rate of $60^{\circ} \mathrm{C} / \mathrm{s}$, the microstructure should be completely martensitic, which is approximately the microstructure found experimentally. On the other hand the curves of Wilson et al. (Ref. 4) would indicate that at the slow cooling rate of 5 ${ }^{\circ} \mathrm{C} / \mathrm{s}$, the microstructure would also be completely martensitic. Experimentally the microstructure at the slow cooling rate was found to be dependent on the peak temperature and varied from $95 \%$ martensite at a peak temperature of $1400{ }^{\circ} \mathrm{C}$ to $35 \%$ martensite at a peak temperate of 900

\section{${ }^{\circ}$ C.CALCULA TION RESULTS}

A comparison of the volume fractions of microstructure derived from the calculation and the experimental results is shown in Table 3. The table gives the volume percentage of austenite that was transformed to ferrite (both acicular and polygonal) before the $\mathrm{MS}$ of $420^{\circ} \mathrm{C}$ was reached, and the corresponding volume percentage of martensite. Table 3 clearly indicates that at the fast cooling rate all three models give the same result and are comparable to the experimental results. 


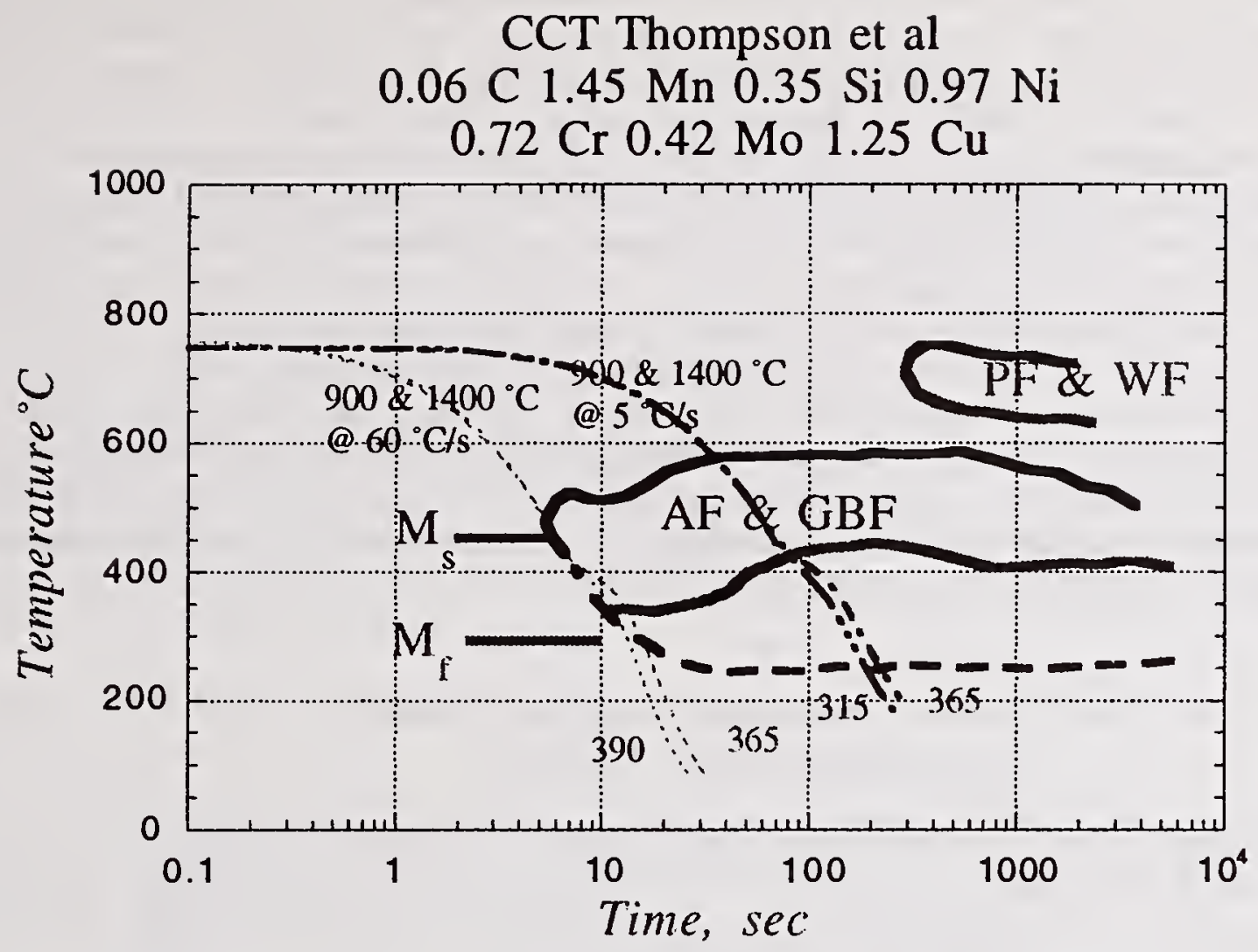

Fig. 5 Continuous cooling curves from dilatometer and continuous cooling curve diagram for HSLA-100 steel after Wilson et al. 4.

Neither Kirkaldy's nor Ion's model result in any decomposition of austenite at the slower cooling rate. Bhadeshia's model gives values comparable to the experimental results at the slower cooling rate and a peak temperature of $900{ }^{\circ} \mathrm{C}$ but not at a peak temperature of $1400{ }^{\circ} \mathrm{C}$. The experimental and the calculated (based on Bhadeshia's model) hardness values are shown in Table 4.

Table 3 Comparison of Volume percentages, Experiment and Calculations

$\begin{array}{llllll}\text { Peak Temp } & \text { Cooling Rate } & \begin{array}{l}\text { Experiment } \\ \text { Trans, Mart }\end{array} & \begin{array}{l}\text { Kirkaldy } \\ \text { Trans, Mart }\end{array} & \begin{array}{l}\text { Ion } \\ \text { Trans, Mart }\end{array} & \begin{array}{l}\text { Bhades } \\ \text { Trans, }\end{array} \\ { }^{\circ} \mathrm{C} & { }^{\circ} \mathrm{C} / \mathrm{s} & & & & \\ 1400 & 60 & 1,99 & 0,100 & 0,100 & 0,100 \\ 900 & 60 & 10,90 & 0,100 & 0,100 & 0,100 \\ 1400 & 5 & 5,95 & 0,100 & 0,100 & 85,15 \\ 900 & 5 & 65,35 & 0,100 & 0,100 & 75,25\end{array}$

Table 4 Experimental and Calculated (Bhadeshia's model) Hardness Values

Peak Temp

${ }^{\circ} \mathrm{C}$

1400

900

1400

900
Cooling Rate

${ }^{\circ} \mathrm{C} / \mathrm{s}$

5

5

60

60
Hardness values,

VHN

Measured

365

315

365

390
Calculated

286

284

320

324

\section{DISCUSSION}


As stated earlier, Bhadeshia's model calculates the transformation start time for a time temperature transformation (TTT) diagram for the steel based only on its chemistry. Although not a set of continuous cooling curves, this TTT diagram can be utilized, with the cooling curves from the dilatometer, to identify the different phases that may be present as a result of each cooling curve. Such a diagram is shown in Fig. 6. Assuming the validity of the start time on the TTT curve and realizing that the comparable CCT curve would be at longer times and lower temperatures above the nose of the curve, the fast cooling curves indicate that the austenite would convert completely to martensite regardless of the peak temperature. The slower cooling curves would indicate the presence of a considerable amount of acicular ferrite/bainite as was found in the $900{ }^{\circ} \mathrm{C}$ peak temperature curve. The discrepancy between the $1400^{\circ} \mathrm{C}$ experimental results and the calculation based on Bhadeshia's model can not be explained easily. Spanos et al. (Ref. 2) explained the differences in the microstructure at the slow cooling rate and different peak temperatures in terms of the significant difference in prior austenite grain size, the variation of precipitate distributions (especially niobium-carbonitrides), and banding in the steel, none of which would be changed significantly at the low peak temperature of $900^{\circ} \mathrm{C}$.

\section{$0.07 \mathrm{C} 0.80 \mathrm{Mn} 0.37 \mathrm{Si} 3.49 \mathrm{Ni}$ \\ $0.57 \mathrm{Cr} 0.58 \mathrm{Mo} 1.61 \mathrm{Cu}$}

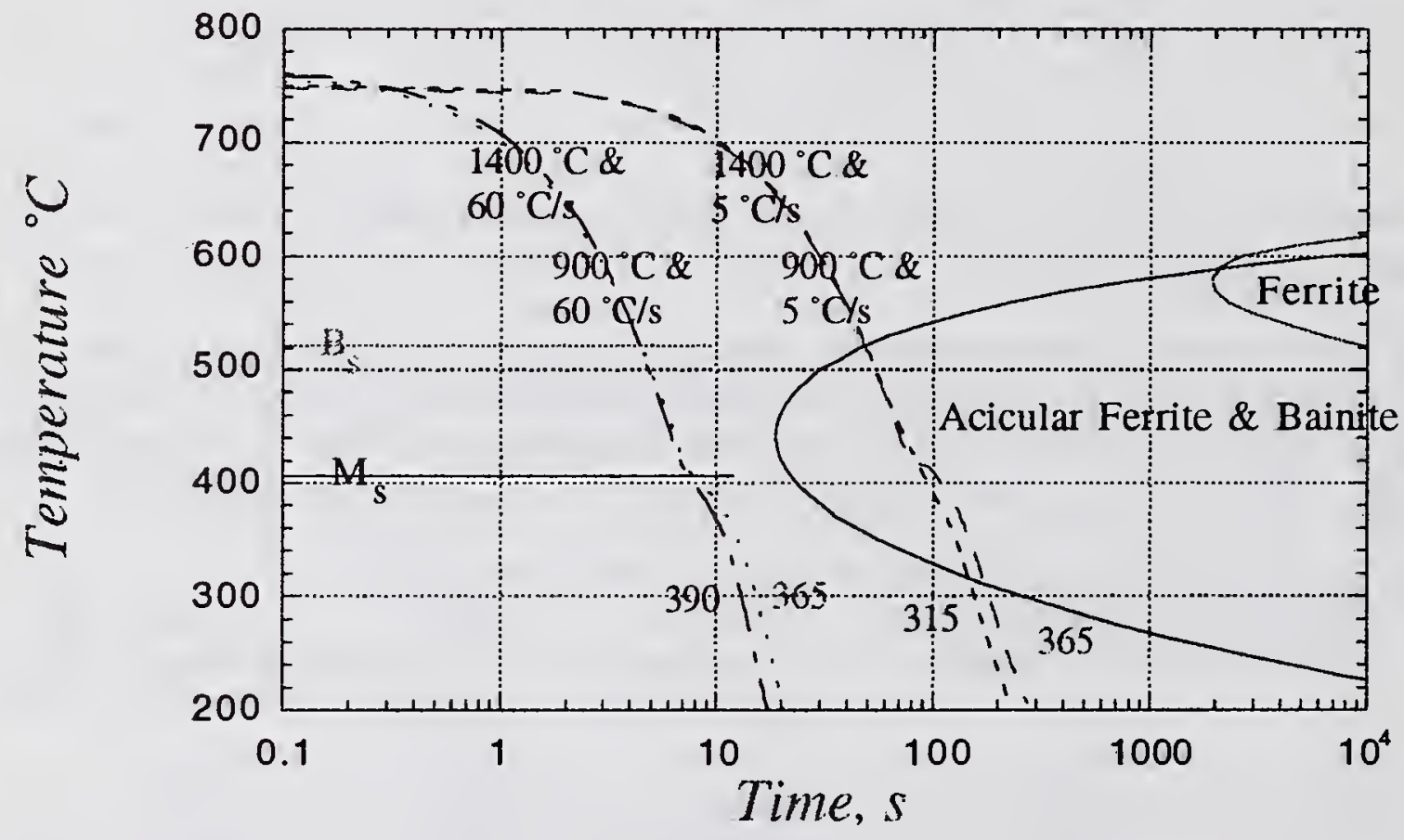

Fig. 6 Thermal Profiles from the dilatometer overlaid on the Time Temperature Transformation Diagram for this HSLA-100 steel.

The calculated hardness values in Table 4 are based on the rule of mixtures and are only given for the volume fractions of microstructure based on Bhadeshia's model. The calculation of the hardness is based on the work of Maynier et al. (Ref. 21) where the copper content was much lower than in the HSLA100 steel with the result that the hardness of martensite as determined through the work of Maynier et al. (Ref. 21) is significantly higher than the experimental value. This is demonstrated in the case of the $900{ }^{\circ} \mathrm{C}$ peak temperature cooled at $60{ }^{\circ} \mathrm{C} / \mathrm{s}$ where the experimental value is $390 \mathrm{VHN}$ for an almost completely martensitic microstructure, whereas the model calculation also indicates a completely martensitic structure and a hardness of only 324 VHN. In HSLA-100 steel a significant fraction of the copper forms a fine dispersion of e-copper which significantly contributes to the excellent strength and toughness of the alloy. In Maynier et al. (Ref. 21) steels, copper is most likely a substitutional alloying element. The difference in 
hardness between the two peak temperatures cooled at $60^{\circ} \mathrm{C} / \mathrm{s}$ is reflected in the microstructure. The higher value $(365 \mathrm{VHN})$ of the $900{ }^{\circ} \mathrm{C}$ peak temperature is a result of its untempered martensitic structure, whereas the $1400{ }^{\circ} \mathrm{C}$ peak temperature has both untempered martensite and coarse autotempered martensite and a hardness value of 315 VHN. The difference in hardness between the two peak temperatures cooled at $5{ }^{\circ} \mathrm{C} / \mathrm{s}$ is reflected again by their microstructure. The higher value $(365 \mathrm{VHN})$ of the $1400^{\circ} \mathrm{C}$ peak temperature is a result of its untempered martensitic structure, whereas the $900{ }^{\circ} \mathrm{C}$ peak temperature has more than $60 \%$ ferrite and a hardness value of 315 VHN.

\section{CONCLUSIONS}

Several conclusions can be drawn from this study.

1. The semi-empirical TTT curve generated by Bhadeshia's model is generally realistic in identifying the phases when compared to the CCT curve in the literature and the experimental results of Spanos et al. (Ref. 2).

2. For HSLA-100 steel the decomposition of austenite at fast cooling rates can generally be explained by all three models.

3. For HSLA-100 steel the decomposition of austenite at slow cooling rates is best explained by Bhadeshia's model, but there is considerable difference between measured and calculated volume fractions which cannot be easily rationalized.

4. The effect of copper and nickel on the decomposition of low carbon austenite is not well understood. The models need to be refined and critical experiments need to be completed.

5. The effect of copper on the hardness of HSLA-100 steel needs to be reformulated in terms of how much copper is in the lattice as a substitutional atom and how much is in the steel as fine precipitates.

\section{ACKNOWLEDGMENT}

The authors are grateful to the Office of Naval Research for the funding to carry out this research.

\section{REFERENCES}

1. Holsberg, P. W.; Gudas, J. P.; and Caplan, I. L. 1990. Navy's Welding Research Picks Up Steam. Adv. Mater Processes, 138 (1): 45-49.

2. Spanos, G.; Fonda, R. W.; Vandermeer, R. A.; and Matuszeski A. 1995. Microstructural Changes in HSLA-100 Steel Thermally Cycled to Simulate the Heat-affected Zone during Welding. Met. Mat. Trans. 26A (12): 3277-3293.

3. Andrews, K. W. 1956. Empirical Formulae for the Calculation of Some Transformation Temperatures. J. Iron Steel Inst.. 203(7): 721-727.

4. Wilson, A. D.; Hamburg, E. G.; Colvin, D. J.; Thompson, S. W.; and Krauss, G. 1988. Properties and Microstructures of Copper Precipitation Aged Plate Steels. Proceedings of Microalloying '88. World Materials Congress. 259-275, ASM International, Materials Park, Ohio.

5. Kirkaldy, J. S.; and Venugopalan, D. 1984. Prediction of Microstructure and Hardenability in Low Alloy Steels. Proc. Intern. Conf. Phase Transformations in Ferrous Alloys eds. A. R. Marder and J. I. Goldenstein: 125-148, Am Inst. Min. Engrs. 
6. Kirkaldy, J. S.; and Sharma, R. C. 1982. A New Phenomenology for Steel IT and CCT Curves. Scripta Metall. 16: 1193-1198.

7. Kirkaldy, J. S. 1973. Prediction of Alloy Harenability from Thermodynamic and Kinetic Data. Metall. Trans., 4(10): 2327-2333.

8. Kirkaldy, J. S.; and Baganis, E. A.; 1978. Thermodynamic Predictions of the $\mathrm{Ae}_{3}$ Temperature of Steels with Additions of Mn, Si, Ni, Mo, Cu. Metall. Trans. 9A(4): 495-501.

9. Hashiguchi, K.; Kirkaldy, J. S.; Fukuzumi, T.; and Pavaskar, V. 1984. Prediction of the Equilibium, Paraequilibrium and No-partition Local Equilibrium Phase Diagrams for Multicomponent Fe-C Base Alloys. CALPHAD, 8: 173.

10. Watt, D. F.; Coon, L.; Bibby, M.; Goldak, J.; and Henwood, C. 1986. An Algorithm for Modelling Microstructural Development in Weld Heat-Affected Zones (Part A) Reaction Kinetics. Acta metall, 36(11): 3029-3035.

11. Henwood, C.; Bibby, M.; Goldak, J.; and Watt, D. F. 1988. Coupled Transient Heat Transfer - Microstructure Weld Computations (Part B). Acta metall, 36(11): 3037-3046.

12. Cahn, J. W. 1956. Transformation Kinetics During Continuous Cooling. Acta metall., 4:572575.

13. Ion, J. C.; Easterling. K. E.; and Ashby, M. F. 1984. A Second Report on Diagrams of Microstructure and Hardness for Heat-affected Zones in Welds. Acta metall. 32(11): 1949-1962.

14. Inagaki, M.; and Sekiguchi, H. 1960. Continuous Cooling Transformation Diagrams of Steels for Welding and Their Applications. Trans. Natn. Res. Inst. Metals, Japan.2(2): 102-125.

15. Johnson, W. A.; and Mehl, R. F. 1939. Reaction Kinetics in Processes of Nucleation and Growth. Trans. Am. Inst. Min. Engrs, 135: 416-442.

16. Ion, J. C.; Salminen, A. S.; and Sun, Z. 1996. Process Diagrams for Laser Beam Welding of Carbon Manganese Steels. Welding Journal, 75(7): 225-s to 322-s.

17. Bhadeshia, H. K. D. H.; Svensson, L.-E.; and Gretoft, B. 1985. A Model for the Development of Microstructure in Low Alloy Steel (Fe-Mn-Si-C) Weld Deposits. Acta Metall., 33(7): 1271-1283.

18. Bhadeshia, H. K. D. H. 1992. Modelling of Steel Welds. Mater. Sci.Technol, 8: 123-133.

19. Bhadeshia, H. K. D. H. 1985. Diffusion-controlled Growth of Ferrite Plates in Plain-carbon Steels. Mater. Sci. Technol. 1(7): 497-504.

20. Takahashi, M.; and Bhashedia, H. K. D. H. 1991. A Model for the Microstucture of Some Advanced Bainitic Steels. Trans. Jap. Inst. Met. 32(8): 689-696.

21. Maynier, Ph.; Jungmann, B.: and Dollet, J. 1978. Creusot-Loire System for the Orediction of mechanical Properties of Low Alloy Steel Products. Hardenability Concepts with Applications to Steel. eds.D. V. Doane and J. S. Kirkaldy: 518-544. AIME, Warendale, PA.

22. Rosenthal, D. 1941. Mathematical Theory of Heat Distribution During Welding and Cutting. Weld. J. Res. Suppl., 20: 220-s to 234-s. 
23. Rosenthal, D. 1946. The Theory of Moving Heat Sources and its Application to Metal Treatments. Trans. ASME 68(11): 849-866.

24. Aaronson, H. I.; Domian, H.A.; and Pound, G. M. 1966. Thermodynamics of the Austenite Proeutectoid Ferrite Transformation. II, Fe-C-X Alloys. Trans. TMS AIME, 236(5): 768-781.

25. Eldis, G. T. A Critical Review of Data Sources for Isothermal and Continuous Cooling Curves. Hardenability Concepts with Applications to Steel. eds.D. V. Doane and J. S. Kirkaldy: 126-148. AIME, Warendale, PA. 


\title{
MATHEMATICAL MODEL OF PREDICTION OF PHASE COMPOSITION, STRUCTURE AND PROPERTIES OF WELD METAL
}

\author{
N.V. Korolev*, V.N. Boronenkov*, O.V. Pimenova*
}

\begin{abstract}
A mathematical model permitting to prognosticate type and quantity of the initial and eutectic hardening phases as well as chemical and structural composition of the matrix of surfacing alloys has been described. Wear resistance of alloys is being evaluated by specific work of destruction of phase and structural components. The calculation results for a number of alloys have been given as an example. The model permits to significantly facilitate the choice of the optimal composition of surfaced metal.
\end{abstract}

\section{INTRODUCTION}

It is known that a wide range of materials that provide surfaced metal of various structural classes (Ref. 1-4) exists and is used for surfacing of parts exposed in work to abrasive and impactabrasive affects: austenite and austenite-martensite steels with carbide, carboboride and carbonitride hardening, alloyed cast irons and composites. A certain optimal structural state of the surfaced metal corresponds to every concrete condition of working loading. That is why it is necessary to establish the relation between chemical composition of the surfaced metal and its structural-phase composition.

One of the methods of prognosis of workability of the surfaced metal and choice of its optimal composition for conditions of impact and abrasive-impact affects based upon the structuralenergetic approach to evaluation of its properties (Ref. 5,3) has been considered below. The method is based on successive calculation of the type and quantity of resulting initial carbides, borides and nitrides, the quantity of hardening phases of the eutectic origin, the structural composition of the matrix of the alloy, and, on the basis of the above data, the evaluation of power-consumption of destruction of the alloy.

\section{TYPE AND QUANTITY OF INITIAL PHASES}

We write down the reaction of phases formation in the general form:

$$
\begin{aligned}
& x\left[E_{i}\right]+[C]=\left(E_{i_{X}} C\right) \\
& y\left[E_{i}\right]+[N]=\left(E_{i_{Y}} N\right) \\
& z\left[E_{i}\right]+[B]=\left(E_{i_{Z}} B\right)
\end{aligned}
$$

where $\mathrm{Ei}, \mathrm{C}, \mathrm{N}, \mathrm{B}$, are the elements dissolved in the metal. The possibility of formation of several * Ural State Technical University, 19 Mira st., Ekaterinburg, Russia, 620002 
types of the phases comprising one element is being taken into consideration by different values of $\mathrm{x}, \mathrm{y}$ and $\mathrm{z}$. We note that the state of equilibrium of the system is being completely described by the set of reactions (1-3) therefore there is no need to consider other possible reactions between substances included into the set (1-3). Mutual influence of reactions is automatically being considered through the common reagent.

The state of equilibrium is being described by the constants of equilibrium:

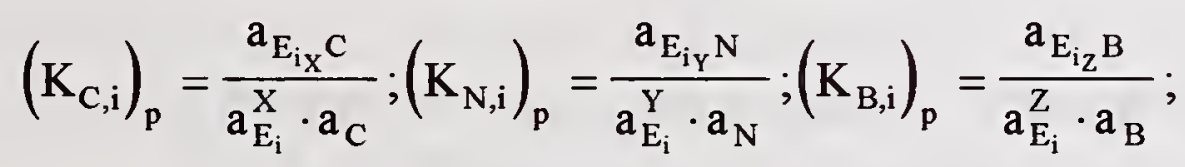

where $a_{i}=N_{i} \cdot \gamma_{i} ; a_{i}, N_{i}, \gamma_{i}-$ are activity, mole fraction and activity coefficient of the i-th component.

The "kinetic" method (Ref. 6) based on the use of the law of acting masses with successive calculation in time of approximation of solutions' concentration to equilibrium was used for the calculations. In the case of multi-component solutions the necessity of solution of the systems of equations in so doing is eliminated. The method has been modified for the multi-phase systems under consideration: the law of acting masses is being expressed through the activities and the elaboration of thermodynamic constants, masses of all phases, the list of possible reactions etc. is being performed at each stage.

The equations of velocity of reactions of formation of carbides, nitrides and borides are expressed in the following form:

$$
\begin{aligned}
& \mathrm{V}_{\mathrm{C}, \mathrm{i}}=\left(\mathrm{K}_{\mathrm{C}, \mathrm{i}}\right)_{0} \cdot\left(\left[\mathrm{E}_{\mathrm{i}}\right]^{\mathrm{X}} \cdot[\mathrm{C}]-\mathrm{K}_{\mathrm{C}, \mathrm{i}}\right) \cdot \mathrm{m} ; \\
& \mathrm{V}_{\mathrm{N}, \mathrm{i}}=\left(\mathrm{K}_{\mathrm{N}, \mathrm{i}}\right)_{0} \cdot\left(\left[\mathrm{E}_{\mathrm{i}}\right]^{\mathrm{Y}} \cdot[\mathrm{N}]-\mathrm{K}_{\mathrm{N}, \mathrm{i}}\right) \cdot \mathrm{m} ; \\
& \mathrm{V}_{\mathrm{B}, \mathrm{i}}=\left(\mathrm{K}_{\mathrm{B}, \mathrm{i}}\right)_{0} \cdot\left(\left[\mathrm{E}_{\mathrm{i}}\right]^{\mathrm{Z}} \cdot[\mathrm{B}]-\mathrm{K}_{\mathrm{B}, \mathrm{i}}\right) \cdot \mathrm{m} ;
\end{aligned}
$$

where $\mathrm{K}_{\mathrm{i} 0}$ - are formal constants of velocity, their values are chosen for reasons of stability and swiftness of solution, $K_{I}$ are balanced products of concentrations found in accordance with (4) from equations:

$$
\begin{aligned}
& \mathrm{K}_{\mathrm{C}, \mathrm{i}}=\mathrm{M}_{\mathrm{E}_{\mathrm{i}}}^{\mathrm{X}} \cdot \mathrm{M}_{\mathrm{C}} \cdot\left(\sum\left[\mathrm{E}_{\mathrm{i}}\right] / \mathrm{M}_{\mathrm{i}}\right)^{\mathrm{X}+1} /\left(\left(\mathrm{K}_{\mathrm{C}, \mathrm{i}}\right)_{\mathrm{P}} \cdot \gamma_{\mathrm{E}_{\mathrm{i}}}^{\mathrm{X}} \cdot \gamma_{\mathrm{C}}\right) \\
& \mathrm{K}_{\mathrm{N}, \mathrm{i}}=\mathrm{M}_{\mathrm{E}_{\mathrm{i}}}^{\mathrm{Y}} \cdot \mathrm{M}_{\mathrm{N}} \cdot\left(\sum\left[\mathrm{E}_{\mathrm{i}}\right] / \mathrm{M}_{\mathrm{i}}\right)^{\mathrm{Y}+1} /\left(\left(\mathrm{K}_{\mathrm{N}, \mathrm{i}}\right)_{\mathrm{P}} \cdot \gamma_{\mathrm{E}_{\mathrm{i}}}^{\mathrm{Y}} \cdot \gamma_{\mathrm{N}}\right) \\
& \mathrm{K}_{\mathrm{B}, \mathrm{i}}=\mathrm{M}_{\mathrm{E}_{\mathrm{i}}}^{\mathrm{Z}} \cdot \mathrm{M}_{\mathrm{B}} \cdot\left(\sum\left[\mathrm{E}_{\mathrm{i}}\right] / \mathrm{M}_{\mathrm{i}}\right)^{\mathrm{Z}+1} /\left(\left(\mathrm{K}_{\mathrm{B}, \mathrm{i}}\right)_{\mathrm{P}} \cdot \gamma_{\mathrm{E}_{\mathrm{i}}}^{\mathrm{Z}} \cdot \gamma_{\mathrm{B}}\right)
\end{aligned}
$$

Here $\mathbf{M}_{\mathrm{i}}$ are molar masses of elements. 
The thermodynamic data for the calculations of constants of equilibrium $\left(\mathrm{K}_{\mathrm{C}, \mathrm{i}}\right)_{\mathrm{P}},\left(\mathrm{K}_{\mathrm{N}, \mathrm{i}}\right)_{\mathrm{P}}$ and $\left(\mathrm{K}_{\mathrm{B}, \mathrm{i}}\right)_{\mathrm{P}}$ are taken from works (Ref. 7 and 8 ). The coefficients of components activity were calculated by Wagner:

$$
\ln \gamma_{i}=\ln \gamma_{i}^{0}+\sum_{j=2}^{j} \varepsilon_{i}^{j} \cdot N_{i}
$$

The values of $\gamma_{\mathrm{i}}^{0}$ at $1873 \mathrm{~K}$ and $\varepsilon_{\mathrm{i}}^{\mathrm{j}}$ were set in accordance with (Ref.4). For other temperatures the values of $\gamma_{i}^{0}$ are to be determined from equation $\ln \gamma_{i}(T)=\frac{T}{1873} \ln \gamma_{i}^{0}$.

After the span of time $\Delta \tau$ of one cycle of calculations from $k \Delta \tau$ to $(\mathrm{k}+1) \Delta \tau$ masses of carbon, nitrogen, boron and alloying elements participating in the reaction as well as carbides, nitrides and borides will reach the value

$$
\begin{gathered}
\mathrm{m}_{\mathrm{C},(\mathrm{k}+1)}=\mathrm{m}_{\mathrm{C}, \mathrm{k}}-\mathrm{m}_{\mathrm{k}} \cdot \sum \mathrm{V}_{\mathrm{C}, \mathrm{i}} \cdot \mathrm{M}_{\mathrm{C}} \cdot \Delta \tau \\
\mathrm{m}_{\mathrm{N},(\mathrm{k}+1)}=\mathrm{m}_{\mathrm{N}, \mathrm{k}}-\mathrm{m}_{\mathrm{k}} \cdot \sum \mathrm{V}_{\mathrm{N}, \mathrm{i}} \cdot \mathrm{M}_{\mathrm{N}} \cdot \Delta \tau \\
\mathrm{m}_{\mathrm{B},(\mathrm{k}+1)}=\mathrm{m}_{\mathrm{B}, \mathrm{k}}-\mathrm{m}_{\mathrm{k}} \cdot \sum \mathrm{V}_{\mathrm{B}, \mathrm{i}} \cdot \mathrm{M}_{\mathrm{B}} \cdot \Delta \tau \\
\mathrm{m}_{\mathrm{E}_{\mathrm{i}},(\mathrm{k}+\mathrm{i})}=\mathrm{m}_{\mathrm{E}_{\mathrm{i}}, \mathrm{k}}-\mathrm{m}_{\mathrm{k}} \cdot\left(\sum \mathrm{x} \cdot \mathrm{V}_{\mathrm{C}, \mathrm{i}}+\sum \mathrm{y} \cdot \mathrm{V}_{\mathrm{N}, \mathrm{i}}+\sum \mathrm{z} \cdot \mathrm{V}_{\mathrm{B}, \mathrm{i}}\right) \cdot \mathrm{M}_{\mathrm{E}_{\mathrm{i}}} \cdot \Delta \tau \\
\mathrm{m}_{\mathrm{E}_{\mathrm{iX}} \mathrm{C},(\mathrm{k}+1)}=\mathrm{m}_{\mathrm{E}_{\mathrm{iX}} \mathrm{C}, \mathrm{k}}-\mathrm{m}_{\mathrm{k}} \cdot \sum \mathrm{V}_{\mathrm{C}, \mathrm{i}} \cdot \mathrm{M}_{\mathrm{E}_{\mathrm{ix}} \mathrm{C}} \cdot \Delta \tau \\
\mathrm{m}_{\mathrm{E}_{\mathrm{iY}} \mathrm{N},(\mathrm{k}+1)}=\mathrm{m}_{\mathrm{E}_{\mathrm{iY}} \mathrm{N}, \mathrm{k}}-\mathrm{m}_{\mathrm{k}} \cdot \sum \mathrm{V}_{\mathrm{N}, \mathrm{i}} \cdot \mathrm{M}_{\mathrm{E}_{\mathrm{i} \mathrm{Y}} \mathrm{N}} \cdot \Delta \tau \\
\mathrm{m}_{\mathrm{E}_{\mathrm{i}_{\mathrm{Z}} \mathrm{B},(\mathrm{k}+1)}}=\mathrm{m}_{\mathrm{E}_{\mathrm{iz}} \mathrm{B}, \mathrm{k}}-\mathrm{m}_{\mathrm{k}} \cdot \sum \mathrm{V}_{\mathrm{B}, \mathrm{i}} \cdot \mathrm{M}_{\mathrm{E}_{\mathrm{i}_{\mathrm{Z}} \mathrm{B}} \mathrm{B}} \cdot \Delta \tau
\end{gathered}
$$

where $m_{k}$ is the melt mass by the beginning of the cycle of calculation.

In the process relative change of the melt mass during one cycle will be equal to

$$
\begin{aligned}
\mathrm{S}_{\mathrm{k}+1}= & \mathrm{m}_{\mathrm{k}+1} / \mathrm{m}_{\mathrm{k}}=1-\left(\sum \mathrm{V}_{\mathrm{C}, \mathrm{i}} \cdot \mathrm{M}_{\mathrm{C}}+\sum \mathrm{V}_{\mathrm{N}, \mathrm{i}} \cdot \mathrm{M}_{\mathrm{N}}+\sum \mathrm{V}_{\mathrm{B}, \mathrm{i}} \cdot \mathrm{M}_{\mathrm{B}}+\right) \\
& +\left(\sum \mathrm{x} \cdot \mathrm{V}_{\mathrm{C}, \mathrm{i}} \cdot \mathrm{M}_{\mathrm{E}_{\mathrm{i}}}+\sum \mathrm{y} \cdot \mathrm{V}_{\mathrm{N}, \mathrm{i}} \cdot \mathrm{M}_{\mathrm{E}_{\mathrm{i}}}+\sum \mathrm{z} \cdot \mathrm{V}_{\mathrm{B}, \mathrm{i}} \cdot \mathrm{M}_{\mathrm{E}_{\mathrm{i}}}\right)
\end{aligned}
$$

The change of the melt mass by the moment $\mathrm{k} \Delta \tau$ relative to the initial one we determine as

$$
\mathrm{S}_{0, \mathrm{k}}=\mathrm{S}_{1} \cdot \mathrm{S}_{2} \cdot \ldots \cdot \mathrm{S}_{\mathrm{k}}
$$

The carbon, nitrogen, boron and the interacting elements concentrations in the melt at each successive moment are determined by equations 


$$
\begin{gathered}
{[\mathrm{C}]_{\mathrm{k}+1}=\left([\mathrm{C}]_{\mathrm{k}}-\sum \mathrm{V}_{\mathrm{C}, \mathrm{i}} \cdot \mathrm{M}_{\mathrm{C}} \cdot \Delta \tau \cdot 100\right) / \mathrm{S}_{\mathrm{k}+1} ;} \\
{[\mathrm{N}]_{\mathrm{k}+1}=\left([\mathrm{N}]_{\mathrm{k}}-\sum \mathrm{V}_{\mathrm{N}, \mathrm{i}} \cdot \mathrm{M}_{\mathrm{N}} \cdot \Delta \tau \cdot 100\right) / \mathrm{S}_{\mathrm{k}+1}} \\
{[\mathrm{~B}]_{\mathrm{k}+1}=\left([\mathrm{B}]_{\mathrm{k}}-\sum \mathrm{V}_{\mathrm{B}, \mathrm{i}} \cdot \mathrm{M}_{\mathrm{B}} \cdot \Delta \tau \cdot 100\right) / \mathrm{S}_{\mathrm{k}+1} ;} \\
{\left[\mathrm{E}_{\mathrm{i}}\right]_{\mathrm{k}+1}=\left(\left[\mathrm{E}_{\mathrm{i}}\right]_{\mathrm{k}}-\left(\sum \mathrm{x} \cdot \mathrm{V}_{\mathrm{C}, \mathrm{i}}+\sum \mathrm{y} \cdot \mathrm{V}_{\mathrm{N}, \mathrm{i}}+\sum \mathrm{z} \cdot \mathrm{V}_{\mathrm{B}, \mathrm{i}}\right) \cdot \mathrm{M}_{\mathrm{E}_{\mathrm{i}}} \cdot \Delta \tau \cdot 100\right) / \mathrm{S}_{\mathrm{k}+1}}
\end{gathered}
$$

The carbides, nitrides and borides concentrations relative to the system mass we find as

$$
\begin{aligned}
& \left(E_{i x} C\right)_{k+1}=\left(E_{i x} C\right)_{k}+S_{0, k} \cdot V_{C, i} \cdot M_{E_{i x} C} \cdot \Delta \tau \cdot 100 \\
& \left(E_{i y} N\right)_{k+1}=\left(E_{i y} N\right)_{k}+S_{0, k} \cdot V_{N, i} \cdot M_{E_{i Y N}} \cdot \Delta \tau \cdot 100 \\
& \left(E_{i z} B\right)_{k+1}=\left(E_{i B} B\right)_{k}+S_{0, k} \cdot V_{B, i} \cdot M_{E_{i_{Z} B}} \cdot \Delta \tau \cdot 100
\end{aligned}
$$

We assumed the concentration distinction of not more than $0.01 \%$ rel. for all reactions as a criterion of the attainment of equilibrium, this corresponding to the conditions of:

$$
1-\frac{\left[\mathrm{E}_{\mathrm{i}}\right]_{\mathrm{k}+1} \cdot[\mathrm{C}]_{\mathrm{k}+1}}{\mathrm{~K}_{\mathrm{C}, \mathrm{i}}} \leq 10^{-4} ; 1-\frac{\left[\mathrm{E}_{\mathrm{i}}\right]_{\mathrm{k}+1} \cdot[\mathrm{N}]_{\mathrm{k}+1}}{\mathrm{~K}_{\mathrm{N}, \mathrm{i}}} \leq 10^{-4} ; 1-\frac{\left[\mathrm{E}_{\mathrm{i}}\right]_{\mathrm{k}+1} \cdot[\mathrm{B}]_{\mathrm{k}+1}}{\mathrm{~K}_{\mathrm{B}, \mathrm{i}}} \leq 10^{-4}
$$

The calculation practice has demonstrated that at $\Delta \tau=1-5 \mathrm{~s}$ this condition is satisfied at $\mathrm{K}=50$ 200 .

\section{PHASES OF EUTECTIC ORIGIN}

The melt with balanced concentrations of alloying elements $[C]_{p},[B]_{p}$ и $\left[\mathrm{E}_{\mathrm{i}}\right]_{\mathrm{p}}$, determined as a result of the previous calculation for the temperature close to the temperature of crystallization of the alloy is being considered at this stage.

We assume that in alloys close to the eutectic ones the fraction of dendrite cells and difference of their composition from that of the solid solution of eutectic is small that is why the system at the moment of completion of crystallization consists of two phases, i.e. solid solution and caroboride phase. Influence of liquation processes in this case is insignificant while the distribution of alloying elements among phases is close to equilibrium and can be found from the respective diagrams of state and from the results of experiments. In particular, after averaging we assume the following values of the distribution coefficients (Ref. 9): $\eta_{\mathrm{Cr}_{\mathrm{r}}}=3,5 ; \eta_{\mathrm{Ni}}=0 ; \eta_{\mathrm{Mn}}=1,6 ; \eta_{\mathrm{Mo}}=10 ; \eta_{\mathrm{V}}=7,5 ; \eta_{\mathrm{Ti}}=50$. The carbon distribution coefficient depends on concentration of other alloying elements and can be assumed as constant $\left(\eta_{\mathrm{C}}=10\right)$ only at the initial stage of the calculation when evaluating concentration of alloying elements in the solid solution, after that its value is to be defined more precisely. Distribution of boron among the phases can be taken into consideration provided that this element is contained only in the carboboride phase as its solubility in austenite is low $(0.02 \%$ 
at $1150 \mathrm{C})$ (Ref.10). Taking into account of carboboride phase content was executed by parameter A (Ref.9):

$$
A=(C)_{C}+(B)_{C}=\frac{m \cdot M_{C B} \cdot 100}{n \cdot M_{E}+m \cdot M_{C B}}
$$

where $(C)_{C},(B)_{C}$ are concentrations of carbon and boron, respectively, in the carboboride phase, $\%$ by mass; $n, m$ are stoichiometric coefficients of the $E_{n}(C B)_{m} ; M_{C B}=\left(M_{C}+M_{B}\right) / 2=11,5$ is the average atomic mass of carbon and boron; $\mathrm{M}_{\mathrm{E}}$ is the average atomic mass of metals included into the carboboride phase (it is close to the atomic mass of iron $\mathrm{M}_{\mathrm{Fe}}=56$ ).

At the carbon concentration up to $1.5 \%$ and the $[\mathrm{B}] /[\mathrm{C}]$ ratio $<1.4$ in general the phase of the $\mathrm{Me}_{23}(\mathrm{C}, \mathrm{B})_{6}$ type is being formed, for which $\mathrm{A}=5.1$. At $[\mathrm{B}] /[\mathrm{C}]>1.4$ the $\mathrm{Me}_{2}(\mathrm{~B}, \mathrm{C})$ phase, for which $A=9.3$, appears alongside with the $\mathrm{Me}_{23}(\mathrm{C}, \mathrm{B})_{6}$ phase. With content of carbon in the melt of more than $1.5 \%$ and the $[\mathrm{B}] /[\mathrm{C}]$ ratio $<0.1$ the phase of the $\mathrm{Me}_{7} \mathrm{C}_{3}$ type is being formed generally while at $[\mathrm{B}] /[\mathrm{C}]>0.1$ the phases $\mathrm{Me}_{7} \mathrm{C}_{3}$ and $\mathrm{Me}_{2} \mathrm{~B}$ are being formed (Ref.11). For the areas characterized by the presence of two types of caroboride phases we assume equivalent values of the A parameter that change linearly with the change of the $[\mathrm{B}] /[\mathrm{C}]$ ratio. Thus, at $[\mathrm{C}]<1.5 \%$ and $[\mathrm{B}] /[\mathrm{C}]=1.4-3 \mathrm{~A}=3[\mathrm{~B}] /[\mathrm{C}]$, at $[\mathrm{C}]>1.5 \%$ and $[\mathrm{B}] /[\mathrm{C}]=0.1-1.4$ and $[\mathrm{B}] /[\mathrm{C}]-1.5>3 \mathrm{~A}=$ $5+3[\mathrm{~B}] /[\mathrm{C}]$. If in the last case $[\mathrm{B}] /[\mathrm{C}]-1.5<3$, than $\mathrm{A}=8.2+[\mathrm{B}] /[\mathrm{C}]$. (Ref. 5).

The calculation of the type and quantity of eutectic phases and the composition of solid solution is to be performed in two stages. First, assuming that $\eta_{\mathrm{c}}=10$, we calculate the carbon concentration in solid solution in accordance with the formula (Ref. 9):

$$
[C]_{\gamma}=\left(D-\left(D^{2}-4 \cdot \eta_{C} \cdot A \cdot[C]_{P}\right)^{1 / 2}\right) / 2 \cdot \eta_{C}
$$

where $D=\left(\eta_{C}-1\right) \cdot[B]_{P}+\eta_{C} \cdot[C]_{P}+A$

The mass fraction of the carboboride phase we find from equation:

$$
\mathbf{P}=\frac{[\mathrm{C}]_{\mathrm{P}}-[\mathrm{C}]_{\gamma}}{[\mathrm{C}]_{\gamma} \cdot\left(\eta_{\mathrm{C}}-1\right)}
$$

Concentrations of other alloying elements in the solid solution are determined by equation

$$
\left[\mathrm{E}_{\mathrm{i}}\right]_{\gamma}=\frac{\left[\mathrm{E}_{\mathrm{i}}\right]_{\mathrm{P}}}{1+\mathrm{P} \cdot\left(\eta_{\mathrm{i}}-1\right)}
$$

At the second stage we assume that the carbon concentration in the solid solution is equal to the limit calculated by the equation obtained as a result of the analysis of the state diagrams: 


$$
\ln [\mathrm{C}]_{1}=0,70-44,2 \cdot[\mathrm{B}]_{\gamma}-0,06 \cdot[\mathrm{Cr}]_{\gamma}-0,028 \cdot[\mathrm{Ni}]_{\gamma}-0,2 \cdot[\mathrm{Mn}]_{\gamma}^{-0,3}-0,19 \cdot[\mathrm{Mo}]_{\gamma}-0,82 \cdot[\mathrm{V}]
$$

More precise value of the fraction of the carboboride phase we determine as (Ref. 9)

$$
\mathrm{P}=\frac{[\mathrm{C}]_{\mathrm{P}}+[\mathrm{B}]_{\mathrm{P}}-[\mathrm{C}]_{1}}{\mathrm{~A}-[\mathrm{C}]_{1}}
$$

and concentrations of boron and carbon in the carboboride phase are determined by equations

$$
[\mathrm{B}]_{\mathrm{f}}=[\mathrm{B}]_{\mathrm{P}} / \mathrm{P} ;[\mathrm{C}]_{\mathrm{f}}=\mathrm{A}-[\mathrm{B}]_{\mathrm{f}}
$$

The concentration of alloying elements in the solid solution we find by Equation (28) with the help of the precise value of $P$.

\section{STRUCTURE OF THE MATRIX SOLID SOLUTION}

The main factors that determine the structure of the matrix of wear-resistant alloys are its chemical composition and rate of surfaced metal cooling in the process of surfacing, that can vary in the wide range. As a result of our experiments and calculations it was established that in the process of electric arc surfacing with the optimal modes the rate of cooling of the second layer of the surfaced metal can vary in the range from 20 to 60 degree/sec. Further the cooling rate of 40 degrees/sec was assumed to be a characteristic one.

The field of application of the Potak-Sagalevich structural diagram (Ref. 13) was expanded on the basis of the analysis of a number of works (Ref. 11,12), anisothermal diagrams of austenite transformation at the cooling rate 40 degrees/sec for carbon and alloyed steels as well as on the basis of the authors' research. In particular, the areas of formation of bainite and perlite structure were revealed together with more precise defining of boundaries of martensite, austenite and $\delta$ ferrite areas. This made possible the use of this diagram for the evaluation of structural composition of the matrix of high carbon surfacing alloys in the wide range of their alloying.

On the basis of the analysis of dependence of the matrix structure of more than 200 alloys upon the concentration of alloying elements equations for the calculation of ferrite formation $\left(\mathrm{K}_{\mathrm{f}}\right)$ and martensite formation $\left(\mathrm{K}_{\mathrm{s}}\right)$ coefficients were written in the following form:

$$
\begin{gathered}
\mathrm{K}_{\mathrm{f}}=[\mathrm{Cr}]_{\gamma}-1,5 \cdot[\mathrm{Ni}]_{\gamma}-0,75 \cdot[\mathrm{Mn}]_{\gamma}-\mathrm{K}_{1} \cdot\left([\mathrm{C}]_{\gamma}+[\mathrm{N}]_{\gamma}\right)+[\mathrm{Mo}]_{\gamma}+4 \cdot\left([\mathrm{Ti}]_{\gamma}+[\mathrm{Al}]_{\gamma}\right)+ \\
+1,5 \cdot[\mathrm{V}]_{\gamma}+0,5 \cdot[\mathrm{W}]_{\gamma}+0,9 \cdot[\mathrm{Nb}]_{\gamma}+0,2 \cdot[\mathrm{Si}]_{\gamma} \\
\mathrm{K}_{\mathrm{M}}=18-\mathrm{K}_{2} \cdot\left([\mathrm{C}]_{\gamma}+[\mathrm{N}]_{\gamma}\right)-\mathrm{K}_{3} \cdot[\mathrm{Cr}]_{\gamma}-\mathrm{K}_{4} \cdot[\mathrm{Ni}]_{\gamma}-\mathrm{K}_{5} \cdot[\mathrm{Mn}]_{\gamma}-0,6 \cdot[\mathrm{Mo}]_{\gamma}-1,5 \cdot[\mathrm{V}]_{\gamma}- \\
-1,9 \cdot[\mathrm{Ti}]_{\gamma}-1,1 \cdot[\mathrm{W}]_{\gamma}+0,1 \cdot[\mathrm{Al}]_{\gamma}-0,7 \cdot[\mathrm{Si}]_{\gamma}
\end{gathered}
$$


where $\mathrm{K}_{1}=5 \cdot\left([\mathrm{C}]_{\gamma}+[\mathrm{N}]_{\gamma}\right)^{-1,5}+8$;

$\mathrm{K}_{2}=6 \cdot\left([\mathrm{C}]_{\gamma}+[\mathrm{N}]_{\gamma}\right)^{-1,5}-10$;

$\mathrm{K}_{\mathbf{3}}=3-0,17 \cdot[\mathrm{Cr}]_{\gamma}$ if $[\mathrm{Cr}]_{\gamma}<10$;

$\mathrm{K}_{3}=1,3$ if $[\mathrm{Cr}]_{\gamma} \geq 10$;

$\mathrm{K}_{4}=0,6-0,03 \cdot[\mathrm{Ni}]_{\gamma}$ if $[\mathrm{Ni}]_{\gamma}<10$;

$\mathrm{K}_{4}=1,2-0,06 \cdot[\mathrm{Ni}]_{\gamma}$ if $10 \leq[\mathrm{Ni}]_{\gamma} \leq 13$

$\mathbf{K}_{4}=0,4$ if $[\mathrm{Ni}]_{\gamma}>13$

$\mathrm{K}_{5}=1-0,065 \cdot[\mathrm{Mn}]_{\gamma}$.

\section{EVALUATION OF WEAR RESISTANCE OF ALLOYS}

In the works of a number of authors (Ref. 3,14 ) it was noted that the metal resistance to abrasive wear can be evaluated by the quantity of energy spent in the process of wear for removal of a unit of its volume. This permits to use a structural-energetic approach to the evaluation of wear resistance of alloys (Ref. 3,5,15).

With difference in power-consumption of distraction of the strengthening phases and structural components of the alloy matrix it is possible to assume the additivity of each component contribution to the general power-consumption of the alloy destruction:

$$
\Delta \mathrm{U}=\sum \mathrm{P}_{\mathrm{i}}^{\prime} \cdot \Delta \mathrm{U}_{\mathrm{i}}
$$

where $\Delta \mathrm{U}$ is the specific power of alloy destruction; $\mathrm{P}_{\mathrm{i}}^{\prime}$ is the volume fraction of the $\mathrm{i}$-th phase in the alloy; $\Delta \mathrm{Ui}$ is the specific power of distraction of the $\mathrm{i}$-th phase, $\mathrm{kJ} /$ cubic sm.

The value of specific work of distraction in the process of abrasive wear is in good correlation (Ref. 3,5) with the heat of melting.

Low ductility and deformation ability of carbide, nitride and boride phases permit to consider the condition of their crystal lattice to be analog to the utmost distorted crystal lattice of ductile materials at the melting temperature. In this case it is possible to presume that specific power of distraction of these phases with sufficient precision can be determined through their latent heat of melting. Thus, according to the data of (Ref. 3) specific work of distraction for the most frequently used strengthening phases calculated by the melting heat is (kJ/cubic sm): TiC-24,7; $\mathrm{VC}-23,7 ; \mathrm{NbC}-26,55 ; \mathrm{WC}-17,2 ; \mathrm{Cr}_{3} \mathrm{C}_{2}-12,75 ; \mathrm{Cr}_{23} \mathrm{C}_{6}-1,535 ; \mathrm{Cr}_{7} \mathrm{C}_{3}-9,23 ; \mathrm{MnC}-13,3 ; \mathrm{Fe}_{3} \mathrm{C}-13,3$; $\mathrm{B}_{4} \mathrm{C}-15,3$.

It is known that complex carbides are being formed in iron-carbon alloys. In these carbides a fraction of atoms of the main carbide-forming element is replaced by iron. Because of the absence 
of thermodynamic data for mixed carbides, the work of distraction of simple carbides of the given type for the leading element was used for estimation of wear resistance.

We determined experimentally (Ref. 16) specific work of distraction of separate structural components and it was, depending on alloying: $3-5 \mathrm{~kJ} /$ cubic $\mathrm{cm}$ fir stable austenite; $4-8 \mathrm{~kJ} / \mathrm{cubic}$ $\mathrm{cm}$ for metastable austenite; and $6-8 \mathrm{~kJ} / \mathrm{cubic} \mathrm{cm}$ for martensite.

\section{CHECK OF THE MATHEMATICAL MODEL}

A program for the calculation of the phase composition, structure and wear resistance of the alloys alloyed with 14 chemical elements has been developed to realize the described model.

Table I. Composition of the surfaced metal (numerator) and calculated concentration of alloying elements in the matrix (denominator), $\%$ by mass.

\begin{tabular}{|c|c|c|c|c|c|c|c|c|c|c|}
\hline Alloy & $\mathrm{C}$ & $\mathrm{Cr}$ & $\mathrm{Mn}$ & Mo & $\mathrm{V}$ & $\mathrm{Ti}$ & $\mathrm{Nb}$ & $\mathrm{B}$ & $\mathrm{Si}$ & Others \\
\hline 1 & $\frac{0,92}{0,29}$ & $\frac{19,10}{13,61}$ & $\frac{0,60}{0,53}$ & - & - & $\frac{0,20}{<0,01}$ & - & $\underline{3,00}$ & $\frac{0,60}{0,72}$ & - \\
\hline 2 & $\begin{array}{l}2,00 \\
0,33\end{array}$ & $\frac{15,00}{11,70}$ & $\frac{1,00}{0,92}$ & - & - & $\frac{0,30}{<0,01}$ & - & $\underline{0,70}$ & $\frac{1,50}{1,70}$ & - \\
\hline 3 & $\frac{1,80}{0,25}$ & $\frac{12,00}{9,64}$ & $\frac{0,60}{0,56}$ & - & $\frac{0,25}{0,10}$ & - & - & - & $\frac{0,60}{0,65}$ & $\begin{array}{c}\mathrm{W}, 1,00 \\
0,32\end{array}$ \\
\hline 4 & $\begin{array}{l}3,00 \\
0,18\end{array}$ & $\frac{14,45}{10,13}$ & $\begin{array}{l}0,50 \\
0,44 \\
\end{array}$ & $\begin{array}{l}1,30 \\
0,34\end{array}$ & $\begin{array}{l}0,10 \\
0,03 \\
\end{array}$ & $\frac{0,20}{<0,01}$ & - & - & $\underline{0,90}$ & - \\
\hline 5 & $\begin{array}{l}2,60 \\
0,15 \\
\end{array}$ & $\frac{14,00}{12,25}$ & - & $\frac{3,00}{1,25}$ & - & $\underline{3,00}$ & $\begin{array}{l}6,00 \\
0,16 \\
\end{array}$ & - & - & - \\
\hline 6 & $\frac{1,90}{0,38}$ & $\frac{6,90}{6,16}$ & - & $\frac{2,78}{1,35}$ & - & $\frac{1,55}{0,01}$ & $\frac{3,90}{0,10}$ & - & - & $\begin{array}{l}\mathrm{Ni} \\
\underline{0,50} \\
0,64\end{array}$ \\
\hline
\end{tabular}

Table II. Phase composition, structure and wear resistance of alloys

\begin{tabular}{|c|c|c|c|c|c|}
\hline \multirow[t]{2}{*}{ Alloy } & \multicolumn{2}{|c|}{ Hardening phases, $\%$} & \multirow{2}{*}{$\begin{array}{l}\text { Composition of the } \\
\text { matrix, } \%\end{array}$} & \multirow{2}{*}{$\begin{array}{l}\text { Wear } \\
\text { resist. }\end{array}$} & \multirow{2}{*}{$\begin{array}{c}\Delta \mathrm{U} \\
\mathrm{kJ} / \mathrm{cm}^{3}\end{array}$} \\
\hline & initial & eutectic & & & \\
\hline 1 & $\frac{0,37 \% \mathrm{TiC}}{-}$ & $\begin{array}{c}\frac{43,2 \% \mathrm{E}_{2}(\mathrm{~B}, \mathrm{C})}{44,0 \% \mathrm{Cr}_{2}(\mathrm{~B}, \mathrm{C})^{*}} \\
4\end{array}$ & $\begin{array}{r}21,1 \% \mathrm{~A}+78,9 \% \mathrm{M} \\
23,0 \% \mathrm{~A}+77,0 \% \mathrm{M}^{*}\end{array}$ & $7,48^{*}$ & 9,56 \\
\hline 2 & $\frac{0,56 \% \mathrm{TiC}}{-}$ & $\frac{30,5 \% \mathrm{E}_{7}(\mathrm{~B}, \mathrm{C})_{3}}{15 \%(\mathrm{Cr}, \mathrm{Fe})_{7}(\mathrm{~B}, \mathrm{C})_{3}}$ & $\begin{array}{l}11,4 \% \mathrm{~A}+88,6 \% \mathrm{M} \\
15,0 \% \mathrm{~A}+85,0 \% \mathrm{M}\end{array}$ & $3,75^{*}$ & 7,55 \\
\hline 3 & - & $\begin{array}{c}\underline{26,0 \% \mathrm{E}_{3} \mathrm{C}} \\
15,0 \%(\mathrm{Cr}, \mathrm{Fe})_{3} \mathrm{C}^{*}\end{array}$ & $\frac{9,1 \% \mathrm{~A}+90,9 \% \mathrm{M}}{30,0 \% \mathrm{~A}+70,0 \% \mathrm{M}^{*}}$ & $3,72 *$ & 6,62 \\
\hline 4 & $\frac{0,37 \% \mathrm{TiC}}{-}$ & 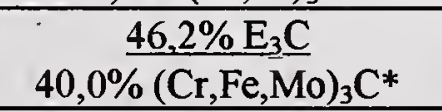 & $\frac{14,9 \% \mathrm{~A}+85,1 \% \mathrm{M}}{15, \% \mathrm{~A}+85,0 \% \mathrm{M}}$ & $5,96^{*}$ & 7,80 \\
\hline 5 & $\frac{6,27 \% \mathrm{TiC}+4,54 \% \mathrm{NbC}}{15,0 \%(\mathrm{TiC}+\mathrm{NbC})^{* *}}$ & $\begin{array}{c}\underline{28,7 \% \mathrm{E}_{23} \mathrm{C}_{6}} \\
24,0 \%(\mathrm{Cr}, \mathrm{Fe}, \overline{\mathrm{Mo}})_{23} \mathrm{C}_{6} * *\end{array}$ & $\frac{42,4 \% \mathrm{~A}+57,6 \% \mathrm{M}}{\mathrm{A}+\mathrm{M}}$ & $6,65 * *$ & 8,01 \\
\hline 6 & $\frac{2,83 \% \mathrm{TiC}+3,32 \% \mathrm{NbC}}{8,00 \%(\mathrm{TiC}+\mathrm{NbC})^{* *}}$ & $\frac{20,0 \% \mathrm{E}_{3} \mathrm{C}_{6}}{25,0 \%(\mathrm{Cr}, \mathrm{Mo}, \mathrm{Fe})_{23} \mathrm{C}_{6} * *}$ & $\frac{3,6 \% \mathrm{~A}+96,4 \% \mathrm{M}}{\mathrm{A}+\mathrm{M}}$ & 5,27 ** & 7,96 \\
\hline
\end{tabular}

${ }^{*}$ Ref. $3,{ }^{* *}$ Ref. 17. 
The program accounts for the possibility of forming of 27 independent carbides and borides $\left(\mathrm{B}_{4} \mathrm{C}\right.$, $\mathrm{Cr}_{23} \mathrm{C}_{6}, \mathrm{Cr}_{7} \mathrm{C}_{3}, \mathrm{Mn}_{23} \mathrm{C}_{6}, \mathrm{Mo}_{2} \mathrm{C}, \mathrm{MoC}, \mathrm{VC}, \mathrm{V}_{2} \mathrm{C}, \mathrm{TiC}, \mathrm{NbC}, \mathrm{SiC}, \mathrm{ZrC}, \mathrm{W}_{2} \mathrm{C}, \mathrm{WC}, \mathrm{Fe}_{3} \mathrm{C}, \mathrm{CrB}_{2}, \mathrm{CrB}$, $\mathrm{NiB}, \mathrm{Ni}_{4} \mathrm{~B}_{3}, \mathrm{VB}, \mathrm{TiB}, \mathrm{NbB}_{2}, \mathrm{ZrB}_{2}, \mathrm{AlB}_{12}, \mathrm{FeB}_{2}, \mathrm{FeB}$ ) as well as complex carbides and carboborides. Depending on alloying the matrix structure can de defined as austenite, austenitemartensite, martensite, austenite-bainite, bainite or perlite with the evaluation of quantitative ration of structural components. Table I gives compositions of the surfaced metal (numerator) and calculated composition of their matrix. Table II shows the results of calculation (numerator) of the phase composition and structure of the surfaced metal as compared with experimental data contained in references (Ref. 3, 17)

\section{CONCLUSIONS}

The developed mathematical model precisely enough prognosticates the phase composition, structure and wear resistance of the surfaced metal in the wide range of alloying and can be used for selection and development of new surfacing alloys. It is necessary additionally to take into account the heat conditions of surfacing and the degree of stability of components to increase the precision of the prognosis.

\section{REFERENCES}

1. Livshits, L.S.; Grinberg, N.A.; and Kurkumelli, E.G. 1969. Fundamentals of alloying of the surfaced metal: 188 Moscow: Mashinostroyenie.

2. Frumin, I.I. 1977 Modern types of surfaced metal and their classification. Theoretical and technological aspects of surfacing. Surfaced metal: Kiev: Naukova dumka: 3-s to 17-s.

3. Popov, V.S.; Brikov, N.N.; Dmitrienwko, N.S.; and Pristupa, P.G. 1978. Long term service life of equipment for refractory production: 272 Moscow: Metallurgiya.

4. Hasun, A.; and Morigaki, O. 1985. Surfacing and spray coating: 240 Moscow: Mashinostroyenie.

5. Fedorov, V.V. 1979 Thermodynamic aspects of strength and destruction of solid state objects: 168 Tashkent: FAN.

6. Glushko, V.V. (edit.) 1971. Thermodynamic and heat physical properties of products of burning: vol. 1266 Moscow: VNITI.

7. Turkdogan, E.T. 1980. Physical chemistry of high temperature technology: 344 New Work: Academic Press. Inc.

8. Kulikov, I.S. 1988. Thermodynamics of carbides and nitrides: 320 Chelyabinsk: Metallurgiya (Chelyabinsk departament).

9. Korolev, N.V.; Grigoruev, S.L.; and Logakina, I.S. 1986. Calculation of the phase composition of surfaced metal with carboboride strengthening. Avtomaticheskaya svarka 8: 18 -s to 22 -s.

10. Hudremon, E. 1959. Special steels vol.2: 952 Moscow: Metallurgizdat.

11. Grinberg, N.A.; and Mongait, N.A. 1978. Affect of boron on the phase structure of surfaced metal at abrasive wearing. Metallovedenie i termicheskaya obrabotka metallov 12: 48-s to 60-s.

12. Hrushev, M.N. 1978. Wear resistance and structure of solid surfacing: 132 Moscow: Mashinostroyenie. 
13. Potak, Y.M.: and Sagalevich, E.A. 1972. Structural diagram of low carbon stainless steels as applied to cast and surfaced metal. Avtomaticheskaya svarka 5: 10-s to 13-s.

14. Giltrow, J.P. 1970 Wear vol. 15(1): 71-s to 78-s.

15. Korolev, N.V.; Logakina, I.S;Grigoruev, S.L.; and Abdulin, E.B. 1990 Principles of choice of surfacing materials resistant under conditions of abrasive and impact-abrasive wearing. Equipment and materials for surfacing. IES of E.O.Paton: 74-s to 77-s.

16. Korolev, N.V.; Grigoruev, S.L ; Logakina, I.S;. and Abdulin, E.B 1988 Structural-energetic approach to the evaluation of wear resistance of alloys at abrasive wear. Theoretical and technological fundamentals of surfacing. Surfacing in metallurgy and mining: IES of E.O.Paton: 49-s to 50-s.

17. Mosok, V.M; Danilets, A.I.; and others. 1991. Affect of composition and structure of complex-alloyed carbon-chrome-niobium alloys on iron base upon resistance to impactabrasive wear. Avtomaticheskaya svarka 12: 43-s to 45-s. 


\title{
PREDICTION OF FERRITE CONTENT IN THE HEAT AFFECTED ZONE OF DUPLEX AND SUPER DUPLEX STAINLESS STEELS
}

\author{
S. M. Khafagy", J. C. Suárez", F. Molleda*
}

\begin{abstract}
The fusion welding zone and heat-affected zone (HAZ), heated above the ferrite solvus temperature and followed by a rapid cooling to room temperature, have been shown to exhibit an increase in the ferrite content compared with the base metal. This increase in ferrite/austenite balance is known to promote deterioration of both the corrosion resistance and toughness of the joint. Concerning this problem, a mathematical model based on empirical relations between the maximum ferrite content in HAZ and logarithm of cooling rate has been developed, in order to predict the ferrite content and the width of HAZ at high temperatures. On the other hand, this model enables to determine approximately the heat input required for welding plates of defined thickness and targeted ferrite content.
\end{abstract}

\section{KEYWORDS}

Duplex stainless steel, super duplex stainless steel, modelling, HAZ, ferrite, heat input.

\section{INTRODUCTION}

Duplex stainless steels (DSS) usually consist of approximately equal percentages of austenite and ferrite. This microstructure enables to take advantage of the properties of both phases. Compared with ferritic stainless steels, they can offer improved formability, weldability and toughness; compared with austenitic stainless steels, higher strength, better corrosion resistance, and improved resistance to corrosion cracking. For that reason this type of steel is used in chemical and petrochemical industries.

During the weld process, the microstructure completely changes in the HAZ, i.e., from temperatures just below the melting point to room temperature. These changes are controlled by the weld thermal cycle, that is a function of several weld variables such as arc speed, voltage and current intensity. Therefore, it is necessary to find a physical model able to predict the microstructural changes happening in the HAZ during the welding process, when a certain set of welding parameters are used.

A review of the related literature shows two ways to investigate the effect of welding variables on the microstructural changes. Some researchers [ref. 1,2,3] studied the ferritic grain growth of this zone by utilizing the Ahrrenius equation, in which grain growth is function of temperature and

\footnotetext{
* Escuela Técnica Superior de Ingenieros Navales. Universidad Politécnica de Madrid. Ciudad Universitaria, 28040 Madrid, Spain
} 
time during the weld thermal cycle. Actually, HAZ consist of a few ferritic grain rows which surround the fusion zone and in some cases it is difficult to find complete grains like in the TIG welding process. Therefore, most of the researches have used different weld simulators to produce specimens with a microstructure similar to the real HAZ. They also related grain growth to the cooling time from $1200-800^{\circ} \mathrm{C}$ and this value is then correlated to the heat input to provide a method for prediction of $\mathrm{HAZ}$ grain size based on welding parameters.

In other studies, variations in the ratio of phases $(\delta / \gamma)$ in the $\mathrm{HAZ}$ were experimentally determined and the correlation between weld parameters and the change in the relative proportion of phases established. For example, Hannerz et al [ref. 4] studied the effect of the cooling rate on the simulated HAZ for different types of duplex stainless steels. They have used a regression analysis to find out a mathematical model that determines the austenite content in the HAZ, starting from the weld conditions and physical properties of the base metal.

\section{THEORETICAL MODEL}

\section{Prediction of ferrite content}

To obtain a good predictive analysis, it is very important to use suitable equations for the calculation of the weld thermal cycle. Rosenthal [ref.5] was the first to use an analytical solution of the heat flow differential equation. His solution gives the temperature $(T)$ in a point located at a distance (r) from the welding line as a function of the time $(\mathrm{t})$. For a given heat input, two limit solutions have been used: thin plate and thick plate. But this solution has been lately modified by some other [ref. 6,7,8,9,10].

To calculate the thermal cycle, a mobile heat source (electrode) is assumed, displacing at a constant speed (v). Therefor, heat input is calculated as:

$$
Q=\frac{\eta V I}{v}
$$

where:

$$
\begin{aligned}
& \eta=\text { efficiency of the welding process. } \\
& V=\operatorname{arc~voltage}(V) \text {. } \\
& I=\operatorname{arc} \text { current intensity (A). }
\end{aligned}
$$

Energy losses by radiation and convection from the hot surface have been included in the efficiency factor, $\eta$. Therefore, the equation for heat flow conduction is given by:

$$
\frac{\partial T}{\partial t}=\frac{\lambda}{c \rho}\left(\frac{\partial^{2} T}{\partial x^{2}}+\frac{\partial^{2} T}{\partial y^{2}}+\frac{\partial^{2} T}{\partial z^{2}}\right)
$$

where:

$$
\begin{aligned}
& T=\text { temperature }(K) . \\
& t=\text { time }(s)
\end{aligned}
$$


$\lambda=$ thermal conductivity $\left(\mathrm{J} \mathrm{m}^{-1} \mathrm{~s}^{-1} \mathrm{~K}^{-1}\right)$

$\mathrm{c} \rho=$ specific heat per unit volume $\left(\mathrm{J} \mathrm{m}^{-3} \mathrm{~K}^{-1}\right)$.

To solve the previous differential equation, the following assumptions were assumed:

- Thermal conductivity $(\lambda)$ and specific heat (co) do not change with temperature.

- Fixed coordinate system $(x, y, z)$ is replaced by a movable coordinate system $(\xi, y, z)$, where the coordinate $\xi$ is given by:

$$
\xi=x-v t
$$

Therefore, equation (2) yields the following limit solutions:

For thick plate:

$$
T=T_{0}+\frac{Q / v}{2 \pi \lambda t} \exp \left(-\frac{r^{2}}{4 a t}\right)
$$

For thin plate:

$$
T=T_{0}+\frac{Q / v}{d \sqrt{4 \pi \lambda c \rho t}} \exp \left(-\frac{r^{2}}{4 a t}\right)
$$

where:

$$
\begin{aligned}
& T_{0}=\text { initial temperature }(K) . \\
& r=\text { distance from the heat source to the point in which } T \text { is calculated }(m) . \\
& a=\lambda / c \rho .
\end{aligned}
$$

For thick plate, equations have been slightly modified to take into consideration that the heat source has a finite radius $\left(\mathrm{r}_{\mathrm{B}}\right)$, transforming into:

$$
T=T_{0}+\frac{Q / v}{2 \cdot \pi \cdot \lambda \sqrt{t\left(t+t_{0}\right)}} \exp \left\{-\frac{1}{4 a}\left[\frac{\left(z+z_{0}\right)^{2}}{t}+\frac{y^{2}}{t+t_{0}}\right]\right\}
$$

where :

$$
\begin{gathered}
t_{0}=\frac{r_{B}}{4 a} \\
z_{0}=\frac{r_{B}}{e} \sqrt{\frac{\pi a r_{B}}{v}}
\end{gathered}
$$

In the case of thick plate, part of the heat input is absorbed by the liquid metal as a latent heat of fusion (L). Subsequently, during solidification, this heat is given back to the base metal. For calculation of the actual heat input, the latent heat should be subtracted from the total heat input. If the fusion zone is modeled as a semicylinder of radius $\left(z_{m}\right)$, actual heat input is: 


$$
Q^{\prime}=Q-\frac{\pi z_{m}^{2} \nu L}{2}
$$

A linear relationship has been found between the maximum ferrite content and the logarithm of the cooling rate at $700^{\circ} \mathrm{C}$. This relationship has been confirmed by the works of some other researchers [ref. 11,12], as shown by the following equation:

$$
\% \text { Ferrite }=\zeta \log V_{700}+\psi
$$

where:

$$
\begin{aligned}
& \zeta=\text { constant related to the nitrogen content in the base metal. } \\
& \psi=\text { constant. } \\
& V_{700}=\text { cooling rate at } 700^{\circ} \mathrm{C}(\mathrm{K} / \mathrm{s}) .
\end{aligned}
$$

To determine the thermal cycle of the HAZ experiencing high temperatures, i.e., in which the austenite phase transforms totally to ferrite during heating, it is necessary to calculate the distance $\left(r_{\alpha}\right)$ between this zone and the heat source using the previous equations for the thermal cycle. For the thin plate, value of ferrite solvus temperature $\left(\mathrm{T}_{\alpha}\right)$ is replaced in equation $(4 \mathrm{~b})$, determining this distance using equation (8):

$$
r_{\alpha}=\frac{Q / v}{2 d c \rho\left(T_{\alpha}-T_{0}\right)} \sqrt{\frac{2}{\pi e}}
$$

For the thick plate, value of ferrite solvus temperature $\left(T_{\alpha}\right)$ is replaced in equation $(4 a)$, determining this distance using equation (9):

$$
r_{\alpha}=\frac{I}{c \rho} \sqrt{\frac{2 Q / v}{e\left(T_{\alpha}-T_{0}\right)}}
$$

Once the distance $r_{\alpha}$ is known, the cooling rate at $700^{\circ} \mathrm{C}$ can be determined in this zone, where the amount of ferrite is maximum, by differentiating equation (4b) respect to the time. For thin plate:

$$
V_{700}=\frac{A}{\theta^{3 / 2}}\left(\frac{B}{\theta}-\frac{1}{2}\right) \exp \left(-\frac{B}{\theta}\right)
$$

where:

$\theta=$ time at which the temperature reaches to precisely $700^{\circ} \mathrm{C}$ during the cooling (s).

$$
\begin{gathered}
A=\frac{Q / v}{d \sqrt{4 \pi \lambda c \rho}} \\
B=\frac{r_{\alpha}^{2}}{4 a}
\end{gathered}
$$


To simplify equation ( $5 \mathrm{a}$ ), it has been assumed that the point where the maximum ferrite content is calculated remains just below the axis of arc displacement $\left(y=0, z=r_{\alpha}\right)$. Therefore, equation (10) can be rewritten as:

$$
V_{700}=-C \frac{\exp \frac{G}{\theta}}{\sqrt{\theta\left(\theta+t_{0}\right)}}\left\{\frac{2+t_{0}}{2\left[\theta\left(\theta+t_{0}\right)\right]}+\frac{G}{t^{2}}\right\}
$$

where :

$$
\begin{gathered}
C=\frac{Q / v}{2 \pi \lambda} \\
G=-\frac{r_{\alpha}+z_{0}}{4 a}
\end{gathered}
$$

Substituting the value of $V_{700}$ into equation (7), the maximum ferrite content in HAZ can be predicted.

\section{Prediction of suitable heat input}

Figure (1) shows the result of this model, explaining the role of heat input and thickness of thin plate of duplex stainless steel (SAF2205) on the maximum ferrite content in the HAZ. At the highest heat inputs, equations switch from the case of thin plate to thick plate, corresponding to the gray area in the drawing.

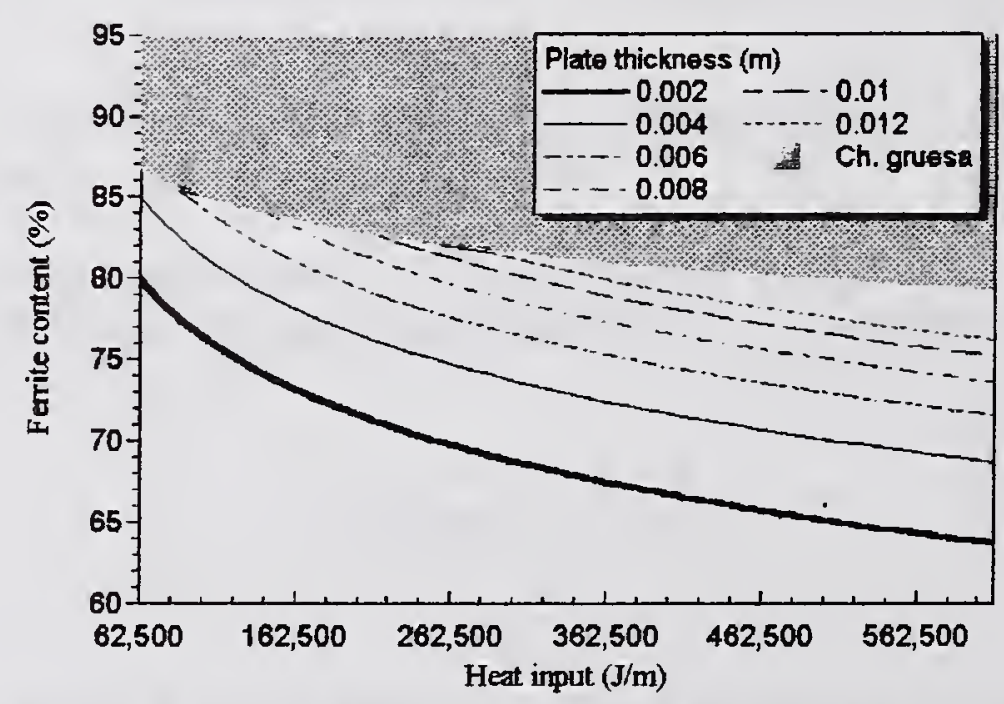

Figure 1. Effect of heat input and plate thickness on ferrite zontent in the HAZ.

From this figure, we can obtain a relationships between the maximum ferrite content $(\alpha)$ and heat input (HI) applied for welding plate of thickness (d), without no need of determining the cooling rate at $700^{\circ} \mathrm{C}$, as shown in the following equation: 


$$
H I=(\tau d+\varepsilon) \exp (\alpha \chi)
$$

where: $\tau, \varepsilon$ and $\chi$ are constants depending on the base metal.

Repeating the previous steps by using physical properties and constants $(\zeta, \Psi)$ obtained from reference 11 , we can determine the constants for different types of duplex and super-duplex stainless steels, as showing in table (1).

\begin{tabular}{|c|c|c|c|}
\hline Material & $\chi$ & $\tau$ & $\varepsilon$ \\
\hline UNS 31803 (SAF 2205) & -0.0841 & $7.933 \mathrm{E} 10$ & $3.731 \mathrm{E} 06$ \\
URANUS 45N & -0.140 & $2.261 \mathrm{E} 12$ & $4.736 \mathrm{E} 08$ \\
UNS 32550 & -0.0845 & $8.582 \mathrm{E} 10$ & $6.059 \mathrm{E} 06$ \\
URANUS 52N & -0.185 & $2.394 \mathrm{E} 13$ & $8.574 \mathrm{E} 08$ \\
\hline
\end{tabular}

Table 1. Values of constants of equation (12) for several duplex and super duplex stainless steels.

\section{MODEL RESULTS}

The first part of this model determines the weld thermal cycle, constant temperature profile and cooling rate, specially at $700^{\circ} \mathrm{C}$, in any point of the joint. Figures (2-5) show the calculated thermal cycle and the variation of heating and cooling rates during welding in a point located at $4.5 \mathrm{~mm}$ from the heating source. For this example, thin plate (6 mm) of DSS (SAF 2205) is welded with TIG, using a heat input of $0.42 \mathrm{MJ} / \mathrm{m}$. Figures (2) and (4) show the heating half of the weld thermal cycle and variation in heating rate. This is divided into two region. In the first region, at low temperature, the heating rate increases up to a maximum value. But in the second region, heating rate decreases with increasing heating temperature until it reaches zero at peak temperature. During cooling, figures (3) and (5), the variation in cooling rate behaves in the same manner as the heating rate but with higher and negative value.

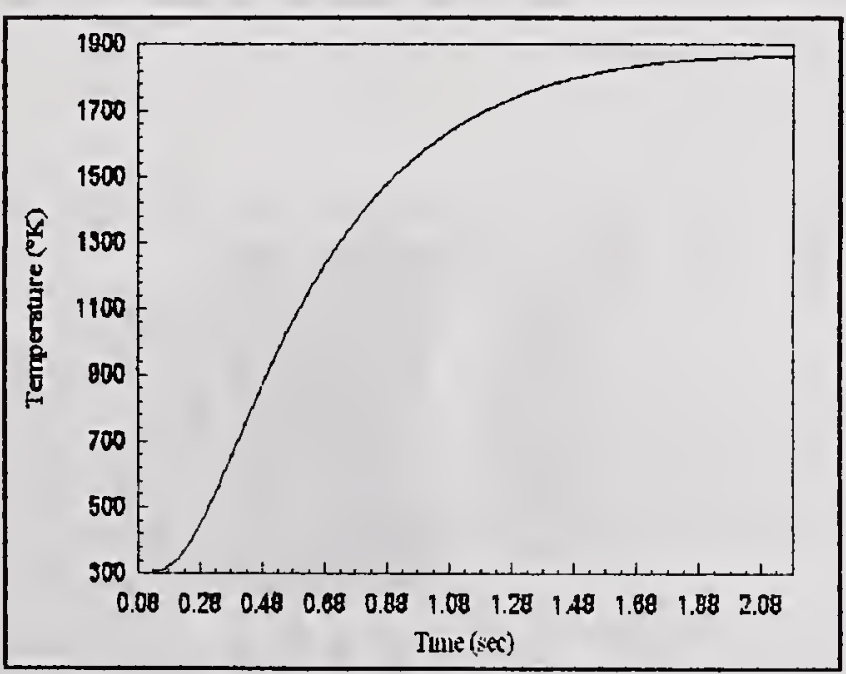

Figure 2. Half thermal cycle, during the heating period of the weld process.

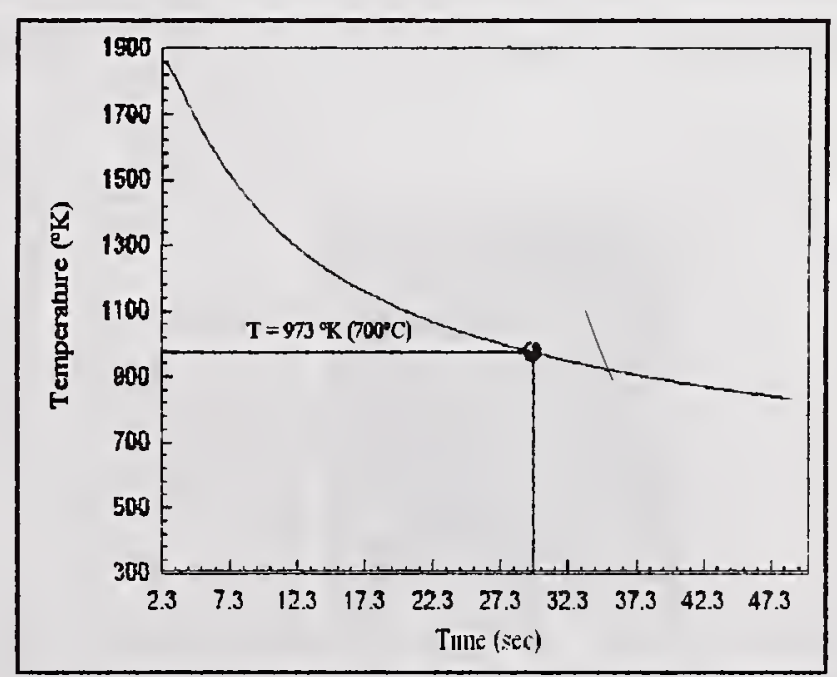

Figure 3. Half thermal cycle, during the cooling period of the weld process. 


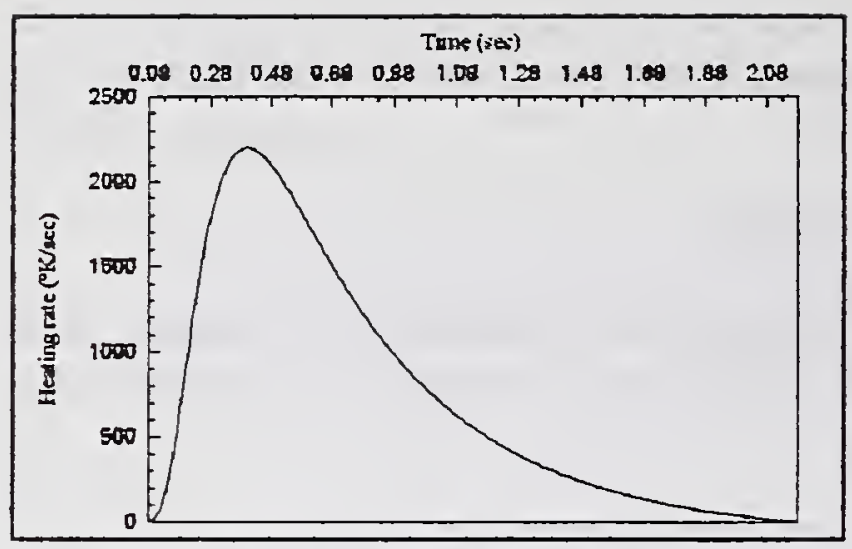

Figure 4. Variation in heating rate.

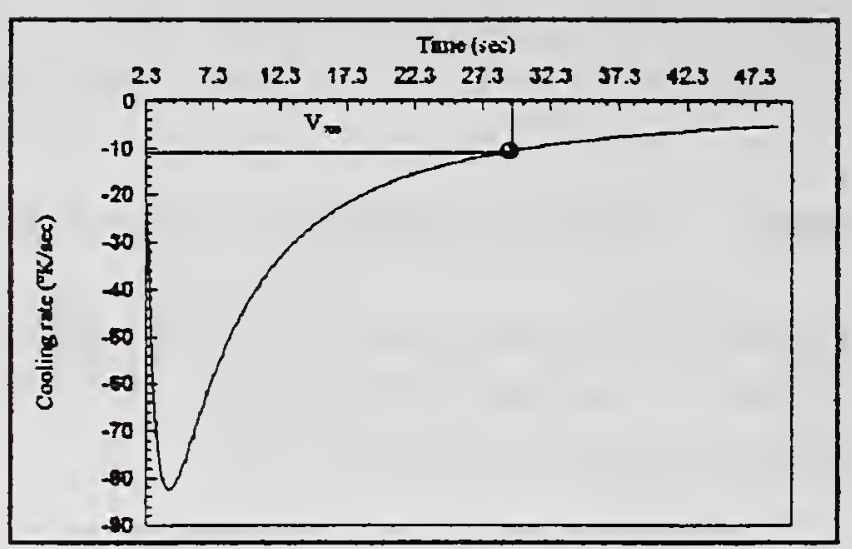

Figure 5. Variaticn in cooling rate.

Velocity of arc displacement is an important factor that determines the heat input, as explained in the equation (2). Consequently, figures (6) and (7) show four thermal cycles and cooling rate diagrams calculated for different values of arc velocity. Data for these curves correspond to points located just below the fusion line, where the peak temperature is $1650 \mathrm{~K}$. Dash line represents the variation of cooling rate at $700^{\circ} \mathrm{C}$ as welding time advances.

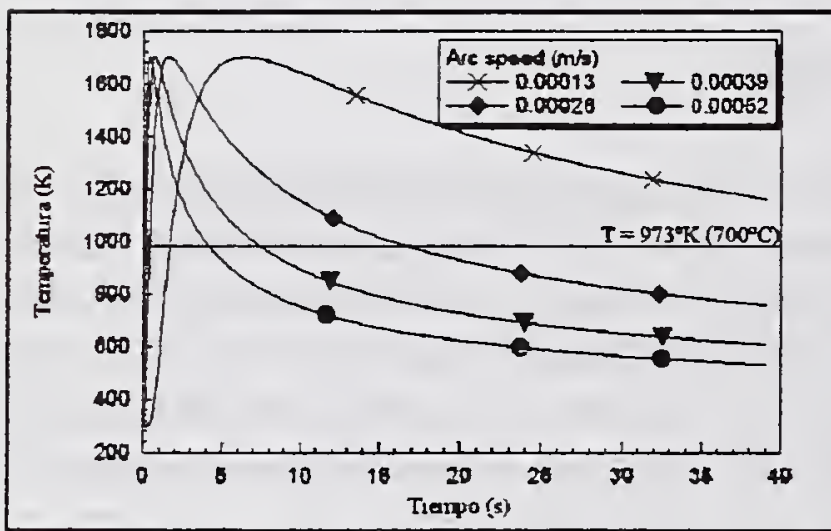

Figure 6. Effect of arc speed on the thermal cycle.

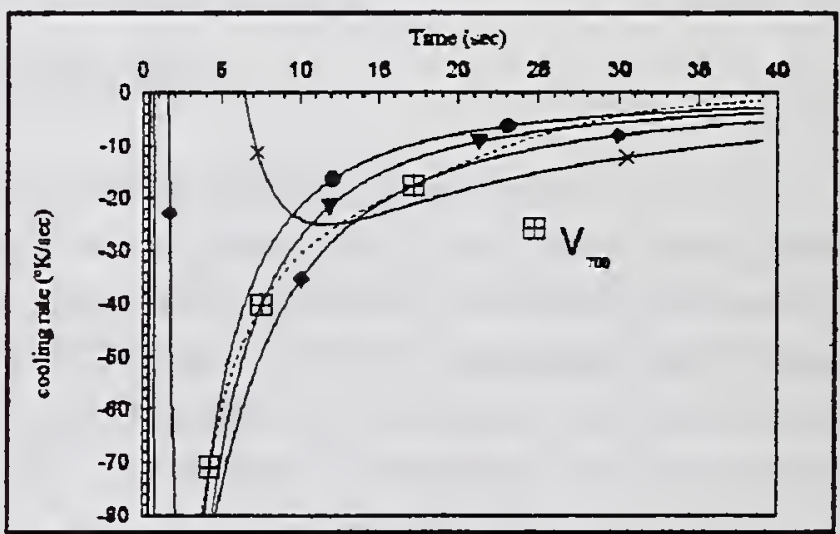

Figure 7. Effect of arc speed on the variation of cooling rate.

Figures (8) and (9) show isothermal diagrams for thin and thick plates respectively, with the same process variables. It can be observed that the Heat Affected Zone for thin plate is spread in a wider area and so is for the Fusion Zone, as compare with a thick plate.

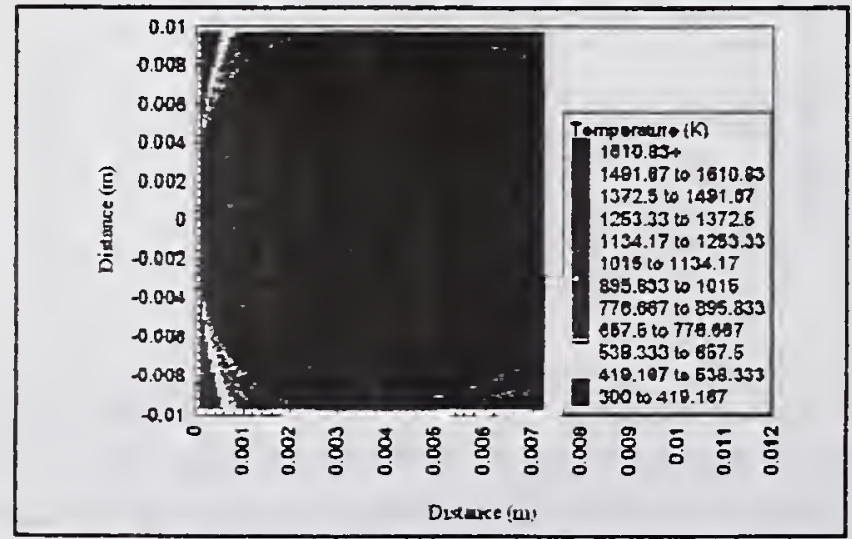

Figure 8. Isothermal diagram for thin plate.

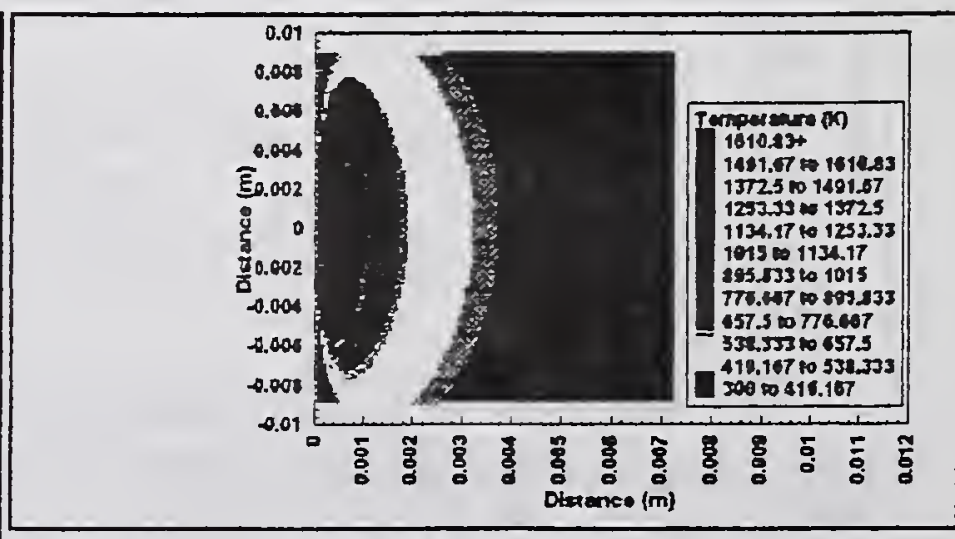

Figure 9. Isothermal diagram in the case of thick plate 
The second part of model can predict not only maximum ferrite content in the HAZ but HAZ width also. Figure (10) show the effect of heat input on HAZ width for DSS thin plate with different thicknesses. It should be noted that $\mathrm{HAZ}$ width diminish with increasing plate thickness and decreasing heat input, as heat transfer condition approaches to thick plate, where model equation predicts that HAZ width is virtually independent of plate thickness.

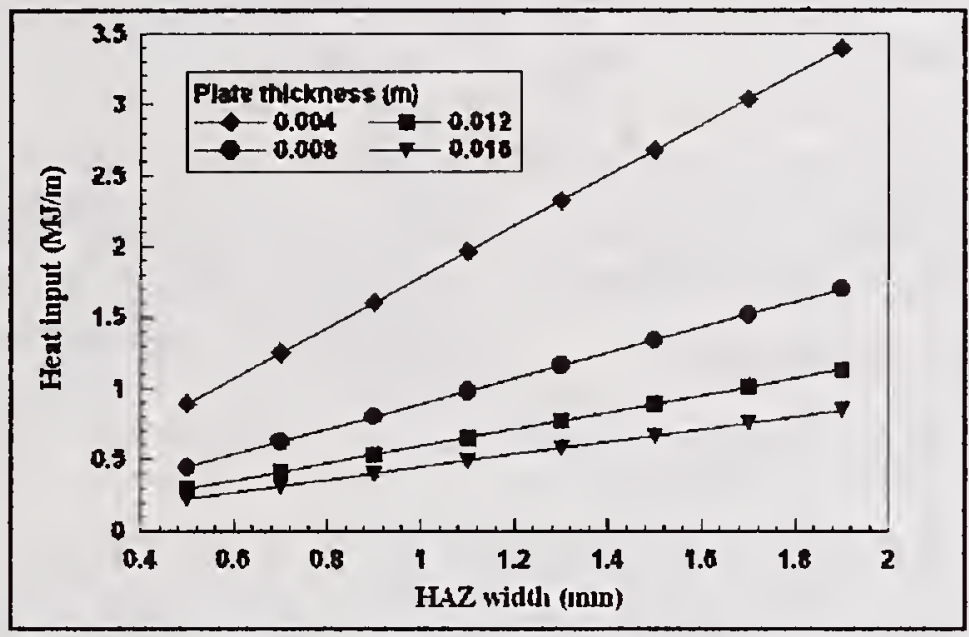

Figure 10. Effect of Heat input on the HAZ width of DSS (SAF 2205).

Figure (11) explains the variations in the maximum ferrite content of the HAZ for different types of duplex and superduplex stainless steels, both for thick and thin plates. The graphic shows the difference in ferrite content between all these types of duplex stainless steels. It is observed that ferrite content is higher for thick plate than for thin plate as a result of higher cooling rate at $700^{\circ} \mathrm{C}$.

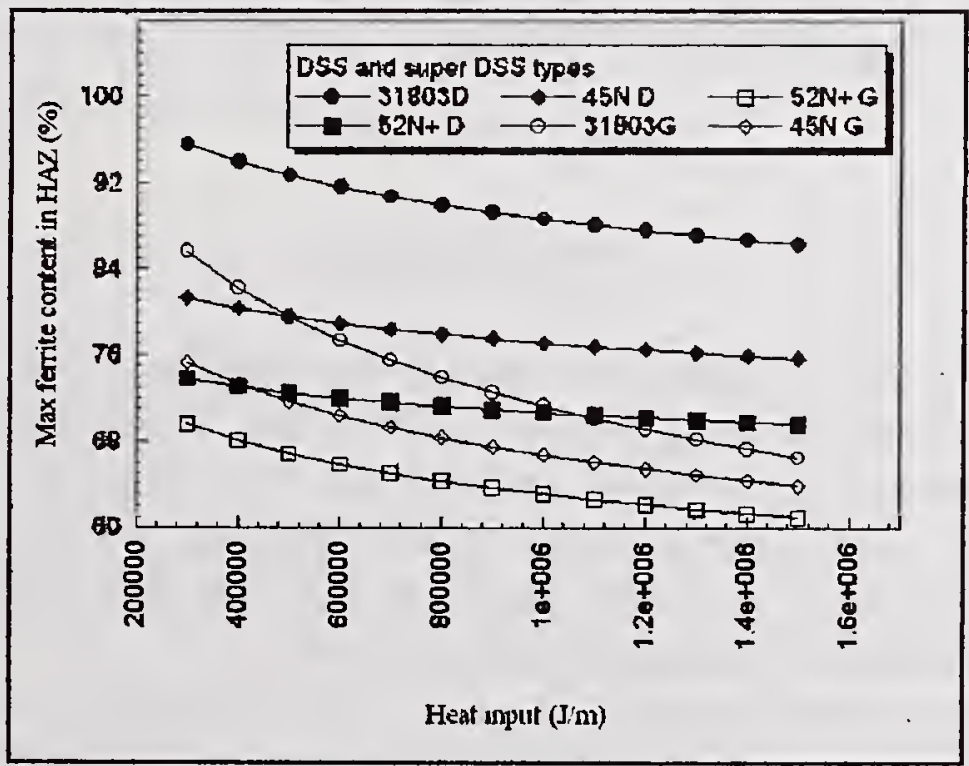

Figure 12, Maximum ferrite content in the $\mathrm{HAZ}$ as a function of heat input.

From equation (12), heat input needed to weld thin plate of known thickness can be calculated. Figures (13-15) present the results obtained by the referred equation. These graphics are easily used to select the optimum heat input. On the other hand, they can also predict ferrite content in the HAZ from chosen welding variables. 


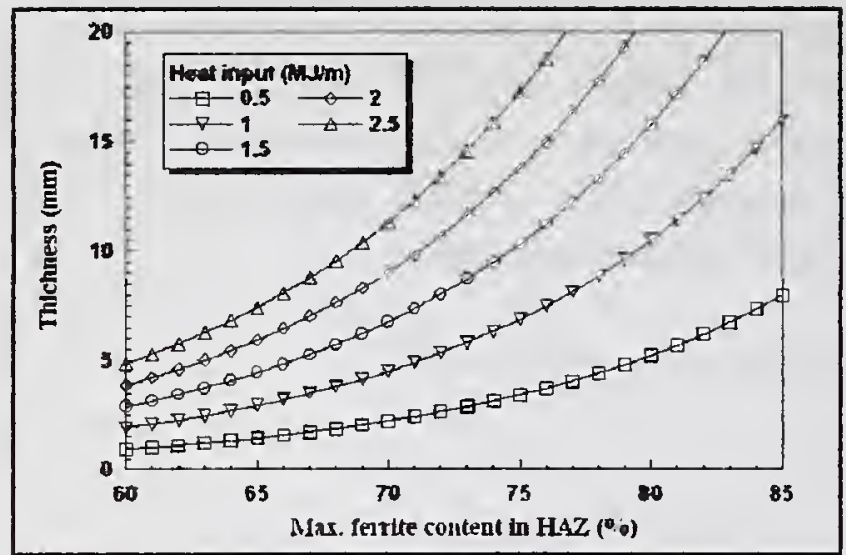

Figure 13. Correlation between heat input and ferrite content in the HAZ of DSS (SAF-2205) with different thicknesses .

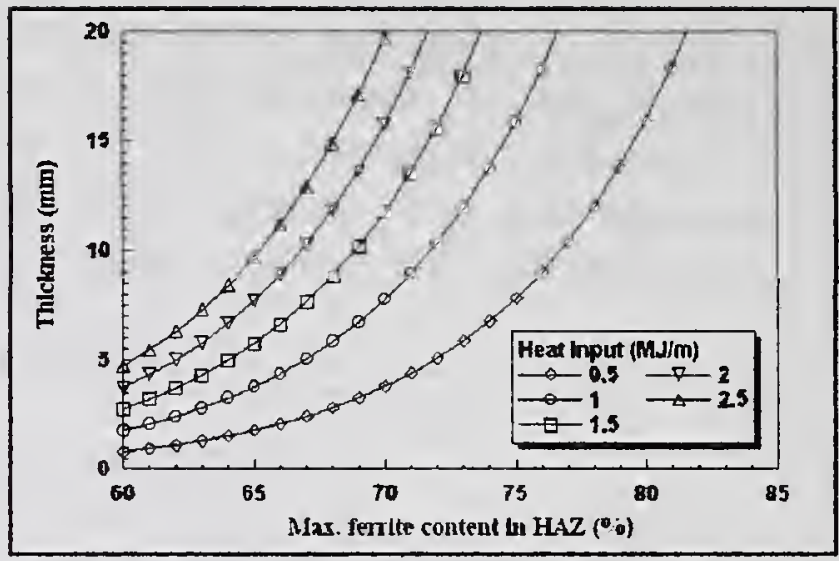

Figure 14. Correlation between heat input and ferrite content in the HAZ of DSS (Uranus-45N) with different thicknesses.

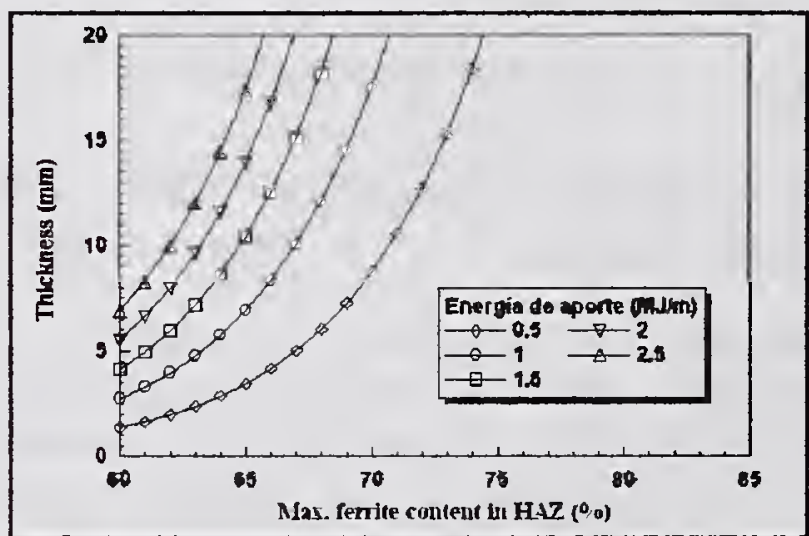

Figure 15. Correlation between heat input and ferrite content in the HAZ of DSS (Uranus-52N) with different thicknesses.

\section{CONCLUSION}

1. It has been presented a mathematical modeling for calculation of the thermal cycle, cooling and heating rates during welding process in DSS and super DSS. This modeling also enables to predict the maximum ferrite content in the Heat Affected Zone.

2. It is necessary to determine several constants for every type of DSS and super DSS. In this paper, these constants have been determined for different types of DSS by using physical properties and other constants obtained from references.

3. For a pre-determined ferrite/austenite balance, the model enables to predict the welding variables, i.e. heat input, necessary to obtain the desired microstructure.

\section{REFERENCES}

1. Kivinera, I.; and Hannerz, N, E. 1994. The properties of Gleeble simulated heat affected zone of 2205 and 2507 duplex stainless steels. Proc. DSS'94: paper 7 Glasgow: 
2. Lindblom, B. E.; Lundqvist, B.; and Hannerz, N, E. 1991. Grain growth in HAZ of duplex stainless steels. Proc. DSS'91 conf. eds. J.Charles, and S. Bernhardsson: 373-382. Les Editions de Physique. France.

3. 3Ferreira, P. J.; and Hertzman, S. Delta-ferrite grain growth in simulated high temperature of three duplex stainless steels.Proc. DSS'91 conf. eds. J.Charles, and S. Bernhardsson: 959-966. Les Editions de Physique. France.

4. Lindblom, B. E.; and Hannerz, N, E. 1991. Austenite reformation in HAZ of ferritic austenitic stainless steels. Proc. DSS'91 conf. eds. J. Charles, and S. Bernhardsson: 951958. Les Editions de Physique. France.

5. Rosenthal, D. 1941. Mathematical theory of heat distribution during cutting and welding. Welding Journal 20 (5).

6. Ashby, M. F.; and Easterling, K. E. 1982. First report on diagrams for grain growth in welding. Acta, Metall. 30: 1969-1982.

7. Ion, J. C.; Ashby, M. F.; and Easterling, K. E. 1984. A second report on diagrams of microstructure and hardness for heat-affected zone in welding. Acta. Metall. 32: 19491962.

8. Senk, I.; and Martinak, M. 1992. Mathematical simulation on thermal cycle in the heat affected zone in narrow-gap. Welding International 6 (8): 654-660.

9. Easterling, K. E. 1993. Modelling the weld thermal cycle and transformation behaviour in the heat affected zone. Mathematical modelling of weld phenomena. eds. H. Cerjak, K. E. Easterling, Institute of Material.

10.Suárez, J. C. 1990. Modelización numérica de los cambios microestructurales en la soldadura de aceros HSLA microaleados. Ph thesis. Univ. Complutense de Madrid.

11.Bonnefois, B.; Charles, J.; Dupoiron, F.; and Soulignac, P. 1991. How to predict welding properties of duplex stainless steel?. Proc. DSS'91 conf. eds. J.Charles, and S. Bernhardsson: 347-361. Les Editions de Physique. France.

12.Khafagy, S. M.; Suárez, J.C.; and Molleda, F. 1996. Simulation of heat affected zone in welded joints of duplex stainless steels. Proc. Inter. MDP conf. eds M. E. Elarabi, and A. S. Wifi: 273-280. Cairo Univ. 


\title{
COMPUTATIONAL MODELING OF MICROSTRUCTURE EVOLUTION IN WELD HEAT AFFECTED ZONE OF LOW ALLOY STEEL
}

\author{
M. V. Li, ${ }^{1}$ L. L. Meekisho, ${ }^{2}$ and D. G. Atteridge ${ }^{2}$
}

\begin{abstract}
Preliminary studies by the authors revealed some serious limitations of the Watt algorithm in the prediction of microstructure evolution in the weld heat affected zone of low alloy steels. The core of the Watt algorithm was the reaction kinetics model for austenite decomposition originally developed by Kirkaldy and Venugopalan. Although theoretically sound, the KirkaldyVenugopalan model was found to underestimate steel hardenability significantly and it has to be modified to give more reliable predictions.

The pioneering work of Kirkaldy and his associates has laid down the foundation for the computational prediction of phase transformations under non-isothermal conditions. Based on that, a new model has been developed for the prediction of austenite decomposition reactions under arbitrary cooling conditions. The new model was empirically calibrated with CCT diagrams. This paper presents some fundamental aspects of predicting microstructure development in weld heat affected zone of low alloy steels and the development and validation of the new model. This new model was applied for the prediction of microstructure evolution in the HAZ of electroslag cladding onto carbon steel. The predicted HAZ microstructure was found in good agreement with the metallographic examinations. The predicted HAZ microhardness was found in excellent agreement with the hardness measurements.
\end{abstract}

\section{INTRODUCTION}

Weld heat affected zone (HAZ) has both intriguing metallurgical complexities and considerable commercial significance. Mechanical properties of this area are different from, and usually inferior to, those of the base metal. It is the most common region of weld failures with failures generally related directly to the HAZ microstructure. Prediction of microstructure evolution and mechanical properties in the weld HAZ has thus become an important subject in modern computational weld mechanics (Ref. 1). The objective of this study was to predict the microstructure evolution in the HAZ induced by electroslag cladding of nickel alloys onto high strength low alloy steel.

In the preliminary studies by the authors, the algorithm originally proposed by Watt $e t$ al. (Ref. 2) was used for the prediction of weld heat affected zone microstructure and hardness of a hardenable steel (close to HY80 type). The Watt algorithm was one of the few open literature

\footnotetext{
' Center for Welded Structures Research, Battelle Memorial Institute, Columbus, Ohio

${ }^{2}$ Materials Science and Engineering Department, Oregon Graduate Institute, Portland, Oregon
} 
methods that predict complete microstructure evolution as a function of arbitrary thermal history. It incorporated the austenite grain growth model by Ashby and Easterling (Refs. 3,4), and the reaction kinetics model for austenite decomposition by Kirkaldy and Venugopalan (Ref. 5). The computed results led to unexpected amount of ferrite and bainite, whereas metallographic examination showed that the microstructure of the heat affected zone is primarily martensite and bainite. There was no martensite predicted even in the coarse grain heat affected zone of such hardenable steel.

To further verify the computational results, the Watt algorithm was then applied to predict the hardness distribution in end quench bars, commonly known as the Jominy hardness. The reason for doing so for the model validation was that, end quench test is a well known hardenability test, and there are lots of measured end-quench hardness data readily available in the open literature. Again, the Watt algorithm was found to underestimate steel hardenability by a factor of 3 to 5 for selected steels with different hardenability.

The core of the Watt algorithm is the austenite decomposition kinetics model by Kirkaldy and Venugopalan. Studies by the authors (Ref. 6) as well as others (Ref. 7) have shown that the Kirkaldy-Venugopalan model gives reasonably accurate predictions for low hardenable steels but gives less than desirable predictions for steels with moderate to high alloying element content. These results led to the decision to develop a modified prediction methodology that would be applicable to medium and high hardenability steels as well as low hardenability steels.

This paper presents the computational model and its application in the prediction of microstructure development in the HAZ induced by electroslag cladding onto low alloy carbon steels.

\section{MATERIALS AND EXPERIMENT}

This study involved prediction of the microstructure evolution in the heat affected zone induced by multipass electroslag cladding of nickel alloys onto carbon steel propeller shafts of submarines. The compositions of materials included MIL-S-23284, Class 1. The strip electrodes were $30 \mathrm{~mm}$ wide and $0.5 \mathrm{~mm}$ thick and were made of $\mathrm{Ni}$ Alloy $625,70 \mathrm{Cu}-30 \mathrm{Ni}$, $20 \mathrm{Cu}-70 \mathrm{Ni}$ and $\mathrm{Ni}$. Compositions of materials used in this study are listed in Table 1.

The optimal cladding parameters were determined by experiments. Current was found to be 650 amperes and the voltage 27 volts. The cladding speed was $7 \mathrm{in} / \mathrm{min}(2.96 \mathrm{~mm} / \mathrm{sec})$. The strip feed rate was $70 \mathrm{in} / \mathrm{min}(2.96 \mathrm{~cm} / \mathrm{sec})$ during the cladding. All clads were made at a heat input level of $59 \mathrm{~kJ} / \mathrm{cm}(150 \mathrm{~kJ} / \mathrm{in})$.

Cladding onto one shaft was analyzed in this study. The shaft was uniformly preheated to the predetermined preheating temperatures, $150{ }^{\circ} \mathrm{C}$. The shaft was of a dimension of $61 \mathrm{~cm}(24 \mathrm{in}$.) in diameter, $12.7 \mathrm{~cm}$ (5 in.) in wall thickness, and $104.1 \mathrm{~cm}$ (41 in.) in length. Transition from pass to pass was accomplished by a stepping technique with a slope of 1/7. 


\section{DESCRIPTION OF MICROSTRUCTURE EVOLUTION IN THE HAZ}

The microstructural evolution in the weld $\mathrm{HAZ}$ of carbon steels is primarily determined by the thermal cycle and steel composition. From a thermodynamics standpoint, any low alloy steel can be regarded as a multicomponent Fe-C-X system. Shown in Fig. 1 is a schematic illustration of phase diagram of multicomponent Fe-C-X system along with a typical thermal cycle in the weld HAZ. It should be noted that the equilibrium phase diagram of a low alloy steel differs from commonly known binary $\mathrm{Fe}-\mathrm{C}$ equilibrium diagram. The eutectoid reaction in such a multicomponent system would occur in a temperature range, which is represented by a multidimensional region. The authors have designated $\mathrm{Ae}_{1}$ and $\mathrm{As}_{1}$ temperatures as the upper and lower limits the eutectoid reaction region.

During the heating cycle in the weld HAZ, it is reasonable to assume that phase changes in weld HAZ follow equilibrium condition. HAZ microstructure would remain virtually unchanged until temperature reaches $\mathrm{As}_{1}$ temperature. At $\mathrm{As}_{1}$ temperature both ferrite and pearlite in the HAZ start to change into austenite. All pearlite is completely transformed to austenite at $\mathrm{Ae}_{1}$ temperature and all ferrite is completely transformed into austenite at $\mathrm{Ae}_{3}$ temperature.

At temperatures slight above $\mathrm{Ae}_{3}$ temperature, only austenite exists, newly formed austenite has very fine grains. For most low alloy steels, the average size of newly formed austenite grains is approximately 5 microns. These grains have strong tendency to grow larger so that the surface energy at grain boundaries would be reduced. Growth of austenite grains is very stagnant at temperature slightly above the $\mathrm{Ae}_{3}$ temperature due to insufficient thermal activation and the pinning effect of carbide and nitride precipitates. The pinning effect of carbide and nitride precipitates would disappear when the precipitate dissolution temperature, $T_{\text {DISS }}$, is reach. Austenite grains would grow at temperature higher than the dissolution temperature. Austenite grain growth is stopped when either $\mathrm{Ae}_{4}$ temperature is reached or certain maximum value has been reached which is associated with the dispersion of oxide precipitates and the temperature gradients.

As the temperature goes below $\mathrm{Ae}_{3}$ during cooling, austenite starts to decompose into ferrite phase. Ferrite has a body-centered crystal structure. In the welding HAZ, ferrite normally appears as a network or in parallel laths. It is a fairly soft and ductile structure.

At temperature below $\mathrm{Ae}_{1}$, ferrite transformation continues and pearlite transformation also starts. Pearlite normally appears in colonies and each colony consists of alternate plates of $(\mathrm{FeM})_{3} \mathrm{C}$ and ferrite, with ferrite being the continuous phase. Pearlite is not a phase, but a mixture of two phases: $(\mathrm{FeM})_{3} \mathrm{C}$ and ferrite.

Both ferrite and pearlite reactions are nucleation and growth transformations controlled by the diffusion of elements. Ferrite and pearlite are usually recognized as austenite decomposition products at high temperatures. In the weld HAZ of low alloy steels, it is very rare for these two reactions to reach completion. The lower the welding heat input and/or the better the steel hardenability, the harder it is for these two reactions reach completion. 
At temperatures below $\mathrm{B}_{\mathrm{s}}$ temperature, the formations of bainite would take precedence over ferrite and pearlite. The untransformed austenite would start to transform into bainite. Like pearlite, bainite is not a phase, but a mixture of ferrite and carbides and it is slightly harder than ferrite or pearlite structure. Bainite reaction is also a nucleation and growth transformation.

As the temperature goes below $\mathbf{M}_{\mathrm{s}}$ temperature, the untransformed austenite starts to transform into martensite and bainite formation would continue until there is no untransformed austenite. Martensite reaction is known to be athermal. The transformation is a function of temperature only. It is produced by shear movements of the austenite lattice, which produce a body-centered tetragonal lattice. Martensite is considered a phase and is the hardest of all austenite decomposition products in steels.

\section{COMPUTATIONAL MODEL}

To predict the microstructure evolution in the weld heat affected zone, one has to know the thermal history experienced by the weld HAZ. The thermal history needed for the microstructure prediction was obtained from the results of previously reported finite element analyses. The methodology of the finite element model developed for analyzing the heat transfer induced by multipass electroslag cladding has been presented in (Ref. 8). Its accuracy has been verified with temperature measurements. The finite element mesh used in the heat transfer analysis is presented in Fig. 2. Commercial finite element code ANSYS was used in the heat transfer analysis (Ref. 8). The computed nodal temperature results were written into ASCII files for later prediction of microstructure development. Temperature history in the HAZ was used as the thermal input for the subsequent analysis of microstructure evolution.

The methodology adopted by the authors in this research is similar to that in the Watt algorithm. Some subtle but important modifications have been made (Ref. 9). This model development effort concentrated in three major areas: 1) prediction of equilibrium phase composition and transformation characteristics; 2) reaction kinetics model of austenite decomposition on cooling; and 3) kinetic model for the austenite grain growth. The model for the prediction of microstructure development was coded in a Fortran program. The input for this program is the temperature history and the compositions of the materials. The output from this program includes the history of microstructure evolution and the resultant room temperature hardness distribution.

In the Watt algorithm, empirical formulas were used to estimate the temperatures of equilibrium phase transformations as well as the equilibrium amount of phase transformation products. In this study, it was found that empirical formulas were adequate for the estimation of phase transformation temperatures. Watt et al. combined empirical equations for $\mathrm{Ac}_{3}$ and $\mathrm{Ac}_{1}$ to estimate the eutectoid point and used the level rule to estimate the equilibrium amount of ferrite and pearlite. This approach often leads to erroneous results. In this study, temperatures of equilibrium phase transformations were computed using a rigorous thermodynamics model. The accuracy of the thermodynamics model was verified with experimentally measured phase 
transformation temperatures in the open literature (Ref. 10). A typical comparison between the predicted and measured $\mathrm{Ae}_{3}$ temperatures is presented in Fig. 3.

Also developed was a new reaction kinetics model for austenite decomposition. Two distinctions should be made between the new reaction kinetics model and the KirkaldyVenugopalan model. First, this new model was formulated in the way that reflects the wellaccepted hypothesis that the effects of alloying elements on steel hardenability are multiplicative. The original Kirkaldy-Venugopalan model implies the effect of alloying elements were additive. Second, the new reaction kinetics model for austenite decomposition was calibrated with continuous cooling transformation (CCT) diagrams. The KirkaldyVenugopalan model was calibrated with isothermal transformation (IT) diagrams.

It is rather difficult, if not impossible, to verify the accuracy of the model in a straightforward manner. An indirect approach was taken to verify the reliability of this new model against experimentally measured Jominy hardness of end-quench bars. This new austenite decomposition reaction model was found to give reasonably accurate predictions for Jominy hardness curves of low as well as medium and high hardenable steels. The predicted Jominy hardness curves using both the new model and the Kirkaldy-Venugopalan model are presented in Fig. 4 along with the experimentally measured ones.

The approach adopted in the current computational model for the prediction of austenite grain growth is similar to what was in the Watt algorithm. The difference is that, in the current program, computation of austenite grain growth was performed only during the heating cycle. As soon as the peak temperature is reached, computation of austenite grain growth was terminated. This approach was validated by the experimental observation of Ikawa et al. (Ref. 11), which says, austenite grain growth in the weld heat affected zone only takes place during the heating cycle.

\section{RESULTS AND DISCUSSION}

Computations showed that prior austenite grain size is rather uniform in the coarse grain region of the heat affected zone induced by electroslag cladding and the average diameter of prior austenite grains was around $105 \mu \mathrm{m}$. This was confirmed with experimental examinations.

The predicted HAZ microstructure consisted primarily with martensite and some amount of bainite in the coarse grain region. In the grain refined region, the microstructure is primarily bainite plus some martensite. The predicted martensite and bainite distributions are presented in Fig. 5 and 6 . The predicted microstructure is found in qualitative agreement with the metallographic examination (Ref. 12).

The predicted hardness distribution along the centerline of the HAZ is presented in Fig. 7 in comparison with the measured microhardness. The agreement between the prediction and 
measurements is very good. The predicted final room-temperature microhardness distribution in the clad is presented in Fig. 8.

\section{SUMMARY}

A fundamentally based and empirically calibrated reaction kinetics model for austenite decomposition has been developed, and it is the core of the computational algorithm used in this study for the prediction of microstructure development in weld HAZ of low alloy steels. This study has demonstrated that this algorithm gives more reliable predictions than the Watt algorithm. The predicted microstructure and hardness distributions in weld HAZ as well as Jominy end-quench bars are found in good agreement with experimental measurements.

\section{REFERENCES}

1. Easterling, K. E. 1990. Keynote address: microstructure and properties of the heat affected zone. Recent trends in welding science and technology ed. S. A. David: 177188.

2. Watt, D. F.; Coon, L.; Bibby, M.; Goldak, J.; and Henwood, C. 1988. An algorithm for modeling microstructural development in weld heat-affected zones (part A) reaction kinetics. Acta Metallurgica 36(11): 3029 to 3035.

3. Ashby, M. F. and Easterling, K. E. 1982. A first report on diagrams for grain growth in welds. Acta Metallurgica 30(11): 1969 to 1978.

4. Ion, J. C.; Easterling, K. E.; and Ashby, M. F. 1984. A second report on diagrams of microstructure and hardness for heat-affected zones in welds. Acta Metallurgica 32(11): 1949 to 1962.

5. Kirkaldy, J. S. and Venugopalan, D. 1984. Prediction of microstructure and hardenability in low alloy steels. Phase Transformation in Ferrous Alloys eds. A. R. Marder and J. I. Goldstein: 125-148.

6. Li, M. V.; Neibuhr, D.; Atteridge, D.; and Meekisho, L. 1995. Computing Jominy hardness curves of steels. Phase transformations during the thermal/mechanical processing of steel eds. E. B. Hawbolt and S. Yue: 485-501. Canadian Institute of Mining, Metallurgy and Petroleum.

7. Lee, J. L. and Bhadeshia, H. K. D. H. 1992. Assessment of TTT diagrams: examination of existing models. China Steel Technical Report (7): 19 to 29.

8. Li, M.; Atteridge, D. G.; Dikshit, V.; and Meekisho, L. 1993. Finite element modeling of heat transfer in multipass electroslag cladding. Proc. international conference on modeling and control of joining processes ed. T. Zacharia: 541-548. AWS/ORNL. 
9. Li, M. V.; Atteridge, D. G.; and Meekisho, L. 1995. Mathematical modeling of microstructure evolution in the heat affected zone of electroslag cladding. Proc. 4th international conference on trends in welding research eds. H. B. Smartt, J. A. Johnson, and S. A. David: 283-288, ASM.

10. Grange, R. A. 1961. Estimating critical ranges in heat treatment of steels. Metal Progress 70(4): 73 to 75 .

11. Ikawa, H.; Oshige, H.; and Nio, S. 1977. Austenite grain growth of steel in weld-heat affected zone. Transactions of the Japan Welding Society 8(2): 52 to 57.

12. Zhao, Q. H. 1993. Microstructural analysis of ni alloy cladding onto low alloy steel. M.S. dissertation, Portland, Or.: Oregon Graduate Institute of Science \& Technology.

Table 1 Composition of base metal steels and strip electrode

\begin{tabular}{|c|c|c|c|c|c|c|c|c|}
\hline Material & $\mathrm{C}$ & $\mathrm{Mn}$ & $\mathrm{Si}$ & $\mathrm{Ni}$ & $\mathrm{Cr}$ & $\mathrm{Mo}$ & $\mathrm{W}$ & $\mathrm{Fe}$ \\
\hline $\mathrm{A} 36$ & 0.20 & 1.01 & 0.10 & 0.02 & 0.02 & - & 0.002 & $\mathrm{Bal}$. \\
\hline A588 & 0.24 & 0.98 & 0.10 & 0.02 & 0.02 & - & 0.001 & Bal. \\
\hline 4140 & 0.38 & 0.81 & 0.28 & 0.11 & 0.98 & 0.22 & 0.003 & Bal. \\
\hline Class 1 & 0.24 & 0.31 & 0.20 & 3.04 & 0.39 & 0.44 & 0.011 & Bal. \\
\hline Ni 625 & 0.03 & 0.08 & 0.09 & Bal. & 21.50 & 9.00 & - & 4.25 \\
\hline
\end{tabular}




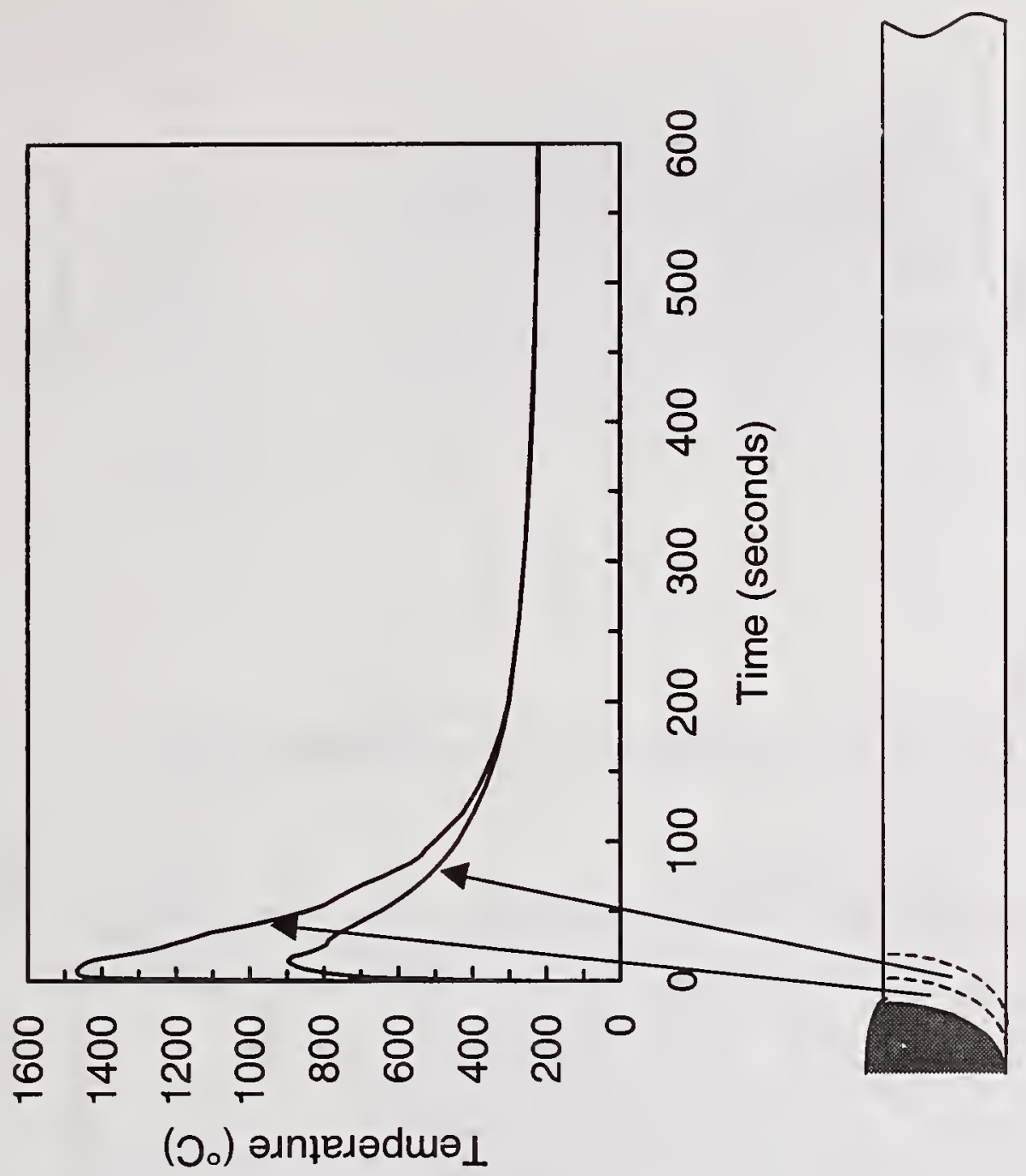

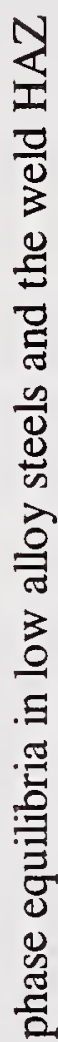

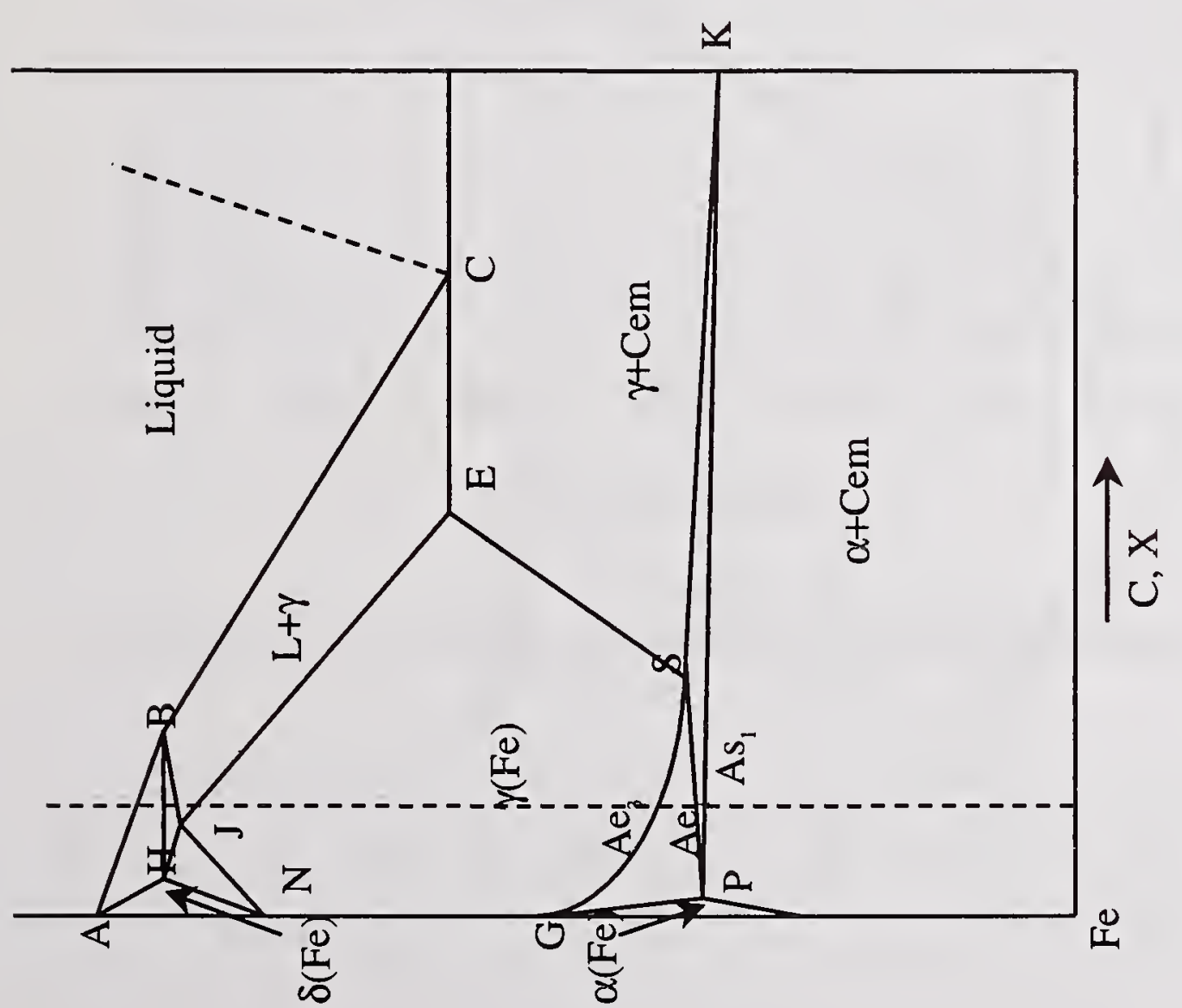

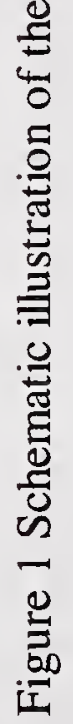




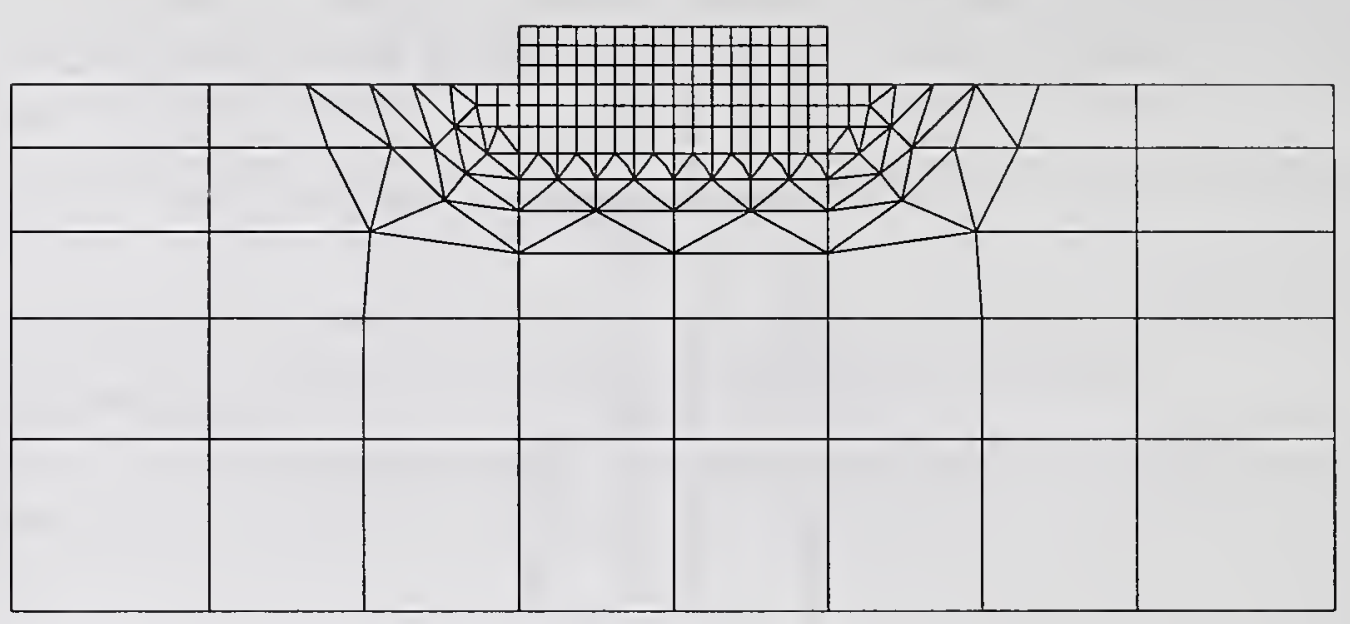

Figure 2 Finite element mesh used in the heat transfer analysis

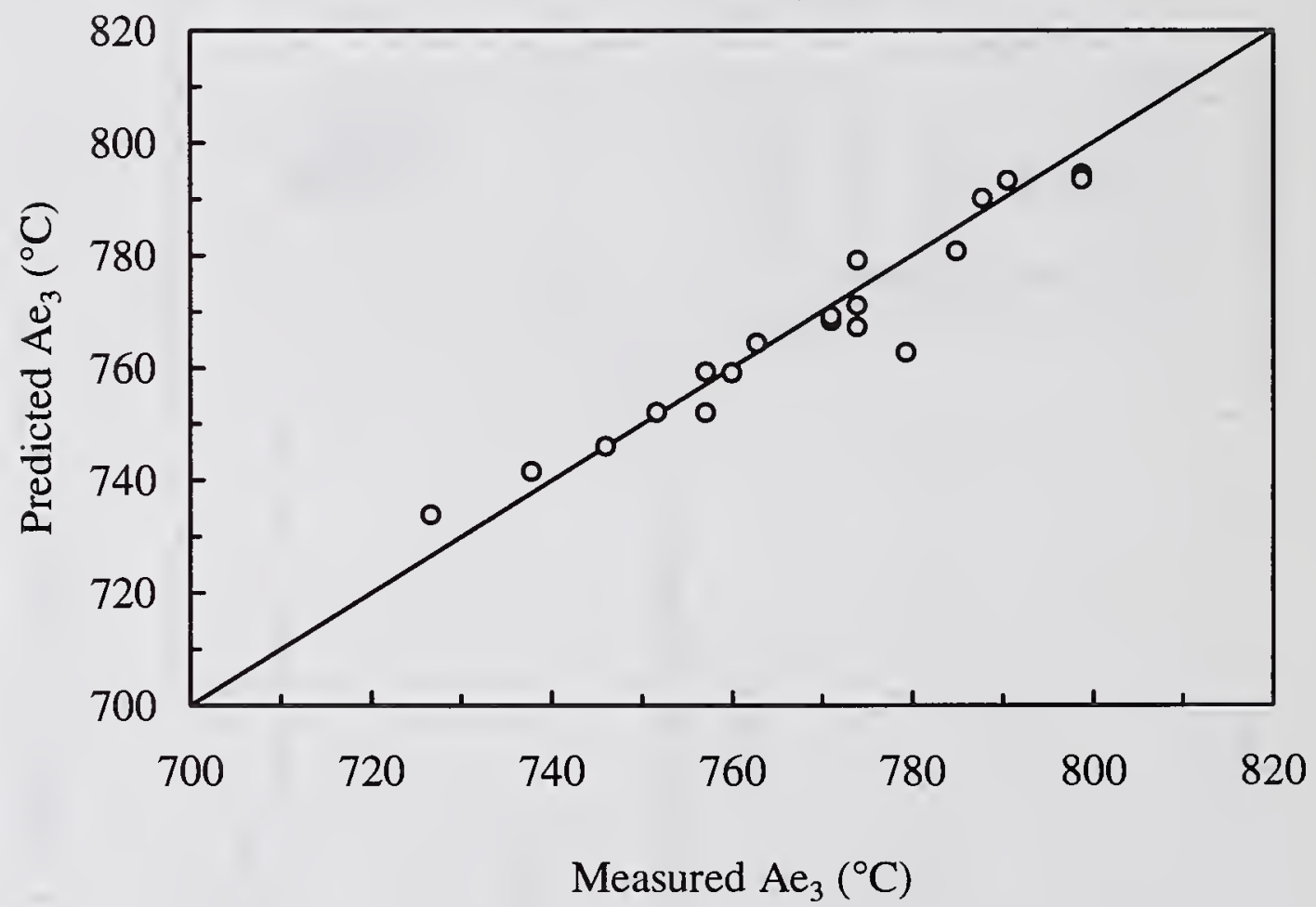

Figure 3 Comparison between the predicted and measured $\mathrm{Ae}_{3}$ temperatures 


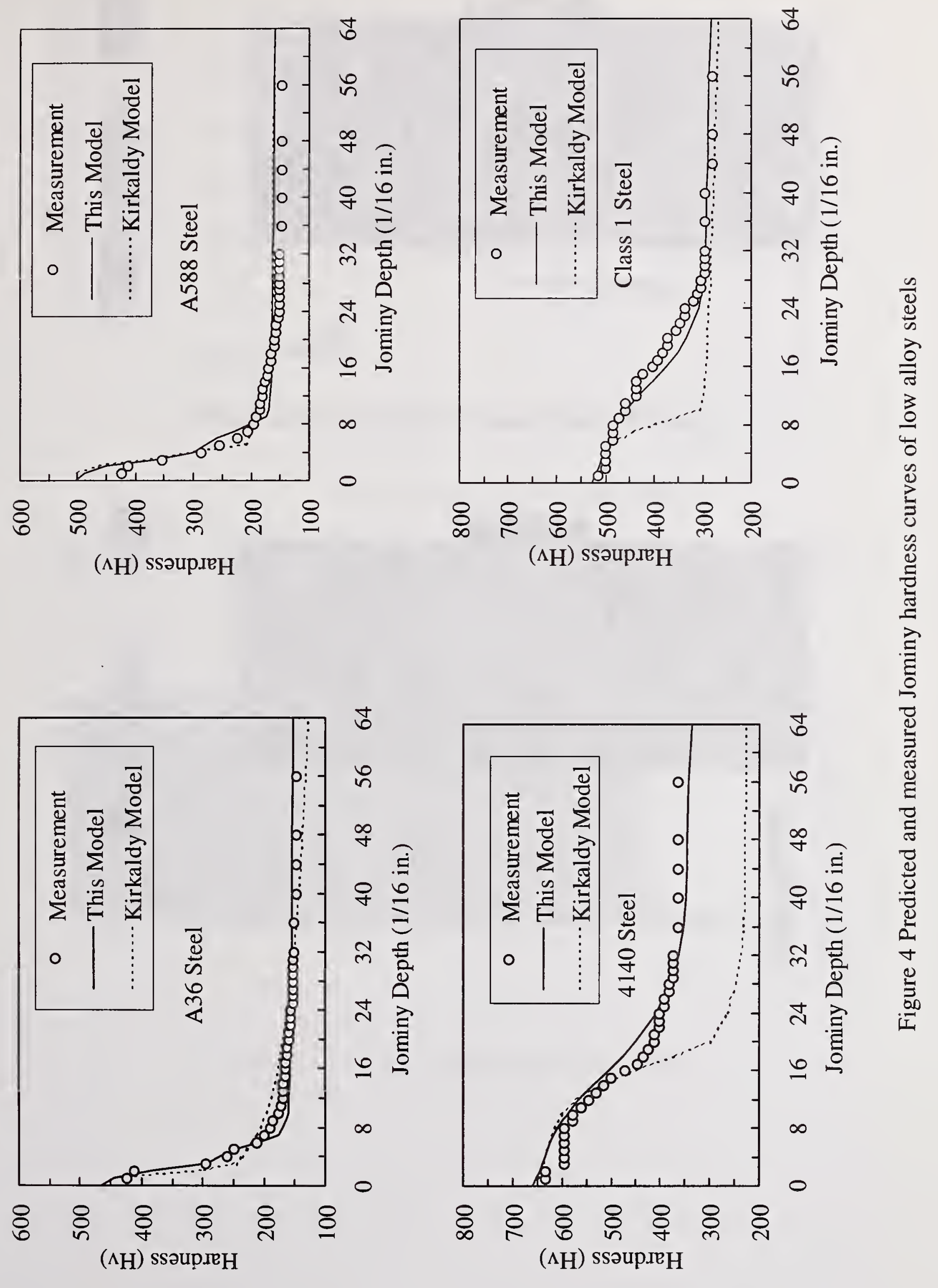




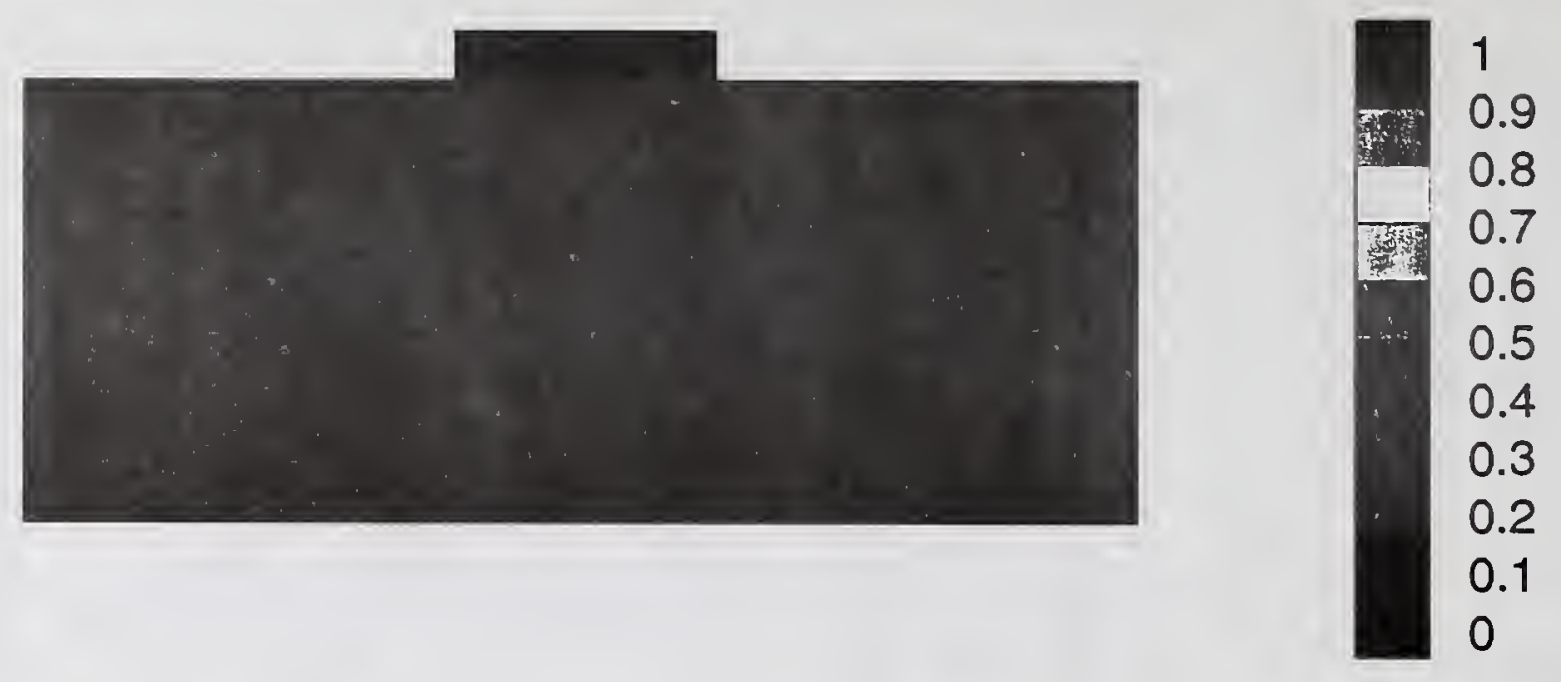

Figure 5 Predicted bainite distribution (volume fraction)

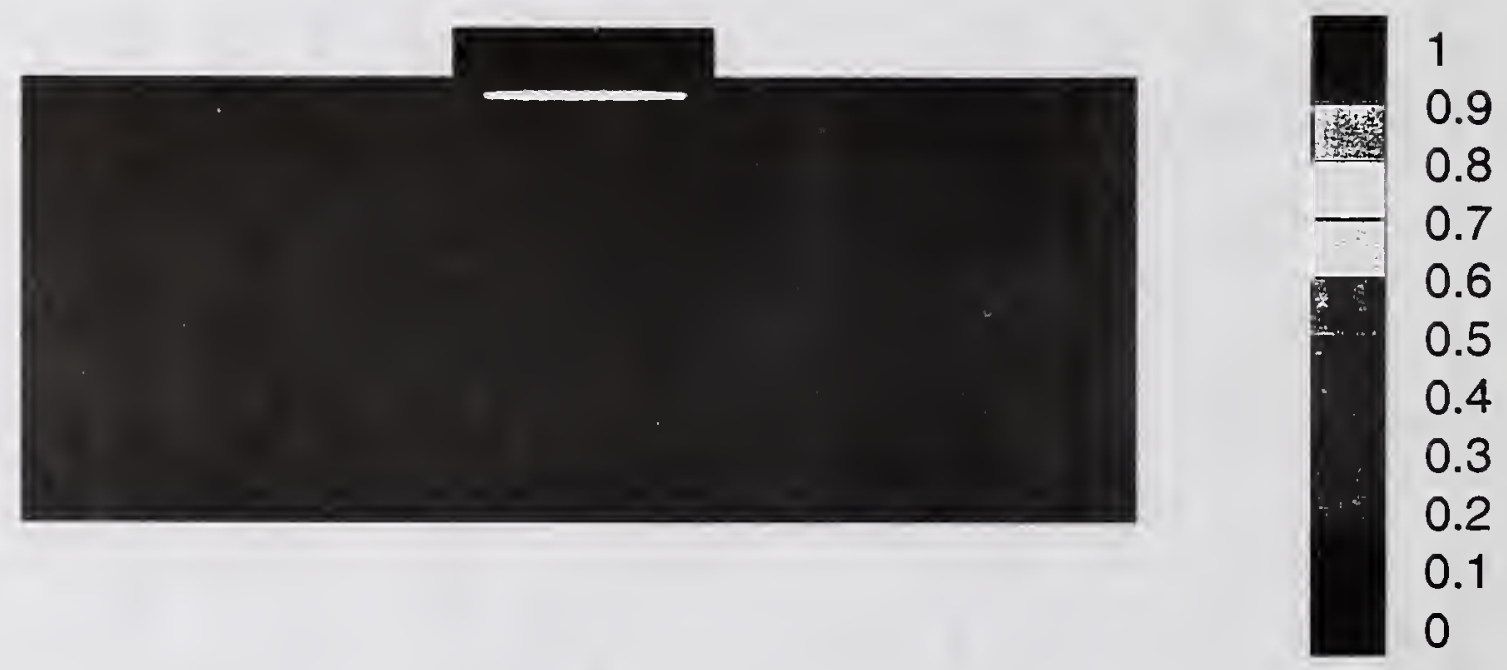

Figure 6 Predicted martensite distribution (volume fraction) 


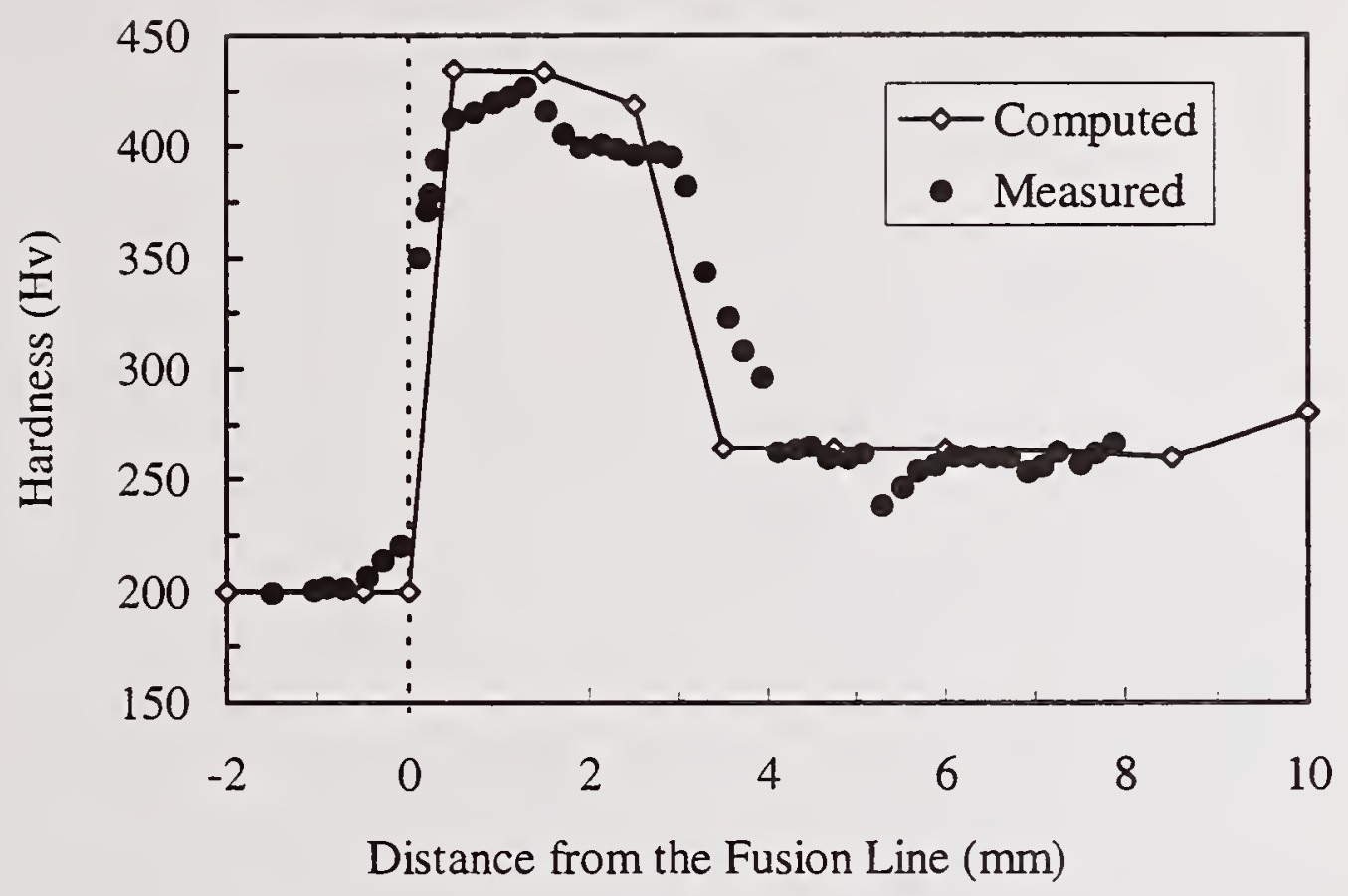

Figure 7 Predicted and measured microhardness along the centerline of the HAZ

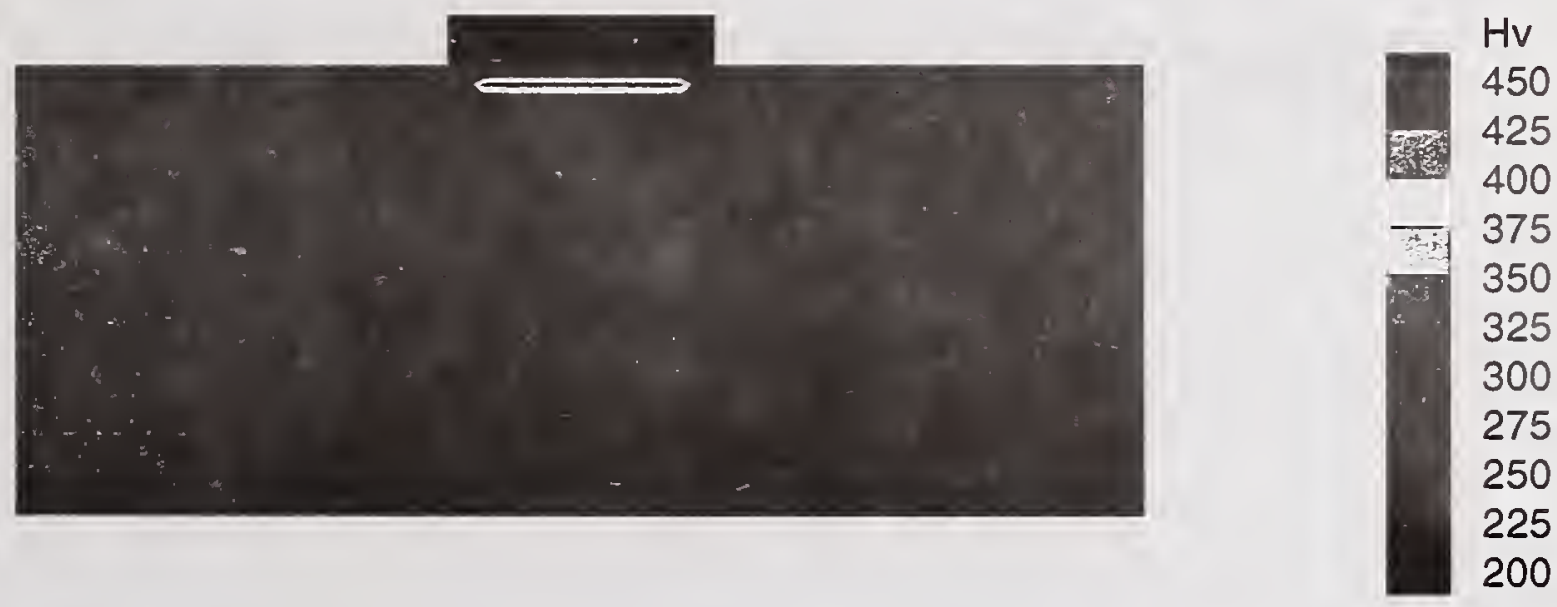

Figure 8 Predicted hardness (Hv) distribution 

MODELING - RESIDUAL and MECHANICAL STRESSES 


\title{
COMPUTER SIMULATION OF WELDED CONNECTIONS IN SEISMIC TESTS
}

\author{
O. Blodgett* and T. Spear*
}

\begin{abstract}
A Windows based PC program has been developed to simulate an actual seismic test of a beamto-column connection. A number of technical publications have addressed the wide range of variables that may affect the performance of the connection. The relative influence of the potential variables is a subject of continuing research, and it is extremely important that these variables be individually evaluated before assigning any particular relevance to their degree of influence.

This computer model evaluates the variables of connection geometry, and the steel properties of the beam and column. It assumes a perfect weld between the beam and column flanges, and therefore does not consider the following parameters: workmanship, notch effects, uneven stress distribution along the weld length, fatigue properties, notch toughnesses of the weld metal, base metal, or heat affected zone, or the effects of triaxial stress on ductility. These issues are the subject of research currently underway. The model will help researchers and practitioners alike to select details for research or for testing of actual structural applications that have a high probability of success, providing the other contributing variables are also addressed.
\end{abstract}

\section{INTRODUCTION}

The unexpected fractures that occurred in many of the welded connections of steel framed buildings during the Northridge earthquake caused the engineering community to reassess the basic concepts that had been employed for the typical connections used in Special Moment Resisting Frames (SMRFs). Much of the research has focused on geometric modifications to the connection. These changes have, in general, resulted in better performance in the test assemblies. Geometric modifications have included options that strengthen the connection, such as cover plates, ribs or haunches, as well as designs that reduce the demand on the connection such as the reduced beam section approach, commonly called the "dogbone." The computer model presented here allows each of these alternate geometries to be evaluated relative to its expected behavior.

When the test specimen is loaded into the plastic range, that is, beyond the yield point, the behavior of the specimen is significantly affected by the strain hardening properties of the steel in the beam and column. This model allows for the evaluation of various strength levels of steel for the beam and column in order to determine expected deflection.

*The Lincoln Electric Company, Weld Technology Center, Cleveland, Ohio. 


\section{MODELING PRINCIPLE AND TECHNIQUE}

An actual seismic test of a beam-to-column connection is modeled (see Figure 1). The top and bottom portions of the test column are held with a pin. The end of the test beam is forced to move vertically up and down at a given distance.

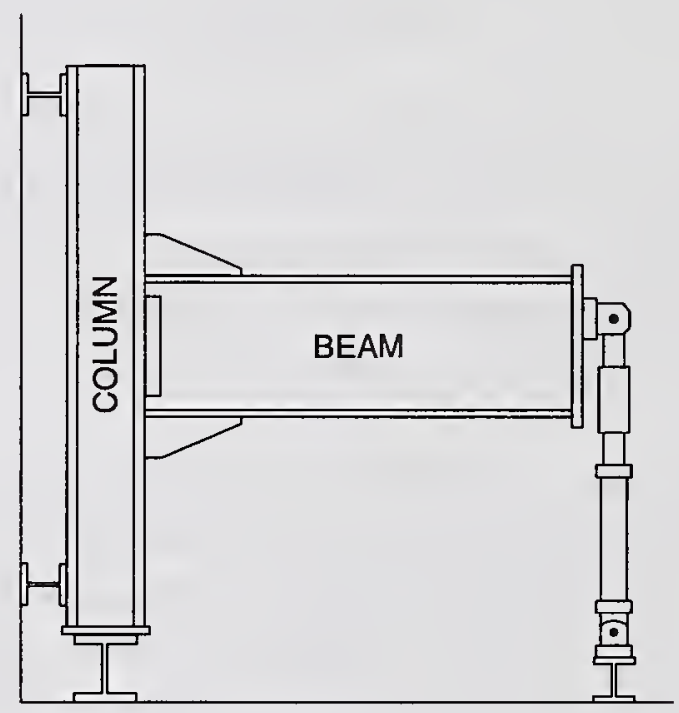

Figure 1. Beam-to-column seismic test setup used by Michael Englehardt of the University of Texas at Austin.

Since the differential equations needed to consider the elastic as well as the plastic regions would be very complicated, numerical integration, in which very small segments of the beam are applied, is used. The web is divided into one hundred segments, fifty segments above, and fifty segments below the neutral axis of the beam. The beam flange (and cover plate, if used) are each divided into five segments. The entire length of the beam is divided into one-inch-long sections.

As it is modeled, the vertical force placed on the end of the beam causes bending moments (MX) along the length of the beam, as shown in Figure 2. These bending moments set up bending stresses across all sections of the beam. It is usually assumed that the bending stress is zero at the neutral axis and then increases linearly to a maximum at the outer fibers. One side of the outer fiber is under compression, while the other is under tension. As illustrated in Figure 2, the moment which is used to find the deflection of the beam varies along the length of the beam. The

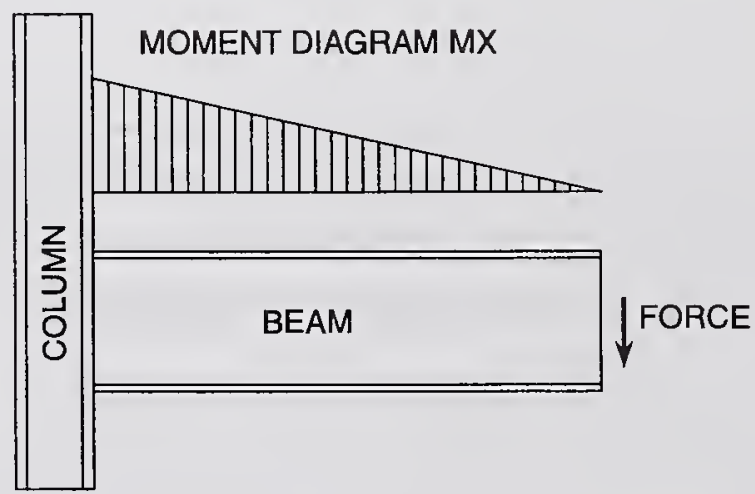

Figure 2. Moment distribution of test beam. 
beam and column resist this moment. The resisting moment (MEX) of the section is calculated based upon a linear increase in stress (SX) for each segment. This stress is increased until the resisting moment (MEX) is equal to the bending moment (MX). When i.ecessary, the stress will be increased above the yield point. This occurs because the steel will strain harden into the plastic region. Strain hardening is accounted for by comparing the actual strain to a true stress-micro strain curve. After the resisting moment (MEX) is made equal to the applied moment (MX) through a trial and error method, the strain in the outer fiber of the beam is used to calculate the angular rotation of the section. The resulting angle of rotation per inch of length of the section is multiplied by the particular length (LX) to give the increment of deflection at the end of the beam.

For every inch-long section of the beam, an initial force of 40 kips is used to calculate the rotation of the section. Then the cumulative deflection from the entire length of the beam at 40 kips is compared with the desired deflection input by the user. In each iteration, if the calculated deflection is smaller than the desired deflection, the force is increased by an increment of 40 kips until it is greater than the desired deflection. Then, the load is returned back to the previous setting and a smaller increment of force is then used. The iteration process goes on in a similar fashion until the desired deflection reaches within a range of $1 \mathrm{kip}$ in force.

Within each section of the beam, the total resisting moment (MEX) is calculated by integrating twice (above and below the neutral axis) the stress over the area multiplied by the distance to the neutral axis (DH), as shown in Figure 3. The applied moment (MX) is obtained by the product
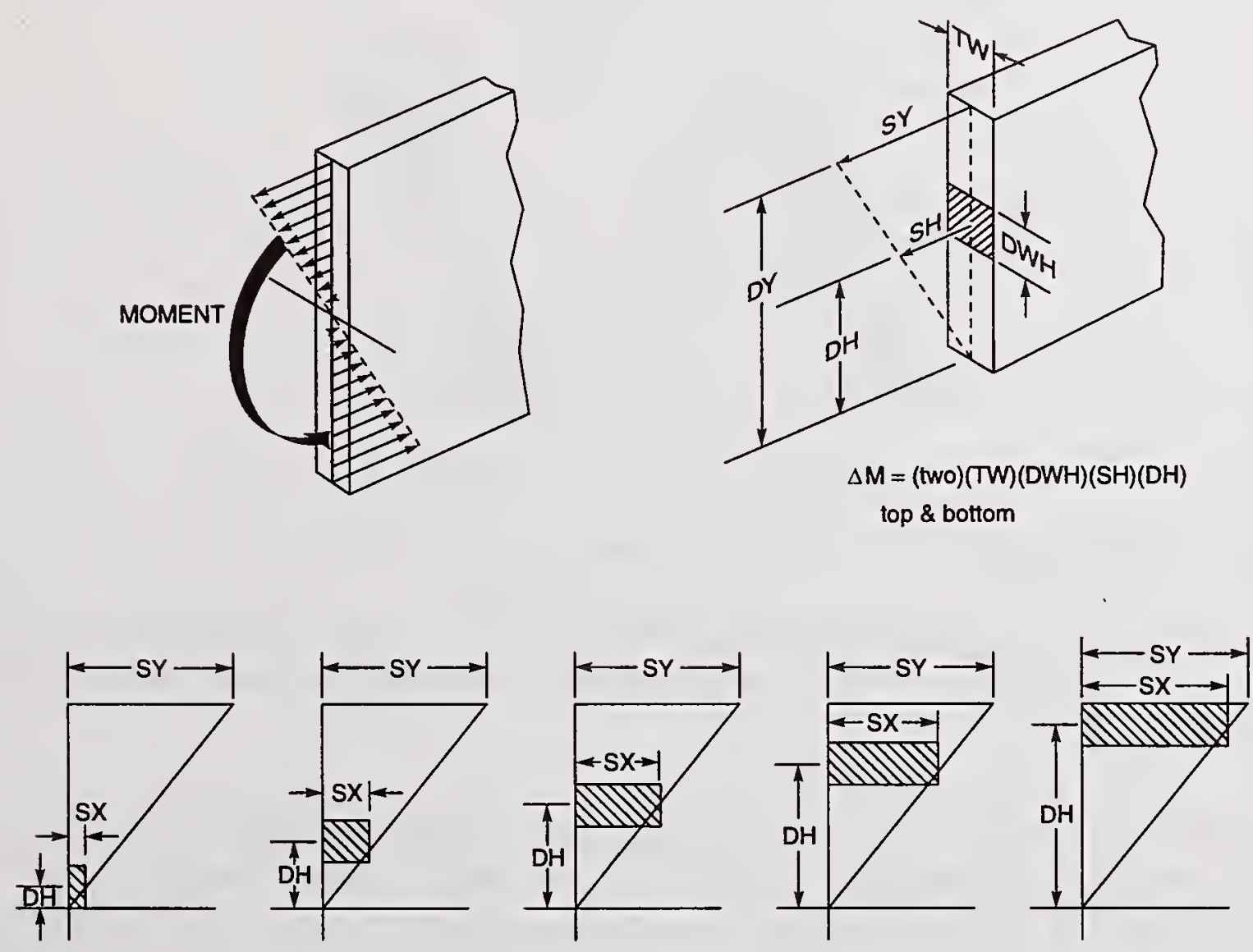

Figure 3. Developing resisting moment (MEX) of beam section. 
of the starting load (40 kips) and the distance to the end of the beam (LX). Within the elastic limit, the bending stress (SX) is equal to $\mathrm{SY}^{*}(\mathrm{MX} / \mathrm{MEX})$ as shown in Figure 4. Strain is equal

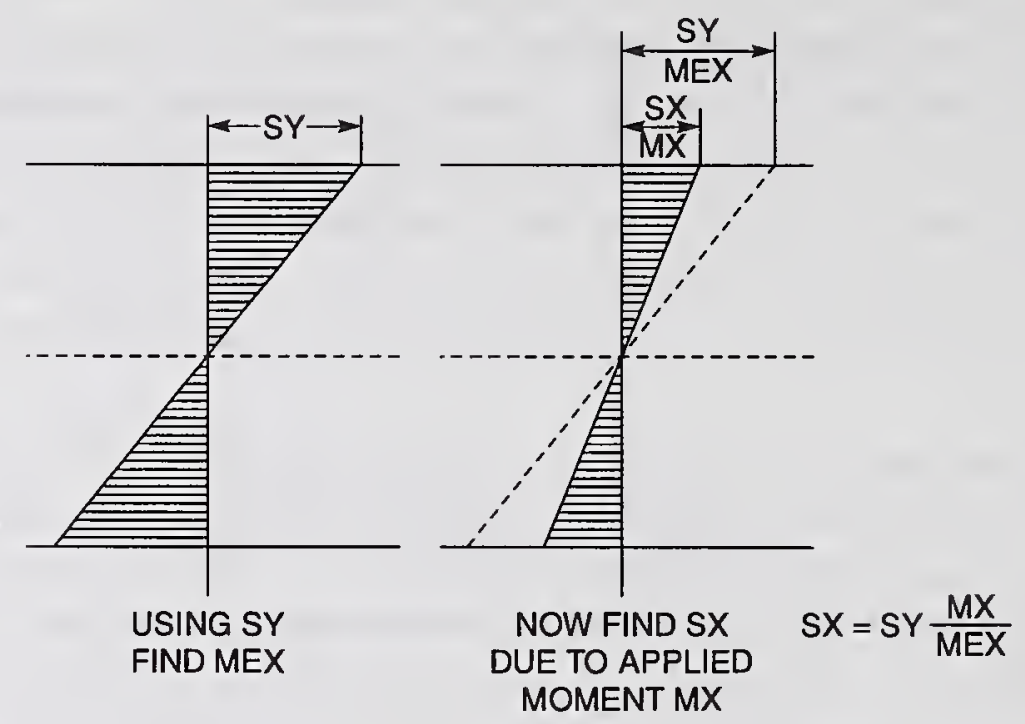

Figure 4. Finding bending stress in beam section (sx) if below yield strength.

to the stress divided by the modulus of elasticity. As Figure 5 illustrated, the rotation (TH) is equal to $\mathrm{ET} /(\mathrm{DB} / 2)$. The incremental deflection is obtained by multiplying the rotation by the length to the end of the beam. As the moment arm increases, it may reach the yield point. Above the elastic limit, the total resisting moment (MEX) is made equal to the applied moment (MX) by
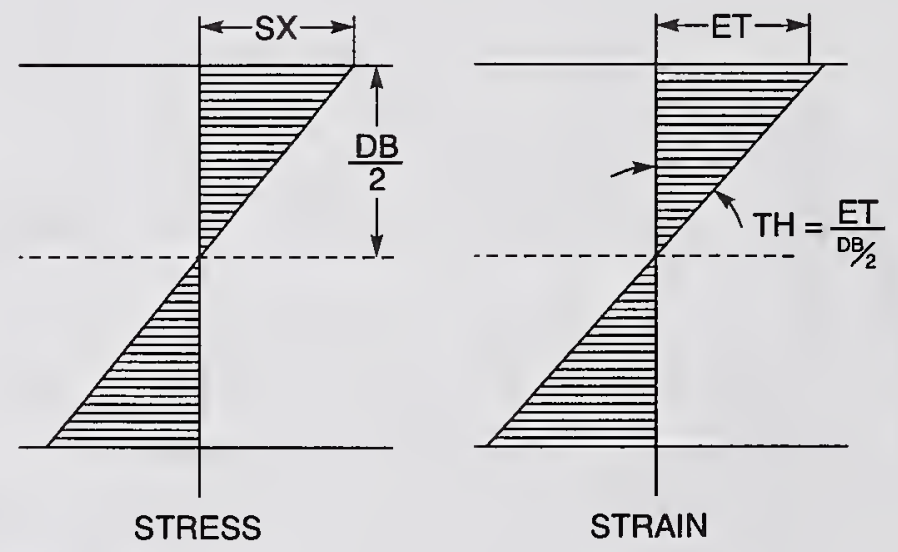

Figure 5. Finding angle of rotation (TH) of beam section.

reducing DY in another iteration cycle. DY is the point at which yield stress is reached. Once DY is found, a true strain (ET), and the angle of rotation at the outer fiber, are determined as shown in Figure 6.

Consider the strain hardening in the plastic region. The usual stress-strain (engineering stressstrain) curve for steel has little value because the results depend upon the gage length used. However, a gage length approaching zero must be applied in this case. A better method is to assume that a given volume of steel remains constant as it is being pulled in tension. The actual diameter in the necked down zone is then measured for various tensile loads. The true stress- 


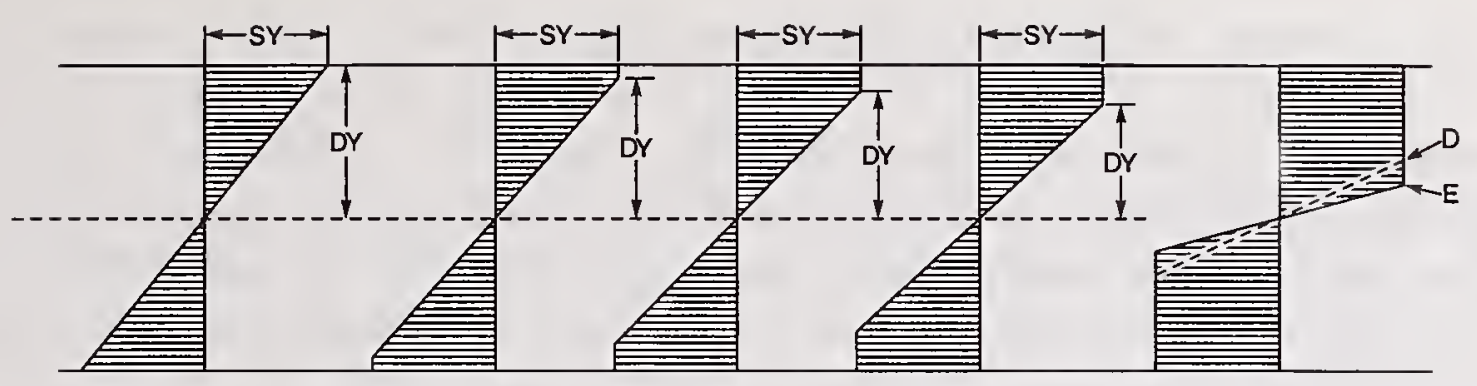

Figure 6. Finding point (DY) which will develop resisting moment (MEX) when above yield strength.

micro strain curve is then found by dividing the applied force $(F)$ by the true cross sectional area of the necked down specimen. Figure 7 will compare a common stress-strain curve with the true stress-micro strain curve. Now the computer program will use this true stress-micro strain rela-

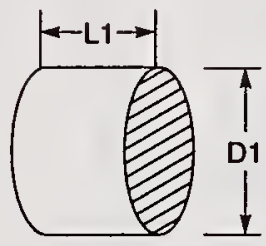

VOL $=\frac{\pi D 1^{2}}{4} L 1$

$\mathrm{L}_{2}=\frac{4 \mathrm{VOL}}{\pi \mathrm{D} 2^{2}} \quad \mathrm{~L} 1=\frac{4 \mathrm{VOL}}{\pi \mathrm{D} 1^{2}}$

$\varepsilon=\frac{L 1-L 2}{L 1}=\frac{\frac{4 V O L}{\pi D^{2}}-\frac{4 \mathrm{VOL}}{\pi \mathrm{NO} 1^{2}}}{\frac{4 \mathrm{VOL}}{\pi D 1^{2}}}=\frac{D 1^{2}}{D 2^{2}}-\frac{D 1^{2}}{D 1^{2}} \quad O R \quad \varepsilon=\frac{D 1^{2}}{D 2^{2}}-1$

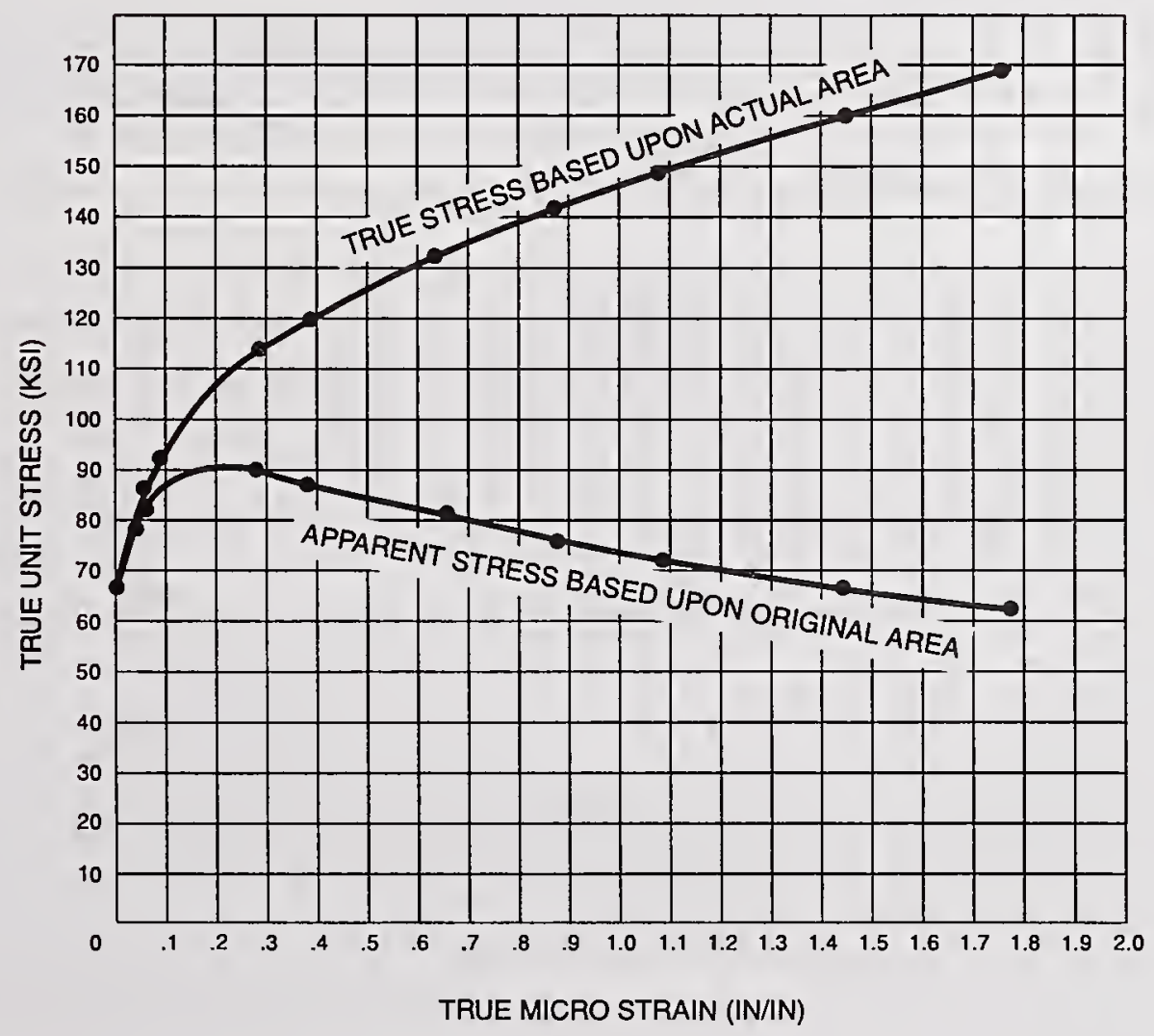

Figure 7. Using micro strain to find true stress of stress-strain curve. 
tionship for every section and segment of the beam stressed above its yield strength to determine the new strain hardened stress. When the beam is "unloaded" to zero, the previously calculated stresses in each segment and section are recalled from the arrays. This stress is used to give the elastic energy (inch kips) stored. This is accumulated to give the total inch kips of internal energy. Upon loading, the beam absorbs elastic energy up to the yield strength, and plastic energy above the yield strength. When unloading, the energy is given up as elastic. Therefore, the total energy is set equal to half of force (F) times deflection (DXR). From this, one can solve for the elastic movement (DXR) and know the position of the beam when unloaded (DXI). Figure 8 illustrates this released elastic energy and its effect on the beam deflection.
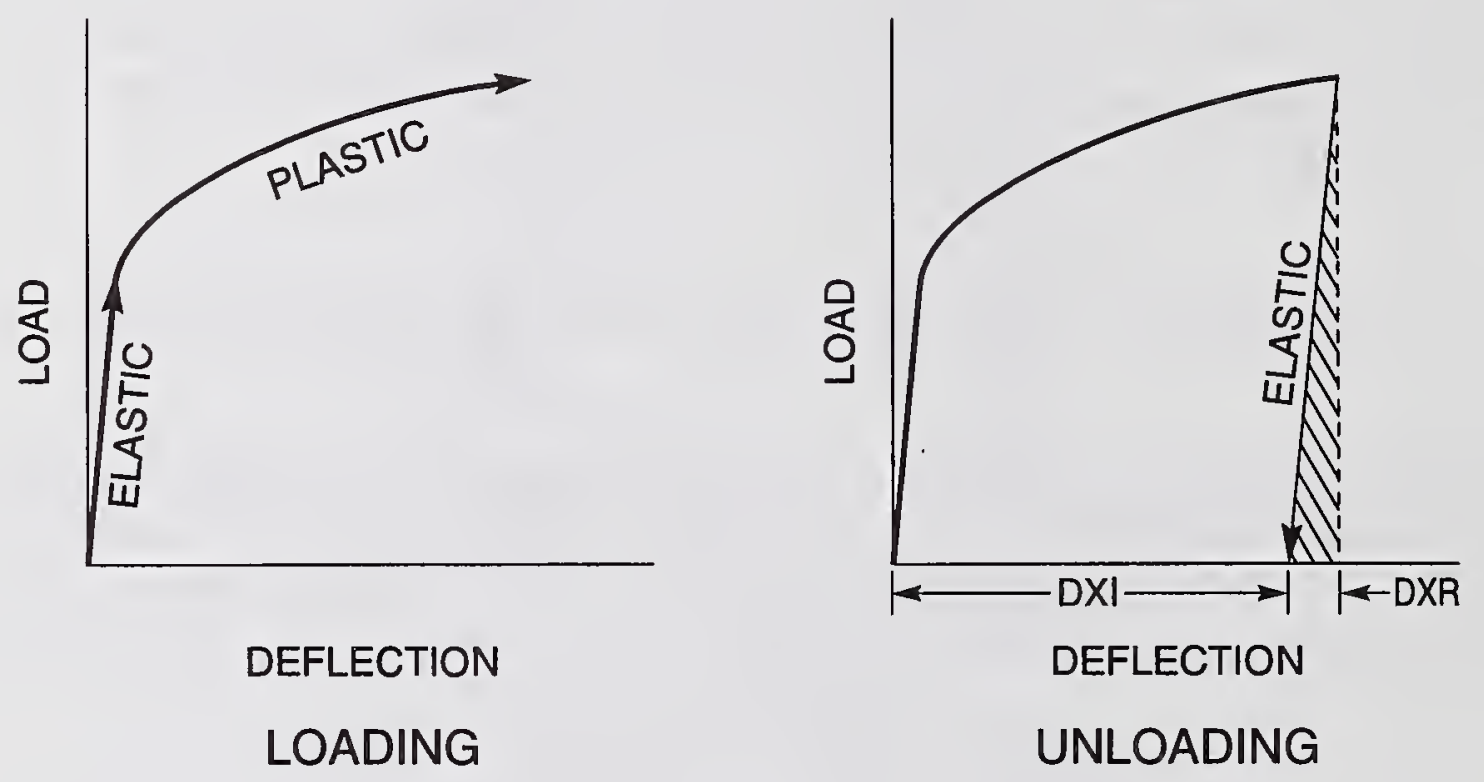

$$
\begin{aligned}
& \text { STORED ELASTIC ENERGY }=\frac{(S X)(\varepsilon)(V O L)}{2} \\
& \text { OR } \quad E \rho=\frac{(S X)^{2}(V O L)}{2 E} \\
& \text { INTERNAL ENERGY } \\
& \text { ALSO } E \rho=\frac{(F)(\Delta)}{2} \\
& \text { EXTERNAL ENERGY }
\end{aligned}
$$$$
\text { UNLOADING }
$$

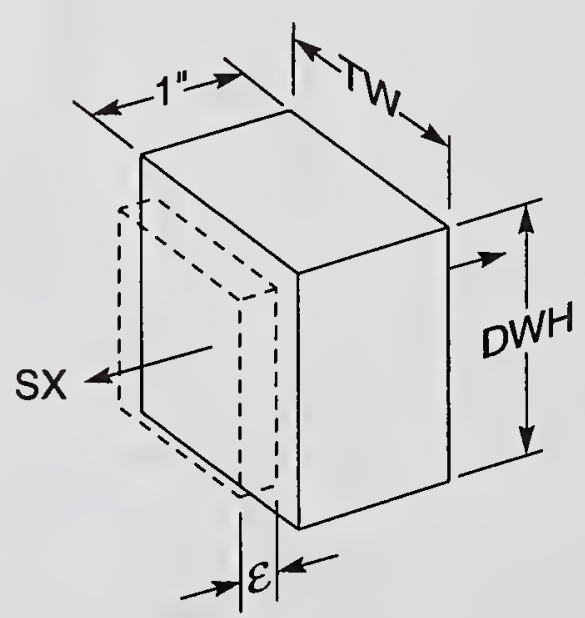

Figure 8. Finding spring back distance (DXR) when beam is unloaded. 


\section{SOFTWARE IMPLEMENTATION}

This program, converted from Basic into Delphi, is a 16 bit Microsoft Windows based application. It is designed to run under Microsoft Windows 3.1, 3.11, Windows for Workgroups, Windows 95 and Windows NT operating systems. The graphical user interface, which complies with the Microsoft Windows' GUI standards, will minimize the need to train experienced PC users.

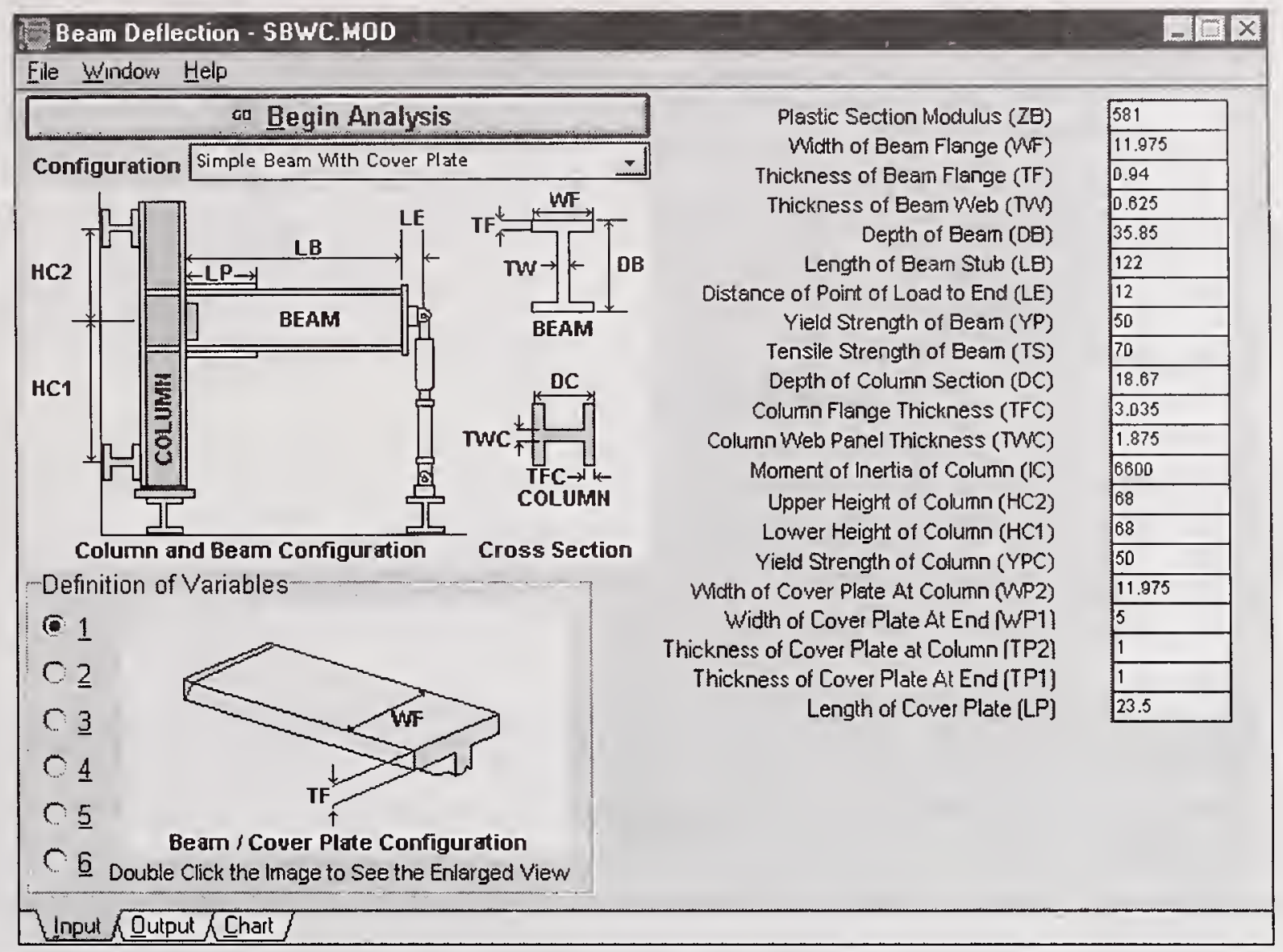

Figure 9. The screen capture of the Beam Deflection Application.

The Input page (in Figure 9) allows the user to model various geometrical and the material properties of the beam and column connection. The top image field is a representation of the beam and column configuration while the bottom one illustrates the definition of the variables in the drawings. As the beam configuration is selected from the dropdown list, unrelated input fields will be hidden and initialized to zero. If a previously saved file is loaded, the configuration information will also be extracted. Once a valid set of data is entered, a click at the start button will automatically take the user to the Output page. The Output page displays a list of calculated data, such as load, moment, deflection, angle, stresses in the connection beam flange, and panel zone. The Chart page automatically displays the force vs. deflection in a graphic form. This charting tool has some friendly functions that will allow the user to alter the scales, select grid options, specify legends and titles, as well as save, retrieve and print the chart. If more elaborate functions are desired, the force vs. deflection data set can be saved in a standard comma delimited type file, which allows the data set to be imported into more advanced scientific or graphing tools for better illustration or comparison. 


\section{RESULTS}

The results of actual tests performed at the University of Texas-Austin (Ref. 1) were compared with the behavior predicted by the model. In each case, the correlation was quite good. For example, Figure 10 represents a "dogbone" detail.
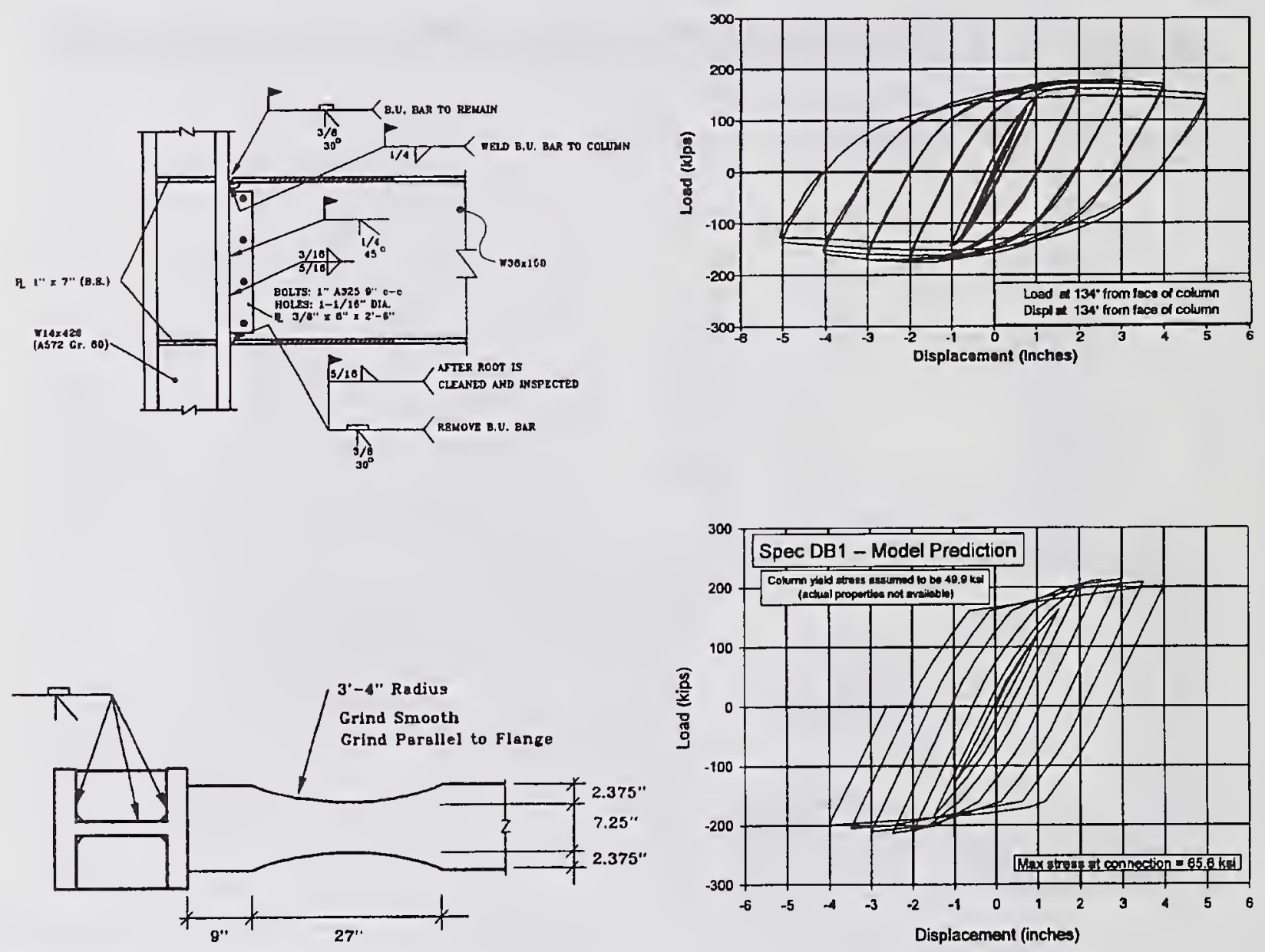

Figure 10. The "dogbone" configuration.

The model permits quick evaluation of the effects of individual variables. For example, in Figure 11 , the behavior of the beam with coverplates is evaluated by changing the yield strength of the beam. The higher yield strength increases the demand on the connection. Other variables, such as the shear stress in the panel and the compression stress in the flange of the beam can be examined along with the critical buckling stresses in the panel and the flange of the beam to gauge the buckling potential of the structure. 

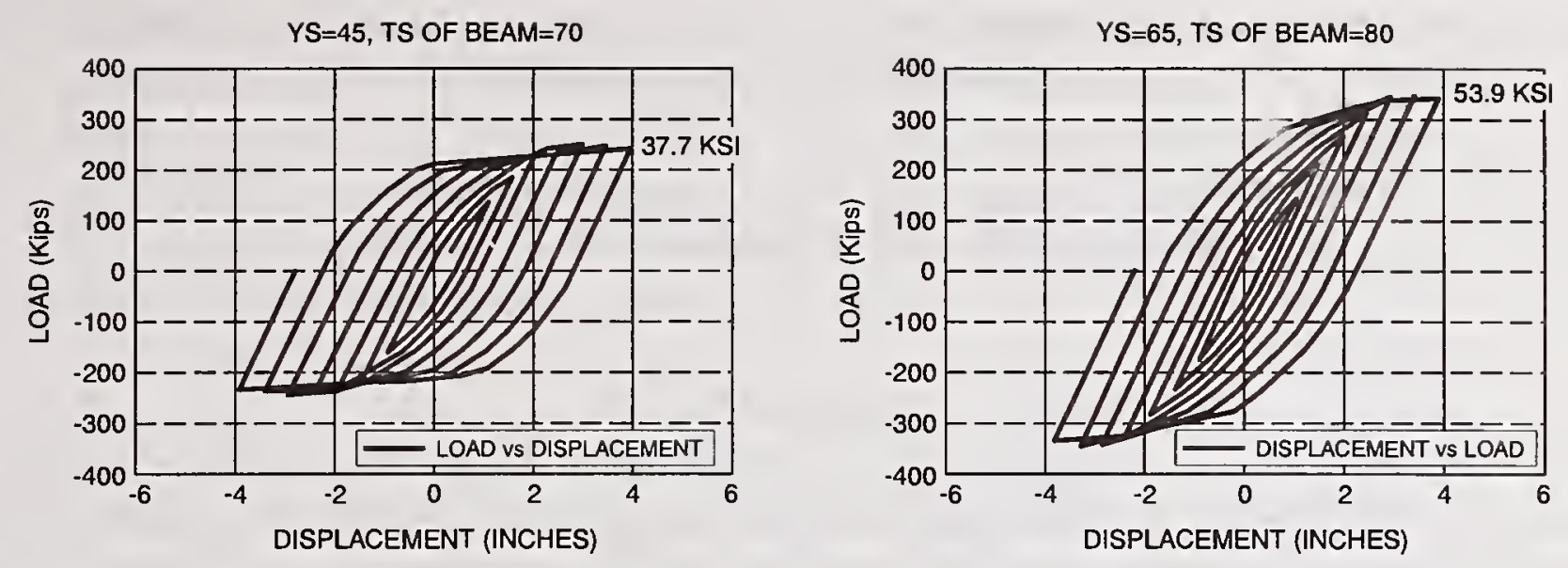

Figure 11. Shows how higher yield strength beams will result in higher stresses in beam-tocolumn connections.

\section{CONCLUSIONS}

The computer model facilitates a quick selection of various parameters in the beam-column connections including geometric modifications and strength levels of the base metal. Most significantly, a more accurate evaluation is made possible through the strain hardening derived from the true stress-micro strain curve.

While this computer program has demonstrated good ability to replicate the behavior of actual specimens that have been tested, the model is not to be used as a replacement for analysis or testing. Post-Northridge specifications require that the configuration anticipated for construction be physically tested to determine its ability to perform as expected. This model permits the engineer to quickly evaluate various possible options and to select the best candidates for testing, reducing the risk of testing details with lower probabilities of success.

\section{REFERENCE}

1. Engelhardt, M.D.; and Sabol, T.A. 1994. Testing of Welded Steel Moment Connections In Response to the Northridge Earthquake. Progress Report to the AISC Advisory Subcommittee on Special Moment Resisting Frame Research. 


\title{
MEASUREMENT OF RESTRAINING FORCES FOR A GAS METAL ARC WELDED MILD STEEL BUTT JOINT
}

\author{
M. A. Wahab ${ }^{*}$, M. J. Painter ${ }^{* *}$, P. E. Stafford ${ }^{*}$
}

\begin{abstract}
Distortion is the change in shape experienced by metals as a result of the fusion welding process. This arises from the non-uniform thermal field generated by welding which causes non-uniform expansion. Numerical methods such as finite element analysis are now being used to predict the effects of distortion. In order to fine tune, debug and validation of the finite element models, experimental results are needed. A jig was designed and built which uses load cells to measure the force required to restrain two steel plates while they are being welded After checking for reliability, tests were carried out to check the effects of various welding speed and heat inputs on the restraining forces. The results showed that welding speed has little effect on the restraining force and increasing heat input increases the restraining force. The most useful information on the effects of the welding conditions are derived from the slope of the graph of "force versus distance".
\end{abstract}

Keywords: Gas Metal Arc Welding, Post-Weld Residual Stress, Distortion, Restraining Force, Butt-Welded Joints, Welding Jigs.

\section{INTRODUCTION}

Distortion is the change in shape experienced by metals as a result of welding. To generate input information and fine-tune finite element models which predict distortion, experimental results are needed. The aim of this study was to produce experimental results which could be used by such finite element models. A jig was designed and built which could restrain two steel plates in the same way as the finite element model and measure the forces applied to the restraints as a function of time while being welded.

Objective: The objective of this project were:

- to design and build an instrumented jig capable of measuring the forces required to restrain two steel plates while being butt welded;

- to check that the results of the jig are reliable and repeatable;

- to use the jig to record some results for various welding conditions;

- and to analyse the results and find any specific pattern.

\footnotetext{
*The University of Adelaide. Adelaide. SA 5005 . Australia

*** CSIRO-DMT.. Woodville North. SA 5012. Australia

The University of Adelaide and CSIRO-Division of Manufacturing Technology are Core partners of CRC-MWJ (Co-Operative Research Center for Materials Welding \& Joining).
} 
Background: Distortion is caused by an expansion and shrinkage of the base metal due to the heat of the arc as well of the shrinkages of the weld on solidification. When two parts are welded under condition of restraints, the compressive stress applied by the restraints, change the stress distribution. Therefore the results from unrestrained distortion can not easily be used to predict restraining forces in restrained welding. The distorted shape is the result of a combination of the following three types of distortion:

- Perpendicular to the direction of the weld (transverse distortion)

- Parallel to the direction of the weld (longitudinal distortion)

- Transverse to the surface of the deposit (angular distortion)

\section{METHODS FOR MEASURING RESIDUAL STRESS AND DISTORTION}

There have been significant amount of research on the measurement of residual stress and distortion. These methods either measure free-distortion of an unrestrained plate or residual stress of a restrained plate after post weld cooling. No literature has indicated measurement of restraining forces or distortion of restrained plates. Some methods for measuring residual stress and distortion found in the literature and discussed as follows:

Strain gauges can be placed on the surface of a plate to measure strain while welding or used to measure residual stress in a welded plate. The laser speckle method reflects a laser off the surface of the steel and collects the reflection with a linear image sensor (Ref.1). Displacement transducers contact the surface of the steel and measure the displacement in one direction (Ref.2). The distorted shape can be measured and compared to the undistorted shape. (Ref.3)

Design: To generate the experimental data required, a jig was designed and built. The aim of the jig was to hold two steel plates in position while being butt-welded and measure the restraining forces in positions which can be simulated by the finite element model.

The jig was designed to comply with the following constraints:

- to hold two hot rolled mild steel plates, $10 \mathrm{~mm} \times 100 \mathrm{~mm} \times 500 \mathrm{~mm}$.

- to allow measurement of restraining forces typically in the vertical and horizontal directions during simple butt -welding.

- to allow access to a Gas Metal Arc Welding robot.

- to allow flexible placement of the restraint positions.

To measure the restraining forces, it was necessary to use some suitable force measuring device. The two types of force measuring device available are strain gauges and load cells. For ease of interpretation of output and simplicity of design a load cells were selected.

As a result of thermal expansion and the restraints, the welded steel plates will shrink in the direction perpendicular to the direction of the weld. The jig was designed to measure only in compression which includes the restraining forces for expansion and contraction down to 0.0 $\mathrm{kN}$. This is sufficient to meet the aims of the project because all of the changes to the structure of the steel happen in this range. 
Configuration: The aim of the design of the jig is to hold the load cells against the plates so that they can prevent the plates from distorting and measure the restraining forces. The load cells are attached to studs which tighten against slotted beams above and to the side of the plates. An important part of the design of the jig was making sure that the bed and restraint supports were stiff enough. If either the bed or restraint supports deflected significantly, the results would become inaccurate. The layout of the jig consists of two end plates with the bed and restraint supports between them.

Because the two plates being welded were identical, the restraining forces for both are the same. For this reason, the jig is designed to only restrain one plate with load cells. The plate restrained with load cells will be referred to as the measured plate.

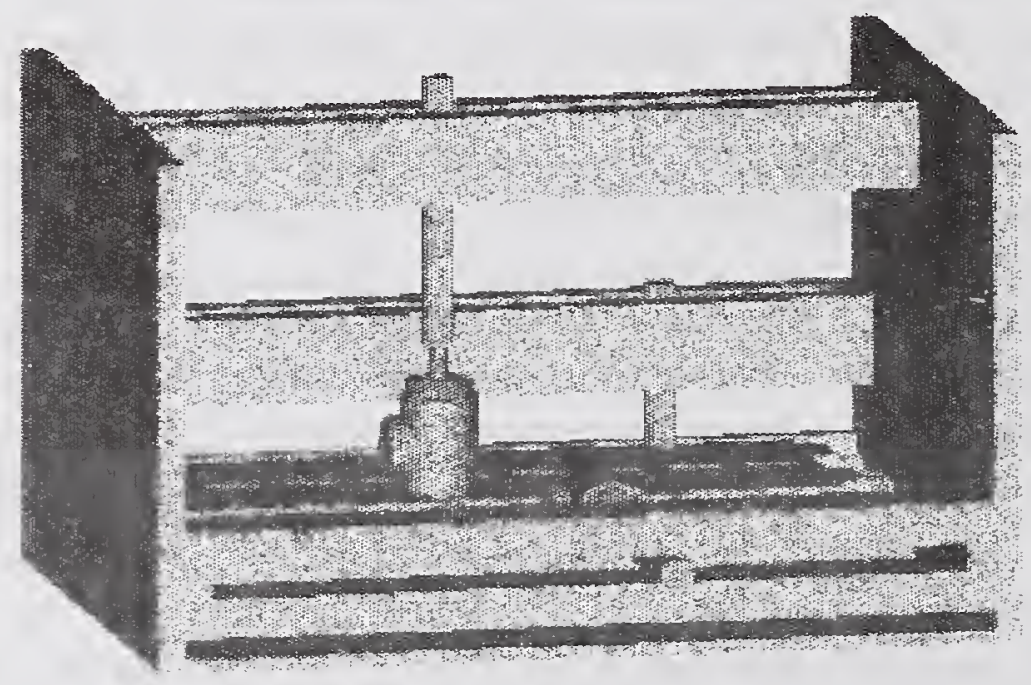

Figure 1: CAD drawing of the jig (some restraints not shown).

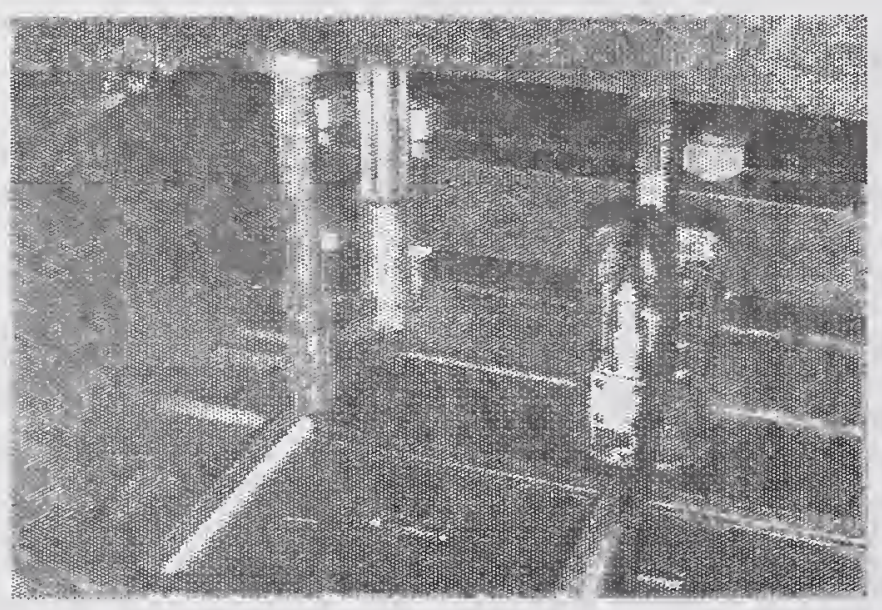

Figure 2: Close up of the jig. Note the load cell to the left and the welding torch to the right at the end of the weld.

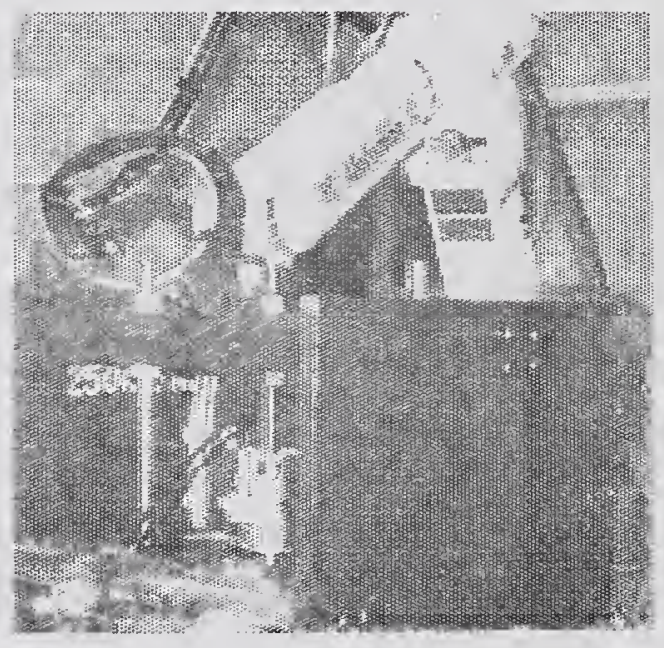

Figure 3: The jig with the robot welder in the background 
An important part of the design of the restraint supports was to make them versatile enough to restrain the plates with any number of restraints in any position. This was achieved by the slots in the beams which allow the studs to slide to any position.

\section{INSTRUMENTATION AND TESTING}

The data was collected with two load cells connected to a load cell amplifier with the output to a data logger. A $20 \mathrm{kN}$ load cell was used in low force positions on the jig. The $100 \mathrm{kN}$ load cell (Tokyo Sokki Kenkyujo CLP 10B) was used in high force positions. The load cells were calibrated by compressing them in an MTS testing machine and charting the output voltage from the amplifier against the measured load on the MTS

The restraints can be tightened against the plate with a compressive force, called preloading. The effects of preloading had to be determined to find the best experimental procedure and ensure that the experiments could be reliably repeated. A large pre-load greatly decreased the restraining force before and after the peak force value (Figure 4). This can be explained by the restrained expansion, free contraction model described by Cornu (Ref.4) When the yield stress of the base metal is exceeded in the high temperature region near the weld, the restraining force is relieved by plastic deformation. In the case of a large pre-load, the pre-load is also relieved by plastic deformation. This results in greater shrinkage so the restraining force drops.

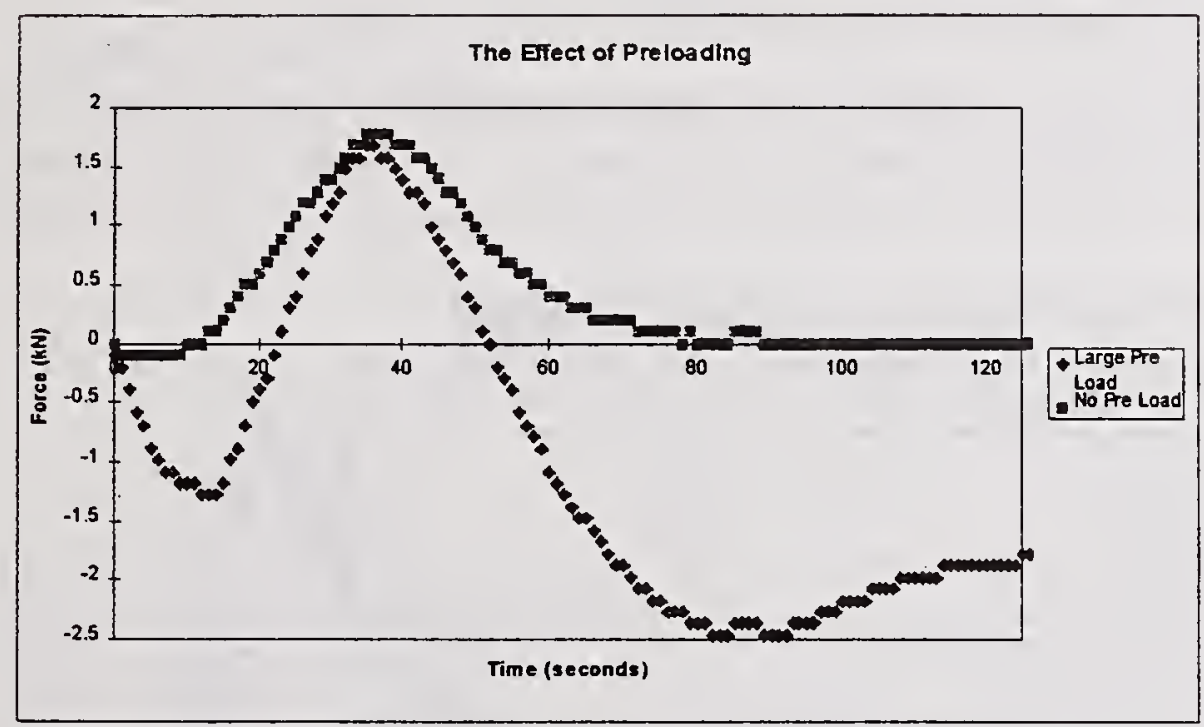

Figure 4: The effects of side pre loading measured from the midpoint of the side of the plate.

For the force values taken from the side positions to be true, there must be no sideways force resisted by the top restraints. Increasing the force on the top of the plates did not reduce the force measured by a load cell on the side of the plate. 


\section{DEVELOPMENT OF EXPERIMENTAL PROCEDURE}

The aim of the experiments is to gain an understanding of what factors influence distortion by undertaking two main sets of experiments, varying one parameter at a time. As this research aims to look at the overall trends for each parameter rather than calculation of the effects of a small change; only three samples were taken, from the upper, central and lower bounds of what may be used in commercial practice.

Two restraints on each of the top and edge of each plate were used. This gives cleaner and easier to understand results than those for three restraints tried earlier. This gives a total of four positions which data will be collected from: Top Start, Top End, Side Start and Side End.

Welding Conditions: The first set of experiments consider the effect of holding the welding speed constant while changing the heat input. The range in heat inputs covered spans from the minimum to maximum which could be used on the $10 \times 100 \mathrm{~mm}$ mild steel.

Table 1: Welding conditions for constant speed, varied heat input.

\begin{tabular}{|l|l|l|l|}
\hline Current (Amps) & Voltage (V) & Heat Input $(\mathrm{kJ} / \mathrm{mm})$ & Speed $(\mathrm{mm} / \mathrm{min})$ \\
\hline 124 & 20 & 0.5 & 300 \\
\hline 232 & 23 & 1.0 & 300 \\
\hline 278 & 27 & 1.5 & 300 \\
\hline
\end{tabular}

The next set of experiments examines the effect of applying the same heat input at various speeds.

Table 2: Welding conditions for constant heat input, varied speed.

\begin{tabular}{|l|l|l|l|}
\hline Current (Amps) & Voltage (V) & Heat Input (kJ/mm) & Speed (mm/min) \\
\hline 228 & 23 & 1.5 & 200 \\
\hline 278 & 27 & 1.5 & 300 \\
\hline 334 & 31 & 1.5 & 400 \\
\hline
\end{tabular}

Table 3: Constant Welding Parameters

\begin{tabular}{|l|l|}
\hline Filler Wire & $1.2 \mathrm{~mm}$ diameter LW1 \\
\hline Gas & Argon with $1.5 \%$ Oxygen \\
\hline Air Temperature & $18-25$ degrees Celsius \\
\hline Stick Out & $16 \mathrm{~mm}$ (except $0.5 \mathrm{~kJ} / \mathrm{mm}: 11 \mathrm{~mm})$ \\
\hline
\end{tabular}




\section{EXPERIMENTAL RESULTS}

The results presented in this study are for the position "at the side of the plate at the end of the weld" as shown in Figure 5, as this position gave the highest restraining forces and the clearest correlations.

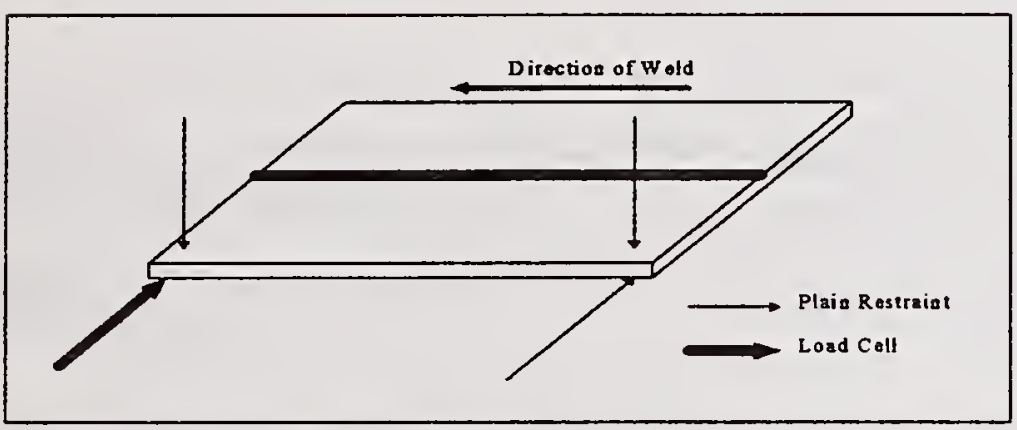

Figure 5: Position of load cell for side of plate, end of weld.

Varied Speed: Figure 6 shows that as the speed increases, the peak occurs earlier and occurs at a higher force. The reason for the peak occurring earlier is because as the speed increases, the weld finishes earlier. The peaks occur at the finish of the weld in all cases this is shown by the black line which is drawn where the welding current drops to 0 for each weld.

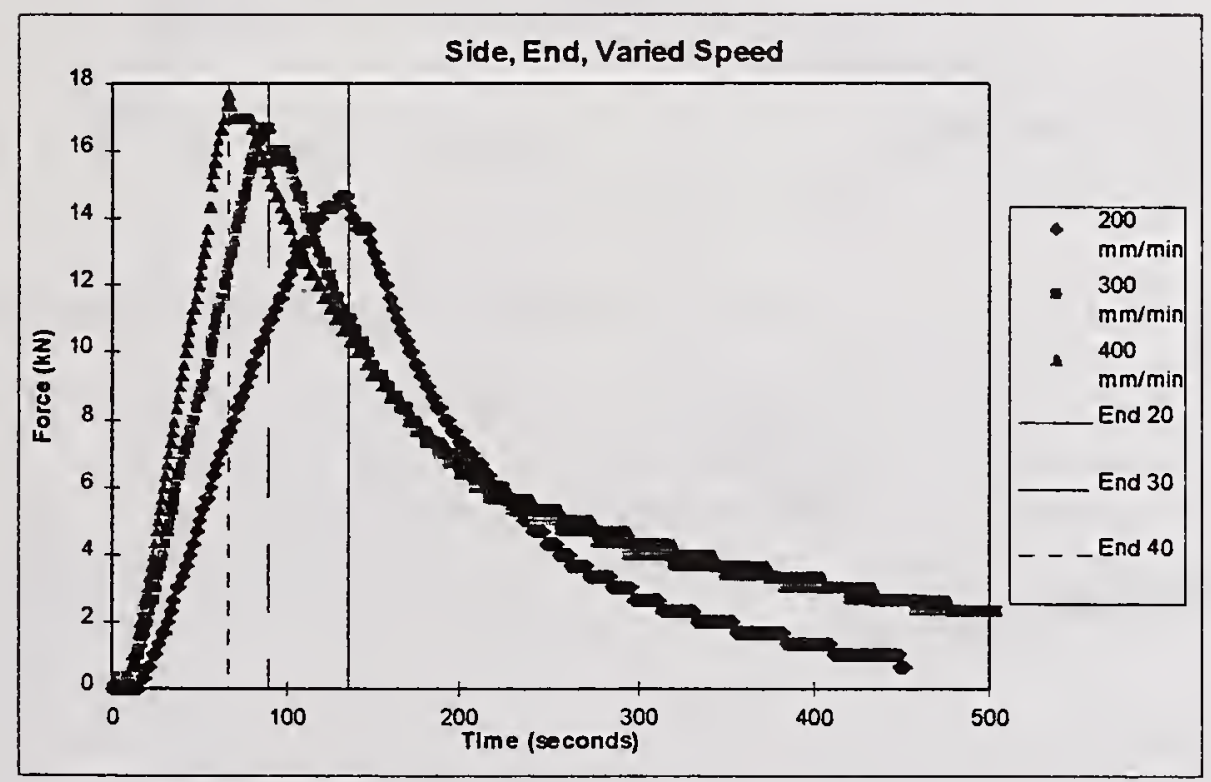

Figure 6: Side, End, Varied Speed.

An easier to understand graph uses a distance scale rather than a time scale (Figure 7). This has all of the welds starting and ending at the same position on the $\mathrm{x}$-axis and only shows the force while welding is happening. 


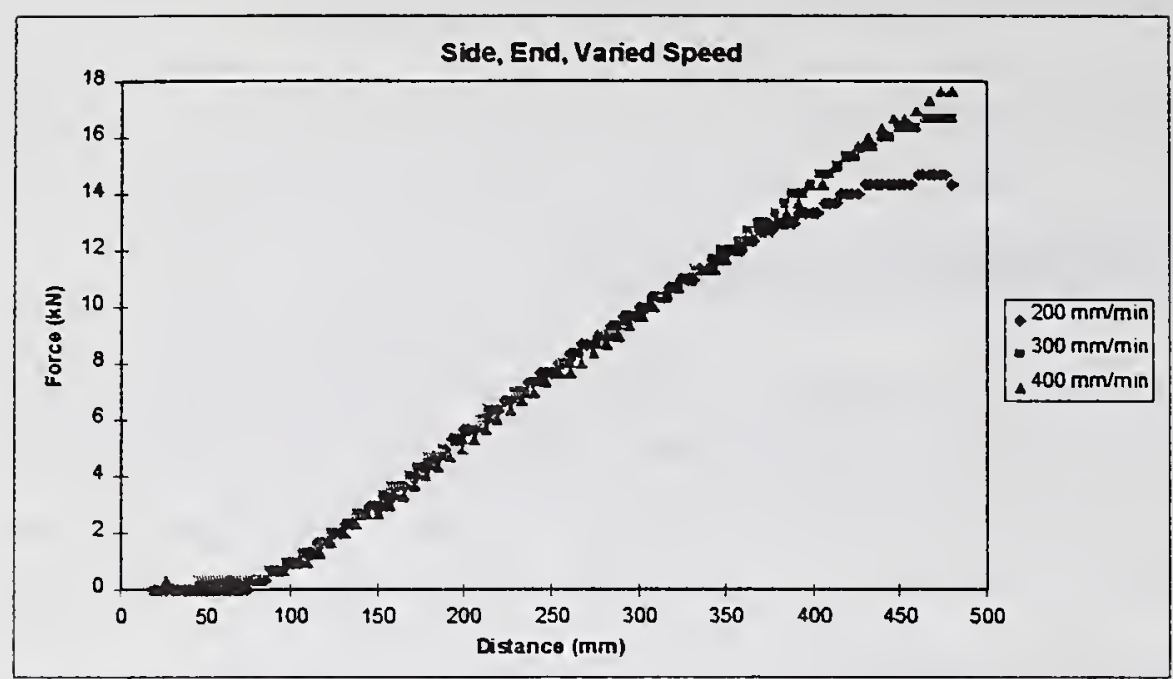

Figure 7: Time Scale

Figure 7 shows that the restraining force is independent of speed for most of the graph and only gains a dependence on speed in the region close to the restraint. This indicates that at a certain welding speed, the graph will be linear and any further increase in speed will have no effect on the restraining force at this position.

The linear rise in restraining force with distance indicates that the maximum force reached is a linear function of the length of the welded plates, so as a means of comparing two alternative welding processes, the slope of the linear part of the graph would be more useful than the maximum force.

After the arc cuts out, the force against the restraint relaxes with time. The pattern is not immediately visible in Figure 6 so the origin is placed at the point where the arc stops for Figure 8.

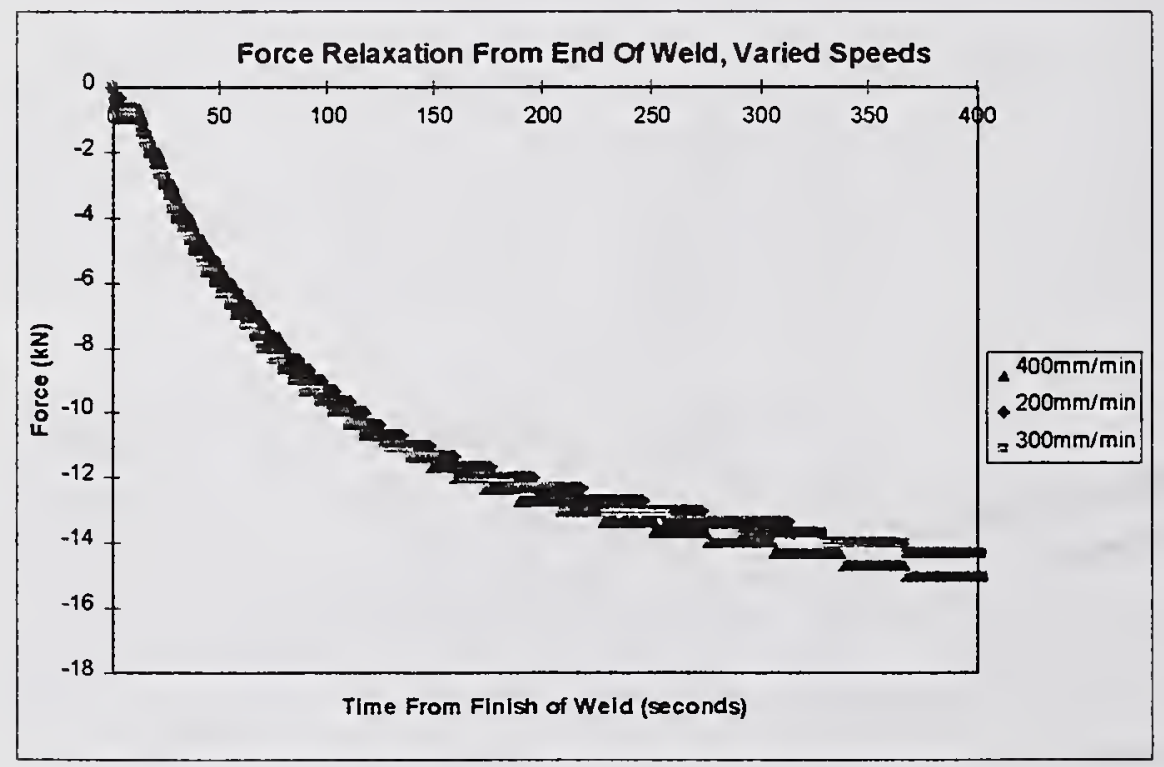

Figure 8: Relaxation of restraining force with time. The origin for each graph is taken from where the weld finishes. 
The result shows that the force relaxation is a function of time, which is not affected by welding speed or the restraining force at the end of the weld.

This graph shows that the relaxation of the restraining force while cooling is determined by deformations which take place at stresses above the yield stress while the welding is taking place. The stress relaxation is simply the contraction of the welded plates with no plastic deformation. The fact that the contraction for all three welding speeds is the same indicates that all three welds must have undergone the same deformations in the expansion stage and therefore have the same structure. This indicates that the departure from linearity of the force-distance curve resulting in a lower peak force has little effect on the deformations of the plate, reinforcing the idea that the slope of the curve is more important than the peak force.

This is an important result for modelling because once the force relaxation curve for a given heat input is known and the force at the end of the weld is known; the restraining force can easily be calculated.

Varied Heat Input: Figure shows the restraining forces for varied heat input and constant speed.

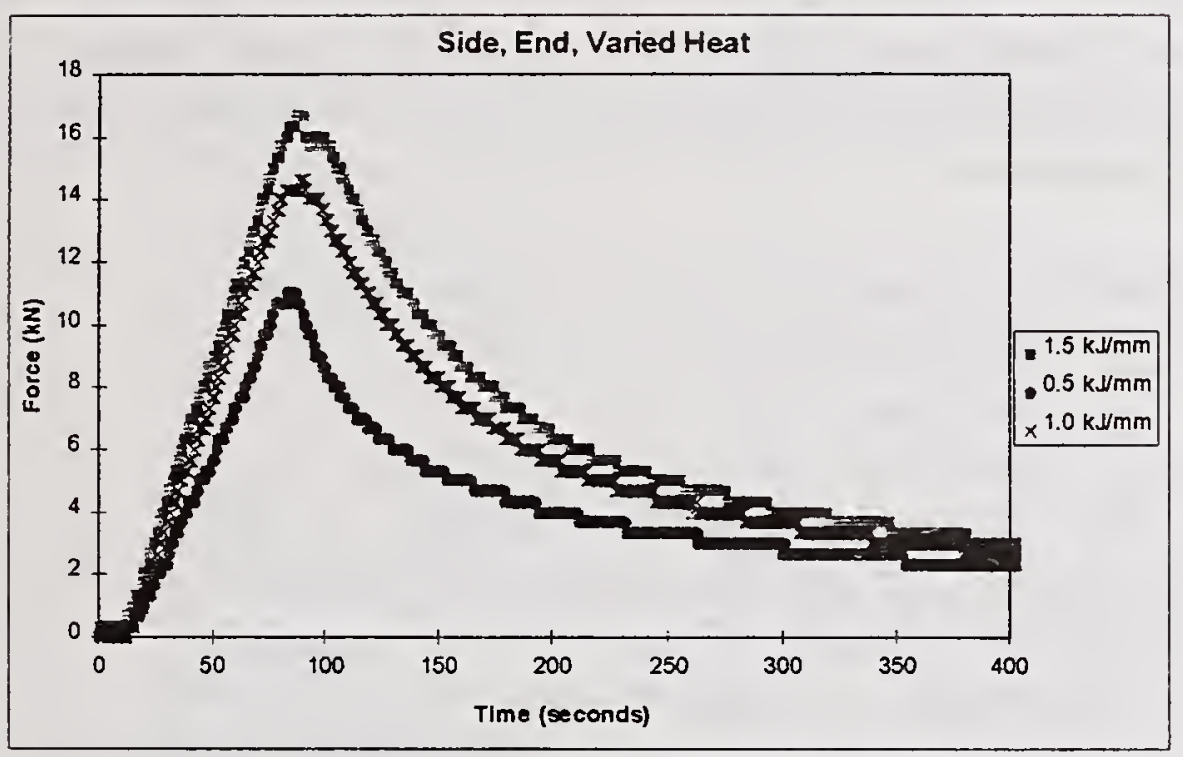

Figure 9: Variation of restraining forces with heat input

As a means of comparing Figure to Figure 6 , the graph for $1.5 \mathrm{~kJ} / \mathrm{mm}, 300 \mathrm{~mm} / \mathrm{min}$ is the same on both charts. Figure 9 has four distinct sections which will be considered separately. These are: a flat heating time, a linear rise, a non-linear rise and force relaxation. To make the first three sections easier to see, Figure 10 is plotted for the time from the start to the end of the weld. 


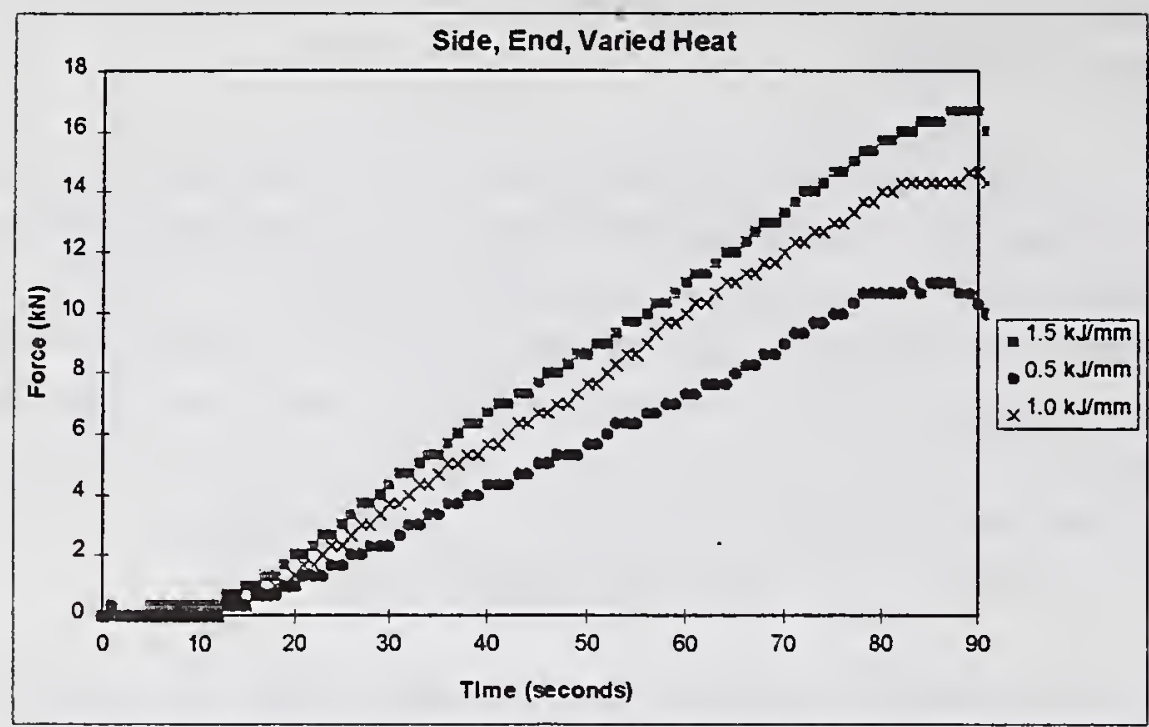

\section{Figure 10: Detail of force rise while welding.}

On this chart the flat heating time is clearly visible. The heating time is the same for all three heat inputs. The linear rise starts at the end of the heating zone and extends to the non-linear zone near the end restraint. This is the most important part of the graph because the slope of the linear section gives a direct means of comparison of the effect of different heat inputs. For a clearer comparison of the effects of heat input on restraining force, Figure 11 shows the slope of the force-time curve plotted against heat input.

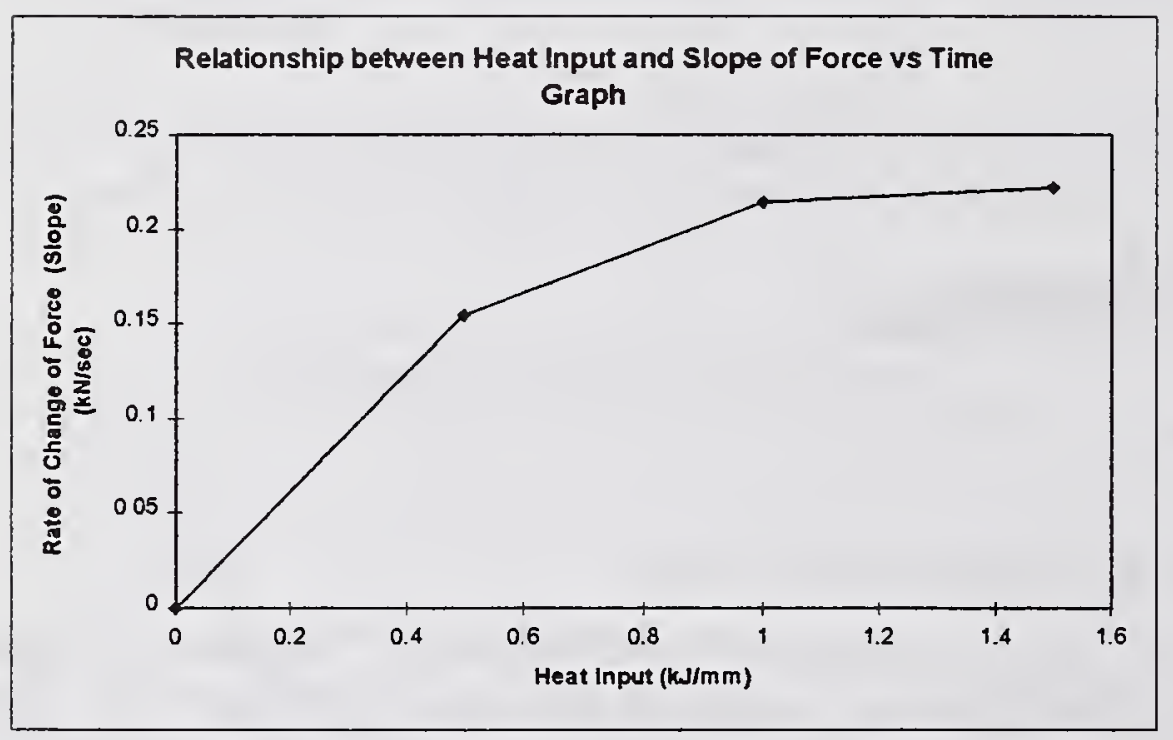

\section{Figure 11: Heat Input and Slope of Force versus Time Graph}

This plot shows that the relationship is certainly non-linear but only has three data points so it can not be conclusive at this stage. This graph can be used as a rough guide as to what the slope of the linear part of the force - time graph will be. It should be mentioned once again that the slope of the force - time graph is dependent on the shape and size of the two plates being welded and therefore the numbers presented in this paper can not be applied directly to other sized plates without investigating further. 


\section{CONCLUSIONS}

1. For a given heat input, the restraining force will be the same as a function of distance except close to the end of the plate while it is being welded, regardless of welding speed.

2. For a given heat input, the force relaxation after welding will have the same shape regardless of welding speed and maximum restraining force reached.

3. Decreasing welding speed makes the force versus distance graph depart from linearity earlier.

4. Increasing the heat input, increases the slope of the force versus time graph and the maximum restraining force.

5. The slope of the force versus time graph gives a better indication of the effects of the welding conditions than maximum restraining force.

6. The restraining force for the top of the plate increases with increased welding speed

7. The restraining force for the top of the plate increases with increased heat input.

\section{REFERENCES}

1. Muramatsu Y and Kuroda S: (1992) Application of the Laser Speckle Method to Strain Measurement in Welding: Basic Evaluation and Examination of Effects of Strain Rate. Welding International vol.6, no.12.

2. Wohlfahrt $\mathrm{H}$ and Zhang F: (1994) Experimental Evaluation and Calculation of Transverse Distortions due to Welding in Butt Joints and T-joints. Welding in the World / Soudage dans le Monde. Vol.34.. pp.397-406.

3. Goglio L and Gola M M: (1993) Shrinkage in butt welded joints: Measurement and prediction. Welding International 7 (10) 776-787.

4. Cornu Jean: (1988) Advanced Welding Systems - Fundamentals of Fusion Welding Technology. Bedford, UK, IFS (Publications) Ltd.

5. Cary H B: (1979) Modern Welding Technology. Eaglewood Cliffs, N.J. 07632, Prentice - Hall

6. Juvinall R C and Marshek K M: (1993) Fundamentals of Machine Component Design. New York, John Wiley \& Sons.

7. Makhnenko V I, Korotynskii A E, Kirillov S A, Statnikov E Sh and Abramov S N: (1981) A Four-Channel Device for Measuring the Deformations of Welded Joints. Automatic Welding, vol.34, no.9.

8. Radaj Dieter: (1992) Heat Effects of Welding. Berlin Heidelberg, Spriger - Verlag,

\section{ACKNOWLEDGMENTS}

The work reported herein was undertaken as part of a Research Project of the Cooperative Research Center for the Materials Welding \& Joining (CRC-MWJ) of Australia. The authors gratefully acknowledges the financial support received the CRC-MWJ and from the University of Adelaide. 


\title{
FINITE ELEMENT MODELING OF SPOT WELDING SIMILAR AND DISSIMILAR METALS
}

\author{
W. Zhang, H. H. Jensen and N. Bay
}

\begin{abstract}
Numerical modeling of resistance welding is carried out using the finite element method with coupled electrical and thermal analyses considering the dynamics of the welding current and force as well as the influence of temperature on the properties of the materials. Models for contact resistance and thermal conductivity at the interfaces are developed based on the friction theory of Wanheim and Bay, by which the welding force is included as a process parameter. An FEM program is developed for simulation of resistance welding processes with an animated display of the development of the electrical field and the temperature field. In order to verify the simulations, experiments of spot welding stainless steel to stainless steel and stainless steel to mild steel with identical and different thicknesses were carried out. After welding the weld interface was investigated in a microscope to determine the size and shape of the weld nugget. Results of simulation show the influence of the basic parameters, such as the process parameters, the material properties and the geometries of the workpieces, and are generally in good agreement with the experimental results.
\end{abstract}

Keywords: Spot welding, FEM modeling, stainless steel, mild steel

\section{INTRODUCTION}

Resistance welding is a complicated process, which involves interactions of electrical, thermal, mechanical and metallurgical phenomena. Many parameters are of influence in the process including the welding current, the welding time, the welding force, the geometry and material properties of the workpieces, the electrodes and the contact interfaces, as well as the dynamic behavior of the welding machines. For welding of dissimilar metals, the process becomes further complex. In order to determine the process parameters, to design the electrodes or to chose the capacity of the welding machine while developing a new product or maintaining an old process in industrial production, a great number of running-in experiments have often to be carried out. This increases the cost of products and in many cases delays the production operations.

Applying numerical methods may not only reduce the costs and time required for product and process development, but also reveal some internal phenomena in the materials which may be otherwise difficult to see thus facilitating the understanding of the resistance welding processes. The first numerical model for the temperature development in resistance welding was attempted by 
Roberts et al. (Ref. 1) using an analytical method. A number of other studies have followed in the recent years. However, most of the published work dealt only with the temperature development in spot welding of similar metals. The electrical analysis was usually not carried out coupling with the thermal analysis, and arbitrary assumptions were made on the contact resistance. The numerical methods applied were generally analytical methods (Refs. 1-2) and the finite difference method (Refs. 3-5), although in a few publications commercial FEM code for general purposes have been used for modeling of spot welding of similar materials (Refs. 6-7).

In order to facilitate the industrial application of resistance welding, numerical modeling of the resistance welding process has been carried out using the finite element method(FEM). Considering the physical process of resistance welding, the main tasks of numerical modeling are divided into three models, namely the electrical model, the thermal model and the mechanical model for deformation and stress analyses, whereas the metallurgical phenomena are included in the thermal model. These models are strongly interrelated with each other. The electrical model outputs the heat generation but requires the temperature distribution from the thermal model in order to determine the material properties, and the stress distribution and the geometry of the workpieces from the mechanical model in order to determine the contact properties and the distribution of parameters. The thermal model outputs the temperature distribution but requires the heat generation from the electrical model and the stress distribution and the geometry of the workpieces from the mechanical model. The mechanical model outputs the stress distribution and the geometry of the workpieces, but requires the temperature distribution to determine the materials properties.

It is very difficult to couple all these three models simultaneously, since it implies a very complicated, sometimes impossible numerical modeling. Instead, a strong coupling is made only between the electrical and thermal models, whereas the mechanical model will be coupled stepwise with the other two models. In this way, the non-linearity of the electrical and thermal models is limited to only materials properties and the time parameter. Convergence of these two models can be easily achieved simultaneously. The more complex non-linearity of geometry and materials properties is confined to the mechanical model. It is much easier to achieve convergence of this model independently.

The finite element equations of all three models have been formulated. So far, a software package, named SORPAS, has been developed for simulation of resistance welding processes, where the electrical and the thermal models are strongly coupled ensuring the nonlinear influences of temperature on the material properties and time on the temperature development. The mechanical FEM model has been developed independently but not coupled with the other two models yet.

\section{BASIC EQUATIONS OF THE ELECTRICAL AND THERMAL MODELS}

There are basically four physical phenomena involved in resistance welding, namely the electrical, the thermal, the mechanical and the metallurgical phenomena influenced by numerous parameters involved in the process, the materials and the welding machine. According to the physical process of resistance welding, it is understood that the electrical phenomena concern the electric potential distribution, the current flow and the heat generation due to Joule heating; the thermal phenomena 
concern the heat transfer and the temperature distribution; the mechanical phenomena concern the stress distribution and the deformation of the workpieces, the electrodes and the contact interfaces; whereas the metallurgical phenomena concern the variation of material properties, the phase transformation especially the melting and solidification.

The complexity of the problem is that all of these phenomena occur simultaneously under strong influence of the dynamic parameters of the process and the welding machine. At the first stage the electrical and thermal models are developed considering the variation of material properties with temperature and the liquid-solid phase transformation as well as the dynamic process parameters. The mechanical model for the deformation of workpieces and electrodes are under development.

\section{Basic equations of the electric model}

In two-dimensional problems, the governing differential equation for the electric potential distribution is expressed by Laplace's equation:

$$
\frac{\partial^{2} \Phi}{\partial x^{2}}+\frac{\partial^{2} \Phi}{\partial y^{2}}=0
$$

where $\Phi=\Phi(x, y, t)$ is the electric potential as a function of coordinates and time. There are two kinds of boundary conditions to be specified in the electrical analysis:

$$
\Phi=\Phi_{0}
$$

on the boundaries in contact with the power supply with known potential $\Phi_{0}$, and

$$
\frac{\partial \Phi}{\partial n}=0
$$

on the free boundaries, where $n$ denotes the normal direction of the boundary.

After determining the potential distribution, the current density can then be calculated as:

$$
J_{x}=-\frac{1}{\rho} \frac{\partial \Phi}{\partial x}, \quad J_{y}=-\frac{1}{\rho} \frac{\partial \Phi}{\partial y}
$$

where $J$ is the current density, and $\rho$ is the electric resistivity, thus the heat generation rate per unit volume is obtained:

$$
\dot{Q}=\rho J^{2}
$$




\section{Basic equations of the thermal model}

The governing differential equation for the transient heat conduction with internal heat source is expressed as:

$$
\frac{\partial}{\partial x}\left(k \frac{\partial T}{\partial x}\right)+\frac{\partial}{\partial y}\left(k \frac{\partial T}{\partial y}\right)+\dot{Q}=\gamma C \frac{\partial T}{\partial t}
$$

where $T=T(x, y, t)$ is the temperature as a function of coordinates and time, $k$ is the thermal conductivity, $\dot{Q}=\dot{Q}(x, y, t)$ is the internal heat generation rate per unit volume, $\gamma$ is the mass density and $C$ is the heat capacity per unit mass. There are three kinds of boundary conditions involved in the thermal analysis:

$$
T=T_{0}
$$

on boundaries with specified temperatures,

$$
-k \frac{\partial T}{\partial n}=0
$$

on the lines of symmetry, and

$$
-k \frac{\partial T}{\partial n}=h\left(T-T_{e}\right)
$$

on the free surfaces taking the convective heat exchange into account, $h$ is the convection heat transfer rate of the surrounding air and $T_{e}$ is the temperature of the air.

\section{Contact properties at interfaces}

The contact resistivity $\rho_{\text {contact }}$ at the interfaces is calculated according to Wanheim and Bay's model for plastic deformation of surface asperities determining the real contact area between rough surfaces (Ref. 8):

$$
\rho_{\text {connact }}=3\left(\frac{\sigma_{s+\text { soff }}}{\sigma_{n}}\right)\left(\frac{\rho_{1}+\rho_{2}}{2}\right)+\rho_{\text {contraninamt }}
$$

where $\sigma_{s_{s} \text { soft }}$ is the flow stress of the softer metal, $\sigma_{n}$ is the contact normal pressure at the interface, and the subscripts 1 and 2 indicate the two metals in contact. The contact resistance is influenced not only by the constraint contact between the rough surfaces but also by the surface contamination. An extra term $\rho_{\text {contaminum }}$ is therefore introduced in the equation to include the influence of the surface contaminants, such as oxides, water vapor, grease etc. 
Following a similar procedure as regards the contact thermal conductivity $k_{\text {contact }}$ at the interfaces this is calculated as:

$$
k_{\text {contuct }}=\frac{1}{3}\left(\frac{\sigma_{n}}{\sigma_{s_{-} \text {svft }}}\right)\left(\frac{k_{1}+k_{2}}{2}\right)
$$

\section{Latent heat during melting or solidification}

In order to simulate the nugget development in resistance welding realistically, the latent heat during melting or solidification is included by estimating an effective heat capacity in the temperature range of melting (Ref. 9):

$$
\widetilde{C}=C+\frac{L}{T_{l}-T_{s}} \quad \text { when }\left(T_{s}<T<T_{l}\right)
$$

where $L$ is the latent heat and $T_{s}$ and $T_{1}$ are the solidus and liquidus temperatures of the material.

\section{FINITE ELEMENT FORMULATIONS}

The domain or solution region can be discretized into a set of subareas or finite elements. The solution of the domain can be obtained by analyzing each element and then solving the assemblage of the elemental equations over the entire domain. In the present analysis, the linear triangular elements are used. Within an element the electric potential can be expressed approximately by the values at the three corner points:

$$
\Phi(x, y, t)=\sum_{i=1}^{3} N_{i}(x, y) \Phi_{i}(t)
$$

Similarly, the temperature can be expressed by:

$$
T(x, y, t)=\sum_{i=1}^{3} N_{i}(x, y) T_{i}(t)
$$

where $N_{i}(x, y)$ are the shape functions of the elements.

Introducing eqn. (13) into eqn. (1) and integrating over the entire domain by applying the Galerkin method and the rule of integration by parts, the system equations for the potential distribution are derived:

$$
[A]\{\Phi\}=\{\stackrel{n \times 1}{\prime \prime}\}
$$


where $n$ is the total number of nodes, $[A]$ is the electric conductance matrix, $\{F\}$ is the boundary conditions vector and $\{\Phi\}$ is the unknowns vector for the nodal potential values.

Similarly, the matrix system equations for temperature distribution are obtained:

$$
[A]\{T\}+[C]\left\{\frac{\partial T}{\partial t}\right\}=\{F\}
$$

where $[A]$ is the thermal conductance matrix, $[C]$ is the heat capacity matrix, $\{F\}$ is the vector of heat source and boundary conditions and $\{T\}$ is the unknowns vector for the nodal temperatures.

The Crank-Nicolson method is applied for the time stepping:

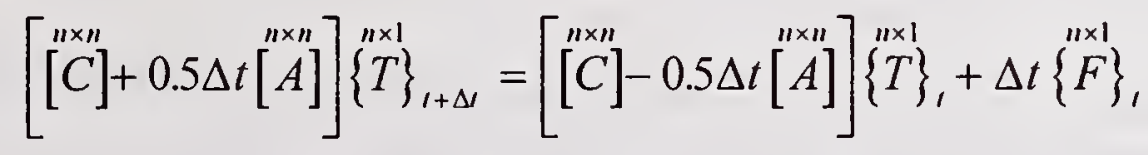

Solving eqns. (15), (4) and (5), the potential distribution, the current distribution and the heat generation will be obtained. Solving eqn. (17) the temperature distribution will be obtained as a function of time.

\section{EXPERIMENTS OF SPOT WELDING}

In order to verify the numerical models, experiments were carried out with an $\mathrm{AC}$ welding machine spot welding stainless steel (Type 304) and mild steel (Tibnor SS 1142) in two different thicknesses of $2 \mathrm{~mm}$ and $1 \mathrm{~mm}$ with the following combinations:

a) $2 \mathrm{~mm}$ stainless steel to $2 \mathrm{~mm}$ stainless steel,

b) $2 \mathrm{~mm}$ stainless steel to $2 \mathrm{~mm}$ mild steel,

c) $2 \mathrm{~mm}$ stainless steel to $1 \mathrm{~mm}$ mild steel,

d) $1 \mathrm{~mm}$ stainless steel to $2 \mathrm{~mm}$ mild steel.

The welding current and the welding force were measured during spot welding. The weld nuggets were observed in a microscope after welding. Micrographs of the cross section of the welds were made in order to compare with the numerical results.

Fig. 1 shows an overview of the weld nuggets in all the 4 metal combinations. It is noticed that a symmetric weld nugget is formed in spot welding of stainless steel to stainless steel with identical thickness, as shown in Fig. 1 (a). In spot welding of stainless steel to mild steel with identical thickness, as seen in Fig. 1 (b) the weld nugget is formed in an unsymmetric shape with a larger diameter in the stainless steel due to its higher resistance. In spot welding of stainless steel to mild steel with different thicknesses, as seen in Figs. 1 (c) and (d), the weld nuggets formed are very 
different when the thickness of the two metals are reversed. It is seen that the formation of the weld nuggets is strongly influenced by the geometries and the material properties of the workpieces.

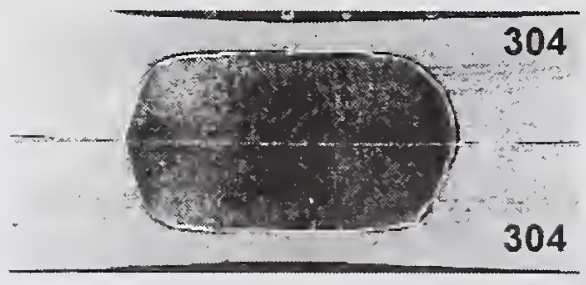

(a)

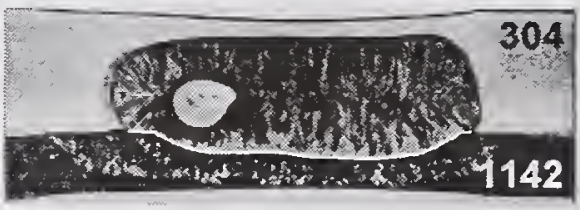

(c)

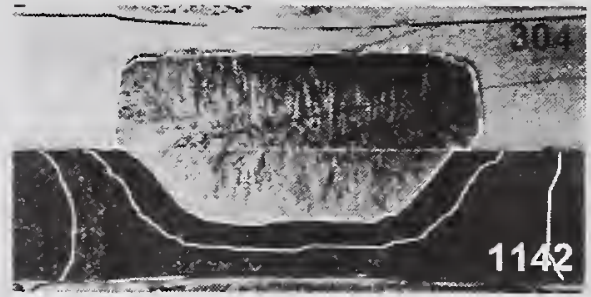

(b)

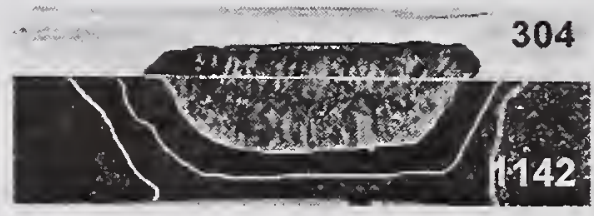

(d)

Fig. 1: Weld nuggets in spot welding, (a) stainless steel to stainless steel with identical thickness of $2 \mathrm{~mm}$, (b) stainless steel to mild steel with identical thickness of $2 \mathrm{~mm}$, (c) $2 \mathrm{~mm}$ stainless steel to 1 $\mathrm{mm}$ mild steel and (d) $1 \mathrm{~mm}$ stainless steel to $2 \mathrm{~mm}$ mild steel.

\section{RESULTS OF SIMULATION AND DISCUSSIONS}

An FEM program has been specially developed for numerical modeling of resistance welding with coupled electrical and thermal analyses. The numerical simulation requires the welding voltage, welding force, welding time and geometry and material properties of the workpieces and electrodes as input. The output covers the following parameters: the welding current, energy consumption, total resistance, potential and current distribution and temperature distribution in workpieces as well as electrodes.

\section{Spot welding of stainless steel to stainless steel with identical thickness}

Fig. 2 shows a comparison between the simulated and the experimental weld nuggets in spot welding of stainless steel to stainless steel with identical thickness. The welding force was $2250 \mathrm{~N}$, the welding current was $8.8 \mathrm{kA}$ and the welding time was 10 periods as measured in the experiments. The welding current applied in the numerical modeling was increased to $12 \mathrm{kA}$ in order to get a similar size of the weld nugget. This is because the mechanical model was not coupled with the electrical and thermal models thus assuming the stress distribution to be uniform over the interface. This will introduce some error in calculation of the contact properties.

It is also noticed that the thickness of the simulated weld nugget is smaller than that in the micrograph. This is probably due to the calculated contact thermal conductivity being too high. The heat developed in the workpieces has transferred quickly into the electrodes, thereby reducing the thickness of the weld nugget. 


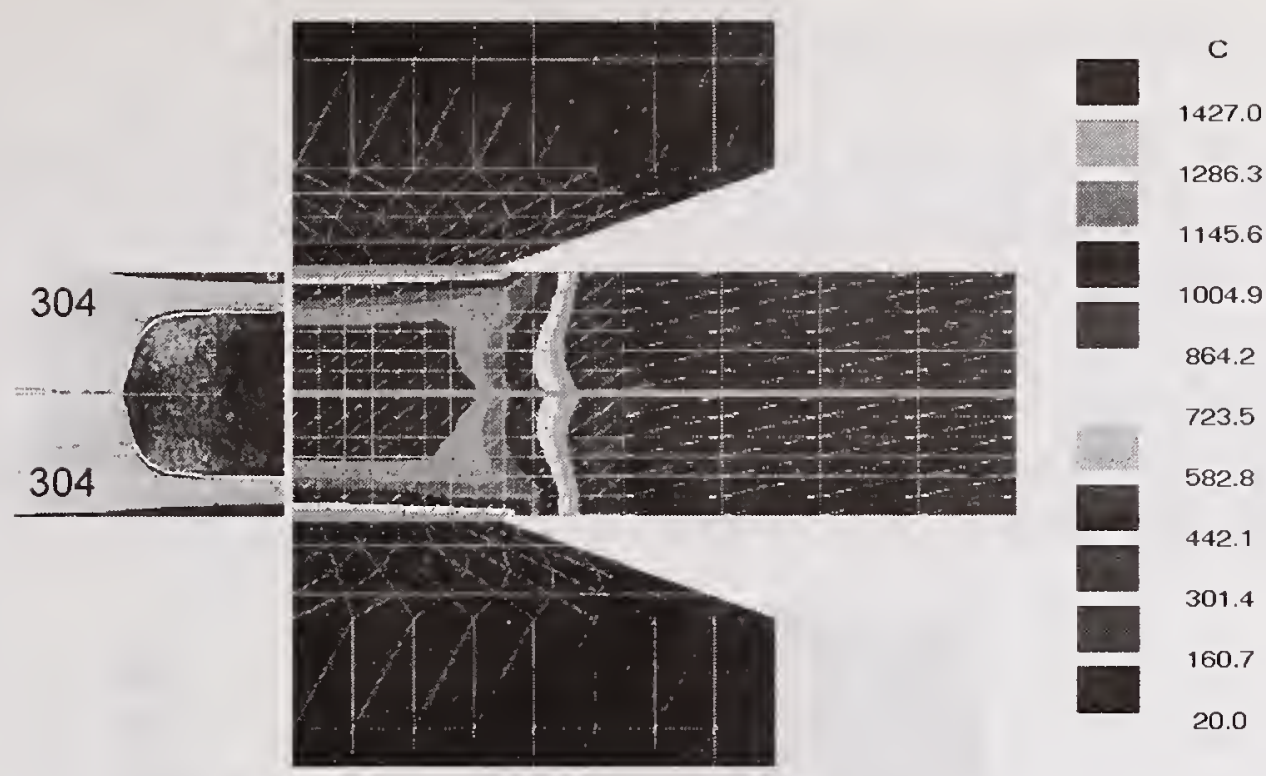

Fig. 2: Comparison of the simulated and experimental weld nuggets in spot welding of stainless steel to stainless steel with identical thickness of $2 \mathrm{~mm}$.

\section{Spot welding of stainless steel to mild steel with identical thickness}

Fig. 3 shows a comparison of the simulated and experimental weld nuggets in spot welding of stainless steel to mild steel with identical thickness. The welding force was $2250 \mathrm{~N}$, the welding current was $7.1 \mathrm{kA}$ and the welding time was 10 periods as measured in the experiments. The welding current in the numerical modeling was increased to $11 \mathrm{kA}$. It is found that the simulated weld nugget for dissimilar metals is developed in the same way as in the experiments. This implies that the numerical simulation can successfully demonstrate the influence of material properties on the formation of a weld nugget.

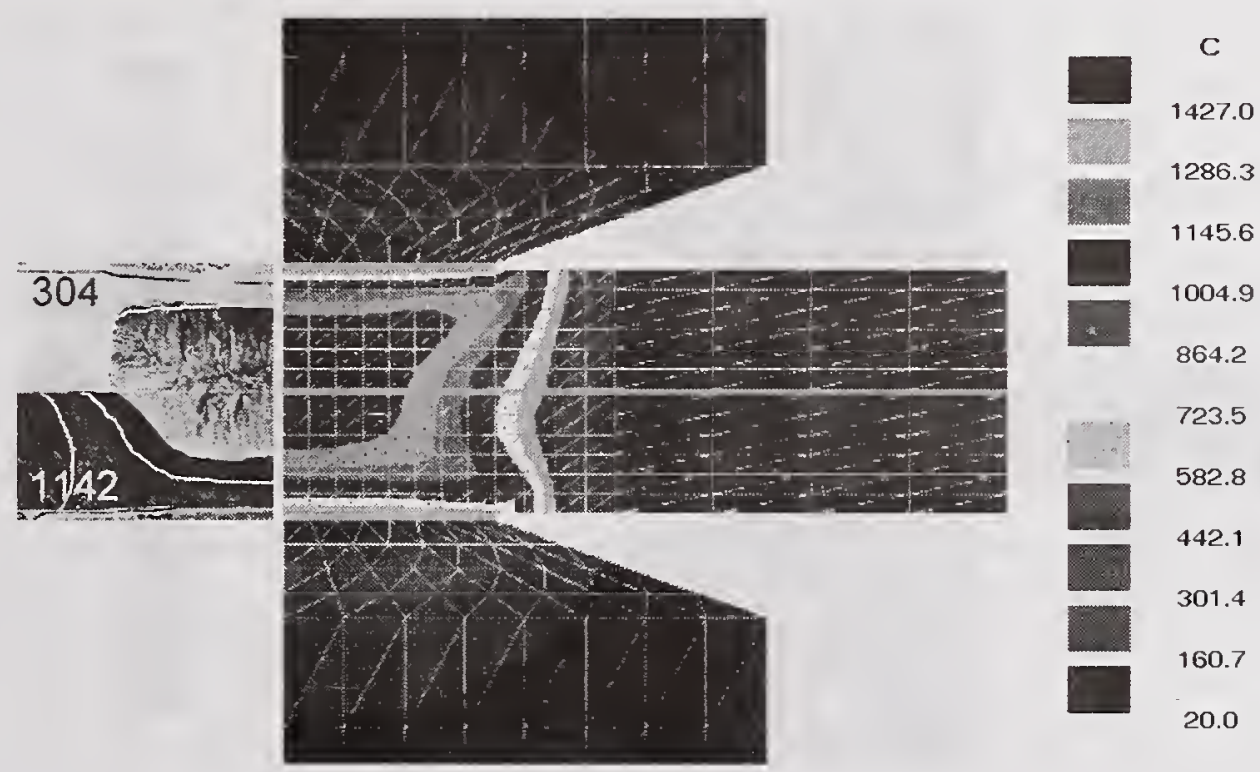

Fig. 3: Comparison of the simulated and experimental weld nuggets in spot welding of stainless steel to mild steel with identical thickness of $2 \mathrm{~mm}$. 


\section{Spot welding of stainless steel to mild steel with different thickness}

Figs. 4 and 5 show the comparisons of the simulated and experimental weld nuggets in spot welding of $1 \mathrm{~mm}$ stainless steel to $2 \mathrm{~mm}$ mild steel and $2 \mathrm{~mm}$ stainless steel to $1 \mathrm{~mm}$ mild steel respectively. The welding time was 10 periods, the welding force was $2250 \mathrm{~N}$ and the welding current was $7.9 \mathrm{kA}$ in both cases. The welding current in the numerical modeling was increased to $10 \mathrm{kA}$. It is found that the simulated weld nuggets are in good accordance with the micrographs implying that the program can demonstrate not only the influence of the material properties but also the influence of the geometries of the workpieces.

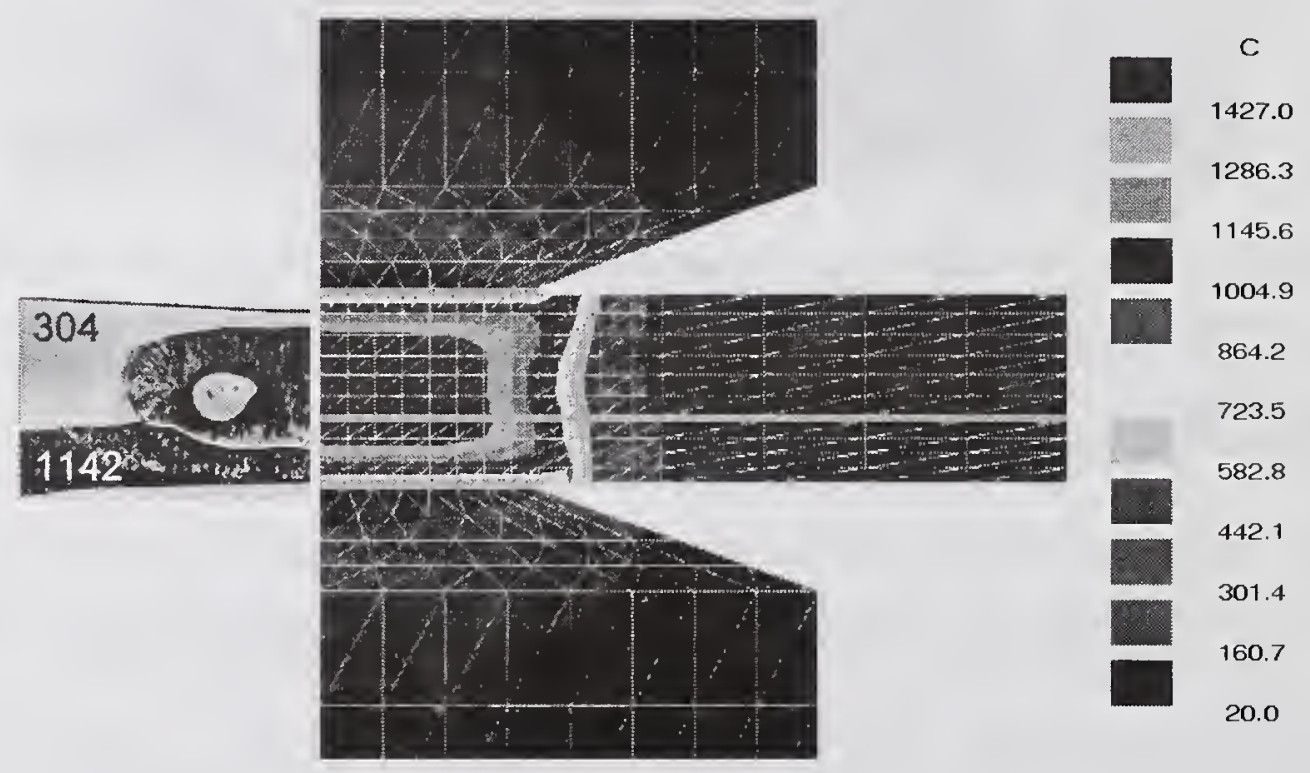

Fig. 4: Comparison of the simulated and experimental weld nuggets in spot welding of $2 \mathrm{~mm}$ stainless steel to $1 \mathrm{~mm}$ mild steel.

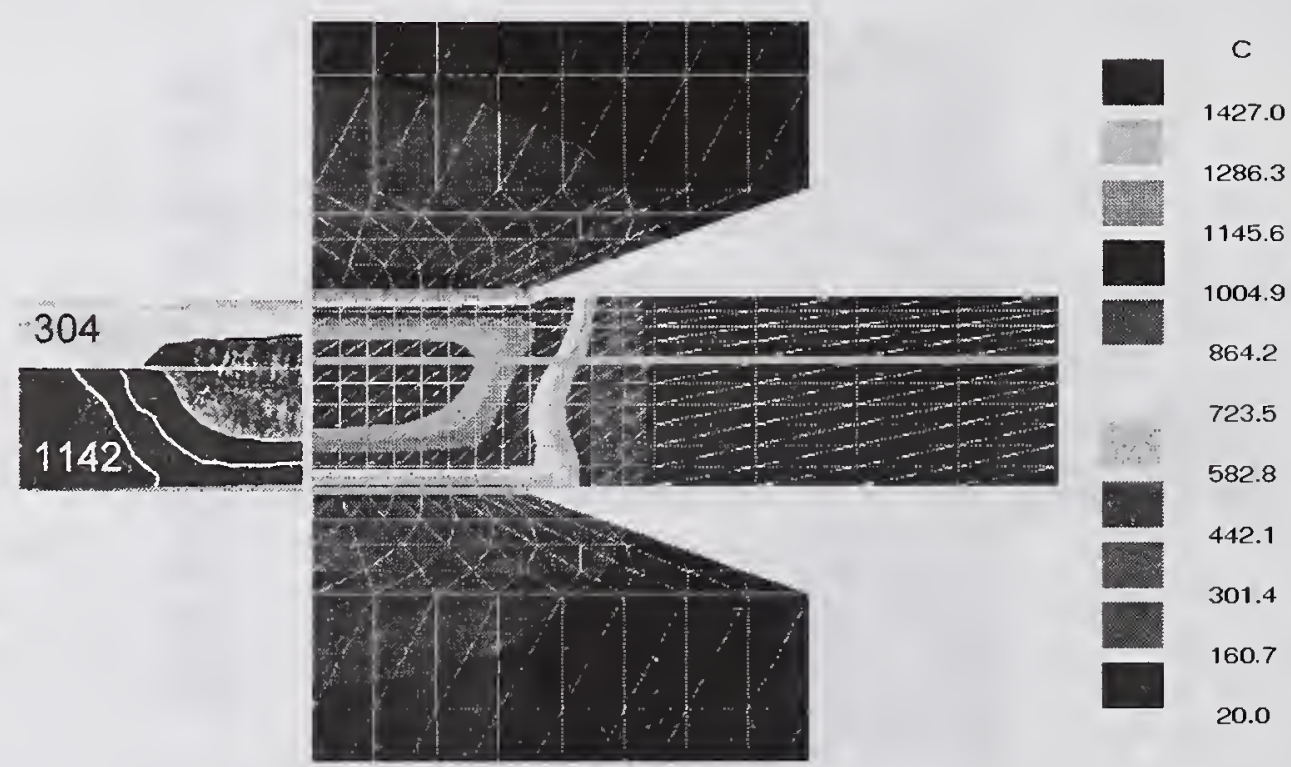

Fig. 5: Comparison of the simulated and experimental weld nuggets in spot welding of $1 \mathrm{~mm}$ stainless steel to $2 \mathrm{~mm}$ mild steel. 


\section{CONCLUSIONS}

A finite element program has been developed for numerical modeling of resistance welding with coupled electrical and thermal analyses. The simulated weld nuggets are compared with the experimental results. Good agreement is generally obtained. Results of simulation show the influence of basic parameters involved in spot welding of similar and dissimilar metals including the welding current, force and time as well as the materials properties and geometries of the workpieces. This implies that the program is useful in supporting the understanding of the process parameters thereby facilitating process development. More accurate simulations are expected to be achieved when the mechanical model is coupled with the electrical and the thermal models.

\section{ACKNOWLEDGMENTS}

The authors would like to thank Mr. E. Østgaard from the FORCE Institute, Denmark, for his help on the spot welding experiments and Dr. T. Funder-Kristensen, Danfoss A/S, Denmark, for his help on the metallographic investigations.

\section{REFERENCES}

1. Roberts, D. K.; Roberts, J. E.; and Wells, A. A. 1958. Fundamental Resistance Welding Investigations, British Welding Journal, (3): 117-126.

2. Archer, G. R. 1960. Calculations for Temperature Response in Spot Welding, Welding Journal (8): $327 \mathrm{~s}$ to $330 \mathrm{~s}$.

3. Greenwood, J. A. 1961. Temperatures in Spot Welding, British Welding Journal (3):316-322.

4. Rice, W.; and Funk., E. J. 1967. An Analytical Investigation of the Temperature Distributions during Resistance Welding, Welding Journal (4): $175 \mathrm{~s}$ to $186 \mathrm{~s}$.

5. Cho, H. S.; and Cho, Y. J. 1989. A Study of the Thermal Behavior in Resistance Spot Welds, Welding Journal (6): $236 \mathrm{~s}$ to $244 \mathrm{~s}$.

6. Nied, H. A. 1984. The Finite Element Modeling of the Resistance Spot Welding Process, Welding Journal (4): 123 s to $132 \mathrm{~s}$.

7. Tsai, C. L.; Jammal, O. A.; Papritan, J. C.; and Dickinson, D. W. 1992. Modeling of Resistance Spot Weld Nugget Growth, Welding Journal (2): $47 \mathrm{~s}$ to $54 \mathrm{~s}$.

8. Wanheim, T.; and Bay, N. 1978. A model for friction in metal forming processes. Annals of the CIRP 27(1): 189-194.

9. Sahm, P. R.; and Hansen, P. N. 1984. Numerical Simulation and Modeling of Casting and Solidification Processes for Foundry and Cast-House. CIATF - International Committee of Foundry Technical Associations. 190-192. 


\title{
TWO DIMENSIONAL FINITE ELEMENT ANALYSIS OF RESIDUAL STRESS IN ELECTROSLAG BUTT WELDS
}

\author{
Leilei Zhang*, David G. Atteridge*, Lemmy Meekisho*, Martin Becker*
}

\begin{abstract}
The electroslag welding process is an attractive process for welding of thick plates which includes construction of ships, storage tanks, pressure vessels, bridges, buildings and other heavy structures. Computational models developed to calculate the magnitude and distribution of residual stresses for electroslag welded plates are presented in this paper. Two two-dimensional idealized models using finite element methods were developed to evaluate the residual stress distribution along the length and through the thickness of the welded plates. Mid-plate residual stress distribution was found to be influenced by the presence of cooling shoe induced accelerated surface cooling, and to be a complex function of distance from weld centerline. Tensile residual stresses were found perpendicular to the welding direct while a combination of tensile and compressive stress were found parallel to the welding direction. Residual stresses at weld centerline as a function of plate thickness were found to be tensile perpendicular to the welding direction and compressive stresses parallel to the welding direction.
\end{abstract}

Key Words: Finite Element Analysis, Residual Stress, Electroslag Welding ( ESW )

\section{INTRODUCTION}

Electroslag welding (ESW) was developed by Paton and coworkers in the Paton Electric Welding Institute, USSR, in the early 1950's. (Ref. 1) It is a unique welding process that is an arc-less process and is, in general, a single pass process no matter what the thickness of the material. The weld wire is added into, and melted in, a slag bath covering the molten pool, as illustrated in Figure 1. The molten slag and weld pool are restricted from flowing out between the plates to be welded by the use of (water-cooled) shoe "dams", see Figure 1.

This results in essentially welding a cast "plug" between the two plates to be joined. The large heat input associated with the process results in a coarse cast structure and an extended coarse grained heat affected zone (HAZ) region unless special plate and consumable alloying chemistry are invoked. This typical coarse grain structure can lead to low fracture toughness and hard to detect weld defects which are the main reasons for ESW not being used more extensively for welding thick tension-member plates. In addition, cenerline grain orientation and segregation can lead to hot cracking. Bridge members fabricated using ESW have had a history of brittle failure. ( Ref. 2)

* Oregon Graduate Institute of Science and Technology, Department of Materials Science and Engineering , P.O. Box 91000, Portland, OR 97291-1000) 


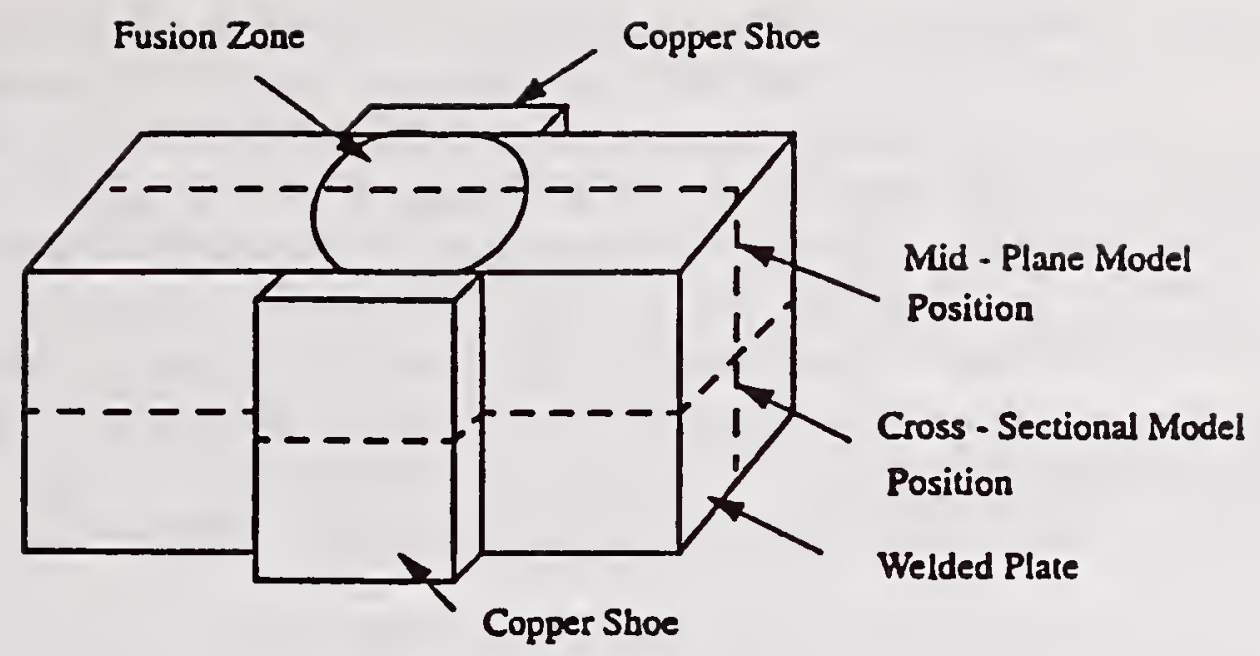

(a)
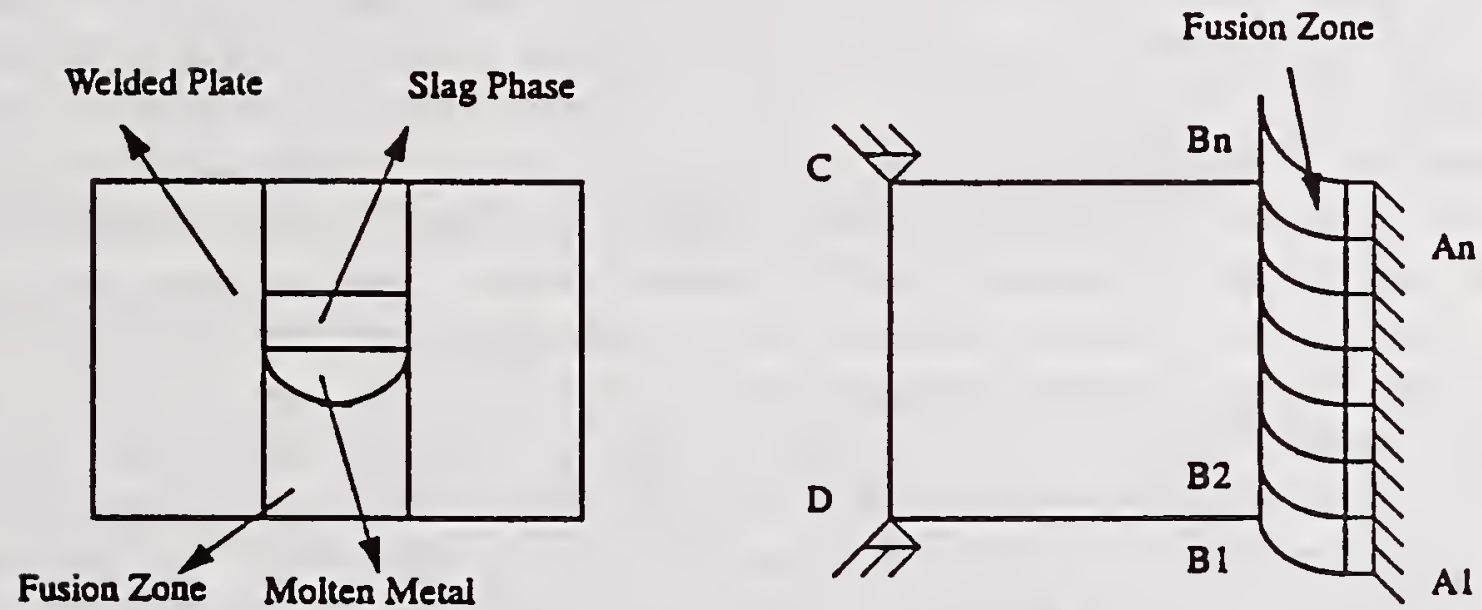

(b)
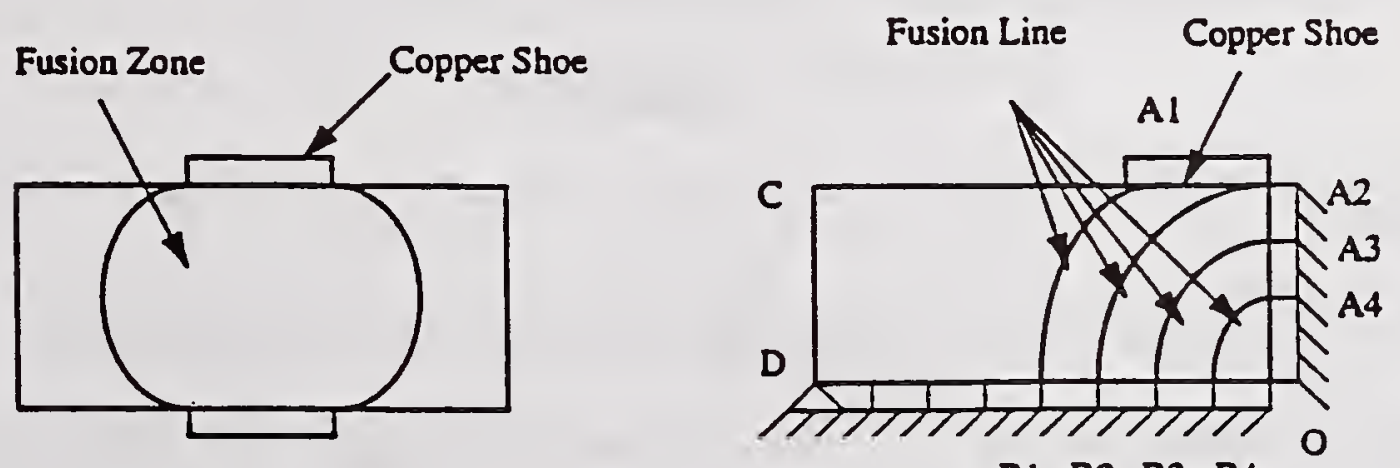

(c)

Figure 1 ( a ) Illustration of Electroslag Welding Processes ( b ) Mid - Plane Model Position and Shape (c) Cross - Section Model Position and Shape 
The unique combination of welding thick plates with a single pass technique and accelerated surface cooling due to the cooper shoes presents the potential that the weld-induced residual stress state for ESW will be different from that associated with conventional multi-pass welding. Initial study results on ESW-induced residual stress distribution is presented herein.

The first modeling work on the ESW process was presented by Dallier, Szekely, and Eagar ( Ref. 3 ). They calculated the heat and fluid flow in the liquid slag and liquid metal regions in an idealized two dimensional systems using finite differential methods. After that they used a three dimensional model to calculate the temperature distribution associated with different ESW operating parameters. ( Ref. 4-6) They didn't consider phase transformation effects on thermal distribution during welding process and did not predict resultant residual stress distributions.

The total stress state at the tip of a crack or crack-like defect is one of the most important factors influencing th: mechanism of fatigue, brittle fracture, or stress corrosion cracking. In all of these failure mechanism, the residual stress of weldments can make a major contribution to the stress field at a crack tip. Detailed research work or modeling on residual stress of the ESW processing hasn't been done yet. Results from computational models developed to calculate the magnitude and distribution of residual stresses for ESW welded plates are presented in this paper. Two dimensional idealized models were used to evaluate the residual stress distribution along the length and through the thickness of the ESW welded plates. Residual stress analysis was done based on the results of thermal analysis, i.e., a decouping analysis was used in this study. Because of the decouping of the thermal and stress processes, the same geometry was used in the residual stress analysis as in the thermal analysis. At the same time, because of the complexities of the underlying phenomena, unavailability of a material property database and limited computer configuration, simplifying assumptions were necessary. It should be noted that these simplifications are only expected to affect the magnitude of residual stresses but not the residual stress distribution. Elastic-plastic temperature dependent mechanical properties were included in this model as were phase transformation effects.

\section{MODEL}

\section{Assumptions}

Multiple modeling simplifications were necessary to allow model development. They are listed below.

1) The weld pool remains in a steady state condition during the ESW process.

2) The weld pool shape is simplified to be rectangular for the slag phase and a half circle for the metal phase.

3) The melting point of the metal is used as the boundary conditions at the contact layers of the molten metal/base metal, molten metal/plate, and molten slag/plate. A convection boundary condition has been used for the remaining sides of the welded plate.

4) The heat generated by the deformed solid is neglected. 
5) The actual non-linear thermoplastic-viscoplastic problem was assumed to be a linear thermoplastic problem. A linear elastic-plastic stress-strain relation was assumed. The elastic and plastic regions were defined by elastic modules and plastic modules, respectively. The yield stress defined the separation between these two regions. The thermal coefficient is assumed to be constant.

6) Potential defect or crack formation was ignored, as well as creep and hardening. Simplifying yield strength laws were used. The Von-Mises yield criterion and associated flow rule with kinematic hardening were assumed to consider the Bauschinger effect. As in most welding problems, creep effects were neglected on the basis that time spent at high temperature is short for a weld.

7) The phase transformation expansion effect was considered to be similar during heating as well as during cooling because of the lack of available thermal expansion coefficient values. Plastic strain due to the change in volume during allotropic phase transformation is neglected in this study. A more representative treatment would involve incorporating the transformation plasticity effect for weld metal as well as different HAZ regions.

8) The same material properties were used for base metal, weld metal and HAZ. This will change the magnitude of the resulting residual stresses. Although, the overall residual stress distribution are expected to be unchanged. Since the objective of the research work was to study the residual stress distribution instead of specific stress values, the final conclusions will not be affected dramatically with this assumption.

\section{Initial and Boundary Conditions}

The heat transfer field equation is solved under the given initial and boundary conditions. Room temperature ( $T=298 \mathrm{~K}$ ) is prescribed to the all points in the domain except the heat source boundary as the initial condition for the calculation. During the ESW process, the weld pool was considered as the heat source. The heat flow behavior in welded plate is affected by the shape of weld pool. The heat source distribution could be modeled using the essential boundary condition $\left(T(x, y)=T_{s}(x, y)\right)$. Melting temperature of the welded plate is used for $T_{s}$ $(x, y)$. That is to say, a temperature boundary versus a heat flux heat source model was used in this study. This greatly simplifies the numerical simulation work.

The surface defined as $A_{1} B_{1} B_{2}$ in the mid-plane model, Figure $1 b$, is the heat source at a certain time step. $A_{1} B_{1}$ defines the fusion line of the molten pool (modeled as a quarter circle of radius $0.04 \mathrm{~mm}$ (1.6 in.) while $B_{1} B_{2}$ defines the slag pool to plate boundary. Note that the temperature of the complete $A_{1} B_{1} B_{2}$ surface is defined as the steel melting point, $1773 \mathrm{~K}$. This heat source configuration is stepped from the bottom to the top of the plate at a given welding speed. Equilibrium configuration of the weld pool is assumed as starting and stopping blocks are used during the process to allow pool development prior to plate welding initiation and to assure the pool is above the top of the plate prior to weld termination.

The weld pool in the mid-plane model retains a constant configuration throughout the model calculation. This is not true for the cross-sectional model pool. Here the pool starts at a maximum dimension and then continuously decreases in cross section until it completely 
disappears, as illustrated in Figure 1c. This is because the molten pool approaches, intersects, and then passes through the cross-sectional model plane position. Note that the effect of the copper shoe was simulated by an increase in surface heat transfer coefficient, as mentioned above.

Only symmetry boundary conditions were used during the residual stress analysis. The residual stress was modeled under self-restrain only. No external restrains were applied in the analysis. To prevent rigid body motion during the finite element analysis, only two nodes on the domain edge were fully restrained. This suggested that the welded plate is not restrained but is pivoted around these restrained nodes and the restraint free condition is realized. This is illustrated in Figures $1 \mathrm{~b}$ and $\mathrm{c}$.

\section{Mesh and Time Discretization}

The mesh needs to be sufficiently fine to model the heat source accurately. Therefore, weld metal and HAZ regions should be discretized with smaller elements than regions far away from the heat source. At the same time, use of element types such as linear or quadratic elements will also affect the mesh coarseness.

Because of the symmetry nature of the ESW weldment, only half of the weldment for the midplane model and one quarter of the weldment for the cross-sectional model,with one end at the weld center plane, were modeled. For both of the models, the modeled plate thickness was $0.08 \mathrm{~m}(3.15 \mathrm{in}$.) and the length was $0.8 \mathrm{~m}(31.5 \mathrm{in}$.), as shown in Figure 2, where the $\mathrm{x}=0$ line is the weld center line. Note that the "effective" plate length of each welded plate was $6.12 \mathrm{~m}$ (20 ft) due to use of a length-specific modified heat conductivity as a plate end boundary condition. A nonuniform mesh with 1419 elements and 1456 nodes was generated over the domain for the mid-plane model. 154 elements and 183 nodes were generated for cross sectional model. The density of elements varies as a function of distance from the weld center line. The mesh for both mid-plane model and cross-sectional model are given in Figure 2.

Short time increments and relatively large temperature convergence tolerance should were used due to the sharp temperature rise in the initial heating period, while longer time increments and smaller tolerances were used during cooling.

\section{SOLUTION PROCEDURE}

The ANSYS 53 finite element code was used for the analysis. A transient thermal analysis was first performed to obtain a nodal temperature solution. The nodal thermal history was used as input as the thermal load in the structural analysis. The same geometry and finite element mesh was employed in both the thermal and structural analysis. The temperature dependent thermal properties and mechanical properties of A36 steel were used in the model. For thermal analysis, thermal conductivity and enthalpy were required. ( Ref. 7-8 ) 


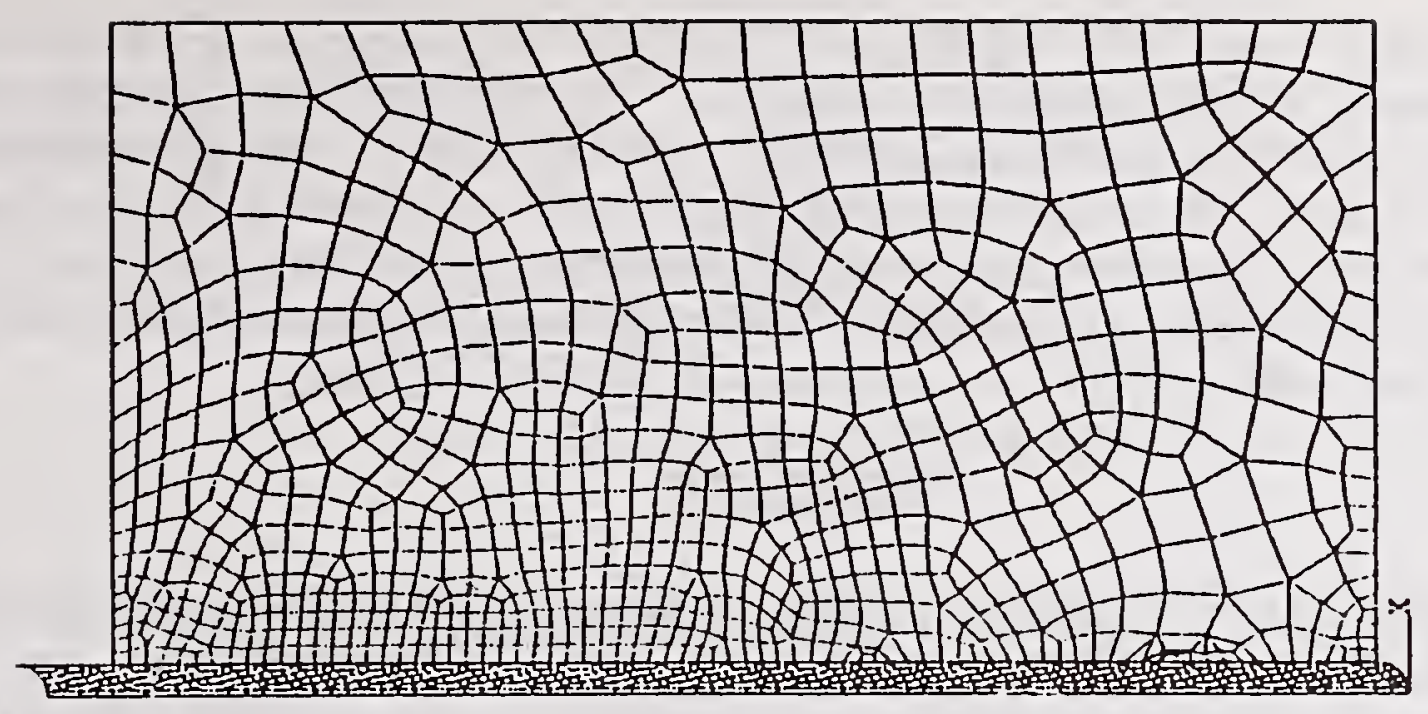

Figure 2 (a ) Mesh of Mid - Plane Model

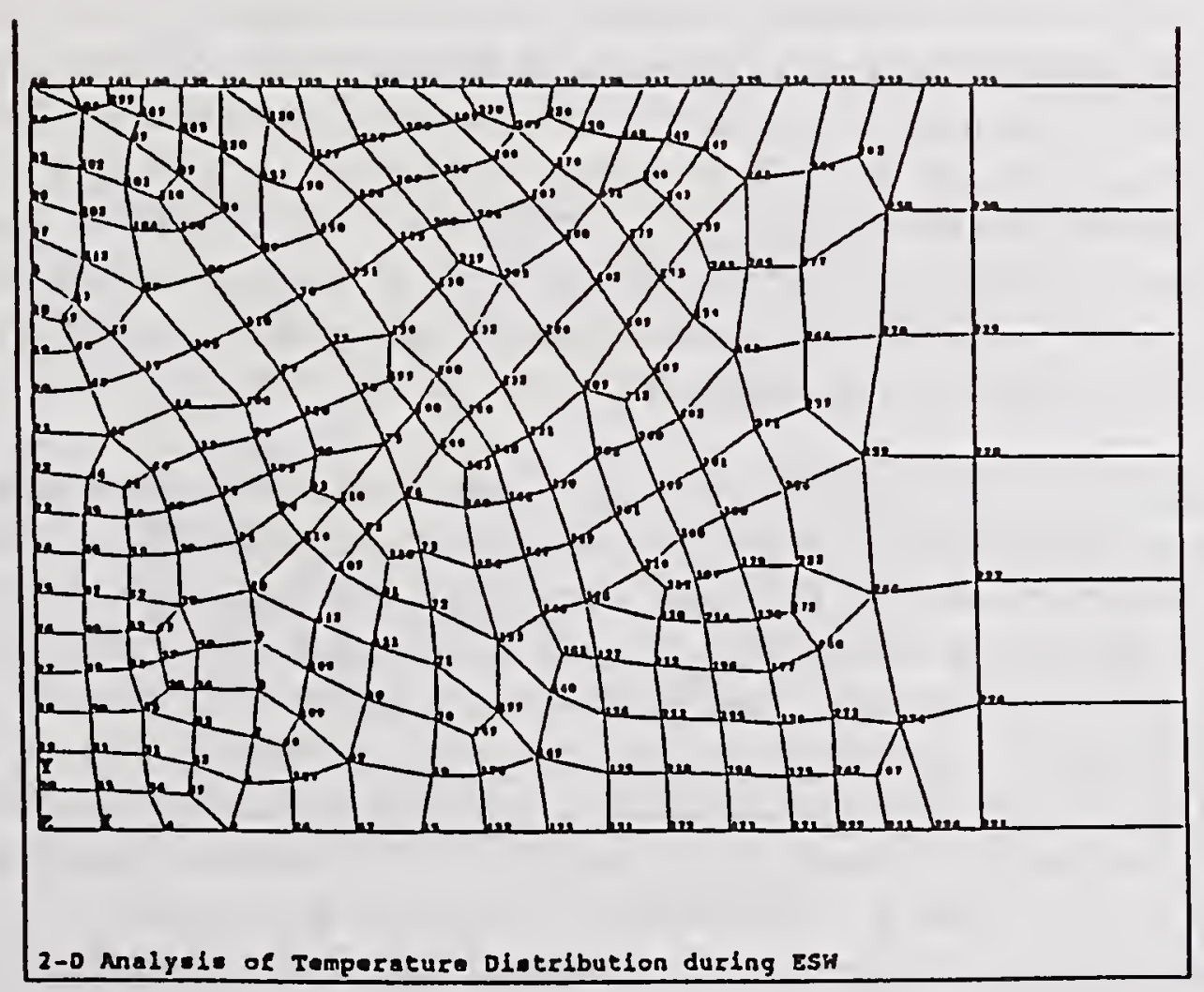

Figure 2 (b ) Mesh of Cross - Section Model 
A static stress analysis was used during the solution part of ANSYS analysis. Large strain option and Newton-Raphson solution procedures were used. The input loads were chosen from the thermal analysis to represent the thermal history as close as possible. Different load steps were defined by reading nodal temperatures corresponding to certain time intervals. This load stepping scheme was essentially the same as the temperature history from the thermal analysis, i.e., the time stepping was much finer in the initial part of the thermal cycle and gradually coarsened in the later part of the weld cooling.

\section{RESULTS}

The thermal models yielded reasonable temperature profiles as a function of distance from the WCL, as illustrated for the mid-plane model in Figure 3; note that increasing node number relates to increasing distance from WCL. The two models did, however, exhibit different heating and cooling characteristics at equal distances from WCL/fusion line, as illustrated in Figure $3 \mathrm{~b}$ and c. The mid-plane results yielded higher peak temperature and longer cooling time than the cross-sectional model. The residual stress distribution results from the two models were also different.

The mid-plane model results for residual stress distribution along the length of the weldment are illustrated in Figure 4. Residual stress perpendicular to the weld line (Figure 4a) begins at a high tensile value at weld centerline, decreases slightly and then increases into the fusion zone/HAZ, and then decreases to zero with increasing distance down the weldment. Its initial value is approximately at plate material yield strength level of $250 \mathrm{MPa}(36 \mathrm{ksi})$ with its maximum approximately $0.025 \mathrm{~m}(\sim 1 \mathrm{in})$ from weld centerline (WCL), and decreases to zero around $0.2 \mathrm{~m}$ ( -8 in). Residual stress parallel to weld centerline, Figure $4 \mathrm{~b}$, begins at a tensile stress approximately 170 percent of plate material yield strength at weld centerline, decreases to a compression value near 33 percent of yield strength, and then increases to zero as distance from weld centerline increases. Maximum compression residual stress occurs around $0.15 \mathrm{~m}$ ( $~ 6 \mathrm{in}$ ) from WCL, and zero residual stress is approached near $0.4 \mathrm{~m}$ ( 16 in) from WCL.

The cross-sectional plane model results for residual stress distribution at mid-plane along the length of the weldment are illustrated in Figure 5. Residual stress perpendicular to the weld line (Figure 5a) begins at a tensile value at WCL, decreases rapidly and then increases into the fusion zone/HAZ, and then decreases to zero with increasing distance down the weldment. Its initial value is approximately 45 percent of the plate yield strength, decreases to a tensile stress of 15 percent of the yield strength, rises to a tensile value of approximately 90 percent of yield approximately $0.05 \mathrm{~m}$ ( $\sim 1.6$ in) from weld centerline, and then decreases to zero around $0.2 \mathrm{~m}$ ( $\sim 8$ in). Residual stress parallel to weld centerline (Figure $5 \mathrm{~b}$ ) begins at a compressive stress approximately 50 percent of yield at weld centerline, decreases to a compressive stress of 90 percent of yield, increases to a tensile stress of approximately 110 percent of plate yield $0.05 \mathrm{~m}$ ( 2 in.) from weld centerline, decreases to a compressive strength of 30 percent of yield and then increases to zero as distance from weld centerline increases ( $0.14 \mathrm{~m}, 0.55 \mathrm{in})$. 


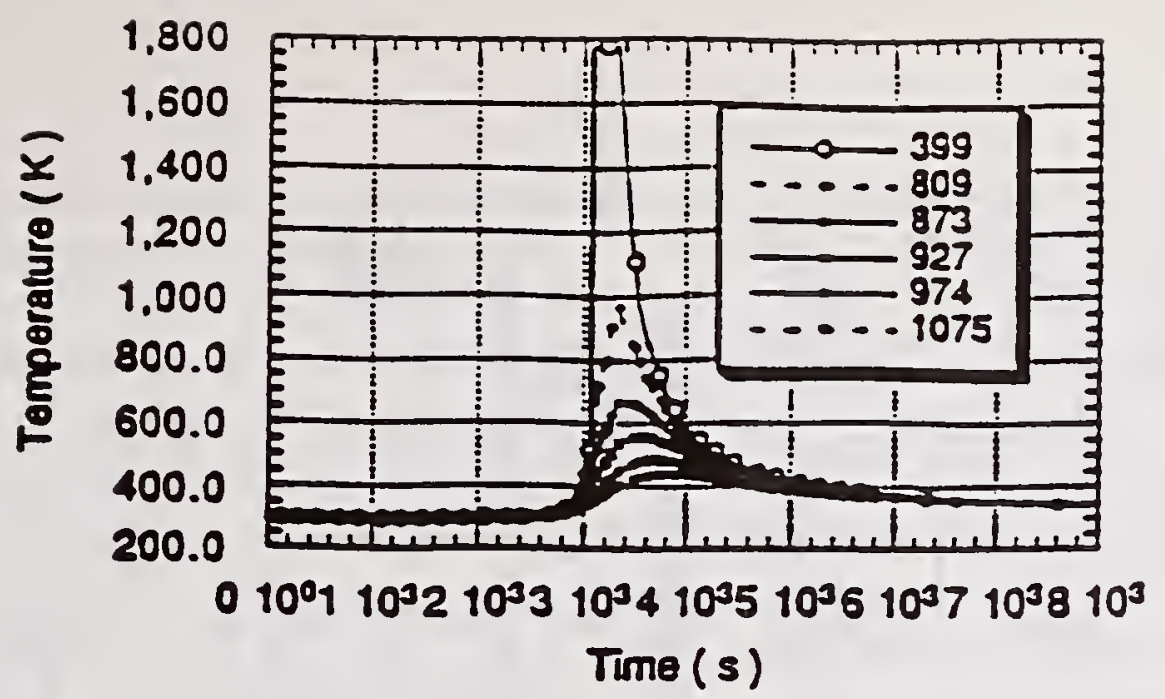

Figure 3 (a) Dlustration of Temperature as a Function of Time of Node Point 399,809,873,972,974 and 1075 in Mid - Plane Model

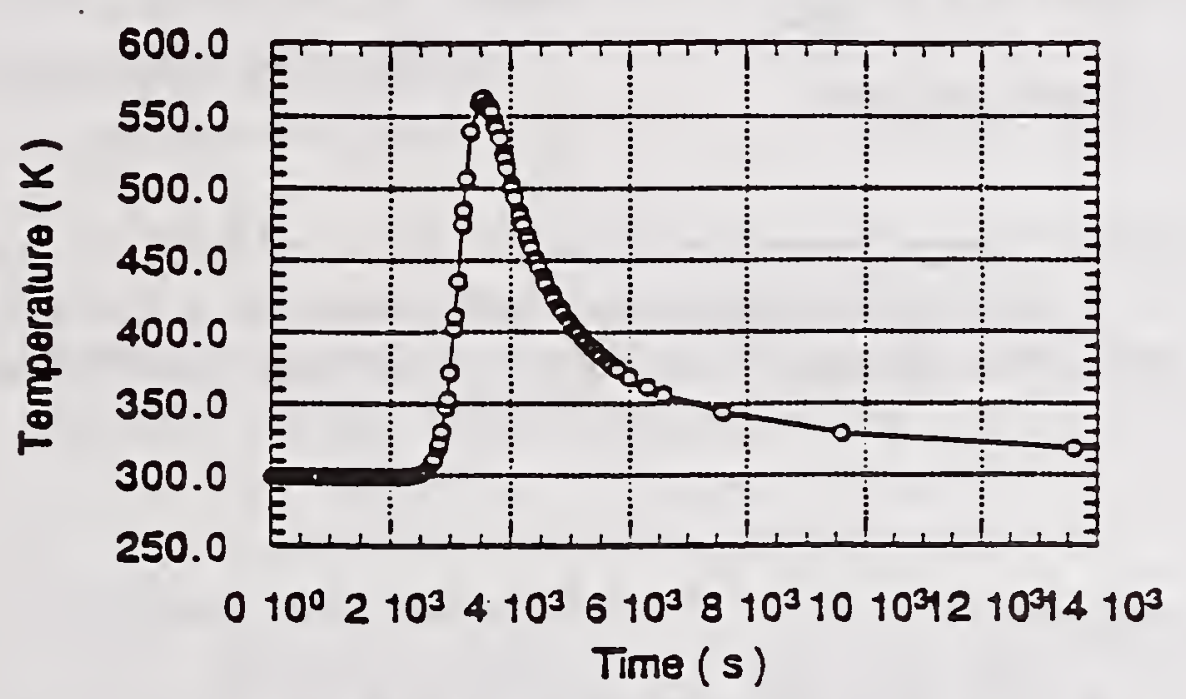

Figure 3 (b ) Illustration of Temperature as a Function of Time of Node Point 927 in Mid - Plane Model

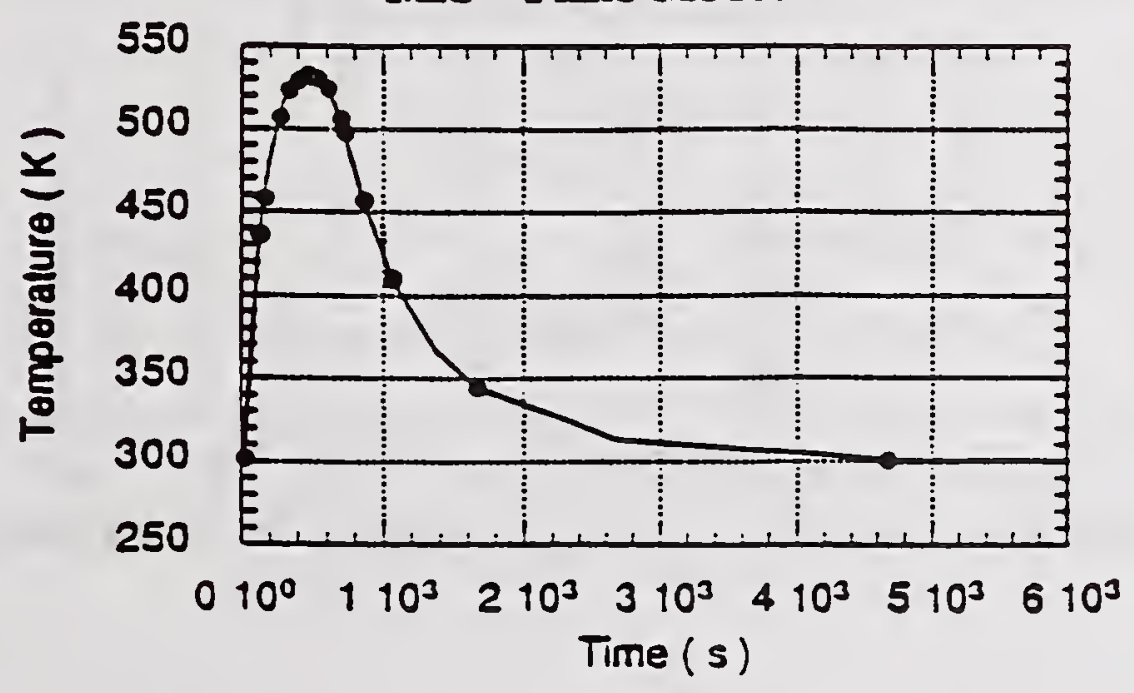

Figure 3 (c) Illustration of Temperature as a Funcion of Time of Node Point Equivalent to Mid - Plane Node Point 927 in Cross - Section Model 

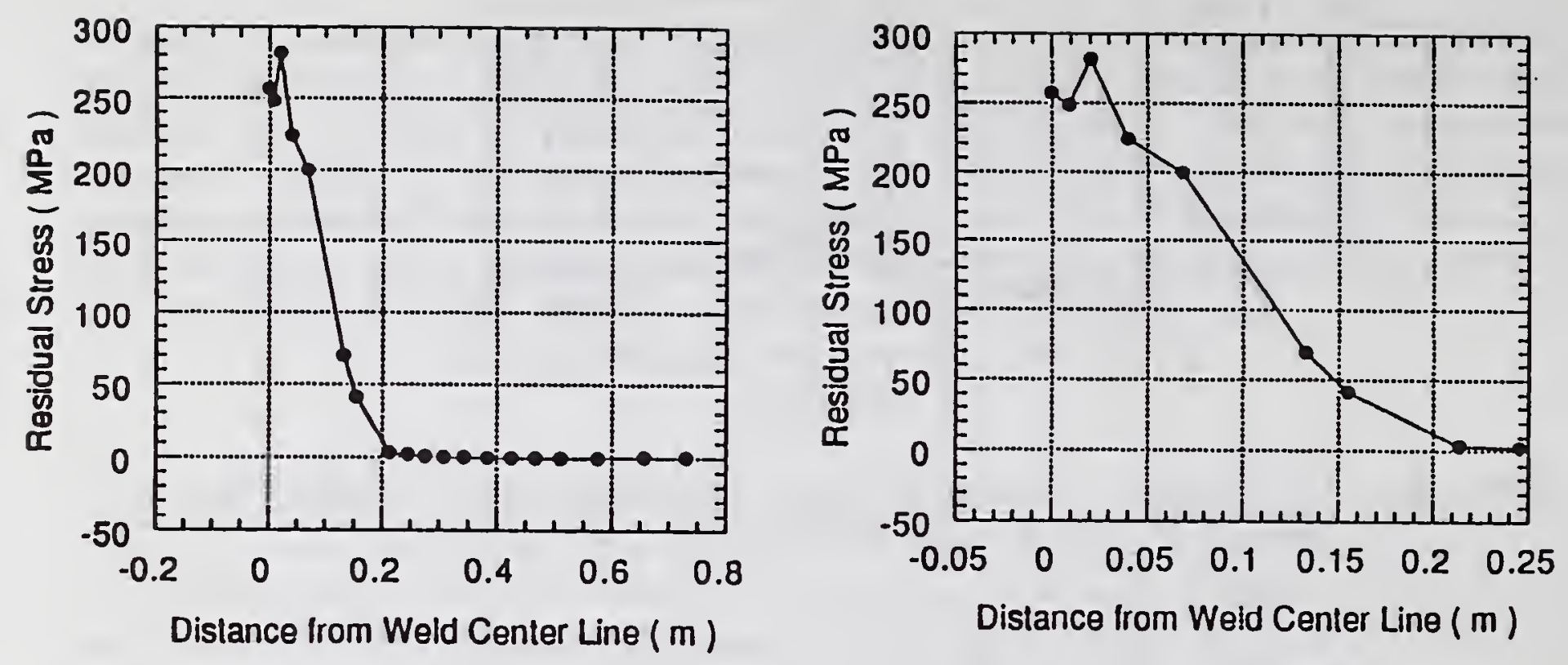

Figure 4 (a ) Residual Stress Perpendicular to Weld Line of ESW Processes as a Function of Distance from Weld Centerline ( Mid - Plane Model, $v=3 \times 10^{-4} \mathrm{~m} / \mathrm{s}, \mathrm{l}=6.12 \mathrm{~m}, \mathrm{t}=0.08 \mathrm{~m}$ )
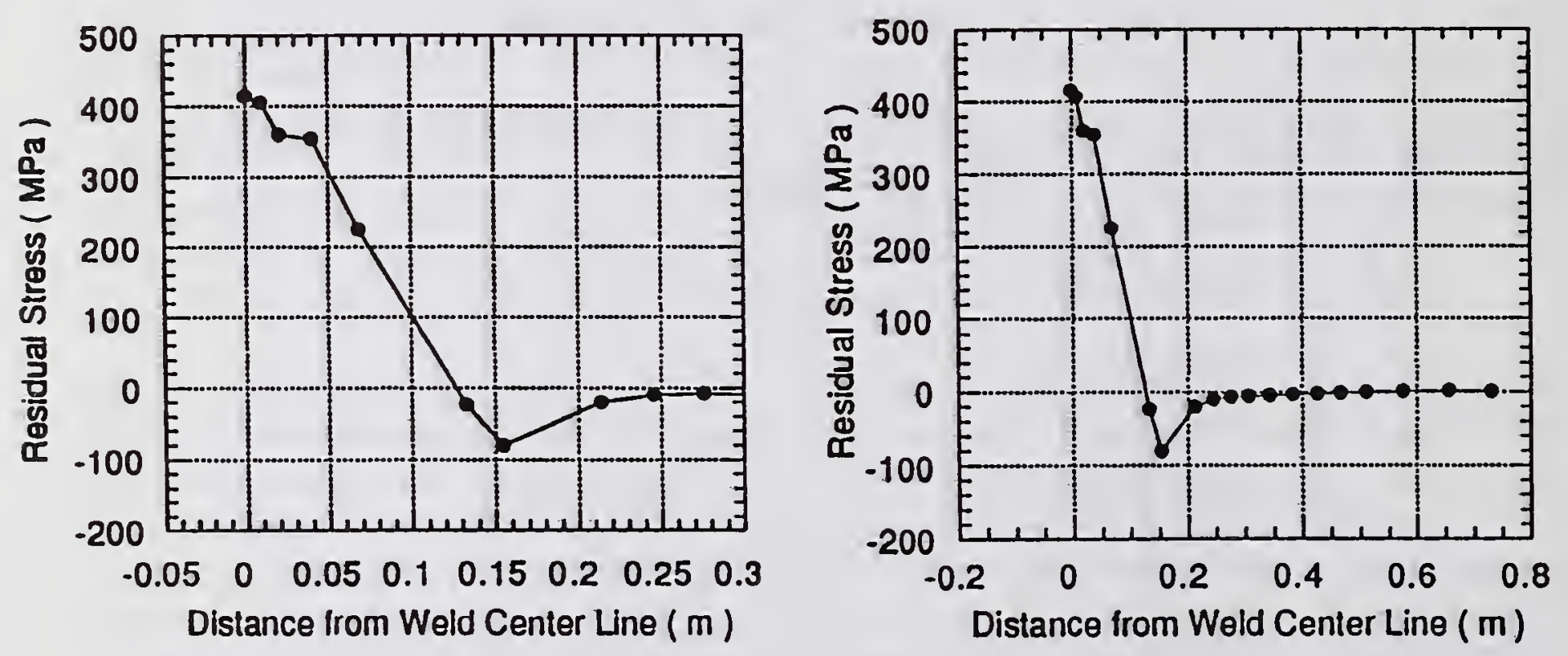

Figure 4 ( b ) Residual Stress Parallel to Weld Line of ESW Process as a Function of Distance from Weld Center Line

( Mid-Plane Model, $v=3 \times 10^{-4} \mathrm{~m} / \mathrm{s}, 1=6.12 \mathrm{~m}, \mathrm{t}=0.08 \mathrm{~m}$ ) 


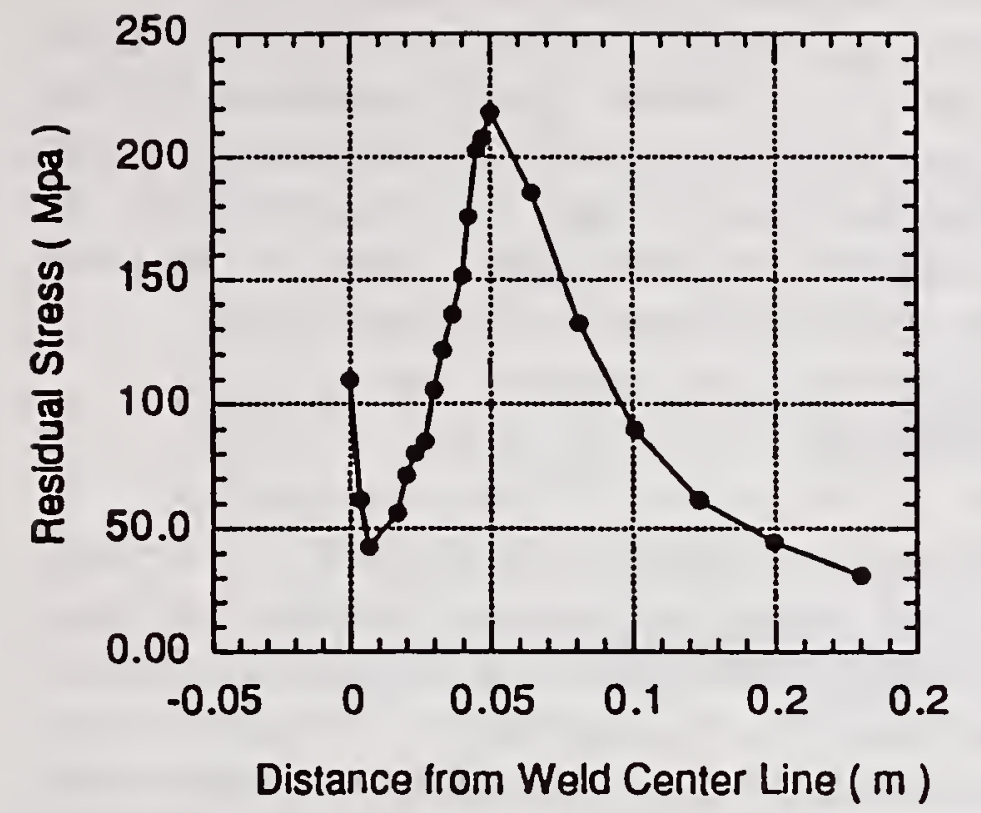

(a)

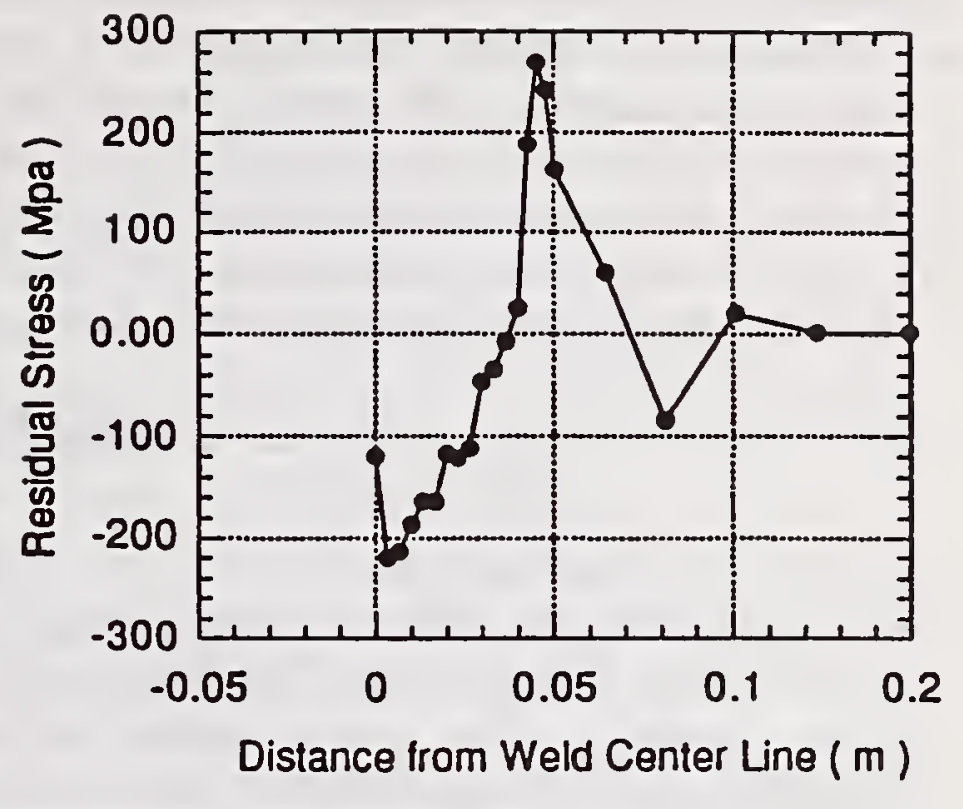

(b)

Figure 5 Residual Stress along Middle Plane of Welded Plate (a ) Perpendicular (b ) Parallel to Weld Direction as a Function of Distance from Weld Center Line ( Cross - Section Model, $v=3 \times 10^{-4} \mathrm{~m} / \mathrm{s}, \mathrm{l}=6.12 \mathrm{~m}, \mathrm{t}=0.08 \mathrm{~m}$ )

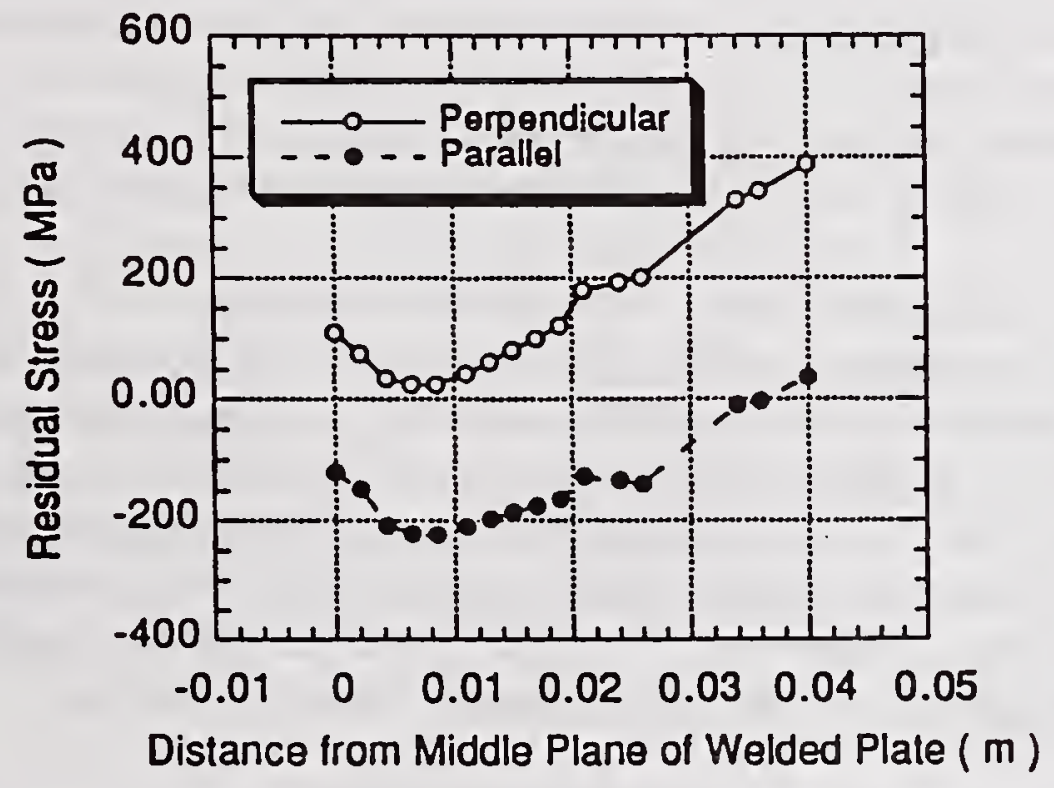

Figure 5 (c) Residual Stress along Weld Center Line Parallel and Perpendicular to Weld Direction as a Function of Distance from Weld Center Line (Cross - Section Model, $v=3 \times 10^{-4} \mathrm{~m} / \mathrm{s}, \mathrm{l}=6.12 \mathrm{~m}, \mathrm{t}=0.08 \mathrm{~m}$ ) 
Cross-sectional model residual stresses along the weld center line as a function of distance from the middle of the welded plate are shown in Figure 5c. Residual stresses perpendicular to the welding direction are tensile at mid-plate ( 40 percent of plate yield). They then decrease to near zero and then increase with increasing distance from the middle plane of welded plate to a tensile value near 160 percent of plate yield at the plate surface. Residual stresses parallel to the welding direction are compressive at mid-plate ( 50 percent of plate yield). They then decrease to 90 percent of plate yield and then increase to slightly over zero at the plate surface.

\section{DISCUSSION}

The above described mid-plane residual stress distribution results from both models are directly comparable. The two different model results exhibit similar general trends while at the same time exhibiting different model-specific results, as should be expected as they model different cooling scenarios. Both models exhibit similar trends for the distribution of residual stress perpendicular to weld centerline (Figure $4 \mathrm{a}$ and $5 \mathrm{a}$ ) with the slower-cooling mid-plane model exhibiting higher tensile stresses and the cross-sectional model exhibiting fusion/HAZ peak tensile stresses farther from the WCL.

All mid-plane residual stress distributions appear to exhibit a "dip" in residual stress near WCL with the parallel residual stress distributions exhibiting two dips as a function of distance from WCL. The cross-sectional model dips near weld centerline are more pronounced, with deeper "troughs" and wider "mouths". This appears to result in lower residual stress at/near WCL. Cross-sectional model perpendicular residual stress at WCL are greatly reduced and parallel residual stresses change from tensile to compression, with respect to the mid-plane model. Thus accelerated plate surface cooling induced by the cooling shoes, which is only modeled in the cross-sectional model, appears to be beneficial to the final residual stress distribution.

Assessment of the over-all results of the mid-plane analysis indicates that the effect of the copper cooling shoes/surface cooling can not be ignored even at mid-thickness for this thickness weld. Thus a significant portion of the heat transfer in ESW for plates (at least) less than or equal to $0.08 \mathrm{~m}(3.15 \mathrm{in}$.) thick is in the welded plate thickness direction due to the cooling effect off the cooper shoes, instead of down the length of the plate, as would be expected in other (multi-pass) welding techniques. A considerably greater thickness plate would need to be welded by ESW to yield true 2D cooling at mid-plane. Thus it is expected that there will be high compressive residual stresses parallel to weld centerline present at weld centerline.

\section{CONCLUSIONS}

1) Modeling of the effect of accelerated surface cooling in ESW is important even at midthickness.

2) Tension residual stresses perpendicular to the welding direction are present at mid- 
thickness. The highest tension stress value is in the yield stress range.

3) A combination of tension and compression residual stresses are present parallel to the welding direction at mid-thickness. The prediction of the presence of tension or compression stresses at WCL depends on the type of model assumptions used.

4) The modeling results indicate two stress reversals take place in the fusion/HAZ region; i.e., stress initially decreases, then increases, and then decreases again, with increasing distance from WCL.

5) Residual stress decreases and then increases from WCL as a function of plate thickness. Residual stress perpendicular to the welding direction starts at WCL and ends at the plate surface at a high tensile value while residual stress parallel to the welding direction starts in compression at WCL and ends near zero stress at the plate surface.

\section{ACKNOWLEDGEMENTS}

The authors gratefully acknowledge research support for this work from the Oregon Department of Transportation.

\section{REFERENCES}

1. B.E. Paton. 1962. Electroslag Welding, American Welding Society : New York.

2. A.W. Pense, J.D. Wood, J.W. Fisher. Recent Experiences with Electroslag Welded Bridges. Welding Journal : 12: 33 to 42.

3. A.H. Dallier, J. Szekely, and T.W. Eager. 1978. Electromagnetically and Thermally Driven Flow Phenomena in Electroslag Welding : Metall. Trans. B : 9 B : 371 to 381.

4. A.H. Dilawari, T.W. Eager and J. Szekely. 1978. An Analysis of Heat and Fluid Flow Phenomena in Electroslag Welding : Welding Journal : 1: 20 s to $30 \mathrm{~s}$.

5. T. Debroy, J. Szekely and T.W. Eagar. 1985. Temperature profiles, the size of the heataffected zone and dilution in electroslag welding: Materials Science and Engineering: 56 : 181 to 193.

6. T. Debroy, J. Szekely and T.W. Eagar. 1980. Heat generation patterns and temperature profiles in electroslag welding : 11B: 593 to 604

7. Y.S. Touloukin et al. 1970. Thermal Conductivity, Metallic Elements and Alloys. Thermophysical Properties of Matter: IFI/Plenum

8. Y.S. Touloukin et al. 1970. Specific Heat, Metallic Elements and Alloys, Thermophysical Properties of Matter: IFI/Plenum 



\section{MODELING - THERMOMECHANICAL EFFECTS}





\title{
FINITE ELEMENT MODELING OF RESISTANCE SPOT WELDING OF GALVANIZED STEEL
}

\author{
M. V. Li, ${ }^{1}$ P. Dong, ${ }^{1}$ and M. Kimchi ${ }^{2}$
}

\begin{abstract}
Resistance spot welding of galvanized steel has been analyzed with advanced finite element analysis procedures. The predictions are verified with experimental measurements. Some intriguing observations can be made. First, the contact resistances at both the electrode to sheet and sheet to sheet interfaces are much smaller comparing to those of bare steel, their effects in the early stage of the welding process can not be ignored. They provide localized heating at the interfaces, which leads to the reduction of contact areas in the first few cycles. They increase the current density during the early stage of the welding process. Second, free-zinc coating is melted after one cycle at the faying surface and three cycles at electrode to sheet interfaces. At certain stage of the welding process, the areas of melted zinc at the interfaces may become larger than the mechanical contact areas. The molten zinc is then pushed to the periphery of mechanical contact areas, which enlarges the effective area for the conduction of electricity and hence reduce the current density. Both experimental studies and numerical analyses revealed that the effect of molten zinc is primarily dependent upon the ratio between the electrode face diameter and sheet thickness. The thickness of free-zinc coating also contributes to the molten zinc effect.
\end{abstract}

\section{INTRODUCTION}

Demands for increased product performance in automotive industry have motivated the steel making industry to develop a wide range of new steel products. One major innovation is to apply various coatings designed for improved corrosion resistance. Coated steels have introduced considerable impacts on the weldability of the steels and have led to reduced electrode lives. Many experimental studies have been conducted to investigate the effects of different coatings on the weldability and on electrode lives (Refs. 1-10). These studies have established the current knowledge base for the resistance spot welding of coated steels.

Hot-dip galvanized steels are commonly used in the current automotive industry. The key issues regarding the resistance spot welding of galvanized steels include weld quality and electrode wear. Manufacturing issues include electrode sticking and weld surface appearance.

Resistance spot welding of galvanized steels has never been analyzed with previous modeling prior to this study. This might have been hindered by three reasons. Firstly, decoupled

\footnotetext{
${ }^{1}$ Center for Welded Structures Research, Battelle Memorial Institute, Columbus, Ohio

${ }^{2}$ Edison Welding Institute, Columbus, Ohio
} 
thermoelectric analyses have been used for a long time without incorporating the interaction of the thermomechanical effects (Refs. 11-13). Only recently, there are a few coupled numerical analysis procedures based on finite element method which effectively take the thermal, mechanical, and electrical interactions in to consideration (Refs. 14,15). Secondly, there was no realistic model to characterize the contact resistance for the welding of galvanized steels (Ref. 16). Thirdly, because of the lack of a realistic contact resistance model, most of the modeling work to date has used contact resistance values on the trial-and-error basis to get optimal fit between the predicted nugget sizes with the experimentally measured ones. The effect of molten zinc on the effective contact area for the conduction of electricity has not been recognized and simulated.

Finite element analysis was conducted in this study to simulate the resistance spot welding of galvanized steel. The three major difficult problems associated in the finite element analysis of resistance spot welding of galvanized steels are tackled in this study. The results presented in this paper revealed some intriguing interactions between thermal, mechanical, and electrical aspects of resistance spot welding of galvanized steels, and particularly the importance of incorporating molten zinc effect into the finite element model.

\section{FINITE ELEMENT ANALYSIS}

In this study, the incrementally coupled finite element analysis procedure previously developed for the analysis of resistance spot welding of bare steel (Ref. 15), was adopted for the analysis of resistance spot welding of galvanized steel. The finite element model used in this study is shown in Fig. 1.

In this paper, three cases were simulated using the welding parameters employed in the experimental validation test. In the first case, the contact resistance was ignored. It was widely perceived that contact resistance played a negligible role in the resistance spot welding of galvanized steels. This was because the measured static contact resistance values are much lower than those encountered in the welding of bare steels. A case study was then performed to evaluate the legitimacy of this perception. In the numerical implementation, the contact resistance was ignored by assigning the electric resistivity values of steel substrate to the contact elements.

In the second case, the contact resistance was taken into consideration and the electric contact areas were assumed to be the same as the mechanical contact areas. This case study was to further understand how contact resistance affects the heating process during spot welding. The contact resistance during the welding of galvanized steel was simulated using the contact resistance model presented in Refs. 16 and 17. In this contact resistance model, the contact resistance during the spot welding process is estimated from the characteristic relationship between the voltage drop across the interface and the maximum interface temperature.

$$
V^{2}=4 L\left(T_{S}{ }^{2}-T_{0}^{2}\right)
$$


In the numerical implementation of this contact resistance model, the maximum interface temperature, $T_{s}$, for both the electrode/sheet and sheet/sheet interfaces to maintain solid contacts were assumed to be the melting temperature of pure zinc, $420^{\circ} \mathrm{C}$.

In both case I and II, the effective contact areas in the thermoelectric analysis were assumed equal to the mechanical contact areas. As indicated earlier by Howe (Ref. 8), the molten zinc at the periphery of mechanical contact areas may enlarge the effective electric contact area. Therefore, in case three, the molten zinc effect was incorporated into the numerical analysis procedure. In this analysis, the temperature at the periphery of the mechanical contact area was checked after each time increment during the thermomechanical analysis. If this temperature fell within the temperature range for liquid zinc to exist, $420{ }^{\circ} \mathrm{C}$ (melting point) to $911{ }^{\circ} \mathrm{C}$ (evaporation point), the effective contact area for the thermoelectric analysis was thus determined by the radius where solid to solid contact was established.

\section{EXPERIMENTAL VALIDATION}

The material used for this study was $0.8 \mathrm{~mm}$ thick hot-dip galvanized steel, sheared into $44 \times 106$ $\mathrm{mm}(1.75 \times 4 \mathrm{in}$.) coupons. The substrate is AISI-SAE 1008 type steel and is coated on both sides with coating weight of $130 \mathrm{~g} / \mathrm{m}^{2}$.

Experimental studies were conducted to study the nugget growth and to verify the finite element model. Welds were made with a $100 \mathrm{KVA}$ Taylor-Winfield pedestal-type resistance spot welder. The welding setup and conditions were taken from Ford Motor Specifications BA 13-1 and 13-4. The electrode caps used in the experiment were made of a RWMA Class II copper alloy. The electrode caps have the A-nose configuration specified by the American Welding Society and the face diameter of the electrodes is $6.37 \mathrm{~mm}(0.25 \mathrm{in}$.). The applied electrode force was $2.4 \mathrm{kN}(550 \mathrm{lb}$.) during the squeeze, weld, and hold cycles. The welding current was $13.1 \mathrm{kA}$ (RMS). The weld time was 12 cycles. The hold time was 12 cycles.

To study nugget development, welding was interrupted after each cycle. Two weld specimens were made at each set of welding conditions. One specimen was used for metallographic examinations, and a second for peel test to measure the button sizes. The experimental measurements were then compared with those predicted by the model.

\section{RESULTS}

\section{Case I}

When the contact resistance was ignored, the simulation ended up with no melting by the end of 12 weld cycles. By the end of 12 cycles, the maximum temperatures reached at the faying surface and the electrode tip surface were $1102{ }^{\circ} \mathrm{C}$ and $443{ }^{\circ} \mathrm{C}$ respectively. The predicted radii 
of contact areas are plotted in Fig. 2. Note that contact radii have been normalized to the radius of the electrode tip surface, $R_{E}$.

Apparently this simulation has demonstrated that the effects of contact resistance in spot welding of galvanized steels are not negligible despite its small magnitude comparing to those encountered in the welding of bare steels. The results of this simulation confirmed that contact resistances play an important role in the resistance spot welding of galvanized steel. Accurate analysis of the heat generation in the welding process has to incorporate the contact resistance into the model.

\section{Case II}

The predicted radii of contact areas during the process are presented in Fig. 3. Fig. 3 demonstrates that the contact resistances at both the electrode/sheet and sheet/sheet interfaces have significant impact on the changes of contact areas and the current density in the early stage of welding process.

The predicted nugget sizes are presented in Fig. 4 in comparison with the experimentally measured nugget sizes from metallographic examination and the button sizes from peel tests. It is clearly shown that failing to incorporate the molten zinc effect would lead to overprediction of heat generation in the welding process. In this case simulation, initial melting was predicted to take place by the end of 5th cycle at the faying surface, whereas the actual nugget was not formed until the end of 7th cycle. In fact, expulsion was predicted to occur by the end of 10 cycles.

\section{Case III}

When molten zinc effect was properly accounted for in the numerical procedure, the predicted contact areas are shown in Fig. 5 and 6 for the electrode/sheet and sheet/sheet interfaces respectively. The predicted nugget sizes are presented in Fig. 7 in comparison with the experimentally measured ones. Excellent agreement is found between the predictions and the measurements.

\section{DISCUSSION}

The effects of contact resistance on the resistive heating during spot welding should be understood in two perspectives. Firstly, it directly generates localized heating in the vicinity of the contact interfaces. Secondly, the localized heating leads to thermal expansion and reduces the contact area. Consequently, it indirectly increases the current density. Increased current density would further intensify localized heating. The actual contact area during the welding process would be determined by the competing effects of thermal expansion due to localized heating and softening of materials. 
When molten zinc is in effect, it enlarges the area for the conduction of electricity across interfaces. As a result, it decreases the current density and slows down the heating process. Fig. 4 and 6 clearly demonstrated that the molten zinc effect delayed the nugget formation by two cycles. Molten zinc at the periphery of electrode tip surface also slows down the nugget growth. Molten zinc effect has only one advantage, that is, welds can be made with wider current range. The disadvantage is that, as shown by Howe (Ref. 8), it shortens the electrode life. It also requires higher current to make welds.

\section{SUMMARY}

Although the contact resistance values at both the electrode/sheet and sheet/sheet resistance are low, much lower in comparison with those encountered in the welding of bare steel, their effects on the resistive heating during the welding process can not be ignored. They provided the initial heating at the contact interfaces, which reduced the contact areas and increased the current density.

At certainly stage of the welding process, the zinc coating at the periphery of mechanic contact areas was melted. The molten zinc was pushed outward and formed annulus at the periphery of mechanical contact areas. The molten zinc annulus thus enlarged the effective contact area for the conduction of electricity and reduced the current density and heating rate at the interfaces. Numerical simulation demonstrated that the nugget would have been formed by the end of 5 th cycle if the molten zinc were not present. In actual welding experiment, the nugget was formed by the end of 7 th cycle. Apparently the molten zinc effect had delayed the nugget formation by 2 cycles.

Numerical procedures have developed to incorporate the effect of molten zinc on the effective contact area for the conduction of electricity. Excellent agreement has been obtained between the predicted nugget sizes with the experimentally measured ones after incorporating the molten zinc effect into the finite element analysis.

\section{ACKNOWLEDGEMENTS}

The authors are grateful to the financial support provided by the Auto-Steel Partnership, Battelle, and EWI for this work.

\section{REFERENCES}

1. Freytag, N. A. 1965, A Comprehensive Study of Spot Welding Galvanized Steel. Welding Journal 34(4): 145-s to 156-s.

2. Friedman, L. M. and McCauley, R. B. 1969, Influence of Metallurgical Characteristics on Resistance Welding of Galvanized Steel. Welding Journal 38(10): 454-s to 462-s. 
3. Lavery, R. C. and Williams, N. T. 1969, Resistance spot welding spot and seam welding of galvanized steel. Metal Construction and British Welding Journal (2): 79-87.

4. Williams, N. T. 1972, Problems in the resistance welding of coated steels. Sheet Metal Industries (10): 625-630.

5. Natale, T. V. 1986, A comparison of the resistance spot weldability of hot-dip and electrogalvanized sheet steels. $\underline{\text { SAE Technical Paper } 860435 .}$

6. Howe, P. and Kelley, S. C. 1988, A comparison of resistance spot welding of bare, hotdipped, galvannealed, and electrogalvanized DQSK sheet steel. SAE Technical Paper 880280.

7. Kimchi, M. and Gould, J. E. 1986, Effects of coating weight on the resistance spot weldability of galvanized steel, SAE Technical Paper 860436

8. Howe, P. and Kelley, S. C. 1988, Coating weight effect on resistance spot weldability of electrogalvanized sheet steels, Welding Journal 67(12): 271-s to280-s.

9. Holiday, R.; Parker, J. D.; and Williams, N. T. 1995, Electrode deformation when spot welding coated steels. Welding in the World 35(3): 160-164.

10. Holiday, R.; Parker, J. D.; and Williams, N. T. 1996, Relative contribution of electrode tip growth mechanisms in spot welding zinc coated steels. Welding in the World 37(4): 186-193.

11. Nied, H. A. 1984, The finite element modeling of the resistance spot welding process. Welding Journal 63(4): 123-s to 132-s.

12. Cho, H. S. and Cho, Y. J. 1989, A study of the thermal behavior in resistance spot welds. Welding Journal 68(6): 236-s to 244-s.

13. Tsai, C. L.; Dai, W. L.; Dickinson, D. W.; and Papritan, J. C. 1991, Analysis and development of a real-time control methodology in resistance spot welding. Welding Journal 70(12): 339 s to $351 \mathrm{~s}$.

14. Browne, D. J.; Chandler, H. W; Evans, J. T.; and Wen, J. 1995. Computer simulation of resistance spot welding in aluminum: Part I. Welding Journal 74(10): 339s to 344s.

15. Dong, P.; Li, M. V.; and Kimchi, M. 1997, Analysis of electrode wear mechanisms: face extrusion and pitting effects. to appear in Science and Technology of Welding and Joining.

16. Dong, P., Kimchi, M., and Holmes, S., 1996, Thermomechanical analysis of electrode wear mechanisms: a finite element study. Proc. sheet metal welding conference VII. Paper E5, AWS Detroit Section.

17. Li, M. V.; Dong, P.; and Kimchi, M. 1997, Modeling of contact resistance during resistance spot welding. This conference proceedings. 


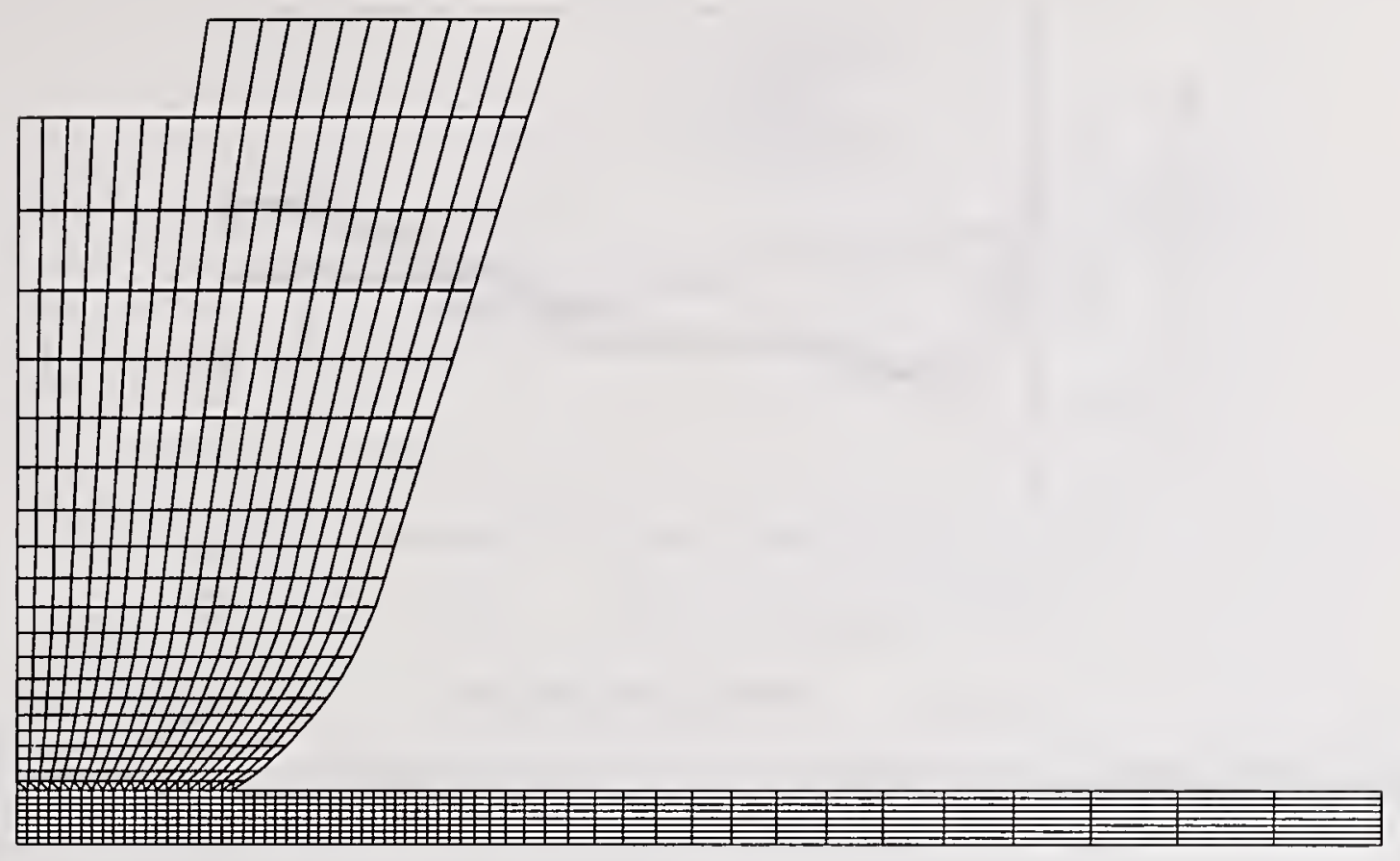

Figure 1 Finite element mesh for the incrementally coupled analysis.

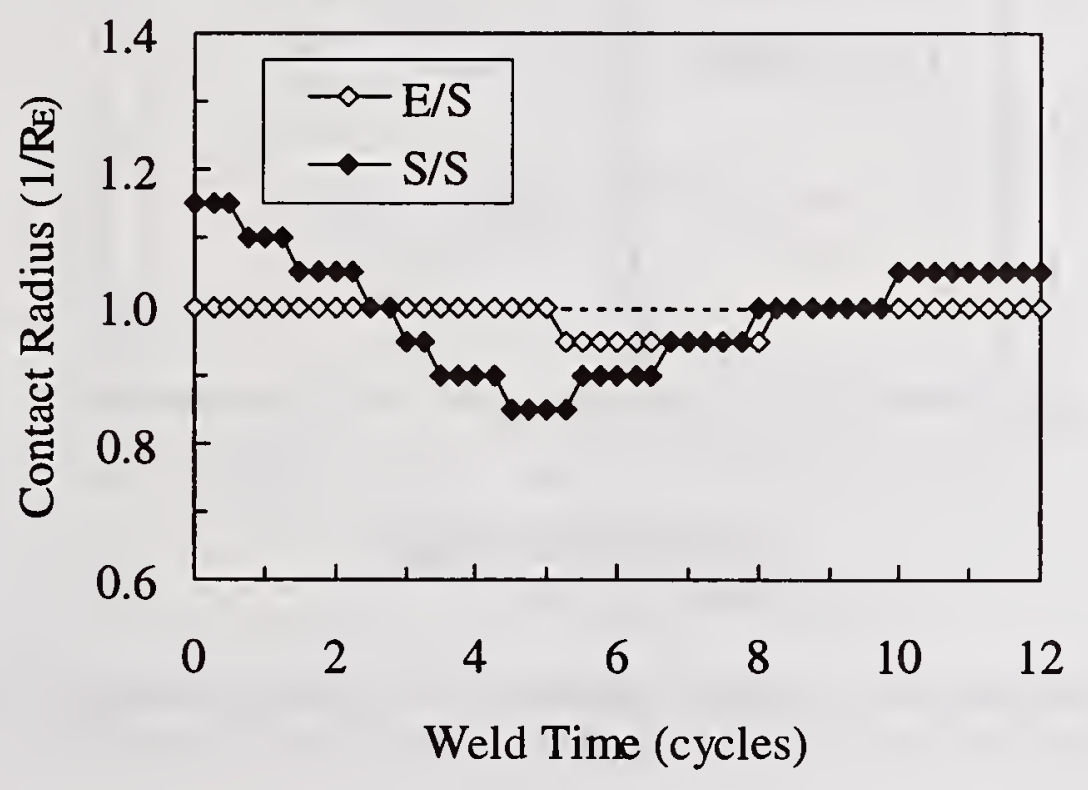

Figure 2 Predicted contact radius in the Case I (contact resistance ignored) 


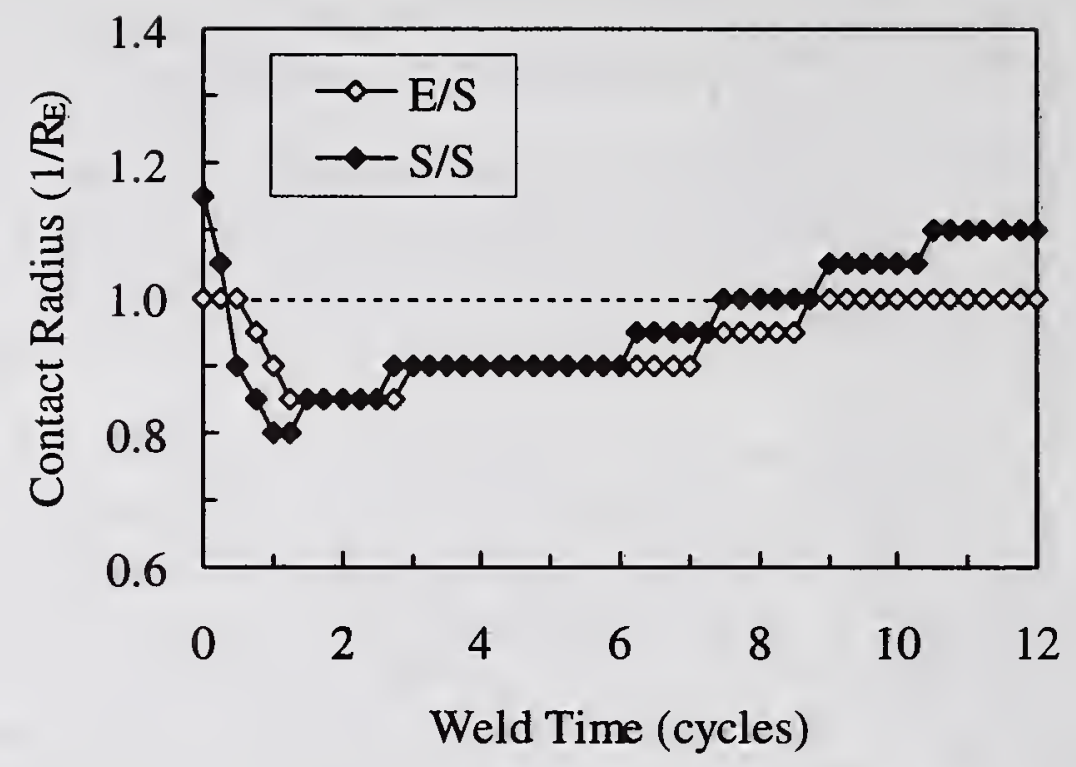

Figure 3 Predicted contact radius in Case II (contact resistance incorporated)

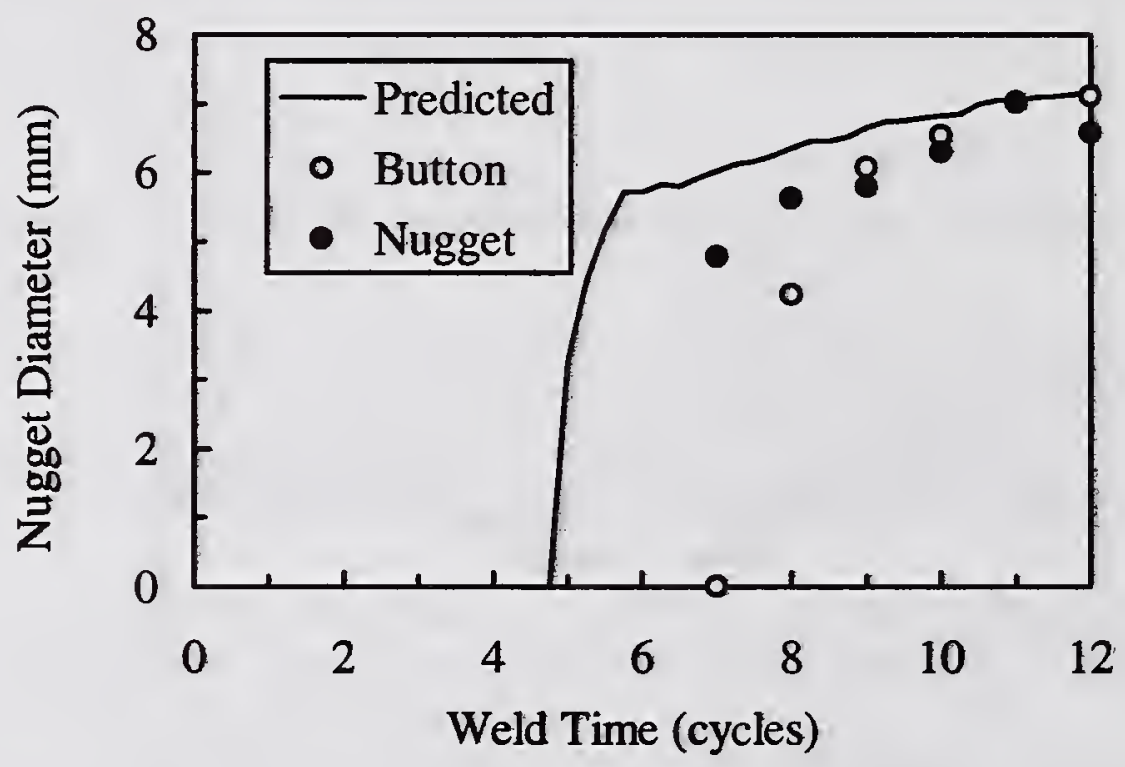

Figure 4 Predicted and measured nugget sizes in Case II (contact resistance incorporated) 


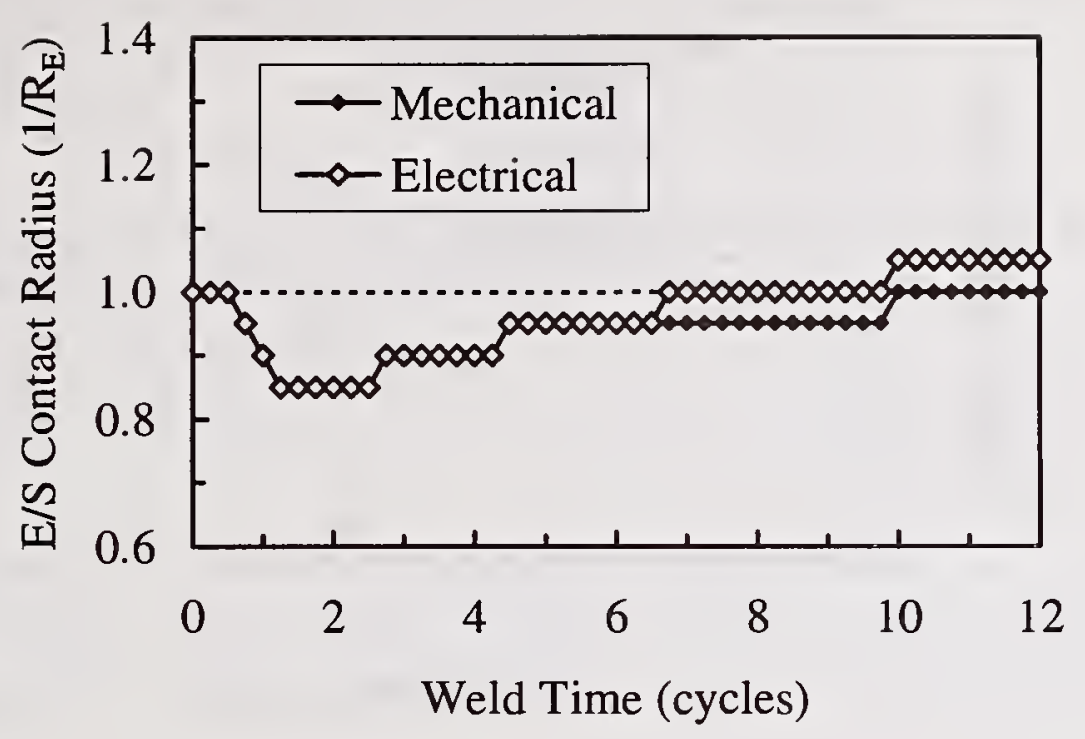

Figure 5 Predicted electrode/sheet contact radius in Case III

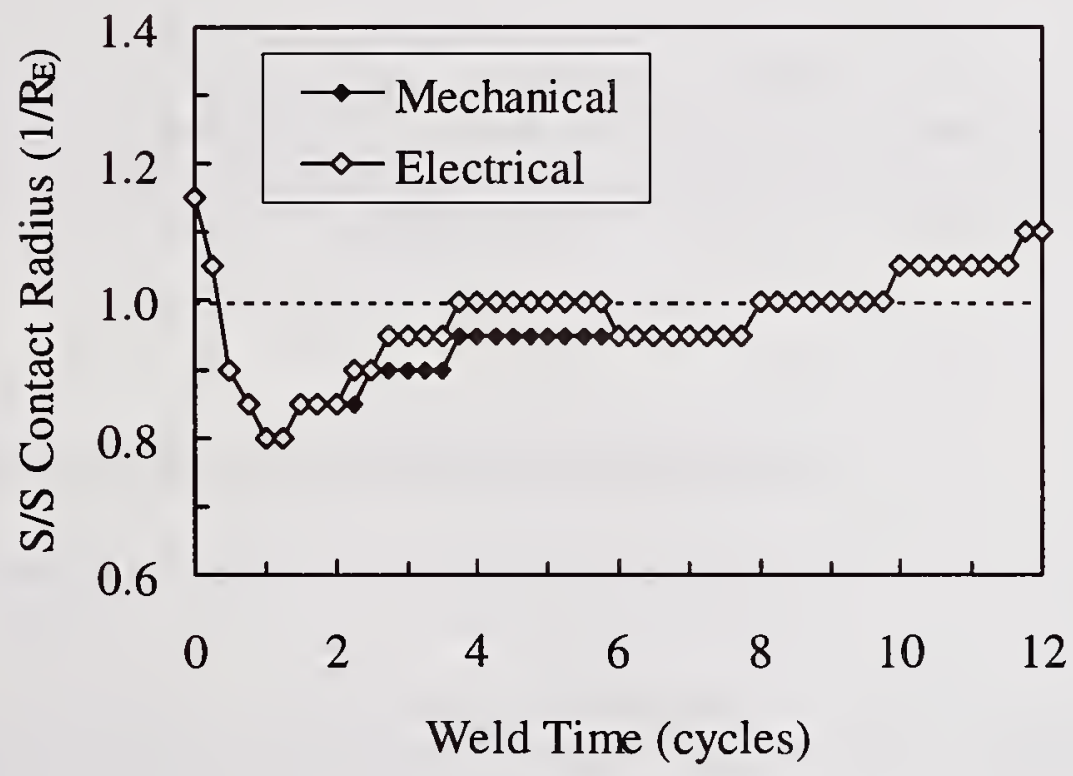

Figure 6 Predicted sheet/sheet contact radius in Case III 


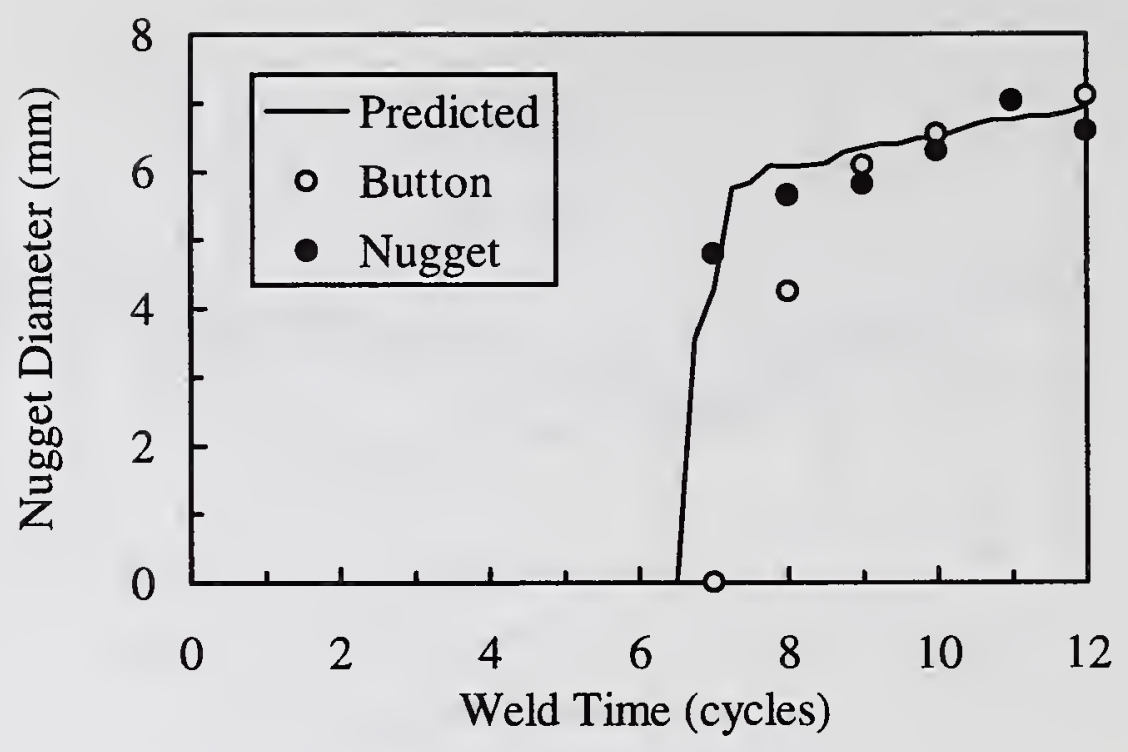

Figure 7 Predicted and measured nugget sizes in Case III. 


\title{
THE NUMERICAL SIMULATION OF WELDING. DESCRIPTION OF REQUIRED FUNCTIONS. INDUSTRIAL APPLICATIONS WITH THE SOFTWARE SYSWELD+
}

\author{
F. Boitout, G. Mangialenti, J.M. Bergheau \\ FRAMASOFT (ESI Group) \\ 84, Bd Vivier Merle \\ 69485 LYON Cedex 03, \\ FRANCE
}

\section{INTRODUCTION}

The increased power of computer resources, combined with the development of scientific software products, has opened the way for numerical simulation of manufacturing processes such as welding. These processes induce metallurgical transformations and mechanical state modifications which have a major impact on the behaviour of the structure. That means that the modelling of these processes requires to take into account several physical phenomena (thermics, metallurgy and mechanics) and their interactions.

Numerical simulations provide a complement to experimental techniques, which can be cumbersome and costly in their execution. Moreover, numerical simulation provides rich and comprehensive information concerning all the quantities involved (temperatures, metallurgical phase proportions, strains, stresses, etc.), and consequently makes it possible to achieve clear comprehension of the processes concerned.

\section{DESCRIPTION OF THE REQUIRED FUNCTIONS}

Three physical phenomena (thermics, metallurgy and mechanics) and their interactions are involved.

The influence of mechanics on thermics and metallurgy can be neglected. Major interactions resulting in residual stresses and strains concern the link between thermics and metallurgy, and their influence in mechanics.

\subsection{Thermo-metallurgical link}

There are three types of interaction between the thermal and metallurgical analyses :

1. Metallurgical transformations depend directly on the evolution of temperatures in time.

2. Metallurgical transformations modify temperature distribution by latent heat effect.

3. The thermo-physical properties of materials depend on the various phases of their constitution.

The solution of the heat equation based on an enthalpic approach guarantee a rigorous consideration of latent heat effects. 
A purely phenomenon related approach of metallurgical transformation modelling has been proposed by J.B. Leblond. It can be extended to Johnson-Mehl-Avrami law. These models can be used for transformation occuring during heating and cooling. They can be applied to steels and alloys. They are particularly suitable for diffusion-controlled transformations.

Other laws are dedicated to transformations involving other mechanisms.

The martensitic transformation in steels generally follows the law of KoïstinenMarburger.

Although tempering is not a genuine metallurgical transformation, the Leblond approach can be used. A transformation corresponding to (as-quenched structure -- $>$ tempered structure) is introduced.

Two kinds of metallurgical transformations can occur in Aluminum alloys : recrystallization or dissolution/coarsening of precipitates. The corresponding kinetics can be taken as the form : $\mathrm{dP} / \mathrm{dt}=\mathrm{f}(\mathrm{P}, \mathrm{T})$ where $\mathrm{T}$ is the temperature, $\mathrm{t}$ the time and $\mathrm{P}$ a metallurgical parameter.

\subsection{Mechanical analysis}

The following capabilities are required :

- usual thermoplastic effects,

- dependence of material properties (yield stress, strain hardening) upon temperature and metallurgical phases or parameter,

- volume changes arising from transformations, which always play a role in the generation of residual stresses -for steels only-,

- the transformation plasticity phenomenon (plastic flow resulting from a variation of the proportions of the phases, which occurs even for very small stresses applied)-for steels only-:

\section{INDUSTRIAL APPLICATIONS WITH THE SOFTWARE SYSWELD+}

The SYSWELD+ finite elements software developed by Framatome then Framasoft+CSI over the last fifteen years for the simulation of welding fully integrates the functions described above.

Several industrial applications will be presented :

- the welding modelling of aluminum alloys at AEROSPATIALE : welding simulation of ARIANE 5 main cryogenic tank

- FRAMATOME : welding of ferritic and stainless steel pipes 


\section{PROCESS AUTOMATION}




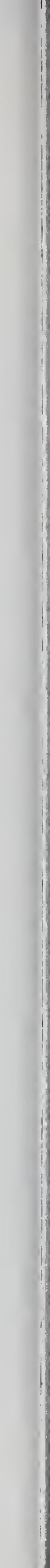




\title{
FULLY AUTOMATIC GMAW SYSTEM
}

\author{
A. Kolasa*, P. Cegielski*
}

\begin{abstract}
Equipment used for fully automatic gas metal arc welding (GMAW) systems has to meet high technical requirements. It becomes more and more complex, microprocessor controlled apparatus with its own programming units, data memories, etc. Modern fully automatic welding installations consisting of a welding power supply, an industrial robot, a positioning table, a wire feeder and a number of auxiliary devices become a multicomputer systems. In order to assure proper functioning of all such system components, they have to be connected using an interface with relevant input and output sets. Since in some cases the installation has to be completed of apparatus of various make, the problem of their proper connection is of a great importance. In this paper the concept of fully automatic GMAW system assembled of industrial robot and synergic GMA welding equipment connected by an especially designed interface is described.
\end{abstract}

\section{INTRODUCTION}

Automation was once thought to be most applicable to the automotive industry and other highly productive industries, but it is now realized that it can be a feasible means of increasing productivity and quality in many types of manufacturing operations. In recent years, it has become particularly attractive to the welding operations. Beside a number of advantages resulted from an automated welding system application, such as increased productivity and high repeatability, a significant improvement of an operator natural environment is a vital factor. A human labor from the role of a direct manufacturer is replaced by the role of operator and/or supervisor. In the case of welding processes the removal of man from dangerous and hazardous environment is of great importance.

An automation of welding processes creates high requirements to be met by both personnel and the equipment used. In case of personnel the most important could be change of mentality. The high accuracy of an automated welding setup operation requires better preparation of parts to be joined. The welding equipment becomes more and more complex, computer controlled apparatus. Under such conditions the optimal solution of automatic welding system should fulfill the following requirements: (i) to be simple in operation, (ii) to be reliable, (iii) to be safe for personnel, equipment installed, and manufacturing process being applied, (iv) to be low cost investment. The concept of a welding robotic installation being presented in this paper meets all above requirements. The installation consists of commercially available two major component parts: industrial robot Irp-6 and microprocessor controlled GMAW equipment Optymag 500. In order to connect both parts and assure proper communication between them, an especially designed interface has been employed. All component parts are made in Poland. Additionally the istallation has been equipped with a number of auxiliary devices to make it fully productive.

\footnotetext{
* Department of Welding Engineering, Warsaw University of Technology, Narbutta St. 85, 02-524 Warsaw, Poland
} 


\section{MULTICOMPUTER SYSTEMS}

All industrial robots are equipped with a microprocessor control system owing both programming and data memory units. Similarly, recent welding power supplies are also controlled by microcomputer systems with its own programming and memory units. Therefore modern robotic welding installations become integrated multicomputer systems (Ref. 1). In order to assure proper communication between these microcomputer controllers, they must be connected with an interface using their own input and output systems, shown in fig. 1. In this solution both microcomputers are of an homogenous, equivalent configuration and they can communicate with each other.

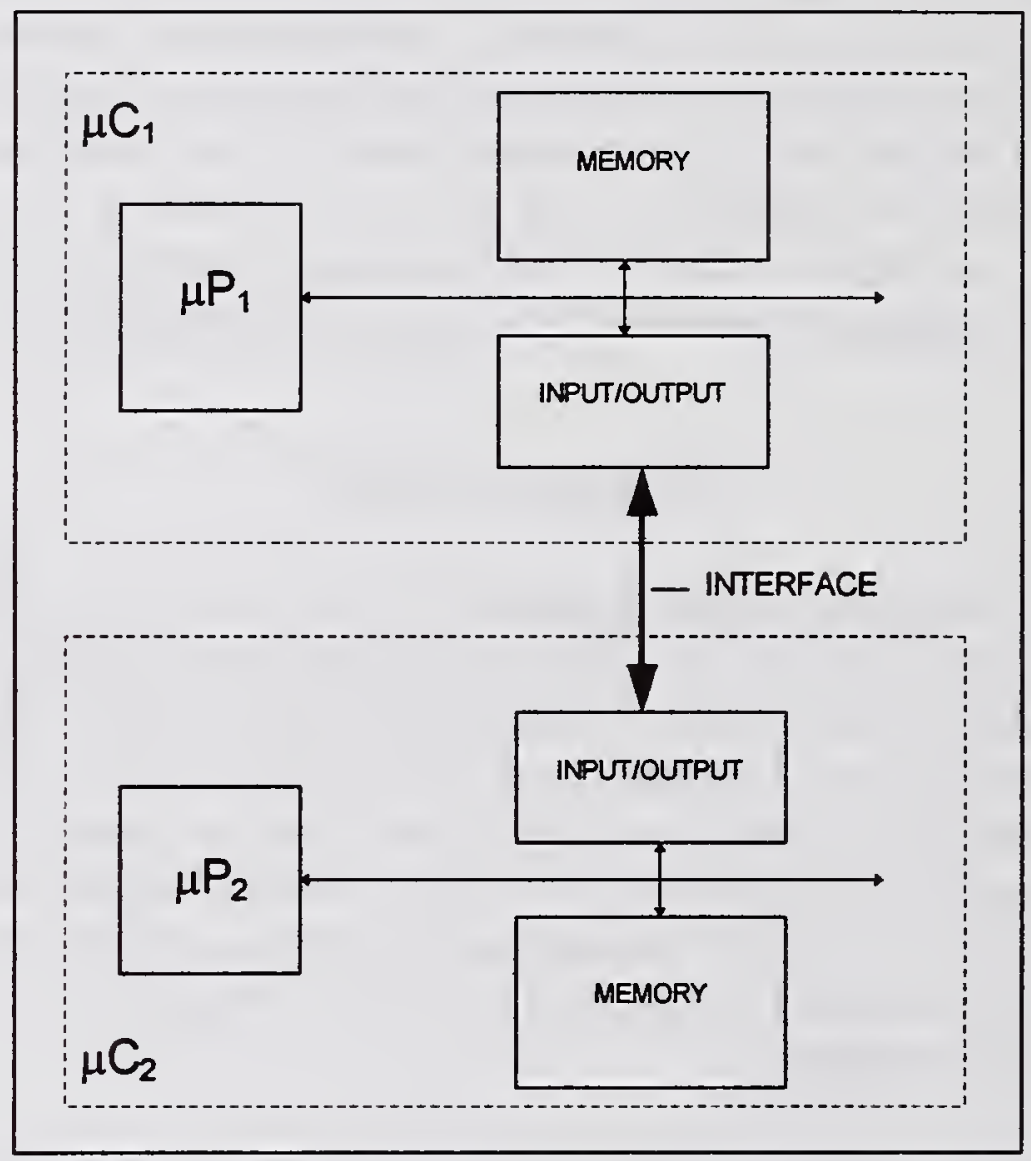

Figure 1. Multicomputer system: $\mu \mathrm{P}_{1}, \mu \mathrm{P}_{2}$ - microprocessors, $\mu \mathrm{C}_{1}, \mu \mathrm{C}_{2}$ - microcomputers.

The interface is used to transmit signals between them and, if necessary, to convert signals from the source microcomputer to a form usable by the microcomputer receiver. Those signals could be of either control and alarm mode.

\section{CONTROL SYSTEMS}

The control of any apparatus is an action the aim of which is to assure its functioning in a required manner. There are two kinds of controllers: open-loop unable to make needed compensating changes that influence the course and quality of a given process and closed-loop to produce high quality products in spite of variations. 
The major components of all arc welding robotic systems are industrial robot and welding equipment. There are a wide variety of both robots and welding machines offered on the market. Taking into account demands of welding process, very often there is a need to combine in one welding installation these two main components made by two different manufacturers. Therefore the main problem to be solved is a proper connection between both controllers and programming units ,i.e. a connection that assures proper functioning of the entire installation. This is usually done by an interface which transmits all communication signals necessary to produce high quality welds. There are control and alarm signals. Signals of the first kind are used to select proper welding parameters and directly control tool and work motion devices, while signals of the second kind have to warn each of the component against any malfunctions that might occur and cause a defense reaction.

In practical applications there are three types of signals used for welding power supplies control. They could be analog, digital (on-off), and both analog and digital used together. The use of analog output/inputs may control such process parameters as welding voltage and wire feed speed during GMAW or welding current during GTAW process. The use of digital output/inputs is possible with welding power supplies equipped with programmable control units and memories. In early construction of semiautomatic welding power supplies, the control of welding parameters was done by switching sets of potentiometers (each used for adjusting one particular parameter) with use the of electromagnetic relays. This system was adopted in $\mathrm{A} 30 \mathrm{~A}$ robotic system of ESAB, shown in fig. 2. In the case of modern welding machines with synergic control having the possibility to program and store welding process parameters, the robot controller, being a source digital output signals can directly activate previously stored sets of parameters including pulsed current welding.

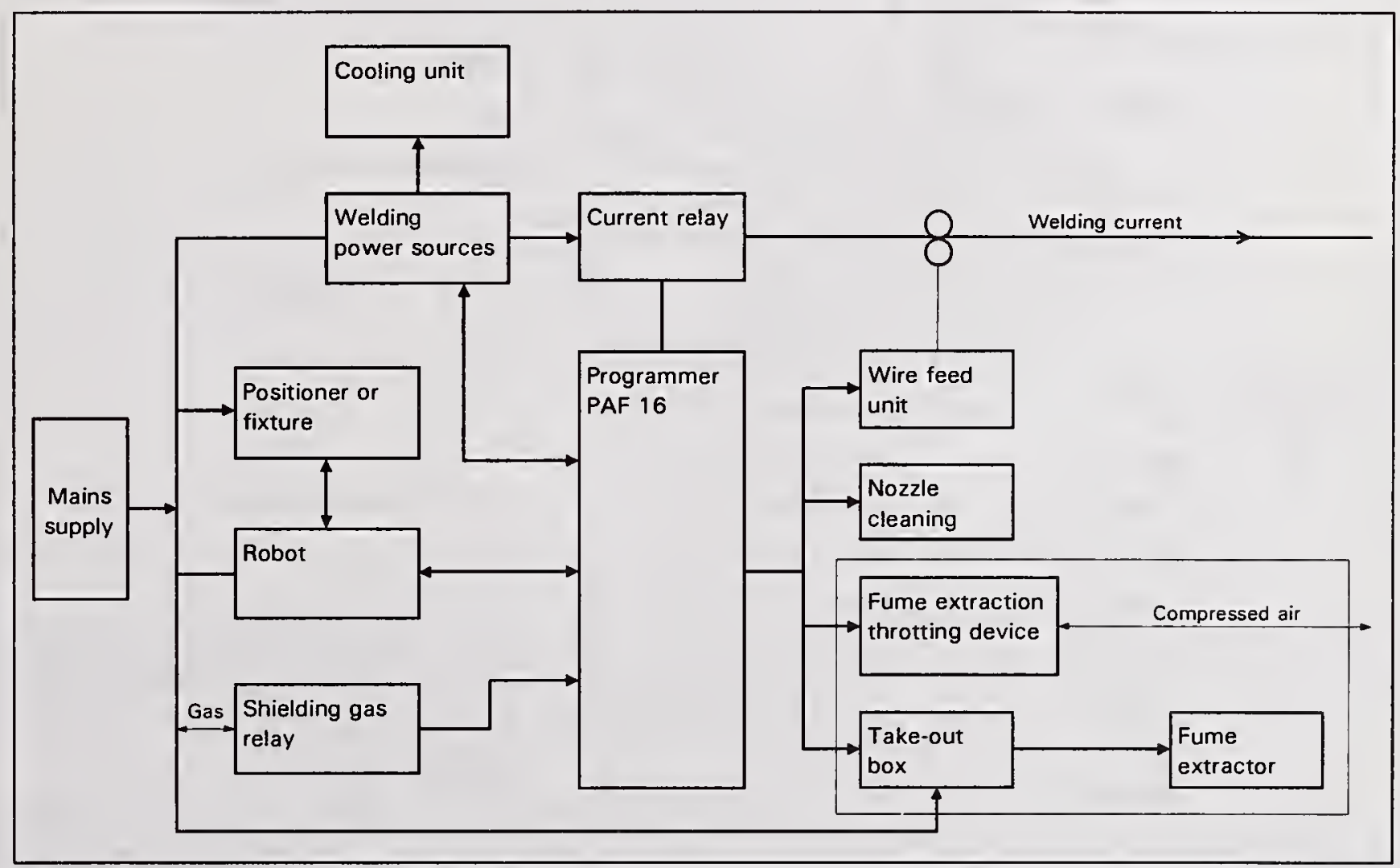

Figure 2. Block diagram of ESAB A30A welding equipment for robot operation (Ref. 2). 
The most advanced controllers use both techniques. The analog output/input signals are used for continuos welding parameters control, while digital on - off signals are reserved for auxiliary functions such as emergency status. This solution has been employed by Fronius Co. in TSST 153 robotic system, shown in fig. 3 .

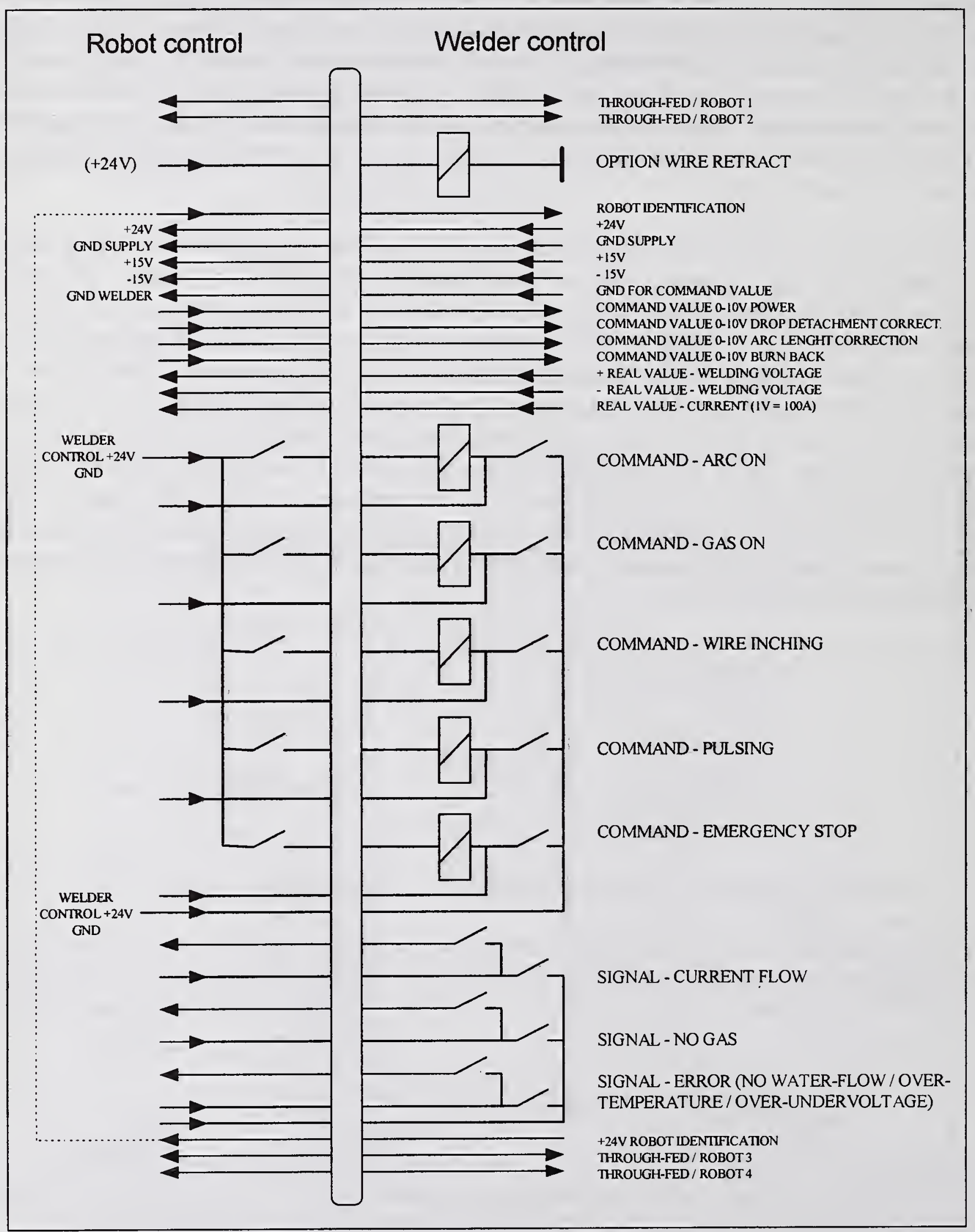

Figure 3. Fronius TSST 153 interface (Ref. 3). 


\section{ROBOTIC WELDING INSTALLATION}

The proposed, typical robotic GTAW arrangement is shown in fig. 4. All components are controlled by the Irp- 6 robot controller with digital output/inputs. For more complex applications or including the whole setup in an automatic line or flexible manufacturing system, the application of a master controller is also possible.

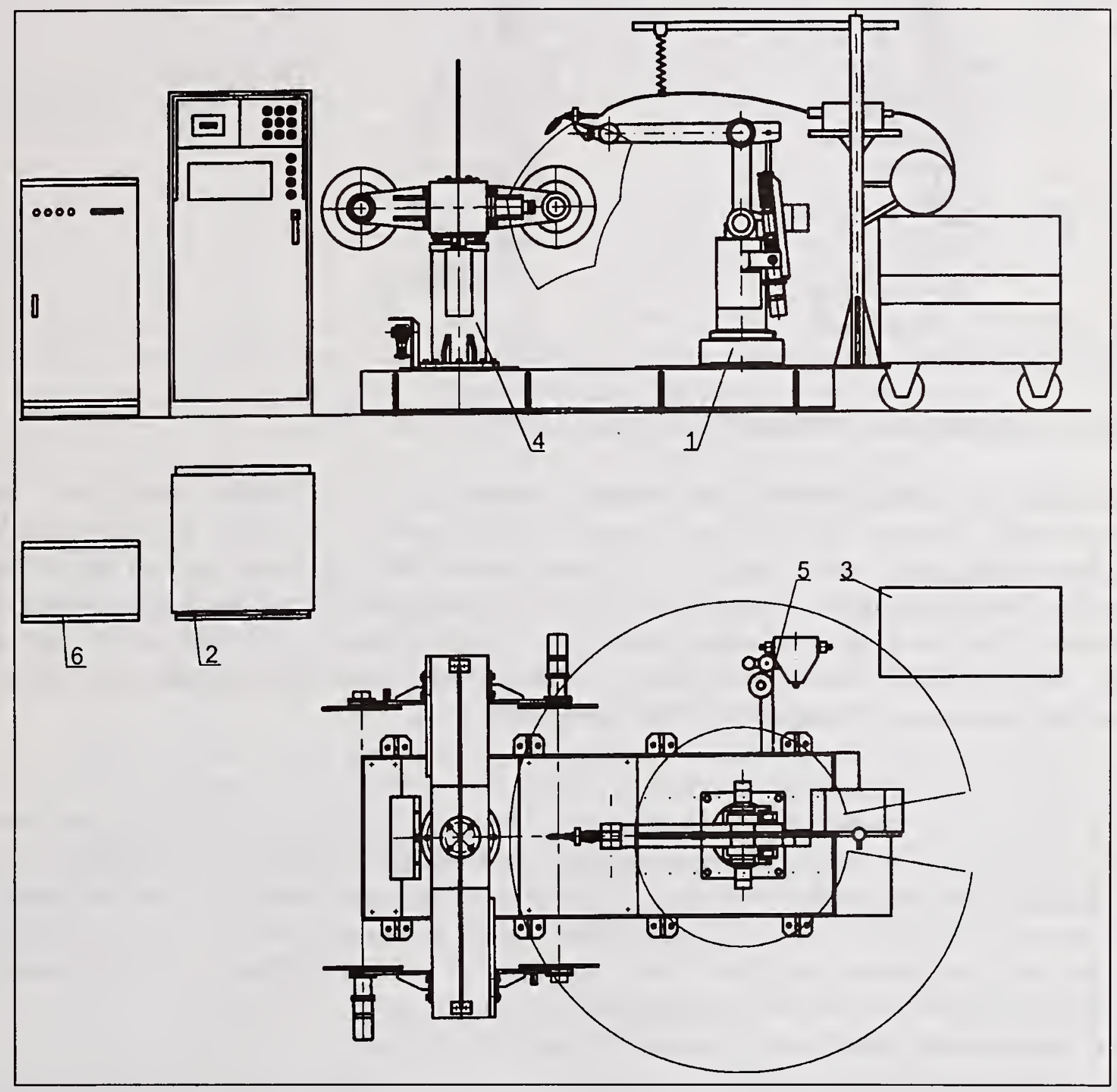

Figure 4. Robotic welding installation: (1) IRp-6 welding robot, (2) robot control unit USR-6, (3) welding power source OPTYMAG-500R, (4) positioning table PH-2-100, (5) torch cleaner with wire cutter, (6) positioning table control unit.

The robot that was used in the presented installation is of an anthropomorphic type, manufactured in Poland. It consists of three major component systems: the supplying system, the mechanical system (manipulator), and the controller. It has five servo-driven motion axes (fig. 5) with repeatability less than $\pm 0.20 \mathrm{~mm}$ and the handling capacity of $6.0 \mathrm{~kg}$. 


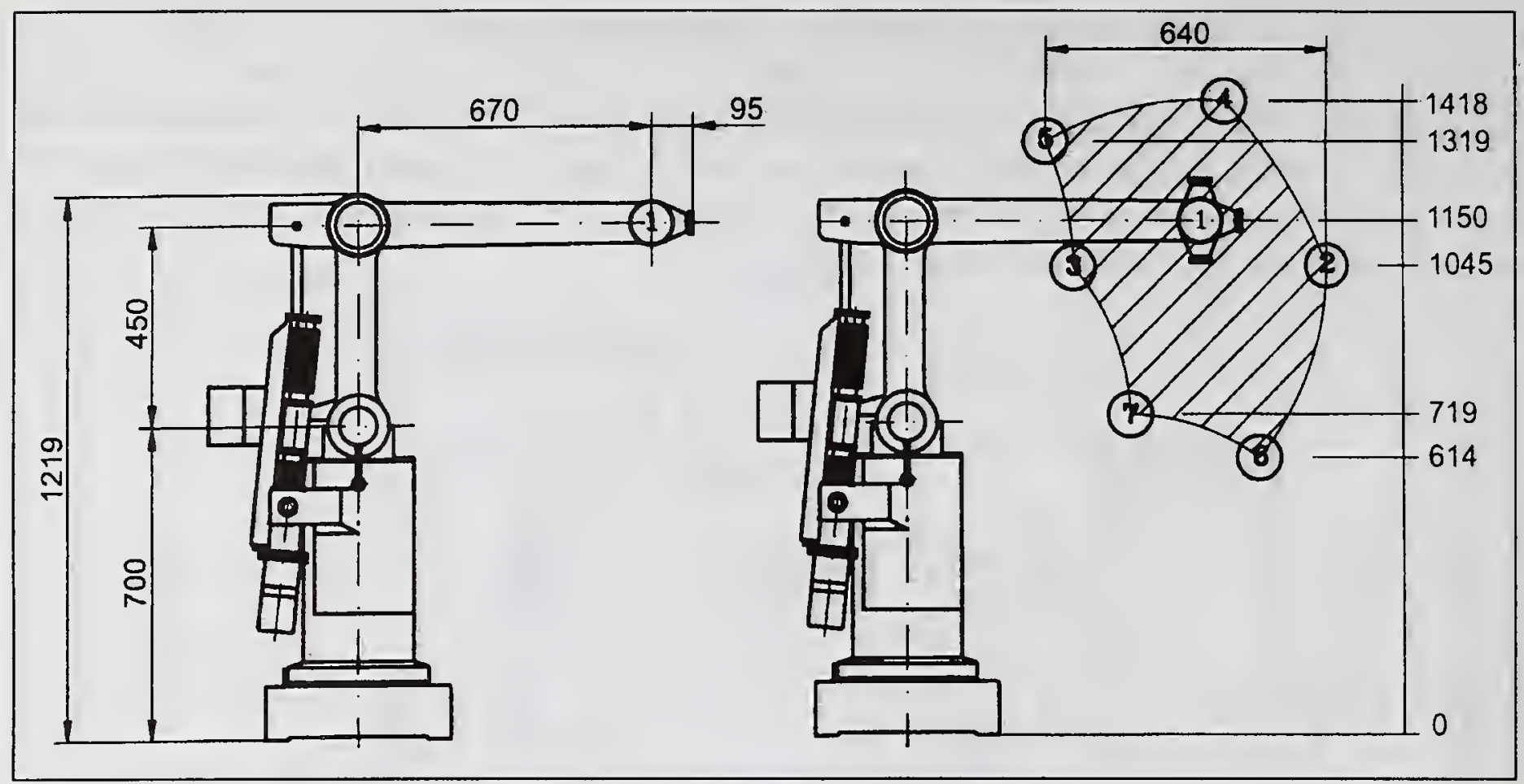

Figure 5. Dimensions and working range (in $\mathrm{mm}$ ) of IRp-6 robot (Ref. 4).

The next main component of the robotic installation is the welding equipment. The semiautomatic, synergic GMAW power supply with separate wire feeder and water cooled machine welding torch, called Optymag 500, was applied (Ref. 5). It can be used for welding low-carbon and low-alloyed steels with an active shielding gases as well as stainless steels and aluminum alloys with inert shielding gases. The microprocessor controlled synergic power source can supply both constant and pulsed welding current with an option to store up to 49 sets of welding parameters, referred to as welding programs.

Having these two commercial machines, i.e. robot and welding equipment, our task was to design and implement the mutual connection of them as well as to equip the constructed installation with all necessary auxiliary devices to make it fully productive. Additionally it has been assumed that the installation should fulfill the following requirements: (i) to be simple in both construction and operation, (ii) to be reliable, (iii) to be low-cost, and (iv) to be safe for the operator, all installation equipment and welding processes performed. To reach project objectives, the following tasks have been undertaken:

$\checkmark$ the adaptation of welding equipment for continuous duty,

$\checkmark$ the adaptation of robot controller,

$\checkmark$ the design and implementation of an interface connecting the robotic controller and the welding equipment,

$\checkmark$ the completion of auxiliary devices, such as positioning table, torch cleaning device, electrode wire cutter, etc.

The adaptation of semiautomatic welding equipment was aimed to increase its output parameters stabilization and operation reliability. Also it has been decided to locate the interface inside the power supply cabinet providing a necessary connector to be joined with the robotic controller. 
The necessary adaptation of the robotic controller enabled it to have control of the welding equipment and to maintain emergency cases. As an addition to the controller circuitry a function called "stop welding program" can immediately, in case of any emergency, put out of action the robot and all other active devices. However, the most important for the proper communication between robot and welding equipment was the interface.

The control unit of the welding power source allows a direct access to any set of parameters stored in a memory by simply pointing out the number of the welding program. This was the main point to the choice digital signals for controlling welding equipment. The robotic controller was equipped with 16 digital output/inputs that could be easily used for this purpose. Such a solution made the interface structure simple and low-cost in manufacturing. The designed interface uses two-directional digital signals for mutual communication between the robotic controller and the welding equipment. Assisted with relevant software the robotic controller played the master role in the controlling and the supervising of the system operation. Control signals are limited to the most significant for the GMAW process as follows.

The "Welding program" signal is used for selection by the robot control unit through the interface of one out of fifteen sets of parameters which are stored in the welding power source memory. This signal is coded on four lines and decoded on one-out-of-fifteen basis.

The "Wire extension" signal is used for wire inching after the welding operation in order to cut off the end of the wire to a required length by an automatic wire cutting unit.

The "Welding start" signal actuates the power source, the wire feeder and the shielding gas valve.

The "Error" signal is sent from the robotic controller that monitors all malfunctions and resets the power source and the wire feeder, i.e. the welding operation may be resumed once the error has been remedied and a new start instruction has been given. This signal may be generated by any of the following malfunctions:

(i) overtemperature; if the duty cycle is exceeded or if the cooling system malfunctions,

(ii) no program; if the program selector does not act correctly or the parameters set is incomplete,

(iii) power on reset; for $2 \mathrm{sec}$. after the power source is switched on,

(iv) robot reset; if an emergency stop command is given for the robot.

The "No water" signal is generated if the cooling water pressure/level transmitter reports an insufficient water flow while welding is being carried on. It effects a reset of the power source and wire feeder.

The "No gas" signal appears if the shielding gas flow transmitter reports an insufficient gas flow while welding is being carried on. This signal effects a reset of both the power source and the wire feeder similarly to "no water" and "error" signals.

The "No wire" signal is generated by the electrode wire detector when the whole wire has been consumed. This signal acts as "no water" and "no gas" signals.

The "No arc" signal is generated by the currant flow sensor after a sudden welding arc extinguishing and effects a reset of both the power source and the wire feeder. This signal is also used as a confirmation signal of the arc ignition while the welding operation is being initiated.

The "Thermal overloading" signal appears when the operation temperature of the thermally protected component of the installation exceeds the limited value and immediately stops the welding process. 
Fig. 6 shows the scheme of interface connections with the robotic controller and the welding equipment.

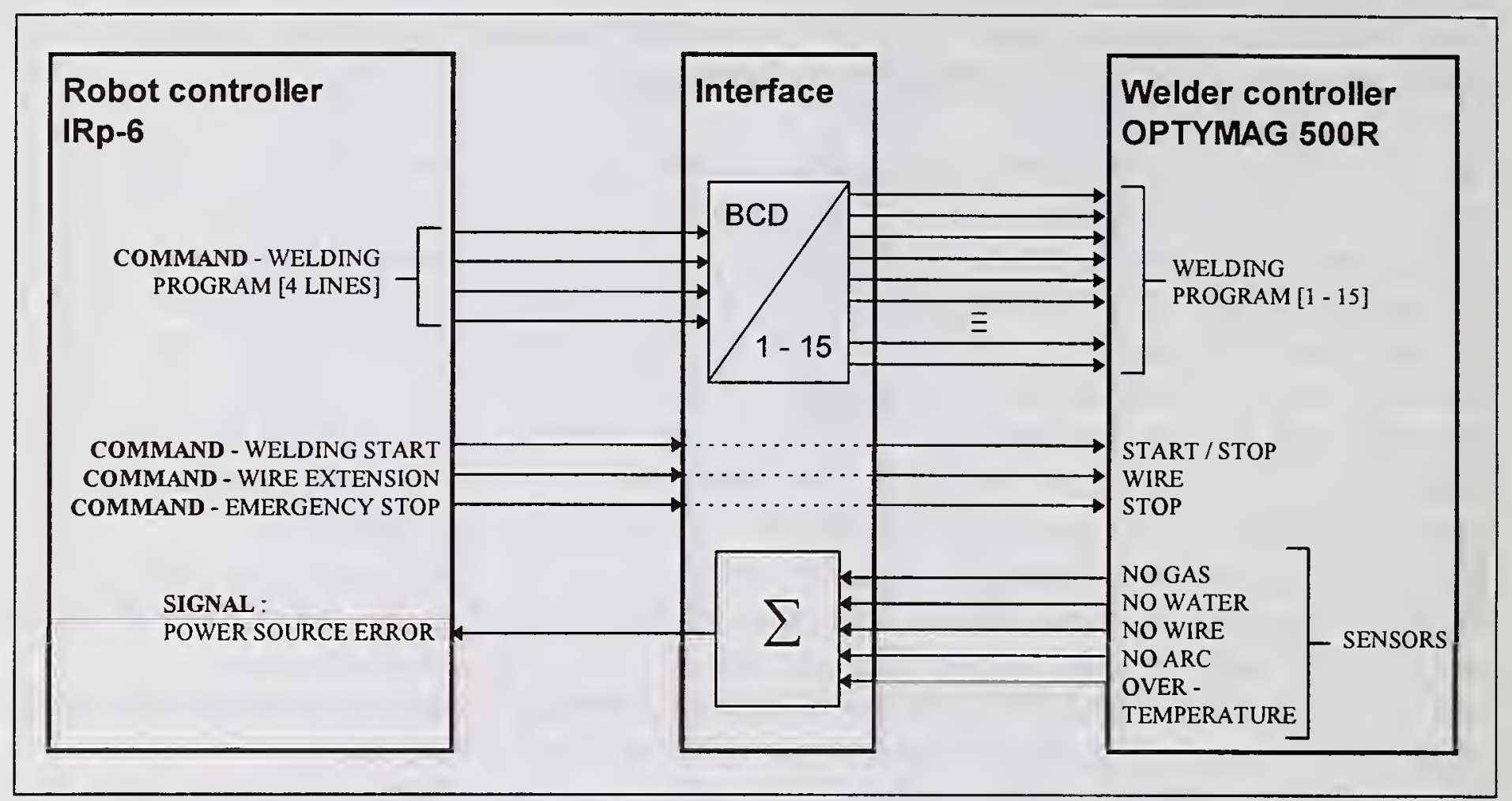

Figure 6. Block diagram: robot control unit (IRp-6) - welder control (OPTYMAG-500R) interface.

\section{CONCLUSIONS}

Presented here the concept of robotic installation is a compromise between the requirements concerning the industrial robot - welding equipment system employed to a typical welding application and the technical specification of these two component units. The implementation of the installation done so far approved the correctness of design assumptions and practical solutions.

\section{REFERENCES}

1. Misiurewicz P. 1991. Podstawy techniki mikroprocesorowej: WNT: Warsaw (in Polish).

2. Robot Welding System A30A. ASEA Pamphlet. Västeras. Sweden.

3. Operating instructions. Roboter Interface TSST 153. Fronius Schweissmachinen. Austria.

4. Industrial Robot Irp-6. Operating instructions. Zakłady Automatyki Przemysłowej ZAP S.A. Ostrów Wielkopolski. Poland (in Polish).

5. Optymag 500. Operating instructions. Zakłady Urządzeń Sterujących BESTER. Bielawa. Poland (in Polish). 


\title{
INTELLIGENT AUTOMATIC PIPE WELDING
}

\section{Ole Madsen $^{*}$, Rune Larsen ${ }^{* *}$, Lars Overgaard ${ }^{* *}$, Niels J. Jacobsen ${ }^{* *}$, Ingvar Hafsteinsson ${ }^{* * *}$, Carsten Bro Sørensen}

\begin{abstract}
An automatic production system for welding large diameter pipe structures is described. The system integrates: 1) Off-line programming, 2) Motion planning for redundant robots, 3) Collision-free motion planning, and 4) Sensor-based control. The paper outlines the software architecture and the production system. An implementation made at Odense Steel Shipyard Ltd, Denmark demonstrates the system can be used for automatic welding of complex structures in small batches.
\end{abstract}

\section{INTRODUCTION}

Generally, welding of large pipe structures involves many quite labour intensive manual operations. Because of the high degree of human involvement in the welding process and the high demands on kinematic manipulation associated with pipe welding, automation by robots offers a great potential to improve welding rates and in particular improve welding quality.

However, there are only few reports on industrial implementations of automatic robot-based methods for welding of large diameter pipe structures. The main reasons behind this are believed to be: 1) the total quantity of equal products are usually low, often one-of-a-kind, 2) the welding seam forms a rather complex shape, and 3) often there are relatively large tolerances on the workpiece shape.

Hence, there are a number of challenges involved in applying robots for pipe welding. First of all, due to the small batch sizes, the costs and time associated with generating the necessary programs to the robots are critical. Thus, efficient automatic off-line programming techniques must be developed and applied.

Secondly, due to the complex geometry, a need exists for complex manipulation of the welding torch as well as the workpiece. Hence, robots with more than 6 degrees-of-freedom must in general be applied. Furthermore, the complex geometry as well as the application of highly redundant robots increases the need for automatic collision avoidance schema's.

Finally, compensations of the off-line generated robot programs must be made, due to the large workpiece tolerances. To carry out the compensations, deviations between the physical world

\footnotetext{
* Dep. of Production, Aalborg University, Fibigerstræde 16, 9220 Aalborg, Denmark. email: i9om@iprod.auc.dk

** AMROSE Ltd, Forskerparken 10, DK-5230 Odense M, Denmark.

*** Odense Steel Shipyard Ltd, P. O. Box 176, DK-5100 Odense C, Denmark.
} 
and the models after which the robot is programmed must be identified by a sensing system. Compensations can then be computed by an appropriate control strategy.

Hence, automatic pipe welding can be performed by combining: 1) Off-line programming, 2) Control of redundant robots, 3) Collision free motion planning and 4) Sensor-based control. In literature a number of references can be found to work focusing on one or two of these areas (e.g. Ref. 1-5). However, for a large scale penetration of robots into the area of pipe welding, systems coping with all the four areas must be available.

In this paper the outline of a system is presented where all the elements are integrated. The system is currently running-in as a production-cell at Odense Steel Shipyard Ltd., Denmark.

\section{THE WELDING TASK.}

The task of the robot cell at Odense Steel Shipyard Ltd. is to assemble pipe structures used in ship sections. The diameters of the pipe structures welded in the robot cell are typically in the range of $250 \mathrm{~mm}-600 \mathrm{~mm}$ ( 10 inches - 24 inches). The lengths of the pipe structures are up to 6 meters ( 20 feet). Figure 1 shows some examples of the pipes welded in the cell.

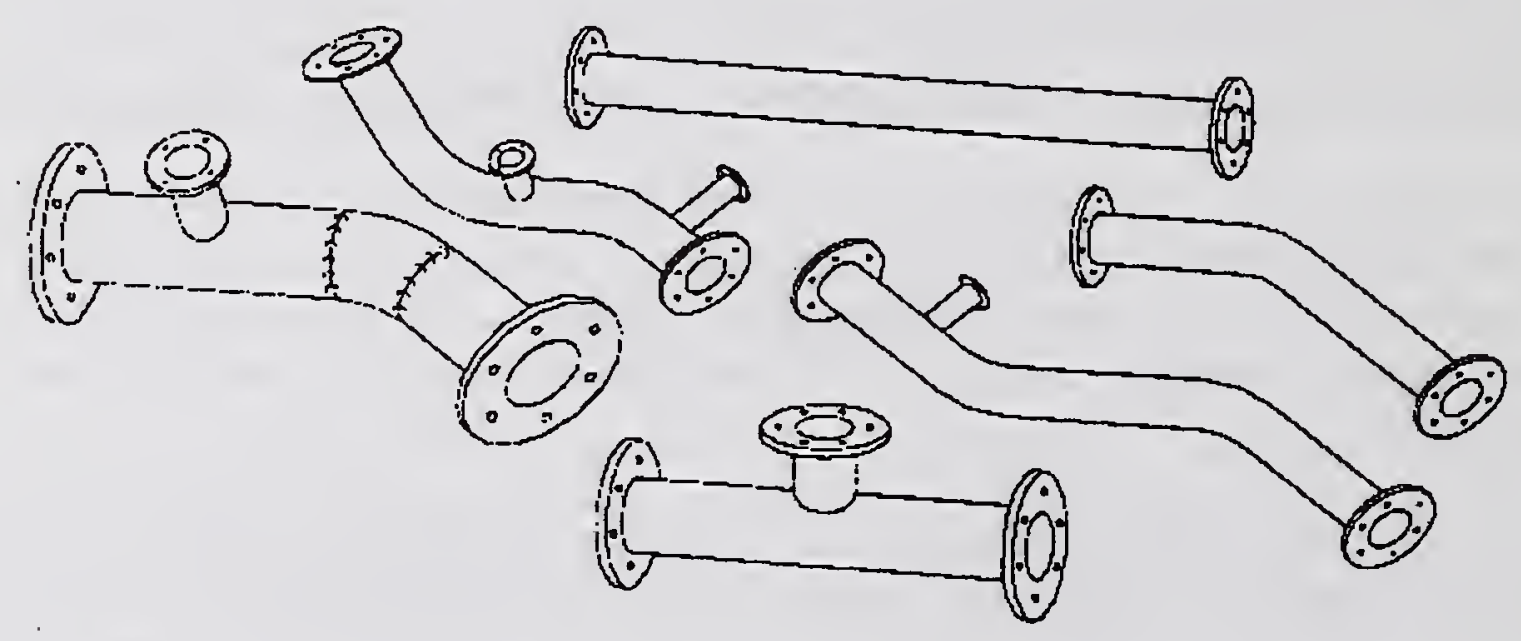

Figure 1. Examples of workpieces to be welded in the robot cell (Ref. 5).

The pipe structures have been tack welded before they arrive to the robot cell. I.e. nozzles have been tacked to the main pipe, flanges have been tacked to pipe ends, and pipe segment ends have been tacked to other pipe segment ends.

Gas metal arc welding (MAG-welding) is used, and in most cases multiple passes (2-3 passes) are needed to fill the welding seams.

\section{SYSTEM HARDWARE}

A picture of the robot cell is shown in figure 2 . 


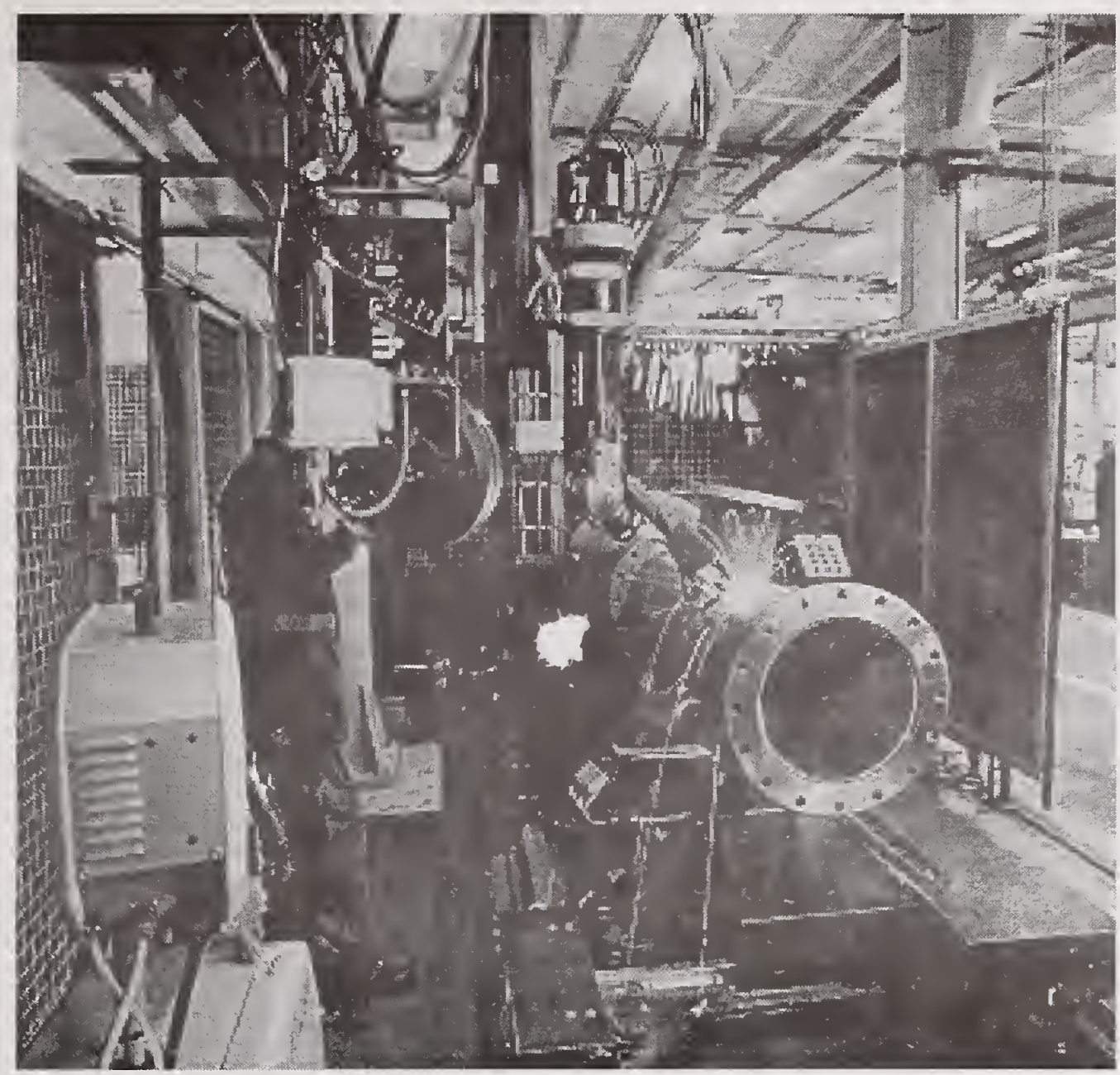

Figure 2. The robot cell.

The system consist of a 6-degree-of-freedom robot RV80 robot from REIS-robotics in Germany. The robot is equipped with a so-called "coordinate interface" making it possible to control the robot on-line from an external computer. In the cell, coordinates are transmitted to the robot via the coordinate interface with a frequency of app. $30 \mathrm{~Hz}$.

The pipes are mounted on a 2-degree-of-freedom workpiece positioner RDK1100 from REISrobotics. The pipes are mounted such that the positioner can rotate the pipes about their center axis. The positioner is also controllable via the coordinate interface.

A welding torch is mounted on the robot arm. The welding machine providing the necessary welding power and feeding the electrode material is a MIGATRONIC BDH 550. This welding machine is controllable from an extern computer via a digital and analogue interface.

A laser scanner is mounted approximately $35 \mathrm{~mm}$ ahead of the welding torch. The laser scanner used in the system is a MVS 30 from Modular Vision Systems in Canada. During welding the laser scanner is positioned such that it can provide an external computer with profile measurements with a frequency of approximately $10 \mathrm{~Hz}$.

The robot, workpiece positioner, laser scanner and welding machine is controlled from a PC with a Win-NT operating system. All necessary reference data (more about this below) are transmitted to the PC via the shipyard network. 


\section{SYSTEM ARCHITECTURE.}

Figure 3 shows the general software architecture of the system controlling the robot, workpiece positioner and the welding machine. In the figure functional entities of the system are represented by circles, to and from which data enters and leaves (in the figure represented by arrows). The sources and destinations (terminators) of the data (represented by boxes) represent other systems which interact with the system. In the following, first the system terminators are presented, followed by a presentation of the functional entities. Finally the principal mode of operation of the system is presented.

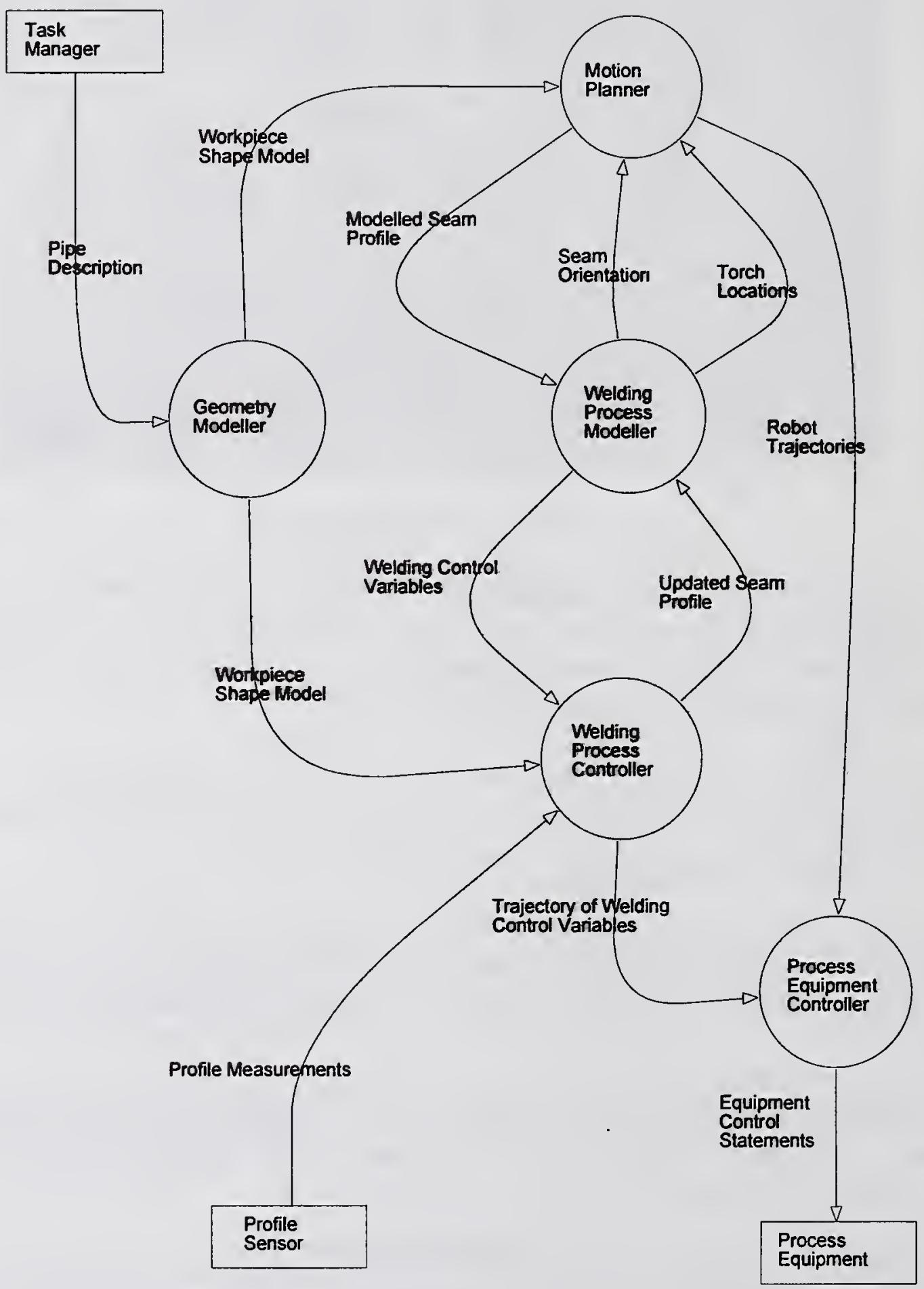

Figure 3. System outline. 


\section{System Terminators.}

As it appears from the figure the system interacts with three terminators.

The main task of the Task Manager is to control the sequence and modes in which the functional entities of the system are triggered. Furthermore, the Task Manager initiates the system by sending a pipe specification to the Geometry Modeller. The pipe description contains a basic specification (e.g. pipe length, number and placement of nozzles, thickness etc.) of the pipe to be welded. The pipe description is generated in a ship design system HICADEC-PIPE and stored in a database containing the overall ship design. From here it is retrieved by the Task Manager. The Task Manager used in the robot cell has been implemented by a team, ROB-EX, from Odense Steel Shipyard Ltd.

The task of the Profile Sensor is to provide the control system with profile measurements.

The Process Equipment consists of the robot, the workpiece positioner, and the welding machine.

\section{Functional Entities.}

As it appears from figure 3 the system contains five functional entities: Geometry Modeller, Welding Process Modeller, Motion Planner, Welding Process Controller, and Process Equipment Controller.

The task of the Geometry Modeller is to provide the Welding Process Controller and the Motion Planner with an shape model of the workpiece to be processed (see figure 4).

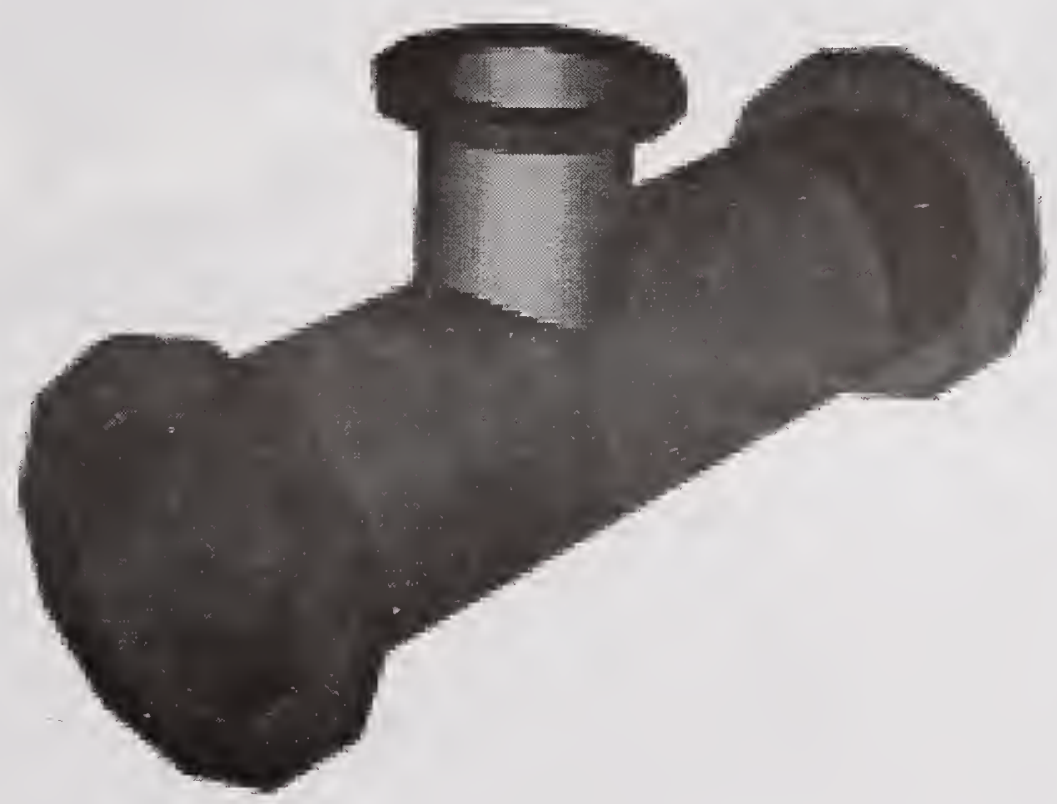

Figure 4. An example of an workpiece shape model from the Geometry Modeller. 
To generate the required data, the pipe description from the Task Manager is transformed into a 3D spatial NURBS geometry model. To obtain a sufficiently accurate geometry model, general knowledge of cutting procedures and bevel angles are the necessary.

The main task of the Welding Process Modeller is on request to provide the Motion Planner and the Welding Process Controller with welding control variables (torch angles, torch positions, travel speed, oscillation patterns etc.) to be used is certain positions along the welding seam (only torch positions and orientations are transmitted to the Motion Planner). These variables are computed as a function of parameters describing the seam profile. To perform this computation a number of welding process models have been developed. E.g. for welding of nozzles, a model has been developed relating the bevel angles and the root-gap to travel speed, torch position and torch orientation (Ref. 6).

It is also the task of the Welding Process Modeller to compute the desired orientation of the seam relatively to gravity in the point of welding. This is computed such that a satisfactory weld quality can be obtained along the seam.

The Motion Planner plan the motion of both the workpiece manipulator and the robot. The Motion Planner applied in this project is developed and provided by AMROSE Ltd., Denmark.

It generates an optimal trajectory for the workpiece manipulator. Given the actual mounting of the workpiece, the workpiece manipulator trajectory is planned in a such a way that the instantaneous orientation of the grove in the point being welded is optimal relative to gravity.

The collision-free motion planning for the robot is split into three phases (see Figure 5):

1) To plan inter-task robot trajectories, i.e. plan a trajectory that brings the robot from the end of one welding seam to the beginning of the next seam.

2) To plan start-point detection trajectories, that place the profile sensor relative to the seam, such that the start-point of the seam can be detected.

3) To plan task trajectories. The collision-free motion is designed in such a way that the weld torch observes the specified position and orientation along the welding seam.

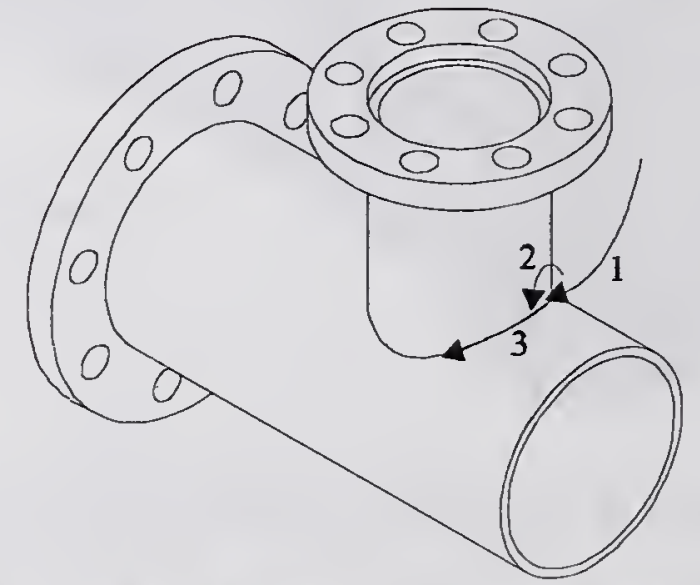

Figure 5. The three robot motion planning phases. 
The resulting robot and manipulator motions are specified as joint trajectories.

The AMROSE motion planner is based on very new mathematical principles allowing so-called forward dynamic calculations of the robots collision-free motion in environments of any complexity (Ref. 7).

Provided with a CAD model of the workpiece the AMROSE Motion Planner guides the robot through the structure, regardless of the number of degrees of freedom of the robot. The Motion Planner applies a multi-agent system (Ref. 8) in order to continuously ensure avoidance of collisions between the robot and it's environment, as well as prevent problems with reachability and singularities. Thus, every robot trajectory generated by the AMROSE Motion Planner is guarantied to be collision-free.

The result of the Motion Planner can be visualised in a 3D-simulation system developed by AMROSE Ltd (see figure 6).

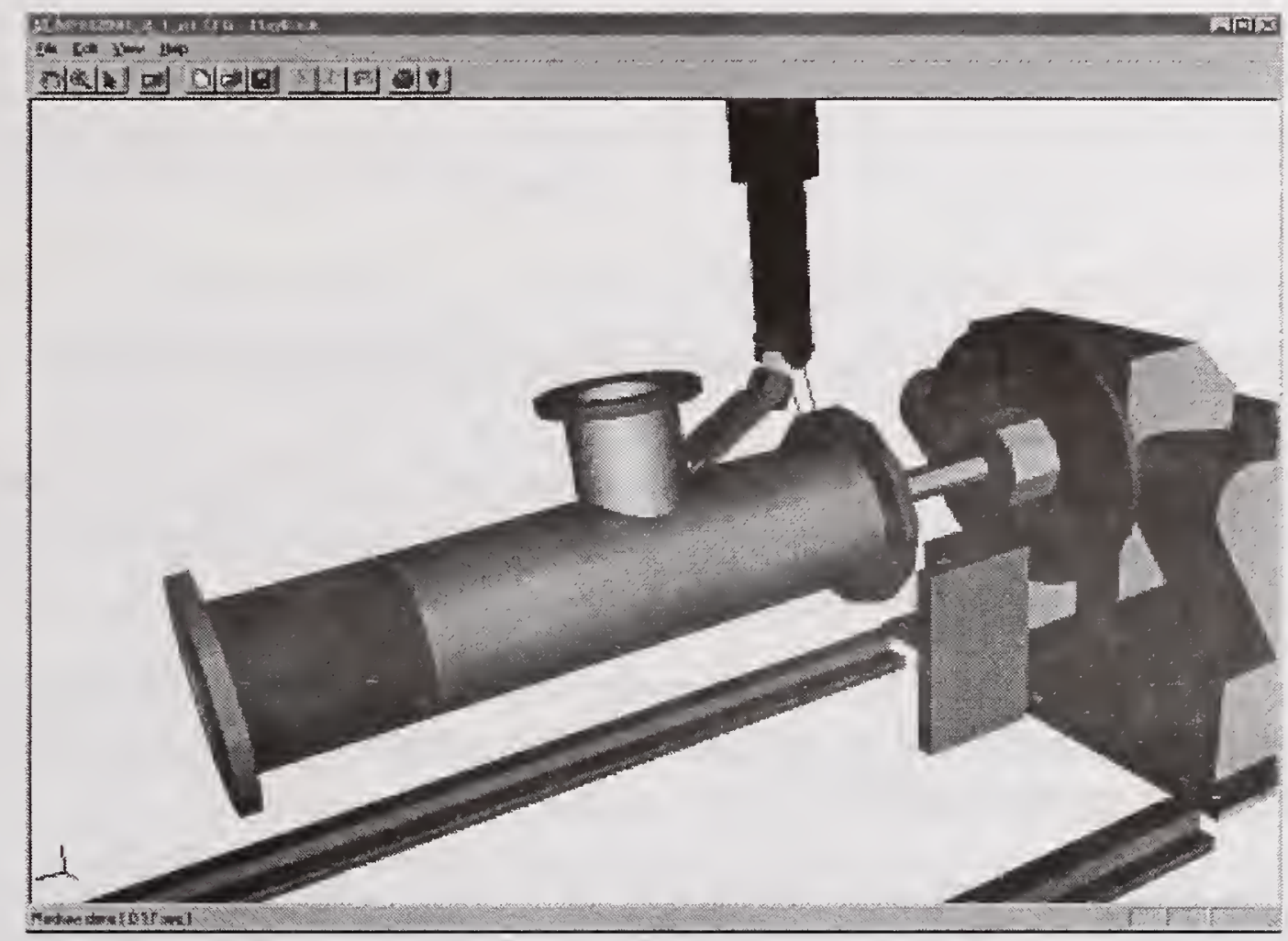

Figure 6. Example of a simulation of the result from the Motion Planner.

The task of the Welding Process Controller is to compute welding control variables matching the shape of the physical workpiece. The computation is based on measurements from the profile sensor. An example of a measurement is shown in figure 7. Since the sensor is mounted on the robot arm and moved along the seam while welding, a measured 3D-model of the workpiece can be generated by combining a number of profile measurements as shown in figure 7 .

The measured 3D-model is used to update the workpiece shape model so it corresponds to the shape of the physical workpiece. The measured 3D-model is not used directly because there are a number of features which cannot be measured sufficiently accurately by means of profile information (e.g. plate thickness and bevel angles). 


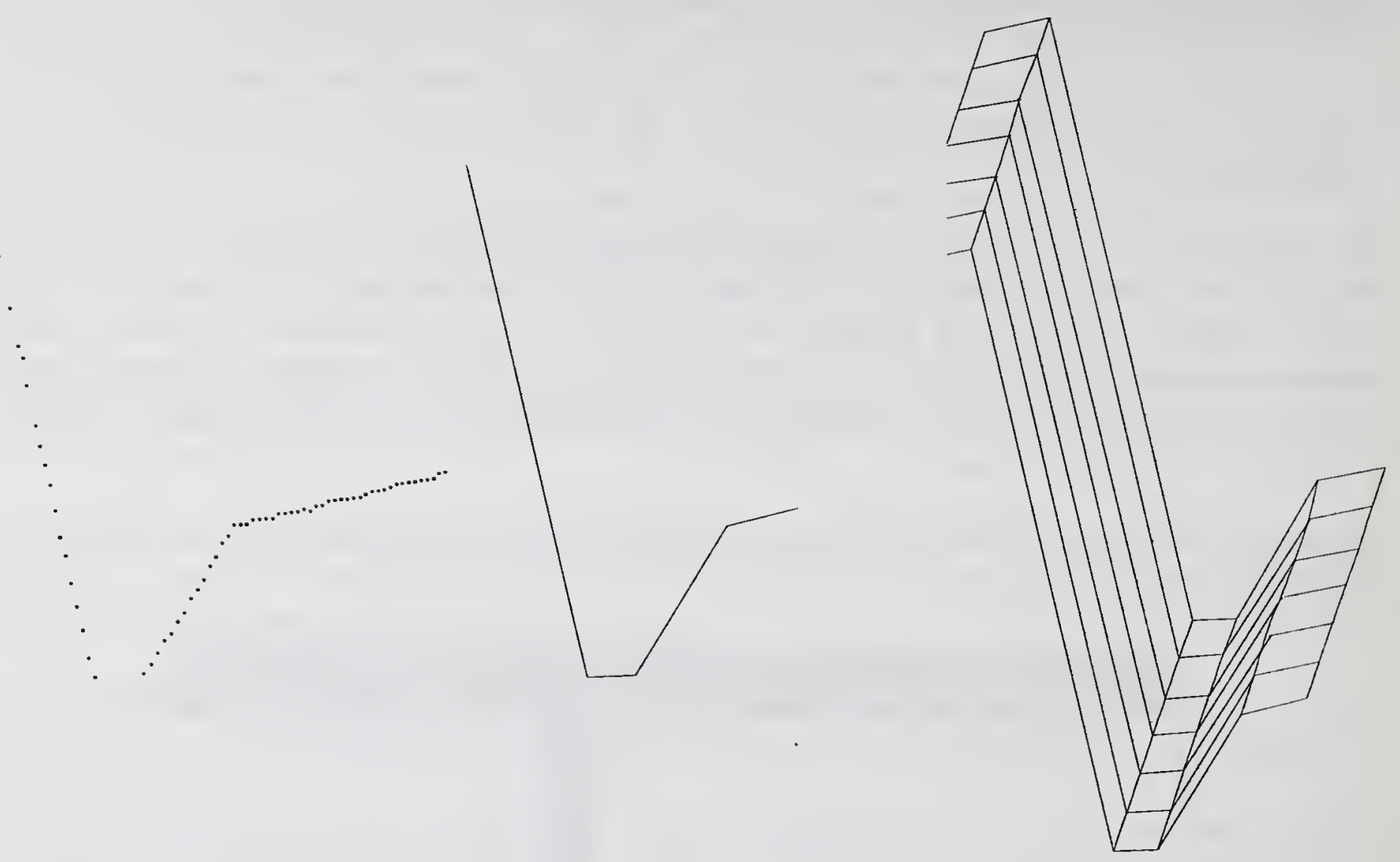

Figure 7. Different steps in the interpretation of signals from the profile sensor.

Profiles generated from the updated workpiece shape are transmitted to the Welding Process Modeller which uses these to compute welding control variables. These are then transmitted to the Process Equipment Controller.

The Welding Process Controller has been developed at the Department of Production, Aalborg University, Denmark in collaboration with the MultiPro-group at Odense Steel Shipyard Ltd. See Ref. 5 and Ref. 9 for more information.

The task of the Process Equipment Controller is to control the execution of the trajectories from the Motion Planner and the Welding Process Controller. It transforms the trajectories to control statements understandable to the Process Equipment, and it controls the transmission of the control statements to the Process Equipment. The Process Equipment Controller can operate in two different modes:

1. It can directly execute the robot trajectories from the Motion Planner.

2. It can execute the trajectories of welding control variables from the Welding Process Controller. In this mode the Process Equipment Controller performs inverse kinematic transformations of the cartesian torch positions and orientations specified in the welding control variables. To aid the selection of solutions in joint space, the Process Equipment Controller uses the collision-free robot trajectory computed by the Motion Planner. 
The Process Equipment Controller is operating in the first mode when performing inter-task movements and in the latter when performing the actual welding.

The Process Equipment Controller has been implemented by a team, CON-OSS, from Odense Steel Shipyard Ltd.

\section{Mode of operation.}

The fundamental mode of operation of the system is as follows:

1. The Geometry Modeller generates workpiece shape models of the seam to be welded.

2. Based on these models, the Motion Planner computes a collision-free trajectory for the intertask motion and for the welding operation. The trajectory of the workpiece manipulator is computed such that the instantaneous groove orientation relative to gravity is as specified by the Welding Process Modeller, which also specifies the torch positions and orientation along the seam.

3. The Process Equipment Controller executes the inter-task motion, which will bring the sensor to the modelled starting point of the seam.

4. The Welding Process Controller performs a search for the starting point of the physical seam by acquiring measurements from the profile sensor. If an acceptable profile can be measured, the seam is found. Otherwise, the Welding Process Controller will compute a new robot location, which is transmitted to the Process Equipment Controller. After reaching the new position it will attempt to make a new profile measurement. It will continue moving the robot and making new profile measurements until the seam is found or until the robot is moved more than a predetermined distance from the modelled start point. In the latter case an error signal is transmitted to the Task Manager.

5. Having found the starting point of the seam, the Welding Process Controller computes the starting location of welding torch by means of a profile measured in the starting point. The measured profile is used to update the profile of the workpiece shape model. The updated profile is transmitted to the Welding Process Modeller, which computes welding control variables. The torch position and orientation is then transmitted to the Process Equipment Controller such that the torch can be moved to the starting point.

6. The motion of the torch to its starting point can now be performed. While the torch is being moved, a number of profile measurements are acquired by the Welding Process Controller. These are used to update the workpiece shape model, and to compute welding control variables.

7. When the torch reaches the starting point, the welding control variables computed while making the initial motion are transmitted to the Process Equipment Controller and the welding can be started. While welding the sensor is being moved along the seam, profile 
measurements are acquired by the Welding Process Controller, the workpiece shape model is updated, and new welding control variables computed.

8. When reaching the end of the seam, the computation of welding control variables is stopped. The welding is finished when the torch reaches the point of the last welding control variables.

\section{CONCLUSIONS}

In this paper a robot based automatic pipe welding system has been described. The system integrates: 1) Off-line programming, 2) Control of redundant robots, 3) Collision free trajectory planning, and 4) Sensor based control. The integration enables automatic welding of complex pipe structures in small batches. A task which can not be solved with traditional off-line programming and/or welding control systems. Instead the system is based on highly advanced technologies, and we believe that this system is unique.

\section{REFERENCES}

1. Holm, H., 1988, Off-line Programming of Robots for High Strength Welding of Tubular Joints. Main report from the ROPS-project, April 1988.

2. Koguchi, T. et. al., 1990, Development of Automatic Welding Method for Large Diameter Nozzle Attached to Piping With GMAW. IIW Doc. XII-1162-90. July 1990.

3. Madsen, O., Holm, H., Lauridsen, J. K, 1995, Control System Architecture for Robotic Welding of Tubular Joints. In proceeding of 4 th International Conference on Trends in Welding Research, Gatlingburg, USA, June 1995.

4. Huissoon, J. P., Strauss, D. L., 1994, Automatic Control of a Robotic Gas Metal Arc Welding Cell, 5 th World Conference on Robotics Research, Cambridge, Massachusetts, Sep. 27-29, 1994.

5. Overby, J., Simonsen, S., Nielsen, C. P., 1996, Through-the-Arc Sensor Based Welding Control System, 9th semester report, Department of Production, Jan 1996.

6. Madsen, O., Holm, H., Boelskifte, J., Hafsteinsson, I., 1995, Model of Root-bead Welding for Off-line Programming and Control, CAPE'95, Beijing, China, 16-18 May 1995.

7. Overgaard, L., Petersen, H. G., Perram, J. W., 1995, A General Algorithm for Dynamic Control of Multilink Robots, International Journal of Robotics Research, Volume 14, Number 3, p. 281-294.

8. Overgaard, L., Petersen, H. G., Perram, J. W., 1996, Reactive Motion Planning: A Multiagent Approach, Applied Artificial Intelligence, Volume 10, Number 1, p. 35-51.

9. Madsen, O., Holm, H., 1997, Model and Sensor Based Control of Industrial Robots, International Conference on Manufacturing Automation (ICMA'97), Hong-Kong, April 28-30, 1997. 


\section{RESISTANCE WELDING}





\title{
MODELING OF CONTACT RESISTANCE DURING RESISTANCE SPOT WELDING
}

\author{
M. V. Li, ${ }^{1}$ P. Dong, ${ }^{1}$ and M. Kimchi ${ }^{2}$
}

\begin{abstract}
A fundamental-based contact resistance model is proposed for the analysis of resistance spot welding. The contact resistance model was formulated based on the characteristics of voltage and resistance of electric contacts and the well-established theory by Khlrausch, which has been experimentally validated by many sources. The numerical implementation of this contact resistance model is presented in the context of finite element modeling procedure for the analysis of resistance spot welding. To demonstrate the effectiveness of this contact resistance model, resistance spot welding of bare steel was analyzed. The predicted nugget sizes were found in excellent agreement with the experimental measurements. The corresponding electrical, thermal, mechanical interactions associated with the welding process are also discussed in light of the contact resistance model.
\end{abstract}

\section{INTRODUCTION}

Resistance spot welding is a strongly coupled electrical, thermal, mechanical, and metallurgical process. The physics associated with the process is very complex. Over the recent years, there has been a growing interest in achieving a better understanding of the welding process, particularly for the development of reliable control and monitoring system (Ref. 1). The key to achieve such a goal is to quantitatively characterize the mechanical, electrical, thermal, and interactions during the welding process. Computational modeling has been proven to be a very powerful tool in gaining the insights on the process physics associated with welding processes including resistance spot welding (Refs. 2-4).

One of the major challenges in analyzing resistance spot welding is the modeling of contact phenomena at various interfaces, especially the electrical contacts (Ref. 5). It should be noted that the majority of the modeling works to date on the analysis of resistance spot welding were conducted by prescribing contact resistance values on the trial-and-error basis to achieve optimal fit between the predictions and experimental measurements.

Although there are lots of experimentally measured static and dynamic resistance data readily available in the open literature, they were of little use for numerical studies (Ref. 5). Normally, static resistance is measured with search currents of relatively small magnitudes, which generate insufficient heat in the vicinity of the contacts. Static resistance measurements are often

\footnotetext{
' Center for Welded Structures Research, Battelle Memorial Institute, Columbus, Ohio

${ }^{2}$ Edison Welding Institute, Columbus, Ohio
} 
performed using a DC bridge, which may provide a current, for measurement purposes, of the order of 1 ampere. Examinations of the reported contact resistance values in the open literature revealed that the measured static contact resistance values are mostly attributed to the film effects (Ref. 5) and are dependent on the surface condition, search current used in the measurements, temperature, and applied force. Electric contacts under such conditions are normally characterized as quasimetallic. The measured static contact resistance values can not be directly used in the numerical studies because it is now well known that film breakdown occurs very early in the welding process, typically in a fraction of the first quarter cycle. After film breakdown, the contacts become almost purely metallic and the measured static resistance prior to the film breakdown does not represent the contact resistance in the rest of the welding process. Dynamic resistance, on the other hand, is defined as the ratio of the instantaneous voltage to the current passing through a purely resistive conductor at the same instant in time, during actual welding operations. It is believed that the measured dynamic resistance values are more representative to the resistive heating during an actual welding process. However, there are many factors that would affect the dynamic resistance values. These factors include electrode geometry, welding current, sheet thickness, electrode force, etc. and their effects are difficult to be quantified.

To overcome this difficulty in computational modeling of resistance spot welding, a fundamental based contact resistance model was proposed. The contact resistance model was derived from the characteristics of voltage and resistance of electric contacts. In this paper, the contact resistance model and its numerical implementation are presented. The contact resistance model was applied to the analysis of resistance spot welding of $0.8 \mathrm{~mm}$ bare steel. The predictions by the finite element analysis were validated with experimental measurements. The electrical, thermal, and mechanical changes encountered in the welding process are discussed in light of the contact resistances.

\section{CONTACT RESISTANCE MODEL}

In this analysis, contact resistance prior to the film breakdown was ignored because it takes place at the very early stage of the welding process, typically in a fraction of the first quarter cycle. After the film breakdown, contact resistance during the resistance spot welding becomes metallic and it can be estimated based on Khlrausch model (Ref. 6). This model was developed based on the relationship between the voltage drop across a metallic contact interface and its resistance. Diesselhorst (Ref. 7), Bowden and Williamson (Ref. 8), Greenwood and Williamson (Ref. 9), published strict proofs under well defined conditions to substantiate this model. This model simply states that, the voltage drop across the contact interface can be estimated by

$$
V^{2}=4 L\left(T_{S}{ }^{2}-T_{0}^{2}\right)
$$

In this equation, $V$ is the voltage drop across the contact interface, $T_{s}$ and $T_{0}$ are the maximum temperature at the interface and the bulk temperature respectively. $L$ is Lorentz constant and its value is about $2.4 \times 10^{-8}\left(\mathrm{~V} /{ }^{\circ} \mathrm{K}\right)^{2}$ for most metals. This model is valid for metallic contacts and contact members obey the Wiedemann-Franz-Lorentz law 


$$
k \rho=L T
$$

where $k$ is the thermal conductivity in $\mathrm{W} / \mathrm{m}^{\circ} \mathrm{K}, \rho$ the electric resistivity in $\Omega / \mathrm{m}, T$ the temperature in ${ }^{\circ} \mathrm{K}$. Details of the mathematical derivation of Kohlrausch model can be found in the book by Holms (Ref. 10). Holms also discussed deviations from Kohlrausch model caused by geometric and material variations.

For the analysis of resistance spot welding of bare steels, we have specified $T_{s}$ to be the solidus of bare steel at the faying surface. For the E/S interface, $T_{s}$ was set to $814{ }^{\circ} \mathrm{C}$, which is the eutectoid temperature of $\mathrm{Fe}-\mathrm{Cu}$ alloy. Consequently, we were able to obtain the voltage drop across the interface at any bulk temperature $\left(T_{0}\right)$ below $T_{s}$, and thus the equivalent contact resistance/resistivity as a function of temperature by dividing the voltage drop with the welding current. The voltage drops evaluated from Eqn. 1 for the welding of bare steel are presented in Fig. 1. At temperatures above $T_{s}$, electric resistivity values of the steel sheets are used for the contact elements.

\section{NUMERICAL IMPLEMENTATION}

A coupled finite element analysis procedure (Ref. 4) was used to model resistance spot welding. This modeling procedure is automated using an input file prepared with the Applied Parametric Design Language of ANSYS. In this modeling procedure, the mechanical, thermal, and electrical interactions are taken into account during the resistance spot welding and the temperature and stress distribution in the weld are tracked down at specified time instant.

Due to the geometric symmetry, only a quarter axisymmetric model was used in this study to analyze the resistance spot welding process. Commercial finite element code ANSYS is used in this study. Almost identical mesh was used for the thermomechanical and thermoelectric analyses. The only difference between the finite element meshes for thermomechanical and thermoelectric analyses was the surface elements. ANSYS treats mechanical contact as a surface problem whereas thermal and electrical contacts as a volumetric problem. In reality, this is true. Consequently, nodes on surfaces of contact members shared the same coordinates in the thermomechanical model. Whereas solid elements with finite thickness of $0.02 \mathrm{~mm}$ to simulate the electric contact in the thermoelectric analysis. Matching nodal numbers were used in these two models.

The finite element mesh for the thermomechanical analysis is shown in Fig. 2. Twodimensional quadrilateral thermoelectric solid elements were used for thermoelectric analysis. The nodal degrees of freedom of these elements are voltage potential and temperature. Twodimensional isoparametric solid elements and point to point contact elements were used in the thermomechanical analysis. The nodal degrees of freedom of these elements are in-plane displacements.

In the mechanical analysis, the application of electrode force was assumed to remain constant during the squeeze, weld and hold cycles. A normalized current waveform, as shown in Fig. 3, 
was used in the thermoelectric analysis. In a normalized current waveform, the current is assumed to be sinusoidal during the conducting time. There exists a relationship between the peak current value and the RMS value (Ref. 11)

$$
I_{\text {Max }}=\sqrt{2 t / t_{0}} I_{R M S}
$$

where $I_{R M S}$ and $I_{M a x}$ are the root-mean-square and peak values of current, $t$ and $t_{0}$ are the total and conducting time in a cycle. From this relationship, one can expect that, to provide the same RMS current, the less the conducting time, the higher the peak current.

The inner surface of the cooling chamber is subjected to forced convection induced by the flow of cooling water. Empirical relationships readily available in the heat transfer textbooks (Refs. 12,13 ) were used to quantify the heat transfer coefficients at the cooling water chamber surface subjected to forced convection and other surfaces subjected to free convection in ambient air. The merit of using such relationship is that the cooling effect in the cooling chamber of electrode caps can be related to the water flow rate and temperature in a rational manner.

Thermal, electrical, and mechanical properties of copper electrode and carbon steels commonly used in the automotive industry are readily available in materials handbooks (Refs. 14-16). In this study, all the materiel properties are treated as nonlinear, temperature dependent properties. Latent heat of fusion is also incorporated into the enthalpy change of materials.

Based on the dependence of voltage drop across a contact interface on the bulk temperature of the interface, Eqn. 1, the temperature dependent contact resistance of each interface was calculated using the Ohm's law and the welding current. Furthermore, the temperaturedependent contact resistance values were converted to the equivalent electrical resistivity for the contact elements.

\section{EXPERIMENTAL VERIFICATION}

Experimental studies were conducted to study the nugget growth and to verify the finite element model. Welds were made with a $100 \mathrm{KVA}$ Taylor-Winfield pedestal-type resistance spot welder. The welding setup and conditions were taken from Ford Motor Specifications BA 13-1 and 13-4. The electrode caps used in the experiment were made of a RWMA Class II copper alloy. They were machined into $45^{\circ}$ truncated cones with $6.0 \mathrm{~mm}(0.24 \mathrm{in}$.) face diameters.

Bare steel sheets of automotive grade carbon steel AISI-SAE 1008 were used. The thickness of steel sheets was $0.8 \mathrm{~mm}(0.031 \mathrm{in}$.). The RMS current values used in the experiments were $7 \mathrm{kA}$ with $74 \%$ conducting time. Weld time was 12 cycles $(60 \mathrm{~Hz})$. The applied electrode force was $2000 \mathrm{~N}$ (450 lb.). Squeeze time was 60 cycles. The hold time after terminating electric current was 12 cycles.

To study nugget development, welding was interrupted after each cycle. Two weld specimens were made at each set of welding conditions. One specimen was used for metallographic 
examinations, and a second for peel test to measure the button sizes. The experimental measurements were then compared with the prediction by the model.

\section{RESULTS AND DISCUSSION}

For the experimental case simulated in this study, the pressure distribution after squeeze cycle at both the E/S and S/S interfaces are presented in Fig. 4. It is clear that after squeezing, the electrode tip surface is in full contact with the steel sheet. The diameter of the S/S contact area is approximately $15 \%$ larger than that of the electrode face diameter. For both the E/S and S/S interfaces, pressure is concentrated at the periphery of the contact areas.

The sizes of contact areas and pressure distribution pattern are changed soon after the welding current started passing through. The changes of contact radii at the E/S and S/S interface, normalized to the radius of the electrode tip surface, are presented in Fig. 5. As one would expect, heat generation during the first cycle of electric current was very localized at the interfaces. Localized heating caused localized thermal expansion, which pushed the contact members apart and reduced the contact area. The reduction of contact areas further increase the current density and thus intensify localized heating until considerable volume of metal are heated up and softened. The contact areas at the E/S and S/S interfaces reached their minimums by the end of the first cycle. As the welding process proceeded, more materials were heated up and softened and the contact areas gradually increased. Also the contact resistance decreased as the temperature increased. By the end of 12 weld cycles, the contact areas at both the E/S and S/S interfaces were still smaller than the electrode tip surface.

Accompanied with the change of contact area at the S/S interface, the pressure distribution pattern was also changed as shown in Fig. 6. After one cycle, the pressure is concentrated at the center of the contact area. Pressure concentration at the center reaches its maximum at around 3 to 4 cycles right before the nugget was formed. After nugget formation, pressure became less concentrated at the center. When nugget reached a decent size, the periphery of the contact area, which contained the molten nugget started picking up some pressure concentration. This pressure distribution pattern is considered beneficial for the nugget containment.

The temperature profile by the end of 12 cycles is shown in Fig. 7, plotted on the distorted geometry. From this plot, an interesting observation one may make is that the electrode tip surface was no longer perfectly flat during the weld cycle. The tip surface resumed its flatness after cooling down. A more significant observation is that, by the end of the weld cycle, contact areas at both the E/S and S/S interfaces are still smaller than the electrode tip surface. This implies that, at the given force level, the maximum nugget size one can obtain without causing expulsion would not be larger than the face diameter of the electrode for the welding of bare steel. If larger nugget diameter is desired, one should either change electrodes with larger face diameter or increase the electrode force. 
Temperature distribution at the faying surface during the welding cycle is presented in Fig. 8. Heating rate at the faying surface is very fast in the first three cycles. This is primarily due to the contact resistance. The contact resistance has two effects: first, it directly contributes to the heat generation in the vicinity of the interface, second, localized heating results in thermal expansion and reduced contact area, which in turn increases current density and heat generation. The predicted nugget growth profile is shown in Fig. 9. Lines in this figure represent the isothermal lines with temperature equal to the solidus of the steel. After 4 cycles, nugget was formed. The newly formed nugget has an appreciable penetration in the thickness direction. There is a drastic growth of the nugget in the first three cycles after the nugget formation (4-7 cycles). The volume increases in these three cycles are approximately the same. By the end of eighth cycle, the nugget has reached its full penetration. In the last four cycles, there were slow increases of nugget diameter. This is accompanied with the increase of contact area at the S/S interface.

The comparison between the predicted nugget diameter and measured button size is plotted in Fig. 10. They are in excellent agreement. Close examination of the nugget formation revealed that initial melting did not occur at the center of the contact area at the faying surface, but at a radius of about approximately $2 / 3$ of the contact radius. The newly formed nugget was found in the shape of a toroid. This molten zone expands rapidly to fill in the center region of the toroid within a fraction of a quarter cycle after its formation.

This contact resistance model presented in this study has a great advantage of being a generic model. It suggests that the static contact resistance have negligible effect on the resistance spot welding process. It also implies that the contact resistance after film breakdown is solely dependent upon the welding current. The applied electrode force does not affect the contact resistance itself. It does, however, affect the size of contact area, the current density, and measured dynamic resistance during the welding process. Sensitivity studies using this model have revealed that the applied electrode force has significant effect on the weldability lobe. For the same current level, low electrode forces favor the nugget formation but also lead to small nugget size. Increasing the electrode force delays the nugget formation and often requires higher welding current or longer weld time, but larger nugget size can be obtained.

\section{CONCLUSIONS}

This study proposed to a fundamental-based contact resistance model for the modeling of resistance spot welding. This contact resistance model has been incorporated into the finite element analysis and it was applied in this study to analyze resistance spot welding of bare steel. The predicted nugget sizes were found in good agreement with the experimental measurements.

The proposed contact resistance model offers a number of unique features in comparison with typical trial-and-error approach in the past. Being a generically applicable model, it allows sensitivity studies to be performed in a systematic manner, which offers insightful understanding of the effects of welding parameters on the nugget formation. 


\section{ACKNOWLEDGEMENTS}

The authors are grateful to the financial support provided by the Auto-Steel Partnership, Battelle, and EWI for this work.

\section{REFERENCES}

1. Tsai, C. L.; Dai, W. L.; Dickinson, D. W.; and Papritan, J. C. 1991, Analysis and development of a real-time control methodology in resistance spot welding. Welding Journal 70(12): 339s to 351s.

2. Dong, P.; Li, M. V.; and Kimchi, M. 1997, Analysis of electrode wear mechanisms: face extrusion and pitting effects. submitted to Science and Technology of Welding and Joining.

3. Browne, D. J.; Chandler, H. W; Evans, J. T.; and Wen, J. 1995. Computer simulation of resistance spot welding in aluminum: Part I. Welding Journal 74(10): 339s to 344s.

4. Dong, P.; Li, M. V.; and Sun, X. 1997, An incrementally coupled finite element procedure for the analysis of resistance spot welding. to be submitted to Science and Technology of Welding and Joining.

5. Li, M. V.; Dong, P.; 1997, Computational modeling of resistance spot welding process. to be submitted to Science and Technology of Welding and Joining.

6. Kohlrausch, F. 1900. Unber den stationaren temperaturzustand eines elektrisch geheizten leiters. Ann. Phys. Lpz 1: 132-158.

7. Diesselhorst, H. 1900: Uber das problem eines elektrisch erwarmten leiters. Ann. Phys. Lpz 1: 312.

8. Bowden, F. P. and Williamson, J. B. P. 1958. Electrical conduction in solids I. influence of the passage of current on the contact between solids. Proc. Roy. Soc. of London 246(8): $1-12$.

9. Greenwood, J. A. and Williamson, J. B. P. 1958. Electrical conduction in solids II. theory of temperature-dependent conductors. Proc. Roy. Soc. of London 246(8): 13-31.

10. Holm, R. and Holm, E. 1967. Electric contacts: theory and application 4th edition, Springer-Verlag New York Inc.

11. Evrard, M. and Hasle, C. 1958, Resistance welding parameters. British Welding Journal, 9 (1): $18-20$.

12. Chapman, A. J. Fundamentals of heat transfer: New York, Macmillan Publishing Company.

13. Holman, J. P. 1986, Heat transfer: $6^{\text {th }}$ edition, McGraw-Hill.

14. Metals handbook, vol. 1 properties and selections: irons and steels: 1978, 9th Edition, ASM International.

15. Brandes, E. A. and Brook, G. B. 1992, Smithells metals reference book: 7th Edition, Butterworths-Heinemann.

16. Physical constants of some commercial steels at elevated temperatures: 1953, The British Iron and Steel Research Association, London, Butterworths Scientific Publications. 


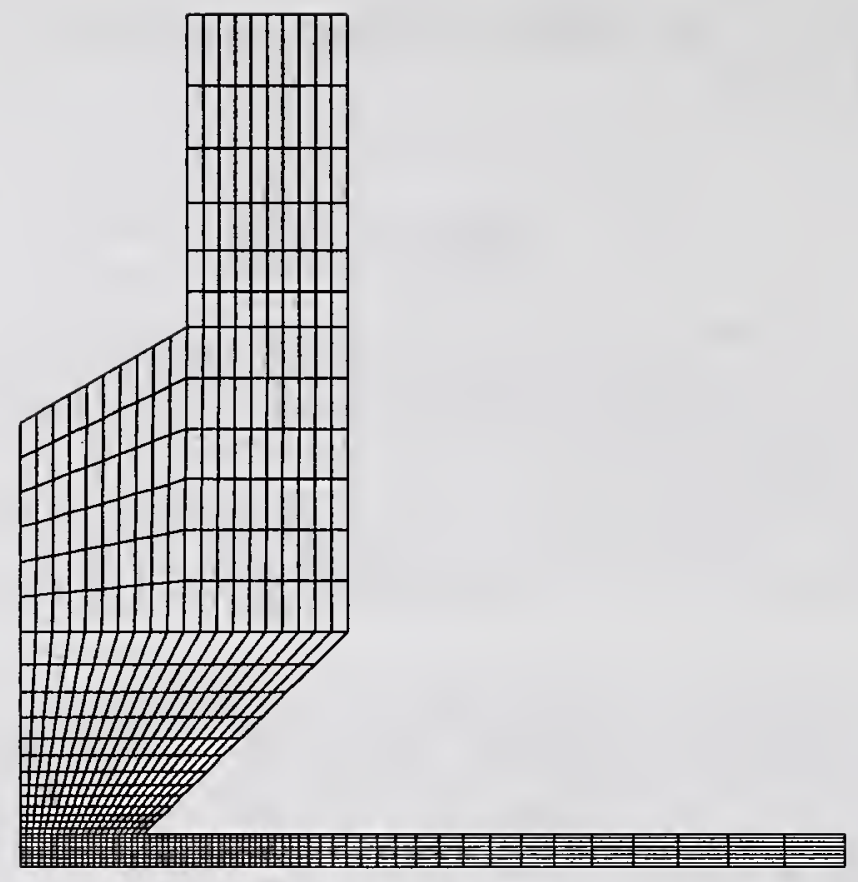

Figure 1 Finite element model for the simulation of resistance spot welding

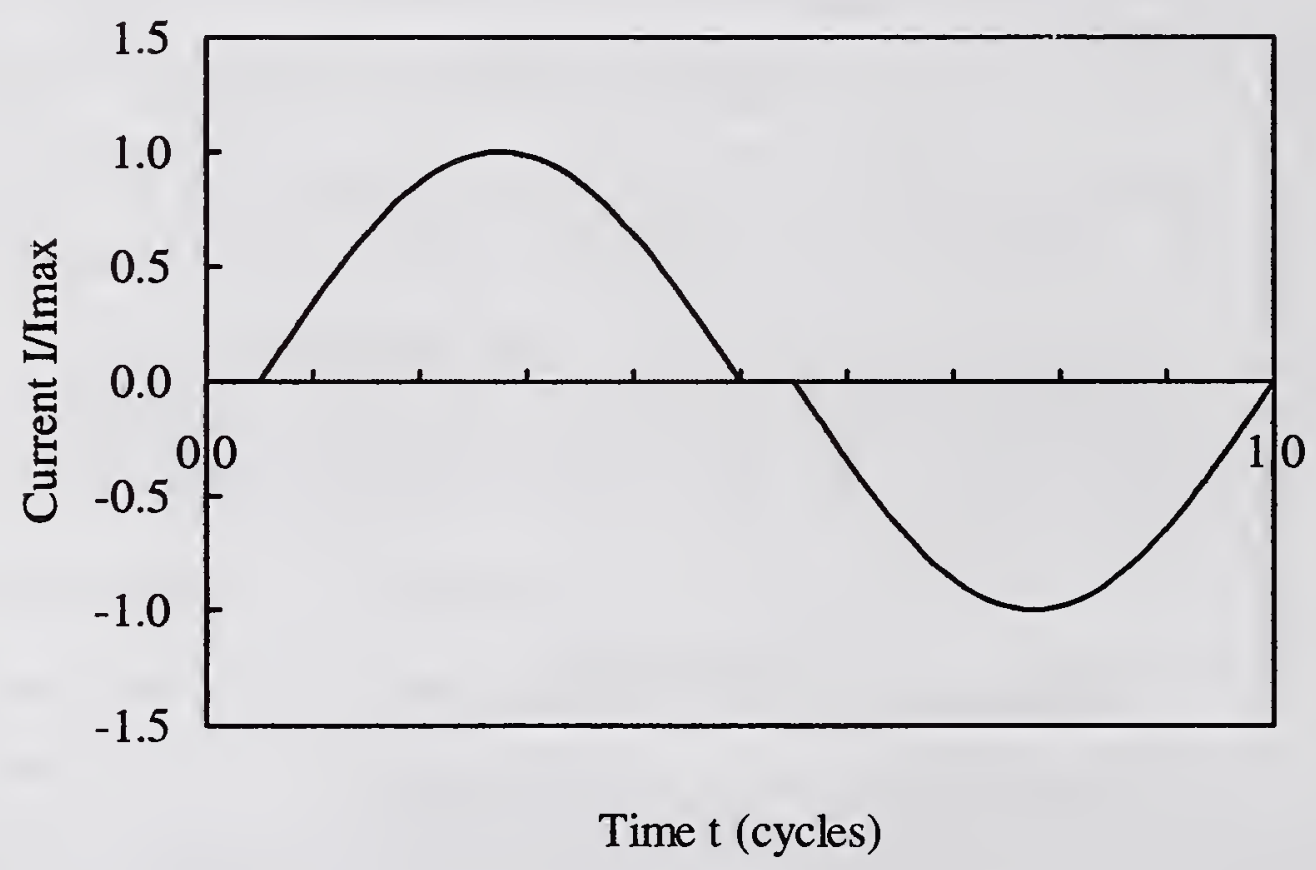

Figure 2 Schematic illustration of a normalized secondary current waveform 


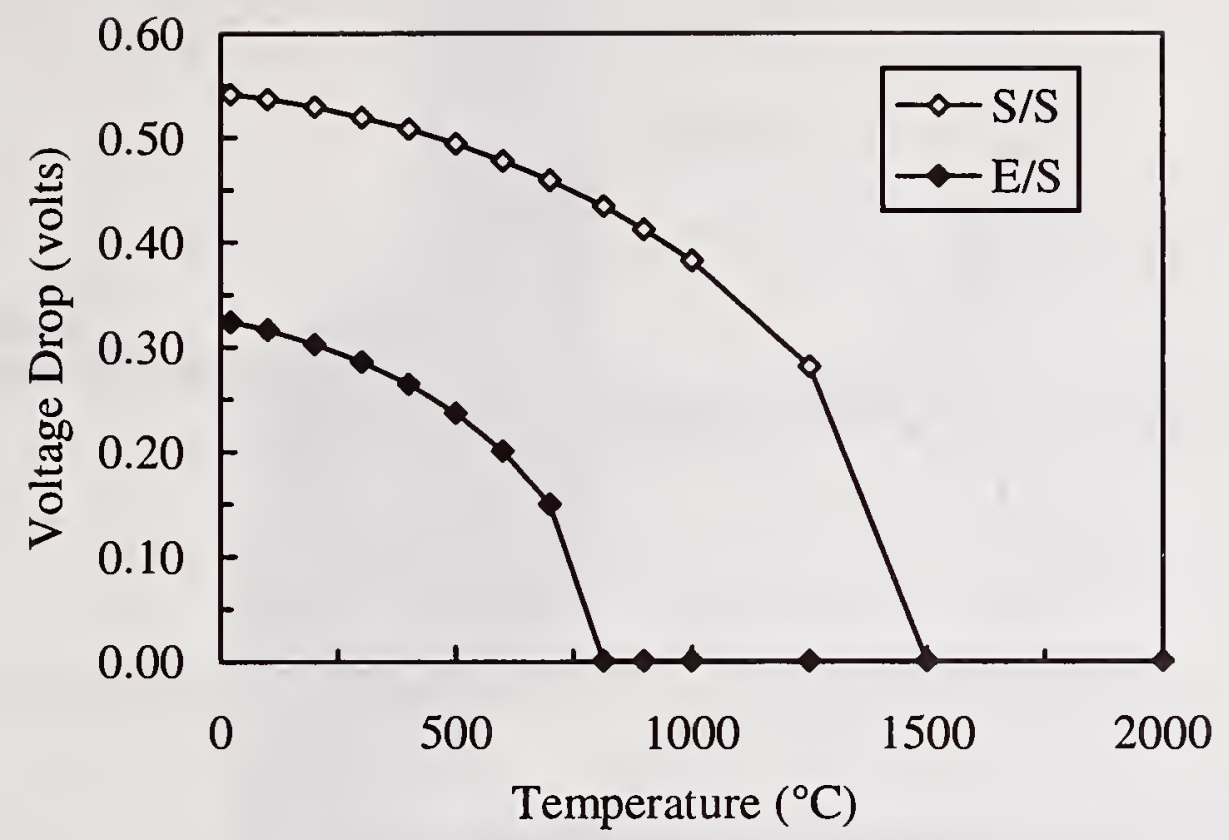

Figure 3 Voltage drops across the contact interfaces vs. the interfacial temperature

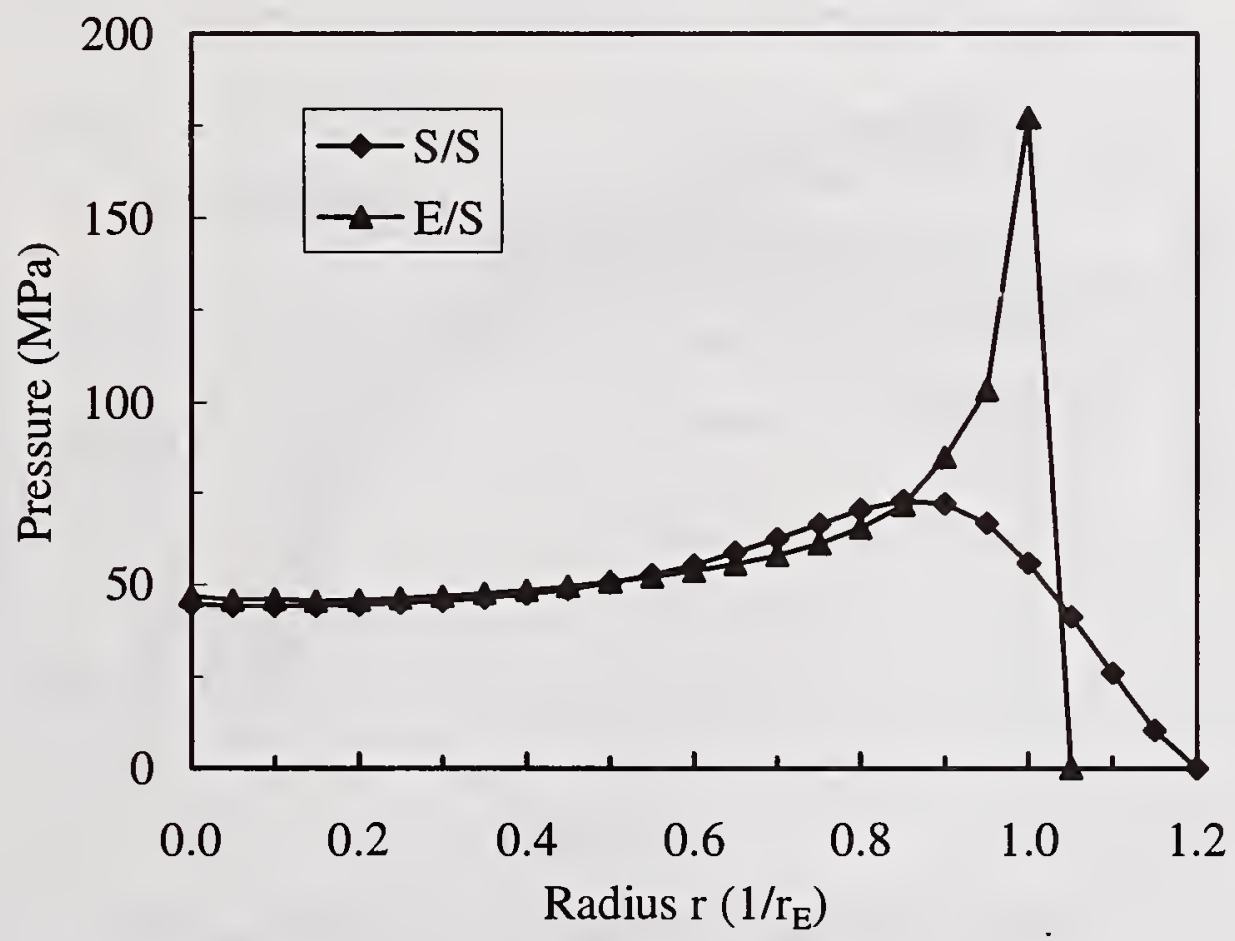

Figure 4 Pressure distribution after squeezing 


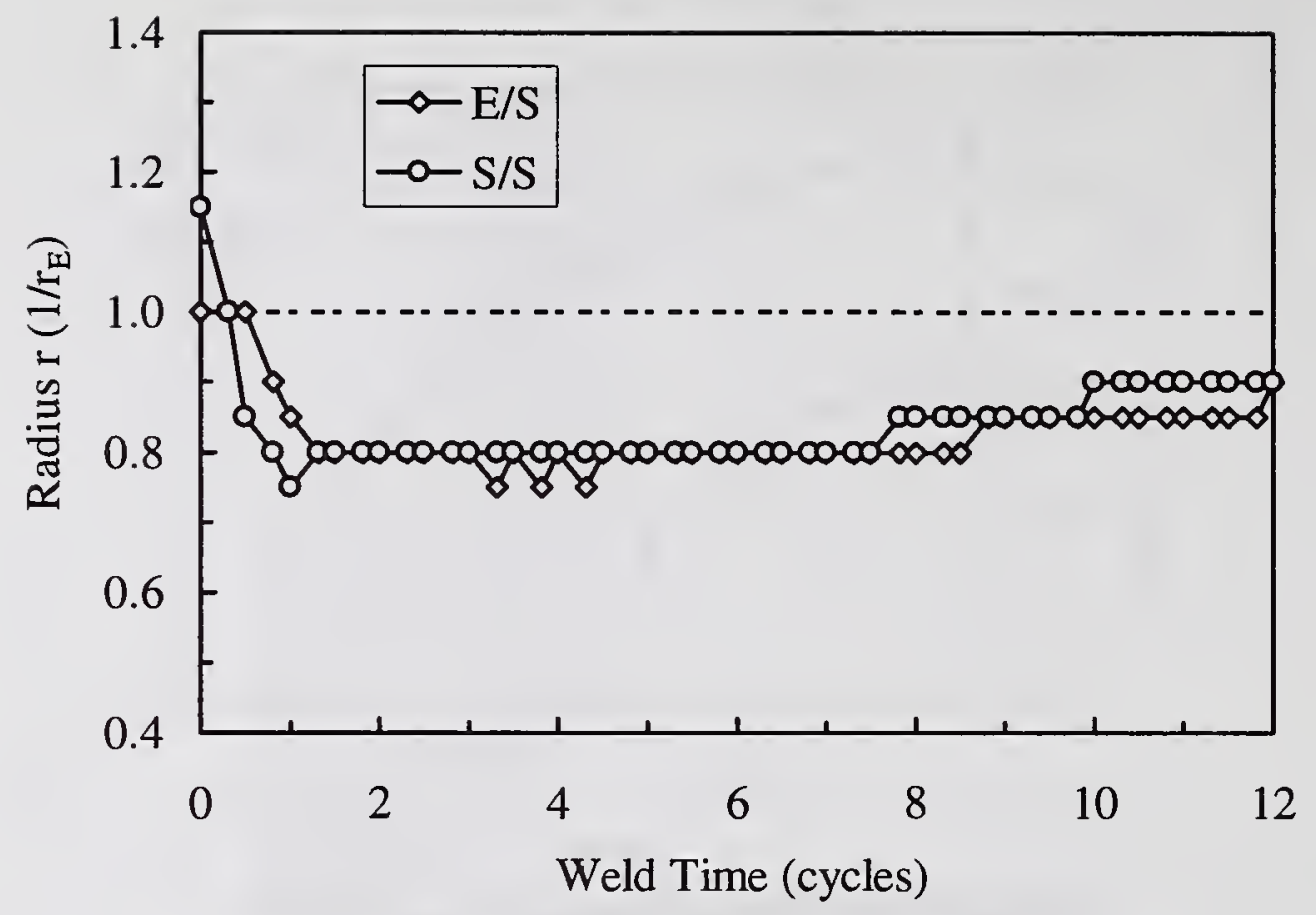

Figure 5 Change of contact areas during the welding process

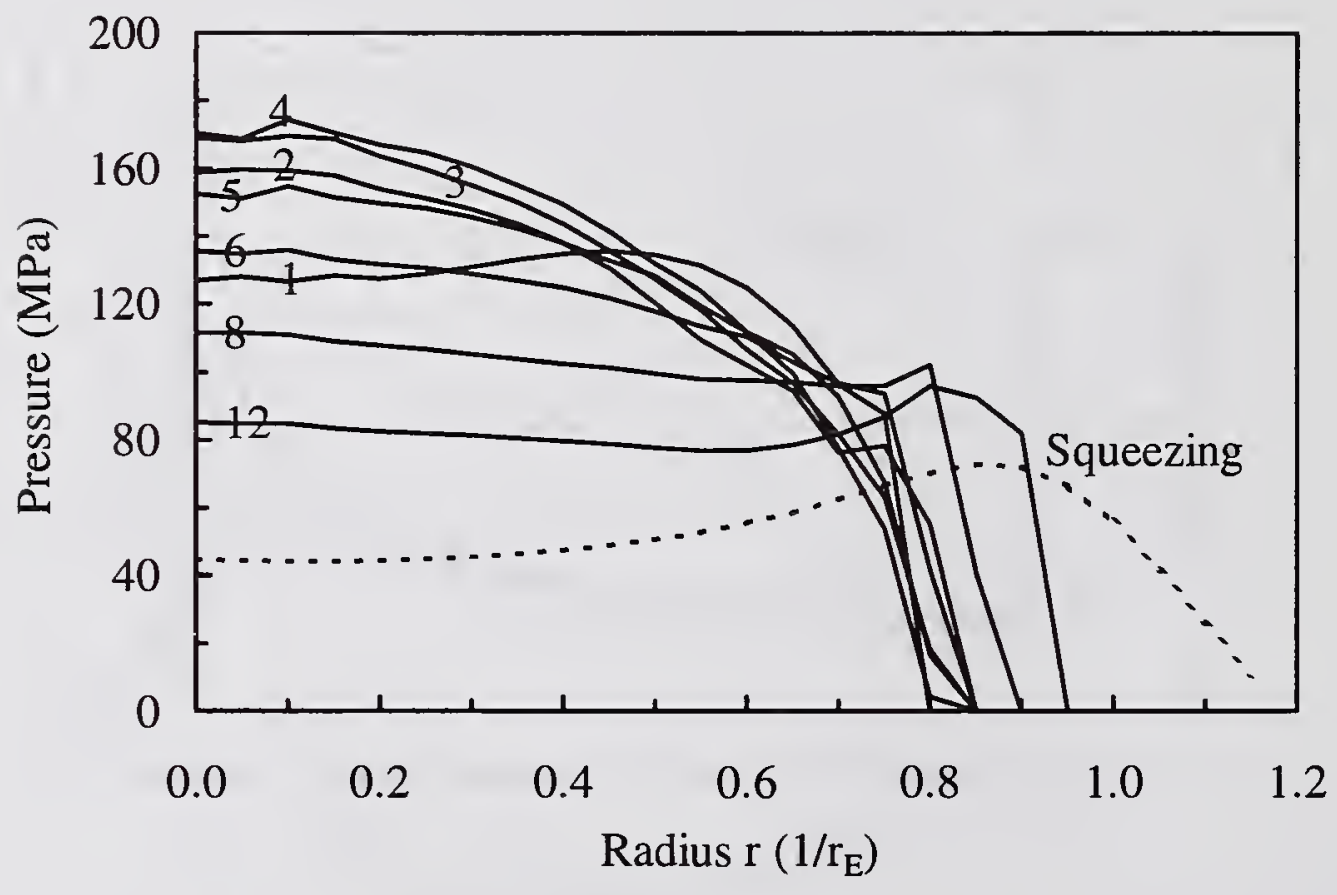

Figure 6 Pressure distribution at the S/S interface 


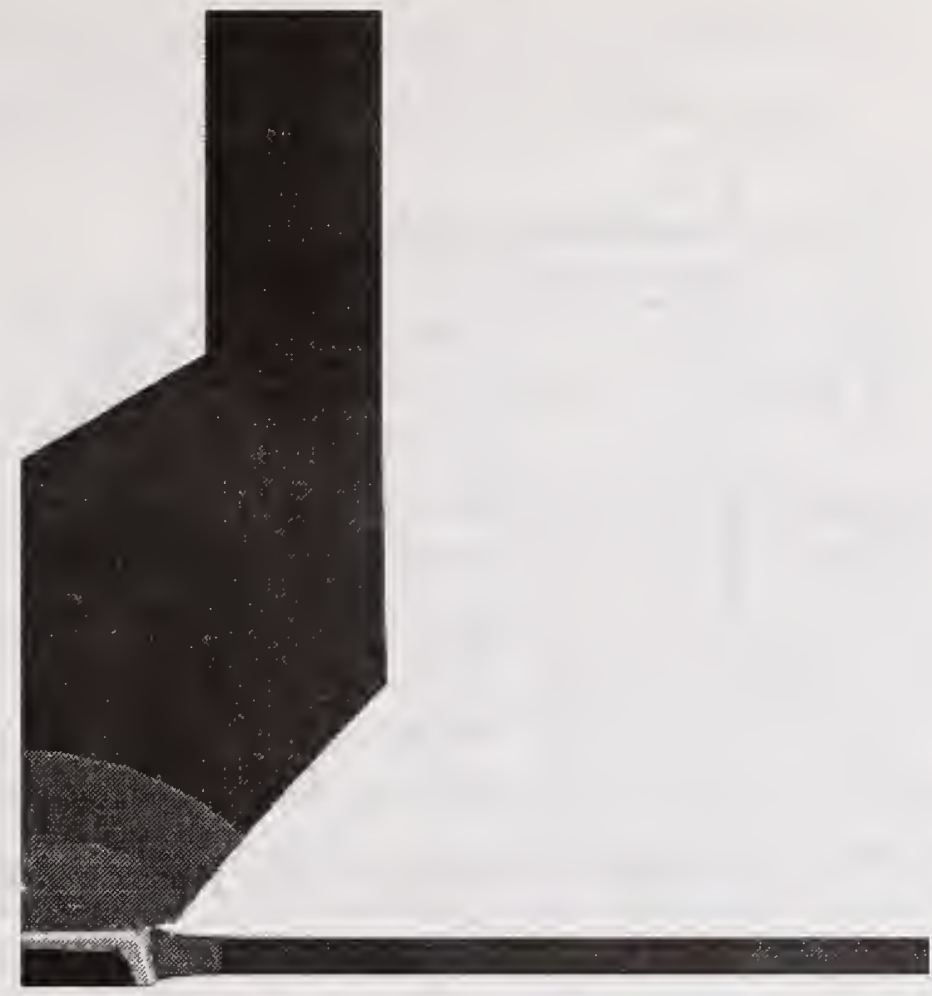

Figure 7 Temperature distribution by the end of 12 cycles

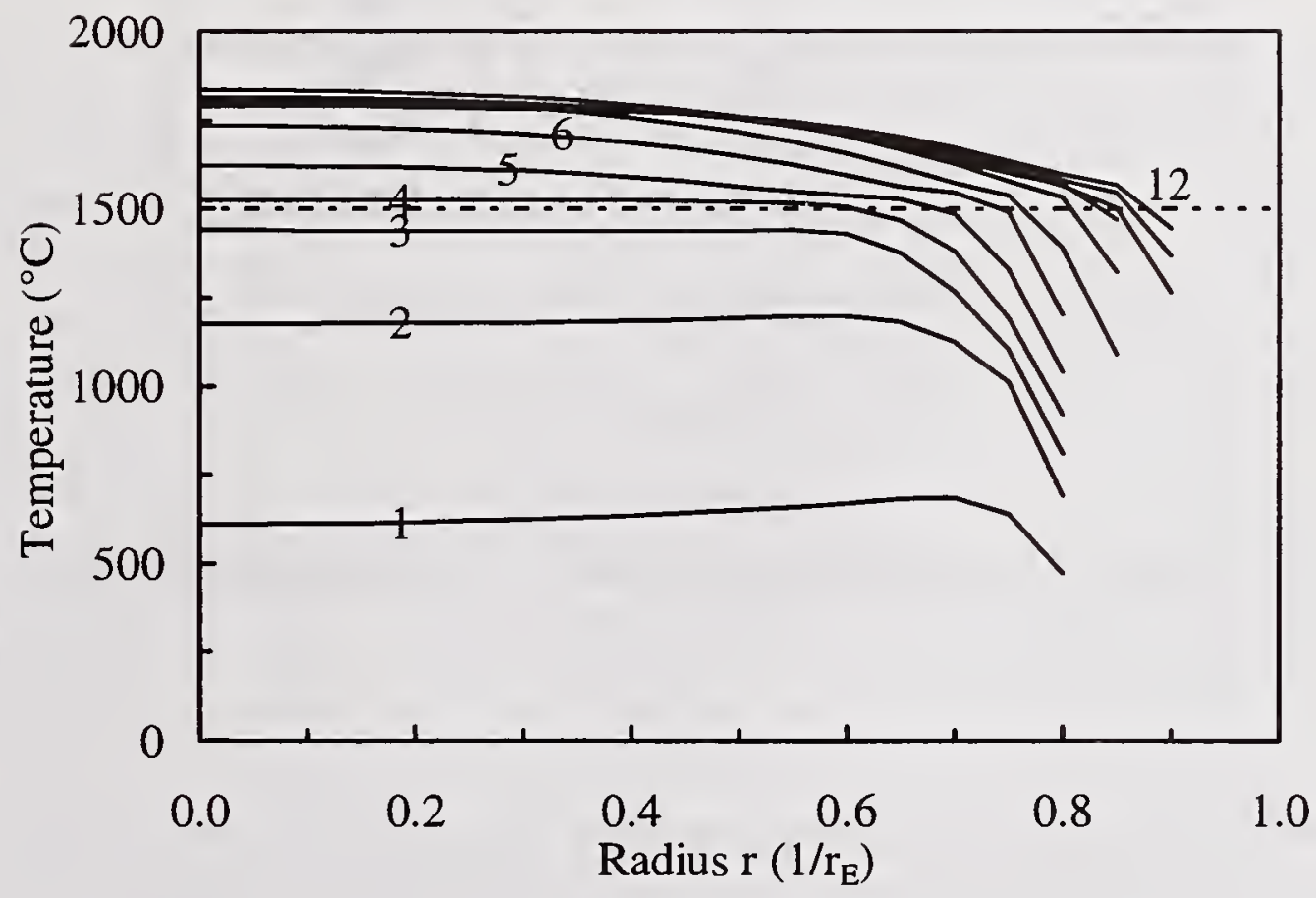

Figure 8 Temperature distribution at the $\mathrm{S} / \mathrm{S}$ interface (Numbers denote the weld time in cycles) 


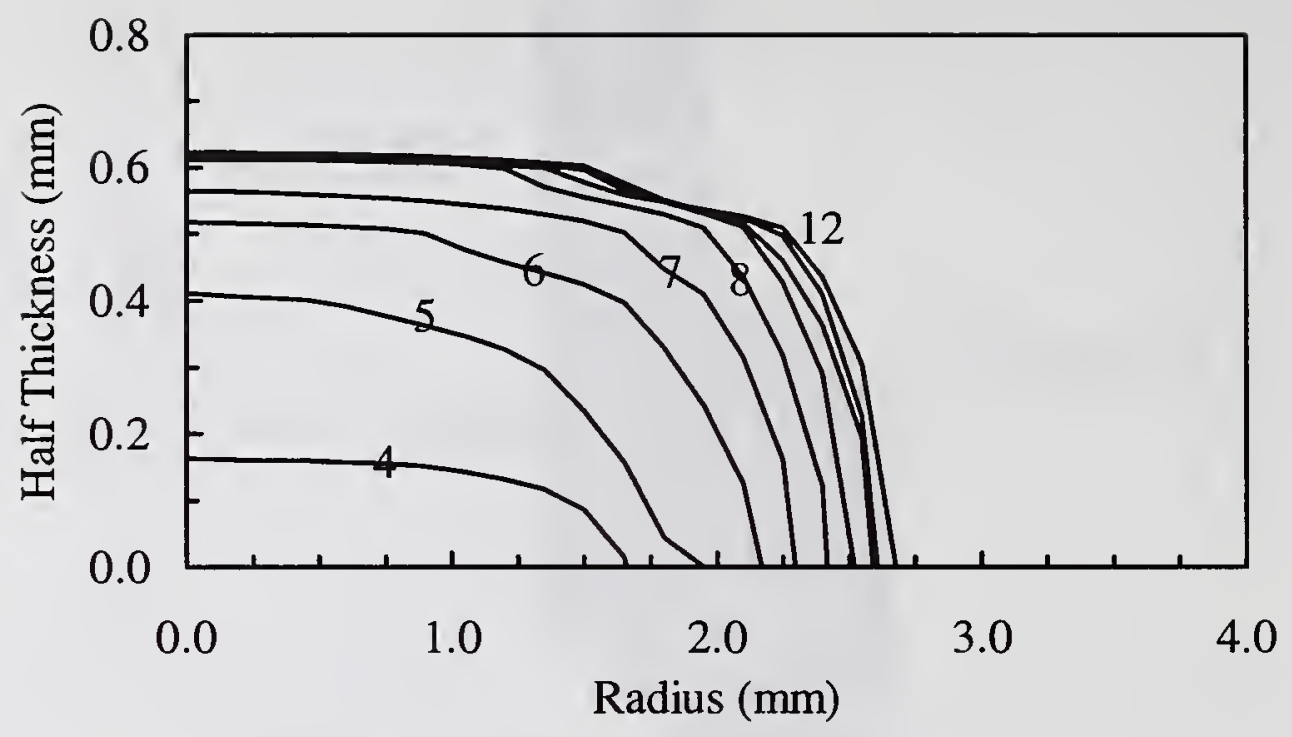

Figure 9 Predicted nugget growth profile

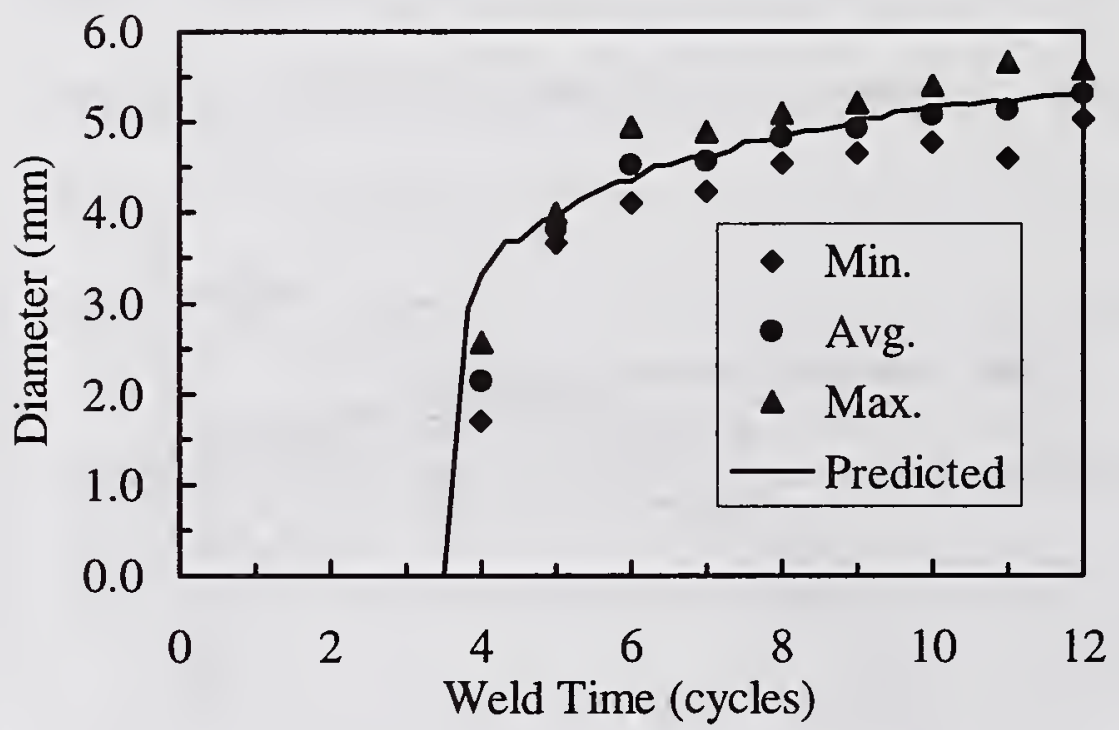

Figure 10 Predicted nugget diameter and measured button size 


\title{
MODELING OF NUGGET FORMAION PROCESS IN RESISTANCE SPOT WELDING
}

\author{
K. Matsuyama*
}

\begin{abstract}
A numerical simulation procedure has been developed based on a dynamic increasing model of contact areas (contact diameters) at electrode-to-sheet interface and at faying one during each resistance spot welding, in which the contact diameters were calculated with a fast estimation algorithm determined by many calculation results for the contact problem of electrode-to-sheet and sheet-to-sheet obtained by FEM with elas-plastic deformation model. And then, the interface (surface) contact resistance existed at each interface has been examined, which is socalled contact resistance. As a result, it is clarified that the interface contact resistance is not so important to make nugget in resistance spot welding, and that the initial contact resistance phenomenon can be explained only by the contact diameter model. Finally, the simulation procedure with the varying contact diameter model and with zero contact resistance can be suitably applied to estimate nugget formation process.
\end{abstract}

\section{INTRODUCTION}

Many investigations have been performed to be developed various types of numerical simulation program in order to examine and predict the nugget formation process in resistance spot welding. The first famous research had been reported by J. A. Greenwood (Ref.1). In the report, only potential and temperature distribution patterns had been computed, where the contact diameter during each resistance spot welding kept constant in given values for electrode-to-sheet interface and faying one, respectively. After that, T. Okuda et al. proposed that an increasing contact diameter patter during welding at each interface is very important to perform highly accurate simulation of actual nugget formation process with numerical simulation (Ref.2), because the generated heat density within weld part is governed by four powers of contact diameter, and only square of welding current value. No modeling of varying contact diameter during welding, however, was used in his study. Only measured contact diameters obtained from actual spot welds were input as given boundary condition to achieve the accurate numerical simulations of nugget growth pattern.

Fully self-consistent model of the nugget formation process had not appeared before the 1980's. In the middle of $1980^{\prime} \mathrm{s}$, simple self-consistent model was developed and applied to explain the influence of current wave forms on the nugget formation process in resistance spot welding steel sheets by K. Nishiguchi et al. (Ref.3). The simulation program consisted of four parts; one is an estimation part of contact diameters at each interface, another is a calculation part of potential

\footnotetext{
* Faculty of Engineering, Osaka University, 2-1 Yamada-Oka, Suita, Osaka 565 Japan
} 
distribution in workpiece to estimate current density distribution, the others are a calculation part of temperature distribution in workpieces and electrodes and modifying part of physical properties for next time step. These calculations were cyclically repeated in each time step till given end time, and it was shown that relatively accurate simulation results could be easily obtained without the so-called contact resistance model.

Most researchers except our group, however, have believed that the contact resistance model is important, and supposed that the contact diameters keep constant during each spot welding in order to run the simulator (Ref.4-6). Especially, in resistance spot welding aluminum alloy sheets, it has been understood that the contact resistance is very important and strongly affects the nugget formation process, although rapidly decreasing phenomenon of the contact resistance less than several ms to about ten ms had been well known in Japan, and same results were reported in USA, too (Ref.7).

So, the author has developed a combined model of the previous contact diameter model and the contact resistance one at electrode-to-sheet and faying interfaces, and examines the effect of contact resistance on the nugget formation process. Moreover, the details of our dynamic increasing model of contact diameter are also introduced for understanding the nugget formation process in resistance spot welding.

\section{A SIMULATION PROCEDURE BASED ON DYNAMIC INCREASING MODEL OF CONTACT DIAMETERS DURING EACH WELDING}

\section{A modeling of nugget formation process and its simulation algorithm}

Two stack sheet model is examined here as a typical target of the numerical simulation. A schematic illustration of the weld part is shown in Fig.1(a). An axisymmetric coordinate system was used to make easy the computation. Current distributions were calculated with a Laplace equation after contact diameters at electrode-to-sheet interface $(d e)$ and at faying interface $(d c)$ were fixed. And then temperature distribution patterns were computed from a thermal conduction equation by FDM. Therefore, the decision of all contact diameters has to be achieved as a first step of the computation. The flowchart is shown in Fig. 1(b).

The computation procedure is separated into eight stages. After input of welding current $i$ and electrode force $P$ at each calculation time step $t$, the contact diameters $d e$ and $d \mathrm{c}$ are estimated with FEM results. And then, the potential distribution is calculated to estimate current density distribution and Joule's heat one not only in workpiece but also in electrode. After that, temperature distribution in both of the workpiece and the electrode are computed to estimate nugget shape and size, etc. At the end of this sequence, material properties are modified for next time step calculation, because the all physical properties have strong temperature dependency. Especially, influence of melting of weld part is remarkable, and moreover the increasing the value of resistivity is also remarkable influence on the calculate result, and stability of such a time step calculation. These stages are repeated by a given end time. The computation was done for quarter area of the illustration in Fig. $\mathrm{l}(\mathrm{a})$. 


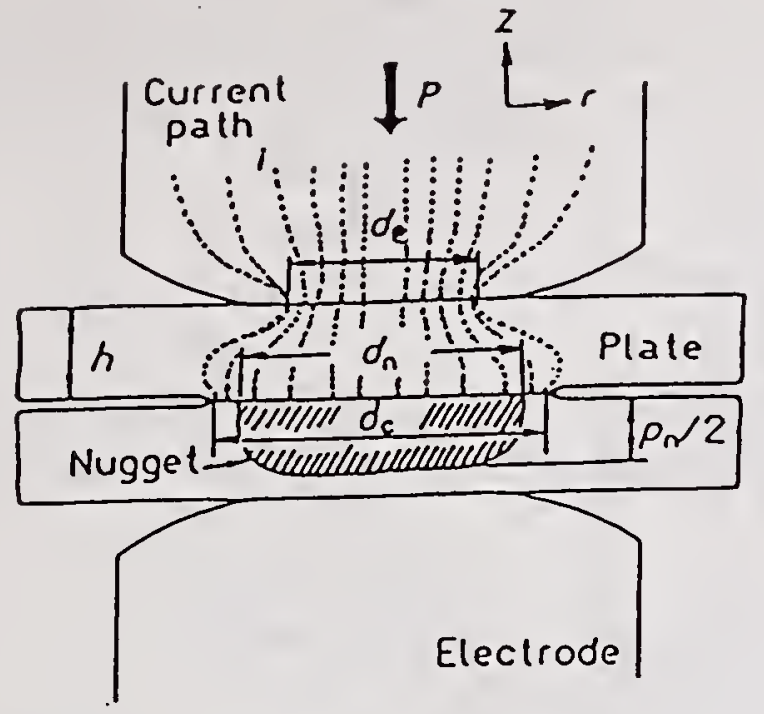

(a) Weld part model

(b) Flowchart for simulation

Fig. 1 Simulation model and its procedure

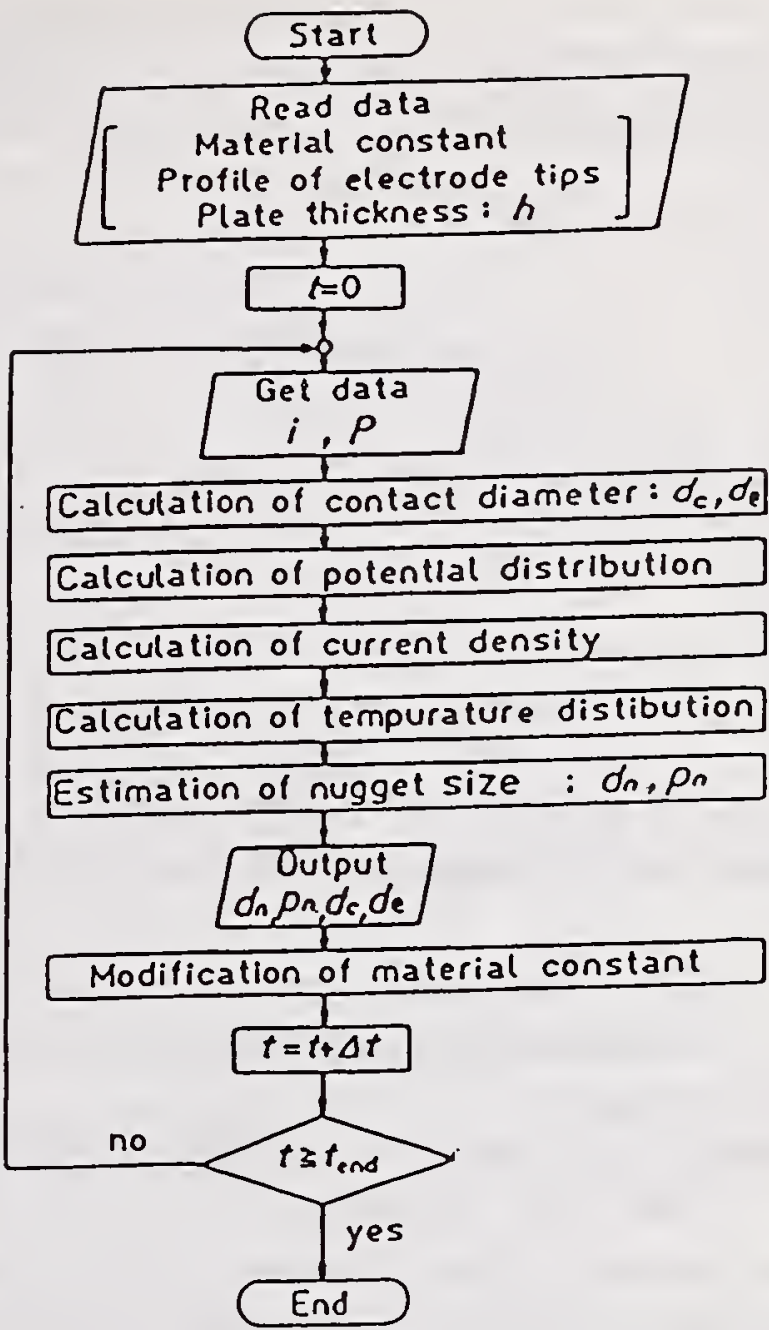

Fast estimation procedure of contact diameters during each welding

Contact diameters at electrode-sheet interface $d e$ and at faying one $d \mathrm{c}$ were originally computed with an elastic and plastic deformation contact model by FEM since the calculated contact diameters change depending on applied electrode force $P$, flow stress curve (yield stress curve in low temperature), electrode tip profile, and Young's modulus $E$. In the calculation, the correct contact diameters were determined by changing the fixed boundary area and checking both of the stress distribution and geometrical fitness at each interface. Very long computation time, however, requires to estimate one nugget formation process if the FEM part is installed in the flowchart to estimate the contact diameters at each time step. So, making some approximation equations was tried to reduce the computation time.

A typical calculated result of the contact diameters is shown in Fig.2. The calculation was achieved for a combination of steel sheets and $\mathrm{Cr}$-copper electrodes, where $R \mathrm{t}$ is initial tip radius before contact. The contact diameter de at electrode-sheet interface increases to be almost proportionate to square root of the applied electrode force $P$. The relation between $d e$ and $P$ is almost same in hardness test to estimate the strength of target material. This means that a simple equation can be made in order to estimate contact diameter with the information of material strength by using various calculation results obtained by FEM. So, relationships between the mean value of yield stress $\sigma y$ at each part in weld and mean contact stress $\overline{\sigma z}$ applied at electrode-sheet interface were examined. An arranged result is shown in Fig.3. Each 


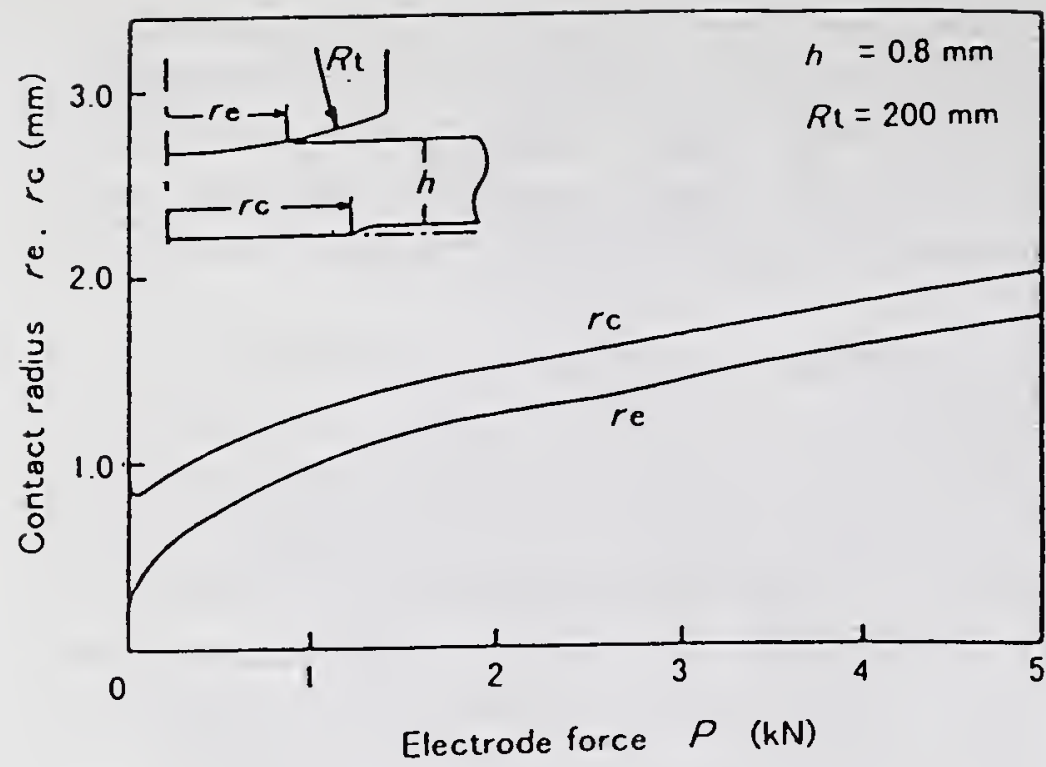

Fig.2 A calculation of contact diameters with an elas-plastic deformation contact model by FEM

Fig.3 Relationship between mean yield stress of weld part and mean axial stress on the interface

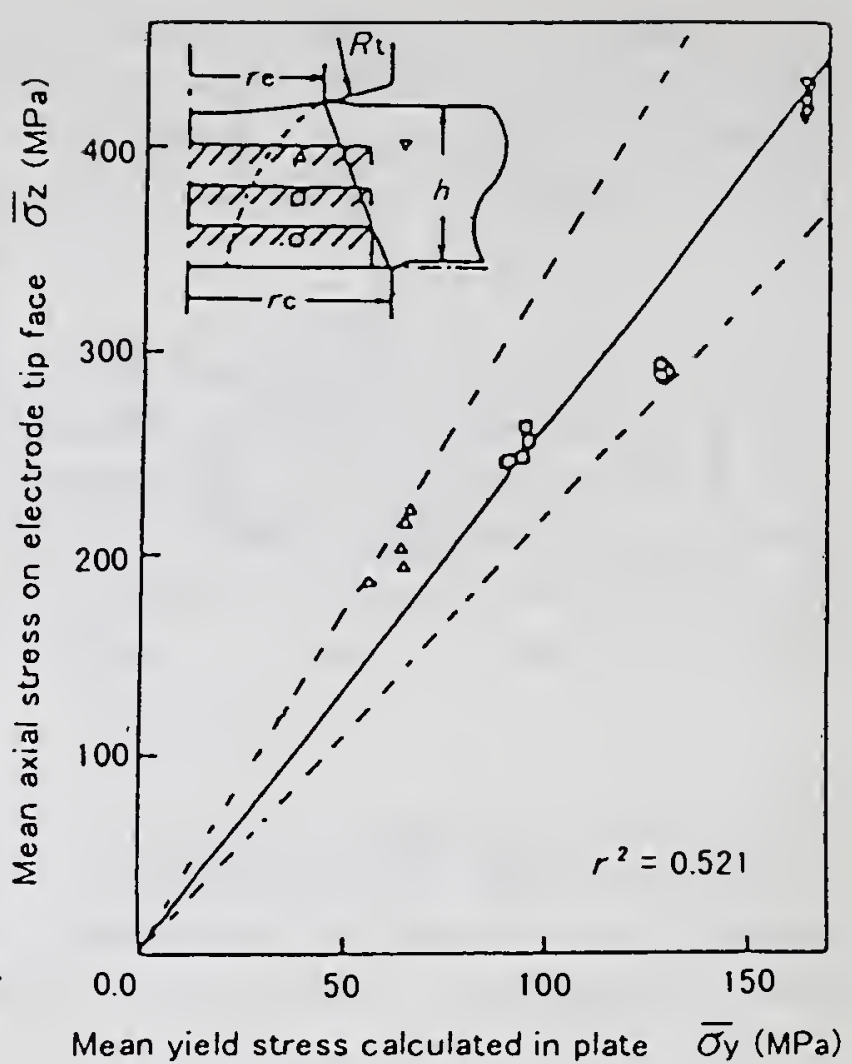

hatched area schematically illustrated in the figure indicates each soften area boundary in weld part. In this case, averaging $\bar{\sigma}$ y was done within the solid line connected from the re radius point at electrode-sheet interface to the $r c$ one at faying interface, and with an exponential function as a weight function toward thickness direction of the sheet.

The result suggests that the contact diameter $d e$ in plastic deformation contact condition can be calculated only with Eq.1. The limit of integration range was a little shifted toward outer side than the solid line shown in Fig. 3 in order to improve the estimated accuracy of contact diameter.

$$
\frac{P}{\pi \frac{d \mathrm{e}^{2}}{4}}=2.6 \bar{\sigma}=2.6 \frac{\int_{0}^{h} \int_{0}^{r(z)} W r \sigma \mathrm{d} d r \mathrm{~d} z}{\int_{0}^{h} \int_{0}^{r(x)} W r \mathrm{~d} r \mathrm{~d} z}
$$

Where, $\sigma_{y}$ : Flow stress or yield stress of workpiece at each location in weld

$W:$ A weight function

$$
W=\exp \left\{-\frac{2(z-h)}{d e}\right\}
$$

$r(z)$ : Limit of integration range at each $z$ position

$$
r(z)=\frac{d \mathrm{c}}{2}-\frac{(d \mathrm{c}-d \mathrm{e}) z}{2 h}+\frac{3}{8} h
$$

$h$ : Sheet thickness of workpiece

Moreover, the contact diameter $d c$ at faying interface changes just like only shifting the de curve 
toward upper side in almost constant value except near zero electrode force condition as shown in Fig.2. Such shifting phenomena can be also recognized in the other calculation results, and the shifting value was almost proportionate to the sheet thickness $h$. So, the contact diameter $d \mathrm{c}$ at faying interface was calculated with Eq.2 as an approximate equation.

$$
d \mathrm{c}=d \mathrm{e}+\alpha h
$$

Where, $\alpha: 0.7-1.0 \quad(0.7-0.8$ for steel, 1.0 for aluminum alloy $)$

The contact diameter $d \mathrm{c}$, however, can be alternatively estimated with following equation made by a concept where a certain soften area contacts at faying interface.

$$
\begin{gathered}
d \mathrm{c}^{2}=8 P / \pi \sigma_{\mathrm{B}} \\
\text { Where, } \sigma_{\mathrm{B}}: \text { Flow stress or yield stress at the end of contact area } \\
P: \text { Actual force applied at the faying surface }
\end{gathered}
$$

This equation can be applicable in a three stack sheet case or more beside the two stack sheet case., and selected instead of Eq.2 when needed high accuracy.

In addition, contact diameter de at electrode-sheet interface in elastic deformation contact condition, which usually appears just at initial period in each welding, was estimated with Eq.4 if the electrode tip shape is dome, and the value of initial radius $R_{\mathrm{t}}$ is given. And then $d \mathrm{c}$ for faying interface was calculated with the Eq.2.

$$
\frac{d \mathrm{e}}{2}=f(h)\left\{\frac{3}{4}\left(\frac{1-v^{2}}{E_{1}}+\frac{1-v^{2}}{E_{2}}\right) R_{\mathrm{t}} P\right\}^{1 / 3}
$$

Where, $P$ : Applied electrode force $\quad v$ : Poison ratio $E_{1}, E_{2}$ : Young's modus of electrode and sheet materials

$$
f(h)=0.5 h+0.81 \quad \text { (correction factor for two stack condition) }
$$

These equations are deduced from elastic and plastic deformation model, but actual behavior of the contact problem has to be treated as a viscoelastic-plastic model. The effect was involved as added following value $\Delta d_{\mathrm{cp}}$ to the contact diameters calculated with the above equations.

$$
\begin{aligned}
& \Delta d_{\mathrm{cp}}=\mathrm{C}_{\mathrm{cp}} h d \mathrm{n} \sqrt{P} \cdot \Delta t \\
& \text { Where, } \mathrm{C}_{\mathrm{cp}}: \text { Constant (tiny value) } \\
& d \mathrm{n}: \text { Nugget diameter } \\
& \Delta t: \text { Time interval from now step to next step in calculation }
\end{aligned}
$$

\section{Potential distribution and current distribution in workpieces and electrodes}

Potential distribution $V$ in workpiece and electrode is calculated with a finite deference equation yielded from the following Poison equation with the control volume method.

$$
\begin{aligned}
& \nabla(\kappa \nabla V)=0 \\
& \text { Where, } \kappa: \text { Electric conductivity }
\end{aligned}
$$




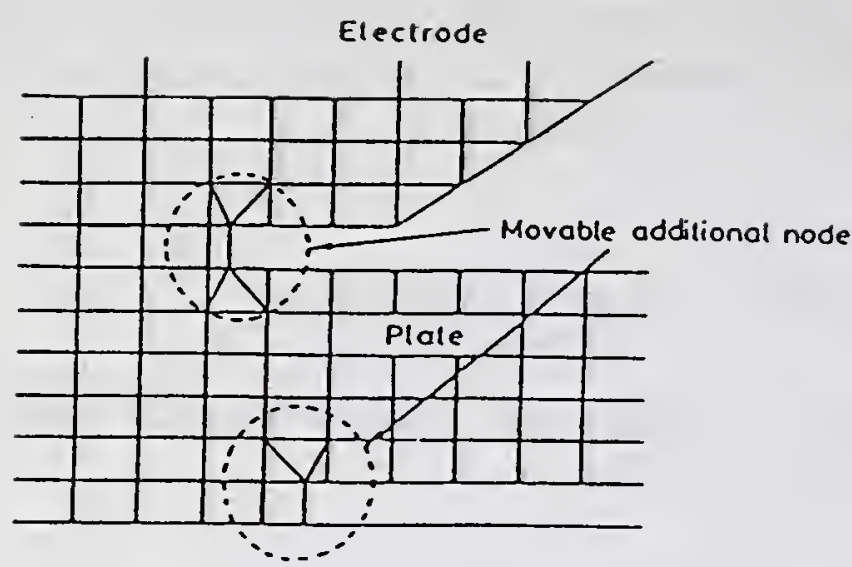

Fig.4 Additional node used to improve the simulated accuracy

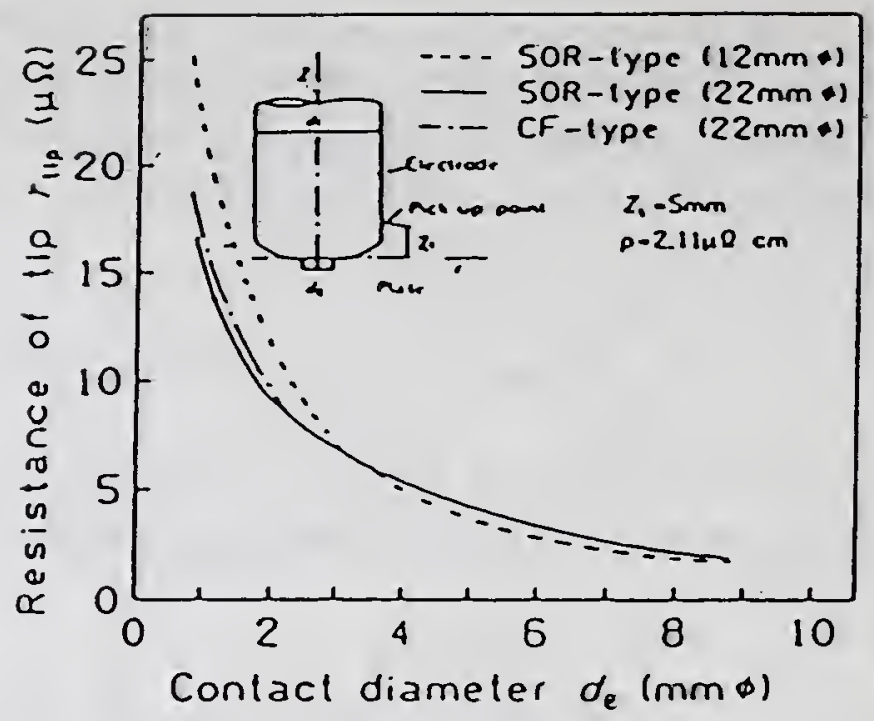

Fig.5 Calculated resistance of electrode tip

The calculation was achieved with a movable node system as shown in Fig.4, since the value of contact diameters is remarkably influence on the generated heat density in resistance welding. Variable size mesh system was also used to decrease the total mesh number within electrode tip, and reduce computing time in keeping high accuracy. Moreover, the elimination method was employed for the solver, since the simulation program can also apply in a condition with shunting current to some previous spot welds.

The value of voltage drop in electrode is not so small. Some calculated results are shown in Fig.5. The results are indicated with resistance value for easy understanding, and arranged as a function of the contact diameter at electrode-sheet interface. The resistance value in small contact diameter is almost same order as that in resistance spot welding aluminum alloy sheets.

In addition, a conformal mapping technique was employed to suppress the influence of current concentration at the edge of contact area at each interface, and improve the calculated accuracy of generated heat quantity at and near the edge part of each contact area. The conductance matrix values near the contact edge part were modified with correction factors determined by the conformal mapping calculation.

Current distribution within sheets and electrode tips are calculated from the potential distribution patterns. Each calculation was achieved at each time step to determine Joule's heat distribution in electrode tips and workpieces.

\section{Temperature calculation at each time step}

Temperature distribution in workpieces and electrode tips at each time step was calculated with another finite difference equation deduced with the following heat conduction equation.

$$
C \sigma \frac{\partial T}{\partial t}=\nabla(K \nabla T)+\rho \delta^{2}
$$

Where, $\mathrm{C}:$ Specific heat, $\sigma$ : Density, $K$ : Thermal conductivity,

$\rho$ : Resistivity, $\delta:$ Current density, $T:$ Temperature 
Latent heat at melting temperature was treated at each grid, and convection effect within molten nugget was treated as an increment of thermal conductivity. The calculation formula were fixed up with an implicit scheme, and the calculations were carried out with the Gauss-Seidel iteration method for the same mesh system in the potential calculation. 1/300s (for steel) to $1 / 600 \mathrm{~s}$ (for aluminum alloy) was selected for the time step duration. Moreover, the conductance matrix value near the contact edge part were also modified with the same manner in the electric conductance matrix.

\section{Modification of material properties for the next time step}

After such calculations, the calculated results of potential distribution, current distribution, temperature distribution, the consumption state of latent heat, and contact diameters were recorded on a floppy disk. Nugget size and shape were determined with the temperature distribution and consumption state of latent heat at each grid. And then, all material properties; thermal conductivity, specific heat, density, resistivity, and flow stress, were updated for the next time step calculation with a predicted temperature pattern, which was estimated with the present and the previous temperature distributions. Moreover, strain rate for determination of the flow stress value was supposed to be equal to the volume expansion rate caused by temperature rise.

\section{EXAMINATION ON THE INFLUENCE OF INTERFACE CONTACT RESISTANCE}

\section{Estimation of the interface contact resistance caused by surface roughness}

First, the interface contact resistance, so-called surface resistance, was theoretically examined to make a contact resistance model inserted into each interface as one dimensional element because the concentration resistance, which is one of contact resistance, can be automatically treated with the simulation program.

Now, sheet surface is assumed to be covered with many corns of same size as shown in Fig.6(a). In this case, the collapse state of corns can be estimated with the perfect plastic deformation theory(Ref.8), and the interface contact resistance can be calculated as only within truncated corn. The calculated results are shown in Fig.6. Figure 6(a) shows the relationship between nondimensional load applied on the corns and collapse ratio of the corns which was determined with the reducing ratio of height. The collapse ratio decreases with decreasing corn number $N$ existed in same contact area. Figure 6(b) indicates the relative surface (interface) contact resistance value calculated only within the truncated corn. The value remarkably increases with decreasing the collapse ratio.

At first sight, it seems to be suggest that the interface contact resistance is important, but the actual value is not so high, since the reference value $R \infty$ shown in Fig. $6(\mathrm{~b})$ is very small. For example, the value is only $26 \mathrm{n} \Omega$ in usual contact condition for steel sheet in room temperature, and $5 \mathrm{n} \Omega$ for aluminum alloy sheet. These results means that the interface contact resistance caused by workpiece surface is not so high, and tiny.

Of course, the interface contact resistance becomes high value if there is high resistivity film layer on the workpiece surface. No large difference by brushing the workpiece surface, 


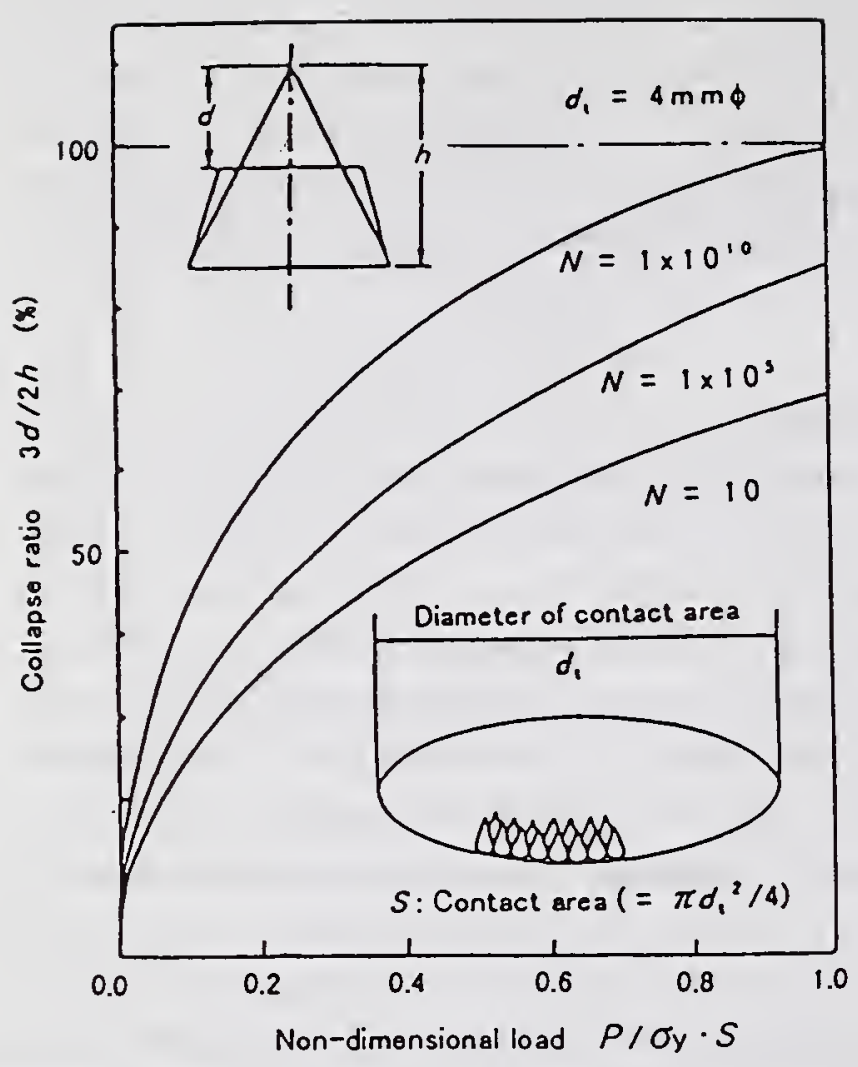

(a) Load vs. collapse ratio

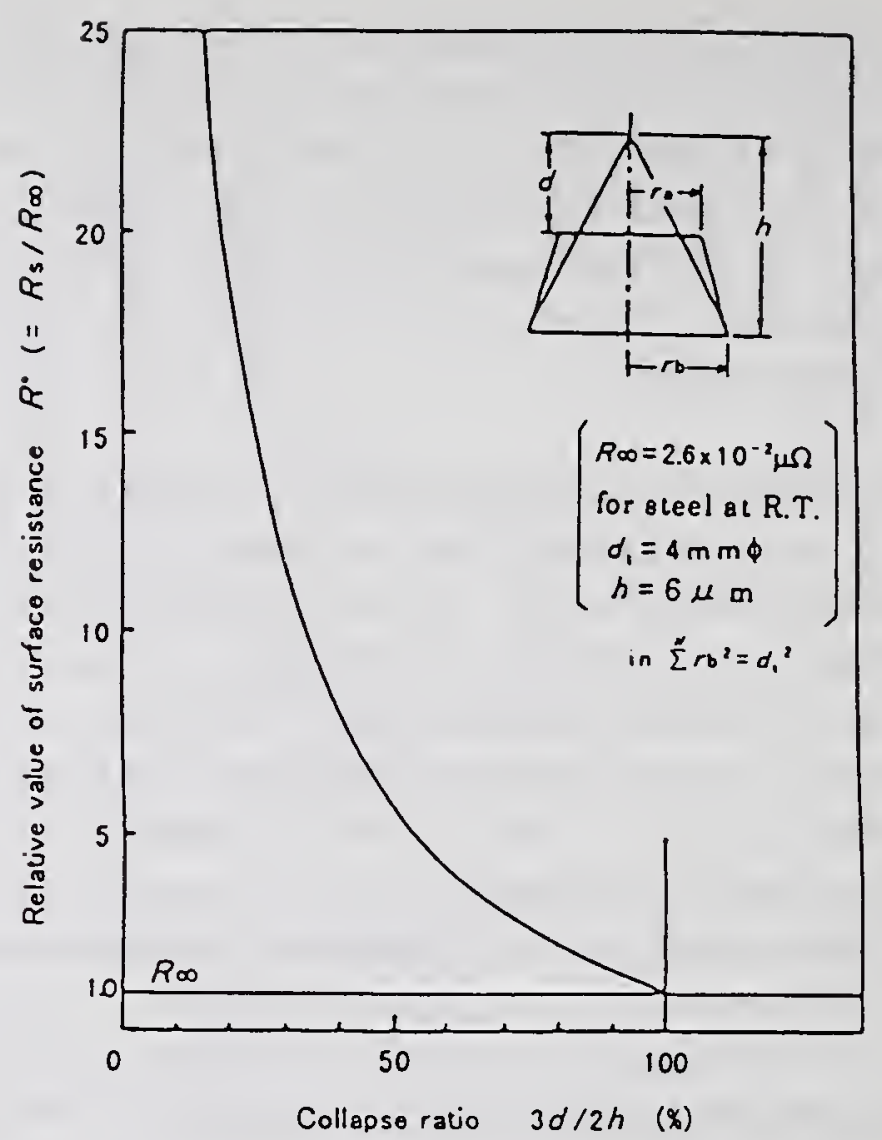

(b) Collapse ratio vs. surface resistance

Fig.6 Estimation of interface contact resistance with perfect plastic deformation model when the surface profile can be approximated to be gathered many small corns

however, can be observed even in nugget formation process and in dynamic voltage between tips (Ref.9). This means that the high resistance film on workpiece surface should not be considered in usual welding condition and materials.

On the contrary, roughness height on electrode tip surface is not so low. For example, the height after many resistance spot welds of aluminum alloy sheets was measured in almost $20 \mu \mathrm{m}$ - $50 \mu \mathrm{m}$ or more. Moreover, the copper electrode surface is allied by the workpiece material, and the resistivity increases after many welds. If the resistivity becomes five times, and roughness height is $50 \mu \mathrm{m}$, the reference value $R \infty$ can be supposed to be about $150 \mathrm{n} \Omega$ in $d_{\mathrm{t}}=4$ $\mathrm{mm} \phi$. This result suggests that electrode tip roughness is important for understanding the interface contact resistance.

\section{Influence of the interface contact resistance on the nugget formation process}

The above described interface contact resistance was installed into the numerical simulation program as a one dimensional element without volume, the value was set up as a function of temperature as shown in Fig.7. The interface contact resistance value is defined as a specific contact resistance, and the value in low temperature was determined by the above described reference resistance value $R \infty$ and estimated collapse ratio value $(=40 \%)$. The decreasing pattern of the curve was also determined with the temperature dependence of the flow stress curve for the workpiece, since the aluminum alloy becomes soft at lower temperature than that of electrode copper. Moreover, it was supposed that the same interface contact resistance exists at the 


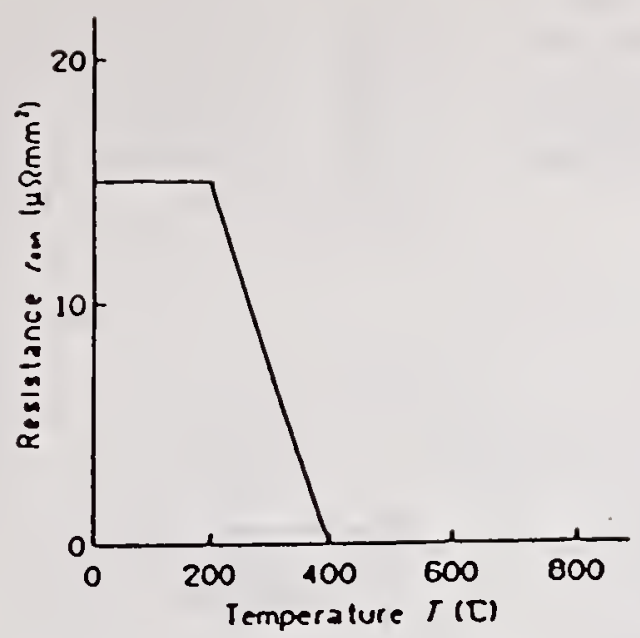

Fig. 7 Specific contact resistance used to calculate interface contact resistance for aluminum alloy sheet

faying interface to enlarge the contact resistance effect although it is over-estimation.

Figure 8 shows a typical simulated results with the interface contact resistance model. One-dotted chain line shown in Fig.8(a) indicates the interface contact resistance calculated for the electrode-sheet interface, and a broken line for the faying interface. Both values are pretty small, and rapidly reduced comparing the total resistance value $r_{\text {all }}$ displayed with a solid line. The nugget growth patterns calculated with and without the contact resistance model

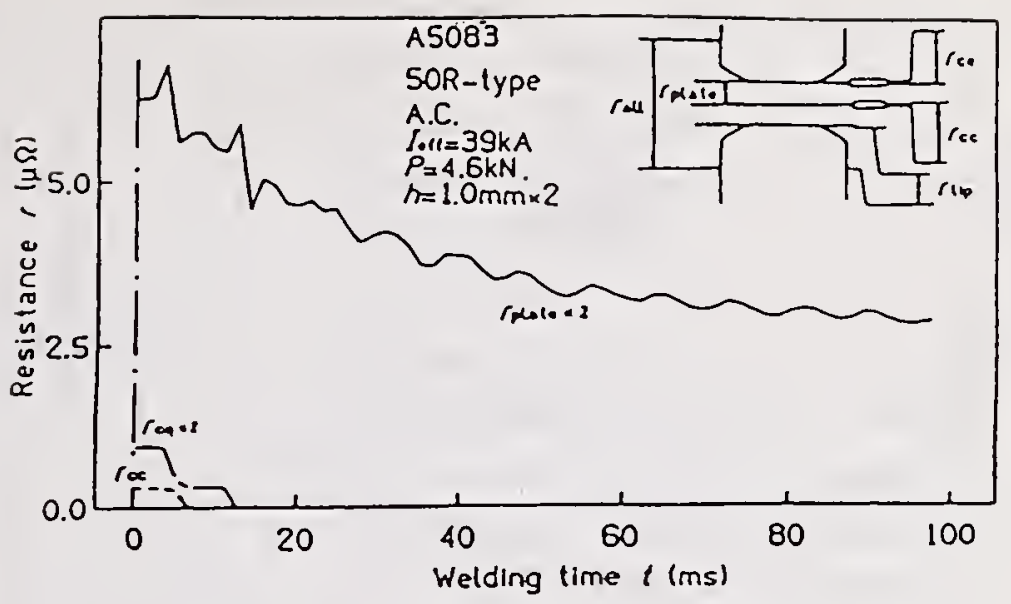

(a) Computed dynamic resistance

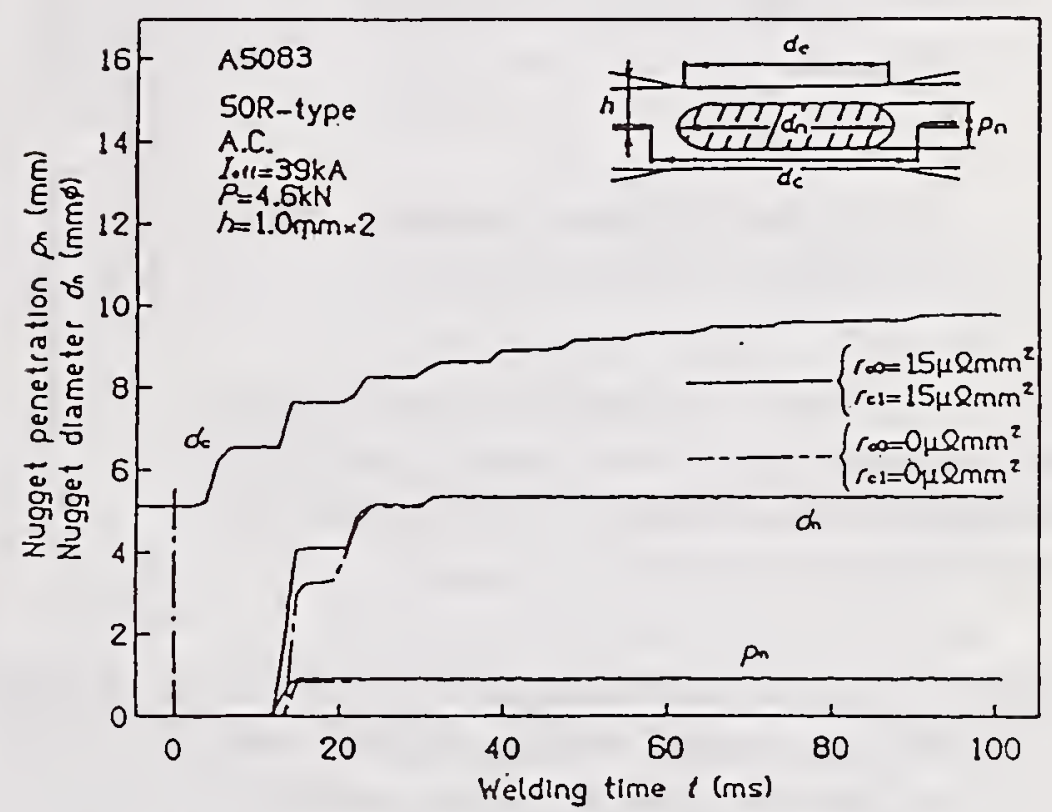

(b) Computed nugget formation process

Fig.8 Influence of interface contact resistance on nugget formation process

are shown in Fig.8(b). Solid line indicates the result with the interface contact resistance, and one-dotted chain line is in that without the contact resistance model. $0 \mu \Omega$ is corresponding to the no contact resistance model in the simulation. There is a little difference in the nugget growth patter, and the difference appears only at the initiation period of nugget growth. The interface contact resistance did not affect the maximum nugget size. Moreover, any difference in increasing pattern of contact diameter could not be observed in this case.

This result suggests that the contact resistant is not so important for simulation of the nugget formation process in normal resistant spot welding conditions except the case that the electrode tip surface is remarkably rough, there are some deep dimples, and the surface material is highly alloyed by the workpiece material.

\section{Understanding of the initial contact resistance by the new concept}

The reason why the high initial resistance appears in the resistance spot welding aluminum alloy sheets was examined with the dynamic increasing model of contact diameters. The simulation 

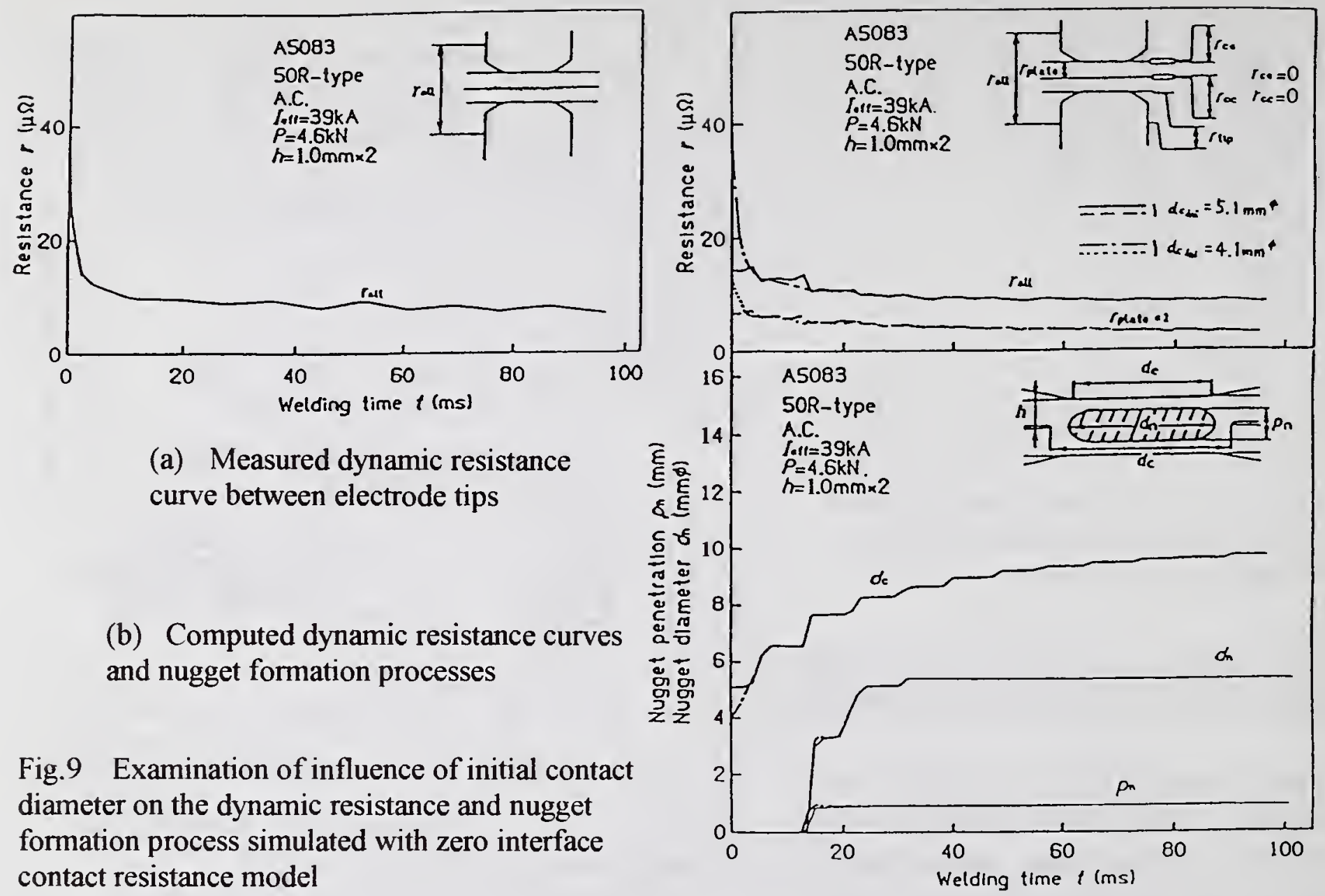

Fig.9 Examination of influence of initial contact diameter on the dynamic resistance and nugget formation process simulated with zero interface contact resistance model

(a) Measured dynamic resistance curve between electrode tips

(b) Computed dynamic resistance curves and nugget formation processes

results without the interface contact resistance model are displayed in Fig.9(a), and observed result of the resistance between tips is shown in Fig.9(b). The experiment was carried out in the same welding condition as in the simulation. The calculations were achieved in two initial contact diameter conditions. A one-dotted chain line and a dotted line indicated in the upper figure of Fig.9(a) show the calculated result for the resistance between tips and twice value of the resistance of workpiece, and simulated in the $d_{c, \text { ini }}=4.1 \mathrm{~mm} \phi$. A solid line and a broken line in the upper figure also show the same resistance values obtained in the $d_{\mathrm{c} \text {,ini }}=5.1 \mathrm{~mm} \phi$. Two solid lines and two broken line in lower figure of Fig. 9(a) show the calculated results of contact diameter $d c$ at faying interface, nugget diameter $d_{\mathrm{n}}$ and nugget penetration $p_{\mathrm{n}}$

This difference of initial contact diameter only influenced on the initial dynamic resistance, which scarcely affected the nugget growth pattern. The high initial resistance curve observed in actual resistance spot welding of aluminum alloy sheets was explained only with the dynamic increasing model of contact diameter during welding. This means that the numerical simulation of resistance spot welding can be achieved with no contact resistance model.

\section{ADAPUTABILITY OF THE SIMULATION MODEL BASED ON THE CONTACT DIAMETER CONCEPT}

Adaptability of the present simulation procedure made of varying contact diameter concept with 


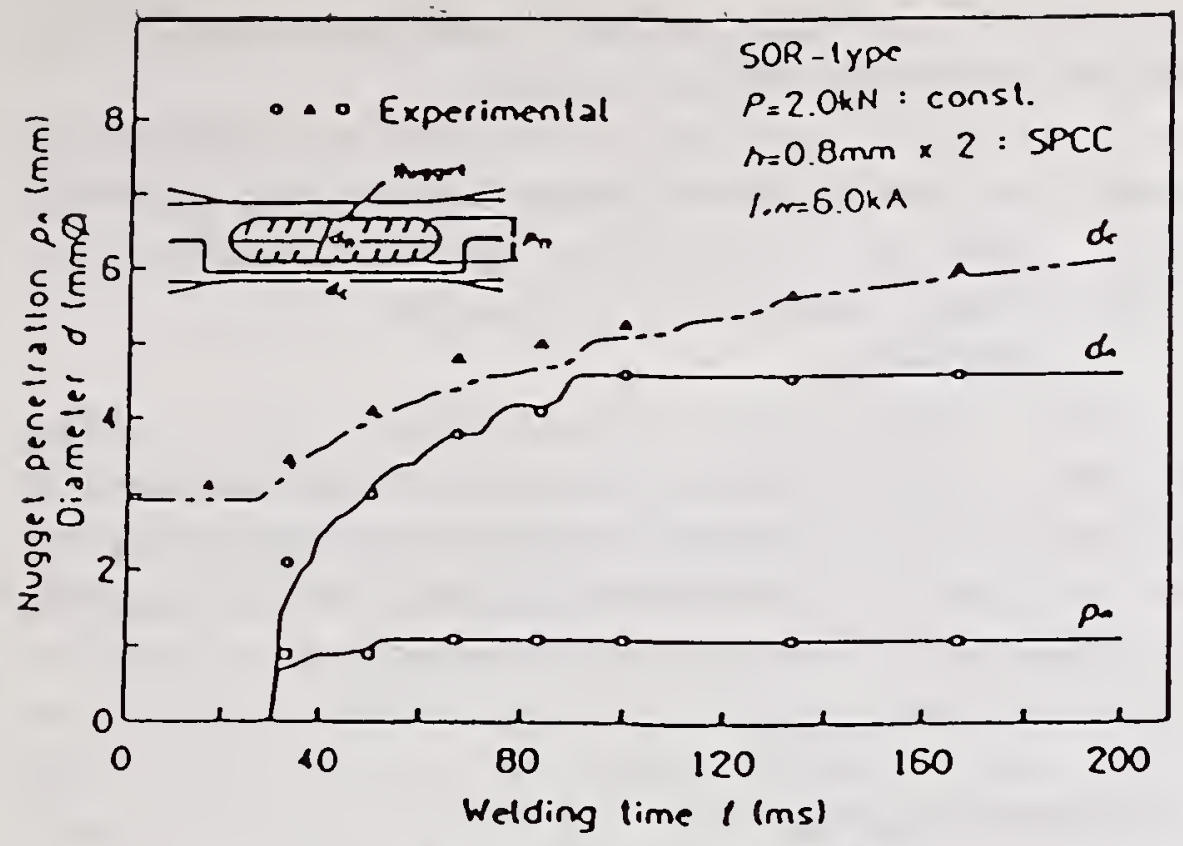

(a) Nugget growth pattern

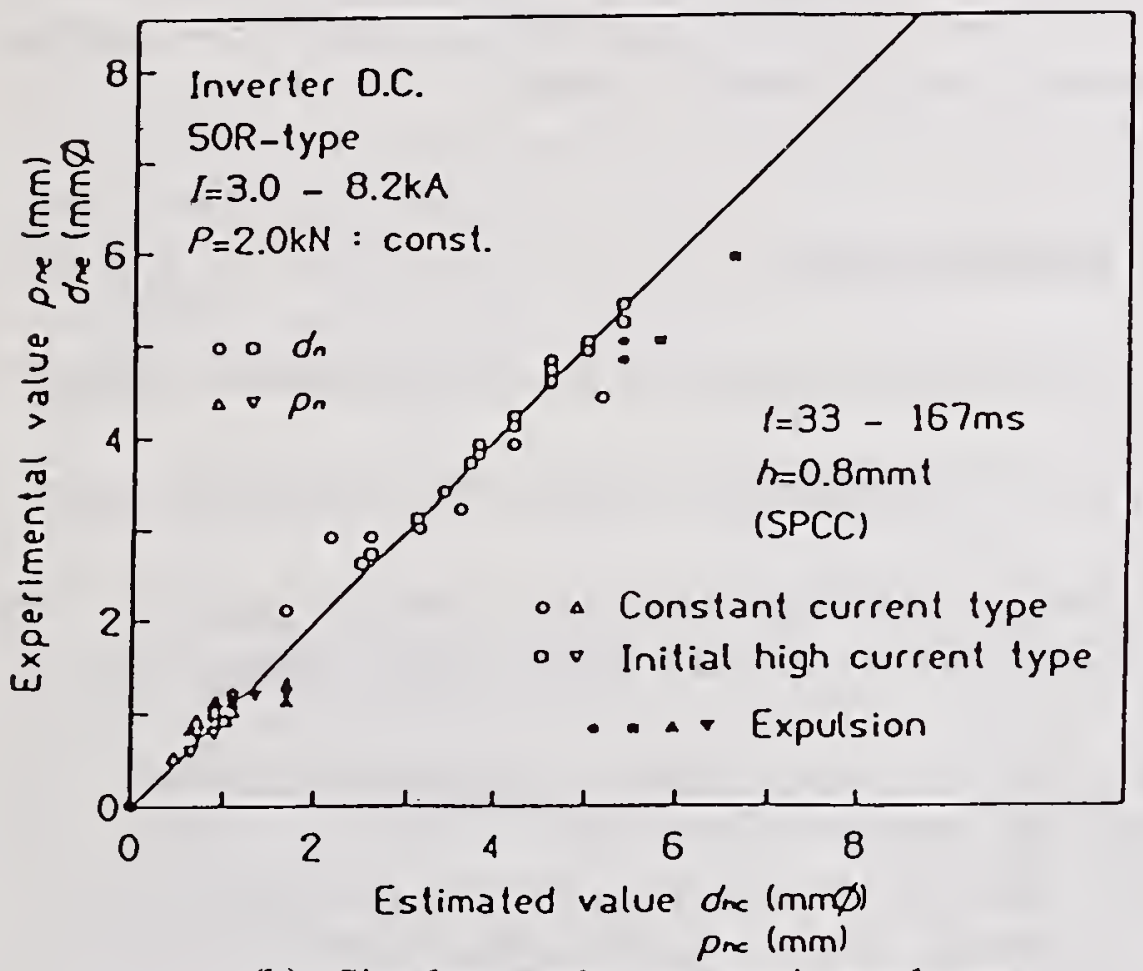

(b) Simulate results vs. experimental ones

Fig. 10 Adaptability of the present simulation procedure with dynamic increasing model of contact diameter zero interface contact resistance model was examined with some experiments. The experiments were carried out with AC spot welding machine for aluminum alloy sheets, and HF-DC spot welding machine for steel sheets. Nugget diameter $d_{\mathrm{n}}$ and nugget penetration $p_{\mathrm{n}}$ were measured from etched cross-section at center of weld part, and the contact diameter $d c$ at faying interface was measured from the corona bond diameter after removing one side sheet by a torsion tool.

Typical comparison results between the estimated and experimental ones are shown in Fig.10. Figure 10(a) indicates the nugget growth pattern, and Fig. 10(b) shoes the relationship between the estimated results and experimental ones for nugget size. Both figures indicate that the estimated and experimental results are good agreement with each other. Similarly good agreement was observed for the aluminum alloy sheet case although the results does not shown here.

So, it is concluded that the simulation can be successful if contact diameters are correctly treated.

\section{SUMMARY AND CONCLUSIONS}

A numerical simulation procedure was developed based on a dynamic increasing concept of contact diameter to examine the influence of contact resistance on the nugget formation process in resistance spot welding. As a result, the contact resistance is not so important in usual resistance spot welding not only steel sheets but also aluminum alloy ones. And then, the adaptability without the contact resistance model was made clear by comparing experimental results. In addition, the simulation program can be applied to estimate the critical expulsion 
current condition in resistance spot welding if it is supposed that the molten nugget diameter becomes larger than the contact diameter at the faying interface.

This simulation program, however, does not almighty, since the accurate flow stress data must be needed to run program. The flow stress data in high temperature near melting point over softening temperature are not completed. There are only few data. So, the simulation has to be used for qualitative examination of the resistance spot welding phenomena.

This problem can be resolved with some monitoring date during welding. One is voltage between tips, and the other is welding current. If both data are measured as continuous data, the dynamic increasing pattern of contact diameters can be estimated with these data and the present described simulation program (Ref.10-11). This method can be also applied to in-line quality monitoring of weld, and it is useful to examination adequacy of the material properties used.

\section{ACKNOLEDGEMENT}

I would like to express my thanks to Dr. K. Nishiguchi for his good advise, and Mr. A. Ishihara and Mr. K. Uchida for their help in calculation and experimental work.

\section{REFERENCES}

1. Bently, K. P.; Greenwood, J. A. et al. 1963, Temperature distribution in spot welds, British Welding Journal, 10(12), 613 to 619

2. Yamamoto, T; and T. Okuda, T 1970, A study of spot welding of heavy gage mild steel, IIW Document, No.III-389-70

3. Nishiguchi, K.; and Matsuyama, K.1987, Influence of current wave form on nugget formation phenomena when spot welding thin steel sheets, Welding in the World, $25(11 / 12), 222$ to 245

4. Tsai, C. L. et al. 1992, Analysis and development of a real-time control methodology in resistance spot welding, Welding Journal, 69(12), 319-s to 351-s

5. Browne, D. J. et al. 1995, Computer simulation of resistance spot welding in aluminum: Part 1 and 2, Welding Journal, 74(10), 339-s to 344-s \& 74(11), 417-s to 422-s

6. Na, S. J.; and Park, S. W. 1996, A theoretical study on electrical and thermal response in resistance spot welding, Welding Journal, 75(10), 233-s to 241-s

7. Thorton, P. H.; Krause, A. R.; and Davies, R.G. 1996, Contact resistance in spot welding, Welding Journal, 75(12), 402-s to 412-s

8. Ling, F. 1975, Surface mechanics, John Milky Sons

9. Matsuyama, K. 1996, Numerical simulation of nugget formation process in resistance spot welding aluminum alloys and its application to the quality monitoring of spot welds, IIW Document, III-1060-96

10. Matsuyama, K; Satoh, H.; and Nishiguchi, K 1990, Computer-aided monitoring system of nugget formation process, Proc. of Fifth International Symposium of JWS, 577 to 582

11. Matsuyama, K. 1994, Procedure for reducing the solution time by using numerical simulation techniques for measurement of weld nugget diameter,IIW Document, III-1028-94 


\title{
THE COUPLED ELECTRICAL-THERMAL-MECHANICAL PROCESS ASSOCIATED WITH ALUMINUM RSW
}

\author{
Xin Sun", Pingsha Dong", Menachem Kimchi ${ }^{\dagger}$
}

\begin{abstract}
A comprehensive analysis procedure has been developed to perform the incrementally coupled thermal-electrical-mechanical analysis to simulate the resistance spot welding process of aluminum alloys. Since aluminum has high thermal conductivity, low melting temperature and low yield strength, distortions resulting from resistance spot welding are expected to be more severe than that for steel. Compared with most of the published work in this area, this paper takes into account the incremental changes in sheet deformed shape, contact area and current density profile as well as large deformation effect. It describes electrical and thermal contact resistivities to be not only functions of contact temperature, but also functions of pressure.

This new procedure is suitable for predicting many important parameters such as contact area changes, electrode movement, dynamic resistance as well as other factors that affect the weld quality such as weld size, weld indentation, sheet separation and weld residual stresses. It can also be a very powerful tool for studying nugget development and for analyzing the mechanisms of electrode wear and weld cracking.
\end{abstract}

\section{INTRODUCTION}

Spot welding is the major joining technique used in the automotive industry. With the rapidly increasing use of aluminum alloys for automotive body and structural components, robustness and control of aluminum spot welding have received considerable attention. Spot welding for steel and for aluminum alloys share the same principles. However, the productivity of aluminum spot welding is relatively low than steel and the control of weld quality is much more difficult. Since aluminum alloys have higher thermal and electrical conductivity, higher welding current and electrode force are required for the weld nugget to form. The resulting higher stress and current density concentration would in turn lead to faster electrode wear.

Because the RSW process is so fast (often done within $200 \mathrm{msec}$ ), it is very difficult to experimentally monitor the important transient data such as current density distribution and temperature distribution, etc. Furthermore, the intrinsic nature of the process variation itself gives rise to large scatter of the experimental measurement data on nugget growth and size.

Numerical modeling can be a very effective and inexpensive tool to study the RSW process in quantitative details. Greenwood (Ref. 1) investigated the temperature distributions in spot welding by solving a two dimensional boundary value problem using finite difference method.

\footnotetext{
* Battelle Memorial Institute, Columbus, OH 43201.

${ }^{\dagger}$ Edison Welding Institute, Columbus, OH 43210.
} 
Han et. al (Ref. 2) conducted a similar study but allowed for variations in the physical properties of the workpiece. Cho and Cho (Ref. 3) developed a finite difference scheme to predict the temperature and voltage distribution incorporating the thermoelectric interaction at the interface in the weldment. More recently, the similar problem has been addressed by several authors using the advanced finite element method. Among them, are Nied (Ref. 4), Browne et al. (Ref. 5 and Ref. 6), Tsai et al. (Ref. 7), Sheppard (Ref. 8), Murakawa (Ref. 9) and Dong et al. (Ref. 10).

The complete spot welding process is a physical coupling of electrical-thermal and mechanical processes. Since aluminum has high thermal conductivity, low melting temperature and low yield strength, the distortions resulting from RSW are expected to be more severe than that for steel. Therefore, the conventional decoupled approaches that model squeezing cycle and welding cycle in sequential order will no longer be sufficient to model this complex behavior. Since there is no commercial software in the market that is able to handle these simultaneous coupling effects, an incrementally coupled finite element analysis procedure has been developed in this study. The commercial FEA code ABAQUS is used and an infrastructure has been set up to automate the modeling process. As a final product of the procedure development, a simulation run can be done by typing one command on the UNIX command line.

The modeling procedure is then validated with nugget growth data obtained from experimental measurements. Excellent predictions are obtained compared with experimentally observed nugget size. Final weld residual stresses and weld indentation are also obtained using this analysis procedure. It is shown that the simulation will not only provide useful insight on nugget growth history, it also provides valuable information on the electrode wear mechanisms.

\section{MODELING PROCEDURE}

A typical 2" radius faced truncated cone electrode is used to weld $2.0 \mathrm{~mm}$ gauge AA5754 sheets. Axisymmetric models as shown in Figure 1 are used in both thermal-electrical and thermalmechanical analyses. Different welding currents and electrode forces are used in two sets of experiment and the model validation will be discussed in the following sections.

To simulate the spot welding process, an incrementally coupled approach is used. First, the squeeze cycle is modeled by a mechanical analysis. Contact surface interactions between the electrode-sheet interfaces and faying interface are modeled by the concept of contact pair. Results from the squeezing cycle mechanical analysis, including deformed shape, contact pressure, contact radius, gap opening and etc. are then passed into the next step electrical-thermal analysis in which the welding current is applied. The temperature distribution resulting from the above mentioned electrical-thermal analysis is then used as initial conditions for the subsequent thermal-mechanical analysis. This updating procedure repeats itself for every half cycle until the entire welding cycle is totally completed. The flow chart of the analyses procedure is shown in Figure 2. Modeling details for each analysis module are discussed next.

Following the mechanical analysis for the $10^{\text {th }}$ welding cycle, a sequentially coupled thermalmechanical analysis is performed to determine the weld residual stresses. To simulate the joining effect, nodes on the faying interface which have experienced temperature higher than 
solidus of AA5754 are joined together using multiple point constraints (MPC). With these nodes being tied, the weldment is cooled down to room temperature and the final weld residual stresses are predicted.

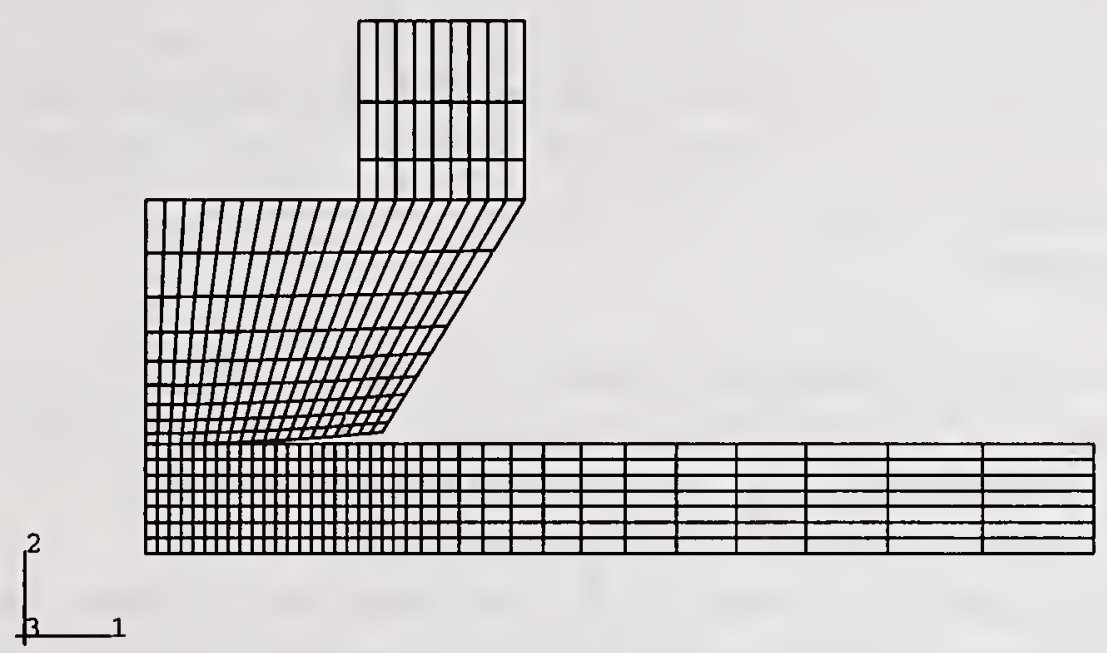

Figure 1. Model geometry

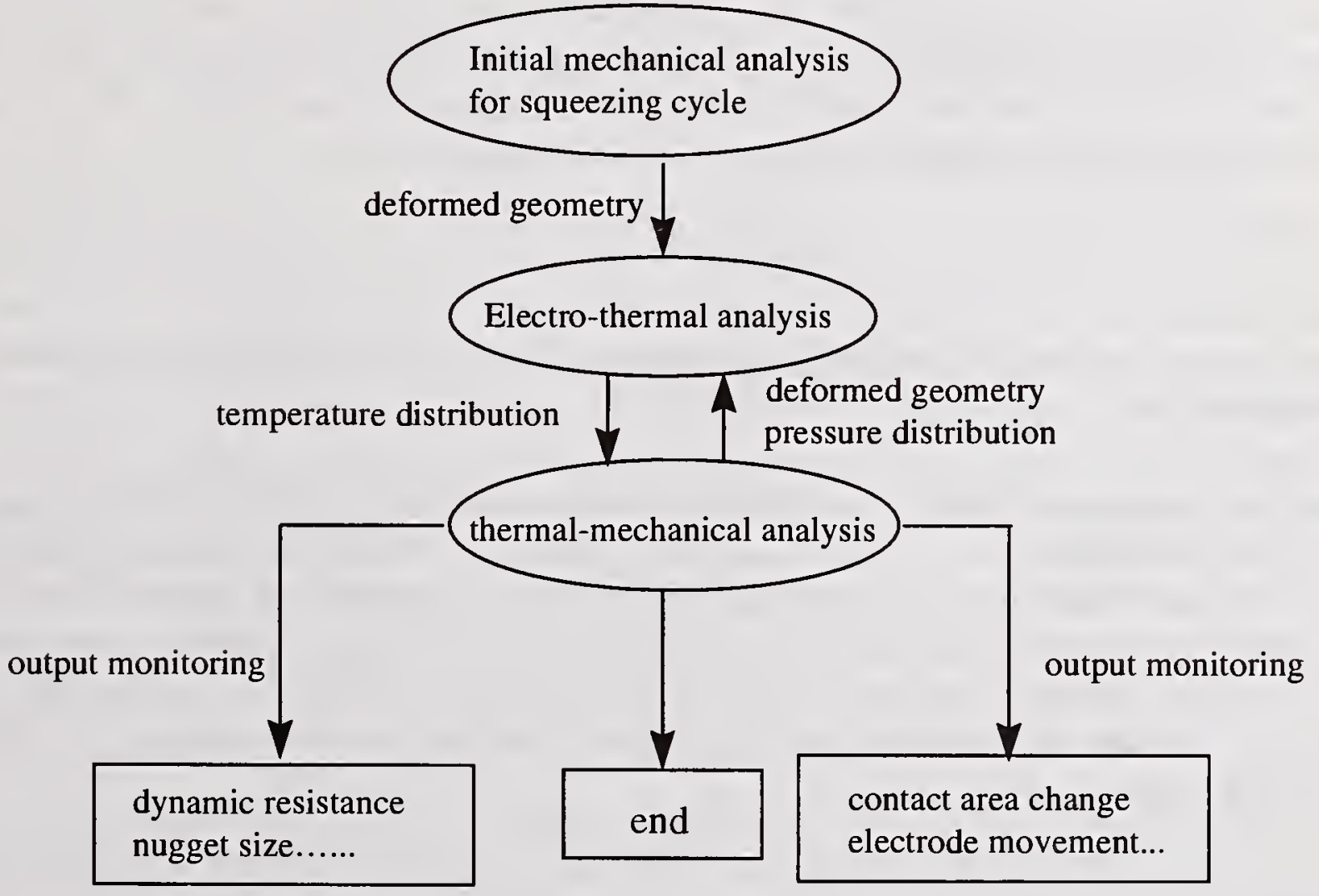

Figure 2. Analysis flow chart of the coupled electrical-thermal mechanical analysis 


\section{Thermal-Mechanical Analysis}

The 4-node axisymmetric element CAX4 is used for mechanical analysis. Temperature dependent material properties are used for both the AA5754 sheets and the copper electrodes (Ref. 11), large deformation effect is also considered in the model. Nodes on the base of the lower electrode is constrained from movement in the vertical direction and uniformly distributed electrode force is applied to the upper electrode. Three contact pairs are set up between the upper electrode to sheet interface, faying interface and the lower electrode to sheet interface respectively. This ensures that the surfaces in contact will not interpenetrate each other. To improve convergence, softened surface properties are used and small sliding effect is included.

\section{Electrical-Thermal Analysis}

The electrical-thermal analysis is done using element DCAX4E. Material properties are again dependent upon temperature. The cooling water temperature is set to be $25^{\circ} \mathrm{C}$ and convection effect is modeled by film coefficient.

The deformed configuration of the electrode and sheet assembly obtained from the previous mechanical analysis step is used as the input for electrical thermal analysis. Contact radius and contact pressure obtained in the mechanical analysis are used to determine the width of the current passage and the electrical conductivity of the interfaces. The electrical and thermal conductivity of the electrode to sheet interfaces are set to be the same as the bulk properties of aluminum once physical contact is established (Ref. 5 and Ref. 6). Properties of the faying interface are functions of both temperature and pressure with the electrical conductivity being proportional to the contact pressure (Ref. 12). Unlike other approaches where a layer of fictitious contact elements has to be introduced with a physical thickness (Ref. 4 and Ref. 7), the thermalelectrical surface interactions are modeled using the concept of contact pairs. The electrical current flowing between the contact surface is modeled by (Ref. 13):

$$
J=\sigma_{g}\left(\varphi_{A}-\varphi_{B}\right)
$$

where $\sigma_{g}$ is the gap electrical conductance, $\varphi_{\mathrm{A}}$ and $\varphi_{\mathrm{B}}$ are the electrical potentials at the two points on the contact surfaces.

As discussed by Browne et al. (Ref. 5 and Ref. 6), contact resistance plays a very important role in RSW process for aluminum. In this study, the contact electrical and thermal conductivity input is first tuned to match one set of the experimentally measured nugget size data. The obtained contact pair properties are then used for the prediction of nugget growth for the other set of experiment under different current and electrode force. It needs to be noticed that the present study does not include any material coating effects, neither does it consider the effect of aluminum-oxide layer. 


\section{RESULTS AND DISCUSSION}

Two sets of experiments are carried out and the resulted nugget growth data are used to validate the analysis procedure. The welding condition for these two sets of experiments are shown in Table 1:

\begin{tabular}{|c|c|}
\hline WELDING CURRENT(KA) & ELECTRODE FORCE (LBS) \\
\hline 29.5 & 800 \\
\hline 26.0 & 1100 \\
\hline
\end{tabular}

*squeeze time: 60 cycles, weld time: 10 cycles, hold time 30 cycles

Table 1. Welding parameters for the two experiment cases

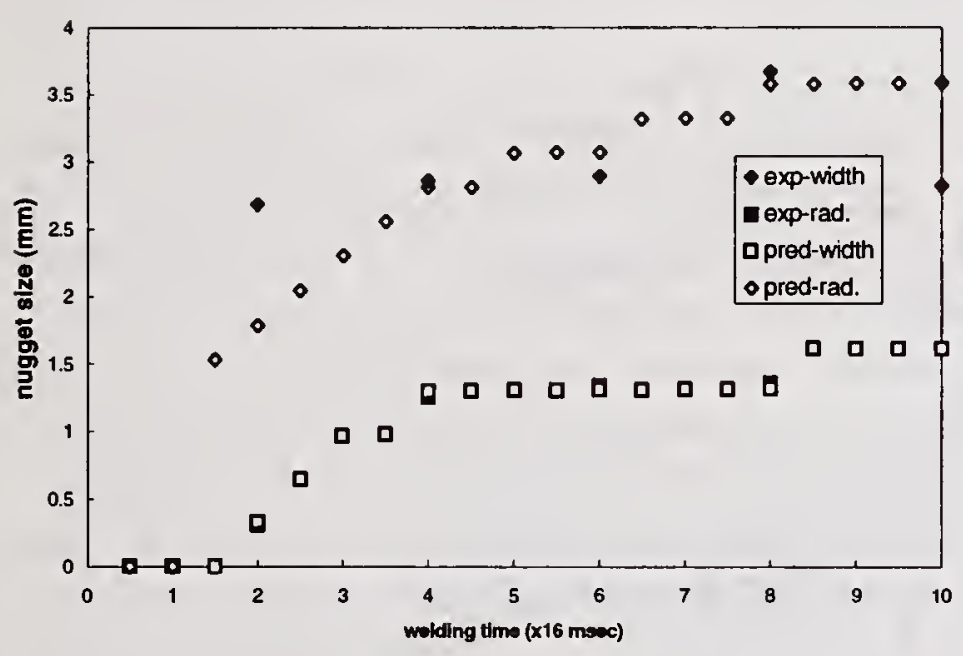

Case I: $29.5 \mathrm{kA}, 800 \mathrm{lbs}$

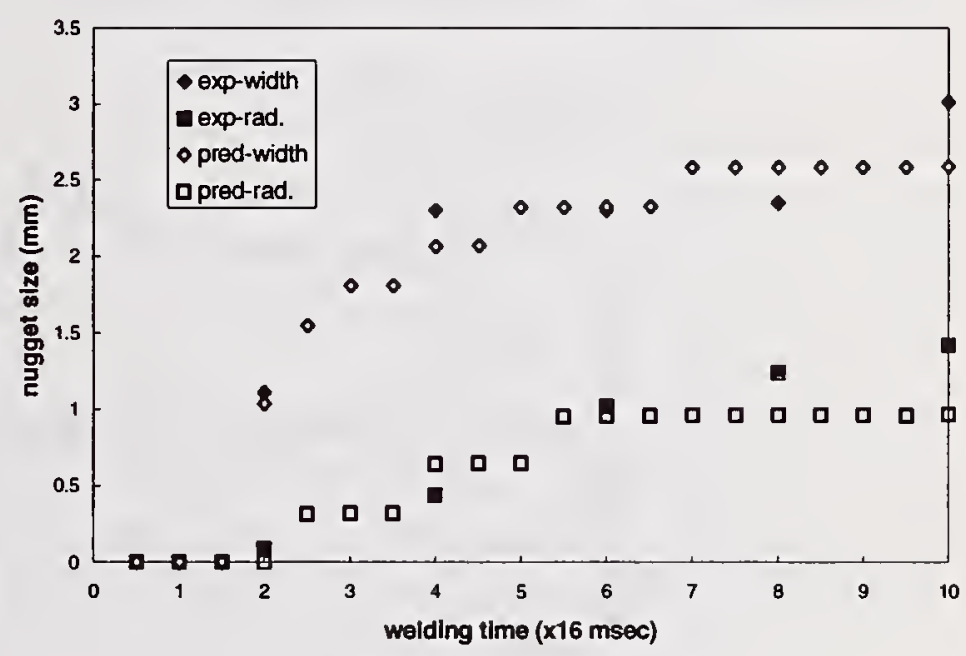

Case II: $26 \mathrm{kA}, 1100 \mathrm{lbs}$

Figure 3 Comparison of prediction and measurement for nugget growth

Figure 3 shows the comparison of nugget growth history for the two welding cases. The solid symbols represent cross section measurements of the fusion zone size from experiments; while the hollow symbols represents the analysis predictions for each half cycle. Considering the process variation of aluminum RSW, excellent overall correlation has been achieved using the current modeling procedure to predict nugget formation and growth. This proves that the finite element analysis procedure can be effective and predictive. As a general trend, higher current produces bigger nugget. Lower electrode force yields bigger nugget in the range tested. However, it should be noted that sufficient electrode force is needed to generate enough faying interface pressure that can contain the molten metal and prevent expulsion. The underlying principle for RSW is Joule heating, in which the heat generated Q can be express as:

$$
Q=\int I^{2} R d t
$$

Therefore, higher current generates more heat and forms a bigger nugget. On the other hand, the decrease of electrode force would reduce the contact area and in turn would increase the current density. Plus the contact electrical conductivity of the faying interface will also decrease for a smaller electrode force, these factors will jointly lead to a bigger nugget. 
Some detailed results for Case I are discussed next. Figure 4 depicts the temperature and pressure distribution at the end of the first half cycle. At this moment in time, the area near the location of $x=2.0 \mathrm{~mm}$ experiences higher temperature and higher contact pressure than that of the surrounding locations. The combination of this higher temperature and higher pressure provides a favorable condition for some surface alloys to be formed. This in turn suggests that electrode wear is more likely to initiate at this ring shape location starting from the middle of the electrode diameter rather than the electrode periphery. The result may be used to explain the electrode alloying and pitting phenomenon. It is also worthwhile to mention that this analysis is only done for the first weld of the electrode. With more subsequent welds, this effect may become more prominent. Figure 5 shows the photograph of a worn electrode face. Clearly, surface alloying and pitting occur at the ring location discussed above. To this end, the analytical prediction is further supported by experimental observations.

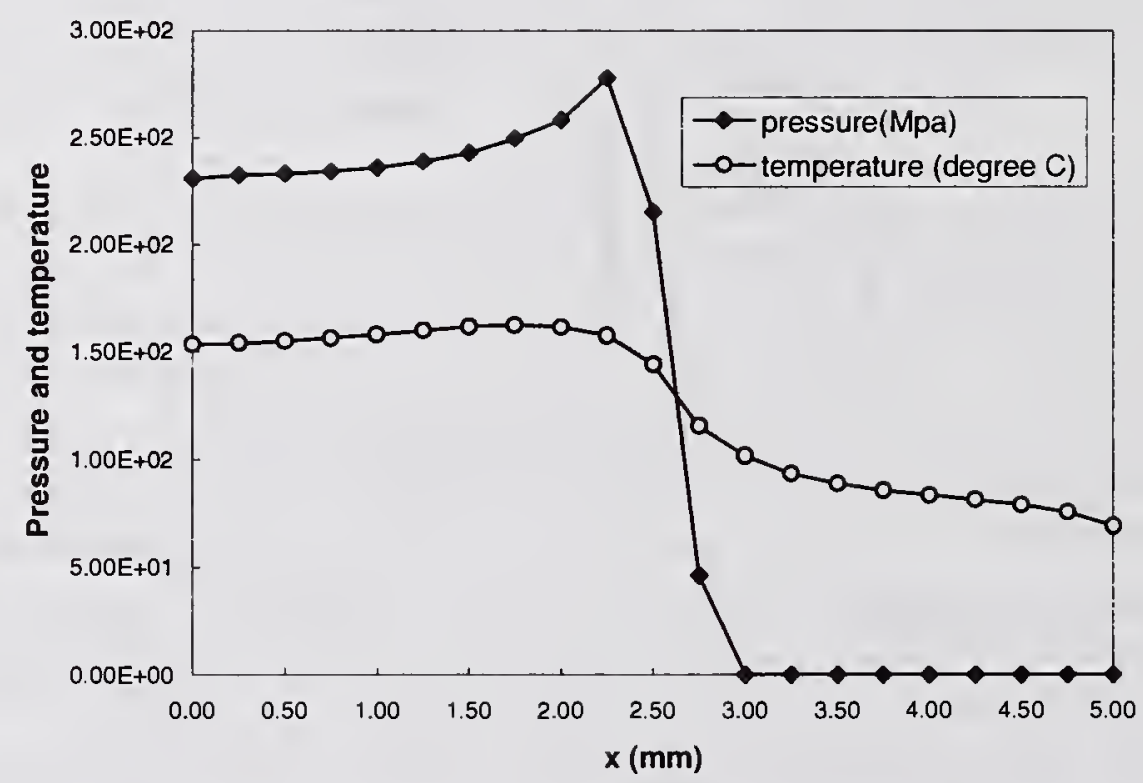

Figure 4. Temperature and pressure distribution on the electrode sheet interface

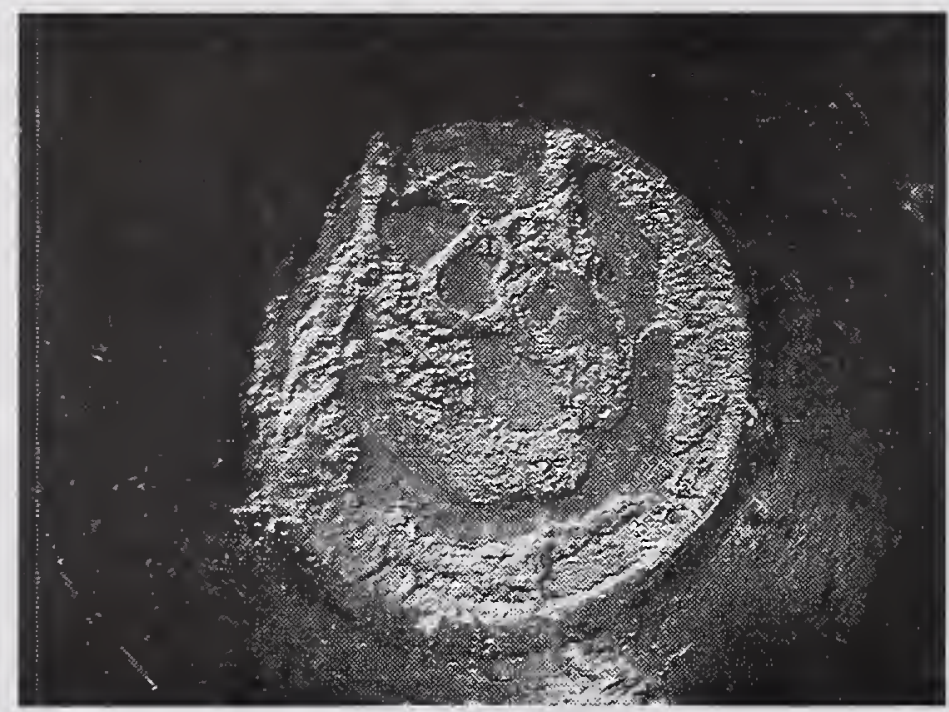

Figure 5. Photograph of a worn electrode face 
The contact area change during welding is plotted in Figure 6. Using this dome electrode, gradual increase of the contact area between the faying interface and the electrode-sheet interface is obtained as a result of the continue softening of the aluminum sheets as temperature increases. The final contact radii are almost the same for the two interfaces at the value around $5 \mathrm{~mm}$.

In the following sections, equivalent plastic strain and weld residual stresses for Case II will be discussed. Figure 8 shows the equivalent plastic strain plotted on the final weld shape after cooling. Noticeable weld indentation and sheet to sheet separation are observed. The final plastic strain on the sheet is concentrated at the periphery of the electrode to sheet contact. The predicted sheet indentation is $6.3 \%$ versus the experimental result of $6.8 \%$. The final weld cross section from the same set of experiment is shown in Figure 9 as a comparison to the prediction.

Tensile radial residual stress (S11) of magnitude around $70 \mathrm{MPa}$ is predicted for the center of the nugget and the areas outside the electrode periphery on the sheet surface, see Figure 10. This tensile residual stress at the weld center may cause grain boundary opening which would promote through thickness nugget cracking (Ref. 14). Vertical residual stress S22 is found to be of a smaller magnitude (around $17 \mathrm{Mpa}$ ). The tensile regions are at the nugget periphery on the faying interface, and the locations just beneath the electrode periphery in the sheets. This distribution is similar in qualitative manner to the residual stresses obtained by Dong et al. (Ref. 15) for steel, although the residual stress magnitude for aluminum is much smaller. Circumferential tensile residual stress S33 of magnitude around $125 \mathrm{MPa}$ is observed at the region just outside the electrode periphery, and its distribution is almost uniform through the sheet thickness. These tensile residual stresses form some favorable conditions for crack initiation and they deserve further investigations to thoroughly evaluate the weld quality and its engineering performance.

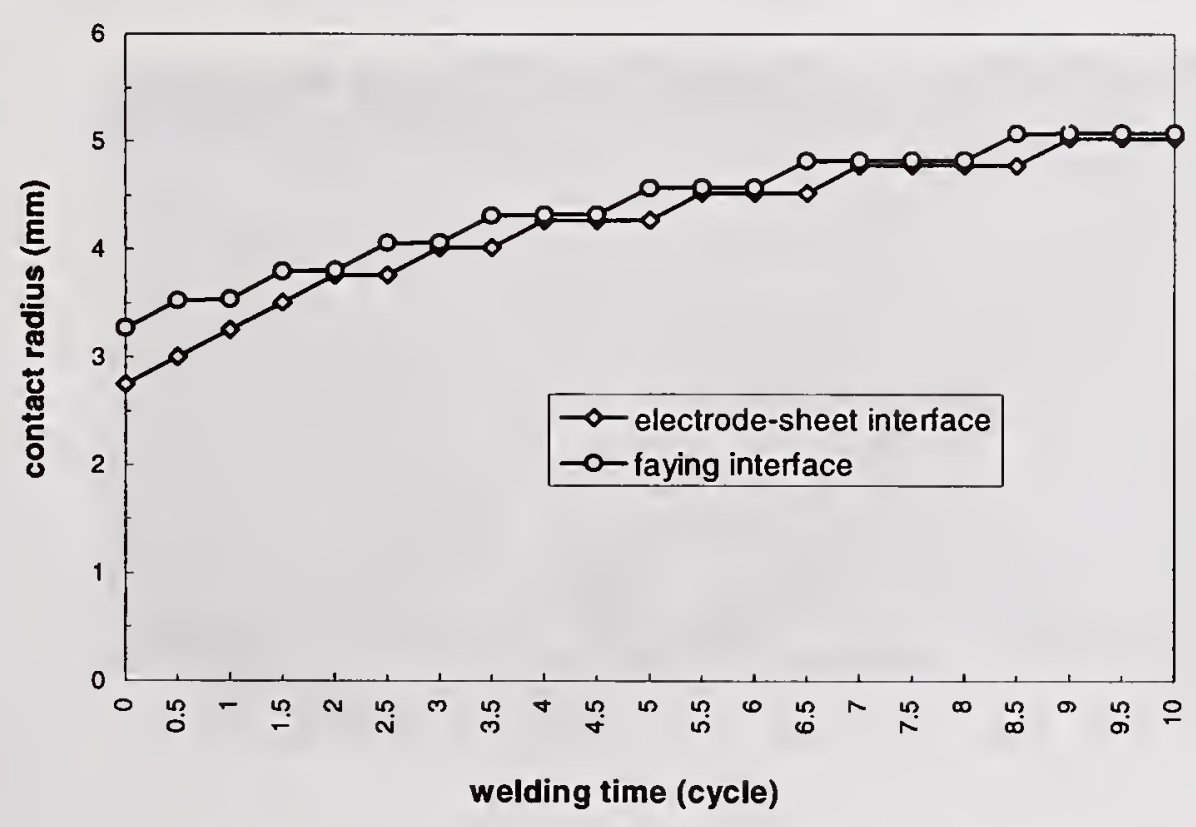

Figure 6. Predicted contact radius changes during welding 

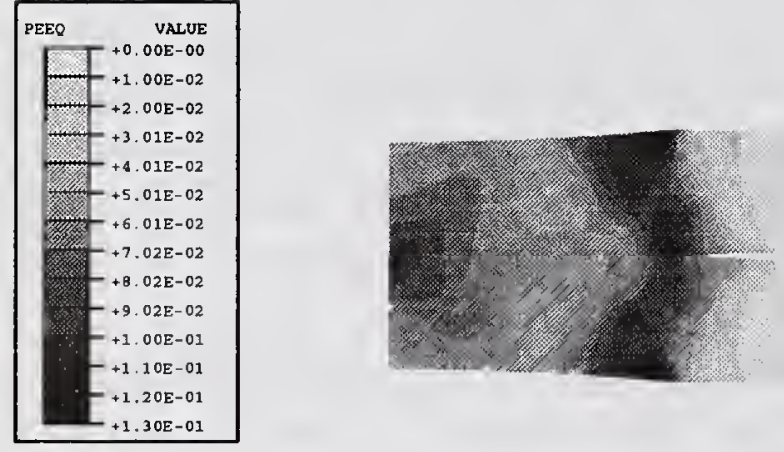

Figure 8. Equivalent plastic strain distribution and final weld shape

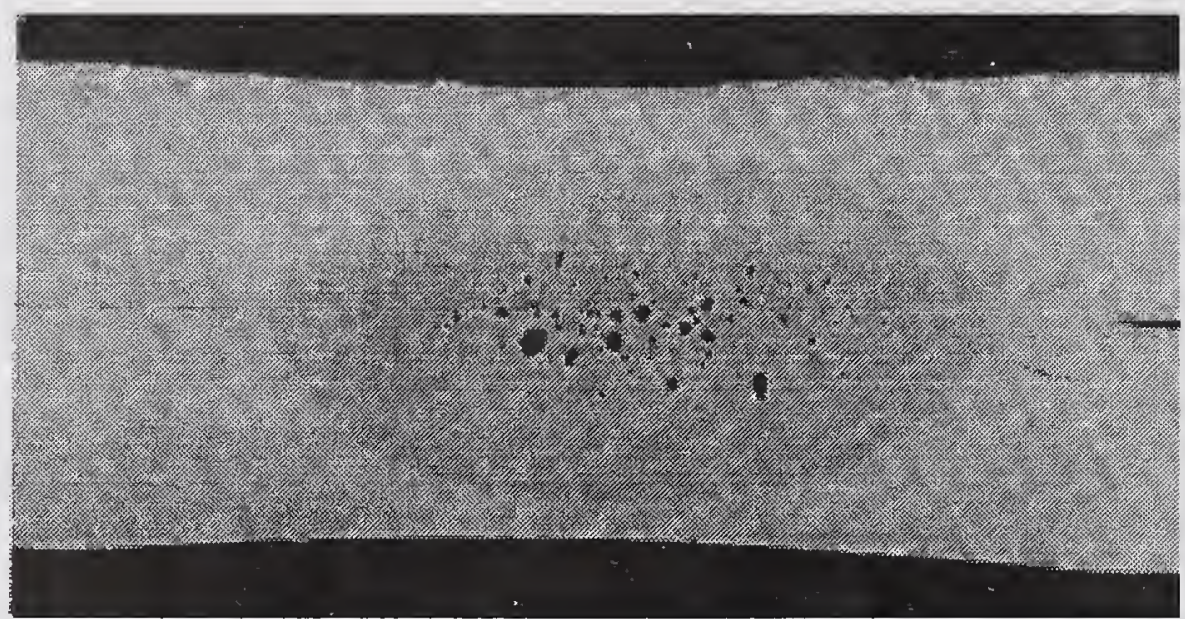

Figure 9 Nugget cross section from experiment for Case II 

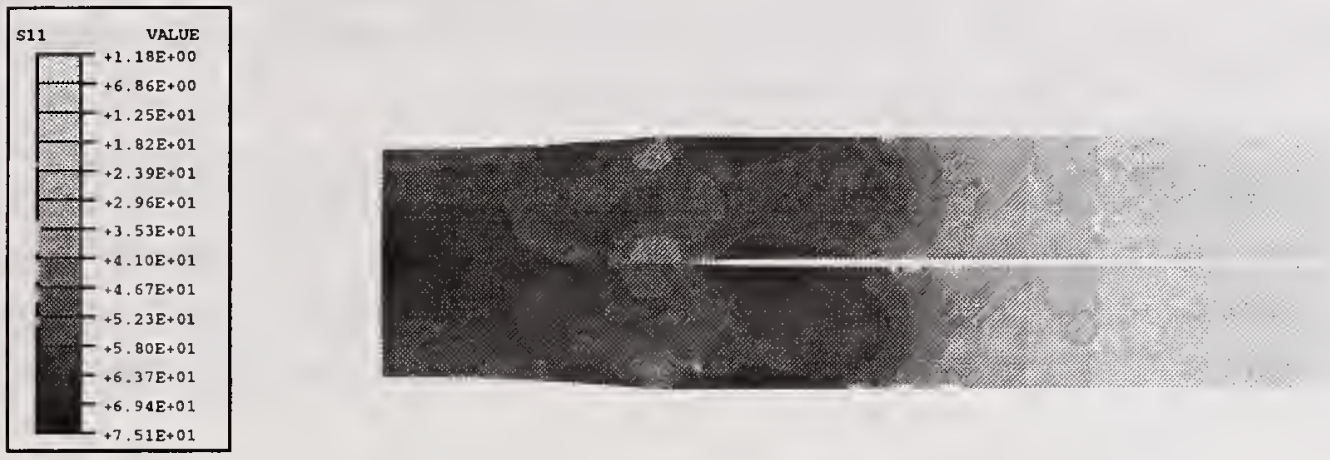

residual stress $s 11$
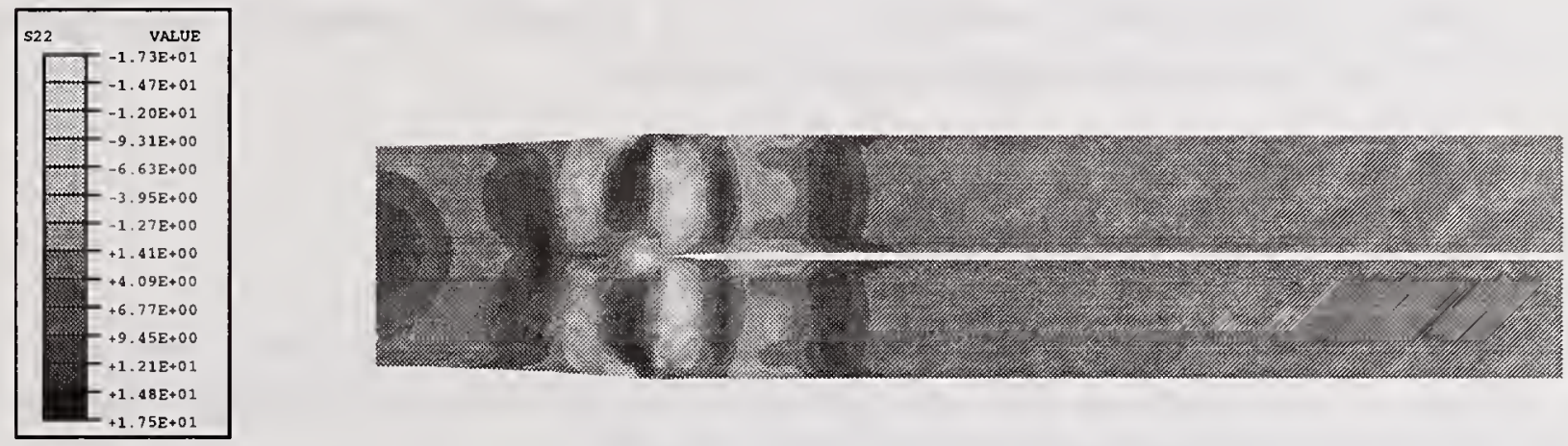

residual stress $s 22$
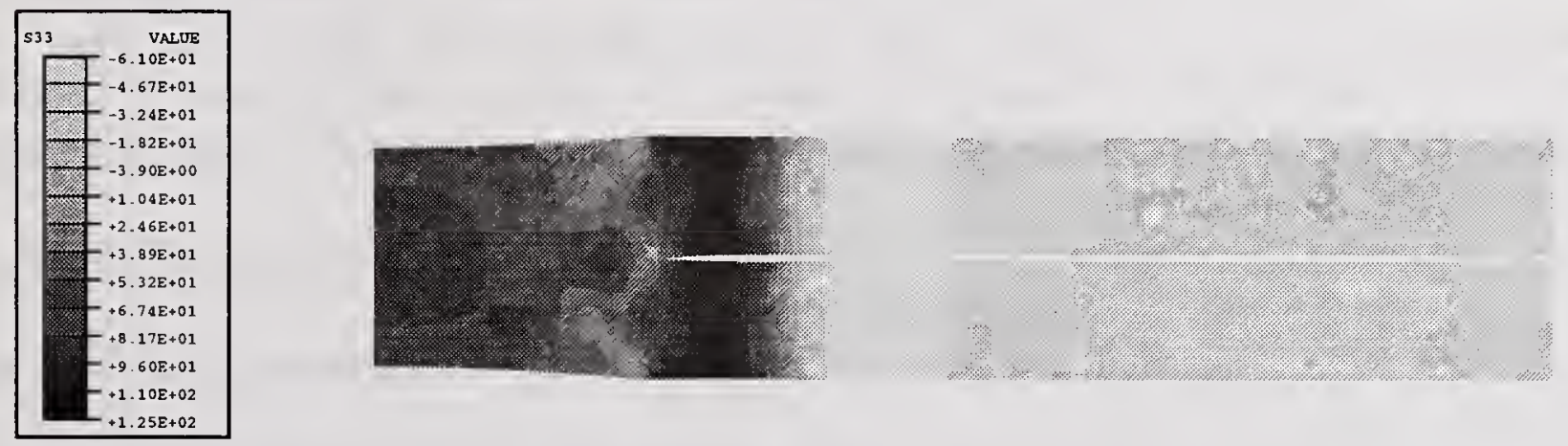

residual stress s33

Figure 10. Weld residual stress distributions 


\section{CONCLUSIONS}

In this study, an incrementally coupled finite element analysis procedure is used to study the RSW process of AA5754. Unlike most of the previous works in this area that either do not include updating effect or just update the contact area change, this study takes into account the contact area changes as well as the change of the deformed geometry of the sheet assembly being welded. This is particularly important for simulating the spot welding process of aluminum alloys because of its large deformation due to low Young's modulus.

Model validation is carried out by comparing the predictions with the experimentally measured nugget growth data. Excellent agreement have been achieved. Comparisons have also been made for the weld indentation, final weld shape and size. It is found that higher welding current generates bigger nugget, and within the range tested, low electrode force generates bigger nugget.

Variations of process parameters during welding, such as temperature and pressure distributions, electrode movements and dynamic resistance can also be monitored during the welding simulation. These predictions offer insight information on the mechanisms for nugget formation and electrode wear. Furthermore, parameters such as electrode movement and dynamic resistance could be used as in-process welding quality monitors.

\section{REFERENCES}

1. Greenwood, J.A.: 1961. Temperatures in Spot Welding. BWRA Report, 1961: 316-322.

2. Han, Z., Orozco, J., Indacochea, J.E. and Chen, C.H.: 1989: Resistance Spot Welding: A Heat Transfer Study. Welding Journal. Sept. 1989, 363s-371s.

3. Cho, H.S. and Cho, Y.J.: 1989. A Study of the Thermal Behavior in Resistance Spot Welds. Welding Journal. June 1989, 236s-244s.

4. Nied, H.A.: 1984. The Finite Element Modeling of the Resistance Spot Welding Process. Welding Journal, April 1984: 123s-132s.

5. Browne, D.J., Chandler, H.W., Evans, J.T. and Wen, J.: 1995. Computer Simulation of Resistance Spot Welding in Aluminum: Part I. Welding Journal. Oct. 1995, 339s-344s.

6. Browne, D.J., Chandler, H.W., Evans, J.T., James, P.S., Wen, J. and Newton, C.J.: 1995. Computer Simulation of Resistance Spot Welding in Aluminum: Part II. Welding Journal. Dec. $1995,417 \mathrm{~s}-422 \mathrm{~s}$.

7. Tsai, C.L., Jammal, O.A., Papritan, C., and Dickinson, D.W.: 1992. Modeling of Resistance Spot Weld Nugget Growth. Welding Journal, Feb. 1992, 47s-54s.

8. Sheppard, S.D.:1990. Thermal and Mechanical Simulations of Resistance Spot Welding. WRC Bulletin, Aug. 1990, 34-41.

9. Murakawa, H., Kimura, F. and Ueda, Y.: 1997. Weldability Analysis of Spot Welding on Aluminum using FEM. Mathematical Modeling of Weld Phenomena, edited by Cerjak, H., The Institute of Materials, 1997, 944-966.

10. Dong, P., Li, M.V. and Kimchi, M.: 1997. Analysis of Electrode Wear Mechanisms: Face Extrusion and Pitting Effects. To appear in Science and Technology of Welding and Joining. 
11. Properties and Selections: Nonferrous Alloys and Pure Metals. Metal's Handbook, Ninth Edition, Volume 2, 1979.

12. Resistance and Solid-State Welding and Other Joining Processes. Edited by Kearn, W.H. Welding Handbook, 1980. Seventh Edition, Volume 3: 2-23.

13. ABAQUS Theory Manual, Version 5.5. Hibbitt, Karlsson \& Sorensen, Inc. 1995.

14. Watanabe, G. and Tachikawa, H.: 1995. Behavior of Cracking Formed in Aluminum Alloy sheets on Spot Welding. IIW Doc. No. III-1041-95.

15. Dong, P. and Kimchi, M. and Holmes, S.: 1995. Thermomechanical Analysis of Electrode Wear Mechanisms: A Finite Element Study. Proceedings of the $11^{\text {th }}$ Annual North American Welding Research Conference: Advances in Welding Technology, Nov. 1995, 145-162. 


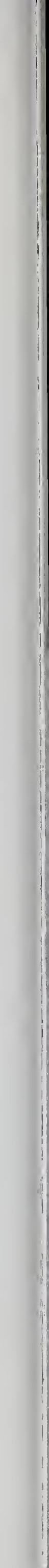




\section{SENSING}





\title{
GMA PROCESS IDENTIFICATION USING ARC ACOUSTICS
}

\author{
A.M. Mansoor ${ }^{1}$, J.P. Huissoon ${ }^{2}$
}

\section{ABSTRACT}

In developing automated Gas Metal ARC Welding (GMAW) systems it is insightful to study the sources of process feedback utilized by human welders. One such source of feedback is the arc sound.

A high speed data acquisition system was developed to record the arc sound produced during GMA welding. The data so collected was processed to obtain time domain, frequency domain and time-frequency domain descriptions. Relationships between these descriptors and the originating weld parameter levels and metal transfer mode were sought. The relationship between the electrical power supplied to the weld and the arc sound was investigated.

The results indicate that the arc sound exhibits distinct charcateristics for each welding mode. The occurrence of spatter and short cicuits is also clearly detectable in the arc sound record.

\section{INTRODUCTION}

The automation of manufacturing processes usally results in increased productivity and quality. As such, automated systems are now often used to perform many of the tasks traditionally performed by human operators. This is especially so if the task or process is hazardous or unpleasant. Gas Metal Arc Welding is a manufacturing process that can be difficult to automate properly, since replicating the level of skill required to produce a quality weld by an automatic machine relies heavily on an understanding of the relationship between feedback from the process and action to be taken in response.

Skilled human welders represent extremely extremely sophisticated and effective feedback control systems. Visual, audio and tactile feedback cues are sensed, a highly sophisticated non-linear adaptive controller processes this data and recruits muscles in response. In attempting to develop a feedback control system for automated GMA welding, it is useful to study the sources of feedback utilized by human welders. One such source of feedback is the arc sound produced during welding [1,2]. This present study was undertaken to determine if and how this sound can be used as a source of feedback for automated GMA welding.

\footnotetext{
${ }^{1}$ Graduate Student, Dept. of Mech. Eng. University of Waterloo, Ontario Canada, N2L 3G1

${ }^{2}$ Associate Professor, Dept. of Mech. Eng. University of Waterloo, Ontario Canada, N2L 3G1
} 

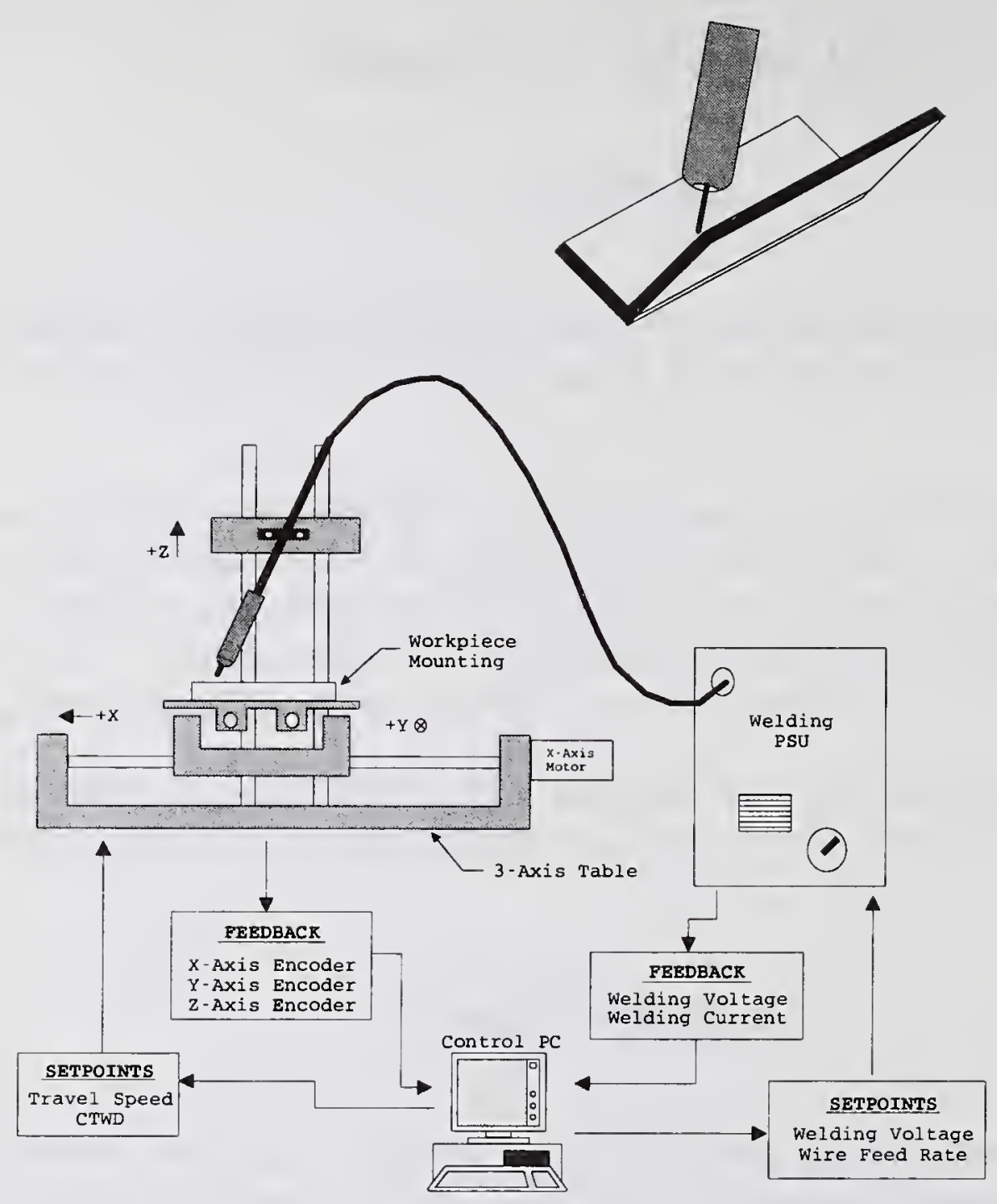

Figure 1: Schematic of the Automated GMA Welder.

\section{EXPERIMENTAL SETUP}

The experimental equipment consisted of a computer controlled GMA welding system, synchronized with a PC based data acquisition system for collecting acoustic data while welding.

\section{The Automated Welder}

The welding equipment used for the experiments is shown in Figure 1. This consists of a Fronius $500 \mathrm{~A}$ welding power source and wire feed unit, a three axis motion control system, and a control computer. This setup provides computer control of the wire feed rate (WFR), the torch travel speed (TS), the contact tube to workpiece distance (CTWD), and the weld voltage (V) measured at the torch. The shielding gas flow (SGF) rate could be manually set, and a standard Ar-15 shielding gas composition was used. 


\section{The Data Acquisition System}

A block diagram representation of the data acquisition system developed in this study is shown in Figure 2. This consists of a sound level meter (SLM), low-pass filtering and preamplification circuitry, shielding for the SLM and circuitry, a PC compatible high speed four channel analog to digital converter board, and a host PC.
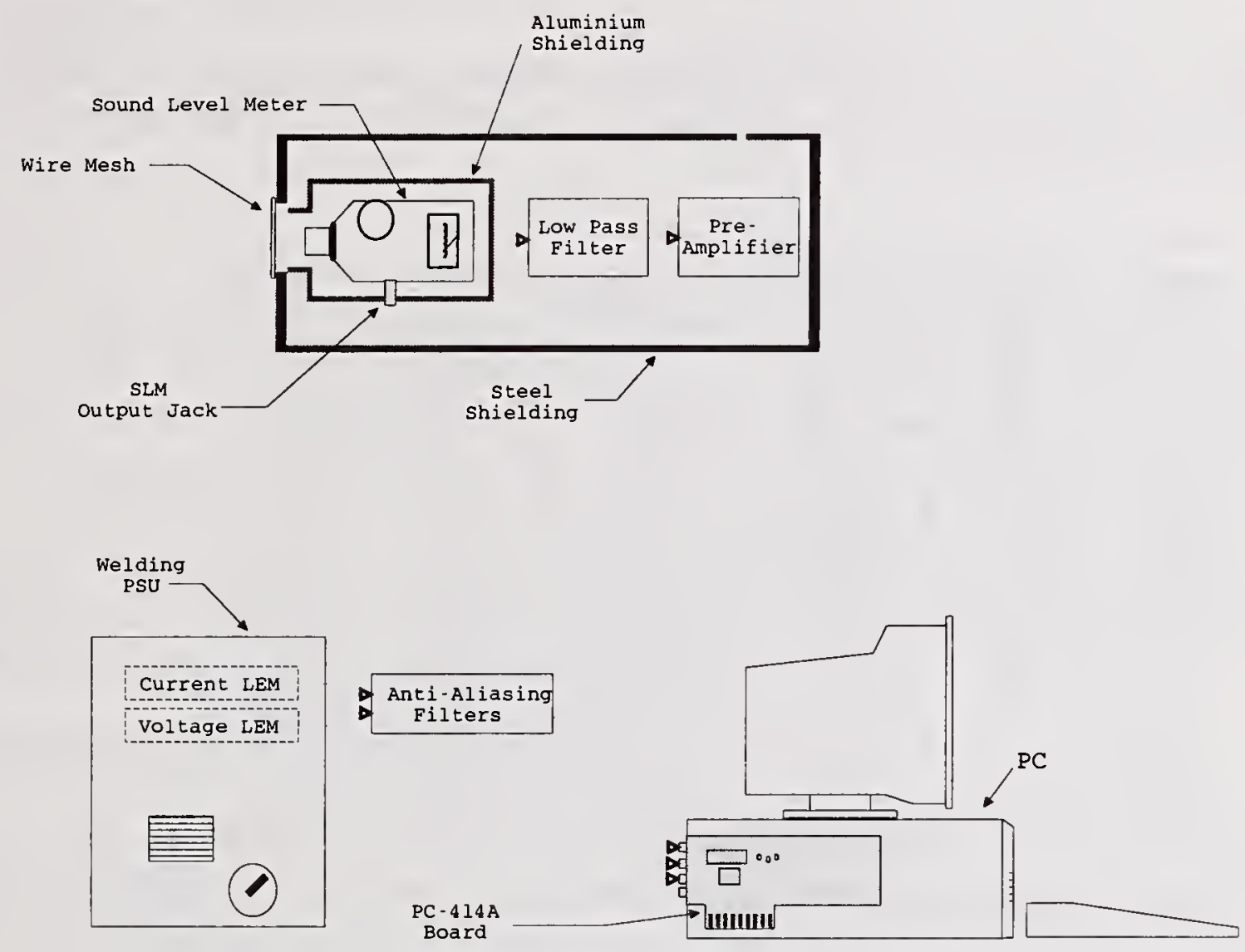

Figure 2: Schematic of the Data Acquisition Hardware.

The SLM is capable of measuring sound pressure levels from $50 \mathrm{~dB}$ to $126 \mathrm{~dB}$ with an accuracy of $2 \mathrm{~dB}$. The SLM frequency response conforms to the " C weighted response. The output from the SLM was conditioned using a 6 th order low-pass Butterworth filter with a break frequency of $10 \mathrm{kHz}$, followed by an $20 \mathrm{~dB}$ gain low-noise amplification stage. This effectively limited the range of study from $32 \mathrm{~Hz}$ to $10 \mathrm{kHz}$. The conditioned SLM analog output was connected to one channel of the analog to digital converter board. The SLM and conditioning cicuitry were enclosed in a $20 \mathrm{GA}$ steel casing, thus shielded from electric arc noise and other e.m.i. The SLM was placed $2.13 \mathrm{~m}$ behind the welding torch at a height of $20 \mathrm{~cm}$ above the seam of the weld. It should be noted that with this equipment configuration, the welding torch is stationary and the workpiece is moved while welding (see Figure 1). This arrangment allows the SLM to be maintined at a constant distance from the arc.

Isolated voltage and current feedback signals from the welding power supply were also recorded using the analog to digital converter board. This board (Datel 414A) is capable of 
simultaneously sampling the inputs and providing 12-bit data from three input channels at up to $350 \mathrm{kHz}$ per channel. For this application, the acoustic, welding voltage and current signals were sampled at $40 \mathrm{kHz}$ per channel. An $8 \mathrm{Mb}$ RAM drive enabled up to 35 seconds of continuous data to be recorded.

\section{Background Sound}

Aside form the welding arc, there are many other sound sources during welding. These include the the power supply unit, the servo motors on the 3 -axis table, ventilation and shielding gas flow. The sound from these background sources was measured and found to have very constast characteristics, with a mean level of $68.33 \mathrm{~dB}$ and a very consistent time averaged frequency response.

\begin{tabular}{|c|c|c|c|c|c|}
\hline Weld No. & Voltage (V) & WFR (m/min) & CTWD (mm) & TS (mm/s) & SGF (cfm) \\
\hline 1 & 21 & 6.0 & 20 & 8.0 & 50 \\
\hline 2 & 24 & 6.0 & 20 & 8.0 & 50 \\
\hline 3 & 27 & 6.0 & 20 & 8.0 & 50 \\
\hline 4 & 30 & 6.0 & 20 & 8.0 & 50 \\
\hline 5 & 33 & 6.0 & 20 & 8.0 & 50 \\
\hline 6 & 27 & 3.6 & 20 & 8.0 & 50 \\
\hline 7 & 27 & 4.8 & 20 & 8.0 & 50 \\
\hline 8 & 27 & 6.0 & 20 & 8.0 & 50 \\
\hline 9 & 27 & 7.2 & 20 & 8.0 & 50 \\
\hline 10 & 27 & 8.0 & 20 & 8.0 & 50 \\
\hline 11 & 27 & 6.0 & 16 & 8.0 & 50 \\
\hline 12 & 27 & 6.0 & 20 & 8.0 & 50 \\
\hline 13 & 27 & 6.0 & 24 & 8.0 & 50 \\
\hline 14 & 27 & 6.0 & 28 & 8.0 & 50 \\
\hline 15 & 27 & 6.0 & 32 & 8.0 & 50 \\
\hline 16 & 27 & 6.0 & 20 & 8.5 & 50 \\
\hline 17 & 27 & 6.0 & 20 & 8.0 & 50 \\
\hline 18 & 27 & 6.0 & 20 & 9.5 & 50 \\
\hline 19 & 27 & 6.0 & 20 & 11.0 & 50 \\
\hline 20 & 27 & 6.0 & 20 & 14.0 & 50 \\
\hline 21 & 27 & 6.0 & 20 & 8.0 & 50 \\
\hline 22 & 27 & 6.0 & 20 & 8.0 & 5 \\
\hline
\end{tabular}

Table 1: Experiments for Investigation of Weld Parameters on Arc Sound

\section{EXPERIMENTAL PROCEDURE AND RESULTS}

Experiments were performed by welding at prescribed conditions while acquiring the sound signal, weld voltage, and weld current as described above. All welds were 90deg fillet geometry on 2 pieces of $51 \mathrm{~mm} \times 6.4 \mathrm{~mm}$ hot rolled flat steel cut to $0.6 \mathrm{~m}$ lengths. All analyses were performed on 5.1456 seconds of weld data. This 5.1456 data sample was taken at least 2 seconds into the data record from a weld, so that any transient effects caused by the arc start could be ignored.

\section{Preliminary Study}

To provide a base-line, nominal welding conditions were chosen to be $27 \mathrm{~V}, 6.0 \mathrm{~m} / \mathrm{min}$ wire feed rate, $20 \mathrm{~mm}$ CTWD, $8.0 \mathrm{~mm}$ travel speed, and $50 \mathrm{cfm}$ gas flow rate. An initial set of experiments to investigate the primary effect of the five controllable parameters was performed. Twenty two welds were made, using the parameter combinations given in Table 1 


\begin{tabular}{|c|c|c|c|c|c|}
\cline { 2 - 6 } \multicolumn{1}{c|}{} & \multicolumn{5}{c|}{ Weld No. } \\
\cline { 2 - 6 } & 8 & 17 & 3 & 12 & 21 \\
\hline Mean Snd Level (V) & $\mathbf{0 . 2 3 2}$ & $\mathbf{0 . 2 2 2}$ & $\mathbf{0 . 1 4 7}$ & $\mathbf{0 . 3 0 0}$ & 0.149 \\
\hline Peak Snd Level (V) & 3.427 & 2.756 & 2.280 & 3.294 & 2.788 \\
\hline Standard Dev. (V) & 0.199 & 0.189 & $\mathbf{0 . 1 2 8}$ & 0.330 & 0.250 \\
\hline Coeff. Variation \% & 85.60 & 85.20 & 87.24 & 87.81 & 83.28 \\
\hline Mean Snd Level (dB) & 86.40 & 82.72 & 78.91 & 87.01 & 85.38 \\
\hline Peak Snd Level (dB) & 113.93 & 108.73 & 107.07 & 110.53 & 108.82 \\
\hline
\end{tabular}

Table 2: Characteristic Values for Same Source Parameter Values

above. Welds 1 through 5 were intended to characterize the effect of weld voltage, as these welds were produced at varying levels of arc voltage while the remaining weld parameters were held constant. Similarly welds 6 to 10 investigate the effect of wire feed rate, welds 11 to 15 focus on the influence of CTWD (standoff), welds 16 to 20 consider the impact of travel speed, while welds 21 and 22 investigate the difference between adequate and inadequate shielding gas flow. The collected data was subjected to both time and time-frequency analysis. Welds $3,8,12,17$ and 21 from Table 1 were produced using the same weld parameter levels. The characteristics of the data from these welds were compared in order to assess how consistent the arc sound characteristics are over several non-consecutive tests for identical parameter levels. Time averaged frequency spectra for these welds are shown in Figure 3, while time domain descriptors are tabulated in Table 2. These preliminary results, especially those of Figure 3 and Table 2, would seem to indicate that the arc sound is a poor indicator of the source weld parameter levels.

\section{Arc Sound vs. Metal Transfer Mode}

The metal transfer mode is the characteristic most commonly associated with arc sound, and therefore warrants investigation. An experienced professional welder was consulted to determine weld parameters which resulted in spray, globular and short arc transfer. Using these parameters, five welds were performed in each transfer mode. Typical sound records from each transfer mode are shown on a compressed time scale in Figure 4 . These records are consistent with those found by other researchers [3,4]. From Figure 4 it is clear that the time domain characteristics vary greatly with transfer mode. Spray transfer produces a low amplitude signal, while globular and short arc transfer produce louder signals. The sound from short arc transfer is distinguished from that of spray and globular transfer by abrupt and short lived spikes in the sound signal, these spikes being associated with the occurence of a short circuit. In order to summarize the nature of the acquired sound signals, time domain descriptor values were calculated. Three of these descriptors, Peak Sound level, Coefficient of Variation, and Maximum Rise are plotted versus transfer mode in Figures 5,6 and 7. The Maximum Rise is found as the largest absolute difference between consecutive samples in a sound record. These Figures show that the values of all three of the plotted time descriptors increase from spray to globular to short arc transfer. Time averaged frequency spectra were computed for each of the five sound records associated with each transfer mode. These spectra are plotted in Figures 8,9 and 10. From these plots it can be seen that even though the frequency spectra for a given transfer mode exhibit some variation, the general shape of the spectra is quite constant. Spray transfer exhibits an almost monotone spectrum, while 

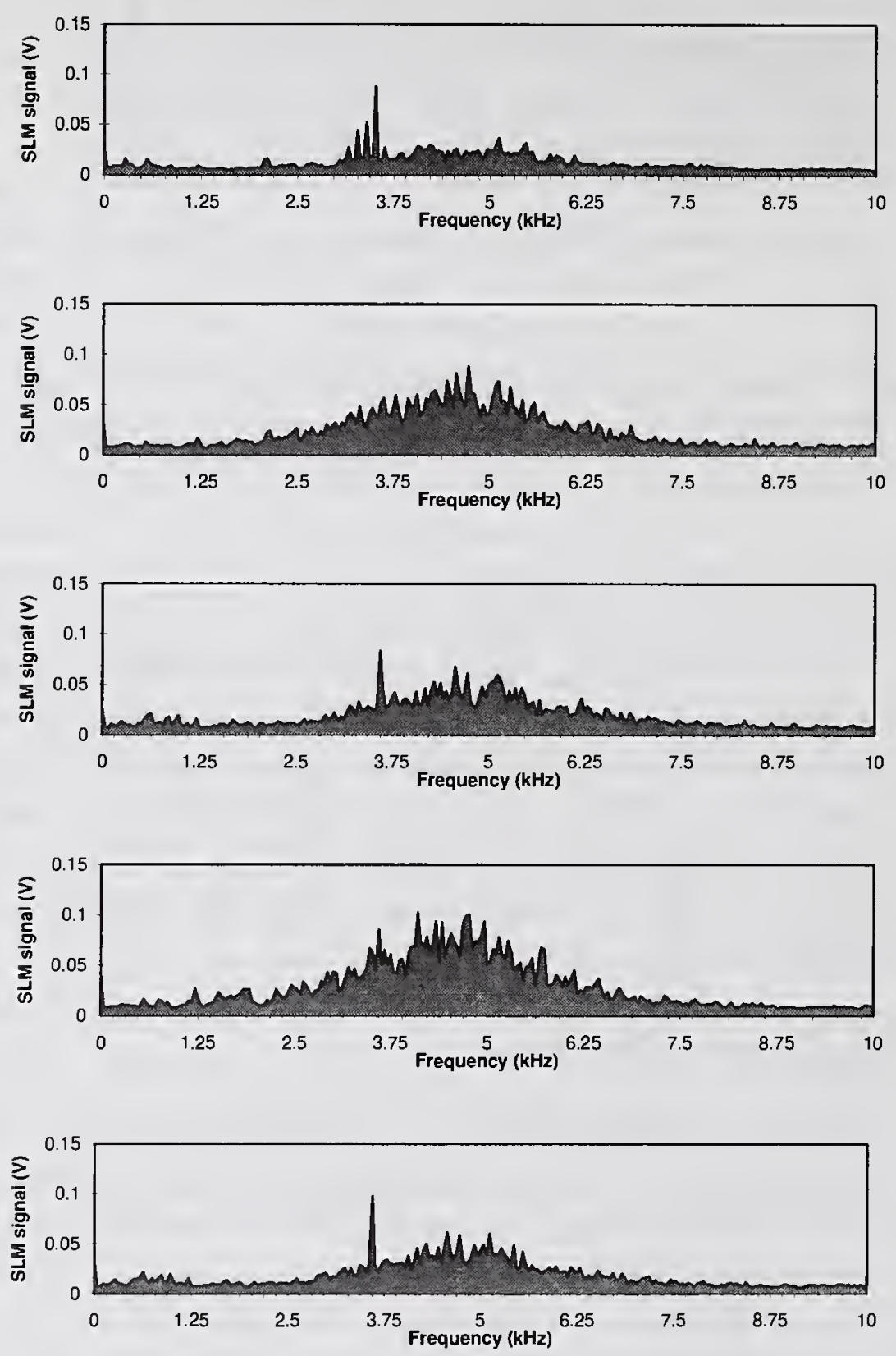

Figure 3: Time Avg'd Frequency Spectra for Same Source Parameter Values.

globular and short arc transfer exhibit more broad-band signals. The spectra for globular and short arc transfer are similar, but the short arc spectra can be distiguished by smaller amplitudes and with a more trapezoidal shape.

Spectrograms were obtained for the 15 sound records discussed above, and these were also found to exhibit distinct characteristics for each transfer mode.

\section{Comparison of Arc sound and Power}

Previous work done in this area suggests that the sound produced by an electrical arc is 

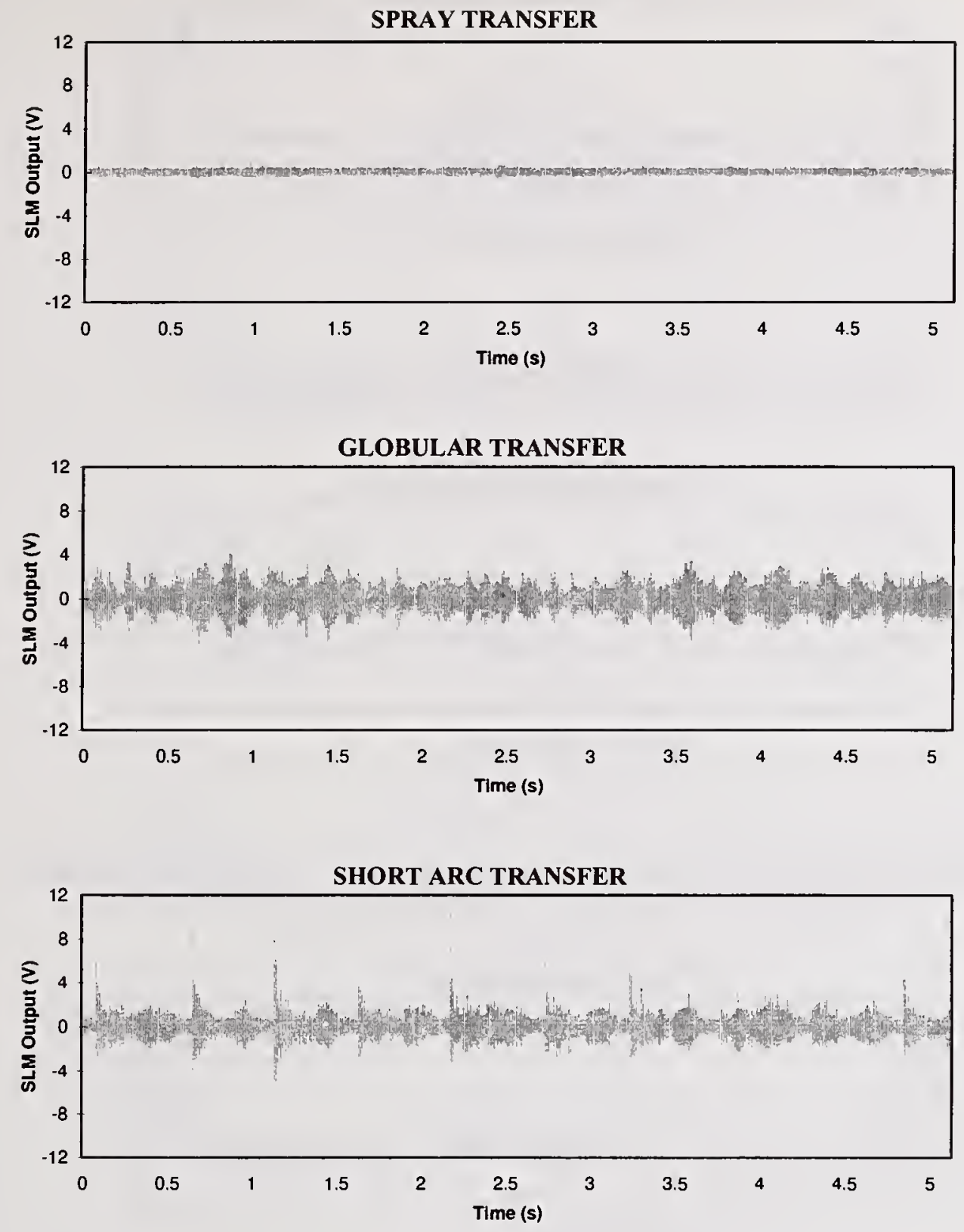

Figure 4: Typical Compressed Sound Records for each Transfer Mode.

related to the electrical power supplied to the arc as described in $(1)[5,6]$.

$$
S_{a}(t)=k \frac{d}{d t}(V(t) I(t))
$$

Where:

- $S_{a}(\mathrm{t})$ is the amplitude of the sound signal at time $\mathrm{t}$,

$-V(t)$ is the voltage across the arc at time $t$, 


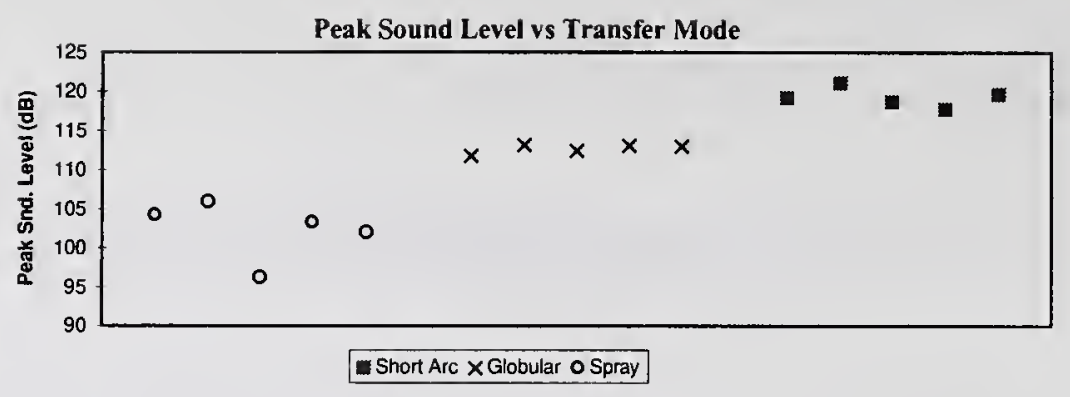

Figure 5: Peak Sound Levels vs. Transfer Mode.

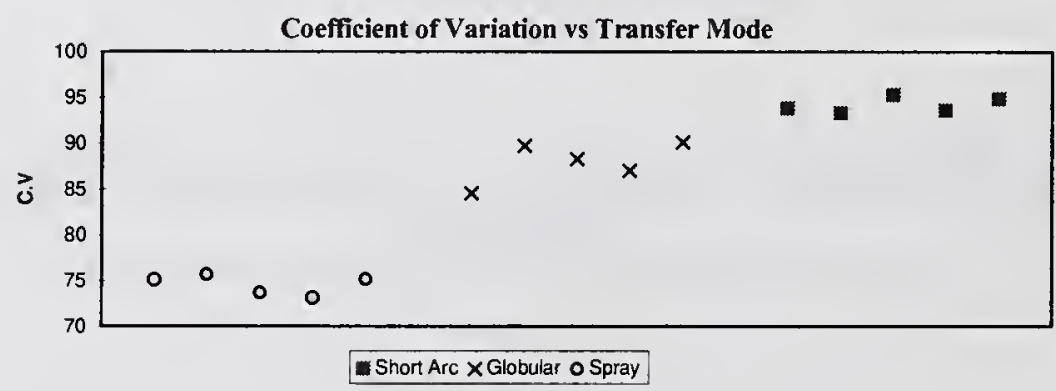

Figure 6: Coefficient of Variation of Sound Level vs. Transfer Mode.

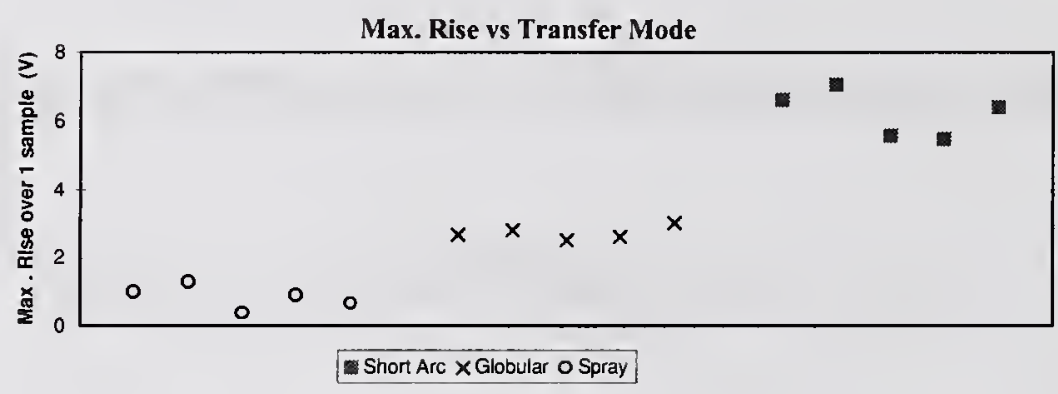

Figure 7: Max. Rise vs. Transfer Mode.

- $I(t)$ is the current flowing through the arc at time $t$, and

$-k$ is a constant of proportionality .

There is evidence that this relationship also holds for the GTA welding arc [7]. In order to investigate this relationship for the GMA welding arc, the sound and power derivative signals for welds were compared in the time and frequency domains. The electrical power supplied to the arc was calculated as the product of the measured welding current and voltage. This power signal was then differentiated using a 2 nd order backward difference approximation. Comparisons of the sound and power differential in the time domain and the frequency 

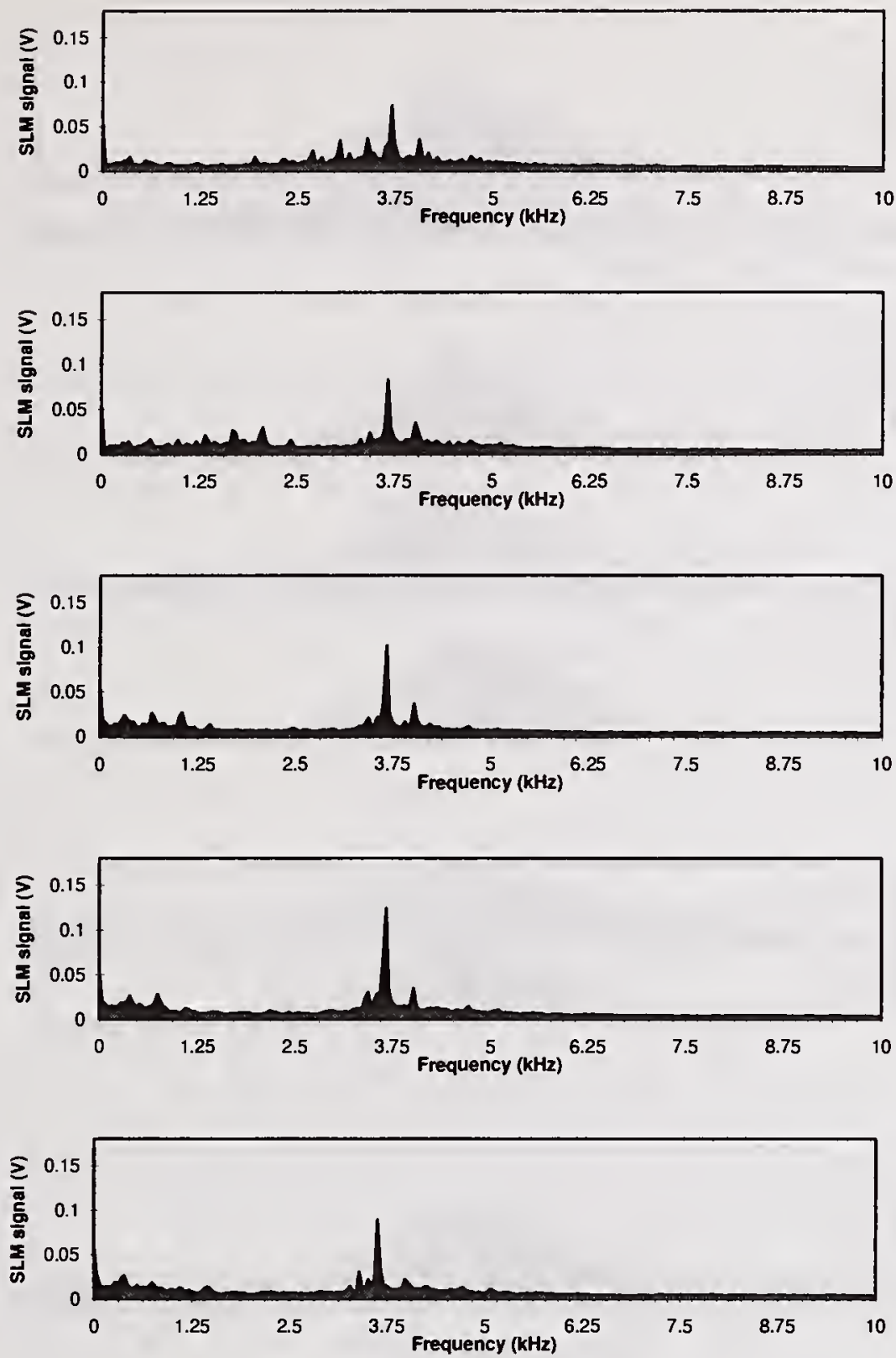

Figure 8: Time Avg'd. Frequency Spectra for Sound from Spray Transfer.

domain did not indicate any strong relationship. More encouraging results were obtained when a low pass filtered version of the power derivative was used (see Figure 11).

\section{CONCLUSIONS}

The results of this study indicate that the arc sound produced during the GMA welding process can be used as a source of process feedback. Specifically, this study has shown that the metal transfer mode, and events such as the occurrence of spatter or a short circuit can be discerned from the arc sound. Such feedback has many potential applications in the monitoring of the GMA welding process. 

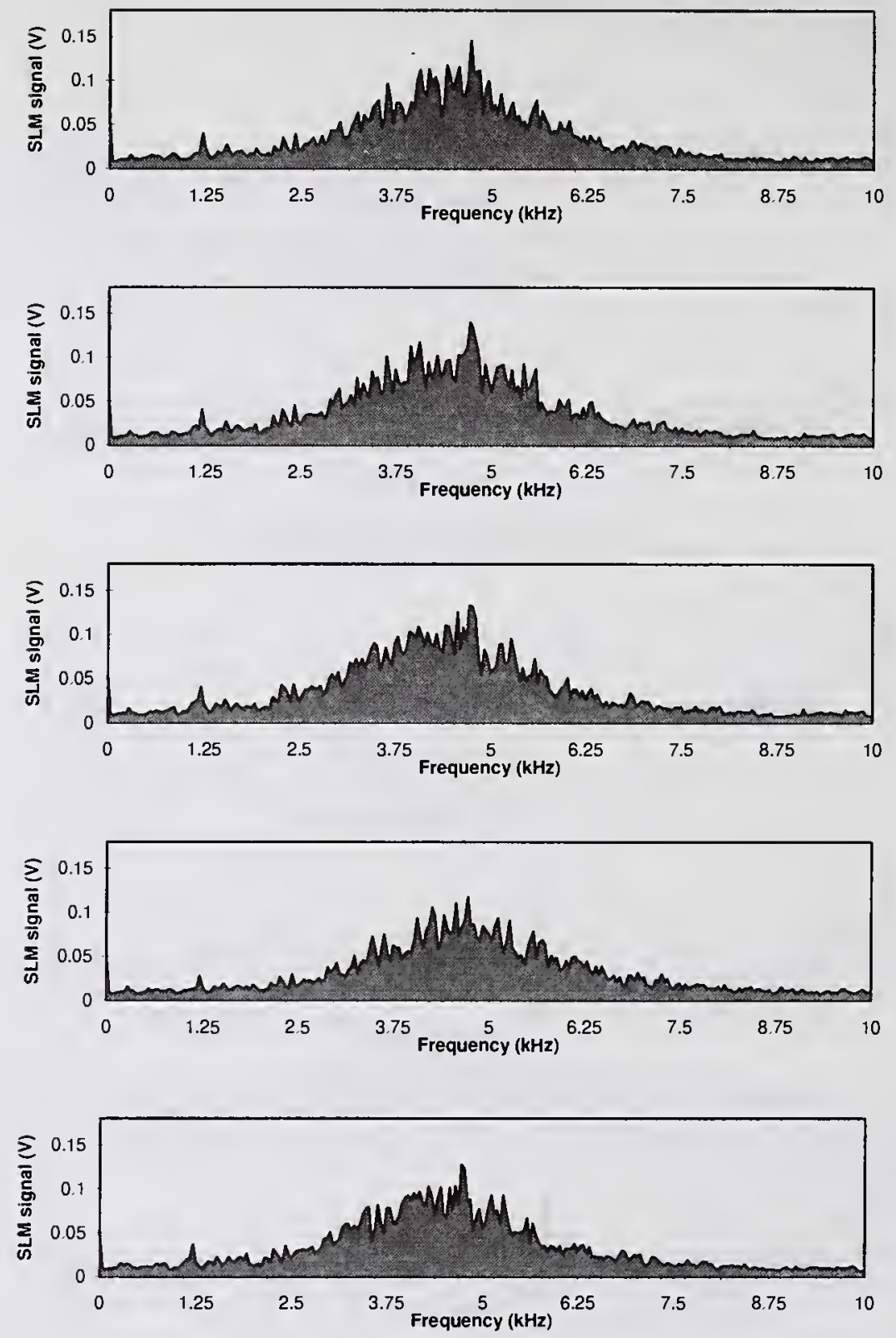

Figure 9: Time Avg'd. Frequency Spectra for Sound from Globular Transfer.

\section{FUTURE WORK}

Further work in this area will concentrate on incorporating the arc sound descriptors developed in this study into weld monitoring systems. Such systems would acquire the sound from the arc and calculate time domain and frequency domain and frequency-time domain descriptors. These descriptors would then be further analyzed using a set of heuristics or a pattern recognition tool such as a neural network to extract useful information about the weld process. Possible applications are spatter detection and automatic arc tuning. 

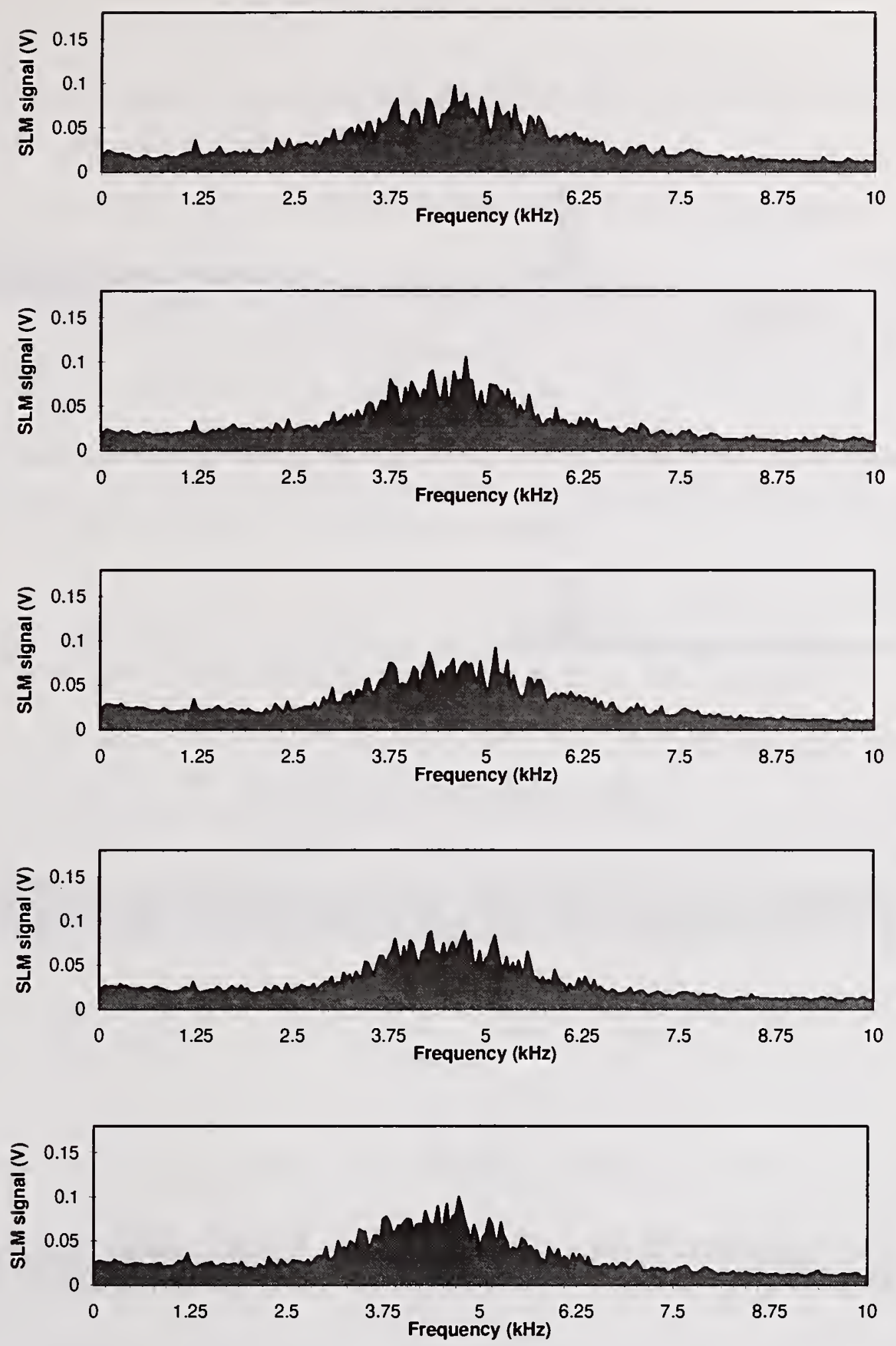

Figure 10: Time Avg'd. Frequency Spectra for Sound from Short Arc Transfer.

\section{ACKNOWLEDGEMENTS}

This research was supported by the Manufacturing Research Corporation of Ontario. 


\section{GLOBULAR TRANSFER}
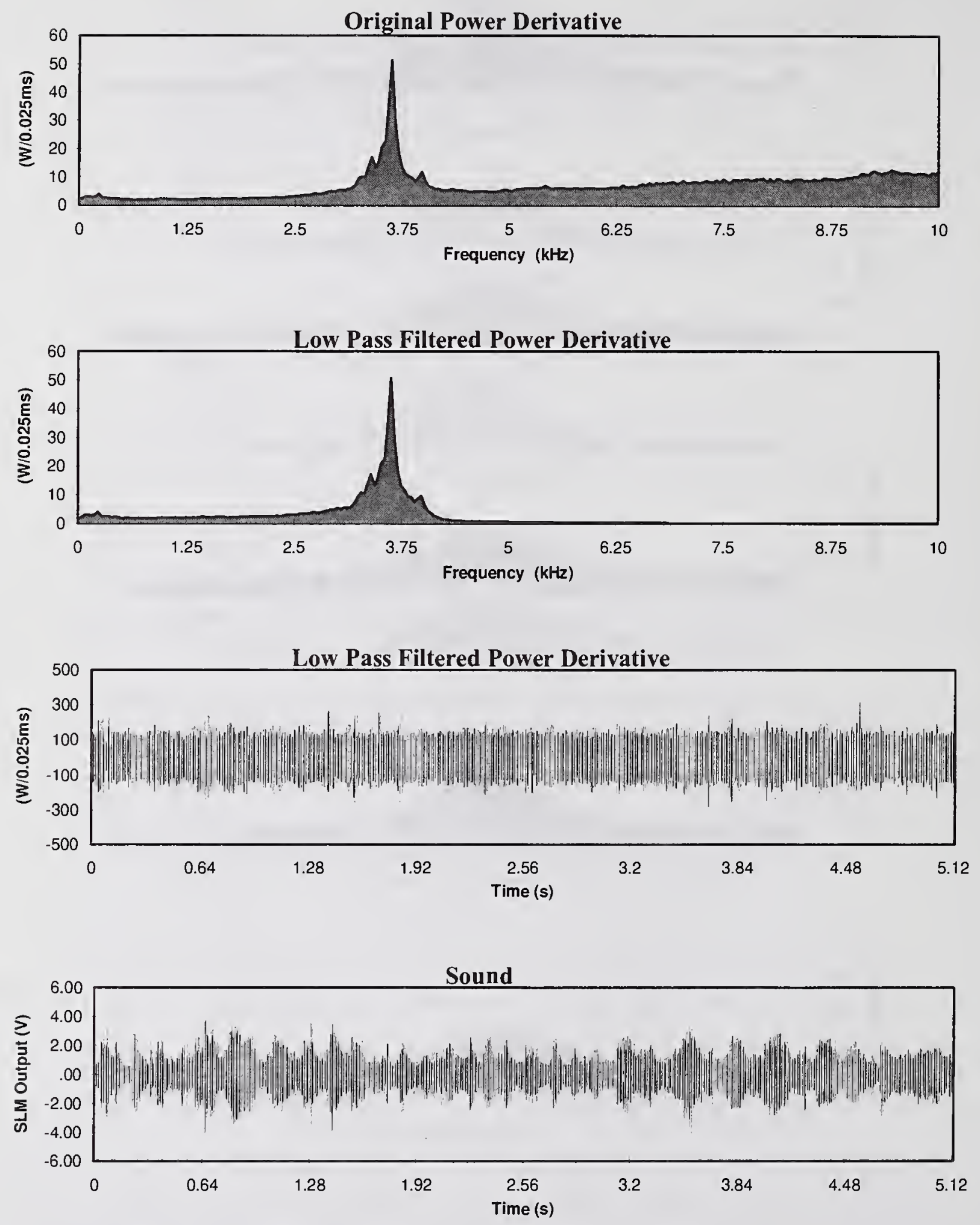

Figure 11: Low Pass Filtered Power Derivative for Globular Transfer Data. 


\section{REFERENCES}

1. Y. Arata, K. Inoue, M. Futama and T. Toh, "Investigation on Welding Arc Sound(Report II), Evaluation by Hearing Acuity and Some Characteristics of Arc Sound", Transactions of JWRI, Vol 8, No. 2, pp 3-38, 1979.

2. C. Wu. "Microcomputer Based Welder Training Simulator", Computers in Industry, Vol. 20, pp 321-325, Oct 1992.

3. T. Morita, Y. Ogawa and T Sumitomo. "Analysis of Acoustic Signals on Welding and Cutting", Proceedings of the 14 th International Conference on Offshore Mechanics and Arctic Engineering, ASME, Part 3,pp 547-552, 1995.

4. E. Blumschein, C. Werner, J Blumschein and U Schik. "A Concept for Cognitive Control of Welding Processes", Proceedings of 6th International Conference on Computers in Welding, Lanaken, Belgium, paper 33, June 1996

5. H. Dadgar, A. Pilorget and M. Fitaire. "Acoustic Noise Excited by Electric Arcs", The IEEE 1977 International Conference on Plasma Science : Conference Record Abstracts, Rensselaer Polytechnic Institute Troy N.Y, pp 117, May 1977.

6. Drouet and F. Nadeau. "Acoustic Measurement of the Arc Voltage Applicable to Arc Welding and Arc Furnaces",Journal of Physics E: Sci. Instrum., Vol. 15, 1982.

7. P. Kaskinen. G. Mueller. "Acoustic Arc Length Control", Advances in Welding Science and Technology :Proceedings of an International Conference on Trends in Welding Research, Gatlinburg, TN, USA, pp 763-765, 1986. 


\title{
FAST DETECTION OF ESSENTIAL CHANGES IN GMAW PROCESSES
}

\author{
E. Blumschein*
}

\begin{abstract}
An investigation has been performed into fast detection of sudden changes being considered as seriously affecting regularity of GMA welding processes, welding control, and eventually weld quality. It includes case studies in how the plasma changes into an unstable mode and return, in transition among different metal transfer modes, in sudden failures like arcs between wire and contact-tube, and in how large droplets emerge. Pattern selection and recognition have been based on electrical, acoustic and video signals which were recorded synchronously and with a sufficient in most cases sample rate. In order to cope with a plenty of irrelevant or even misleading information, some principles of expert welder's intelligence have been adapted. They will allow to monitor that process more distinctively and to improve it on condition it is quickly controlled by an inverter type power supply.
\end{abstract}

\section{INTRODUCTION}

Arc welding, GMAW in particular, behaves rather chaotic due to a variety of poorly understood plasma physical phenomena (Ref. 1). Therefore, weld quality is affected by random spattering and other disturbances. Traditional control systems are not able to react against them. Advanced welding systems try to mimic human intelligence in order to associate weld quality with both observable deviations from optimal process and adequate countermeasures. Though, the brain works quite different from today's computers, cognitive control (Ref. 2) is likely to become the preferred approach during the next century, at least. Imitation of human cognitive performance should not stick for details. Technical sensory systems have specific advantages and drawbacks. They are capable for sensing electrical quantities. They may be specialized by exchangeable software. On the other hand, they will perhaps never reach the astonishing performance of the slow but very complex signal processing by neurons in the brain.

However, power electronics can react much faster than any human muscle. Even the most skilled performer of welding is not able to take full advantage of already existing fast controllable power electronics. Mitigation of trouble requires its detection in-time. This task can definitely not be fulfilled by statistical signal analysis based on a much longer span of time being indispensable for getting a useful representation of a typical welding process. For that reason, there is no chance for creating any controller that uses unspecifically averaged data to recognize failures much faster than the human brain. The only promising solution is a combination of proper conditioning, knowledge of physical scenarios to be expected, presage and attention, fast detection of features that mark essential changes, and an appropriate, well-calculated response to them. This paper starts with an exemplary review of some observed physical phenomena.

* IELE, Otto von Guericke University, PSF 4120, D-39016 Magdeburg / blumschein@et.uni-magdeburg.de 


\section{STRIKING FEATURES OF PLASMA MODE CHANGES}

Voltage of an ordinary arc is fairly smooth. It varies only a little around $20 \mathrm{~V}$ with shielding gas, wire extension, current, etc. This stable behavior might be explained by a balancing effect of competition between several invisible, restless moving to fresh oxides cathode spots of very small size and short life span.

There is an unstable mode of plasma, too (Ref. 3). Figs. 1 to 14 show distinctive features.

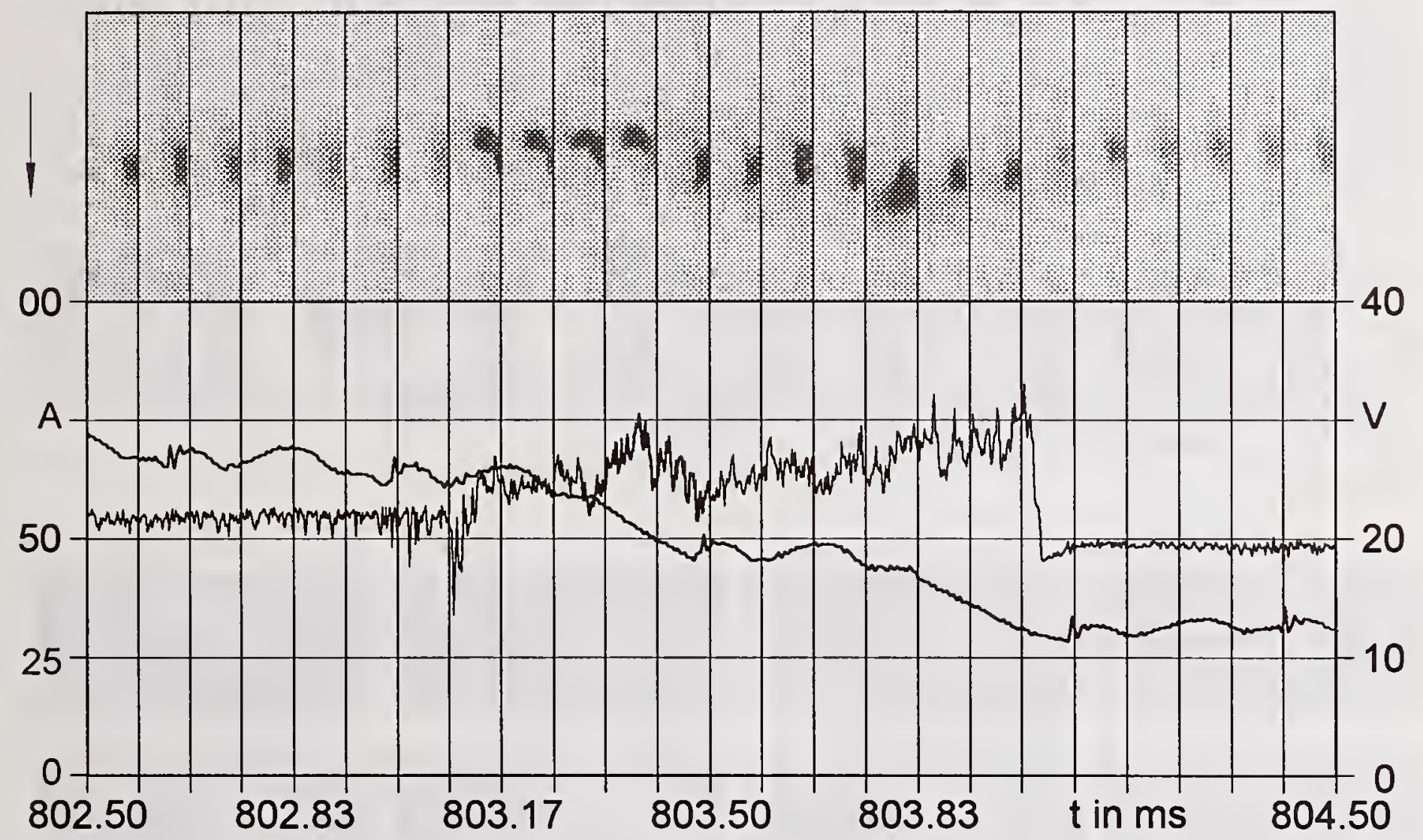

Figure I - Video (12 $000 \mathrm{fps,} \mathrm{inverted,} \mathrm{positive} \mathrm{wire} \mathrm{electrode} \mathrm{left,} \mathrm{arrow:} \mathrm{direction} \mathrm{of} \mathrm{welding),}$ voltage, and current marking transient unstable plasma mode within carbon dioxide.
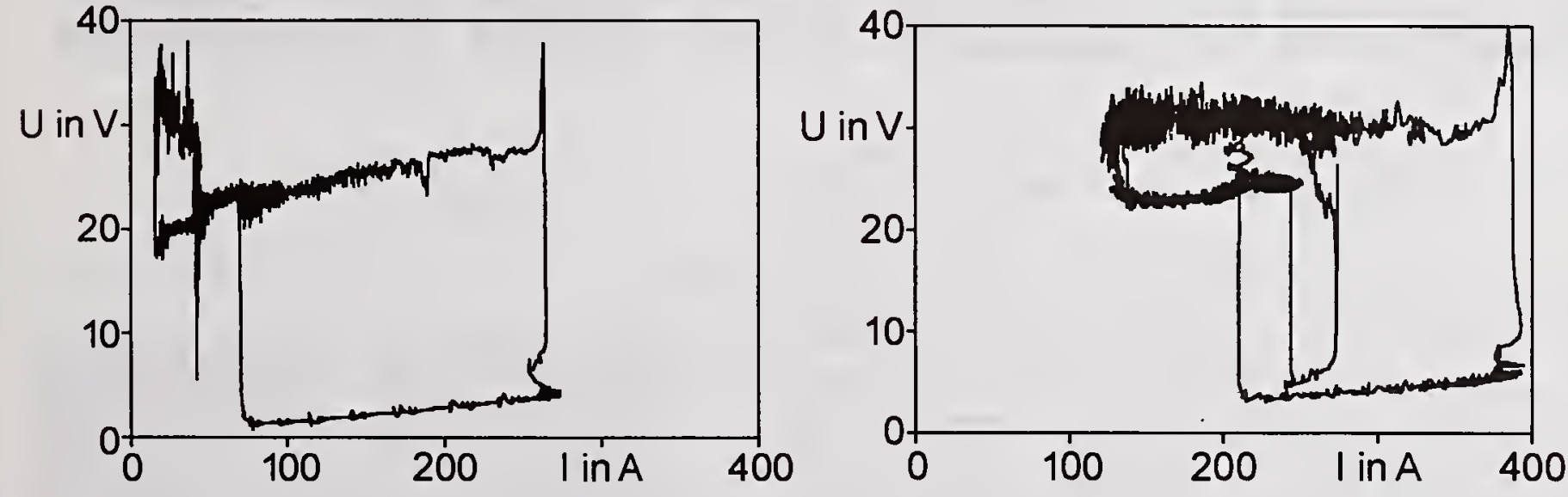

Figure 2 - Unstable mode (upper traces) within carbon dioxide (left) and 98\% argon (right). 
Measured voltage fluctuates heavily in unstable mode (Fig. 1). Mean value of voltage is, as a rule, increased by up to $10 \mathrm{~V}$ (Fig. 2). Consequently, current drops (Figs. 1, 3, 10, and 14). Also, the emitted light is much brighter (Figs. 1,4), the arc's shape and location are changed (Fig. 4), and the droplet of liquid metal, hanging at the tip of electrode is obviously rejected and deflected ahead the direction of welding. Fig. 3 shows how the unstable arc comes out from dip transfer.

It disappears also pretty abruptly after a few milliseconds or less. One can see how little the welding process is muddled up. Notice the voltage dropping and returning instantly before transition into unstable mode being connected with only a negative sound pulse due to implosion. Surprisingly, sound emission during the unstable mode is this time even weaker than that from ordinary arc, although the unstable plasma is exceptionally bright and moves quickly around as to be seen from video frames (Fig. 4). The isthmus of liquid metal bridge is to be seen in frame (a) and marked by a cross as a reference point.

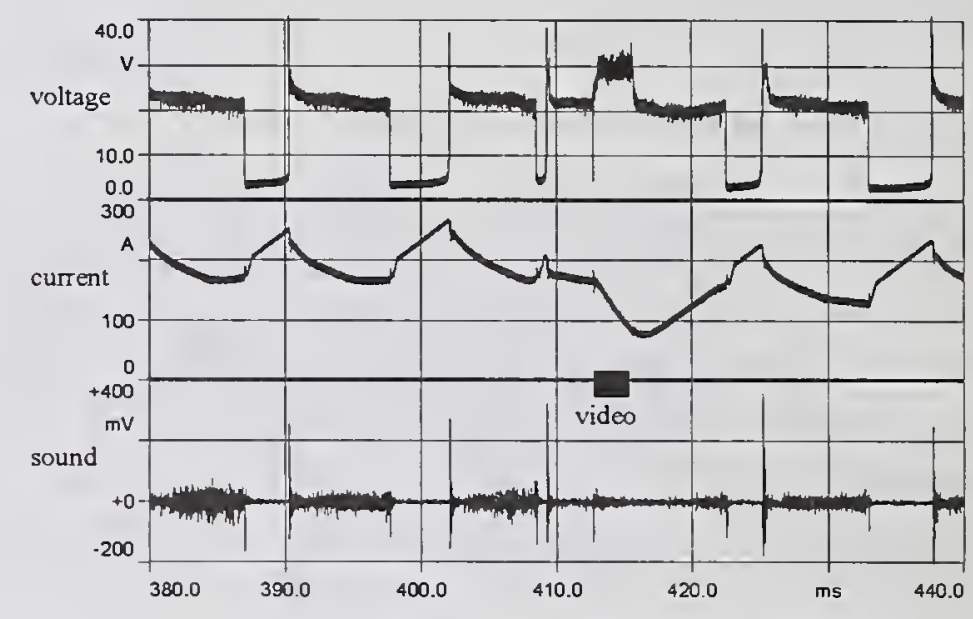

Figure 3 - Effect of unstable mode on dip transfer.

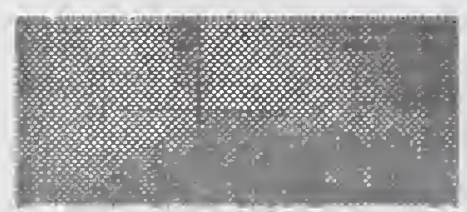

(a) $412.25 \mathrm{~ms}$

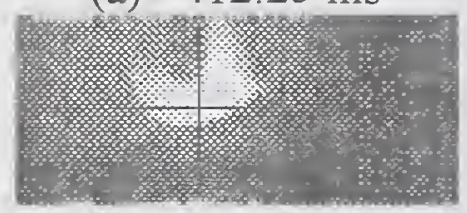

(e) $413.25 \mathrm{~ms}$

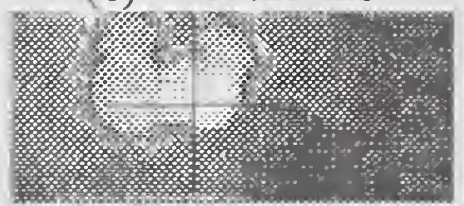

(i) $414.25 \mathrm{~ms}$

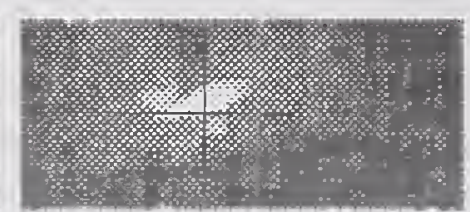

(b) $412.5 \mathrm{~ms}$

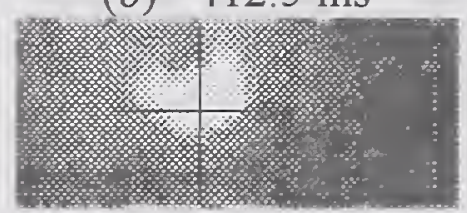

(f) $413.5 \mathrm{~ms}$

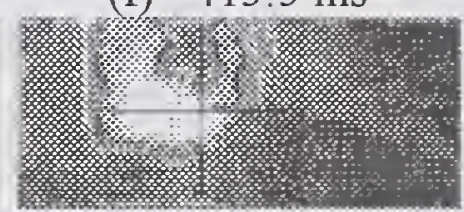

(j) $414.5 \mathrm{~ms}$

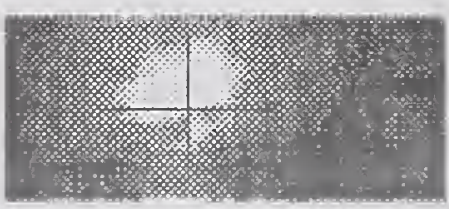

(c) $412.75 \mathrm{~ms}$

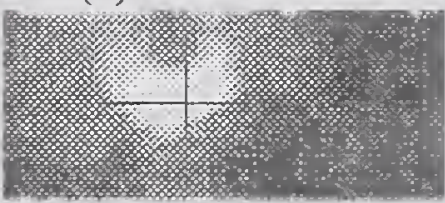

(g) $413.75 \mathrm{~ms}$

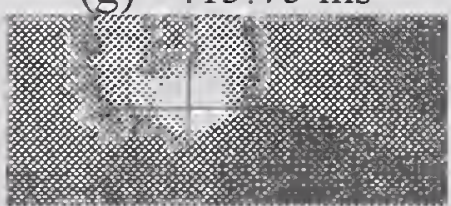

(k) $414.75 \mathrm{~ms}$

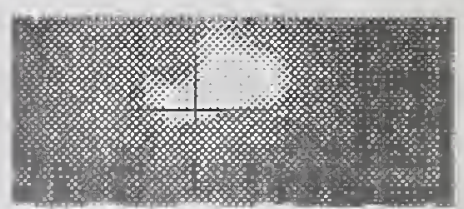

(d) $413.0 \mathrm{~ms}$

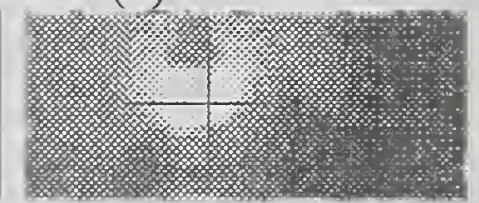

(h) $414.0 \mathrm{~ms}$

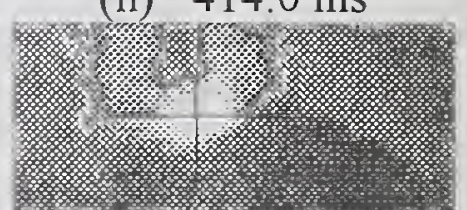

(1) $415.0 \mathrm{~ms}$

Figure 4-Wiggling plasma corresponding to a marked section of Fig. 3.

Arc plasma expands sidewards and upwards. It flicks off the molten metal, and it quickly shapes a rather large droplet by touching them from all sides like with baker's hand. The restless jiggling is a striking feature of unstable plasma mode. Separate and less intense light patches below the main one are certainly no indications of cathode spots but reflections on surface of molten pool. Workpiece was moved to the right in all experiments. As a result, bead has built up on right side, and large droplets were deflected to the left. While the normal arc tends to locate its cathode spots on a crescent shaped cool area ahead the molten pool providing fresh oxides, this behavior is entirely lost during unstable mode. 
The large droplet in Fig. 5 is already deflected. Plasma mode changes within a quarter of a millisecond from normal (a) over unstable (b) back to normal (c). Correspondingly, location is stepwise changed, too. The unstable mode seems to have a preference for hot metal surfaces. It mostly starts from a tiny hot spot, as a rule after a short circuit.

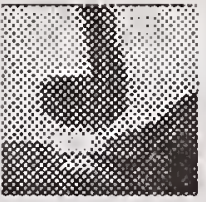

(a) $0.0 \mathrm{~ms}$

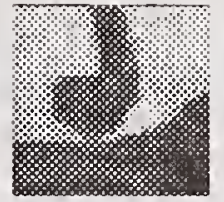

(b) $0.25 \mathrm{~ms}$

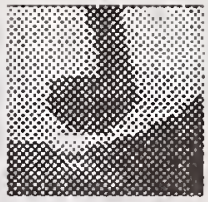

(c) $0.5 \mathrm{~ms}$

Figure 5-Stepping plasma.

Any kind of voltage drop is always preceding, at least for a very short time. As expected, there was not any occurrence of an unstable mode in dip transfer unless arc length was very small before. Repetitive forerunners of increasingly deeper dropping voltage, as sketchy to be seen in Fig. 1, were often noticed. Maybe, initial wire-to-pool distance can be larger at higher current as to conclude from in Fig. 6. The left images (a), (b) belong to a slowly but continuously broadening and declining in height, bell shaped, transitional but ordinary arc. The other show a rapidly and jerky expanding plasma just at the beginning of unstable and rejecting plasma mode. A visible piece of electrode seems not to reach the pool. Anyway, between (b) and (c) there was a full short circuit lasting over 75 microseconds.
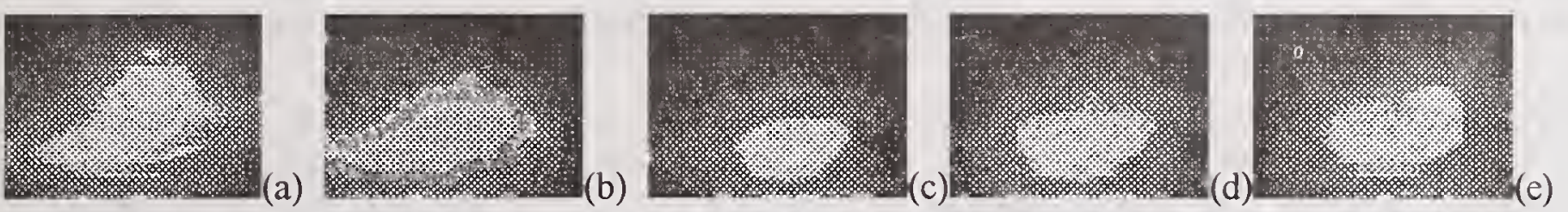

Figure 6 - Transition into unstable plasma mode.

During unstable mode the gap between wire tip and molten pool is increasingly widened to an extend preventing immediate transition into a new short circuit. Fig. 7 gives evidence for an early possible exception from that. Immediate transition into a longer lasting short circuit happens rarely.

The sudden return from unstable into stable mode is probably forced by two reasons for cooling: dilution of extending plasma volume and decrease of current. This transition is highly striking in videos which were recorded from welding under conditions known for causing unstable mode for a remarkable share

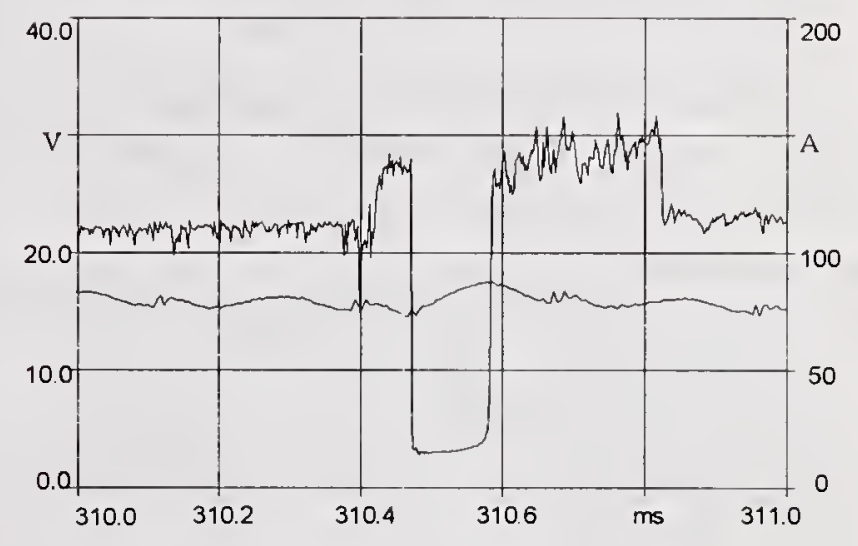

Figure 7 - Interrupted unstable mode.

of time (Ref. 4). In that case, the droplet is repelled during unstable mode to the left and upwards. It becomes very large. In Fig. 8(a), the neck is illuminated by a wiggling unstable plasma being located by chance just behind. Plasma suddenly steps to left (i. e. ahead in direction of welding) onto a previously dark area. Its shape simultaneously changes into a stretched ahead conic one, to be seen in Fig. 8(b). It
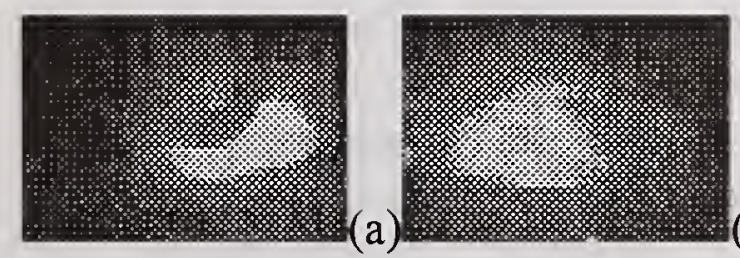

Figure 8-Return from repulsive unstable mode (a) to normal arc (b). also contrasts by quiet behavior. The droplet is no longer rejected. It falls down into a short circuit soon afterwards. Uncertainty of such preceding return to normal arc before metal transfer could provide a plausible explanation why the so called 'globular' metal transfer is a highly erratic one. Perhaps, the necessary cooling can be stimulated by deliberate reduction of electric power. 
Signs of unstable plasma mode were detected at almost all arc welding processes including pulsed spray metal transfer (Fig. 9).

A transitional unstable for a short time plasma is normally not considered as a serious disturbance, since fast power electronic control is able to quickly output more power in order to prevent the current to fall below a given limit.

Nevertheless, diagnosis of welding processes and control will benefit a lot from detection and separation of time

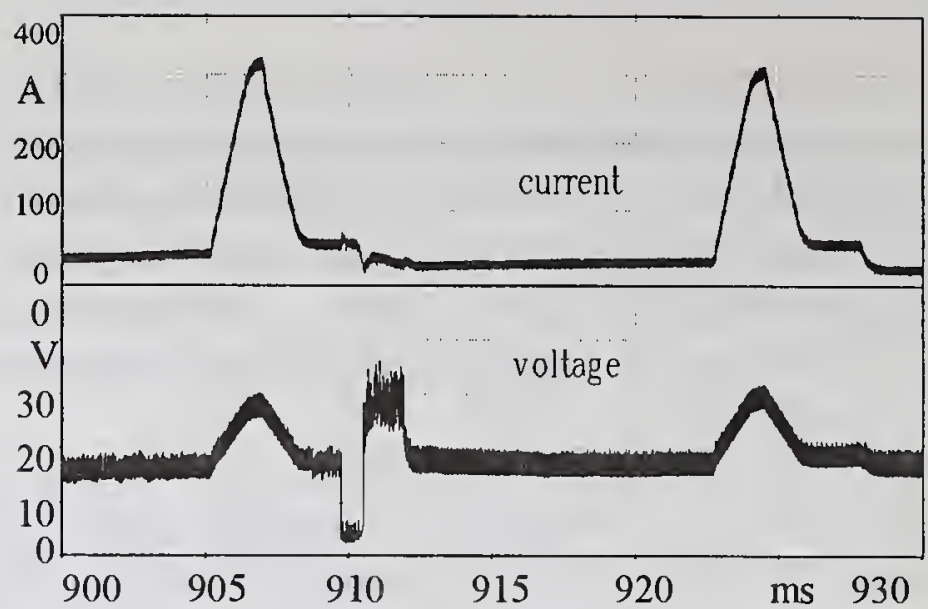
intervals in which measured signals do not correctly mirror the main process but an abnormal transitional deviation from anticipated behavior. It would be possible to detect the unstable mode from a variety of discernible features. Restriction to the voltage signal alone is also possible, and it is kept for the best option in this case.

However, the unstable mode can last for tens of milliseconds or even longer. Conditions to Fig. 10 were stainless steel of $1 \mathrm{~mm}$ diameter, TPS adjusted at $28 \mathrm{~V}$ (recommended value $30 \mathrm{~V}$ ), $250 \mathrm{~mm} / \mathrm{s}$ wfs, $98 \% \mathrm{Ar}, 2 \% \mathrm{CO}_{2}$.

As a result of long time unstable plasma mode, regular short circuiting transfer of molten electrode is obstructed. The accumulated volume forms a huge hanging droplet which is sidewards and upwards deflected. Because transfer of it requires almost $10 \mathrm{~ms}$, current reaches 300 Amps causing unacceptable heavy sputtering.

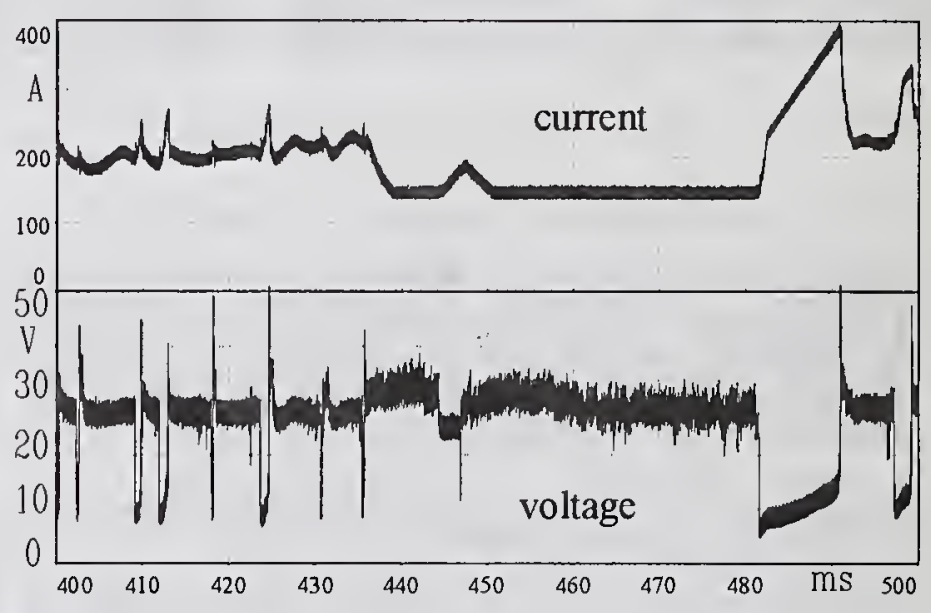

Moreover, the essentially unstable plasma mode is very likely linked with an obvious deterioration of weld bead quality. In particular, GMAW of aluminum alloys is known to suddenly fall into puckering if temperature of molten pool becomes too high.

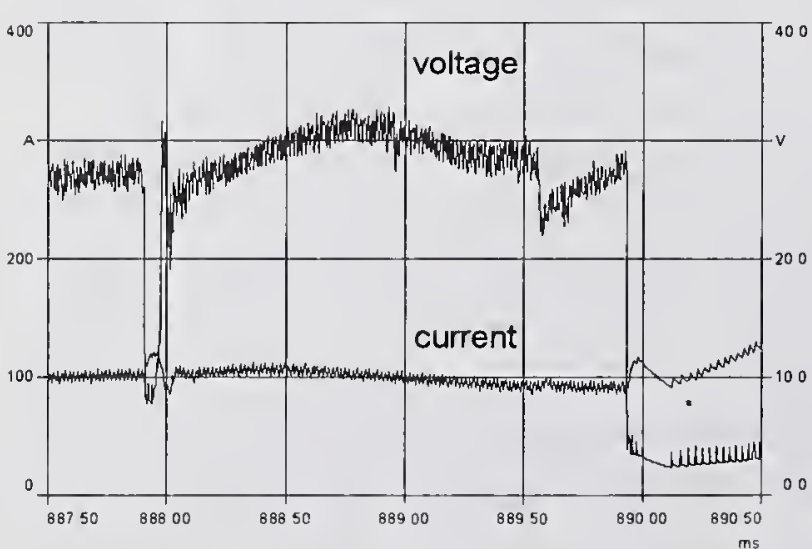

Figure 11 - Lack of shielding gas.
Puckering means not only a bad surface but also porosity, oxidation, and nitrification. It is believed to be caused by air entrainment (Ref. 5).

Experiments with totally unshielded welding were performed in order to study indications of lacking shielding gas. Arc welding in pure air was found to be accompanied by an almost permanently unstable plasma mode as illustrated in Figs. 11, 12. The first two frames show an ordinary arc, burning ahead, corresponding to smooth voltage in Fig. 11. 
Then the molten wire tip is hooked and very irregularly shaped by an unstable backwards directed plasma, until finally a taper causes a durable short circuit.

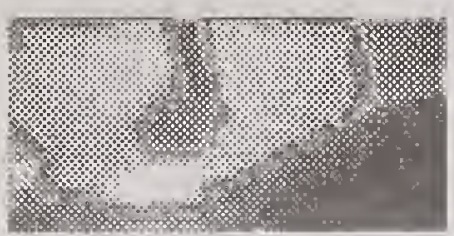

$887,0 \mathrm{~ms}+\mathrm{K}$

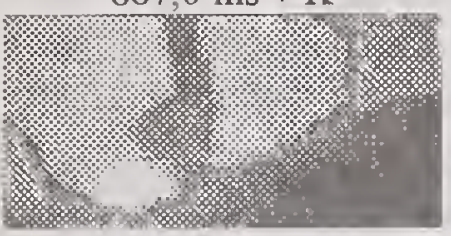

$887,25 \mathrm{~ms}+\mathrm{K}$

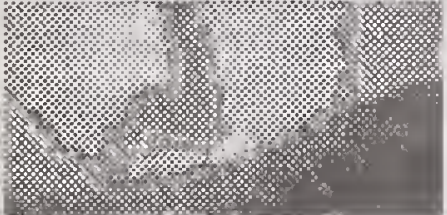

$887,5 \mathrm{~ms}+\mathrm{K}$

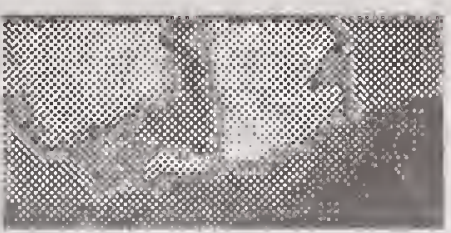

$887,75 \mathrm{~ms}+\mathrm{K}$

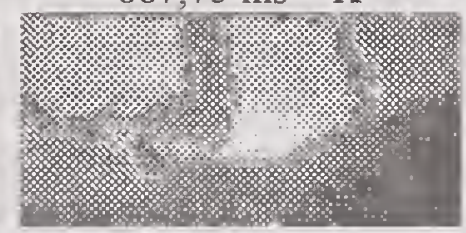

$888,0 \mathrm{~ms}+\mathrm{K}$

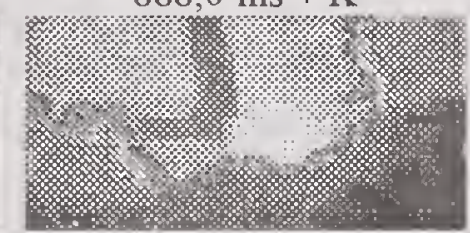

$888,25 \mathrm{~ms}+\mathrm{K}$

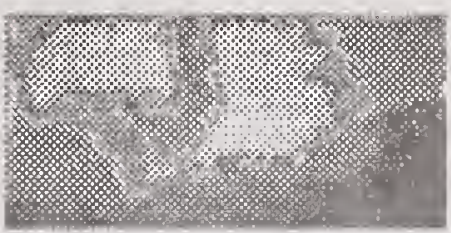

$888,5 \mathrm{~ms}+\mathrm{K}$

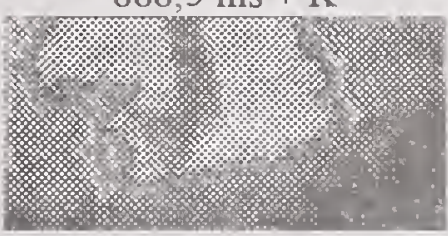

$888,75 \mathrm{~ms}+\mathrm{K}$

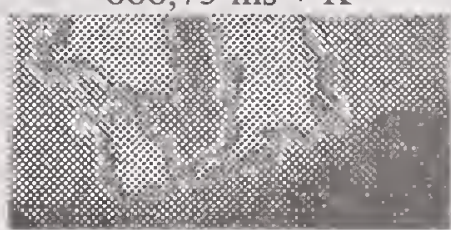

$889,0 \mathrm{~ms}+\mathrm{K}$

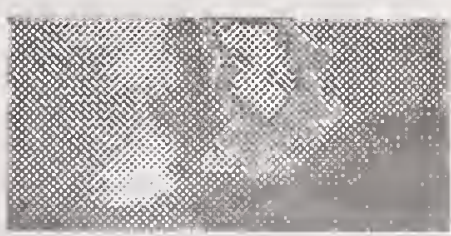

$889,25 \mathrm{~ms}+\mathrm{K}$

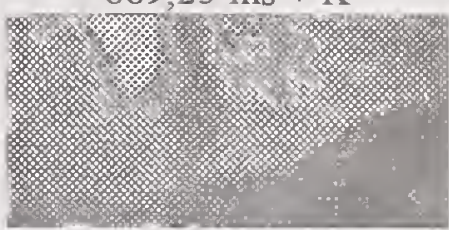

$889,5 \mathrm{~ms}+\mathrm{K}$

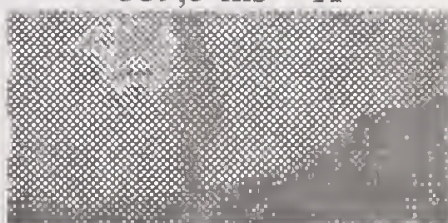

$889,75 \mathrm{~ms}+\mathrm{K}$

$\mathrm{K} \approx 0,6 \mathrm{~ms}$

Figure 12 -Video images of unstable plasma mode in air corresponding to Fig. 11.

There is a remarkable stabilizing effect of a small portion of titanium added to the wire. It can presumably be attributed to desoxydation ability of titanium (Ref. 6). Occurrence of a conspicuous black trace from condensed material and some effects of oxygen on arc stability and spatter (Ref. 7) might also be related to the unstable plasma mode.

Probably, plasma mode will also turn out as important outside arc welding on applications like lamps and switchgear. For that reason, the neutral denotation 'unstable plasma mode' has been preferred as long as it is not entirely clarified whether or not this type of plasma reckons among arcs. A seemingly similar unstable plasma is called spark, in ED machining.

\section{DETECTION OF TRANSITION BETWEEN TRANSFER MODES}

As generally accepted, GMAW can be divided into modes of molten metal transition as follows: Short circuiting or dip transfer relates to low voltage and low current. It changes with increasing power into globular transfer. Beyond critical values and only with argon-rich shielding gas the arc constricts, tapers the wire tip, and actively transfers metal as a rapid sequence of small detached and in line accelerated droplets, projected to the molten pool. Rotational spray transfer is the mode at highest power and large stand off length.
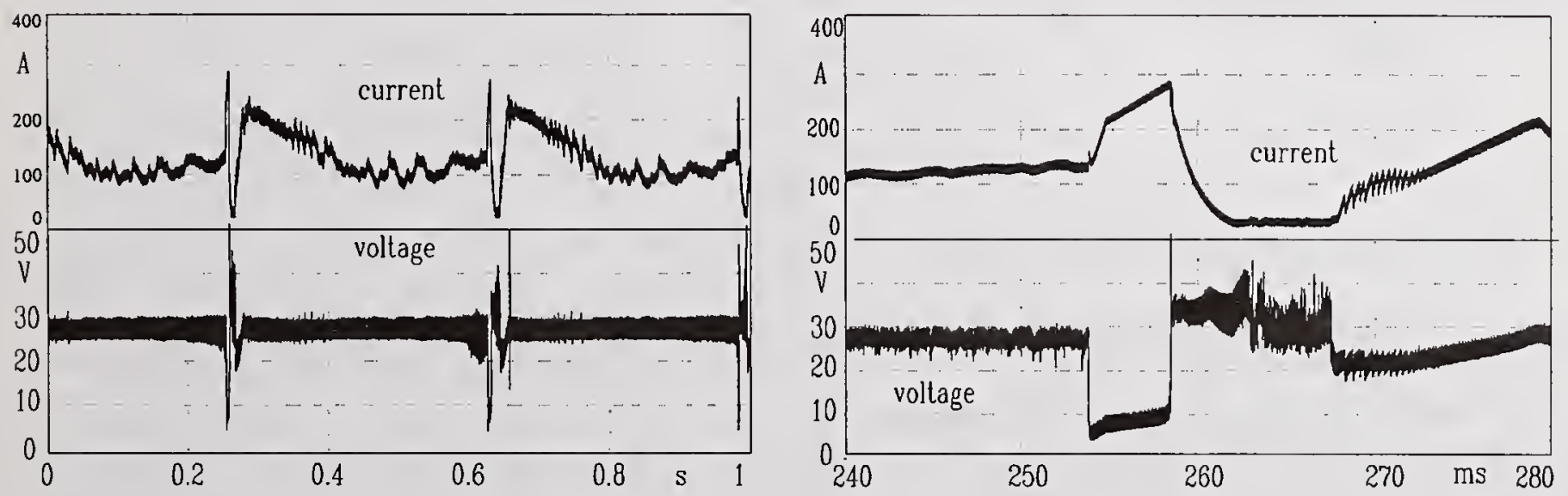

Figure 13-Periodically changing transfer.

Figure 14-Detail from Fig. 14. 
Why has mapping of boundaries between transfer modes (Ref. 8) proven a rather difficult task? Is somewhat wrong with this classification? Well, 'globular' can be mistaken as free falling, and one might verbatim imagine 'spray' as scattering, too. However, the real problems are practical ones. Figs. 13, 14 show for instance a mixed, periodically alternating transfer.

Each of the two short circuits transfers within about 5 milliseconds a pretty large droplet and generates much sputter. Thereafter, an unstable rejecting plasma mode creates a long arc gap. Extinction of plasma is prevented by control, ensuring a constant minimal current. Then, as long as current is high enough, so called spray transfers fine droplets. This may happen without any short circuit. But spray might be mixed with short circuits due to pre-formed droplets, too. Fig. 15 shows a typical detachment of such a droplet. The short circuit would be identified as instantaneous from its duration, even though it

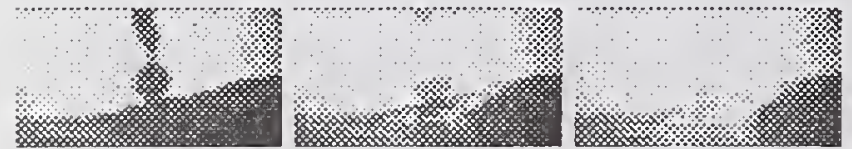

Figure 15 - Transfer of pre-formed droplet. transfers the droplet. Since the neck is already thin, a new plasma expands from the neck. The droplet is not rejected. It is first detached from wire, then incorporated. Normally, incorporation goes before detachment in dip transfer.

If voltage is somewhat increased, the large short circuits vanish, but, as evident from Fig. 16, there is still a periodic repetition of metal transferring short circuits, alternating with periods of spray transfer. Because small droplets are transferred anyway, it does almost no matter whether there is a transferring short circuit or no short circuit at all. Quite regular distances between the events correspond to time required for droplet formation. Electronics clamps voltage. So current slightly floats. A periodic change between audible sputtery and inaudible genuine spray generates a rather strange soft noise. It sounds like shaking a tin with some peas. Further transition into pure spray may also be precisely perceived by ear. The less crackle happen, the more each single one stands out.

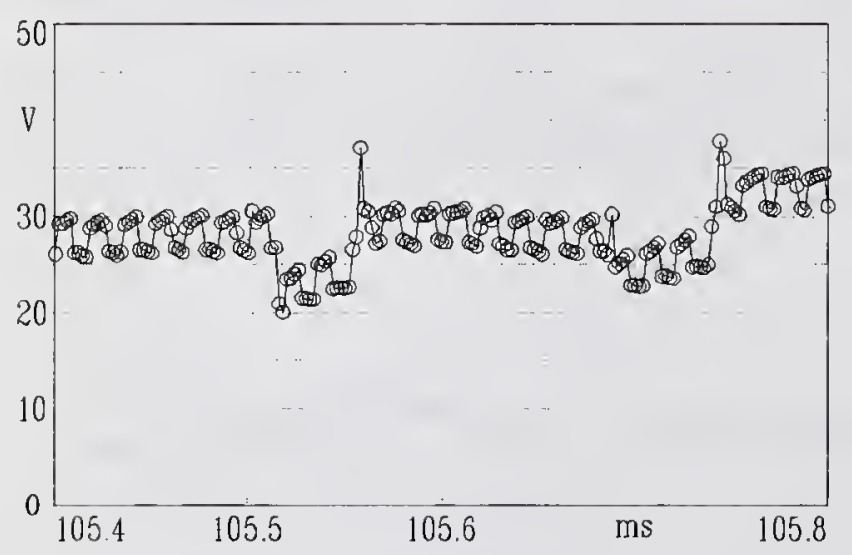

Figure 17 - Disturbance in spray mode.

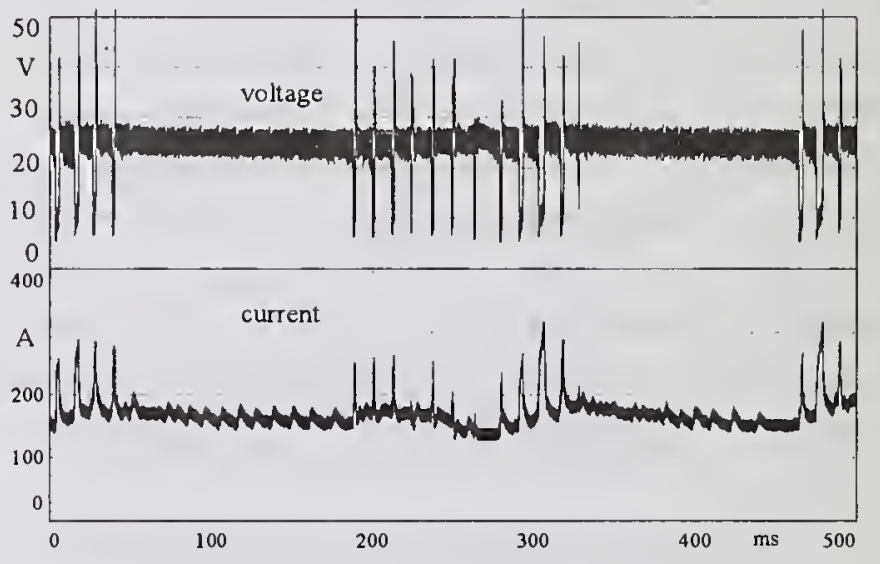

Figure 16 - Genuine and sputtery spray.

Fig. 17 focuses on two successive disturbances. They were audible as only a single one. Else the voltage was smooth for seconds. The $60 \mathrm{kHz}$ voltage ripple is also beyond the frequency limit of human hearing. Superior human performance is based on several principles very worth to study. One of them is diversification of inputs. Auditory and visual perceptions of changes in transfer mode complement each other. Vision provides a clear picture of arc shape and of corresponding penetration profile to be expected. Audition provides a more gradual
suickly as possible by purely empirical means. orientation and warning about risk of change as quickly as possible by purely empirical means.
It benefits in that from an other principle. Recognition of trained temporal pattern is generally more versatile and faster than identification by statistical values. However, dynamic pattern recognition is still in its initial stage. Human intelligence includes permanently adaptive pursuit. 
Matching pattern sequences are activated from memory on demand. So a broad temporal horizon does not contradict to ability for reaction within a tiny span of time. Actively adapting sensation is a rather general key to efficiency. Active sensation (Ref. 9) includes targeting, focusing and preprocessing. An example reveals considerable deficiency of today's sensory systems:

A serious problem is detection of uncontrolled transition from rotational spray into ordinary spray transfer. Sudden standstill is unacceptable during so called T. I. M. E. process due to the digging effect of constricted arc. Although rotational spray transfer was not within the scope of this investigation, occasional rotation of wire tip like a garden hose was observed (Fig. 18).
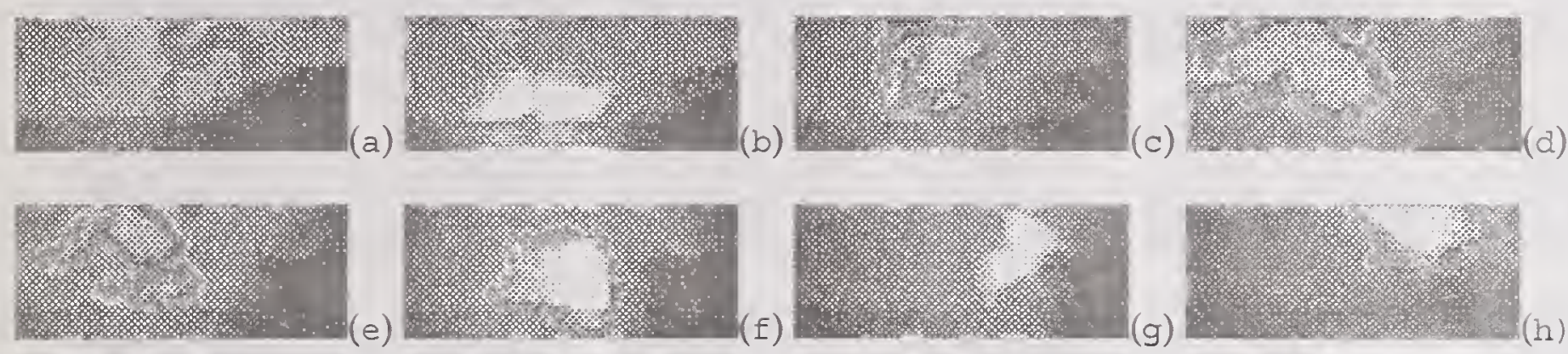

Figure 18 - Transition from stubbing wire into rotation of wire tip.

Rotation seems to be an usual failure, ending either badly in extinction of arc or favorably in sudden standstill with detachment of a somewhat larger droplet immersed into the arc. Unfortunately, there was no sufficient indication of rotation available from voltage or current signals. Visual monitoring is complicated because of fast spatial movement and changing brightness.

\section{TRACKING AND INTERPRETATION OF SELECTED DETAILS}

Prospect seems to be bleak for designing an artificial neural network or any other classifier as a comprehensive and general one-one imitation of how operator's intelligence copes with failures in complex welding processes. In our time, even a most dilettante copy of brain would be too expensive. Also, it would never act as fast as possible with sensors and necessary for welding. More promising progress has been achieved i identification of single selected details fro signals sampled with a high rate providing hig resolution beyond human perception.

There is still a lot to discover. For instance, strange cluster (Fig. 19) was revealed to consis of irregularly repeating with rather high frequenc voltage drops. It indicates a tiny gap betwee molten pool and hanging droplet almost dippin into it but finally failing to do so. A regula pattern of lower frequent voltage ripple is visibl before and after that phenomenon. The calculabl

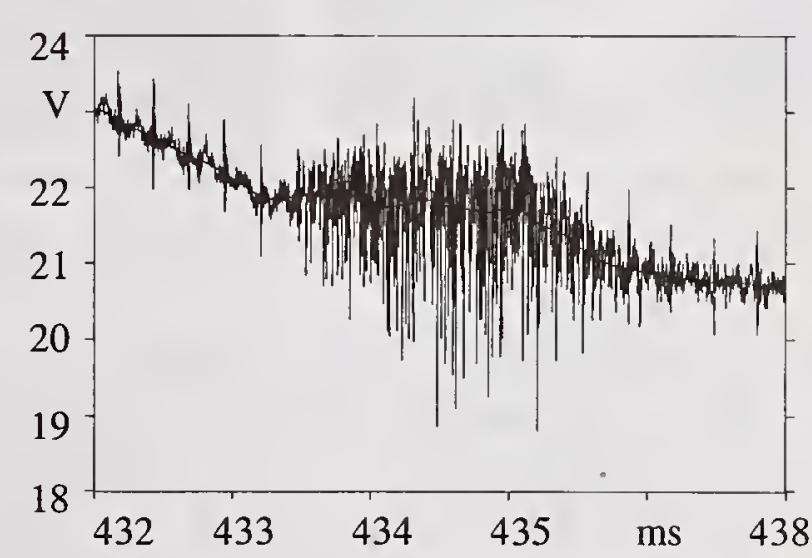

Figure 19 - Cloud of voltage dips. ripple can easily be separated despite its type considerably differs from that shown in Fig. 17. Maybe, such detail is marginal and useless. In that case it might be considered as a possible cause of irritation. Possibly all peculiar features of a signal were pre-interpreted to a certain extend in order to exclude irrelevant or misleading information. Our final aims have been, of course, first tracking down in reliable signs of essential primary events to react to quickly and then implementing that intuitively found way by means of digital signal processing. 
Since there is currently much upcoming progress on cheap and robust microphones and video cameras, acoustic and visible signals, in particular, were searched for indications of such events with special emphasis on origin of excessive spatter. Unexpected emergence of some exceptional large droplets was observed as shown in Fig. 20.
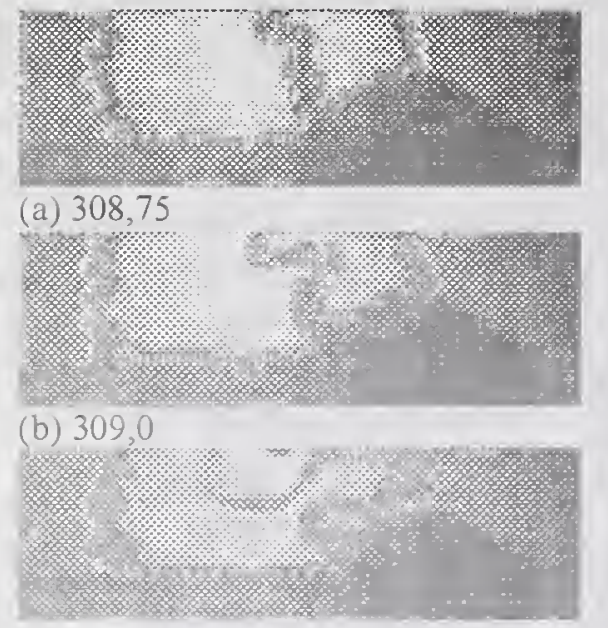

(c) 309,25
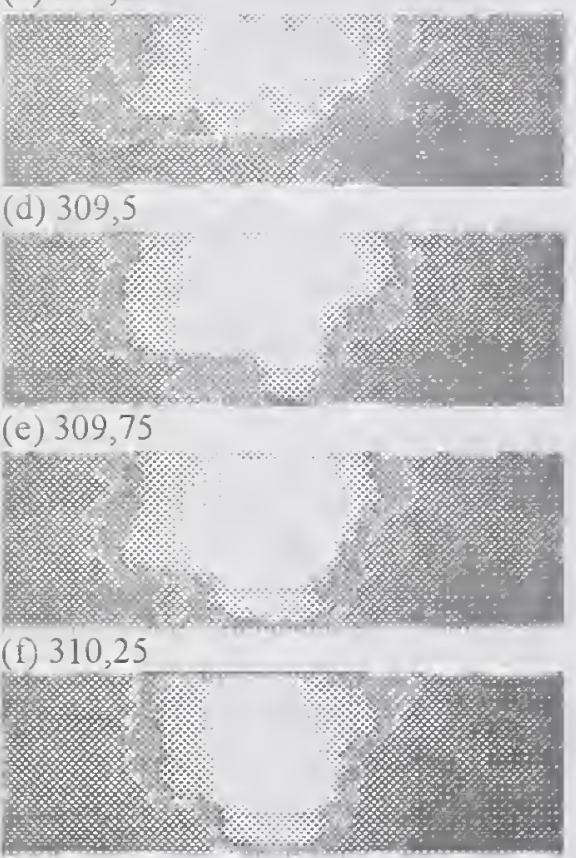

(a) 311,0

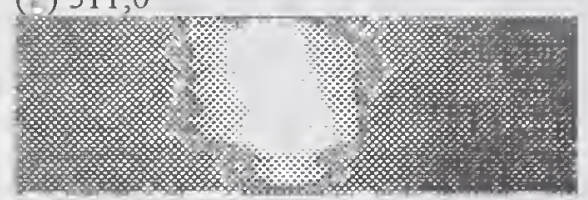

(h) 311,5
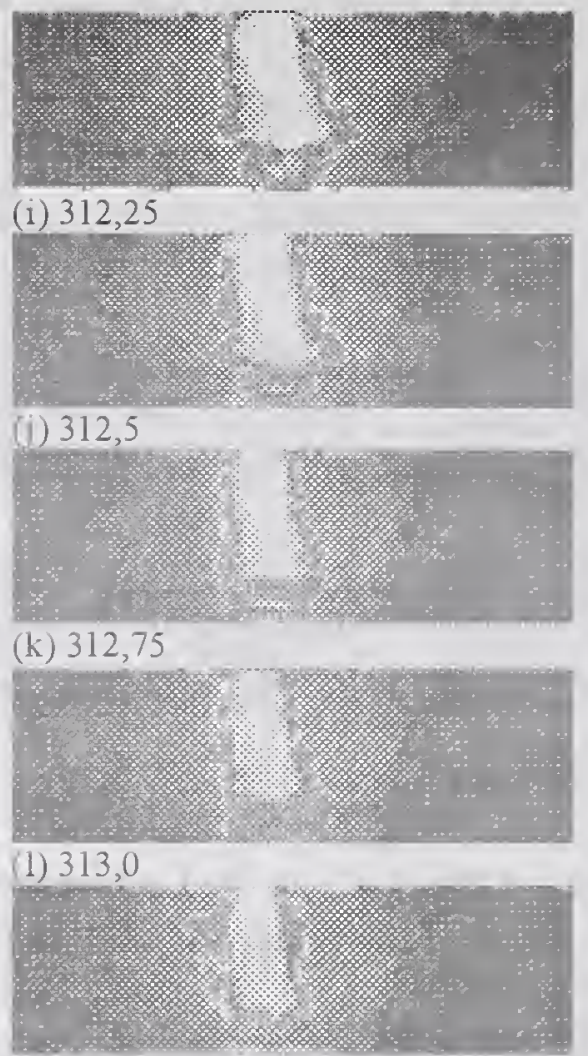

(m) 313,25
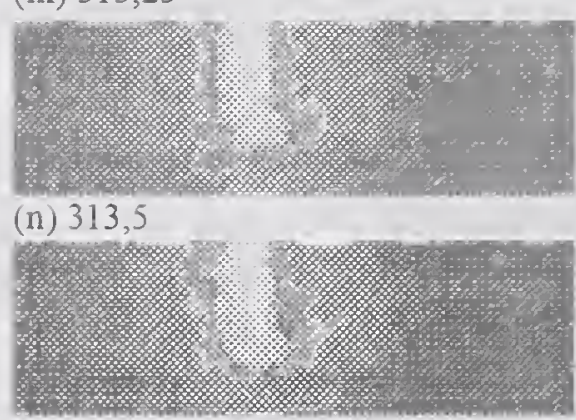

(o) 313,75

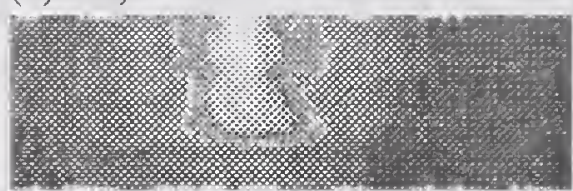

(p) 314,0

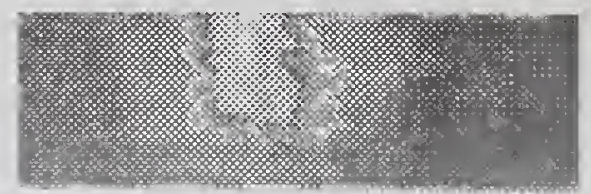

(q) 314,25
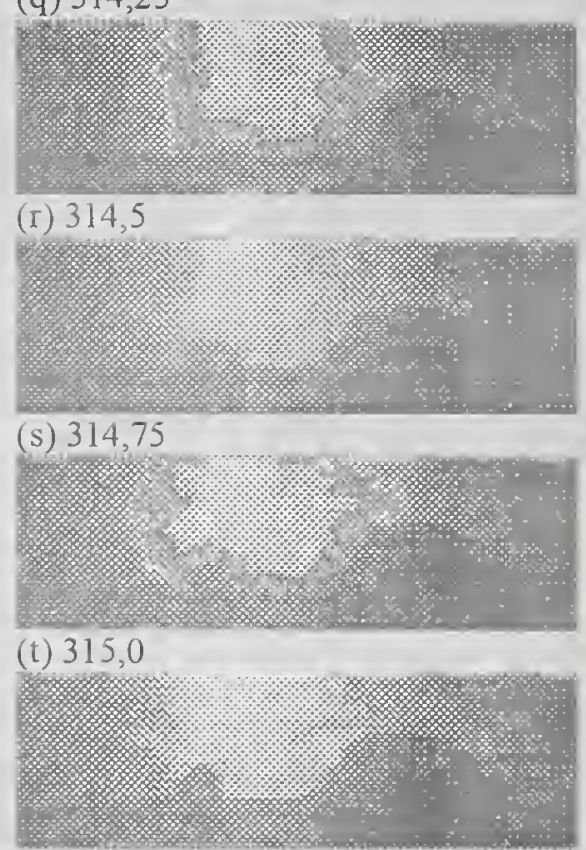

(u) 315,5

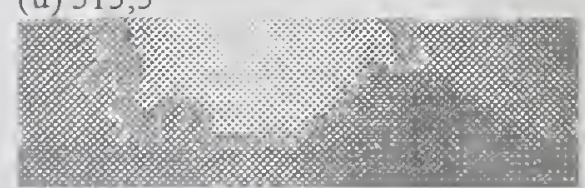

(v) 316,5
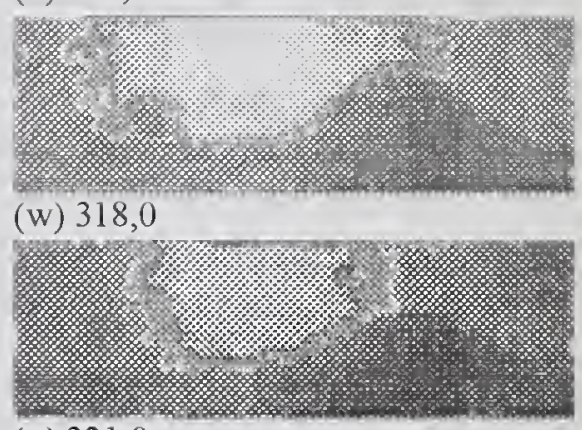

(x) 321,0

Figure 20 - Large droplet emerging below the arc after stubbing.

Rejection of droplets is known (Refs. 10, 11) in principle. In above case the droplet was definitely not rejected after hanging at the wire tip before. It seems to emerge (at $p$ ) from the middle of molten pool, but it might originate from snake shaped rest of wire (c). Given, acceleration of the droplet depends on electromagnetic force until it has detached, then early reduction of current could help, maybe. However, it is not realistic to identify on-line from video signal all rising droplets for several reasons. This would require an additional back light. Only a small part of spatter is visible because focus is fine tuned on centre of arc with small depth of field. Success of image processing is questionable due to brightness of unstable plasma mode and strong reflection in that case. Successive stripes in Fig. 21 point at very fast expansion.

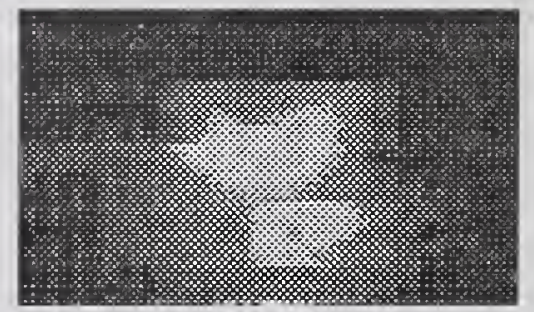

Figure 21 - Reflection. 
Moreover, there is no need for droplet detection since all exceptional current peaks turned out to cause one or more spatter made visible as shadows from arc itself. Consequently, it will be better to prevent in time conditions leading to excessive spatter. Therefore short circuiting transfer has been studied and moments of transition from arc to short circuit and reverse have been precisely determined. Sample rates were adapted to requirements and limitations in a laboratory, not yet tailored on application. OneMHz summary sample rate and at least one second record length were chosen for most of the reported measurements in order to avoid worst errors.

Study in peculiar arc behavior required the voltage to be sampled with a much higher rate. Figure 22 illustrates difficulties to decide when a short circuit starts and ends. Twin-spiked arc voltage is typical for reignition after stubbing. Usually, the first arc appears near to contact tube where the stubbed wire was mostly bent. Accordingly, the belonging sound signal may not be used this time for monitoring distance between torch and surface of molten pool (Ref. 3). Figure 23 also relates to stubbing. On the left, wire has just

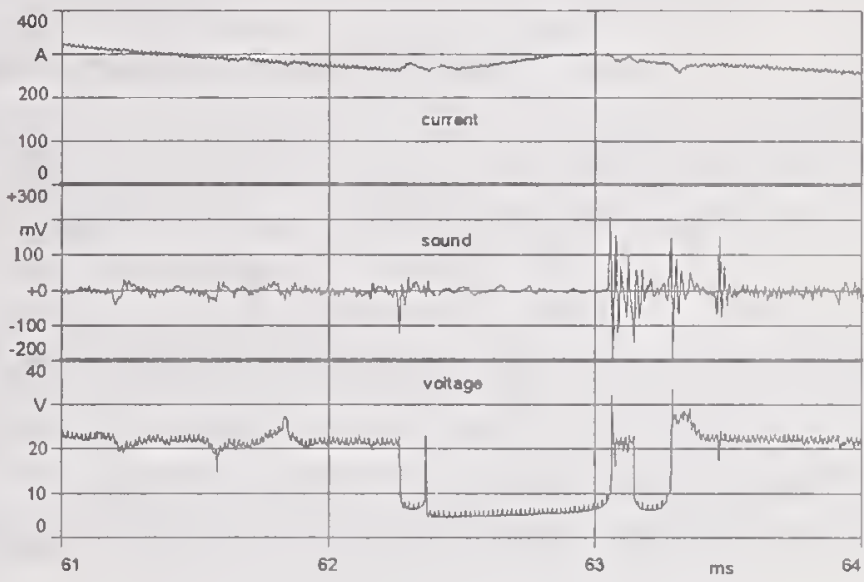

Figure 22 - Splitted voltage signals.

stubbed on solid ground of molten pool. Movement of torch trucks it sidewards inside contact tube. By chance, contact has to change from leading to lagging side. Current has to flow from contact tube to wire through an arc during that short time. That hidden arc could be detected from potential of wire but it is also evident from an inconspicuous voltage step by about 10 Volt for half a millisecond in combination with missing of sound signal. Although energy amounts only as few as $3 \mathrm{Ws}$, a wire of $1 \mathrm{~mm}$ diameter can locally melt and may or may not stick within contact tube. The sticking point was observed to be broken by powerful wire

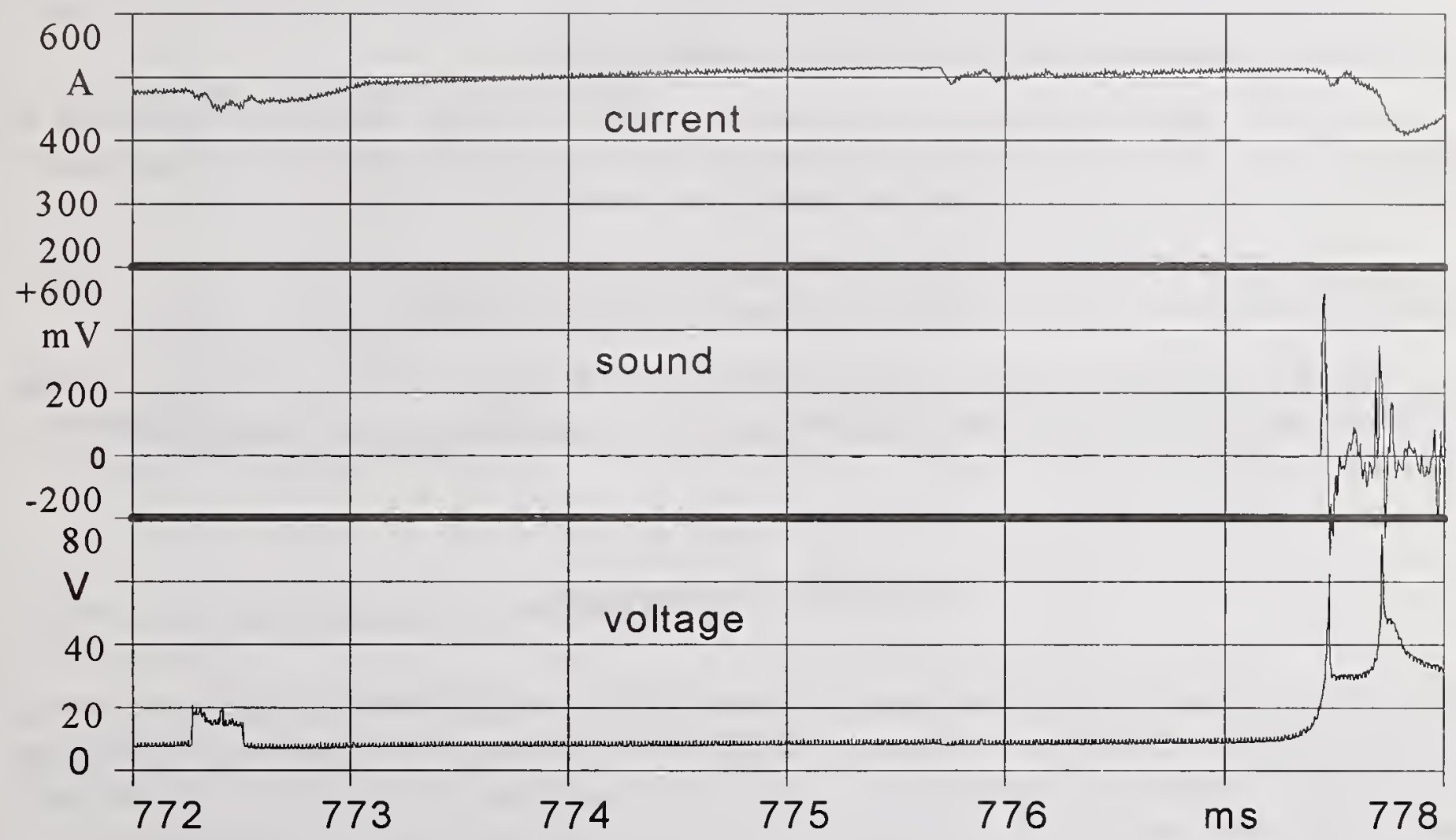

Figure 23 - Hidden arc between wire and contact tube. 
feeder after totally some 20 milliseconds. This is a while after reignition happened which is to be seen as twin-peaked voltage on right. Then wire shoots out with very high speed.

Although random coincidence led to the crucial event of such hidden arc, a lot of implications were reduced to that one plausible cause and confirmed by recognition. For that reason, there is no danger of confusion between the increased voltage due to hidden arc and the similar pattern due to unstable plasma mode on condition of careful identification.

Stingy calculating engineers might balance whether hidden arcs should be recognized by the additional means of wire potential or by pattern recognition from voltage alone. Nature recommends to us using both options mutually confirming each other. A plurality of signals and methods of preprocessing has proven most efficiently.

The hidden arc was discovered during regular stubbing being forced by combination of low voltage setting and high wire feed speed. Stubbing is unacceptable but it is also a kind of metal transfer mode the welder can feel as a force acting on torch in his hand and he can markedly hear each single burst for physical reasons (Ref. 12). Twin-spiked voltage may serve the blind and deaf welding machine a substitute feature among others.

\section{CONCLUSIONS}

1) A plurality of synchronously recorded signals and its computerized analysis have proven to be best in broadening the necessary basis for detection of essential changes in GMAW processes.

2) A lot of striking features reckon among indications of an unstable plasma mode. An investigation into that mode led to better understanding the chaotic behavior of so called globular transfer.

3) Common conceptions of so called instantaneous short circuits should also be revised, in order to improve diagnosis and control based on voltage signal.

4) Some transitions between metal transfer modes can easily be detected but there are deficiencies, too. Unfortunately, in most cases, there are no simple transitions between transfer modes but several variants of cyclically alternating mixed transfer.

5) Human intelligence gives good examples how to efficiently select and process data by attention, diversification, active sensation, expectation, and confirmation.

6) Fast recognition of failures remains the bottleneck in fast control of welding processes, and focusing on crucial details selected by reasoning from a plurality of signals has confirmed to be the most promising way to go through.

\section{ACKNOWLEDGMENTS}

This work was granted by the Land Saxony-Anhalt. DFG is also appreciated. The author would like to acknowledge U. Dilthey, T. Eagar, J. Heberlein, B. Juettner, A. Matsunawa, P. Modenesi, G. denOuden, D. Rehfeldt, J. Reece Roth, J. Schein, M. Schellhase, T. Shinoda, V. Stenke, and J. Wendelstorf for encouragement, kind information, and pieces of advice, respectively. Last not least, he thanks for assistance by E. Hettkamp, Ch. Werner, and some students. 


\section{REFERENCES}

1. Jönsson, P. G., Murphy, A. B., Szekely, J. 1995. The Influence of Oxygen Additions on Argon-Shielded Gas Metal Arc Welding Processes. Welding Journal 74(2): 48-s to 49-s.

2. Blumschein, E., Werner, Ch. a. o. 1996. A concept for cognitive control of welding processes. Proc. 6th Internat. Conf. Computer Technology in Welding. Lanaken, Belgium, 9-12 June 1996, paper 22, Abington Publishing Cambridge, UK 1996.

3. Blumschein, E. 1994. Acoustic tuning versus predetermination of parameters for short arc welding. Proc. 5th Internat. Conf. Computer Technology in Welding. Paris, France, 15-16 June 1994, ed. W. Lucas paper 46: Abington Publishing Cambridge UK 1994.

4. Modenesi, P. J., Nixon, J. H. 1994. Arc Instability Phenomena in GMA Welding. Welding Journal 73(9): 219-s to 224-s.

5. Miyazaki, H., Miyauchi, H., Sugiyama, Y., Shinoda, T. 1994. Puckering Phenomenon and its Prevention in GMA Welding of Aluminium Alloys. Welding Journal 73(12): 227-s to 284-s.

6. Shinoda, T., Nishikawa, H., Shimizu, T. 1996. The development of data processing algorithms and assessment of arc stability as affected by the titanium content of GMAW wires during metal transfer. Proc. 6th Internat. Conf. Computer Technology in Welding. Lanaken, Belgium, 9-12 June 1996, ed. W. Lucas, paper 57, Abington Publishing 1996.

7. Chen, J. H., Sun, Z. C., Fan, D. 1996. Study on the Mechanism of Spatter Produced by Basic Welding Electrodes. Welding Journal 75(10): 311 -s to 316-s.

8. Siewers, T. A., Liu, S., Heald, P. R., Madigan, R. B. 1994. Mapping the Droplet Transfer Modes for an ER100s-1 GMAW Electrode. Welding Journal 73(1994): 38-s to 45-s.

9. Slaney, M. 1995. A Critique of Pure Audition. Proc. Computational Auditory Scene Analysis Workshop, Montreal 1995.

10. Eickhoff, S. T., Eagar, T. W. 1990. Characterization of Spatter in Low-Current GMAW of Titanium Alloy Plate. Welding Journal 69(10): 382-s to 388-s.

11. Kim, Y. S., Eagar, T. 1993. Analysis of Metal Transfer in Gas Metal Arc Welding. Welding Journal 72(1993): 269-s to 278-s.

12. Blumschein, E. 1993. Multimedia Modeling and Monitoring of GMAW Processes. Proc. Int. Conf. Modeling and Control of Joining Processes. December 8-10, 1993, Orlando, ed. Th. Zacharia: 624-630.

Accessory video and sound files are available. 


\title{
NUGGET SIZE SENSING OF SPOT WELD BASED ON NEURAL NETWORK LEARNING
}

\author{
K. Matsuyama*
}

\begin{abstract}
A new quality monitoring system has been developed based on a neural network learning procedure to guarantee the resistance spot weld quality without mechanical tests such as chisel test in automobile industry. A dynamic voltage between tips and a welding current wave form are measured for monitoring parameters to predict the nugget size, and to detect the occurrence of expulsion during welding with each neural network learning result determined by some preliminary experiments. As a result, it is clarified that the new system can be applicable not only to estimate the nugget size but also to find the occurrence of expulsion during welding in high accuracy and high speed with relatively low CPU power system for monitoring system. This means that low cost sensing system of resistance spot welds could be easily realized for a real time monitoring and an adaptive control.
\end{abstract}

\section{INTRODUCTION}

About thirty years have passed after a study on the quality monitoring in resistance spot welding began by using regression equations determined with many preliminarily experimental data (Ref.1), where the monitoring was achieved with the dynamic electrode movement caused by thermal expansion of workpieces and electrodes. After that, other monitoring systems also investigated with voltage between tips, resistance between tips calculated with the voltage and welding current, or surface temperature of weld measured by thermo-sensor etc. as a sensing parameter. These monitoring systems have not been practically used without trial use because the electrode movement system has some problems in applying to over-edge welding of electrode tip at workpiece edge and pressed parts welding with mis-fitness at the faying interface, the system with voltage or resistance between tips cannot apply to coated steel sheets for the workpiece, and the measurement of surface temperature is not so easy in actual production lines.

Re-attracting the quality monitoring system of resistance spot welding to public is after the end of 1980's when a quality monitoring system was developed by the authors (Ref.2). In the quality monitoring system, virtual resistance welding machine was built up and run in computer, some differences between actual monitoring values and corresponded virtual ones were detected to control the working condition of the virtual machine, and welding state in actual welding part was estimated from the virtual working state. Although this method has some merits of that the system can apply to be predicted the contact diameters representing the current flow area and electrode tip temperature etc. besides the nugget size in high accuracy with no preliminary

\footnotetext{
* Faculty of Engineering, Osaka University, 2-1 Yamada-Oka, Suita, Osaka 565 Japan
} 
experiments, it also has a weak point of that high CPU power is required for the simulation of nugget formation process because the simulator run in a long program codes made by a finite difference method with many grids.

So, the author has tried to investigate a new quality monitoring system with a neural network learning procedure to resolve the problem of CPU power in keeping high accuracy for estimated results, and realize easy adjustment of pre-set parameters by users, since the new procedure can be considered to be one of approximate some equations with sigmoid function as basic function series, and to be adaptable and suitable for nonlinear problem such as resistance spot welding phenomena. Moreover, the neural network learning system can be considered to be applicable to detect the occurrence of expulsion during welding if the adaptability for the nonlinear problem is attracted. Therefore, nugget size sensing system included the detecting one for expulsion has been examined and developed with a neural network learning procedure.

\section{OUTLINE OF NEURAL NETWORK LEARNING PROCEDURE USED}

\section{Network structure and learning procedure}

A three layer type was used for the neural network system where artificial neurons are combined in two steps as shown in Fig.1. Predicting the nugget size was achieved with welding current value and dynamic resistance between tips for the input data, which have been used and successfully applied to be predicted the nugget formation process in resistance spot welding assisted with the numerical simulation program (Ref. $2 \& 3$ ). Detecting the occurrence of expulsion during welding was done with the welding current and reducing rate of the dynamic resistance during

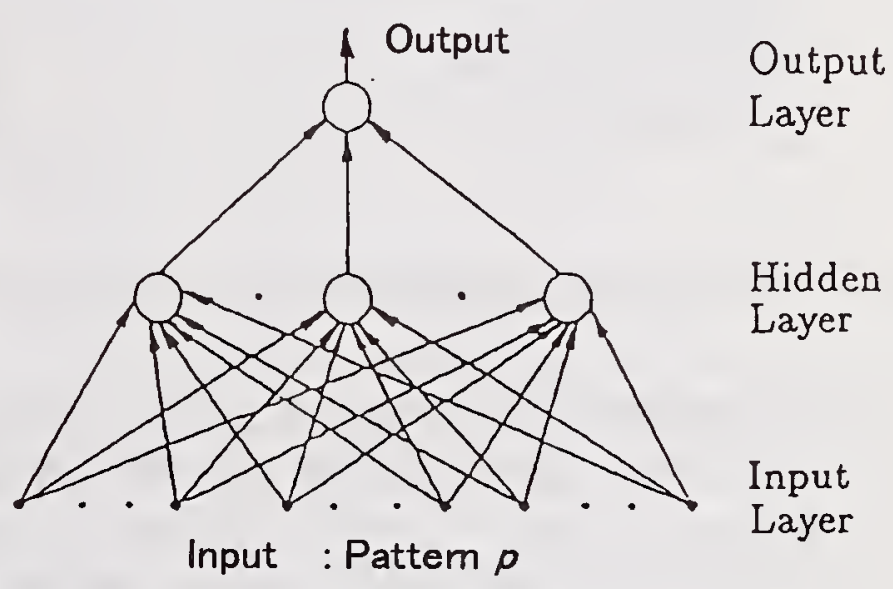

Fig. 1 A neural network with three layers each one welding current cycle. And then, it was supposed that the following relation can be applied at each artificial neuron;

$$
\begin{aligned}
& u_{j}^{(n+1)}(p)=\sum_{i} w_{j i}^{(n)} \cdot v_{i}^{(n)}(p)+\theta_{j}^{(n)} \\
& v_{j}^{(n+1)}(p)=h\left(u_{j}^{(n+1)}(p)\right)
\end{aligned}
$$

$$
\begin{aligned}
\text { Where, } v_{i}^{(n)}(p) & ; \text { Output from unit } i \text { of the } n^{\text {th }} \text { layer } \\
u_{j}^{(n+1)}(p) & \text {; Input for unit jof the } n+1^{\text {th }} \text { layer } \\
w_{j i}^{(n)} & ; \text { Weight, } \theta_{j}^{(n)} ; \text { Threshold or offset } \\
h\left(u_{j}^{(n+1)}(p)\right) & ; \text { Nonlinear activation function }
\end{aligned}
$$

For input layer, a following equation was used ;

$$
v_{j}^{(1)}(p)=u_{j}^{(1)}(p)
$$




\section{System data}

Number of input layer units Number of hidden layer units Number of output layer units Number of teach data

Parameter data

Slope of sigmoid function
Initial value of weight
Initial value of offset
Lerning cofficient of weight
Leming coficient of offset
Initial value of inertia coefficient
Increacing quantity of inertia coefficient
Limit of inertia coefficient
Limit of error
Limit of iterations

Input data

Welding current
Resistance between eletrode tips
at each cycle

Teach data

Nugget diameter

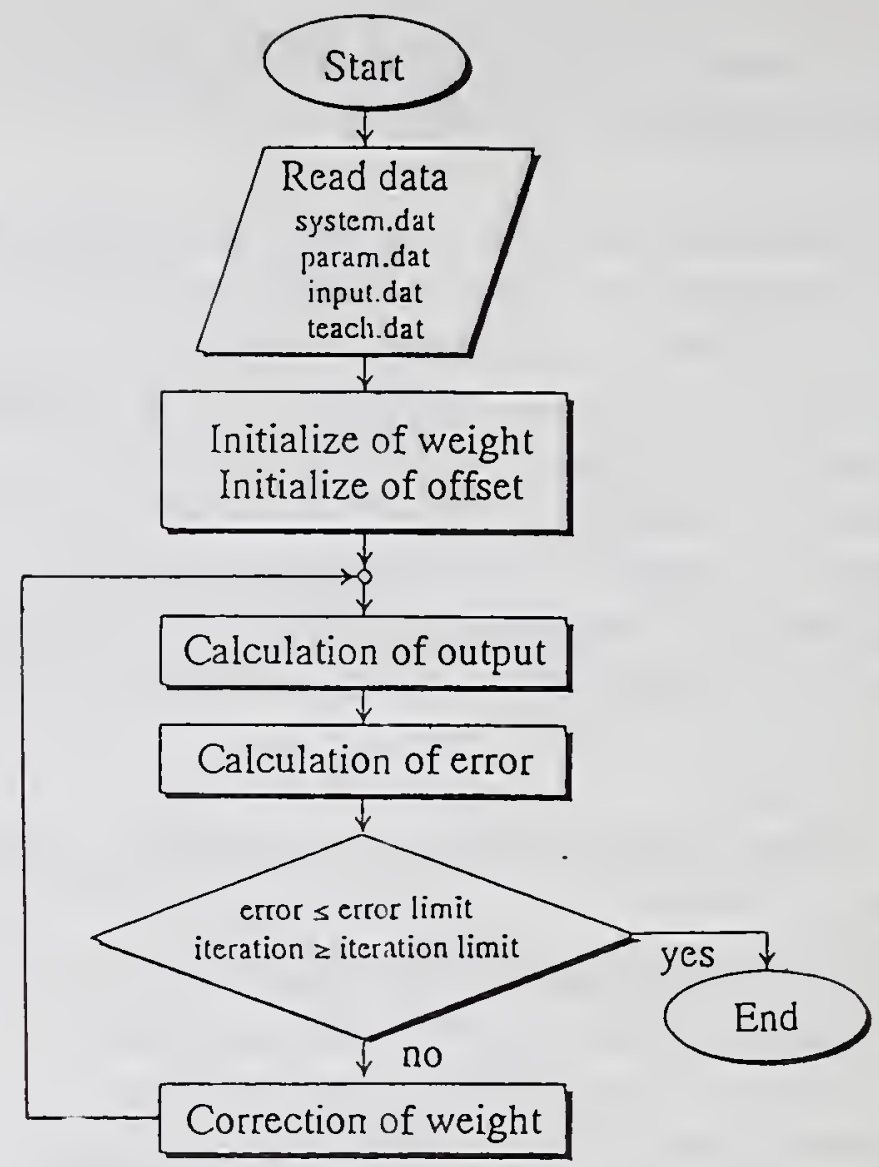

Fig. 2 A flowchart to adjust the weights and offset with backpropargation algorithm, and parameters used

A backpropagation algorithm was utilized for adjustment of the weights and the offset shown in Eq.1 ( Ref. 4). The flowchart with the backpropargation algorithm is shown in Fig.2. Four system data to set up the artificial neural network structure, ten parameter data to fix initial and end conditions for the calculation, and several pairs of input and teach data are read at the first step of calculation. A list utilized in prediction of the nugget size is shown at the left side of Fig.2.

\section{Nonlinear activation function as input-output relation}

Not only the sigmoid function but also Gaussian distribution function and others can be utilized for a nonlinear activation function at each artificial neuron, and the function requires only to be continuous and differentiable. This means that many functions can be applied to the activation function, and the selection of suitable one is important to improve the adaptability and conversion speed.

Some relationships, in which the welding current and welding time are used for input data and nugget diameter for output ones, are shown in Fig.3. The nugget diameters rapidly increase only over a certain value in horizontal axis, and saturate in spite of welding current or welding time, and the profiles are almost similar to that of the sigmoid function. Therefore, the following sigmoid functions were selected for the input-output relation at each artificial neuron;

$$
h(u)=1 /\left[1+\exp \left(-u / u_{0}\right)\right]
$$




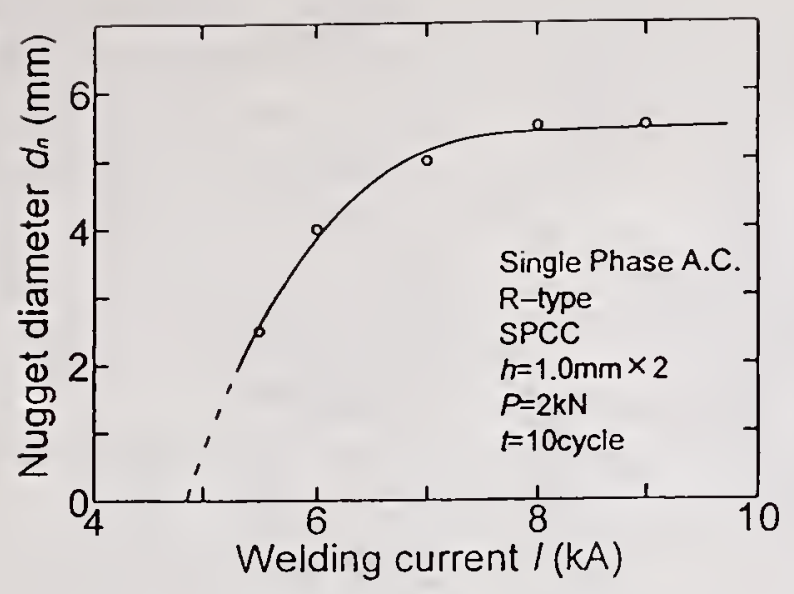

(a)

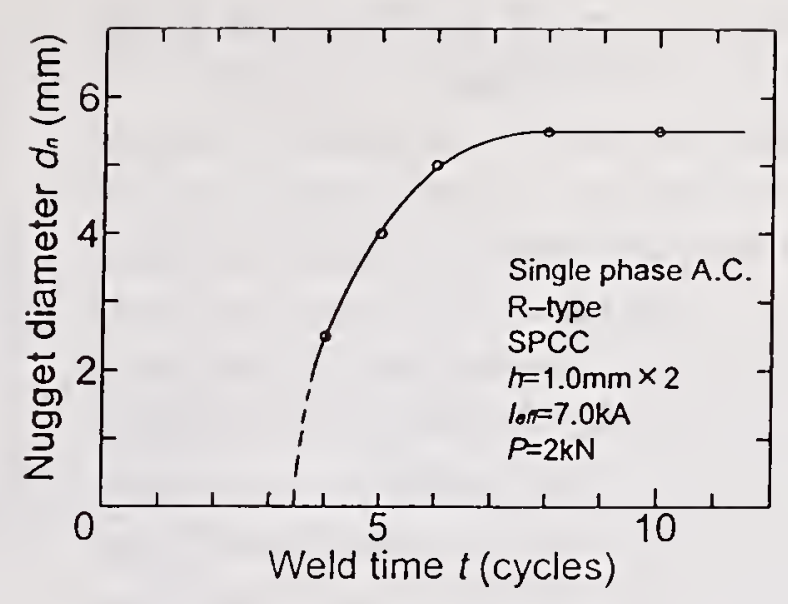

(b)

Fig. 3 Relationship between welding current or welding time and nugget. diameter

The initial setting value of the constant $u_{0}$ in the nonlinear activation function was remarkably influence on the convergency of updated weight values in the backpropargation algorithm. Figure 4 shows the relationship between the initial constant value $u_{0}$ and iteration number for convergence of the adjusted weights. In the present study, a modifying procedure for the constant $u_{0}$ was installed to improve the convergency of solver algorithm.

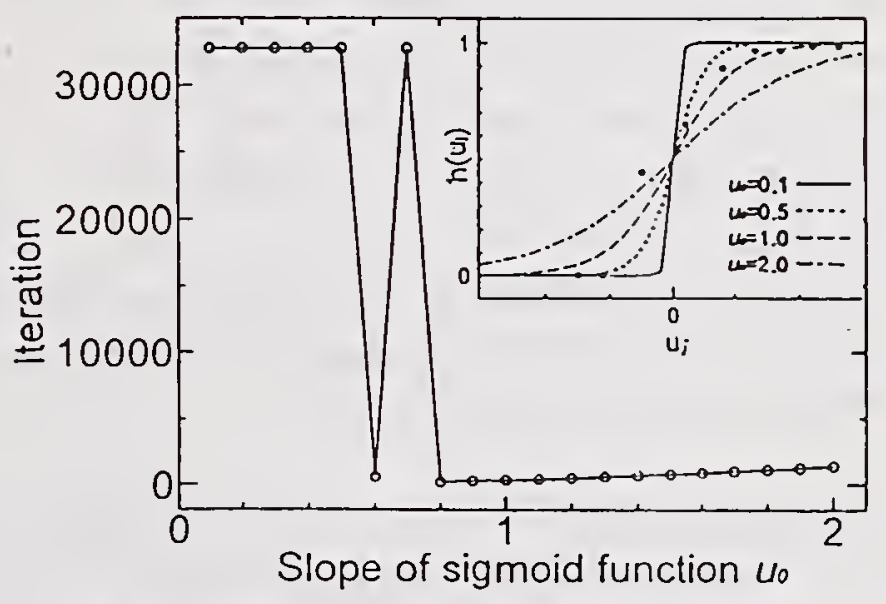

Fig. 4 Influence of initial slope of sigmoid function on the convergence rate

\section{Treatment of input data error included in experiments}

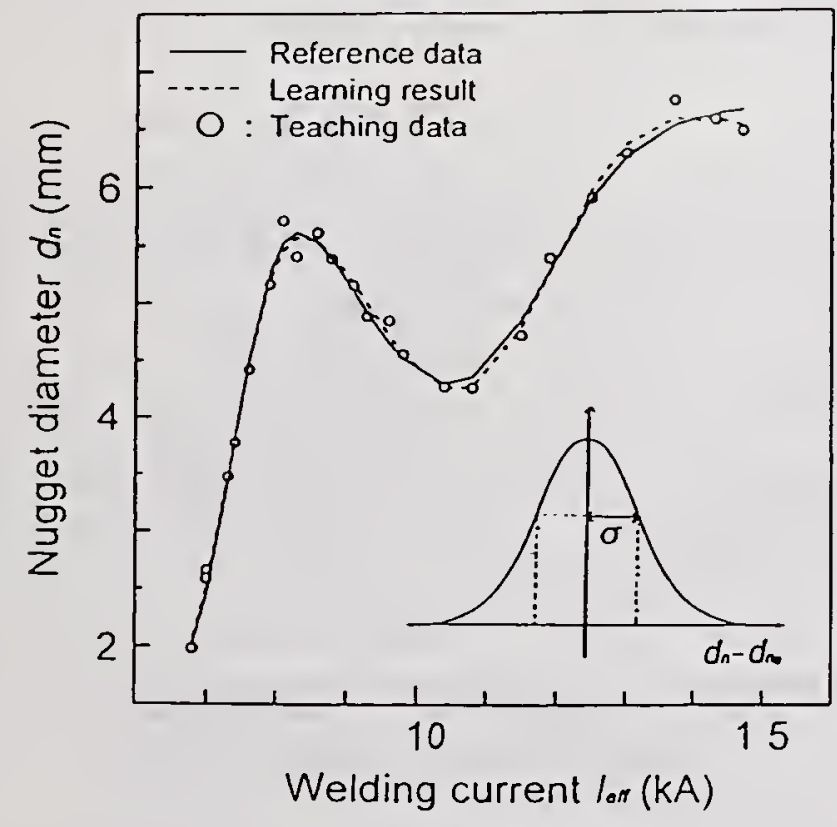

(a) $\sigma=0.12 \mathrm{~mm}$

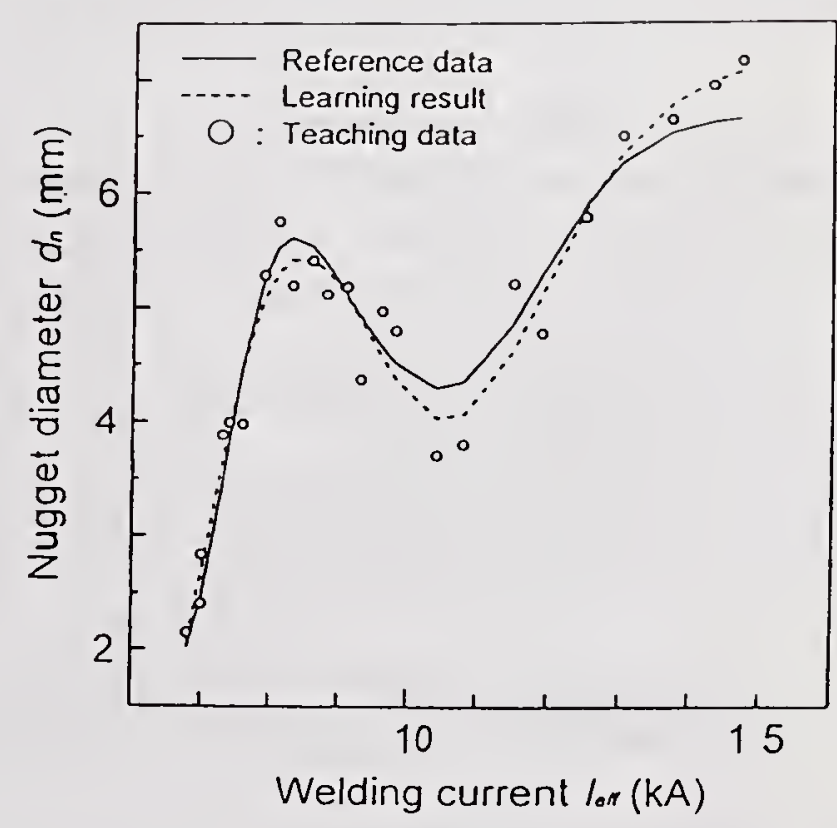

(b) $\sigma=0.36 \mathrm{~mm}$

Fig. 5 Influence of teach data error on the learning results 
The influence of the input and output data error on teaching results can be preliminarily examined from viewpoint of making the approximate equation. Simulated sample data are shown with open circle marks in Fig.5, that were calculated by adding random error to the reference data shown with solid lines in the figure, and teaching results with these simulated data including the random error are shown with dotted lines in the Fig.5. The solid line shape was determined from some experimental results in usual resistance spot welding newly thin steel sheets. In the present examination, only welding current value is used for the input data, and simulated nugget diameter for output data. Randomly calculated error value with the normal distribution function of standard

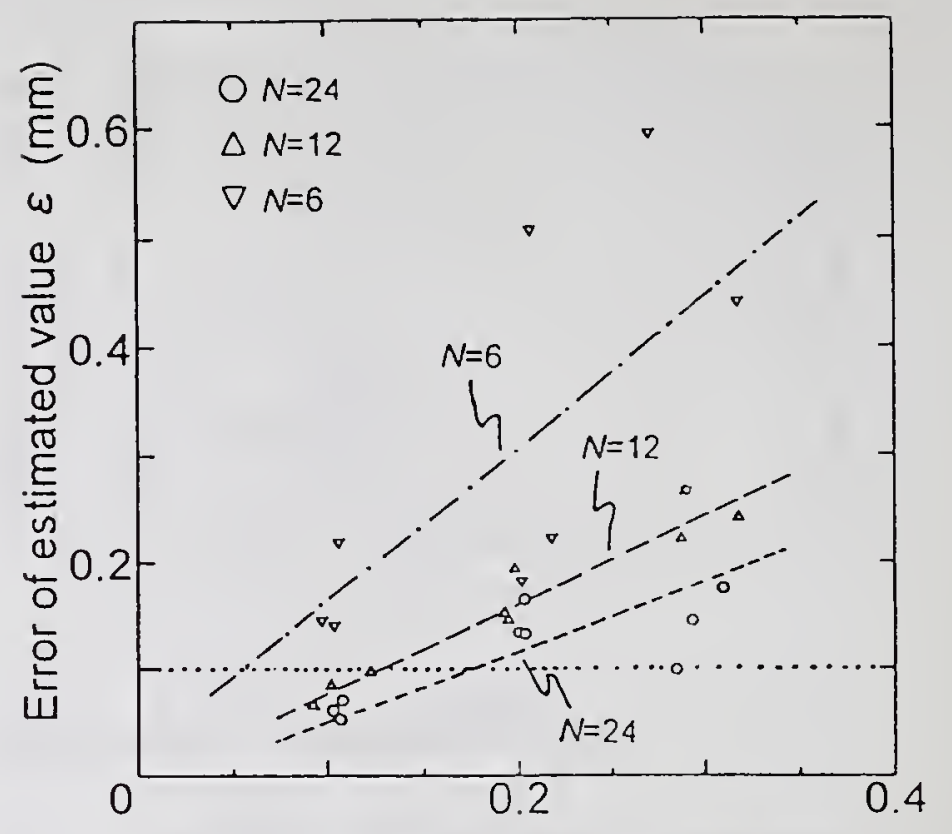

Standard deviation of teach data $\sigma(\mathrm{mm})$

Fig. 6 Influence of teach data error on the accuracy of estimated result deviation $\sigma$ was added to the solid line value, and obtained the simulated input data shown with the open circle marks. The error treatment was applied only for 24 output data for teach data. Small data error case is shown in Fig.5(a), and relatively large one in Fig.5(b).

The figures indicate that the teaching results shifts from the reference curve in the case of that the standard deviation enlarges about more than $0.4 \mathrm{~mm}$ even in such large number of sampling data. Figure 6 shows the relationships between the standard deviation and the error estimated for various sampling data numbers. The calculation was done in the same manner in Fig.5. The results suggest that it is almost sufficient in only 10 data par about $10 \mathrm{kA}$ range in usual resistance spot welding condition for thin sheets such as $1.0 \mathrm{~mm}$ thickness. In addition, the teach data error was reduced by increasing measurement numbers for each nugget .

\section{Examination on the hidden layer}

The unit number of hidden layer also influence on the taught results. Therefore, the initial unit number is commonly set almost same or a little small as the unit number of input layer, and the optimal unit number of hidden layer has been usually found by decreasing procedure of the unit number from maximum number state. On the contrary, if the system is considered to be one of approximate equation, the unit number of hidden layer has to be determined with an increasing procedure of the unit number from one that is set up for an initial state. In the present study, an increasing unit number procedure of hidden layer was employed.

\section{ADAPTBILITY OF THE NEURAL NETWORL LEARNING RESULTS TO PREDICT NUGGET SIZE AND DETECT OCCURRENCE OF EXPULSION}

Input and the output data used

Table 1 shows an example of the input and teach (output) data used for neural network learning 
in resistance spot welding. The experiments were carried out mainly with uncoated steel sheets of $1 \mathrm{~mm}$ thickness. Character " $A$ " and " $B$ " shown in the first row in Table 1 classify the electrode type used. Character " $A$ " corresponds to use a relatively good pair of electrode tips which has been used for welding steel sheets less than about 1000 welds, and a small projection can be observed on the tip surfaces. Character " $B$ " means that a pair of old electrode tips used more than 5000 welds, and the projection has already disappeared on the tip surfaces. Welding time was counted with cycles in AC of $60 \mathrm{~Hz}$, "* " marks at the row of nugget diameter means that the expulsion was occurred in its condition. $\mathrm{Cr}-\mathrm{Cu}$ electrode tips of $50 \mathrm{R}$ type were used for the experiments.

Welding current and dynamic voltage between tips were measured through A/D converter with a personal computer. The sampling was done in high speed of $1000 \mathrm{~s} / \mathrm{s}$, square of the instantaneous welding current was integrated to obtain the effective value at each one cycle. The dynamic resistance $R$ between tips was calculated by using the following equation at near peak welding current period in each half cycle, and the resistance value at each $n$ cycle was determined by arithmetic average of the values at $n .25$ cycle and at $n .75$ cycle;

$$
R=\left\{v-(L+M) \frac{\mathrm{d} i}{\mathrm{~d} t}\right\} / i
$$




\section{Where, $v$; Measured instantaneous voltage between tips \\ $i$; Measured instantaneous current \\ $L$; Self-inductance of welding circuit between tips \\ $M$; Mutual inductance between welding circuit and measured one}

In the present study, an effective value of welding current calculated during whole weld time and 10 dynamic resistance values determined at each one welding cycle during initial 10 cycles of weld time are employed for the input data, because the welding current could be considered to be constant during the welding. Still more, the electrode force and the weld time were not used for the input data because of little or no relation to the learning results

The nugget diameter used for the teach data and non-teach ones was determined with a square root of the product of both radii measured on transverse axis and on conjugate one although the arithmetic mean of them has been usually used until now because the nugget diameter has to be determined with the square root value in the theoretical.

\section{Examination on the suitable unit number of hidden layer}

The suitable unit number of hidden layer was examined for 37 non-teach data obtained at a little different welding current values or weld time from each welding condition shown in Table 1. The examination was achieved according to a flowchart shown in Fig.7.

A typical result is shown in Fig.8. A broken line and a dotted one indicate error for the learning result with the 37 teach data shown in Table 1. A solid line and a one-dotted chain one also

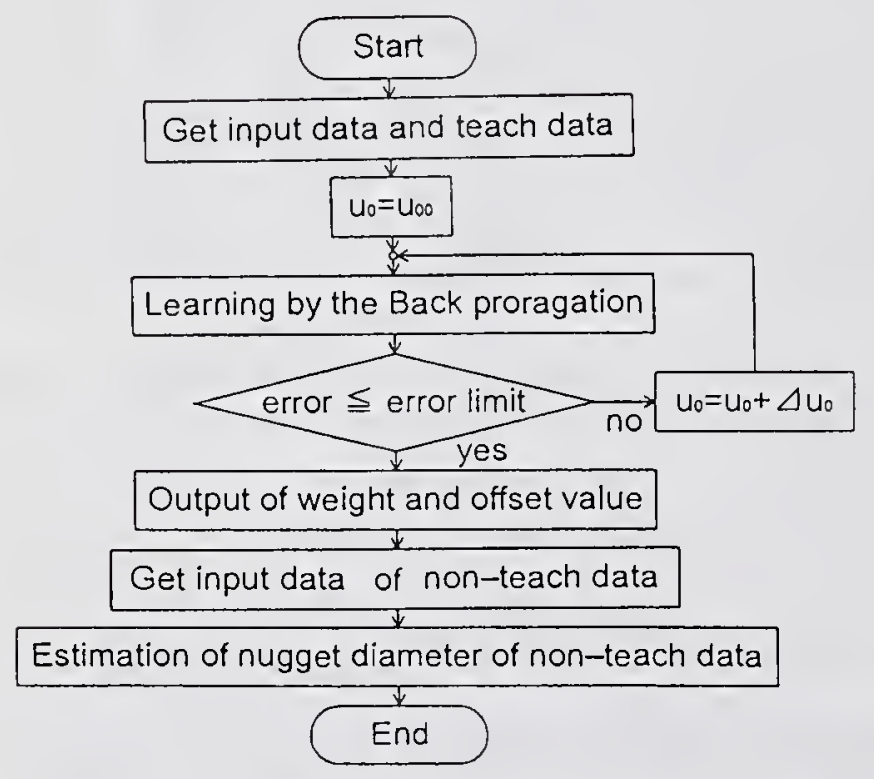

Fig. 7 A flowchart for examination of the optimal unit number of hidden layer

Fig. 8 Examination of optimal unit number of hidden layer with non-teach data

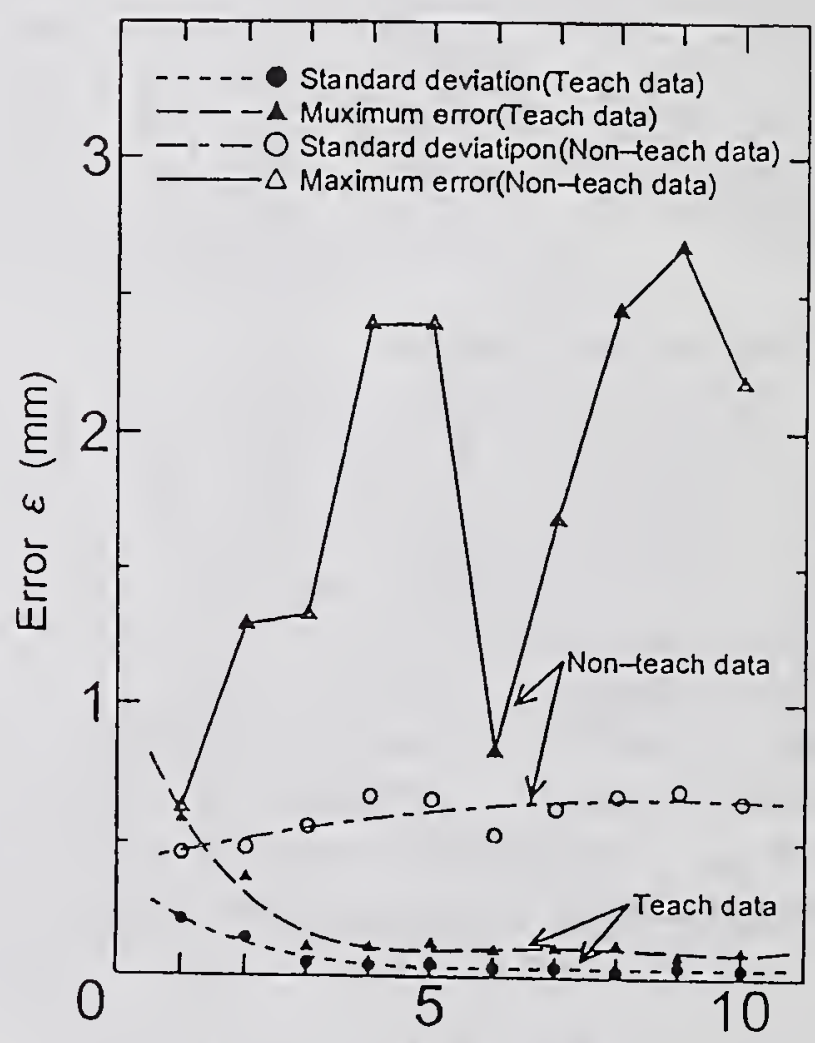

Number of hidden layer units $H U$ 
indicate the error for estimated results of the 37 non-teach data. Moreover, the solid line and the broken one indicate the maximum error, and one-dotted chain line and dotted one are standard deviation of the error, respectively.

Although the error for the teach data rapidly decreased with increasing the unit number of hidden layer, on the contrary, error for the non-teach data increased with the increasing number. This means that the optimal unit number of hidden layer cannot be decided only with the teach data. In the present study, one unit model for hidden layer was utilized for the artificial neural network to estimate the nugget diameter and find the occurrence of expulsion during welding.

\section{Adaptability of the learning results}

Several nugget diameters in different welding condition from the teaching ones were estimated with the learning results, where the artificial neural network consist of one output unit, one hidden unit, one input unit for welding current, and 10 input units for dynamic resistance at each welding cycle. The typical results are shown in Fig.9. Closed circle marks indicate the learning results with the teach data obtained from center part welding tests of lap sheet specimen. The open circle marks indicate the estimated nugget diameter with test specimens welded at the center part in a little different welding conditions from the teach data. Open triangle marks show the estimated result for near edge position illustrated on the Fig.9.

The estimated results by the artificial neural network model with the adjusted only with the 37 teach data were good agreement with the experimental ones in spite of the learning with only center part data. The error is

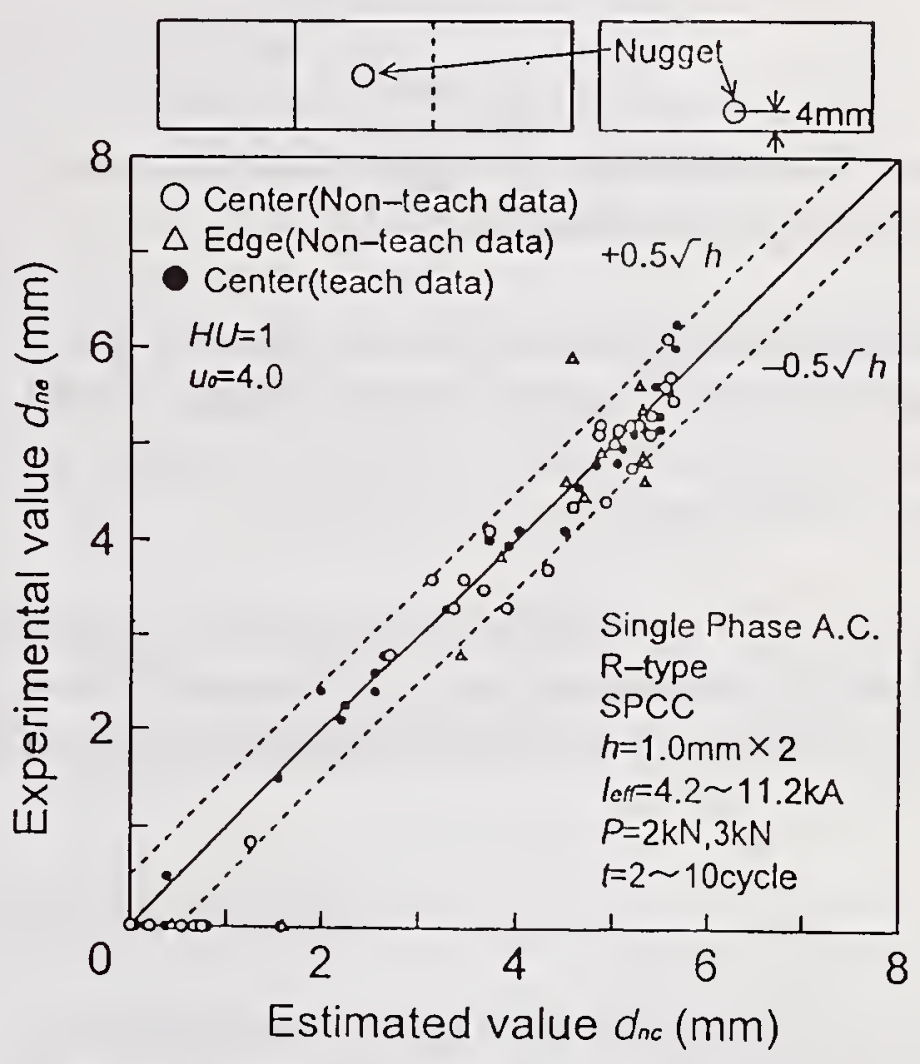

Fig. 9 Adaptability of the learning results with teach data welded at center part of workpieces to the estimation of nugget diameter for non-teach data

remained almost less than $0.5 \sqrt{h}$ ( $h$ : Plate thickness in $\mathrm{mm}$ ) The accuracy of estimated results is almost same as that with the previous numerical simulator (Ref.3). Moreover, similarly good arrangement were also obtained in spot welding galvanized steel sheets although the results do not shown here.

Such good agreements can be recognized in the detecting the occurrence of expulsion during welding. The learning has been achieved with the same experiment data shown in Table 1 , and only the structure of input layer was changed. In the detecting of the expulsion, welding current during whole weld time and maximum value of instantaneous dropping rate of the dynamic resistance were utilized for the input data. The adjusted neural network model only with the 
Table 2 Detecting expulsion in edge welding with a neural network learned for the center part welding of workpieces

\begin{tabular}{|c|c|c|c|c|c|}
\hline Electrode type & $\begin{array}{l}\text { Welding current } \\
\text { lodhal }\end{array}$ & $\begin{array}{c}\text { Electione } \\
\text { prossure /qkN] }\end{array}$ & $\begin{array}{l}\text { Wolding time } \\
\text { (feycle) }\end{array}$ & Estimaled & Experimenlal \\
\hline \multirow{6}{*}{ A } & 4.7 & \multirow{6}{*}{2} & \multirow{6}{*}{10} & 0 & 0 \\
\hline & 5.6 & & & 0 & 0 \\
\hline & 6.2 & & & 0 & 0 \\
\hline & 6.6 & & & 0 & 0 \\
\hline & 7.3 & & & 1 & 1 \\
\hline & 7.8 & & & 1 & 1 \\
\hline \multirow{7}{*}{$\wedge$} & 4.2 & \multirow{7}{*}{3} & \multirow{7}{*}{10} & 0 & 0 \\
\hline & 6.0 & & & 0 & 0 \\
\hline & 6.8 & & & 0 & 0 \\
\hline & 7.9 & & & 0 & 0 \\
\hline & 8.6 & & & 1 & $T$ \\
\hline & 8.9 & & & 1 & 1 \\
\hline & 9.3 & & & 1 & 1 \\
\hline
\end{tabular}

center part data were also applied for the welds formed not only at the center part but also at the near edge part as shown in Table 2.

In addition, the system can be applied to predict the nugget size and detect the occurrence of expulsion even in case of an over-edge welding such as a part of weld is overhanged from the edge of workpieces, that is normal in automobile factory, if the learning would be carried out for the over-edge welding

Therefore, it is concluded that the neural network system adjusted with teach data is useful and effective to estimate the nugget formation state in resistance spot welding although the adaptable range should be restricted within the teaching data zone.

\section{SUMMARY AND CONCLUSIONS}

A new sensing system of nugget formation state in resistance spot welding has been developed with an artificial neural network learning procedure with welding current and dynamic resistance measured between electrode tips for monitoring parameters, and it was clarified that the new system can be applicable to predict the nugget size, and detect the occurrence of expulsion during welding.

The new system with the neural network learning procedure does not required high CPU power to estimate the nugget size and detect the occurrence of expulsion in high speed comparing the previous monitoring system with the numerical simulator so that the system can be easily applied to sensing unit of some adaptive control system if the system is applied within restricted zone examined and determined with preliminary experiments.

If the neural network system, however, will be applied to estimating the welding state for unknown welding condition zone not examined with the preliminary experiments, any output from the system does not guaranteed whether it is correct. In this case, the previous monitoring system with numerical simulator will help dynamically modifying weights of the 
neural network.

\section{ACKNOWLEDGMENTS}

I would like to express my thanks to Mr. H. Sakamoto for his help in calculation and experimental work.

\section{REFERENCES}

1. Waller, D. N; and Knowlson, P. M. 1965, Electrode separation applied to quality control in resistance welding, Welding Journal, 12(4), 168-s to 174-s

2. Matsuyama, K.; Nishiguchi, K.; and Satoh, H. 1988, Applying to quality monitoring system assisted with model-referenced observer for contact diameters and nugget ones in resistance welding, MP-5-88, The document presented at the Technical committee on Joining and materials processing of light structure of Japan Welding Society

Matsuyama, K.; Nishiguchi, K.; and Satoh, H. 1990, Computer-aided monitoring system of nugget formation process, Proc. of the $5^{\text {th }}$ Welding Symposium of Japan Welding Society in Makuhari. 577-582

3. Matsuyama, K. 1994, Procedure for reducing the solution time by using numerical simulation

techniques for the measurement of weld nugget, IIW Document, III-1024-94

4. Rumelhart, D. E.; Hilton, G. R.; Williams, R. J. 1986, Learning internal representation by error propagation, MIT-press, Parallel Distributed Processing, Rumelhalt at al. eds. Vol.1, 


\title{
WELD DEFECT DECISIONS USING
}

\section{THROUGH-THE-ARC SENSOR DATA ${ }^{1}$}

\author{
T.P. Quinn", R.B. Madigan*, C.B. Smith ${ }^{\dagger}$, \\ E.G. Blachowiak ${ }^{\ddagger}$
}

\begin{abstract}
A defect detection algorithm was developed to allow real-time quality sensing of arc welding. The algorithm constructs a baseline record by averaging the output from the sensor from several acceptable welds. Subsequent welds can be compared to the baseline record at each time increment. A threshold value is set as some fraction of the standard deviation of the output relative to the mean value of the baseline record. In this manner, the threshold value tracks intentional changes in weld inputs. If a production weld exceeds the threshold for a certain fraction of a specific interval, the weld is flagged as defective. The algorithm was used with a through-the-arc sensor to monitor welds made on the production floor. The constants used to tune the algorithm were found from the first 82 welds that included 4 defective welds. Another 444 welds were monitored with 2 more defective welds. The sensor flagged 5 of the 6 defective welds and did not incorrectly flag any defect-free welds.
\end{abstract}

\section{INTRODUCTION}

This paper presents a method for detecting defects in automatic arc welds made in a massproduction environment. In automatic welding cells, the operator usually cannot directly monitor the arc because of the danger of being in the workspace of the robotic arm. Welds may not be inspected until several stations further down the production line; the value added may be lost if the part must be scrapped because of a defective weld. Weld inspection stations add another processing step that adds cost. Therefore, there is a need for sensors to indicate when a part has a welding defect while the weld is being made so that immediate action can be taken. However, if a sensor incorrectly flags a weld as defective, the part may be scrapped or the production line may be stopped unnecessarily.

There has been much work on real-time sensors to diagnose problems in arc welding (for example see Refs. 1-7 or the reviews in Refs. 8 and 9), but with the volumes of output from realtime sensors, it is often difficult to decide whether a defect has occurred. The most common

1 Product of NIST; not subject to U.S. copyright.

* National Institute of Standards and Technology, Boulder, CO

$\dagger$ Tower Automotive, Milwaukee, WI

$\ddagger$ A.O. Smith Corporation, Milwaukee, WI 
technique for making this decision is to compare the output of the sensor, the "quality parameter," to an absolute threshold. For example, an absolute threshold used for a through-thearc sensor might be "If the current exceeds 450 A for more than $1 / 2 \mathrm{~s}$ then a defect has occurred." However, on parts with complex geometries, the weld schedule may change automatically, or changing torch positions may cause variations in the weld inputs (for example, the current changes as the torch rounds a corner); this intentional variation in the welding inputs will be reflected in the measured quality parameters. An absolute threshold will have to be set above (or below), the highest desired point of the weld, and sensitivity will be lost for the parts of the weld that produce lower (or higher) values of the parameters. In this paper we develop a method that tracks the intentional variations to increase the sensitivity for predicting defects.

\section{METHODS}

The defect detection method is suited for a mass production environment and is straightforward. A baseline record is constructed from the recordings of the quality parameter(s) made during a number of defect-free welds. A threshold is set based on the standard deviation of the quality parameter(s) or on a fraction of the value of the quality parameter(s). Since excursions of the quality parameter(s) beyond the threshold for short periods of time may be acceptable, a weld is declared defective if the quality parameter is out of the threshold for some part of a window that moves with the data.

To construct a baseline record, the quality parameter(s) $Q$ is recorded at sampling frequency $F$ for at least $N_{b}$ defect-free welds resulting in ${ }^{j} Q_{i}$ where $i$ refers to the $i$ th sample in time and $j$ refers to the $j$ th weld. The standard deviation of the quality parameter $S$ is also recorded for the $N_{b}$ defectfree welds if it is available. To construct the baseline weld, the average over the $N_{b}$ welds $\hat{Q}$ of the quality parameter at each time increment is first found:

$$
\hat{Q}_{i}=\frac{1}{N_{b}} \sum_{j}^{N_{b}}{ }^{j} Q_{i}
$$

The baseline weld series $Q_{i}^{b}$ is finally found by smoothing $\hat{Q}_{i}$ using a moving average:

$$
Q_{i}^{b}=\sum_{j=i-i_{s} / 2}^{i+N_{s} / 2} \hat{Q}_{j}
$$

$N_{s}$ is the duration of the moving window and is set to a value large enough so that the series is smoothed but not so large that intended changes in $Q$ are smeared out. For example, if the quality parameter is the welding current and the current is reduced as the torch welds around a corner, $N_{s}$ cannot be made so large that this intentional dip in the current is smoothed. For the start of the 
weld, $i<N_{s} / 2, Q_{i}^{b}=\hat{Q}_{i}$; a similar procedure can be used for the end of the weld.

If $S$ is available, the threshold is first found by computing the average $\hat{S}$ over the $N_{b}$ welds and then smoothing the result:

$$
\begin{gathered}
\hat{S}_{i}=\frac{1}{N_{b}} \sum_{j}^{N_{b}}{ }^{j} S_{i} ; \\
S_{i}^{b}=\sum_{j=i-i_{S} / 2}^{i+N_{s} / 2} \hat{S}_{j} .
\end{gathered}
$$

The thresholds are then constructed as

$$
\begin{aligned}
& T_{i}^{u}=Q_{i}^{b}+\alpha S_{i}^{b}, \\
& T_{i}^{l}=Q_{i}^{b}-\alpha S_{i}^{b},
\end{aligned}
$$

where $T_{i}^{u}$ and $T_{i}^{l}$ are the upper and lower threshold, and $\alpha$ is a positive number used to set the threshold width. If $S$ is not available then the thresholds are set as

$$
\begin{aligned}
& T_{i}^{u}=Q_{i}^{b}+\beta Q_{i}^{b}, \\
& T_{i}^{l}=Q_{i}^{b}-\beta Q_{i}^{b},
\end{aligned}
$$

where $\beta$ is used to tune the threshold width. For some quality parameters one of the thresholds may not be needed.

To allow for short excursions of the quality parameter(s) out of the tolerance band, a weld is not flagged as defective unless $M_{f}$ out of $N_{f}$ consecutive $Q_{i}$ are out of tolerance. For example, if $M_{f}=$ 3 and $N_{f}=10$, and $Q_{12}, Q_{14}, Q_{19}$ are out of the tolerance band, then the weld is flagged as defective.

Software that uses this method of defect detection was used with a through-the-arc sensor for gas metal arc welding (GMAW). The through-the-arc sensor samples the current and voltage, and processes the signals through algorithms to output the quality parameters. The sensor is detailed in Refs. 1 and 2 but is described here briefly. The current and voltage signals are digitally sampled at frequency $f$ (usually in excess of 5000 samples/s). The sensor processes the signals in intervals $N$ samples long, giving sensor data at $N / f$; here we used $N=375$ samples and $f=6000$ samples/s for a sensor rate of $1 / 16 \mathrm{~s}$. The sensor outputs 7 parameters for constant voltage GMAW: average voltage, average current, average resistance, arc condition number, short frequency, current trend, and voltage trend. The average voltage $V$ and current $I$ are calculated as the $N$-point average of the signals for each interval; the resistance is the quotient $R=V / I$. The arc condition number is a dimensionless measure of the low-frequency stability of the arc. The 
voltage trend and current trend are the slopes of the lines fit to $M_{s}$ points of the average current and voltage. The short frequency is found by identifying the short circuit events in the interval and dividing by the length of the interval.

\section{EXPERIMENTS}

\section{$\underline{\text { Series I }}$}

Experiments were conducted in two series on the production floor. In the first series the raw current and voltage signals were recorded at 6000 samples/s. A robotic GMAW torch with a constant-voltage power supply welded a fillet weld in a lap joint of $3 \mathrm{~mm}$ thick mild steel. Mild steel wire, $1.2 \mathrm{~mm}$ in diameter was used with an $85 \% \mathrm{Ar}-15 \% \mathrm{CO}_{2}$ shielding gas. The effective travel speed was approximately $35 \mathrm{~mm} / \mathrm{s}$; the wire feed speed was approximately $201 \mathrm{~mm} / \mathrm{s}$; the voltage was set at approximately $22 \mathrm{~V}$ for a "buried arc" transfer mode. The welds lasted about $32 \mathrm{~s}$. We monitored 63 welds for each of two kinds of parts which had slightly different geometry: "Part A" and "Part B." The welds monitored were made on three different shifts of the production floor operation. Part A and Part B were made on the first shift; only Part A was made on the second shift and only Part B was made on the third shift. The weld path for both parts involved compound curves. The parts were visually inspected for defects such as surface porosity and burn-through in a manner similar to production quality checks.

The raw data was passed through the sensor algorithms offline. The constants needed for the defect detection algorithm $N_{f}, M_{f}$, and $\alpha$ or $\beta$ for each of the 7 quality parameters were found by processing the first 82 welds; the parameters were set so that defective welds were flagged and defect-free welds were not. The optimal constants were found by cut-and-try using batch processing. The defect detection algorithm was then tested for robustness by applying it to the final 44 welds using the constants found for the first 82 welds. A baseline series was constructed with $N_{b}=10$ with defect-free welds made at the start of each shift. $N_{s}$ was chosen as 10 for a smoothing window of $0.625 \mathrm{~s}$ based on the travel speed and changes in geometry of the part. The first and last $1.3 \mathrm{~s}$ of the weld were ignored due to the variability in the starts and stops.

\section{$\underline{\text { Series II }}$}

A software package implementing the defect detection algorithm was coupled with the sensing hardware and brought to a second production floor. In this case, defects were flagged on-line. The constants found in the first series of tests were used in the defect detection algorithm for a weld that lasted approximately $2 \mathrm{~s}$. The joint, power source and welding conditions were approximately the same as for Part A and Part B above. 400 welds were monitored on three production shifts. A baseline series was constructed (as above) with the first 10 welds which were defect-free and used for all of the shifts. The first $0.5 \mathrm{~s}$ of weld were ignored; no data were ignored at the end of the weld. 


\section{RESULTS AND DISCUSSION}

\section{Series I}

Of the 126 welds monitored in the first series of tests, 5 defects were detected. One defect (surface porosity) was about $70 \mathrm{~mm}$ in length; the other 4 were small skips or pores about $3 \mathrm{~mm}$ in diameter. The other 121 welds were defect-free on visual inspection. The first 82 welds with which the defect detection constants were found had 4 defective welds. The defect detection constants (Table 1) flagged 3 of the 4 defective welds of the first 82 and did not flag any of the defect-free welds. There was a single small defect in the final 44 welds. The algorithm flagged this weld (Fig. 1) and did not flag any of the 43 others.

Table 1: The best defect detection constants found using the first 82 welds of Series I.

\begin{tabular}{|ccccc|}
\hline Quality Parameter & $\mathrm{M}_{\mathrm{f}}$ & $\mathrm{N}_{\mathrm{f}}$ & $\alpha$ & $\beta$ \\
\hline Average Current & 3 & 5 & 2 & - \\
Average Voltage & 3 & 3 & 0.4 & - \\
Average Resistance & 2 & 2 & 1.75 & - \\
Arc Condition Number & 2 & 2 & - & 2.9 \\
Short Circuit Frequency & 10 & 10 & 1.5 & \\
Voltage Trend & 6 & 10 & - & 20 \\
Current Trend & 6 & 10 & - & 140 \\
\hline
\end{tabular}

For the reference series shown in Fig.1, the arc condition number is about 4 times greater in the last $20 \mathrm{~s}$ of weld than it is from $4 \mathrm{~s}$ to $10 \mathrm{~s}$. If an absolute limit on the arc condition number were used for defect detection, it would have to be set around 60 to accommodate this intentional change in the welding parameters. The defective weld shown in Fig. 1 would not have been flagged with this absoluté limit.

One of the difficulties with the defect detection algorithm is that, with several quality parameters, there are many constants to find. To speed the selection of the defect detection constants, the $2 \mathrm{~s}$ of the current and voltage data during which the defect occurred for each of the 4 defective welds was concatenated together with $2 \mathrm{~s}$ worth of data from defect-free welds that showed large excursions from the baseline series to form a single composite series. The corresponding sections of the baseline series were put together to form a composite baseline series. The software we wrote allowed us to plot the 7 quality parameters of the composite series against the 7 quality parameters of the baseline composite together with the thresholds. Using the composite series, the constants were found within a few $(<10)$ iterations.

\section{$\underline{\text { Series II }}$}

Since the first 358 welds monitored were free of defects, the torch position was changed on number 359 to cause a burn-through and returned to its original position for the rest of the welds. No defects were observed on the subsequent 41 welds. The defect detection algorithm flagged the single defective weld and did not flag any of the defect-free welds. 
The overall statistics for the algorithm were 5 of 6 welds with defects were flagged, 520 of 520 defect-free welds were not flagged. The defect that was not flagged was caused by a piece of spatter build-up on the gas cup falling into the weld pool causing a small pore and a lump in the weld bead.
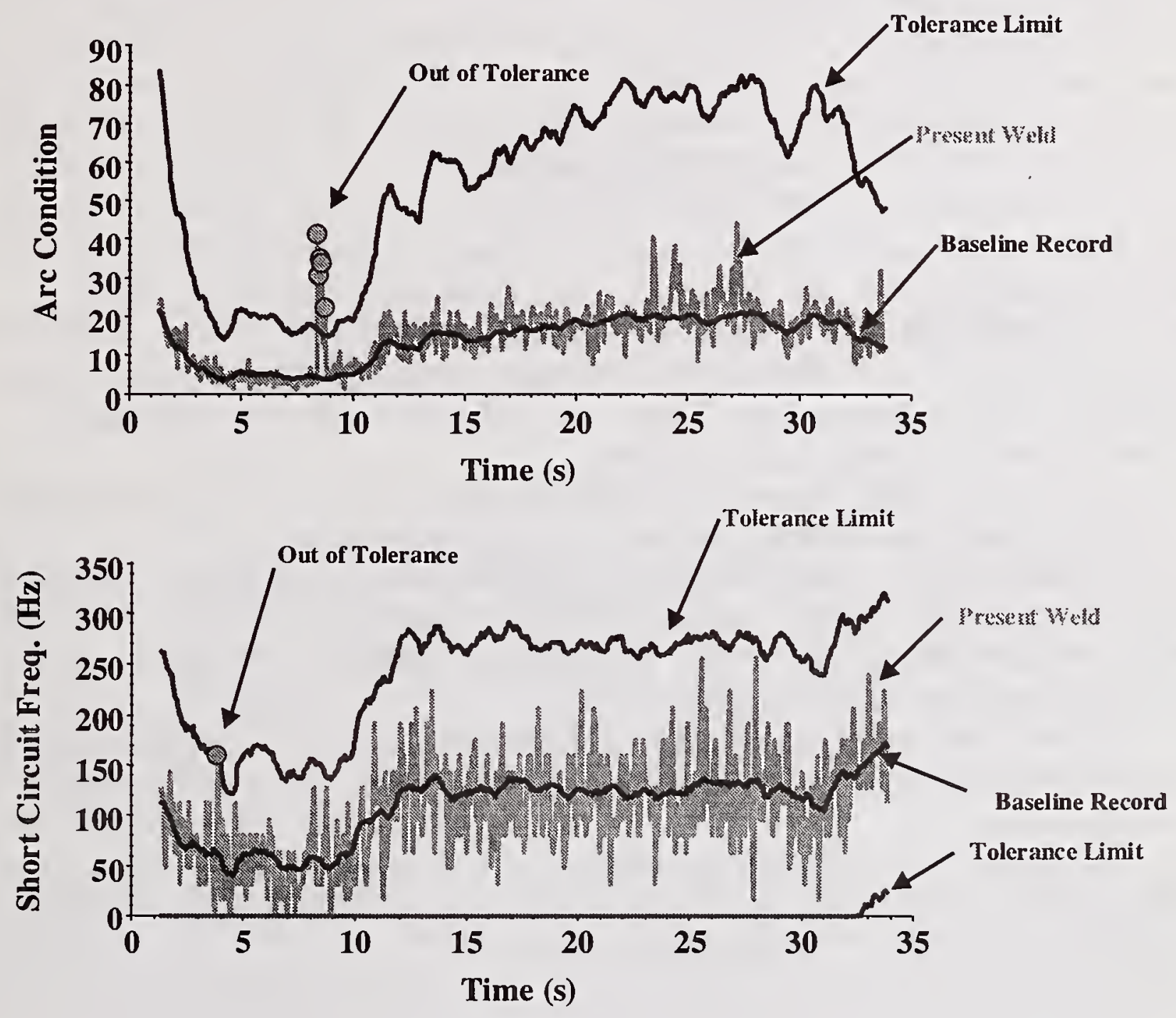

Figure 1: The arc condition and short circuit frequency quality parameters for a defective weld shown here as the "Present Weld." Because of the settings of the constants in Table 1, the defect detection algorithm flagged the weld because of the arc condition number (more than 2 out of 2 out) but not because of the short circuit frequency (only 1 out of 10 out). 


\section{CONCLUSION}

A defect detection algorithm was developed and used with a through-the-arc sensing system. The algorithm was tested on data from the production floor. The constants used to tune the algorithm were found from the first 82 welds which included 4 defective welds. Another 444 welds were monitored with 2 more defective welds. The sensor flagged 5 of the 6 defective welds and did not flag any defect-free welds.

\section{REFERENCES}

1. Quinn, T.P., and Madigan, R.B. 1997. United States Patent Pending.

2. Siewert, T.A., Madigan, R.B., and Quinn, T.P. 1993. United States Patent 5,221,825.

3. Madigan, R.B., and Quinn, T.P. 1994. United States Patent 5,349,156.

4. Kovacevic, R., Zhang, Y.M., and Ruan, S. 1993. Three-dimensional Measurement of Weld

Pool Surface. Proc. International Conference on Modeling and Control of Joining

Processes, ed. T. Zacharia: 600-607. AWS.

5. Johnson, J.A., Carlson, N.M., Smartt, H.B., and Clark, D.E. 1991. Process Control of GMAW:

Sensing of Metal Transfer Mode. Welding Journal 70 (4):91s-99s.

6. Song, J.-B., and Hardt, D.E. 1993. Closed-Loop Control of Weld Depth Using A Thermally

Based Depth Estimator. Welding Journal 72 (10):471s-478s.

7. Ludewig, H.W., Siwicke, J.H., Kilty, A.L., Wallace, B.L., Haytcher, S.A., and Rees, D.E.

1996. United States Patent 5,521,354.

8. Smartt, H.B. 1992. Intelligent Sensing and Control of Arc Welding. Proc. 3rd International

Conference on Trends in Welding Research, eds. S. A. David and J. M. Vitek: 843-851.

ASM International.

9. Araya, T., and Saukawa, S. 1992. Recent Activities on Sensing and Adaptive Control of Arc

Welding. Proc. 3rd International Conference on Trends in Welding Research, eds. S. A.

David and J. M. Vitek: 833-842. ASM International. 


\title{
THROUGH-THE-WIRE SENSOR FOR SEAM TRACKING IN GMA/CO ${ }_{2}$ AUTOMATIC WELDING*
}

\author{
P. Orszagh*, Y. C. Kim** and K. Horikawa**
}

\section{ABSTRACT}

The paper describes results, which have been achieved in development of a new type of welding torch position sensor. The new sensor enabled a real-time measurement of the torchto-workpiece position in GMA welding with short-circuiting metal transfer. The sensor was based on a unique detection algorithm, which detected the torch-to-workpiece distance from welding current and voltage waveforms, regardless huge variations of instant welding parameters. It was found that torch-to-workpiece distance could be reliably detected from wire resistance during short-circuit state of the welding process. The sensor was tested under various welding parameters and conditions and its applicability was confirmed by real time monitoring of the sensor signal.

KEY WORDS : (GMA Welding) (Short-circuit) (Metal Transfer) (Liquid Bridge)

(Drop Profile) (Position Sensor) (Seam Tracking)

\section{INTRODUCTION}

One of the basic hurdles in GMAW automation is insufficient flexible adaptation of the welding system to a huge variety of weld joint geometry, welding trajectory and torch-toworkpiece positions and orientations. Weld joint detection and seam tracking functions represent basic and imperative features of automatic welding systems. Small, low-cost, reliable and precise torch-to-workpiece position sensors, which are capable to resist extremely rash welding environment, become crucial elements for flexible automation and robotisation of arc welding processes in this way.

For the past 30 years various types of position sensors have been developed for arc welding and some of them have been used in industrial manufacturing. One can find one/two dimensional contact probe sensors (Ref. 1-3) with or without memory delay playback function, electrode contact sensors (Ref. 4,5), electromagnetic sensors (Ref. 6), arc sensors (Ref. 7-11), optical sensors (Ref. 12-16), sound sensors (Ref. 17), etc. Excellent review of the sensors, and their industrial applications in arc welding processes can be found in literature (Ref. 6,17).

Both reviews show that only contact (probe and electrode type) and arc sensors are widely used in welding practice. The arc sensors, due to their advantages (such as measurement and arc position coincidence, durability, real time control capability, reliability, no space requirements in the weld pool vicinity, low installation and maintenance cost, etc.) are extra desirable for seam tracking and adaptive welding control systems. Arc sensors, based on the arc length feedback control by means of AVC or ACC principle, are primarily used in GTAW, GMAW with spray metal transfer mode and plasma welding and cutting.

\footnotetext{
* Slovak Technical University, Bratislava, Slovakia

** Joining \& Welding Research Institute, Osaka University, Japan
} 
In GMAW with short-circuiting metal transfer mode, especially in GMAW with $\mathrm{CO}_{2}$ shielding gas, arc sensor applications are very rarely (Ref. 18). Extreme variations of instant welding parameters strictly limit a torch-to-workpiece distance detection sensitivity of the arc sensor. Some attemps (Ref. 19,20) of using the arc sensor in $\mathrm{GMAW} / \mathrm{CO}_{2}$ have been carried out, but they are still in the R\&D stage.

The paper describes principle and basic characteristics of a new position sensor for $\mathrm{GMA} / \mathrm{CO}_{2}$ welding. The sensor is based on a unique detection algorithm, which enables to detect the torch-to-workpiece distance from welding current and voltage waveforms, regardless huge variations of instant values. The new sensor was tested under various welding parameters and conditions.

\section{EXPERIMENTAL TECHNIQUES AND MATERIALS}

GMAW experiments were carried out in horizontal position. Series of single pass beads were deposited on mild steel plates at constant welding speed under various welding parameters and conditions. A block diagram of experimental set-up can be seen in Figure 1.

During the tests welding current and voltage were monitored by high speed data acquisition system and data were stored to files for post-process analysis. Technical parameters are summarised in Table 1 and 2.

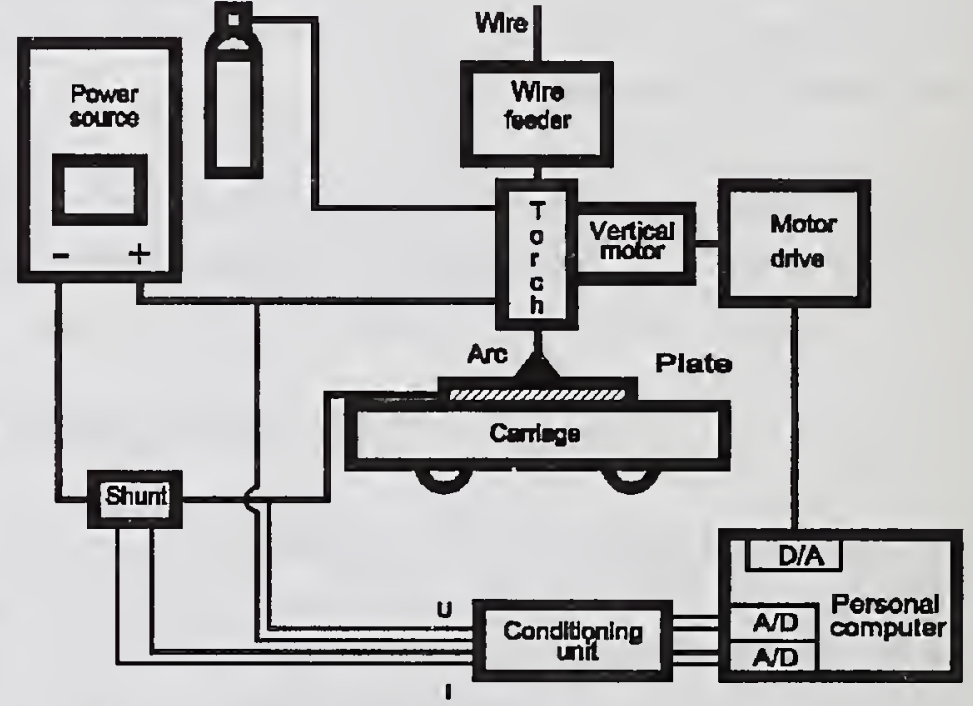

Figure 1. Welding experimental set-up

\begin{tabular}{|l|l||l|c|}
\hline \multicolumn{1}{|c|}{ Parameter } & \multicolumn{1}{c|}{ Value } & \multicolumn{1}{c|}{ Parameter } & Value \\
\hline Base material & MS, $500 \times 100 \times 12 \mathrm{~mm}$ & Scan & 2 channels \\
\hline Wire material & MS, $\varnothing 1.2 \mathrm{~mm}$ & Scanning period & $20 \mu \mathrm{s}$ \\
\hline Welding speed & 1 to $25 \mathrm{~mm} / \mathrm{s}$ & Scanning mode & Burst \\
\hline Torch height & 11 to $30 \mathrm{~mm}$ & Internal \\
\hline Wire feeding rate & 2 to $25 \mathrm{~m} / \mathrm{min}$ & Trigger & $2 \times 10^{6}$ \\
\hline Shielding gas & $\mathrm{CO}_{2,15} 1 / \mathrm{min}$ & Data volume & DMA \\
\hline Welding voltage & 15 to $29 \mathrm{~V}$ & Data transfer & $20 \mathrm{~s}$ \\
\hline Welding current & 0 to $800 \mathrm{~A}$ & Sampling activity & DAP95 \\
\hline Current slope & 31 to $200 \mathrm{kA} / \mathrm{s}$ & Acquisition control & ANA95 \\
\hline
\end{tabular}

Table 1. Welding parameters and conditions

Table 2. Data acquisition parameters

In GMAW with short-circuiting metal transfer mode a liquid drop touches a weld pool surface and transient liquid bridge profile can be observed. To study bridging phenomena simulated drop-pool "touching" experiments were carried out. Digital high speed photography with backlighting technique was used for recording transient drop/bridge profiles. Figure 2 shows experimental set-up for simulated "touching" tests. 
Experiments were carried out with various solid steel wire rods, of different diameters, several kinds of liquid and drop volumes (heights). Only homophase drop-pool bridging phenomena were studied. Four liquids with significantly different physical properties, e.g. mass density, surface tension, contact angle and kinematic viscosity were selected. The values were mostly taken from literature for pure materials, some values were directly measured or calculated from experimental data. Basic properties of the liquids used in experiments are summarised in Table 3.

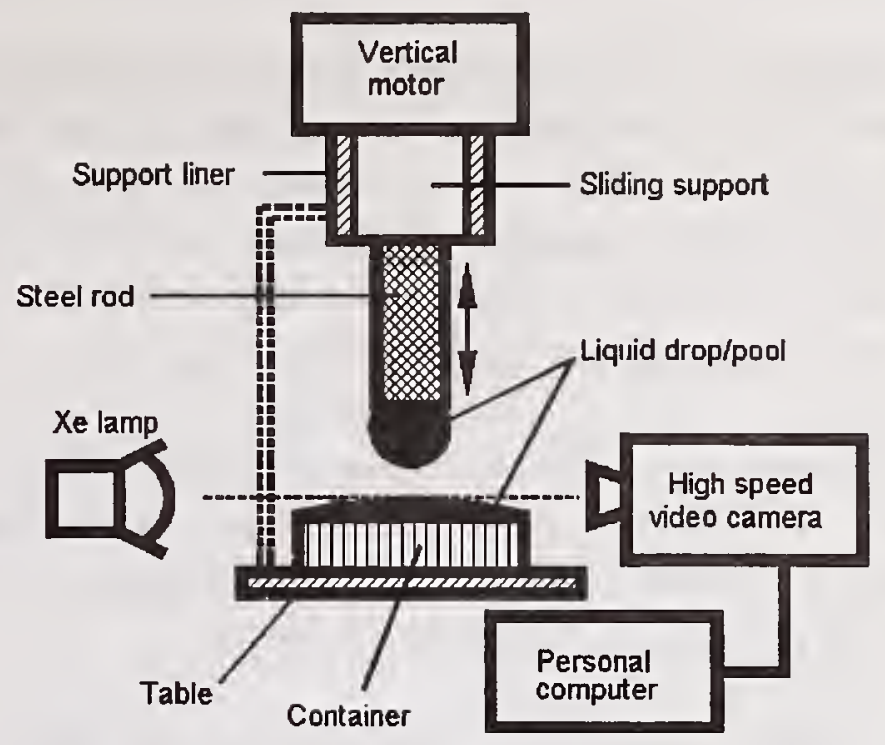

Figure 2. Simulated "touching". apparatus

\begin{tabular}{|c|c|c|c|c|}
\hline $\begin{array}{l}\text { Liquid } \\
\text { type }\end{array}$ & $\begin{array}{l}\text { Mass density } \\
\rho\left(\mathrm{kg} / \mathrm{m}^{3}\right)\end{array}$ & $\begin{array}{c}\text { Surface tension } \\
\sigma\left(10^{-3} \mathrm{~N} / \mathrm{m}\right)\end{array}$ & $\begin{array}{c}\text { Contact angle }^{1} \\
\Theta(\mathrm{deg})\end{array}$ & $\begin{array}{c}\text { Typical length }{ }^{2} \\
\mathrm{~L}_{\mathrm{t}}(\mathrm{mm})\end{array}$ \\
\hline Water & 1000 & 73 & $60^{*}$ & 2.72 \\
\hline Ethyl & $820^{*}$ & 22 & 0 & 1.65 \\
\hline Oil & $921^{*}$ & $29^{*}$ & 0 & 1.79 \\
\hline Mercury & 13546 & 475 & 120 & 1.89 \\
\hline $\operatorname{Iron}^{+}$ & 7000 & 1200 & 0 & 4.18 \\
\hline \multicolumn{3}{|c|}{${ }^{+}$liquid iron added } & uid/steel pair & ${ }^{2} L_{t}=(\sigma / \rho / g)^{1 / 2}$ \\
\hline
\end{tabular}

Table 3 Physical properties of liquids used in simulated experiments

\section{RESULTS AND DISCUSSION}

\section{Liquid bridge resistance}

Figure 3 shows typical pattern of the liquid bridge resistance in simulated homophase drop-pool "touching" experiments. It shows the liquid bridge resistance as a function of time. At the beginning the bridge resistance is high due to small crosssection area of the drop-pool contact. A sharp drop of resistance is well-marked at the beginning of the graph. The curve behaviour indicates a contact creation between the drop and the pool $(t<5 \mathrm{~ms})$, when fast radial contact spreading occurs on the surface of the pool (Ref. 21). During the first stage of the short-circuit the resistance of the liquid bridge has a negative first derivative $(\mathrm{dR} / \mathrm{dt}<0)$.

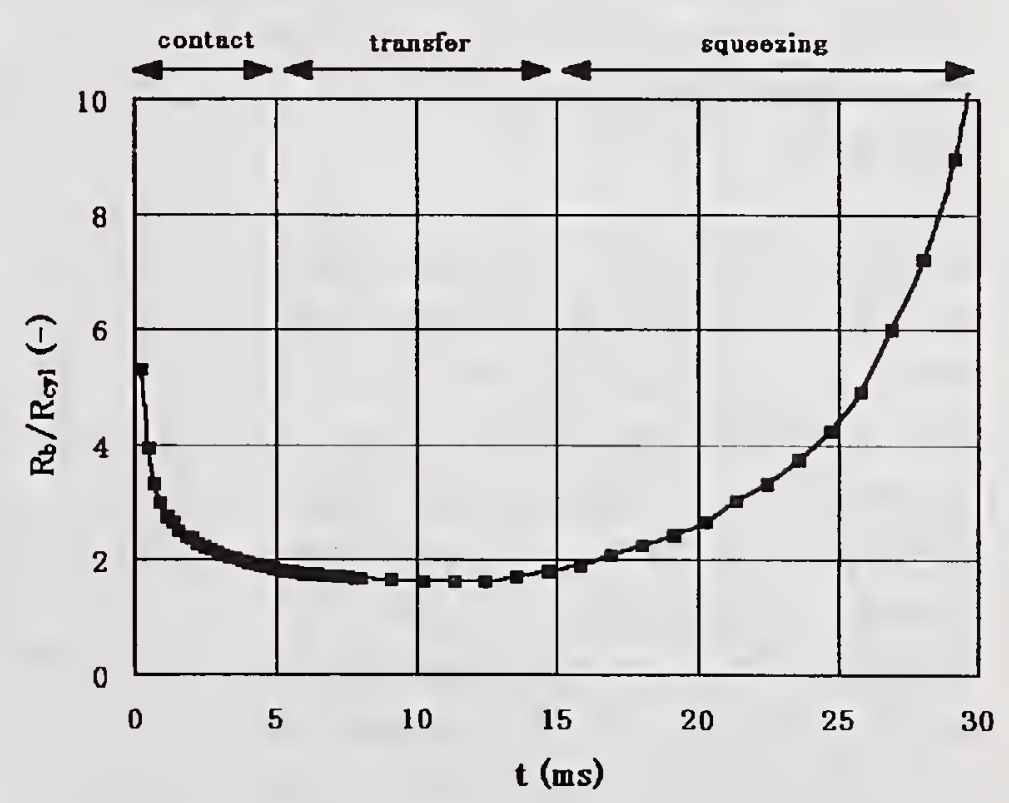

Figure 3. Resistance of the liquid bridge (water simulation) $R_{d}$-bridge resistance $R_{c}$-equivalent cylinder resistance 
The centre part $(5<\mathrm{t}<15 \mathrm{~ms})$ of the resistance shows a relatively flat plateau, indicating small change of the resistance. During this stage the neck radius is not significantly changed and it only moves up from the pool vicinity closer to the solid wire tip. The resistance of the bridge is almost constant, because it depends on the neck radius, not on the neck position within the bridge. The second stage of the bridging is characteristic by the first derivative of the bridge resistance close to zero $(\mathrm{dR} / \mathrm{dt} \approx 0)$.

In the last stage of the bridging the liquid bridge is gradually squeezed by capillary and gravity force and the neck radius decreases. The narrower the neck is, the higher resistance of the bridge becomes. During the squeezing stage $(t>15 \mathrm{~ms})$ the resistance increases and the curve has a positive first derivative $\mathrm{dR} / \mathrm{dt}>0$.

The resistance curve is not symmetric. The absolute value of the first derivative is higher in the contacting stage than that in the squeezing stage. The trend of the curve indirectly indicates that the contact radius spreading process was faster than the neck squeezing one.

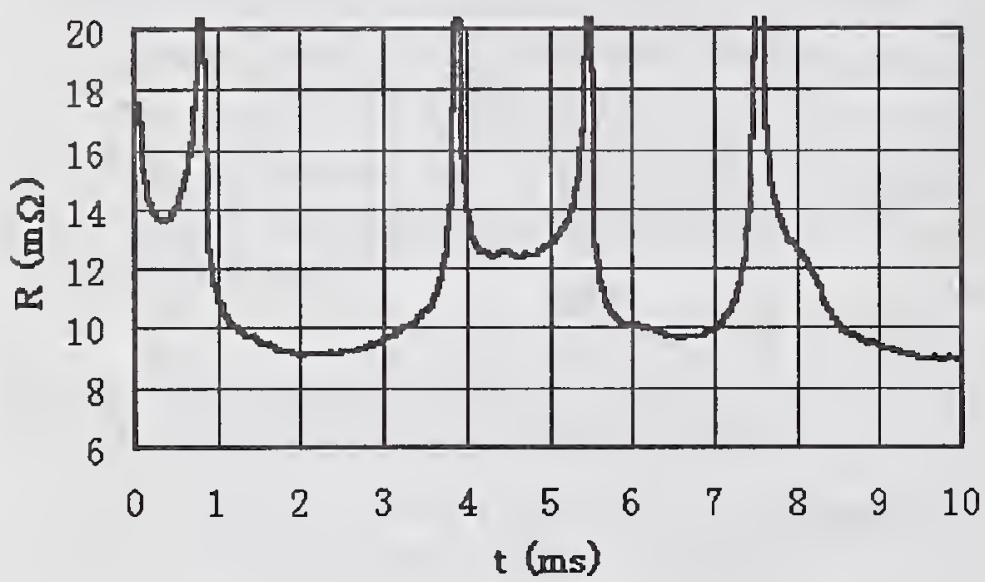

Figure 4. Resistance waveforms in short-circuiting state (arc burning state removed)

$\left(I=190 \mathrm{~A}, v_{d}=5.2 \mathrm{~m} / \mathrm{min}, L=14 \mathrm{~mm}, \mathrm{MS}\right.$ wire $\varnothing 1.2 \mathrm{~mm}, \mathrm{CP}$ mode, $\left.K_{I}=60 \mathrm{kA} / \mathrm{s}\right)$
Figure 4 shows typical measured waveforms of welding resistance in several sequential short-circuits. The arc burning cycles were removed from data. Detail of the $2^{\text {nd }}$ short-circuit can be seen in Figure 5 , where $2.5 x$ time zoom scale is applied.

It is obvious from the graphs that the resistance curve has a typical U-shape, which is characteristic for homophase liquid drop-pool "touching" phenomena (see Figure 3). At the beginning of the short-circuit the resistance rapidly falls following the drop-pool contact radial expansion on the weld pool surface. From Fig. 5 it can be found that the fast contact spreading (A) only lasted $1.3 \mathrm{~ms}(0.7<\mathrm{t}<2 \mathrm{~ms})$ and after a very short flat plateau (B) duration $0.5 \mathrm{~ms}(2<\mathrm{t}<2.5 \mathrm{~ms})$ the resistance started to rise $(\mathrm{t}>2.5 \mathrm{~ms})$, indicating the beginning of the neck squeezing stage $(\mathrm{C})$. The whole metal transfer took place about $3 \mathrm{~ms}$.

Taking into account characteristics of homophase drop-pool touching phenomena a new detection method of the torch-to-workpiece distance can be proposed as follows. In shortcircuiting state the torch-to-workpiece distance corresponds to the wire extension. The wire extension (length) can be determined by means of measurement of its resistance, when the wire is in a good and stable electrical contact with the weld pool.

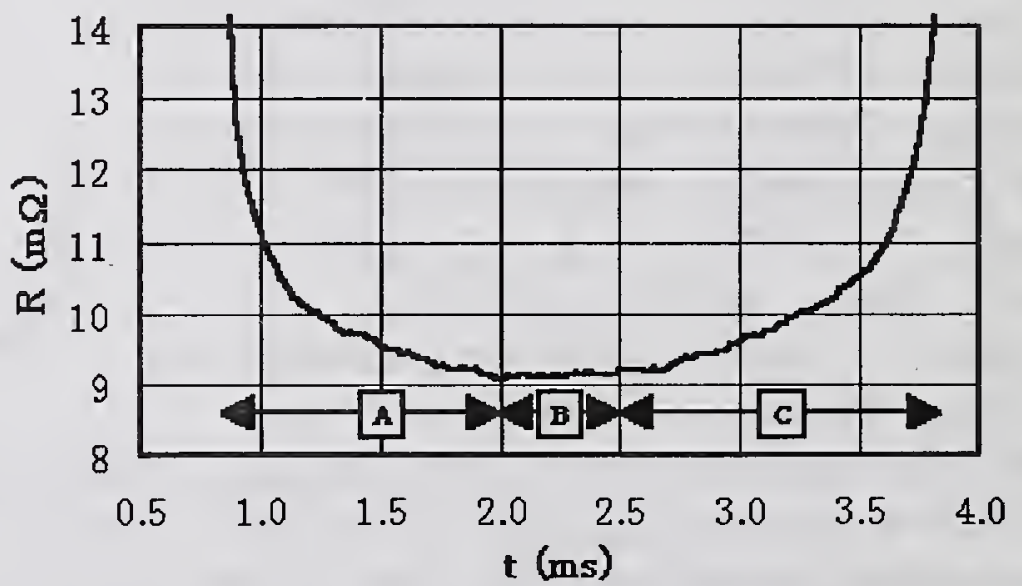

Figure 5. Detail of resistance waveform in short-circuiting state $\left(I=190 \mathrm{~A}, v_{d}=5.2 \mathrm{~m} / \mathrm{min}, L=14 \mathrm{~mm}, M S\right.$ wire $\varnothing 1.2 \mathrm{~mm}, C P$ mode, $K_{I}=60 \mathrm{kA} / \mathrm{s}$ ) 
To avoid a high resistance of the drop-pool contact region at the beginning of short-circuit, the resistance should be sampled after the contact radius is wide enough $(t>1 \mathrm{~ms})$. The contribution of the contact region to the total welding resistance will be reduced in this way and the measured value will better correspond to the torch-to-workpiece distance L (Ref. 21).

On the other hand the welding resistance can not be measured too late. At the end of the short-circuiting state the resistance becomes high again. The neck is very narrow and its resistance becomes dominant (Ref. 22).

\section{Sensor principle}

A typical U-type resistance waveform automatically suggests to pick the data in the "centre" part of the curve, where the welding resistance reaches local minimum $R_{\text {smin }}$ with first derivative equal to zero $(\mathrm{dR} / \mathrm{dt}=0)$. At these moments both conditions are satisfied: the contact area is wide enough and the neck region is not significantly developed yet. The total welding resistance will the most reflect the torch-to-workpiece distance.

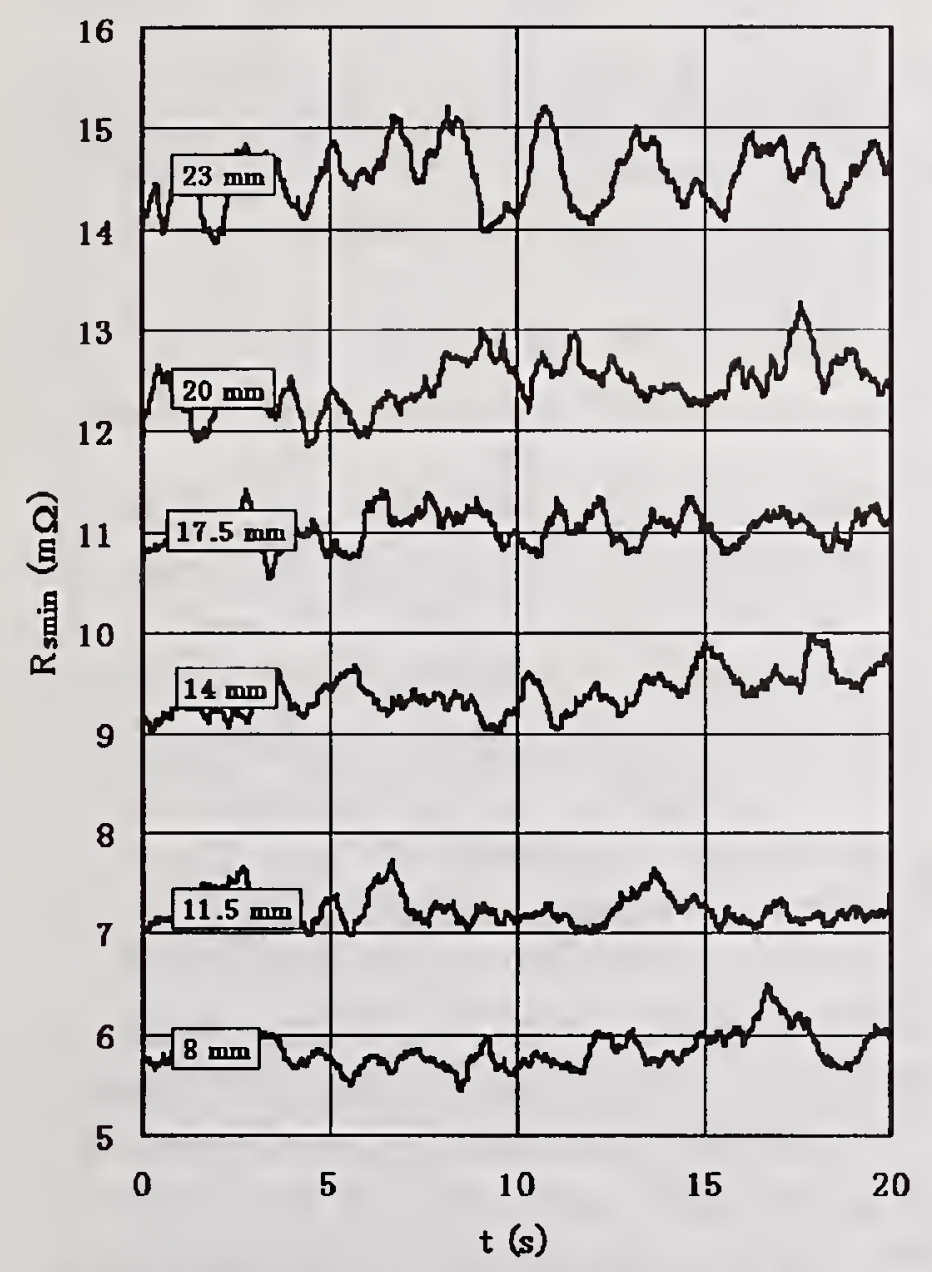

Figure 7. Sensor output at various torch heights $a=165 \mathrm{~A}, U=20 \mathrm{~V}, L=8-23 \mathrm{~mm}, v_{d}=4 \mathrm{~m} / \mathrm{min}$, MS wire, $\varnothing 1.2 \mathrm{~mm}, C P$ mode, $K_{I}=60 \mathrm{kA} / \mathrm{s}$ )

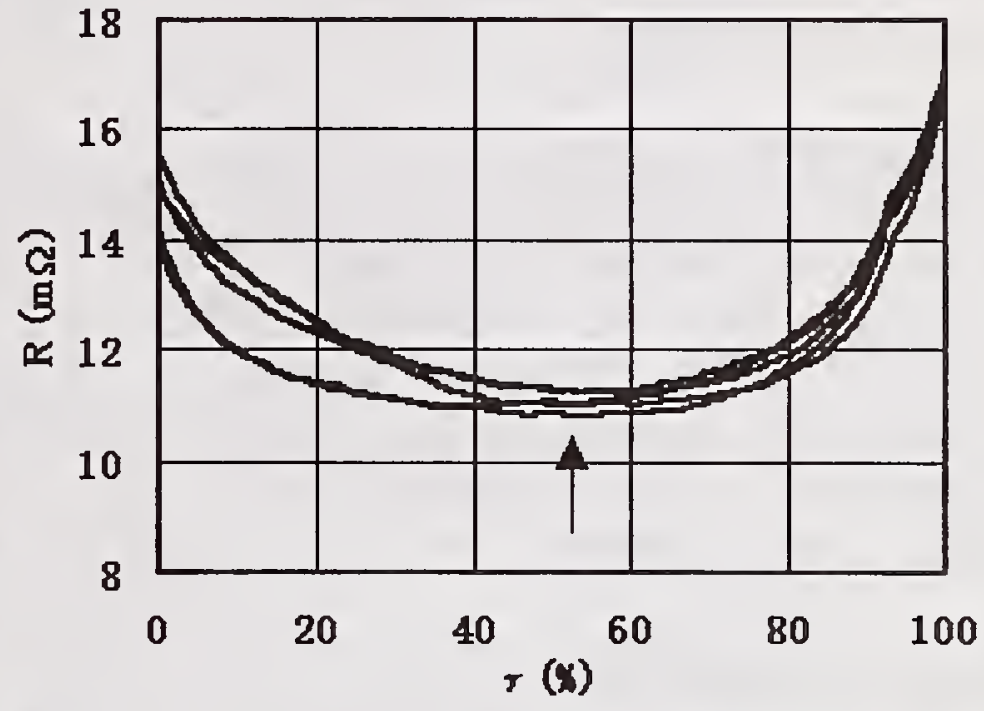

Figure 6. Resistance waveform in short-circuiting state $\left(I=190 \mathrm{~A}, v_{d}=5.2 \mathrm{~m} / \mathrm{min}, L=17 \mathrm{~mm}, M S\right.$ wire, $01.2 \mathrm{~mm}, C P$ mode, $\left.K_{I}=60 \mathrm{kA} / \mathrm{s}\right)$

Figure 6 shows welding resistance waveforms of 6 short-circuits with duration $\mathrm{t}_{\mathrm{s}}>2 \mathrm{~ms}$. The short-circuit time was normalised to dimensionless variable in $0-100 \%$ scale. All curves show a typical U-type shape with very close local minimum values of resistance $\mathrm{R}_{\mathrm{smin}} \approx 11 \mathrm{~m} \Omega$ in the centre part. Taking account previous discussions on liquid bridge resistance waveform in homophase drop-pool touching, the value $R_{\text {smin }}$ was selected as the sensor output for torch-to-workpiece distance detection. The sensor was tested under various welding parameters and conditions.

Figure 7 shows the results of test series when torch-to-workpiece distance L was gradually changed in a range from 8 to $23 \mathrm{~mm}$. It is obvious from the graph that the sensor provides a stable signal of the torch position. The torch-to-workpiece distance can be reliably extracted from the welding resistance signal. The bigger the torch height is, the higher sensor signal level becomes. 
Figure 8 shows the sensor static characteristics. It is obvious from the graph that the sensor output $R_{\text {smin }}$ is sufficiently sensitive to the torch-toworkpiece distance L. Moreover the input-output function is linear in the stated range of the torch height. For mild steel wire $\varnothing 1.2 \mathrm{~mm}$ the sensor sensitivity $\Pi$ was $0.6 \mathrm{~m} \Omega / \mathrm{mm}$.

It is generally known that welding parameters, mainly welding voltage and short-circuiting current slope, have a substantial effect on short-circuiting phenomena of the $\mathrm{GMA} / \mathrm{CO}_{2}$ welding process. That is why the next interest was focused into a sensor stability under various welding conditions.

\section{Sensor characteristics}

Figure 9 shows a dependency of a shortcircuit duration, short-circuit frequency and measu-rement frequency of the sensor on the welding voltage. All parameters are strongly affected by the welding voltage. The higher voltage $U$ is the lower values $t_{s}, f_{s}$ and $f_{m}$ are observed. When welding voltage is changed from $19 \mathrm{~V}$ to $25 \mathrm{~V}$ the short-circuit duration is reduced 4 times (from 4 to $1 \mathrm{~ms}$ ) and short-circuit frequency decreases from 60 to $35 \mathrm{~Hz}$. The sensor measurement frequency was reduced from 40 to $12 \mathrm{~Hz}$.

Regardless huge process characteristics changes, the sensor output is stable, not significantly affected by the welding voltage (see Figure 10).

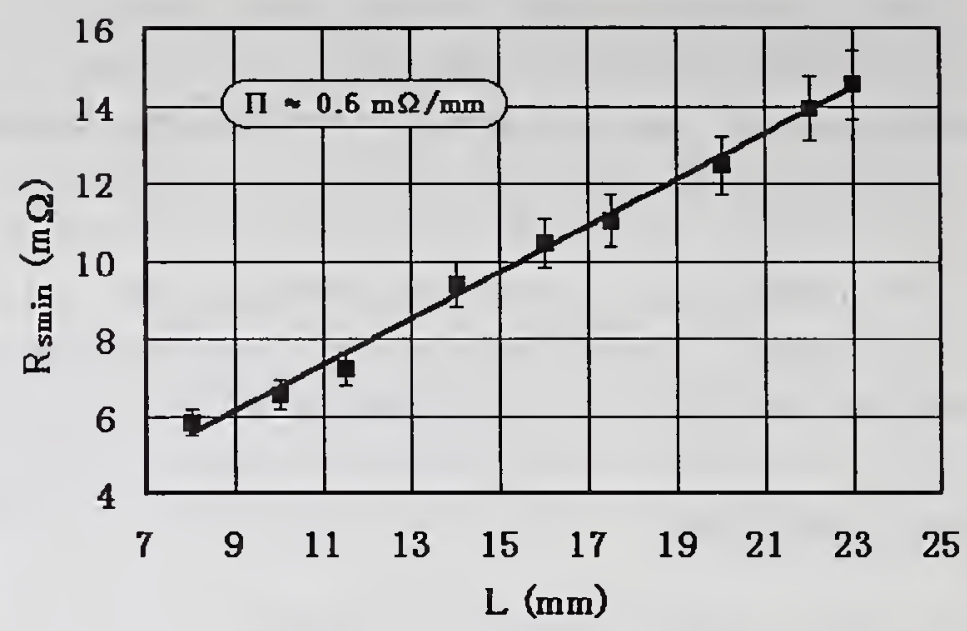

Figure 8. Sensor output vs. torch-to-workpiece distance $\left(I=165 \mathrm{~A}, U=20 \mathrm{~V}, v_{d}=4 \mathrm{~m} / \mathrm{min}, \mathrm{MS}\right.$ wire, $\varnothing 1.2 \mathrm{~mm}, C P$ mode, $K_{I}=60 \mathrm{kA} / \mathrm{s}$ )

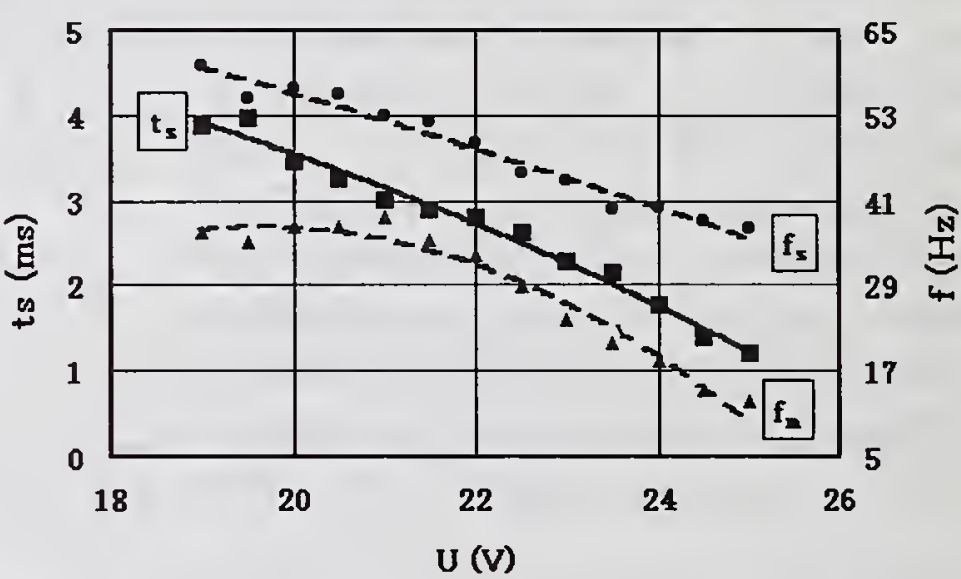

Figure 9. Short-circuit duration $\left(t_{s}\right)$ and frequency $\left(f_{s}\right)$ and sensor measurement frequency $\left(f_{m}\right)$ vs. welding voltage $(U)$ $\left(I=190 \mathrm{~A}, L=14 \mathrm{~mm}, v_{d}=5.2 \mathrm{~m} / \mathrm{min}, \mathrm{MS}\right.$ wire, $\varnothing 1.2 \mathrm{~mm}, C P$ mode, $\left.K_{1}=60 \mathrm{kA} / \mathrm{s}\right)$

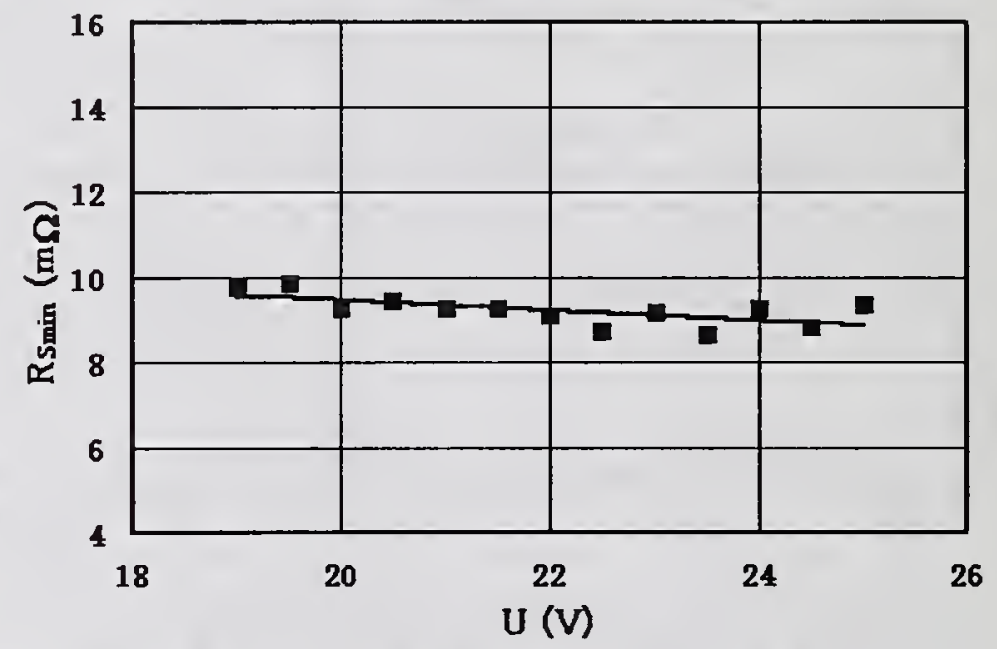

Figure 10. The sensor output vs. welding voltage

$\left(I=190 \mathrm{~A}, L=14 \mathrm{~mm}, v_{d}=5.2 \mathrm{~m} / \mathrm{min}, \mathrm{MS}\right.$ wire, $\varnothing 1.2 \mathrm{~mm}, C P$ mode, $\left.K_{1}=60 \mathrm{kA} / \mathrm{s}\right)$

Figure 11 shows a strong effect of short-circuiting current slope $K_{I}$ on basic process characteristics. When the current slope is increased from 31 to $200 \mathrm{kA} / \mathrm{s}$ the short-circuit duration $t_{s}$ is decreased almost 6 times, from 6 to $1 \mathrm{~ms}$. The short-circuiting frequency changes in the range $35-75 \mathrm{~Hz}$ and the measurement frequency $15-60 \mathrm{~Hz}$, respectively. In spite of such tremendous changes of metal transfer characteristics, the sensor provides a relatively stable signal for torch height detection (see Figure 12). 


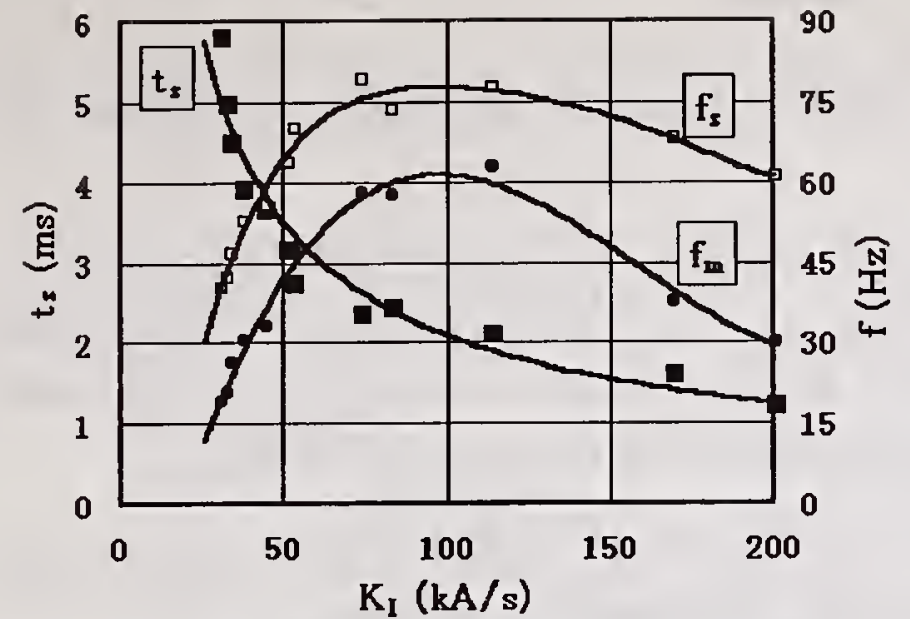

Figure 11. Short-circuit duration $\left(t_{s}\right)$ and frequency $\left(f_{s}\right)$ and sensor measurement frequency $\left(f_{m}\right)$ vs. current slope $\left(\mathrm{K}_{\mathrm{I}}\right) I=175 \mathrm{~A}, U=20.5 \mathrm{~V}, L=14 \mathrm{~mm}, v_{d}=5.2 \mathrm{~m} / \mathrm{min}, M S$ wire, $\varnothing 1.2 \mathrm{~mm}, C P$ mode, $\left.K_{t}=31-200 \mathrm{kA} / \mathrm{s}\right)$

Figures 13 and 14 show the sensor signal $\mathrm{R}_{\text {smin }}$ when torch-to-workpiece distance $\mathrm{L}$ is continuously changed during the welding operation. In both experiments the welding speed was $5.3 \mathrm{~mm} / \mathrm{s}$.

In downhill test the torch height $\mathrm{L}$ was increased from 13 to $23 \mathrm{~mm}$. The graph indicates the sensor output from 8.5 to $15.5 \mathrm{~m} \Omega$. From the sensor calibration characteristics (Fig. 8) the detected torch-to-workpiece distance $\mathrm{L}_{\text {det }}$ is in a range $12.5-23.5 \mathrm{~mm}$. The values detected by means of sensor $\mathrm{L}_{\mathrm{det}}$ are very close to real torch-to-workpiece values $\mathrm{L}$.

In uphill test (Fig. 14) the torch-toworkpiece distance linearly changed from 23 to $9 \mathrm{~mm}$. The sensor output gradually decreased from 14.5 to $7 \mathrm{~m} \Omega$ and corresponding torch height $\mathrm{L}_{\text {det }}$, measured by the sensor, reduced from 22.5 to $10 \mathrm{~mm}$. The values are very close to real torch-to-workpiece distance.

A small difference $(\approx 1 \mathrm{~mm})$ between uphill and downhill sensor values were caused by a different weld bead formation in the welding tests. The weld bead reinforcement difference was detected by the sensor as the sensor principle is exactly based on a torch-to-pool distance measurement technique.

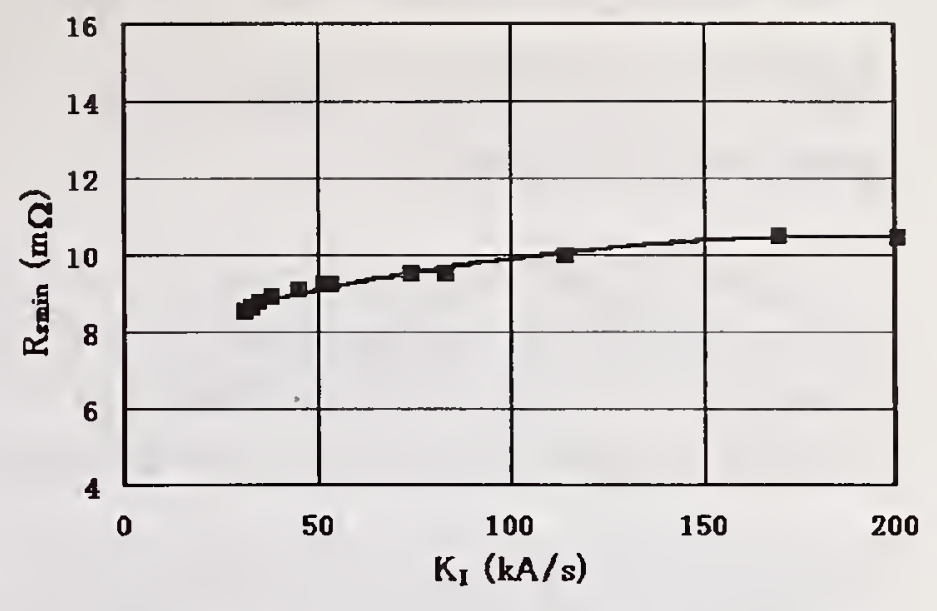

Figure 12. The sensor output $\left(\mathrm{R}_{\mathrm{smin}}\right)$ vs. short-circuit current slope $\left(\mathrm{K}_{\mathrm{I}}\right)$

$\left(I=175 \mathrm{~A}, U=20.5 \mathrm{~V}, \mathrm{~L}=14 \mathrm{~mm}, v_{d}=5.2 \mathrm{~m} / \mathrm{min}, M S\right.$ wire, $\varnothing 1.2 \mathrm{~mm}, \mathrm{CP}$ mode, $K_{1}=31-200 \mathrm{kA} / \mathrm{s}$ )

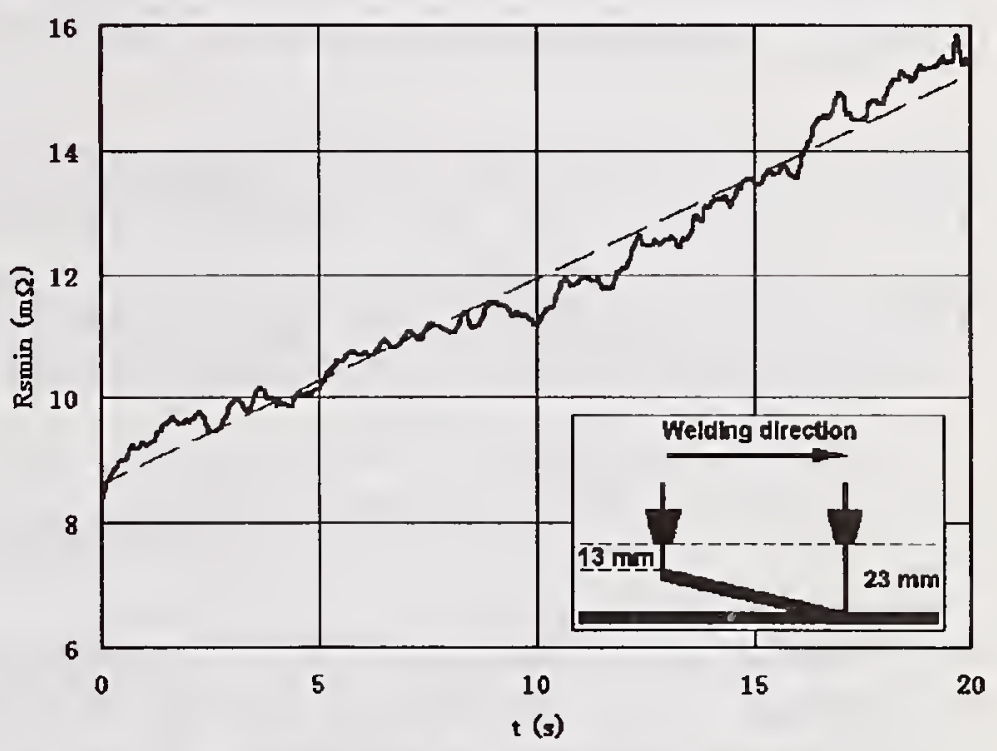

Figure 13. Sensor output during downhill welding $\left(I=180 \mathrm{~A}, U=19 \mathrm{~V}, v_{d}=5.2 \mathrm{~m} / \mathrm{min}, \mathrm{MS}\right.$ wire $\varnothing 1.2 \mathrm{~mm}, \mathrm{CP}$ mode, $\left.K_{I}=60 \mathrm{kA} / \mathrm{s}\right)$

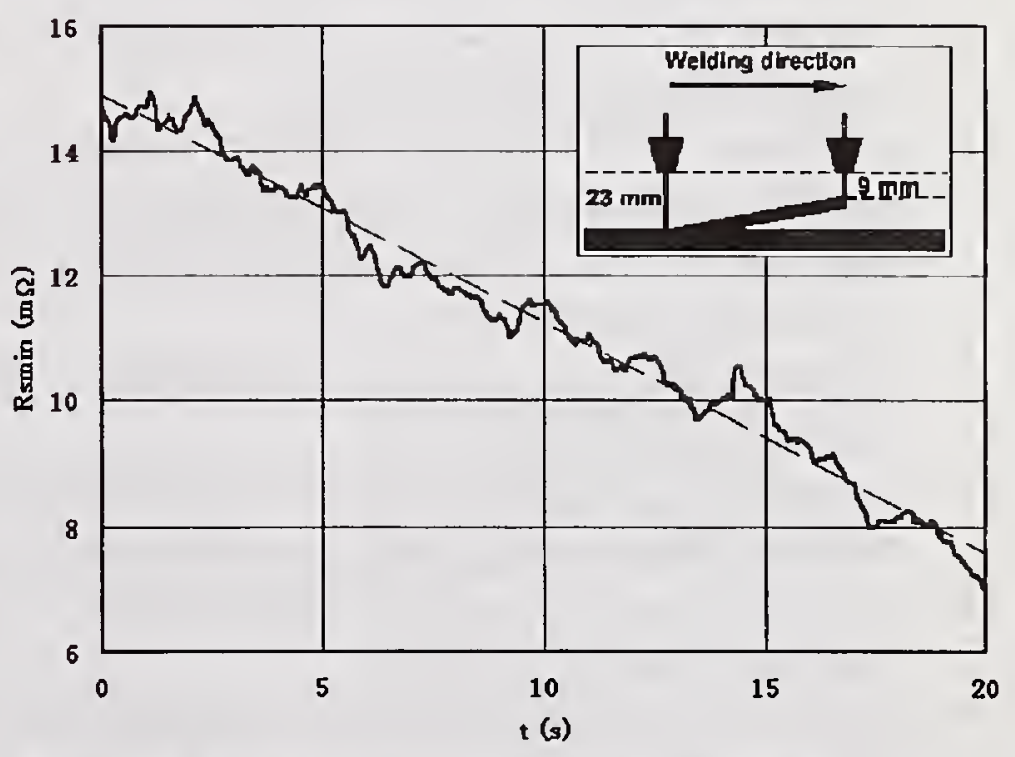

Figure 14. Sensor output during uphill welding $\left(I=180 \mathrm{~A}, U=19 \mathrm{~V}, v_{d}=5.2 \mathrm{~m} / \mathrm{min}, \mathrm{MS}\right.$ wire $\varnothing 1.2 \mathrm{~mm}, \mathrm{CP}$ mode, $\left.K_{I}=60 \mathrm{kA} / \mathrm{s}\right)$ 


\section{CONCLUSIONS}

Study on short-circuiting metal transfer in GMAW has lead to results, which can be summarised as follows:

1. In short-circuiting state the liquid bridge resistance has a typical U-type shape profile in time with a local minimum more-less in the centre part of the curve. At the beginning and the end of the bridging the resistance is high due to a small cross-section of the liquid bridge, caused by drop-pool contact formation and neck squeezing, respectively.

2. When high resistivity welding wires are used (e.g. mild steel, stainless steel, etc.., the liquid bridge resistance can be used for a short-circuiting phenomena monitoring.

3. The liquid bridge resistance can be used for a torch-to-workpiece distance detection in GMAW with short-circuiting metal transfer mode. The through-the-wire sensor, based on a minimum value of short-circuit welding resistance, provides a reliable torch-to-workpiece distance signal for a feedback control of the torch position in seam tracking systems.

\section{ACKNOWLEDGEMENTS}

The authors are grateful to Prof. M. Ushio and Dr. T. Ueyama from R\&D division of Daihen Corp. for technical support of the research project.

\section{REFERENCES}

1. Masumoto, I.; Nomura; H. 1982. Development and Application of Sensors and Sensor Systems for Arc Welding in Japan. Doc. IIW XII-C-031-82.

2. Heitmayer, U.; Weman, K. 1981. Adaptive Sensing : Key to the Adult Robot. Welding and Metal Fabrication (12): 592-596.

3. Nomura, H. et al. 1982. Automatic Welding of the Corrugated Membrane of an LNG Tank. Metal Construction (7): 391-395.

4. Towata, T. 1979. Outline and Its Application of Shin Meiwa of Sensorless Sensing System. Robot (25): 16-20.

5. Fukuoka, H. 1994. Application of a Touch Sensor to an Arc Welding Robot. Sensors and Control Systems in Arc Welding, Chapman\&Hall Publishing, London: 292-299.

6. Nomura, H. 1994. Sensors and Control Systems in Arc Welding, Chapman\&Hall Publishing, London: 40-43.

7. Fujimura, H.; Ide, E. and Inoue, H. 1987. Joint Tracking Control Sensor of GMAW. Trans. JWRI (1): 32-40.

8. Nomura, H.; Sugitani, Y. and Mutayama,M. 1987. Development of Automatic Fillet Welding Process with High Speed Rotating Arc. Trans. JWRI (2): 26-34.

9. Orszagh, P. and Sencak, V. 1990. Adaptive Control of Torch Position with Arc Sensor. IIW Doc SG 212-763-90.

10. Ushio, M. and Mao, W. 1994. Modelling and Characteristics of Through-the-arc sensor. Trans. JWRI (1): 13-19.

11. Dilthey, U.; Stein, L. and Oster,M. 1996. Through-the-arc Sensing - An Universal and Multipurpose Sensor for Arc Welding Automation, JOM (1): 6-12. 
12. Arata, Y. and Inoue, K. 1976. Application of Digital Picture Processing Technique to Automatic Control. Doc. IIW XII-K-65-76.

13. Pan, J. L. and Wang, J. L. 1983. Development of Two-directional Seam Tracking System with Laser Sensor. Welding Journal (2): 28-35.

14. Drews, P. and Starke,G. 1985. Development Approaches for Advanced Adaptive Control in Automated Arc Welding. Proc. IIW Int. Conference: 115-124, Pergamon Press, Oxford.

15. Orszagh, P. and Vyjidak, O. 1985. System for Feedback Control of Circumferential TIG Welding of Pipes. Proceeding of Conf. On Advanced Welding Systems, London.

16. Boillot, J. P.; Yu, X. and Fontaine, J. C. 1994. Automatic Welding Using 3D Laser Vision System. Welding in the World (2): 173-182.

17. Ushio, M. and Mao, W. 1994. Sensors for Arc Welding : Advantages and Limitations. Trans. JWRI (2): 135-141.

18. Technical Commission on Welding Process of JWS 1991. Sensors and Control Systems in Arc Welding, Kuroki Publish.

19. By, J.; Kim, W. and Na, J. 1991. A Study on an Arc Sensor for Gas Metal Arc Welding of Horizontal Fillets. Welding Journal (8): 216-s to 221-s.

20. Ushio, M.; Liu, W. and Mao, W. 1995. Experimental Investigation of Dynamic Behaviour of Arc Sensor in GMAW in Short Circuit Transfer Mode. Trans. JWRI (1): 25-30.

21. Orszagh, P.; Kim, Y. C. and Horikawa, K. 1997. Short-circuiting Transient Phenomena in GMA $/ \mathrm{CO}_{2}$ Welding Process -Report I. Trans. JWRI, 26 (1): in print / IIW Doc.212908-97:in print

22. Orszagh, P.; Kim, Y. C. and Horikawa, K. 1997. Real-time Torch Height Detection Technique for GMA/CO $/ \mathrm{CO}_{2}$ Welding. Scien. And Tech. of Weld. and Join.: in print.

\section{APPENDIX}

Greek letters

$\begin{array}{ll}\rho & \text { mass density } \\ \sigma & \text { surface tension } \\ \tau & \text { dimensionless time }\left(\tau=\mathrm{t} / \mathrm{ts}^{*} 100\right) \\ \Pi & \text { sensor sensitivity } \\ \varnothing & \text { wire diameter }\end{array}$

\section{Roman letters}

$f_{m} \quad$ measurement frequency of the sensor

$f_{s} \quad$ short-circuit frequency

g gravity constant

I welding current

$\mathrm{K}_{\mathrm{I}}$ dynamic characteristics of the power source

$$
\left(\mathrm{K}_{\mathrm{I}}=\mathrm{d} \mathrm{I} / \mathrm{dt}\right)
$$

$\mathrm{L}$ torch-to-workpiece distance

weld pool is part of the workpiece

torch bottom is the bottom end of contact tip

$L_{t} \quad$ typical length of the liquid

$r$ radius

$\mathrm{R}$ resistance in short-circuiting state

$\mathrm{R}_{\mathrm{smin}}$ minimum resistance in short-circuiting state

S cross-section of the wire/bridge $\left(S=\pi r^{2}\right)$ t time

$t_{\min } \quad$ time when $R=R_{\text {amin }}$

$\mathrm{t}_{3} \quad$ short-circuiting duration

$\mathrm{T}_{\mathrm{o}} \quad$ temperature profile in solid wire

$\mathrm{U}$ welding voltage

$\mathrm{v}_{\mathrm{d}} \quad$ wire feeding rate

$\mathrm{Z} \quad$ wire axis from weld pool to torch tip

Special symbols

$\underline{x} \quad$ average value of $x$

Shortcuts

ACC

AVC

AVD

D/A

DMA

CP

GMA

GMAW

$\mathrm{GMA} / \mathrm{CO}_{2}$

MS

$\mathrm{SC}$ automatic current control automatic voltage control analog/digital converter digital/analog converter direct memory access constant potential power source gas metal arc gas metal arc welding GMA in $\mathrm{CO}_{2}$ shielding gas mild steel MIX-50S short-circuit 



\section{WELD PLAN SIMULATION}





\title{
DESIGN OF A WELDING APPLICATION PROGRAMMING INTERFACE
}

\author{
T.E. Doyle*
}

\begin{abstract}
The effective use of automated welding systems is dependent upon efficient equipment programming methods. This is especially true in small to medium batch operations where excessive equipment programming time can be an economic barrier to automated equipment utilization. A number of tools exist which attempt to address these concerns. Notably, robotic off-line programming systems can reduce the amount of time required for programming. However, these systems typically lack methods for including process information in the automated planning methods. This information is typically manually inserted in order to complete the planning operations. The true benefits of technologies such as off-line programming will only be achieved when totally automated planning can be realized. This will not be possible until methods for integrating welding process knowledge with the application planning can be realized.
\end{abstract}

A development effort is currently underway which addresses the issue of automated access to welding procedure information. This program is defining a Welding Application Programming Interface that will address the issues for automated welding application planning.

\section{OVERVIEW}

The Welding Application Programming Interface (API) defines standardized methods by which other systems (e.g., OLP, CAD, etc.) can interact with a welding procedure database. Figure 1 depicts a typical information flow model for an automated planning system. The Welding API defines the methods to obtain, create, modify, and deposit welding procedure information within the database without requiring knowledge of the database design. The information content supported by the API is based on the Welding Neutral File Format Specification. The ability to interact with a welding procedure database allows external system to obtain welding knowledge to assist with decisionmaking operations.

The Welding API is intended to support the access, creation, modification, and storage of information within disparate databases of undetermined structure. The Welding API is based on a messaging scheme and a command set that can be used to link these dissimilar databases with external systems.

\footnotetext{
"McDermott Technologies Incorporated, Alliance, Ohio
} 


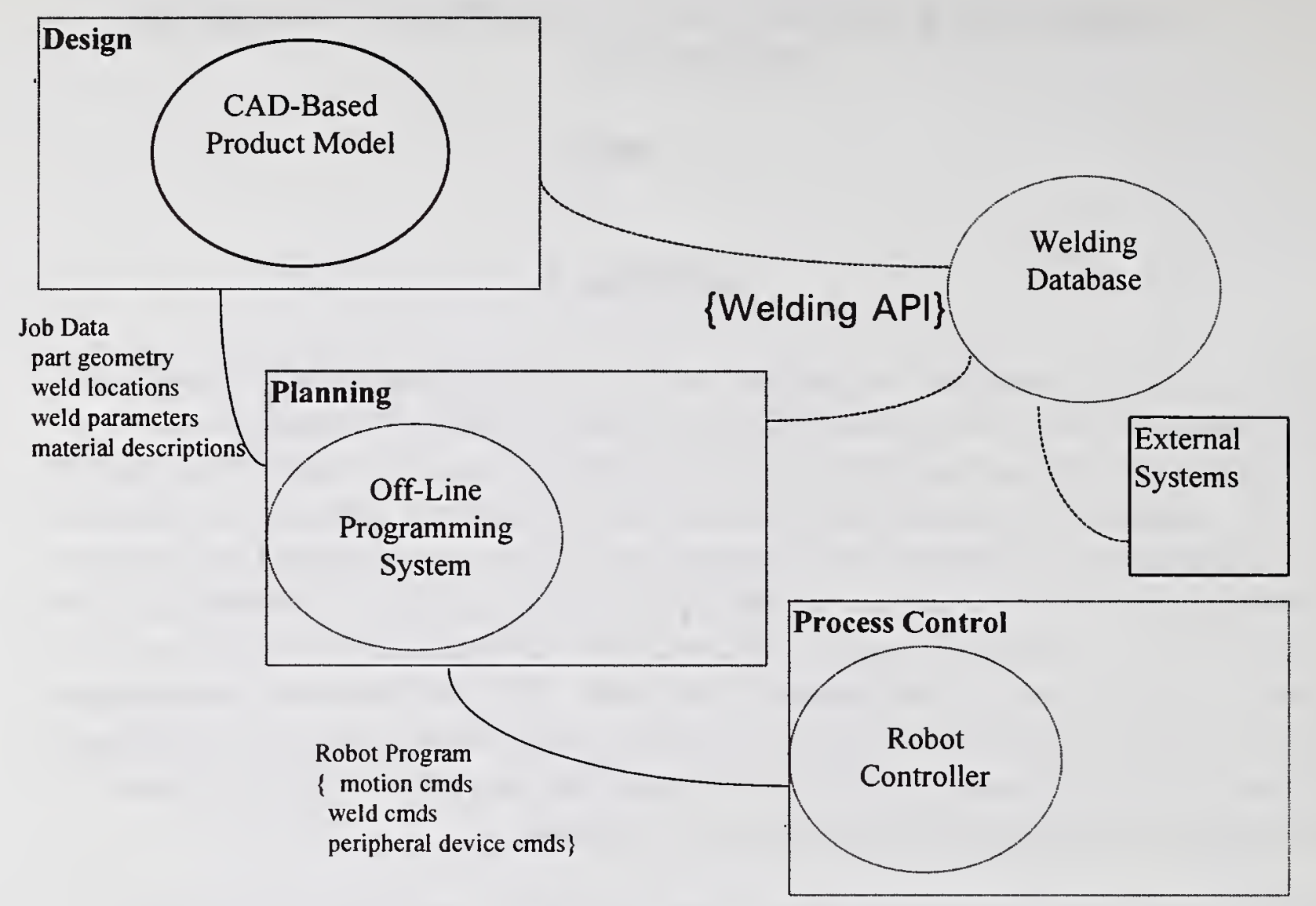

Figure 1. OLP Environment

The mechanism used to implement the Weld API is the TCP/IP "sockets" services. Sockets are end points of communications channels and are identified by a unique IP address and port number. A user wishing to access a weld database site must know the IP address of the site's Weld API server and its port number. IP addresses are assigned by an Internet committee, but the Weld API Specification can recommend a port number to be used by all Weld API servers.

The role of the Weld API in supporting the CAD-to-OLP relationship is depicted in Figure 2. The Weld API is supported by a standard client-server model in which the API Server is attached to a native welding database. Each native database site that wishes to be Weld API accessible must implement its own Weld API server. The Weld API specification does not address the design of Weld API servers because they are specific to each native data base management system.

Other devices which require information in the welding database use Weld API commands to access the data. Figure 2 illustrates the relationship between the API Server and other devices such as an OLP or Weld Process Editor. However, any intelligent device that requires access to welding data could access it through this strategy, as long as it adheres to the rules stated in the Welding API Specification. 


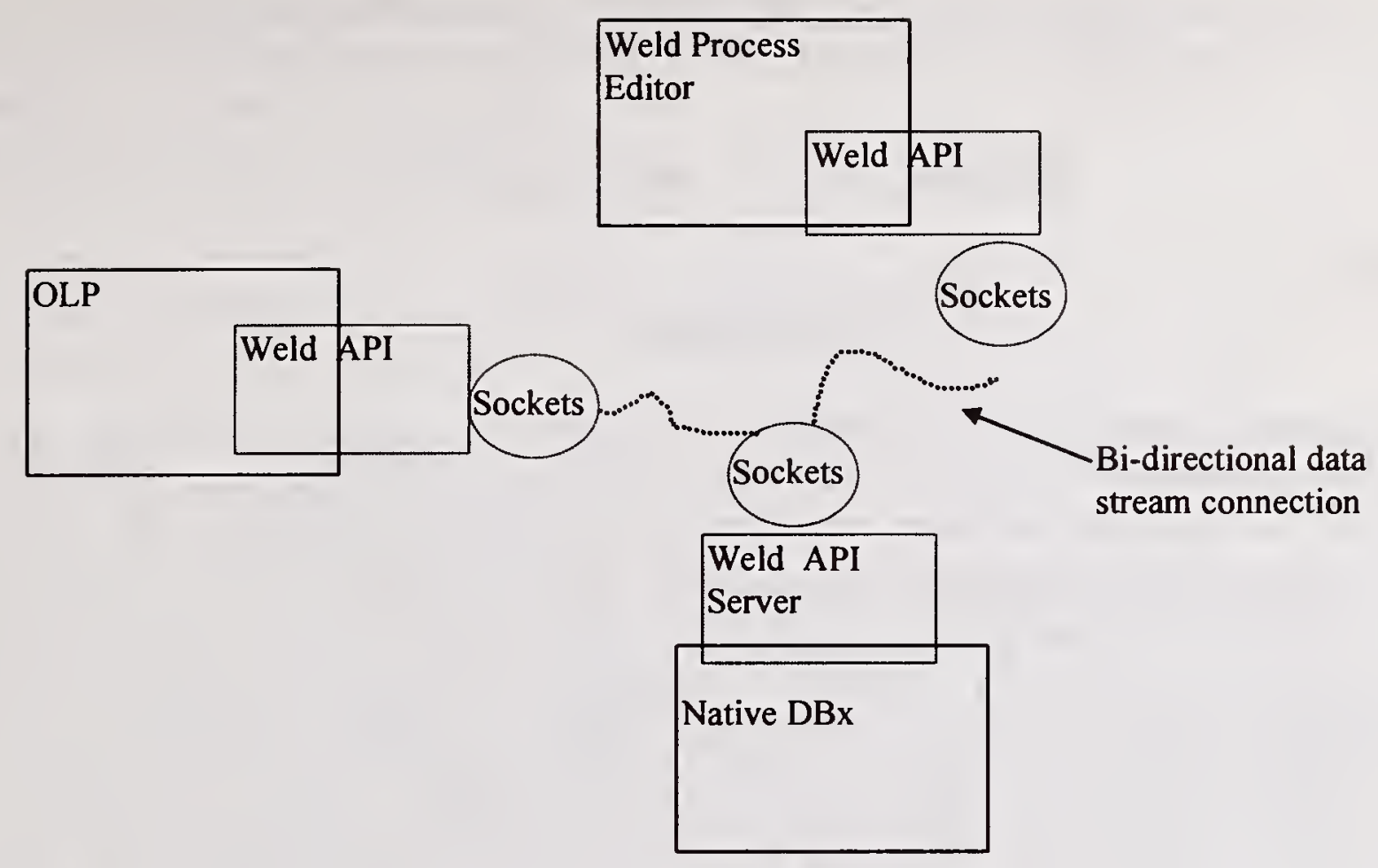

Figure 2. Weld API Utilization

The Welding API has been developed in conjunction with the Weld Neutral File Format (WNFF) Specification. The WNFF specifies database content in a generic manner. The WNFF proposes that a database consist of Procedures, Fragments, and Subroutines. Procedures are made up of Fragments that subsequently reference Subroutines. These Fragments may in-turn reference any number of Subroutines.

In a typical CAD-to-OLP interface the Weld API will be utilized to supply weld process related information to the planning process. The CAD-based design system will provide geometry information that is annotated with a list of feature nodes and a list of connectivity for these nodes. These feature nodes and the list of connectivity will denote where welding is to be performed. The OLP will utilize the Weld API to search the entire collection of possible Procedures for those appropriate to performing each weld. The list of connectivity identifies the order in which the nodes are to be connected, thus identifying the weld segments to be planned. The Weld API will allow the OLP to access the Procedures stored in external data repositories and assign the appropriate weld process related information. 


\title{
ESTIMATING OPTIMAL WELDING PARAMETERS USING ARTIFICIAL NEURAL NETWORK TECHNOLOGY
}

\author{
B. Chan", J. Pacey ${ }^{* *}$ and M. Bibby ${ }^{* * *}$
}

\begin{abstract}
An inverse backpropagation network (BPN) is presented to estimate optimal gas metal arc (GMA) welding parameters for bead-on-plate welds. In particular, the system is used to determine the arc current, voltage and travel speed to achieve a pre-determined weld bead size and shape. For the purposes of this investigation, the weld bead size and shape are defined by bead width, bead height, penetration depth and bay length at $22.5^{\circ}$, all measured from the plate surface. In addition, workpiece thickness is a variable that is taken into consideration in this investigation. Deposit and plate fusion areas may be selected as input parameters if so desired. The electrode diameter is assumed fixed at $0.9 \mathrm{~mm}$, the extension at $19 \mathrm{~mm}$, the DCEP polarity and C-25 shielding, all consistent with the experimental data base from which this model is derived. The data base consists of some ninety-six welds which were used to train the network and test the accuracy of the output.
\end{abstract}

\section{INTRODUCTION}

Weld geometry directly affects the complexity of a welding procedure and thereby the manufacturing cost of fabricating steel structures. To a large extent, the size and shape of a weld bead can be controlled by the welding parameters such as arc current, arc voltage, travel speed, electrode extension, electrode diameter, electrode polarity and workpiece thickness. Estimating optimal welding parameters for a given weld geometry is difficult because of the number of competing variables involved. Furthermore, the problem is non-trivial because there may be more than one set of welding parameters that corresponds to a given weld shape. In fact, relatively extensive field trials are often necessary to fix welding specifications in most cases. The ability to estimate welding parameters given the weld geometry would reduce the number of such trial runs for determining weld schedules in practice.

Various authors [Ref. 1,2,3] have generated algorithms for determining weld size and shape given the welding parameters. Most of these are based on regression analyses of large experimental data bases. Recently, artificial neural network (ANN) technology and more specifically, the backpropagation network (BPN) method has been applied to the problem [Ref. 4,5]. For example, it has been shown that the weld geometry of gas metal arc (GMA) welds can be estimated from the operational parameters (arc current, voltage, travel speed, electrode diameter, polarity and extension). The geometry parameters estimated are bead height, width, penetration

\footnotetext{
"MIL Systems Engineering, 1150 Morrison Drive, Ottawa, Ont., Canada K2H 8S9

"* Mech. \& Weld. Tech. Dept., Northern College, Kirkland Lake, Ont., Canada P2N 3L8

${ }^{* * *}$ Faculty of Engineering, Carleton University, Ottawa, Ont., Canada K1S 5B6
} 
and "bay length $\left(22.5^{\circ}\right.$ from the plate surface); see Figure 1. A series of elliptical and parabolic functions are then fitted to these dimensions to generate the weld shape [Ref. 5,6]. Good size and shape correlation with experiment is shown in these works to provide a high confidence level for applying this technology to the welding process.

While the accuracy of generating weld shapes with the regression technique is comparable to that of the BPN method in this regard, there is still good reason to use ANN technology [Ref. 5,6]. In practice, both

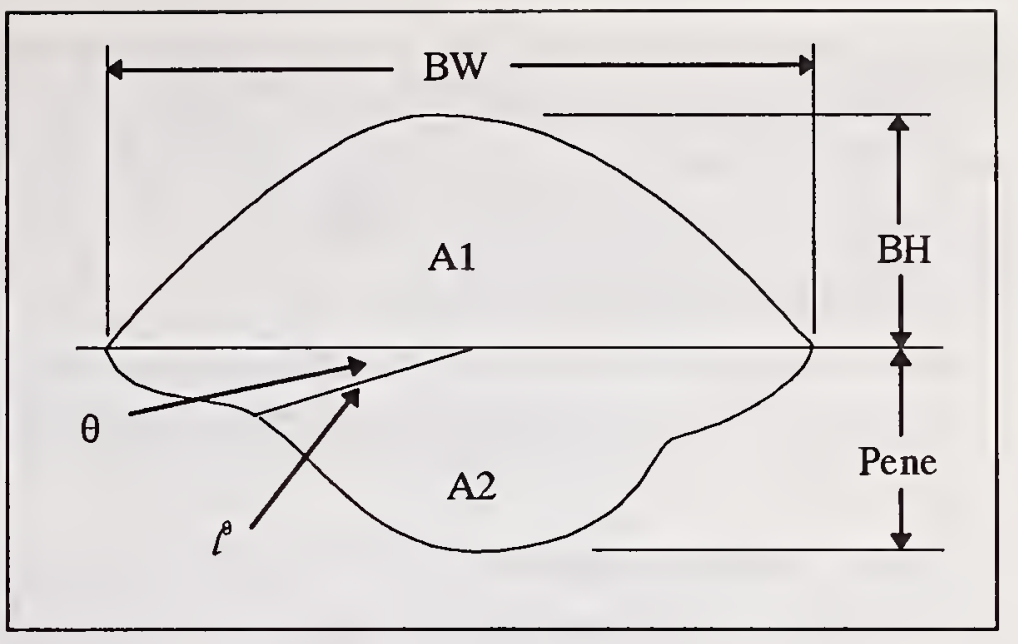

Figure 1 Experimental weld bead shape trace with weld dimensions definitions. methods are limited to the range of the experimental experience of the data base upon which they are based. However, the BPN method is more easily extended beyond the limits of the original data base as new data are accumulated. In principle, this simply involves new network learning, whereas the analysis must be completely redone if regression methods are used. Moreover, parametric relationships (at least the trends) must be determined before analysis in the regression situation whereas, this is less important (although helpful) with BPN technology. Therefore, there would seem to be good reason to adopt a BPN approach for this problem in any event.

In this publication, the reverse situation is presented, i.e., BPN technology is applied to the welding process to predict operational parameters, given a weld shape. As mentioned above, this is otherwise a difficult problem and yet it is important in practice. Welding engineers need to have some idea (an estimate) of operational welding parameters to achieve a given weld size and shape before commencing a "job", i.e., at the planning stage. The BPN method applied to this problem offers promise.

\section{EXPERIMENTAL}

Ninety-six bead-on-plate GMA welds with C-25 (25\% carbon dioxide and 75\% argon) shielding and ER70S-6 consumable electrodes were prepared for this study [Ref. 7]. The electrode extension $(19.05 \mathrm{~mm})$, diameter $(0.89 \mathrm{~mm})$ and polarity (DCEP), and C-25 shielding gas flow rate (35 cubic feet per hour) were held constant for all runs. The workpieces measured 6" (152 mm) by $16 "(406 \mathrm{~mm})$ in all cases. The input parameters (variables) were arc current (I), voltage (V), travel speed $(\mathrm{S})$, and plate thickness $(\mathrm{ht})$. The welding parameter and plate thickness ranges are listed in Table 1.

Table 1. Range of Arc Current, Voltage, Travel Speed and Plate Thickness Used in the GMAW Bead Geometry Investigation.

\begin{tabular}{|c|c|c|c|c|c|}
\hline & $\begin{array}{c}\text { Current } \\
\text { (amperes) }\end{array}$ & $\begin{array}{c}\text { Voltage } \\
(\text { volts })\end{array}$ & $\begin{array}{c}\text { Speed } \\
(\mathrm{mm} / \mathrm{s})\end{array}$ & $\begin{array}{c}\text { Thickness } \\
(\mathrm{mm})\end{array}$ & $\begin{array}{c}\text { Heat Input } \\
(\mathrm{kJ})\end{array}$ \\
\hline Minimum & 160 & 20 & 3.4 & 6.4 & 0.53 \\
\hline Maximum & 320 & 42 & 11 & 15.9 & 1.22 \\
\hline
\end{tabular}


Metallographic cross sections were used to determine weld bead shapes and sizes. Several critical weld dimensions were taken from weld shape traces as shown in Figure 1: bead width (BW), bead height $(\mathrm{BH})$, penetration (Pene), bay angle from the surface $(\theta)$ and bay length $(L)$. The size of the weld was represented in terms of deposit (A1) and fusion (A2) areas and these parameters were also measured and documented.

\section{BACKPROPAGATION NETWORK ALGORITHM}

A basic backpropagation network [Ref. 8,9] consists of a collection of nodes organized in layers (input, hidden and output,) as shown in Figure 2. Nodes in different layers are interconnected by weighted links, $\mathrm{W}_{\mathrm{hi}}$ or $\mathrm{W}_{\mathrm{ij}}$. All nodes in the hidden and output layers have two functions. They gather weighted incoming signals (Equation 1) and relay the summation to nodes in the next layer based on a transfer function (Equation 2):

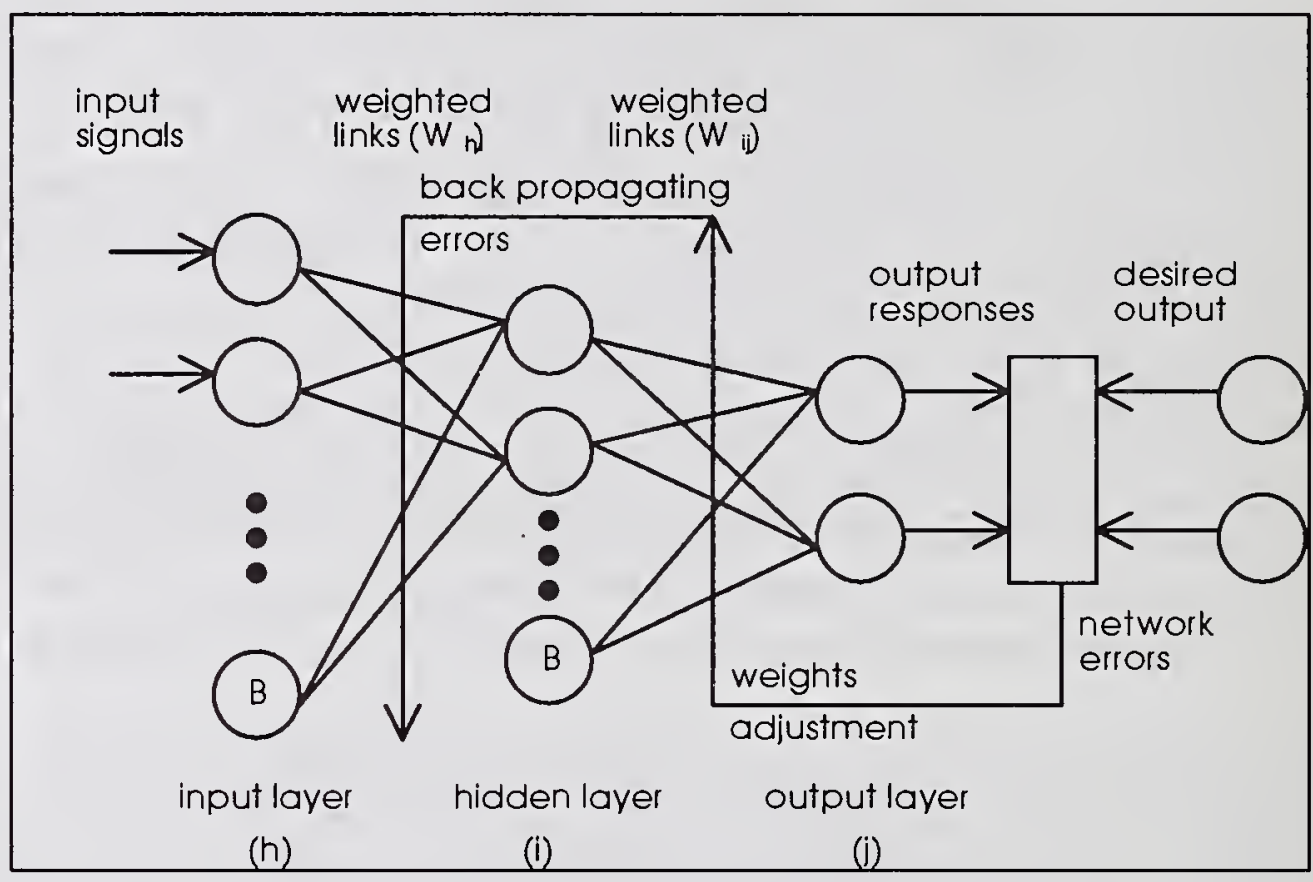

Figure 2 A backpropagation network.

$$
\begin{aligned}
& \text { Sum }=\sum_{\text {for all } i} W_{i j} \cdot x_{i} \\
& F(\text { Sum })=\frac{1}{1+e^{- \text {Sum }}}
\end{aligned}
$$

where $\mathrm{x}_{\mathrm{i}}$ is the incoming signal and $\mathrm{F}(\mathrm{Sum})$ is the transfer function. The function of the nodes in the input layer is to distribute the input signals to the hidden layer. A bias node is added to each layer (expect for the output layer) to stabilize the network [Ref. 9]. It always has an output value of one and is not connected to the previous layer; see Figure 2.

Network prediction can be achieved by moving the input data through the network, i.e., input data are submitted to the network through the input layer and processed by nodes in the hidden and the output layers. Because the values of output nodes are limited to a range between zero and one $\left(0 \leq y_{j}{ }^{n} \leq 1\right)$ by the transfer function, all network input and output data are normalized accordingly. In this study, all network input and output data were normalized between 0.1 and 0.9 since the output equals zero or one when $\mathrm{F}(\mathrm{Sum})$ approaches $\pm \infty$. 
Network learning is achieved by exposing data from experimental measurements to the system. For BPN, the initial weights of the network are set randomly between -1 and +1 . During BPN learning, the weights are adjusted according to errors, $E_{j}$, computed by comparing the results of the network prediction (as described previously), $y_{j}{ }^{n}$, and the desired results, $y_{j}^{d}$ (provided in advance from the experimental data base); see Figure 2. The network prediction and error backpropagation activities repeat until the root mean squared error (RMS error) of the network is less than a pre-defined tolerance level and the learning is said to be successful. The RMS error is calculated as follow:

$$
\text { RMS error }=\sqrt{\frac{\sum_{a=1}^{p} \sum_{b=1}^{\tau}\left(y_{b}^{d a}-y_{b}^{n a}\right)^{2}}{p \cdot J}}
$$

where $p$ is the number of patterns in the training set and $\tau$ is the number of output parameters. In this study, a tolerance level of 0.05 was used. A tighter tolerance may ensure a more accurate system but the learning time (and thereby the computer demands) increase dramatically.

The weight adjustments are based on the least mean square method, otherwise known as the generalized delta rule [Ref. 8,9] as follows:

$$
E_{j}=\frac{1}{2}\left(y_{j}^{d}-y_{j}^{n}\right)^{2}
$$

The weight of the connectors between the hidden and output layers are adjusted by:

$$
\begin{gathered}
\mathrm{W}_{\mathrm{ij}}^{\mathrm{t}}=\mathrm{W}_{\mathrm{ij}}^{\mathrm{t}-1}+\Delta \mathrm{W}_{\mathrm{ij}}^{\mathrm{t}}+\alpha \Delta \mathrm{W}_{\mathrm{ij}}^{\mathrm{t}-1} \\
\Delta \mathrm{W}_{\mathrm{ij}}=\mathrm{nI}_{\mathrm{i}} \delta_{j}^{J} \\
\delta_{j}^{J}=\mathrm{F}\left(\operatorname{Sum}_{\mathrm{j}}\right)\left[1-\mathrm{F}\left(\operatorname{Sum}_{\mathrm{j}}\right)\right]\left[\mathrm{y}_{\mathrm{j}}^{\mathrm{d}}-\mathrm{y}_{\mathrm{j}}^{\mathrm{n}}\right]
\end{gathered}
$$

where $\mathrm{n}$ is the learning rate, $\alpha$ is the momentum coefficient and $\Delta \mathrm{W}^{(t-1)}{ }_{\mathrm{ij}}$ signifies the weight adjustment of the previous pass. The learning time is controlled by the learning rate. Values between zero and one are recommended for the learning rate. The network may learn faster with a large learning rate, e.g., 0.9. However, a large rate tends to destabilize the learning process. The momentum term, $\alpha \Delta \mathrm{W}^{\mathrm{t}-1}$, reduces the learning time by recalling a fraction of the last adjustment. A typical value of 0.25 is used for $\alpha$ in this study.

The weights of the connectors between the input and hidden layers are adjusted by backpropagating the network errors, $\mathrm{E}_{\mathrm{j}}$ :

$$
\begin{gathered}
\mathrm{W}_{\mathrm{hi}}^{\mathrm{t}}=\mathrm{W}_{\mathrm{hi}}^{\mathrm{t}-1}+\Delta \mathrm{W}_{\mathrm{hi}}^{\mathrm{t}}+\alpha \Delta \mathrm{W}_{\mathrm{hi}}^{\mathrm{t}-1} \\
\Delta \mathrm{W}_{\mathrm{hi}}=\mathrm{nH}_{\mathrm{h}} \delta_{\mathrm{i}}
\end{gathered}
$$




$$
\delta_{i}^{I}=\mathrm{F}\left(\operatorname{Sum}_{\mathrm{i}}\right)\left[1-\mathrm{F}\left(\operatorname{Sum}_{\mathrm{i}}\right)\right] \sum_{\mathrm{a}=1}^{\mathrm{T}}\left(\delta_{a}^{J} \mathrm{~W}_{\mathrm{ia}}\right)
$$

\section{Learning Enhancements}

It is possible for learning to become saturated when Sum approaches $\pm \infty$ and $\delta$ becomes zero which leads to a value of $\Delta \mathrm{W}=0$ and it stops. To avoid saturated learning, Equations 7 and 10 are modified as follows:

$$
\begin{gathered}
\delta_{j}^{J}=\left\{0.1+\mathrm{F}\left(\operatorname{Sum}_{\mathrm{j}}\right)\left[1-\mathrm{F}\left(\operatorname{Sum}_{\mathrm{j}}\right)\right]\right\}\left[\mathrm{y}_{\mathrm{j}}^{\mathrm{d}}-\mathrm{y}_{\mathrm{j}}^{\mathrm{n}}\right] \\
\delta_{i}^{I}=\left\{0.1+\mathrm{F}\left(\operatorname{Sum}_{\mathrm{i}}\right)\left[1-\mathrm{F}\left(\operatorname{Sum}_{\mathrm{i}}\right)\right]\right\} \sum_{\mathrm{a}=1}^{\tau}\left(\delta_{a}^{J} \mathrm{~W}_{\mathrm{ia}}\right)
\end{gathered}
$$

as suggested by Fahlman et al [Ref. 10]. The value 0.1 is arbitrary but it is a pragmatic assignment to ensure continuous network learning.

Furthermore the learning rate can be adjusted according to the RMS error. A so called stepdeclining learning rate [Ref. 6] is used in this study which is a modification of the dynamic learning originally proposed by Jacobs [Ref. 11]. In step-declining learning, the rate is reduced by $1 \%$ whenever the RMS error increases. In addition, it is limited to a value of 0.005 to avoid a zero learning rate. By employing the step-declining method, a large initial learning rate, e.g., 0.5, can be applied and the stability of the network is not jeopardized.

\section{NETWORK EXPERIMENTATION}

Different practitioners may want to define one or another weld bead differently. For example, instead of defining a weld bead by its width, height, penetration and bay length, deposit or fusion areas may be of interest. In all cases, plate thickness is submitted to the network as one of the input parameters to correspond with that available in a given situation, rather than view it as an output.

It is conventional to define a weld bead by its width, height and penetration. However, with extra lower-bead bay information $\left({ }^{22.5}\right)$, the weld bead shape description is more complete and better results would normally be expected [Ref. 5,6$]$. Therefore, these shape parameters together with plate thickness are the input parameters for network A.

Sometimes in practice, the filler metal (deposited material) is eliminated (ground off) to provide a smooth surface. Therefore, the bead height and deposit area become less important. In this case, the weld bead characteristics - bead width, penetration and plate fusion area together with plate thickness can be used for defining the input space to the network. This is the configuration of network B which is selected when there is no interest in the geometry developed above the plate surface. 
In some cases, deposit area together with bead width, penetration, plate fusion area and plate thickness are convenient in practice to describe a desired weld bead (network $\mathrm{C}$ ). The input space to this network is similar to the one mentioned in the previous paragraph with the addition of the deposit area constraint.

Finally, where weld areas are the principal concern (perhaps a filler situation), the parameters bead width, deposit area and plate fusion area can be used to define the weld bead. This is believed to be one of the least complex networks since there are no constraints regarding the height or penetration. In terms of this investigation, this is network D. A summary of the network input parameters for solving the inverse weld geometry problem is listed in Table 2.

Table 2. Summary of Network Input Parameters for Solving the Inverse Weld Geometry Problem.

\begin{tabular}{|c||c|c|c|c|c|c|c|}
\hline Network & BW & BH & Pene & $2^{2.5}$ & A1 & A2 & $\mathrm{h}_{\mathrm{t}}$ \\
\hline \hline $\mathrm{A}$ & $\checkmark$ & $\checkmark$ & $\checkmark$ & $\checkmark$ & & & $\checkmark$ \\
\hline $\mathrm{B}$ & $\checkmark$ & & $\checkmark$ & & & $\checkmark$ & $\checkmark$ \\
\hline $\mathrm{C}$ & $\checkmark$ & & $\checkmark$ & & $\checkmark$ & $\checkmark$ & $\checkmark$ \\
\hline $\mathrm{D}$ & $\checkmark$ & & & & $\checkmark$ & $\checkmark$ & $\checkmark$ \\
\hline
\end{tabular}

A schematic diagram of the basic network structure used in this part of the study is shown in Figure 3. The input layer is defined by the weld bead characteristics and plate thickness. The hidden structure is composed of one or two hidden layers with various numbers of hidden nodes. The output layer contains arc current, voltage and travel speed. Bias nodes are included in all hidden and input layers. Twenty-one out of ninety-six weld bead data sets (welding parameters weld dimensions) were used for training. The remainder ( 75 sets) were used for testing the accuracy of the trained networks. The normalization range for all data is between 0.1 and 0.9 . Learning rate, momentum coefficient and RMS error tolerance used were 0.5, 0.25 and 0.05 respectively. Fahlman's derivative and step-declining learning were used to improve the learning time. Only the results of the best networks are reported.

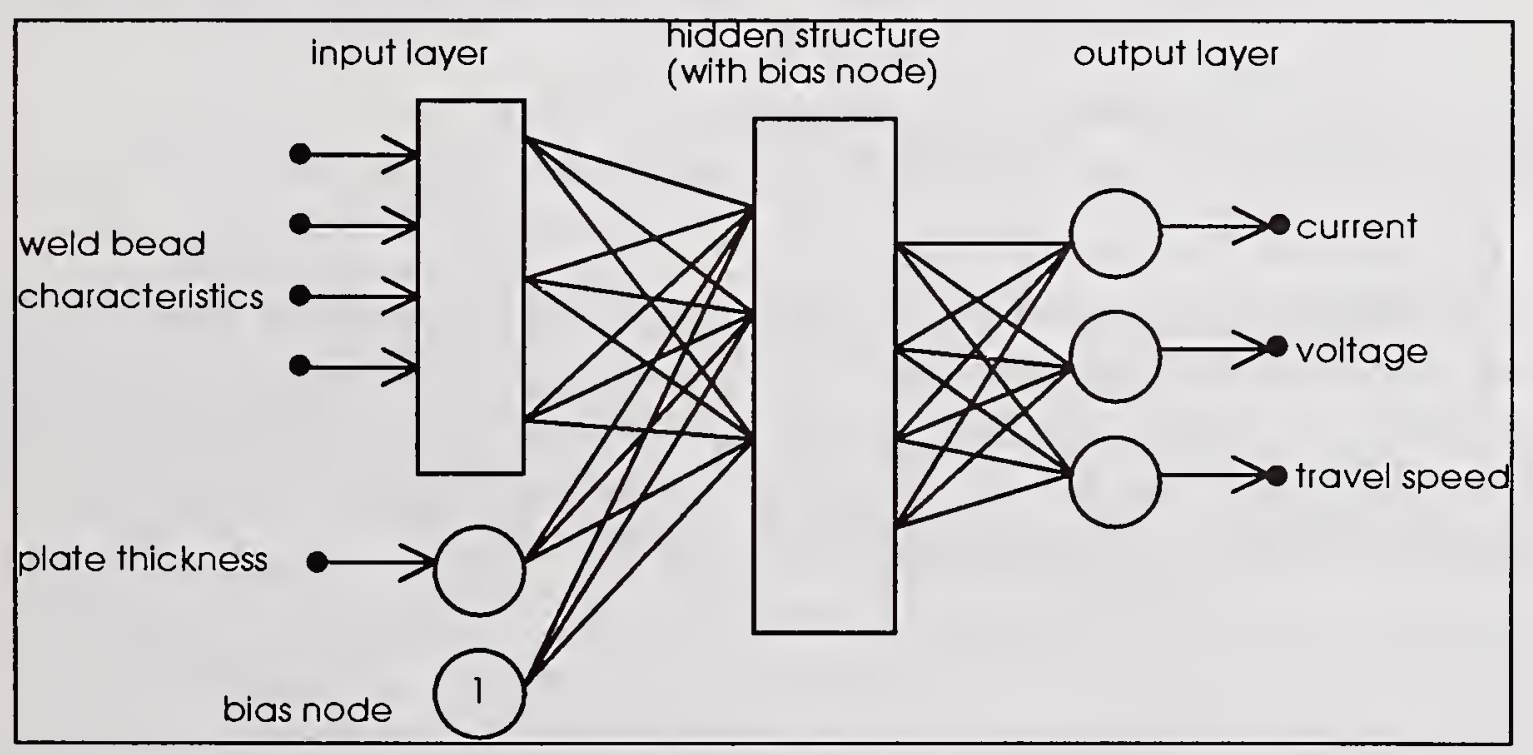

Figure 3 A schematic diagram of the BPN tested for solving the inverse weld bead problem. 


\section{RESULTS}

For networks A, B \& C, a 6\&4-hidden node 2-hidden layer network structure was believed to be the most suitable. A 6-hidden node single-hidden layer network was found to be the most appropriate for network D. All networks were able to solve the inverse weld geometry problem with reasonable accuracy; see Table 3. Network B was found to provide the most accurate arc current, voltage and travel speed estimates although no reinforcement information is presented to the network as input.

Table 3. Summary of Estimates of Welding Parameters for Various Networks.

\begin{tabular}{|c|c|c|c|c|}
\hline Network & $\begin{array}{c}\text { \# of Hidden } \\
\text { Layers (nodes) }\end{array}$ & $\begin{array}{c}\text { Arc Current } \\
\text { (amperes) }\end{array}$ & $\begin{array}{c}\text { Arc Voltage } \\
\text { (volts) }\end{array}$ & $\begin{array}{c}\text { Wire Travel } \\
\text { Speed } \\
\text { (mm/s) }\end{array}$ \\
\hline \multicolumn{5}{|c|}{ Correlation Coefficient } \\
\hline A & $2(6 / 4)$ & 0.839 & 0.862 & 0.773 \\
$B^{*}$ & $2(6 / 4)$ & 0.876 & 0.860 & 0.759 \\
C & $2(6 / 4)$ & 0.848 & 0.854 & 0.750 \\
D & $1(6)$ & 0.766 & 0.855 & 0.705 \\
\hline \multicolumn{5}{|c|}{ Mean } \\
\hline \multicolumn{6}{|c|}{ Mean of Absolute Difference } \\
\hline A & $2(6 / 4)$ & 20.3 & 2.2 & 0.7 \\
B & $2(6 / 4)$ & 18.7 & 2.0 & 0.89 \\
C & $2(6 / 4)$ & 22.5 & 2.4 & 1.09 \\
D & $1(6)$ & 24.3 & 2.3 & 1.03 \\
\hline \multicolumn{6}{|c|}{ Average Percentage Error (\%) } \\
\hline A & $2(6 / 4)$ & 9.2 & 6.8 & 12.5 \\
B & $2(6 / 4)$ & 7.7 & 6.1 & 13.4 \\
C & $2(6 / 4)$ & 10.1 & 7.1 & 16.4 \\
D & $1(6)$ & 11.0 & 7.0 & 15.5 \\
\hline
\end{tabular}

Notes: Refer to Table 2 for definitions of networks A, B, C \& D. Results of network B are plotted in Figures 4, 5 \& 6.

A summary of the results is listed in Table 3 which includes the correlation factors, the mean absolute difference and the average percentage error. The average percentage error is obtained by taking the ratio of the absolute difference and the mean value for each individual entry. The estimated and experimental values for network B are plotted in Figures $4,5 \& 6$ to provide a pictorial representation of the trends and scatter involved in the results. 


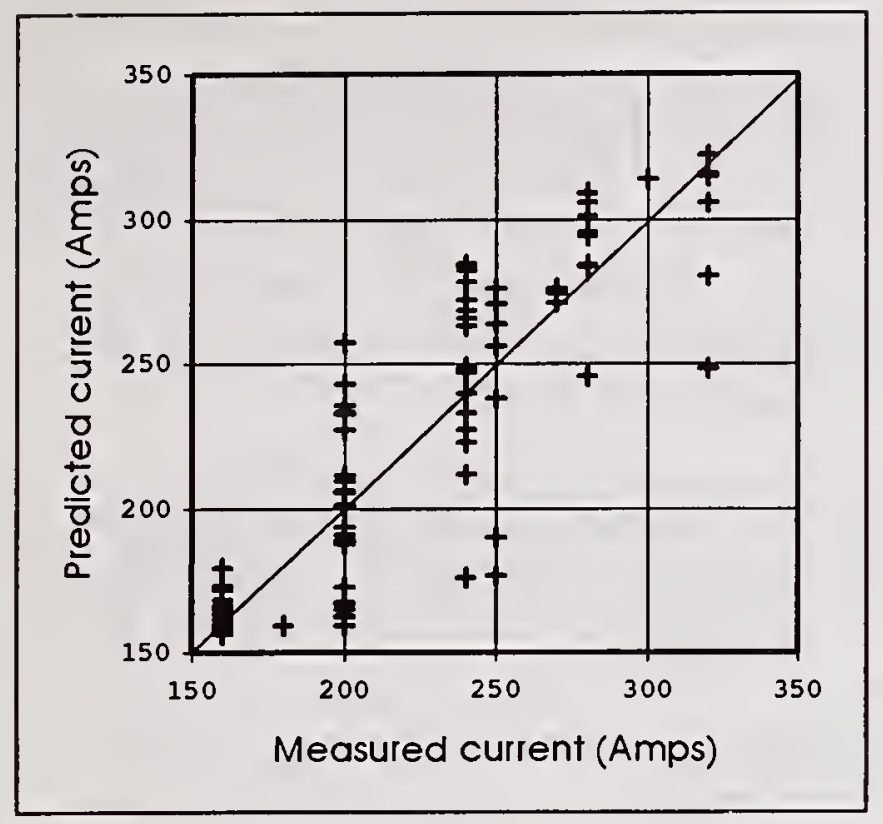

Figure 4 Comparison of measured currents with corresponding values estimated from the BPN analysis using BW, Pene, $A 2$ and $h_{t}$.

\section{DISCUSSION}

In general the results are encouraging. It seems that reasonable welding parameters can be estimated for a given weld size and shape at least for GMA welding and within the limits of this study. Nonetheless, there is no reason to believe that BPN could not be applied to situations beyond the range of this study for other welding processes and for a wide range of variables.

The least accurate results were encountered from the results of network D where the weld geometry was defined by width, deposit area, plate fusion area and plate thickness. This is not unexpected because of the rather loose definition of bead geometry. On the other hand, a more complete weld bead definition (bead width, bead height, penetration, bay length at $22.5^{\circ}$ and plate thickness) was used for network $\mathrm{A}$ and the results are more accurate since the definition of weld shape in this case is more descriptive. The use of bead width, penetration, deposit area, plate fusion area and plate thickness (network C) to estimate the welding parameters generates comparable results because deposit area is somewhat coupled to bead height and plate fusion area is closely related to penetration and bay length at $22.5^{\circ}$.

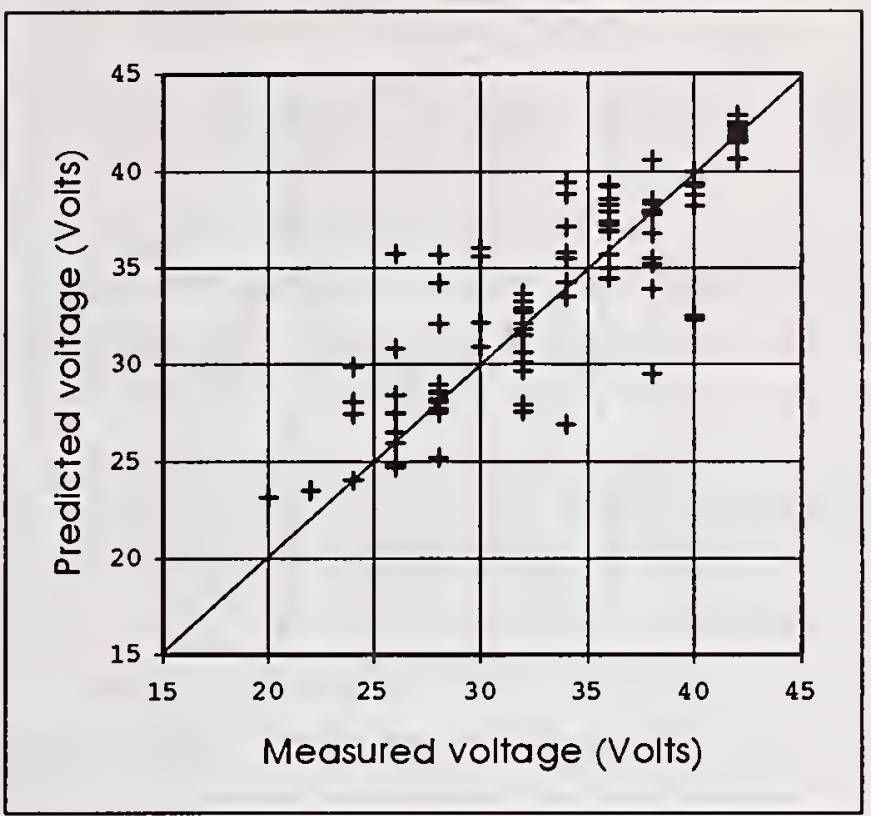

Figure 5 Comparison of measured voltages with corresponding values estimated from the BPN analysis using BW, Pene, A2 and $h_{t}$.

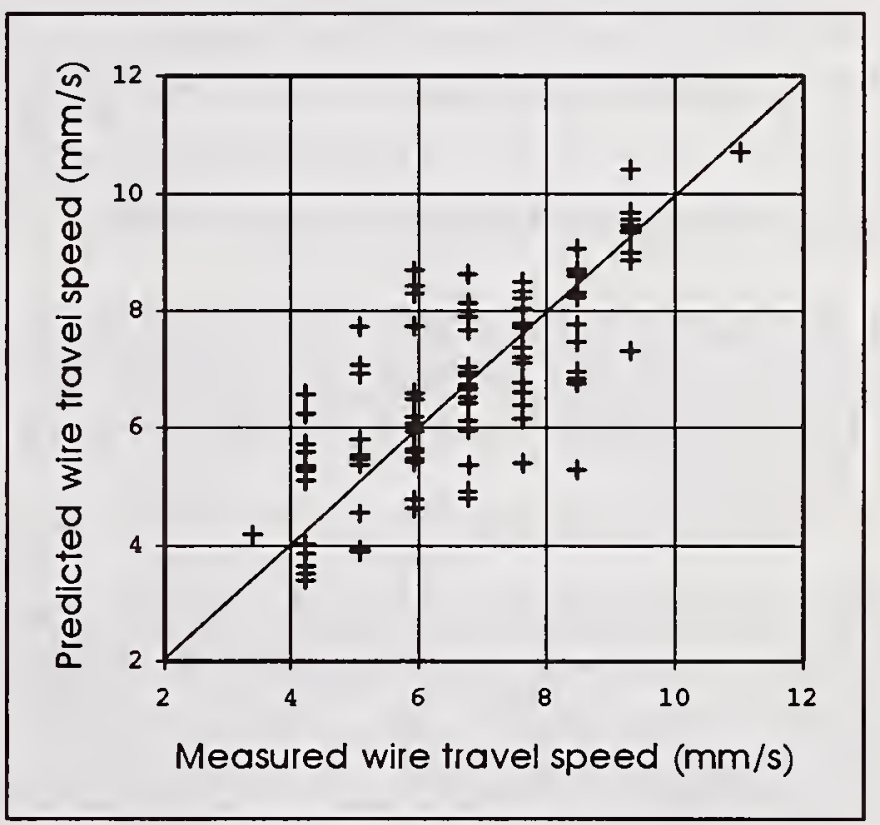

Figure 6 Comparison of measured wire travel speeds with corresponding values estimated from the BPN analysis using BW, Pene, A2 and $h_{t}$. 
The most accurate welding parameter estimates are provided by using only bead width, penetration and plate fusion area (network B) where the upper-bead is not included. Eliminating the upper-bead for a flat plate situation (ground bead) gives the network more flexibility in selecting the most appropriate welding conditions. Furthermore, experimental information for the upper-bead is somewhat uncertain. By neglecting the upper-bead information, some of the uncertainties of this feature can be avoided and hence better results are achieved.

Because of the complexity of the input-output relationships of the inverse weld bead geometry problem, correlation coefficients are lower than would normally be accepted. Nonetheless, network estimates such as these can no doubt reduce the number of trial runs required for specifying a weld procedure in practice. More accurate heat affected zone hardness and weld cooling time predictions are reported by these authors [Ref. 12,13$]$ which strongly suggested that $\mathrm{BPN}$ can also be used as an alternative method to conventional regression analysis for the welding process in a general sense.

The use of BPN to estimate gas tungsten arc (GTA) welding conditions (arc current, voltage and wire travel speed) given the weld bead characteristics (bead height, bead width and penetration) is reported in another investigation [Ref. 4]. The GTA work was limited to a single plate thickness (0.125 inches) and narrowly defined welding conditions. Furthermore, the use of bead width, height and penetration in general is not descriptive enough to be useful. Bay shape is important and appears to have been neglected in the GTA study, although it may not be a factor for the relatively smaller weld beads produced with that process.

It is important that there are now two independent investigations to confirm the potential of BPN for the inverse situation regarding the welding process [Ref. 6,11]. It is not obvious beforehand that the inverse situation would be useful, given the complexity of estimating welding parameters from bead geometry.

Designing a backpropagation network is more an art than science because there are no firm rules for defining the structure or the appropriate number of training patterns. An optimal network is always achieved by trial and error. Backpropagation networks such as those used in this study can be viewed as systems for mapping the input to the output. The complexity of a network structure depends on the input-output relationship to be determined. Moreover, the appropriate number of training patterns depends on the complexity of the relationship. As a minimum, the training pattern set should cover the entire problem domain. As a rough rule of thumb, the appropriate number of training sets should be about twice the number of total connections. However, this is only a guide. Having said all of this, it is difficult to provide guidance for those who might follow this work. The network experimentation procedure documented in this presentation would provide some guide but practitioners should not be surprised to find significant departures from this experience as new data or extended applications (e.g., other welding processes) are considered.

The BPN technique is useful for solving non-trivial and highly coupled problems, such as the inverse weld geometry problem. It is almost impossible to find a physical solution to this problem 
any other way. The principal disadvantage of the BPN technique is that reasoning about the solution is not available because knowledge is stored as weights and distributed in the entire network. Therefore, the physical basis for the solution is not apparent and this is a major limitation.

\section{CONCLUSION}

Given the weld bead geometry dimensions and plate thickness, gas metal arc welding parameters (arc current, voltage and travel speed) with C-25 shielding can be estimated with reasonable accuracy by using backpropagation network technology.

\section{ACKNOWLEDGEMENT}

The authors would like to acknowledge the support of the National Science and Engineering Research Council, grant NSERC A4601. Valuable comments concerning the backpropagation network technique by Dr. H.M. Hafez, Department of Systems and Computer Engineering, Carleton University are greatly appreciated.

\section{REFERENCE}

1. Chandel R. S. 1988. Mathematical modelling of GMAW features. Modelling and Control of Casting and Welding Process IV: 109 to 120.

2. McGlone J. C.; Chadwick B. D. 1978. The submerged arc butt welding of mild steel, Part I: The influence of procedure parameters on weld bead geometry. The Welding Institute Report No. 80/1978/PR: 26 pgs.

3. Raveendra J.; Parmar R. S. 1978. Mathematical models to predict weld bead geometry of flux cored arc. Welding Metal Construction Vol. 19, No. 1: pp 31R.

4. Madden S.; Rock A.; Xu X.; Jones J. 1992. Neural networks for sensor data processing in welding application. American Welding Institute, Internal Report: 24 pgs.

5. Andersen K.: Cook G.E.; Ramaswamy K.; Karsai G. 1989. Artificial neural networks applied to arc welding process modeling and control. Conference Record - 24th IAS Annual Meeting (IEEE Industry Applications Society), ISSN: 0160-8592: 2327 to 2331.

6. Chan B. 1996. Predicting weld features using artificial neural network technology. Doctorate Thesis, Ottawa, Canada : Carleton University.

7. Beaudoin M. 1993. Expanding the Carleton University Weldsoft package. Graduate Technical Report, Kirkland Lake, Ontario: Northern College.

8. Rumelhart D.E.; Hinton G.; Williams R.J. 1986. Learning internal representation by error propagation. Parallel Distributed Processing Vol. I, edited by Rumelhart and McClelland, Cambridge MA, the MIT Press: 318 to 362.

9. Eberhat R.C.; Dobbins R.W. (editors). 1990. Neural network PC tools: a practical guide. Academic Press, Inc., San Diego, CA: 40 to 43 \& 240 to 242. 
10. Hoehfeld M.; Fahlman S.E. 1992. Learning with limited numerical precision using the cascade-correlation algorithm. IEEE Trans. Neural Networks, v3, n4: 602 to 611.

11. Jacobs R. 1988. Increased rates of convergence through learning rate adaptation. Neural Networks, Vol. 1: 295 to 307.

12. Chan B.; Bibby M.; Holtz N. 1995. Predicting HAZ hardness with artificial neural networks. Canadian Metallurgical Quarterly, Vol. 34, No. 4: 353 to 356.

13. Chan B.; Bibby M.; Holtz N. 1996. Predicting 800 to $500{ }^{\circ} \mathrm{C}$ welding cooling times by using backpropagation networks. Transactions of Canadian Society of Mechanical Engineering, Vol. 20, No. 1: 75 to 86 . 


\title{
COMPUTER AIDED HARDFACING TECHNOLOGY PLANNING
}

\author{
B. Palotás*, L. Béres\#, B. Keskeny*, R. Pap*
}

\section{ABSTRACT}

Hardfacing has many economical advantages in the technical practice. However the planning of its technology demands special knowledge. There are not many specialists who have considerable experience in the field of hardfacing. Consequently a software would be useful which helps technologist on this field.

The paper offers a possible computer system for the planning of hardfacing technology. From typified basic tools and component's geometry the system gives advises for given tasks such as the welding sequence, the weld build-up, the welding consumable(s), the welding parameters and the heat treatment.

The depth of planning is adjustable, and pre-calculation of cost is also available. The computer system creates the welding procedure specification in an interactive way. The system can save and read WPS. The database of the base materials and the welding consumable are included in the technology planning system, which can be upgraded, from time to time as needed. Also, TTT diagrams and data of heat treatments can be saved in the base material's database. The welding consumable database can substitute consumable catalogues, therefor one can compare welding materials of different sources. The software will be useful in factories working with hardfacing. Significant technical and economical advantages expected in practice.

\section{INTRODUCTION}

Hardfacing is a very important technology in the practice. The hardfacing is generally used for making layers onto surfaces of elements, to change properties of surface. Using this technology is not only economical but a technical question, too. In many cases the technologist hasn't got enough experience to make a correct WPS for hardfacing. A computer aided advisory system would be well usable in these kinds of factories and workshops.

The authorizes show a computerized system for hardfacing planning, which is based on practical experiences, knowledge and experiences of computer using in the field of welding.

\section{THE TASK OF HARDFACING}

Typical hardfacing tasks shown in figure 1. (Ref. 1.). The figure shows that we can use hardfacing in many fields of practice. Of course as many elements there are in the practice as many requirements there are for surface layers.

If we are looking for requirements of surface layers, we have to take into consideration:

- The hardness ( HRC, HV, HB ),

- Wear resistance,

- Toughness,

- Corrosion resistance,

- Operation temperature $\left(\mathrm{T},{ }^{\circ} \mathrm{C}\right)$,

- Oscillation of temperature. 
When there is no numerical requirement (for example corrosion resistance), we have to make a scale of them. We can assign rcquirements from 1 to 15 , where 1 is low and 15 is high (Ref. 2.). About requirements we made Table 1., for an example where the typical chemical composition of welding materials are given, too. We have made 47 groups of materials by requirement and have selected the suitable welding materials, too. In Table 1., the welding materials are give with DIN Code (DIN 8555). The DIN code contains information about the group of materials and it's hardness. On the basis of Table 1., one can select the welding materials. If we made an analysis of the element operation then we can decide which group is needed in Table 1, from the 47.

Hardfacing requires special techniques, some examples are shown in Figure 2., by Ref. 2.

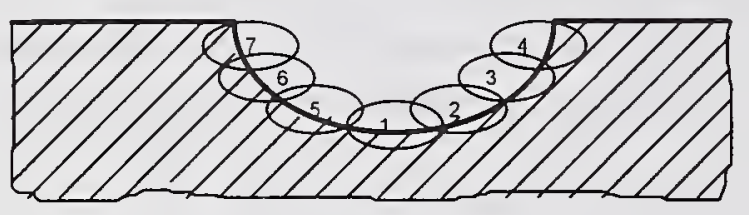

Simmetricaly up on the wall

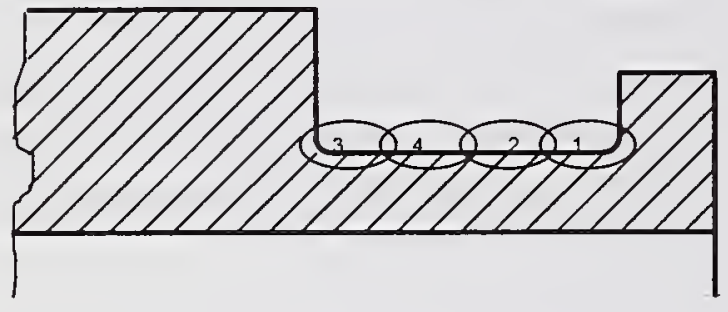

From edge to inside, simmetricaly

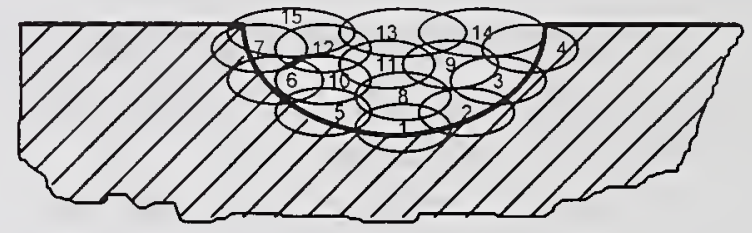

Up on the wall, from centrum to out

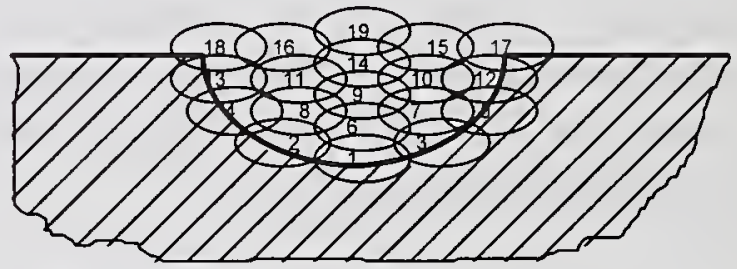

Up on the wall simmetricaly, from centrum to out

Figure 2.

The technics of surfacing

Generally we have to us preheating in hardfacing. The preheating temperature calculation elements are shown in Ref. 3., and Ref. 4. The system of preheating temperature calculation is in Ref. 5.

After this short theoretical basis, we can show that, how we can use the computer for aiding hardfacing technology planning. 
Figure 1.

Tipical hardfacing tasks

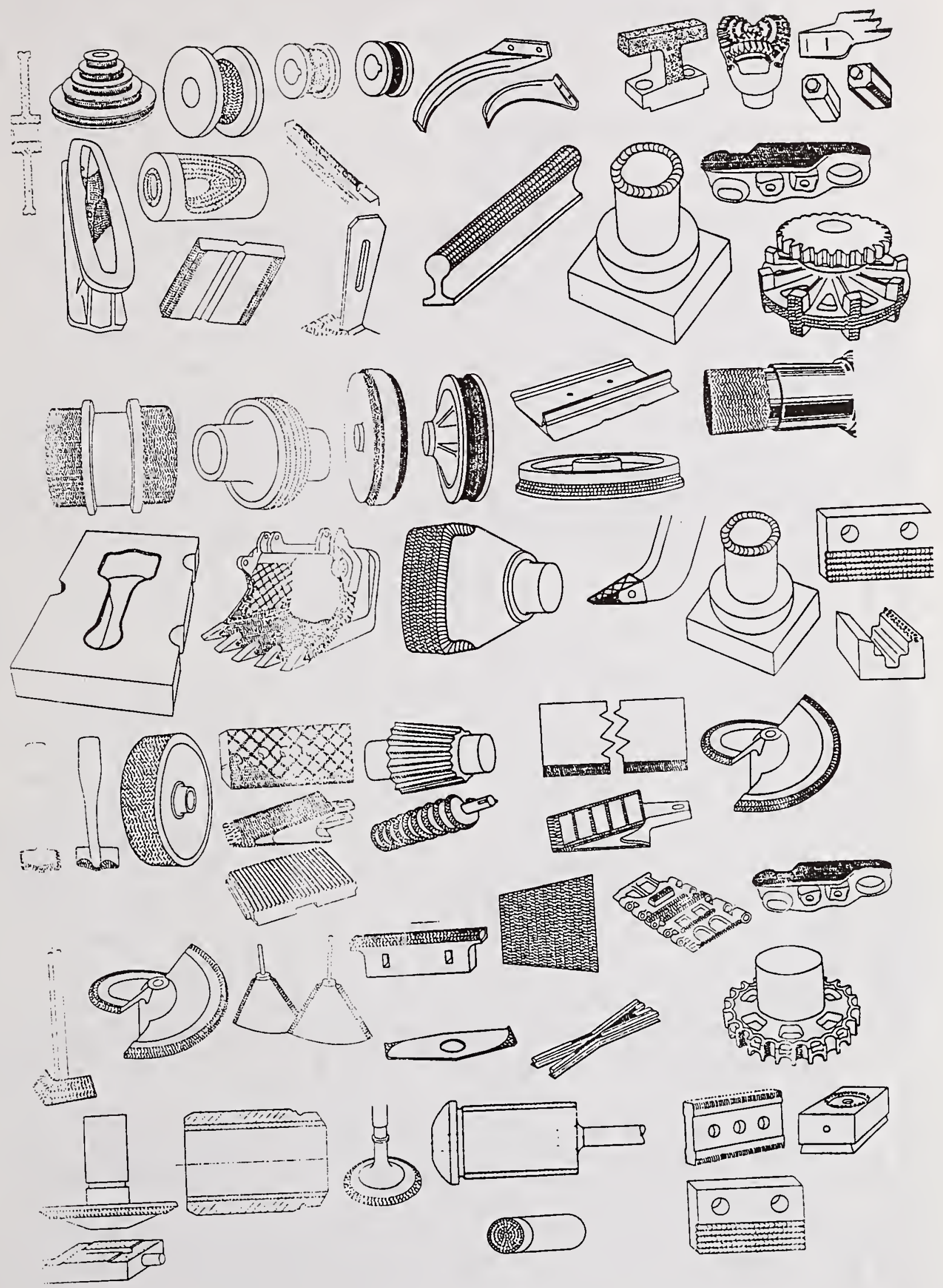




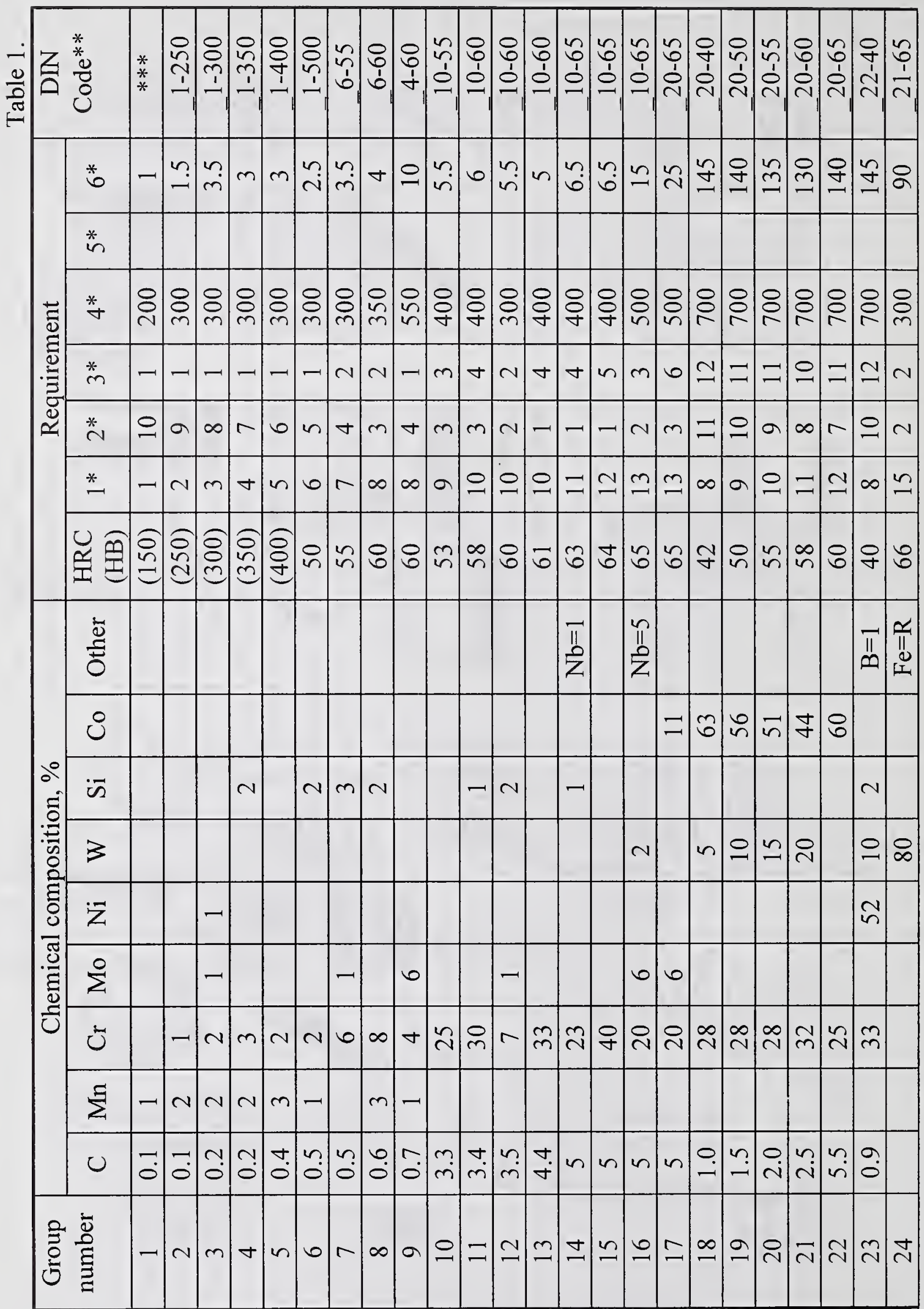




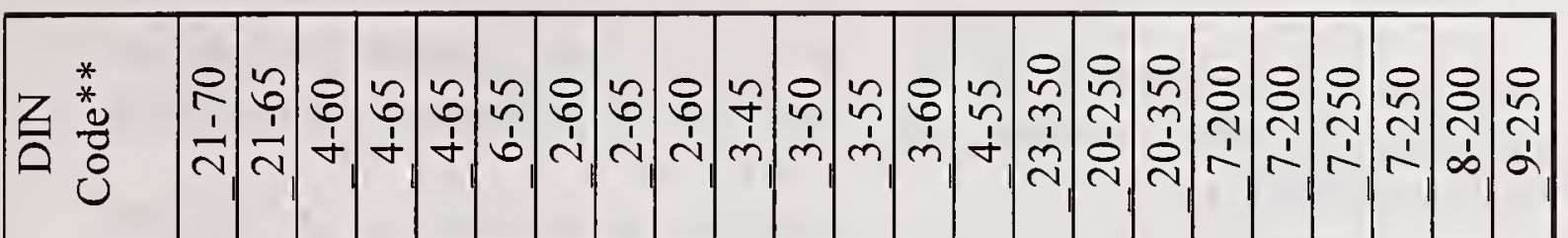

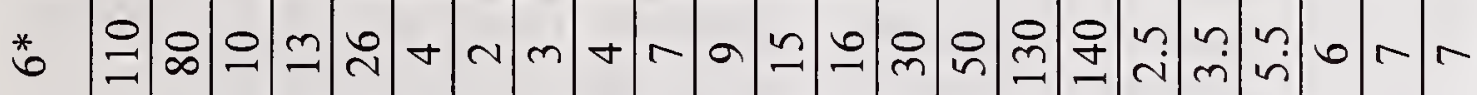

*

$\infty a$ 은 $\because n$

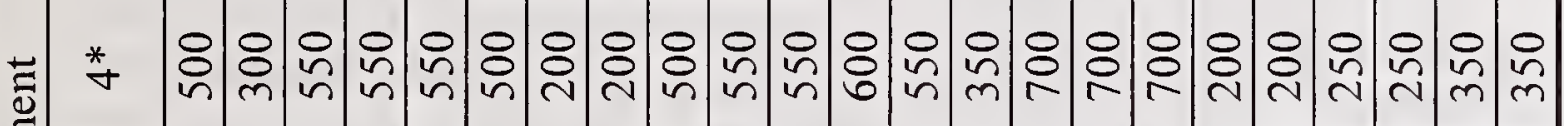

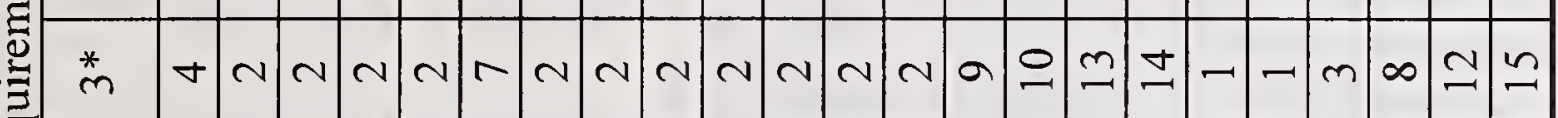

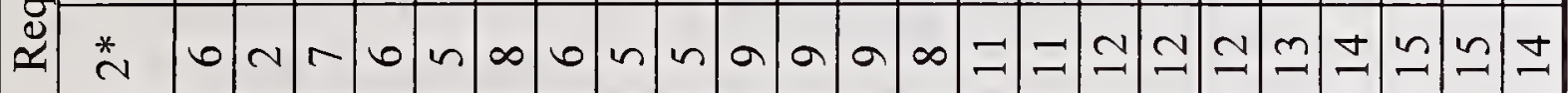

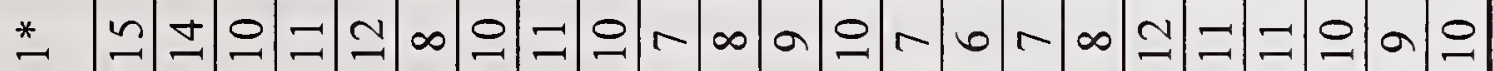

น

焉

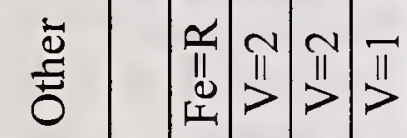

$\stackrel{\pi}{>}$

$\frac{\pi}{Z}$

ن

2

:⿱一𫝀)

is

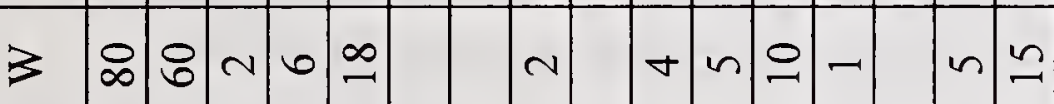

Z

드요의

$\pm \operatorname{nก}$

(1)

(

证

芯

$\Xi$

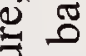

a

。

$*$

ธี

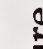

ᄋ

$\ddot{\forall}$

8

E

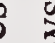

ธี

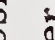

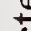

ส

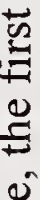




\section{SYSTEM PLAN FOR COMPUTER}

In Figure 3., a system plan of computer aided hardfacing technology planning is shown. When we have made the system plan, we took into consideration experiences of computer using in the field of welding (Ref.6.).

The system plan shows an interactive system. We can edit the data and see the data on the screen, after every change. Figure 4., shows the modulated system plan, which gives the modules a hierarchy. After data input the computer searches data of base material, if it is not in the database of course there is a possibility to load it into the database. In the geometrical routine, we can edit some examples of elements or tools (dies) and can make a new scheme of them. As above were shown the correct definition of requirements is very important. From them there is a possibility to select the welding material group. It might determine the welding procedure and we could select the procedure and material(s), right after. The preheating temperature can be calculated as Ref. 5., describes it.

The planning of technology means many steps, some of these are planning of preparation, planning of build-up of weld, calculation of welding parameters, calculation of sequence of welding and after-treatment of weld. Of course we have to make the calculation algorithms depending on the welding procedure. When we calculate the welding parameters, we can start from suggested algorithms (Ref.7.) A correction in the technology has to be made if there is sensitivity for cracking, it means that after iteration we get the correct results. After postwelding heat-treatment and quality assurance planning, the estimation of costs and output of data gives the results of computer aided planning. After planning we can find the output data on the screen and we can print the Welding Procedure Specification (WPS), Procedure Qualification Record (PQR) and Welder Qualification Record (WQR) onto paper. As well known, in the computer technology the qualities of results are depending on exacting of data input. The parts of data input are shown in Figure 5. It is possible and a good help if one can edit previous jobs changing only some of the requirements. Of course, in the definition of tasks, also helps if we can search by a name of an element, because many literatures are giving examples about usage of welding materials. This information could be also.

Of course the modules presented are just the main modules. The program plan contains several parts. 


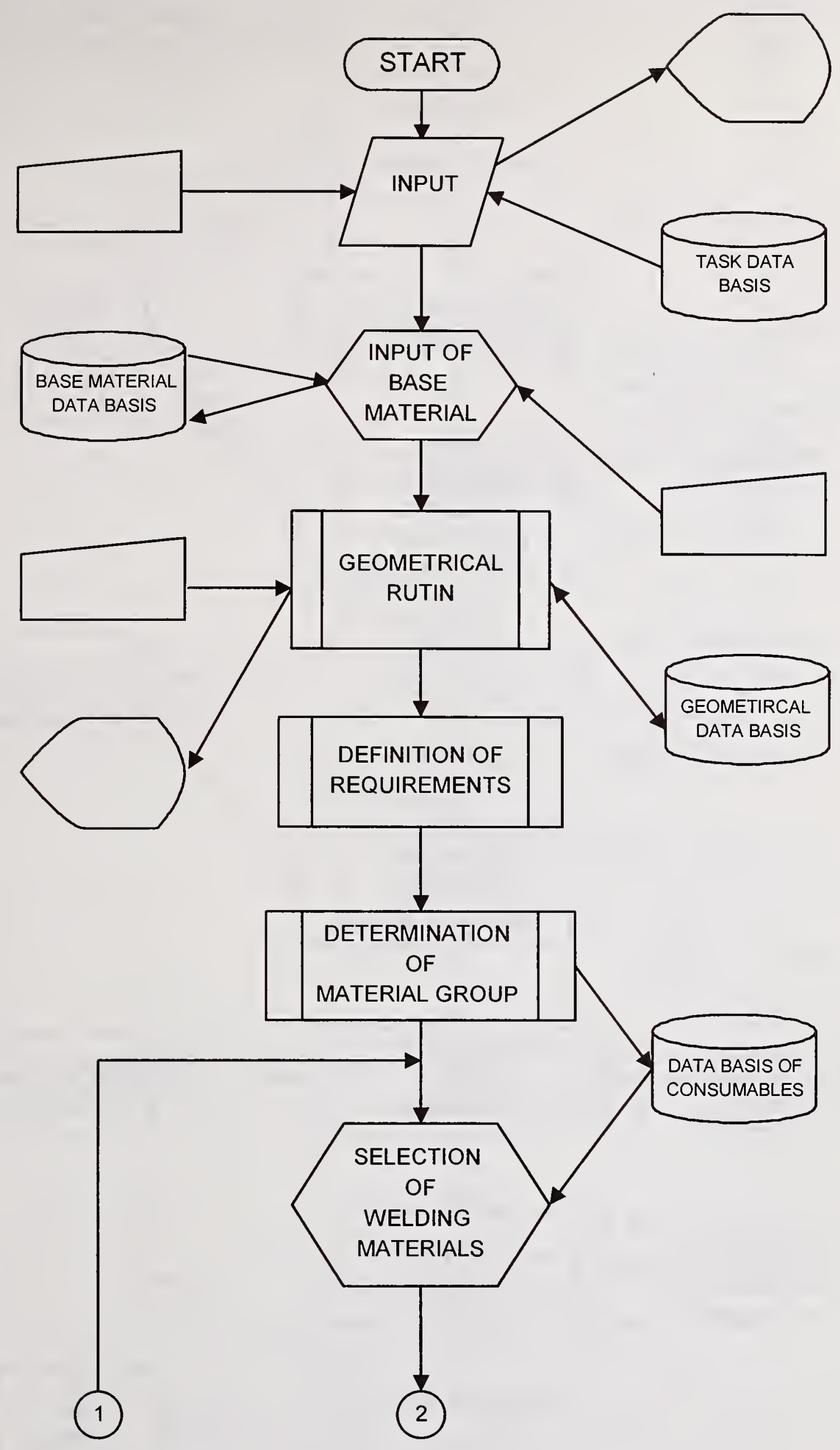

Figure 3. System plan of CAPP for hardfacing $\left(1^{\text {st }}\right.$ part $)$ 


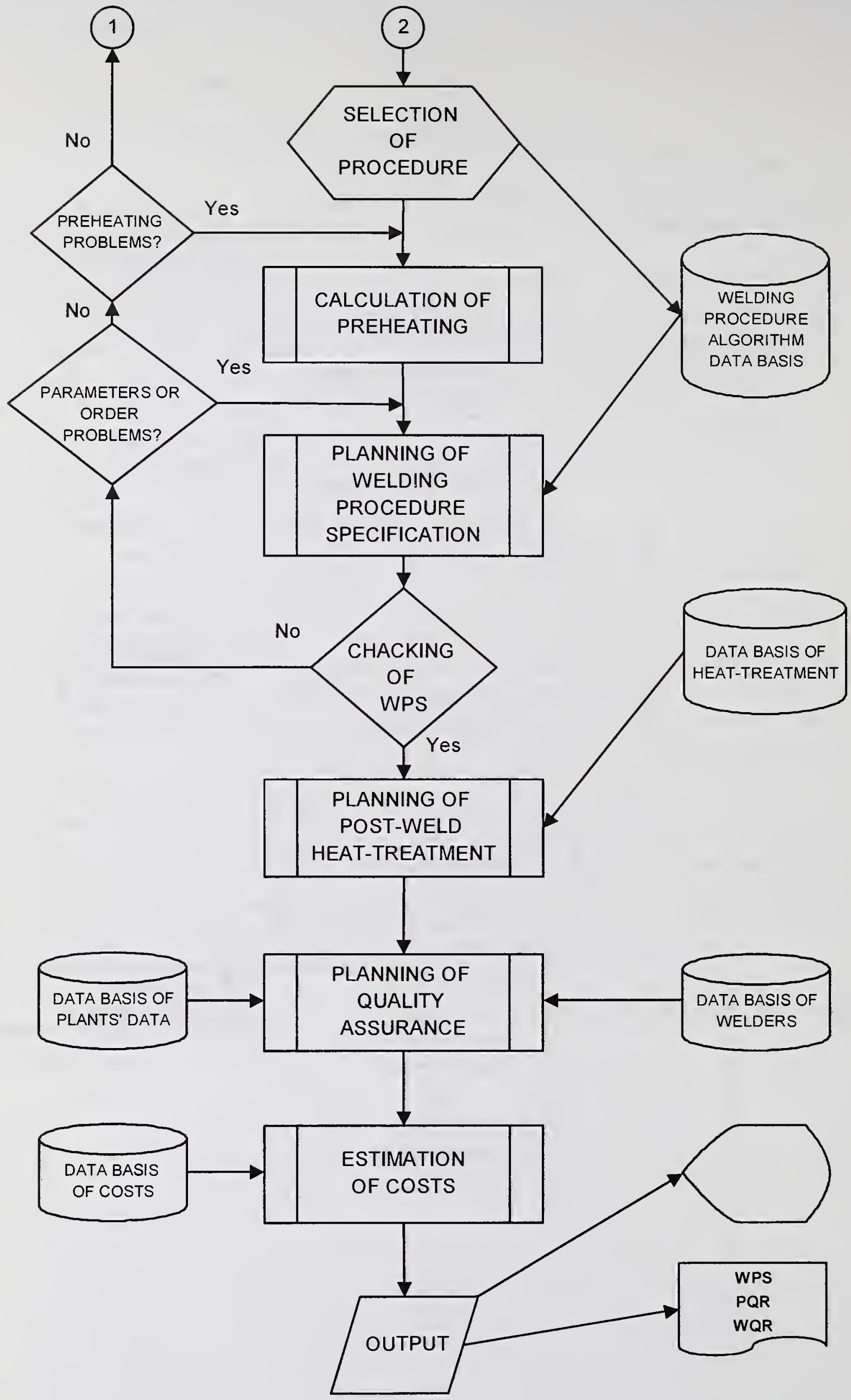

Figure 3. System plan of CAPP for hardfacing $\left(2^{\text {nd }}\right.$ part $)$ 
"HARDWELD"

Computer Aided Advisory System for

HARDFACING

INPUT DATA
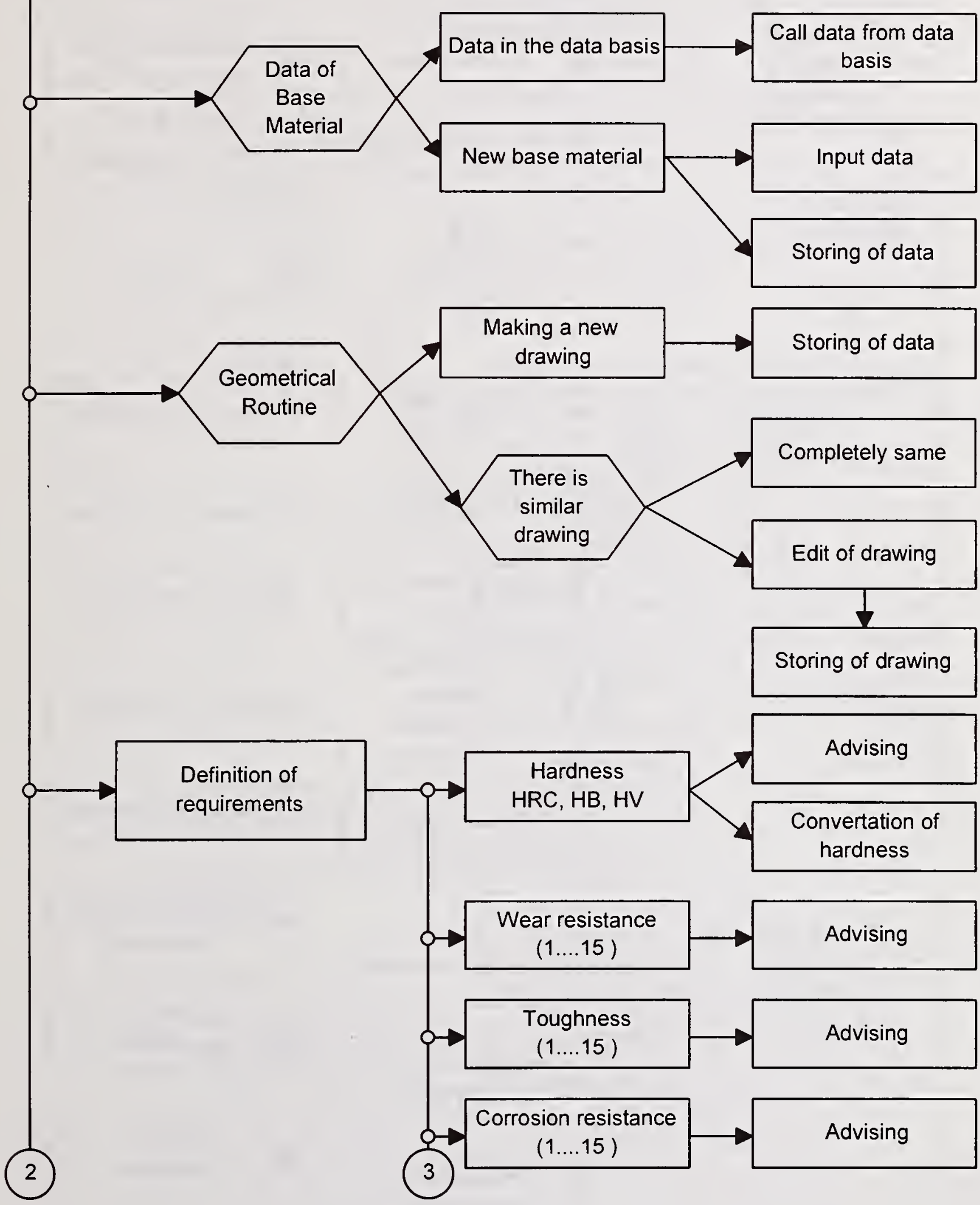

Figure 4. Hierarchy of Modules ( $1^{\text {st }}$ part) 


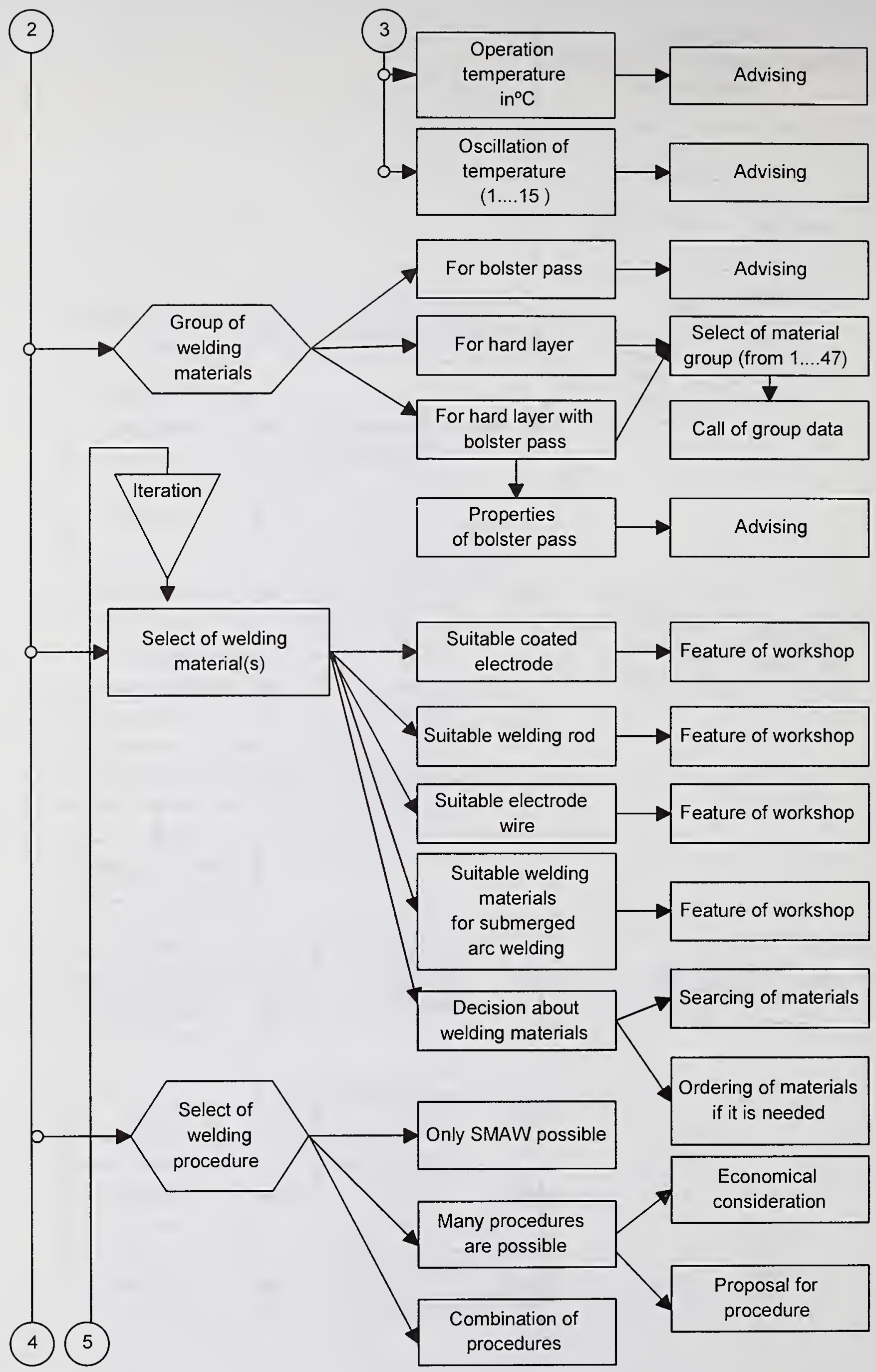

Figure 4. Hierarchy of Modules ( $2^{\text {nd }}$ part $)$ 


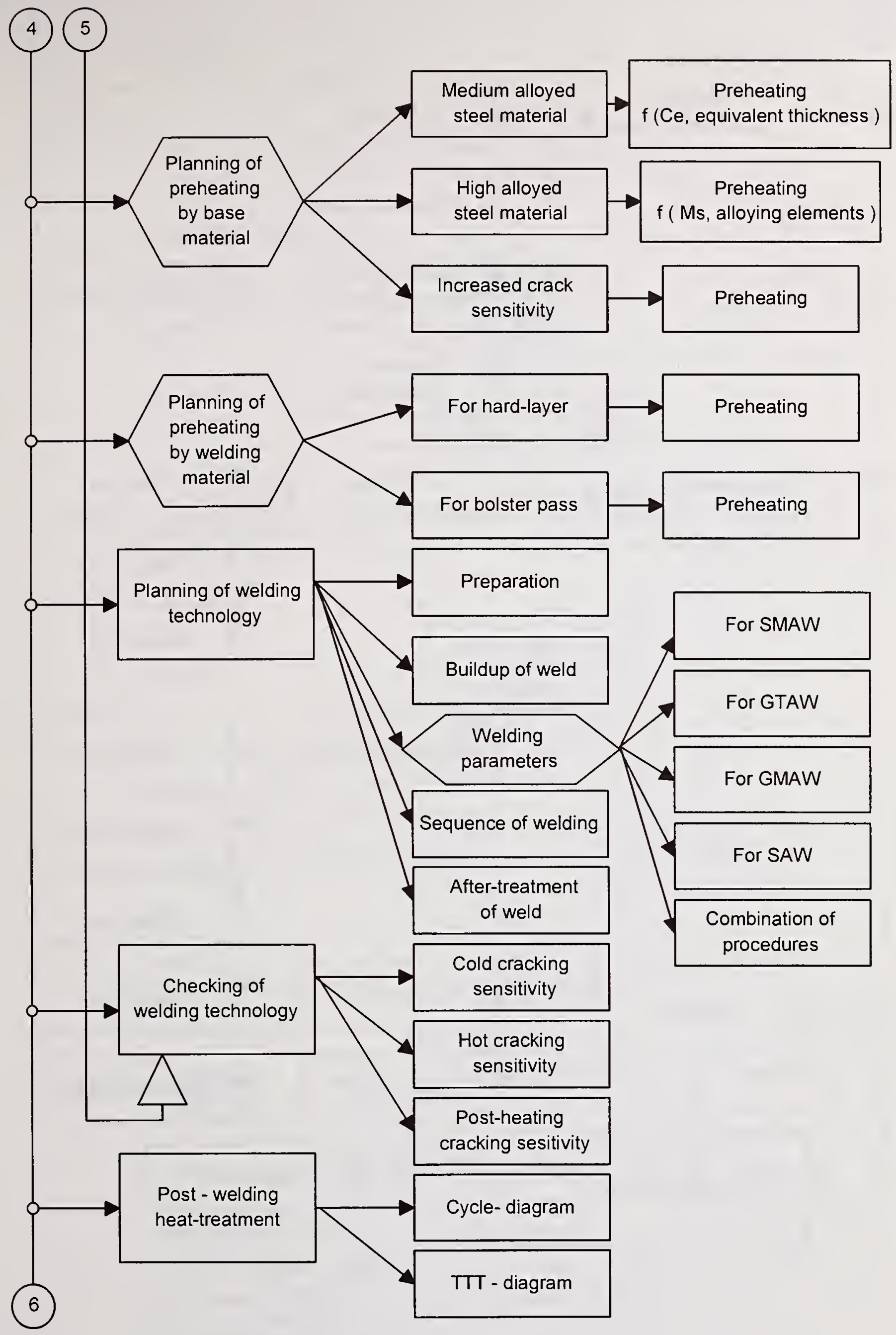

Figure 4. Hierarchy of Modules ( $3^{\text {rd }}$ part) 


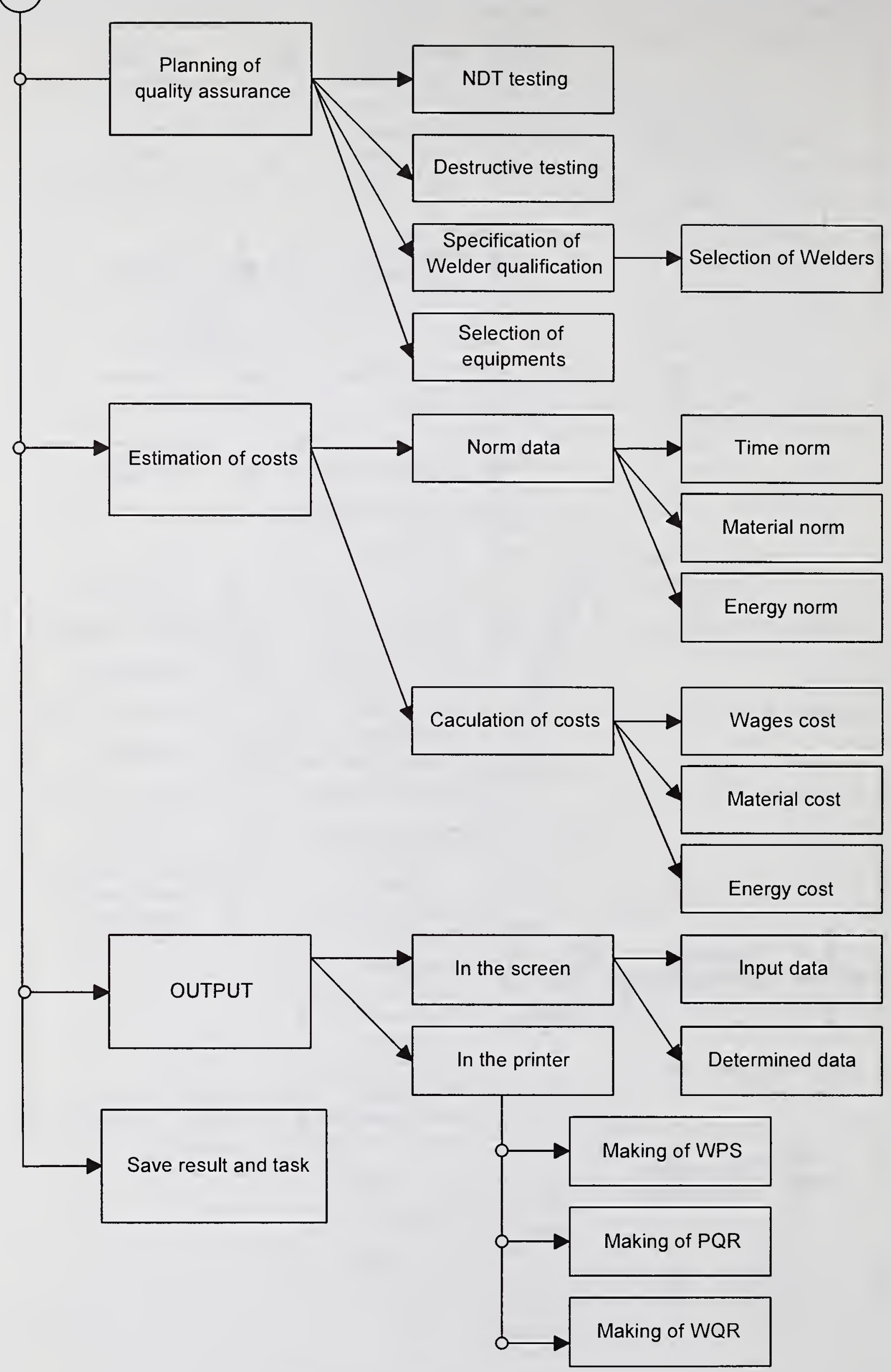

Figure 4 . Hierarchy of Modules ( $4^{\text {th }}$ part) 


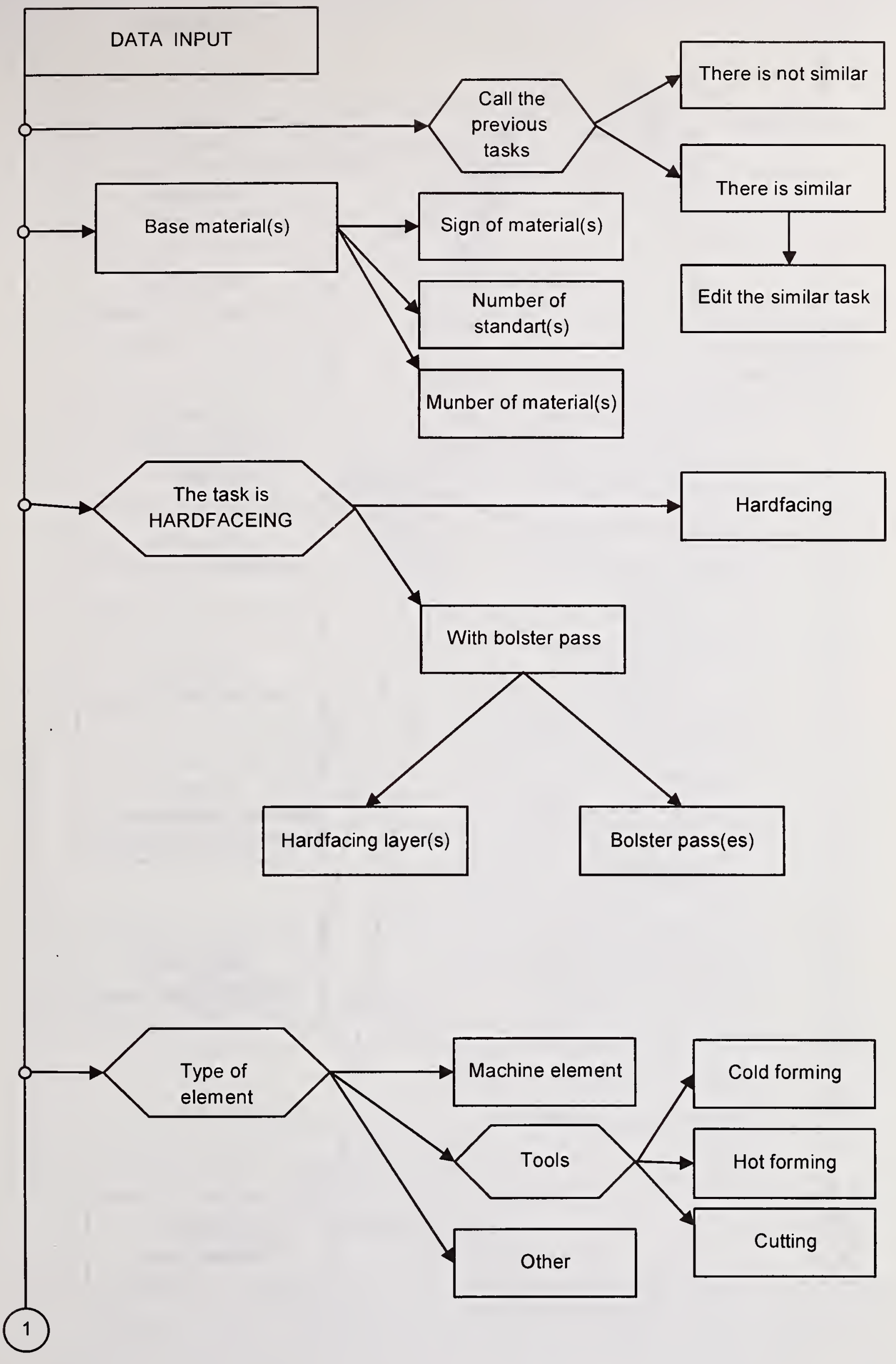

Figure 5. Input modules ( $1^{\text {st }}$ part $)$ 


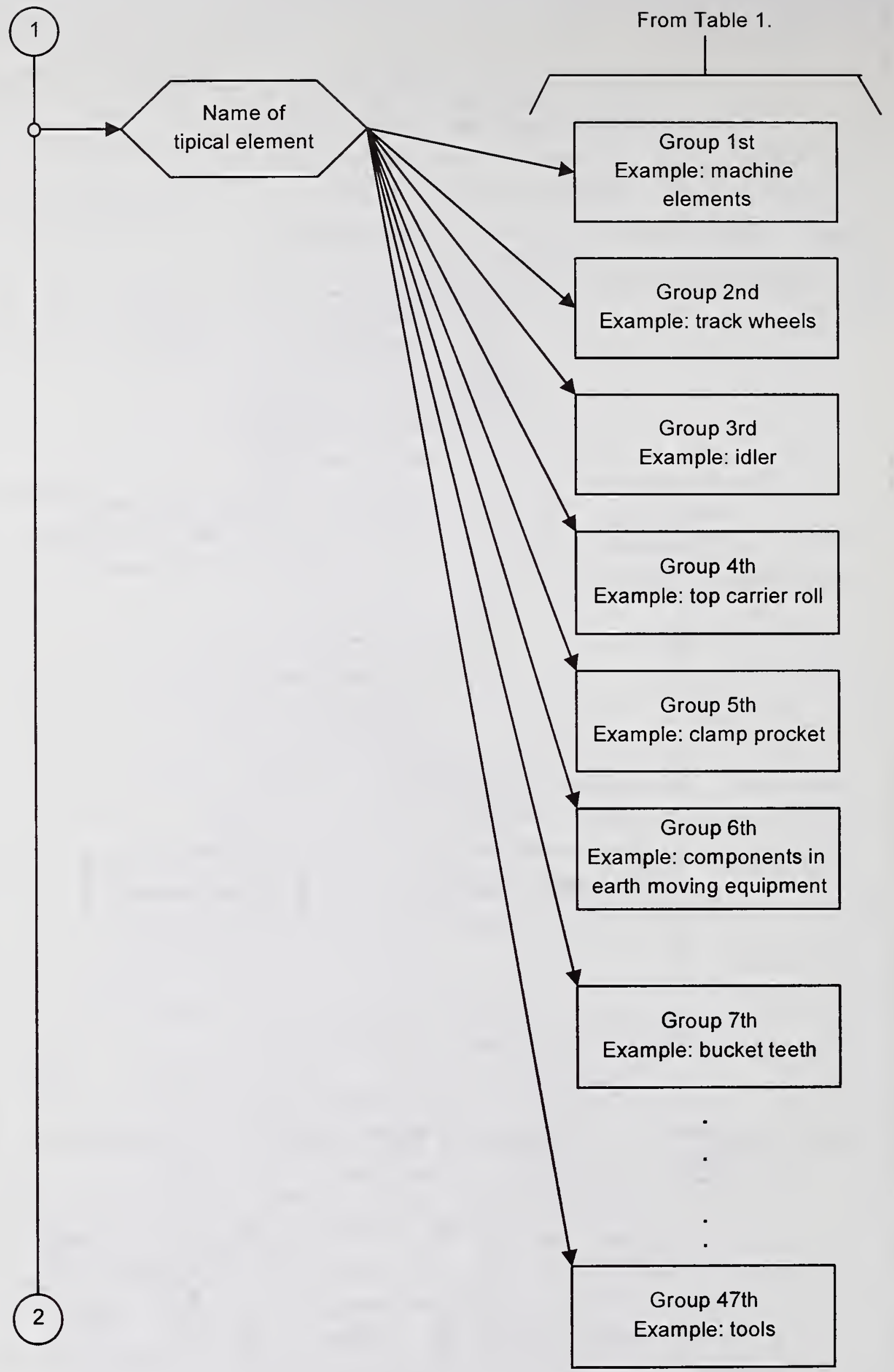

Figure 5. Input modules ( $2^{\text {nd }}$ part $)$ 


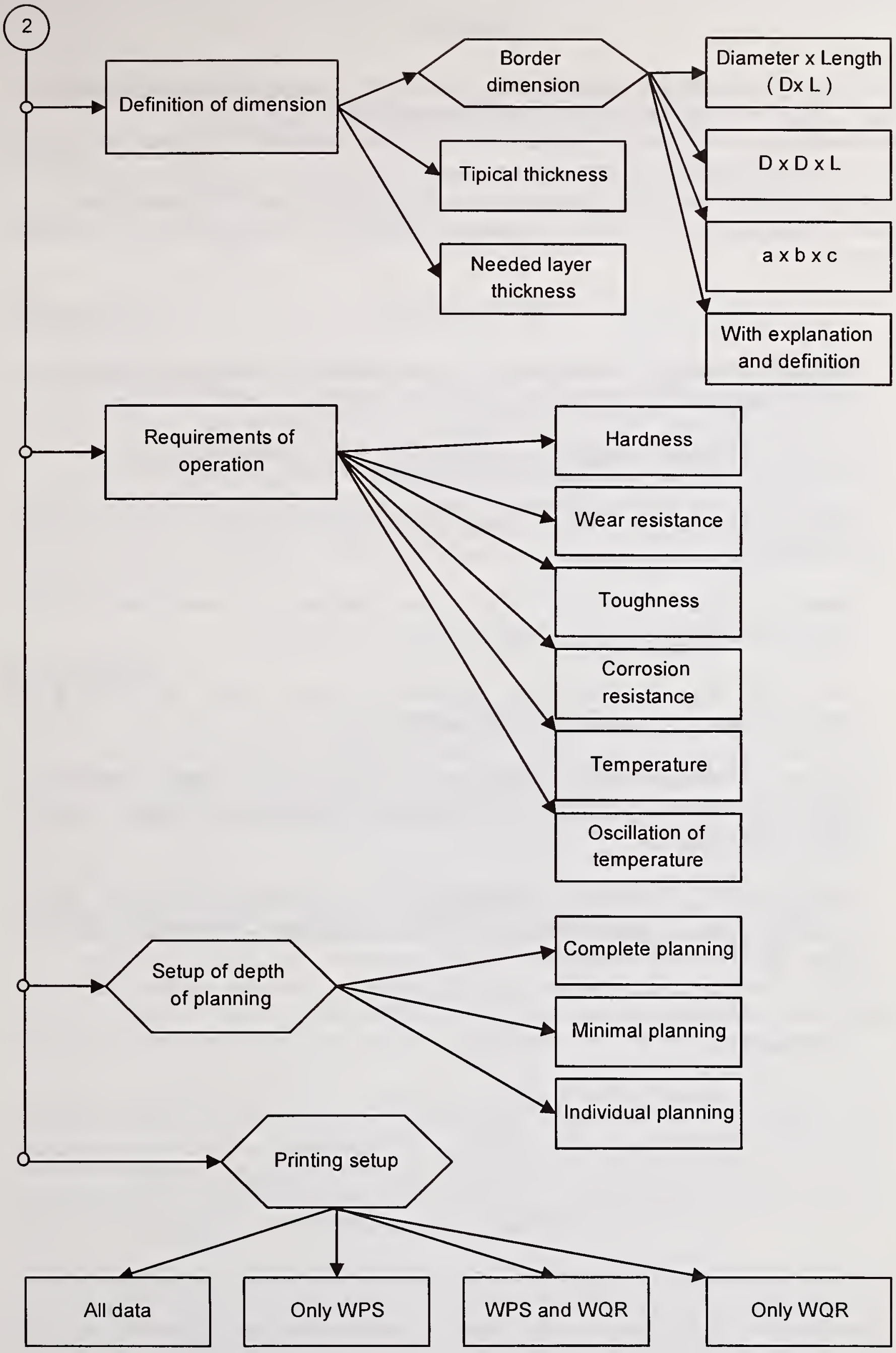

Figure 5. Input modules ( $3^{\text {rd }}$ part $)$ 


\section{SUMMARY}

The planning of hardfacing technology can be aided with computer. An advisory system for this technology is well usable in factories and in workshops.

The authorizes show the base of hardfacing. Gives some examples of elements, which were hardfaced and a method for selecting the welding materials.

On the base of theory, they show a computer system plan and a modulated system plan.

By these system plans one can make a computer program for aiding hardfacing technology planning.

\section{REFERENCES}

1. BÖHLER Welding Consumables for Hard Surfacing.Vereinigte Edelstahlwerke Aktiengesellschaft. VEW $828 \mathrm{D}, \mathrm{E}, \mathrm{Sp},-4.81-5000 \mathrm{G}$

2. Romvári, P. - Béres, L.1984.:Repair welding and surfacing*: Budapest, MK.

3. Thieme, G.; Jahre, H. 1988. Instandsetzungsschweissen :DVS - Verlag GmbH., Düsseldorf

4. Béres, L.; Béres, Zs.; Irmer,W. 1994 : Neue Gleichung zur Berechnung der Ms Temperatur. Schweissen und Schneiden 46 (8) : 372 -s to 374-s.

5. Palotás, B.; Béres, L.1996 : Computer Aided Advisory System for Surfacing and Repair Welding. Gép XLVIII (9) : 44-s to 50-s.

6. Palotás, B.; Becker, L.1988. Designing the manufacture of welded constructions. Proc.2nd International Conference on Computer Technology in Welding :Paper 5. TWI. Cambridge, UK.

7. Palotás, B.1992. Computer determination of welding parameters for arc welding processes. Proc. $4^{\text {th }}$ International Conference on Computer Technology in Welding . Paper 15. TWI. Cambridge, UK.,

*: In Hungarian 


\title{
Automatic Extraction of CAD Geometry Data in an Automated Intelligent Weld Off-Line Planning System
}

\author{
Paul A. Oberly, Patrick T. Sewell, Valerie L. Rhoades, Jerry E. Jones \\ N. A. Technologies Company \\ Golden, Colorado
}

\section{Introduction}

Design changes are inevitable in the fabrication of various products and may result in better or less-expensive items. There is a cost associated with these changes and it is related to when the change is introduced into the design process. Changes made early in the design cost much less than those made later - sometimes very significantly less1 (Figure 1). If it were possible to test out a welding manufacturing process before the parts, robot and associated fixturing were finalized, design changes could be made at virtually no cost to the production and may even save money, time or both. The current work relates to such as system where CAD drawings of welded parts, and their robotic fabrication workcell are accessed via software to plan and test the robotic welding path.

\section{Background}

Historically, robots have been very manual machines during their programming or "training" phase. Many various methods have been developed to help the operator to "teach" the points to the robot that make up the path to be welded (these include other points for approaching the part, leaving, etc.). A collection of these "teach points" and associated tasks to be done at or between points was referred to as a robot program2. Most programs represented a great deal of work by technicians, and engineers. Design changes at this stage meant re-training the robot to adjust to new positions on newly shaped parts. These robot programs could be stored, but until recently were very limited to floppy storage in proprietary formats. Modifications at the program level were difficult and often resulted in errors due to miscalculations of new positions for the teach points. During the teaching and programming stages, the particular robot cell that was being programmed was also not doing any production work and was, for all practical purposes, considered "down". Various off-line planning systems have been proposed 3 to deal with these problems, but until recently, they have not been fully integrated into the work flow.

The advent of personal computers and computer graphics enabled a new technique of robot programming where the robot workcell was simulated on the computer screen in a graphical and sometimes animated computer program. These simulators, when used correctly could enhance the task of assigning teach points for the robot welding path, but now instead of a technician doing the training, is likely a welding engineer. There is a trade-off here in money spent on engineers programming via simulators or technicians programming interactively.

Traditional fixturing of weldments was designed mainly in terms of constraining parts, with no concern of referencing or calibrating to the robotic task frame. Since these were not integrated into the path planning phases, there were often potential collisions between the robot and fixtures that needed to be discovered and averted prior to any production welding. These corrections are 
required at the workcell and usually involve the technician adding teach points or adjusting approach angles or other process related parameters. Unfortunately, these adjustments may not conform to the intended welding procedure and are usually not passed back "upstream" to the welding engineer or design stages. These "changes on the fly" could result in process variations that are hard to track down should a problem arise. The graphical simulator helps to predict collisions and also usually does not pass any feedback information upstream when modifications are required to avoid collisions.

\section{Enabling Technologies}

New technologies have recently become available that help usher in a more intelligent approach to the simulation and planning dilemma. The first technologies are in CAD. Standardization has been a big problem in the $\mathrm{CAD}$ world as many drawing formats are emerging that could not talk to each other's CAD program. STEP (ISO 10303), the International Standard for the Exchange of Product Data is an ISO industrial standard for communicating design \& manufacturing information over an entire product life-cycle including fabrication. Since it was developed by an international community to be applicable to all engineering data, and was designed to be open and extensible, it should meet most design and manufacturing needs well into the next century4,5. Modeling the weldments and their fixtures must be done with accuracy as the robot will need to follow welding paths without collisions or deviation for the prepared joint. The CAD drawings need to be created with the best accuracy possible while at the same time they need to be integrated with drawings from other CAD systems. Solid modeling is the new trend in $\mathrm{CAD}$ that allows the designer to fully model parts and assemblies with knowledge of the material and the volume it occupies. This data is actually stored in the drawing file. One company is spearheading the solid modeling CAD movement is Spatial Technologies, with their ACIS format and CAD programming tools. Solid models created in an ACIS-based modeler can be transferred to any other solid modeler capable of reading the general ACIS file. The Spatial Technologies modeling technology allows creation of models with extremely tight tolerances which means accurate models for robot path plaining6.

\section{Automated intelligent Concurrent Engineering System (AiCES)}

The new technologies in CAD, robot controllers, and neural networks allow us to develop a more "intelligent" approach to the weld robot path planning task. The newly developed AiCES (Automated Intelligent Concurrent Engineering System) has been created to take advantage of these new technologies and provide a better approach to path planning. We can model parts, fixtures and the robot workcell in an ACIS solid model (with some drawings possibly imported as STEP files), plan the path in the CAD environment based on the geometry of the parts, test the path with a simulator, choose welding procedures with the assistance of a neural network and send the robot program to the robot over a computer network. This process will soon be enabled as a two-way interaction of data, where the changes made at the workcell or in the simulator can pass back up to the CAD system so that the path can be correctly represented there also.

The CAD part of the path planning starts with the solid model of the weldment. The program establishes an interactive dialog with the designer or engineer and gathers pertinent information allowing the creation of: 1) a part coordinate system (figure 1), 2) weld path points/coordinates (figure 2), Approach angle (Figure 3), and 3) procedure data (figure 4). The program can then 
save the data for the off-line planner/ simulator. The off-line planner translates the path into fixture coordinates, writes the robot motion program and integrates the optimum welding procedures - automatically. It then communicates this information to the robot controller electronically.

\section{Benefits of CAD Extraction}

The use of an automated system such as AiCES can greatly reduce the time and cost associated with initiation or design changes in robotic welded part production. Since the path is defined from the CAD drawing of the part, there is no more assigning teachpoints from the robot in the workcell. Revision of the path due to manufacturability can be automatically captured in revised $\mathrm{CAD}$ part drawings as well as involving designers more. The planning can either be done on-line or off-line making efficient use of the robot and engineer's time.

The newer AiCES system can reduce the impact of design changes. In comparison the older way involves 1) changes to CAD drawing, 2) new manual simulation planning, and 3) many manual iterations that may not be integrated with CAD. The newer way all of the changes are made automatically.

This system represents a wonderful combination of technologies, but requires some paradigm shifts among its users. The first is that they need to represent weldments and fixtures as Solid Models to increase geometric accuracy and location of parts. The second is to integrate computerized process planning into the standard workflow. Third, is the need to move manufacturing engineering "up-stream" - changes made at the production level need to move back up to engineering. Lastly, the concept that robot controllers can be integrated with PCbased path planners and simulators.

\section{$\underline{\text { Summary }}$}

The use of an intelligent weld planner can save time and money through many efficiencies. Part production can be planned off-line, reducing the time to the first part. A welding engineer can plan the weld from the computer and identify welds in a fast-changing welding cell. The first part will be good since the location is more accurate due to a more accurate CAD model and optimum weld procedure. Performing "virtual welds" with the simulator can help eliminate shop floor "surprises".

\section{References:}

1. JCI communication - "Cost of Design Changes"

2. Deisenroth, Michael, "Robot Teaching", Article in Industrial Handbook of Robotics, Wiley \& Sons, 1985

3. Yong, Y..F., et. al., "Off-Line Programming of Robots", Article in Industrial Handbook $r f$ Robotics, Wiley \& Sons, 1985

4. STEP Tools, Incorporated. "The ISO STEP Standards" http://www.steptools.com/library/standard/ (30 June 1997)

5. National Institute of Standards and Technology. "The STEP Project" http://www.nist.gov/sc4/stepdocs.htm (30 June 1997)

6. Spatial Technology, Incorporated. "ACIS . Customers, Standards" http://www.spatial.com/corporat.htm (30 June 1997) 


\title{
MAGSIM AND SPOTSIM - PC AIDED SIMULATION OF GMA AND SPOT WELDING PROCESSES
}

\author{
U. Dilthey*, S. Roosen ${ }^{\#}$, V. Sudnik ${ }^{+}$
}

\section{SUMMARY}

For fast simulation with a response time not more then a few seconds on standard Personal Computers optimised algorithms were developed for the simulation of welding processes. The graphical user interface gives an easy and self-explaining definition of the task, the equipment and the welding conditions and parameters.

The MAGSIM program simulates the GMA welding process calculating the weld shape and the thermo cycle at various points. A graphical display of the cross-sections of shape and seam and a three-dimensional view visualises the simulation results. All calculations are available as number values too. The result is rated according to the European Standard EN25817. Other objectives allow the statistical prediction of quality at welding parameters with tolerances. The automated look for optimal welding parameters for a specific welding task is possible as well. Under development are extensions to other joint types and pulse arc welding. The simulation of self-regulation in pulsed GMAW processes is discussed

SPOTSIM calculates the forming of the spot lens between two sheet metals for spot welding numerically, based upon a differential equation system on electrical potential, thermal conductivity, and stress-strain condition. Many spot welding parameters and machine characteristics can be defined for the simulation of the welding task as exactly as possible.

Both programs will be available for running on WINDOWS95 shortly.

\section{INTRODUCTION}

Welding as a technological process concerns special processes, the results of which cannot be verified completely by the subsequent control, production inspection or, for example, can only be revealed during operation of the product (Ref. 1). Therefore the measures quality assurance made during engineering preparation of production, should use modern designing methods for welded constructions and the latest manufacturing techniques.

One of the assurance quality model of robotics arc welding (Ref. 2) covers three groups of problems. Figure 1 depicts the main phases: Education of the stuff and engineering preparation of the production (before welding), operation of the arc robot (during welding) and quality surveillance of welded seams (after welding).

\footnotetext{
*Welding Institute (ISF) Aachen University, Germany

\#German Welding Society (DVS), Düsseldorf, Germany

${ }^{+}$Welding Institute, Tula University, Russia
} 
The new welding standards for quality assurance in fusion welding according to EN 729 confront the engineer in the welding production area with tasks that can be solved by practical weld tests on the one hand, and, increasingly, by the assistance of expert systems with integrated data bases and by means of computer simulation on the other hand.

If suitable computer simulation programs are made available at engineer's working place due to their solution attachment and their structure, they achieve a better quality product with greater speed and economy.

\section{PC AIDED SIMULATION OF GMAW PROCESS WITH MAGSIM}

\section{$\underline{\text { State of Development }}$}

Using the commercial FEM program ABAQUS, Tekriwal \& Mazumder (Ref. 3) have simulated the 3D transient thermal conditions in the arc welding process. The analysis by Pardo \& Weckman (Ref. 4) is based on the finite element formulation for the calculation of steady state temperatures and overfill height in the GMAW process without taking into account the pool surface.

Dilthey \& Sudnik with assistants (Ref. 5-7) published the first 3D steady state model of GMAW process which included ranging from the power source setting to the weld seam evaluation (the quality level) as per EN 25817 . The analysis comprises "thermohydrostatic" weld pool modelling: conservation of energy and mass, a two-phase boundary zone, a gap with transverse shrinkage, a surface heat source distribution, evaporation, convection and radiation at the surface, the force balance on top-side and root-side comprising surface tension, hydrostatic pressure and arc pressure.

Weiss et al (Ref. 8) have shown, that the knowledge of the temperature distribution in the workpiece during arc welding is necessary in order to predict the change in material structure as well as strength of the joint. The simulation has not only to consider the heat effect of the arc but also the weld pool deformation influenced by arc pressure and gravitation. A coupled non-linear system based on the description of the energy transport and minimisation of surface energy by GMAW has been employed. The experience described by the authors in (Ref 5,6), that the iterative calculated solution of the system of equations resulting in the thermal conduction and surface differential equation neglecting the thermal conductivity in welding direction will be speeded up essentially, is confirmed in (Ref. 8).

Kumar \& Bhaduri (Ref. 9) presented a 3D finite-element model for prediction of transient temperature distribution in the workpiece at the GMAW process. The model considers the physical reality that kinetic energy and the heat content of transferred metal droplets play an important role in shaping the weld bead and also in the making of finger penetration, usually encountered in GMA welding at high currents.

Despite various publications in this field there is not any commercially obtainable simulation software for the GMAW process with exception of the following described MAGSIM. 


\section{$\underline{\text { Object }}$}

One-sided welding of thin sheets without backing causes typical problems, from the sensitive reaction of the welding process to variations in the welding parameters causing weld defects such as incomplete fusion or burn-through. To analyse these problems and optimise the process parameters, several programs may be surveyed quickly to help technologists and designers overcome this daily problems. The integration of modules with algorithms, which are based on the theory of heat transfer, as well as on probabilistic evaluation complements, the knowledge- and data base of the expert systems for process diagnosis and optimisation of welding. This improves evaluation of the influences of afflicting parameter variations effecting the weld geometry. The simulation program MAGSIM has been developed in order to fulfil the above-mentioned tasks by means of computer.

\section{The Program Design}

The authors have taken into account their trouble solving experience in the field of GMA welding of thin sheets (butt square, gravity position) when structuring the software of the simulation program.

- Description of the task (material, sheet thickness, evaluation group)

- Description of the installation (electric resistance of power source and cable, location of voltage measuring, wire diameter, contact tube distance, shielding gas)

- Description of the technological conditions (gap width, backing, workpiece temperature)

- Resolution objectives (process analysis, diagnosis or optimisation).

Altogether, for calculating analysis, process diagnosis and -optimisation, 25 parameters are taken into account.

\section{UI Diagram for GMAW}

During the input or the alteration of values for welding current and voltage, the respective operating point is found, by means of the software module "UI-diagram".

The electrical (current, voltage) and energetic (efficiency of arc and droplet heat flow) parameters are determined by the potential, mass and energy balance at the weld outline. The calculated data are required for solving the equation of heat conduction.

\section{Analysing of Butt Joint Welding}

The data acquired by the module "UI-diagram" are used by the software module "Analysis" in order to calculate the thermo physical processes at the square butt joint. The results of 
"Analysis" are depicted in Fig. 2. The mathematical and physical model based upon solving the differential equations system of heat distribution and weld pool surface is described in (Ref. 5) and (Ref. 6) in detail.

The calculated results were subjected to investigations by comparative weld tests and by literature data and they showed throughout well up to very well conformity in the weld formation. The input of data shift values for the specification of GMA welding data for square butt welds, carried out by a well-known welding company, gave a generally good evaluation of the weld results, according to EN 25817.

\section{Optimisation Strategies}

To begin with, this section presents some ideas on how quality assurance problems in the production preparation can be solved theoretically. Newly developed algorithms form the basis for the optimisation of GMAW process parameters as well as a numerical 3D-model of the process. The optimisation criteria should be chosen for the maximum achievable, reproducible quality production, even if parameter variations are prevalent (Ref. 7).

In preparation of technological production, the welding parameters such as current I, welding voltage $U$ as well as welding speed $v$ have to be selected by means of a suited optimisation method in such a manner that the weld geometry can meet certain demands and, likewise, fulfil the optimum value of a previously defined objective function, (e.g. the number of weld defects, weld time). As an example, the solution of the inverse task of mathematical physics will be considered: The parameters of GMA-welding in the spray-arc range of mild steel sheets, with the thickness s, without backing, have to be chosen by the optimisation method thus that the seam geometry meets the demands of the evaluation group B (EN 25 817) and, likewise, the minimum value of the objective function. A suitable objective function is, in this case, the minimum probability of weld defects.

The fixed input data are: material (thermo physical properties), sheet thickness, gap width, wire diameter $D_{w}$, contact tube distance $Z_{c}$, and shielding gas type (voltage gradient in the arc column $E_{S G}$ ). Variable input data are the welding parameters $I, U$ and $v$. The output data are top and root reinforcements or weld and root width and undercuts.

The parameters $\mathrm{I}, \mathrm{U}$ and $\mathrm{v}$ within a limited range shall be located by means of the optimisation method: $I_{\min }<I<I_{\max } ; \quad U=f\left(I, D_{w r}, Z_{c}, E_{S G}\right) ; \quad v>0$.

Moreover, the following conditions have to be met in order to find a solution:

$b_{W}>\varepsilon_{W}$ (sufficient weld-through), $\mathrm{d}^{2} Z_{W} / d y^{2}>\varepsilon_{Z}$ (no burn-through) and $\boldsymbol{F} \rightarrow \max$.

The values $I_{\min }$ and $I_{\max }$ are calculated from the UI-diagram taking account of contact tube distance, wire diameter, shielding gas type as well as critical current data during the transfer to another process region. The values $\varepsilon_{W}$ (good fusion) and $\varepsilon_{Z}$ (no burn through) are limiting values. When they are reached, further calculation for the actual operating point stops. 


\section{Prevention of quality disturbing sources}

The solution of different practical optimisation tasks entails the problem of taking into account parameter variations. Substantial information about scattering of the weld geometry can be derived from the statistical analysis e.g. the Monte-Carlo technique (Ref. 10).

The characteristics for the statistical distribution of the process parameters (current, voltage, speed, etc.) around the operating point serve as input data for the statistical analysis. The calculated result includes the statistical characteristics of the distribution and the weld geometry resulting thereof.

Generating values for the tolerance-dependent welding results based on the randomly generated welding parameters $U, I$ and $v$ (normal distribution around the operating point) is done by means of an algorithm that is based on the method of an inverted function as well as the spline interpolation.

While the 3D-simulation of the GMA welding process takes only approx. 1 minute of computing time, sampling by means of the Monte-Carlo technique takes substantially more time, even on a computer that works considerably faster. A possible way is therefore the conversion of the 3D-micro-model by means of numerical convolution into the polynomial form of the macro-model.

From a factor plan $2^{3}$ by means of the following calculation of the 3D-micro-model the appropriate weld results are determined.

In order to achieve the linear relation between the geometrical characteristics of the weld and the process parameters, the results of the 3D-micro-model are used for the set-up of a valid macro-model (valid within the limits of the tolerance zone) by creating a polynomial relation between input and output data $Y_{j}=a_{o j}+a_{1 j} X_{1}+a_{2 j} X_{2}+a_{3 j} X_{3}$.

This formula shows the dependence between weld size $y_{j}$ and the process parameters $\mathrm{x}_{\mathrm{k}}$, in correspondence to $I, U$ and $v$. The determined 5 equations for seam- and root-width, top- and root-reinforcement as well as undercut depth, stand for the reduced models of the analysed process in the zone of parameter tolerances around the selected operating point. Subsequently, the process simulation is carried out by means of the polynomial five macro-model equations, derived from the 9 simulations. The parameters of the five macro-model equations are statistical values within the zones of $I \pm \Delta I, v \pm \Delta v$ and $U \pm \Delta U$.

For the statistical probability evaluation by means of the Monte-Carlo technique, a sufficiently high number of simulation runs are carried out. This is, thanks to the specified macromodel equations, practicable relatively fast on a commercial PC. The possible results as well as their display on the computer screen was depicted in (Ref. 6) and (Ref. 7) in detail. 


\section{UI Diagram for pulse arc welding}

Within the scope of a research project between the Tula University (Russia) and the Aachen University (Germany) a model of the impulse arc heat source was developed. A new module for MAGSIM, based upon this efficient model, allows the prediction of pulsed GMAW processes and their weld results.

An energetic analysis of the whole welding circuit in consideration of the static (base and pulse phase) and dynamic (leading and trailing edge of pulse current) characteristics of the power source, the dynamic of electrode wire burn-off and the change of arc length was carried out.

With it the effective power of the pulse heat source results from the product of the effective current $i_{w}(t)$ and voltage $u_{w}(t)$.

$$
Q_{e f f}=\frac{\eta_{e f f}}{t_{P}+t_{B}} \int_{0}^{t_{M P P}} i_{w}(t) u_{w}(t) d t
$$

$\eta_{\text {eff }}$ is the effective efficiency of the pulse arc determined by experimental found data (11). It depends on the actual welding parameters and amounts to about $80 \ldots 85 \%$.

The mean values of current and voltage results from:

$$
I_{M}=\frac{1}{t_{P}+t_{B}} \int_{0}^{t_{P}+t_{B}} i_{w}(t) d t \text { and } U_{M}=\frac{1}{t_{P}+t_{B}} \int_{0}^{t_{P}+t_{B}} u_{w}(t) d t
$$

The effective current and voltage are defined by:

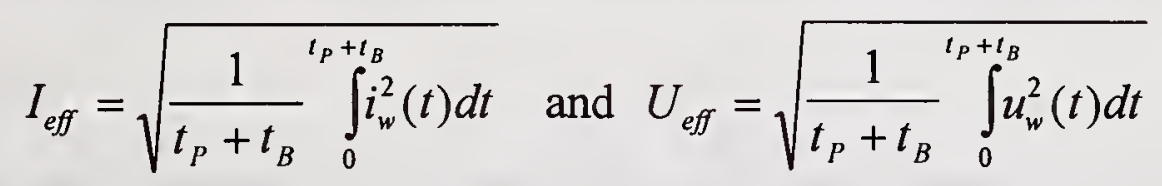

The partial model of the non-short-circuiting material transfer takes the electromagnetic forces, the forces resulting from the surface tension and the gravitational force into consideration. The nonlinear interaction among the parameters makes a numerical solution of the problem necessary. As result of the computer simulation is shown the current and voltage curve of the process on the screen, Fig. 3.

Depicted are samples of calculating the influences of the input parameters on the output characteristics of the GMA pulse processes welding unalloyed steels with shielding gas $82 \%$ Argon and $18 \% \mathrm{CO}_{2}$.

The already developed model not only allows the simulation at constant contact tube distance, but also the behaviour of welding across a step with following self-regulation, Fig. 4. As can be seen, first the process is in stability at a contact tube distance of $15 \mathrm{~mm}$, Pos. 1 . When 
running over the step with an height of $5 \mathrm{~mm}$ the arc length is shortened directly very much, Pos. 2. As one can see from the calculated oscillograms belonging to it, the process after this tends to a stable condition, Pos. 3. Reaching the end of the step the arc length increases discontinuous, Pos. 4. Here as well the changing and the following self-regulation can be seen in the oscillograms, Pos. 5.

The heat source model for pulsed arc welding describes the distribution in time and space of the effective arc power as well as the droplets power of the wire material in a mixed gas shielding atmosphere. For this the dependencies of effective arc efficiency and radius (hot spot) and the drop formation on the impulse arc process parameters were found.

\section{Expansion of MAGSIM2 to Filled Welds in Future}

Even more complicated than the simulation of butt welding is setting up the physical and mathematical model of the heat source for filled welds and lap joints. Because in practice such welds are more frequent, the program MAGSIM will be expanded to simulate such joint geometries.

The peculiarity describing the weld shape of such non symmetric weld geometries is, that

- the task cannot be solved symmetrical,

- the description of the power source distribution depends not only on the radius, but also on the distance between wire electrode and weld zone.

Figure 5 depicts first results of the analysis module under development for filled weld joints.

\section{PC AIDED SIMULATION OF SPOT WELDING WITH SPOTSIM}

\section{$\underline{\text { Object }}$}

The training of specialists for spot welding occupies itself with topics like the regularities at the forming of welds welding sheets of different thickness and alloys by means of several spot welding machines. In addition various kinds of welding electrodes of different construction determine the welding result.

The aspects mentioned above make it clear that the use of a computer program for simulation of various aspects of spot welding can make a contribution to increasing the effectiveness of training and production scheduling effectiveness and reducing the costs.

A simulation program for spot welding for training and production scheduling has to fulfil the following tasks:

\section{Prediction of lens forming:}

- by spot welding of several alloys,

- with various machine types,

- using different electrode geometries. 
2. Definition of the parameter field guaranteeing adequate lens forming.

3. Analysis of process stability with various machines, tolerances of sheet thickness, electrode wear, variation of electrode pressure and by-pass current.

Consequently a model of spot welding processes was developed at the State University in Tula. It is designed to find parameters for a specific lens formed by spot welding various sheet thicknesses of unequal and equal materials. The quality criterion for the process result are the dimensions of the lens and the press welding zone and thermal effect, geometry of electrode mark at the surface and gap between the sheets after joining.

\section{Regarded Input Data}

The input data and parameters being considered during simulation are the thickness of both sheets, thermo physical and mechanical properties of the welded alloys, electrical properties of the welding machine (thyristor controlled a.c. current, d.c. current with secondary circuit rectifier), electrode geometry, electrode material and electrode pressure, the welding cycle time, the no-load voltage of the power source, the shunt characteristics of the welding current and the surface condition of sheets and electrodes.

\section{Modelling the Process}

The spot welding model is based upon a system of differential equations describing the distribution of the electrical tension and current, the heat distribution, the temperature, the plastic deformation of the sheets and the components' interaction with the electrical machine parameters in the sheets to be welded (Ref. 12). The system was realised numerically by the finite volume method.

\section{$\underline{\text { Simulation Results }}$}

For simulation the machine characteristics and material properties provided by the data base are used. Additionally there is an experimental data base for the model's use in a real application. The model was verified by experimental data gained welding of St14 sheets with various thickness, welding parameters welding equipment and electrode geometry, Fig. 6. One can see the good correspondence between practical experiment and the simulated result by referring to the forming of spot lens and electrode mark.

The model allows:

- Definition of the welding parameter field (no-load voltage of power source - welding cycle time - electrode pressure), guaranteeing the lens forming (required diameter) at given sheet materials and thickness' at a selected equipment and electrode geometry,

- estimation of spot welding process stability and the appearance probability of inadmissible defects in the lens shape at given parameter tolerances. 


\section{CONCLUSION}

During many years of co-operation between the Welding Institutes at the Universities in Aachen and Tula the simulation software MAGSIM was developed and put into a commercial release. It is supplied as a tool for the training in welding technology and for the production scheduling of GWAW processes. The software was published in several languages. New versions will cover the simulation of pulsed processes and various joint types.

Software for simulation of spot welding processes, named SPOTSIM, is under preparation. It will be similar to the MAGSIM program concerning the objective and user interface. A first beta version is already available.

\section{ACKNOWLEDGEMENTS}

The results reported in this paper were promoted partly by the German society "Deutsche Forschungsgemeinschaft DFG". We also thank Dr. Erofeev and Ing. Kudinov for his data on spot welding, and Ing. Ivanov and Ing. Mokrov for numerical calculations of the GMAW processes.

\section{REFERENCES}

1 EN 729. Quality requirements for welding. Part $1-4$.

2 Xie M., Bolmsjö G.: Quality Assurance and Control for Robotics GMA Welding - Part 1: QA Model and Welding Procedure Specification. Joining Sciences. 1993. Vol. 1. No 4. P. 212.

3 Tekrival P., Mazumder J.: Welding Journal. 1988. Vol 67. No 7. P. 150s - 156s.

4 Pardo E., Weckman D.C.: Prediction of Weld Pool and Reinforcement Dimensions of GMA Welds using a Finite-Element Model. Met. Trans., 1989, Vol. 20B, No. 12. P. $937-$ 947.

5 Dilthey U., Habedank G., Reichel T., Sudnik W., Iwanow A.: Numerische Simulation des Metall-Aktivgasschweißprozesses. Schweißen und Schneiden, 1993, No 3. S. 148153.

6 Dilthey U., Reichel T., Sudnik W.A., Iwanow A.W., Mokrow O.A., Habedank G.: MAGSIM: Anforderungsgerechtes MAG-Schweißen von Dünnblechteilen mit Unterstützung durch Computersimulation. Düsseldorf: DVS-Berichte, Band 156, 1993, S.8791.

7 Dilthey U., Habedank G., Reichel T., Sudnik W., Mokrow O.A.: Analytical system for metal-arc active gas welding of thin-walled steel sheet in conformance with requirement. Welding and cutting, 1994, No 2, P. E24 - E25.

8 Weiß D., Franz U., Quaissa, B., Schmidt, J.: Rechnergestützte Simulation von Temperaturfeld und Nahtausbildung beim MAG-Schweißen in senkrechter Position. DVSBerichte, Band 179, DVS-Verlag, Düsseldorf 1996 
9 Kumar S., Bhaduri S.C.: Three-Dimensional Finite Element Modeling of Gas Metal Arc Welding. Metallurgical and Materials Transactions, 1994, Vol. 25B, No 6, P.435 - 441.

10 Dilthey U., Habedank G., Reichel T., Sudnik W., Mokrow O.: Numerische Prozeßdiagnose der Nahtausbildung beim Metall-Aktivgasschweißen von dünnwandigen Stahlblechen. Schweissen und Schneiden, 1996, No. 12. S. 938-942.

11 Boswort M.R.: Effective Heat Input in Pulsed Current GMAW with Solid Wire Electrodes. Welding Journal, 1991, No 5. P.111-117.

12 Erofeev V.A., Kudinov R.A.: Computer Model of the Resistance Spot Welding Process for the Analysis Welded Joints Quality. In: CAD and Expert Systems in Welding: Transactions of Tula State Technical University . Edited by V. Sudnik Tula, 1995. Pp. 84 - 91.

\section{FIGURES}

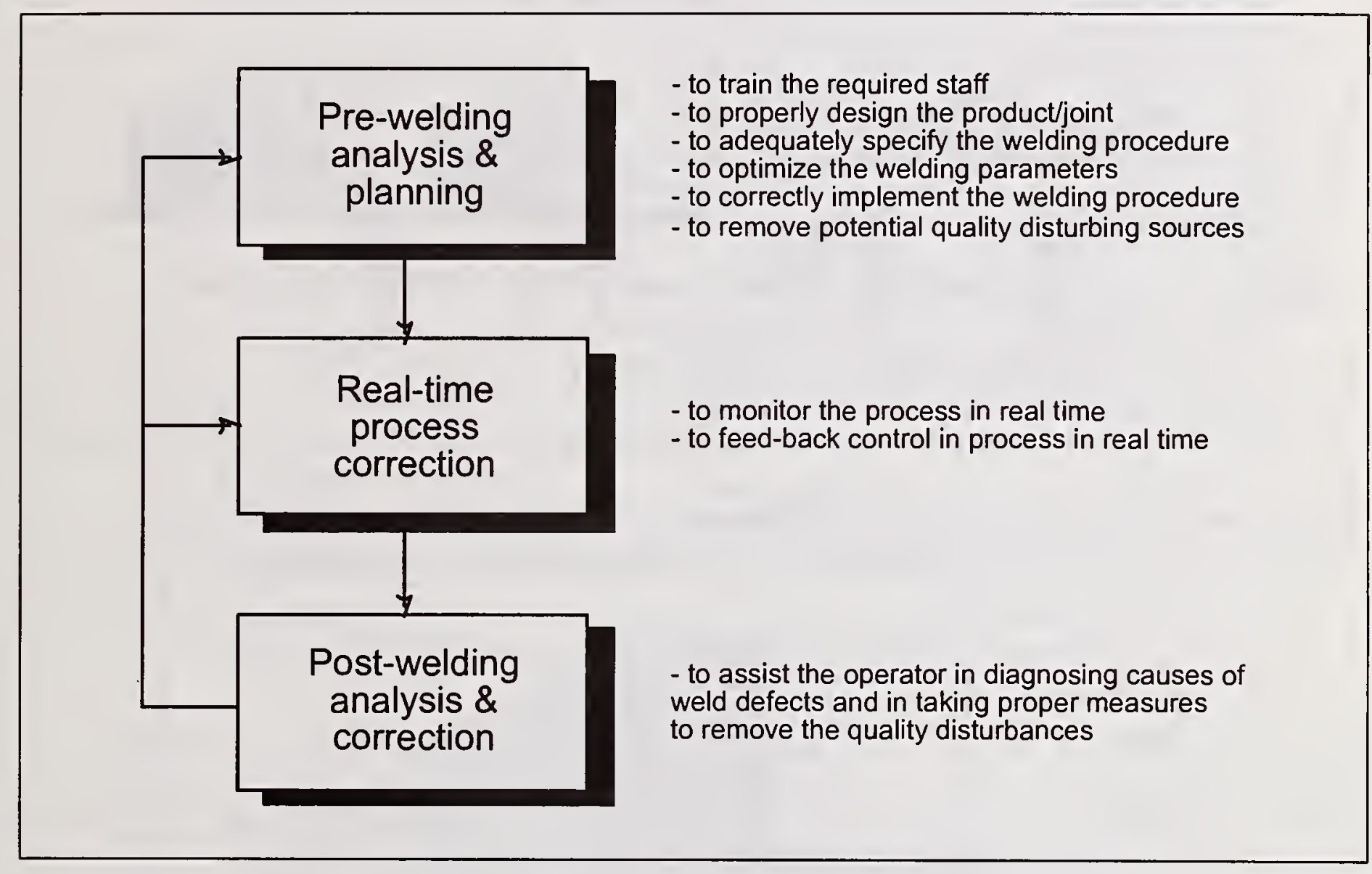

Figure 1 Assurance quality model 


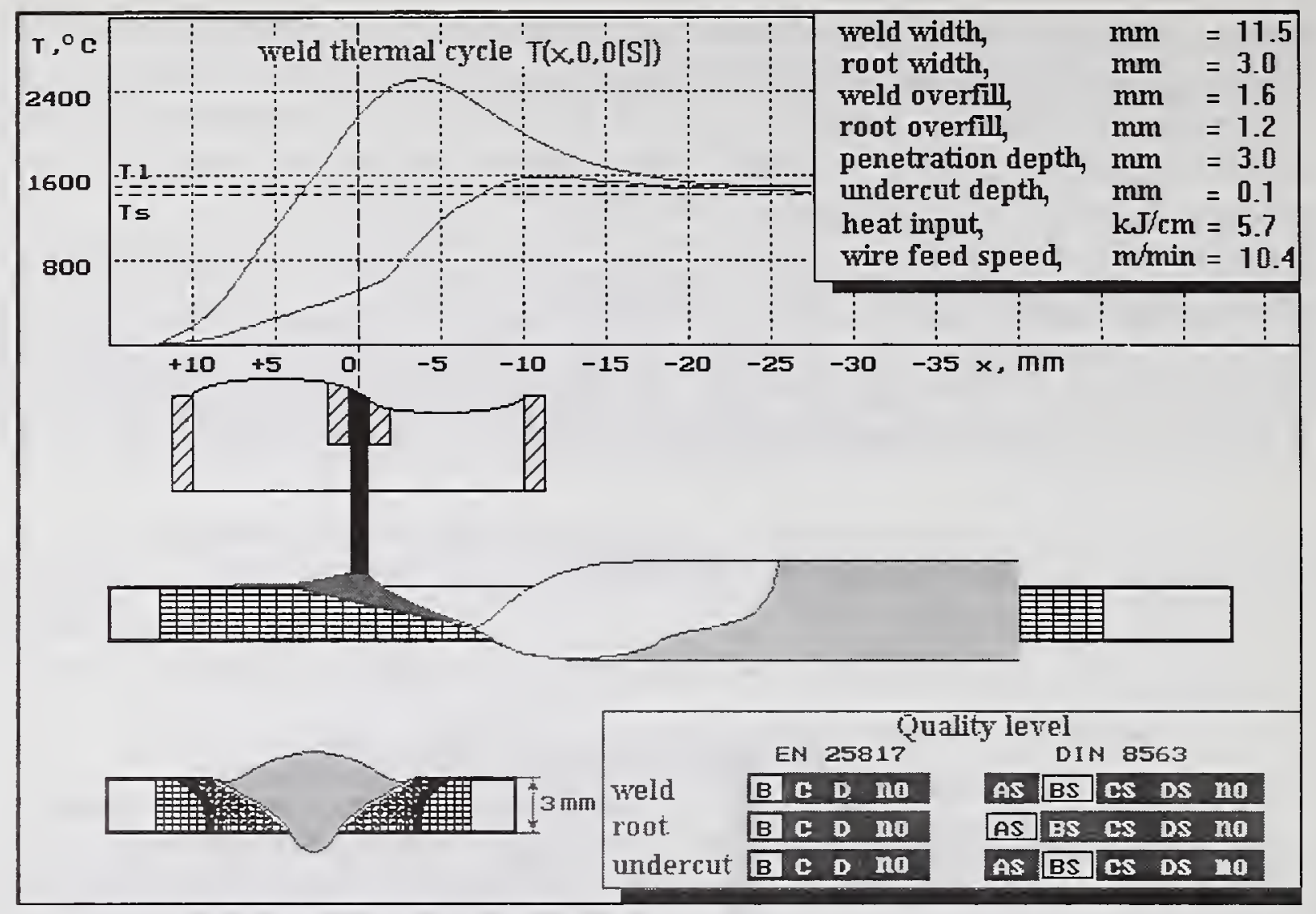

Figure 2 Calculated weld pool and weld shape with estimation of quality level

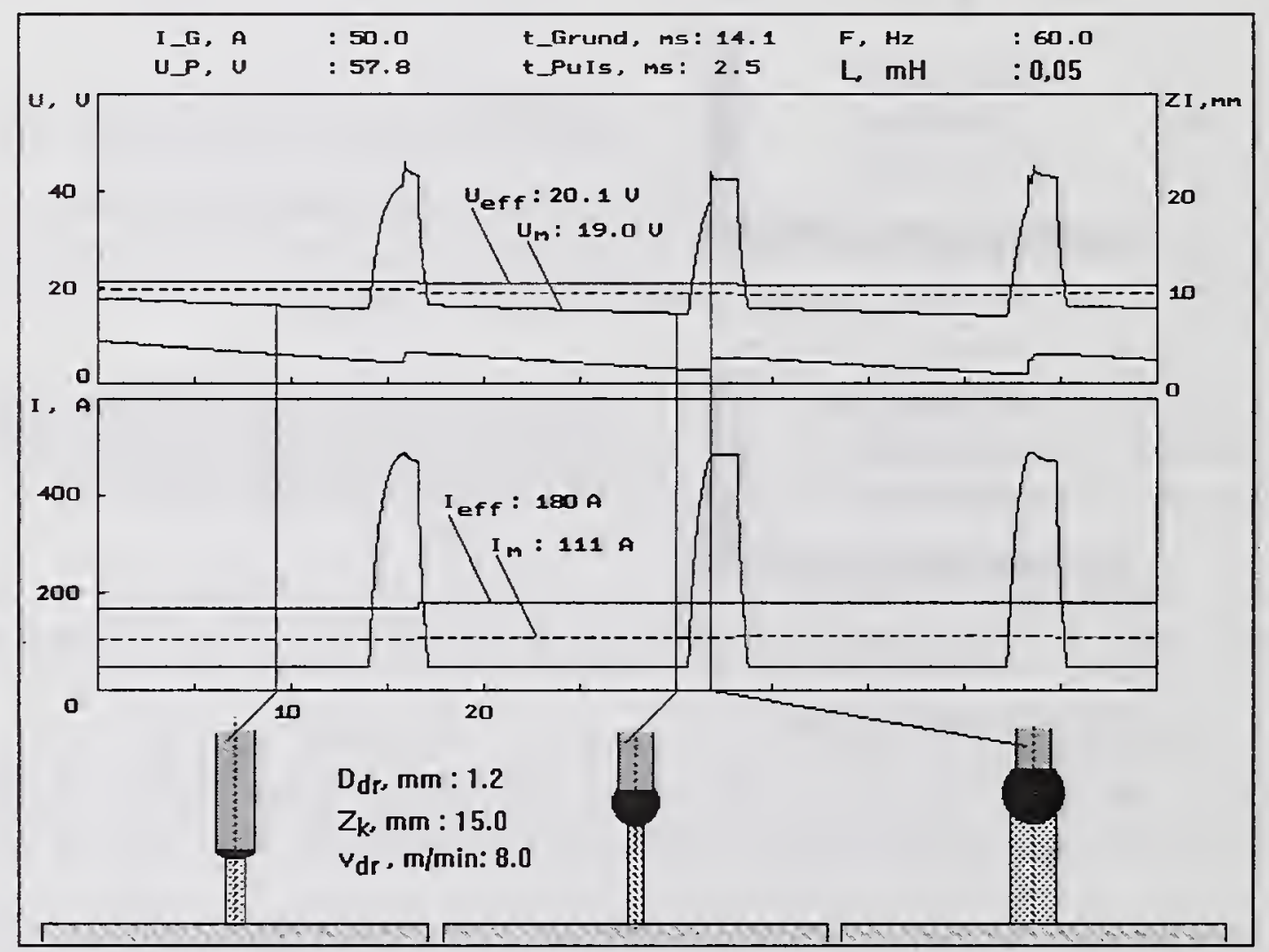

Figure 3 Results of the numerically calculated impulse process course with IB-UPmodulation.

Parameters: Shielding gas $82 \% \mathrm{Ar}+18 \% \mathrm{CO}_{2}$, wire diameter $\mathrm{D}_{\mathrm{wr}} 1,2 \mathrm{~mm}$, contact tube distance $Z_{\mathrm{c}} 15 \mathrm{~mm}$, wire speed $\mathrm{v}_{\mathrm{wr}} 8.0 \mathrm{~m} / \mathrm{min}$ 


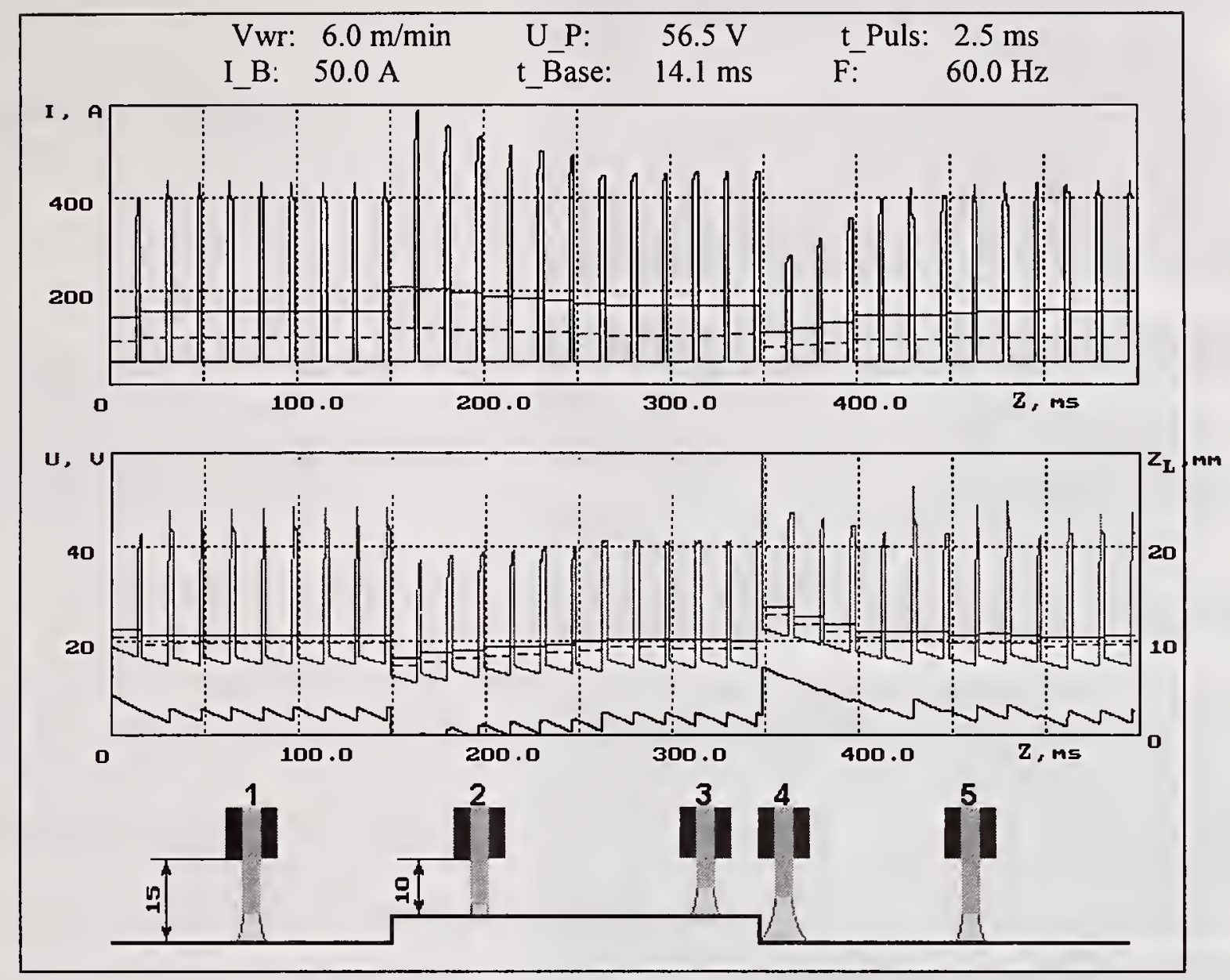

Figure 4 Simulation of self-regulation welding across a $5 \mathrm{~mm}$ thick step with IB-UP modulated impulse arc.

Parameters: Shielding gas $82 \% \mathrm{Ar}+18 \% \mathrm{CO}_{2}$, wire diameter $\mathrm{D}_{\mathrm{wr}} 1,2 \mathrm{~mm}$, contact tube distance $Z_{c} 15 \mathrm{~mm}$, wire speed $\mathrm{v}_{\mathrm{wr}} 6.0 \mathrm{~m} / \mathrm{min}$

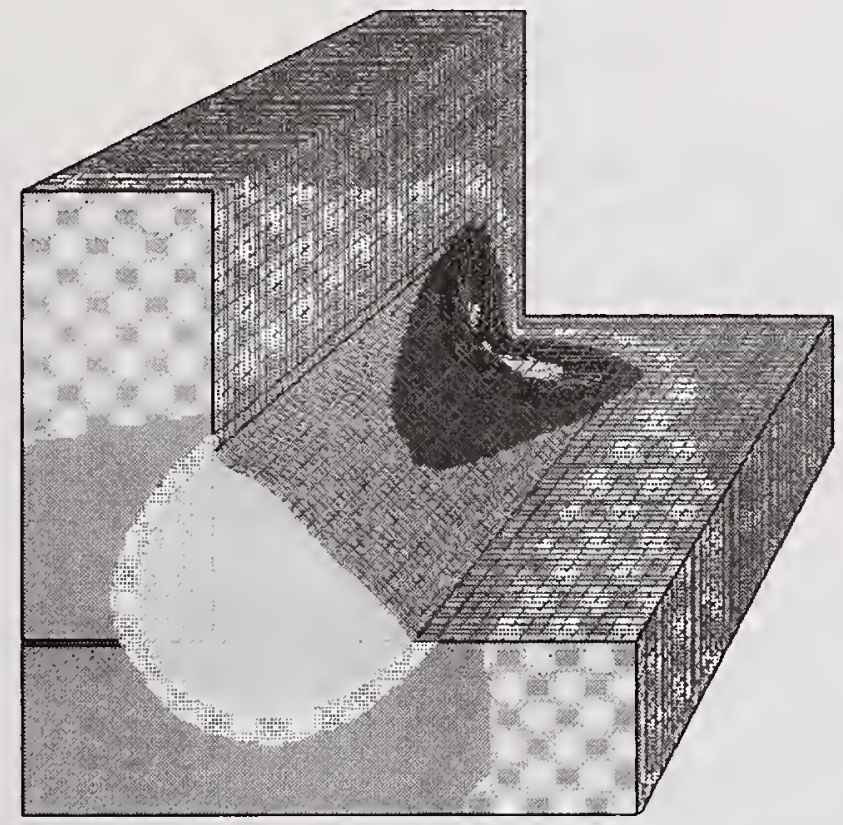

Figure 53 3D display of a simulated fillet weld with MAGSIM2 


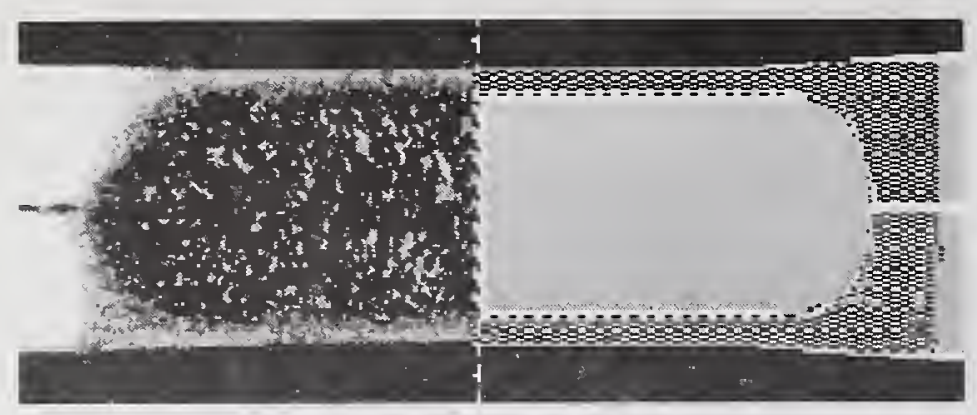

$$
\begin{gathered}
U=2.6 \mathrm{~V} ; \mathrm{t}=6 \text { periods; } \\
\mathrm{d}_{\mathrm{L}}=5.39 \mathrm{~mm} ; \mathrm{h}_{\mathrm{L}}=0.59 \mathrm{~mm}
\end{gathered}
$$

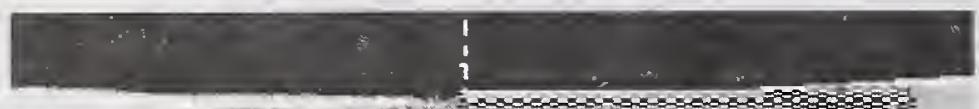

$$
\begin{gathered}
U=2.4 \mathrm{~V} ; \mathrm{t}=10 \text { periods; } \\
\mathrm{d}_{\mathrm{L}}=5.07 \mathrm{~mm} ; \mathrm{h}_{\mathrm{L}}=0.6 \mathrm{~mm}
\end{gathered}
$$

$$
\begin{gathered}
U=2.2 \mathrm{~V} ; t=10 \text { periods; } d_{L}=5.70 \mathrm{~mm} ; h_{L}= \\
0.64 \mathrm{~mm}
\end{gathered}
$$

Figure 6 Comparison of spot welding experiment and its simulation Common parameters: Material St 14; Electrode pressure $\mathrm{F}_{\mathrm{El}}=2 \mathrm{kN}$; Sheet thickness $0.8 \mathrm{~mm}$ 
2. Investigations into alternative fusion techniques with a view to establishing depth boundaries for methods. An investigation is already underway inconjunction with Cranfield University in the UK to look into the performance of deep water plasma and MIG welding.

3. Investigations into practical penetration control without invalidating certified procedures.

4. Improving the performance of demagnetism systems to minimise arc deflections. This will facilitate the use of laser following strategies and reduce the stress on the operator.

5. Automate the replacement of tungsten welding electrodes.

6. Automate the grinding of irregularities in the groove.

7. Automatically map the groove geometry by use of digital cameras and lasers and use the information for optimum parameter settings and torch positioning.

8. Further investigation into the remote installation and remote operation of mechanical couplings.

\section{CONCLUSION.}

In an attempt to reliably deliver large quantities of oil \& gas to Europe, the Norwegian petroleum industry have invested heavily in new technology to solve the problem of subsea pipeline joining and repair. A consortium of companies led by Statoil and Norsk-Hydro have combined to produce the innovative Pipeline Repair Spread (PRS), which is capable of remotely controlling all aspects of hyperbaric welding and mechanical coupling installation. The equipment is continually being improved to operate in deeper waters to support oil and gas recovery into the next century. 


\title{
THE ROBOTIC EASY TEACHING SYSTEM IN COMPUTER AIDED WELDING
}

\author{
H. Handa*, S. Okumura*, S. Nio\#
}

\begin{abstract}
The Welding robots have been mainly applied to the mass production of the automotive Industry representatively. More recently, however, welding applications are trending toward the field of the multi-kind and small-quantity production. The Arc Welding Robot should give the key experts to effectively respond to problems in the field. In the circumstances, Computer Aided Welding (CAW) is contributing efficiently to solve the subject.

The first step of YASKAWA CAW system, which we have named Easy- Teaching PAD or ET-PAD, consists of Algorithm aided Welding Data Base and Off-Line Robotic Programming. The Algorithm aided Welding Data Base consists of the Algorithm, which automatically generates welding parameters for the 6 kinds of the joint, the base metal thickness ranging from 0.8 to $6.0 \mathrm{~mm}$, and the Welding Data Bank related to robotic welding for GMAW.

Off-Line Robotic Programming provides optimized torch orientation, automatic programming of the robotic job and simulation of robotic motion on surface model. These functions can be easily operated through Graphical User Interface (GUI). And these software architecture utilizes OLE (Object Linking and Embedding). These functions are seamless in the human interface as well.

As a result, ET-PAD CAW system sets the operator free from the skill in welding and complex programming of robotic welding. We have developed ET-PAD which automatically calculates not only the welding current, voltage, travel speed, but also the torch and arm orientation etc. for the robot by only setting the joint type and the base metal thickness in pre-welding.

This paper focuses on an outline of our CAW philosophy and the significance of the positioning of ET-PAD within this system. The Algorithm Aided Welding Data Base and Easy Teaching System (ET-PAD) are described in detail, and additionally the Algorithm validity are experimentally evaluated.
\end{abstract}

\section{INTRODUCTION}

With continuing progress in computer technology, computer-aided welding (CAW) has become a key research topic in a genre of welding automation through robots. CAW is a technology of broad category, because CAW is defined as merely being computer-aided. This allows an excessively large range of systems from a single purpose (narrow sense) to fully automated welding system (broad sense). As narrow definition, therefore, welding systems for academic research theme implemented with the assistance of a computer have emerged (Refs. 1, 2, 3, 4, 5).

Setting aside this mainly academic research trends, the research and development of CAW as a robot manufacturer should be enhanced up to the final target in broad sense, namely, fully automated robotic arc welding system with practical application by use of CAW method.

\footnotetext{
* Research Laboratory, Robotics Research Section, YASKAWA ELECTRIC CO., Kitakyushu, Japan. \# General Manager, Research Laboratory, Robotics Business Sector, YASKAWA ELECTRIC CO.. Kitakyushu, Japan.
} 
The CAW philosophy of the authors is the construction of robotic arc welding system in multikind \& small-quantity production, providing high quality and high productivity. This concept is described in detail in Chapter 2.

One of the key constituent technologies in the implementation of CAW is the expert welding system. In this system, information equivalent to the expertise possessed by a skilled welding technician is stored in the computer, as hard data or inferences. As the first step toward the realization of this system, we have productized the ET (Easy Teaching)-PAD CAW system to be applicable to GMAW, integrating the algorithm-aided welding database and off-line robot programming.

This system applies the latest computer technology in arc welding robot teaching, making set-up and control simple.

Chapter 3 discusses primarily the algorithm-aided welding database, off-line robot programming, the results of robotic arc welding tests with the system, and the system's practical utility.

\section{PHILOSOPHY OF CAW AT YASKAWA}

There have been two major approaches to automating the process from design through manufacturing, not only with application to arc welding systems. The first approach is automation of off-line system based on CAD/CAM in a CIM system, and the second is automation of the shop floor, such as through robotic systems.

The final target of Yaskawa CAW system is flexible welding automation, seamlessly integrating these two approaches. Part of this is technology to fully utilize information from upstream processes (planning and design), improving production efficiency through organic linkage with downstream processes. Another part of this is a research way to the realization of a humancooperative autonomous robot which generates motion plans semi-automatically from job plans, by providing the system itself with the ability to analyze and understand the welding process, minimizing human interposition and enhancing human factor. More concretely, the arc welding robot system itself analyzes the arc welding process, which is a non-linear phenomenon, by utilizing in-process sensors and the arc process model base, and compensates external disturbances such as workpiece deviation, heat deformation, etc. in realtime, by providing the optimum welding conditions while preventing welding defects (burn through, undercut, etc.).

The application of such an adaptive control system to the robotic welding process assures accurate bead profile, bead appearance and penetration profile control, contributing to higher quality and high-productivity automation. Breakthroughs in the constituent technologies involved are difficult, but it is a challenge that must be faced. Our future target is the realization of flexible welding automation, which we define as an integration of CIM-based robotics and APO (arc process oriented) based robotics. This is the Yaskawa CAW philosophy, as indicated in Fig. 1.

We believe that this concept is essential to ensure that arc-welding robots will be able to be applied to "true" multi-kind and small-quantity production in the future, continuing to meet diversifying user needs. The authors will continue research and development into the constituent technologies of arc welding robotics, based on our CAW philosophy and with the goal of continuing contribution to arc-welding robot systems.

The CAD/CAM systems positioned upstream of CIM-based robotics (the office environment) are not developed by the robot manufacturer, but on the user side. The robot manufacturer must 
develop more efficient interfaces between this system and the robot system, and between the systems and human operators, in the form of simple man:machine interfaces and feedback to enhance the CAD/CAM system from the viewpoint of robotics. Technical development must focus on these two sectors in order to establish CIM-based robotics as the core of CAW. Noncooperative relationship would mean that CIM-based robotics would only become the establishment of functions for automation tools for each individual job, and would not provide seamless integration between individual processes. As an example, the simple and precise expression of design and pre-manufacturing processes in CAD/CAM shows an improvement for individual job performance, but there is insufficient information breakdown between the upstream and downstream processes. Concretely, information such as welding path sequence and welding conditions hidden in the welding workpiece drawings are not clearly spelled out, which means that skilled technicians must intervene to compensate. The intervention of a skilled operator is undesirable in CIM-based robotics. If a human operator is unavoidable, then the provision of a human-guided man:machine interface instead of a machine-guided interface should make it possible to compensate for these problems even when the operator has no welding skill. This type of interface will be required.

Shop floor (downstream) technology related to existing robots and welding control is trapped in systems which rely on human skills because the robots lack sufficient intelligence. This is a considerable distance yet from human-cooperative autonomous robots which require only a minimum of human interference. Industry eagerly awaits optimization of welding control, including welding power source, wire melting and welding tip, and the appearance of semiautonomous robots.

To promote integration of the entire process from upstream to downstream, the welding expert system is thought to be essential.

The first step is to convert the welding expertise of the skilled welder into software, and develop an algorithm-aided welding database designed to optimize welding conditions at the design level. As long as there are no external disturbances (fabrication error, set-up error, etc.) in the manufacturing process, this system will provide, at least at the design level, job and motion plans to the robot, making possible automated welding with the skill of a expert welder.

The second step is the development of a welding simulator based on the technology acquired from the analysis and identification of the welding process. This simulator will allow simulation of welding results (penetration profile, bead profile) by changing virtual welding conditions prior to actual welding. Flexibility will be enhanced because simulation can be used to check set-up for multi-kind and small-quantity workpieces, and handle changes of welding parameters in design and fabrication method without actual welding.

The third step is to integrate the job information from upstream processes, the welding database and the welding results simulator, to take tact time and weld distortion into account, to optimize welding conditions and sequence, and to provide the motion plan to the manufacturing process, through intellectual job simulation technology. If the system can be enhanced to this level, then the system will be able to respond to disturbances other than unexpected external one, from the design through the manufacturing processes.

What remains is the most important issue of welding process control. The welding process is a non-linear phenomenon, and it is clear that much of the process remains to be scientifically and technologically clarified. The key issues for CAW are (1) provision of semi-autonomous control 
of the robot's own actions (physical), including job and motion plans, (2) control of welding, including peripheral equipment, (3) modelling based on welding process heat transfer theory, molten metal convection (hydrodynamics), and surface tension theory, (4) sensorless in-process sensors extracted from information sources such as waveform analysis of welding process operation quanties and states vectors (variables), including welding current, voltage and wire feed speed, (5) the construction of an adaptive control system as described above, based on the constituent welding process identification technologies, through realtime inference of welding state.

The identification of the welding process is therefore step 1 as outlined above. The authors are involved in research on this theme, but have not yet reached the point of publication.

As described above, the target CAW is flexible welding automation. This paper introduces a fundamental milestone in the pursuit of this target: Easy-Teaching system.

\section{EASY TEACHING SYSTEM: ET-PAD, ET-HANDLE}

Industry requirements to robots within the production system are changing. Major volume production users, primarily in the automotive industry, have shown tremendous growth thanks to the use of robots in their production lines, but as market needs diversify, the single-model production line is being replaced by mixed-model production lines handling multiple models at the same time, although all are based on a single basic model. It is expected that automotive industry will accelerate toward CIM-based robotics in the future. The Easy Teaching System, which is one key of CIM-based robotics as illustrated in Fig. 1, will be extensively used on the line in the automotive industry. Especially in the small-kind, multi-quantity manufacturing industry, generally small-and medium-scale enterprises, problems are being caused by the decreasing amount of skilled labor available. Within this situation, robot users in such production system are demanding easy teaching techniques for robotic welding systems with increasing stridency. Industrial robots today generally require dozens of times longer for teaching as they do for actual welding, and the conventional teach-playback approach is not applicable to emerging needs for small-kind, multi-quantity manufacturing. Until a system can be developed which makes it possible for even untrained personnel to teach, there is little hope for major growth in arc welding robots.

When teaching a welding robot, it is known that teaching welding conditions and torch orientation required to achieve a specific quality account for more weight of the total than teaching the robot trajectory path (Ref. 6).

Fig. 2 indicates the results of an analysis of how much time is required for each process within the teaching task. Four processes account for essentially all: position teaching, position modification, welding condition teaching, and welding condition modification. Excluding the position teaching, about $80 \%$ of the total is accounted for by position modification, welding condition teaching and welding condition modification, which all require welding skill.

Based on these analysis results, we have made efforts to minimize position teaching time by replacing indirect operation methods such as axis operation using buttons in conventional teach pendant and joysticks with direct methods, such as an impedance-controlled direct teaching system (ET-HANDLE -.Fig.2), which provides enhanced flexibility and safety. Teaching with ET-HANDLE operates teaching only the welding torch tip position, with torch orientation data generated automatically by Off-line Programming related to ET-PAD. The ET-PAD provides simplification of the position modifications, welding condition teaching and welding condition 
modification processes which account for about $80 \%$ of the total: automatic torch orientation generation, automatic welding condition generation, and adjustment of welding conditions to match plate thickness, joint and gas parameters (welding assistant function).

Fig. 3 is a concept diagram of our first target being developed for an automation solution to multi-kind, small-quantity production systems, combining an Easy Teaching System with robotic welding GMAW in-process control (existing functions at YASKAWA such as seam tracking with arc sensor, contact tip cap method quaranteeing long electrode extension for ultra-thin plate welding. switchback welding method, etc.). By integrating the Easy Teaching System with a range of other existing technologies, the goal is to construct a robotic arc welding system which can be used easily and conveniently by anyone.

The human interface technology is also critical in the implementation of this technology, such as the view (external specification) and document (internal specification).

Under above-mentioned background. the authors developed the CAW ET-PAD intelligent teaching tool applying pen-input computing technology with reliability-improvement functions that can be used in the shop environment. Results indicate that the new system reduced teaching time to a tenth of that with conventional systems, even for novices (in-house data comparison $\cdots$ Fig. 2). The ET-PAD intelligent teaching tool uses the system configuration indicated in Fig. 4. All teaching informations (automatic welding condition set-up, automatic torch orientation generation, welding assistant. etc.) are automatically made on the teaching sheet in ET-PAD (pen-input computer) through GUI as illustrated in the figure. By incorporating the algorithmaided welding database with the automatic generating-functions of orientation and path conditions (torch and arm), ET-PAD automatically generates superimposed welding conditions and the sequences on a 3D display of robot trajectory. implementing a human interface that is easy to understand even for novices. Unlike the conventional teaching interface where welding conditions and trajectory conditions are input separately, the operator can input a variety of welding lines directly on the teaching sheet referenced to a virtual workpiece identical to the real workpiece, along with joint type and material specifications, and the system automatically generates optimum welding conditions from the algorithm-aided welding database, which can then be checked on-screen. Torch orientation is also generated automatically, based on the database. Icons are used to notify the operator of various precautions and remarks before andafter welding, through a welding assistant which displays YES/NO dialog boxes to implement Easy Teaching for even novice operators without welding skill. The human interface utilizes icons and graphic user interface (GUI) to allow the operator to complete complex jobs (documents) through simple operations (views). This system is constituted through OLE (object linking and embedding) method to enhance software productivity, efficiency and flexibility, implementing the significant features of functions indicated in Fig. 4.

The ET-PAD Easy Teaching System, which is the key to integration from upstream through downstream processes, is discussed below. with particular emphasis on the technical features: algorithm-aided welding database and off-line robotic programming.

\subsection{THE ALGORITHM-AIDED WELDING DATABASE}

This algorithm-aided welding database is designed for gas metal arc welding (GMAW), which is the most prevalent type of robotic arc welding used. With a minimum input of joint type and plate thickness (Figs. 2. 4) to the teaching sheet. it provides functions to automatically generate welding conditions for six types of joints (lap. T. butt. flare. corner and edge) even when the wire 
electrode diameter, extension or shielding gas are changed, for a range of base metal thickness from 0.8 to $6.0 \mathrm{~mm}$.

This section describes the basic approach to condition determination used in the algorithm-aided welding database.

This algorithm-aided welding database, as indicated in Fig. 5, uses a discrete welding databank as lattice points with representative information, while the algorithm is used to interpolate between them. Fig. 6 indicates a concrete example of interconnection between ET-PAD data processing and the algorithm. Based on preliminary welding information including welding machine, shield gas, wire diameter, wire extension, base metal material, as well as workpiece information from the teaching sheet such as joint type, base metal thickness and joint gap, welding conditions are searched within the welding databank. If the corresponding data cannot be found in the databank, welding conditions are calculated by the algorithm, meaning that the operator can obtain welding conditions no matter what the workpiece. Welding conditions (necessary welding current, voltage, travel speed, torch target position and travel angle, etc.) and welding results (leg length, penetration, weld reinforcement, etc.) can be registered in the databank, so that if the databank is enhanced the flexibility of the entire system improves.

Below the details of the welding condition algorithm are discussed.

The system is designed for use with gas metal arc (GMA) welding where the amount of deposited metal to the base metal is basically equivalent to the amount of filler metal consumed by heat and transferred to the base metal. The authors focused on the quantitative relationship between filler metal consumed and deposited metal, and developed a basic GMA welding model, coupled with experimental confirmation. By focusing on filler metal consumed, it was possible to apply the experimental approach to develop a filler metal consumed model based on the Halmoy (Ref. 7) and Lesnewich (Ref. 8) expressions.

For a welding bead cross-sectional area $S_{B}$, a welding travel speed $v_{t s}$, a wire corss-sectional area $S W$ and a wire melting rate $v_{M R}$, expression (1) can be established.

$$
S_{W} v_{M R}=S_{B} v_{T S}
$$

The arc welding phenomenon, however, is an extremely complex non-linear phenomenon dependent on a number of factors including base metal material, shield gas composition, wire material, welding Torch Orientation, joint gap, welding current, and welding travel speed. The welding results, namely weld bead shape and penetration shape, vary considerably depending on the specific phenomena and factors. As a result, it is in $i_{i}$ : sible to merely set the weld bead cross-section area to the required value.

To resolve this problem, the authors assumed that the weld bead cross-sectional area $S_{B}$ could be expressed by multiplying the cross-section of a quarter-circle with radius $t$ (plate thickness) $S_{B}^{\prime}$ by a weighted coefficient $\alpha$ for representing various factors, as indicated in expressions (2) and (3).

$$
\begin{aligned}
& S_{B}=\alpha S_{B}^{\prime} \\
& S_{B}^{\prime}=\pi t^{2 / 4}
\end{aligned}
$$

Lesnewich experimental expression was used to define the wire melting rate with the current density in the welding wire, yielding expression (4). Note that the physical interpretation of Halmoy applies expression (4). (Refs. 7, 8)

$$
v_{M R}=\xi j+\zeta L j^{2}
$$




\section{$(\xi, \zeta$ :constants, L:wire extension, $\mathrm{j}=\mathrm{I} / \mathrm{SW}$ (current density), I:welding current)}

Expression (4) was verified experimentally, and is explained here. The experiment used solid wire with diameters of 0.9 and $1.2 \mathrm{~mm}$, and measured wire melting rate to welding current. Fig. 7 indicates the welding current, and wire melting rate against current density (welding current over wire cross-sectional area). The relation between current density and wire melting rate in Fig. 7 closely approximates that of expression (5).

$$
\mathrm{V}_{M R}=0.0232 j+3.01 \times 10^{-6} L j^{2}
$$

The correlation between expression (5) and experimental data is a high 0.997 , demonstrating the validity of expression (4).

From expressions (1), (2), (3) and (4), welding current. I can thus be expressed as indicated in expression (6).

$$
I=\frac{-\xi+\sqrt{\zeta^{2}+4 \zeta L \alpha S_{B}^{\prime} \nu_{\mathrm{Ts}} / S_{W}}}{2 \zeta L} \cdot S W
$$

Based on experimental data, it is evident that the value: $\alpha$ in expressions (2) and (6) can be approximated by a function for plate thickness and joint gap.

Welding voltage $\mathrm{V}$ (voltage between tip and base metal) can be expressed as indicated in expression (7), derived through statistical methods from experimental data.

$$
V=f\left(I, v_{\mathrm{is}}, \theta, t\right)
$$

where $\theta$ is the inclination.

As a result of the above, the welding current for changes in wire diameter, wire extension and joint gap (in response to joint type and plate thickness specifications) can be calculated through expression (6), and the welding current from expression (7).

\subsection{VERIFICATION AND VALIDITY OF EXPERIMENTAL RESULTS}

This section discusses the results of welding tests performed using the welding conditions generated by the algorithm.

There are a variety evaluation criteria, but Fig. 8 compares the welding condition values generated by the algorithm with actual welding conditions, based on welding current. The results are expressed as the deviation between the calculated and actual values.

Welding tests were performed for a variety of conditions, namely shield gas (MAG [Ar80\%+ $\left.\mathrm{CO} 220 \%], \mathrm{CO}_{2} 100 \%\right)$, wire diameter $(0.8 \mathrm{~mm}, 1.0 \mathrm{~mm}, 1.2 \mathrm{~mm})$, wire extension $(10.0 \mathrm{~mm}$, $15.0 \mathrm{~mm})$, joint type (lap, T, butt, flare, corner, edge), plate thickness $(0.8 \mathrm{~mm}, 1.2 \mathrm{~mm}, 1.6 \mathrm{~mm}$, $2.0 \mathrm{~mm}, 3.2 \mathrm{~mm}, 4.5 \mathrm{~mm}, 6.0 \mathrm{~mm})$, gap $(0 \mathrm{~mm}$ to half plate thickness), and slope angle $(0$ and $-90^{\circ}$ ), for a total of 267 tests, $85 \%$ of the tests showed a deviation from calculated values of under $10 \%$, indicating that the algorithm has reached the level of practical utility.

A, B, C and D in Fig. 8 are examples of welding beads formed under welding conditions calculated by the algorithm (all with shield gas Ar80\% + CO220\%). Conditions for A (bead figure) were lap joint, plate thickness $0.8 \mathrm{~mm}$, wire diameter $0.9 \mathrm{~mm}$, wire extension $10.0 \mathrm{~mm}$, welding current $76 \mathrm{~A}$, voltage $15.0 \mathrm{~V}$, and travel speed $500 \mathrm{~mm} / \mathrm{min}$.

$\mathrm{B}$ is a bead figure of $\mathrm{A}$ in flip side. Conditions for $\mathrm{C}$ (cross-section) were butt joint, plate thickness $2.0 \mathrm{~mm}$, wire diameter $1.2 \mathrm{~mm}$, wire extension $15.0 \mathrm{~mm}$, welding current $212 \mathrm{~A}$, voltage $19.5 \mathrm{~V}$, and travel speed $1500 \mathrm{~mm} / \mathrm{min}$. Conditions for D (cross-section) were T joint, 
plate thickness $6.0 \mathrm{~mm}$, wire diameter $1.2 \mathrm{~mm}$, wire extension $15.0 \mathrm{~mm}$, welding current $278 \mathrm{~A}$, voltage $25.8 \mathrm{~V}$, and travel speed $400 \mathrm{~mm} / \mathrm{min}$.

The plate thickness is extremely thin in A and B, for which reason bead figure of both sides were shown instead of cross-sectional macro-photograph. From these photograph is evident that the algorithm is fully applicable for CAW as interpreted by the authors.

\subsection{OFFLINE ROBOT PROGRAMMING (TEACHING SHEET AND AUTOMATIC ARM/TORCH ORIENTATION GENERATION)}

This section does not discuss the flow of CAD/CAM to ET-PAD, but rather the ET-PAD Easy Teaching System as an offline programming system in an environment closer to the manufacturing process. The term "Easy Teaching" means that untrained operators, unskilled at computers, welding or robot operation, must be able to operate the system easily. As a result, the off-line programming system available to Easy Teaching must be considerably different from conventional offline programming system, which are operated by trained personnel.

First, the operator must be able to intuitively understand the human interface. Second, the system must automatically generate inherent and complex information to specified work. Third, the architecture must provide for ease of use and simple upgrading of the offline programming system. From the view point of these three requirements, the authors developed the following offline programming concept.

The first point, the human interface is fundamentally a graphical user interface (GUI), using icons instead of language. The teaching sheet indicated in Fig. 4 displays a 3D representation of the robot motion path, with robot action commands, workpiece information such as joint type and plate thickness, and job information such as welding conditions displayed as icons along the motion path. In accordance with preset properties, line type and colors can be used to make clear differences in interpolation method, welding region or air cut, for example. Based on the workpiece information set on the teaching sheet, welding conditions for each step, such as welding current, voltage and travel speed, are automatically generated as described in section 3.1 above. This allows a simple operation (View) to be used to process a complex work (Document).

The second point, automatic generation of commands to the robot, is handled by special functions developed for the purpose, which (1) automatically set the optimum torch orientation, replacing conventional time-consuming methods, and (2) automatically set the optimum robot arm orientation, based on torch target position and angle, torch travel angle, and angle information of individual robot articulations, with consideration for interference between torch and work, or between articulations. The generated program can be checked by the operator in the job simulation, as a 3D surface model.

The use of the GUI and the integration of existing PC software resources for 3D offline robot programming (Robot Offline Teaching System of Yaskawa;ROTSY) with arc welding technology, through object linking and embedding (OLE), results in a system which offers high internal processing efficiency and reliability. The introduction of this object-oriented programming method contributed significantly to the attainment of the third requirement, namely system flexibility.

OLE is a method by which independent software packages can be combined efficiently, and the 
linking between ROTSY, the algorithm-aided welding database and the torch/arm orientation generation system is implemented through OLE. The entire process is controlled by ET-PAD, making more easily possible an integrated system configuration and simplifying new function addition. ROTSY, the algorithm-aided welding database and the torch/arm orientation generation system are servers, and ET-PAD is a client (Fig. 10). The characteristics of the individual software packages are inherited, and used to implement required functions for the overall system internally.

As an example, Fig. 11 indicates how ROTSY functions are inherited to the ET-PAD. The primitive 3D model management function is inherited to the viewpoint control function, which controls how images of robots, peripheral equipments and work are drawn. Other, higher functions are inherited in turn from this function to robot dynamics calculation, robot program management, robot program interpretation, and finally the teaching sheet.

Because this type of software architecture has been adopted, it is expected that the integration with the welding expert system, including welding result simulation and intelligent programming, will be much more efficient.

\section{CONCLUSION}

As outlined above, CAW as interpreted by the authors is flexible welding automation.

This article introduced the ET-PAD Easy Teaching System, as fundamental milestone in the development of CAW, which integrates the algorithm-aided welding database and offline robot programming. The authors believe that the early development of a welding result simulator will significantly enhance the function of the ET-PAD. The adaptive control system, which is the ultimate goal of APOT and welding expert systems, which is the core of CIM-based robotics, was discussed in Chapter 2. The authors will continue to resolve the many problems outstanding in the development of CAW one at a time, in pursuit of the ideal system.

\section{REFERENCES}

1. Lucas, W. Computer Aided Welding Software in the UK. The International Institute of Welding Document XII-1449-96.

2. Siewert, T.A. CAW '96 : Computer Aided Welding Software in the Americas. The International Institute of Welding Document XII-1450-96.

3. Prof. Dr. -Ing. Dilthey, U. ; and Dipl-Ing. Roosen, S. Computer Aided Welding (CAW) in Central Europe. The International Institute of Welding Document XII-1451-96.

4. Jinning, P. ; and Bingsen, C. Computer Aided Welding in China. The International Institute of Welding Document XII-1453-96.

5. Kutsuna, M ; and Ohji, T. Computer Aided Welding (CAW) System in Japan. The International Institute of Welding Document XII-1472-96.

6. Inoue, Y. ; and Takaoka, K. 1996. Teaching Method for Industrial Robot. Journal of Robotics Society of Japan 14 (6) : 780-783.

7. Halmoy, E. 1979. Wire Melting Rate, Dropet Temperature, and Effective Anode Melting Potential. The Welding Institute, Int. Conf. Arc Physics and Weld Pool Behavior, London. 49-57.

89. Lesnewich, A. 1958. Control of Melting Rate and Metal Transfer in Gas-Shielded Metal-Arc Welding part-1 control of electrode melting rate. Wclding Journal 37 (8) : 343s-353s. 


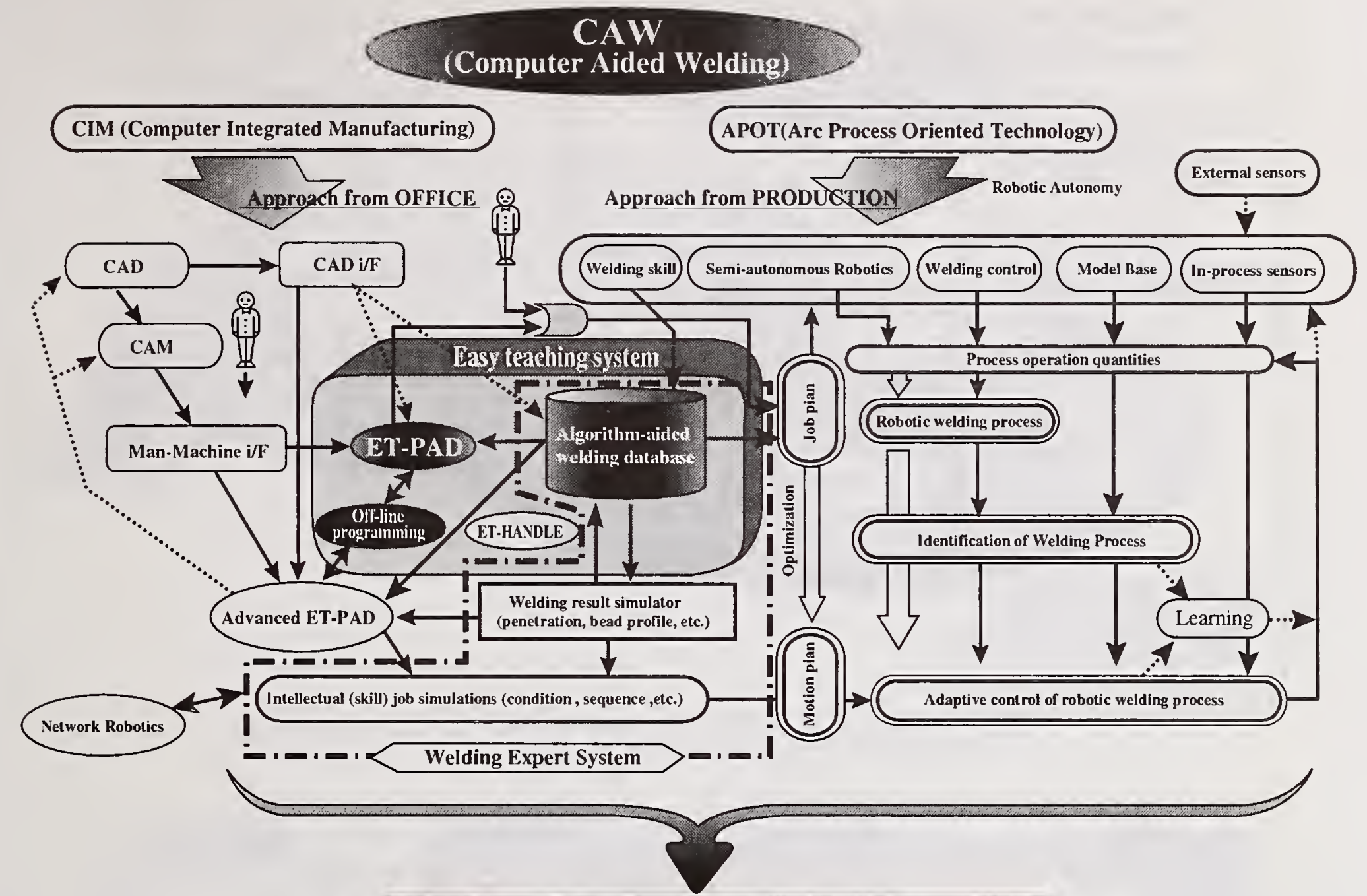

\section{Flexible Welding Automation}

Fig.1 Final Target of CAW at YASKAWA

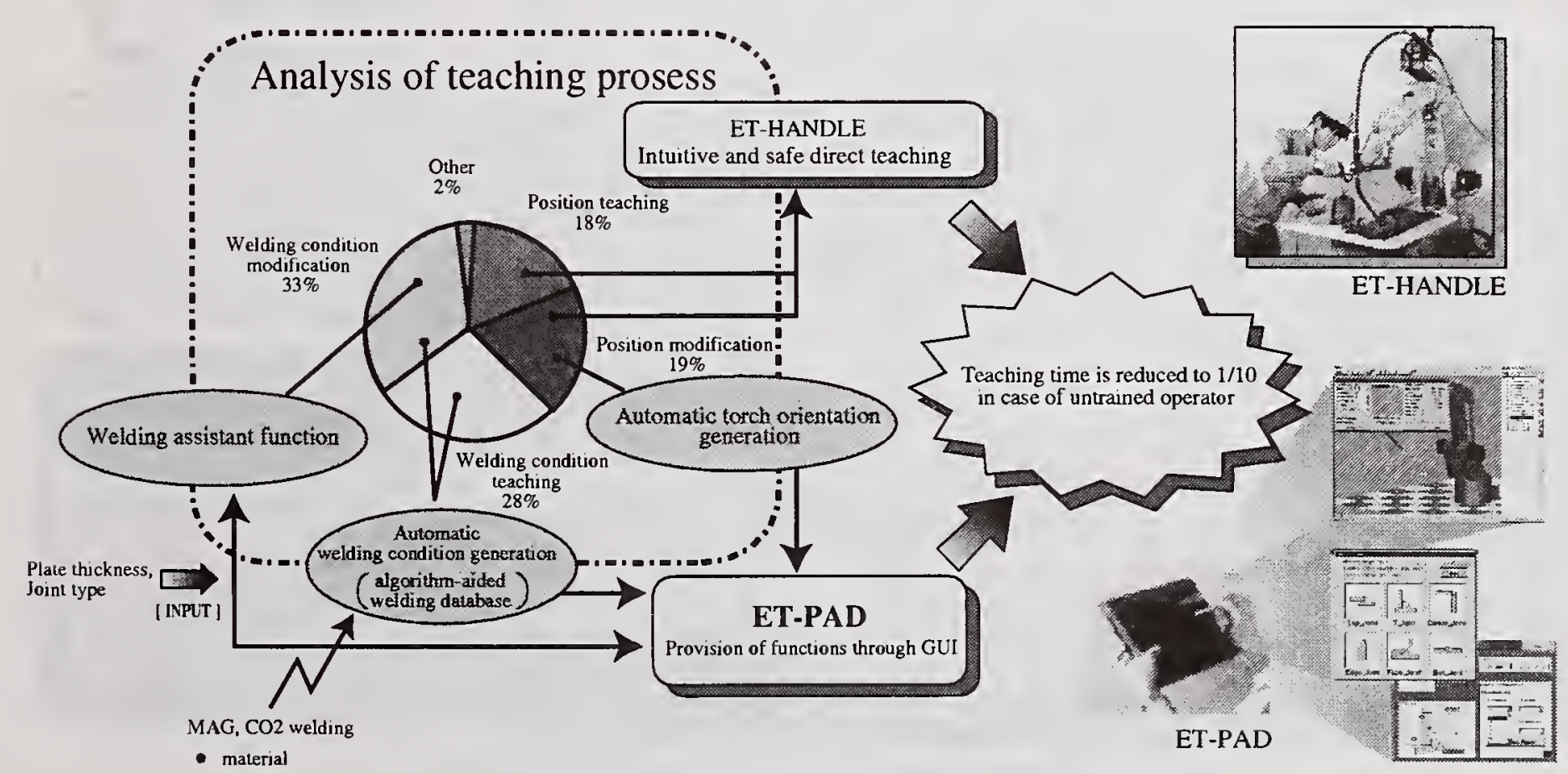

- materia

- shield gas

- ...

Fig.2 Analysis of Teaching Process 


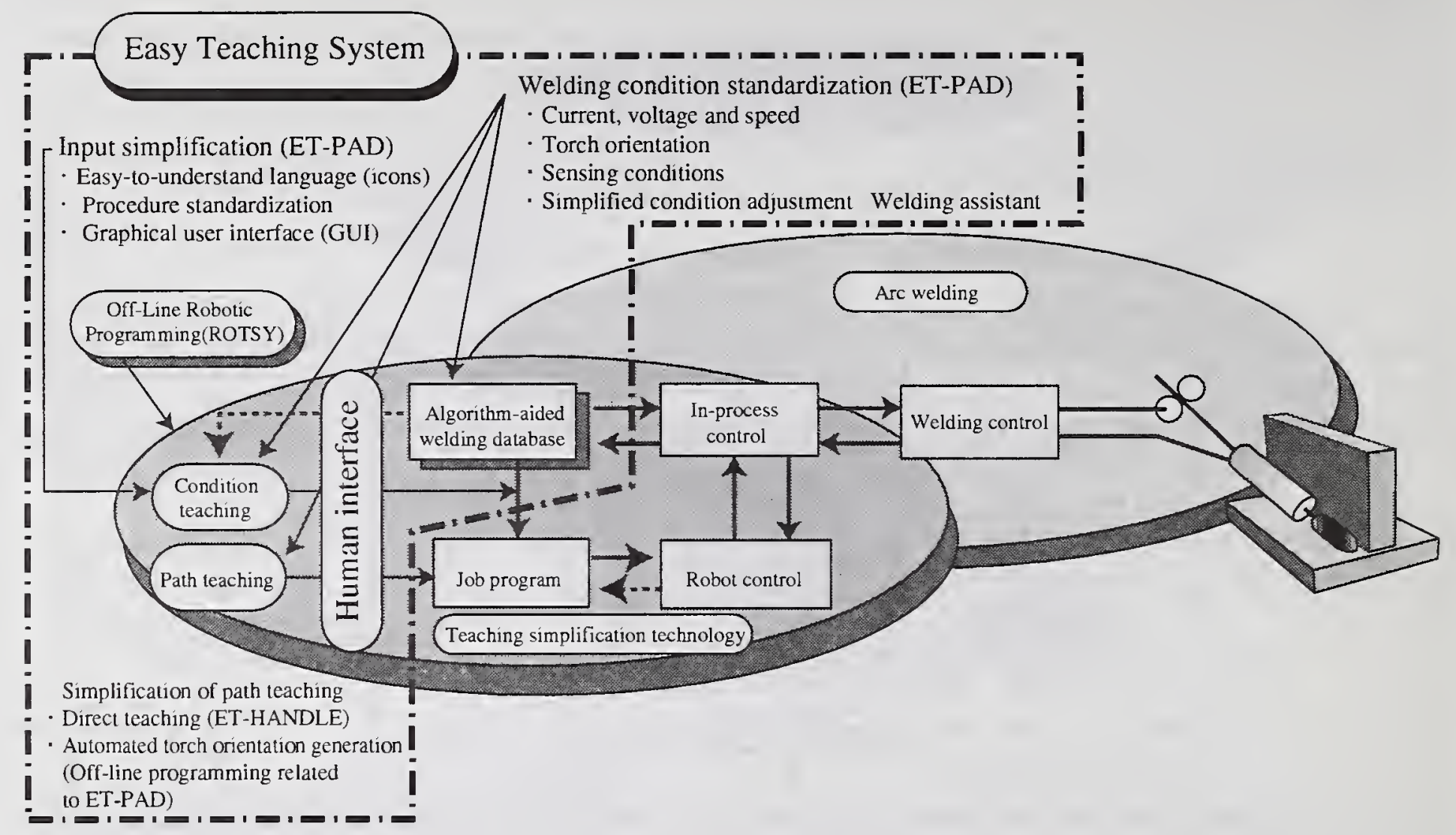

Fig. 3 Positioning of Easy Teaching System, and 1st Target Image

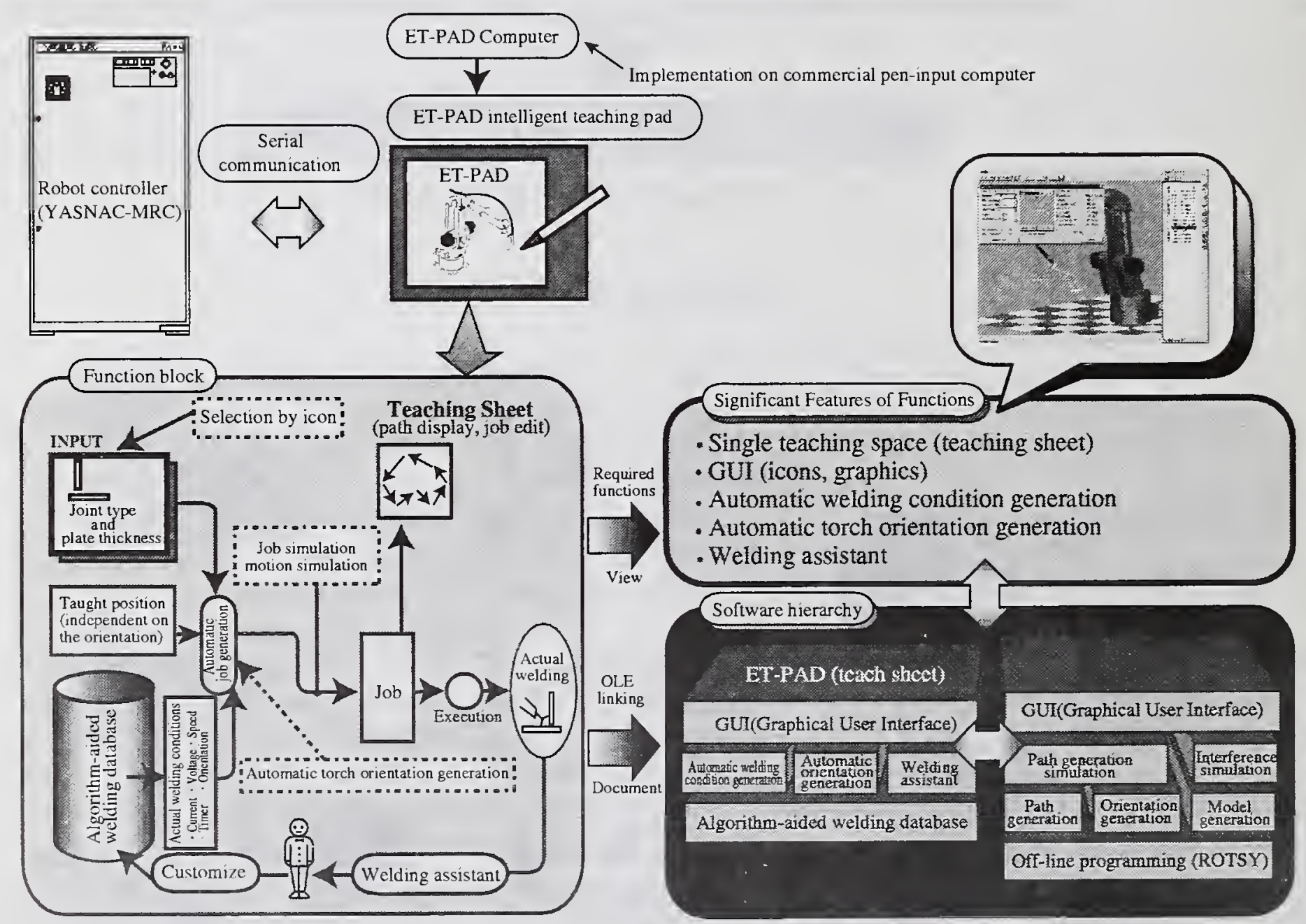

Fig. 4 Configuration of ET-PAD Intelligent Teaching Tool 


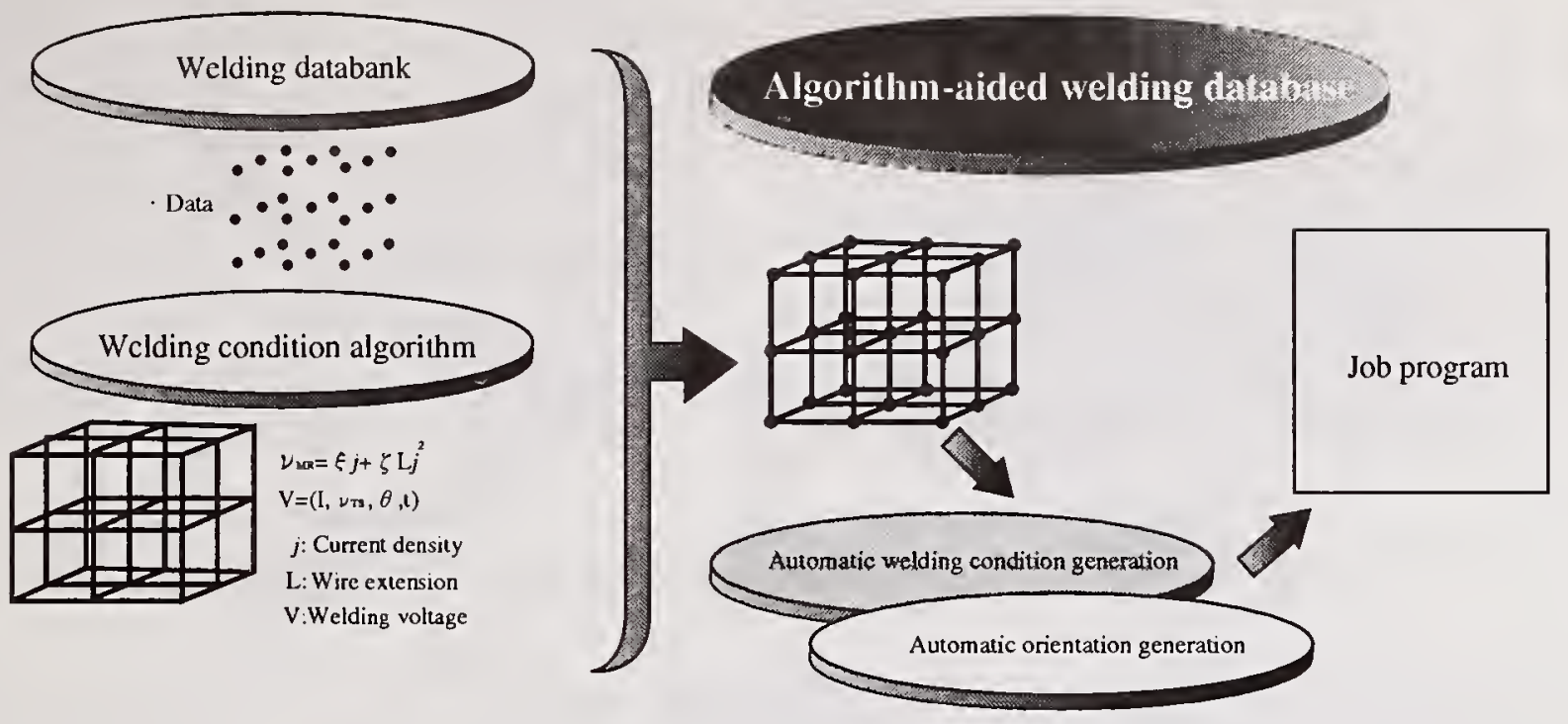

FIG. 5 Algorithm-Aided Welding Database

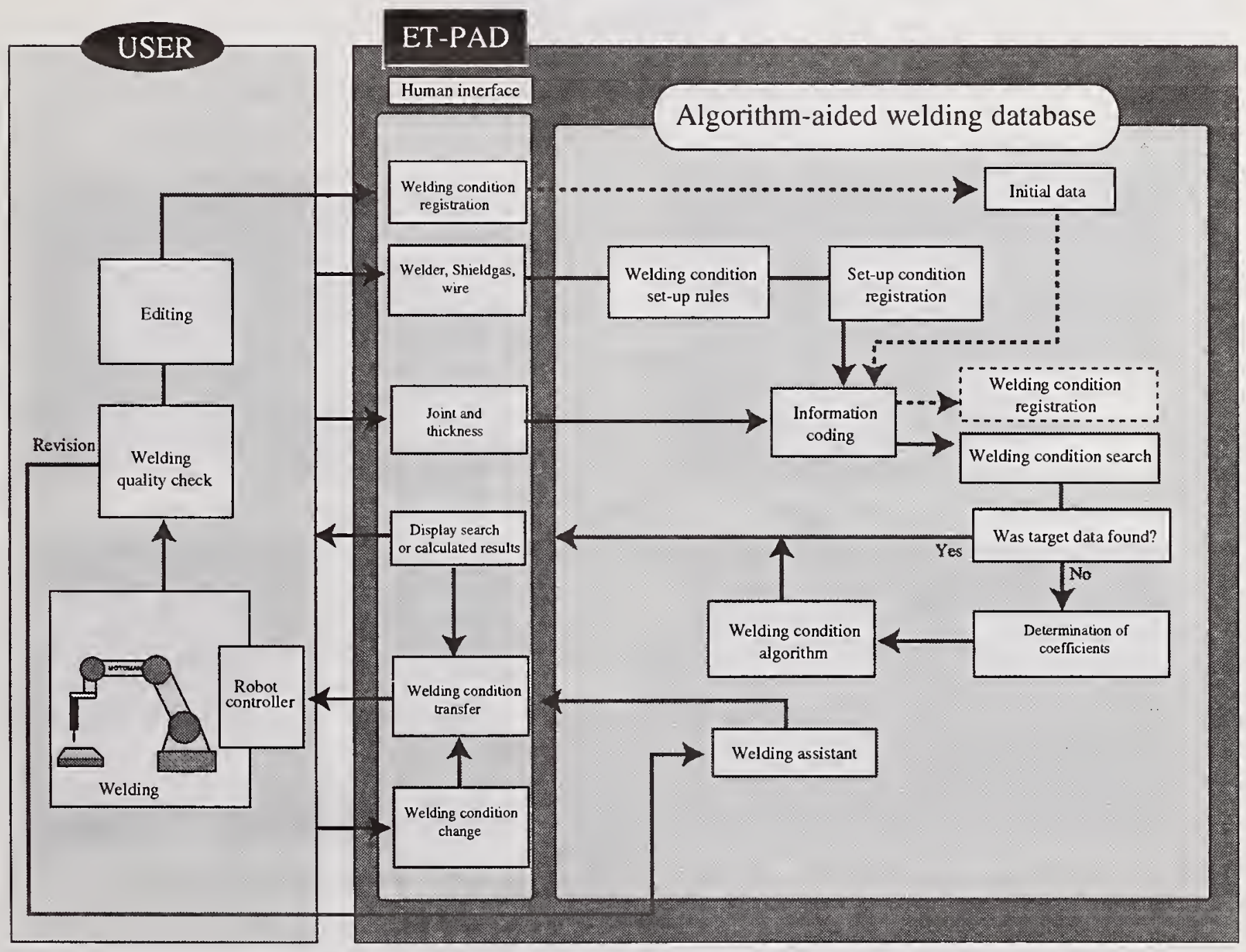

Fig. 6 Algorithm-Aided Welding Database Flow 

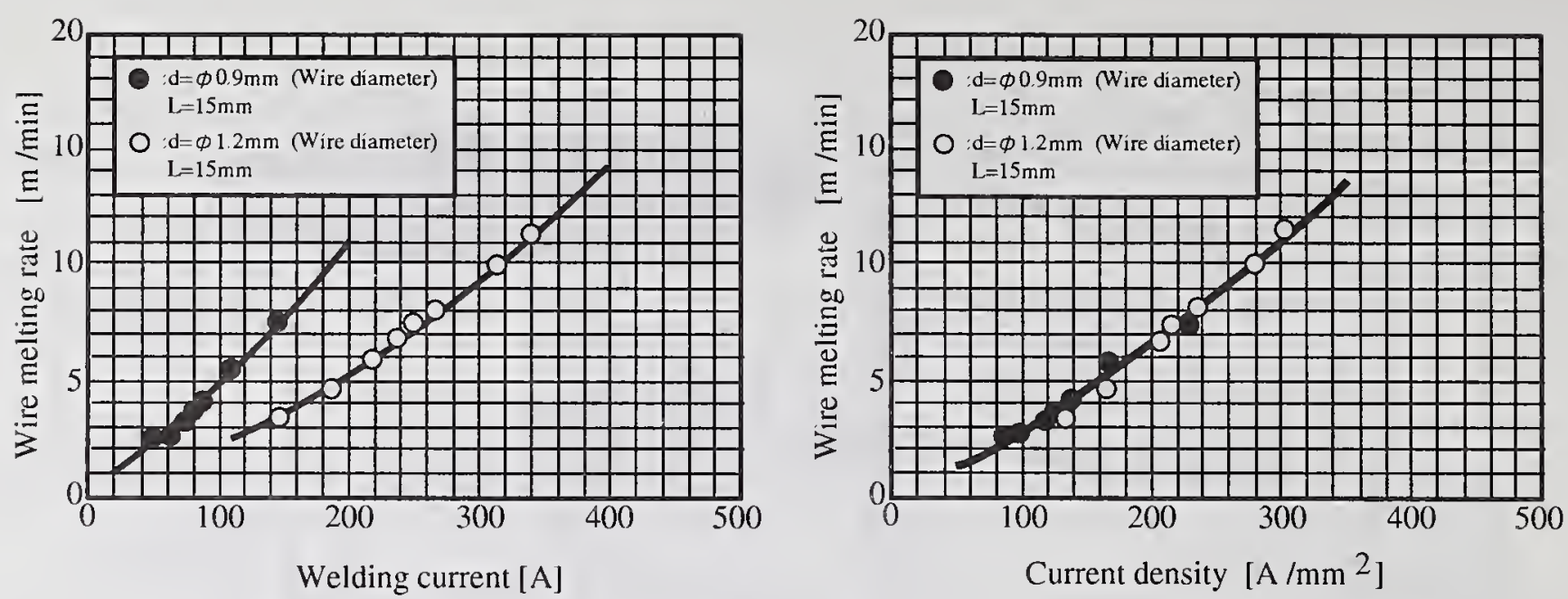

Fig. 7 Relation between wire melting rate and Welding Current/Current Density

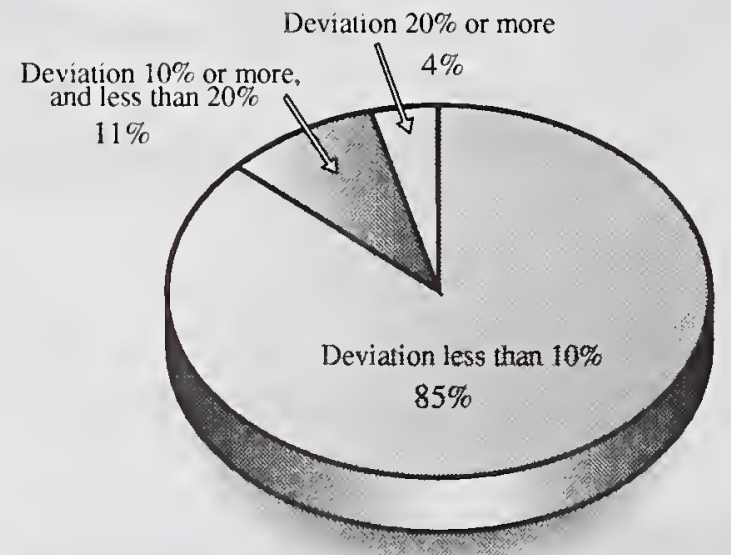

Fig. 8 Deviation between Calculated and Actual Values

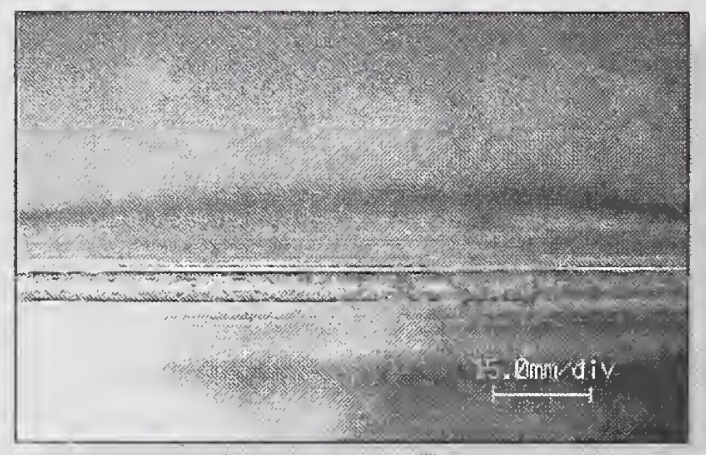

A: Front Side [LAP Joint]

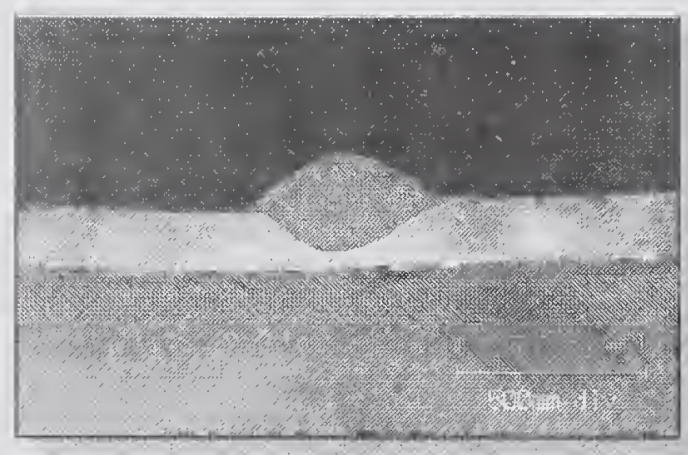

C: Cross-Section [BUTT Joint]

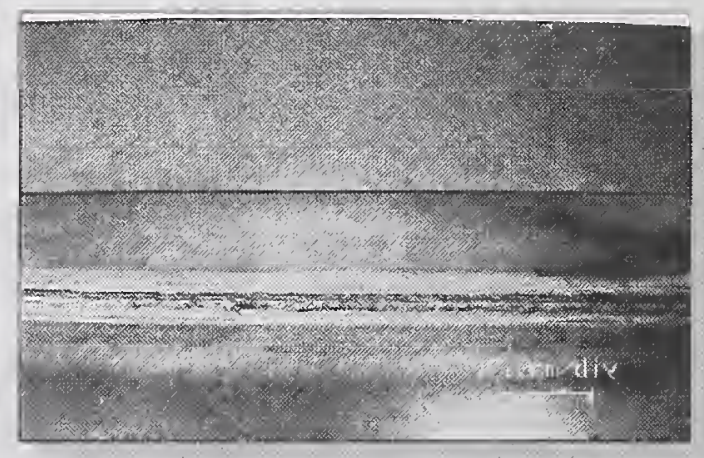

B: Flip Side [LAF Joint]

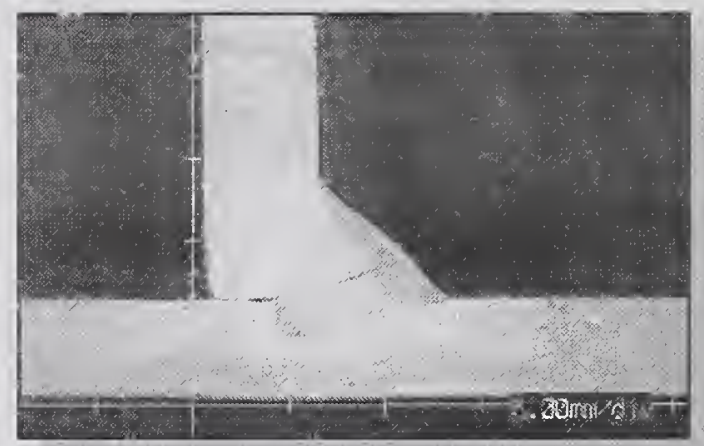

D: Cross-Section [T Joint]

Fig. 9 Results of Welding to Calculated Values for Photograph 


\section{Client}

ET-PAD(integration of individual applications)

- Linking between job editing and 3Ddisplay

- Linking between job and algorithm-aided database

- Linking with automatic job generation

\section{Server}

ROTSY(robot simulator)

- 3D robot model display

- Job display (3D)

- Viewpoint control

- Interference simulater

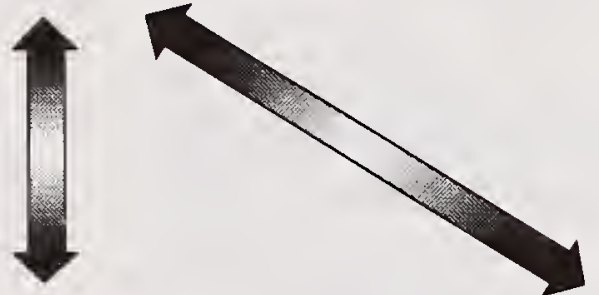

\section{Server}

Algorithm-aided welding database

(Automatic welding condition generation)

- Algorithm calculation

- Database search

- Registration, update,delete

\section{Server}

Automatic torch/arm orientation generation (optimum position and orientation)

- Orientation calculation function

- Position adjustment calculation function

Fig. 10 Division of Function between Applications in ET-PAD

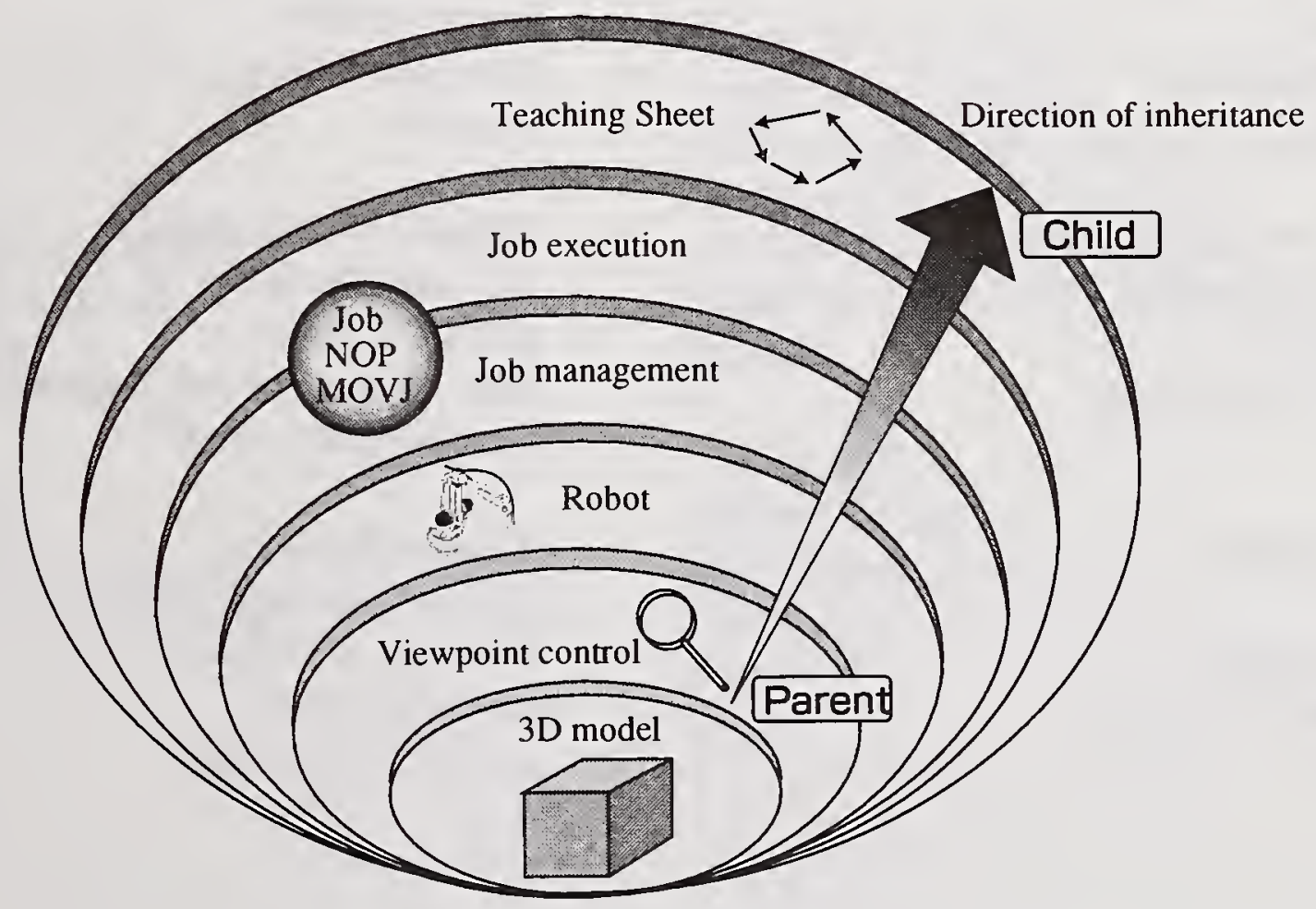

Fig. 11 Example of Inheritance of ROTSY Functions by ET-PAD (conceptual diagram) 


\section{Appendix A: Participants in Seventh International Conference on Computer Technology in Welding}

\section{A.1 Speakers}

H J Andersen

Department of Production

Fibigerstrade 16

Mlborg East 9220

Denmark

Martin Becker

Professor

Oregon Graduate Institute

PO Box 91000

Portland, OR 97291

Omer Blodgett

Senior Design Consultant

The Lincoln Electric Company

22801 St. Clair Ave.

Cleveland, $\mathrm{OH} 44117$

Eckard Blumschein

Guericke University

PSF 4120

39016 Magdeburg

Germany

Vladislav N. Boronenkov

The Urals Technical State Univ.

19 Mira Str.

920002 Ekaterinburg

Russia

Andy Brightmore

TWI

Abington Hall

Cambridge CB1 6AL

U.K.

Pawel Cegielski

Warsaw University of Tech.

U1 Narbutta 85

02-524 Warsaw

Poland

Billy Chan

Mil Systems

1150 Morrison Dr.

Ottawa, Ontario

Canada

Ron Citterberg

EWI

1250 Arthur E. Adams Dr.

Columbus, $\mathrm{OH} 43221$

Ulrich Dilthey

Professor

Inst. Fuer Schweisstechnik

Pontstrasse 49

Munchen 52062

Germany

Charalabos Doumanidis

Assistant Professor

Tufts University

Dept. of Mechanical Engineering

Medford, MA 02155

Thomas E. Doyle

Tech. Adv./Auto. Tech.

Babcock \& Wilcox

1562 Beeson St.

Alliance, OH 44601 
Richard G. Eisler

Sandia National Labs

PO Box 5800, MS 0439

Albuquerque, NM 87185-0439

Erik Engh

Manager

4X Software AS

Grini Naeringspark 1

Osteras 1345

Norway

Lance Flitter

Computer Scientist

NSWC Carderock Division

Code 251

9500 MacArthur Blvd.

West Bethesda, MD 20817-5700

Charles L. Foster

397 Camellia Way

Vacaville, CA 95688

Phillip W. Fuerschbach

Sandia National Labs

PO Box 5800

$\mathrm{M} / \mathrm{S} 0387$

Albuquerque, NM 87185-0367

Tore Habrekke

Sintef Materials Technology

Richard Birkelandsv 2b

Trondheim 7034

Norway

Hiroyuki Handa

Yaskawa Electric Corp.

Yahatanishi - Ku Kitakyusha

Japan
Grant K. Hicken

Weld Engineer

Sandia National Labs

PO Box 969

Livermore, CA 94550

Yoshinori Hirata

Associate Professor

Dept. of Adaptive Machine Systems

Osaka University

2-1 Yamada-oka, Suita

Osaka 565

Japan

Torbjorn Johnsson

4X Software AS

Grini Naeringspark 1

Osteras 1345

Norway

B Keskeny

Technical University of Budapest

Irinyi I. 9-11

Budapest 1111

Hungary

Sameh M.M. Khafagy

Universidad Technical

ETSI Navales Laboratorio De Soldadura

Avda. Arco De La Victoria

S-N. (Ciudad Universitaria)

28040 Madrid

Spain

You Chul Kim

Osaka University

11-1 Mihogaokaibaraki

Osaka 567

Japan 
Gerald A. Knorovsky

Senior Member of Tech. Staff

Sandia National Labs

PO Box 5800, Org 1833

MS 0367

Albuquerque, NM 87185
A. Kolasa
Warsaw University of Tech.
U1 Narbutta 85
02-524 Warsaw
Poland

M. V. Li

Battelle

505 King Ave.

Columbus, OH 43201

Bruce Madigan

Materials Reliability

NIST

325 Broadway

Boulder, CO 80303

Ole Madsen

Department of Production

Fibigerstrade 16

Mlborg East 9220

Denmark

Kim W. Mahin

Manager for Smart Processing

Sandia National Labs

PO Box 5800, MS 0336

Albuquerque, NM 87185

A.M. Mansoor

University of Waterloo

Dept. of Mechanical Engineering

Waterloo, Ontario N21 3G1

Canada
Kin-ichi K. Matsuyama

MIT, DMSE

77 Massachusetts Ave.

Room 4-047

Cambridge, MA 02139

Edward A. Metzbower

Head Welding Met. Section

U.S. Naval Research Lab

Code 6320

4555 Overlook Ave. S.W.

Washington, DC 20375

S.-J. Na

Korea Advanced Institute of Science

Kusongdong 373-1 Yusonggu

Taejon 305701

Korea

Valerian A. Nemchinsky

The Esab Group

PO Box 100

545 Ebenezer Rd.

Florence, SC 29501

Donald J. Newman

PO Box 50225

Colorado Springs, CO 80949-0225

Paul Oberly

NA Technologies

1317 Washington Ave., Suite 1

Golden, CO 80401

Babatunde Ogunbiyi

7 Maltings Close

Cranfield Bedford

United Kingdom MK43 OAZ

England

Peter Orszagh

Karloveska 45

84104 Bratislava

Slovakia 
Roland Pap

Technical University of Budapest

Irinyi I. 9-11

Budapest 1111

Hungary

Steven R. Potter

Sales Manager

Sciaky Inc

4915 W. 67 St.

Chicago, IL 60638

Timothy P. Quinn

Mechanical Engineer

NIST

325 Broadway

Boulder, CO 80303

William Rippey

NIST

220/B124

Gaithersburg, MD 20899

Sabine Roosen

German Welding Society

Michener Str. 172

Dusseldorf 40223

Germany

Wolfgang Scholz

New Zealand Welding Center

PO Box 76-134

Manukau City, Auckland

New Zealand

Patrick T. Sewell

$1450 \mathrm{~S}$. Youngfield Ct.

Lakewood, CO 80228

Hassan I. Shaaban

Atomic Energy Authority

PO Box 183 Side Gaber

Alexandria

Egypt
Tom Siewert

Materials Reliability Division

NIST

325 Broadway

Boulder, CO 80303

Gerald D. Simpson

858 Back Valley Rd.

Sweet Springs, WV 24941

J.E. Sims

Consulting Engineer

12114 Dakar

Houston, TX 77065

Chris Smith

Tower Automotive

3533 N. 27th St.

Milwaukee, WI 53216

Theresa M. Spear

The Lincoln Electric Co.

22801 St. Clair Ave.

Cleveland, $\mathrm{OH} 44117$

Xin Sun

Battelle

505 King Ave.

Columbus, OH 43201

M.A. Wahab

University of Adelaide

Mechanical Engineering Deptartment

Adelaide, S.A. 5005

Australia

Wenqi Zhang

Technical Univ. of Denmark

Inst. of Meg. Engn. Bldg. 425

DK2800 Lyngby

Denmark

Michael Zinigrad

College of Judea and Samaria

PO Box 3

Ariel 44837

Israel 


\section{A.2 Attendees}

Claude Albert

Bechtel Group

111 De La Barre, Apt. 218

Longuevil, Quebec J4K 2T8

Canada

V. Ananthanarayanan

Advanced Manufacturing Engineer

Delphi Chassis Systems

1420 Wisconsin Blvd.

Dayton, OH 45401

M. Armstrong

Managing Director

Isotek Electronics Ltd.

Claro House, Servia Road

Leeds LS7-1NL

England

Paul Blomquist

The Pennsylvania State University

PO Box 30

State College, PA 16804-0030

Chun Chen

Institute of Materials Science \& Engineering

1 Roosevelt Road Section

Taipei 106

R.O.C.

Andrew Debiccari

Senior Methods Specialist

Pratt and Whitney

Aircraft Rd., M/S 401-36

Middletown, CT 06457

Richard Dickens

South African Insttitute of Welding

PO Box 527, Crown Mines

Johannesburg 2025

South Africa
Todd E. Holverson

Staff Engineer

Miller Electric

1138 West Elsie St.

Appleton, WI 54914

Oussama A. Jammal

N \& C Tech.

Caterpillar Inc.

Rte. 31, PO Box 348

Aurora, IL 60507

Carl F. Klein

Manager New Technology

Johnson Controls

170.1 West Civic Center

Milwaukee, WI 53209

Kishore N. Lankalapalli

Fanuc Robotics NA, Inc.

3900 West Hamlin Rd.

Rochester Hills, MI 48309-3253

William C. LaPlante

304 St. Nicholas Ave.

Worcester, MA 01606

Marilyn D. Levine

Conference Coordinator

AWS

500 N.W. LeJeune Rd.

Miami, FL 33126

Bryan H. Lyons

Staff Engineer

AWS

550 N.W. LeJeune Rd.

Miami, FL 33126 
S. Doug Mauk

President

SDM Consulting Inc.

P O Box 928

New Market, MD 21774-0928

John Mitchiner

Sandia National Labs

PO Box 580

Albuquerque, NM 87185

Thomas M. Mustaleski, Jr.

Welding Engineer

Lockheed Martin

PO Box 2009

Oak Ridge, TN 37831

Bob Pow

POW Technologies

P O Box 24006 CDO

63 Ridgeway $\mathrm{Cr}$.

Woodstock, Ontario N4S 8Y4

Canada

Robert A. Riddle

Lawrence Livermore National Labs

PO Box 808, L-125

Livermore, CA 94551
David Russler

1801 Nemoke Ter., Apt. 11

Haslett, MI 48840

Roger Wilson

Code 138.1

1400 Farragut Ave.

Naval Shipyard Puget Sound

Bremerton, WA 98314

Edwin L. Wolf

Project Manager

High Purity Systems

8432 Quarry Rd.

Manassas, VA 20110

Nannette Zapata

Conference Director

AWS

550 N.W. LeJeune Rd.

Miami, FL 33126 


\section{A.3 Exhibitors}

Frederic Boitout

Sysweld Prod. Manager

ESI Group

5330 Carroll Canyon Rd., Ste. 201

San Diego, CA 91212

Ta Chieh Huang

TWI/EWI

1250 Arthur E. Adams

Columbus, OH 43221

Jerald E. Jones

Chief Scientist

NA Technologies, Co.

1317 Washington Ave., Ste. 1

Golden, CO 80401
Don Schwemmer

V.P. Engineering

AMET Inc.

7176 North River Rd.

Idaho Falls, ID 83402

Kevin M. Rigby

ESI North America

5230 Carroll Canyon Rd., Ste 224

San Diego, CA 91212 


\section{AUTHOR INDEX}

Aksyutin, $\mathrm{O}$.

Andersen, H.J.

Armstrong, $\mathrm{M}$.

Atteridge, E.

Atteridge, D.G.

Bay, N.

Becker, M.

Beres, L.

Berge, J.O.

Bergheau, J.M.

Bibby, M.

Blachowiak, E.G.

Blodgett, $\mathrm{O}$.

Blumschein, E.

Boitout, F.

Boronenkov, V.N.

Carvalho, G.C.

Cegielski, P.

Chan, B.

Chang, W.-S.

Chen, X.

Citterberg, R.G.

Dilthey, U.

Dong, $P$.

Doumanidis, C.C.

Doyle, T.E.

$222,237,364,374$

529

31

399

518

496

345

474

399

310

101

403

518

213

237

195

187,548

$389,423,447$

89

515

Eisler, G.R.

El-Ashram, A.

Engh, E.

Falco, J.A.

Fonda, R.W.

Foster, C.L.

Fuerschbach, P.W.

Gershanok, G.A.

Gomez, N.B.

Goto, N.
Håbrekke, T.

Korolev, N.V.

Larsen, R.

Madigan, R.B.

Mangialenti, G.

Matsuyama, K.

Mazurovsky, V.

\section{McMaster, R.}

Metzbower, E.A.

Molleda, F.

Moon, H.-S.

$\mathrm{Na}$, S.J.

Nemchinsky, V.A. 
Newman, D.J.

Nio, S.

Noruk, J.

Oberly, P.A.

Ogunbiyi, T.E.B.

Ohji, T.

Okumura, S.

Orszagh. P.

Osamura, $\mathrm{T}$.

Overgaard, L.

Pacey, J.

Painter, M.J.

Palotas, B.

Pap, R.

Pimenova, O.V.

Pinn, T.

Potter, S.R.

Quinn, T.P.

Rhoades, V.

Rhoades, V.L.

Rippey, W.G.

Roosen, S.
101

279

562

503

279

411

518

354

529

529

310

139

112

496

171

545

203

$3,187,548$
Sakata, C.

Scholz, W.

139

171,545

Shaaban, E.A.

152

152

Shaaban, H.I.

Siewert, T.A.

3

Simpson, G.D.

Smith, C.B.

496

411

298

345

354

320

548

447

Sun, $\mathrm{X}$.

112

298

Vandermeer, R.A.

354

Wahab, M.A.

291

364

237, 374

291 


\section{Technical Publications}

\section{Periodical}

Journal of Research of the National Institute of Standards and Technology-Reports NIST research and development in those disciplines of the physical and engineering sciences in which the Institute is active. These include physics, chemistry, engineering, mathematics, and computer sciences. Papers cover a broad range of subjects, with major emphasis on measurement methodology and the basic technology underlying standardization. Also included from time to time are survey articles on topics closely related to the Institute's technical and scientific programs. Issued six times a year.

\section{Nonperiodicals}

Monographs-Major contributions to the technical literature on various subjects related to the Institute's scientific and technical activities.

Handbooks-Recommended codes of engineering and industrial practice (including safety codes) developed in cooperation with interested industries, professional organizations, and regulatory bodies.

Special Publications-Include proceedings of conferences sponsored by NIST, NIST annual reports, and other special publications appropriate to this grouping such as wall charts, pocket cards, and bibliographies.

Applied Mathematics Series-Mathematical tables, manuals, and studies of special interest to physicists, engineers, chemists, biologists, mathematicians, computer programmers, and others engaged in scientific and technical work.

National Standard Reference Data Series-Provides quantitative data on the physical and chemical properties of materials, compiled from the world's literature and critically evaluated. Developed under a worldwide program coordinated by NIST under the authority of the National Standard Data Act (Public Law 90-396). NOTE: The Journal of Physical and Chemical Reference Data (JPCRD) is published bimonthly for NIST by the American Cremical Society (ACS) and the American Institute of Physics (AIP). Subscriptions, reprints, and supplements are available from ACS, 1155 Sixteenth St., NW, Washington, DC 20056.

Building Science Series-Disseminates technical information developed at the Institute on building materials, components, systems, and whole structures. The series presents research results, test methods, and performance criteria related to the structural and environmental functions and the durability and safety characteristics of building elements and systems.

Technical Notes-Studies or reports which are complete in themselves but restrictive in their treatment of a subject. Analogous to monographs but not so comprehensive in scope or definitive in treatment of the subject area. Often serve as a vehicle for final reports of work performed at NIST under the sponsorship of other government agencies.

Voluntary Product Standards-Developed under procedures published by the Department of Commerce in Part 10, Title 15, of the Code of Federal Regulations. The standards establish nationally recognized requirements for products, and provide all concerned interests with a basis for common understanding of the characteristics of the products. NIST administers this program in support of the efforts of privatesector standardizing organizations.

Consumer Information Series-Practical information, based on NIST research and experience, covering areas of interest to the consumer. Easily understandable language and illustrations provide useful background knowledge for shopping in today's technological marketplace.

Order the above NIST publications from: Superintendent of Documents, Government Printing Office, Washington, DC 20402.

Order the following NIST publications-FIPS and NISTIRs-from the National Technical Information Service, Springfield, VA 22161.

Federal Information Processing Standards Publications (FIPS PUB)-Publications in this series collectively constitute the Federal Information Processing Standards Register. The Register serves as the official source of information in the Federal Government regarding standards issued by NIST pursuant to the Federal Property and Administrative Services Act of 1949 as amended, Public Law 89-306 (79 Stat. 1127), and as implemented by Executive Order 11717 (38 FR 12315, dated May 11, 1973) and Part 6 of Title 15 CFR (Code of Federal Regulations).

NIST Interagency Reports (NISTIR)-A special series of interim or final reports on work performed by NIST for outside sponsors (both government and non-government). In general, initial distribution is handled by the sponsor; public distribution is by the National Technical Information Service, Springfield, VA 22161, in paper copy or microfiche form. 
U.S. Department of Commerce

National Institute of Standards and Technology

325 Broadway

Boulder, Colorado 80303-3328

\section{Official Business}

Penalty for Private Use, $\$ 300$ 